BAYESIAN METHODS

for Statistical Analysis 



\title{
BAYESIAN METHODS for Statistical Analysis
}

\author{
BY BOREK PUZA
}

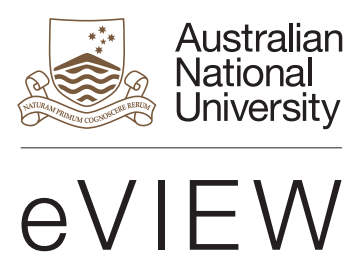




\section{ANU \\ eVIEW}

Published by ANU eView

The Australian National University

Acton ACT 2601, Australia

Email: enquiries.eview@anu.edu.au

This title is also available online at http://eview.anu.edu.au

National Library of Australia Cataloguing-in-Publication entry

Creator: $\quad$ Puza, Borek, author.

Title: Bayesian methods for statistical analysis / Borek Puza.

ISBN: $\quad 9781921934254$ (paperback) 9781921934261 (ebook)

Subjects: $\quad$ Bayesian statistical decision theory.

Statistical decision.

Dewey Number: $\quad 519.542$

All rights reserved. No part of this publication may be reproduced, stored in a retrieval system or transmitted in any form or by any means, electronic, mechanical, photocopying or otherwise, without the prior permission of the publisher.

Cover design and layout by ANU Press

Printed by Griffin Press

This edition (C) 2015 ANU eView 


\section{Contents}

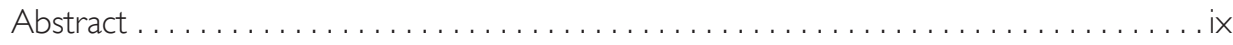

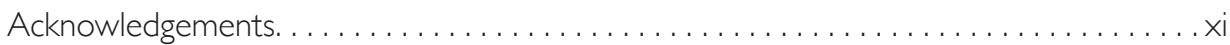

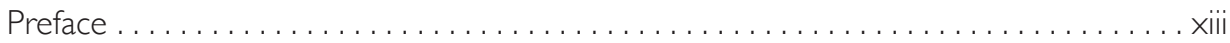

Overview.........................................

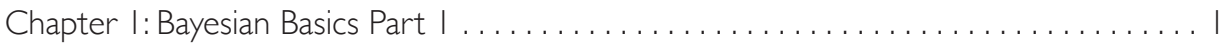

I.I Introduction .....................................

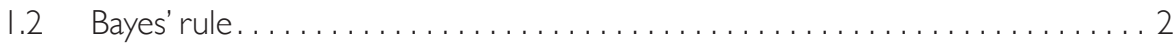

I.3 Bayes factors. .................................. 9

1.4 Bayesian models................................... I

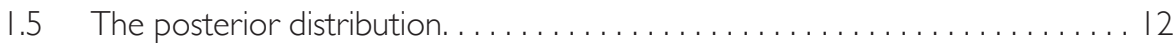

I.6 The proportionality formula. .......................... 13

1.7 Continuous parameters .............................. 19

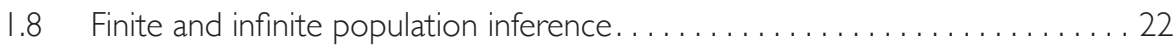

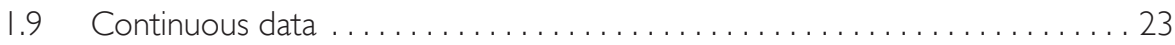

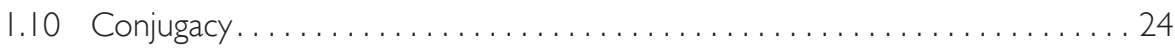

I.II Bayesian point estimation .................................. 25

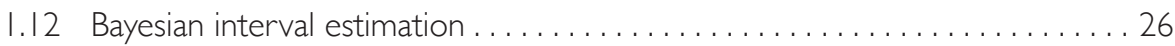

I.13 Inference on functions of the model parameter ................. 3 |

I.14 Credibility estimates ..................................... 34

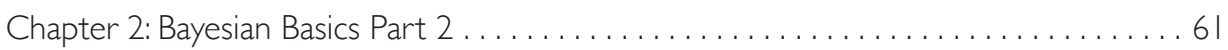

2.I Frequentist characteristics of Bayesian estimators...............6 6 |

2.2 Mixture prior distributions ............................. 74

2.3 Dealing with a priori ignorance $\ldots \ldots \ldots \ldots \ldots \ldots \ldots \ldots \ldots \ldots \ldots$

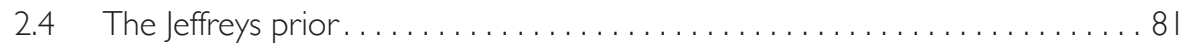

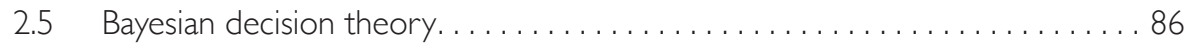

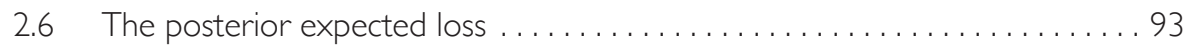

2.7 The Bayes estimate . . . . . . . . . . . . . . . . . . . . . . . . . . . . . . 98

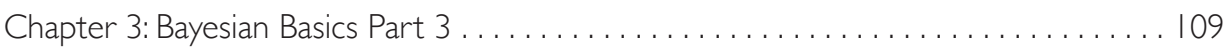

3.I Inference given functions of the data. ...................... 09

3.2 Bayesian predictive inference $\ldots \ldots \ldots \ldots \ldots \ldots \ldots \ldots \ldots \ldots \ldots \ldots \ldots$

3.3 Posterior predictive $p$-values . . . . . . . . . . . . . . . . . . . . . . 130

3.4 Bayesian models with multiple parameters .................... 35 
Chapter 4: Computational Tools

4.I Solving equations.

4.2 The Newton-Raphson algorithm. .......................... 55

4.3 The multivariate Newton-Raphson algorithm .................... 6 |

4.4 The Expectation-Maximisation (EM) algorithm .................. 65

4.5 Variants of the NR and EM algorithms .................... 175

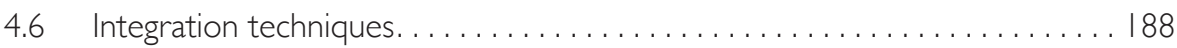

4.7 The optim() function. ............................. 94

Chapter 5: Monte Carlo Basics ................................ 20 I

5.I Introduction ....................................... 20 I

5.2 The method of Monte Carlo integration for estimating means. . . . . . . . . . 202

5.3 Other uses of the $\mathrm{MC}$ sample . . . . . . . . . . . . . . . . . . . . . 205

5.4 Importance sampling. .................................. 209

$5.5 \mathrm{MC}$ estimation involving two or more random variables ............. 213

5.6 The method of composition .............................. 214

5.7 Monte Carlo estimation of a binomial parameter.................. 216

5.8 Random number generation . . . . . . . . . . . . . . . . . . . . 227

5.9 Sampling from an arbitrary discrete distribution . . . . . . . . . . . . . 228

5.10 The inversion technique .................................... 23 I

5.11 Random number generation via compositions .................... 234

5.12 Rejection sampling.................................. 236

5.13 Methods based on the rejection algorithm .................... 240

5.14 Monte Carlo methods in Bayesian inference ...................... 24I

$5.15 \mathrm{MC}$ predictive inference via the method of composition.............. 25 I

5.16 Rao-Blackwell methods for estimation and prediction . . . . . . . . . . . . . . . 253

5.17 MC estimation of posterior predictive p-values. . . . . . . . . . . . . . 258

Chapter 6: MCMC Methods Part I . . . ............................ 263

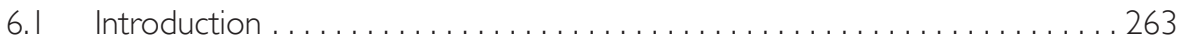

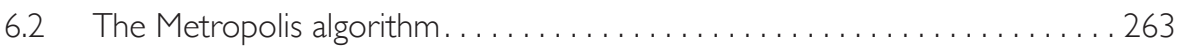

6.3 The batch means method ................................ 274

6.4 Computational issues .................................. 285

6.5 Non-symmetric drivers and the general Metropolis algorithm . . . . . . . . . 286

6.6 The Metropolis-Hastings algorithm . . . . . . . . . . . . . . . . . . . . . . . . 290

6.7 Independence drivers and block sampling . . . . . . . . . . . . . . . . . . 305

6.8 Gibbs steps and the Gibbs sampler........................... 306 


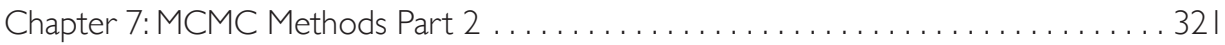

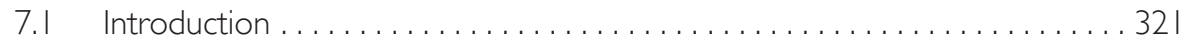

7.2 Data augmentation ...................................... 32 I

Chapter 8: Inference via WinBUGS. . . . . . . . . . . . . . . . . . . . . . . . . 365

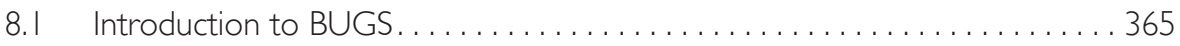

8.2 A first tutorial in BUGS. . . . . . . . . . . . . . . . . . . . . . . . . . 367

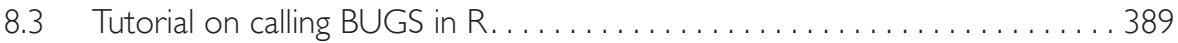

Chapter 9: Bayesian Finite Population Theory . . . . . . . . . . . . . . . . . . . . . . . . . 407

9.I Introduction ........................................ 407

9.2 Finite population notation and terminology .................... 408

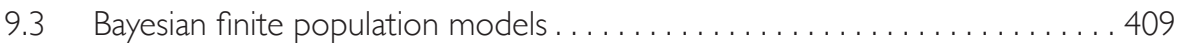

9.4 Two types of sampling mechanism ......................... 4 I I

9.5 Two types of inference .......................... 412

9.6 Analytic inference. . . . . . . . . . . . . . . . . . . . . . . . . . . . . . . 413

9.7 Descriptive inference. ................................ 4/4

Chapter 10: Normal Finite Population Models . ............................. 467

10.1 The basic normal-normal finite population model . . . . . . . . . . . . . . . 467

10.2 The general normal-normal finite population model . . . . . . . . . . . . . . . . 477

10.3 Derivation of the predictive distribution of the nonsample vector . . . . . . . 480

10.4 Alternative formulae for the predictive distribution of the nonsample vector48 I

10.5 Prediction of the finite population mean and other linear combinations . . . 483

10.6 Special cases including ratio estimation. . . . . . . . . . . . . . . . . . . . . . 484

10.7 The normal-normal-gamma finite population model . . . . . . . . . . . . . . . 494

10.8 Special cases of the normal-normal-gamma finite population model . . . . . . 497

10.9 The case of an informative prior on the regression parameter......... 50 I

Chapter I I:Transformations and OtherTopics..................... 515

II.I Inference on complicated quantities ......................... 5 I5

I I.2 Data transformations. . . . . . . . . . . . . . . . . . . . . . . . . . . . . 526

I I.3 Frequentist properties of Bayesian finite population estimators . . . . . . . . 536

Chapter 12: Biased Sampling and Nonresponse . . . . . . . . . . . . . . . . . . . . . 559

12.I Review of sampling mechanisms . . . . . . . . . . . . . . . . . . . . . . . 559

12.2 Nonresponse mechanisms ....................................... 560

12.3 Selection bias in volunteer surveys . . . . . . . . . . . . . . . . . . . 578

12.4 A classical model for self-selection bias. . . . . . . . . . . . . . . . . . . . . . . . . . 578

I2.5 Uncertainty regarding the sampling mechanism . . . . . . . . . . . . . 583

12.6 Finite population inference under selection bias in volunteer surveys . . . . . 588 


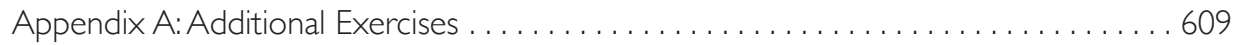

Exercise A. I Practice with the Metropolis algorithm . . . . . . . . . . . . . . . . . . . . 609

Exercise A.2 Practice with the $\mathrm{MH}$ algorithm .....................619

Exercise A.3 Practice with a Bayesian finite population regression model. . . . . . . 626

Exercise A.4 Case study in Bayesian finite population models

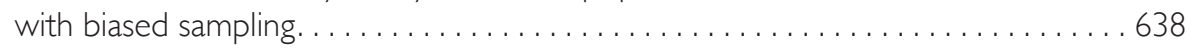

Appendix B: Distributions and Notation . . . . . . . . . . . . . . . . . . . 667

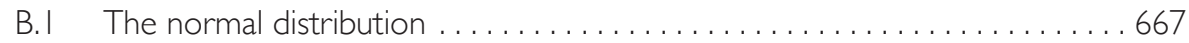

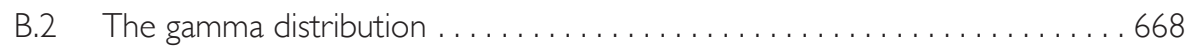

B.3 The exponential distribution ............................. 669

B.4 The chi-squared distribution. . . . . . . . . . . . . . . . . . . . . . . . . 669

B.5 The inverse gamma distribution. . . . . . . . . . . . . . . . . 670

B.6 The t distribution. ................................... 670

B.7 The F distribution. . . . . . . . . . . . . . . . . . . . . . . . 67

B.8 The (continuous) uniform distribution ................... 67 I

B.9 The discrete uniform distribution ..................... 67 I

B. 10 The binomial distribution ............................... 672

B.II The Bernoulli distribution .............................6 672

B.12 The geometric distribution........................... 672

Appendix C: Abbreviations and Acronyms . . . . . . . . . . . . . . . . . . . . 673

Bibliography .........................................6 677 


\section{Abstract}

'Bayesian Methods for Statistical Analysis' is a book on statistical methods for analysing a wide variety of data. The book consists of 12 chapters, starting with basic concepts and covering numerous topics, including Bayesian estimation, decision theory, prediction, hypothesis testing, hierarchical models, Markov chain Monte Carlo methods, finite population inference, biased sampling and nonignorable nonresponse. The book contains many exercises, all with worked solutions, including complete computer code. It is suitable for self-study or a semester-long course, with three hours of lectures and one tutorial per week for 13 weeks. 



\section{Acknowledgements}

'Bayesian Methods for Statistical Analysis' derives from the lecture notes for a four-day course titled 'Bayesian Methods', which was presented to staff of the Australian Bureau of Statistics, at ABS House in Canberra, in 2013. Lectures of three hours each were held in the mornings of 11, 18 and 25 November and 9 December, and three-hour tutorials were held in the mornings of 14, 20 and 27 November and 11 December.

Of the 30-odd participants, some of whom attended via video link from regional ABS offices, special thanks go to Anura Amarasinghe, Rachel Barker, Geoffrey Brent, Joseph Chien, Alexander Hanysz, Sebastien Lucie, Peter Radisich and Anthony Russo, who asked insightful questions, pointed out errors, and contributed to an improved second edition of the lecture notes. Thanks also to Siu-Ming Tam, First Australian Statistician of the Methodology and Data Management Division at ABS, for useful comments, and for inviting the author to present the course in the first place, after having read Puza (1995). Last but not least, special thanks go to Kylie Johnson for her excellent work as the course administrator.

Further modifications to 'Bayesian Methods' led to the present work, which is published as an eView textbook by the ANU Press, Canberra. Many thanks to David Gardiner, Brian Kennett, Teresa Prowse, Emily Tinker and two anonymous referees for useful comments and suggestions which helped to further improve the quality of the book. Thanks also to Yi (James) Zhang for his proofreading of the book whilst learning the material as part of his Honours studies. 



\section{Preface}

'Bayesian Methods for Statistical Analysis' is a book which can be used as the text for a semester-long course and is suitable for anyone who is familiar with statistics at the level of 'Mathematical Statistics with Applications' by Wackerly, Mendenhall and Scheaffer (2008). The book does not attempt to cover all aspects of Bayesian methods but to provide a 'guided tour' through the subject matter, one which naturally reflects the author's particular interests gained over years of research and teaching.

For a more comprehensive account of Bayesian methods, the reader is referred to the very extensive literature on this subject, including 'Theory of Probability' by Jeffreys (1961), 'Bayesian Inference in Statistical Analysis' by Box and Tiao (1973), 'Markov Chain Monte Carlo in Practice' by Gilks et al. (1996), 'Bayesian Statistics: An Introduction' by Lee (1997), 'Bayesian Methods: An Analysis for Statisticians and Interdisciplinary Researchers' by Leonard and Hsu (1999), 'Bayesian Data Analysis' by Gelman et al. (2004), 'Computational Bayesian Statistics' by Bolstad (2009) and 'Handbook of Markov Chain Monte Carlo' by Brooks et al. (2011). See also Smith and Gelfand (1992) and O'Hagan and Forster (2004).

The software packages which feature in this book are R and WinBUGS.

$\mathrm{R}$ is a general software environment for statistical computing and graphics which compiles and runs on UNIX platforms, Windows and MacOS. This software is available for free at www.r-project.org/ Two useful guides to $\mathrm{R}$ are 'Bayesian Computation With R' by Albert (2009) and 'Data Analysis and Graphics Using R: An Example-Based Approach' by Maindonald and Braun (2010).

BUGS stands for 'Bayesian Inference Using Gibbs Sampling' and is a specialised software environment for the Bayesian analysis of complex statistical models using Markov chain Monte Carlo methods. WinBUGS, a version of BUGS for Microsoft Windows, is available for free at www.mrc-bsu.cam.ac.uk/software/bugs/ Two useful guides to WinBUGS are 'Bayesian Modeling Using WinBUGS' by Ntzoufras (2009) and 'Bayesian Population Analysis Using WinBUGS' by Kéry and Schaub (2012). 
The present book includes a large number of exercises, interspersed throughout and each followed by a detailed solution, including complete computer code. A student should be able to reproduce all of the numerical and graphical results in the book by running the provided code. Although many of the exercises are straightforward, some are fairly involved, and a few will be of interest only to the particularly keen or advanced student. All of the code in this book is also available in the form of an electronic text document which can be obtained from the same website as the book.

This book is in the form of an Adobe PDF file saved from Microsoft Word 2013 documents, with the equations as MathType 6.9 objects. The figures in the book were created using Microsoft Paint, the Snipping Tool in Windows, WinBUGS and R. In the few instances where color is used, this is only for additional clarity. Thus, the book can be printed in black and white with no loss of essential information.

The following chapter provides an overview of the book. Appendix A contains several additional exercises with worked solutions, Appendix B has selected distributions and notation, and Appendix C lists some abbreviations and acronyms. Following the appendices is a bibliography for the entire book.

The last four of the 12 chapters in this book constitute a practical companion to 'Monte Carlo Methods for Finite Population Inference', a largely theoretical manuscript written by the author (Puza, 1995) during the last year of his employment at the Australian Bureau of Statistics in Canberra. 


\section{Overview}

\section{Chapter I: Bayesian Basics Part I (pages I-60)}

Introduces Bayes' rule, Bayes factors, Bayesian models, posterior distributions, and the proportionality formula. Also covered are the binomial-beta model, the Jeffreys' famous tramcar problem, the distinction between finite population inference and superpopulation inference, conjugacy, point and interval estimation, inference on functions of parameters, credibility estimation, the normal-normal model, and the normal-gamma model.

\section{Chapter 2: Bayesian Basics Part 2 (pages $6 \mathrm{I}-108$ )}

Covers the frequentist characteristics of Bayesian estimators including bias and coverage probabilities, mixture priors, uninformative priors including the Jeffreys prior, and Bayesian decision theory including the posterior expected loss and Bayes risk.

\section{Chapter 3: Bayesian Basics Part 3 (pages 109-152)}

Covers inference based on functions of the data including censoring and rounded data, predictive inference, posterior predictive p-values, multiple-parameter models, and the normal-normal-gamma model including an example of Bayesian finite population inference.

\section{Chapter 4: Computational Tools (pages I53-200)}

Covers the Newton-Raphson (NR) algorithm including its multivariate version, the expectation-maximisation (EM) algorithm, hybrid search algorithms, integration techniques including double integration, optimisation in $\mathrm{R}$, and specification of prior distributions.

\section{Chapter 5: Monte Carlo Basics (pages 20I-262)}

Covers Monte Carlo integration, importance sampling, the method of composition, Buffon's needle problem, testing the coverage of Monte Carlo confidence intervals, random number generation including the inversion technique, rejection sampling, and applications to Bayesian inference including prediction in the normal-normal-gamma model, RaoBlackwell estimation, and estimation of posterior predictive p-values. 


\section{Chapter 6: MCMC Methods Part I (pages 263-320)}

Covers Markov chain Monte Carlo (MCMC) methods including the Metropolis-Hastings algorithm, the Gibbs sampler, specification of tuning parameters, the batch means method, computational issues, and applications to the normal-normal-gamma model.

\section{Chapter 7: MCMC Methods Part 2 (pages 32I-364)}

Covers stochastic data augmentation, a comparison of classical and Bayesian methods for linear regression and logistic regression, respectively, and a Bayesian model for correlated Bernoulli data.

\section{Chapter 8: MCMC Inference via WinBUGS (pages 365-406)}

Provides a detailed tutorial in the WinBUGS computer package including running WinBUGS within R, and shows how WinBUGS can be used for linear regression, logistic regression and ARIMA time series analysis.

\section{Chapter 9: Bayesian Finite Population Theory (pages 407-466)}

Introduces notation and terminology for Bayesian finite population inference in the survey sampling context, and discusses ignorable and nonignorable sampling mechanisms. These concepts are illustrated by way of examples and exercises, some of which involve MCMC methods.

\section{Chapter I0: Normal Finite Population Models (pages 467-5I4)}

Contains a generalisation of the normal-normal-gamma model to the finite population context with covariates. Useful vector and matrix formulae are provided, special cases such as ratio estimation are treated in detail, and it is shown how MCMC methods can be used for both descriptive and analytic inferences. 


\section{Chapter I I: Transformations and Other Topics (pages 5 I 5-558)}

Shows how MCMC methods can be used for inference on complicated functions of superpopulation and finite population quantities, as well for inference based on transformed data. Frequentist characteristics of Bayesian estimators are discussed in the finite population context, with examples of how Monte Carlo methods can be used to estimate model bias, design bias, model coverage and design coverage.

\section{Chapter I 2: Biased Sampling and Nonresponse (pages 559-608)}

Discusses and provides examples of ignorable and nonignorable response mechanisms, with an exercise involving follow-up data. The topic of selfselection bias in volunteer surveys is studied from a frequentist perspective, then treated using Bayesian methods, and finally extended to the finite population context.

\section{Appendix A: Additional Exercises (pages 609-666)}

Provides practice at applying concepts in the last four chapters.

\section{Appendix B: Distributions and Notation (pages 667-672)}

Provides details of some distributions which feature in the book.

\section{Appendix C: Abbreviations and Acronyms (pages 673-676)}

Catalogues many of the simplified expressions used throughout.

\section{Computer Code in Bayesian Methods for Statistical Analysis}

Combines all of the $\mathrm{R}$ and WinBUGS code interspersed throughout the 679-page book. This separate 126-page PDF file is available online at: http://eview.anu.edu.au/bayesian_methods/pdf/computer_code.pdf. 



\section{CHAPTER I}

\section{I.I Introduction}

Bayesian methods is a term which may be used to refer to any mathematical tools that are useful and relevant in some way to Bayesian inference, an approach to statistics based on the work of Thomas Bayes (1701-1761). Bayes was an English mathematician and Presbyterian minister who is best known for having formulated a basic version of the well-known Bayes' Theorem.

Figure 1.1 (page 3) shows part of the Wikipedia article for Thomas Bayes. Bayes' ideas were later developed and generalised by many others, most notably the French mathematician Pierre-Simon Laplace (1749-1827) and the British astronomer Harold Jeffreys (1891-1989).

Bayesian inference is different to classical inference (or frequentist inference) mainly in that it treats model parameters as random variables rather than as constants. The Bayesian framework (or paradigm) allows for prior information to be formally taken into account. It can also be useful for formulating a complicated statistical model that presents a challenge to classical methods.

One drawback of Bayesian inference is that it invariably requires a prior distribution to be specified, even in the absence of any prior information. However, suitable uninformative prior distributions (also known as noninformative, objective or reference priors) have been developed which address this issue, and in many cases a nice feature of Bayesian inference is that these priors lead to exactly the same point and interval estimates as does classical inference. The issue becomes even less important when there is at least a moderate amount of data available. As sample size increases, the Bayesian approach typically converges to the same inferential results, irrespective of the specified prior distribution.

Another issue with Bayesian inference is that, although it may easily lead to suitable formulations of a challenging statistical problem, the types of calculation needed for inference can themselves be very complicated. Often, these calculations take on the form of multiple 
integrals (or summations) which are intractable and difficult (or impossible) to solve, even with the aid of advanced numerical techniques.

In such situations, the desired solutions can typically be approximated to any degree of precision using Monte Carlo (MC) methods. The idea is to make clever use of a large sample of values generated from a suitable probability distribution.

How to generate this sample presents another problem, but one which can typically be solved easily via Markov chain Monte Carlo (MCMC) methods. Both MC and MCMC methods will feature in later chapters of the course.

\section{I.2 Bayes' rule}

The starting point for Bayesian inference is Bayes' rule. The simplest form of this is

$$
P(A \mid B)=\frac{P(A) P(B \mid A)}{P(A) P(B \mid A)+P(\bar{A}) P(B \mid \bar{A})},
$$

where $A$ and $B$ are events such that $P(B)>0$. This is easily proven by considering that:

$$
\begin{aligned}
& P(A \mid B)=\frac{P(A B)}{P(B)} \text { by the definition of conditional probability } \\
& P(A B)=P(A) P(B \mid A) \quad \text { by the multiplicative law of probability } \\
& P(B)=P(A B)+P(\bar{A} B)=P(A) P(B \mid A)+P(\bar{A}) P(B \mid \bar{A}) \\
& \text { by the law of total probability. }
\end{aligned}
$$

We see that the posterior probability $P(A \mid B)$ is equal to the prior probability $P(A)$ multiplied by a factor, where this factor is given by $P(B \mid A) / P(B)$.

As regards terminology, we call $P(A)$ the prior probability of $A$ (meaning the probability of $A$ before $B$ is known to have occurred), and we call $P(A \mid B)$ the posterior probability of $A$ given $B$ (meaning the probability of $A$ after $B$ is known to have occurred). We may also say that $P(A)$ represents our a priori beliefs regarding $A$, and $P(A \mid B)$ represents our a posteriori beliefs regarding $A$. 


\section{Figure I.I Beginning of the Wikipedia article on Thomas Bayes}

Source: en.wikipedia.org/wiki/Thomas_Bayes, 29//0/20।4

\section{Thomas Bayes}

From Wikipedia, the free encyclopedia

Thomas Bayes (/ beiz/; c. $1701-7$ April 1761) ${ }^{[1][2][\text { note a] }}$ was an English statistician, philosopher and Presbyterian minister, known for having formulated a specific case of the theorem that bears his name: Bayes' theorem. Bayes never published what would eventually become his most famous accomplishment; his notes were edited and published after his death by Richard Price. ${ }^{[3]}$

Contents [hide]
1 Biography
2 Bayes' theorem
3 Bayesianism
4 See also
5 Notes
6 References
7 External links

Biography [edit]

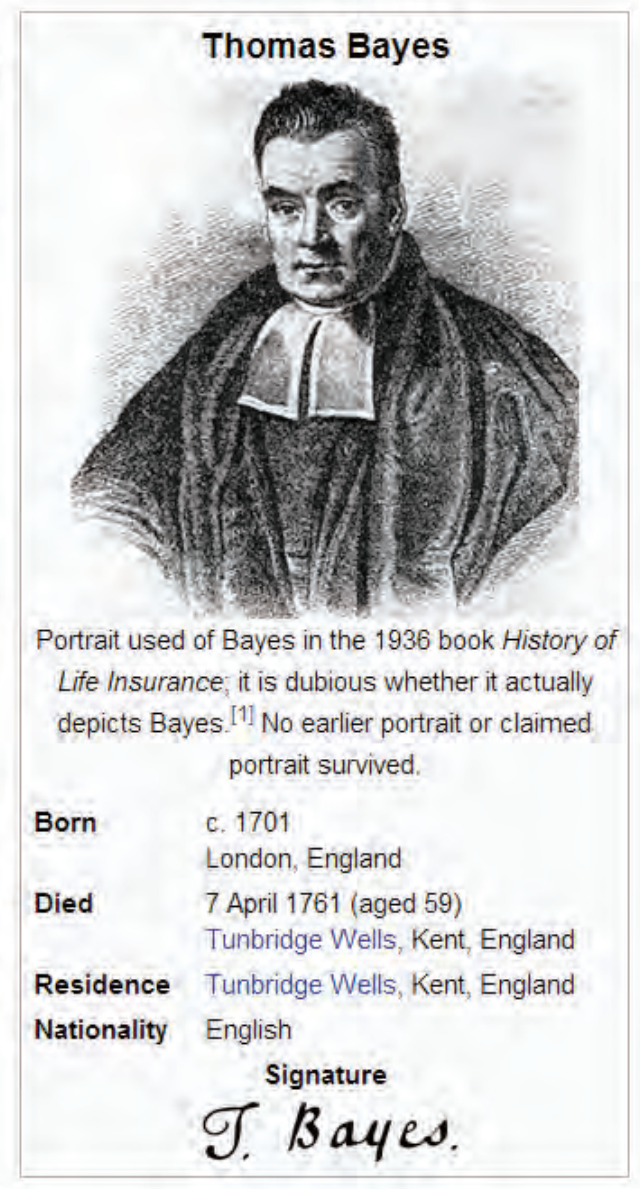

Thomas Bayes was the son of London Presbyterian minister Joshua Bayes, ${ }_{1}^{[4]}$ and was possibly born in Hertfordshire. ${ }^{[5]}$ He came from a prominent nonconformist family from Sheffield. In 1719, he enrolled at the University of Edinburgh to study logic and theology. On his return around 1722 , he assisted his father at the latter's chapel in London before moving to Tunbridge Wells, Kent, around 1734 . There he was minister of the Mount Sion chapel, until 1752. ${ }^{[6]}$ 
More generally, we may consider any event $B$ such that $P(B)>0$ and $k>1$ events $A_{1}, \ldots, A_{k}$ which form a partition of any superset of $B$ (such as the entire sample space $S$ ). Then, for any $i=1, \ldots, k$, it is true that

$$
P\left(A_{i} \mid B\right)=\frac{P\left(A_{i} B\right)}{P(B)}
$$

where $P(B)=\sum_{j=1}^{n} P\left(A_{j} B\right)$ and $P\left(A_{j} B\right)=P\left(A_{j}\right) P\left(B \mid A_{j}\right)$.

\section{Exercise I.I Medical testing}

The incidence of a disease in the population is $1 \%$. A medical test for the disease is $90 \%$ accurate in the sense that it produces a false reading $10 \%$ of the time, both: (a) when the test is applied to a person with the disease; and (b) when the test is applied to a person without the disease.

A person is randomly selected from population and given the test. The test result is positive (i.e. it indicates that the person has the disease).

What is the probability that the person actually has the disease?

\section{Solution to Exercise I.I}

Let $A$ be the event that the person has the disease, and let $B$ be the event that they test positive for the disease. Then:

$$
\begin{aligned}
& P(A)=0.01 \quad \text { (the prior probability of the person having the disease) } \\
& P(B \mid A)=0.9 \text { (the true positive rate, also called } \\
& \text { the sensitivity of the test) } \\
& P(\bar{B} \mid \bar{A})=0.9 \text { (the true negative rate, also called } \\
& \text { the specificity of the test). }
\end{aligned}
$$

So: $\quad P(A B)=P(A) P(B \mid A)=0.01 \times 0.9=0.009$

$$
P(\bar{A} B)=P(\bar{A}) P(B \mid \bar{A})=0.99 \times 0.1=0.099 \text {. }
$$

So the unconditional (or prior) probability of the person testing positive is $\quad P(B)=P(A B)+P(\bar{A} B)=0.009+0.099=0.108$.

So the required posterior probability of the person having the disease is

$$
P(A \mid B)=\frac{P(A B)}{P(B)}=\frac{0.009}{0.108}=\frac{1}{12}=0.08333 .
$$


Figure 1.2 is a Venn diagram which illustrates how $B$ may be considered as the union of $A B$ and $\bar{A} B$. The required posterior probability of $A$ given $B$ is simply the probability of $A B$ divided by the probability of $B$.

\section{Figure I.2 Venn diagram for Exercise I.I}

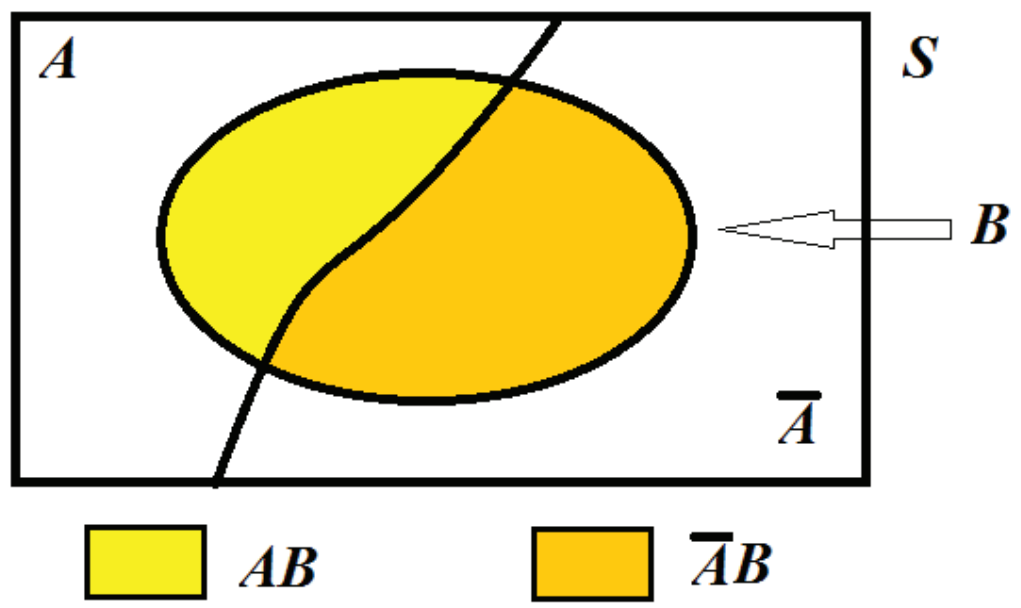

\section{Discussion}

It may seem the posterior probability that the person has the disease $(1 / 12)$ is rather low, considering the high accuracy of the test (namely $P(B \mid A)=P(\bar{B} \mid \bar{A})=0.9)$.

This may be explained by considering 1,000 random persons in the population and applying the test to each one. About 10 persons will have the disease, and of these, 9 will test positive. Of the 990 who do not have the disease, 99 will test positive. So the total number of persons testing positive will be $9+99=108$, and the proportion of these 108 who actually have the disease will be 9/108 $=1 / 12$. This heuristic derivation of the answer shows it to be small on account of the large number of false positives (99) amongst the overall number of positives (108).

On the other hand, it may be noted that the posterior probability of the person having the disease is actually very high relative to the prior probability of them having the disease $(P(A)=0.01)$. The positive test result has greatly increased the person's chance of having the disease (increased it by more than $700 \%$, since $0.01+7.333 \times 0.01=0.08333$ ). 
It is instructive to generalise the answer (1/12) as a function of the prevalence (i.e. proportion) of the disease in the population, $p=P(A)$, and the common accuracy rate of the test, $q=P(B \mid A)=P(\bar{B} \mid \bar{A})$.

We find that

$$
P(A \mid B)=\frac{P(A) P(B \mid A)}{P(A) P(B \mid A)+P(\bar{A}) P(B \mid \bar{A})}=\frac{p q}{p q+(1-p)(1-q)} .
$$

Figure 1.3 shows the posterior probability of the person having the disease $(P(A \mid B))$ as a function of $p$ with $q$ fixed at 0.9 and 0.95, respectively (subplot (a)), and as a function of $q$ with $p$ fixed at 0.01 and 0.05 , respectively (subplot (b)). In each case, the answer (1/12) is represented as a dot corresponding to $p=0.01$ and $q=0.9$.

Figure I.3 Posterior probability of disease as functions of $p$ and $q$
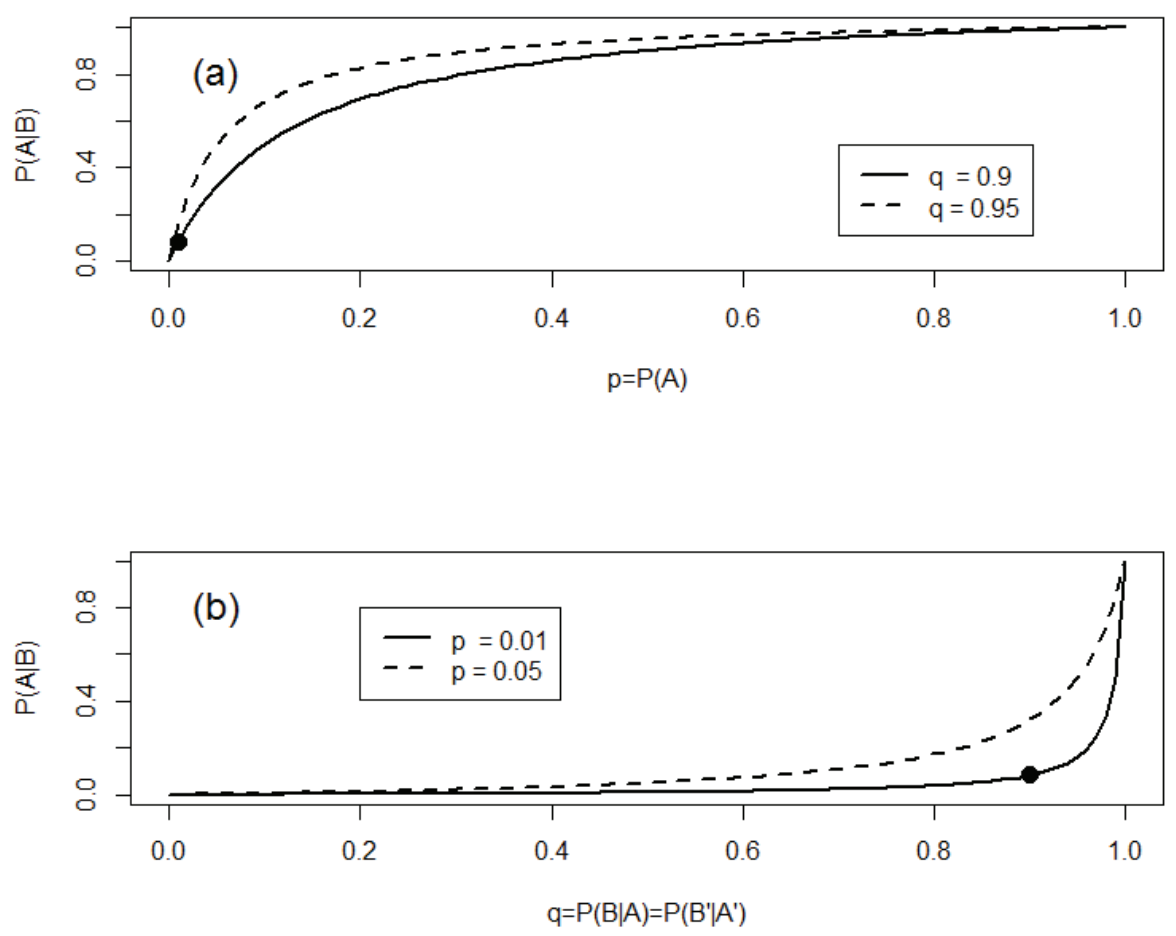


\section{R Code for Exercise I.I}

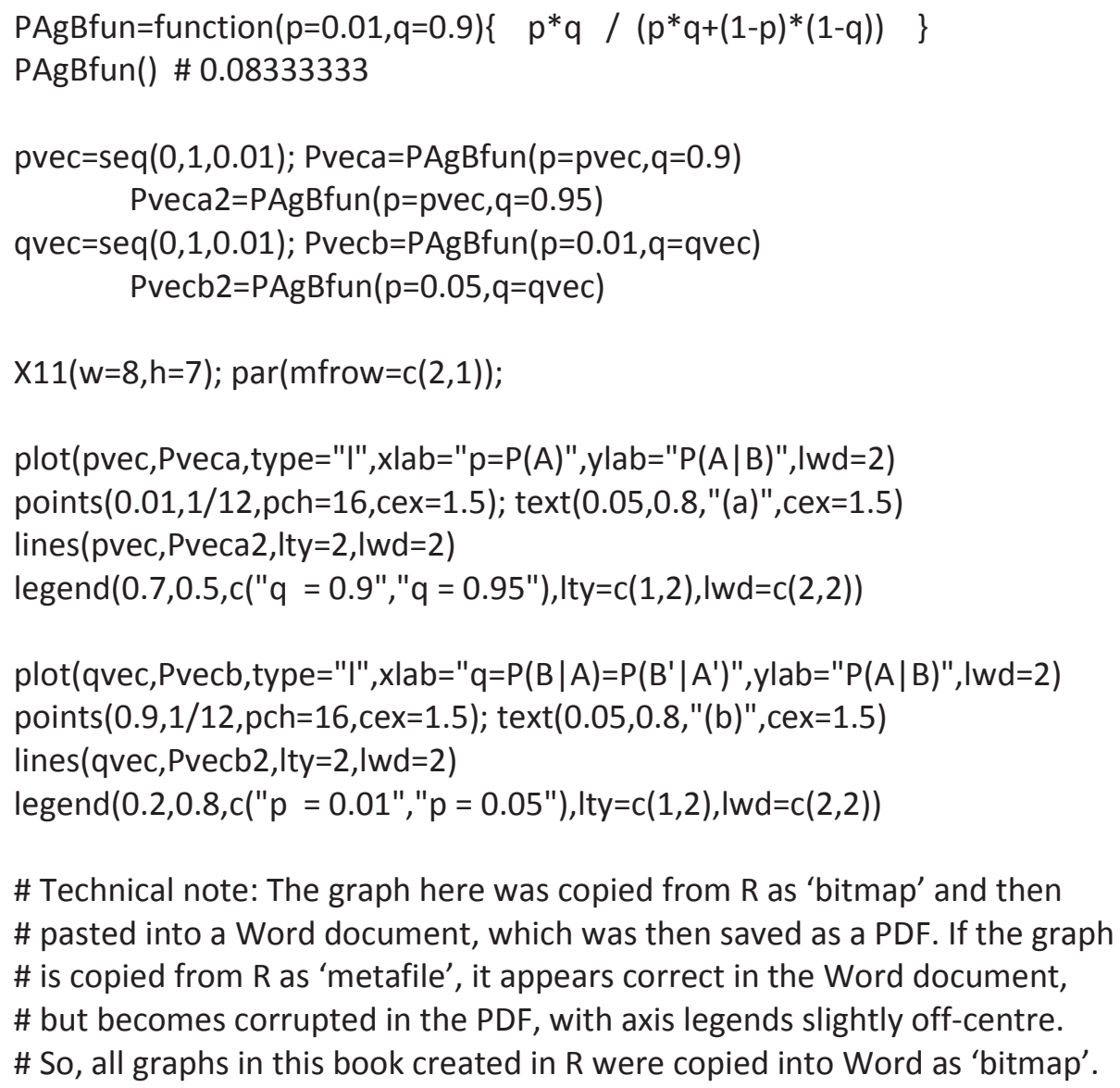

\# Technical note: The graph here was copied from R as 'bitmap' and then \# pasted into a Word document, which was then saved as a PDF. If the graph \# is copied from R as 'metafile', it appears correct in the Word document, \# but becomes corrupted in the PDF, with axis legends slightly off-centre. \# So, all graphs in this book created in R were copied into Word as 'bitmap'.

\section{Exercise I.2 Blood types}

In a particular population:

$10 \%$ of persons have Type 1 blood, and of these, $2 \%$ have a particular disease;

$30 \%$ of persons have Type 2 blood, and of these, $4 \%$ have the disease;

$60 \%$ of persons have Type 3 blood, and of these, $3 \%$ have the disease.

A person is randomly selected from the population and found to have the disease.

What is the probability that this person has Type 3 blood? 


\section{Solution to Exercise I.2}

Let: $\quad A=$ 'The person has Type 1 blood'

$B=$ 'The person has Type 2 blood'

$C=$ 'The person has Type 3 blood'

$D=$ 'The person has the disease'.

Then: $\quad P(A)=0.1, \quad P(D \mid A)=0.02$

$$
\begin{array}{ll}
P(B)=0.3, & P(D \mid B)=0.04 \\
P(C)=0.6, & P(D \mid C)=0.03 .
\end{array}
$$

So: $\quad P(D)=P(A D)+P(B D)+P(C D)$

$$
\begin{aligned}
& =P(A) P(D \mid A)+P(B) P(D \mid B)+P(C) P(D \mid C) \\
& =0.1 \times 0.02+0.3 \times 0.04+0.6 \times 0.03 \\
& =0.002+0.012+0.018=0.032 .
\end{aligned}
$$

Hence: $P(C \mid D)=\frac{P(C D)}{P(D)}=\frac{0.018}{0.032}=\frac{9}{16}=56.25 \%$.

Figure 1.4 is a Venn diagram showing how $D$ may be considered as the union of $A D, B D$ and $C D$. The required posterior probability of $C$ given $D$ is simply the probability of $C D$ divided by the probability of $D$.

Figure I.4 Venn diagram for Exercise I.2

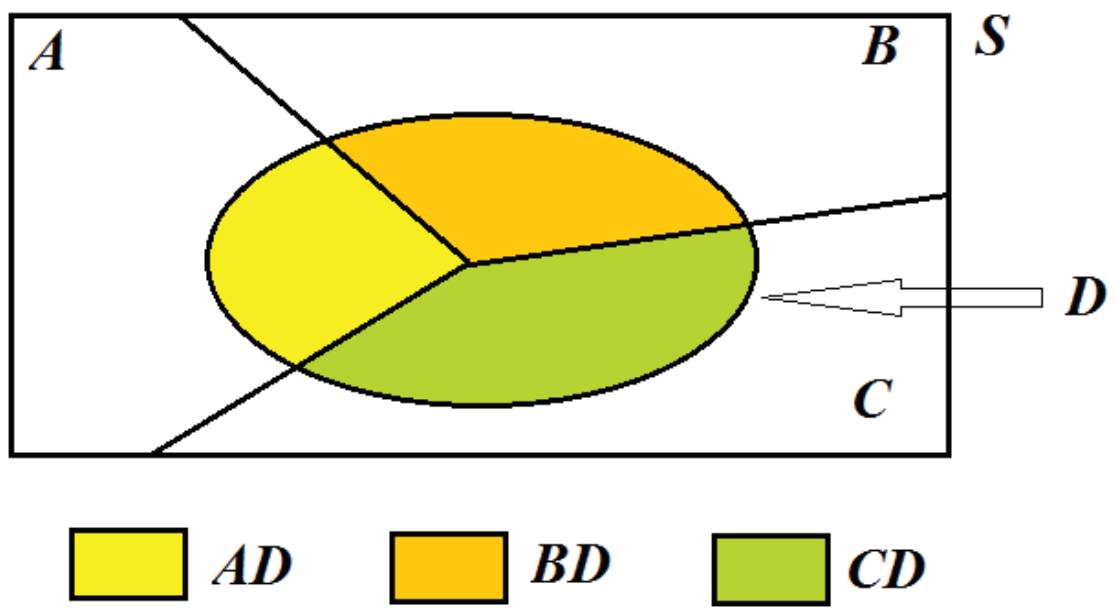




\section{I.3 Bayes factors}

One way to perform hypothesis testing in the Bayesian framework is via the theory of Bayes factors. Suppose that on the basis of an observed event $D$ (standing for data) we wish to test a null hypothesis

$$
H_{0}: E_{0}
$$

versus an alternative hypothesis

$$
H_{1}: E_{1} \text {, }
$$

where $E_{0}$ and $E_{1}$ are two events (which are not necessarily mutually exclusive or even exhaustive of the event space).

Then we calculate:

$$
\begin{aligned}
& \pi_{0}=P\left(E_{0}\right)=\text { the prior probability of the null hypothesis } \\
& \pi_{1}=P\left(E_{1}\right)=\text { the prior probability of the alternative hypothesis } \\
& P R O=\pi_{0} / \pi_{1}=\text { the prior odds in favour of the null hypothesis } \\
& p_{0}=P\left(E_{0} \mid D\right)=\text { the posterior probability of the null hypothesis } \\
& p_{1}=P\left(E_{1} \mid D\right)=\text { the posterior probability of the alternative hypothesis } \\
& P O O=p_{0} / p_{1}=\text { the posterior odds in favour of the null hypothesis. }
\end{aligned}
$$

The Bayes factor is then defined as $B F=P O O / P R O$. This may be interpreted as the factor by which the data have multiplied the odds in favour of the null hypothesis relative to the alternative hypothesis. If $B F>1$ then the data has increased the relative likelihood of the null, and if $B F<1$ then the data has decreased that relative likelihood. The magnitude of $B F$ tells us how much effect the data has had on the relative likelihood.

Note 1: Another way to express the Bayes factor is as

$$
\begin{aligned}
B F=\frac{p_{0} / p_{1}}{\pi_{0} / \pi_{1}}=\frac{P\left(E_{0} \mid D\right) / P\left(E_{1} \mid D\right)}{P\left(E_{0}\right) / P\left(E_{1}\right)} & =\frac{P(D) P\left(E_{0} \mid D\right) / P\left(E_{0}\right)}{P(D) P\left(E_{1} \mid D\right) / P\left(E_{1}\right)} \\
& =\frac{P\left(D \mid E_{0}\right)}{P\left(D \mid E_{1}\right)} .
\end{aligned}
$$

Thus, the Bayes factor may also be interpreted as the ratio of the likelihood of the data given the null hypothesis to the likelihood of the data given the alternative hypothesis. 
Note 2: The idea of a Bayes factor extends to situations where the null and alternative hypotheses are statistical models rather than events. This idea may be taken up later.

\section{Exercise I.3 Bayes factor in disease testing}

The incidence of a disease in the population is $1 \%$. A medical test for the disease is $90 \%$ accurate in the sense that it produces a false reading $10 \%$ of the time, both: (a) when the test is applied to a person with the disease; and (b) when the test is applied to a person without the disease.

A person is randomly selected from population and given the test. The test result is positive (i.e. it indicates that the person has the disease).

Calculate the Bayes factor for testing that the person has the disease versus that they do not have the disease.

\section{Solution to Exercise I.3}

Recall in Exercise 1.1, where $A=$ 'Person has disease' and $B=$ 'Person tests positive', the relevant probabilities are $P(A)=0.01, P(B \mid A)=0.9$ and $P(\bar{B} \mid \bar{A})=0.9$, from which can be deduced that $P(A \mid B)=1 / 12$.

We now wish to test $H_{0}: A$ vs $H_{1}: \bar{A}$. So we calculate:

$$
\begin{aligned}
& \pi_{0}=P(A)=0.01, \pi_{1}=P(\bar{A})=0.99, P R O=\pi_{0} / \pi_{1}=1 / 99, \\
& p_{0}=P(A \mid B)=1 / 12, p_{1}=P(\bar{A} \mid B)=11 / 12, P O O=p_{0} / p_{1}=1 / 11 .
\end{aligned}
$$

Hence the required Bayes factor is $B F=P O O / P R O=(1 / 11) /(1 / 99)=9$.

This means the positive test result has multiplied the odds of the person having the disease relative to not having it by a factor of 9 or $900 \%$. Another way to say this is that those odds have increased by $800 \%$.

Note: We could also work out the Bayes factor here as

$$
B F=\frac{P(B \mid A)}{P(B \mid \bar{A})}=\frac{0.9}{0.1}=9,
$$

namely as the ratio of the probability that the person tests positive given they have the disease to the probability that they test positive given they do not have the disease. 


\section{I.4 Bayesian models}

Bayes' formula extends naturally to statistical models. A Bayesian model is a parametric model in the classical (or frequentist) sense, but with the addition of a prior probability distribution for the model parameter, which is treated as a random variable rather than an unknown constant. The basic components of a Bayesian model may be listed as:

- the data, denoted by $y$

- the parameter, denoted by $\theta$

- the model distribution, given by a specification of

$f(y \mid \theta)$ or $F(y \mid \theta)$ or the distribution of $(y \mid \theta)$

- the prior distribution, given by a specification of $f(\theta)$ or $F(\theta)$ or the distribution of $\theta$.

Here, $F$ is a generic symbol which denotes cumulative distribution function (cdf), and $f$ is a generic symbol which denotes probability density function (pdf) (when applied to a continuous random variable) or probability mass function (pmf) (when applied to a discrete random variable). For simplicity, we will avoid the term pmf and use the term pdf or density for all types of random variable, including the mixed type.

Note 1: A mixed distribution is defined by a cdf which exhibits at least one discontinuity (or jump) and is strictly increasing over at least one interval of values.

Note 2: The prior may be specified by writing a statement of the form ' $\theta \sim \ldots$. ', where the symbol ' $\sim$ ' means 'is distributed as', and where '...' denotes the relevant distribution. Likewise, the model for the data may be specified by writing a statement of the form ' $(y \mid \theta) \sim \ldots$ ' .

Note 3: At this stage we will not usually distinguish between $y$ as a random variable and $y$ as a value of that random variable; but sometimes we may use $Y$ for the former. Each of $y$ and $\theta$ may be a scalar, vector, matrix or array. Also, each component of $y$ and $\theta$ may have a discrete distribution, a continuous distribution, or a mixed distribution.

In the first few examples below, we will focus on the simplest case where both $y$ and $\theta$ are scalar and discrete. 


\section{I.5 The posterior distribution}

Bayesian inference requires determination of the posterior probability distribution of $\theta$. This task is equivalent to finding the posterior $p d f$ of $\theta$, which may be done using the equation

$$
f(\theta \mid y)=\frac{f(\theta) f(y \mid \theta)}{f(y)} .
$$

Here, $f(y)$ is the unconditional (or prior) pdf of $y$, as given by

$$
f(y)=\int f(y \mid \theta) d F(\theta)= \begin{cases}\int f(\theta) f(y \mid \theta) d \theta & \text { if } \theta \text { is continuous } \\ \sum_{\theta} f(\theta) f(y \mid \theta) & \text { if } \theta \text { is discrete. }\end{cases}
$$

Note: Here, $\int f(y \mid \theta) d F(\theta)$ is a Lebesgue-Stieltjes integral, which may need evaluating by breaking the integral into two parts in the case where $\theta$ has a mixed distribution. In the continuous case, think of $d F(\theta)$ as $\frac{d F(\theta)}{d \theta} d \theta=f(\theta) d \theta$.

\section{Exercise I.4 Loaded dice}

Consider six loaded dice with the following properties. Die A has probability 0.1 of coming up 6, each of Dice B and C has probability 0.2 of coming up 6, and each of Dice D, E and F has probability 0.3 of coming up 6.

A die is chosen randomly from the six dice and rolled twice. On both occasions, 6 comes up.

What is the posterior probability distribution of $\theta$, the probability of 6 coming up on the chosen die.

\section{Solution to Exercise I.4}

Let $y$ be the number of times that 6 comes up on the two rolls of the chosen die, and let $\theta$ be the probability of 6 coming up on a single roll of that die. Then the Bayesian model is: 


$$
\begin{aligned}
& (y \mid \theta) \sim \operatorname{Bin}(2, \theta) \\
& f(\theta)= \begin{cases}1 / 6, & \theta=0.1 \\
2 / 6, & \theta=0.2 \\
3 / 6, & \theta=0.3 .\end{cases}
\end{aligned}
$$

In this case $y=2$ and so

$$
f(y \mid \theta)=\left(\begin{array}{l}
2 \\
y
\end{array}\right) \theta^{y}(1-\theta)^{2-y}=\left(\begin{array}{l}
2 \\
2
\end{array}\right) \theta^{2}(1-\theta)^{2-2}=\theta^{2} .
$$

So $f(y)=\sum_{\theta} f(\theta) f(y \mid \theta)=\frac{1}{6}(0.1)^{2}+\frac{2}{6}(0.2)^{2}+\frac{3}{6}(0.3)^{2}=0.06$.

So $f(\theta \mid y)=\frac{f(\theta) f(y \mid \theta)}{f(y)}=\left\{\begin{array}{cc}(1 / 6) 0.1^{2} / 0.06=0.02778, & \theta=0.1 \\ (2 / 6) 0.2^{2} / 0.06=0.22222, & \theta=0.2 \\ (3 / 6) 0.3^{2} / 0.06=0.75, & \theta=0.3 .\end{array}\right.$

Note: This result means that if the chosen die were to be tossed again a large number of times (say 10,000) then there is a 75\% chance that 6 would come up about $30 \%$ of the time, a $22.2 \%$ chance that 6 would come up about $20 \%$ of the time, and a $2.8 \%$ chance that 6 would come up about $10 \%$ of the time.

\section{I.6 The proportionality formula}

Observe that $f(y)$ is a constant with respect to $\theta$ in the Bayesian equation

$$
f(\theta \mid y)=f(\theta) f(y \mid \theta) / f(y),
$$

which means that we may also write the equation as

$$
f(\theta \mid y)=\frac{f(\theta) f(y \mid \theta)}{k},
$$

or as

$$
f(\theta \mid y)=c f(\theta) f(y \mid \theta),
$$

where $k=f(y)$ and $c=1 / k$.

We may also write

$$
f(\theta \mid y) \propto f(\theta) f(y \mid \theta),
$$

where $\propto$ is the proportionality sign. 
Equivalently, we may write

$$
f(\theta \mid y) \stackrel{\theta}{\propto} f(\theta) f(y \mid \theta)
$$

to emphasise that the proportionality is specifically with respect to $\theta$.

Another way to express the last equation is

$$
f(\theta \mid y) \propto f(\theta) \times L(\theta \mid y),
$$

where $L(\theta \mid y)$ is the likelihood function (defined as the model density $f(y \mid \theta)$ multiplied by any constant with respect to $\theta$, and viewed as a function of $\theta$ rather than of $y$ ).

The last equation may also be stated in words as:

\section{The posterior is proportional to the prior times the likelihood.}

These observations indicate a shortcut method for determining the required posterior distribution which obviates the need for calculating $f(y)$ (which may be difficult).

This method is to multiply the prior density (or the kernel of that density) by the likelihood function and try to identify the resulting function of $\theta$ as the density of a well-known or common distribution.

Once the posterior distribution has been identified, $f(y)$ may then be obtained easily as the associated normalising constant.

\section{Exercise I.5 Loaded dice with solution via the proportionality formula}

As in Exercise 1.4, suppose that Die A has probability 0.1 of coming up 6, each of Dice B and C has probability 0.2 of coming up 6, and each of Dice D, E and F has probability 0.3 of coming up 6.

A die is chosen randomly from the six dice and rolled twice. On both occasions, 6 comes up.

Using the proportionality formula, find the posterior probability distribution of $\theta$, the probability of 6 coming up on the chosen die. 


\section{Solution to Exercise I.5}

With $y$ denoting the number of times 6 comes up, the Bayesian model may be written:

$$
\begin{aligned}
& f(y \mid \theta)=\left(\begin{array}{l}
2 \\
y
\end{array}\right) \theta^{y}(1-\theta)^{2-y}, y=0,1,2 \\
& f(\theta)=10 \theta / 6, \theta=0.1,0.2,0.3 .
\end{aligned}
$$

Note: $10 \theta / 6=1 / 6,2 / 6$ and 3/6 for $\theta=0.1,0.2$ and 0.3 , respectively.

Hence $f(\theta \mid y) \propto f(\theta) f(y \mid \theta)$

$$
\begin{aligned}
& =\frac{10 \theta}{6} \times\left(\begin{array}{l}
2 \\
y
\end{array}\right) \theta^{y}(1-\theta)^{2-y} \\
& \propto \theta \times \theta^{2} \quad \text { since } y=2 .
\end{aligned}
$$

Thus $f(\theta \mid y) \propto \theta^{3}=\left\{\begin{array}{c}0.1^{3}=1 / 1000, \theta=0.1 \\ 0.2^{3}=8 / 1000, \theta=0.2 \\ 0.3^{3}=27 / 1000, \theta=0.3\end{array}\right\} \propto\left\{\begin{array}{c}1, \theta=0.1 \\ 8, \theta=0.2 \\ 27, \theta=0.3 .\end{array}\right.$

Now, $1+8+27=36$, and so $f(\theta \mid y)=\left\{\begin{array}{c}1^{3} / 36=0.02778, \theta=0.1 \\ 2^{3} / 36=0.22222, \theta=0.2 \\ 3^{3} / 36=0.75, \theta=0.3,\end{array}\right.$

which is the same result as obtained earlier in Exercise 1.4.

\section{Exercise I.6 Buses}

You are visiting a town with buses whose licence plates show their numbers consecutively from 1 up to however many there are. In your mind the number of buses could be anything from one to five, with all possibilities equally likely.

Whilst touring the town you first happen to see Bus 3.

Assuming that at any point in time you are equally likely to see any of the buses in the town, how likely is it that the town has at least four buses? 


\section{Solution to Exercise I.6}

Let $\theta$ be the number of buses in the town and let $y$ be the number of the bus that you happen to first see. Then an appropriate Bayesian model is:

$$
\begin{aligned}
& f(y \mid \theta)=1 / \theta, y=1, \ldots, \theta \\
& f(\theta)=1 / 5, \theta=1, \ldots, 5 \quad \text { (prior). }
\end{aligned}
$$

Note: We could also write this model as:

$$
\begin{aligned}
& (y \mid \theta) \sim D U(1, \ldots, \theta) \\
& \theta \sim D U(1, \ldots, 5),
\end{aligned}
$$

where $D U$ denotes the discrete uniform distribution. (See Appendix B.9 for details regarding this distribution. Appendix B also provides details regarding some other important distributions that feature in this book.)

So the posterior density of $\theta$ is

$$
\begin{aligned}
f(\theta \mid y) & \propto f(\theta) f(y \mid \theta) \\
& \propto 1 \times 1 / \theta, \quad \theta=y, \ldots, 5 .
\end{aligned}
$$

Noting that $y=3$, we have that

$$
f(\theta \mid y) \propto\left\{\begin{array}{l}
1 / 3, \theta=3 \\
1 / 4, \theta=4 \\
1 / 5, \theta=5 .
\end{array}\right.
$$

Now, $1 / 3+1 / 4+1 / 5=(20+15+12) / 60=47 / 60$, and so

$$
f(\theta \mid y)=\left\{\begin{array}{l}
\frac{1 / 3}{47 / 60}=\frac{20}{47}, \theta=3 \\
\frac{1 / 4}{47 / 60}=\frac{15}{47}, \theta=4 \\
\frac{1 / 5}{47 / 60}=\frac{12}{47}, \theta=5 .
\end{array}\right.
$$

So the posterior probability that the town has at least four buses is

$$
\begin{aligned}
P(\theta \geq 4 \mid y) & =\sum_{\theta: \theta \geq 4} f(\theta \mid y)=f(\theta=4 \mid y)+f(\theta=5 \mid y) \\
& =1-f(\theta=3 \mid y)=1-\frac{20}{47}=\frac{27}{47}=0.5745 .
\end{aligned}
$$




\section{Discussion}

This exercise is a variant of the famous 'tramcar problem' considered by Harold Jeffreys in his book Theory of Probability and previously suggested to him by M.H.A. Newman (see Jeffreys, 1961, page 238). Suppose that before entering the town you had absolutely no idea about the number of buses $\theta$. Then, according to Jeffreys' logic, a prior which may be considered as suitably uninformative (or noninformative) in this situation is given by $f(\theta) \propto 1 / \theta, \theta=1,2,3, \ldots$.

Now, this prior density is problematic because it is improper (since $\left.\sum_{\theta=1}^{\infty} 1 / \theta=\infty\right)$. However, it leads to a proper posterior density given by

$$
f(\theta \mid y)=\frac{1}{c \theta^{2}}, \theta=3,4,5, \ldots,
$$

where $c=\frac{1}{3^{2}}+\frac{1}{4^{2}}+\frac{1}{5^{2}}+\ldots=\frac{\pi^{2}}{6}-\left(\frac{1}{1^{2}}+\frac{1}{2^{2}}\right)=0.394934$.

So, under this alternative prior, the probability of there being at least four buses in the town (given that you have seen Bus 3) works out as

$$
P(\theta \geq 4 \mid y)=1-P(\theta=3 \mid y)=1-\frac{1}{9 c}=0.7187 \text {. }
$$

The logic which Jeffreys used to come up with the prior $f(\theta) \propto 1 / \theta$ in relation to the tramcar problem will be discussed further in Chapter 2 .

\section{R Code for Exercise I.6}

options(digits=6); c=(1/6)*(pi^2)-5/4; c \# 0.394934

$1-\left(1 / 3^{\wedge} 2\right) / c$ \# 0.718659

\section{Exercise I.7 Balls in a box}

In each of nine indistinguishable boxes there are nine balls, the $i$ th box having $i$ red balls and $9-i$ white balls $(i=1, \ldots, 9)$.

One box is selected randomly from the nine, and then three balls are chosen randomly from the selected box (without replacement and without looking at the remaining balls in the box).

Exactly two of the three chosen balls are red. Find the probability that the selected box has at least four red balls remaining in it. 


\section{Solution to Exercise I.7}

Let: $\quad N=$ the number of balls in each box (9)

$n=$ the number of balls chosen from the selected box (3)

$\theta=$ the number of red balls initially in the selected box

$(1,2, \ldots, 8$ or 9$)$

$y=$ the number of red balls amongst the $n$ chosen balls (2).

Then an appropriate Bayesian model is:

$(y \mid \theta) \sim \operatorname{Hyp}(N, \theta, n) \quad$ (Hypergeometric with parameters

$N, \theta$ and $n$, and having mean $n \theta / N$ )

$\theta \sim D U(1, \ldots, N) \quad$ (discrete uniform over the integers $1,2, \ldots, N$ ).

For this model, the posterior density of $\theta$ is

$$
\begin{aligned}
f(\theta \mid y) & \propto f(\theta) f(y \mid \theta)=\frac{1}{N} \times\left(\begin{array}{l}
\theta \\
y
\end{array}\right)\left(\begin{array}{c}
N-\theta \\
n-y
\end{array}\right) /\left(\begin{array}{c}
N \\
n
\end{array}\right) \\
& \propto \frac{\theta !(N-\theta) !}{(\theta-y) !(N-\theta-(n-y)) !}, \quad \theta=y, \ldots, N-(n-y) .
\end{aligned}
$$

In our case,

$$
f(\theta \mid y) \propto \frac{\theta !(9-\theta) !}{(\theta-2) !(9-\theta-(3-2)) !}, \theta=2, \ldots, 9-(3-2),
$$

or more simply,

$$
f(\theta \mid y) \propto \theta(\theta-1)(9-\theta), \quad \theta=2, \ldots, 8 .
$$

Thus $f(\theta \mid y) \propto\left\{\begin{array}{l}14, \theta=2 \\ 36, \theta=3 \\ 60, \theta=4 \\ 80, \theta=5 \\ 90, \theta=6 \\ 84, \theta=7 \\ 56, \theta=8\end{array}\right\} \equiv k(\theta)$,

where

$$
C \equiv \sum_{\theta=1}^{8} k(\theta)=14+36+\ldots+56=420 .
$$


So $f(\theta \mid y)=\frac{k(\theta)}{c}=\left\{\begin{array}{l}14 / 420=0.03333, \theta=2 \\ 36 / 420=0.08571, \theta=3 \\ 60 / 420=0.14286, \theta=4 \\ 80 / 420=0.19048, \theta=5 \\ 90 / 420=0.21429, \theta=6 \\ 84 / 420=0.20000, \theta=7 \\ 56 / 420=0.13333, \theta=8 .\end{array}\right.$

The probability that the selected box has at least four red balls remaining is the posterior probability that $\theta$ (the number of red balls initially in the box) is at least 6 (since two red balls have already been taken out of the box). So the required probability is

$$
P(\theta \geq 6 \mid y)=\frac{90+84+56}{420}=\frac{23}{42}=0.5476 \text {. }
$$

\section{R Code for Exercise I.7}

$\mathrm{tv}=2: 8 ; \mathrm{kv}=\mathrm{tv} *(\mathrm{tv}-1) *(9-\mathrm{tv}) ; \mathrm{c}=\mathrm{sum}(\mathrm{kv}) ; \mathrm{c} \# 420$

options(digits=4); cbind(tv,kv,kv/c,cumsum(kv/c))

\#[1,] $2 \quad 140.03333 \quad 0.03333$

\#[2,] $3 \quad 36 \quad 0.08571 \quad 0.11905$

\#[3,] $4 \quad 60 \quad 0.14286 \quad 0.26190$

\#[4,] $5 \quad 80 \quad 0.19048 \quad 0.45238$

\#[5,] $\begin{array}{llll}6 & 90 & 0.21429 & 0.66667\end{array}$

\#[6,] $7 \quad 84 \quad 0.20000 \quad 0.86667$

\#[7,] $8 \quad 56 \quad 0.13333 \quad 1.00000$

$23 / 42 \# 0.5476$

1-0.45238 \# 0.5476 (alternative calculation of the required probability) $\operatorname{sum}((\mathrm{kv} / \mathrm{c})[\mathrm{tv}>=6]) \# 0.5476$

\# (yet another calculation of the required probability)

\section{I.7 Continuous parameters}

The examples above have all featured a target parameter which is discrete. The following example illustrates Bayesian inference involving a continuous parameter. This case presents no new problems, except that the prior and posterior densities of the parameter may no longer be interpreted directly as probabilities. 


\section{Exercise I.8 The binomial-beta model (or beta-binomial model)}

Consider the following Bayesian model:

$$
\begin{aligned}
& (y \mid \theta) \sim \operatorname{Binomial}(n, \theta) \\
& \theta \sim \operatorname{Beta}(\alpha, \beta) \quad \text { (prior). }
\end{aligned}
$$

Find the posterior distribution of $\theta$.

\section{Solution to Exercise I.8}

The posterior density is

$$
\begin{aligned}
f(\theta \mid y) & \propto f(\theta) f(y \mid \theta) \\
& =\frac{\theta^{\alpha-1}(1-\theta)^{\beta-1}}{B(\alpha, \beta)} \times\left(\begin{array}{l}
n \\
y
\end{array}\right) \theta^{y}(1-\theta)^{n-y} \\
& \propto \theta^{\alpha-1}(1-\theta)^{\beta-1} \times \theta^{y}(1-\theta)^{n-y} \quad \text { ignoring constants which } \\
& =\theta^{(\alpha+y)-1}(1-\theta)^{(\beta+n-y)-1}, 0<\theta<1 .
\end{aligned}
$$

This is the kernel of the beta density with parameters $\alpha+y$ and $\beta+n-y$. It follows that the posterior distribution of $\theta$ is given by

$$
(\theta \mid y) \sim \operatorname{Beta}(\alpha+y, \beta+n-y),
$$

and the posterior density of $\theta$ is (exactly)

$$
f(\theta \mid y)=\frac{\theta^{(\alpha+y)-1}(1-\theta)^{(\beta+n-y)-1}}{B(\alpha+y, \beta+n-y)}, 0<\theta<1 .
$$

For example, suppose that $\alpha=\beta=1$, that is, $\theta \sim \operatorname{Beta}(1,1)$.

Then the prior density is $f(\theta)=\frac{\theta^{1-1}(1-\theta)^{1-1}}{B(1,1)}=1,0<\theta<1$.

Thus the prior may also be expressed by writing $\theta \sim U(0,1)$.

Also, suppose that $n=2$. Then there are three possible values of $y$, namely 0,1 and 2, and these lead to the following three posteriors, respectively:

$$
\begin{aligned}
& (\theta \mid y) \sim \operatorname{Beta}(1+0,1+2-0)=\operatorname{Beta}(1,3) \\
& (\theta \mid y) \sim \operatorname{Beta}(1+1,1+2-1)=\operatorname{Beta}(2,2) \\
& (\theta \mid y) \sim \operatorname{Beta}(1+2,1+2-2)=\operatorname{Beta}(3,1) .
\end{aligned}
$$

These three posteriors and the prior are illustrated in Figure 1.5. 
Note: The prior here may be considered uninformative because it is 'flat' over the entire range of possible values for $\theta$, namely 0 to 1 . This prior was originally used by Thomas Bayes and is often called the Bayes prior. However, other uninformative priors have been proposed for the binomial parameter $\theta$. These will be discussed later, in Chapter 2 .

Figure I.5 The prior and three posteriors in Exercise I.8

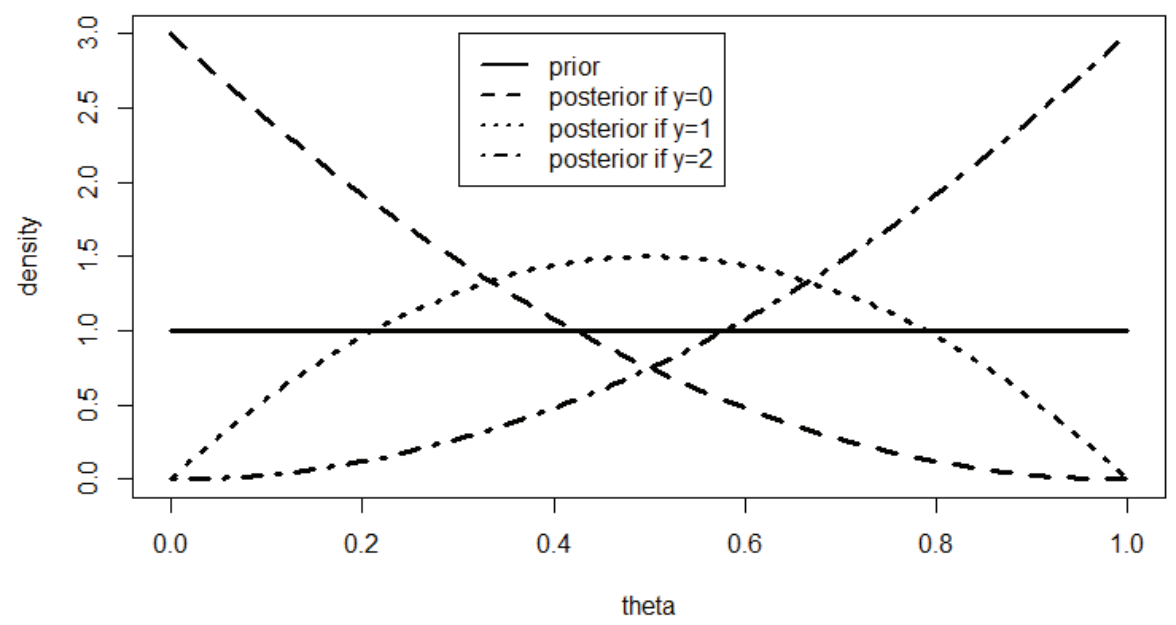

R Code for Exercise I.8

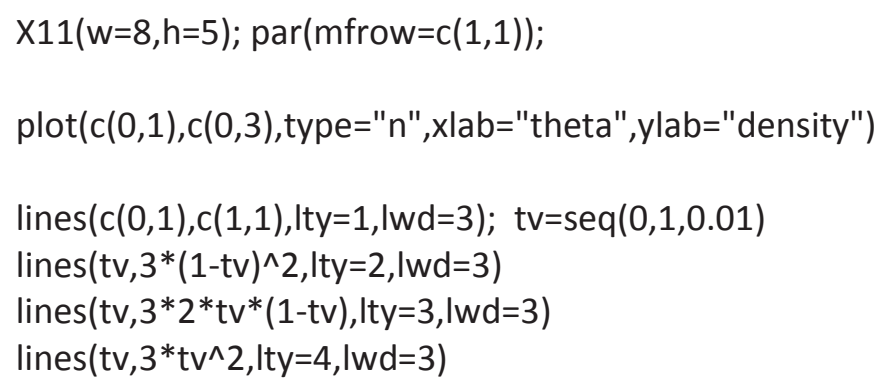




\section{I.8 Finite and infinite population inference}

In the last example (Exercise 1.8), with the model:

$$
\begin{aligned}
& (y \mid \theta) \sim \operatorname{Binomial}(n, \theta) \\
& \theta \sim \operatorname{Beta}(\alpha, \beta),
\end{aligned}
$$

the quantity of interest $\theta$ is the probability of success on a single Bernoulli trial.

This quantity may be thought of as the average of a hypothetically infinite number of Bernoulli trials. For that reason we may refer to derivation of the posterior distribution,

$$
(\theta \mid y) \sim \operatorname{Beta}(\alpha+y, \beta+n-y),
$$

as infinite population inference.

In contrast, for the 'buses' example further above (Exercise 1.6), which involves the model:

$$
\begin{aligned}
& f(y \mid \theta)=1 / \theta, y=1, \ldots, \theta \\
& f(\theta)=1 / 5, \theta=1, \ldots, 5,
\end{aligned}
$$

the quantity of interest $\theta$ represents the number of buses in a population of buses, which of course is finite.

Therefore derivation of the posterior,

$$
f(\theta \mid y)=\left\{\begin{array}{l}
20 / 47, \theta=3 \\
15 / 47, \theta=4 \\
12 / 47, \theta=5
\end{array}\right.
$$

may be termed finite population inference.

Another example of finite population inference is the 'balls in a box' example (Exercise 1.7), where the model is:

$$
\begin{aligned}
& (y \mid \theta) \sim \operatorname{Hyp}(N, \theta, n) \\
& \theta \sim \operatorname{DU}(1, \ldots, N),
\end{aligned}
$$

and where the quantity of interest $\theta$ is the number of red balls initially in the selected box $(1,2, \ldots, 8$ or 9$)$.

And another example of infinite population inference is the 'loaded dice' example (Exercises 1.4 and 1.5), where the model is:

$$
\begin{aligned}
& f(y \mid \theta)=\left(\begin{array}{l}
2 \\
y
\end{array}\right) \theta^{y}(1-\theta)^{2-y}, y=0,1,2 \\
& f(\theta)=10 \theta / 6, \theta=0.1,0.2,0.3,
\end{aligned}
$$


and where the quantity of interest $\theta$ is the probability of 6 coming up on a single roll of the chosen die (i.e. the average number of $6 \mathrm{~s}$ that come up on a hypothetically infinite number of rolls of that particular die).

Generally, finite population inference may also be thought of in terms of prediction (e.g. in the 'buses' example, we are predicting the total number of buses in the town). For that reason, finite population inference may also be referred to as predictive inference. Yet another term for finite population inference is descriptive inference. In contrast, infinite population inference may also be called analytic inference. More will be said on finite population/predictive/descriptive inference in later chapters of the course.

\section{I.9 Continuous data}

So far, all the Bayesian models considered have featured data which is modelled using a discrete distribution. (Some of these models have a discrete parameter and some have a continuous parameter.) The following is an example with data that follows a continuous probability distribution. (This example also has a continuous parameter.)

\section{Exercise I.9 The exponential-exponential model}

Suppose $\theta$ has the standard exponential distribution, and the conditional distribution of $y$ given $\theta$ is exponential with mean $1 / \theta$. Find the posterior density of $\theta$ given $y$.

\section{Solution to Exercise I.9}

The Bayesian model here is: $f(y \mid \theta)=\theta e^{-\theta y}, y>0$

$$
f(\theta)=e^{-\theta}, \theta>0 \text {. }
$$

So $f(\theta \mid y) \propto f(\theta) f(y \mid \theta) \propto e^{-\theta} \times \theta e^{-\theta y}=\theta^{2-1} e^{-\theta(y+1)}, y>0$.

This is the kernel of a gamma distribution with parameters 2 and $y+1$, as per the definitions in Appendix B.2. Thus we may write

$$
(\theta \mid y) \sim \operatorname{Gamma}(2, y+1),
$$

from which it follows that the posterior density of $\theta$ is

$$
f(\theta \mid y)=\frac{(y+1)^{2} \theta^{2-1} e^{-\theta(y+1)}}{\Gamma(2)}, \theta>0 .
$$




\section{Exercise I.I 0 The uniform-uniform model}

Consider the Bayesian model given by:

$$
\begin{aligned}
& (y \mid \theta) \sim U(0, \theta) \\
& \theta \sim U(0,1) .
\end{aligned}
$$

Find the posterior density of $\theta$ given $y$.

\section{Solution to Exercise I.I0}

Noting that $0<y<\theta<1$, we see that the posterior density is

$$
\begin{aligned}
f(\theta \mid y)=\frac{f(\theta) f(y \mid \theta)}{f(y)} & =\frac{1 \times(1 / \theta)}{\int_{y}^{1} 1 \times(1 / \theta) d \theta} \\
& =\frac{1 / \theta}{\log 1-\log y}=\frac{-1}{\theta \log y}, y<\theta<1 .
\end{aligned}
$$

Note: This is a 'non-standard' density and strictly decreasing. To give a physical example, a stick of length 1 metre is cut at a point randomly located along its length. The part to the right of the cut is discarded and then another cut is made randomly along the stick which remains. Then the part to the right of that second cut is likewise discarded. The length of the stick remaining after the first cut is a random variable with density as given above, with $y$ being the length of the finally remaining stick.

\section{I 0 Conjugacy}

When the prior and posterior distributions are members of the same class of distributions, we say that they form a conjugate pair, or that the prior is conjugate. For example, consider the binomial-beta model:

$$
\begin{array}{rlrl} 
& (y \mid \theta) \sim \operatorname{Binomial}(n, \theta) & \\
& \theta \sim \operatorname{Beta}(\alpha, \beta) & & \text { (prior) } \\
\Rightarrow & (\theta \mid y) \sim \operatorname{Beta}(\alpha+y, \beta+n-y) & & \text { (posterior). }
\end{array}
$$

Since both prior and posterior are beta, the prior is conjugate.

Likewise, consider the exponential-exponential model:

$$
\begin{array}{rll} 
& f(y \mid \theta)=\theta e^{-\theta y}, y>0 \\
& f(\theta)=e^{-\theta}, \theta>0 \quad(\text { i.e. } \theta \sim \operatorname{Gamma}(1,1)) & \\
\Rightarrow & (\theta \mid y) \sim \operatorname{Gamma}(2, y+1) & \text { (posterior) }
\end{array}
$$


Since both prior and posterior are gamma, the prior is conjugate.

On the other hand, consider the model in the buses example:

$$
\begin{gathered}
(y \mid \theta) \sim D U(1, \ldots, \theta) \\
\theta \sim D U(1, \ldots, 5) \\
\Rightarrow \quad f(\theta \mid y=3)=\left\{\begin{array}{l}
20 / 47, \theta=3 \\
15 / 47, \theta=4 \\
12 / 47, \theta=5
\end{array} \quad\right. \text { (prior) }
\end{gathered}
$$

The prior is discrete uniform but the posterior is not. So in this case the prior is not conjugate.

Specifying a Bayesian model using a conjugate prior is generally desirable because it can simplify the calculations required.

\section{I.I I Bayesian point estimation}

Once the posterior distribution or density $f(\theta \mid y)$ has been obtained, Bayesian point estimates of the model parameter $\theta$ can be calculated. The three most commonly used point estimates are as follows.

- The posterior mean of $\theta$ is

$$
E(\theta \mid y)=\int \theta d F(\theta \mid y)=\left\{\begin{array}{cl}
\int \theta f(\theta \mid y) d \theta & \text { if } \theta \text { is continuous } \\
\sum_{\theta} \theta f(\theta \mid y) & \text { if } \theta \text { is discrete. }
\end{array}\right.
$$

- The posterior mode of $\theta$ is

$\operatorname{Mode}(\theta \mid y)=$ any value $m \in \mathfrak{R}$ which satisfies

$$
\begin{aligned}
& \qquad f(\theta=m \mid x)=\max _{\theta} f(\theta \mid x) \\
& \text { or } \lim _{\theta \rightarrow m} f(\theta \mid x)=\sup f(\theta \mid x), \\
& \text { or the set of all such values. }
\end{aligned}
$$

- The posterior median of $\theta$ is

$\operatorname{Median}(\theta \mid y)=$ any value $m$ of $\theta$ such that

$$
P(\theta \leq m \mid y) \geq 1 / 2
$$

and $P(\theta \geq m \mid y) \geq 1 / 2$,

or the set of all such values.

Note 1: In some cases, the posterior mean does not exist or it is equal to infinity or minus infinity. 
Note 2: Typically, the posterior mode and posterior median are unique. The above definitions are given for completeness.

Note 3: The integral $\int \theta d F(\theta \mid y)$ is a Lebesgue-Stieltje's integral. This may need to be evaluated as the sum of two separate parts in the case where $\theta$ has a mixed distribution. In the continuous case, it is useful to think of $d F(\theta \mid y)$ as $\frac{d F(\theta \mid y)}{d \theta} d \theta=f(\theta \mid y) d \theta$.

Note 4: The above three Bayesian point estimates may be interpreted in an intuitive manner. For example, $\theta$ 's posterior mode is the value of $\theta$ which is 'made most likely by the data'. They may also be understood in the context of Bayesian decision theory (discussed later).

\section{I.I 2 Bayesian interval estimation}

There are many ways to construct a Bayesian interval estimate, but the two most common ways are defined as follows. The $1-\alpha$ (or $100(1-\alpha) \%$ ) highest posterior density region (HPDR) for $\theta$ is the smallest set $S$ such that:

$$
\begin{aligned}
& \quad P(\theta \in S \mid y) \geq 1-\alpha \\
& \text { and } f\left(\theta_{1} \mid y\right) \geq f\left(\theta_{2} \mid y\right) \text { if } \theta_{1} \in S \text { and } \theta_{2} \notin S .
\end{aligned}
$$

Figure 1.6 illustrates the idea of the HPDR. In the very common situation where $\theta$ is scalar, continuous and has a posterior density which is unimodal with no local modes (i.e. has the form of a single 'mound'), the 1- $\alpha$ HPDR takes on the form of a single interval defined by two points at which the posterior density has the same value. When the HPDR is a single interval, it is the shortest possible single interval over which the area under the posterior density is $1-\alpha$.

The 1- $\alpha$ central posterior density region (CPDR) for a scalar parameter $\theta$ may be defined as the shortest single interval $[a, b]$ such that:

$$
\begin{aligned}
& P(\theta<a \mid y) \leq \alpha / 2 \\
\text { and } \quad & P(\theta>b \mid y) \leq \alpha / 2 .
\end{aligned}
$$




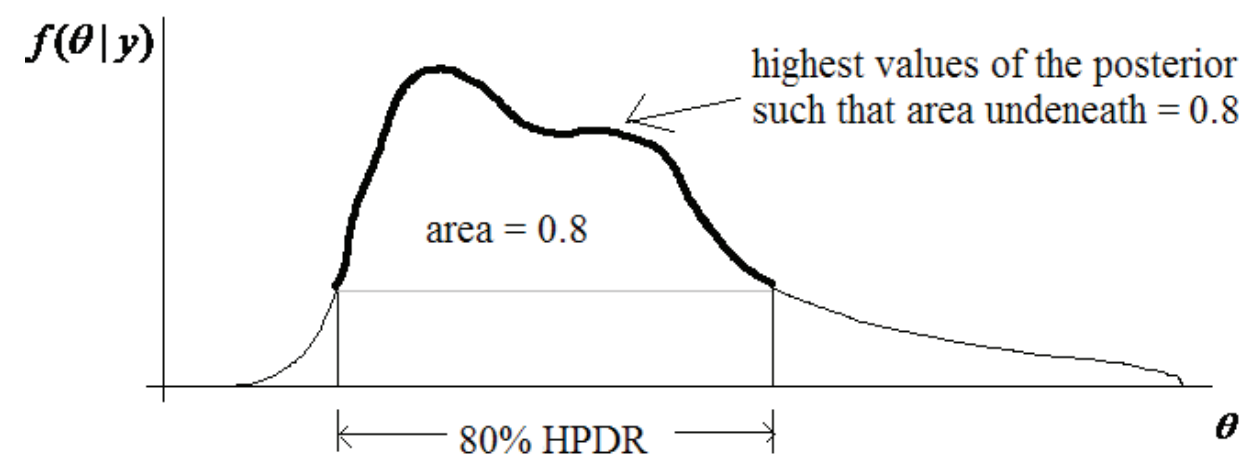

Figure 1.7 illustrates the idea of the CPDR. One drawback of the CPDR is that it is only defined for a scalar parameter. Another drawback is that some values inside the CPDR may be less likely a posteriori than some values outside it (which is not the case with the HPDR). For example, in Figure 1.7, a value just below the upper bound of the $80 \%$ CPDR has a smaller posterior density than a value just below the lower bound of that CPDR. However, CPDRs are typically easier to calculate than HPDRs.

In the common case of a continuous parameter with a posterior density in the form of a single 'mound' which is furthermore symmetric, the CPDR and HPDR are identical.

Note 1: The $1-\alpha$ CPDR for $\theta$ may alternatively be defined as the shortest single open interval $(a, b)$ such that:

$$
P(\theta \leq a \mid y) \leq \alpha / 2
$$

and $P(\theta \geq b \mid y) \leq \alpha / 2$.

Other variations are possible (of the form $[a, b)$ and $(a, b])$; but when the parameter of interest $\theta$ is continuous these definitions are all equivalent. Yet another definition of the $1-\alpha$ CPDR is any of the CPDRs as defined above but with all $a$ posteriori impossible values of $\theta$ excluded.

Note 2: As regards terminology, whenever the HPDR is a single interval, it may also be called the highest posterior density interval (HPDI). Likewise, the CPDR, which is always a single interval, may also be called the central posterior density interval (CPDI). 


\section{Figure I.7 An 80\% CPDR}

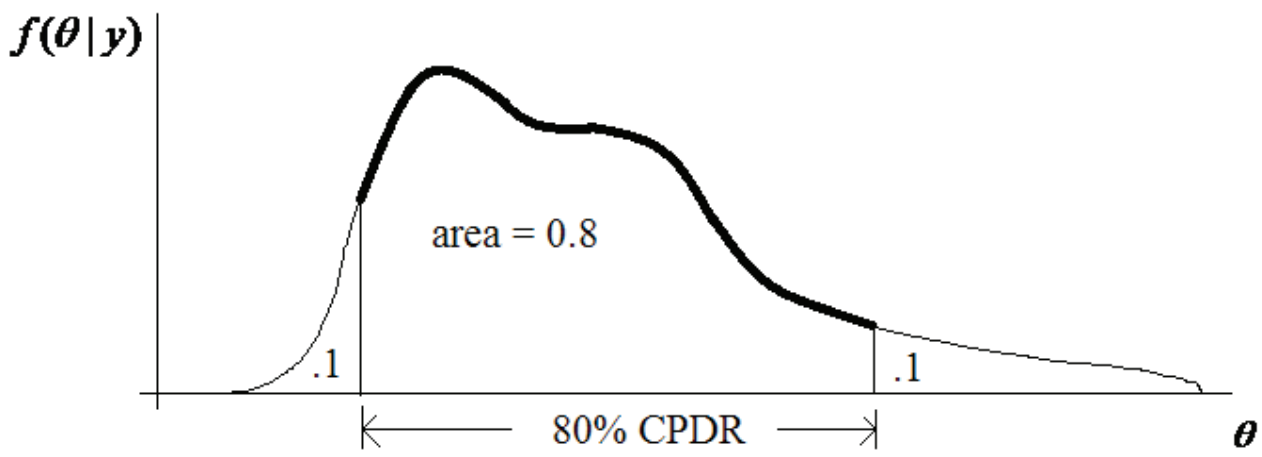

\section{Exercise I.I I A bent coin}

We have a bent coin, for which $\theta$, the probability of heads coming up, is unknown. Our prior beliefs regarding $\theta$ may be described by a standard uniform distribution. Thus no value of $\theta$ is deemed more or less likely than any other.

We toss the coin $n=5$ times (independently), and heads come up every time.

Find the posterior mean, mode and median of $\theta$. Also find the $80 \%$ HPDR and CPDR for $\theta$.

\section{Solution to Exercise I.I I}

Recall the binomial-beta model:

$$
\begin{aligned}
& (y \mid \theta) \sim \operatorname{Binomial}(n, \theta) \\
& \theta \sim \operatorname{Beta}(\alpha, \beta),
\end{aligned}
$$

for which $(\theta \mid y) \sim \operatorname{Beta}(\alpha+y, \beta+n-y)$.

We now apply this result with $n=y=5$ and $\alpha=\beta=1$ (corresponding to $\theta \sim U(0,1))$, and find that:

$$
\begin{aligned}
& (\theta \mid y) \sim \operatorname{Beta}(1+5,5-5+1)=\operatorname{Beta}(6,1) \\
& f(\theta \mid y)=\frac{\theta^{6-1}(1-\theta)^{1-1}}{B(6,1)}=6 \theta^{5}, \quad 0<\theta<1 \\
& F(\theta \mid y)=\int_{0}^{\theta} 6 t^{5} d t=\theta^{6}, \quad 0<\theta<1 .
\end{aligned}
$$


Therefore: $\quad E(\theta \mid y)=\frac{6}{6+1}=\frac{6}{7}=0.8571$

$$
\begin{aligned}
\operatorname{Mode}(\theta \mid y) & =\frac{6-1}{(6-1)+(1-1)}=1 \\
\operatorname{Median}(\theta \mid y) & =\text { solution in } \theta \text { of } F(\theta \mid y)=1 / 2, \text { i.e. } \theta^{6}=0.5 \\
& =(0.5)^{1 / 6}=0.8909 .
\end{aligned}
$$

Also, the $80 \%$ HPDR is $\left(0.2^{1 / 6}, 1\right)=(0.7647,1)$ (since $f(\theta \mid y)$ is strictly increasing), and the $80 \%$ CPDR is $\left(0.1^{1 / 6}, 0.9^{1 / 6}\right)=(0.6813,0.9826)$. The three point estimate and two interval estimates just derived are shown in Figure 1.8.

\section{Figure I.8 Inference in Exercise I.I I}

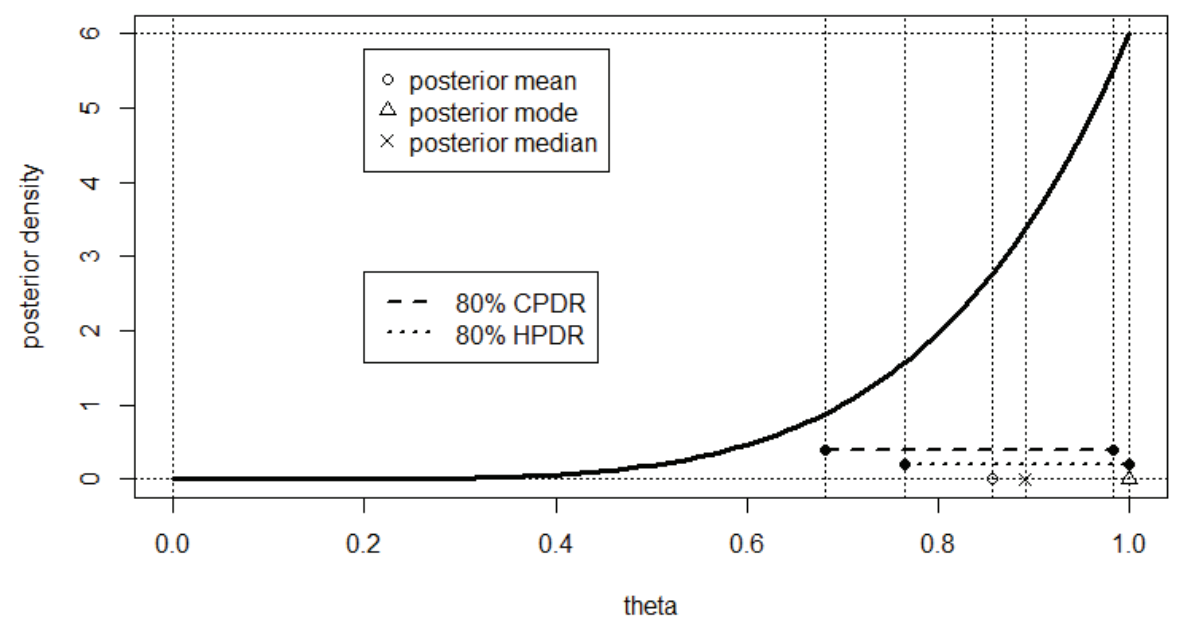

\section{R Code for Exercise I.I I}

options(digits=4); postmean=6/7; postmode $=1$; postmedian $=0.5^{\wedge}(1 / 6)$ c(postmean, postmode, postmedian) \# 0.85711 .00000 .8909 $\mathrm{hpdr}=\mathrm{c}\left(0.2^{\wedge}(1 / 6), 1\right) ; \mathrm{cpdr}=\mathrm{c}(0.1,0.9)^{\wedge}(1 / 6)$ c(hpdr,cpdr) \# 0.76471 .00000 .68130 .9826

$\mathrm{X} 11(\mathrm{w}=8, \mathrm{~h}=5) ; \operatorname{par}(\operatorname{mfrow}=\mathrm{c}(1,1)) ; \mathrm{tv}=\mathrm{seq}(0,1,0.01) ; \mathrm{fv}=\mathrm{dbeta}(\mathrm{tv}, 6,1)$ plot(tv,fv,type="l",Iwd=3,xlab="theta",ylab="posterior density") points(c(postmean, postmode, postmedian), $c(0,0,0), p c h=c(1,2,4))$ points(hpdr, rep(0.2,2), $p c h=16)$; lines(hpdr, rep(0.2,2), Ity=3, Iwd=2) 
points(cpdr, rep(0.4,2), pch=16); lines(cpdr, $\operatorname{rep}(0.4,2)$, Ity=2, Iwd=2)

abline $(v=c($ postmean, postmode, postmedian), Ity=3)

abline $(v=c(0, h p d r, c p d r)$, Ity=3); abline $(h=c(0,6)$, Ity=3)

legend(0.2,5.8,c("posterior mean","posterior mode", "posterior median"), $\mathrm{pch}=\mathrm{c}(1,2,4))$

legend(0.2,2.8,c("80\% CPDR","80\% HPDR"), Ity=c(2,3), Iwd=c(2,2))

\section{Exercise I.I 2 HPDR and CPDR for a discrete parameter}

Consider the posterior distribution from Exercise 1.7 (Balls in a box):

$$
f(\theta \mid y)=\left\{\begin{array}{l}
14 / 420=0.03333, \theta=2 \\
36 / 420=0.08571, \theta=3 \\
60 / 420=0.14286, \theta=4 \\
80 / 420=0.19048, \theta=5 \\
90 / 420=0.21429, \theta=6 \\
84 / 420=0.20000, \theta=7 \\
56 / 420=0.13333, \theta=8 .
\end{array}\right.
$$

Find the 90\% HPDR and 90\% CPDR for $\theta$. Also find the 50\% HPDR and $50 \%$ CPDR for $\theta$. For each region, calculate the associated exact coverage probability.

\section{Solution to Exercise I.I2}

The $90 \%$ HPDR is the set $\{3,4,5,6,7,8\}$;

this has exact coverage $1-14 / 420=0.9667$.

The 90\% CPDR is the closed interval [3, 8];

this likewise has exact coverage 0.9667 .

The $50 \%$ HPDR is $\{5,6,7\}$;

this has exact coverage $(80+90+84) / 420=0.6047$.

The $50 \%$ CPDR is $[4,7]$;

this has exact coverage $(60+80+90+84) / 420=0.7476$.

Note: The lower bound of the $50 \%$ CPDR cannot be equal to 5 . This is because $P(\theta<5 \mid y)=(14+36+60) / 420=0.2619$, which is not less than or equal to $\alpha / 2=0.25$, as required by the definition of CPDR. 


\section{Exercise I.I 3 Illustration of the definition of HPDR}

Suppose that the posterior probabilities of a parameter $\theta$ given data $y$ are exactly $10 \%, 40 \%$ and $50 \%$ for values 1, 2 and 3, respectively. Find $S$, the $40 \%$ HPDR for $\theta$.

\section{Solution to Exercise I.13}

The smallest set $S$ such that $P(\theta \in S \mid y) \geq 0.4$ is $\{2\}$ or $\{3\}$. With the additional requirement that $f\left(\theta_{1} \mid y\right) \geq f\left(\theta_{2} \mid y\right)$ if $\theta_{1} \in S$ and $\theta_{2} \notin S$, we see that $S=\{3\}$ (only). That is, the $40 \%$ HPDR is the singleton set $\{3\}$.

\section{I.I 3 Inference on functions of the model parameter}

So far we have examined Bayesian models with a single parameter $\theta$ and described how to perform posterior inference on that parameter. Sometimes there may also be interest in some function of the model parameter, denoted by (say)

$$
\psi=g(\theta) \text {. }
$$

Then the posterior density of $\psi$ can be derived using distribution theory, for example by applying the transformation rule,

$$
f(\psi \mid y)=f(\theta \mid y)\left|\frac{d \theta}{d \psi}\right|,
$$

in cases where $\psi=g(\theta)$ is strictly increasing or strictly decreasing.

Point and interval estimates of $\psi$ can then be calculated in the usual way, using $f(\psi \mid y)$. For example, the posterior mean of $\psi$ equals

$$
E(\psi \mid y)=\int \psi f(\psi \mid y) d \psi .
$$

Sometimes it is more practical to calculate point and interval estimates another way, without first deriving $f(\psi \mid y)$.

For example, another expression for the posterior mean is

$$
E(\psi \mid y)=E(g(\theta) \mid y)=\int g(\theta) f(\theta \mid y) d \theta .
$$


Also, the posterior median of $\psi$, call this $M$, can typically be obtained by simply calculating

$$
M=g(m),
$$

where $m$ is the posterior median of $\theta$.

Note: To see why this works, we write

$$
\begin{aligned}
P(\psi<M \mid y) & =P(g(\theta)<M \mid y) \\
& =P(g(\theta)<g(m) \mid y)=P(\theta<m \mid y)=1 / 2 .
\end{aligned}
$$

\section{Exercise I.I4 Estimation of an exponential mean}

Suppose that $\theta$ has the standard exponential distribution, and $y$ given $\theta$ is exponential with mean $1 / \theta$. Find the posterior density and posterior mean of the model mean, $\psi=E(y \mid \theta)=1 / \theta$, given the data $y$.

\section{Solution to Exercise I.I4}

Recall that the Bayesian model

$$
\begin{aligned}
& f(y \mid \theta)=\theta e^{-\theta y}, y>0 \\
& f(\theta)=e^{-\theta}, \theta>0
\end{aligned}
$$

implies the posterior $(\theta \mid y) \sim \operatorname{Gamma}(2, y+1)$.

So, by definition, $(\psi \mid y) \sim$ InverseGamma $(2, y+1)$,

with density $f(\psi \mid y)=\frac{(y+1)^{2} \psi^{-(2+1)} e^{-(y+1) / \psi}}{\Gamma(2)}=\frac{(y+1)^{2}}{\psi^{3} e^{(y+1) / \psi}}, \psi>0$,

and mean $E(\psi \mid y)=\frac{y+1}{2-1}=y+1$.

Note: This mean could also be obtained as follows:

$$
\begin{aligned}
E(\psi \mid y) & =E\left(\frac{1}{\theta} \mid y\right)=\int_{0}^{\infty} \frac{1}{\theta} f(\theta \mid y) d \theta \\
& =\int_{0}^{\infty} \frac{1}{\theta} \times \frac{(y+1)^{2} \theta^{2-1} e^{-\theta(y+1)}}{\Gamma(2)} d \theta \\
& =\frac{\Gamma(1)(y+1)^{2}}{\Gamma(2)(y+1)^{1}} \int_{0}^{\infty} \frac{1}{\theta} \times \frac{(y+1)^{1} \theta^{1-1} e^{-\theta(y+1)}}{\Gamma(1)} d \theta \\
& =y+1 \quad \text { (using the fact that the last integral equals } 1) .
\end{aligned}
$$




\section{Exercise I.I5 Inference on a function of the binomial parameter}

Recall the binomial-beta model given by:

$$
\begin{aligned}
& (y \mid \theta) \sim \operatorname{Binomial}(n, \theta) \\
& \theta \sim \operatorname{Beta}(\alpha, \beta),
\end{aligned}
$$

for which $(\theta \mid y) \sim \operatorname{Beta}(\alpha+y, \beta+n-y)$.

Find the posterior mean, density function and distribution function of $\psi=\theta^{2}$ in the case where $n=5, y=5$, and $\alpha=\beta=1$.

Note: In the context where we toss a bent coin five times and get heads every time (and the prior on the probability of heads is standard uniform), the quantity $\psi$ may be interpreted as the probability of the next two tosses both coming up heads, or equivalently, as the proportion of times heads will come up twice if the coin is repeatedly tossed in groups of two tosses a hypothetically infinite number of times.

\section{Solution to Exercise I.I5}

Here, $(\theta \mid y) \sim \operatorname{Beta}(1+5,1+5-5) \sim \operatorname{Beta}(6,1)$

with pdf $f(\theta \mid y)=6 \theta^{5}, 0<\theta<1$.

Now $\theta=\psi^{1 / 2}$ and so, by the transformation method, the posterior density function of $\psi$ is

$$
f(\psi \mid y)=f(\theta \mid y)\left|\frac{d \theta}{d \psi}\right|=6 \psi^{5 / 2}\left|-\frac{1}{2} \psi^{-\frac{1}{2}}\right|=3 \psi^{2}, 0<\psi<1 .
$$

It follows that the posterior mean of $\psi$ is

$$
\hat{\psi}=E(\psi \mid y)=\int_{0}^{1} \psi\left(3 \psi^{2}\right) d \psi=0.75,
$$

and the posterior distribution function of $\psi$ is

$$
F(\psi \mid y)=\int_{0}^{\psi} f(\psi=t \mid y) d t=\int_{0}^{\psi} 3 t^{2} d t=\psi^{3}, 0<\psi<1 .
$$


Note 1: The posterior mean of $\psi=\theta^{2}$ can also be obtained by writing

$$
\hat{\psi}=E\left(\theta^{2} \mid y\right)=\int_{0}^{1} \theta^{2}\left(6 \theta^{5}\right) d \theta=0.75
$$

or

$$
\hat{\psi}=E\left(\theta^{2} \mid y\right)=V(\theta \mid y)+\{E(\theta \mid y)\}^{2}
$$

$$
=\frac{6 \times 1}{(6+1)^{2}(6+1+1)}+\left(\frac{6}{6+1}\right)^{2}=0.75
$$

or

$$
(\psi \mid y) \sim \operatorname{Beta}(3,1) \Rightarrow \hat{\psi}=E(\psi \mid y)=3 /(3+1)=0.75 \text {. }
$$

Note 2: The distribution function of $\psi=\theta^{2}$ can also be obtained by writing

$$
\begin{aligned}
F(\psi=v \mid y) & =P(\psi \leq v \mid y)=P\left(\theta^{2} \leq v \mid y\right)=P\left(\theta \leq v^{1 / 2} \mid y\right) \\
& =F\left(\theta=v^{1 / 2} \mid y\right)=\left[\left.\theta^{6}\right|_{\theta=v^{1 / 2}}\right]=v^{3}, 0<v<1 .
\end{aligned}
$$

Note 3: In the above, $f(\psi=t \mid y)$ denotes the pdf of $\psi$ given $y$, but evaluated at $t$. This pdf could also be written as $f_{\psi}(t \mid y)$ or as $\left[\left.f(\psi \mid y)\right|_{\psi=t}\right]$. Likewise, $F(\psi=v \mid y) \equiv F_{\psi}(v \mid y) \equiv\left[\left.F(\psi \mid y)\right|_{\psi=v}\right]$.

\section{I.I 4 Credibility estimates}

In actuarial studies, a credibility estimate is one which can be expressed as a weighted average of the form

$$
C=(1-k) A+k B \text {, }
$$

where:

A is the subjective estimate (or the collateral data estimate)

$B \quad$ is the objective estimate (or the direct data estimate)

$k \quad$ is the credibility factor, a number that is between 0 and 1 (inclusive) and represents the weight assigned to the objective estimate.

A high value of $k$ implies $C \cong B$, representing a situation where the objective estimate is assigned 'high credibility'. A primary aim of credibility theory is to determine an appropriate value or formula for $k$, as is done, for example, in the theory of the Bühlmann model (Bühlmann, 1967). Many Bayesian models lead to a point estimate which can be expressed as an intuitively appealing credibility estimate. 


\section{Exercise I.16 Credibility estimation in the binomial-beta model}

Consider the binomial-beta model: $\quad(y \mid \theta) \sim \operatorname{Binomial}(n, \theta)$

$$
\theta \sim \operatorname{Beta}(\alpha, \beta) \text {. }
$$

Express the posterior mean of $\theta$ as a credibility estimate and discuss.

\section{Solution to Exercise I.16}

Earlier we showed that

$$
(\theta \mid y) \sim \operatorname{Beta}(\alpha+y, \beta+n-y),
$$

and hence that the posterior mean of $\theta$ is

$$
\hat{\theta}=E(\theta \mid y)=\frac{(\alpha+y)}{(\alpha+y)+(\beta+n-y)}=\frac{\alpha+y}{\alpha+\beta+n} .
$$

Observe that the prior mean of $\theta$ is $E \theta=\alpha /(\alpha+\beta)$, and the maximum likelihood estimate (MLE) of $\theta$ is $y / n$. This suggests that we write

$$
\begin{aligned}
\hat{\theta} & =\frac{\alpha}{\alpha+\beta+n}+\frac{y}{\alpha+\beta+n} \\
& =\frac{\alpha}{\alpha+\beta+n}\left(\frac{\alpha+\beta}{\alpha}\right)\left(\frac{\alpha}{\alpha+\beta}\right)+\frac{n}{\alpha+\beta+n}\left(\frac{y}{n}\right) \\
& =\frac{\alpha+\beta}{\alpha+\beta+n}\left(\frac{\alpha}{\alpha+\beta}\right)+\frac{n}{\alpha+\beta+n}\left(\frac{y}{n}\right) .
\end{aligned}
$$

Thus $\hat{\theta}=(1-k) A+k B$

where: $\quad A=\frac{\alpha}{\alpha+\beta}, \quad B=\frac{y}{n}, \quad k=\frac{n}{\alpha+\beta+n}$.

We see that the posterior mean $\hat{\theta}$ is a credibility estimate in the form of a weighted average of the prior mean $A=E \theta=\alpha /(\alpha+\beta)$ and the MLE $B=y / n$, where the weight assigned to the MLE is the credibility factor given by $k=n /(n+\alpha+\beta)$. Observe that as $n$ increases, the credibility factor $k$ approaches 1 . This makes sense: if there is a lot of data then the prior should not have much influence on the estimation.

Figure 1.9 illustrates this idea by showing relevant densities, likelihoods and estimates for the following two cases, respectively: 
(a) $n=5, y=4, \alpha=2, \beta=6$

(b) $n=20, y=16, \alpha=2, \beta=6$.

In both cases, the prior mean is the same $(A=2 /(2+6)=0.25)$, as is the MLE $(B=4 / 5=16 / 20=0.8)$. However, due to $n$ being larger in case (b) (i.e. there being more direct data), case (b) leads to a larger credibility factor (0.714 compared to 0.385 ) and hence a posterior mean closer to the MLE (0.643 compared to 0.462).

Note: Each likelihood function in Figure 1.9 has been normalised so that the area underneath it is exactly 1 . This means that in each case (a) and (b), the likelihood function $L(\theta)$ as shown is identical to the posterior density which would be implied by the standard uniform prior, i.e. under $f_{U(0,1)}(\theta)=f_{\text {Beta }(1,1)}(\theta)$. Thus, $L(\theta)=f_{\text {Beta }(1+y, 1+n-y)}(\theta)$.

\section{Figure I.9 Illustration for Exercise I.16}

Legend: solid line $=$ prior , dashed line $=$ likelihood, dotted line $=$ posterior, circle $=$ prior mean, triangle $=$ MLE, cross $=$ posterior mean
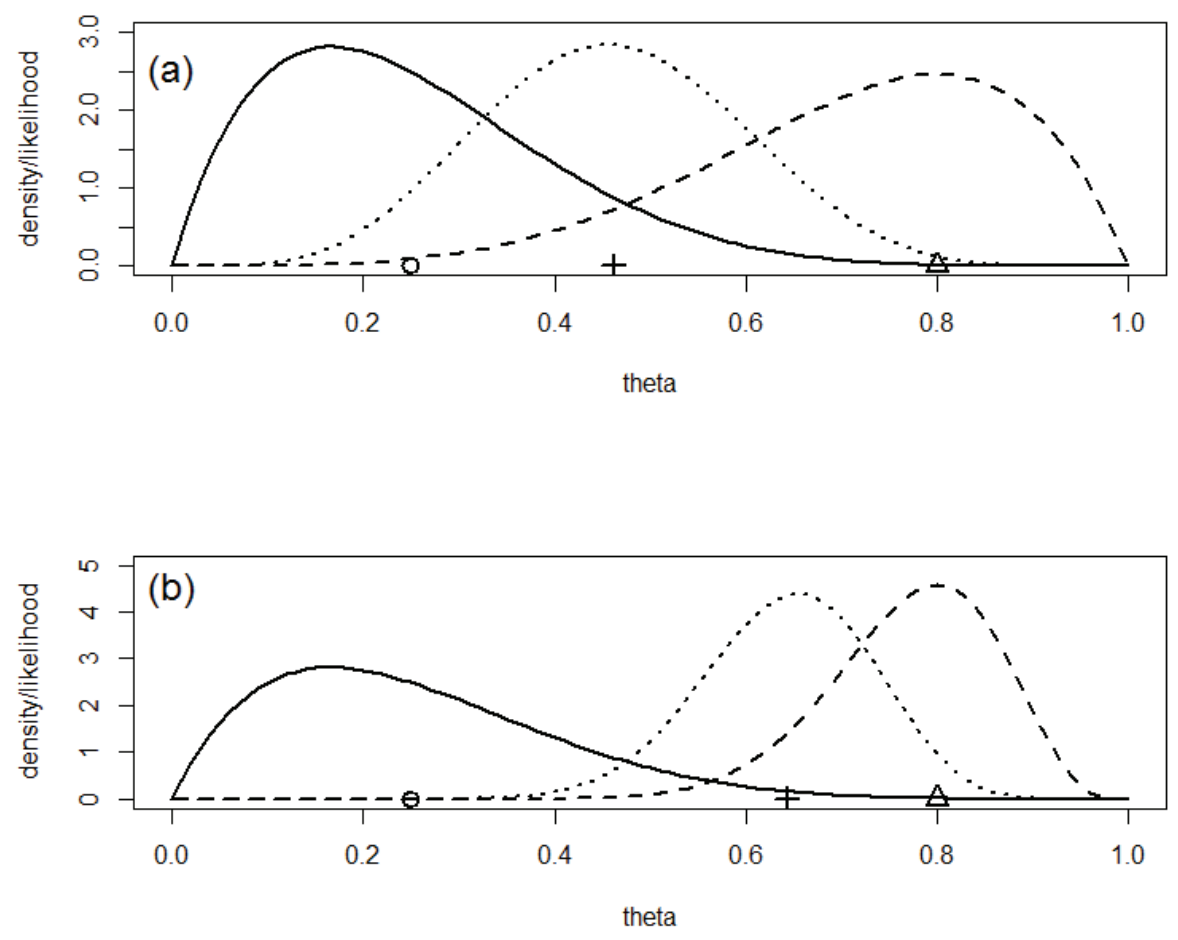


\section{R Code for Exercise I.I6}

$\mathrm{X} 11(\mathrm{w}=8, \mathrm{~h}=7) ; \operatorname{par}(\operatorname{mfrow}=\mathrm{c}(2,1))$

alp=2; bet $=6 ; n=5 ; y=4 ; \operatorname{pvec}=\operatorname{seq}(0,1,0.01)$

plot $(c(0,1), c(0,3)$, type="n",xlab="theta",ylab="density/likelihood")

lines(pvec,dbeta(pvec,alp,bet), Ity=1,lwd=2)

lines(pvec,dbeta(pvec, $1+y, n-y+1)$, Ity=2,lwd=2)

lines(pvec,dbeta(pvec,alp+y,n-y+bet), Ity=3,lwd=2)

points(c(alp/(alp+bet), y/n,(alp+y)/(alp+bet+n)),c(0,0,0), pch=c(1,2,3), cex=rep(1.5,3),lwd=2); text(0,2.5,"(a)",cex=1.5)

$c($ alp/(alp+bet), y/n,(alp+y)/(alp+bet+n)) \# 0.25000000 .80000000 .4615385

$\mathrm{n} /($ alp+bet+n) \# 0.3846154

alp=2; bet=6; $n=20 ; y=16 ;$ pvec=seq $(0,1,0.01)$

plot $(c(0,1), c(0,5)$, type="n",xlab="theta",ylab="density/likelihood")

lines(pvec,dbeta(pvec,alp, bet), Ity=1,lwd=2)

lines(pvec,dbeta(pvec,1+y,n-y+1), Ity=2,lwd=2)

lines(pvec,dbeta(pvec,alp+y,n-y+bet), Ity=3,lwd=2)

points(c(alp/(alp+bet), y/n,(alp+y)/(alp+bet+n)),c(0,0,0),pch=c(1,2,3),

cex=rep(1.5,3),lwd=2); text(0,4.5,"(b)",cex=1.5)

$c($ alp/(alp+bet), y/n,(alp+y)/(alp+bet+n)) \# 0.25000000 .80000000 .6428571

$\mathrm{n} /($ alp + bet $+\mathrm{n}) \# 0.7142857$

\section{Exercise I.I7 Further credibility estimation in the binomial- beta model}

Consider the binomial-beta model:

$$
\begin{aligned}
& (Y \mid \theta) \sim \operatorname{Binomial}(n, \theta) \\
& \theta \sim \operatorname{Beta}(\alpha, \beta) .
\end{aligned}
$$

If possible, express the posterior mode of $\theta$ as a credibility estimate.

\section{Solution to Exercise I.I7}

Since $(\theta \mid y) \sim \operatorname{Beta}(\alpha+y, \beta+n-y)$, the posterior mode of $\theta$ is

$$
\operatorname{Mode}(\theta \mid y)=\frac{(\alpha+y-1)}{(\alpha+y-1)+(\beta+n-y-1)}=\frac{\alpha+y-1}{\alpha+\beta+n-2} \text {. }
$$


Now, the prior mode of $\theta$ is $\operatorname{Mode}(\theta)=\frac{(\alpha-1)}{(\alpha-1)+(\beta-1)}=\frac{\alpha-1}{\alpha+\beta-2}$.

So we write $\operatorname{Mode}(\theta \mid y)=\frac{\alpha-1}{\alpha+\beta+n-2}+\frac{y}{\alpha+\beta+n-2}$

$$
=\frac{\alpha-1}{\alpha+\beta+n-2}\left(\frac{\alpha+\beta-2}{\alpha-1}\right)\left(\frac{\alpha-1}{\alpha+\beta-2}\right)+\frac{n}{\alpha+\beta+n-2}\left(\frac{y}{n}\right) \text {. }
$$

We see that the posterior mode is a credibility estimate of the form

$$
\operatorname{Mode}(\theta \mid y)=(1-c) \operatorname{Mode}(\theta)+c \hat{\theta},
$$

where: $\operatorname{Mode}(\theta)=\frac{\alpha-1}{\alpha+\beta-2}$ is the prior mode

$$
\begin{aligned}
& \hat{\theta}=\frac{y}{n} \quad \text { is the maximum likelihood estimate } \\
& \text { (mode of the likelihood function) } \\
& c=\frac{n}{n+\alpha+\beta-2} \text { is the credibility factor }
\end{aligned}
$$

(assigned to the direct data estimate, $\hat{\theta}$ ).

\section{Exercise I.I8 The normal-normal model}

Consider the following Bayesian model:

$$
\begin{aligned}
& \left(y_{1}, \ldots, y_{n} \mid \mu\right) \sim \text { iid } N\left(\mu, \sigma^{2}\right) \\
& \mu \sim N\left(\mu_{0}, \sigma_{0}^{2}\right),
\end{aligned}
$$

where $\sigma^{2}, \mu_{0}$ and $\sigma_{0}^{2}$ are known or specified constants.

Find the posterior distribution of $\mu$ given data in the form of the vector $y=\left(y_{1}, \ldots, y_{n}\right)$.

\section{Solution to Exercise I.18}

The posterior density of $\mu$ is

$$
\begin{aligned}
f(\mu \mid y) & \propto f(\mu) f(y \mid \mu) \\
& \propto \exp \left(-\frac{1}{2}\left(\frac{\mu-\mu_{0}}{\sigma_{0}}\right)^{2}\right) \times \prod_{i=1}^{n} \exp \left\{-\frac{1}{2}\left(\frac{y_{i}-\mu}{\sigma}\right)^{2}\right\}
\end{aligned}
$$




$$
=\exp \left(-\frac{1}{2}\left[\frac{1}{\sigma_{0}^{2}}\left(\mu^{2}-2 \mu \mu_{0}+\mu_{0}^{2}\right)+\frac{1}{\sigma^{2}}\left(\sum_{i=1}^{n} y_{i}^{2}-2 \mu n \bar{y}+n \mu^{2}\right)\right]\right),
$$

where $\bar{y}=\left(y_{1}+\ldots+y_{n}\right) / n$ is the sample mean.

We see that the posterior density of $\mu$ is proportional to the exponent of a quadratic in $\mu$. That is,

$$
f(\mu \mid y) \propto \exp \left(-\frac{1}{2 \sigma_{*}^{2}}\left(\mu-\mu_{*}\right)^{2}\right),
$$

which then implies that

$$
(\mu \mid y) \sim N\left(\mu_{*}, \sigma_{*}^{2}\right),
$$

for some constants $\mu_{*}$ and $\sigma_{*}^{2}$.

It remains to find the normal mean and variance parameters, $\mu_{*}$ and $\sigma_{*}^{2}$. (These must be functions of the known quantities $n, \bar{y}, \sigma, \mu_{0}$ and $\sigma_{0}$.)

One way to obtain these parameters which completely define $\mu$ 's posterior distribution is to complete the square in the exponent of (1.2). To this end we write

$$
f(\mu \mid y) \propto \exp \left(-\frac{1}{2} q\right)
$$

where

$$
\begin{aligned}
q & =\frac{1}{\sigma_{0}^{2}}\left(\mu^{2}-2 \mu \mu_{0}\right)+\frac{1}{\sigma^{2}}\left(-2 \mu n \bar{y}+n \mu^{2}\right) \\
& =\mu^{2}\left(\frac{1}{\sigma_{0}^{2}}+\frac{n}{\sigma^{2}}\right)-2 \mu\left(\frac{\mu_{0}}{\sigma_{0}^{2}}+\frac{n \bar{y}}{\sigma^{2}}\right)+c \\
& \quad \text { (where } c \text { is a constant with respect to } \mu) \\
& =a \mu^{2}-2 b \mu+c \quad \text { where } \quad a=\frac{1}{\sigma_{0}^{2}}+\frac{n}{\sigma^{2}} \quad \text { and } \quad b=\frac{\mu_{0}}{\sigma_{0}^{2}}+\frac{n \bar{y}}{\sigma^{2}} \\
& =a\left(\mu^{2}-2 \frac{b}{a} \mu\right)+c \quad=a\left(\mu^{2}-2\left(\frac{b}{a}\right) \mu+\left(\frac{b}{a}\right)^{2}\right)+c^{\prime} \\
& =\frac{1}{1 / a}\left(\mu-\frac{b}{a}\right)^{2}+c^{\prime} . \quad\left(\text { where } c^{\prime} \text { is a constant with respect to } \mu\right)
\end{aligned}
$$


Thus, $\quad f(\mu \mid y) \propto \exp \left(-\frac{1}{2(1 / a)}\left(\mu-\frac{b}{a}\right)^{2}\right)$.

So, equating (1.2) and (1.3), we obtain:

$$
\begin{aligned}
& \sigma_{*}^{2}=\frac{1}{a}=\frac{1}{\frac{1}{\sigma_{0}^{2}}+\frac{n}{\sigma^{2}}}=\frac{\sigma^{2} \sigma_{0}^{2}}{\sigma^{2}+n \sigma_{0}^{2}} \\
& \mu_{*}=\frac{b}{a}=\frac{\frac{\mu_{0}}{\sigma_{0}^{2}}+\frac{n \bar{y}}{\sigma^{2}}}{\frac{1}{\sigma_{0}^{2}}+\frac{n}{\sigma^{2}}}=\frac{\sigma^{2} \mu_{0}+n \sigma_{0}^{2} \bar{y}}{\sigma^{2}+n \sigma_{0}^{2}} .
\end{aligned}
$$

Note 1: A little algebra (left as an additional exercise) shows that the posterior mean can also be written as

$$
\mu_{*}=(1-k) \mu_{0}+k \bar{y},
$$

and the posterior variance can be written as

$$
\sigma_{*}^{2}=k \frac{\sigma^{2}}{n}
$$

where

$$
k=\frac{n}{n+\frac{\sigma^{2}}{\sigma_{0}^{2}}} .
$$

We see that $\mu$ 's posterior mean is a credibility estimate in the form of a weighted average of the prior mean $\mu_{0}$ and the sample mean $\bar{y}$ (which is also the maximum likelihood estimate), with the weight assigned to $\bar{y}$ being the credibility factor, $k$. More will be said on this further down.

Note 2: Another way to derive $\mu_{*}$ and $\sigma_{*}^{2}$ is to write (1.2) as

$$
f(\mu \mid y) \propto \exp \left(-\frac{1}{2 \sigma_{*}^{2}}\left(\mu^{2}-2 \mu \mu_{*}+\mu_{*}^{2}\right)\right)
$$

and then equate coefficients of powers of $\mu$ in (1.1) and (1.5). This logic leads to $\frac{1}{\sigma_{*}^{2}}=\frac{1}{\sigma_{0}^{2}}+\frac{n}{\sigma^{2}}$ and $\frac{\mu_{*}}{\sigma_{*}^{2}}=\frac{\mu_{0}}{\sigma_{0}^{2}}+\frac{n \bar{y}}{\sigma^{2}}$ and ultimately the same formulae for $\mu_{*}$ and $\sigma_{*}^{2}$ as given by (1.4). 
Note 3: Since both prior and posterior are normal, the prior is conjugate.

Note 4: The posterior mean, mode and median of $\mu$ are the same and equal to $\mu_{*}$. The $1-\alpha$ CPDR and 1- $\alpha$ HPDR for $\mu$ are the same and equal to $\left(\mu_{*} \pm z_{\alpha / 2} \sigma_{*}\right)$.

Note 5: The posterior distribution of $\mu$ depends on the data vector $y=\left(y_{1}, \ldots, y_{n}\right)$ only by way of the sample mean, i.e. $\bar{y}=\left(y_{1}+\ldots+y_{n}\right) / n$. Therefore, the main result, $(\mu \mid y) \sim N\left(\mu_{*}, \sigma_{*}^{2}\right)$, also implies that $(\mu \mid \bar{y}) \sim N\left(\mu_{*}, \sigma_{*}^{2}\right)$.

That is, if we know only the sample mean $\bar{y}$, the posterior distribution of $\mu$ is the same as if we know $y$, i.e. all $n$ sample values. Knowing the individual $y_{i}$ values makes no difference to the inference.

Note 6: The formula for the credibility factor in Note 1, namely

$$
k=\frac{n}{n+\frac{\sigma^{2}}{\sigma_{0}^{2}}}=\frac{1}{1+\frac{\sigma^{2} / n}{\sigma_{0}^{2}}},
$$

makes sense in the following ways:

(i) If the prior standard deviation $\sigma_{0}$ is small then $k \approx 0$, so that $\mu_{*} \approx \mu_{0}$ and $\sigma_{*} \approx \sigma_{0}$. Therefore $(\mu \mid y) \dot{\sim} N\left(\mu_{0}, \sigma_{0}^{2}\right)$.

That is, if the prior information is very 'precise' or 'definite', the data has little influence on the posterior. So the posterior is approximately equal to the prior; i.e. $f(\mu \mid y) \approx f(\mu)$, or equivalently, $(\mu \mid y) \dot{\sim} \mu$. In this case the posterior mean, mode and median of $\mu$ are approximately equal to $\mu_{0}$. Also, the $1-\alpha$ CPDR and $1-\alpha$ HPDR for $\mu$ are approximately equal to $\left(\mu_{0} \pm z_{\alpha / 2} \sigma_{0}\right)$.

(ii) If $\sigma_{0}$ is large then $k \approx 1$, so that $\mu_{*} \approx \bar{y}, \sigma_{*}^{2} \approx \sigma^{2} / n$, and so $(\mu \mid y) \dot{\sim} N\left(\bar{y}, \sigma^{2} / n\right)$. 
That is, a large $\sigma_{0}$ corresponds to a highly disperse prior, reflecting little prior information and so little influence of the prior distribution (as specified by $\mu_{0}$ and $\sigma_{0}$ ) on the inference. In this case the posterior mean, mode and median of $\mu$ are approximately equal to $\bar{y}$. Also, the $1-\alpha \mathrm{CPDR}$ and $1-\alpha$ HPDR for $\mu$ are approximately equal to $\left(\bar{y} \pm z_{\alpha / 2} \sigma / \sqrt{n}\right)$. Thus, inference is almost the same as implied by the classical approach.

(iii) If the sample size $n$ is large then $k \approx 1$, so that $\mu_{*} \approx \bar{y}$ and $\sigma_{*}^{2} \approx \sigma^{2} / n$. Therefore $(\mu \mid y) \dot{\sim} N\left(\bar{y}, \sigma^{2} / n\right)$.

So, in this case, just as when $\sigma_{0}$ is large, the prior distribution has very little influence on the posterior, and the ensuing inference is almost the same as that implied by the classical approach.

Note 7: In the case of a priori ignorance (meaning no prior information at all) it is customary to take $\sigma_{0}=\infty$, which implies that

$$
\mu \sim N(0, \infty) \text {. }
$$

This prior on $\mu$ appears to be problematic, because it is improper. However, it meaningfully leads to a proper posterior, namely

$$
(\mu \mid y) \sim N\left(\bar{y}, \sigma^{2} / n\right)
$$

which then leads to the same point and interval estimates implied by the classical approach, namely the MLE $\bar{y}$ and $1-\alpha \operatorname{CI}\left(\bar{y} \pm z_{\alpha / 2} \sigma / \sqrt{n}\right)$.

The improper prior $\mu \sim N(0, \infty)$ may be described as 'flat' or 'uniform over the whole real line' and can also be written as

$$
\begin{gathered}
\mu \sim U(-\infty, \infty) \\
\text { or } f(\mu) \propto 1, \mu \in \mathfrak{R} .
\end{gathered}
$$

In some cases (more complicated models not considered here), using an improper prior may lead to an improper posterior, which then becomes problematic. For more information on this topic, see Hobert and Casella (1996). 
Summary: For the normal-normal model, defined by:

$$
\begin{aligned}
& \left(y_{1}, \ldots, y_{n} \mid \mu\right) \sim \text { iid } N\left(\mu, \sigma^{2}\right) \\
& \mu \sim N\left(\mu_{0}, \sigma_{0}^{2}\right),
\end{aligned}
$$

the posterior distribution of the normal mean $\mu$ is given by

$$
(\mu \mid y) \sim N\left(\mu_{*}, \sigma_{*}^{2}\right),
$$

where: $\mu_{*}=(1-k) \mu_{0}+k \bar{y}$

$$
\begin{aligned}
\sigma_{*}^{2} & =k \frac{\sigma^{2}}{n} \\
k & =\frac{n}{n+\sigma^{2} / \sigma_{0}^{2}} \quad \text { (the normal-normal model credibility factor). }
\end{aligned}
$$

The posterior mean, mode and median of $\mu$ are all equal to $\mu_{*}$, and the $1-\alpha$ CPDR and HPDR for $\mu$ are both $\left(\mu_{*} \pm z_{\alpha / 2} \sigma_{*}\right)$.

In the case of a priori ignorance it is appropriate to set $\sigma_{0}=\infty$.

This defines an improper prior

$$
f(\mu) \propto 1, \mu \in \mathfrak{R}
$$

and the proper posterior

$$
(\mu \mid y) \sim N\left(\bar{y}, \sigma^{2} / n\right) .
$$

\section{Exercise I.19 Practice with the normal-normal model}

In the context of the normal-normal model, given by:

$$
\begin{aligned}
& \left(y_{1}, \ldots, y_{n} \mid \mu\right) \sim \text { iid } N\left(\mu, \sigma^{2}\right) \\
& \mu \sim N\left(\mu_{0}, \sigma_{0}^{2}\right),
\end{aligned}
$$

suppose that $y=(8.4,10.1,9.4), \sigma=1, \mu_{0}=5$ and $\sigma_{0}=1 / 2$.

Calculate the posterior mean, mode and median of $\mu$.

Also calculate the 95\% CPDR and 95\% HPDR for $\mu$.

Create a graph which shows these estimates as well as the prior density, prior mean, likelihood, MLE and posterior density. 


\section{Solution to Exercise I.I9}

Here: $n=3$,

$$
\begin{aligned}
& \bar{y}=(8.4+10.1+9.4) / 3=9.3 \\
& k=\frac{1}{1+\frac{1^{2} / 3}{(1 / 2)^{2}}}=\frac{3}{7}=0.4285714 \\
& \mu_{*}=\left(1-\frac{3}{7}\right) 5+\frac{3}{7} \times 9.3=6.8428571 \\
& \sigma_{*}^{2}=\frac{3}{7} \times \frac{1^{2}}{3}=\frac{1}{7}=0.1428571 .
\end{aligned}
$$

So the posterior mean/mode/median is

$$
\mu_{*}=6.84286 \text {, }
$$

and the $95 \%$ CPDR/HPDR is

$$
\begin{aligned}
\left(\mu_{*} \pm z_{0.025} \sigma_{*}\right) & =(6.84286 \pm 1.96 \sqrt{0.14286}) \\
& =(6.102,7.584) .
\end{aligned}
$$

Figure 1.10 shows the various densities and estimates here, as well as the normalised likelihood. Note that the likelihood function as shown is also the posterior density if the prior is taken to be uniform over the whole real line, i.e. $\mu \sim U(-\infty, \infty)$.

\section{Discussion}

If we change $\sigma_{0}$ from 0.5 to 2 we get $k=0.923$ and results as illustrated in Figure 1.11.

If we change $\sigma_{0}$ from 0.5 to 0.25 we get $k=0.158$ and results as illustrated in Figure 1.12 (page 46).

If we keep $\sigma_{0}$ as 0.5 but change $\sigma$ from 1 to 2 we get $k=0.158$ and results as illustrated in Figure 1.13 (page 46).

Note that the posteriors in Figures 1.12 and 1.13 have the same mean but different variances. 
Figure I. I 0 Results if $\sigma_{0}=0.5, \sigma=1, k=n /\left(n+\sigma^{2} / \sigma_{0}^{2}\right)=0.429$

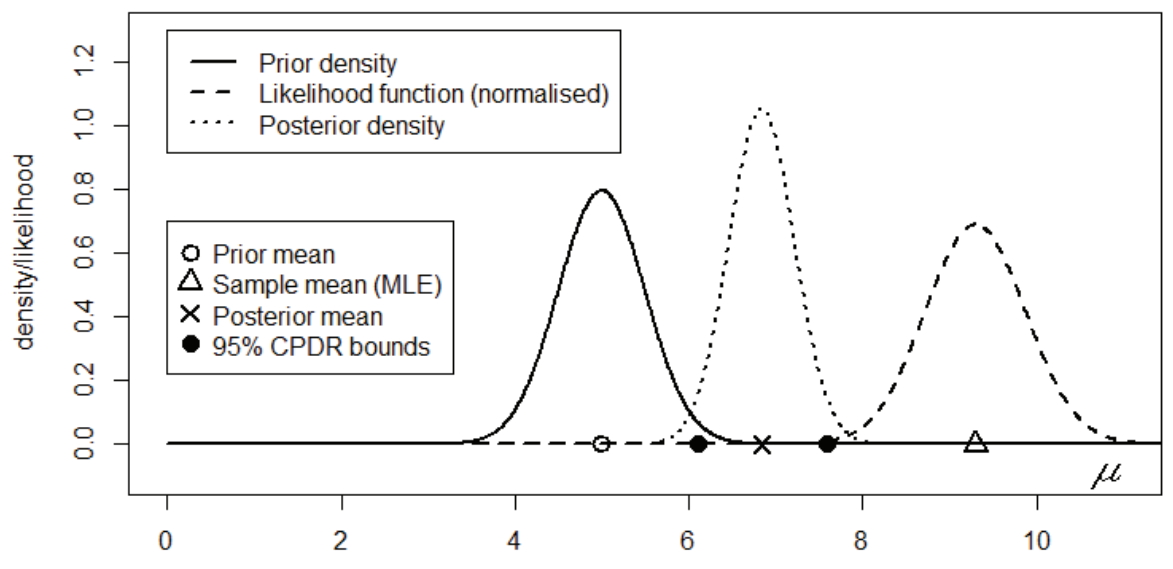

Figure I.I I Results if $\sigma_{0}=2, \sigma=1, k=n /\left(n+\sigma^{2} / \sigma_{0}^{2}\right)=0.9223$

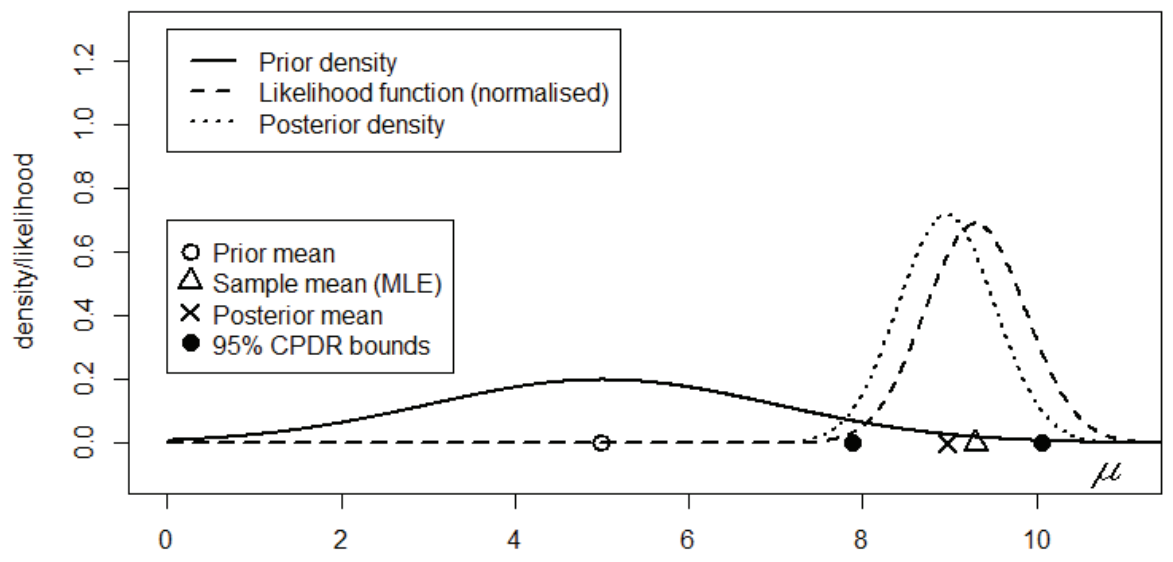


Figure I.I 2 Results if $\sigma_{0}=0.25, \sigma=1, k=n /\left(n+\sigma^{2} / \sigma_{0}^{2}\right)=0.158$

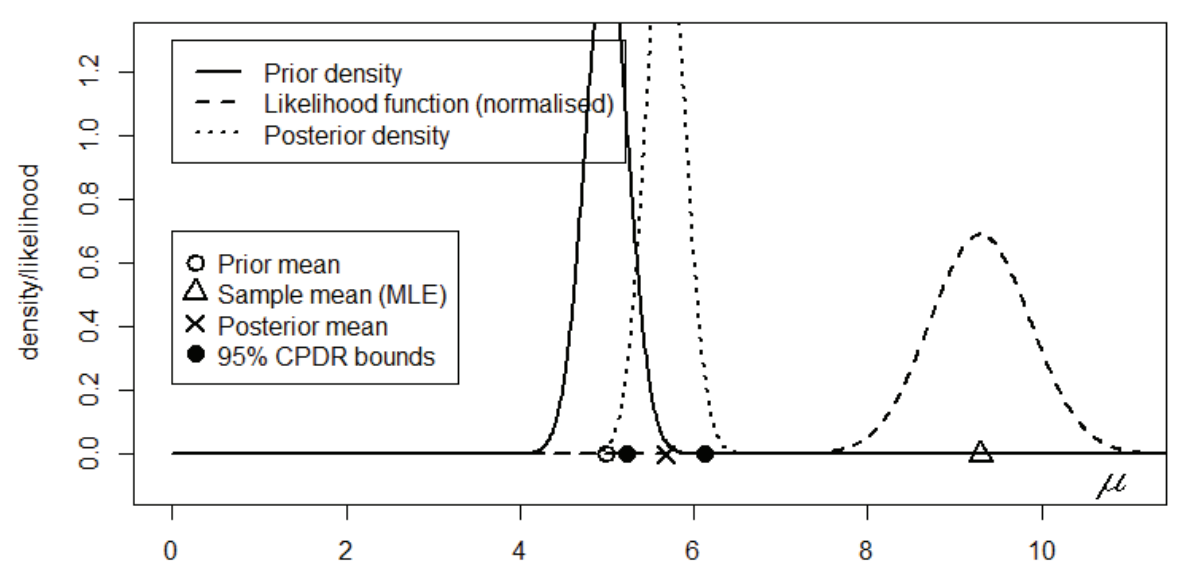

Figure I.I3 Results if $\sigma_{0}=0.5, \sigma=2, k=n /\left(n+\sigma^{2} / \sigma_{0}^{2}\right)=0.158$

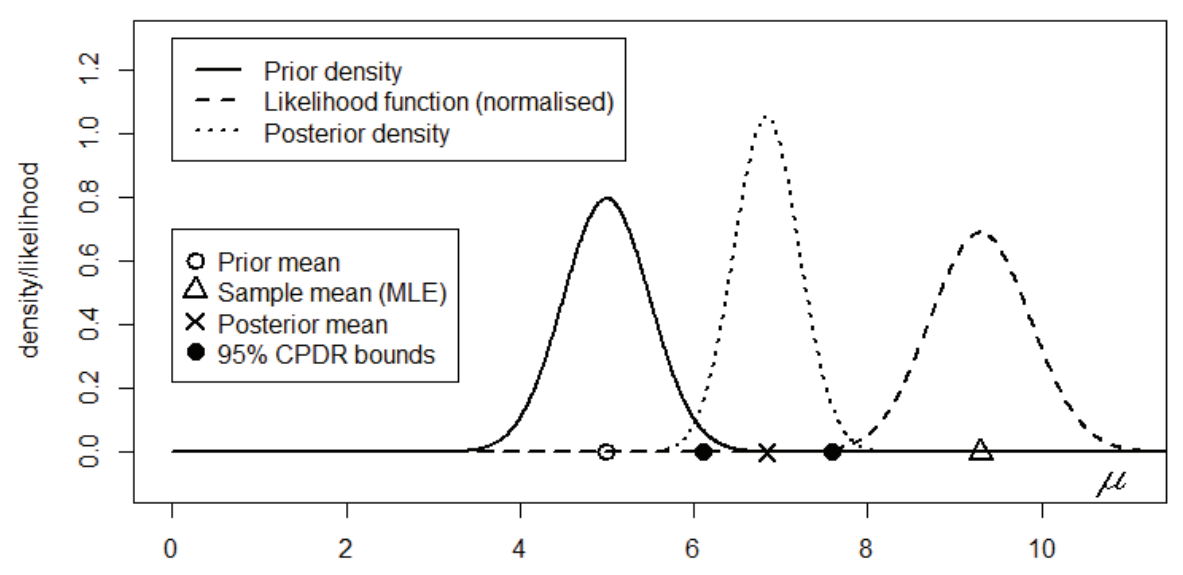




\section{R Code for Exercise I.19}

$\mathrm{X} 11(\mathrm{w}=8, \mathrm{~h}=5) ; \operatorname{par}(\mathrm{mfrow}=\mathrm{c}(1,1)) ; \mathrm{mu} 0=5 ; \operatorname{sig} 0=0.5 ; \operatorname{sig}=1$

$y=c(8.4,10.1,9.4) ; n=$ length $(y) ; k=1 /\left(1+\left(\operatorname{sig}^{\wedge} 2 / n\right) / \operatorname{sig} 0^{\wedge} 2\right) ; k \# 0.4285714$ ybar=mean $(y)$; ybar \# 9.3

mus $=(1-k)^{*} m u 0+k^{*}$ ybar; sigs $2=k^{*} \operatorname{sig} \wedge 2 / n$

c(mus,sigs2) \# 6.84285710 .1428571

muv=seq $(0,15,0.01)$

prior $=\operatorname{dnorm}($ muv, mu0, sig0); post $=$ dnorm $($ muv, mus,sqrt(sigs 2$))$

like $=\operatorname{dnorm}($ muv, ybar, sig/sqrt(n))

$c p d r=$ mus $+c(-1,1) *$ qnorm $(0.975) *$ sqrt(sigs 2$)$

cpdr \# 6.1020607 .583654

plot(c(0,11),c(-0.1,1.3),type="n",xlab="",ylab="density/likelihood")

lines(muv, prior, Ity=1,lwd=2); lines(muv, like, Ity=2,lwd=2)

lines(muv, post, Ity=3,lwd=2)

points(c(mu0,ybar, mus), $c(0,0,0), p c h=c(1,2,4), \operatorname{cex}=\operatorname{rep}(1.5,3), \operatorname{lwd}=2)$

points $(c p d r, c(0,0), p c h=r e p(16,2), \operatorname{cex}=\operatorname{rep}(1.5,2))$

legend $(0,1.3$,

c("Prior density", "Likelihood function (normalised)","Posterior density"), Ity $=c(1,2,3), \operatorname{lwd}=c(2,2,2))$

legend $(0,0.7, c($ "Prior mean","Sample mean (MLE)","Posterior mean",

"95\% CPDR bounds"), pch=c(1,2,4,16),pt.cex=rep(1.5,4),pt.Iwd=rep(2,4))

text(10.8,-0.075,"m", vfont=c("serif symbol","italic"), cex=1.5)

\# Repeat above with sig0=2 to obtain Figure 1.11

\# Repeat above with sig0 $=0.25$ to obtain Figure 1.12

\# Repeat above with sig0 $=0.5$ and sig=2 to obtain Figure 1.13

\section{Exercise I.20 The normal-gamma model}

Consider the following Bayesian model:

$$
\begin{aligned}
& \left(y_{1}, \ldots, y_{n} \mid \lambda\right) \sim \text { iid } N(\mu, 1 / \lambda) \\
& \lambda \sim G(\alpha, \beta) .
\end{aligned}
$$

Find the posterior distribution of $\lambda$ given $y=\left(y_{1}, \ldots, y_{n}\right)$. 
Note 1: In the normal-normal model, the normal mean $\mu$ is unknown and the normal variance $\sigma^{2}$ is known. Now we consider the same Bayesian model but with those roles reversed, i.e. with $\mu$ known and $\sigma^{2}$ unknown. For an example of where this kind of situation might arise, see Byrne and Dracoulis (1985).

Note 2: For reasons of mathematical convenience and conjugacy, we parameterise the normal distribution here via the precision parameter

$$
\lambda=1 / \sigma^{2}
$$

rather than using $\sigma^{2}$ directly as before in the normal-normal model.

Note 3: An equivalent formulation of the normal-gamma model being considered here is:

$$
\begin{aligned}
& \left(y_{1}, \ldots, y_{n} \mid \sigma^{2}\right) \sim \text { iid } N\left(\mu, \sigma^{2}\right) \\
& \sigma^{2} \sim \operatorname{IG}(\alpha, \beta),
\end{aligned}
$$

where this may be called the normal-inverse-gamma model.

\section{Solution to Exercise I.20}

The posterior density of $\lambda$ is

$$
\begin{aligned}
f(\lambda \mid y) & \propto f(\lambda) f(y \mid \lambda) \\
& \propto \lambda^{\alpha-1} e^{-\beta \lambda} \times \prod_{i=1}^{n} \frac{1}{1 / \sqrt{\lambda}} \exp \left\{-\frac{1}{2}\left(\frac{y_{i}-\mu}{1 / \sqrt{\lambda}}\right)^{2}\right\} \\
& =\lambda^{\alpha-1} e^{-\beta \lambda} \times \lambda^{n / 2} \exp \left\{-\frac{\lambda}{2} \sum_{i=1}^{n}\left(y_{i}-\mu\right)^{2}\right\} \\
& =\lambda^{a-1} e^{-b \lambda} \quad \text { for some } a \text { and } b .
\end{aligned}
$$

We see that

$$
(\lambda \mid y) \sim G(a, b),
$$

where: $a=\alpha+\frac{n}{2}$

$$
\begin{aligned}
& b=\beta+\frac{n}{2} s_{\mu}^{2} \\
& s_{\mu}^{2}=\frac{1}{n} \sum_{i=1}^{n}\left(y_{i}-\mu\right)^{2} .
\end{aligned}
$$


Note 1: The posterior mean of $\lambda$, namely

$$
E(\lambda \mid y)=\frac{a}{b}=\frac{\alpha+n / 2}{\beta+n s_{\mu}^{2} / 2},
$$

converges to $\hat{\lambda}=\frac{1}{s_{\mu}^{2}}$ (the MLE of $\lambda$ ) as $n \rightarrow \infty$.

If $\alpha=\beta=0$ then $E(\lambda \mid y)=\hat{\lambda}$ exactly for all $n$.

Note 2: Unlike the posterior mean of $\mu$ in the normal-normal model, the posterior mean of $\lambda$ cannot be expressed as a credibility estimate of the form

$$
(1-c) \lambda_{0}+c \hat{\lambda}
$$

where: $\lambda_{0}=E \lambda=\frac{\alpha}{\beta}$ (the prior mean of $\lambda$ )

$$
\hat{\lambda}=\frac{1}{s_{\mu}^{2}}(\text { the MLE of } \lambda) \text {. }
$$

Note 3: We may write the posterior as

$$
(\lambda \mid y) \sim G\left(\frac{2 \alpha+n}{2}, \frac{2 \beta+n s_{\mu}^{2}}{2}\right) .
$$

It can then be shown via the method of transformations that

$$
(u \mid y) \sim G\left(\frac{2 \alpha+n}{2}, \frac{1}{2}\right) \sim \chi^{2}(2 \alpha+n),
$$

where $u=\left(2 \beta+n s_{\mu}^{2}\right) \lambda$.

So the $1-A$ CPDR for $u$ is $\left(\chi_{1-A / 2}^{2}(2 \alpha+n), \chi_{A / 2}^{2}(2 \alpha+n)\right)$.

So the $1-A$ CPDR for $\lambda=\frac{u}{2 \beta+n s_{\mu}^{2}}$ is $\left(\frac{\chi_{1-A / 2}^{2}(2 \alpha+n)}{2 \beta+n s_{\mu}^{2}}, \frac{\chi_{A / 2}^{2}(2 \alpha+n)}{2 \beta+n s_{\mu}^{2}}\right)$.

So the $1-A$ CPDR for $\sigma^{2}=\frac{1}{\lambda}$ is $\left(\frac{2 \beta+n s_{\mu}^{2}}{\chi_{A / 2}^{2}(2 \alpha+n)}, \frac{2 \beta+n s_{\mu}^{2}}{\chi_{1-A / 2}^{2}(2 \alpha+n)}\right)$.

If $\alpha=\beta=0$, this is exactly the same as the classical $1-A$ CI for $\sigma^{2}$. 
Note 4: The classical 1-A CI for $\sigma^{2}$ may be derived as follows. First consider all parameters fixed as constants. Then

$$
\frac{y_{1}-\mu}{\sigma}, \ldots, \frac{y_{n}-\mu}{\sigma} \sim \text { iid } N(0,1) \text {. }
$$

So

$$
\left(\frac{y_{1}-\mu}{\sigma}\right)^{2}, \ldots,\left(\frac{y_{n}-\mu}{\sigma}\right)^{2} \sim \text { iid } \chi^{2}(1)
$$

So

$$
\sum_{i=1}^{n}\left(\frac{y_{i}-\mu}{\sigma}\right)^{2}=\frac{n s_{\mu}^{2}}{\sigma^{2}} \sim \chi^{2}(n) .
$$

So

$$
\begin{aligned}
1-A & =P\left(\chi_{1-A / 2}^{2}(n)<\frac{n s_{\mu}^{2}}{\sigma^{2}}<\chi_{A / 2}^{2}(n)\right) \\
& =P\left(\frac{n s_{\mu}^{2}}{\chi_{A / 2}^{2}(n)}<\sigma^{2}<\frac{n s_{\mu}^{2}}{\chi_{1-A / 2}^{2}(n)}\right) .
\end{aligned}
$$

Note 5: Notes 1 to 3 indicate that in the case of a priori ignorance, a reasonable specification is

$$
\alpha=\beta=0,
$$

or equivalently,

$$
f(\lambda) \propto 1 / \lambda, \quad \lambda>0 .
$$

This improper prior may be thought of as the limiting case as $\varepsilon \rightarrow 0$ of the proper prior

$$
\lambda \sim \operatorname{Gam}(\varepsilon, \varepsilon),
$$

where $\varepsilon \approx 0$.

Observe that

$$
E \lambda=\varepsilon / \varepsilon=1
$$

for all $\varepsilon$, and

$$
\begin{aligned}
& V \lambda=\varepsilon / \varepsilon^{2} \rightarrow \infty \\
\text { as } \varepsilon \rightarrow & 0 \text {. }
\end{aligned}
$$


Summary: For the normal-gamma model, defined by:

$$
\begin{aligned}
& \left(y_{1}, \ldots, y_{n} \mid \lambda\right) \sim \text { iid } N(\mu, 1 / \lambda) \\
& \lambda \sim G(\alpha, \beta),
\end{aligned}
$$

the posterior distribution of $\lambda$ is given by

$$
(\lambda \mid y) \sim G(a, b),
$$

where: $\quad a=\alpha+\frac{n}{2}, \quad b=\beta+\frac{n}{2} s_{\mu}^{2}, \quad s_{\mu}^{2}=\frac{1}{n} \sum_{i=1}^{n}\left(y_{i}-\mu\right)^{2}$.

The posterior mean of $\lambda$ is $a / b$. The posterior median is $F_{G(a, b)}^{-1}(1 / 2)$. The posterior mode of $\lambda$ is $(a-1) / b$ if $a>1$; otherwise that mode is 0 .

The $1-A$ CPDR for $\lambda$ is $\left(F_{G(a, b)}^{-1}(A / 2), F_{G(a, b)}^{-1}(1-A / 2)\right)$ and may also be written as $\left(\frac{\chi_{1-A / 2}^{2}(2 \alpha+n)}{2 \beta+n s_{\mu}^{2}}, \frac{\chi_{A / 2}^{2}(2 \alpha+n)}{2 \beta+n s_{\mu}^{2}}\right)$.

The $1-A$ CPDR for $\sigma^{2}=1 / \lambda$ is $\left(\frac{2 \beta+n s_{\mu}^{2}}{\chi_{A / 2}^{2}(2 \alpha+n)}, \frac{2 \beta+n s_{\mu}^{2}}{\chi_{1-A / 2}^{2}(2 \alpha+n)}\right)$.

In the case of a priori ignorance it is appropriate to set $\alpha=\beta=0$. This defines an improper prior with density

$$
f(\lambda) \propto 1 / \lambda, \lambda>0,
$$

and a proper posterior distribution given by

$$
\left(n s_{\mu}^{2} \lambda \mid y\right) \sim \chi^{2}(n) .
$$

\section{Exercise I.2I Practice with the normal-gamma model}

In the context of the normal-gamma model, given by:

$$
\begin{aligned}
& \left(y_{1}, \ldots, y_{n} \mid \lambda\right) \sim \text { iid } N(\mu, 1 / \lambda) \\
& \lambda \sim \operatorname{Gamma}(\alpha, \beta),
\end{aligned}
$$

suppose that $y=(8.4,10.1,9.4), \mu=8, \alpha=3$ and $\beta=2$.

(a) Calculate the posterior mean, mode and median of the model precision $\lambda$. Also calculate the 95\% CPDR for $\lambda$. Create a graph which shows these estimates as well as the prior density, prior mean, likelihood, MLE and posterior density. 
(b) Calculate the posterior mean, mode and median of the model variance $\sigma^{2}=1 / \lambda$. Also calculate the 95\% CPDR for $\sigma^{2}$. Create a graph which shows these estimates as well as the prior density, prior mean, likelihood, MLE and posterior density.

(c) Calculate the posterior mean, mode and median of the model standard deviation $\sigma$. Also calculate the 95\% CPDR for $\sigma$. Create a graph which shows these estimates as well as the prior density, prior mean, likelihood, MLE and posterior density.

(d) Examine each of the point estimates in (a), (b) and (c) and determine which ones, if any, can be easily expressed in the form of a credibility estimate.

\section{Solution to Exercise I.2 I}

(a) The required posterior distribution is $(\lambda \mid y) \sim \operatorname{Gamma}(a, b)$, where:

$$
a=\alpha+\frac{n}{2}=4.5, b=\beta+\frac{n}{2} s_{\mu}^{2}=5.265, s_{\mu}^{2}=\frac{1}{n} \sum_{i=1}^{n}\left(y_{i}-\mu\right)^{2}=2.177 \text {. }
$$

So:

- the posterior mean of $\lambda$ is $E(\lambda \mid y)=a / b=0.8547$

- the posterior mode is $\operatorname{Mode}(\lambda \mid y)=(a-1) / b=0.6648$

- the posterior median is the 0.5 quantile of the $G(a, b)$ distribution and works out as $\operatorname{Median}(\lambda \mid y)=0.7923$

(as obtained using the qgamma() function in R; see below)

- the 95\% CPDR for $\lambda$ is $(0.2564,1.8065)$ (where the bounds are the 0.025 and 0.975 quantiles of the $G(a, b)$ distribution).

Also:

- the prior mean is $E \lambda=\alpha / \beta=1.5$

- the prior mode is $\operatorname{Mode}(\lambda)=(\alpha-1) / \beta=1$

- the prior median is $\operatorname{Median}(\lambda)=1.3370$

- the MLE of $\lambda$ is $\hat{\lambda}=1 / s_{\mu}^{2}=0.4594$

(note that this estimate is biased).

Figure 1.14 shows the various densities and estimates here, as well as the normalised likelihood function. 
Note: The normalised likelihood function (with area below equal to 1 ) is the same as the posterior density of $\lambda$ if the prior is taken to be uniform over the positive real line, i.e. $\lambda \sim U(0, \infty)$. This prior is specified by taking $\alpha=1$ and $\beta=0$, because then $f(\lambda) \propto \lambda^{1-1} e^{-0 \lambda} \propto 1$.

\section{Figure I.I4 Results for Exercise I.2 I(a)}

Inference on the model precision parameter

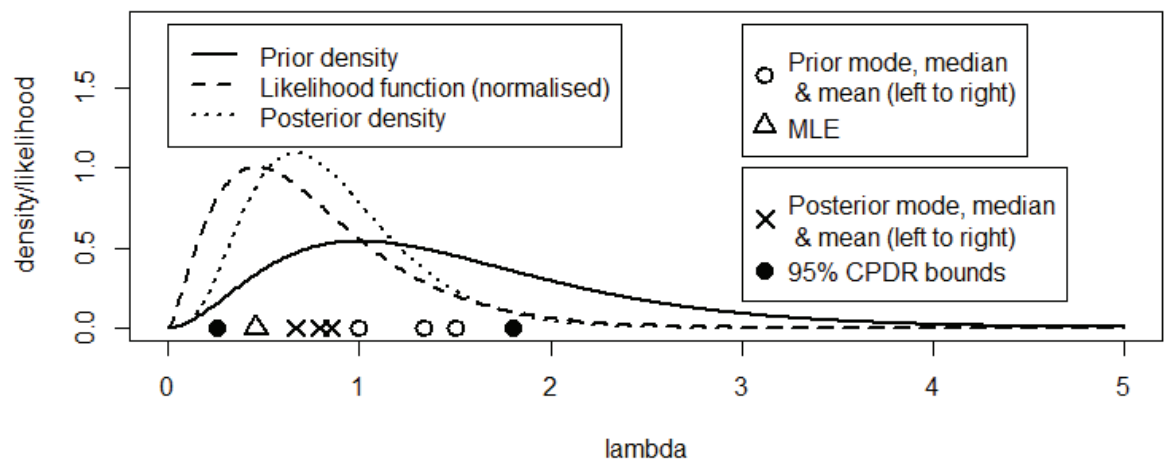

(b) As regards the model variance $\sigma^{2}=1 / \lambda$ we note that $\sigma^{2} \sim \operatorname{IG}(\alpha, \beta)$ with density

$$
\begin{aligned}
f\left(\sigma^{2}\right) & =f(\lambda)\left|\frac{d \lambda}{d \sigma^{2}}\right| \text { where } \lambda=\left(\sigma^{2}\right)^{-1} \\
& \left.=\frac{\beta^{\alpha}\left[\left(\sigma^{2}\right)^{-1}\right]^{\alpha-1} e^{-\beta\left(\sigma^{2}\right)^{-1}}}{\Gamma(\alpha)} \mid-\left(\sigma^{2}\right)^{-2}\right) \mid \\
& =\frac{\beta^{\alpha}}{\Gamma(\alpha)}\left(\sigma^{2}\right)^{-\alpha-1} e^{-\beta / \sigma^{2}}, \sigma^{2}>0 .
\end{aligned}
$$

Then, by well-known properties of the inverse gamma distribution and maximum likelihood theory:

- the prior mean of $\sigma^{2}$ is $E \sigma^{2}=\beta /(\alpha-1)=1$

- the prior mode is $\operatorname{Mode}\left(\sigma^{2}\right)=\beta /(\alpha+1)=0.5$

- the prior median is $\operatorname{Median}\left(\sigma^{2}\right)=1 / \operatorname{Median}(\lambda)=0.7479$

- the MLE of $\sigma^{2}$ is $\hat{\sigma}^{2}=1 / \hat{\lambda}=s_{\mu}^{2}=2.1767$

(note that this estimate is unbiased). 
By analogy with the prior (1.6), we find that $\left(\sigma^{2} \mid y\right) \sim \operatorname{IG}(a, b)$ with density

$$
f\left(\sigma^{2} \mid y\right)=\frac{b^{a}}{\Gamma(a)}\left(\sigma^{2}\right)^{-a-1} e^{-b / \sigma^{2}}, \sigma^{2}>0,
$$

and hence that:

- the posterior mean of $\sigma^{2}$ is $E\left(\sigma^{2} \mid y\right)=b /(a-1)=1.5043$

- the posterior mode is $\operatorname{Mode}\left(\sigma^{2} \mid y\right)=b /(a+1)=0.9573$

- the posterior median is

$$
\operatorname{Median}\left(\sigma^{2} \mid y\right)=1 / \operatorname{Median}(\lambda \mid y)=1.2622
$$

(since $1 / 2=P\left(\sigma^{2}<m \mid y\right)=P(1 / \lambda<m \mid y)=P(1 / m<\lambda \mid y)$ )

- the 95\% CPDR for $\sigma^{2}$ is $(0.5535,3.8994) \quad$ (where the lower and upper bounds are the inverses of the 0.975 and 0.025 quantiles of the $G(a, b)$ distribution, respectively).

Figure 1.15 shows the various densities and estimates here, as well as the normalised likelihood function.

Note: The normalised likelihood function is the same as the posterior density of $\sigma^{2}$ if the prior on $\sigma^{2}$ is taken to be uniform over the positive real line, i.e. $f\left(\sigma^{2}\right) \propto 1, \sigma^{2}>0$. This prior is specified by $\lambda \sim G(-1,0)$, i.e. by $\alpha=-1$ and $\beta=0$ as is evident from (1.6) above.

\section{Figure I. I 5 Results for Exercise I.2 I(b)}

\section{Inference on the model variance parameter}

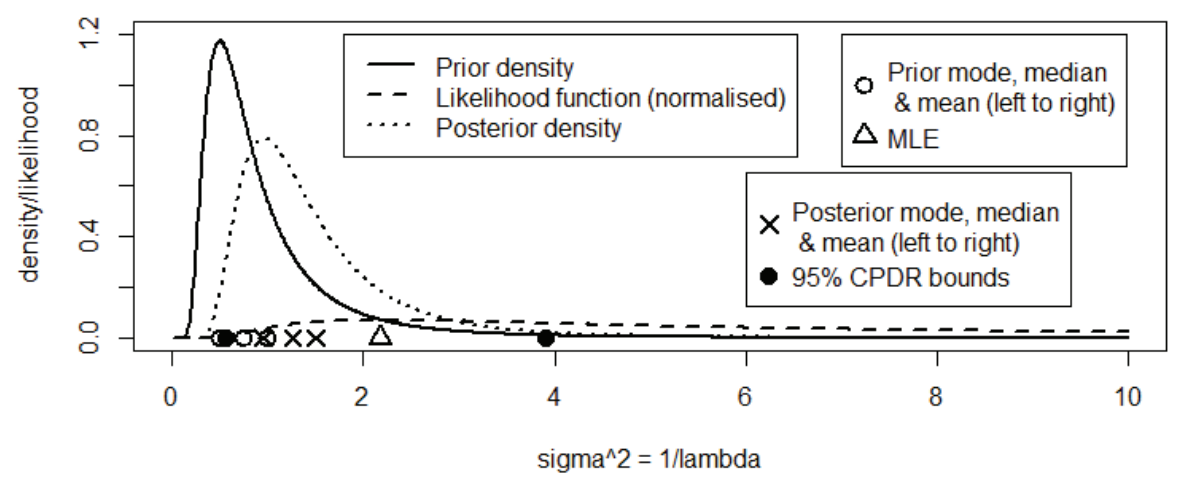


(c) As regards the model standard deviation $\sigma=1 / \sqrt{\lambda}$, observe that the prior density of this quantity is

$$
\begin{aligned}
f(\sigma) & =f(\lambda)\left|\frac{d \lambda}{d \sigma}\right| \quad \text { where } \lambda=\sigma^{-2} \\
& =\frac{\beta^{\alpha}\left(\sigma^{-2}\right)^{\alpha-1} e^{-\beta \sigma^{-2}}}{\Gamma(\alpha)}\left|-2 \sigma^{-3}\right|=\frac{2 \beta^{\alpha}}{\Gamma(\alpha)} \sigma^{-2 \alpha-1} e^{-\beta / \sigma^{2}}, \sigma>0 .
\end{aligned}
$$

We find that:

- the prior mean of $\sigma$ is

$$
\begin{aligned}
E \sigma & =E \lambda^{-1 / 2}=\int_{0}^{\infty} \lambda^{-1 / 2} \frac{\beta^{\alpha} \lambda^{\alpha-1} e^{-\beta \lambda}}{\Gamma(\alpha)} d \lambda \\
& =\frac{\beta^{\alpha} \Gamma(\alpha-1 / 2)}{\beta^{\alpha-1 / 2} \Gamma(\alpha)} \int_{0}^{\infty} \frac{\beta^{\alpha-\frac{1}{2}} \lambda^{\alpha-\frac{1}{2}-1} e^{-\beta \lambda}}{\Gamma(\alpha-1 / 2)} d \lambda \\
& =\beta^{1 / 2} \frac{\Gamma(\alpha-1 / 2)}{\Gamma(\alpha)}=0.9400
\end{aligned}
$$

- the prior mode of $\sigma$ is $\operatorname{Mode}(\sigma)=\sqrt{\frac{2 \beta}{2 \alpha+1}}=0.7559$

(obtained by setting the derivative of the logarithm of (1.7)

to zero, where that derivative is derived as follows:

$$
\begin{aligned}
& l(\sigma)=\log f(\sigma)=-(2 \alpha+1) \log \sigma-\beta \sigma^{-2}+\text { constant } \\
\Rightarrow & \left.l^{\prime}(\sigma)=-\frac{2 \alpha+1}{\sigma}+2 \beta \sigma^{-3} \stackrel{\text { set }}{=} 0 \Rightarrow \sigma^{2}=\frac{2 \beta}{2 \alpha+1}\right)
\end{aligned}
$$

- the prior median of $\sigma$ is $\operatorname{Median}(\sigma)=\sqrt{\operatorname{Median}\left(\sigma^{2}\right)}=0.8648$

- the MLE of $\sigma$ is $\hat{\sigma}=\sqrt{s_{\mu}^{2}}=1.4754$ (which is biased).

By analogy with the above, $f(\sigma \mid y)=\frac{2 b^{a}}{\Gamma(a)} \sigma^{-2 a-1} e^{-b / \sigma^{2}}, \sigma>0$.

So we find that:

- the posterior mean of $\sigma$ is $E(\sigma \mid y)=b^{1 / 2} \frac{\Gamma(a-1 / 2)}{\Gamma(a)}=1.1836$

- the posterior mode is $\operatorname{Mode}(\sigma \mid y)=\sqrt{\frac{2 b}{2 a+1}}=1.0262$ 
- the posterior median is

$$
\begin{aligned}
& \operatorname{Median}(\sigma \mid y)=\sqrt{\operatorname{Median}\left(\sigma^{2} \mid y\right)}=1.1235 \\
& \quad\left(\text { since } 1 / 2=P\left(\sigma^{2}<m \mid y\right)=P(\sigma<\sqrt{m} \mid y)\right)
\end{aligned}
$$

- the 95\% CPDR for $\sigma$ is $(0.7440,1.9747)$ (where these bounds are the square roots of the bounds of the 95\% CPDR for $\sigma^{2}$ ).

Figure 1.16 shows the various densities and estimates here, as well as the normalised likelihood function.

Note: The normalised likelihood function is the same as the posterior density of $\sigma$ if the prior on $\sigma$ is taken to be uniform over the positive real line, i.e. $f(\sigma) \propto 1, \sigma>0$. This prior is specified by $\lambda \sim G(-1 / 2,0)$, i.e. by $\alpha=-1 / 2$ and $\beta=0$, as is evident from (1.7) above.

Figure I.16 Results for Exercise I.2 I(c)

\section{Inference on the model standard deviation parameter}

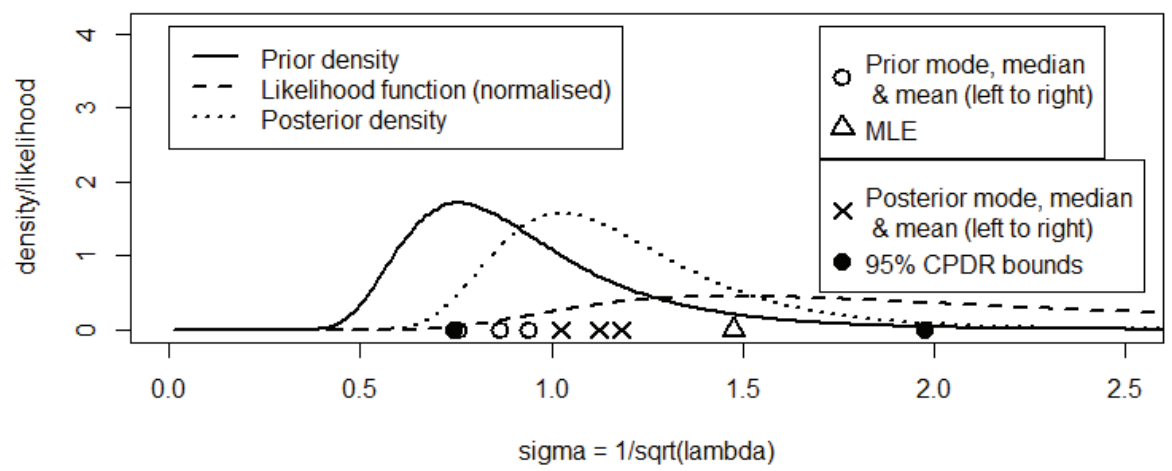

(d) Considering the various point estimates of $\lambda, \sigma^{2}$ and $\sigma$ derived above, we find that two of them can easily be expressed as credibility estimates, as follows. First, observe that

$$
\begin{aligned}
E\left(\sigma^{2} \mid y\right)=\frac{b}{a-1} & =\frac{\beta+n s_{\mu}^{2} / 2}{\alpha+(n / 2)-1}=\frac{2 \beta+n s_{\mu}^{2}}{2 \alpha+n-2} \\
& =\left(\frac{n}{n+2 \alpha-2}\right) s_{\mu}^{2}+\frac{2 \beta}{n+2 \alpha-2},
\end{aligned}
$$

where

$$
\frac{2 \beta}{n+2 \alpha-2}=\frac{2 \not \beta}{n+2 \alpha-2} \times \frac{\alpha-1}{\not \beta} \times \frac{\beta}{\alpha-1}=\frac{2 \alpha-2}{n+2 \alpha-2} \times E \sigma^{2} .
$$


We see that the posterior mean of $\sigma^{2}$ is a credibility estimate of the form

$$
E\left(\sigma^{2} \mid y\right)=(1-c) E \sigma^{2}+c s_{\mu}^{2},
$$

where:

$$
\begin{aligned}
& E \sigma^{2}=\frac{\beta}{\alpha-1} \text { is the prior mean of } \sigma^{2} \\
& s_{\mu}^{2}=\frac{1}{n} \sum_{i=1}^{n}\left(y_{i}-\mu\right)^{2} \text { is the MLE of } \sigma^{2} \\
& c=\frac{n}{n+2 \alpha-2} \text { is the credibility factor (assigned to the MLE). }
\end{aligned}
$$

Likewise,

$$
\begin{aligned}
\operatorname{Mode}\left(\sigma^{2} \mid y\right) & =\frac{b}{a+1}=\frac{\beta+n s_{\mu}^{2} / 2}{\alpha+(n / 2)+1}=\frac{2 \beta+n s_{\mu}^{2}}{2 \alpha+n+2} \\
& =\left(\frac{n}{n+2 \alpha+2}\right) s_{\mu}^{2}+\frac{2 \beta}{n+2 \alpha+2},
\end{aligned}
$$

where

$$
\begin{aligned}
\frac{2 \beta}{n+2 \alpha+2} & =\frac{2 \not \beta}{n+2 \alpha+2} \times \frac{\alpha+1}{\not \beta} \times \frac{\beta}{\alpha+1} \\
& =\frac{2 \alpha+2}{n+2 \alpha+2} \times \operatorname{Mode}\left(\sigma^{2}\right) \\
& =\left(1-\frac{n}{n+2 \alpha+2}\right) \operatorname{Mode}\left(\sigma^{2}\right) .
\end{aligned}
$$

We see that the posterior mode of $\sigma^{2}$ is a credibility estimate of the form

$$
\operatorname{Mode}\left(\sigma^{2} \mid y\right)=(1-d) \operatorname{Mode}\left(\sigma^{2}\right)+d s_{\mu}^{2},
$$

where:

$$
\begin{aligned}
& \operatorname{Mode}\left(\sigma^{2}\right)=\frac{\beta}{\alpha+1} \text { is the prior mode of } \sigma^{2} \\
& s_{\mu}^{2}=\frac{1}{n} \sum_{i=1}^{n}\left(y_{i}-\mu\right)^{2} \text { is the MLE of } \sigma^{2} \\
& \text { (i.e. mode of the likelihood function) } \\
& d=\frac{n}{n+2 \alpha+2} \text { is the credibility factor (assigned to the MLE). }
\end{aligned}
$$




\section{R Code for Exercise I.2I}

\# (a) Inference on lambda

$y=c(8.4,10.1,9.4) ; n=$ length(y); $m u=8 ;$ alp=3; bet=2; options(digits=4)

$a=a l p+n / 2 ; \operatorname{sigmu} 2=\operatorname{mean}\left((y-m u)^{\wedge} 2\right) ; b=\operatorname{bet}+(n / 2)^{*} \operatorname{sigmu} 2$

c(a,sigmu2,b) \# 4.5002 .1775 .265

lampriormean=alp/bet; lamlikemode=1/sigmu2; lampriormode=(alp-1)/bet lampriormedian $=$ qgamma(0.5,alp,bet $)$ lampostmean=a/b; lampostmode=(a-1)/b; lampostmedian=qgamma(0.5,a,b) lamcpdr=qgamma(c(0.025,0.975),a,b)

c(lampriormean,lamlikemode,lampriormode,lampriormedian, lampostmode,lampostmedian, lampostmean,lamcpdr)

\# 1.50000 .45941 .00001 .33700 .66480 .79230 .85470 .25641 .8065

lamv=seq(0,5,0.01); prior=dgamma(lamv,alp, bet $)$ post=dgamma(lamv,a,b); like=dgamma(lamv,a-alp+1,b-bet+0)

$\mathrm{X} 11(\mathrm{w}=8, \mathrm{~h}=4) ; \operatorname{par}(\mathrm{mfrow}=\mathrm{c}(1,1))$

$\operatorname{plot}(c(0,5), c(0,1.9)$, type $=" n "$, main="Inference on the model precision parameter", xlab="lambda",ylab="density/likelihood")

lines(lamv, prior,lty=1,lwd=2); lines(lamv,like,lty=2,lwd=2);

lines(lamv, post, Ity=3,lwd=2)

points(c(lampriormean,lampriormode, lampriormedian, lamlikemode,lampostmode,lampostmedian,lampostmean), $\operatorname{rep}(0,7), \operatorname{pch}=c(1,1,1,2,4,4,4), \operatorname{cex}=\operatorname{rep}(1.5,7), \operatorname{lwd}=2)$

points(lamcpdr,c(0,0),pch=rep(16,2),cex=rep(1.5,2))

legend(0,1.9,

c("Prior density","Likelihood function (normalised)","Posterior density"), $\operatorname{lty}=c(1,2,3), \operatorname{lwd}=c(2,2,2))$

legend(3,1.9,c("Prior mode, median \n \& mean (left to right)", "MLE"), pch=c(1,2),pt.cex=rep(1.5,4),pt.Iwd=rep(2,4)) legend(3,1,c("Posterior mode, median\n \& mean (left to right)", "95\% CPDR bounds"), pch=c(4,16),pt.cex=rep(1.5,4),pt.Iwd=rep(2,4)) 
\# (b) Inference on sigma2 = 1/lambda

sig2priormean=bet/(alp-1); sig2likemode=sigmu2; sig2priormode=bet $/(a l p+1)$ sig2postmean=b/(a-1); sig2postmode $=b /(a+1)$;

sig2postmedian=1/lampostmedian

sig2cpdr=1/qgamma(c(0.975,0.025),a,b); sig2priormedian= 1/lampriormedian

c(sig2priormean, sig2likemode, sig2priormode, sig2priormedian,

sig2postmode, sig2postmedian, sig2postmean, sig2cpdr)

\# 1.00002 .17670 .50000 .74790 .95731 .26221 .50430 .55353 .8994

sig2v=seq(0.01,10,0.01); prior=dgamma(1/sig2v,alp,bet $) / \operatorname{sig} 2 v^{\wedge} 2$

post=dgamma $(1 / \operatorname{sig} 2 v, a, b) / \operatorname{sig} 2 v^{\wedge} 2$;

like=dgamma $(1 / \operatorname{sig} 2 v$,a-alp-1,b-bet+0)/sig2v^2

$\operatorname{plot}(c(0,10), c(0,1.2)$, type="n",

main="Inference on the model variance parameter",

xlab="sigma^2 = 1/lambda",ylab="density/likelihood")

lines(sig2v, prior, Ity=1,lwd=2); lines(sig2v, like, Ity=2,lwd=2)

lines(sig2v, post, Ity=3,lwd=2)

points(c(sig2priormean, sig2priormode, sig2priormedian, sig2likemode, sig2postmode, sig2postmedian,sig2postmean), $\operatorname{rep}(0,7), p c h=c(1,1,1,2,4,4,4)$, cex=rep(1.5,7),lwd=2)

points(sig2cpdr,c(0,0),pch=rep(16,2),cex=rep(1.5,2))

legend(1.8,1.2,

c("Prior density","Likelihood function (normalised)","Posterior density"), Ity $=c(1,2,3), \operatorname{lwd}=c(2,2,2))$

legend(7,1.2,c("Prior mode, median $\backslash n$ \& mean (left to right)",

"MLE"), pch=c(1,2),pt.cex=rep(1.5,4),pt.Iwd=rep(2,4))

legend(6,0.65,c("Posterior mode, median \n \& mean (left to right)", "95\% CPDR bounds"), pch=c(4,16),pt.cex=rep(1.5,4),pt.Iwd=rep(2,4))

\# abline(h=max(like),Ity=3) \# Checking likelihood and MLE are consistent \# fun=function(t)\{ dgamma(1/t,a-alp-1,b-bet+0)/t^2 \}

\# integrate(f=fun,lower=0, upper=Inf) $\$$ value

\# 1 Checking likelihood is normalised 
\# (c) Inference on sigma $=1 /$ sqrt(lambda)

sigpriormean=sqrt(bet)*gamma(alp-1/2)/gamma(alp);

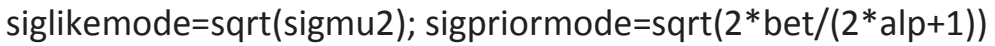

sigpostmean $=\operatorname{sqrt}(\mathrm{b}) * \operatorname{gamma}(\mathrm{a}-1 / 2) / \operatorname{gamma}(\mathrm{a})$

sigpostmode $=\operatorname{sqrt}(2 * b /(2 * a+1)) ;$ sigpostmedian=sqrt(sig2postmedian)

sigcpdr=sqrt(sig2cpdr); sigpriormedian= sqrt(sig2priormedian)

c(sigpriormean, siglikemode, sigpriormode, sigpriormedian,

sigpostmode, sigpostmedian, sigpostmean, sigcpdr)

\# 0.94001 .47540 .75590 .86481 .02621 .12351 .18360 .74401 .9747

sigv=seq(0.01,3,0.01); prior=dgamma(1/sigv^2,alp,bet $)^{*} 2 / \operatorname{sigv}^{\wedge} 3$

post=dgamma(1/sigv^2,a,b)*2/sigv^3;

like=dgamma(1/sigv^2,a-alp-1/2,b-bet+0)*2/sigv^3

$\operatorname{plot}(c(0,2.5), c(0,4.1)$, type=" $n "$, main="Inference on the model standard deviation parameter", xlab="sigma = 1/sqrt(lambda)",ylab="density/likelihood")

lines(sigv, prior, Ity=1,lwd=2)

lines(sigv,like, $\mid$ ty $=2, \mathrm{lwd}=2$ )

lines(sigv, post, lty=3,lwd=2)

points(c(sigpriormean, sigpriormode, sigpriormedian, siglikemode, sigpostmode, sigpostmedian, sigpostmean), $\operatorname{rep}(0,7), p c h=c(1,1,1,2,4,4,4)$, cex=rep $(1.5,7), l w d=2)$

points(sigcpdr,c(0,0),pch=rep(16,2),cex=rep(1.5,2))

legend(0,4.1,

c("Prior density", "Likelihood function (normalised)","Posterior density"), $\operatorname{lty}=c(1,2,3), \operatorname{lwd}=c(2,2,2))$

legend(1.7,4.1,c("Prior mode, median \n \& mean (left to right)", "MLE"), pch=c(1,2),pt.cex=rep(1.5,4),pt.Iwd=rep(2,4))

legend(1.7,2.3,c("Posterior mode, median\n \& mean (left to right)", "95\% CPDR bounds"), pch=c(4,16),pt.cex=rep(1.5,4),pt.Iwd=rep(2,4)) 


\section{Bayesian Basics Part 2}

\section{I Frequentist characteristics of Bayesian estimators}

Consider a Bayesian model defined by a likelihood $f(y \mid \theta)$ and a prior $f(\theta)$, leading to the posterior

$$
f(\theta \mid y)=\frac{f(\theta) f(y \mid \theta)}{f(y)} .
$$

Suppose that we choose to perform inference on $\theta$ by constructing a point estimate $\hat{\theta}$ (such as the posterior mean, mode or median) and a $(1-\alpha)$-level interval estimate $I=(L, U)$ (such as the CPDR or HPDR).

Then $\hat{\theta}, I, L$ and $U$ are functions of the data $y$ and may be written $\hat{\theta}(y)$, $I(y), L(y)$ and $U(y)$. Once these functions are defined, the estimates which they define stand on their own, so to speak, and may be studied from many different perspectives.

Naturally, the characteristics of these estimates may be seen in the context of the Bayesian framework in which they were constructed. More will be said on this below when we come to discuss Bayesian decision theory.

However, another important use of Bayesian estimates is as a proxy for classical estimates. We have already mentioned this in relation to the normal-normal model:

$$
\begin{aligned}
& \left(y_{1}, \ldots, y_{n} \mid \mu\right) \sim \text { iid } N\left(\mu, \sigma^{2}\right) \\
& \mu \sim N\left(\mu_{0}, \sigma_{0}^{2}\right),
\end{aligned}
$$

where the use of a particular prior, namely the one specified by $\sigma_{0}=\infty$, led to the point estimate $\hat{\mu}=\hat{\mu}(y)=\bar{y}$ and the interval estimate

$$
I(y)=(L(y), U(y))=\left(\bar{y} \pm z_{\alpha / 2} \sigma / \sqrt{n}\right) .
$$

As we noted earlier, these estimates are exactly the same as the usual estimates used in the context of the corresponding classical model, 


$$
y_{1}, \ldots, y_{n} \sim \text { iid } N\left(\mu, \sigma^{2}\right),
$$

where $\mu$ is an unknown constant and $\sigma^{2}$ is given.

Therefore, the frequentist operating characteristics of the Bayesian estimates are immediately known. In particular, we refer to the fact that the frequentist bias of $\hat{\mu}$ is zero, and the frequentist coverage probability of $I$ is exactly $1-\alpha$. These statements mean that the expected value of $\bar{y}$ given $\mu$ is $\mu$ for all possible values of $\mu$, and that the probability of $\mu$ being inside $I$ given $\mu$ is $1-\alpha$ for all possible values of $\mu$.

More generally, in the context of a Bayesian model as above, we may define the frequentist bias of a Bayesian point estimate

$$
\hat{\theta}=\hat{\theta}(y)
$$

as

$$
B_{\theta}=E\{\hat{\theta}(y)-\theta \mid \theta\}
$$

Also, we may define the frequentist relative bias of $\hat{\theta}$ as

$$
R_{\theta}=E\left(\frac{\hat{\theta}(y)-\theta}{\theta} \mid \theta\right)=\frac{B_{\theta}}{\theta} \quad(\theta \neq 0) .
$$

Furthermore, we may define the frequentist coverage probability (FCP) of a Bayesian interval estimate

as

$$
I(y)=(L(y), U(y))
$$

$$
C_{\theta}=P\{\theta \in I(y) \mid \theta\} .
$$

Thus, for the normal-normal model with $\sigma_{0}=\infty$, we may write:

$$
\begin{aligned}
B_{\mu} & =E\{\hat{\mu}(y)-\mu \mid \mu\}=E(\bar{y} \mid \mu)-\mu=\mu-\mu=0 \quad \forall \mu \in \mathfrak{R} \\
R_{\mu} & =\frac{0}{\mu}=0 \quad(\mu \neq 0) \\
C_{\mu} & =P\{\mu \in I(y) \mid \mu\} \\
& =P\left(\bar{y}-z_{\alpha / 2} \frac{\sigma}{\sqrt{n}}<\mu<\bar{y}+z_{\alpha / 2} \frac{\sigma}{\sqrt{n}} \mid \mu\right)=1-\alpha \forall \mu \in \mathfrak{R} .
\end{aligned}
$$

The above analysis is straightforward enough. However, in the case of an informative prior (one with $\sigma_{0}<\infty$ ), or in the context of other 
Bayesian models, the frequentist bias of a Bayesian point estimate $\left(B_{\theta}\right)$ and the frequentist coverage probability of a Bayesian interval estimate $\left(C_{\theta}\right)$ may not be so obvious. Working out these functions may be useful for adding insight to the estimation process as well as for deciding whether or not to use a set of Bayesian estimates as frequentist proxies.

\section{Exercise 2.I Frequentist characteristics of estimators in the normal-normal model}

Consider the normal-normal model:

$$
\begin{aligned}
& \left(y_{1}, \ldots, y_{n} \mid \mu\right) \sim \text { iid } N\left(\mu, \sigma^{2}\right) \\
& \mu \sim N\left(\mu_{0}, \sigma_{0}^{2}\right) .
\end{aligned}
$$

Work out general formulae for the frequentist and relative bias of the posterior mean of $\mu$, and for the frequentist coverage probability of the $1-\alpha$ HPDR for $\mu$.

Produce graphs showing a number of examples of each of these three functions.

\section{Solution to Exercise 2.I}

Recall that

$$
(\mu \mid y) \sim N\left(\mu_{*}, \sigma_{*}^{2}\right),
$$

where:

$$
\begin{aligned}
& \mu_{*}=(1-k) \mu_{0}+k \bar{y} \text { is } \mu \text { 's posterior mean } \\
& \sigma_{*}^{2}=k \frac{\sigma^{2}}{n} \text { is } \mu \text { 's posterior variance } \\
& k=\frac{n}{n+\sigma^{2} / \sigma_{0}^{2}} \text { is a credibility factor. }
\end{aligned}
$$

Also, recall that $\mu$ 's HPDR (and CPDR) is

$$
\left(\mu_{*} \pm z_{\alpha / 2} \sigma_{*}\right) \text {. }
$$

Using these results, we find that the frequentist bias of the posterior mean of $\mu$ is

$$
\begin{aligned}
B_{\mu}=E\left(\mu_{*}-\mu \mid \mu\right) & =(1-k) \mu_{0}+k E(\bar{y} \mid \mu)-\mu \\
& =(1-k) \mu_{0}+k \mu-\mu \\
& =(1-k)\left(\mu_{0}-\mu\right) .
\end{aligned}
$$


Also, the frequentist relative bias of that mean is

$$
\begin{aligned}
R_{\mu}=\frac{R_{\mu}}{\mu} & =\frac{(1-k)\left(\mu_{0}-\mu\right)}{\mu} \\
& =(1-k)\left(\frac{\mu_{0}}{\mu}-1\right) \quad(\mu \neq 0) .
\end{aligned}
$$

Further, the frequentist coverage probability of the $1-\alpha$ HPDR for $\mu$ is

$$
\begin{aligned}
& C_{\mu}=P\left\{\mu \in\left(\mu_{*} \pm z_{\alpha / 2} \sigma_{*}\right) \mid \mu\right\} \\
& \quad=P\left(\mu_{*}-z_{\alpha / 2} \sigma_{*}<\mu<\mu_{*}+z_{\alpha / 2} \sigma_{*} \mid \mu\right) \\
& \quad=P\left(\mu_{*}-z_{\alpha / 2} \sigma_{*}<\mu, \mu<\mu_{*}+z_{\alpha / 2} \sigma_{*} \mid \mu\right) \\
& =P\left((1-k) \mu_{0}+k \bar{y}-z_{\alpha / 2} \sigma_{*}<\mu, \mu<(1-k) \mu_{0}+k \bar{y}+z_{\alpha / 2} \sigma_{*} \mid \mu\right) \\
& =P\left(\bar{y}<\frac{\mu-(1-k) \mu_{0}+z_{\alpha / 2} \sigma_{*}}{k}, \frac{\mu-(1-k) \mu_{0}-z_{\alpha / 2} \sigma_{*}}{k}<\bar{y} \mid \mu\right) \\
& =P(\bar{y}<b(\mu), a(\mu)<\bar{y} \mid \mu),
\end{aligned}
$$

where:

$$
\begin{aligned}
& b(\mu)=\frac{\mu-(1-k) \mu_{0}+z_{\alpha / 2} \sigma_{*}}{k} \\
& a(\mu)=\frac{\mu-(1-k) \mu_{0}-z_{\alpha / 2} \sigma_{*}}{k} .
\end{aligned}
$$

Thus, we find that

$$
\begin{aligned}
C_{\mu} & =P(a(\mu)<\bar{y}<b(\mu) \mid \mu) \\
& =P\left(\frac{a(\mu)-\mu}{\sigma / \sqrt{n}}<\frac{\bar{y}-\mu}{\sigma / \sqrt{n}}<\frac{b(\mu)-\mu}{\sigma / \sqrt{n}} \mid \mu\right) \\
& =P\left(\frac{a(\mu)-\mu}{\sigma / \sqrt{n}}<Z<\frac{b(\mu)-\mu}{\sigma / \sqrt{n}}\right) \\
& \quad \text { where } Z \sim N(0,1), \text { since }\left(\frac{\bar{y}-\mu}{\sigma / \sqrt{n}} \mid \mu\right) \sim N(0,1) \\
& =\Phi\left(\frac{b(\mu)-\mu}{\sigma / \sqrt{n}}\right)-\Phi\left(\frac{a(\mu)-\mu}{\sigma / \sqrt{n}}\right) .
\end{aligned}
$$

Note: Here, $\Phi$ denotes the standard normal cdf. 
Figures 2.1, 2.2 and 2.3 (pages 66 and 67) show $B_{\mu}, R_{\mu}$ and $C_{\mu}$ for selected values of $\sigma_{0}$, with $n=10, \mu_{0}=1, \sigma=1$ and $\alpha=0.05$ in each case. The strength of the prior belief is represented by $\sigma_{0}$, with large values of this parameter indicating relative ignorance.

In Figure 2.1, we see that, for any given value of $\mu$, the frequentist bias $B_{\mu}$ of the posterior mean $\mu_{*}=E(\mu \mid y)$ converges to zero as the prior belief tends to total ignorance, that is, in the limit as $\sigma_{0} \rightarrow \infty$.

Also, $B_{\mu} \rightarrow \mu_{0}-\mu$ as the prior belief tends to complete certainty, that is, in the limit as $\sigma_{0} \rightarrow 0$.

Note: One of the thin dotted guidelines in Figure 2.1 shows the function $B_{\mu}=\mu_{0}-\mu$ in this latter extreme case of 'absolute' prior belief that $\mu=\mu_{0}$. In all of the examples, $\mu_{0}=1$.

In Figure 2.2, we see that, for any given value of $\mu$, the frequentist relative bias $R_{\mu}$ of the posterior mean $\mu_{*}=E(\mu \mid y)$ converges to zero as $\sigma_{0} \rightarrow \infty$. Also, $R_{\mu} \rightarrow\left(\mu_{0} / \mu\right)-1$ as $\sigma_{0} \rightarrow 0$.

Note: The curved thin dotted guidelines in Figure 2.2 shows the function $R_{\mu}=\left(\mu_{0} / \mu\right)-1$ in this latter extreme case of 'absolute' prior belief that $\mu=\mu_{0}$.

In Figure 2.3, we see that, for any given value of $\mu$, the frequentist coverage probability $C_{\mu}$ of the $1-\alpha$ (i.e. 0.95 or $95 \%$ ) HPDR, namely $\left(\mu_{*} \pm z_{\alpha / 2} \sigma_{*}\right)$, converges to $1-\alpha$ as $\sigma_{0} \rightarrow \infty$.

Also, $C_{\mu} \rightarrow 0$ as $\sigma_{0} \rightarrow 0$, except at exactly $\mu=\mu_{0}$ where $C_{\mu} \rightarrow 1$; thus, $C_{\mu} \rightarrow I\left(\mu=\mu_{0}\right)$ as $\sigma_{0} \rightarrow 0$ (where $I$ denotes the standard indicator function).

Note: In Figure 2.3, the thin dotted horizontal guidelines show the values $0,0.95$ and 1 . 
Figure 2.I Frequentist bias in Exercise 2.I

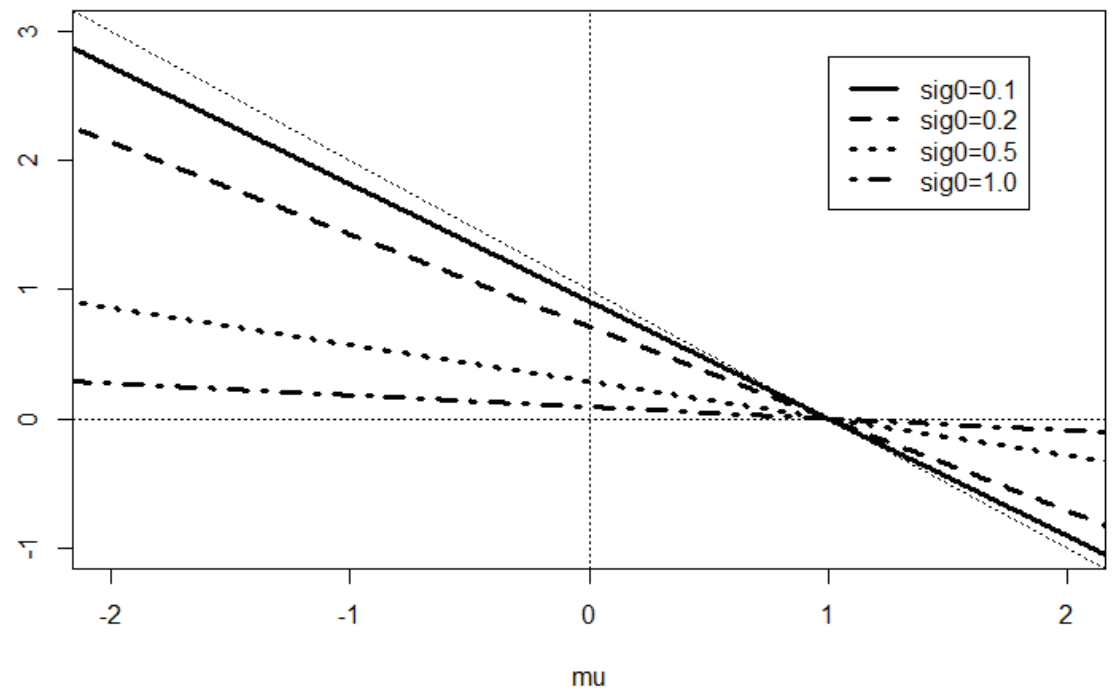

Figure 2.2 Frequentist relative bias in Exercise 2.I

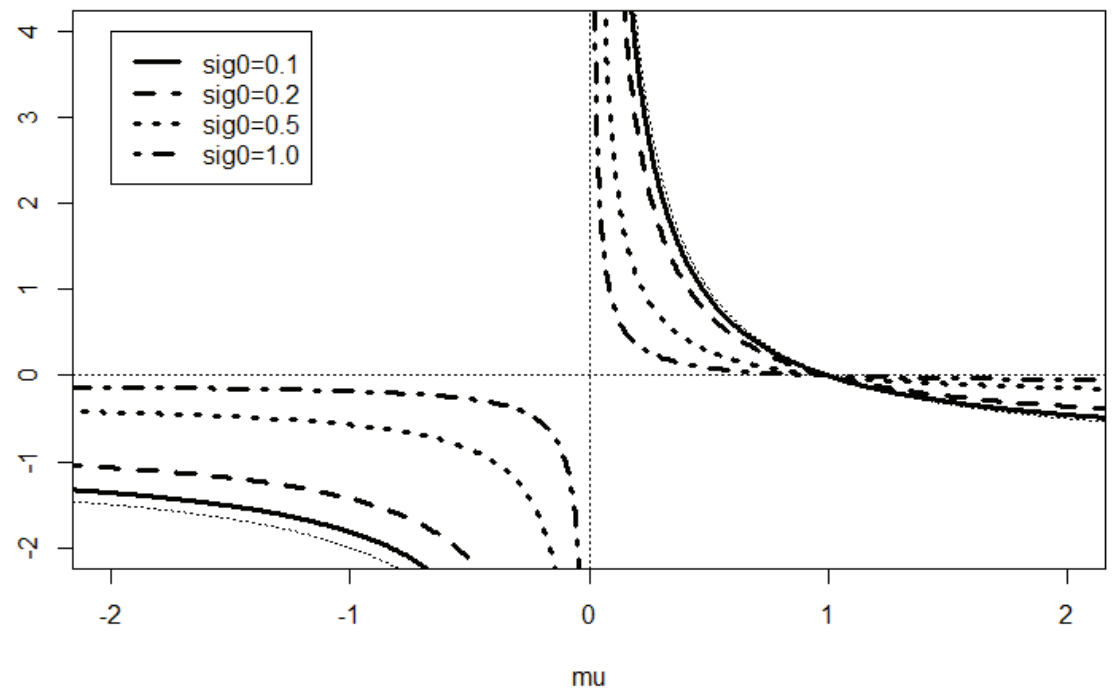


Figure 2.3 Frequentist coverage probability in Exercise 2. I

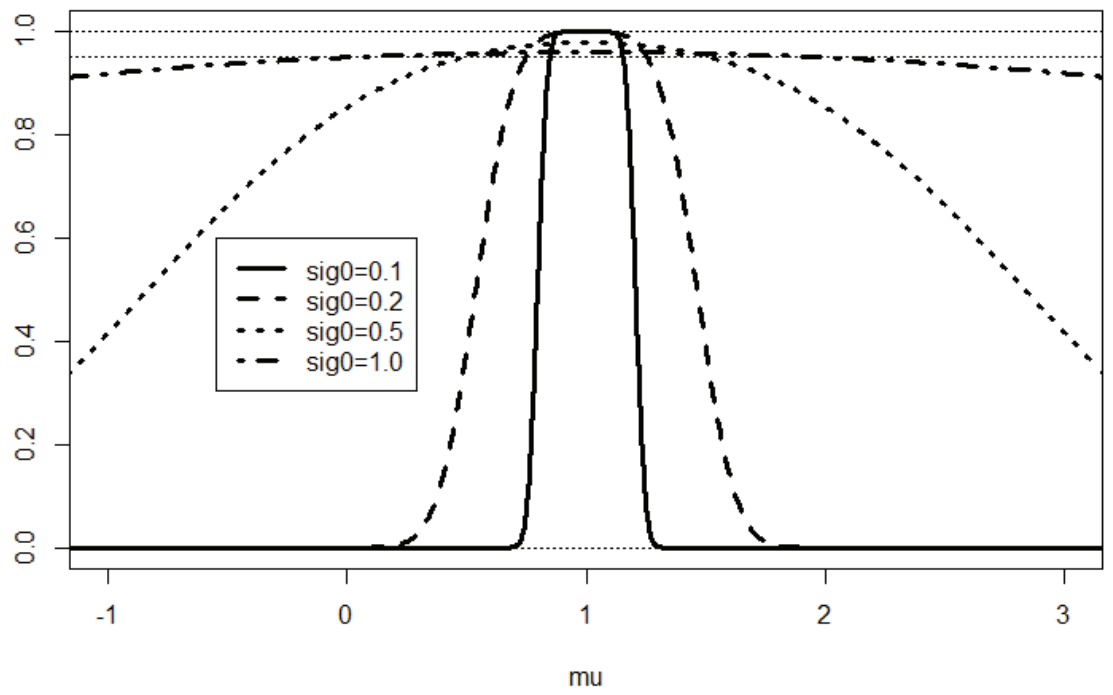

\section{R Code for Exercise 2.I}

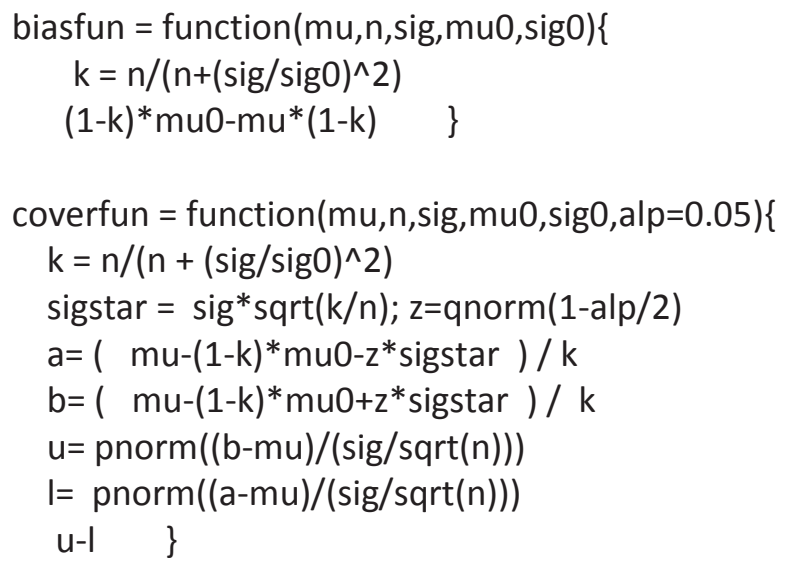

$\mathrm{X} 11(\mathrm{w}=8, \mathrm{~h}=5.5) ; \operatorname{par}(\mathrm{mfrow}=\mathrm{c}(1,1))$

muvec=seq $(-5,5,0.01) ; \mathrm{mu0}=1 ; \operatorname{sig}=1 ; \mathrm{n}=10 ; \operatorname{sig} 0 \mathrm{v}=\mathrm{c}(0.1,0.2,0.5,1)$

plot(c(-2,2),c(-1,3),type="n",xlab="mu",ylab="',,main=" ") abline (1,-1,lty=3); abline( $v=0$, Ity=3); abline( $h=0$, lty=3)

lines(muvec, biasfun(mu=muvec, $n=n$, sig=sig, mu0=mu0,sig0=sig0v[1]), Ity=1,lwd=3) 
lines (muvec, biasfun (mu=muvec, $n=n, s i g=s i g, m u 0=m u 0, \operatorname{sig} 0=s i g 0 v[2])$, Ity $=2, \mathrm{lwd}=3$ )

lines (muvec, biasfun( $m u=$ muvec, $n=n$, sig=sig, mu0=mu0,sig0=sig0v[3]), Ity $=3, \operatorname{lwd}=3$ )

lines(muvec, biasfun( $m u=m u v e c, n=n, s i g=s i g, m u 0=m u 0, \operatorname{sig} 0=s i g 0 v[4]$ ), Ity $=4, \mathrm{lwd}=3$ )

legend $(1,2.8, c(" \operatorname{sig} 0=0.1$ ","sig0=0.2","sig0=0.5","sig0=1.0"), Ity $=1: 4, \operatorname{lwd}=\operatorname{rep}(3,4))$

plot(c(-2,2),c(-2,4),type="n",xlab="mu",ylab="',main=" ") abline( $\mathrm{v}=0$, Ity=3); abline( $\mathrm{h}=0$,lty=3); lines(muvec, mu0/muvec-1,lty=3) lines(muvec, biasfun(mu=muvec, $n=n$, sig=sig,mu0=mu0, sig0=sig0v[1])/muvec, Ity $=1, \mathrm{lwd}=3$ )

lines(muvec, biasfun(mu=muvec, $n=n$, sig=sig,mu0=mu0, sig0=sig0v[2])/muvec, Ity $=2, \operatorname{lwd}=3$ )

lines(muvec, biasfun(mu=muvec, $n=n$, sig=sig,mu0=mu0, sig0=sig0v[3])/muvec, Ity $=3, \operatorname{lwd}=3$ )

lines(muvec, biasfun(mu=muvec, $n=n, s i g=s i g, m u 0=m u 0$, sig0=sig0v[4])/muvec, Ity $=4, \operatorname{lwd}=3$ ) legend(-2,4,c("sig0=0.1","sig0=0.2","sig0=0.5","sig0=1.0"), Ity $=1: 4, \operatorname{lwd}=\operatorname{rep}(3,4))$

plot(c(-1,3),c(0,1),type="n",xlab="mu",ylab="",main=" ") abline $(\mathrm{h}=\mathrm{c}(0,0.95,1)$, tty $=3)$

lines(muvec, coverfun (mu=muvec, $n=n, s i g=s i g, m u 0=m u 0, \operatorname{sig} 0=\operatorname{sig} 0 v[1]$ ), Ity $=1, \mid \mathrm{w} d=3)$ lines(muvec, coverfun (mu=muvec, $n=n$, sig=sig, mu0=mu0,sig0=sig0v[2]), Ity $=2, \operatorname{lwd}=3$ )

lines(muvec, coverfun(mu=muvec, $n=n$, sig=sig,mu0=mu0,sig0=sig0v[3]), Ity $=3, \mid w d=3)$

lines(muvec, coverfun(mu=muvec, $n=n$, sig=sig,mu0=mu0,sig0=sig0v[4]), Ity $=4, \operatorname{lwd}=3$ )

legend(-0.55,0.6,c("sig0=0.1","sig0=0.2","sig0=0.5","sig0=1.0"), Ity $=1: 4, \operatorname{lwd}=\mathrm{rep}(3,4))$

\section{Exercise 2.2 Frequentist characteristics of estimators in the normal-gamma model}

Consider the normal-gamma model given by:

$$
\begin{aligned}
& \left(y_{1}, \ldots, y_{n} \mid \lambda\right) \sim \text { iid } N(\mu, 1 / \lambda) \\
& \lambda \sim \operatorname{Gamma}(\alpha, \beta) .
\end{aligned}
$$


(a) Work out general formulae for the frequentist bias and relative bias of the posterior mean of $\sigma^{2}=1 / \lambda$, and for the frequentist coverage probability of the $1-\alpha$ CPDR for $\sigma^{2}$.

Produce graphs showing examples of each of these three functions.

(b) Attempt to find a single prior under this model (that is, a single suitable pair of values $\alpha, \beta$ ) which results in both:

(i) a Bayesian posterior mean of $\sigma^{2}$ that is unbiased (in the frequentist sense) for all possible values of $\sigma^{2}$; and

(ii) a CPDR for $\sigma^{2}$ that has frequentist coverage probabilities exactly equal to the desired coverage for all possible values of $\sigma^{2}$.

\section{Solution to Exercise 2.2}

(a) Recall that the posterior mean of $\sigma^{2}$ is

$$
\hat{\sigma}^{2}=E\left(\sigma^{2} \mid y\right)=\frac{b}{a-1},
$$

where: $\quad a=\alpha+\frac{n}{2}, \quad b=\beta+\frac{n}{2} s_{\mu}^{2}, \quad s_{\mu}^{2}=\frac{1}{n} \sum_{i=1}^{n}\left(y_{i}-\mu\right)^{2}$.

Thus, $\hat{\sigma}^{2}=\frac{\beta+(n / 2) s_{\mu}^{2}}{\alpha+(n / 2)-1}=\frac{2 \beta+n s_{\mu}^{2}}{2 \alpha+n-2}$.

So the frequentist bias of $\hat{\sigma}^{2}$ is

$$
B_{\sigma^{2}}=E\left(\hat{\sigma}^{2}-\sigma^{2} \mid \sigma^{2}\right)=\frac{2 \beta+n E\left(s_{\mu}^{2} \mid \sigma^{2}\right)}{2 \alpha+n-2}-\sigma^{2}=\frac{2 \beta+n \sigma^{2}}{2 \alpha+n-2}-\sigma^{2} .
$$

Note: This follows because, conditional on $\sigma^{2}$, it is true that

$$
\frac{n s_{\mu}^{2}}{\sigma^{2}}=\sum_{i=1}^{n}\left(\frac{y_{i}-\mu}{\sigma}\right)^{2} \sim \chi^{2}(n) \quad \text { (with mean } n \text { ). }
$$

Therefore the frequentist relative bias of $\hat{\sigma}^{2}$ is

$$
R_{\sigma^{2}}=\frac{B_{\sigma^{2}}}{\sigma^{2}}=\frac{\left(2 \beta / \sigma^{2}\right)+n}{2 \alpha+n-2}-1 .
$$


Note: We see that for any fixed $\sigma^{2}, \alpha$ and $\beta$ it is true that

$$
B_{\sigma^{2}}, R_{\sigma^{2}} \rightarrow 0 \text { as } n \rightarrow \infty \text {. }
$$

Thus the posterior mean of $\sigma^{2}$ is asymptotically unbiased, in the frequentist sense.

Next, recall that the $1-A$ CPDR for $\sigma^{2}=1 / \lambda$ is

$$
I=I(y)=\left(\frac{2 \beta+n s_{\mu}^{2}}{v}, \frac{2 \beta+n s_{\mu}^{2}}{u}\right),
$$

where: $\quad v=\chi_{A / 2}^{2}(2 \alpha+n)=F_{\chi^{2}(2 \alpha+n)}^{-1}(1-A / 2)$

$$
u=\chi_{1-A / 2}^{2}(2 \alpha+n)=F_{\chi^{2}(2 \alpha+n)}^{-1}(A / 2) \text {. }
$$

So the frequentist coverage probability of $I$ is

$$
\begin{aligned}
C_{\sigma^{2}} & =P\left\{\sigma^{2} \in I(y) \mid \sigma^{2}\right\} \\
& =P\left(\frac{2 \beta+n s_{\mu}^{2}}{v}<\sigma^{2}<\frac{2 \beta+n s_{\mu}^{2}}{u} \mid \sigma^{2}\right) \\
& =P\left\{\sigma^{2} \in I(y) \mid \sigma^{2}\right\} \\
& =P\left(\frac{n s_{\mu}^{2}}{\sigma^{2}}<v-\frac{2 \beta}{\sigma^{2}}, u-\frac{2 \beta}{\sigma^{2}}<\frac{n s_{\mu}^{2}}{\sigma^{2}} \mid \sigma^{2}\right) \\
& =F_{\chi^{2}(n)}\left(v-\frac{2 \beta}{\sigma^{2}}\right)-F_{\chi^{2}(n)}\left(u-\frac{2 \beta}{\sigma^{2}}\right) .
\end{aligned}
$$

Figures 2.4, 2.5 and 2.6 (pages 72 and 73) show $B_{\sigma^{2}}, R_{\sigma^{2}}$ and $C_{\sigma^{2}}$ for selected values of $\alpha$ and $\beta$, with $n=10$ and $A=0.05$ in each case.

(b) Observe that under the prior given by $\alpha=1$ and $\beta=0$ (that is, $f(\lambda) \propto \lambda^{1-1} e^{-0 \lambda} \propto 1$ ), it is true that:

- the posterior mean of $\sigma^{2}$ equals the MLE, namely $s_{\mu}^{2}$, and so is unbiased

- the $1-A$ CPDR for $\sigma^{2}$ is $\left(\frac{n s_{\mu}^{2}}{\chi_{A / 2}^{2}(n+2)}, \frac{n s_{\mu}^{2}}{\chi_{1-A / 2}^{2}(n+2)}\right)$,

which has coverage probability less than $1-A$ for all $\sigma^{2}$. 
Also, under the prior given by $\alpha=\beta=0$ (i.e. $f(\lambda) \propto \lambda^{0-1} e^{-0 \lambda} \propto 1 / \lambda$ ), it is true that:

- the posterior mean of $\sigma^{2}$ equals $s_{\mu}^{2} /(1-2 / n)$ and so is biased

- the $1-A$ CPDR for $\sigma^{2}$ is the same as the classical CI, namely $\left(\frac{n s_{\mu}^{2}}{\chi_{A / 2}^{2}(n)}, \frac{n s_{\mu}^{2}}{\chi_{1-A / 2}^{2}(n)}\right)$, and so has coverage exactly $1-A$ for all $\sigma^{2}$.

We see that there is no single gamma prior for $\lambda=1 / \sigma^{2}$ which results in both:

(i) a Bayesian posterior mean of $\sigma^{2}$ that is unbiased (in the frequentist sense) for all possible values of $\sigma^{2}$; and

(ii) a CPDR for $\sigma^{2}$ that has frequentist coverage probabilities exactly equal to the desired coverage for all possible values of $\sigma^{2}$.

Note: It is easy to modify or 'correct' the posterior mean under $\alpha=\beta=0$ so that it becomes unbiased. Explictly, if $\alpha=\beta=0$, then

$$
E\left(\hat{\sigma}^{2} \mid \sigma^{2}\right)=\frac{n \sigma^{2}}{n-2} \text {. }
$$

So an unbiased estimate of $\sigma^{2}$ is

$$
\tilde{\sigma}^{2}=\frac{n-2}{n} \hat{\sigma}^{2}=\frac{n-2}{n} \times \frac{0+(n / 2) s_{\mu}^{2}}{0+(n / 2)-1}=s_{\mu}^{2} \quad \text { (i.e. the MLE). }
$$


Figure 2.4 Frequentist bias in Exercise 2.2

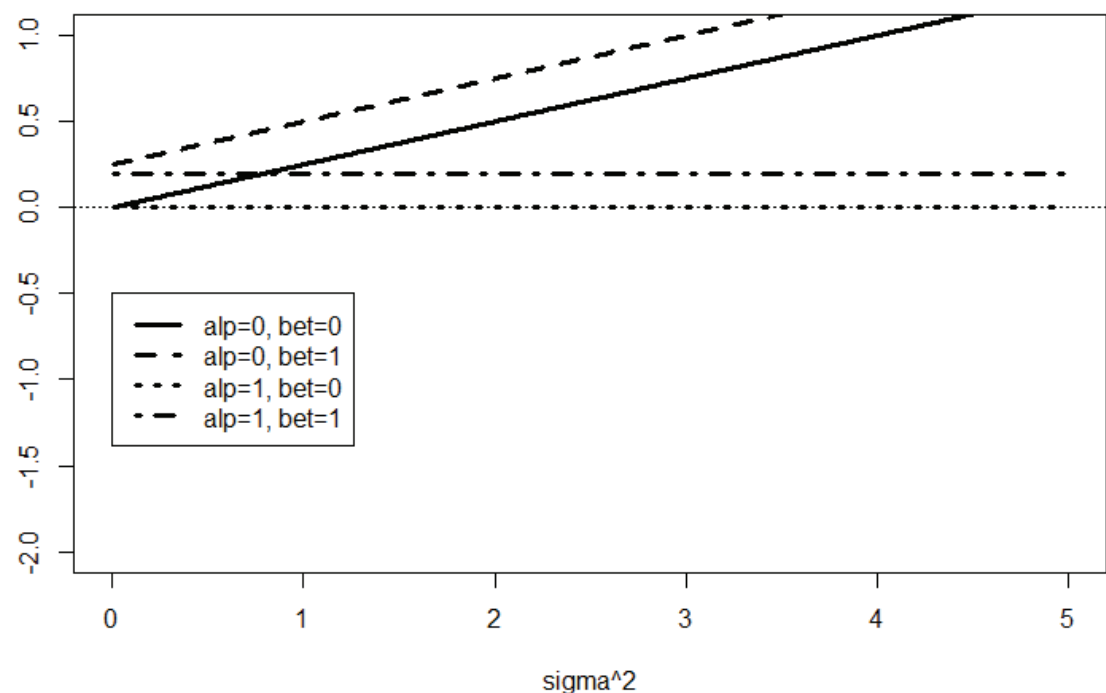

Figure 2.5 Frequentist relative bias in Exercise $\mathbf{2 . 2}$

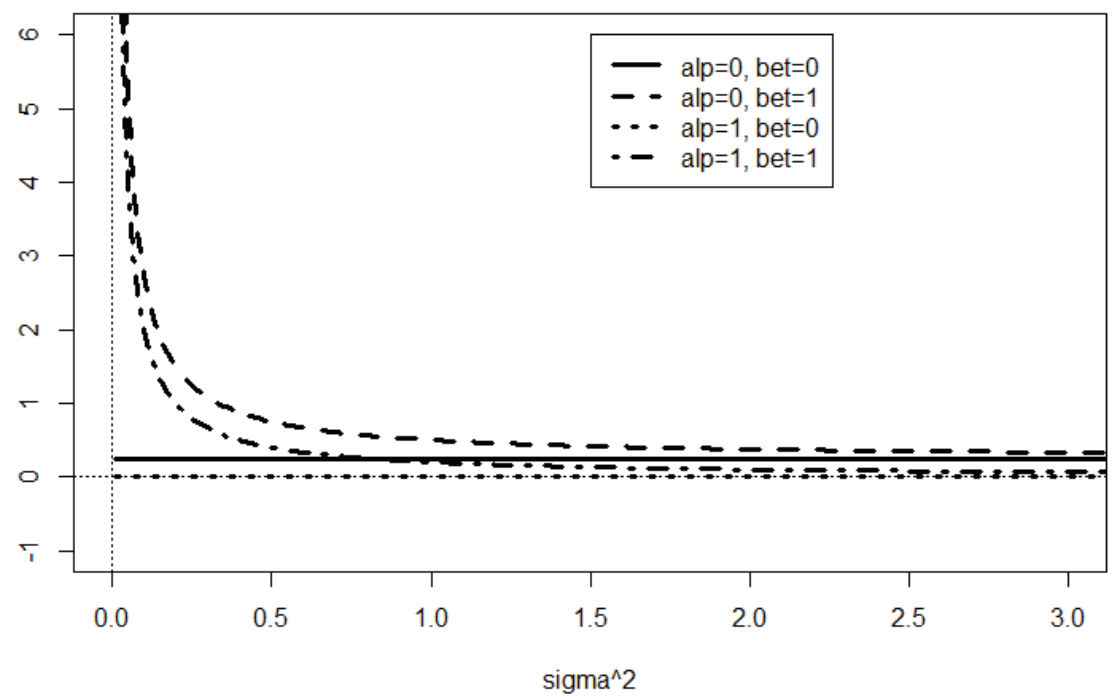


Figure 2.6 Frequentist coverage probability in Exercise $\mathbf{2 . 2}$

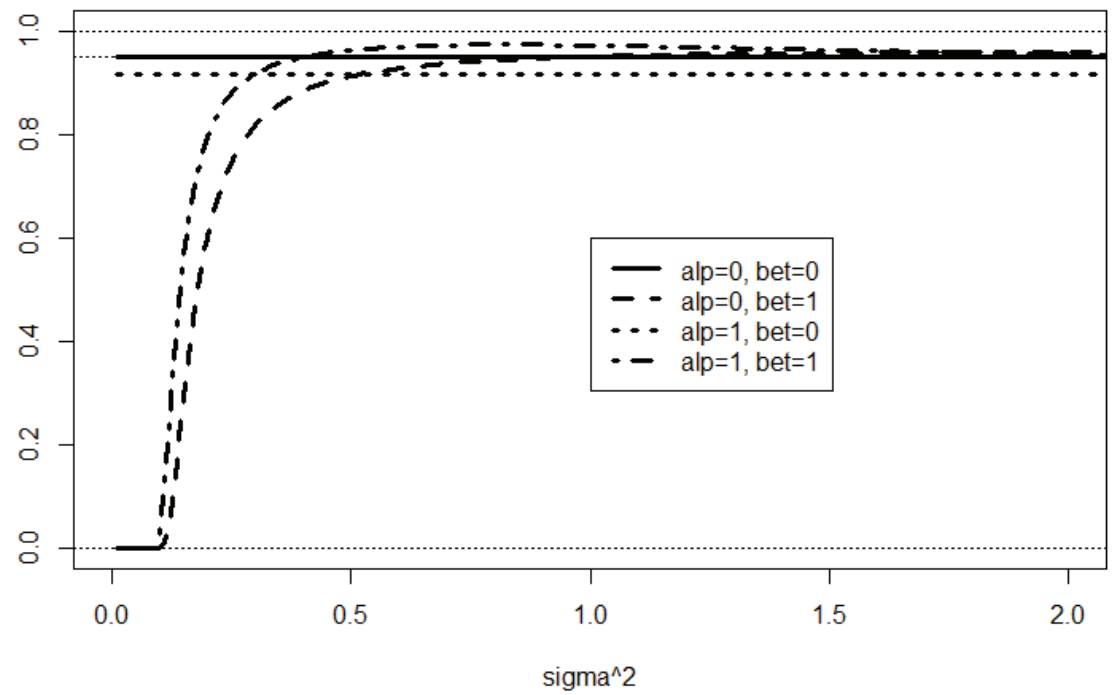

\section{R Code for Exercise 2.2}

biasfun $=$ function $(\operatorname{sig} 2, n=10$, alp $=0$, bet $=0)\{(2 *$ bet $+n * \operatorname{sig} 2) /(2 *$ alp $+n-2)$-sig2 $\}$

coverfun $=$ function $(\operatorname{sig} 2, n=10$, alp $=0$, bet $=0, A=0.05)\{$

$u=q \operatorname{chisq}(A / 2,2 * a l p+n) ; v=$ qchisq $(1-A / 2,2 * a l p+n)$

pchisq(v-2*bet/sig2, n) - pchisq(u-2*bet/sig2, n) \}

$\mathrm{X} 11(\mathrm{w}=8, \mathrm{~h}=5.5) ; \operatorname{par}(\operatorname{mfrow}=\mathrm{c}(1,1))$

sig2vec=seq $(0.01,5,0.01) ; n=10 ; \quad \operatorname{lpv}=c(0.1,1,5) ;$ betv $=c(0.1,1,5)$

plot(c(0,5),c(-2,1),type="n",xlab="sigma^2",ylab="',main=" ") abline $(\mathrm{h}=0, \mathrm{lt} y=3)$

lines(sig2vec, biasfun(sig2=sig2vec, alp=0,bet=0), Ity=1,lwd=3) lines(sig2vec, biasfun(sig2=sig2vec, alp=0, bet=1), Ity=2,lwd=3) lines(sig2vec, biasfun(sig2=sig2vec, alp=1, bet $=0$ ), Ity=3,lwd=3) lines(sig2vec, biasfun(sig2=sig2vec, alp=1, bet $=1$ ), Ity=4, Iwd=3) legend $(0,-0.5, c(" a l p=0$, bet=0","alp=0, bet=1","alp=1, bet=0","alp=1, bet=1"), Ity=1:4,lwd=rep $(3,4))$ 
plot(c(0,3),c(-1,6),type="n",xlab="sigma^2",ylab=" ",main=" ")

abline(h=0,lty=3); abline $(v=0$, tty=3)

lines(sig2vec, biasfun(sig2=sig2vec,alp=0, bet=0)/ sig2vec, Ity=1,lwd=3)

lines(sig2vec, biasfun(sig2=sig2vec,alp=0, bet=1)/ sig2vec, Ity=2,lwd=3)

lines(sig2vec,biasfun(sig2=sig2vec,alp=1, bet=0)/ sig2vec, Ity=3,lwd=3)

lines(sig2vec,biasfun(sig2=sig2vec,alp=1,bet=1)/ sig2vec, Ity=4,lwd=3)

legend(1.5,6,c("alp=0, bet=0","alp=0, bet=1","alp=1, bet=0","alp=1, bet=1"), Ity=1:4, Iwd=rep $(3,4))$

plot $(c(0,2), c(0,1)$, type="n",xlab="sigma^2",ylab="",main=" ")

abline $(h=c(0,0.95,1)$, lty=3)

lines(sig2vec, coverfun(sig2=sig2vec, $n=10$, al $p=0$, bet $=0, A=0.05),(t y=1, l w d=3$ ) lines(sig2vec, coverfun(sig2=sig2vec, $n=10$, al $p=0$, bet $=1, A=0.05$ ), Ity=2,lwd=3) lines(sig2vec, coverfun(sig2=sig2vec, $n=10$, alp $=1$, bet $=0, A=0.05$ ), Ity=3,lwd=3) lines(sig2vec, coverfun(sig2=sig2vec, $n=10$, alp=1, bet=1,A=0.05), Ity=4,lwd=3) legend(1,0.6,c("alp=0, bet=0","alp=0, bet=1","alp=1, bet=0","alp=1, bet=1"), Ity=1:4, Iwd=rep $(3,4))$

\subsection{Mixture prior distributions}

So far we have considered Bayesian models with priors that are limited in the types of prior information that they can represent. For example, the normal-normal model does not allow a prior for the normal mean which has two or more modes. If a non-normal class of prior is used to represent one's complicated prior beliefs regarding the normal mean, then that prior will not be conjugate, and this will lead to difficulties down the track when making inferences based on the nonstandard posterior distribution.

Fortunately, this problem can be addressed in any Bayesian model for which a conjugate class of prior exists by specifying the prior as a mixture of members of that class.

Generally, a random variable $X$ with a mixture distribution has a density of the form

$$
f(x)=\sum_{m=1}^{M} c_{m} f_{m}(x),
$$

where each $f_{m}(x)$ is a proper density and the $c_{m}$ values are positive and sum to 1 . 
If our prior beliefs regarding a parameter $\theta$ do not follow any single well-known distribution, those beliefs can in that case be conveniently approximated to any degree of precision by a suitable mixture prior distribution with a density having the form

$$
f(\theta)=\sum_{m=1}^{M} c_{m} f_{m}(\theta) .
$$

It can be shown (see Exercise 2.3 below) that if each component prior $f_{m}(\theta)$ is conjugate then $f(\theta)$ is also conjugate. This means that $\theta$ 's posterior distribution is also a mixture with density of the form

$$
f(\theta \mid y)=\sum_{m=1}^{M} c_{m}^{\prime} f_{m}(\theta \mid y),
$$

where $f_{m}(\theta \mid y)$ is the posterior implied by the $m$ th prior $f_{m}(\theta)$ and is from the same family of distributions as that prior.

\section{Exercise 2.3 Binomial-beta model with a mixture prior}

(a) Consider the following Bayesian model:

$$
\begin{aligned}
& (y \mid \theta) \sim \operatorname{Bin}(n, \theta) \\
& f(\theta)=k f_{\text {Beta }\left(a_{1}, b_{1}\right)}(\theta)+(1-k) f_{\text {Beta }\left(a_{2}, b_{2}\right)}(\theta),
\end{aligned}
$$

where $n, k$ and the $a_{i}, b_{i}$ are specified constants.

Note: Here, $f_{\text {Beta(a,b) }}(t)$ denotes the density at $t$ of the beta distribution with parameters $a$ and $b$ (and mean $a /(a+b)$ ).

Find the posterior distribution of $\theta$ and shows that $\theta$ 's prior is conjugate. Then create a figure showing the prior, likelihood and posterior for the situation defined by:

$$
n=5, k=3 / 4, a_{1}=8, b_{1}=25, a_{2}=20, b_{2}=20 \text { and } y=4 \text {. }
$$

Also calculate the prior mean of $\theta$, the posterior mean of $\theta$ and the MLE of $\theta$. Then mark these three points in the figure.

(b) Show that any mixture of conjugate priors is also conjugate and derive a general formula which could be used to calculate the mixture weights $c_{m}^{\prime}$ in (2.1) above. 


\section{Solution to Exercise 2.3}

(a) The posterior density is

$$
\begin{aligned}
& f(\theta \mid y) \propto f(\theta) f(y \mid \theta) \\
& =\left(k \frac{\theta^{a_{1}-1}(1-\theta)^{b_{1}-1}}{B\left(a_{1}, b_{1}\right)}+(1-k) \frac{\theta^{a_{2}-1}(1-\theta)^{b_{2}-1}}{B\left(a_{2}, b_{2}\right)}\right) \times \theta^{y}(1-\theta)^{n-y} \\
& =\left[k \frac{B\left(a_{1}+y, b_{1}+n-y\right)}{B\left(a_{1}, b_{1}\right)}\right]\left(\frac{\theta^{\left(a_{1}+y\right)-1}(1-\theta)^{\left(b_{1}+n-y\right)-1}}{B\left(a_{1}+y, b_{1}+n-y\right)}\right) \\
& +\left\{(1-k) \frac{B\left(a_{2}+y, b_{2}+n-y\right)}{B\left(a_{2}, b_{2}\right)}\right\}\left(\frac{\theta^{\left(a_{2}+y\right)-1}(1-\theta)^{\left(b_{2}+n-y\right)-1}}{B\left(a_{2}+y, b_{2}+n-y\right)}\right) .
\end{aligned}
$$

Thus

$$
f(\theta \mid y) \propto c_{1} f_{1}(\theta \mid y)+c_{2} f_{2}(\theta \mid y),
$$

where:

$$
\begin{aligned}
& c_{1}=k \frac{B\left(a_{1}+y, b_{1}+n-y\right)}{B\left(a_{1}, b_{1}\right)} \\
& c_{2}=(1-k) \frac{B\left(a_{2}+y, b_{2}+n-y\right)}{B\left(a_{2}, b_{2}\right)} \\
& f_{i}(\theta \mid y)=f_{\text {Beta }\left(a_{i}+y, b_{i}+n-y\right)}(\theta)=\frac{\theta^{\left(a_{i}+y\right)-1}(1-\theta)^{\left(b_{i}+n-y\right)-1}}{B\left(a_{i}+y, b_{i}+n-y\right)}, 0<\theta<1 \\
& \text { (the posterior density corresponding to } \theta \sim \operatorname{Beta}\left(a_{i}, b_{i}\right) \\
& \text { as prior). }
\end{aligned}
$$

Now,

$$
\int f(\theta \mid y) d \theta=1,
$$

and so

$$
f(\theta \mid y)=c f_{\text {Beta }\left(a_{1}+y, b_{1}+n-y\right)}(\theta)+(1-c) f_{\text {Beta }\left(a_{2}+y, b_{2}+n-y\right)}(\theta),
$$

where

$$
c=\frac{c_{1}}{C_{1}+c_{2}} .
$$


Note: This ensures that $\int f(\theta \mid y) d \theta=c \times 1+(1-c) \times 1=1$.

We see that the prior $f(\theta)$ and posterior $f(\theta \mid y)$ are in the same family, namely the family of mixtures of two beta distributions. Therefore the mixture prior is conjugate.

For the situation where

$$
n=5, k=3 / 4, a_{1}=8, b_{1}=25, a_{2}=20, b_{2}=20 \text { and } y=4,
$$

we find that:

- the prior mean is

$$
E \theta=k\left(\frac{a_{1}}{a_{1}+b_{1}}\right)+(1-k)\left(\frac{a_{2}}{a_{2}+b_{2}}\right)=0.3068
$$

- the maximum likelihood estimate is

$$
y / n=0.8
$$

- the posterior mean is

$$
E(\theta \mid y)=c\left(\frac{a_{1}+y}{a_{1}+b_{1}+n}\right)+(1-c)\left(\frac{a_{2}+y}{a_{2}+b_{2}+n}\right)=0.4772 .
$$

Figure 2.7 shows the prior density $f(\theta)$, the likelihood function $L(\theta)$, and the posterior density $f(\theta \mid y)$, as well as the prior mean, the MLE and the posterior mean.

Note: The likelihood function in Figure 2.7 has been normalised so that the area underneath it is exactly 1 . This means that this likelihood function is identical to the posterior density under the standard uniform prior, i.e. under $f_{U(0,1)}(\theta)=f_{\text {Beta }(1,1)}(\theta)$. Thus, $L(\theta)=f_{\text {Beta }(1+y, 1+n-y)}(\theta)$.

Figure 2.7 also shows the two component prior densities and the two component posterior densities. It may be observed that, whereas the lower component prior has the highest weight, 0.8, the opposite is the case regarding the component posteriors. For these, the weight associated with the lower posterior is only 0.2583 . This is because the inference is being 'pulled up' in the direction of the likelihood (with the posterior mean being between the prior mean and the MLE, 0.8). 
Figure 2.7 Densities and likelihood in Exercise 2.3

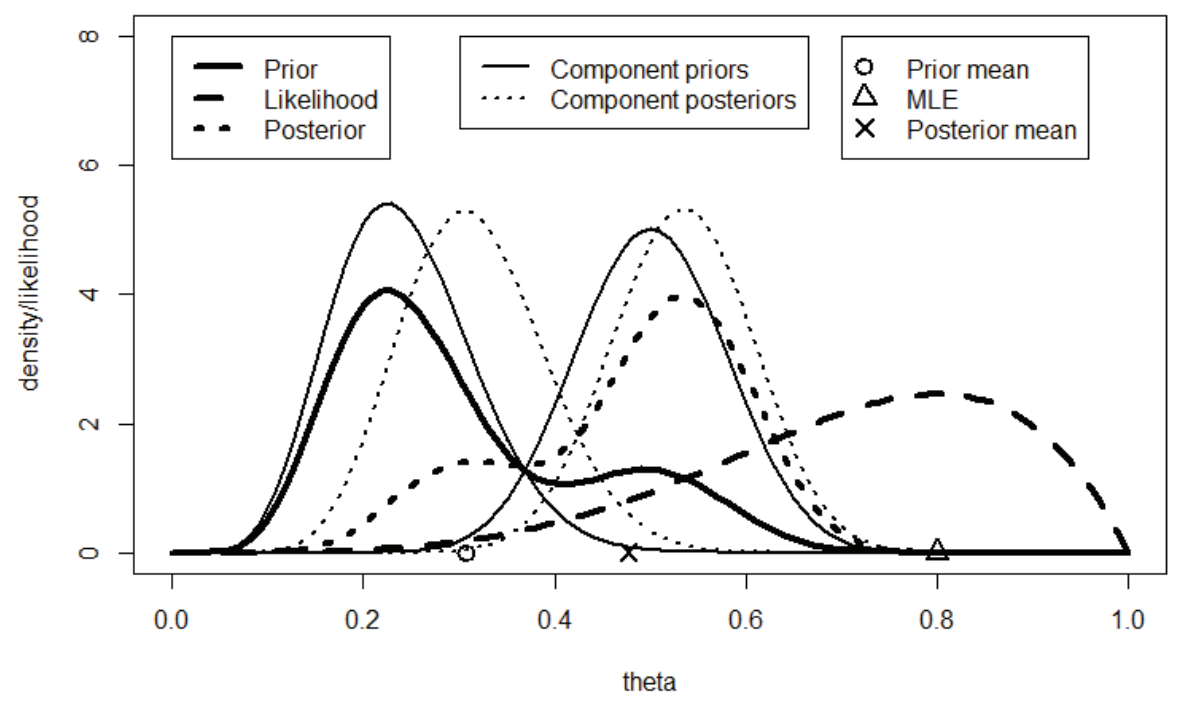

(b) Suppose that $\theta$ has a mixture prior of the general form

$$
f(\theta)=\sum_{m=1}^{M} c_{m} f_{m}(\theta)
$$

where each $f_{m}(\theta)$ is conjugate for the data model.

Then the posterior density is

$$
\begin{aligned}
f(\theta \mid y) & \propto f(\theta) f(y \mid \theta)=\left(\sum_{m=1}^{M} c_{m} f_{m}(\theta)\right) f(y \mid \theta) \\
= & \sum_{m=1}^{M}\left\{c_{m} f_{m}(\theta) f(y \mid \theta)\right\}=\sum_{m=1}^{M}\left\{\left(c_{m} f_{m}(y)\right)\left(\frac{f_{m}(\theta) f(y \mid \theta)}{f_{m}(y)}\right)\right\},
\end{aligned}
$$

where $f_{m}(y)=\int f_{m}(\theta) f(y \mid \theta) d \theta$ is the unconditional density of the data under the $m$ th prior, $f_{m}(\theta)$. Thus

$$
f(\theta \mid y) \propto \sum_{m=1}^{M} k_{m} f_{m}(\theta \mid y),
$$

where

$$
k_{m}=c_{m} f_{m}(y) \text { and } f_{m}(\theta \mid y)=\frac{f_{m}(\theta) f(y \mid \theta)}{f_{m}(y)}
$$

is the posterior density of $\theta$ under the $m$ th prior, $f_{m}(\theta)$. 
It follows that

$$
f(\theta \mid y)=\sum_{m=1}^{M} c_{m}^{\prime} f_{m}(\theta \mid y),
$$

where $c_{m}^{\prime}=k_{m} /\left(k_{1}+\ldots+k_{M}\right)$.

Thus $\theta$ 's posterior is a mixture of distributions from the same families to which the components of $\theta$ 's mixture prior belong, respectively. This shows that $\theta$ 's mixture prior is conjugate. Note that the component prior distributions can be from different classes, so long as each is conjugate in relation to its own class.

\section{R Code for Exercise 2.3}

$n=5 ; k=3 / 4 ; a 1=8 ; b 1=25 ; a 2=20 ; b 2=20 ; y=4$; thetav=seq $(0,1,0.01)$

prior $1=\mathrm{dbeta}($ thetav $, \mathrm{a} 1, \mathrm{~b} 1)$; prior $2=\mathrm{dbeta}($ thetav $, \mathrm{a} 2, \mathrm{~b} 2)$

post $1=d b e t a($ thetav $, a 1+y, b 1+n-y) ;$ post $2=d b e t a($ thetav, $a 2+y, b 2+n-y)$

prior $=k^{*}$ prior $1+(1-k) *$ prior2

$c 1=k^{*}$ beta $(a 1+y, b 1+n-y) /$ beta $(a 1, b 1) ; c 2=(1-k) * b e t a(a 2+y, b 2+n-y) / b e t a(a 2, b 2)$

$\mathrm{c}=\mathrm{c} 1 /(\mathrm{c} 1+\mathrm{c} 2) ;$ post=c*post1 + (1-c)* post2; options(digits=4); $\mathrm{c}$ \# 0.2583

like=dbeta(thetav, $1+y, 1+n-y)$ \# likelihood = post. under $U(0,1)=$ beta $(1,1)$ prior

$\mathrm{X} 11(\mathrm{w}=8, \mathrm{~h}=5.5)$

$\operatorname{plot}(\mathrm{c}(0,1), c(0,8)$,type="n",xlab="theta",ylab="density/likelihood")

lines(thetav, prior, Ity=1,lwd=4)

lines(thetav, like, Ity $=2, I w d=4$ )

lines(thetav, post, Ity $=3$, Iwd $=4$ )

legend(0,8,c("Prior","Likelihood","Posterior"),Ity=c(1,2,3),Iwd=c(4,4,4))

lines(thetav, prior 1, Ity $=1, \operatorname{lwd}=2$ )

lines(thetav, prior $2, \mathrm{lty}=1, \mathrm{lwd}=2$ )

lines(thetav, post1, Ity=3, Iwd=2)

lines(thetav, post2, Ity=3, Iwd $=2$ )

legend( $0.3,8, c(" C o m p o n e n t$ priors","Component posteriors"),

$\operatorname{Ity}=c(1,3), \mid \mathrm{w} d=c(2,2))$

mle $=y / n ;$ priormean $=k^{*} a 1 /(a 1+b 1)+(1-k) * a 2 /(a 2+b 2)$

postmean $=c^{*}(a 1+y) /(a 1+b 1+n)+(1-c) *(a 2+y) /(a 2+b 2+n)$

points (c(priormean, mle, postmean), $c(0,0,0), p c h=c(1,2,4), c e x=c(1.5,1.5,1.5)$, $\operatorname{lwd}=c(2,2,2))$

c(priormean, mle, postmean) \# 0.30680 .80000 .4772

legend(0.7,8,c(" Prior mean"," MLE"," Posterior mean"), $\mathrm{pch}=c(1,2,4)$, pt.cex $=c(1 \cdot 5,1 \cdot 5,1 \cdot 5)$, pt.Iwd=c(2,2,2)) 


\subsection{Dealing with a priori ignorance}

The Bayesian approach requires a prior distribution to be specified even when there is complete (or total) a priori ignorance (meaning no prior information at all). This feature presents a general and philosophical problem with the Bayesian paradigm, one for which several theoretical solutions have been advanced but which does not yet have a universally accepted solution. We have already discussed finding an uninformative prior in relation to particular Bayesian models, as follows.

For the normal-normal model defined by $\left(y_{1}, \ldots, y_{n} \mid \mu\right) \sim$ iid $N\left(\mu, \sigma^{2}\right)$ and $\mu \sim N\left(\mu_{0}, \sigma_{0}^{2}\right)$, an uninformative prior is given by $\sigma_{0}=\infty$, that is, $f(\mu) \propto 1, \mu \in \Re$.

For the normal-gamma model defined by $\left(y_{1}, \ldots, y_{n} \mid \mu\right) \sim$ iid $N(\mu, 1 / \lambda)$ and $\lambda \sim \operatorname{Gamma}(\alpha, \beta)$, an uninformative prior is given by $\alpha=\beta=0$, that is, $f(\lambda) \propto 1 / \lambda, \lambda>0$.

For the binomial-beta model defined by $(y \mid \theta) \sim \operatorname{Binomial}(n, \theta)$ and $\theta \sim \operatorname{Beta}(\alpha, \beta)$ (having the posterior $(\theta \mid y) \sim \operatorname{Beta}(\alpha+y, \beta+n-y)$ ), an uninformative prior is the Bayes prior given by $\alpha=\beta=1$, that is, $f(\theta)=1,0<\theta<1$. This is the prior that was originally advocated by Thomas Bayes.

Unlike for the normal-normal and normal-gamma models, more than one uninformative prior specification has been proposed as reasonable in the context of the binomial-beta model.

One of these is the improper Haldane prior, defined by $\alpha=\beta=0$, or

$$
f(\theta) \propto \frac{1}{\theta(1-\theta)}, \quad 0<\theta<1 .
$$

Under the prior $\theta \sim \operatorname{Beta}(\alpha, \beta)$ generally, the posterior mean of $\theta$ is

$$
\hat{\theta}=E(\theta \mid y)=\frac{(\alpha+y)}{(\alpha+y)+(\beta+n-y)}=\frac{\alpha+y}{\alpha+\beta+n} .
$$

This reduces to the MLE $y / n$ under the Haldane prior but not under the Bayes prior. In contrast, the Bayes prior leads to a posterior mode which is equal to the MLE. 
The Haldane prior may be considered as being most appropriate for allowing the data to 'speak for itself' in cases of a priori ignorance.

However, the Haldane prior leads to an improper and degenerate posterior if the data $y$ happens to be either 0 or $n$. Specifically:

$$
\begin{aligned}
& y=0 \Rightarrow(\theta \mid y) \sim \operatorname{Beta}(0, n), \text { or equivalently, } P(\theta=0 \mid y)=1 \\
& y=n \Rightarrow(\theta \mid y) \sim \operatorname{Beta}(n, 0), \text { or equivalently, } P(\theta=1 \mid y)=1 .
\end{aligned}
$$

So in each case, point estimation is possible but not interval estimation.

No such problems occur using the Bayes prior. This is because that prior is proper and so cannot lead to an improper posterior, whatever the data may be. Interestingly, there is a third choice which provides a kind of compromise between the Bayes and Haldane priors, as described below.

\subsection{The Jeffreys prior}

The statistician Harold Jeffreys devised a rule for finding a suitable uninformative prior in a wide variety of situations. His idea was to construct a prior which is invariant under reparameterisation. For the case of a univariate model parameter $\theta$, the Jeffreys prior is given by the following equation (also known as Jeffreys' rule):

$$
f(\theta) \propto \sqrt{I(\theta)},
$$

where $I(\theta)$ is the Fisher information defined by

$$
I(\theta)=E\left\{\left(\frac{\partial}{\partial \theta} \log f(y \mid \theta)\right)^{2} \mid \theta\right\} .
$$

Note 1: If $\log f(y \mid \theta)$ is twice differentiable with respect to $\theta$, and certain regularity conditions hold, then

$$
I(\theta)=-E\left\{\frac{\partial^{2}}{\partial \theta^{2}} \log f(y \mid \theta) \mid \theta\right\} .
$$

Note 2: Jeffreys' rule also extends to the multi-parameter case (not considered here).

The significance of Jeffreys' rule may be described as follows. Consider a prior given by $f(\theta) \propto \sqrt{I(\theta)}$ and the transformed parameter $\phi=g(\theta)$, 
where $g$ is a strictly increasing or decreasing function. (For simplicity, we only consider this case.) Then the prior density for $\phi$ is

$$
\begin{aligned}
f(\phi) & \propto f(\theta)\left|\frac{\partial \theta}{\partial \phi}\right| \text { by the transformation rule } \\
& \propto \sqrt{I(\theta)\left(\frac{\partial \theta}{\partial \phi}\right)^{2}}=\sqrt{E\left\{\left(\frac{\partial}{\partial \theta} \log f(y \mid \theta)\right)^{2} \mid \theta\right\}\left(\frac{\partial \theta}{\partial \phi}\right)^{2}} \\
& =\sqrt{E\left\{\left(\frac{\partial}{\partial \theta} \log f(y \mid \theta) \frac{\partial \theta}{\partial \phi}\right)^{2} \mid \theta\right\}} \\
& =\sqrt{E\left\{\left(\frac{\partial}{\partial \phi} \log f(y \mid \phi)\right)^{2} \mid \phi\right\}} \\
& =\sqrt{I(\phi)} .
\end{aligned}
$$

Thus, Jeffreys' rule is 'invariant under reparameterisation', in the sense that if a prior is constructed according to

$$
f(\theta) \propto \sqrt{I(\theta)},
$$

then, for another parameter $\phi=g(\theta)$, it is also true that

$$
f(\phi) \propto \sqrt{I(\phi)} .
$$

\section{Exercise 2.4 Jeffreys prior for the normal-normal model}

Find the Jeffreys prior for $\mu$ if $\left(y_{1}, \ldots, y_{n} \mid \mu\right) \sim$ iid $N\left(\mu, \sigma^{2}\right)$, where $\sigma$ is known.

\section{Solution to Exercise 2.4}

Here: $\quad f(y \mid \mu) \propto \prod_{i=1}^{\mu} \exp \left\{-\frac{1}{2 \sigma^{2}}\left(y_{i}-\mu\right)^{2}\right\}=\exp \left\{-\frac{1}{2 \sigma^{2}} \sum_{i=1}^{n}\left(y_{i}-\mu\right)^{2}\right\}$ $\log f(y \mid \mu)=-\frac{1}{2 \sigma^{2}} \sum_{i=1}^{n}\left(y_{i}-\mu\right)^{2}+c$ (where $c$ is a constant) $\frac{\partial}{\partial \mu} \log f(y \mid \mu)=-\frac{1}{2 \sigma^{2}} \sum_{i=1}^{n} 2\left(y_{i}-\mu\right)^{1}(-1)=\frac{n}{\sigma^{2}}(\bar{y}-\mu)$ $\left(\frac{\partial}{\partial \mu} \log f(y \mid \mu)\right)^{2}=\frac{n^{2}}{\sigma^{4}}(\bar{y}-\mu)^{2}$. 
Therefore the Fisher information is

$$
\begin{aligned}
I(\mu)=E\left\{\left(\frac{\partial}{\partial \mu} \log f(y \mid \mu)\right)^{2} \mid \mu\right\} & =E\left\{\frac{n^{2}}{\sigma^{4}}(\bar{y}-\mu)^{2} \mid \mu\right\} \\
& =\frac{n^{2}}{\sigma^{4}} V(\bar{y} \mid \mu)=\frac{n^{2}}{\sigma^{4}} \frac{\sigma^{2}}{n}=\frac{n}{\sigma^{2}} .
\end{aligned}
$$

It follows that the Jeffreys prior is $f(\mu) \propto \sqrt{I(\mu)}=\sqrt{\frac{n}{\sigma^{2}}} \stackrel{\mu}{\propto} 1, \mu \in \mathfrak{R}$.

Note 1: This is the same prior as used earlier in the uninformative case.

Note 2: The Fisher information here can also be derived as follows:

$$
\begin{aligned}
& \frac{\partial^{2}}{\partial \mu^{2}} \log f(y \mid \mu)=-\frac{n}{\sigma^{2}} \\
\Rightarrow & I(\mu)=-E\left\{\frac{\partial^{2}}{\partial \theta^{2}} \log f(y \mid \theta) \mid \theta\right\}=-E\left(-\frac{n}{\sigma^{2}}\right)=\frac{n}{\sigma^{2}} .
\end{aligned}
$$

\section{Exercise 2.5 Jeffreys prior for the normal-gamma model}

Find the Jeffreys prior for $\lambda$ if $\left(y_{1}, \ldots, y_{n} \mid \lambda\right) \sim$ iid $N(\mu, 1 / \lambda)$, where $\mu$ is known.

\section{Solution to Exercise 2.5}

Here: $f(y \mid \lambda))^{\lambda} \prod_{i=1}^{n} \lambda^{1 / 2} \exp \left\{-\frac{\lambda}{2}\left(y_{i}-\mu\right)^{2}\right\}=\lambda^{n / 2} \exp \left\{-\frac{\lambda}{2} \sum_{i=1}^{n}\left(y_{i}-\mu\right)^{2}\right\}$

$$
\begin{aligned}
& \log f(y \mid \lambda)=\frac{n}{2} \log \lambda-\frac{\lambda}{2} \sum_{i=1}^{n}\left(y_{i}-\mu\right)^{2}+c \text { (where } c \text { is a constant) } \\
& \frac{\partial \log f(y \mid \lambda)}{\partial \lambda}=\frac{n}{2 \lambda}-\frac{1}{2} \sum_{i=1}^{n}\left(y_{i}-\mu\right)^{2}, \quad \frac{\partial^{2} \log f(y \mid \lambda)}{\partial \lambda^{2}}=-\frac{n}{2 \lambda^{2}} .
\end{aligned}
$$

So the Fisher information is

$$
I(\lambda)=-E\left\{\frac{\partial^{2} \log f(y \mid \lambda)}{\partial \lambda^{2}} \mid \lambda\right\}=-E\left\{-\frac{n}{2 \lambda^{2}} \mid \lambda\right\}=\frac{n}{2 \lambda^{2}} .
$$


So the Jeffreys prior is $f(\lambda) \propto \sqrt{I(\lambda)}=\sqrt{\frac{n}{2 \lambda^{2}}} \stackrel{\lambda}{\propto} \frac{1}{\lambda}, \lambda \in \mathfrak{R}$.

Note 1: This is the same prior as used earlier in the uninformative case.

Note 2: Another way to obtain the the Fisher information is to first write

$$
\frac{\partial \log f(y \mid \lambda)}{\partial \lambda}=\frac{n}{2 \lambda}-\frac{1}{2 \lambda}\left\{\lambda \sum_{i=1}^{n}\left(y_{i}-\mu\right)^{2}\right\}=\frac{1}{2 \lambda}(n-q)
$$

where: $q=\sum_{i=1}^{n}\left(\frac{y_{i}-\mu}{1 / \sqrt{\lambda}}\right)^{2},(q \mid \lambda) \sim \chi^{2}(n), E(q \mid \lambda)=n, V(q \mid \lambda)=2 n$.

We may then write $\left(\frac{\partial \log f(y \mid \lambda)}{\partial \lambda}\right)^{2}=\frac{1}{4 \lambda^{2}}\left(n^{2}-2 n q+q^{2}\right)$, and so the Fisher information is $I(\lambda)=E\left\{\left(\frac{\partial \log f(y \mid \lambda)}{\partial \lambda}\right)^{2} \mid \lambda\right\}$

$$
=\frac{1}{4 \lambda^{2}}\left\{n^{2}-2 n E(q \mid \lambda)+E\left(q^{2} \mid \lambda\right)\right\}=\frac{1}{4 \lambda^{2}}\left\{n^{2}-2 n n+\left[2 n+n^{2}\right]\right\}=\frac{n}{2 \lambda^{2}} .
$$

\section{Exercise 2.6 Jeffreys prior for the binomial-beta model}

Find the Jeffreys prior for $\theta$ if $(y \mid \theta) \sim \operatorname{Binomial}(n, \theta)$, where $n$ is known.

\section{Solution to Exercise 2.6}

Here: $\quad f(y \mid \theta)=\left(\begin{array}{l}n \\ y\end{array}\right) \theta^{y}(1-\theta)^{n-y}$

$$
\begin{aligned}
& \log f(y \mid \theta)=\log \left(\begin{array}{l}
n \\
y
\end{array}\right)+y \log \theta+(n-y) \log (1-\theta) \\
& \frac{\partial}{\partial \theta} \log f(y \mid \theta)=0+y \theta^{-1}-(n-y)(1-\theta)^{-1} \\
& \frac{\partial^{2}}{\partial \theta^{2}} \log f(y \mid \theta)=-y \theta^{-2}-(n-y)(1-\theta)^{-2} .
\end{aligned}
$$


So the Fisher information is

$$
\begin{aligned}
I(\theta) & =-E\left\{\frac{\partial^{2}}{\partial \theta^{2}} \log f(y \mid \theta) \mid \theta\right\} \\
& =-E\left\{-y \theta^{-2}-(n-y)(1-\theta)^{-2} \mid \theta\right\} \\
& =(n \theta) \theta^{-2}+(n-n \theta)(1-\theta)^{-2} \\
& =n\left(\frac{1}{\theta}+\frac{1}{1-\theta}\right)=n \frac{(1-\theta+\theta)}{\theta(1-\theta)}=\frac{n}{\theta(1-\theta)} .
\end{aligned}
$$

It follows that the Jeffreys prior is given by

$$
f(\theta) \propto \sqrt{I(\theta)}=\sqrt{\frac{n}{\theta(1-\theta)}} \propto \frac{\theta}{\sqrt{\theta(1-\theta)}}, 0<\theta<1 .
$$

Note: We may also write the Jeffreys prior density as

$$
f(\theta) \propto \theta^{\frac{1}{2}-1}(1-\theta)^{\frac{1}{2}-1}, 0<\theta<1 .
$$

Thus the Jeffreys prior can be specified by writing

$$
\theta \sim \operatorname{Beta}(\alpha, \beta)
$$

with $\alpha=\beta=1 / 2$.

We see that the Jeffreys prior may be thought of as 'half-way' between:

- the Bayes prior, defined by $\alpha=\beta=1$; and

- the Haldane prior, defined by $\alpha=\beta=0$.

\section{Exercise 2.7 Jeffreys prior for the tramcar problem}

Recall the discussion of the tramcar problem following Exercise 1.6, in relation to the model $(y \mid \theta) \sim D U(1, \ldots, \theta)$. Find the Jeffreys prior for $\theta$.

\section{Solution to Exercise 2.7}

Here,

$$
\begin{aligned}
& f(y \mid \theta)=1 / \theta=\theta^{-1} \\
\Rightarrow \quad & \log f(y \mid \theta)=-\log \theta \\
\Rightarrow & \frac{\partial}{\partial \theta} \log f(y \mid \theta)=-\frac{1}{\theta}
\end{aligned}
$$




$$
\begin{aligned}
& \Rightarrow \quad\left(\frac{\partial}{\partial \theta} \log f(y \mid \theta)\right)^{2}=\frac{1}{\theta^{2}} \\
& \Rightarrow I(\theta)=E\left\{\left(\frac{\partial}{\partial \theta} \log f(y \mid \theta)\right)^{2} \mid \theta\right\}=\frac{1}{\theta^{2}} .
\end{aligned}
$$

It follows that the Jeffreys prior for $\theta$ is given by

$$
f(\theta) \propto \sqrt{I(\theta)} \propto 1 / \theta, \theta=1,2,3, \ldots
$$

\subsection{Bayesian decision theory}

The posterior mean, mode and median, as well as other Bayesian point estimates, can all be derived and interpreted using the principles and theory of decision theory. Suppose we wish to choose an estimate of $\theta$ which minimises costs in some sense. To this end, let $L(\hat{\theta}, \theta)$ denote generally a loss function (LF) associated with an estimate $\hat{\theta}$.

Note: The estimator $\hat{\theta}$ is a function of the data $y$ and so could also be written $\hat{\theta}(y)$. For example, in the context where $(y \mid \theta) \sim \operatorname{Bin}(n, \theta)$, the sample proportion or MLE is the function given by $\hat{\theta}=\hat{\theta}(y)=y / n$.

The loss function $L$ represents the cost incurred when the true value $\theta$ is estimated by $\hat{\theta}$ and usually satisfies the property $L(\theta, \theta)=0$.

The three most commonly used loss functions are defined as follows:

$$
\begin{aligned}
& L(\hat{\theta}, \theta)=|\hat{\theta}-\theta| \quad \begin{array}{l}
\text { the absolute error loss function (AELF) } \\
L(\hat{\theta}, \theta)=(\hat{\theta}-\theta)^{2} \quad \text { the quadratic error loss function (QELF) }
\end{array} \\
& L(\hat{\theta}, \theta)=I(\hat{\theta} \neq \theta)=\left\{\begin{array}{ll}
0 & \text { if } \hat{\theta}=\theta \\
1 & \text { if } \hat{\theta} \neq \theta
\end{array}\right\} \text { the indicator error loss } \\
& \text { function (IELF), also known as the zero-one loss function } \\
& \text { (ZOLF) or the all-or-nothing error loss function (ANLF). }
\end{aligned}
$$

Figures 2.8 and 2.9 illustrate these three basic loss functions. 
Figure 2.8 The three most important loss functions
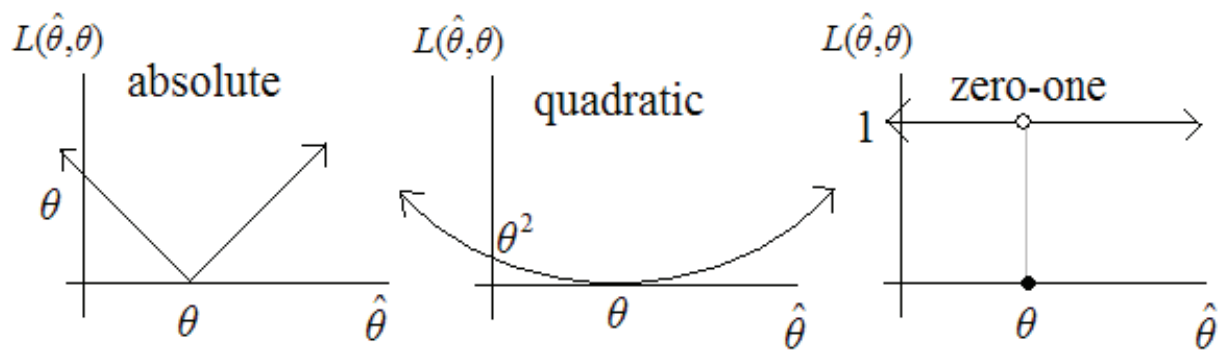

Figure 2.9 Alternative representation of the absolute error loss function

(The other two loss functions can be represented similarly)

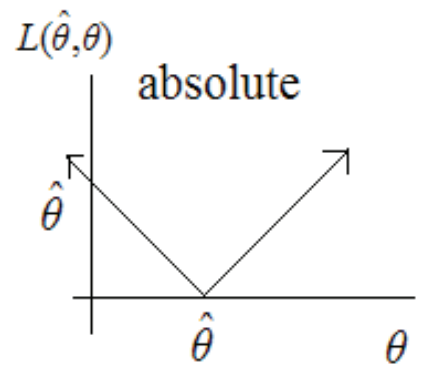

Given a Bayesian model, loss function and estimator, we would like to quantify what the loss is likely to be. However, this loss depends on $\theta$ and $y$, which complicates things. An idea of the expected loss may be provided by the risk function, defined as the conditional expectation

$$
R(\theta)=E(L(\hat{\theta}, \theta) \mid \theta)=\int L(\hat{\theta}(y), \theta) f(y \mid \theta) d y .
$$

The risk function $R(\theta)$ provides us with an idea of the expected loss given any particular value of $\theta$. Figure 2.10 illustrates the idea. 


\section{Figure 2.10 The idea of a risk function}

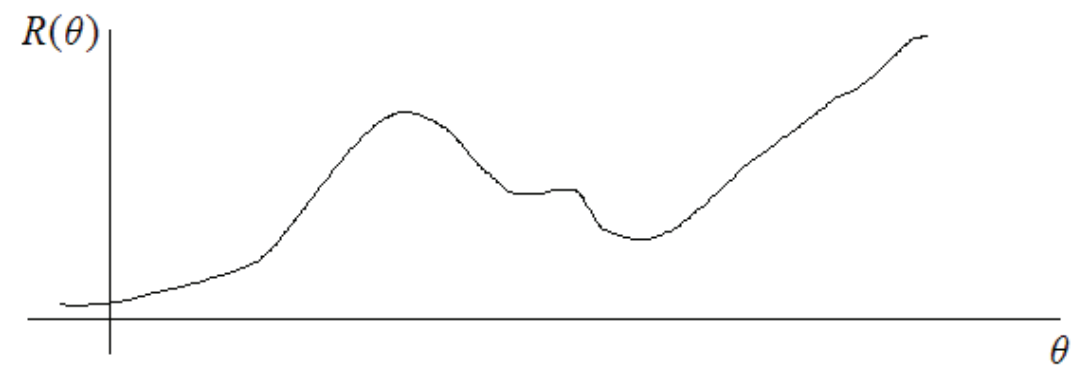

To obtain the overall expected loss we need to average the risk function over all possible values of $\theta$. This overall expected loss is called the Bayes risk and may be defined as

$$
r=E L(\hat{\theta}, \theta)=E E\{L(\hat{\theta}, \theta) \mid \theta\}=E R(\theta)=\int R(\theta) f(\theta) d \theta .
$$

\section{Exercise 2.8 Examples of the risk function and Bayes risk}

Consider the normal-normal model: $\left(y_{1}, \ldots, y_{n} \mid \mu\right) \sim$ iid $N\left(\mu, \sigma^{2}\right)$

$$
\mu \sim N\left(\mu_{0}, \sigma_{0}^{2}\right) \text {. }
$$

For each of the following estimators, derive a formulae for the risk function under the quadratic error loss function:
(a) $\hat{\mu}=\bar{y}=\frac{1}{n}\left(y_{1}+\ldots+y_{n}\right) \quad$ (the sample mean)
(b) $\hat{\mu}=|\bar{y}| \quad$ (the absolute value of the sample mean).

In each case, use the derived risk function to determine the Bayes risk.

\section{Solution to Exercise 2.8}

For both parts of this exercise, the loss function is given by

$$
L(\hat{\mu}, \mu)=(\hat{\mu}-\mu)^{2} .
$$

(a) If $\hat{\mu}=\bar{y}$ then the risk function is

$$
\begin{aligned}
R(\mu)=E\{L(\hat{\mu}, \mu) \mid \mu\}=E\left\{(\bar{y}-\mu)^{2} \mid \mu\right\} & =V(\bar{y} \mid \mu) \\
& =\sigma^{2} / n \text { (a constant). }
\end{aligned}
$$


So the Bayes risk is simply

$$
r=E R(\mu)=E\left(\sigma^{2} / n\right)=\sigma^{2} / n \quad \text { (i.e. the same constant). }
$$

(b) If $\hat{\mu}=|\bar{y}|$ then the risk function is

$$
\begin{aligned}
R(\mu) & =E\left\{(|\bar{y}|-\mu)^{2} \mid \mu\right\}=E\left\{|\bar{y}|^{2}-2 \mu|\bar{y}|+\mu^{2} \mid \mu\right\} \\
& =E\left(\bar{y}^{2} \mid \mu\right)-2 \mu E(\mid \bar{y} \| \mu)+\mu^{2} \\
& =\left(\frac{\sigma^{2}}{n}+\mu^{2}\right)-2 \mu m+\mu^{2} \text {, where } m=E(\mid \bar{y} \| \mu) .
\end{aligned}
$$

Now,

$$
\begin{gathered}
m=\int_{-\infty}^{0}(-\bar{y}) f(\bar{y} \mid \mu) d \bar{y}+\int_{0}^{\infty}(+\bar{y}) f(\bar{y} \mid \mu) d \bar{y} \\
=-\int_{-\infty}^{0} \bar{y} f(\bar{y} \mid \mu) d \bar{y}+\int_{0}^{\infty} \bar{y} f(\bar{y} \mid \mu) d \bar{y}+\int_{-\infty}^{0} \bar{y} f(\bar{y} \mid \mu) d \bar{y}-\int_{-\infty}^{0} \bar{y} f(\bar{y} \mid \mu) d \bar{y} \\
=-2 \int_{-\infty}^{0} \bar{y} f(\bar{y} \mid \mu) d \bar{y}+\int_{-\infty}^{\infty} \bar{y} f(\bar{y} \mid \mu) d \bar{y} \\
=\mu-2 I, \quad \text { where } I=\int_{-\infty}^{0} \bar{y} f(\bar{y} \mid \mu) d \bar{y} .
\end{gathered}
$$

Here,

$$
\begin{aligned}
I & =\int_{-\infty}^{-\mu / c}(\mu+c z) \phi(z) d z \text { after putting } z=\frac{\bar{y}-\mu}{\sigma / \sqrt{n}} \text { with } c=\frac{\sigma}{\sqrt{n}} \\
& =\mu \int_{-\infty}^{-\mu / c} \phi(z) d z+c \int_{-\infty}^{-\mu / c} z \phi(z) d z \\
& =\mu \Phi\left(-\frac{\mu}{c}\right)+c J, \quad \text { where } \quad J=\int_{-\infty}^{-\mu / c} z \phi(z) d z .
\end{aligned}
$$

Note: Here, $\phi(z)=\frac{1}{\sqrt{2 \pi}} e^{-\frac{1}{2} z^{2}}$ and $\Phi(z)=\int_{-\infty}^{z} \phi(t) d t$ are the standard normal pdf and cdf, respectively. 
Now, $\quad J=\int_{-\infty}^{-\mu / c} z \frac{1}{\sqrt{2 \pi}} e^{-\frac{1}{2} z^{2}} d z$

$$
\begin{aligned}
& =\int_{\infty}^{\frac{\mu^{2}}{2 c^{2}}} \frac{1}{\sqrt{2 \pi}} e^{-w} d w \quad \text { after substituting } w=\frac{1}{2} z^{2} \\
& =\frac{1}{\sqrt{2 \pi}}\left[-\left.e^{-w}\right|_{w=\infty} ^{\frac{\mu^{2}}{2 c^{2}}}\right]=\frac{1}{\sqrt{2 \pi}}\left(-e^{-\frac{\mu^{2}}{2 c^{2}}}+e^{-\infty}\right)=-\phi\left(\frac{\mu}{c}\right) .
\end{aligned}
$$

Hence $\quad I=\mu \Phi\left(-\frac{\mu}{c}\right)+c J=\mu \Phi\left(-\frac{\mu}{c}\right)-c \phi\left(\frac{\mu}{c}\right)$,

and so $m=\mu-2 I=\mu-2\left[\mu \Phi\left(-\frac{\mu}{c}\right)-c \phi\left(\frac{\mu}{c}\right)\right]$.

Therefore

$$
R(\mu)=\frac{\sigma^{2}}{n}+2 \mu^{2}-2 \mu m=\frac{\sigma^{2}}{n}+2 \mu^{2}-2 \mu\left\{\not h-2\left[\mu \Phi\left(-\frac{\mu}{c}\right)-c \phi\left(\frac{\mu}{c}\right)\right]\right\} .
$$

Thereby we obtain:

$$
R(\mu)=\frac{\sigma^{2}}{n}+4 \mu^{2} \Phi\left(-\frac{\mu}{\sigma / \sqrt{n}}\right)-4 \mu \frac{\sigma}{\sqrt{n}} \phi\left(\frac{\mu}{\sigma / \sqrt{n}}\right), \mu \in \Re .
$$

The Bayes risk is then given by

$$
r=E R(\mu)=\int R(\mu) f(\mu) d \mu=\int_{-\infty}^{\infty} g(\mu) d \mu,
$$

where

$$
g(\mu)=\left\{\frac{\sigma^{2}}{n}+4 \mu^{2} \Phi\left(-\frac{\mu}{\sigma / \sqrt{n}}\right)-4 \mu \frac{\sigma}{\sqrt{n}} \phi\left(\frac{\mu}{\sigma / \sqrt{n}}\right)\right\} \times \frac{1}{\sigma_{0}} \phi\left(\frac{\mu-\mu_{0}}{\sigma_{0}}\right) .
$$

We see that the Bayes risk $r$ is an intractable integral equal to the area under the integrand, $g(\mu)=R(\mu) f(\mu)$. However, this area can be evaluated numerically (using techniques discussed later). Figures 2.11 and 2.12 show examples of the risk function $R(\mu)$ and the integrand function $g(\mu)$. For the case $n=\sigma=\mu_{0}=\sigma_{0}=1$, we find that $r=1.16$. 
Figure 2. I I Some risk functions in Exercise 2.8

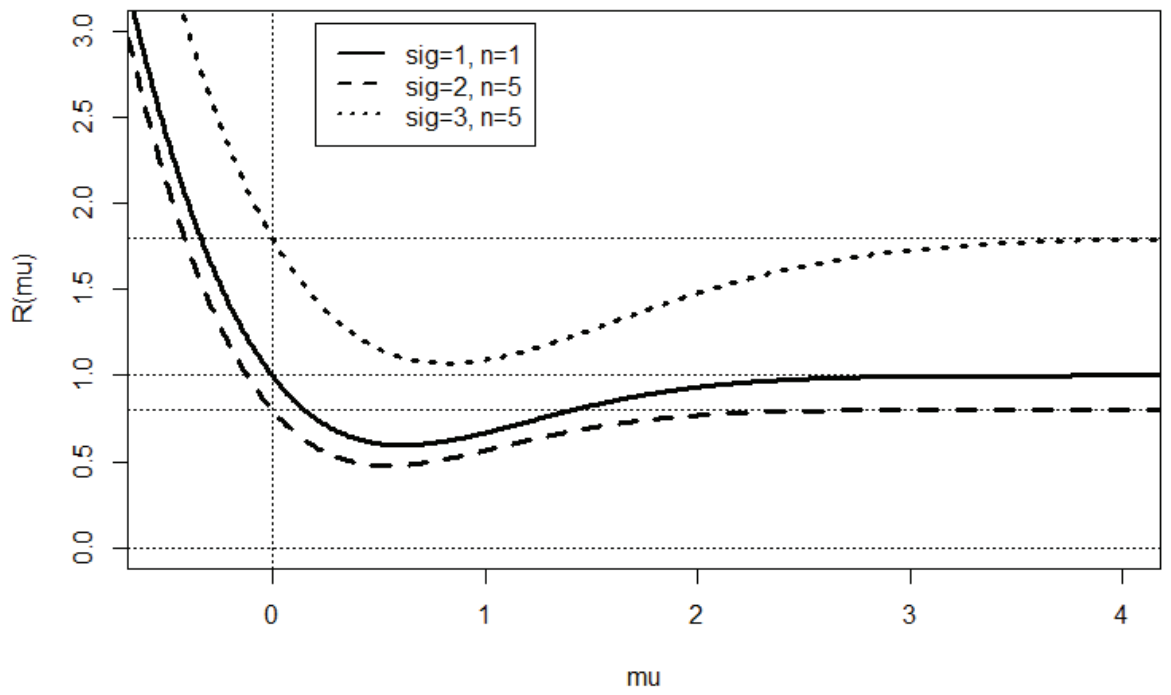

Figure 2.12 Some integrand functions used to calculate the Bayes risk

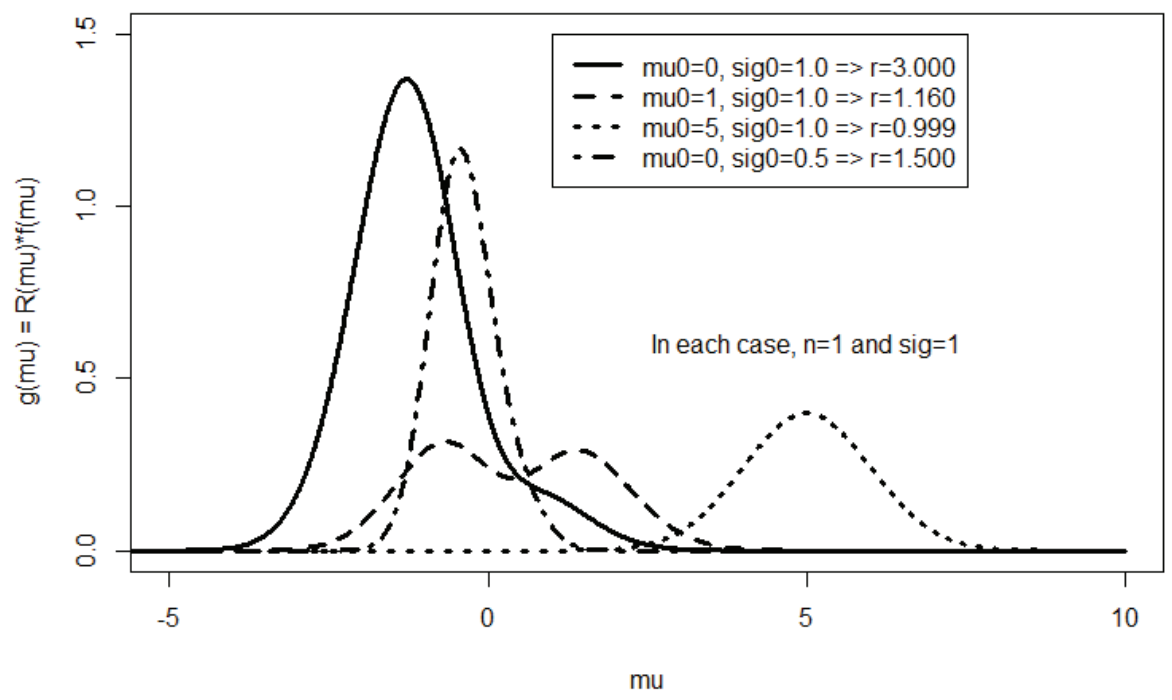




\section{R Code for Exercise 2.8}

Rfun=function(mu,sig,n)\{ sig^2/n+4*mu*( mu*pnorm(-mu/(sig/sqrt(n))) $(\operatorname{sig} / \operatorname{sqrt}(\mathrm{n}))^{*} \operatorname{dnorm}(\mathrm{mu} /(\operatorname{sig} / \mathrm{sqrt}(\mathrm{n}))) \quad$ )

muvec=seq $(-10,10,0.01)$; options $($ digits $=4)$

$X 11(w=8, h=5.5) ; \operatorname{par}(\operatorname{mfrow}=c(1,1)) ;$

plot(c(-0.5,4),c(0,3),type="n",xlab="mu",ylab="R(mu)",main=" ")

$\mathrm{n}=1$; sig=1; lines(muvec, Rfun (muvec,sig=sig, $n=n$ ), Ity=1, Iwd=3);

abline $(v=0$, tty=3); abline $(h=c(0$, sig^ $2 / n)$, tty=3)

$n=5$; sig=2; lines(muvec, Rfun (muvec,sig=sig, $n=n$ ), lty=2,lwd=3);

abline $\left(\mathrm{h}=\operatorname{sig}^{\wedge} 2 / \mathrm{n}\right.$, tty $=3$ )

$n=5$; sig=3; lines(muvec, Rfun(muvec,sig=sig, $n=n$ ), Ity=3,lwd=3);

abline $\left(\mathrm{h}=\operatorname{sig}^{\wedge} 2 / \mathrm{n}, \mathrm{tty}=3\right)$

legend(0.2,3.05,c("sig=1, $n=1 ", " s i g=2, n=5 ", " s i g=3, n=5 ")$,

$\operatorname{lty}=c(1,2,3), \operatorname{lwd}=c(2,2,2))$

Ifun = function(mu,sig, n, mu0,sig0 $)\{$

Rfun(mu=mu,sig=sig, $n=n) * \operatorname{dnorm}(m u, m u 0, \operatorname{sig} 0) \quad\}$

plot $(c(-5,10), c(0,1.5)$, type="n", xlab="mu",ylab="g(mu $=R(m u) * f(m u) "$, main=" ")

$\mathrm{n}=1 ; \operatorname{sig}=1 ; \mathrm{mu} 0=0 ; \operatorname{sig} 0=1$

lines(muvec, Ifun (mu=muvec,sig=sig, $n=n, m u 0=m u 0, \operatorname{sig} 0=\operatorname{sig} 0$ ), Ity=1,Iwd=3)

\# Check range over which to integrate the integrand

integrate $(f=\mid f u n$, lower $=-7$, upper $=7$, sig $=$ sig, $n=n, m u 0=m u 0$, sig0=sig0) $\$$ value \# 3

$\mathrm{n}=1 ; \operatorname{sig}=1 ; \mathrm{mu} 0=1 ; \operatorname{sig} 0=1$

lines(muvec, Ifun (mu=muvec,sig=sig, $n=n, m u 0=m u 0$, sig0=sig0), Ity=2,Iwd=3)

\# Check range over which to integrate the integrand

integrate $(f=\mid f u n$, lower $=-7$, upper $=7$, sig=sig, $n=n, m u 0=m u 0$, sig0=sig0) $\$$ value

\# 1.16

$\mathrm{n}=1 ; \operatorname{sig}=1 ; \mathrm{mu} 0=5 ; \operatorname{sig} 0=1$

lines(muvec, Ifun (mu=muvec,sig=sig, $n=n, m u 0=m u 0$, sig0=sig0), Ity=3,Iwd=3)

\# Check range over which to integrate the integrand

integrate $(f=\mid f u n$, lower $=0$, upper $=10$, sig $=\operatorname{sig}, n=n, m u 0=m u 0$, sig0=sig0) $\$$ value \# 0.9994 
$\mathrm{n}=1 ; \operatorname{sig}=1 ; \mathrm{mu} 0=0 ; \operatorname{sig} 0=0.5$

lines(muvec, Ifun( $m u=m u v e c$, sig=sig, $n=n, m u 0=m u 0$, sig0=sig0), Ity=4, Iwd=3)

\# Check range over which to integrate the integrand integrate ( $\mathrm{f}=$ =Ifun,lower $=-5$, upper $=5$, sig=sig, $\mathrm{n}=\mathrm{n}, \mathrm{mu0}=\mathrm{mu0}$, sig0=sig0) $\$$ value

\# 1.5

legend $(1,1.5, c(" m u 0=0, \operatorname{sig} 0=1.0=>r=3.000 "$, "mu0=1, sig0=1.0 => r=1.160", "mu0=5, sig0=1.0 => r=0.999","mu0=0, sig0=0.5 =>r=1.500"), Ity $=c(1,2,3,4), \operatorname{lwd}=c(3,3,3,3)) ; \operatorname{text}(5,0.6, "$ In each case, $n=1$ and sig=1")

\subsection{The posterior expected loss}

We have defined the risk function as the expectation of the loss function given the parameter, namely

$$
R(\theta)=E(L(\hat{\theta}, \theta) \mid \theta)=\int L(\hat{\theta}(y), \theta) f(y \mid \theta) d y .
$$

Conversely, we now define the posterior expected loss (PEL) as the expectation of the loss function given the data, and we denote this function by

$$
P E L(y)=E\{L(\hat{\theta}, \theta) \mid y\}=\int L(\hat{\theta}(y), \theta) f(\theta \mid y) d \theta .
$$

Then, just as the risk function can be used to compute the Bayes risk according to

$$
r=E L(\hat{\theta}, \theta)=E E\{L(\hat{\theta}, \theta) \mid \theta\}=E R(\theta)=\int R(\theta) f(\theta) d \theta,
$$

so also can the PEL be used, but with the formula

$$
r=E L(\hat{\theta}, \theta)=E E\{L(\hat{\theta}, \theta) \mid y\}=E\{P E L(y)\}=\int P E L(y) f(y) d y .
$$

Note: Both of these formulae for the Bayes risk use the law of iterated expectation, but with different conditionings.

\section{Exercise 2.9 Examples of the PEL and Bayes risk}

Consider the normal-normal model:

$$
\begin{aligned}
& \left(y_{1}, \ldots, y_{n} \mid \mu\right) \sim \text { iid } N\left(\mu, \sigma^{2}\right) \\
& \mu \sim N\left(\mu_{0}, \sigma_{0}^{2}\right) .
\end{aligned}
$$


For each of the following estimators, derive a formula for the posterior expected loss under the quadratic error loss function:
(a) $\hat{\mu}=\bar{y}=\frac{1}{n}\left(y_{1}+\ldots+y_{n}\right) \quad$ (the sample mean)
(b) $\hat{\mu}=|\bar{y}| \quad$ (the absolute value of the sample mean).

In each case, use the derived PEL to obtain the Bayes risk.

Note: This exercise is an extension of Exercise 2.8.

\section{Solution to Exercise 2.9}

(a) If $\hat{\mu}=\bar{y}$ then the PEL function is

$$
\begin{aligned}
\operatorname{PEL}(y) & =E\{L(\hat{\mu}, \mu) \mid y\} \\
& =E\left\{(\bar{y}-\mu)^{2} \mid y\right\} \\
& =\bar{y}^{2}-2 \bar{y} E(\mu \mid y)+E\left(\mu^{2} \mid y\right),
\end{aligned}
$$

where:

$$
\begin{aligned}
& \begin{array}{l}
E(\mu \mid y)=\mu_{*} \\
\begin{aligned}
E\left(\mu^{2} \mid y\right) & =V(\mu \mid y)+\{E(\mu \mid y)\}^{2} \\
& =\sigma_{*}^{2}+\mu_{*}^{2}
\end{aligned} \\
\mu_{*}=(1-k) \mu_{0}+k \bar{y}, \quad \sigma_{*}^{2}=k \frac{\sigma^{2}}{n}, \quad k=\frac{n}{n+\sigma^{2} / \sigma_{0}^{2}} .
\end{array}
\end{aligned}
$$

Thus, more explicitly,

$$
\begin{gathered}
\operatorname{PEL}(y)=\bar{y}^{2}-2 \bar{y}\left\{(1-k) \mu_{0}+k \bar{y}\right\}+\sigma_{*}^{2}+\left\{(1-k) \mu_{0}+k \bar{y}\right\}^{2} \\
=\bar{y}^{2}-2(1-k) \mu_{0} \bar{y}-2 k \bar{y}^{2}+\sigma_{*}^{2}+(1-k)^{2} \mu_{0}^{2}+2(1-k) \mu_{0} k \bar{y}+k^{2} \bar{y}^{2} \\
=\bar{y}^{2}(1-k)^{2}-\bar{y}(1-k)^{2} 2 \mu_{0}+\sigma_{*}^{2}+(1-k)^{2} \mu_{0}^{2} \\
=\sigma_{*}^{2}+(1-k)^{2}\left(\bar{y}-\mu_{0}\right)^{2} .
\end{gathered}
$$

Note: This is a quadratic in $\bar{y}$ with a minimum of $\sigma_{*}^{2}$ at $\bar{y}=\mu_{0}$. 
The Bayes risk is then

$$
\begin{aligned}
r & =E\{\operatorname{PEL}(y)\} \\
& =\sigma_{*}^{2}+(1-k)^{2} E\left\{\left(\bar{y}-\mu_{0}\right)^{2}\right\},
\end{aligned}
$$

where

$$
\begin{aligned}
E\left\{\left(\bar{y}-\mu_{0}\right)^{2}\right\} & =V \bar{y} \\
& =E V(\bar{y} \mid \mu)+V E(\bar{y} \mid \mu) \\
& =E\left(\frac{\sigma^{2}}{n}\right)+V \mu \\
& =\frac{\sigma^{2}}{n}+\sigma_{0}^{2} .
\end{aligned}
$$

Thus $\quad r=\sigma_{*}^{2}+(1-k)^{2}\left(\frac{\sigma^{2}}{n}+\sigma_{0}^{2}\right)$

$$
\begin{aligned}
& \left.=k \frac{\sigma^{2}}{n}+(1-k)^{2}\left(\frac{\sigma^{2}}{n}+\sigma_{0}^{2}\right) \quad \text { (where } k=\frac{n}{n+\sigma^{2} / \sigma_{0}^{2}}\right) \\
& =\frac{\sigma^{2}}{n} \quad \text { (after a little algebra). }
\end{aligned}
$$

Note: This is in agreement with Exercise 2.8, where the result was obtained much more easily by taking the mean of the risk function, as follows:

$$
r=E R(\mu)=E\left(\sigma^{2} / n\right)=\sigma^{2} / n .
$$

(b) If $\hat{\mu}=|\bar{y}|$ then the posterior expected loss function is

$$
\begin{aligned}
\operatorname{PEL}(y) & =E\left\{(|\bar{y}|-\mu)^{2} \mid y\right\} \\
& =\bar{y}^{2}-2|\bar{y}| E(\mu \mid y)+E\left(\mu^{2} \mid y\right) \\
& =\bar{y}^{2}-2|\bar{y}| \mu_{*}+\sigma_{*}^{2}+\mu_{*}^{2} \\
& =\bar{y}^{2}-2|\bar{y}|\left\{(1-k) \mu_{0}+k \bar{y}\right\}+\sigma_{*}^{2}+\left\{(1-k) \mu_{0}+k \bar{y}\right\}^{2} .
\end{aligned}
$$

Some examples of this PEL function are shown in Figure 2.13. In all these examples, $n=\sigma=1$. 
Figure 2.13 Some posterior expected loss functions

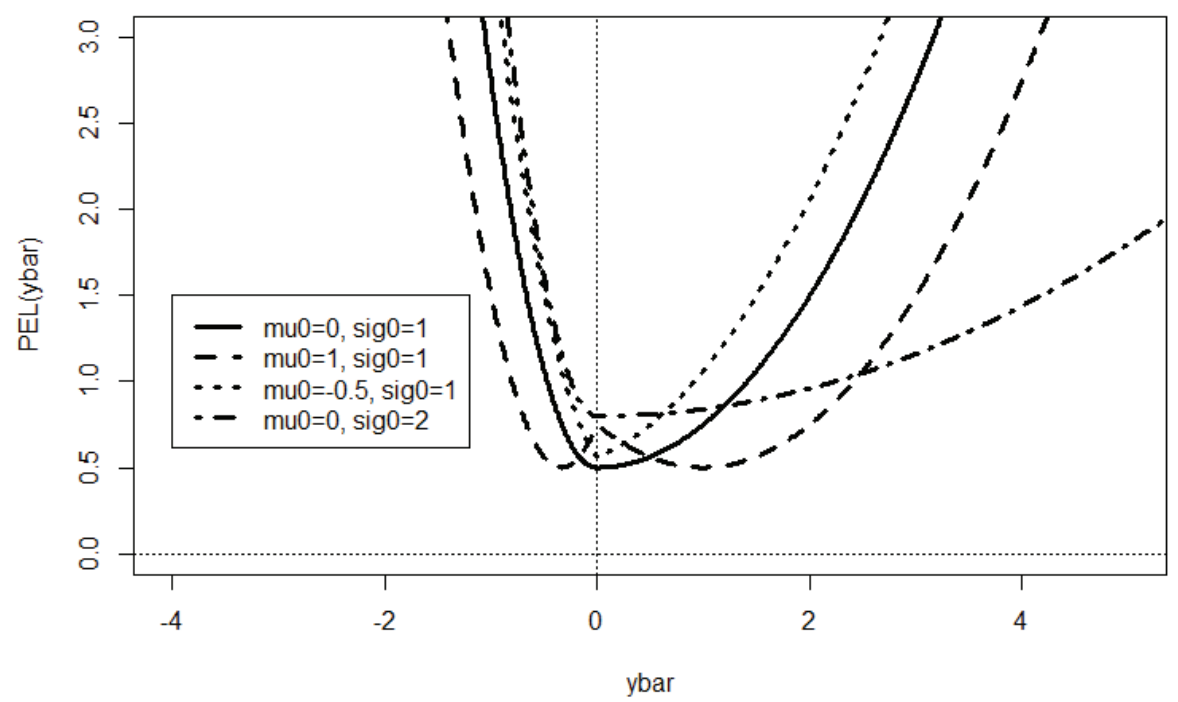

In terms of the PEL function, the Bayes risk can be expressed as

$$
r=E\{\operatorname{PEL}(y)\}=\int_{-\infty}^{\infty} \operatorname{PEL}(\bar{y}) f(\bar{y}) d \bar{y},
$$

where

$$
f(\bar{y})=f_{N\left(\mu_{0}, \sigma_{0}^{2}+\sigma^{2} / n\right)}(\bar{y}),
$$

since

$$
\bar{y} \sim N\left(\mu_{0}, \sigma_{0}^{2}+\frac{\sigma^{2}}{n}\right) .
$$

As an example, we consider the case $n=\sigma=\mu_{0}=\sigma_{0}=1$. Figure 2.14 shows the integrand function $\operatorname{PEL}(\bar{y}) f(\bar{y})$. The area under this function works out as 1.16, in agreement with an alternative working for the Bayes risk in Exercise 2.8 (taking an expectation of the risk function). 
Figure 2.14 An integrand function with area underneath equal to 1.16

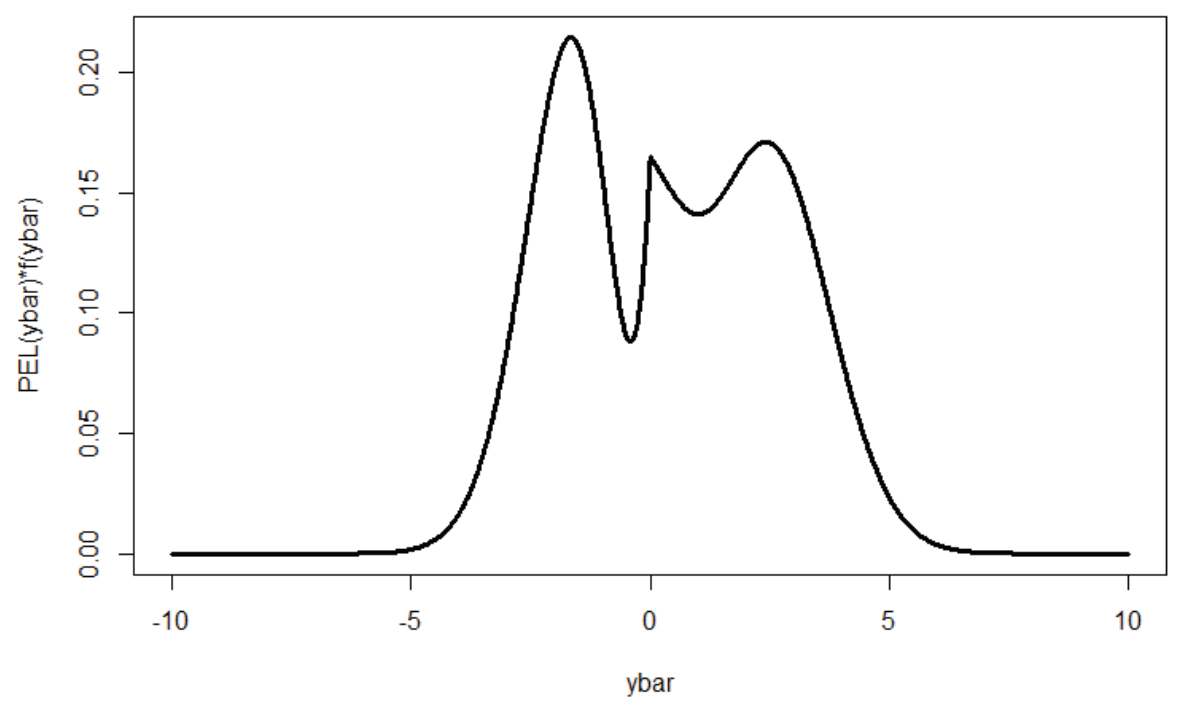

\section{R Code for Exercise 2.9}

PELfun=function(ybar,sig,n,sig0,mu0 $)\{$

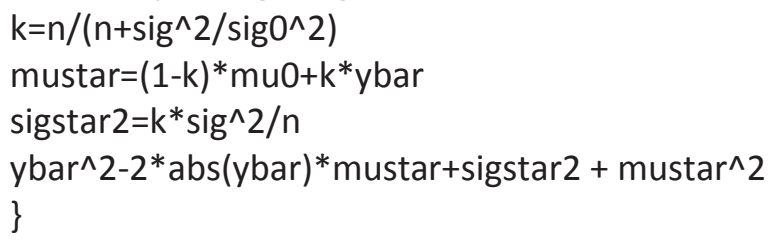

ybarvec=seq $(-10,10,0.01)$; options(digits $=4)$

$\mathrm{X} 11(\mathrm{w}=8, \mathrm{~h}=5.5)$; $\operatorname{par}(\operatorname{mfrow}=\mathrm{c}(1,1))$;

plot $(c(-4,5), c(0,3)$,type="n",xlab="ybar",ylab="PEL(ybar)", main=" ") abline $(v=0$, tty=3); abline $(h=0$, tty=3)

$\mathrm{n}=1 ; \operatorname{sig}=1 ; \mathrm{mu} 0=0 ; \operatorname{sig} 0=1$

lines(ybarvec,PELfun(ybarvec,sig=sig, $n=n, \operatorname{sig} 0=\operatorname{sig} 0, m u 0=m u 0), l t y=1, l w d=3$ );

$\mathrm{n}=1 ; \operatorname{sig}=1 ; \operatorname{mu} 0=1 ; \operatorname{sig} 0=1$

lines(ybarvec,PELfun(ybarvec,sig=sig, $n=n, \operatorname{sig} 0=\operatorname{sig} 0, m u 0=m u 0)$, Ity=2,lwd=3); 
$\mathrm{n}=1 ; \operatorname{sig}=1 ; \mathrm{mu} 0=-0.5 ; \operatorname{sig} 0=1$

lines(ybarvec,PELfun(ybarvec,sig=sig, $n=n, \operatorname{sig} 0=\operatorname{sig} 0$, mu0=mu0), Ity=3,lwd=3);

$\mathrm{n}=1 ;$ sig=1; $\mathrm{mu} 0=0 ; \operatorname{sig} 0=2$

lines(ybarvec,PELfun(ybarvec,sig=sig, $n=n, \operatorname{sig} 0=\operatorname{sig} 0$, mu0=mu0), Ity=4,lwd=3);

legend(-4,1.5,c("mu0=0, sig0=1","mu0=1, sig0=1","mu0=-0.5, sig0=1", "mu0=0, sig0=2"), Ity=c(1,2,3,4), Iwd=c(3,3,3,3))

\# Calculate $r$ when $n=1, \operatorname{sig}=1, m u 0=1, \operatorname{sig} 0=1$ (should get 1.16 as before)

Jfun = function(ybar, sig,n,sig0, mu0 $)\{$

PELfun(ybar=ybar, sig=sig, $n=n, \operatorname{sig} 0=\operatorname{sig} 0, m u 0=m u 0) *$

dnorm(ybar,mu0,sqrt(sig0^2+sig^2/n))

\}

$\mathrm{n}=1 ; \operatorname{sig}=1 ; \mathrm{mu} 0=1 ; \operatorname{sig} 0=1$

plot(ybarvec, PELfun(ybar=ybarvec,sig=sig, $n=n, \operatorname{sig} 0=\operatorname{sig} 0, m u 0=m u 0$ )*

dnorm(ybarvec,mu0,sqrt(sig0^2+sig^2/n)),

type="l", xlab="ybar",ylab="PEL(ybar)*f(ybar)", Iwd=3)

integrate $(\mathrm{f}=\mathrm{Jfun}$,lower $=-10$, upper $=10$, sig=sig, $\mathrm{n}=\mathrm{n}, \mathrm{mu0}=\mathrm{mu0}, \operatorname{sig} 0=\mathrm{sig} 0) \$$ value

\# 1.16 Correct (same as in last exercise)

\subsection{The Bayes estimate}

The Bayes estimate (or estimator) is defined to be the choice of the function $\hat{\theta}=\hat{\theta}(y)$ for which the Bayes risk $r=E L(\hat{\theta}, \theta)$ is minimised. This estimator has the smallest overall expected loss over all estimators under the specified loss function $L(\hat{\theta}, \theta)$.

In many cases, the procedure for finding a Bayes estimate can be considerably simplified by considering which estimate minimises the posterior expected loss function, $\operatorname{PEL}(y)=E\{L(\hat{\theta}, \theta) \mid y\}$.

If we can find an estimate $\hat{\theta}=\hat{\theta}(y)$ which minimises $\operatorname{PEL}(y)$ for all possible values of the data $y$, then that estimate must also minimise the Bayes risk. 
This is because the Bayes risk may be written as a weighted average of the PEL, namely

$$
r=E L(\hat{\theta}, \theta)=E E\{L(\hat{\theta}, \theta) \mid y\}=E\{P E L(y)\}=\int P E L(y) f(y) d y .
$$

\section{Exercise 2.10 Bayes estimate under the QELF}

Find the Bayes estimate under the quadratic error loss function.

\section{Solution to Exercise 2.10}

Observe that $\quad \operatorname{PEL}(y)=E\left\{(\hat{\theta}-\theta)^{2} \mid y\right\}=E\left\{\hat{\theta}^{2}-2 \hat{\theta} \theta+\theta^{2} \mid y\right\}$

$$
\begin{aligned}
& =\hat{\theta}^{2}-2 \hat{\theta} E(\theta \mid y)+E\left(\theta^{2} \mid y\right) \\
& =[\hat{\theta}-E(\theta \mid y)]^{2}-\{E(\theta \mid y)\}^{2}+E\left(\theta^{2} \mid y\right) .
\end{aligned}
$$

Note: We have completed the square in $\hat{\theta}$.

We see that the PEL is a quadratic function of $\hat{\theta}$ which is clearly minimised at the posterior mean, $\hat{\theta}=E(\theta \mid y)$. So the Bayes estimate under the QELF is that posterior mean.

Note 1: This result can also be obtained using Leibniz's rule for differentiating an integral, which is generally

$$
\frac{d}{d x} \int_{a}^{b} G(u, x) d u=\int_{a}^{b} \frac{\partial G(u, x)}{\partial x} d u+G(b, x) \frac{d b}{d x}-G(a, x) \frac{d a}{d x}
$$

and which reduces to $\int_{a}^{b} \frac{\partial G(u, x)}{\partial x} d u+0-0$ if $a$ and $b$ are constants.

Thus we may write $\frac{\partial}{\partial \hat{\theta}} P E L(y)=\frac{\partial}{\partial \hat{\theta}} \int(\hat{\theta}-\theta)^{2} f(\theta \mid y) d \theta$

$$
\begin{gathered}
=\int \frac{\partial}{\partial \hat{\theta}}\left\{(\hat{\theta}-\theta)^{2} f(\theta \mid y)\right\} d \theta+0-0 \\
=\int 2(\hat{\theta}-\theta)^{1} f(\theta \mid y) d \theta=2\left\{\hat{\theta}-\int \theta f(\theta \mid y) d \theta\right\} .
\end{gathered}
$$

Setting this to zero yields $\hat{\theta}=\int \theta f(\theta \mid y) d \theta=E(\theta \mid y)$. 
Note 2: To check that this minimises the PEL (rather than maximises it) we may further calculate

$$
\frac{\partial^{2}}{\partial \hat{\theta}^{2}} \operatorname{PEL}(y)=2 \frac{\partial}{\partial \hat{\theta}}\left\{\hat{\theta}-\int \theta f(\theta \mid y) d \theta\right\}=2\{1-0\}>0 .
$$

Thus the slope of the PEL ( $\partial P E L(y) / \partial \hat{\theta}$ ) is increasing with $\hat{\theta}$, implying that $\operatorname{PEL}(y)$ is indeed minimised at $\hat{\theta}=\hat{\theta}(y)=E(\theta \mid y)$.

\section{Exercise 2.I I Bayes estimate under the AELF}

Find the Bayesian estimate under the absolute error loss function.

\section{Solution to Exercise 2. I I}

Suppose that the parameter $\theta$ is continuous, and let $t$ denote $\hat{\theta}=\hat{\theta}(y)$.

Then $\operatorname{PEL}(y)=\int_{-\infty}^{\infty}|t-\theta| f(\theta \mid y) d \theta$

$$
=\int_{-\infty}^{t}(t-\theta) f(\theta \mid y) d \theta+\int_{t}^{\infty}(\theta-t) f(\theta \mid y) d \theta .
$$

So, by Leibniz's rule for differentiation of an integral (in Exercise 2.10),

$$
\begin{aligned}
\frac{\partial}{\partial t} P E L(y) & =\left\{\int_{-\infty}^{t} \frac{\partial(t-\theta)}{\partial t} f(\theta \mid y) d \theta+\{(t-t) f(\theta=t \mid y)\} \frac{d t}{d t}-(\cdot) \frac{d(-\infty)}{d t}\right\} \\
& +\left\{\int_{t}^{\infty} \frac{\partial(\theta-t)}{\partial t} f(\theta \mid y) d \theta+(\cdot) \frac{d(\infty)}{d t}-\{(t-t) f(\theta=t \mid y)\} \frac{d t}{d t}\right\} \\
& =\left\{\int_{-\infty}^{t} f(\theta \mid y) d \theta+0-0\right\}+\left\{\int_{t}^{\infty}(-1) f(\theta \mid y) d \theta+0-0\right\} \\
& =P(\theta<t \mid y)-P(\theta>t \mid y) .
\end{aligned}
$$

Setting this to zero implies $P(\theta<t \mid y)=P(\theta>t \mid y)$ which yields $t$ as the posterior median. So the Bayes estimate under the AELF is the posterior median. This argument can easily be adapted to the case where $\theta$ is discrete. The idea is to approximate $\theta$ 's discrete prior distribution with a continuous distribution and then apply the result already proved. 


\section{Exercise 2.I 2 Bayes estimate under the IELF}

Find the Bayes estimate under the indicator error loss function.

\section{Solution to Exercise 2.12}

Let $t$ denote $\hat{\theta}=\hat{\theta}(y)$ and first suppose that the parameter $\theta$ is discrete. The indicator error loss function is $L(t, \theta)=I(t \neq \theta)=1-I(t=\theta)$. Therefore

$$
\begin{aligned}
P E L(y)=E\{L(t, \theta) \mid y\}=E\{1-I(t=\theta) \mid y\} & =1-E\{I(t=\theta) \mid y\} \\
& =1-P(t=\theta \mid y) \\
& =1-f(\theta=t \mid y) .
\end{aligned}
$$

Thus PEL $(y)$ is minimised at the value of $t$ which maximises the posterior density $f(\theta \mid y)$. So, when $\theta$ is discrete, the Bayes estimate under the IELF is the posterior mode, $\operatorname{Mode}(\theta \mid y)$.

Now suppose that $\theta$ is continuous. In that case, consider the approximating loss function

$$
L_{\varepsilon}(t, \theta)=1-I(t-\varepsilon<\theta<t+\varepsilon),
$$

where $\varepsilon>0$, and observe that

$$
\lim _{\varepsilon \rightarrow 0} L_{\varepsilon}(t, \theta)=1-I(t=\theta)=L(t, \theta) .
$$

The posterior expected loss under the loss function $L_{\varepsilon}(t, \theta)$ is

$$
\begin{aligned}
P E L_{\varepsilon}(y)=E\left\{L_{\varepsilon}(t, \theta) \mid y\right\} & =1-E\{I(t-\varepsilon<\theta<t+\varepsilon) \mid y\} \\
& =1-P(t-\varepsilon<\theta<t+\varepsilon \mid y) .
\end{aligned}
$$

The value of $t$ which minimises the $P E L_{\varepsilon}(y)$ is the value which maximises the area $P(t-\varepsilon<\theta<t+\varepsilon \mid y)$. But in the limit as $\varepsilon \rightarrow 0$, that value is the posterior mode. So, when $\theta$ is continuous, the Bayes estimate under the IELF is (as before) the posterior mode, $\operatorname{Mode}(\theta \mid y)$.

Note: To clarify the above argument, observe that if $\varepsilon$ is small then

$$
P E L_{\varepsilon}(t) \approx 1-2 \varepsilon f_{\theta}(t \mid y) \text {. }
$$

This function of $t$ is minimised at approximately $t=\operatorname{Mode}(\theta \mid y)$ and at exactly $t=\operatorname{Mode}(\theta \mid y)$ in the limit as $\varepsilon \rightarrow 0$. Figure 2.15 illustrates. 
Figure 2.15 Illustration for the continuous case in Exercise 2.12

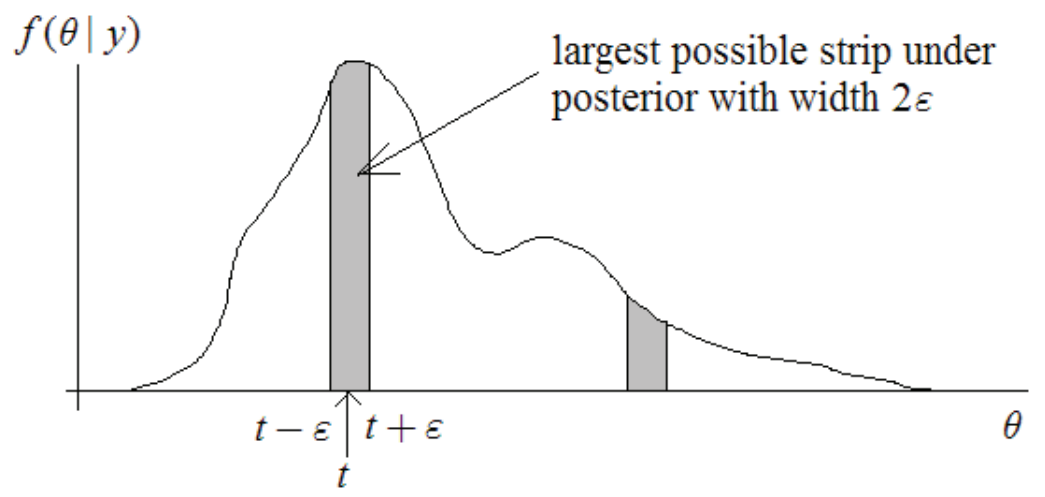

\section{Exercise 2.13 Bayesian decision theory in the Poisson-gamma model}

Consider a random sample $y_{1}, \ldots, y_{n}$ from the Poisson distribution with parameter $\lambda$ whose prior density is gamma with parameters $\alpha$ and $\beta$.

(a) Find the risk function, Bayes risk and posterior expected loss implied by the estimator $\hat{\lambda}=2 \bar{y}$ under the quadratic error loss function.

(b) Assuming quadratic error loss, find an estimator of $\lambda$ with a smaller Bayes risk than the one in (a).

\section{Solution to Exercise 2.13}

(a) The risk function is

$$
\begin{aligned}
R(\lambda)= & E\{L(\hat{\lambda}, \lambda) \mid \lambda\} \\
= & E\left\{(2 \bar{y}-\lambda)^{2} \mid \lambda\right\} \\
= & E\left\{4 \bar{y}^{2}-4 \bar{y} \lambda+\lambda^{2} \mid \lambda\right\} \\
& =4 E\left\{\bar{y}^{2} \mid \lambda\right\}-4 \lambda E\{\bar{y} \mid \lambda\}+\lambda^{2} \\
= & 4\left[V\{\bar{y} \mid \lambda\}+E\{\bar{y} \mid \lambda\}^{2}\right]-4 \lambda E\{\bar{y} \mid \lambda\}+\lambda^{2} \\
= & 4\left(\frac{\lambda}{n}+\lambda^{2}\right)-4 \lambda \lambda+\lambda^{2} \\
= & \lambda^{2}+4 \lambda / n, \lambda>0 \quad \text { (an increasing quadratic). }
\end{aligned}
$$


So the Bayes risk is

$$
\begin{aligned}
r & =E R(\hat{\lambda}, \lambda) \\
& =E\left(\lambda^{2}+4 \lambda / n\right) \\
& =\left\{V \lambda+(E \lambda)^{2}\right\}+4(E \lambda) / n \\
& =\frac{\alpha}{\beta^{2}}+\left(\frac{\alpha}{\beta}\right)^{2}+\frac{4 \alpha}{n \beta} .
\end{aligned}
$$

To find the posterior expected loss, we first derive $\lambda$ 's posterior density:

$f(\lambda \mid y) \propto f(\lambda) f(y \mid \lambda)$

$$
\begin{aligned}
& =\frac{\beta^{\alpha} \lambda^{\alpha-1} e^{-\beta \lambda}}{\Gamma(\alpha)} \times \prod_{i=1}^{n} \frac{e^{-\lambda} \lambda^{y_{i}}}{y_{i} !} \\
& \propto \\
& \propto \lambda^{\alpha+n \bar{y}-1} e^{-\lambda(\beta+n)} .
\end{aligned}
$$

We see that

$$
f(\lambda \mid y) \sim \operatorname{Gam}(\alpha+n \bar{y}, \beta+n) .
$$

It follows that

$$
\begin{aligned}
\operatorname{PEL}(y) & =E\{L(\hat{\lambda}, \lambda) \mid y\} \\
& =E\left\{(2 \bar{y}-\lambda)^{2} \mid y\right\} \\
& =E\left\{4 \bar{y}^{2}-4 \bar{y} \lambda+\lambda^{2} \mid y\right\} \\
& =4 \bar{y}^{2}-4 \bar{y} E(\lambda \mid y)+E\left(\lambda^{2} \mid y\right) \\
& =4 \bar{y}^{2}-4 \bar{y}\left(\frac{\alpha+n \bar{y}}{\beta+n}\right)+\left\{\frac{\alpha+n \bar{y}}{(\beta+n)^{2}}+\left(\frac{\alpha+n \bar{y}}{\beta+n}\right)^{2}\right\} .
\end{aligned}
$$

Note: The Bayes risk could also be computed using an argument which begins as follows:

$$
\begin{aligned}
r & =E\{\operatorname{PEL}(y)\} \\
& =E\left\{4 \bar{y}^{2}-4 \bar{y}\left(\frac{\alpha+n \bar{y}}{\beta+n}\right)+\frac{\alpha+n \bar{y}}{(\beta+n)^{2}}+\left(\frac{\alpha+n \bar{y}}{\beta+n}\right)^{2}\right\},
\end{aligned}
$$

where, for example,

$$
E \bar{y}=E E(\bar{y} \mid \lambda)=E E\left(y_{1} \mid \lambda\right)=E \lambda=\alpha / \beta .
$$


(b) The Bayes estimate under the QELF is the posterior mean,

$$
E(\lambda \mid y)=\frac{\alpha+n \bar{y}}{\beta+n} .
$$

This estimator has the smallest Bayes risk amongst all possible estimators, including the one in (a), which is different. So $E(\lambda \mid y)$ must have a smaller Bayes risk than the estimator in (a).

\section{Discussion}

The last statement could be verified by calculating $r$ according to

$$
E\left(\frac{\alpha+n \bar{Y}}{\beta+n}-\lambda\right)^{2} \text {. }
$$

The result should be an expression for $r$ which is smaller than

$$
\frac{\alpha}{\beta^{2}}+\left(\frac{\alpha}{\beta}\right)^{2}+\frac{4 \alpha}{n \beta},
$$

for all $n=1,2,3, \ldots$, and all $\alpha, \beta>0$.

We leave the required working as an additional exercise.

\section{Exercise 2.14 A non-standard loss function}

Consider the Bayesian model given by:

$$
\begin{aligned}
& (y \mid \mu) \sim N(\mu, 1) \\
& \mu \sim N(0,1) .
\end{aligned}
$$

Then suppose that the loss function is

$$
L(t, \mu)=\left\{\begin{array}{l}
0 \text { if } 0<\mu<t<2 \mu \\
1 \text { otherwise }
\end{array}\right.
$$

(a) Find the risk function and Bayes risk for the estimator $\hat{\mu}=y$.

Sketch the risk function.

(b) Find the Bayes estimate and sketch it as a function of the data $y$.

Explicitly calculate the Bayes estimate at $y=-1,0$ and 1, respectively. 


\section{Solution to Exercise 2.14}

(a) For convenience we will sometimes denote $\hat{\mu}=y$ by $t$. Then, the loss function may be written as

$$
L(t, \mu)=\left\{\begin{array}{rr}
1-I(\mu<t<2 \mu), \mu & >0 \\
1, & \mu \leq 0 .
\end{array}\right.
$$

Now, for $\mu \leq 0$ the risk function is simply

$$
R(\mu)=E\{L(y, \mu) \mid \mu\}=1 \text {. }
$$

For $\mu>0$, the risk function is

$$
\begin{aligned}
R(\mu) & =1-P(\mu<y<2 \mu \mid \mu)=1-P(0<y-\mu<\mu \mid \mu) \\
& =1-P(0<Z<\mu) \quad \text { where } Z \sim N(0,1) \\
& =1-(\Phi(\mu)-1 / 2)=1.5-\Phi(\mu) .
\end{aligned}
$$

In summary, $R(\mu)=\left\{\begin{array}{cc}1, & \mu \leq 0 \\ 1.5-\Phi(\mu), & \mu>0\end{array}\right\}$, as shown in Figure 2.16.

Figure 2.16 Risk function in Exercise 2.14

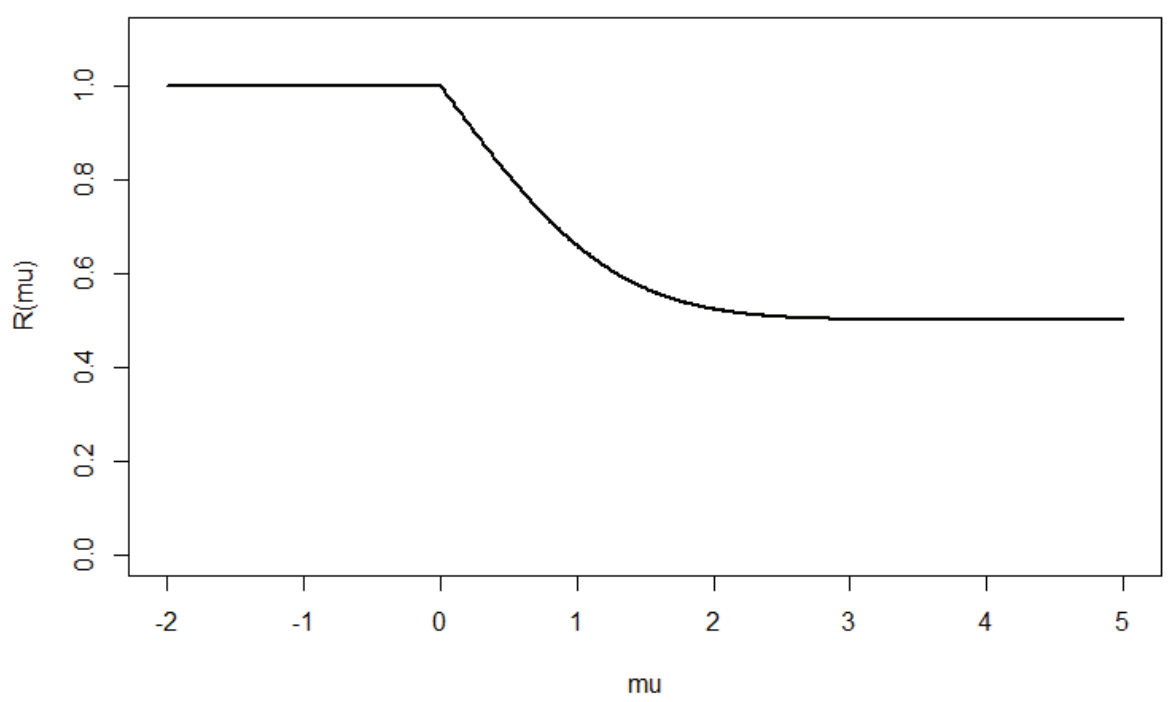


The associated Bayes risk is

$$
\begin{aligned}
r=E R(\mu) & =\int_{-\infty}^{0} \phi(\mu) d \mu+\frac{3}{2} \int_{0}^{\infty} \phi(\mu) d \mu-\int_{0}^{\infty} \Phi(\mu) \phi(\mu) d \mu \\
& =\frac{1}{2}+\frac{3}{2} \times \frac{1}{2}-I,
\end{aligned}
$$

where $I=\int_{1 / 2}^{1} w d w=3 / 8$, after putting $w=\Phi(\mu)$ with $\frac{d w}{d \mu}=\phi(\mu)$.

So, for the estimator $\hat{\mu}=y$, the Bayes risk is

$$
r=\frac{1}{2}+\frac{3}{2} \times \frac{1}{2}-\frac{3}{8}=\frac{7}{8} \text {. }
$$

(b) Here, by the theory of the normal-normal model we have that

$$
(\mu \mid y) \sim N\left(\mu_{*}, \sigma_{*}^{2}\right) \text {, }
$$

where:

$$
\begin{aligned}
& \mu_{*}=(1-k) \mu_{0}+k \bar{y}, \quad \sigma_{*}^{2}=k \sigma^{2} / n, \quad k=1 /\left(1+\sigma^{2} /\left(n \sigma_{0}^{2}\right)\right) \\
& n=1, \quad \mu_{0}=0, \quad \sigma_{0}=1, \quad \bar{y}=y .
\end{aligned}
$$

Thus $k=1 / 2, \mu_{*}=y / 2$ and $\sigma_{*}^{2}=1 / 2$, and so

$$
(\mu \mid y) \sim N(y / 2,1 / 2) \text {. }
$$

The posterior expected loss is

$$
\operatorname{PEL}(y)=E\{L(t, \mu) \mid y\},
$$

where $t$ is a function of $y$ (i.e. $t=t(y)$ ).

Now

and so

$$
L(t, \mu)=1-I(0<\mu<t<2 \mu),
$$

$$
\begin{aligned}
P E L(y) & =E\{1-I(0<\mu<t<2 \mu) \mid y\} \\
& =1-P(0<\mu<t<2 \mu \mid y) .
\end{aligned}
$$

We see that if $t=t(y) \leq 0$ then

$$
\operatorname{PEL}(y)=1 \text {. }
$$


Also, if $t>0$ then

$$
\begin{aligned}
\operatorname{PEL}(t) & =1-E\{I(0<\mu<t<2 \mu) \mid y\} . \\
& =1-P(0<\mu<t<2 \mu \mid y) \\
& =1-P(t / 2<\mu<t \mid y) \\
& =1-\psi(t)
\end{aligned}
$$

where

$$
\psi(t)=F(\mu=t \mid y)-F(\mu=t / 2 \mid y)
$$

is to be maximised.

Now, $\quad \psi^{\prime}(t)=f(\mu=t \mid y)-f(\mu=t / 2 \mid y) \times 1 / 2$

$$
=\frac{1}{(1 / \sqrt{2}) \sqrt{2 \pi}} e^{-\frac{1}{2(1 / 2)}(t-y / 2)^{2}}-\frac{1}{2} \times \frac{1}{(1 / \sqrt{2}) \sqrt{2 \pi}} e^{-\frac{1}{2(1 / 2)}((t / 2)-y / 2)^{2}} .
$$

Setting $\psi^{\prime}(t)$ to zero we obtain

$$
\begin{aligned}
& 2 e^{-(t-y / 2)^{2}}=e^{-((t / 2)-y / 2)^{2}} \\
& \Rightarrow \log 2-\left\{t^{2}-2 t\left(\frac{y}{2}\right)+\left(\frac{y}{2}\right)^{2}\right\}=-\left\{\left(\frac{t}{2}\right)^{2}+2\left(\frac{t}{2}\right)\left(\frac{y}{2}\right)-\left(\frac{y}{2}\right)^{2}\right\} \\
& \Rightarrow \frac{3}{4} t^{2}-\frac{1}{2} t y-\log 2=0 \\
& \Rightarrow t=\frac{\frac{y}{2} \pm \sqrt{\frac{y^{2}}{4}+4 \times \frac{3}{4} \log 2}}{2(3 / 4)} .
\end{aligned}
$$

Hence we find that the Bayes estimate of $\mu$ is given by

$$
\hat{\mu}=\hat{\mu}(y)=\frac{1}{3}\left(y+\sqrt{y^{2}+12 \log 2}\right),
$$

as shown in Figure 2.17.

We see that the Bayes estimate is a strictly increasing function of $y$ and converges to zero as $y$ tends to negative infinity. The required values of the Bayes estimate are:

$$
\begin{aligned}
& \hat{\mu}(-1)=\frac{1}{3}(-1+\sqrt{1+12 \log 2})=0.6842 \\
& \hat{\mu}(0)=\frac{1}{3}(0+\sqrt{0+12 \log 2})=0.9614 \\
& \hat{\mu}(1)=\frac{1}{3}(1+\sqrt{1+12 \log 2})=1.3508 .
\end{aligned}
$$


Figure 2.17 Bayes estimate in Exercise 2.14

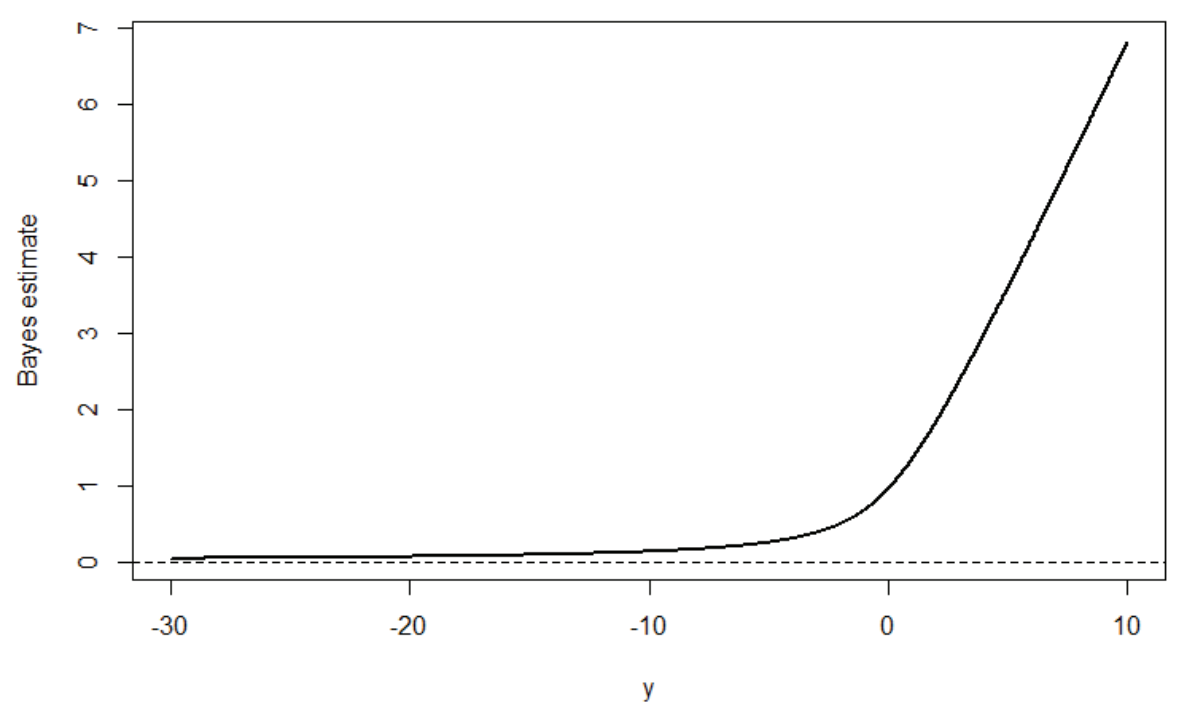

R Code for Exercise 2.14

$X 11(w=8, h=5.5)$

muvec <- seq $(0,5,0.01) ;$ Rvec <- 1.5-pnorm(muvec);

plot $(c(-2,5), c(0,1.1)$,type= "n",xlab="mu",ylab="R(mu)",cex=1.5)

lines(muvec, Rvec, Iwd=2); lines $(c(-2,0), c(1,1), \operatorname{lwd}=2)$

yvec <- seq $(-30,10,0.01) ;$ muhatvec <- $(1 / 3) *\left(\right.$ yvec+sqrt $\left(\right.$ yvec $\left.\left.^{\wedge} 2+12 * \log (2)\right)\right)$ plot(yvec, muhatvec,type="l",xlab="y",ylab="Bayes estimate",cex=1.5, Iwd=2) abline( $\mathrm{h}=0, \mathrm{tty}=2)$

$(1 / 3)^{*}\left(c(-1,0,1)+\operatorname{sqrt}\left(c(-1,0,1)^{\wedge} 2+12 * \log (2)\right)\right)$

\# 0.68416720 .96135131 .3508339 


\section{Bayesian Basics Part 3}

\section{I Inference given functions of the data}

Sometimes we observe a function of the data rather than the data itself. In such cases the function typically degrades the information available in some way. An example is censoring, where we observe a value only if that value is less than some cut-off point (right censoring) or greater than some cut-off value (left censoring). It is also possible to have censoring on the left and right simultaneously. Another example is rounding, where we only observe values to the nearest multiple of $0.1,1$ or 5 , etc.

\section{Exercise 3.I Right censoring of exponential observations}

Each light bulb of a certain type has a life which is conditionally exponential with mean $m=1 / c$, where $c$ has a prior distribution which is standard exponential. We observe $n=5$ light bulbs of this type for 6 units of time, and the lifetimes are:

2.6, 3.2, * $1.2, *$,

where * indicates a right-censored value which is greater than 6. (Only values less than or equal to 6 could be observed.)

Find the posterior distribution and mean of the average light bulb lifetime, $m$.

\section{Solution to Exercise 3.I}

The data here is

$$
D=\left\{y_{1}=2.6, y_{2}=3.2, y_{3}>6, y_{4}=1.2, y_{5}>6\right\},
$$

and the probability of censoring is

$$
P\left(y_{i}>6 \mid c\right)=\int_{6}^{\infty} c e^{-c y_{i}} d y_{i}=e^{-6 c} .
$$

Therefore the posterior density of $c$ is

$$
\begin{aligned}
f(c \mid D) & \propto f(c) f(D \mid c) \\
& \propto f(c) f\left(y_{1} \mid c\right) f\left(y_{2} \mid c\right) P\left(y_{3}>6 \mid c\right) f\left(y_{4} \mid c\right) P\left(y_{5}>6 \mid c\right)
\end{aligned}
$$




$$
\begin{aligned}
& \propto e^{-c}\left(c e^{-c y_{1}}\right)\left(c e^{-c y_{2}}\right)\left(e^{-6 c}\right)\left(c e^{-c y_{4}}\right)\left(e^{-6 c}\right) \\
& =c^{3} \exp \left\{-c\left(1+y_{1}+y_{2}+6+y_{4}+6\right)\right\} \\
& =c^{4-1} \exp \{-c(1+2.6+3.2+6+1.2+6)\} \\
& =c^{4-1} \exp (-20 c) .
\end{aligned}
$$

Hence: $\quad(c \mid D) \sim G(4,20)$

$$
\begin{aligned}
& (m \mid D) \sim I G(4,20) \\
& f(m \mid D)=20^{4} m^{-(4+1)} e^{-20 / m} / \Gamma(4), m>0 \\
& E(m \mid D)=20 /(4-1)=6.667 .
\end{aligned}
$$

It will be observed that this estimate of $m$ is appropriately higher than the estimate obtained by simply averaging the observed values, namely

$$
(1 / 3)(2.6+3.2+1.2)=2.333 \text {. }
$$

The estimate 6.667 is also higher than the estimate obtained by simply replacing the censored values with 6 , namely

$$
(1 / 3)(2.6+3.2+6+1.2+6)=3.8 \text {. }
$$

\section{Exercise 3.2 A uniform-uniform model with rounded data}

Suppose that:

$$
\begin{aligned}
& (y \mid \theta) \sim U(0, \theta) \\
& \theta \sim U(0,2),
\end{aligned}
$$

where the data is

$$
x=g(y)=\text { the value of } y \text { rounded to the nearest integer. }
$$

Find the posterior density and mean of $\theta$ if we observe $x=1$.

\section{Solution to Exercise 3.2}

Observe that:

$$
\begin{aligned}
& x=0 \text { if } 0<y<1 / 2 \\
& x=1 \text { if } 1 / 2<y<3 / 2 \\
& x=2 \text { if } 3 / 2<y<2
\end{aligned}
$$

Therefore, considering $y$ and $\theta$ on a number line from 0 to 2 in each case, we have that: 


$$
\begin{aligned}
& P(x=0 \mid \theta)=P\left(0<y<\frac{1}{2} \mid \theta\right)=\left\{\begin{array}{cc}
1 & \text { if } \theta<1 / 2 \\
\frac{1 / 2}{\theta} & \text { if } \theta>1 / 2
\end{array}\right. \\
& P(x=1 \mid \theta)=P\left(\frac{1}{2}<y<\frac{3}{2} \mid \theta\right)=\left\{\begin{array}{cc}
0 & \text { if } 0<\theta<1 / 2 \\
\frac{\theta-1 / 2}{\theta} & \text { if } \frac{1}{2}<\theta<\frac{3}{2}
\end{array}\right. \\
& P(x=2 \mid \theta)=P\left(\frac{3}{2}<y<2 \mid \theta\right)=\left\{\begin{array}{cc}
\frac{1}{\theta} & \text { if } \frac{3}{2}<\theta<2 \\
0 & \text { if } 0<\theta<3 / 2 \\
\frac{\theta-3 / 2}{\theta} & \text { if } \frac{3}{2}<\theta<2 .
\end{array}\right.
\end{aligned}
$$

Since we observe $x=1$, the posterior density of $\theta$ is

$$
f(\theta \mid x=1) \propto f(\theta) f(x \mid \theta) \propto\left\{\begin{array}{l}
1 \times \frac{\theta-1 / 2}{\theta}, \frac{1}{2}<\theta<\frac{3}{2} \\
1 \times \frac{1}{\theta}, \quad \frac{3}{2}<\theta<2 .
\end{array}\right.
$$

Now, the area under this function is

$$
\begin{aligned}
B & =\int_{1 / 2}^{3 / 2} \frac{\theta-1 / 2}{\theta} d \theta+\int_{3 / 2}^{2} \frac{1}{\theta} d \theta \\
& =\left[\theta-\left.\frac{1}{2} \log \theta\right|_{1 / 2} ^{3 / 2}\right]+\left[\left.\log \theta\right|_{3 / 2} ^{2}\right] \\
& =\left[\frac{3}{2}-\frac{1}{2} \log \frac{3}{2}-\frac{1}{2}+\frac{1}{2} \log \frac{1}{2}\right]+\left[\log 2-\log \frac{3}{2}\right] \\
& =0.7383759 .
\end{aligned}
$$

So the required posterior density is

$$
f(\theta \mid x=1)= \begin{cases}\frac{\theta-1 / 2}{B \theta}, & \frac{1}{2}<\theta<\frac{3}{2} \\ \frac{1}{B \theta}, & \frac{3}{2}<\theta<2\end{cases}
$$

and the associated posterior mean of $\theta$ is 


$$
\begin{aligned}
E_{1}=E(\theta \mid x=1) & =\int_{1 / 2}^{3 / 2} \theta\left(\frac{\theta-1 / 2}{B \theta}\right) d \theta+\int_{3 / 2}^{2} \theta\left(\frac{1}{B \theta}\right) d \theta \\
& =\frac{1}{B}=1.354 \quad \text { (after some working) }
\end{aligned}
$$

\section{Discussion}

In contrast to $f(\theta \mid x)$, the posterior density of $\theta$ given the original data $y$ is

$$
f(\theta \mid y)=\frac{f(\theta) f(y \mid \theta)}{f(y)}=\frac{(1 / 2)(1 / \theta)}{\int_{y}^{2}(1 / 2)(1 / \theta) d \theta}=\frac{1}{\theta(\log 2-\log y)}, y<\theta<2,
$$

and the corresponding posterior mean is

$$
\hat{\theta}=E(\theta \mid y)=\int_{y}^{2} \theta\left(\frac{1}{\theta(\log 2-\log y)}\right) d \theta=\frac{2-y}{\log 2-\log y} .
$$

Figure 3.1 shows $f(\theta \mid x=1)$ and examples of $f(\theta \mid y)$ which are consistent with $x=1$.

Figure 3.I Posteriors given $x=I$ and given $y=0.6, I$, I. I, I.4

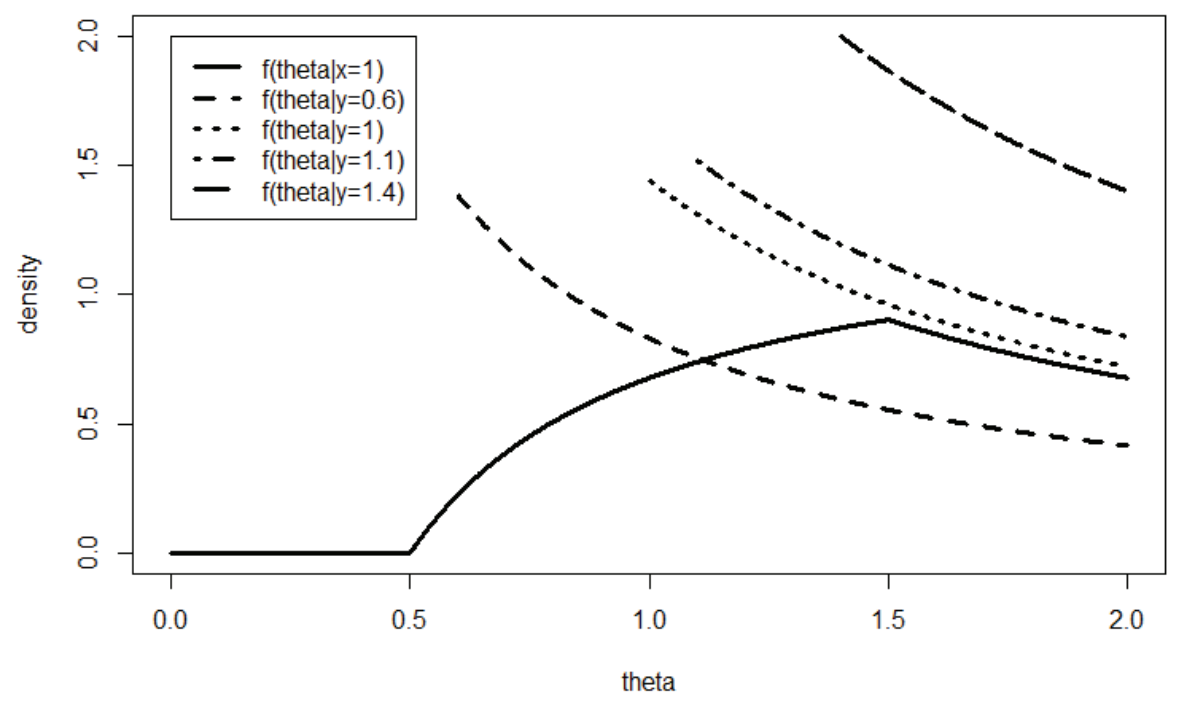


It is now of interest to also calculate $f(\theta \mid x)$ for the other two possible values of $x$, namely 0 and 2 . We find that:

$$
\begin{aligned}
& f(\theta \mid x=0)= \begin{cases}\frac{1}{A}, & 0<\theta<\frac{1}{2} \\
\frac{1}{2 A \theta}, & \frac{1}{2}<\theta<2\end{cases} \\
& \text { where } A=\frac{1}{2}+\frac{1}{2} \log 2-\frac{1}{2} \log \frac{1}{2}=1.1931 \\
& f(\theta \mid x=2)=\frac{1}{C}\left(1-\frac{3}{2 \theta}\right), \frac{3}{2}<\theta<2 \\
& \text { where } C=2-\frac{3}{2} \log 2-\frac{3}{2}+\frac{3}{2} \log \frac{3}{2}=0.068477 \text {. }
\end{aligned}
$$

Figure 3.2 shows these two posteriors, and further examples of $f(\theta \mid y)$.

Figure 3.2 Posteriors given $x=0,1,2$, and given $y=0.1, \ldots, 1.9$

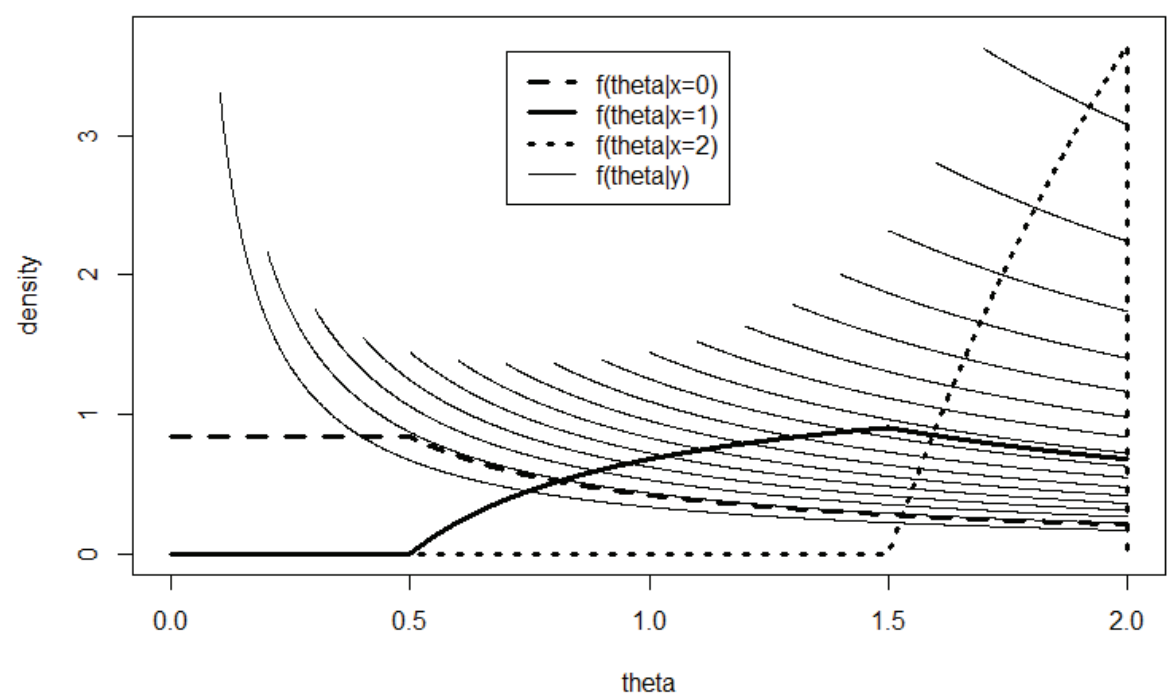

For completeness and checking we now also calculate the other two posterior means:

$$
\begin{aligned}
& E_{0}=E(\theta \mid x=0)=\frac{7}{8 A}=0.7334 \\
& E_{2}=E(\theta \mid x=2)=\frac{1}{8 C}=1.8254,
\end{aligned}
$$


as well as the unconditional probabilities of the data:

$$
\begin{aligned}
& P_{0}=P(x=0)=P\left(y<\frac{1}{2}\right)=E P\left(y<\frac{1}{2} \mid \theta\right)=\int P\left(y<\frac{1}{2} \mid \theta\right) f(\theta) d \theta \\
& \quad=\int_{0}^{1 / 2} 1 \times \frac{1}{2} d \theta+\int_{1 / 2}^{2} \frac{1 / 2}{\theta} \times \frac{1}{2} d \theta=\frac{1}{4}\left(1+\log 2-\log \frac{1}{2}\right)=0.5966 \\
& P_{1}=P(x=1)=0.3692 \\
& P_{2}=P(x=2)=0.0342 .
\end{aligned}
$$

As a check on our calculations, we note that

$$
P_{0}+P_{1}+P_{2}=1 \text { (which is correct). }
$$

We may also calculate the prior mean of $\theta$ (which is obviously 1 ) as

$$
\begin{aligned}
E \theta & =E E(\theta \mid x) \\
=E(\theta \mid x & =0) P(x=0)+E(\theta \mid x=1) P(x=1)+E(\theta \mid x=2) P(x=2) \\
& =E_{0} P_{0}+E_{1} P_{1}+E_{2} P_{2} \\
& =0.7334 \times 0.5966+1.354 \times 0.3692+1.825 \times 0.03424 \\
& =1.000 \text { (correct). }
\end{aligned}
$$

\section{R Code for Exercise 3.2}

$\mathrm{X} 11(\mathrm{w}=8, \mathrm{~h}=5.5) ; \operatorname{par}(\mathrm{mfrow}=\mathrm{c}(1,1)) ;$ options(digits $=7)$

$B=1.5-0.5 * \log (3 / 2)-0.5+0.5 * \log (0.5)+\log (2)-\log (1.5) ; c(B, 1 / B)$

\# 0.73837591 .3543237

postfun $B=$ function(theta, $B=0.7383759)\{$ res $=0$;

if $($ theta $>=1 / 2) \& \&($ theta $<3 / 2))$ res $=1-1 /(2 *$ theta $)$

if $(($ theta $>=3 / 2) \& \&($ theta $<=2))$ res $=1 /$ theta $\mathrm{res} / \mathrm{B} \quad\}$

thetavec $=\operatorname{seq}(0,2,0.001) ;$ postvecB=thetavec;

for(i in 1:length(thetavec)) postvec $B[i]=$ postfun $B($ theta=thetavec $[i])$

plot $(c(0,2), c(0,2)$,type="n",xlab="theta",ylab="density", main=" ")

lines(thetavec, postvecB, Iwd=3)

$y=0.6 ; k=1 /(\log (2)-\log (y))$

lines(thetavec[thetavec $>\mathrm{y}], \mathrm{k} /$ thetavec[thetavec $>\mathrm{y}], \mathrm{Ity}=2, \mathrm{Iwd}=3$ )

$y=1 ; k=1 /(\log (2)-\log (y))$

lines(thetavec[thetavec $>\mathrm{y}], \mathrm{k} /$ thetavec [thetavec $>\mathrm{y}], \mathrm{Ity}=3, \mathrm{Iwd}=3$ )

$y=1.1 ; k=1 /(\log (2)-\log (y))$

lines(thetavec[thetavec $>y$ ], $k$ / thetavec[thetavec $>y$ ], Ity $=4, I w d=3$ )

$y=1.4 ; k=1 /(\log (2)-\log (y))$

lines(thetavec[thetavec $>y$ ], $k$ / thetavec[thetavec $>y$ ], Ity=5,Iwd=3) 
legend $(0,2, c(" f($ theta $\mid x=1)$ ", "f(theta $\mid y=0.6) ", " f($ theta $\mid y=1)$ ","f(theta $\mid y=1.1)$ ", "f(theta $\mid y=1.4) "), \operatorname{Ity}=c(1,2,3,4,5), \operatorname{Iwd}=c(3,3,3,3,3))$

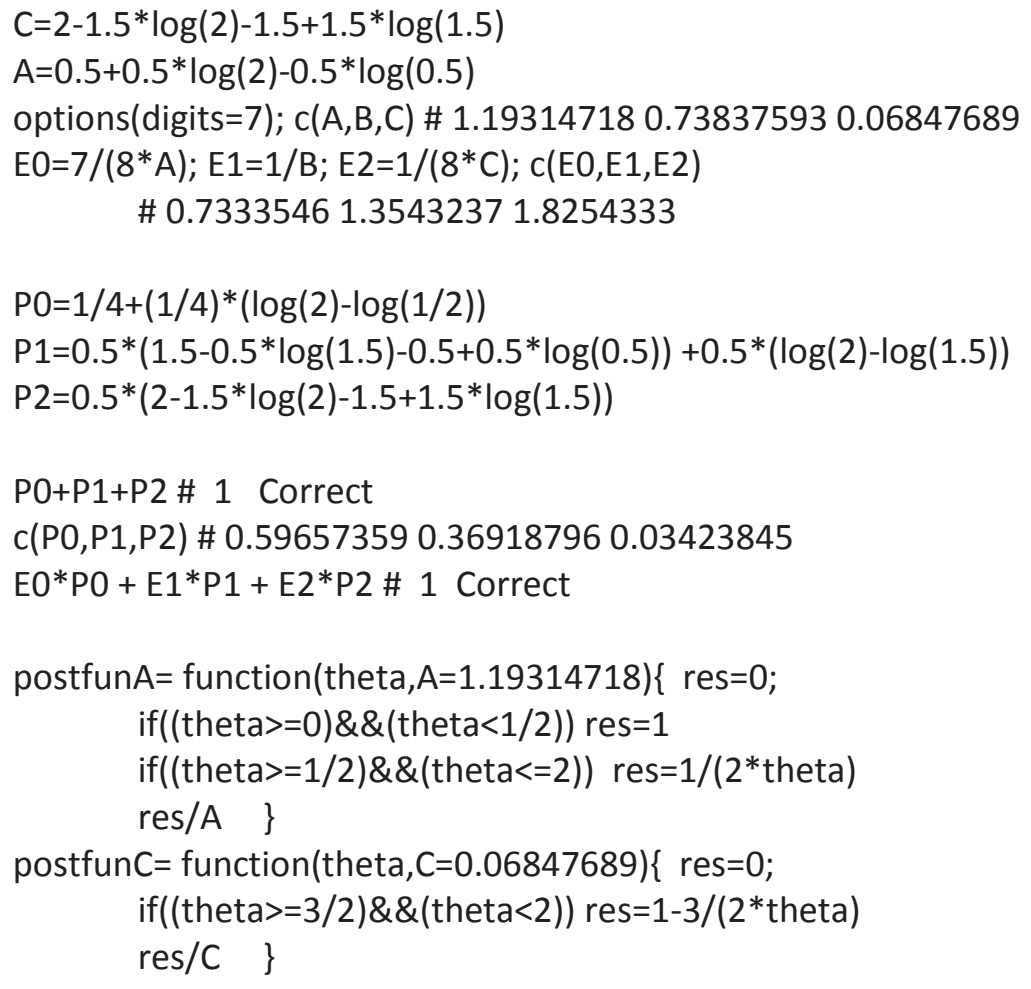




\subsection{Bayesian predictive inference}

In addition to estimating model parameters (and functions of those parameters) there is often interest in predicting some future data (or some other quantity which is not just a function of the model parameters).

Consider a Bayesian model specified by $f(y \mid \theta)$ and $f(\theta)$, with posterior as derived in ways already discussed and given by $f(\theta \mid y)$.

Now consider any other quantity $x$ whose distribution is defined by a density of the form $f(x \mid y, \theta)$.

The posterior predictive distribution of $x$ is given by the posterior predictive density $f(x \mid y)$. This can typically be derived using the following equation:

$$
\begin{aligned}
f(x \mid y) & =\int f(x, \theta \mid y) d \theta \\
& =\int f(x \mid y, \theta) f(\theta \mid y) d \theta .
\end{aligned}
$$

Note: For the case where $\theta$ is discrete, a summation needs to be performed rather than an integral.

The posterior predictive density $f(x \mid y)$ forms a basis for making probability statements about the quantity $x$ given the observed data $y$.

Point and interval estimation for future values $x$ can be performed in very much the same way as that for model parameters, except with a slightly different terminology.

Now, instead of referring to $\hat{x}=E(x \mid y)$ as the posterior mean of $x$, we may instead use the term predictive mean.

Also, the ' $\mathrm{P}$ ' in HPDR, and CPDR may be read as predictive rather than as posterior. For example, the CPDR for $x$ is now the central predictive density region for $x$.

As an example of point prediction, the predictive mean of $x$ is

$$
\hat{x}=E(x \mid y)=\int x f(x \mid y) d x .
$$


Often it is easier to obtain the predictive mean of $x$ using the equation

$$
\begin{aligned}
\hat{x}=E(x \mid y) & =E\{E(x \mid y, \theta) \mid y\} \\
& =\int E(x \mid y, \theta) f(\theta \mid y) d \theta .
\end{aligned}
$$

Note: The basic law of iterated expectation (LIE) implies that $E(x)=E E(x \mid \theta)$. This equation must also be true after conditioning throughout on $y$. We thereby obtain $E(x \mid y)=E\{E(x \mid y, \theta) \mid y\}$.

Likewise, the predictive variance of $x$ can be calculated via the equation

$$
V(x \mid y)=E\{V(x \mid y, \theta) \mid y\}+V\{E(x \mid y, \theta) \mid y\} .
$$

Note: This follows from the basic law of iterated variance (LIV), $V x=E V(x \mid \theta)+V E(x \mid \theta)$, after conditioning throughout on $y$.

An important special case of Bayesian predictive inference is where the quantity of interest $x$ is an independent future replicate of $y$.

This means that $(x \mid y, \theta)$ has exactly the same distribution as $(y \mid \theta)$, which in turn may be expressed mathematically as

$$
(x \mid y, \theta) \sim(y \mid \theta)
$$

or equivalently as

$$
f(x \mid y, \theta)=f(y=x \mid \theta)=\left[\left.f(y \mid \theta)\right|_{y=x}\right] .
$$

Note: The last equation indicates that the pdf of $(x \mid y, \theta)$ is the same as the pdf of $(y \mid \theta)$ but with $y$ changed to $x$ in the density formula.

In the case where $x$ is an independent future replicate of $y$, we may write $f(x \mid y, \theta)$ as $f(x \mid \theta)$, and this then implies that

$$
f(x \mid y)=\int f(x \mid \theta) f(\theta \mid y) d \theta .
$$

\section{Exercise 3.3 Prediction in the exponential-exponential model}

Suppose that $\theta$ has the standard exponential distribution, and the conditional distribution of $y$ given $\theta$ is exponential with mean $1 / \theta$.

Find the posterior predictive density of $x$, a future independent replicate of $y$. 
Then, for $y=2.0$, find the predictive mean, mode and median of $x$, and also the $80 \%$ central predictive density region and $80 \%$ highest predictive density region for $x$.

\section{Solution to Exercise 3.3}

Recall that the Bayesian model given by:

$$
\begin{aligned}
& f(y \mid \theta)=\theta e^{-\theta y}, y>0 \\
& f(\theta)=e^{-\theta}, \theta>0
\end{aligned}
$$

implies the posterior $(\theta \mid y) \sim \operatorname{Gamma}(2, y+1)$.

Now let $x$ be a future independent replicate of the data $y$, so that

$$
f(x \mid y, \theta)=f(x \mid \theta)=f(y=x \mid \theta)=\theta e^{-\theta x}, x>0 .
$$

Then the posterior predictive density of $x$ is

$$
\begin{aligned}
f(x \mid y) & =\int_{\infty} f(x \mid y, \theta) f(\theta \mid y) d \theta \\
& =\int_{0}^{\infty}\left(\theta e^{-\theta x}\right)\left(\frac{(y+1)^{2} \theta^{2-1} e^{-\theta(y+1)}}{\Gamma(2)}\right) d \theta \\
& =\frac{\Gamma(3)(y+1)^{2}}{\Gamma(2)(x+y+1)^{3}} \int_{0}^{\infty} \frac{(x+y+1)^{3} \theta^{3-1} e^{-\theta(x+y+1)}}{\Gamma(3)} d \theta \\
& =\frac{2(y+1)^{2}}{(x+y+1)^{3}}, x>0 .
\end{aligned}
$$

Check: $\int f(x \mid y) d x=\int_{0}^{\infty} \frac{2(y+1)^{2}}{(x+y+1)^{3}} d x$

$$
\begin{array}{r}
\left.=2(y+1)^{2} \int_{0+y+1}^{\infty+y+1} u^{-3} d u \quad \text { (where } u=x+y+1\right) \\
=2(y+1)^{2}\left[\left.\frac{u^{-2}}{-2}\right|_{u=y+1} ^{\infty}\right]=-(y+1)^{2}\left[\frac{1}{\infty^{2}}-\frac{1}{(y+1)^{2}}\right]=1 \quad \text { (correct). }
\end{array}
$$

Next, suppose that $y=2$. Then

$$
f(x \mid y)=18(x+3)^{-3}, x>0 .
$$

This is a strictly decreasing function, and so the predictive mode is zero. 
The predictive mean can be calculated according to the equation

$$
E(x \mid y)=\int_{0}^{\infty} x 18(x+3)^{-3} d x
$$

An easier way to find the predictive mean is to note that

$$
(\theta \mid y) \sim \operatorname{Gamma}(2,3)
$$

and then write

$$
\begin{gathered}
E(x \mid y)=E\{E(x \mid y, \theta) \mid y\}=E\left(\theta^{-1} \mid y\right)=\int_{0}^{\infty} \theta^{-1}\left(\frac{3^{2} \theta^{2-1} e^{-3 \theta}}{\Gamma(2)}\right) d \theta \\
=\frac{3^{2} \Gamma(1)}{3^{1} \Gamma(2)} \int_{0}^{\infty} \frac{3^{1} \theta^{1-1} e^{-3 \theta}}{\Gamma(1)} d \theta=3 .
\end{gathered}
$$

An even easier way to do the calculation is to recall a previous exercise where it was shown that the posterior mean of $\psi=1 / \theta$ is given by

$$
E(\psi \mid y)=y+1 \text {. }
$$

Thus, $E(x \mid y)=E\{E(x \mid y, \theta) \mid y\}=E(\psi \mid y)=y+1=3$ when $y=2$.

One way to find the predictive median of $x$ is to solve $F(x \mid y)=1 / 2$ for $x$, where $F(x \mid y)$ is the predictive cdf of $x$, or equivalently, to calculate $Q(1 / 2)$, where $Q(p)=F^{-1}(p \mid y)$ is the predictive quantile function of $x$.

Now, the predictive cdf of $x$ is

$$
\begin{aligned}
F(x \mid y) & =\int_{0}^{x} 18(t+3)^{-3} d t=\int_{3}^{3+x} 18 u^{-3} d t \quad \text { where } u=3+t \\
& =18\left[\left.\frac{u^{-2}}{-2}\right|_{u=3} ^{3+x}\right]=-9\left[\frac{1}{(3+x)^{2}}-\frac{1}{3^{2}}\right]=1-\frac{9}{(3+x)^{2}} .
\end{aligned}
$$

Setting this to $p$ and solving for $x$ yields the predictive quantile function,

$$
Q(p)=F^{-1}(p \mid y)=3\left(\frac{1}{\sqrt{1-p}}-1\right) \text {. }
$$

So the predictive median is $Q\left(\frac{1}{2}\right)=3\left(\frac{1}{\sqrt{1-1 / 2}}-1\right)=1.2426$. 
The predictive quantile function can now also be used to calculate the 80\% CPDR for $x$,

$$
(Q(0.1), Q(0.9))=(0.1623,6.4868) \text {, }
$$

and the $80 \%$ HPDR for $x$,

$$
(0, Q(0.8))=(0,3.7082) \text {. }
$$

Another way to calculate the predictive median of $x$ is as the solution in $q$ of

$$
1 / 2=P(x<q \mid y)
$$

after noting that the right hand side of this equation also equals

$$
\begin{aligned}
E\{P(x<q \mid y, \theta) \mid y\} & =E\left(1-e^{-\theta q} \mid y\right) \\
& =1-m(-q),
\end{aligned}
$$

where $m(t)$ is the posterior moment generating function (mgf) of $\theta$.

But $(\theta \mid y) \sim \operatorname{Gamma}(2, y+1)$, and so $m(t)=(1-t /(y+1))^{-2}$.

So we need to solve $1 / 2=(1-(-q) /(y+1))^{-2}$ for $q$. The result is $q=(y+1)(\sqrt{2}-1)=1.2426$ when $y=2$ (same as before).

\section{R Code for Exercise 3.3}

Qfun=function(p)\{3*(-1+1/sqrt(1-p)) \}; Qfun(0.5) \# 1.242641

c(Qfun(0.1), Qfun(0.9)) \# 0.16227776 .4868330

$c(0$, Qfun(0.8)) \# 0.0000003 .708204

\section{Exercise 3.4 Predicting a bus number (Extension of Exercise I.6)}

You are visiting a small town with buses whose license plates show their numbers consecutively from 1 up to however many there are. In your mind the number of buses could be anything from 1 to 5, with all possibilities equally likely. Whilst touring the town you first happen to see Bus 3.

Assuming that at any point in time you are equally likely to see any of the buses in the town, how likely is it that the next bus number you see will be at least 4 ?

Also, what is the expected value of the bus number that you will next see? 


\section{Solution to Exercise 3.4}

As in Exercise 1.6, let $\theta$ be the number of buses in the town and let $y$ be the number of the bus you happen to first see. Recall that a suitable Bayesian model is:

$$
\begin{aligned}
& f(y \mid \theta)=1 / \theta, y=1, \ldots, \theta \\
& f(\theta)=1 / 5, \theta=1, \ldots, 5 \quad \text { (prior), }
\end{aligned}
$$

and that the posterior density of $\theta$ works out as

$$
f(\theta \mid y)=\left\{\begin{array}{l}
20 / 47, \theta=3 \\
15 / 47, \theta=4 \\
12 / 47, \theta=5
\end{array}\right.
$$

Now let $x$ be the number on the next bus that you happen to see in the town. Then

$$
\left.f(x \mid y, \theta)=\frac{1}{\theta}, x=1, \ldots, \theta \quad \text { (same distribution as that of }(y \mid \theta)\right) .
$$

This may also be written

$$
f(x \mid y, \theta)=I(x \leq \theta) / \theta, x=1,2,3, \ldots,
$$

and so the posterior predictive density of $x$ is

$$
f(x \mid y)=\sum_{\theta} f(x, \theta \mid y)=\sum_{\theta} f(x \mid y, \theta) f(\theta \mid y)=\sum_{\theta=y}^{5} \frac{I(x \leq \theta)}{\theta} f(\theta \mid y) .
$$

In our case, the observed value of $y$ is 3 and so:

$$
\begin{aligned}
& f(x=1 \mid y)=\frac{1}{3} \times \frac{20}{47}+\frac{1}{4} \times \frac{15}{47}+\frac{1}{5} \times \frac{12}{47}=0.27270 \\
& f(x=2 \mid y)=\frac{1}{3} \times \frac{20}{47}+\frac{1}{4} \times \frac{15}{47}+\frac{1}{5} \times \frac{12}{47}=0.27270 \\
& f(x=3 \mid y)=\frac{1}{3} \times \frac{20}{47}+\frac{1}{4} \times \frac{15}{47}+\frac{1}{5} \times \frac{12}{47}=0.27270 \\
& f(x=4 \mid y)=\frac{1}{4} \times \frac{15}{47}+\frac{1}{5} \times \frac{12}{47}=0.13085 \\
& f(x=5 \mid y)=\frac{1}{5} \times \frac{12}{47}=0.05106 .
\end{aligned}
$$

Check: $\sum_{x=1}^{5} f(x \mid y)=0.27270 \times 3+0.13085+0.05106=1$ (correct). 
In summary, for $y=3$, we have that $f(x \mid y)= \begin{cases}0.27270, & x=1,2,3 \\ 0.13085, & x=4 \\ 0.05106, & x=5\end{cases}$

So the probability that the next bus you see will have a number on it which is at least 4 equals

$$
\begin{aligned}
P(x \geq 4 \mid y)=\sum_{x: x \geq 4} f(x \mid y) & =f(x=4 \mid y)+f(x=5 \mid y) \\
& =0.13085+0.05106=18.2 \% .
\end{aligned}
$$

Also, the expected value of the bus number you will next see is

$$
\begin{aligned}
E(x \mid y)=1(0.27270) & +2(0.27270)+3(0.27270) \\
& +4(0.13085)+5(0.05106)=2.4149
\end{aligned}
$$

Alternatively, $E(x \mid y)=E\{E(x \mid y, \theta) \mid y\}=E\left(\frac{1+\theta}{2} \mid y\right)=\frac{1}{2} E(\theta \mid y)$

$$
=\left\{\frac{1+3(20 / 47)+4(15 / 47)+5(12 / 47)}{2}\right\}=\frac{1+180 / 47}{2}=\frac{227}{94}=2.4149 \text {. }
$$

\section{R Code for Problem 3.4}

$f v=r e p(N A, 5) ; \quad f v[1]=(1 / 3) *(20 / 47)+(1 / 4) *(15 / 47)+(1 / 5) *(12 / 47)$

$f v[2]=f v[1] ; f v[3]=f v[1] ; \quad f v[4]=(1 / 4) *(15 / 47)+(1 / 5) *(12 / 47)$

$\mathrm{fv}[5]=(1 / 5) *(12 / 47) ;$ options(digits $=5)$

fv \# 0.2726950.2726950.2726950.1308510.051064

$\operatorname{sum}(\mathrm{fv}) \# 1$ (OK)

$\operatorname{sum}(f v[4: 5])$ \# 0.18191

$\operatorname{sum}\left((1: 5)^{*} \mathrm{fv}\right) \# 2.4149$

$227 / 94$ \# 2.4149

\section{Exercise 3.5 Prediction in the binomial-beta model}

(a) For the Bayesian model given by $(Y \mid \theta) \sim \operatorname{Bin}(n, \theta)$ and the prior $\theta \sim \operatorname{Beta}(\alpha, \beta)$, find the posterior predictive density of a future data value $x$, whose distribution is defined by $(x \mid y, \theta) \sim \operatorname{Bin}(m, \theta)$.

(b) A bent coin is tossed 20 times and 6 heads come up. Assuming a flat prior on the probability of heads on a single toss, what is the probability that exactly one head will come up on the next two tosses of the same coin? Answer this using results in (a). 
(c) A bent coin is tossed 20 times and 6 heads come up. Assume a Beta $(20.3,20.3)$ prior on the probability of heads.

Find the expected number of times you will have to toss the same coin again repeatedly until the next head comes up.

(d) A bent coin is tossed 20 times and 6 heads come up. Assume a Beta $(20.3,20.3)$ prior on the probability of heads.

Now consider tossing the coin repeatedly until the next head, writing down the number of tosses, and then doing all of this again repeatedly, again and again.

The result will be a sequence of natural numbers (for example $3,1,1,4,2,2,1,5,1, \ldots$.$) , where each number represents a number of$ tails in a row within the sequence, plus one.

Next define $\psi$ to be the average of a very long sequence like this (e.g. one of length 1,000,000). Find the posterior predictive density and mean of $\psi$ (approximately).

Note: In parts (c) and (d) the parameters of the beta distribution (both 20.3) represent a prior belief that the probability of heads is about $1 / 2$, is equally likely to be on either side of $1 / 2$, and is $80 \%$ likely to be between 0.4 and 0.6 . See the R Code below for details.

\section{Solution to Exercise 3.5}

(a) First note that $x$ is not a future independent replicate of the observed data $y$, except in the special case where $m=n$.

Next recall that $(\theta \mid y) \sim \operatorname{Beta}(a, b)$, where:

$$
a=\alpha+y, \quad b=\beta+n-y .
$$

Thus the posterior predictive density of $x$ is

$$
\begin{aligned}
f(x \mid y) & =\int f(x, \theta \mid y) d \theta \\
& =\int f(x \mid y, \theta) f(\theta \mid y) d \theta \\
& =\int_{0}^{1}\left(\begin{array}{l}
m \\
x
\end{array}\right) \theta^{x}(1-\theta)^{m-x} \frac{\theta^{a-1}(1-\theta)^{b-1}}{B(a, b)} d \theta
\end{aligned}
$$




$$
\begin{aligned}
& =\left(\begin{array}{l}
m \\
x
\end{array}\right) \frac{B(x+a, m-x+b)}{B(a, b)} \int_{0}^{1} \frac{\theta^{x+a-1}(1-\theta)^{m-x+b-1}}{B(x+a, m-x+b)} d \theta \\
& =\left(\begin{array}{l}
m \\
x
\end{array}\right) \frac{B(x+\alpha+y, m-x+\beta+n-y)}{B(\alpha+y, \beta+n-y)}, x=0, \ldots, m .
\end{aligned}
$$

Note: The distribution of $(x \mid y)$ here may be called the beta-binomial.

(b) Here, we consider the situation in (a) with $n=20, y=6, m=2$, $\alpha=1, \beta=1$ and $x=0,1$ or 2 . So, specifically,

$$
\begin{aligned}
f(x \mid y) & =\left(\begin{array}{l}
2 \\
x
\end{array}\right) \frac{B(x+1+6,2-x+1+20-6)}{B(1+6,1+20-6)} \\
& =\left(\begin{array}{l}
2 \\
x
\end{array}\right) \frac{\Gamma(7+x) \Gamma(17-x) / \Gamma(24)}{\Gamma(7) \Gamma(15) / \Gamma(22)} \\
& =\frac{2 !}{x !(2-x) !} \frac{(6+x) !(16-x) ! / 23 !}{6 ! 14 ! / 21 !} \\
& =\left\{\begin{array}{l}
0.4743, \quad x=0 \\
0.4150, x=1 \\
0.1107, \quad x=2 .
\end{array}\right.
\end{aligned}
$$

Check: $0.4743+0.4150+0.1107=1$ (correct).

So the (posterior predictive) probability that heads will come up on exactly one of the next two tosses is $f(x=1 \mid y=6)=41.5 \%$.

Note: An alternative way to do the working here is to see that if $y=6$ then

$$
(\theta \mid y) \sim \operatorname{Beta}(1+6,1+20-6) \sim \operatorname{Beta}(7,15),
$$

so that:

$$
\begin{aligned}
& E(\theta \mid y)=\frac{7}{7+15}=\frac{7}{22} \\
& V(\theta \mid y)=\frac{7 \times 15}{(7+15)^{2}(7+15+1)}=0.009432 .
\end{aligned}
$$

Also, $(x \mid y, \theta) \sim \operatorname{Bin}(2, \theta)$ (if $y=6)$. 
It follows that

$$
\begin{aligned}
P(x=1 \mid y) & =E\{P(x=1 \mid y, \theta) \mid y\} \\
& =E\{2 \theta(1-\theta) \mid y\} \\
& =2\left\{E(\theta \mid y)-E\left(\theta^{2} \mid y\right)\right\} \\
& =2\left\{E(\theta \mid y)-\left[V(\theta \mid y)+(E(\theta \mid y))^{2}\right]\right\} \\
& =2\left\{\frac{7}{22}-\left[0.009432+\left(\frac{7}{22}\right)^{2}\right]\right\}=0.415 .
\end{aligned}
$$

(c) Let $z$ be the number of tosses until the next head. Then

$$
(z \mid y, \theta) \sim \operatorname{Geometric}(\theta)
$$

with pdf

$$
f(z \mid y, \theta)=(1-\theta)^{z-1} \theta, z=1,2,3, \ldots .
$$

So the posterior predictive density of $z$ can be obtained via the equation

$$
f(z \mid y)=\int f(z, \theta \mid y) d \theta=\int f(z \mid y, \theta) f(\theta \mid y) d \theta \text {. }
$$

It will be noted that $(z \mid y)$ has a density with a similar form to that of $(x \mid y)$ in $(\mathrm{a})$, but with an infinite range $(z=1,2,3, \ldots)$. If we were to write down $f(z \mid y)$, we could then evaluate the expected number of tosses until the next head according to the equation

$$
E(z \mid y)=\sum_{z=1}^{\infty} z f(z \mid y) \text {. }
$$

More easily, the posterior predictive mean of $z$ can be obtained as

$$
\begin{aligned}
E(z \mid y) & =E\{E(z \mid y, \theta) \mid y\}=E\left(\frac{1}{\theta} \mid y\right)=\int_{0}^{1} \frac{1}{\theta} \times \frac{\theta^{a-1}(1-\theta)^{b-1}}{B(a, b)} d \theta \\
& =\frac{B(a-1, b)}{B(a, b)} \int_{0}^{1} \frac{\theta^{(a-1)-1}(1-\theta)^{b-1}}{B(a-1, b)} d \theta \\
& =\frac{\Gamma(a-1) \Gamma(b) / \Gamma(a-1+b)}{\Gamma(a) \Gamma(b) / \Gamma(a+b)} \times 1=\frac{a+b-1}{a-1} \\
& =\frac{(\alpha+y)+(\beta+n-y)-1}{(\alpha+y)-1}=\frac{\alpha+\beta+n-1}{\alpha+y-1} .
\end{aligned}
$$

For $n=20, y=6$ and $\alpha=\beta=20.3$, we find that $E(z \mid y)=2.356$. 
(d) Here, $\psi$ represents the average of a very large number of independent realisations of the random variable $z$ in (c). Therefore (approximately), $\psi=E(z \mid y, \theta)=1 / \theta$.

It follows that the posterior predictive density of $\psi$ is

$$
f(\psi \mid y)=f(\theta \mid y)\left|\frac{d \theta}{d \psi}\right|,
$$

where $\theta=\psi^{-1}$ and $d \theta / d \psi=-\psi^{-2}$. Thus

$$
\begin{aligned}
f(\psi \mid y) & =\frac{(1 / \psi)^{a-1}(1-1 / \psi)^{b-1}}{B(a, b)}\left|\frac{-1}{\psi^{2}}\right| \\
& =\frac{(\psi-1)^{b-1}}{\psi^{a+b} B(a, b)}, \psi>1 .
\end{aligned}
$$

So the posterior predictive mean of $\psi$ is

$$
\begin{aligned}
E(\psi \mid y) & =\int_{1}^{\infty} \psi \frac{(\psi-1)^{b-1}}{\psi^{a+b} B(a, b)} d \psi \\
& =\frac{B(a-1, b)}{B(a, b)} \int_{1}^{\infty} \frac{(\psi-1)^{b-1}}{\psi^{(a-1)+b} B(a-1, b)} d \psi
\end{aligned}
$$

The last integral is 1 , by analogy of its integrand with $f(\psi \mid y)$. Thus we obtain the same expression as for $E(z \mid y)$ and $E(1 / \theta \mid y)$ in (c), namely

$$
E(\psi \mid y)=\frac{\alpha+\beta+n-1}{\alpha+y-1} .
$$

\section{R Code for Exercise 3.5}

options(digits=4); pbeta $(0.4,20.3,20.3) \# 0.1004$

pbeta $(0.6,20.3,20.3)$ - pbeta $(0.4,20.3,20.3)$ \# 0.7993

$\mathrm{x}=0: 2$

$(2 *$ factorial $(6+x) *$ factorial $(16-x) /$ factorial $(23) \quad$ )/

( factorial $(\mathrm{x}) *$ factorial $(2-\mathrm{x}) *$ factorial $(6) *$ factorial(14)/factorial(21) ) \# 0.47430 .41500 .1107

$7 * 15 /\left(22^{\wedge} 2^{*} 23\right) \# 0.009432$

$2 *\left(7 / 22-\left(0.009432267+(7 / 22)^{\wedge} 2\right)\right) \quad \# 0.415$

$(20.3+20.3+20-1) /(20.3+6-1) \# 2.356$ 


\section{Exercise 3.6 Prediction in the normal-normal model (with variance known)}

Consider the Bayesian model given by:

$$
\begin{aligned}
& \left(y_{1}, \ldots, y_{n} \mid \mu\right) \sim \text { iid } N\left(\mu, \sigma^{2}\right) \\
& \mu \sim N\left(\mu_{0}, \sigma_{0}^{2}\right),
\end{aligned}
$$

and suppose we have data in the form of the vector $y=\left(y_{1}, \ldots, y_{n}\right)$.

Also suppose there is interest in $m$ future values:

$$
\left(x_{1}, \ldots, x_{m} \mid y, \mu\right) \sim \text { iid } N\left(\mu, \sigma^{2}\right) \text {. }
$$

Find the posterior predictive distribution of

$$
\bar{x}=\left(x_{1}+\ldots+x_{m}\right) / m,
$$

both generally and in the case of a priori ignorance regarding $\mu$.

\section{Solution to Exercise 3.6}

By Exercise 1.18 the posterior distribution of $\mu$ is given by

$$
(\mu \mid y) \sim N\left(\mu_{*}, \sigma_{*}^{2}\right),
$$

where: $\mu_{*}=(1-k) \mu_{0}+k \bar{y}, \quad \sigma_{*}^{2}=k \frac{\sigma^{2}}{n}, \quad k=\left(1+\frac{\sigma^{2} / n}{\sigma_{0}^{2}}\right)^{-1}$.

Now, $(\bar{x} \mid y, \mu) \sim N\left(\mu, \sigma^{2} / m\right)$, and therefore

$$
\begin{aligned}
f(\bar{x} \mid y) & =\int f(\bar{x} \mid y, \mu) f(\mu \mid y) d \mu \\
& \propto \int_{-\infty}^{\infty} \exp \left\{-\frac{(\bar{x}-\mu)^{2}}{2 \sigma^{2} / m}\right\} \exp \left\{-\frac{\left(\mu-\mu_{*}\right)^{2}}{2 \sigma_{*}^{2}}\right\} d \mu .
\end{aligned}
$$

This is the integral of the exponent of a quadratic in both $\bar{x}$ and $\mu$ and so must equal the exponent of a quadratic in $\bar{x}$. It follows that

$$
(\bar{x} \mid y) \sim N\left(\eta, \delta^{2}\right)
$$

where $\eta$ and $\delta^{2}$ are to be determined. This final step is easily achieved as follows:

$$
\begin{aligned}
\eta & =E(\bar{x} \mid y) \\
& =E\{E(\bar{x} \mid y, \mu) \mid y\} \\
& =E\{\mu \mid y\}=\mu_{*}
\end{aligned}
$$




$$
\begin{aligned}
\delta^{2} & =V(\bar{x} \mid y) \\
& =E\{V(\bar{x} \mid y, \mu) \mid y\}+V\{E(\bar{x} \mid y, \mu) \mid y\} \\
& =E\left\{\frac{\sigma^{2}}{m} \mid y\right\}+V\{\mu \mid y\}=\frac{\sigma^{2}}{m}+\sigma_{*}^{2} .
\end{aligned}
$$

Thus generally we have that

$$
(\bar{x} \mid y) \sim N\left(\mu_{*}, \sigma_{*}^{2}+\frac{\sigma^{2}}{m}\right) \sim N\left((1-k) \mu_{0}+k \bar{y}, k \frac{\sigma^{2}}{n}+\frac{\sigma^{2}}{m}\right) .
$$

A special case is where there is no prior information regarding the normal mean $\mu$. In this case, assuming it is appropriate to set $\sigma_{0}=\infty$ (so that $f(\mu) \propto 1, \mu \in \mathfrak{R}$ ), we have that $k=1$ and hence

$$
(\bar{x} \mid y) \sim N\left(\bar{y}, \frac{\sigma^{2}}{n}+\frac{\sigma^{2}}{m}\right) .
$$

\section{Exercise 3.7 Prediction in the normal-gamma model (with a known mean)}

Consider the Bayesian model given by :

$$
\begin{aligned}
& \left(y_{1}, \ldots, y_{n} \mid \lambda\right) \sim \text { iid } N(\mu, 1 / \lambda) \\
& \lambda \sim G(\alpha, \beta),
\end{aligned}
$$

and suppose we have data in the form of the vector $y=\left(y_{1}, \ldots, y_{n}\right)$.

Also, suppose we are interested in $m$ future values:

$$
\left(x_{1}, \ldots, x_{m} \mid y, \lambda\right) \sim \text { iid } N(\mu, 1 / \lambda) \text {. }
$$

Find the posterior predictive distribution of

$$
\bar{x}=\left(x_{1}+\ldots+x_{m}\right) / m,
$$

both generally and in the case of a priori ignorance regarding $\lambda$.

\section{Solution to Exercise 3.7}

By Exercise 1.20 the posterior distribution of $\lambda$ is given by

$$
(\lambda \mid y) \sim \operatorname{Gamma}(a, b),
$$

where: $a=\alpha+\frac{n}{2}, \quad b=\beta+\frac{n}{2} s_{y \mu}^{2}, \quad s_{y \mu}^{2}=\frac{1}{n} \sum_{i=1}^{n}\left(y_{i}-\mu\right)^{2}$.

Now, $(\bar{x} \mid y, \lambda) \sim N(\mu, 1 /(m \lambda))$, and therefore 


$$
\begin{aligned}
f(\bar{x} \mid y) & =\int f(\bar{x} \mid y, \lambda) f(\lambda \mid y) d \lambda \\
& \propto \int_{0}^{\infty} \sqrt{\lambda} \exp \left\{-\frac{m \lambda}{2}(\bar{x}-\mu)^{2}\right\} \lambda^{a-1} \exp (-\lambda b) d \lambda \\
& \left.=\int_{0}^{\infty} \lambda^{\left(a+\frac{1}{2}\right)-1} \exp \left\{-\lambda\left[b+\frac{m}{2}(\bar{x}-\mu)^{2}\right]\right\} d \lambda\right] \\
& \propto\left[b+\frac{m}{2}(\bar{x}-\mu)^{2}\right]^{-\left(a+\frac{1}{2}\right)} \propto\left(1+\frac{\left\{\frac{m 2 a(\bar{x}-\mu)^{2}}{2 b}\right\}}{2 a}\right)^{-\frac{1}{2}(2 a+1)}
\end{aligned}
$$

Now let $Q=\sqrt{\frac{m 2 a(\bar{x}-\mu)^{2}}{2 b}}=\frac{\bar{x}-\mu}{(\sqrt{b / a}) / \sqrt{m}}$, so that $\bar{x}=\mu+Q \frac{\sqrt{b / a}}{\sqrt{m}}$.

Then by the transformation rule,

$$
f(Q \mid y)=f(\bar{x} \mid y)\left|\frac{d \bar{x}}{d Q}\right| \propto\left(1+\frac{Q^{2}}{2 a}\right)^{-\frac{1}{2}(2 a+1)}\left|\frac{\sqrt{b / a}}{\sqrt{m}}\right| \propto\left(1+\frac{Q^{2}}{2 a}\right)^{-\frac{1}{2}(2 a+1)} .
$$

This implies that $(Q \mid y) \sim t(2 a)$, or equivalently,

$$
\left.\left|\frac{\bar{x}-\mu}{\sqrt{\frac{s_{y \mu}^{2}+2 \beta / n}{1+2 \alpha / n}} / \sqrt{m}}\right| y\right) \sim t(n+2 \alpha) .
$$

A special case of this general result is when there is no prior information regarding the precision parameter $\lambda$. In that case, and assuming it is then appropriate to set $\alpha=\beta=0$ (so that $f(\lambda) \propto 1 / \lambda, \lambda>0$ ), we have that

$$
\left(\frac{\bar{x}-\mu}{s_{y \mu} / \sqrt{m}} \mid y\right) \sim t(n) \text {. }
$$




\subsection{Posterior predictive p-values}

Earlier, in Section 1.3, we discussed Bayes factors as a form of hypothesis testing within the Bayesian framework. An entirely different way to perform hypothesis testing in that framework is via the theory of posterior predictive p-values (Meng, 1994). As in the theory of Bayes factors, this involves first specifying a null hypothesis

$$
H_{0}: E_{0}
$$

and an alternative hypothesis

$$
H_{1}: E_{1} \text {, }
$$

where $E_{0}$ and $E_{1}$ are two events.

Note: As in Section 1.3, $E_{0}$ and $E_{1}$ may or may not be disjoint. Also, $E_{0}$ and $E_{1}$ may instead represent two different models for the same data.

In the context of a single Bayesian model with data $y$ and parameter $\theta$, the theory of posterior predictive p-values involves the following steps:

(i) Define a suitable discrepancy measure (or test statistic), denoted $T(y, \theta)$,

following careful consideration of both $H_{0}$ and $H_{1}$ (see below).

(ii) Define $x$ as an independent future replicate of the data $y$.

(iii) Calculate the posterior predictive p-value (ppp-value), defined as

$$
p=P\{T(x, \theta) \geq T(y, \theta) \mid y\} .
$$

Note 1: The ppp-value is calculated under the implicit assumption that $H_{0}$ is true. Thus we could also write $p=P\left\{T(x, \theta) \geq T(y, \theta) \mid y, H_{0}\right\}$.

Note 2: The discrepancy measure may or may not depend on the model parameter, $\theta$. Thus in some cases, $T(y, \theta)$ may also be written as $T(y)$.

The underlying idea behind the choice of discrepancy measure $T$ is that if the observed data $y$ is highly inconsistent with $H_{0}$ in favour of $H_{1}$ then $p$ should likely be small. This is the same idea as behind classical hypothesis testing. In fact, the classical theory may be viewed as a special case of the theory of ppp-values. The advantage of the ppp-value framework is that it is far more versatile and can be used in situations where it is not obvious how the classical theory should be applied. 
An example of how ppp-value theory can perform well relative to the classical theory is where the null hypothesis is composite, meaning that it consists of the specification of multiple values rather than a single value (e.g. $H_{0}:|\theta|<\varepsilon$ as compared to $H_{0}: \theta=0$ ). The next exercise illustrates this feature.

\section{Exercise 3.8 Posterior predictive p-values for testing a composite null hypothesis}

Consider the Bayesian model given by:

$$
\begin{aligned}
& (y \mid \lambda) \sim \operatorname{Poisson}(\lambda) \\
& f(\lambda)=e^{-\lambda}, \lambda>0,
\end{aligned}
$$

and suppose that we observe $y=3$.

(a) Find a suitable ppp-value for testing

$$
H_{0}: \lambda=1 \text { versus } H_{1}: \lambda>2 \text {. }
$$

(b) Find a suitable ppp-value for testing

$$
H_{0}: \lambda \in\{1,2\} \text { versus } H_{1}: \lambda>2 \text {. }
$$

\section{Solution to Exercise 3.8}

(a) Here, $(x \mid y, \lambda) \sim \operatorname{Poi}(\lambda)$, and we may define the test statistic as

$$
T(y, \lambda)=y .
$$

Then, the posterior predictive p-value is

$$
\begin{aligned}
p & =P(x \geq y \mid y, \lambda=1) \\
& =1-F_{P o i(1)}(y-1),
\end{aligned}
$$

where $y=3$ and where $F_{P o i(q)}(r)$ is the cumulative distribution function of a Poisson random variable with mean $q$, evaluated at $r$.

Thus a suitable ppp-value is

$$
p=1-\left(\frac{e^{-1} 1^{0}}{0 !}+\frac{e^{-1} 1^{1}}{1 !}+\frac{e^{-1} 1^{2}}{2 !}\right)=0.08030 .
$$

Note: This is just the probability that a Poisson(1) random variable will take on a value greater than 2 , and so is the same as the classical $p$-value which would be used in this situation. 
(b) Here we first observe that

$$
\begin{aligned}
& f\left(\lambda \mid y, H_{0}\right) \propto f\left(\lambda \mid H_{0}\right) f\left(y \mid H_{0}, \lambda\right) \\
& =\frac{e^{-\lambda}}{e^{-1}+e^{-2}} \times \frac{e^{-\lambda} \lambda^{y}}{y !} \propto e^{-2 \lambda} \lambda^{y}, \lambda=1,2 \quad \text { (with } y=3 \text { ). }
\end{aligned}
$$

Thus: $\quad P\left(\lambda=1 \mid y, H_{0}\right)=\frac{e^{-2 \times 1} 1^{3}}{e^{-2 \times 1} 1^{3}+e^{-2 \times 2} 2^{3}}=0.48015$

$$
P\left(\lambda=2 \mid y, H_{0}\right)=1-0.48015=0.51985 \text {. }
$$

So a suitable ppp-value is

$$
\begin{aligned}
p=P\left(x \geq y \mid y, H_{0}\right) & =E\left\{P\left(x \geq y \mid y, H_{0}, \lambda\right) \mid y, H_{0}\right\} \\
& =E\left\{1-F_{\text {Poi }(\lambda)}(y-1) \mid y, H_{0}\right\} \\
= & 0.48015 \times\left(1-F_{\text {Poi }(1)}(2)\right)+0.51985 \times\left(1-F_{\text {Poi }(2)}(2)\right) \\
= & 0.48015\left\{1-\left(\frac{e^{-1} 1^{0}}{0 !}+\frac{e^{-1} 1^{1}}{1 !}+\frac{e^{-1} 1^{2}}{2 !}\right)\right\} \\
& +0.51985\left\{1-\left(\frac{e^{-2} 2^{0}}{0 !}+\frac{e^{-2} 2^{1}}{1 !}+\frac{e^{-2} 2^{2}}{2 !}\right)\right\} \\
= & 0.20664 .
\end{aligned}
$$

\section{R Code for Exercise 3.8}

options(digits=5); 1 -ppois $(2,1) \# 0.080301$

$\mathrm{p} 1=\exp (-2) /\left(\exp (-2)+8^{*} \exp (-4)\right) ; \mathrm{c}(\mathrm{p} 1,1-\mathrm{p} 1) \# 0.480150 .51985$

p1*(1-ppois $(2,1))+(1-p 1) *(1-p p o i s(2,2)) \# 0.20664$

\section{Exercise 3.9 Posterior predictive p-values for testing a normal mean}

Consider a random sample $y_{1}, \ldots, y_{n}$ from a normal distribution with variance $\sigma^{2}$, where the prior on the precision parameter $\lambda=1 / \sigma^{2}$ is given by $\lambda \sim \operatorname{Gamma}(0,0)$, or equivalently by $f(\lambda) \propto 1 / \lambda, \lambda>0$.

We wish to test the null hypothesis

$H_{0}$ : that the normal mean equals $\mu$

against the alternative hypothesis

$H_{1}$ : that the normal mean is greater than $\mu$

(where $\mu$ is a specified constant of interest). 
Derive a formula for the ppp-value under each of the following three choices of the test statistic:
(a) $T(y, \lambda)=\bar{y}$,
(b) $T(y, \lambda)=\frac{\bar{y}-\mu}{\sigma / \sqrt{n}}$,
(c) $T(y, \lambda)=\frac{\bar{y}-\mu}{s_{y} / \sqrt{n}}$,

where: $\quad \bar{y}=\frac{1}{n} \sum_{i=1}^{n} y_{i} \quad$ (the sample mean)

$$
s_{y}^{2}=\frac{1}{n-1} \sum_{i=1}^{n}\left(y_{i}-\bar{y}\right)^{2} \quad \text { (the sample variance). }
$$

For each of these choices of test statistic, report the ppp-value for the case where $\mu=2$ and $y=(2.1,4.0,3.7,5.5,3.0,4.6,8.3,2.2,4.1,6.2)$.

\section{Solution to Exercise 3.9}

(a) Let $\bar{x}=\left(x_{1}+\ldots+x_{n}\right) / n$ be the mean of an independent replicate of the sample values, defined by $\left(x_{1}, \ldots, x_{n} \mid y, \lambda\right) \sim$ iid $N\left(\mu, \sigma^{2}\right)$.

Then, by Exercise 3.7, $\left(\frac{\bar{x}-\mu}{s_{y \mu} / \sqrt{n}} \mid y\right) \sim t(n)$, where $s_{y \mu}^{2}=\frac{1}{n} \sum_{i=1}^{n}\left(y_{i}-\mu\right)^{2}$.

From this, if the test statistic is $T(y, \lambda)=\bar{y}$, then the ppp-value is

$$
p=P(\bar{x}>\bar{y} \mid y)=P\left(\frac{\bar{x}-\mu}{s_{y \mu} / \sqrt{n}}>\frac{\bar{y}-\mu}{s_{y \mu} / \sqrt{n}} \mid y\right)=1-F_{t(n)}\left(\frac{\bar{y}-\mu}{s_{y \mu} / \sqrt{n}}\right) .
$$

Here: $\mu=2, n=10, \quad \bar{y}=\frac{1}{n} \sum_{i=1}^{n} y_{i}=4.370, \quad s_{y \mu}^{2}=\frac{1}{n} \sum_{i=1}^{n}\left(y_{i}-\mu\right)^{2}=2.978$.

Therefore $\frac{\bar{y}-\mu}{s_{y \mu} / \sqrt{n}}=2.51658$, and so $p=1-F_{t(10)}(2.51658)=0.01528$.

(b) If $T(y, \lambda)=\frac{\bar{y}-\mu}{\sigma / \sqrt{n}}$ then the ppp-value is

$$
p=P\left(\frac{\bar{x}-\mu}{\sigma / \sqrt{n}}>\frac{\bar{y}-\mu}{\sigma / \sqrt{n}} \mid y\right)=P(\bar{x}>\bar{y} \mid y) .
$$

We see that the answer here is exactly the same as in (a). 
(c) If $T(y, \lambda)=\frac{\bar{y}-\mu}{s_{y} / \sqrt{n}}$ then the ppp-value is

$$
\begin{aligned}
p & =P\left(\frac{\bar{x}-\mu}{s_{x} / \sqrt{n}}>\frac{\bar{y}-\mu}{s_{y} / \sqrt{n}} \mid y\right) \quad \text { where } s_{x}^{2}=\frac{1}{n-1} \sum_{i=1}^{n}\left(x_{i}-\bar{x}\right)^{2} \\
& =E\left\{P\left(\frac{\bar{x}-\mu}{s_{x} / \sqrt{n}}>\frac{\bar{y}-\mu}{s_{y} / \sqrt{n}} \mid y, \lambda\right) \mid y\right\} \\
& =E\left\{1-F_{t(n-1)}\left(\frac{\bar{y}-\mu}{s_{y} / \sqrt{n}}\right) \mid y\right\} \quad \text { since }\left(\frac{\bar{x}-\mu}{s_{x} / \sqrt{n}} \mid y, \lambda\right) \sim t(n-1) \\
& =1-F_{t(n-1)}\left(\frac{\bar{y}-\mu}{s_{y} / \sqrt{n}}\right) .
\end{aligned}
$$

We see that the ppp-value derived is exactly the same as the classical p-value which would be used in this setting. Numerically, we have that:

$$
s_{y}^{2}=\frac{1}{n-1} \sum_{i=1}^{n}\left(y_{i}-\bar{y}\right)^{2}=1.901, \quad \frac{\bar{y}-\mu}{s_{y} / \sqrt{n}}=3.942645 .
$$

Consequently, the ppp-value is $p=1-F_{t(9)}(3.942645)=0.001696$.

Note: A fourth test statistic which makes sense in the present context is

$$
T(y, \lambda)=\frac{\bar{y}-\mu}{s_{y \mu} / \sqrt{n}} \text { where } s_{y \mu}^{2}=\frac{1}{n} \sum_{i=1}^{n}\left(y_{i}-\mu\right)^{2} \text { (as before). }
$$

This implies a ppp-value given by

$$
p=P\left(\frac{\bar{x}-\mu}{s_{x \mu} / \sqrt{n}}>\frac{\bar{y}-\mu}{s_{y \mu} / \sqrt{n}} \mid y\right) \text { where } s_{x \mu}^{2}=\frac{1}{n} \sum_{i=1}^{n}\left(x_{i}-\mu\right)^{2} .
$$

This ppp-value is more difficult to calculate, and it cannot be expressed in terms of well-known quantities, e.g. the cdf of a t distribution, as in (a), (b) and (c). (Here, $\bar{x}$ and $s_{x \mu}$ are not independent, given $y$ and $\mu$.)

For more details, regarding this exercise specifically and ppp-values generally, see Meng (1994) and Gelman et al. (2004). 


\section{R Code for Exercise 3.9}

options(digits=4); mu=2; y = c(2.1, 4.0, 3.7, 5.5, 3.0, 4.6, 8.3, 2.2 , 4.1, 6.2);

$\mathrm{n}=$ length $(\mathrm{y}) ; \mathrm{ybar}=\operatorname{mean}(\mathrm{y}) ; \mathrm{s}=\mathrm{sd}(\mathrm{y}) ; \operatorname{smu}=\operatorname{sqrt}\left(\operatorname{mean}\left((\mathrm{y}-\mathrm{mu})^{\wedge} 2\right)\right)$

c(ybar,s,smu) \# 4.3701 .9012 .978

arga $=($ ybar-mu) $/($ smu/sqrt(n)); pppa $=1-p t(\operatorname{arga}, n) ; c($ arga,pppa)

\# 2.516580 .01528

$\operatorname{argc}=($ ybar-mu $) /(s /$ sqrt $(n)) ; p p p c=1-p t(\operatorname{argc}, n-1) ; c(\operatorname{argc}, p p p c)$

\# 3.9426450 .001696

\subsection{Bayesian models with multiple parameters}

So far we have examined Bayesian models involving some data $y$ and a parameter $\theta$, where $\theta$ is a strictly scalar quantity. We now consider the case of Bayesian models with multiple parameters, starting with a focus on just two, say $\theta_{1}$ and $\theta_{2}$. In that case, the Bayesian model may be defined by specifying $f(y \mid \theta)$ and $f(\theta)$ in the same way as previously, but with an understanding that $\theta$ is a vector of the form $\theta=\left(\theta_{1}, \theta_{2}\right)$.

The first task now is to find the joint posterior density of $\theta_{1}$ and $\theta_{2}$, according to

$$
f(\theta \mid y) \propto f(\theta) f(y \mid \theta),
$$

or equivalently

$$
f\left(\theta_{1}, \theta_{2} \mid y\right) \propto f\left(\theta_{1}, \theta_{2}\right) f\left(y \mid \theta_{1}, \theta_{2}\right),
$$

where

$$
f(\theta)=f\left(\theta_{1}, \theta_{2}\right)
$$

is the joint prior density of the two parameters.

Often, this joint prior density is specified as an unconditional prior multiplied by a conditional prior, for example as

$$
f\left(\theta_{1}, \theta_{2}\right)=f\left(\theta_{1}\right) f\left(\theta_{2} \mid \theta_{1}\right) \text {. }
$$

Once a Bayesian model with two parameters has been defined, one task is to find the marginal posterior densities of $\theta_{1}$ and $\theta_{2}$, respectively, via the equations:

$$
\begin{aligned}
& f\left(\theta_{1} \mid y\right)=\int f\left(\theta_{1}, \theta_{2} \mid y\right) d \theta_{2} \\
& f\left(\theta_{2} \mid y\right)=\int f\left(\theta_{1}, \theta_{2} \mid y\right) d \theta_{1} .
\end{aligned}
$$


From these two marginal posteriors, one may obtain point and interval estimates of $\theta_{1}$ and $\theta_{2}$ in the usual way (treating each parameter separately). For example, the marginal posterior mean of $\theta_{1}$ is

$$
\hat{\theta}_{1}=E\left(\theta_{1} \mid y\right)=\int \theta_{1} f\left(\theta_{1} \mid y\right) d \theta_{1} \text {. }
$$

Another way to do this calculation is via the law of iterated expectation, according to

$$
\begin{aligned}
\hat{\theta}_{1}=E\left(\theta_{1} \mid y\right) & =E\left\{E\left(\theta_{1} \mid y, \theta_{2}\right) \mid y\right\} \\
& =\int E\left(\theta_{1} \mid y, \theta_{2}\right) f\left(\theta_{2} \mid y\right) d \theta_{2} .
\end{aligned}
$$

Note: The equation $E\left(\theta_{1} \mid y\right)=E\left\{E\left(\theta_{1} \mid y, \theta_{2}\right) \mid y\right\}$ follows from the simpler identity $E \theta_{1}=E E\left(\theta_{1} \mid \theta_{2}\right)$ after conditioning throughout on $y$.

Here, $E\left(\theta_{1} \mid y, \theta_{2}\right)$ is called the conditional posterior mean of $\theta_{1}$ and can be calculated as

$$
E\left(\theta_{1} \mid y, \theta_{2}\right)=\int \theta_{1} f\left(\theta_{1} \mid y, \theta_{2}\right) d \theta_{1} .
$$

Also, $f\left(\theta_{1} \mid y, \theta_{2}\right)$ is called the conditional posterior density of $\theta_{1}$ and may be obtained according to

$$
f\left(\theta_{1} \mid y, \theta_{2}\right) \propto f\left(\theta_{1}, \theta_{2} \mid y\right) .
$$

Note: Equation (3.1) follows after first considering the equation $f\left(\theta_{1} \mid \theta_{2}\right) \propto f\left(\theta_{1}, \theta_{2}\right)$ and then conditioning throughout on $y$.

The main idea of Equation (3.1) is to examine the joint posterior density

$$
f\left(\theta_{1}, \theta_{2} \mid y\right)
$$

(or any kernel thereof), think of all terms in this as constant except for $\theta_{1}$, and then try to recognise a well-known density function of $\theta_{1}$.

This density function will define the conditional posterior distribution of $\theta_{1}$, from which estimates such as the conditional posterior mean of $\theta_{1}$ (i.e. $\left.E\left(\theta_{1} \mid y, \theta_{2}\right)\right)$ will hopefully be apparent.

One may also be interested in some function,

$$
\psi=g\left(\theta_{1}, \theta_{2}\right) \text {, }
$$


of the two parameters (possibly of only one).Then advanced distribution theory may be required to obtain the posterior pdf of $\psi$, i.e. $f(\psi \mid y)$.

This posterior density may then be used to calculate point and interval estimates of $\psi$. For example, the posterior mean of $\psi$ is

$$
\hat{\psi}=E(\psi \mid y)=\int \psi f(\psi \mid y) d \psi .
$$

Alternatively, this mean may be obtained using the equation

$$
\hat{\psi}=E\left(g\left(\theta_{1}, \theta_{2}\right) \mid y\right)=\iint g\left(\theta_{1}, \theta_{2}\right) f\left(\theta_{1}, \theta_{2} \mid y\right) d \theta_{1} d \theta_{2} .
$$

Further, one may be interested in predicting some other quantity $x$, whose model distribution is specified in the form $f(x \mid y, \theta)$.

To obtain the posterior predictive density of $x$ will generally require a double integral (or summation) of the form

$$
f(x \mid y)=\iint f\left(x \mid y, \theta_{1}, \theta_{2}\right) f\left(\theta_{1}, \theta_{2} \mid y\right) d \theta_{1} d \theta_{2} .
$$

Further integrations will then be required to produce point and interval estimates, such as the predictive mean of $x$,

$$
\hat{x}=E(x \mid y)=\int x f(x \mid y) d x .
$$

\section{Exercise 3.10 A bent coin which is tossed an unknown number of times}

Suppose that five heads have come up on an unknown number of tosses of a bent coin.

Before the experiment, we believed the coin was going to be tossed a number of times equal to $1,2,3, \ldots$, or 9 , with all possibilities equally likely. As regards the probability of heads coming up on a single toss, we deemed no value more or less likely than any other value. We also considered the probability of heads as unrelated to the number of tosses.

Find the marginal posterior distribution and mean of the number of tosses and of the probability of heads, respectively. Also find the number of heads we could expect to come up if the coin were to be tossed again the same number of times. 


\section{Solution to Exercise 3.10}

For this problem it is appropriate to consider the following three-level hierarchical Bayesian model:

$$
\begin{aligned}
& (y \mid \theta, n) \sim \operatorname{Binomial}(n, \theta) \\
& (\theta \mid n) \sim U(0,1) \\
& n \sim D U(1, \ldots, k), \quad k=9 \quad \text { (i.e. } f(n)=1 / 9, n=1, \ldots, 9) .
\end{aligned}
$$

Under this model, the joint posterior density of the two parameters $n$ and $\theta$ is

$$
\begin{aligned}
f(n, \theta \mid y) & \propto f(n, \theta) f(y \mid n, \theta) \\
& =f(n) f(\theta \mid n) f(y \mid n, \theta) \\
& =\frac{1}{k} \times 1 \times\left(\begin{array}{l}
n \\
y
\end{array}\right) \theta^{y}(1-\theta)^{n-y} \\
& \propto\left(\begin{array}{l}
n \\
y
\end{array}\right) \theta^{y}(1-\theta)^{n-y}, \quad 0<\theta<1, \quad n=y, y+1, \ldots, 9 .
\end{aligned}
$$

So the marginal posterior density of $n$ is

$$
\begin{aligned}
f(n \mid y) & =\int_{0} f(n, \theta \mid y) d \theta \\
& \left.\propto \int_{0}^{1}\left(\begin{array}{l}
n \\
y
\end{array}\right) \theta^{y}(1-\theta)^{n-y} d \theta, \quad n=y, y+1, \ldots, 9 \quad \text { (since } y=0, \ldots, n\right) \\
& =\left(\begin{array}{l}
n \\
y
\end{array}\right) B(y+1, n-y+1) \int_{0}^{1} \frac{\theta^{y+1-1}(1-\theta)^{n-y+1-1}}{B(y+1, n-y+1)} d \theta, \quad n=5,6,7,8,9 \\
& \left.=\left(\begin{array}{l}
n \\
y
\end{array}\right) \frac{\Gamma(y+1) \Gamma(n-y+1)}{\Gamma(y+1+n-y+1)} \times 1 \quad \text { (since the integral equals } 1\right) \\
& =\frac{n !}{y !(n-y) !} \frac{y !(n-y) !}{(n+1) !} \\
& =\frac{1}{n+1} \quad \begin{array}{ll}
1 / 6, & n=5 \\
1 / 7, & n=6 \\
1 / 8, & n=7 \\
1 / 9, & n=8 \\
1 / 10, & n=9 .
\end{array}
\end{aligned}
$$


After normalising (i.e. dividing each of these five numbers by their sum, 0.6456 ), we find that, to four decimals, $n$ 's posterior pdf is

$$
f(n \mid y)= \begin{cases}0.2581, & n=5 \\ 0.2213, & n=6 \\ 0.1936, & n=7 \\ 0.1721, & n=8 \\ 0.1549, & n=9\end{cases}
$$

Thus, for example, there is a $17.2 \%$ chance a posteriori the coin was tossed 8 times.

It follows that $n$ 's posterior mean is

$$
\begin{aligned}
\hat{n}=E(n \mid y) & =\sum_{n=6}^{9} n f(n \mid y) \\
& =0.2581 \times 5+0.2213 \times 6+\ldots+0.1549 \times 9 \\
& =6.744 .
\end{aligned}
$$

Next, the marginal posterior density of $\theta$ is

$$
\begin{aligned}
f(\theta \mid y) & =\sum_{n} f(n, \theta \mid y) \\
& \propto \sum_{n=y}^{9}\left(\begin{array}{l}
n \\
y
\end{array}\right) \theta^{y}(1-\theta)^{n-y} \\
& =\sum_{n=5}^{9}\left(\begin{array}{l}
n \\
y
\end{array}\right) B(y+1, n-y+1) \frac{\theta^{y+1-1}(1-\theta)^{n-y+1-1}}{B(y+1, n-y+1)} \\
& =\sum_{n=y}^{9} \frac{1}{n+1} f_{\text {Beta }(y+1, n-y+1)}(\theta) .
\end{aligned}
$$

Recall that $f(n \mid y) \propto 1 /(n+1)$. It follows that $\theta$ 's marginal posterior density must be exactly

$$
\begin{aligned}
& f(\theta \mid y)=\sum_{n=5}^{9} f(n \mid y) f_{\text {Beta }(y+1, n-y+1)}(\theta) \\
& =0.2581 \frac{\theta^{5}(1-\theta)^{5-5}}{5 !(5-5) ! /(5+1) !}+\ldots+0.1549 \frac{\theta^{5}(1-\theta)^{9-5}}{5 !(9-5) ! /(9+1) !} .
\end{aligned}
$$

We see that $\theta$ 's posterior is a mixture of five beta distributions. 
Note: This result can also be obtained, more directly, as follows. By considering the 'ordinary' binomial-beta model (from earlier), we see that in the present context the conditional posterior distribution of $\theta$ (given $n$ ) is given by

$$
(\theta \mid y, n) \sim \operatorname{Beta}(y+1, n-y+1) .
$$

It immediately follows that

$$
\begin{aligned}
f(\theta \mid y)=\sum_{n} f(\theta, n \mid y) & =\sum_{n} f(n \mid y) f(\theta \mid y, n) \\
& =\sum_{n=5}^{9} f(n \mid y) f_{\text {Beta }(y+1, n-y+1)}(\theta) .
\end{aligned}
$$

We may now perform inference on $\theta$. The posterior mean of $\theta$ is

$$
\begin{aligned}
& \hat{\theta}=E(\theta \mid y)=E\{E(\theta \mid y, n) \mid y\}=E\left(\frac{y+1}{n+2} \mid y\right) \\
&=(y+1) \sum_{n=5}^{9}\left(\frac{1}{n+2}\right) f(n \mid y) \\
&=6\left\{\left(\frac{1}{7}\right) 0.2581+\left(\frac{1}{8}\right) 0.2213+\left(\frac{1}{9}\right) 0.1936+\left(\frac{1}{10}\right) 0.1721+\left(\frac{1}{11}\right) 0.1549\right\} \\
&=0.7040 .
\end{aligned}
$$

Figures 3.3 and 3.4 (page 141) show the marginal posterior densities of $n$ and $\theta$, respectively, with the posterior means $\hat{n}=6.744$ and $\hat{\theta}=0.7040$ marked by vertical lines.

Finally, we consider $x$, the number of heads on the next $n$ tosses.

The distribution of $x$ is defined by $(x \mid y, n, \theta) \sim \operatorname{Bin}(n, \theta)$.

So the posterior predictive mean of $x$ is

$$
\begin{gathered}
E(x \mid y)=E\{E(x \mid y, n, \theta) \mid y\}=E(n \theta \mid y) \\
=E\{E(n \theta \mid y, n) \mid y\}=E\{n E(\theta \mid y, n) \mid y\} \\
=E\left(n \times \frac{y+1}{n+2} \mid y\right)=(y+1) \sum_{n=5}^{9}\left(\frac{n}{n+2}\right) f(n \mid y) \\
=6\left\{\left(\frac{5}{7}\right) 0.2581+\left(\frac{6}{8}\right) 0.2213+\left(\frac{7}{9}\right) 0.1936+\left(\frac{8}{10}\right) 0.1721+\left(\frac{9}{11}\right) 0.1549\right\} \\
=4.592 .
\end{gathered}
$$


Figure 3.3 Posterior density of $n$

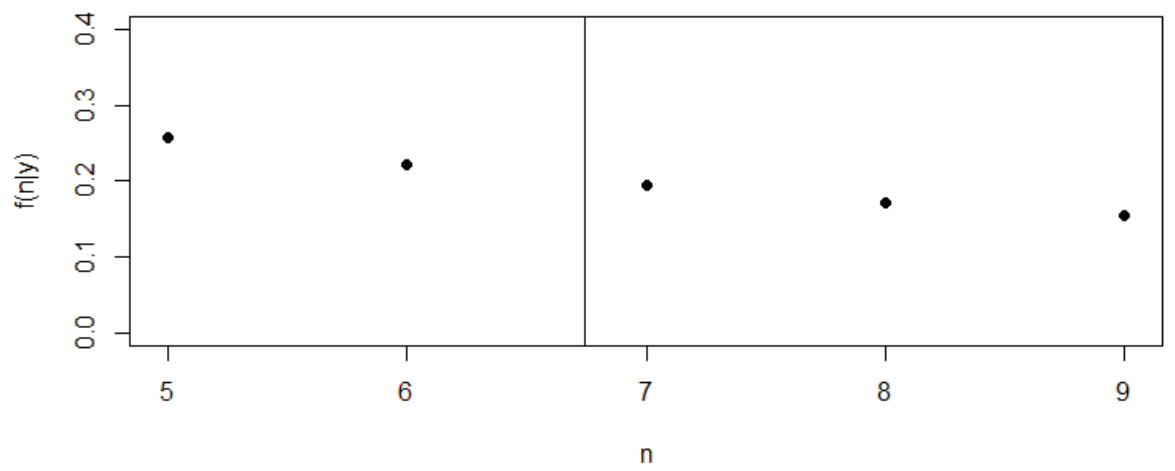

Figure 3.4 Posterior density of $\theta$

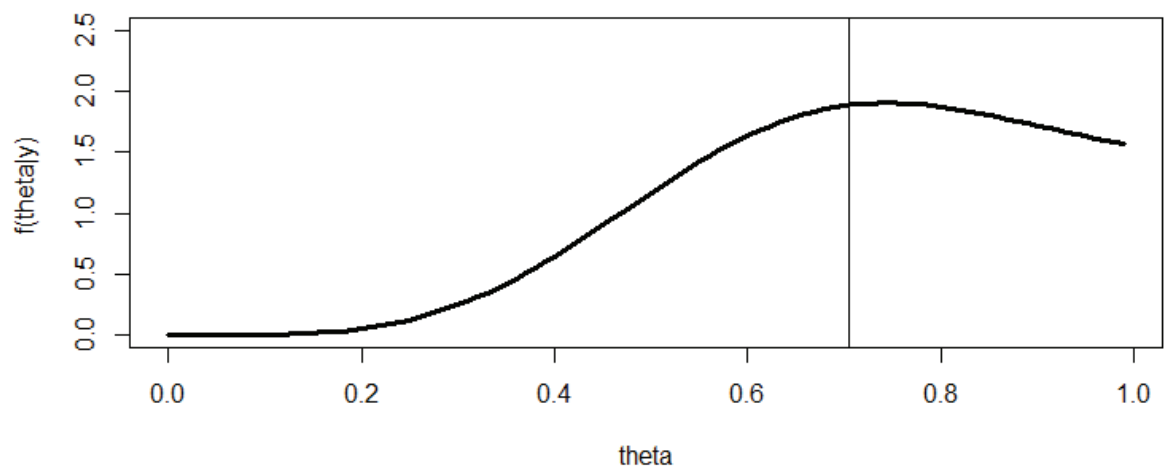

\section{R Code for Exercise 3.10}

$\mathrm{y}<-5 ; \mathrm{k}<-9$; options(digits=4)

nvec <- y:k; avec <- 1/(nvec+1); sumavec <- sum(avec); sumavec \#0.6456 fny <- avec/sumavec; rbind(nvec,avec,fny)

\# nvec 5.00006 .00007 .00008 .00009 .0000

\# avec 0.16670 .14290 .12500 .11110 .1000

\# fny 0.25810 .22130 .19360 .17210 .1549

nhat <- sum(nvec*fny); nhat $\# 6.744$

thhat $<-\operatorname{sum}($ fny * $(y+1) /($ nvec +2$))$; thhat $\# 0.704$

xhat <- sum( fny * nvec* $(y+1) /($ nvec +2$))$; xhat \# 4.592 
thvec <- seq $(0,0.99,0.01)$; fthyvec <- thvec

for(i in 1:length(thvec)) fthyvec[i] <- sum( fny * dbeta(thvec[i],y+1,nvec-y+1) )

$\mathrm{X} 11(\mathrm{w}=8, \mathrm{~h}=4) ; \operatorname{par}(\mathrm{mfrow}=\mathrm{c}(1,1))$

plot(nvec,fny,type="n",xlab="n",ylab="f(n|y)",ylim=c(0,0.4))

points(nvec,fny,pch=16,cex=1); abline(v=nhat)

plot(thvec,fthyvec,type="n",xlab="theta",ylab="f(theta $\mid y)$ ",ylim=c(0,2.5))

lines(thvec,fthyvec,lwd=3); abline(v=thhat)

\section{Exercise 3.I I The uninformative normal-normal-gamma model}

Consider the following Bayesian model:

$$
\begin{aligned}
& \left(y_{1}, \ldots, y_{n} \mid \mu, \lambda\right) \sim \text { iid } N(\mu, 1 / \lambda) \\
& (\mu \mid \lambda) \sim N(0, \infty) \\
& \lambda \sim \operatorname{Gamma}(0,0),
\end{aligned}
$$

with observed data $y=\left(y_{1}, \ldots, y_{n}\right)$.

(a) Find the marginal posterior distribution of $\mu$.

(b) Find the marginal posterior distribution of $\lambda$.

(c) Find the posterior mean of the signal to noise ratio, defined as

$$
\gamma=\mu / \sigma=\mu \sqrt{\lambda} \text {. }
$$

(d) Find the posterior predictive distribution of

$$
\bar{x}=\left(x_{1}+\ldots+x_{m}\right) / m,
$$

where the $x_{i}$ values have a distribution given by

$$
\left(x_{1}, \ldots, x_{m} \mid y, \mu, \lambda\right) \sim N(\mu, 1 / \lambda) .
$$

Note: Both $\mu$ and $\lambda$ are assigned uninformative priors. The joint prior distribution of these two parameters could also be specified by:

$$
\begin{aligned}
& f(\mu \mid \lambda) \propto 1, \mu \in \Re \\
& f(\lambda) \propto 1 / \lambda, \lambda>0,
\end{aligned}
$$

or by the single statement

$$
f(\mu, \lambda) \propto 1 / \lambda, \mu \in \Re, \lambda>0 .
$$




\section{Solution to Exercise 3.I I}

(a) The joint posterior density of the two parameters $\mu$ and $\lambda$ is

$$
\begin{aligned}
f(\mu, \lambda \mid y) & \propto f(\mu, \lambda) f(y \mid \mu, \lambda)=f(\lambda) f(\mu \mid \lambda) f(y \mid \mu, \lambda) \\
& \propto \lambda^{-1} \times 1 \times \prod_{i=1}^{n} \frac{1}{\sqrt{1 / \lambda}} \exp \left(-\frac{\left(y_{i}-\mu\right)^{2}}{2(1 / \lambda)}\right) \\
& =\lambda^{\frac{n}{2}-1} \exp \left(-\frac{\lambda}{2} \sum_{i=1}^{n}\left(y_{i}-\mu\right)^{2}\right) .
\end{aligned}
$$

So the marginal posterior density of $\mu$ is

$$
\begin{aligned}
f(\mu \mid y) & =\int f(\mu, \lambda \mid y) d \lambda \\
& \propto \int_{0}^{\infty} \lambda^{\frac{n}{2}-1} \exp \left(-\lambda\left\{\frac{1}{2} \sum_{i=1}^{n}\left(y_{i}-\mu\right)^{2}\right\}\right) d \lambda \\
& =\frac{\Gamma(n / 2)}{\left\{\frac{1}{2} \sum_{i=1}^{n}\left(y_{i}-\mu\right)^{2}\right\}^{\frac{n}{2}}} \int_{0}^{\infty}\left[\frac{1}{\Gamma(n / 2)}\left\{\frac{1}{2} \sum_{i=1}^{n}\left(y_{i}-\mu\right)^{2}\right\}^{\frac{n}{2}}\right. \\
& \left.=\frac{\Gamma(n / 2)}{\left\{\frac{n}{2}-1\right.} \exp \left(-\lambda\left\{\frac{1}{2} \sum_{i=1}^{n}\left(y_{i}-\mu\right)^{2}\right\}\right)\right] d \lambda \\
& \left.\propto\left\{\sum_{i=1}^{n}\left(y_{i}-\mu\right)^{2}\right\}^{\frac{n}{2}}\left(y_{i}-\mu\right)^{2}\right\}^{-\frac{n}{2}} \cdot
\end{aligned}
$$

Note: The last integral is that of a gamma density and so is equal to 1 .

Now observe that

$$
\begin{aligned}
& \sum_{i=1}^{n}\left(y_{i}-\mu\right)^{2}=\sum_{i=1}^{n}\left\{\left(y_{i}-\bar{y}\right)+(\bar{y}-\mu)\right\}^{2} \\
& =\sum_{i=1}^{n}\left(y_{i}-\bar{y}\right)^{2}+2(\bar{y}-\mu) \sum_{i=1}^{n}\left(y_{i}-\bar{y}\right)+(\bar{y}-\mu)^{2} \sum_{i=1}^{n} 1 \\
& =(n-1)\left\{\frac{1}{n-1} \sum_{i=1}^{n}\left(y_{i}-\bar{y}\right)^{2}\right\}+2(\bar{y}-\mu)(n \bar{y}-n \bar{y})+n(\bar{y}-\mu)^{2} \\
& =(n-1) s^{2}+n(\mu-\bar{y})^{2}, \text { where } s^{2} \text { is the sample variance. }
\end{aligned}
$$


This result implies that

$$
\begin{aligned}
f(\mu \mid y) & \propto\left\{(n-1) s^{2}+n(\mu-\bar{y})^{2}\right\}^{-\frac{n}{2}} \\
& \propto\left\{1+\frac{n(\mu-\bar{y})^{2}}{(n-1) s^{2}}\right\}^{-\frac{1}{2}\{(n-1)+1\}}=\left\{1+\frac{\left(\frac{\mu-\bar{y}}{s / \sqrt{n}}\right)^{2}}{(n-1)}\right\}^{-\frac{1}{2}\{(n-1)+1\}}
\end{aligned}
$$

We now define $r=\frac{\mu-\bar{y}}{s / \sqrt{n}}$, so that $\mu=\bar{y}+\frac{s}{\sqrt{n}} r$ and $\frac{d \mu}{d r}=\frac{s}{\sqrt{n}}$.

By the transformation rule, we then have that

$$
\begin{aligned}
f(r \mid y) & =f(\mu \mid y)\left|\frac{d \mu}{d r}\right| \\
& \stackrel{r}{\propto}\left\{1+\frac{r^{2}}{(n-1)}\right\}^{-\frac{1}{2}\{(n-1)+1\}} \times\left|\frac{s}{\sqrt{n}}\right| \stackrel{r}{\propto}\left\{1+\frac{r^{2}}{(n-1)}\right\}^{-\frac{1}{2}\{(n-1)+1\}} .
\end{aligned}
$$

By definition of the $t$ distribution, we see that $(r \mid y) \sim t(n-1)$.

It follows that the marginal posterior distribution of $\mu$ is given by

$$
\left(\frac{\mu-\bar{y}}{s / \sqrt{n}} \mid y\right) \sim t(n-1) .
$$

Note 1: In result (3.2), the data vector $y$ appears only by way of the sample mean $\bar{y}$ and sample standard deviation $s$. So it is also true that

$$
\left(\frac{\mu-\bar{y}}{s / \sqrt{n}} \mid \bar{y}, s\right) \sim t(n-1) \text {. }
$$

Here, $s$ may not be left out of the conditioning. So it is not true that

$$
\left(\frac{\mu-\bar{y}}{s / \sqrt{n}} \mid \bar{y}\right) \sim t(n-1) .
$$

Note 2: Result (3.2) implies that the marginal posterior mean, mode and median of $\mu$ are all equal to $\bar{y}$, and the $1-\alpha$ CPDR/HPDR for $\mu$ is 


$$
\left(\bar{y} \pm t_{\alpha / 2}(n-1) s / \sqrt{n}\right) .
$$

This inference is identical to that obtained via the classical approach and thereby justifies the use of the joint prior

$$
f(\mu, \lambda) \propto 1 / \lambda, \mu \in \Re, \lambda>0
$$

in cases of $a$ priori ignorance regarding both $\mu$ and $\lambda$.

Note 3: The exact marginal posterior density of $\mu$ is

$$
f(\mu \mid y)=f(r \mid y)\left|\frac{d r}{d \mu}\right|,
$$

where $r=\frac{\mu-\bar{y}}{s / \sqrt{n}}$ and $(r \mid y) \sim t(n-1)$.

Thus $\quad f(\mu \mid y)=\frac{\Gamma(\{(n-1)+1\} / 2)}{\Gamma((n-1) \pi) \Gamma((n-1) / 2)}$

$$
\times\left(1+\frac{1}{n-1}\left(\frac{\mu-\bar{y}}{s / \sqrt{n}}\right)^{2}\right)^{-\frac{1}{2}((n-1)+1)} \frac{\sqrt{n}}{s}, \mu \in \Re .
$$

This density can be calculated in $\mathrm{R}$ at any point $\mu$ by first calculating the corresponding value of $r$ and then returning

$$
d t(r, n-1) * \operatorname{sqrt}(n) / s
$$

(see below for examples).

(b) The marginal posterior density of $\lambda$ is

$$
\begin{aligned}
f(\lambda \mid y) & =\int_{\infty} f(\mu, \lambda \mid y) d \mu \\
& \propto \int_{-\infty}^{\infty} \lambda^{\frac{n}{2}-1} \exp \left(-\frac{\lambda}{2}\left\{(n-1) s^{2}+n(\mu-\bar{y})^{2}\right\}\right) d \mu \\
= & \lambda^{\frac{n}{2}-1} e^{-\lambda\left(\frac{n-1}{2}\right) s^{2}} \sqrt{(1 /(n \lambda))} \sqrt{2 \pi} \\
& \times \int_{-\infty}^{\infty} \frac{1}{\sqrt{(1 /(n \lambda))} \sqrt{2 \pi}} \exp \left(-\frac{1}{2(1 /(n \lambda))}(\mu-\bar{y})^{2}\right) d \mu \\
= & \lambda^{\frac{n}{2}-1} e^{-\lambda\left(\frac{n-1}{2}\right) s^{2}} \sqrt{(1 /(n \lambda))} \sqrt{2 \pi} \stackrel{\lambda\left(\frac{n-1}{2}\right)-1}{\propto} e^{-\lambda\left(\frac{n-1}{2}\right) s^{2}} .
\end{aligned}
$$


Note: The last integral is that of a normal density and so equals 1.

It follows that

$$
(\lambda \mid y) \sim \text { Gamma }\left(\left(\frac{n-1}{2}\right),\left(\frac{n-1}{2}\right) s^{2}\right),
$$

and hence also that

$$
\left((n-1) s^{2} \lambda \mid y\right) \sim \chi^{2}(n-1) .
$$

Note 1: Result (3.4) can be proved as follows. Let

$$
u=(n-1) s^{2} \lambda \text {, }
$$

so that $\lambda=\frac{u}{(n-1) s^{2}}$ and $\frac{d \lambda}{d u}=\frac{1}{(n-1) s^{2}}$.

Then, by the transformation rule,

$$
\begin{aligned}
f(u \mid y) & =f(\lambda \mid y)\left|\frac{d \lambda}{d u}\right| \\
& \stackrel{u}{\propto}\left(\frac{u}{(n-1) s^{2}}\right)^{\left(\frac{n-1}{2}\right)-1} e^{-\frac{u}{(n-1) s^{2}}\left(\frac{n-1}{2}\right) s^{2}}\left|\frac{1}{(n-1) s^{2}}\right| \\
& \stackrel{u}{\propto u^{\left(\frac{n-1}{2}\right)-1}} e^{-\frac{u}{2}} .
\end{aligned}
$$

Thus $(u \mid y) \sim$ Gamma $\left(\frac{n-1}{2}, \frac{1}{2}\right) \sim \chi^{2}(n-1)$, which confirms (3.4).

Note 2: Results (3.3) and (3.4) imply that $\lambda$ has posterior mean $1 / s^{2}$. This makes sense because $\lambda=1 / \sigma^{2}$, and $s^{2}$ is an unbiased estimator of $\sigma^{2}$. We see that the inverse of the posterior mean of $\lambda$ provides us with the classical estimator of $\sigma^{2}$.

Also, result (3.4) implies that the $1-\alpha$ CPDR for $\lambda$ is

$$
\left(\frac{\chi_{1-\alpha / 2}^{2}(n-1)}{(n-1) s^{2}}, \frac{\chi_{\alpha / 2}^{2}(n-1)}{(n-1) s^{2}}\right) \text {. }
$$

It follows that the $1-\alpha$ CPDR for $\sigma^{2}=1 / \lambda$ is 


$$
\left(\frac{(n-1) s^{2}}{\chi_{\alpha / 2}^{2}(n-1)}, \frac{(n-1) s^{2}}{\chi_{1-\alpha / 2}^{2}(n-1)}\right) \text {. }
$$

It will be observed that this is exactly the same as the usual classical $1-\alpha$ CI for $\sigma^{2}$ when the normal mean $\mu$ is unknown.

(c) The posterior mean of $\gamma=\mu / \sigma=\mu \sqrt{\lambda}$ could be calculated using the equation

$$
\hat{\gamma}=\int \gamma f(\gamma \mid y) d \gamma
$$

where $f(\gamma \mid y)$ is the posterior density of $\gamma$.

However, obtaining this density may be difficult. We could use Jacobian theory to find the joint posterior density of $\mu$ and $\gamma$, and then integrate that joint density with respect to $\mu$. The result would be $f(\gamma \mid y)$.

Another approach is to calculate the mean as

$$
\hat{\gamma}=E(\mu \sqrt{\lambda} \mid y)=\int_{\mu=-\infty}^{\infty} \int_{\lambda=0}^{\infty} \mu \sqrt{\lambda} f(\mu, \lambda \mid y) d \mu d \lambda,
$$

where: $f(\mu, \lambda \mid y)=\frac{k(\mu, \lambda)}{c}$

$$
\begin{aligned}
& k(\mu, \lambda)=\lambda^{\frac{n}{2}-1} \exp \left(-\frac{\lambda}{2} \sum_{i=1}^{n}\left(y_{i}-\mu\right)^{2}\right) \\
& c=\int_{\mu=-\infty}^{\infty} \int_{\lambda=0}^{\infty} h(\mu, \lambda) d \mu d \lambda .
\end{aligned}
$$

More simply, we may use the law of iterated expectation to write

$$
\begin{aligned}
\hat{\gamma}=E(\mu \sqrt{\lambda} \mid y) & =E\{E(\mu \sqrt{\lambda} \mid y, \lambda) \mid y\}=E\{\sqrt{\lambda} E(\mu \mid y, \lambda) \mid y\} \\
& =E\{\sqrt{\lambda} \bar{y} \mid y\}=\bar{y} E\left(\lambda^{1 / 2} \mid y\right) \\
& =\bar{y} \frac{\Gamma\left(\frac{n-1}{2}+\frac{1}{2}\right)}{\left(\left(\frac{n-1}{2}\right) s^{2}\right)^{1 / 2} \Gamma\left(\frac{n-1}{2}\right)} \quad \text { by (3.3) } \\
& =\frac{\bar{y}}{s} c_{n},
\end{aligned}
$$


where

$$
c_{n}=\frac{\Gamma\left(\frac{n-1}{2}+\frac{1}{2}\right)}{\left(\frac{n-1}{2}\right)^{1 / 2} \Gamma\left(\frac{n-1}{2}\right)}
$$

Note 1: By a well-known property of the gamma function, $c_{n} \rightarrow 1$ as $n \rightarrow \infty$. So for large $n$ the posterior mean of $\gamma=\mu / \sigma$ is approximately the same as $\gamma$ 's MLE, $\bar{y} / s$.

Note 2: Suppose that we wish to find the posterior median or mode of $\gamma$ or the $95 \%$ CPDR or HPDR for that quantity. Then we first need to determine $f(\gamma \mid y)$. This and subsequent calculations may be difficult. This points to the need for another strategy. As will be seen later, most of these issues can be easily sidestepped using Monte Carlo methods.

(d) Recall from previous exercises that:

$$
\begin{aligned}
& (\bar{x} \mid y, \lambda) \sim N\left(\bar{y}, \frac{1 / \lambda}{m}+\frac{1 / \lambda}{n}\right) \sim N\left(\bar{y}, \frac{n+m}{n m \lambda}\right) \\
& \left.(\lambda \mid y) \sim \text { Gamma }\left(\frac{n-1}{2}\right),\left(\frac{n-1}{2}\right) s^{2}\right) .
\end{aligned}
$$

Hence $f(\bar{x} \mid y)=\int f(\bar{x} \mid y, \lambda) f(\lambda \mid y) d \lambda$

$$
\begin{aligned}
& \propto \int_{0}^{\infty}\left[\lambda^{1 / 2} \exp \left\{-\frac{n m \lambda(\bar{x}-\bar{y})^{2}}{2(n+m)}\right\}\right] \\
& \quad \times\left[\lambda^{\left(\frac{n-1}{2}\right)-1} \exp \left\{-\left(\frac{n-1}{2}\right) s^{2} \lambda\right\}\right] d \lambda \\
& \propto \int_{0}^{\infty} \lambda^{\left(\frac{n}{2}\right)^{-1}} \exp \left\{-\lambda\left[\frac{n m(\bar{x}-\bar{y})^{2}}{2(n+m)}+\left(\frac{n-1}{2}\right) s^{2}\right]\right\} d \lambda \\
& \propto\left[\frac{n m(\bar{x}-\bar{y})^{2}}{2(n+m)}+\left(\frac{n-1}{2}\right) s^{2}\right]^{-\left(\frac{n}{2}\right)}
\end{aligned}
$$




$$
\begin{aligned}
& \propto\left[1+\frac{n m(\bar{x}-\bar{y})^{2}}{(n-1)(n+m) s^{2}}\right]^{-\left(\frac{n}{2}\right)} \\
& \propto\left[1+\frac{\left(\frac{\bar{x}-\bar{y}}{(s / \sqrt{n}) \sqrt{(n+m) / m}}\right)^{2}}{n-1}\right]^{-\left(\frac{n}{2}\right)} .
\end{aligned}
$$

It follows that

$$
\left(\frac{\bar{x}-\bar{y}}{(s / \sqrt{n}) \sqrt{(n+m) / m}} \mid y\right) \sim t(n-1) .
$$

Note 1: Equation (3.5) can be used to derive the predictive distribution of the average of all $n+m$ values considered (both past and future).

That average may be written

$$
a=\frac{1}{n+m}\left(\sum_{i=1}^{n} y_{i}+\sum_{j=1}^{m} x_{i}\right)=\frac{n \bar{y}+m \bar{x}}{n+m} .
$$

Consequently,

$$
\bar{x}=\frac{(n+m) a-n \bar{y}}{m} .
$$

It follows that in (3.5),

$$
\begin{aligned}
\frac{\bar{x}-\bar{y}}{(s / \sqrt{n}) \sqrt{(n+m) / m}} & =\frac{\left(\frac{(n+m) a-n \bar{y}}{m}\right)-\bar{y}}{(s / \sqrt{n}) \sqrt{(n+m) / m}} \\
& =\frac{(a-\bar{y})(n+m) / m}{(s / \sqrt{n}) \sqrt{(n+m) / m}} \\
& =\frac{a-\bar{y}}{(s / \sqrt{n}) \sqrt{m /(n+m)}},
\end{aligned}
$$

and therefore

$$
\left(\frac{a-\bar{y}}{(s / \sqrt{n}) \sqrt{m /(n+m)}} \mid y\right) \sim t(n-1) .
$$


This may look familiar to some readers, the reason being as follows.

Denote the total number of values, $n+m$, as $N$, and write the average of all these observations, $a$, as $\bar{Y}$. Then (3.6) is equivalent to the result

$$
\left(\frac{\bar{Y}-\bar{y}}{\frac{s}{\sqrt{n}} \sqrt{1-\frac{n}{N}}} \mid y\right) \sim t(n-1) .
$$

So the posterior predictive mean of $\bar{Y}$ is the observed sample mean $\bar{y}$, and the 95\% central (and highest) predictive density region for $\bar{Y}$ is

$$
\left(\bar{y} \pm t_{\alpha / 2}(n-1) \frac{s}{\sqrt{n}} \sqrt{1-\frac{n}{N}}\right) .
$$

It will be noted that this inference is exactly the same as implied by the standard approach in the classical survey sampling framework (e.g. see Cochran, 1977).

Recall that in this framework, $\sqrt{1-n / N}$ is the finite population correction (fpc) factor. As $N$ increases, the fpc factor tends to 1 and (3.8) reduces to

$$
\left(\bar{y} \pm t_{\alpha / 2}(n-1) \frac{s}{\sqrt{n}}\right)
$$

which is the 'standard' CI for a normal mean when the normal variance is unknown.

We have here touched on the topic of Bayesian finite population inference. More will be said on this topic later in the book.

Note 2: The exact posterior predictive density of the finite population mean $\bar{Y}$ may be obtained according to

$$
f(\bar{Y} \mid y)=f(q \mid y)\left|\frac{d q}{d \bar{Y}}\right|,
$$

where: $q=\frac{\bar{Y}-\bar{y}}{(s / \sqrt{n}) \sqrt{1-n / N}}$

$$
(q \mid y) \sim t(n-1) \text {. }
$$


We thereby obtain the density $\quad f(\bar{Y} \mid y)=\frac{\Gamma(\{(n-1)+1\} / 2)}{\Gamma((n-1) \pi) \Gamma((n-1) / 2)}$

$$
\times\left(1+\frac{1}{n-1}\left(\frac{\bar{Y}-\bar{y}}{(s / \sqrt{n}) \sqrt{1-n / N}} s\right)^{2}\right)^{-\frac{1}{2}((n-1)+1)} \frac{\sqrt{n}}{s \sqrt{1-n / N}}, \bar{Y} \in \Re .
$$

This density can be calculated in $\mathrm{R}$ at any point $\bar{Y}$ by first calculating the corresponding value of $q$ (as defined above) and then returning $\mathrm{dt}(\mathrm{q}, \mathrm{n}-1) * \operatorname{sqrt}(\mathrm{n}) /\left(\mathrm{s}^{*} \mathrm{sqrt}(1-\mathrm{n} / \mathrm{N})\right)$

(see below for an example).

Note 3: The posterior predictive density of $\bar{Y}$ converges to the marginal posterior density of $\mu$ as $N$ tends to infinity with $n$ fixed. That is,

$$
f(\bar{Y}=c \mid y) \rightarrow f(\mu=c \mid y) \text { as } N \rightarrow \infty .
$$

This is on account of the fpc factor $\sqrt{1-n / N}$ converging to unity. Thus $\mu$ may be interpreted as the average of a hypothetically infinite number of values from the underlying superpopulation, $N(\mu, 1 / \lambda)$.

Figure 3.5 shows the predictive density $f(\bar{Y} \mid y)$ for various values of $N$, as well as the posterior density $f(\mu \mid y)$, corresponding to the limiting case $N=\infty$. In each case, the values of $n, \bar{y}$ and $s$ are (arbitrarily) taken as 5, 10 and 2, respectively. Note that $N=\infty \Leftrightarrow m=\infty$ since $m=N-n$.

Note 4: Consider the following Bayesian model:

$$
\begin{aligned}
& \left(y_{1}, \ldots, y_{n} \mid \mu, \lambda\right) \sim \text { iid } N(\mu, 1 / \lambda) \\
& (\mu \mid \lambda) \sim N\left(\mu_{0}, \sigma_{0}^{2}\right) \\
& \lambda \sim \operatorname{Gamma}(\alpha, \beta),
\end{aligned}
$$

where $\sigma_{0}$ is not necessarily $\infty$ and $\alpha$ and $\beta$ are not necessarily 0 .

This may be called the (general) normal-normal-gamma model, as distinct from the uninformative normal-normal-gamma model, here in Exercise 3.11. In the general model, the inferences typically required are much more difficult to perform. Later in the book, it will be shown how to proceed in this - and similarly difficult-situations using Monte Carlo methods, including Markov chain Monte Carlo (MCMC) methods. 
Figure 3.5 Predictive density of the finite population mean (See Note 3 on page I5I)

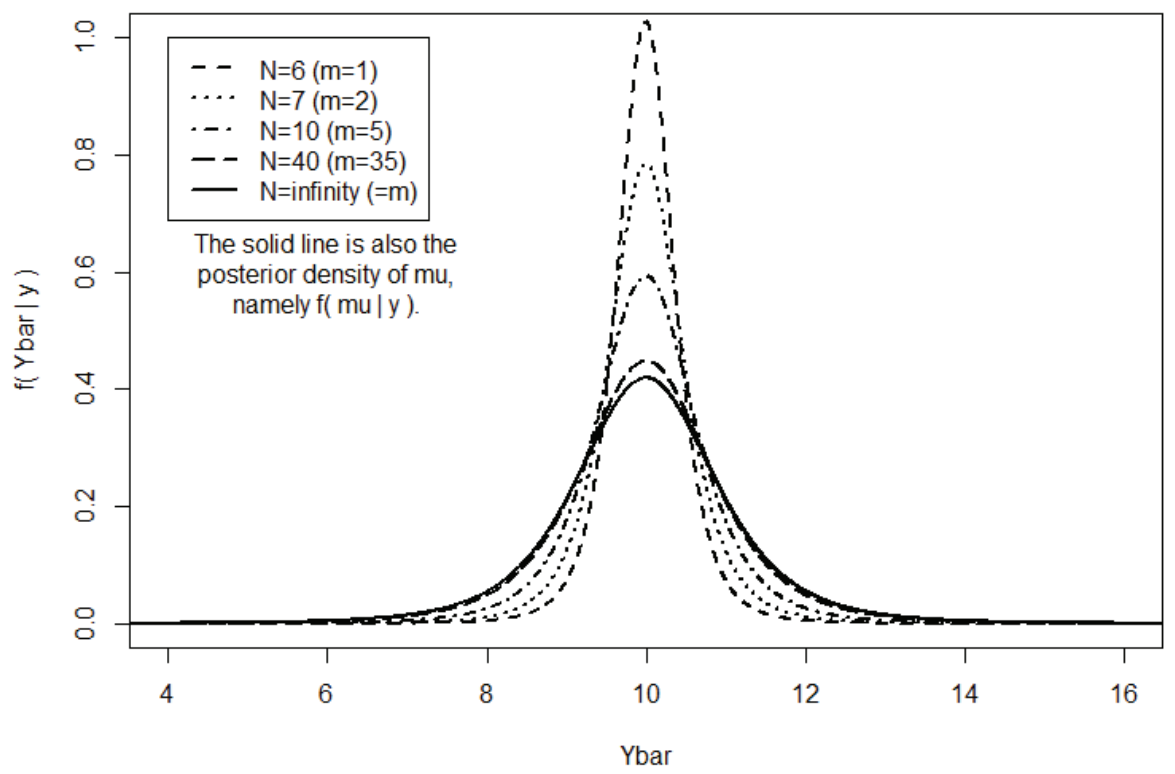

\section{R Code for Exercise 3.I I}

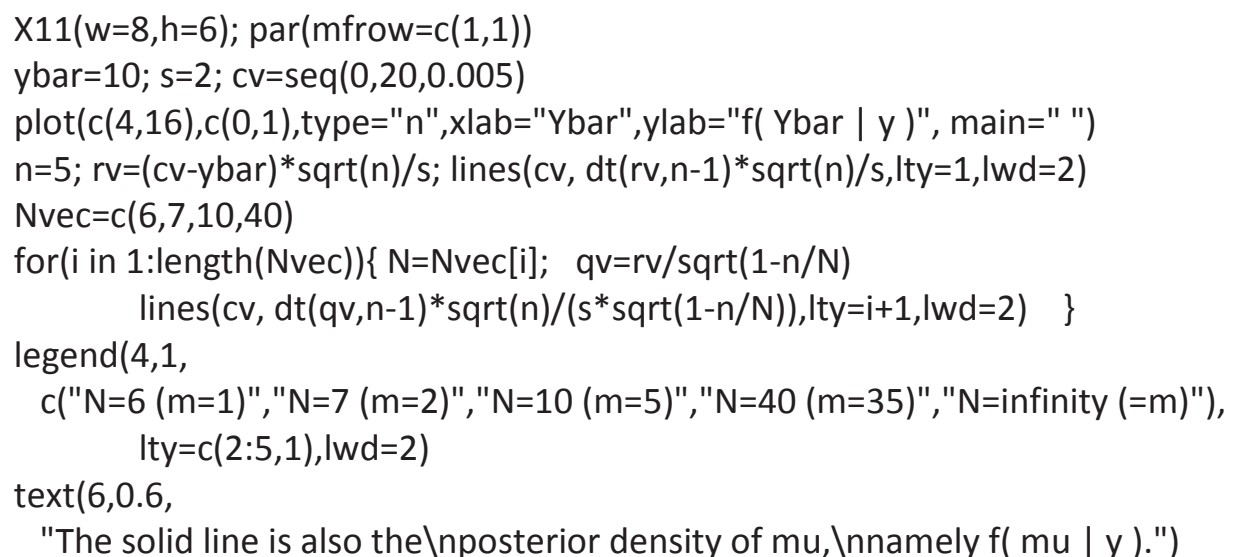

"The solid line is also the \nposterior density of mu, \nnamely $\mathrm{f}(\mathrm{mu} \mid \mathrm{y}) . "$ ) 


\section{Computational Tools}

\section{I Solving equations}

In most of the Bayesian models so far examined, the calculations required could be done analytically. For example, the model given by:

$$
\begin{aligned}
& (Y \mid \theta) \sim \operatorname{Binomial}(5, \theta) \\
& \theta \sim U(0,1),
\end{aligned}
$$

together with data $y=5$, implies the posterior $(\theta \mid y) \sim \operatorname{Beta}(6,1)$. So $\theta$ has posterior pdf $f(\theta \mid y)=6 \theta^{5}$ and posterior cdf $F(\theta \mid y)=\theta^{6}$. Then, setting $F(\theta \mid y)=1 / 2$ yields the posterior median, $\theta=1 / 2^{1 / 6}=0.8909$.

But what if the equation $F(\theta \mid y)=1 / 2$ were not so easy to solve? In that case we could employ a number of strategies. One of these is trial and error, and another is via special functions in software packages, for example using the qbeta() function in R. This yields the correct answer. Yet another method is the Newton-Raphson algorithm, our next topic.

\section{R Code for Section 4.I}

qbeta $(0.5,6,1) \quad \# 0.8908987$

\subsection{The Newton-Raphson algorithm}

The Newton-Raphson (NR) algorithm is a useful technique for solving equations of the form $g(x)=0$.

This algorithm involves choosing a suitable starting value $x_{0}$ and iteratively applying the equation

$$
x_{j+1}=x_{j}-g^{\prime}\left(x_{j}\right)^{-1} g\left(x_{j}\right)
$$

until convergence had been achieved to a desired degree of precision.

How does the NR algorithm work? Figure 4.1 illustrates the idea. 


\section{Figure 4.I The Newton-Raphson algorithm}

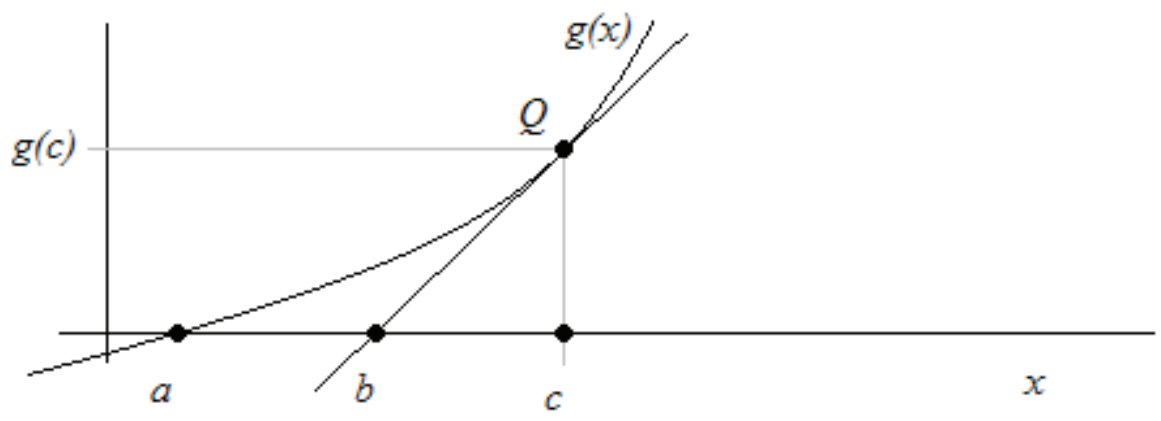

Here, $a$ is the desired solution of the equation $g(x)=0, c$ is a guess at that solution, and $b$ is a better estimate of $a$. Observe that the slope of the tangent at point $Q$ is equal to both $g^{\prime}(c)$ and $g(c) /(c-b)$. Equating these two expressions we get $b=c-g(c) / g^{\prime}(c)$.

Note: Sometimes the NR algorithm takes a long time to converge, and sometimes it converges to the wrong or even impossible value or gets 'stuck' and fails to converge at all. This is a general problem with the NR algorithm, namely its instability and the need to start it off with an initial guess that is sufficiently close to the desired solution.

\section{Exercise 4.I Calculating a posterior median via the Newton- Raphson algorithm}

Suppose that the posterior cdf of a parameter is $F(\theta \mid y)=\theta^{6}$.

Find the posterior median by solving the equation $F(\theta \mid y)=1 / 2$ via the Newton-Raphson algorithm.

Note: The algorithm should converge to the analytical solution, namely $\theta=1 / 2^{1 / 6}=0.8909$.

\section{Solution to Exercise 4.I}

We wish to solve $g(\theta)=0$, where $g(\theta)=F(\theta \mid y)-1 / 2$.

Here, $g^{\prime}(\theta)=f(\theta \mid y)-0$, where $f(\theta \mid y)=6 \theta^{5}$. 
So the algorithm is given by

$$
\theta_{j+1}=\theta_{j}-\frac{g\left(\theta_{j}\right)}{g^{\prime}\left(\theta_{j}\right)}=\theta_{j}-\frac{F\left(\theta_{j} \mid y\right)-1 / 2}{f\left(\theta_{j} \mid y\right)}=\theta_{j}-\frac{\theta_{j}^{6}-1 / 2}{6 \theta_{j}^{5}} .
$$

Starting at the posterior mode, $\theta_{0}=1$ (chosen arbitrarily), we get the sequence shown in Table 4.1.

\section{Table 4.I NR algorithm starting from I}

\begin{tabular}{c|ccccc}
$j$ & 0 & 1 & 2 & 3 & 4 \\
\hline$\theta_{j}$ & 1.0000 & 0.9167 & 0.8926 & 0.8909 & 0.8909 \\
\hline
\end{tabular}

So the posterior median is 0.8909 . The same result is obtained if we start with $\theta_{0}=0.8$, as shown in table 4.2

\section{Table 4.2 NR algorithm starting from 0.8}

\begin{tabular}{c|ccccc}
$j$ & 0 & 1 & 2 & 3 & 4 \\
\hline$\theta_{j}$ & 0.8000 & 0.9210 & 0.8933 & 0.8909 & 0.8909 \\
\hline
\end{tabular}

Note 1: The median must satisfy

$$
\theta=\theta-\frac{\theta^{6}-1 / 2}{6 \theta^{5}} \text {. }
$$

This equation is indeed satisfied at the solution $\theta=0.8909$ (working to four decimals). This illustrates how to check whether or not the NR algorithm has converged properly.

Note 2: In this simple example, one could get the answer by solving the equation $\theta=\theta-g(\theta) / g^{\prime}(\theta)$ analytically. In general, that won't be possible, and iterating the algorithm will be required. Of course, if it is possible to solve that equation analytically, there is no need to iterate. 


\section{R Code for Exercise 4.I}

$N R<-$ function(th, $J=5)\{$

\# This function performs the Newton-Raphson algorithm for J iterations

\# after starting at the value th. It outputs a vector of th values of length $J+1$.

thvec <- th; for(j in 1:J)\{

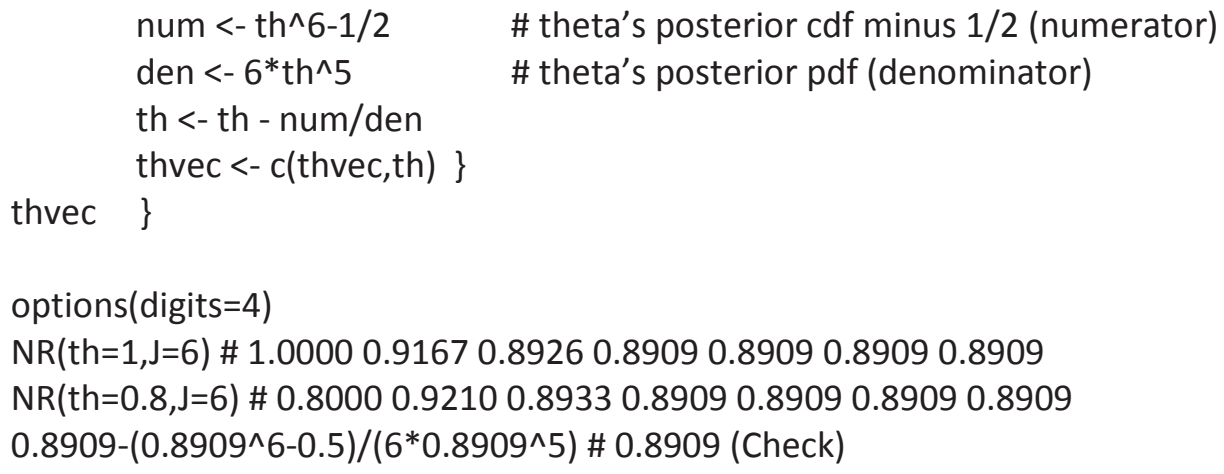

\section{Exercise 4.2 Further practice with the NR algorithm}

Use the Newton-Raphson algorithm to solve the equation $t^{2}=e^{t}$.

Note: In this case there is no analytical solution.

\section{Solution to Exercise 4.2}

We wish to solve $g(t)=0$, where $g(t)=t^{2}-e^{t}$. Now, $g^{\prime}(t)=2 t-e^{t}$.

So we iterate according to $t_{j+1}=t_{j}-\left(\frac{t_{j}^{2}-e^{t_{j}}}{2 t_{j}-e^{t_{j}}}\right)$.

Let us arbitrarily choose $t_{0}=0$. Then we get:

$$
\begin{gathered}
t_{1}=0-\frac{0^{2}-e^{0}}{2(0)-e^{0}}=-1.000000, \quad t_{2}=(-1)-\frac{(-1)^{2}-e^{-1}}{2(-1)-e^{-1}}=-0.733044 \\
t_{3}=(-0.733044)-\frac{(-0.733044)^{2}-e^{-0.733044}}{2(-0.733044)-e^{-0.733044}}=-0.703808 \\
t_{4}=(-0.703808)-\frac{(-0.703808)^{2}-e^{-0.703808}}{2(-0.703808)-e^{-0.703808}}=-0.703467 \\
t_{5}=(-0.703467)-\frac{(-0.703467)^{2}-e^{-0.703467}}{2(-0.703467)-e^{-0.703467}}=-0.703467, \quad \text { etc. }
\end{gathered}
$$


Thus the output of the NR algorithm starting from 0 is:

$0.000000,-1.000000,-0.733044,-0.703808,-0.703467,-0.703467$,

$-0.703467,-0.703467, \ldots .$.

Also, we find that the output of the NR algorithm starting from 1 is:

$1.000000,-1.392211,-0.835088,-0.709834,-0.703483,-0.703467$, $-0.703467,-0.703467, \ldots .$.

From these results we feel confident that the required solution to 6 decimals is -0.703467 . As a check, we calculate

$$
g(-0.703467)=(-0.703467)^{2}-e^{-0.703467}=0.000000803508 \approx 0 .
$$

Figure 4.2 illustrates the function $g$ and the output of the NR algorithm starting from -5 , which is:

$-5.000000,-2.502357,-1.287421,-0.802834,-0.707162,-0.703473$, $-0.703467,-0.703467, \ldots .$.

Figure 4.2 Solution via the NR algorithm starting at -5

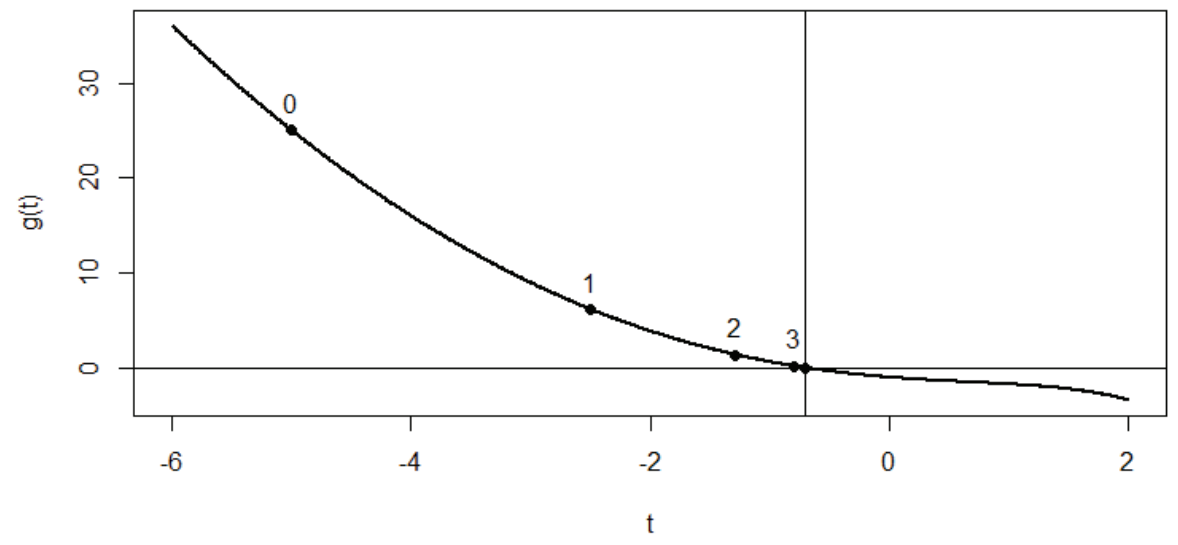

\section{R Code for Exercise 4.2}

options(digits=6); $\mathrm{t}=0$; $\mathrm{tv}=\mathrm{t}$; for(j in 1:7) $\left\{\mathrm{t}=\mathrm{t}-\left(\mathrm{t}^{\wedge} 2-\exp (\mathrm{t})\right) /(2 * \mathrm{t}-\exp (\mathrm{t}))\right.$ $\mathrm{tv}=\mathrm{c}(\mathrm{tv}, \mathrm{t})\} ; \mathrm{tv}$ \# $0.000000-1.000000-0.733044-0.703808-0.703467-0.703467-0.703467$ $\#-0.703467$

\# Check:

$\mathrm{t}^{\wedge} 2-\exp (\mathrm{t}) \quad \# 0$ $(-0.703467)^{\wedge} 2-\exp (-0.703467) \quad \#-8.03508 \mathrm{e}-07$ 
$\mathrm{t}=1 ; \mathrm{tv}=\mathrm{t} ;$ for $(\mathrm{j}$ in $1: 7)\left\{\mathrm{t}=\mathrm{t}-\left(\mathrm{t}^{\wedge} 2-\exp (\mathrm{t})\right) /(2 * \mathrm{t}-\exp (\mathrm{t})) ; \mathrm{tv}=\mathrm{c}(\mathrm{tv}, \mathrm{t})\right\} ; \mathrm{tv}$ \# 1.000000 -1.392211 -0.835088 -0.709834 -0.703483-0.703467 -0.703467 \# -0.703467

$\mathrm{t}=-5 ; \mathrm{tv}=\mathrm{t}$; for(j in 1:7) $\{\mathrm{t}=\mathrm{t}-(\mathrm{t} \wedge 2-\exp (\mathrm{t})) /(2 * \mathrm{t}-\exp (\mathrm{t})) ; \mathrm{tv}=\mathrm{c}(\mathrm{tv}, \mathrm{t})\} ; \mathrm{tv}$ \# -5.000000 -2.502357 -1.287421 -0.802834 -0.707162 -0.703473-0.703467 $\#-0.703467$

tvec=seq(-6,2,0.01); gvec= tvec $^{\wedge} 2-\exp ($ tvec $)$

$\mathrm{X} 11(\mathrm{w}=8, \mathrm{~h}=4.5) ; \operatorname{par}(\mathrm{mfrow}=\mathrm{c}(1,1))$

plot(tvec,gvec,type="l",Iwd=2,xlab="t",ylab="g(t)", main="")

abline(h=0,v=t); points(tv, tv^2-exp(tv),pch=16)

text( $\left.\operatorname{tv}[1: 4], \quad \operatorname{tv}[1: 4]^{\wedge} 2-\exp (\operatorname{tv}[1: 4])+3,0: 3\right)$

\section{Exercise 4.3 Another example of the NR algorithm}

Consider the Bayesian model:

$$
\begin{aligned}
& (x \mid p) \sim \operatorname{Bin}(3, p) \\
& p \sim U(0,1),
\end{aligned}
$$

and suppose the observed value of $x$ is 2. Find the posterior median of $p$.

\section{Solution to Exercise 4.3}

The posterior distribution of $p$ is given by

$$
(p \mid x) \sim \operatorname{Beta}(1+2,1+1),
$$

with density

$$
f(p \mid x)=\frac{p^{3-1}(1-p)^{2-1}}{\Gamma(3) \Gamma(2) / \Gamma(5)}=12 p^{2}(1-p), 0<p<1 .
$$

So, the posterior cdf is

$$
F(p \mid x)=\int_{0}^{p} 12 r^{2}(1-r) d r=12\left(\frac{p^{3}}{3}-\frac{p^{4}}{4}\right)=4 p^{3}-3 p^{4}, 0<p<1 .
$$

To find the posterior median of $p$ we need to solve $F(p \mid x)=1 / 2$, or equivalently $g(p)=0$, where $g(p)=F(p \mid x)-1 / 2=4 p^{3}-3 p^{4}-1 / 2$.

Now, $g^{\prime}(p)=12 p^{2}-12 p^{3}$. So the NR algorithm is defined by iterating

$$
p_{j+1}=p_{j}-\frac{g\left(p_{j}\right)}{g^{\prime}\left(p_{j}\right)}=p_{j}-\left(\frac{4 p_{j}^{3}-3 p_{j}^{4}-1 / 2}{12 p_{j}^{2}-12 p_{j}^{3}}\right) \text {. }
$$


What's a good starting value here? Let's try the MLE, $p_{0}=2 / 3$.

Using this value, we get:

0.666667, 0.614583, 0.614272, 0.614272, 0.614272, 0.614272,

$0.614272,0.61427, \ldots .$.

Starting at other values $(0.5,0.9$ and 0.1$)$, we get the following (three) sequences (respectively):

0.500000, 0.625000, 0.614306, 0.614272, 0.614272, 0.614272,

$0.614272,0.614272, \ldots .$.

0.900000, 0.439403, 0.649191, 0.614501, 0.614272, 0.614272,

$0.614272,0.614272, \ldots .$.

$0.10000,4.69537,3.62690,2.83403,2.25146,1.83195,1.54254$,

$1.36156, \ldots .$.

The last sequence does not seem to have converged. Let's run this for a bit longer. The result is:

$0.10000,4.69537,3.62690,2.83403,2.25146,1.83195,1.54254$,

1.36156, 1.27282, 1.24913, 1.24749, 1.24748, 1.24748, 1.24748,

$1.24748,1.24748,1.24748,1.24748,1.24748,1.24748, \ldots .$.

Thus if we start at 0.1 , the algorithm converges to an impossible value of $p$, namely 1.24748 .

It appears that the required posterior median is 0.61427 . As a check we may calculate

$$
F(p=0.61427 \mid x)=4(0.61427)^{3}-3(0.61427)^{4}=0.499999 \approx 0.5 .
$$

Figures 4.3 and 4.4 show the posterior median 0.61427 , as well as the other solution of $g(p)=0$ (i.e. root of $g$ ), namely 1.24748 . This is not actually a solution of $F(p \mid x)=0.5$, because the values of $F(p \mid x)$ for $p<0$ and $p>1$ are 0 and 1 , respectively.

Thus, the definition of $g$ above is 'deceptive', and a better definition is:

$$
g(p)=F(p \mid x)-1 / 2=\left\{\begin{array}{lr}
0-1 / 2=-1 / 2, & p<0 \\
4 p^{3}-3 p^{4}-1 / 2, & 0 \leq p \leq 1 \\
1-1 / 2=1 / 2, & p>1 .
\end{array}\right.
$$


Figure 4.3 Posterior cdf and median of $p$

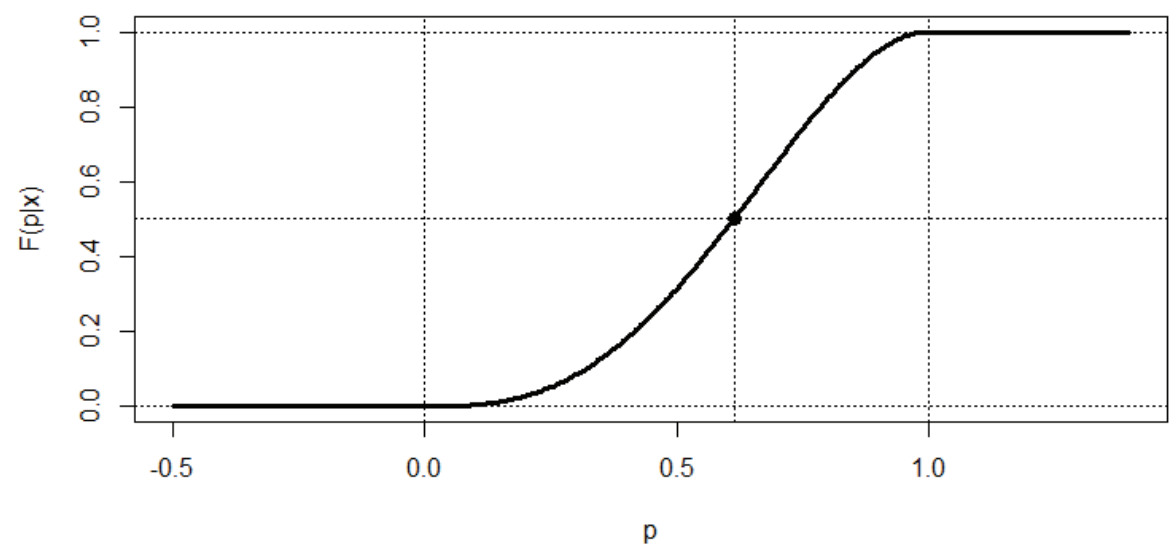

Figure 4.4 Posterior median of $p$ and the other root of $g$

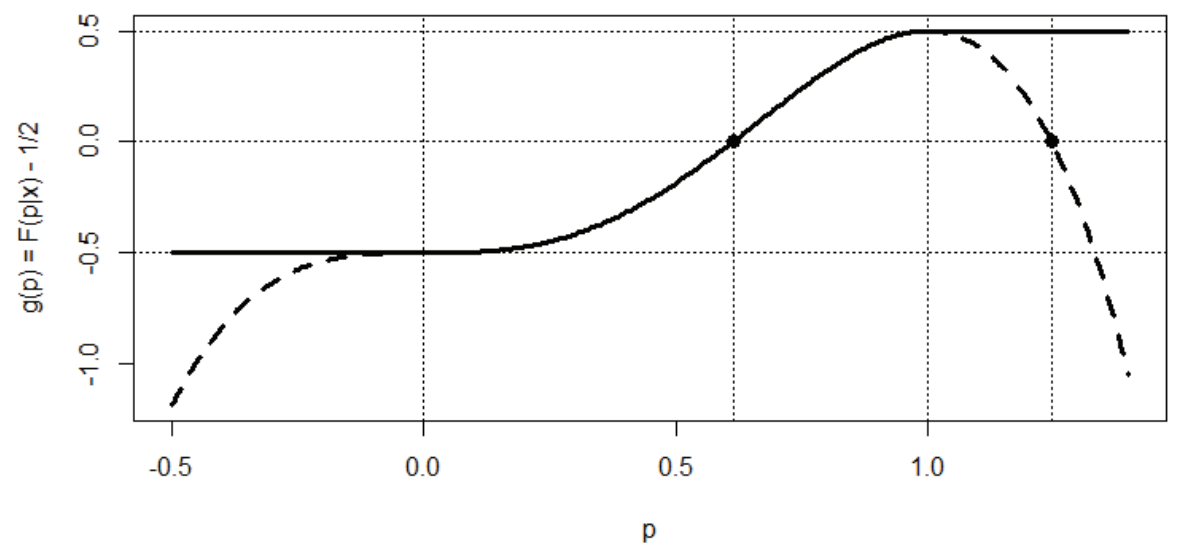

\section{R Code for Exercise 4.3}

options(digits=6); $p=2 / 3 ; p v=p ;$ for $(j$ in $1: 7)\{$

$$
\left.p=p-\left(4^{*} p^{\wedge} 3-3 * p^{\wedge} 4-1 / 2\right) /\left(12 * p^{\wedge} 2-12 * p^{\wedge} 3\right) ; p v=c(p v, p)\right\} ; p v
$$

\# 0.6666670 .6145830 .6142720 .6142720 .6142720 .6142720 .614272

$\# 0.614272$

$p=0.5 ; p v=p$; for $(j$ in $1: 7)\left\{p=p-\left(4^{*} p^{\wedge} 3-3^{*} p^{\wedge} 4-1 / 2\right) /\left(12^{*} p^{\wedge} 2-12^{*} p^{\wedge} 3\right)\right.$;

$p v=c(p v, p)\} ; p v \# 0.5000000 .6250000 .6143060 .6142720 .6142720 .614272$

\# 0.6142720 .614272 
$p=0.9 ; p v=p ;$ for $(j$ in $1: 7)\left\{p=p-\left(4 * p^{\wedge} 3-3 * p^{\wedge} 4-1 / 2\right) /\left(12 * p^{\wedge} 2-12 * p^{\wedge} 3\right)\right.$;

$p v=c(p v, p)\} ; p v \# 0.9000000 .4394030 .6491910 .6145010 .6142720 .614272$

\# 0.6142720 .614272

$p=0.1 ; p v=p ;$ for $(j$ in $1: 7)\left\{p=p-\left(4^{*} p^{\wedge} 3-3^{*} p^{\wedge} 4-1 / 2\right) /\left(12 * p^{\wedge} 2-12 * p^{\wedge} 3\right)\right.$;

$p v=c(p v, p)\} ; p v$

\# 0.100004 .695373 .626902 .834032 .251461 .831951 .542541 .36156

$p=0.1 ; p v=p ;$ for $(j$ in $1: 20)\left\{p=p-\left(4^{*} p^{\wedge} 3-3^{*} p^{\wedge} 4-1 / 2\right) /\left(12 * p^{\wedge} 2-12^{*} p^{\wedge} 3\right)\right.$;

$p v=c(p v, p)\} ; p v$

\# 0.100004 .695373 .626902 .834032 .251461 .831951 .542541 .36156

\# 1.272821 .249131 .247491 .247481 .247481 .247481 .247481 .24748

\# 1.247481 .247481 .247481 .247481 .24748

$4 *(0.614272)^{\wedge} 3-3 *(0.614272)^{\wedge} 4 \quad \# 0.499999$

pvec=seq $(-0.5,1.4,0.005) ;$ Fvec $=4 *$ pvec $^{\wedge} 3-3^{*}$ pvec $^{\wedge} 4$

Fvec $[$ pvec<=0] = 0; Fvec $[$ pvec $>=1]=1$

$\mathrm{X} 11(\mathrm{w}=8, \mathrm{~h}=4.5) ; \operatorname{par}(\operatorname{mfrow}=c(1,1))$

plot(pvec,Fvec,type="l",Iwd=3,xlab="p",ylab="F(p|x)", main=" ")

abline( $h=0.5, v=0.614272$, Ity=3); points $(0.614272,0.5, p c h=16$, cex=1.2)

abline $(h=c(0,1)$, lty $=3)$; abline $(v=c(0,1)$, tty $=3)$

gvecwrong=4* pvec^3-3*pvec^4-0.5

plot(pvec, gvecwrong,type="n",Iwd=2,xlab="p",ylab="g(p) = F(p|x) - 1/2", main=" ")

lines(pvec,Fvec-0.5,lwd=3)

lines (pvec[pvec $<0]$, gvecwrong[pvec $<0]$, Ity=2, Iwd=3)

lines (pvec[pvec $>1]$, gvecwrong[pvec $>1],|t y=2| w d=$,3 )

abline $(v=c(0.614272,1.24748)$, Ity=3); abline $(h=0$, Ity=3)

points $(c(0.614272,1.24748), c(0,0), p c h=16, c e x=1.2)$

abline $(h=c(-0.5,0,0.5)$, Ity=3); abline $(v=c(0,1)$, Ity=3)

\subsection{The multivariate Newton-Raphson algorithm}

The Newton-Raphson algorithm can also be used to solve several equations simultaneously, say

$$
g_{k}\left(x_{1}, \ldots, x_{K}\right)=0, k=1, \ldots, K \text {. }
$$


Let: $\quad x=\left(\begin{array}{c}x_{1} \\ \vdots \\ x_{K}\end{array}\right), g(x)=\left(\begin{array}{c}g_{1}(x) \\ \vdots \\ g_{K}(x)\end{array}\right), 0=\left(\begin{array}{c}0 \\ \vdots \\ 0\end{array}\right)$ (a column vector of length $K$ ).

Then the system of $K$ equations may be expressed as

$$
g(x)=0,
$$

and the NR algorithm involves iterating according to

$$
x^{(j+1)}=x^{(j)}-g^{\prime}\left(x^{(j)}\right)^{-1} g\left(x^{(j)}\right),
$$

where: $x^{(j)}=\left(\begin{array}{c}x_{1}^{(j)} \\ \vdots \\ x_{K}^{(j)}\end{array}\right)$ is the value of $x$ at the $j$ th iteration

$$
\begin{aligned}
& x^{(j+1)}=\left(\begin{array}{c}
x_{1}^{(j+1)} \\
\vdots \\
x_{K}^{(j+1)}
\end{array}\right), \quad g\left(x^{(j)}\right)=\left(\begin{array}{c}
g_{1}\left(x^{(j)}\right) \\
\vdots \\
g_{K}\left(x^{(j)}\right)
\end{array}\right)=\left[\left(\left.\begin{array}{c}
g_{1}(x) \\
\vdots \\
g_{K}(x)
\end{array}\right|_{x=x^{(j)}}\right]\right. \\
& g^{\prime}\left(x^{(j)}\right)=\left[\left.g^{\prime}(x)\right|_{x=x^{(j)}}\right] \\
& g^{\prime}(x)=\left(\begin{array}{c}
\partial g_{1}(x) / \partial x^{T} \\
\vdots \\
\partial g_{K}(x) / \partial x^{T}
\end{array}\right)=\left(\begin{array}{ccc}
\partial g_{1}(x) / \partial x_{1} & \cdots & \partial g_{1}(x) / \partial x_{K} \\
\vdots & \ddots & \vdots \\
\partial g_{K}(x) / \partial x_{1} & \cdots & \partial g_{K}(x) / \partial x_{K}
\end{array}\right)
\end{aligned}
$$

\section{Exercise 4.4 Finding a HPDR via the multivariate NR algorithm}

Consider the Bayesian model: $(x \mid \lambda) \sim \operatorname{Poisson}(\lambda)$

$$
f(\lambda) \propto 1, \lambda>0,
$$

and suppose that we observe $x=1$. Find the $80 \%$ HPDR for $\lambda$.

\section{Solution to Exercise 4.4}

First, $\quad f(\lambda \mid x) \propto f(\lambda) f(x \mid \lambda)=1 \times e^{-\lambda} \lambda^{x} / x !=e^{-\lambda} \lambda$, since $x=1$.

Thus $(\lambda \mid x) \sim \operatorname{Gamma}(2,1)$, with $f(\lambda \mid x)=\lambda e^{-\lambda}, \lambda>0$. 
The $80 \%$ HPDR for $\lambda$ is $(a, b)$, where $a$ and $b$ satisfy the two equations:

$$
\begin{aligned}
& F(b \mid x)-F(a \mid x)=0.8 \\
& f(b \mid x)=f(a \mid x) .
\end{aligned}
$$

Note: Here, $f(b \mid x)$ is the posterior pdf of $\lambda$ evaluated at $b, F(b \mid x)$ is the posterior cdf of $\lambda$ evaluated at $b$, etc. Equations (4.1) and (4.2) reflect the requirement that $\lambda \in(a, b)$ with posterior probability 0.8 , and that the posterior density of $\lambda$ must be the same at both $a$ and $b$, considering that $\lambda$ 's posterior pdf is bell-shaped and unimodal.

Thus we wish to solve the equation

$$
g(t)=0 \text {, }
$$

where:

$$
0=\left(\begin{array}{l}
0 \\
0
\end{array}\right), \quad t=\left(\begin{array}{l}
a \\
b
\end{array}\right), \quad g(t)=\left(\begin{array}{c}
g_{1}(t) \\
g_{2}(t)
\end{array}\right)=\left(\begin{array}{c}
F(b \mid x)-F(a \mid x)-0.8 \\
f(b \mid x)-f(a \mid x)
\end{array}\right) .
$$

The Newton-Raphson algorithm for solving this equation is

$$
t^{(j+1)}=t^{(j)}-g^{\prime}\left(t^{(j)}\right)^{-1} g\left(t^{(j)}\right),
$$

where:

$$
\begin{aligned}
& t^{(j)}=\left(\begin{array}{l}
a_{j} \\
b_{j}
\end{array}\right) \\
& g^{\prime}(t)=\left(\begin{array}{ll}
\partial g_{1}(t) / \partial a & \partial g_{1}(t) / \partial b \\
\partial g_{2}(t) / \partial a & \partial g_{2}(t) / \partial b
\end{array}\right)=\left(\begin{array}{cc}
-a e^{-a} & b e^{-b} \\
e^{-a}(a-1) & e^{-b}(1-b)
\end{array}\right) .
\end{aligned}
$$

Starting at

$$
t^{(0)}=\left(\begin{array}{l}
a_{0} \\
b_{0}
\end{array}\right)=\left(\begin{array}{l}
0.5 \\
3.0
\end{array}\right)
$$

(based on a visual inspection of the posterior density $f(\lambda \mid x)=\lambda e^{-\lambda}$ ), we obtain results as shown in Table 4.3.

Table 4.3 Multivariate NR algorithm

\begin{tabular}{c|cccccc}
$j$ & 0 & 1 & 2 & 3 & 4 & 5 \\
\hline$a_{j}$ & 0.5 & 0.0776524 & 0.163185 & 0.167317 & 0.16730 & 0.16730 \\
$b_{j}$ & 3.0 & 2.7406883 & 3.025571 & 3.079274 & 3.08029 & 3.08029 \\
\hline
\end{tabular}


It seems that the $80 \%$ CPDR for $\lambda$ is $(0.16730,3.08029)$. This interval is illustrated in Figure 4.5 and appears to be correct.

As another check on our calculations, we find that:

$$
\begin{aligned}
& f(\lambda=3.08029 \mid x)-f(\lambda=0.16730 \mid x)=0.14153-0.14153=0 \\
& F(\lambda=3.08029 \mid x)-F(\lambda=0.16730 \mid x)=0.81253-0.01253=0.8 .
\end{aligned}
$$

\section{Figure 4.5 An 80\% HPDR}

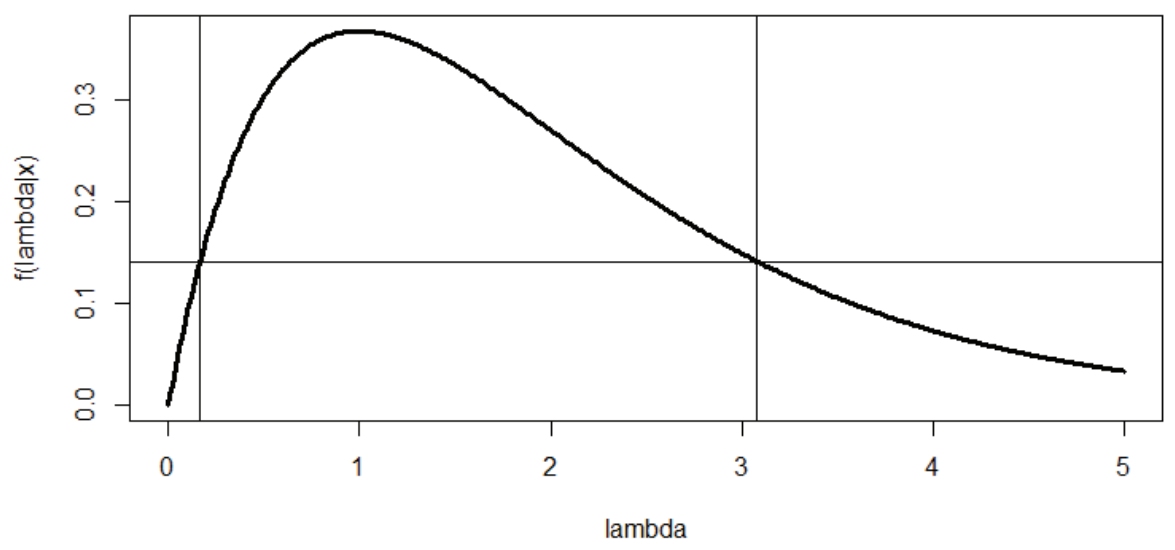

\section{R Code for Exercise 4.4}

gfun $=$ function $(a, b)\{$

g1=pgamma(b,2,1)-pgamma(a,2,1)-0.8; g2=dgamma(b,2,1)-dgamma(a,2,1); $\mathrm{c}(\mathrm{g} 1, \mathrm{~g} 2)\}$

gpfun $=$ function $(a, b)\{\quad m 11=-$ dgamma $(a, 2,1) ; \quad m 12=$ dgamma $(b, 2,1)$

$\mathrm{m} 21=\exp (-\mathrm{a}) *(a-1) ; \quad \mathrm{m} 22=\exp (-b) *(1-b)$

matrix $(\mathrm{c}(\mathrm{m} 11, \mathrm{~m} 12, \mathrm{~m} 21, \mathrm{~m} 22), \mathrm{nrow}=2$, byrow $=\mathrm{T}) \quad$ \}

gvec $=c(0.5,3)$; gmat=gvec; for $(j$ in $1: 7)\{$

$\mathrm{a}=$ gvec[1]; b=gvec[2]

gvec $=$ gvec - solve $($ gpfun $(a, b)) \% * \%$ gfun $(a, b)$

gmat $=$ cbind $($ gmat, gvec $) \quad\}$

options(digits=6); gmat

\# [1,] 0.50 .07765240 .1631850 .1673170 .167300 .167300 .167300 .16730

\#[2,] 3.02 .74068833 .0255713 .0792743 .080293 .080293 .080293 .08029 
lamv $=$ seq $(0,5,0.01) ; \mathrm{fv}=$ dgamma $($ lamv $, 2,1)$

$\mathrm{X} 11(\mathrm{w}=8, \mathrm{~h}=4.5) ; \operatorname{par}(\mathrm{mfrow}=\mathrm{c}(1,1))$

plot(lamv,fv,type="l",Iwd=3,xlab="lambda",ylab="f(lambda|x)", main=" ")

abline $(h=c($ dgamma $(a, 2,1)), v=c(a, b)$, Ity=1)

\# Checks:

$c(a, b$, dgamma(c(a,b),2,1)) \# 0.1673003 .0802910 .1415270 .141527

c(pgamma(a,2,1), pgamma(b,2,1), pgamma(b,2,1) - pgamma(a,2,1))

\# 0.01252750 .81252750 .8000000

\subsection{The Expectation-Maximisation (EM) algorithm}

We have shown how the Newton-Raphson algorithm for solving $g(x)=0$ numerically can be useful for finding the posterior median and the HPDR. That algorithm can also be used for finding the posterior mode, when this is the solution of

$$
\frac{\partial f(\theta \mid y)}{\partial \theta}=0,
$$

or equivalently

$$
\frac{\partial \log f(\theta \mid y)}{\partial \theta}=0 \text {. }
$$

In some situations, finding the posterior mode either analytically or via the NR algorithm may be problematic because the posterior density $f(\theta \mid y)$ has a very complicated form. In that case, one may consider applying the Expectation-Maximisation (EM) algorithm.

This algorithm first requires the specification (i.e. definition by the user) of some suitable latent data, which we will denote by $z$, and then the application of the following two steps iteratively until convergence.

Note: The choice of the latent data $z$ will depend on the particular application.

\section{Step I. The Expectation Step (E-Step)}

Determine the $Q$-function, defined as

$$
\begin{aligned}
Q_{j}(\theta) & =E_{z}\left\{\log f(\theta \mid y, z) \mid y, \theta_{j}\right\} \\
& =\int \log f(\theta \mid y, z) f\left(z \mid y, \theta_{j}\right) d z,
\end{aligned}
$$


or, in words, as

the expectation of the log-augmented posterior density with respect to the distribution of the latent data given the observed data and current parameter estimates.

\section{Step 2. The Maximisation Step (M-Step)}

Find the value of $\theta$ which maximises the Q-function, for example using the Newton-Raphson algorithm.

This value becomes the current parameter estimate in the next iteration.

Note 1: For mathematical convenience, the Q-function may also be defined as at (4.3) but plus and/or multiplied by any constants which do not depend on the parameter $\theta$. This extended definition allows us to ignore terms which have no impact on the final results. If (4.3) is multiplied by a negative constant, the resulting Q-function should be minimised at Step 2 rather than maximised.

Note 2: If there is a choice between using the NR algorithm or the EM algorithm, one should consider the fact that the EM algorithm is slower to converge but far more stable. In fact, under certain regularity conditions, the EM algorithm is guaranteed to move closer to the required solution at each iteration. By contrast, the NR algorithm may not converge at all if started at a value far away from the required solution. Thus, one plausible strategy is to use the EM algorithm to obtain an approximate solution which is sufficiently close to the correct answer, and then to obtain a very high precision using just a few iterations of the NR algorithm.

\section{Exercise 4.5 Illustration of the EM algorithm}

Consider the Bayesian model given by:

$$
\begin{aligned}
& \left(y_{1}, \ldots, y_{n} \mid \lambda\right) \sim \text { iid } \operatorname{Gamma}(1, \lambda) \\
& f(\lambda) \propto 1, \lambda>0 .
\end{aligned}
$$

Suppose that the data, denoted $D$, consists of the observed data vector, denoted by 


$$
y_{o}=\left(y_{1}, \ldots, y_{k}\right),
$$

and the partially observed (or missing) data vector, denoted by

$$
y_{m}=\left(y_{k+1}, \ldots, y_{n}\right) \text {. }
$$

We don't know the values in $y_{m}$ exactly, only that each of those values is greater than some specified constant $c$.

Suppose that $c=10, n=5, k=3$ and $y_{o}=(3.1,8.2,6.9)$.

(a) Find the posterior mode of $\lambda$ by maximising the posterior density directly.

(b) Find the posterior mode of $\lambda$ using the EM algorithm.

\section{Solution to Exercise 4.5}

(a) First, $\quad f(\lambda \mid D) \propto f(\lambda) f(D \mid \lambda)$

$$
\propto 1 \times\left(\prod_{i=1}^{k} f\left(y_{i} \mid \lambda\right)\right)\left(\prod_{i=k+1}^{n} P\left(y_{i}>c \mid \lambda\right)\right),
$$

where: $\quad f\left(y_{i} \mid \lambda\right)=\lambda e^{-\lambda y_{i}}$

$$
P\left(y_{i}>c \mid \lambda\right)=\int_{c}^{\infty} \lambda e^{-y_{i} \lambda} d y_{i}=e^{-c \lambda}
$$

Then $f(\lambda \mid D) \propto\left(\prod_{i=1}^{k} \lambda e^{-\lambda y_{i}}\right)\left(\prod_{i=k+1}^{n} e^{-c \lambda}\right)$

$$
=\lambda^{k} \exp \left\{-\lambda\left[y_{o T}+(n-k) c\right]\right\},
$$

where $y_{o T}=y_{1}+\ldots+y_{k}=18.2 \quad$ (the total of the observed values).

So $\quad l(\lambda) \equiv \log f(\lambda \mid D)=k \log \lambda-\lambda\left[y_{0 T}+(n-k) c\right]$

$$
\Rightarrow l^{\prime}(\lambda)=\frac{k}{\lambda}-\left[y_{o T}+(n-k) c\right] .
$$

Setting $l^{\prime}(\lambda)$ to zero yields the posterior mode,

$$
\frac{k}{y_{o T}+(n-k) c}=0.078534 \text {. }
$$


(b) The latent data here may be defined as $z=y_{m}=\left(y_{k+1}, \ldots, y_{n}\right)$.

Then, the augmented posterior density is

$$
f\left(\lambda \mid y_{o}, y_{m}\right) \propto \prod_{i=1}^{n} \lambda e^{-\lambda y_{i}}=\lambda^{n} \exp \left\{-\lambda\left[y_{o T}+y_{m T}\right]\right\},
$$

where $y_{m T}=y_{k+1}+\ldots+y_{n} \quad$ (the total of the missing values).

So the log-augmented density is

$$
\log f\left(\lambda \mid y_{o}, y_{m}\right)=n \log \lambda-\lambda\left[y_{o T}+y_{m T}\right]+c_{1}
$$

(where $c_{1}$ is a constant with respect to $\lambda$ ).

Now, $f\left(y_{i} \mid y_{i}>c, \lambda\right)=\frac{\lambda e^{-\lambda y_{i}}}{e^{-\lambda c}}=\lambda e^{-\lambda\left(y_{i}-c\right)}, y_{i}>c$

(an exponential pdf shifted to the right by $c$ ).

Therefore, $\quad E\left(y_{i} \mid y_{i}>c, \lambda\right)=c+\frac{1}{\lambda}$.

It follows that the Q-function is given by

$$
Q_{j}(\lambda)=n \log \lambda-\lambda\left[y_{o T}+(n-k)\left(c+\frac{1}{\lambda_{j}}\right)\right]
$$

(note the distinction here between $\lambda$ and $\lambda_{j}$ ).

That concludes the E-Step.

As regards the M-Step, we now calculate the derivative

$$
Q_{j}^{\prime}(\lambda)=\frac{n}{\lambda}-\left[y_{o T}+(n-k)\left(c+\frac{1}{\lambda_{j}}\right)\right] .
$$

Setting this derivative to zero yields a formula for the next value,

$$
\lambda_{j+1}=\frac{n}{y_{o T}+(n-k)\left(c+1 / \lambda_{j}\right)} .
$$

Implementing the above EM algorithm starting at $\lambda_{0}=1$ we get the following sequence:

1.000000, 0.124378, 0.092115, 0.083456, 0.080431, 0.079282,

0.078832, 0.078653, 0.078581, 0.078553, 0.078542, 0.078537,

$0.078535,0.078535,0.078534,0.078534, \ldots \ldots$ 
We see that the EM algorithm has converged correctly to the answer obtained in (a), namely 0.078534 .

Note: Writing (4.4) with $\lambda_{j}=\lambda_{j+1}=\lambda$ (i.e. the limiting value) gives

$$
\lambda=\frac{n}{y_{o T}+(n-k)(c+1 / \lambda)},
$$

and this can be solved easily for the same formula as derived in (a), namely

$$
\lambda=\frac{k}{y_{o T}+(n-k) c} .
$$

Thus, in this exercise it was not necessary to actually perform any iterations of the EM algorithm.

\section{R Code for Exercise 4.5}

$\#(a)$

$n=5 ; k=3 ; c=10 ; y o=c(3.1,8.2,6.9) ;$ yoT=sum(yo); yoT \# 18.2

$\mathrm{k} /(\mathrm{yoT}+(\mathrm{n}-\mathrm{k}) * \mathrm{c}) \# 0.078534$

\# (b)

lam = 1 ; lamv = lam; options $($ digits $=5)$

for $(\mathrm{j}$ in $1: 20)\left\{\operatorname{lam}=\mathrm{n} /\left(\mathrm{yoT}+(\mathrm{n}-\mathrm{k})^{*}(\mathrm{c}+1 / \mathrm{lam})\right) ;\right.$ lamv=c(lamv,lam) $\}$

lamv

\# 1.0000000 .1243780 .0921150 .0834560 .0804310 .0792820 .078832

\# 0.0786530 .0785810 .0785530 .0785420 .0785370 .0785350 .078535

\# 0.0785340 .0785340 .0785340 .0785340 .0785340 .0785340 .078534

\section{Exercise 4.6 EM algorithm for right-censored Gaussian data}

Consider the Bayesian model given by:

$$
\begin{aligned}
& \left(y_{1}, \ldots, y_{n} \mid \lambda\right) \sim \text { iid } N\left(\mu, \sigma^{2}\right) \\
& f(\mu) \propto 1, \mu \in \mathfrak{R} .
\end{aligned}
$$

Suppose that the data, denoted $D$, consists of the observed data vector

$$
y_{o}=\left(y_{1}, \ldots, y_{k}\right)
$$

and the partially observed (or 'missing') data vector

$$
y_{m}=\left(y_{k+1}, \ldots, y_{n}\right) \text {. }
$$


We don't know the values in $y_{m}$ exactly, but only that each of these values is greater than some specified constant $c$.

Suppose that $c=10, n=5, k=3$ and $y_{o}=(3.1,8.2,6.9)$.

(a) Find the log-posterior density of $\mu$ and describe how it could be used to find the posterior mode of $\mu$. (Do not actually find that mode in this way.)

(b) Find the posterior mode of $\mu$ using the EM algorithm. Then check your answer by showing the mode in plots of the likelihood and loglikelihood functions.

\section{Solution to Exercise 4.6}

(a) Observe that $f(\mu \mid D) \propto 1 \times\left(\prod_{i=1}^{k} f\left(y_{i} \mid \mu\right)\right)\left(\prod_{i=k+1}^{n} P\left(y_{i}>c \mid \mu\right)\right)$.

Here, $\prod_{i=1}^{k} f\left(y_{i} \mid \mu\right) \propto \prod_{i=1}^{k} e^{-\frac{1}{2 \sigma^{2}}\left(y_{i}-\mu\right)^{2}}=\exp \left\{-\frac{1}{2 \sigma^{2}} \sum_{i=1}^{k}\left(y_{i}-\mu\right)^{2}\right\}$

$$
=\exp \left\{-\frac{1}{2 \sigma^{2}}\left[(k-1) s_{o}^{2}+k\left(\mu-\bar{y}_{o}\right)^{2}\right]\right\},
$$

where: $\quad \bar{y}_{o}=\frac{1}{k} \sum_{i=1}^{k} y_{i} \quad$ (the observed sample mean)

$$
s_{o}^{2}=\frac{1}{k-1} \sum_{i=1}^{k}\left(y_{i}-\bar{y}_{o}\right)^{2} \quad \text { (the observed sample variance). }
$$

Also, $\quad P\left(y_{i}>c \mid \mu\right)=\int_{c}^{\infty} \frac{1}{\sigma \sqrt{2 \pi}} e^{-\frac{1}{2}\left(y_{i}-\mu\right)^{2}} d y_{i}$

$$
=P\left(Z>\frac{c-\mu}{\sigma}\right)=1-\Phi\left(\frac{c-\mu}{\sigma}\right),
$$

where $Z \sim N(0,1)$ and $\Phi(z)=P(Z \leq z)$ (the standard normal cdf).

Therefore $f(\mu \mid D) \propto \exp \left\{-\frac{k}{2 \sigma^{2}}\left(\mu-\bar{y}_{o}\right)^{2}\right\}\left(1-\Phi\left(\frac{c-\mu}{\sigma}\right)\right)^{n-k}$. 
So the log-posterior is

$$
\log f(\mu \mid D)=-\frac{k}{2 \sigma^{2}}\left(\mu-\bar{y}_{o}\right)^{2}+(n-k) \log \left(1-\Phi\left(\frac{c-\mu}{\sigma}\right)\right)+c_{1}
$$

(where $c_{1}$ is a term which does not depend on $\mu$ ).

To find the posterior mode of $\mu$ we could solve the equation

$$
l^{\prime}(\mu)=0 \text {, }
$$

where $l^{\prime}(\mu)=\frac{\partial \log f(\mu \mid D)}{\partial \mu}$

$$
=-\frac{k}{\sigma^{2}}\left(\mu-\bar{y}_{o}\right)+\frac{(n-k)}{\left(1-\Phi\left(\frac{c-\mu}{\sigma}\right)\right)}\left(-\phi\left(\frac{c-\mu}{\sigma}\right)\right)\left(\frac{-1}{\sigma}\right) .
$$

This solution could be obtained via the NR algorithm defined by

$$
\mu_{j+1}=\mu_{j}-\frac{l^{\prime}\left(\mu_{j}\right)}{l^{\prime \prime}\left(\mu_{j}\right)},
$$

where $l^{\prime \prime}(\mu)=\frac{\partial l^{\prime}(\mu)}{\partial \mu}=-\frac{k}{\sigma^{2}}+\ldots$

As a further exercise, one could complete the formula for $l^{\prime \prime}(\mu)$ above and actually implement the NR algorithm.

Note: The posterior mode here is also the maximum likelihood estimate, since the prior is proportional to a constant.

(b) With $y_{m}=\left(y_{k+1}, \ldots, y_{n}\right)$ as the latent data, the augmented posterior is

$$
\begin{aligned}
f\left(\mu \mid y_{o}, y_{m}\right) & \propto 1 \times\left(\prod_{i=1}^{k} f\left(y_{i} \mid \mu\right)\right)\left(\prod_{i=k+1}^{n} f\left(y_{i} \mid \mu\right)\right) \\
& \propto \exp \left\{-\frac{1}{2 \sigma^{2}} \sum_{i=1}^{k}\left(y_{i}-\mu\right)^{2}\right\} \exp \left\{-\frac{1}{2 \sigma^{2}} \sum_{i=k+1}^{n}\left(y_{i}-\mu\right)^{2}\right\} .
\end{aligned}
$$

So the log-augmented posterior is

$$
\log f\left(\mu \mid y_{o}, y_{m}\right)=-\frac{1}{2 \sigma^{2}} \sum_{i=1}^{k}\left(y_{i}-\mu\right)^{2}-\frac{1}{2 \sigma^{2}} \sum_{i=1}^{k}\left(y_{i}-\mu\right)^{2}+c_{1}
$$




$$
\begin{aligned}
& =-\frac{1}{2 \sigma^{2}} \sum_{i=1}^{k}\left(y_{i}^{2}-2 \mu y_{i}+\mu^{2}\right)-\frac{1}{2 \sigma^{2}} \sum_{i=k+1}^{n}\left(y_{i}^{2}-2 \mu y_{i}+\mu^{2}\right)+c_{1} \\
& =c_{2}\left\{\left(k \mu^{2}-2 \mu n \bar{y}_{o}\right)+\left((n-k) \mu^{2}-2 \mu(n-k) \bar{y}_{m}\right)\right\}+c_{3},
\end{aligned}
$$

where: $\bar{y}_{o}=\frac{1}{k} \sum_{i=1}^{k} y_{i}$ (the sample mean of the observed values)

$$
\bar{y}_{m}=\frac{1}{n-k} \sum_{i=k+1}^{n} y_{i} \quad \text { (the sample mean of the missing values). }
$$

Thus the Q-function may be taken as

$$
\begin{aligned}
Q_{j}(\mu) & =k \mu^{2}-2 \mu k \bar{y}_{o}+(n-k) \mu^{2}-2 \mu(n-k) e_{j} \\
& =2 n \mu-2\left\{k \bar{y}_{o}+(n-k) e_{j}\right\},
\end{aligned}
$$

where $e_{j}=E\left(\bar{y}_{m} \mid D, \mu_{j}\right)=E\left(y_{i} \mid D, \mu_{j}\right) \quad(i>k)$.

We see that $e_{j}=\left[\left.E(X \mid X>c)\right|_{\mu=\mu_{j}}\right]$,

where $X \sim N\left(\mu, \sigma^{2}\right) \quad$ (with $\mu$ taken as a constant).

Now observe that

$$
E(X \mid X>c)=\int_{c}^{\infty} x \frac{f(x)}{P(X>c)} d x=\frac{I}{P(X>c)},
$$

where $P(X>c)=1-P(X<c)=1-P\left(Z<\frac{c-\mu}{\sigma}\right)=1-\Phi\left(\frac{c-\mu}{\sigma}\right)$, and where

$$
\begin{aligned}
I & =\int_{c}^{\infty} x \frac{1}{\sigma \sqrt{2 \pi}} e^{-\frac{1}{2 \sigma^{2}}(x-\mu)^{2}} d x \\
& =\int_{c}^{\infty}(x-\mu) \frac{1}{\sigma \sqrt{2 \pi}} e^{-\frac{1}{2 \sigma^{2}(x-\mu)^{2}}} d x+\int_{c}^{\infty} \mu \frac{1}{\sigma \sqrt{2 \pi}} e^{-\frac{1}{2 \sigma^{2}}(x-\mu)^{2}} d x \\
& =\int_{(c-\mu)^{2} / 2}^{\infty} \frac{1}{\sigma \sqrt{2 \pi}} e^{-\frac{1}{\sigma^{2} t}} d t+\mu P(X>c) \\
& =\frac{\sigma}{\sqrt{2 \pi}} \int_{(c-\mu)^{2} / 2}^{\infty} \frac{1}{\sigma^{2}} e^{-\frac{1}{\sigma^{2}} t} d t+\mu P(X>c)
\end{aligned}
$$




$$
\begin{aligned}
& =\frac{\sigma}{\sqrt{2 \pi}}^{-\frac{1}{\sigma^{2}}(c-\mu)^{2} / 2}+\mu P(X>c)=\sigma\left(\frac{1}{\sqrt{2 \pi}}^{-\frac{1}{2}\left(\frac{c-\mu}{\sigma}\right)^{2}}\right)+\mu P(X>c) \\
& =\sigma \phi\left(\frac{c-\mu}{\sigma}\right)+\mu P(X>c) \quad \text { where } \phi(z) \text { is the standard normal pdf. }
\end{aligned}
$$

Thus $\quad E(X \mid X>c)=\frac{1}{P(X>c)}\left\{\sigma \phi\left(\frac{c-\mu}{\sigma}\right)+\mu P(X>c)\right\}$

$$
=\mu+\sigma \phi\left(\frac{c-\mu}{\sigma}\right) /\left\{1-\Phi\left(\frac{c-\mu}{\sigma}\right)\right\}
$$

and consequently $e_{j}=\mu_{j}+\sigma \phi\left(\frac{c-\mu_{j}}{\sigma}\right) /\left\{1-\Phi\left(\frac{c-\mu_{j}}{\sigma}\right)\right\}$.

That completes the E-Step, which may be summarised by writing

$$
Q_{j}(\mu)=n \mu^{2}-2 \mu\left\{k \bar{y}_{o}+(n-k) e_{j}\right\},
$$

where $e_{j}$ is as given above.

The M-Step then involves calculating

$$
Q_{j}^{\prime}(\mu)=2 n \mu-2\left\{k \bar{y}_{o}+(n-k) e_{j}\right\}
$$

and setting this to zero so as to yield the next parameter estimate,

$$
\begin{aligned}
\mu_{j+1} & =\frac{k \bar{y}_{o}+(n-k) e_{j}}{n} \\
& =\frac{1}{n}\left[k \bar{y}_{o}+(n-k)\left(\mu_{j}+\sigma \phi\left(\frac{c-\mu_{j}}{\sigma}\right) /\left\{1-\Phi\left(\frac{c-\mu_{j}}{\sigma}\right)\right\}\right)\right] .
\end{aligned}
$$

Implementing the above EM algorithm starting at 5 (arbitrarily), we obtain the sequence:

5.000000, 8.137838, 8.371786, 8.395701, 8.398209, 8.398473,

8.398501, 8.398504, 8.398504, 8.398504, 8.398504, .....

We conclude that the posterior mode of $\mu$ is 8.3985 .

Figure 4.6 shows the posterior density (top subplot) and the log-posterior density (bottom subplot). Each of these density functions is drawn scaled, meaning correct only up to a constant of proportionality. In each subplot, the posterior mode is indicated by way of a vertical dashed line. 
Figure 4.6 Posterior and log-posterior densities (scaled)
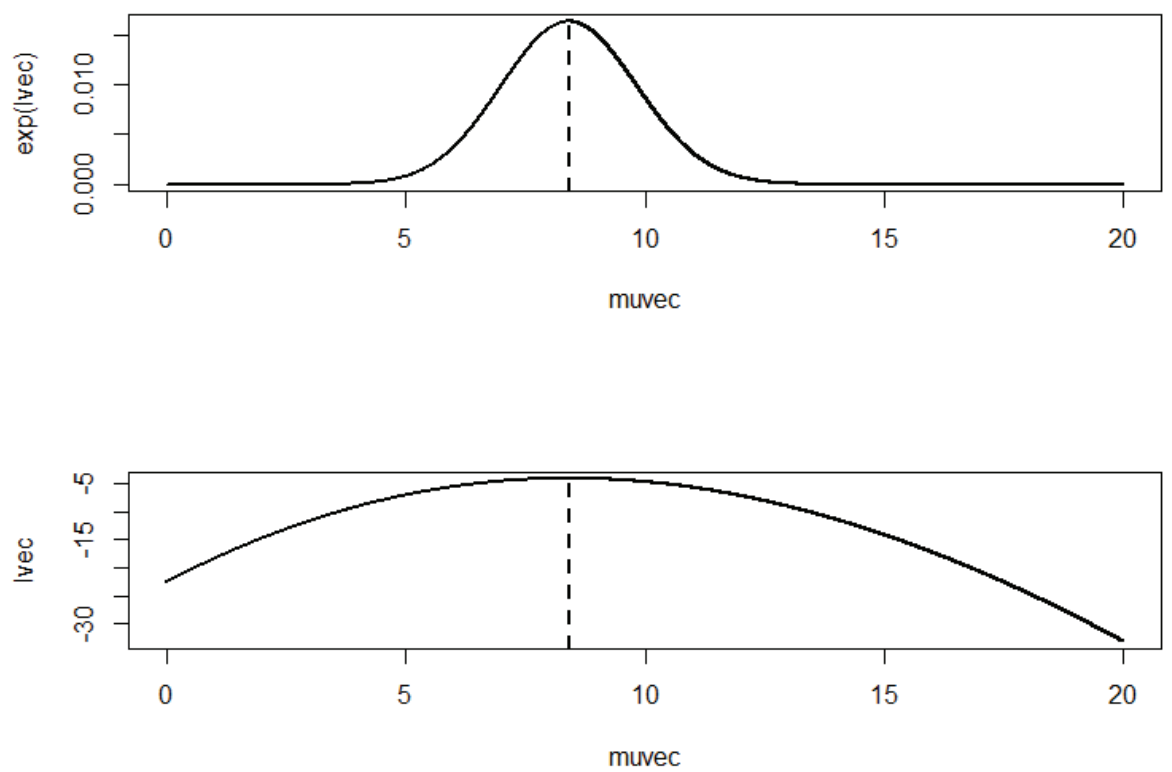

\section{R Code for Exercise 4.6}

\# (b)

options(digits=6); yo =c(3.1, 8.2, 6.9); $n=5 ; k=3 ; c=10 ;$ sig=3;

yoT=sum(yo); c(yoT, yot/3) \# 18.200006 .06667

$\mathrm{mu}=5 ; \mathrm{muv}=\mathrm{mu} ;$ for $(\mathrm{j}$ in $1: 10)\{$

ej $=\mathrm{mu}+\mathrm{sig} *$ dnorm $((c-m u) / s i g) ~ /(1-p n o r m((c-m u) / s i g))$

$m u=($ yoT $+(n-k) *$ ej $) / n$

muv $=c(m u v, m u) \quad\}$

muv \# 5.000008 .137848 .371798 .395708 .398218 .39847

\# 8.398508 .398508 .398508 .398508 .39850

modeval=muv[length(muv)]; modeval \# 8.3985

muvec $=\operatorname{seq}(0,20,0.001) ;$ Ivec=muvec

for(i in 1:length(muvec) $)\{$ muval=muvec[i]

$\operatorname{lvec}[i]=\left(-1 /\left(2 * \operatorname{sig}{ }^{\wedge} 2\right)\right)^{*} \operatorname{sum}\left((\text { yo-muval })^{\wedge} 2\right)+$

$$
(n-k) * \log (1-\text { pnorm ((c-muval)/sig)) \} }
$$

iopt=(1:length(muvec) $)[$ lvec==max(lvec)]; muopt=muvec[iopt]; muopt \# 8.399

$\mathrm{X} 11(\mathrm{w}=8, \mathrm{~h}=6) ; \operatorname{par}(\mathrm{mfrow}=\mathrm{c}(2,1)) ;$

plot(muvec,exp(Ivec),type="I",Iwd=2); abline(v=modeval,lty=2,lwd=2)

plot(muvec,Ivec,type="I",Iwd=2); abline( $v=$ modeval,Ity=2,lwd=2) 


\subsection{Variants of the NR and EM algorithms}

The Newton-Raphson and Expectation-Maximisation algorithms can be modified and combined in various ways to produce a number of useful variants or 'hybrids'. For example, the NR algorithm can be used at each M-Step of the EM algorithm to maximise the Q-function.

If the EM algorithm is applied to find the mode of a parameter vector, say $\theta=\left(\theta_{1}, \theta_{2}\right)$, then the multivariate NR algorithm for doing this may be problematic and one may consider using the ECM algorithm (where $C$ stands for Conditional).

The idea is, at each M-Step, to maximise the Q-function with respect to $\theta_{1}$, with $\theta_{2}$ fixed at its current value; and then to maximise the Q-function with respect to $\theta_{2}$, with $\theta_{1}$ fixed at its current value.

If each of these conditional maximisations is achieved via the NR algorithm, the procedure can be modified to become the ECM1 algorithm. This involves applying only one step of each NR algorithm (rather than finding the exact conditional maximum). In many cases the ECM1 algorithm will be more efficient at finding the posterior mode than the ECM algorithm.

Sometimes, when the simultaneous solution of several equations via the multivariate NR algorithm is problematic, a more feasible solution is to apply a suitable CNR algorithm (where again $C$ stands for Conditional).

For example, suppose we wish to solve two equations simultaneously, say:

$$
\begin{aligned}
& g_{1}(a, b)=0 \\
& g_{2}(a, b)=0,
\end{aligned}
$$

for $a$ and $b$. Then it may be convenient to define the function

$$
g(a, b)=g_{1}(a, b)^{2}+g_{2}(a, b)^{2},
$$

which clearly has a minimum value of zero at the required solutions for $a$ and $b$.

This suggests that we iterate two steps as follows:

Step 1. Minimise $g(a, b)$ with respect to $a$, with $b$ held fixed.

Step 2. Minimise $g(a, b)$ with respect to $b$, with $a$ held fixed. 
The first of these two steps involves solving

$$
\frac{\partial g(a, b)}{\partial a}=0
$$

where $\frac{\partial g(a, b)}{\partial a}=2 g_{1}(a, b) \frac{\partial g_{1}(a, b)}{\partial a}+2 g_{2}(a, b) \frac{\partial g_{2}(a, b)}{\partial a}$.

Assuming the current values of $a$ and $b$ are $a_{j}$ and $b_{j}$, this can be achieved via the NR algorithm by setting $a_{0}^{\prime}=a_{j}$ and iterating until convergence as follows $(k=0,1,2, \ldots)$ :

and finally setting

$$
a_{k+1}^{\prime}=a_{k}^{\prime}-\frac{\left[\frac{\partial g(a, b)}{\partial a} \mid a=a_{k}^{\prime}, b=b_{j}\right]}{\left[\frac{\partial^{2} g(a, b)}{\partial a^{2}} \mid a=a_{k}^{\prime}, b=b_{j}\right]},
$$

$$
a_{j+1}=a_{\infty}^{\prime} \text {. }
$$

The second of the two steps involves solving

$$
\frac{\partial g(a, b)}{\partial b}=0
$$

where $\frac{\partial g(a, b)}{\partial b}=2 g_{1}(a, b) \frac{\partial g_{1}(a, b)}{\partial b}+2 g_{2}(a, b) \frac{\partial g_{2}(a, b)}{\partial b}$.

This can be achieved via the NR algorithm by setting $b_{0}^{\prime}=b_{j}$ and iterating until convergence as follows $(k=0,1,2, \ldots)$ :

$$
b_{k+1}^{\prime}=b_{k}-\frac{\left[\frac{\partial g(a, b)}{\partial b} \mid a=a_{j+1}, b=b_{k}\right]}{\left[\frac{\partial^{2} g(a, b)}{\partial b^{2}} \mid a=a_{j+1}, b=b_{k}\right]},
$$

and finally setting

$$
b_{j+1}=b_{\infty}^{\prime} \text {. }
$$

A variant of the CNR algorithm is the CNR1 algorithm. This involves performing only one step of each NR algorithm in the CNR algorithm.

In the above example, the CNR1 algorithm implies we set $a_{j+1}=a_{1}^{\prime}$ at (4.5) and $b_{j+1}=b_{1}^{\prime}$ at (4.6) (rather than $a_{j+1}=a_{\infty}^{\prime}$ and $b_{j+1}=b_{\infty}^{\prime}$ ). 
This modification will also result in eventual convergence to the solution of $g_{1}(a, b)=0$ and $g_{2}(a, b)=0$.

One application of the CNR and CNR1 algorithms is to finding the HPDR for a parameter.

For example, in Exercise 4.4 we considered the model given by

$$
\begin{aligned}
& (x \mid \lambda) \sim \operatorname{Poisson}(\lambda) \\
& f(\lambda) \propto 1, \lambda>0,
\end{aligned}
$$

with observed data $x=1$.

The $80 \%$ HPDR for $\lambda$ was shown to be $(a, b)$, where $a$ and $b$ are the simultaneous solutions of the two equations:

$$
\begin{aligned}
& g_{1}(a, b)=F(b \mid x)-F(a \mid x)-0.8 \\
& g_{2}(a, b)=f(b \mid x)-f(a \mid x) .
\end{aligned}
$$

Applying the CNR or CNR1 algorithm as described above should also lead to the same interval as obtained earlier via the multivariate NR algorithm, namely $(0.16730,3.08029)$.

For further details regarding the EM algorithm, the Newton-Raphson algorithm, and extensions thereof, see Lachlan and Krishnan (2008).

\section{Exercise 4.7 Application of the EM and ECM algorithms to a normal mixture model}

Consider the following Bayesian model:

$$
\begin{aligned}
& \left(y_{i} \mid R, \mu, \delta\right) \sim \perp\left(\mu+\delta R_{i}, \sigma^{2}\right), i=1, \ldots, n \\
& \left(R_{1}, \ldots, R_{n} \mid \mu, \delta\right) \sim \text { iid Bernoulli }(\pi), i=1, \ldots, n \\
& f(\mu, \delta) \propto 1, \quad \mu \in \mathfrak{R}, \delta>0 .
\end{aligned}
$$

This model says that each value $y_{i}$ has a common variance $\sigma^{2}$ and one of two means, these being: $\quad \mu$ if $R_{i}=0$

$$
\mu+\delta \text { if } R_{i}=1 \text {. }
$$

Each of the 'latent' indicator variables $R_{i}$ has known probability $\pi$ of being equal to 1 , and probability $1-\pi$ of being equal to 0 . 
Note: In more advanced models, the quantity $\pi$ could be treated as unknown and assigned a prior distribution, along with the other two model parameters, $\mu$ and $\delta$. The model here provides a 'stepping stone' to understanding and implementing such more complex models.

(a) Consider the situation where $n=100, \pi=1 / 3, \mu=20, \delta=10$ and $\sigma=3$. Generate a data vector $y=\left(y_{1}, \ldots, y_{n}\right)$ using these specifications and create a histogram of the simulated values.

(b) Design an EM algorithm for finding the posterior mode of $\theta=(\mu, \delta)$. Then implement the algorithm so as to find that mode.

(c) Modify the EM algorithm in part (b) so that it is an ECM algorithm. Then run the ECM algorithm so as to check your answer to part (b).

(d) Create a plot which shows the routes taken by the algorithms in parts (b) and (c).

\section{Solution to Exercise 4.7}

(a) Figure 4.7 shows a histogram of the sampled values which clearly shows the two component normal densities and the mixture density. The sample mean of the data is 23.16. Also, 29 of the $100 R_{i}$ values are equal to 1 , and 71 of them are equal to 0 .

Figure 4.7 Histogram of simulated data

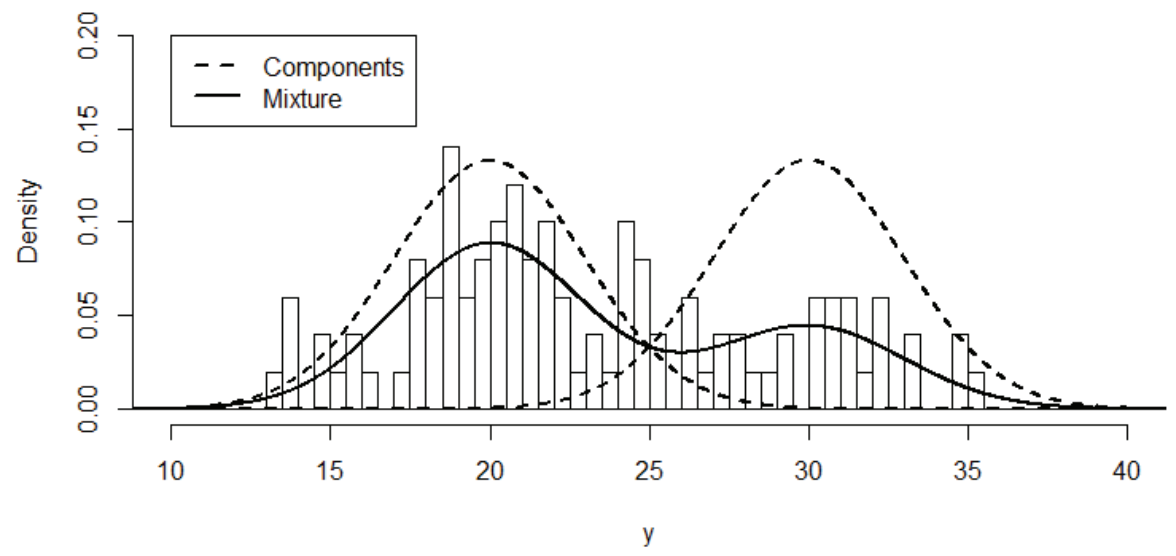


(b) We will here take the vector $R=\left(R_{1}, \ldots, R_{n}\right)$ as the latent data. The conditional posterior of $\mu$ and $\delta$ given this latent data is

$$
\begin{aligned}
f(\mu, \delta \mid y, R) & \propto f(\mu, \delta, y, R) \\
& =f(\mu, \delta) f(R \mid \mu, \delta) f(y \mid R, \mu, \delta) \\
& \propto 1 \times \prod_{i=1}^{n} \pi^{R_{i}}(1-\pi)^{1-R_{i}} \times \prod_{i=1}^{n} \exp \left\{-\frac{1}{2 \sigma^{2}}\left(y_{i}-\left[\mu+R_{i} \delta\right]\right)^{2}\right\} \\
& \propto 1 \times 1 \times \exp \left\{-\frac{1}{2 \sigma^{2}} \sum_{i=1}^{n}\left(y_{i}-\left[\mu+R_{i} \delta\right]\right)^{2}\right\} .
\end{aligned}
$$

So the log-augmented posterior density is

$$
\begin{aligned}
& \log f(\mu, \delta \mid y, R)=-\frac{1}{2 \sigma^{2}} \sum_{i=1}^{n}\left(y_{i}-\left[\mu+R_{i} \delta\right]\right)^{2} \\
&=-\frac{1}{2 \sigma^{2}} \sum_{i=1}^{n}\left(y_{i}^{2}-2 y_{i}\left[\mu+R_{i} \delta\right]+\left[\mu+R_{i} \delta\right]^{2}\right) \\
&=-\frac{1}{2 \sigma^{2}}\left\{\sum_{i=1}^{n} y_{i}^{2}-2 \sum_{i=1}^{n} y_{i}\left[\mu+R_{i} \delta\right]+\sum_{i=1}^{n}\left[\mu+R_{i} \delta\right]^{2}\right\} \\
&=-C_{1}\left\{C_{2}-2 \mu n \bar{y}-2 \delta \sum_{i=1}^{n} y_{i} R_{i}+n \mu^{2}+2 \mu \delta \sum_{i=1}^{n} R_{i}+\delta^{2} \sum_{i=1}^{n} R_{i}^{2}\right\},
\end{aligned}
$$

where $c_{1}$ and $c_{2}$ are positive constants which do not depend on $\mu$ or $\delta$ in any way. We see that

$$
\log f(\mu, \delta \mid y, R)=-c_{1}\left\{c_{2}-2 \mu n \bar{y}-2 \delta \sum_{i=1}^{n} y_{i} R_{i}+n \mu^{2}+2 \mu \delta R_{T}+\delta^{2} R_{T}\right\},
$$

where $R_{T}=\sum_{i=1}^{n} R_{i}$.

Note: Each $R_{i}$ equals 0 or 1 , and therefore $R_{i}^{2}=R_{i}$.

So the Q-function is

$$
\begin{aligned}
Q_{j}(\mu, \delta) & =E_{R}\left\{\log f(\mu, \delta \mid y, R) \mid y, \mu_{j}, \delta_{j}\right\} \\
& =-c_{1}\left\{c_{2}-2 \mu n \bar{y}-2 \delta \sum_{i=1}^{n} y_{i} e_{i j}+n \mu^{2}+2 \mu \delta e_{T j}+\delta^{2} e_{T j}\right\},
\end{aligned}
$$

where: $e_{i j}=E\left(R_{i} \mid y, \mu_{j}, \delta_{j}\right)$

$$
e_{T j}=E\left(R_{T} \mid y, \mu_{j}, \delta_{j}\right)=\sum_{i=1}^{n} e_{i j} .
$$


We now need to obtain formulae for the $e_{i j}$ values. Observe that

$$
\begin{aligned}
& f(R \mid y, \mu, \delta) \propto f(\mu, \delta, y, R) \\
& \quad \propto 1 \times \prod_{i=1}^{n} \pi^{R_{i}}(1-\pi)^{1-R_{i}} \times \prod_{i=1}^{n} \exp \left\{-\frac{1}{2 \sigma^{2}}\left(y_{i}-\left[\mu+R_{i} \delta\right]\right)^{2}\right\} .
\end{aligned}
$$

It follows that

$$
\left(R_{i} \mid y, \mu, \delta\right) \sim \perp \operatorname{Bernoulli}\left(e_{i}\right), i=1, \ldots, n,
$$

where

$$
e_{i}=\frac{\pi \exp \left(-\frac{1}{2 \sigma^{2}}\left(y_{i}-[\mu+\delta]\right)^{2}\right)}{\pi \exp \left(-\frac{1}{2 \sigma^{2}}\left(y_{i}-[\mu+\delta]\right)^{2}\right)+(1-\pi) \exp \left(-\frac{1}{2 \sigma^{2}}\left(y_{i}-\mu\right)^{2}\right)} .
$$

Therefore

$$
e_{i j}=\frac{\pi \exp \left(-\frac{1}{2 \sigma^{2}}\left(y_{i}-\left[\mu_{j}+\delta_{j}\right]\right)^{2}\right)}{\pi \exp \left(-\frac{1}{2 \sigma^{2}}\left(y_{i}-\left[\mu_{j}+\delta_{j}\right]\right)^{2}\right)+(1-\pi) \exp \left(-\frac{1}{2 \sigma^{2}}\left(y_{i}-\mu_{j}\right)^{2}\right)} .
$$

Thereby the E-Step of the EM algorithm has been defined.

Next, the M-Step requires us to maximise the Q-function. We begin by writing:

$$
\begin{aligned}
& \frac{\partial Q_{j}(\mu, \delta)}{\partial \mu}=-c_{1}\left\{0-2 n \bar{y}-0+2 n \mu+2 \delta e_{T j}+0\right\} \\
& \frac{\partial Q_{j}(\mu, \delta)}{\partial \delta}=-c_{1}\left\{0-0-2 \sum_{i=1}^{n} y_{i} e_{i j}+0+2 \mu e_{T j}+2 \delta e_{T j}\right\} .
\end{aligned}
$$

Setting both of these derivatives to zero and solving for $\mu$ and $\delta$ simultaneously, we obtain the next two values in the algorithm:

$$
\mu_{j+1}=\frac{\bar{y}-\frac{1}{n} \sum_{i=1}^{n} y_{i} e_{i j}}{1-\frac{1}{n} e_{T j}}, \quad \delta_{j+1}=\frac{\sum_{i=1}^{n} y_{i} e_{i j}}{e_{T j}}-\mu_{j+1} .
$$

The EM algorithm is now completely defined. 
Starting the algorithm from $\left(\mu_{0}, \delta_{0}\right)=(10,1)$, we obtain the sequence shown in Table 4.4. We see that the algorithm has converged to what we believe to be the posterior mode, $(\hat{\mu}, \hat{\delta})=(20.08,9.72)$.

Running the algorithm from different starting points we obtain the same final results. Unlike the NR algorithm, we find that the EM algorithm always converges, regardless of the point from which it is started.

\section{Table 4.4 Results of an EM algorithm}

\begin{tabular}{c|cc}
$j$ & $\mu_{j}$ & $\delta_{j}$ \\
\hline 0 & 10.000 & 1.000 \\
1 & 21.169 & 3.032 \\
2 & 20.321 & 7.07 \\
3 & 19.843 & 9.139 \\
4 & 19.926 & 9.518 \\
5 & 20.005 & 9.626 \\
6 & 20.046 & 9.674 \\
7 & 20.066 & 9.697 \\
8 & 20.075 & 9.708 \\
9 & 20.08 & 9.713 \\
10 & 20.082 & 9.715 \\
11 & 20.083 & 9.717 \\
12 & 20.084 & 9.717 \\
13 & 20.084 & 9.717 \\
14 & 20.084 & 9.718 \\
15 & 20.084 & 9.718 \\
16 & 20.084 & 9.718 \\
17 & 20.084 & 9.718 \\
18 & 20.084 & 9.718 \\
19 & 20.084 & 9.718 \\
20 & 20.084 & 9.718 \\
\hline
\end{tabular}

(c) The ECM requires us to once again examine the Q-function,

$$
Q_{j}(\mu, \delta)=-c_{1}\left\{c_{2}-2 \mu n \bar{y}-2 \delta \sum_{i=1}^{n} y_{i} e_{i j}+n \mu^{2}+2 \mu \delta e_{T j}+\delta^{2} e_{T j}\right\},
$$

but now to maximise this function with respect to $\mu$ and $\delta$ individually (rather than simultaneously as for the EM algorithm in (c)). 
Thus, setting $\frac{\partial Q_{j}(\mu, \delta)}{\partial \mu}=-c_{1}\left\{0-2 n \bar{y}-0+2 n \mu+2 \delta e_{T j}+0\right\}$

to zero we get $\mu_{j+1}=\bar{y}-\delta_{j} \frac{1}{n} e_{T j}$ (after substituting in $\delta=\delta_{j}$ ).

Then, setting $\frac{\partial Q_{j}(\mu, \delta)}{\partial \delta}=-c_{1}\left\{0-0-2 \sum_{i=1}^{n} y_{i} e_{i j}+0+2 \mu e_{T j}+2 \delta e_{T j}\right\}$

to zero we get $\quad \delta_{j+1}=\frac{\sum_{i=1}^{n} y_{i} e_{i j}}{e_{T j}}-\mu_{j+1} \quad$ (same equation as in (c)).

We see that the ECM algorithm here is fairly similar to the EM algorithm.

Starting the algorithm at $\left(\mu_{0}, \delta_{0}\right)=(10,1)$ we obtain the sequence shown in Table 4.5 (page 184). We see that the ECM algorithm has converged to the same values as the EM algorithm, but along a slightly different route.

(d) Figure 4.8 (page 185) shows a contour plot of the log-posterior density $\log f(\mu, \delta \mid y, R)$ and the routes of the EM and ECM algorithms in parts (b) and (c), each from the starting point $\left(\mu_{0}, \delta_{0}\right)=(10,1)$ to the mode, $(\hat{\mu}, \hat{\delta})=(20.08,9.72)$. Also shown are two other pairs of routes, one pair starting from $(5,30)$, and the other from $(35,20)$.

Note 1: In this exercise there is little difference between the EM and ECM algorithms, both as regards complexity and performance. In more complex models we may expect the EM algorithm to converge faster but have an M-Step which is more difficult to complete than the set of separate Conditional Maximisation-Steps (CM-Steps) of the ECM algorithm.

Note 2: The log-posterior density in Figure 4.8 has a formula which can be derived as follows. First, the joint posterior of all unknowns in the model is

$$
\begin{gathered}
f(\mu, \delta, R \mid y) \propto f(\mu, \delta, y, R) \\
\propto 1 \times \prod_{i=1}^{n} \pi^{R_{i}}(1-\pi)^{1-R_{i}} \times \prod_{i=1}^{n} \exp \left\{-\frac{1}{2 \sigma^{2}}\left(y_{i}-\left[\mu+R_{i} \delta\right]\right)^{2}\right\}
\end{gathered}
$$




$$
=\prod_{i=1}^{n} \pi^{R_{i}}(1-\pi)^{1-R_{i}} \exp \left\{-\frac{1}{2 \sigma^{2}}\left(y_{i}-\left[\mu+R_{i} \delta\right]\right)^{2}\right\} .
$$

So the joint posterior density of just $\mu$ and $\delta$ is

$$
\begin{aligned}
& f(\mu, \delta \mid y)= \sum_{R} f(\mu, \delta, R \mid y) \\
& \propto \prod_{i=1}^{n} \sum_{R_{i}=0}^{1} \pi^{R_{i}}(1-\pi)^{1-R_{i}} \exp \left\{-\frac{1}{2 \sigma^{2}}\left(y_{i}-\left[\mu+R_{i} \delta\right]\right)^{2}\right\} \\
&=\prod_{i=1}^{n}\left(\pi \exp \left\{-\frac{1}{2 \sigma^{2}}\left(y_{i}-[\mu+\delta]\right)^{2}\right\}+(1-\pi) \exp \left\{-\frac{1}{2 \sigma^{2}}\left(y_{i}-\mu\right)^{2}\right\}\right) .
\end{aligned}
$$

So the log-posterior density of $\mu$ and $\delta$ is

$$
\begin{aligned}
l(\mu, \delta) \equiv \log f(\mu, \delta \mid y) & \\
=c+\sum_{i=1}^{n} \log (\pi \exp \{ & \left.-\frac{1}{2 \sigma^{2}}\left(y_{i}-[\mu+\delta]\right)^{2}\right\} \\
& \left.+(1-\pi) \exp \left\{-\frac{1}{2 \sigma^{2}}\left(y_{i}-\mu\right)^{2}\right\}\right),
\end{aligned}
$$

where $c$ is an additive constant and can arbitrarily be set to zero.

Note 3: As an additional exercise (and a check on our calculations above), we could apply the Newton-Raphson algorithm so as to find the mode of $l(\mu, \delta)$. But this would require us to first determine formulae for the following rather complicated partial derivatives:

$$
\frac{\partial l(\mu, \delta)}{\partial \mu}, \frac{\partial l(\mu, \delta)}{\partial \delta}, \frac{\partial^{2} l(\mu, \delta)}{\partial \mu^{2}}, \frac{\partial^{2} l(\mu, \delta)}{\partial \delta^{2}}, \frac{\partial^{2} l(\mu, \delta)}{\partial \delta \partial \mu},
$$

and could prove to be unstable. That is, the algorithm might fail to converge if started from a point not very near the required solution.

Another option is to apply the CNR algorithm (the conditional NewtonRaphson algorithm). This would obviate the need for one of the derivatives above, $\frac{\partial^{2} l(\mu, \delta)}{\partial \delta \partial \mu}$, and might be more stable, albeit at the cost of not converging so quickly as the plain NR algorithm. 
As yet another possibility, we could apply the CNR1 algorithm. This is the same as the CNR algorithm, except that at each conditional step we perform just one iteration of the univariate NR algorithm before moving on to the other of the two conditional steps.

Finally, we could use the $\mathrm{R}$ function optim() to maximise $l(\mu, \delta)$. Although this function will be formally introduced later, we can report that it does indeed find the posterior mode, $(\hat{\mu}, \hat{\delta})=(20.08,9.72)$. For details, see the bottom of the R code below.

\section{Table 4.5 Results of an ECM algorithm}

\begin{tabular}{r|cc}
$j$ & $\mu_{j}$ & $\delta_{j}$ \\
\hline 0 & 10.000 & 1.000 \\
1 & 22.505 & 1.696 \\
2 & 22.566 & 3.882 \\
3 & 21.905 & 6.811 \\
4 & 21.139 & 8.729 \\
5 & 20.611 & 9.501 \\
6 & 20.322 & 9.732 \\
7 & 20.181 & 9.774 \\
8 & 20.118 & 9.764 \\
9 & 20.093 & 9.746 \\
10 & 20.085 & 9.732 \\
11 & 20.083 & 9.725 \\
12 & 20.083 & 9.720 \\
13 & 20.083 & 9.719 \\
14 & 20.084 & 9.718 \\
15 & 20.084 & 9.718 \\
16 & 20.084 & 9.718 \\
17 & 20.084 & 9.718 \\
18 & 20.084 & 9.718 \\
19 & 20.084 & 9.718 \\
20 & 20.084 & 9.718 \\
\hline
\end{tabular}


Figure 4.8 Routes of the EM and ECM algorithms

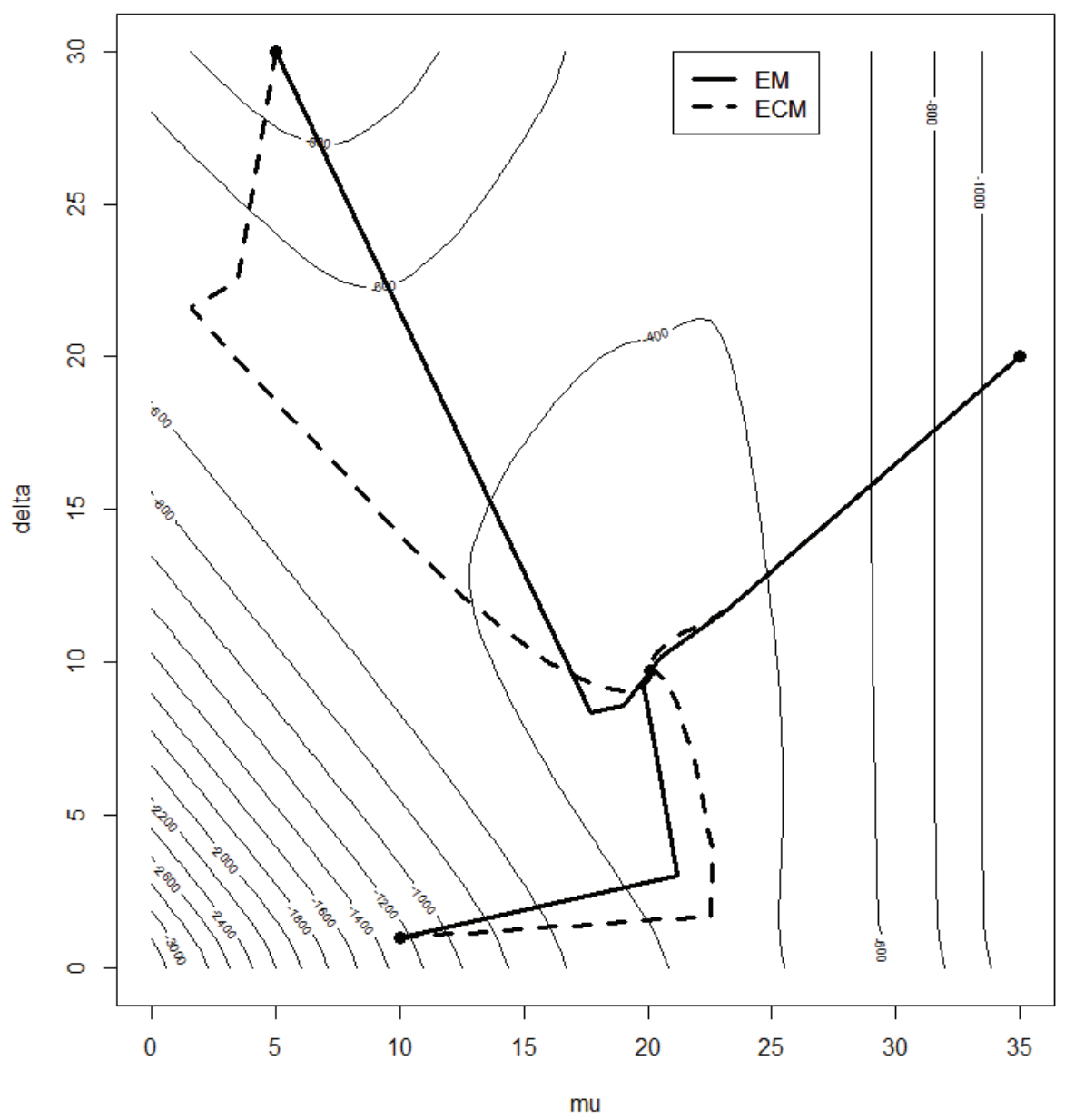

\section{R Code for Exercise 4.7}

\# (a)

$\mathrm{X} 11(\mathrm{w}=8, \mathrm{~h}=4.5)$; $\operatorname{par}(\mathrm{mfrow}=\mathrm{c}(1,1))$; options(digits=4)

ntrue $=100 ;$ pitrue $=1 / 3$; mutrue $=20 ;$ deltrue $=10 ;$ sigtrue $=3$

set.seed(512); Rvec=rbinom(ntrue,1, pitrue); sum(Rvec) \# 29

yvec=rnorm (ntrue, mutrue+deltrue* Rvec,sigtrue)

ybar=mean(yvec); ybar \# 23.16

hist(yvec, prob=T, breaks=seq $(0,50,0.5), x \lim =c(10,40), y \lim =c(0,0.2)$,

$$
\text { xlab="y", main=" ") }
$$


$\mathrm{yv}=\operatorname{seq}(0,50,0.01)$; lines(yv,dnorm(yv, mutrue, sigtrue), Ity=2, Iwd=2)

lines(yv,dnorm(yv, mutrue+deltrue, sigtrue), Ity=2,lwd=2)

lines(yv, (1-pitrue)*dnorm(yv,mutrue,sigtrue)+ pitrue*dnorm(yv, mutrue+deltrue,sigtrue), Ity=1,lwd=2)

legend(10,0.2,c("Components", "Mixture"), Ity=c(2,1), Iwd=c(2,2))

\# (b)

evalsfun= function( $y=y v e c$, pii=pitrue, $m u=$ mutrue,del=deltrue,sig=sigtrue $)\{$

\# This function outputs (e1,e2,...,en)

term1vals=pii*dnorm(y,mu+del,sig)

term0vals $=(1-$ pii $) *$ dnorm(y,mu,sig)

term1vals/(term1vals+term0vals) \}

EMfun=function $(\mathrm{J}=20, \mathrm{mu}=10$, del=1, $\mathrm{y}=\mathrm{yvec}, \mathrm{pii}=$ pitrue, sig=sigtrue $)\{$ muv=mu; delv=del; ybar=mean $(y) ; n=l e n g t h(y)$

for $(\mathrm{j}$ in $1: J)\{$

evals=evalsfun( $y=y, p i i=p i i, m u=m u$, del=del, sig=sig)

sumyevals = sum( $\left(y^{*}\right.$ evals $) ;$ sumevals=sum(evals $)$

mu=(ybar-sumyevals/n) / (1-sumevals/n)

del=sumyevals/sumevals - mu

muv $=c(m u v, m u) ;$ delv $=c($ delv, del $)$

\}

list (muv=muv, delv=delv)

\}

EMres=EMfun(J=20, mu=10, del=1,y=yvec, pii=pitrue, sig=sigtrue)

outmat $=$ cbind $(0: 20$, EMres\$muv, EMres\$delv $)$

print.matrix <- function $(m)\{\quad$ write.table(format(m, justify="right"),

print.matrix(outmat) row.names $=F$, col.names $=F$, quote $=F$ ) \}

\# 0.00010 .0001 .000

\# 1.00021 .1693 .032

\# 2.00020 .3217 .070

\# 3.00019 .8439 .139

\# 4.00019 .9269 .518

\# 5.00020 .0059 .626

$\#$

\# 16.00020 .0849 .718

\# 17.00020 .0849 .718

\# 18.00020 .0849 .718

\# 19.00020 .0849 .718

\# 20.00020 .0849 .718

muhat=EMres\$muv[21]; delhat=EMres\$delv[21];

c(muhat,delhat) \# 20.0849 .718 
\# (c)

CEMfun=function $(J=20, m u=10$, del=1, y=yvec, pii=pitrue, sig=sigtrue $)\{$ muv=mu; delv=del; $y \operatorname{bar}=\operatorname{mean}(\mathrm{y}) ; \mathrm{n}=\operatorname{length}(\mathrm{y})$

for $(j$ in $1: J)\{$

evals=evalsfun( $y=y$, pii=pii, mu=mu, del=del, sig=sig)

sumyevals = sum( $\mathrm{y}^{*}$ evals); sumevals=sum(evals)

mu=ybar-del*sumevals/n

del=sumyevals/sumevals - mu

muv $=c($ muv, mu $) ;$ delv $=c($ delv, del $)$

\}

list (muv=muv, delv=delv)

\}

CEMres=CEMfun( $(\mathrm{J}=20, \mathrm{mu}=10, \mathrm{del}=1, \mathrm{y}=\mathrm{yvec}, \mathrm{pii}=$ pitrue, sig=sigtrue $)$

outmat2 = cbind(0:20, CEMres\$muv, CEMres\$delv)

print.matrix(outmat2)

\# 0.00010 .0001 .000

\# 1.00022 .5051 .696

\# 2.00022 .5663 .882

\# 3.00021 .9056 .811

\# 4.00021 .1398 .729

\# 5.00020 .6119 .501

\#

\# 16.00020 .0849 .718

\# 17.00020 .0849 .718

\# 18.00020 .0849 .718

\# 19.00020 .0849 .718

\# 20.00020 .0849 .718

\# (d)

$\mathrm{X} 11(\mathrm{w}=8, \mathrm{~h}=9) ; \operatorname{par}(\operatorname{mfrow}=\mathrm{c}(1,1))$

logpostfun=function (mu=10, del=10,y=yvec, pii=pitrue, sig=sigtrue) \{ $\operatorname{sum}(\log ($ pii*dnorm(y,mu+del,sig)+(1-pii)*dnorm(y,mu,sig) $))\}$

mugrid $=\operatorname{seq}(0,35,0.5)$; delgrid $=\operatorname{seq}(0,30,0.5)$

logpostmat=as.matrix(mugrid \%*\% t(delgrid))

$\operatorname{dim}$ (logpostmat) \# 4121 OK

for( $\mathrm{i}$ in 1:length(mugrid $)$ ) for( $\mathrm{j}$ in 1 :length(delgrid $)$ ) logpostmat $[\mathrm{i}, \mathrm{j}]=$ logpostfun(mu=mugrid[i],del=delgrid[j],y=yvec, pii=pitrue, sig=sigtrue)

contour( $x=$ mugrid, $y=$ delgrid, $z=$ logpostmat, nlevels=20, xlab="mu", ylab="delta"); points(muhat,delhat, pch=16,cex=1.2)

points $(10,1$, pch=16,cex=1.2) 
EMres=EMfun(J=20, mu=10, del=1,y=yvec, pii=pitrue, sig=sigtrue)

CEMres=CEMfun(J=20, mu=10, del=1,y=yvec, pii=pitrue, sig=sigtrue $)$

lines(EMres\$muv, EMres\$delv, Ity=1, Iwd=3)

lines(CEMres\$muv, CEMres\$delv,lty=2,lwd=3)

points(5,30,pch=16, cex=1.2)

EMres=EMfun( $(\mathrm{J}=50, \mathrm{mu}=5, \mathrm{del}=30, \mathrm{y}=\mathrm{yvec}, \mathrm{pii}=$ pitrue, sig=sigtrue $)$

CEMres=CEMfun( $\mathrm{J}=50, \mathrm{mu}=5$, del=30, y=yvec, pii=pitrue, sig=sigtrue)

lines(EMres\$muv, EMres\$delv, Ity=1,lwd=3)

lines(CEMres\$muv, CEMres\$delv,lty=2,lwd=3)

points(35,20,pch=16, cex=1.2)

EMres=EMfun(J=50, mu=35, del=20,y=yvec, pii=pitrue, sig=sigtrue)

CEMres=CEMfun( $\mathrm{J}=50, \mathrm{mu}=35, \mathrm{del}=20, \mathrm{y}=\mathrm{yvec}$, pii=pitrue, sig=sigtrue $)$

lines(EMres\$muv, EMres\$delv, Ity=1,lwd=3)

lines(CEMres\$muv, CEMres\$delv,lty=2,lwd=3)

legend(21,30,c("EM","ECM"), Ity=c(1,2), Iwd=c(3,3))

\# Note 2. Maximisation of the logposterior density of mu and delta using optim() logpostfun2=function(theta $=c(10,1), y=y v e c, p i i=$ pitrue, sig=sigtrue $)\{$

-sum(log(pii*dnorm(y,theta[1]+theta[2],sig)+

(1-pii)*dnorm(y,theta[1],sig)))

\}

res=optim(par=c(10,1),fn= logpostfun2)\$par; res \# 20.089 .72

res=optim(par=c(5,30),fn= logpostfun2)\$par; res \# 20.0859 .716

res=optim(par=c(35,20),fn=logpostfun2)\$par; res \# 20.0849 .716

res=optim(par=res,fn= logpostfun2)\$par; res \# 20.0849 .718

\# Here we fine-tune the answer by starting at the previous solution.

\subsection{Integration techniques}

Bayesian inference typically involves a great deal of integration (and/or summation). For example, consider the posterior density

$$
f(\theta \mid y)=6 \theta^{5}, 0<\theta<1
$$

(which featured in previous exercise involving the binomial-beta model) and suppose that we wish to find the posterior mean estimate of $\lambda=\theta^{2}$. This estimate is

$$
\hat{\lambda}=E\left(\theta^{2} \mid y\right)=\int_{0}^{1} \theta^{2} \times\left(6 \theta^{5}\right) d \theta=0.75 .
$$

But what if this integral did not have a simple analytical solution? 
In that case, we could consider a number of other strategies. First, we might re-express the posterior mean as

$$
\hat{\lambda}=\int \lambda f(\lambda \mid y) d \lambda
$$

where, using the method of transformation,

$$
f(\lambda \mid y)=f(\theta \mid y)\left|\frac{d \theta}{d \lambda}\right|=6\left(\lambda^{1 / 2}\right)^{5}\left|\frac{1}{2} \lambda^{-1 / 2}\right|=3 \lambda^{2}, \quad 0<\lambda<1,
$$

so that

$$
\hat{\lambda}=\int_{0}^{1} \lambda\left(3 \lambda^{2}\right) d \lambda=0.75 .
$$

If this strategy does not help, we may then consider using a numerical integration technique.

For example, we could apply the integrate() function in $\mathrm{R}$ to get $\hat{\lambda}=0.75$, as follows:

gfun $=$ function $(t)\left\{6^{*} t^{\wedge} 7\right\}$ \# Define the function to be integrated integrate $(\mathrm{f}=$ gfun, lower=0, upper $=1) \$$ value \# 0.75

In some cases the function requiring integration is very complicated or does not have a closed form expression. In that case, direct application of the integrate() function may not work or be practicable, and then it may be useful to apply the trapezoidal rule or Simpson's rule to evaluate the integral.

When working in $\mathrm{R}$, the following is often a convenient strategy:

(i) evaluate $g(\theta)=\theta^{2} \times 6 \theta^{5}$ at each $\theta$ on the grid $0,0.1,0.2, \ldots, 0.9,1$ (say); then

(ii) create a spline through these points, using the fit() and predict() functions; and then

(iii) find the area under this spline using the integrate() function.

Applying this method (see the R code below for details) yields 0.7558 as an estimate of $\hat{\lambda}$. Repeating, but with the evaluations on the grid 0.01 , $0.02, \ldots, 1$ yields 0.7500 . Repeating again, but with evaluations on the grid $0.001,0.002, \ldots, 1$ yields 0.7500 . It appears that a limit has been reached and that using a finer grid would not result in any improvements to the results of this numerical procedure.

We may conclude that $\hat{\lambda}=0.7500$ (to 4 decimals). 


\section{R Code for Section 4.6}

gfun = function(t) $\left\{6^{*} \mathrm{t}^{\wedge} 7\right\}$ \# Define the function to be integrated integrate $(\mathrm{f}=$ gfun,lower=0,upper=1)\$value \# 0.75

INTEG <- function(xvec, yvec, $a=\min (x v e c), b=\max (x v e c))\{$ \# Integrates numerically under a spline through the \# points given by the vectors $x v e c$ and yvec, from $a$ to $b$.

fit <- smooth.spline(xvec, yvec) spline.f <- function( $x)\{$ predict(fit, $x) \$ y$ \} integrate(spline.f, $a, b) \$$ value \}

gfun=function(t) $\left\{6 * \mathrm{t}^{\wedge} 7\right\}$

tvec <- seq $(0,1,0.1) ;$ gvec <- gfun(tvec)

INTEG(tvec,gvec,0,1) \# 0.755803

tvec <- seq(0,1,0.01); gvec <- gfun(tvec)

INTEG(tvec,gvec,0,1) \# 0.75

tvec <- seq(0,1,0.001); gvec <- gfun(tvec)

INTEG(tvec,gvec,0,1) \# 0.75

\section{Exercise 4.8 Numerical integration}

Suppose that $X \sim N\left(\mu, \sigma^{2}\right)$ and $Y=(X \mid X>c)$ where $\mu=8, \sigma=3$ and $c=10$. Find $E Y$ using numerical techniques and compare your answer with the exact value,

$$
\mu+\sigma \phi\left(\frac{c-\mu}{\sigma}\right) /\left\{1-\Phi\left(\frac{c-\mu}{\sigma}\right)\right\},
$$

which was derived analytically in Exercise 4.6.

\section{Solution to Exercise 4.8}

The required integral is

$$
E Y=\int_{c}^{\infty} g(x) d x,
$$

$$
\begin{aligned}
& \text { where: } \quad g(x)=\frac{x f(x)}{P(X>0)} \quad, \quad f(x)=\frac{1}{\sigma} \phi\left(\frac{x-\mu}{\sigma}\right) \\
& P(X>0)=1-\Phi\left(\frac{c-\mu}{\sigma}\right) .
\end{aligned}
$$

Applying the integrate() function directly to $g(x)$ we get $E Y=11.7955$. 
Applying the INTEG() function (defined in Section 4.6) with coordinates given by $(10,10.1,10.2, \ldots, 30)$ and $(g(10), g(10.1), g(10.2), \ldots, g(30))$, we also get $E Y=11.7955$. The exact value of $E Y$ is in fact

$$
\mu+\sigma \phi\left(\frac{c-\mu}{\sigma}\right) /\left\{1-\Phi\left(\frac{c-\mu}{\sigma}\right)\right\}=11.7955
$$

Note: If we use the integrate() function with bounds from 10 to 20 rather than 10 to 30, we get 11.7929, which is slightly in error. Exactly the same happens with the INTEG() function. Thus, when using either of these functions, care must be taken to choose a large enough range. Ideally, we will sketch the integrand function and make sure the range of integration is sufficiently broad to cover all important regions (where the integrand is significantly positive). In practice, it is useful to gradually increase the range of integration until the answer stops changing. Likewise, it is useful to gradually increase the grid density chosen for the INTEG() function until the answer stops changing.

\section{R Code for Exercise 4.8}

\# First declare the function INTEG() as defined in the previous exercise

$m u=8 ;$ sig=3; c = 10; options (digits $=6$ )

PXpos $=(1-$ pnorm $((c-m u) /$ sig $))$

gfun=function $(x)\{x *$ dnorm(x,mu,sig) / PXpos $\}$

integrate(gfun,c,20)\$value \# 11.7929

integrate(gfun,c,30)\$value \# 11.7955

xvec <- seq(c,20,0.1); gvec<- gfun(xvec); INTEG(xvec,gvec,c,20) \#11.7929

xvec <- seq(c,30,0.1); gvec <- gfun(xvec); INTEG(xvec,gvec,c,30) \#11.7955

true=mu + sig*dnorm ((c-mu)/sig)/(1-pnorm((c-mu)/sig)); true \# 11.7955

\section{Exercise 4.9 Double integration}

Use the integrate() and INTEG() functions in at least two different ways so as to calculate the double integral

$$
I=\int_{x=0}^{1}\left(\int_{t=0}^{x^{3}} t^{t} d t\right) d x .
$$

Illustrate your calculations with suitable graphs of the relevant functions involved. 


\section{Solution to Exercise 4.9}

Using the integrate() function alone (and not the INTEG() function), the integral can be worked out as follows:

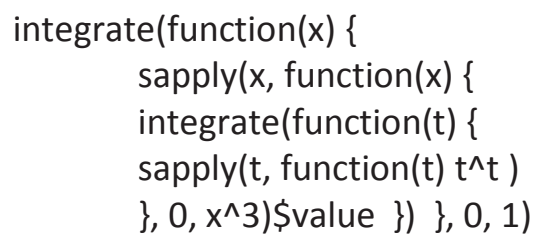

\# 0.192723 with absolute error $<7.8 \mathrm{e}-10$

Another approach is as follows. Observe that

$$
I=\int_{x=0}^{1} g(x) d x,
$$

where

$$
g(x)=\int_{t=0}^{x^{3}} h(t) d t
$$

and

$$
h(t)=t^{t} .
$$

We will now use the integrate() function to obtain $g(x)$ for each value of $x$ in the grid $0,0.01,0.02, \ldots, 1$. We will then apply the INTEG() function to the resulting coordinates.

Figure 4.9 below displays the two functions $h(t)$ and $g(x)$. The value $g(0.8)=0.381116$ is the area under $h(t)$ between 0 and 0.8 . The total area under $h(t)$ (from 0 to 1 ) is 0.78343 .

The total area under $g(x)$ (from 0 to 1 ) is estimated as 0.192723 . Using the grid $0,0.001,0.002, \ldots, 1$ also leads to 0.192723 , whereas using the grid $0,0.1,0.2, \ldots, 1$ leads to 0.193054 .

We conclude that the exact value of the required integral $I$ to 4 decimals is 0.1927, which is in agreement with the first approach above which doesn't make use of the INTEG() function. 
One could also adapt the second approach above so as to calculate the double integral using the INTEG() function only (without using the integrate() function directly). This might be useful if the inner integral

$$
g(x)=\int_{t=0}^{x^{3}} h(t) d t \quad \text { where } h(t)=t^{t}
$$

could not be evaluated easily using integrate() directly, for example if $h(t)$ were a very complicated function which could not be expressed in closed form.

Note: The integrate() function is called within the INTEG() function and so is used at least indirectly in all of the approaches considered here.

\section{Figure 4.9 Two functions}
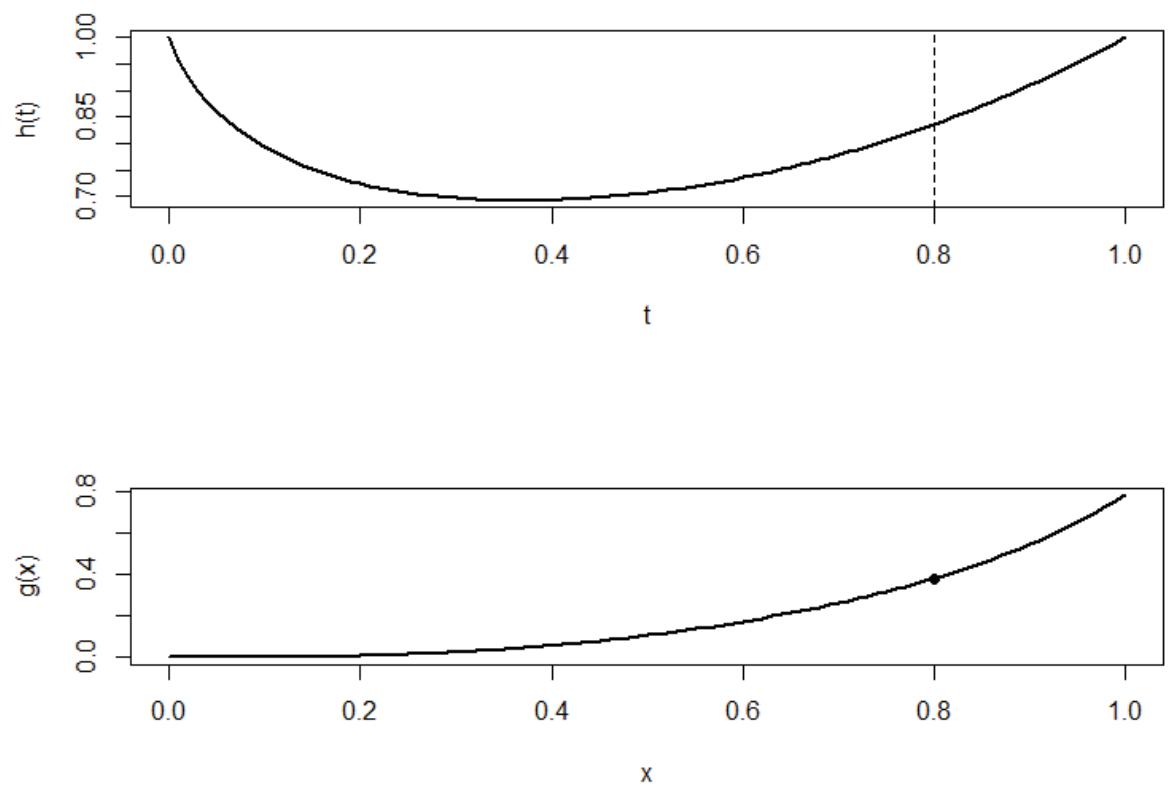

\section{R Code for Exercise 4.9}

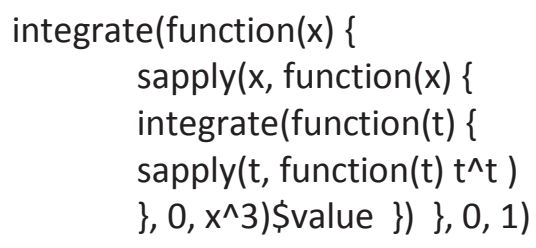

\# 0.192723 with absolute error $<7.8 \mathrm{e}-10$ 
\# Declare the function INTEG() as defined in the previous exercise options(digits=6); $\mathrm{X} 11(\mathrm{w}=8, \mathrm{~h}=6) ; \operatorname{par}(\operatorname{mfrow}=\mathrm{c}(2,1))$

hfun $=$ function $(t)\left\{t^{\wedge} t\right\}$

tvec=seq $(0,1,0.01)$; hvec=hfun(tvec)

plot(tvec,hvec,type="l",xlab="t",ylab="h(t)",Iwd=2); abline(v=0.8,Ity=2)

integrate $\left(\mathrm{f}=\mathrm{hfun}\right.$,lower $=0$, upper $\left.=0.8^{\wedge} 3\right) \$$ value

$\# 0.381116$ This is $g(0.8)=$ area under $h(t)$ to left of 0.8

integrate( $\mathrm{f}=\mathrm{hfun}$, lower=0, upper=1)\$value

\# 0.78343 This is the total areas under $\mathrm{h}(\mathrm{t})$ (from 0 to 1 )

$x v e c=\operatorname{seq}(0,1,0.01) ;$ gvec $=\operatorname{rep}(N A$,length $(x v e c))$

for $(i$ in 1 :length $(x v e c))\{x v a l=x v e c[i]$

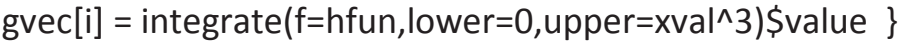

INTEG(xvec,gvec) \# 0.192723

plot(xvec,gvec,type="l",xlab="x",ylab="g(x)",Iwd=2)

points $(0.8,0.381116$, pch=16, cex $=1)$

\# Apply INTEG() using different grids

$x v e c=\operatorname{seq}(0,1,0.001) ;$ gvec $=\operatorname{rep}(N A$,length $(x v e c))$

for $(i$ in 1:length $(x v e c))\{x v a l=x v e c[i]$

gvec $[\mathrm{i}]=$ integrate $(\mathrm{f}=\mathrm{hfun}$, lower=0,upper=xval^3) \$value $\}$

INTEG(xvec,gvec) \# 0.192723

$x v e c=\operatorname{seq}(0,1,0.1) ;$ gvec $=\operatorname{rep}(N A$, length $(x v e c))$

for $(i$ in 1:length $(x v e c))\{x v a l=x v e c[i]$

gvec $[\mathrm{i}]=$ integrate $(\mathrm{f}=\mathrm{hfun}$, lower=0,upper=xval^3) \$value $\}$

INTEG(xvec,gvec) \# 0.193053

\subsection{The optim() function}

The function optim() in $\mathrm{R}$ is a very useful and versatile tool for maximising or minimising functions, both of one and of several variables.

This R function can also be adapted for solving single or simultaneous equations and provides an alternative to other techniques such as trial and error, the Newton-Raphson algorithm and the EM algorithm.

The second of the next two exercises shows how the optim() function can be used to specify a prior distribution. 


\section{Exercise 4. 10 Simple examples of the optim() function}

Use the optim() function to 'find' the mode of each of the following:

(a) $g(x)=x^{2} e^{-5 x}, x>0 \quad($ mode $=2 / 5)$

(b) $g(x)=\frac{|x|^{x} e^{-(x-1)^{2}}}{1+|x|}, x \in \Re \quad$ (the mode has no closed form)

(c) $g(x, y)=y^{3} e^{-y\left\{(x-1)^{2}+(x-3)^{2}\right\}}, x \in \mathfrak{R}, y>0$

$$
\text { (mode }=(x, y)=((1+3) / 2,3 / 2)) \text {. }
$$

\section{Solution to Exercise 4.10}

In each of these cases, the optim() function (which minimises a function by default) may be applied to the negative of the specified function (so as to maximise that function).

(a) The function correctly returns $x=2 / 5$. (NB: The warning message may be ignored.)

(b) The function returns a value of 1.5047. (We presume that this is correct; see below for a verification.)

(c) The mode is correctly computed as $(x, y)=(2,1.5)$. (Note that this solution is obvious by analogy with maximum likelihood estimation of the normal mean and variance.)

Figure 4.10 illustrates these three solutions, with each mode being marked by a dot and vertical line. Subplot (c) shows several examples of the function $g(x, y)$ in part (c) considered as a function of only $x$, with each line defined by a fixed value of $y$ on the grid $0,0.5,1, \ldots, 4.5,5$. 
Figure 4. 10 Maximisation of function $g$ in parts (a), (b) and (c)
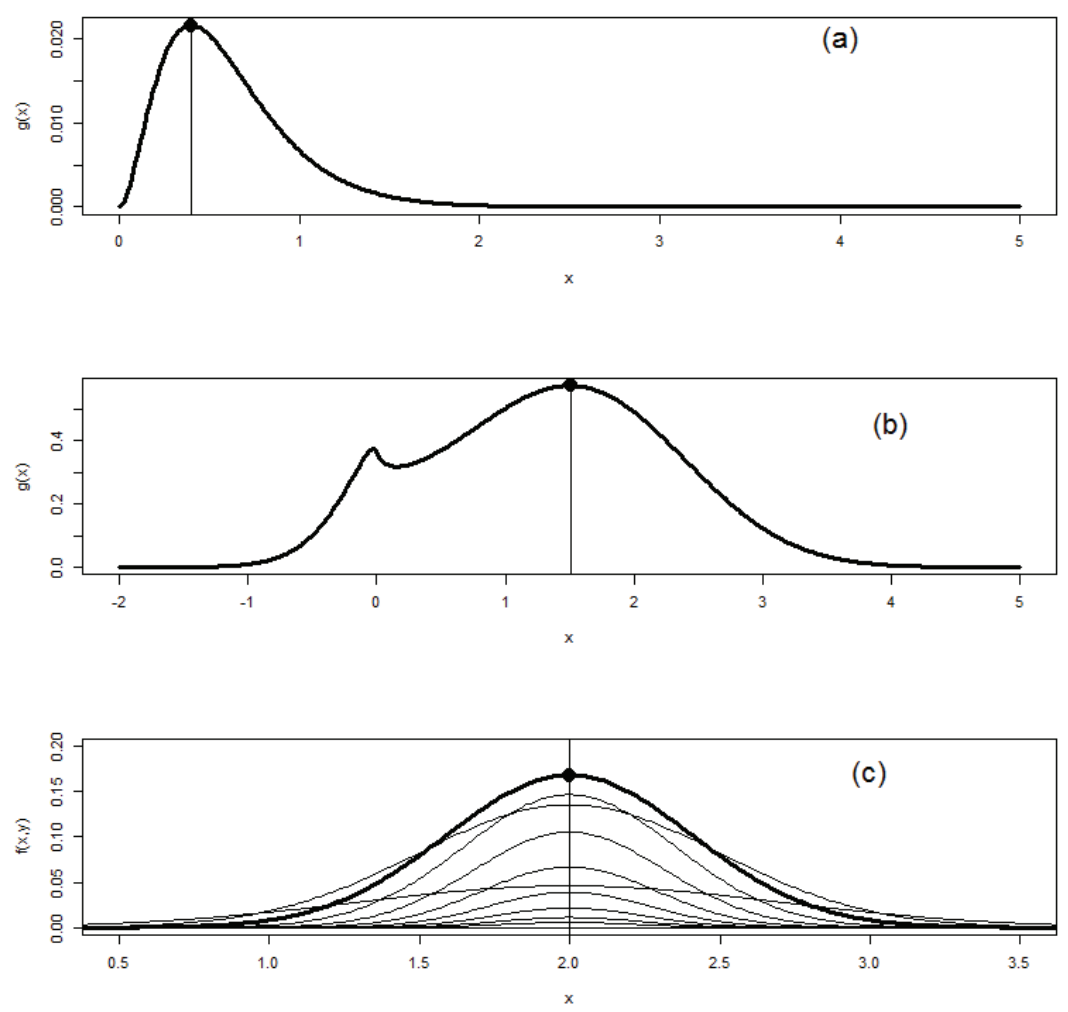

\section{R Code for Exercise 4.10}

help(optim); options(digits=5); $\mathrm{X} 11(\mathrm{w}=8, \mathrm{~h}=8) ; \operatorname{par}(\operatorname{mfrow}=\mathrm{c}(3,1))$

\# (a)

fun=function $(x)\left\{-x^{\wedge} 2 * \exp (-5 * x)\right\}$

res0=optim(par=0.5,fn=fun)\$par; res0 \# 0.4

\# Warning message:

\# In optim(par = 0.5, fn = fun) :

\# one-diml optimization by Nelder-Mead is unreliable:

\# use "Brent" or optimize() directly

plot(seq(0,5,0.01), -fun(seq( $0,5,0.01))$, type="l",Iwd=3,xlab="x",ylab="g(x)");

abline(v=res0); points(res0, -fun(res0), pch=16, cex=2); text(4,0.02,"(a)",cex=2)

\# (b)

fun=function $(x)\left\{-\exp \left(-(x-1)^{\wedge} 2\right)^{*} \operatorname{abs}(x)^{\wedge} x /(1+\operatorname{abs}(x))\right\}$

res $0=$ optim(par=1,fn=fun)\$par; res0 \# 1.5047

plot(seq(-2,5,0.01), -fun(seq(-2,5,0.01)), type="l",Iwd=3, xlab="x",ylab="g(x)");

abline(v=res0); points(res0, -fun(res0), pch=16, cex=2); text(4,0.45,"(b)",cex=2) 
\# (c)

fun=function $(v)\left\{-v[2]^{\wedge} 3 * \exp \left(-v[2] *\left((v[1]-1)^{\wedge} 2+(v[1]-3)^{\wedge} 2\right)\right)\right\}$

res0=optim(par=c(2,2),fn=fun, lower $=c(-\operatorname{Inf}, 0)$, upper $=c(\operatorname{Inf}, \operatorname{Inf})$, method = "L-BFGS-B")\$par; res0 \# 2.01 .5

fun2=function $(x, y)\left\{y^{\wedge} 3 * \exp \left(-y *\left((x-1)^{\wedge} 2+(x-3)^{\wedge} 2\right)\right)\right\}$

$\operatorname{plot}(c(0.5,3.5), c(0,0.2)$, type="n",xlab="x",ylab=" $f(x, y) ")$

for(y in seq $(0,5,0.5))$

lines(seq $(0,5,0.01)$, fun $2(x=\operatorname{seq}(0,5,0.01), y=y)$, Ity=1)

abline(v=res0[1]); points(res0[1],fun2(res0[1],res0[2]), pch=16, cex=2);

lines(seq $(0,5,0.01)$, fun2 $(x=\operatorname{seq}(0,5,0.01), y=\operatorname{res} 0[2])$, Ity=1,lwd=3);

$\operatorname{text}(3,0.17, "(c) ", \operatorname{cex}=2)$

\section{Exercise 4. I I Specification of parameters in a prior distribution using the optim() function}

Consider the normal-gamma model given by:

$$
\begin{aligned}
& \left(y_{1}, \ldots, y_{n} \mid \lambda\right) \sim \text { iid } N(\mu, 1 / \lambda) \\
& \lambda \sim G(\eta, \tau) .
\end{aligned}
$$

Use the optim() function in $\mathrm{R}$ to find the values of $\eta$ and $\tau$ which correspond to a prior belief that the population standard deviation $\sigma=1 / \sqrt{\lambda}$ lies between 0.5 and 1 with 95\% probability, and that $\sigma$ is equally likely to be below 0.5 as it is to be above 1 .

\section{Solution to Exercise 4.I I}

We wish to find the values of $\eta$ and $\tau$ which satisfy the two equations:

$$
P(\sigma<a)=\alpha / 2 \quad \text { and } P(\sigma<b)=1-\alpha / 2,
$$

where $a=0.5, b=1$ and $\alpha=0.05$.

These two equations are together equivalent to each of the following five pairs of equations:

$$
\begin{array}{lll}
P\left(\sigma^{2}<a^{2}\right)=\alpha / 2 & \text { and } & P\left(\sigma^{2}<b^{2}\right)=1-\alpha / 2 \\
P\left(1 / \lambda<a^{2}\right)=\alpha / 2 & \text { and } & P\left(1 / \lambda<b^{2}\right)=1-\alpha / 2 \\
P\left(1 / a^{2}<\lambda\right)=\alpha / 2 & \text { and } & P\left(1 / b^{2}<\lambda\right)=1-\alpha / 2 \\
P\left(\lambda<1 / a^{2}\right)=1-\alpha / 2 & \text { and } & P\left(\lambda<1 / b^{2}\right)=\alpha / 2 \\
F_{G(\eta, \tau)}\left(1 / a^{2}\right)-(1-\alpha / 2)=0 & \text { and } & F_{G(\eta, \tau)}\left(1 / b^{2}\right)-\alpha / 2=0 .
\end{array}
$$


We now focus on the last of these pairs of two equations. Two obvious ways to solve these equations are by trial and error and via the multivariate Newton-Raphson algorithm, as illustrated earlier. But the solution can be obtained more easily by using the optim() function to minimise

$$
g(\eta, \tau)=\left[F_{G(\eta, \tau)}\left(1 / a^{2}\right)-(1-\alpha / 2)\right]^{2}+\left[F_{G(\eta, \tau)}\left(1 / b^{2}\right)-(\alpha / 2)\right]^{2} .
$$

Note: Clearly, this function has a value of zero at the required values of $\eta$ and $\tau$.

With the default settings and starting at $\eta=0.2$ and $\tau=6$, optim() produced some warning messages (which we ignored) and provided the solution, $\eta=8.4764$ and $\tau=3.7679$.

Now, this solution is not exactly correct, because the probabilities of a Gamma(8.4764, 3.7679) random variable lying below $1 / b^{2}=1$ and below $1 / a^{2}=4$, respectively, are 0.025048 and 0.975104 (i.e. not exactly 0.025 and 0.975 as desired).

However, applying the optim() function again but starting at the previous solution, namely $\eta=8.4764$ and $\tau=3.7679$, yielded a 'refined' solution, $\eta=8.4748$ and $\tau=3.7654$.

This solution may be considered correct, because the probabilities of a Gamma(8.4748, 3.7654) random variable being less than $1 / b^{2}=1$ and less than $1 / a^{2}=4$, respectively, are exactly 0.025 and 0.975 .

\section{Discussion}

It is instructive to derive and plot the corresponding density of the precision parameter $\lambda$, and then to do this also for the variance parameter $\sigma^{2}=\lambda^{-1}$ and the standard deviation parameter $\sigma=\lambda^{-1 / 2}$, respectively.

The three densities are plotted in Figure 4.11 (in the stated order from top to bottom). The vertical lines show the 0.025 and 0.975 quantiles of each distribution. The formulae for the three densities are as follows:

$$
f(\lambda)=f_{G(\eta, \tau)}(\lambda)=\frac{\tau^{\eta} \lambda^{\eta-1} e^{-\tau \lambda}}{\Gamma(\eta)}, \lambda>0
$$




$$
\begin{aligned}
& \begin{aligned}
f\left(\sigma^{2}\right)=f_{I G(\eta, \tau)}\left(\sigma^{2}\right)=f(\lambda)\left|\frac{d \lambda}{d\left(\sigma^{2}\right)}\right|=f_{G(\eta, \tau)}\left(\lambda=\sigma^{-2}\right)\left|-\left(\sigma^{2}\right)^{-2}\right|, \\
=
\end{aligned} \\
& \begin{aligned}
f(\sigma)=f(\lambda)\left|\frac{d \lambda}{d \sigma}\right| & \left.=f_{G(\eta, \tau)}\left(\lambda=\sigma^{-2}\right)\left|-2 \sigma^{-3}\right| \quad \text { where } \lambda=\left(\sigma^{2}\right)^{-1}\right)^{\eta-1} e^{-\tau\left(1 / \sigma^{2}\right)} \\
\Gamma(\eta) & \text { where } \lambda=(\sigma)^{-2}
\end{aligned} \\
& =\frac{\tau^{\eta}\left(1 / \sigma^{2}\right)^{\eta-1} e^{-\tau\left(1 / \sigma^{2}\right)}}{\Gamma(\eta)} 2 \sigma^{-3}, \sigma>0 .
\end{aligned}
$$

As a check on the last of these three densities, the integrate() function was used to show that the area under that density is exactly 1 , and that the areas underneath it to the left of 0.5 and to the right of 1 are both exactly 0.025 .

\section{Figure 4.I I Three prior densities}
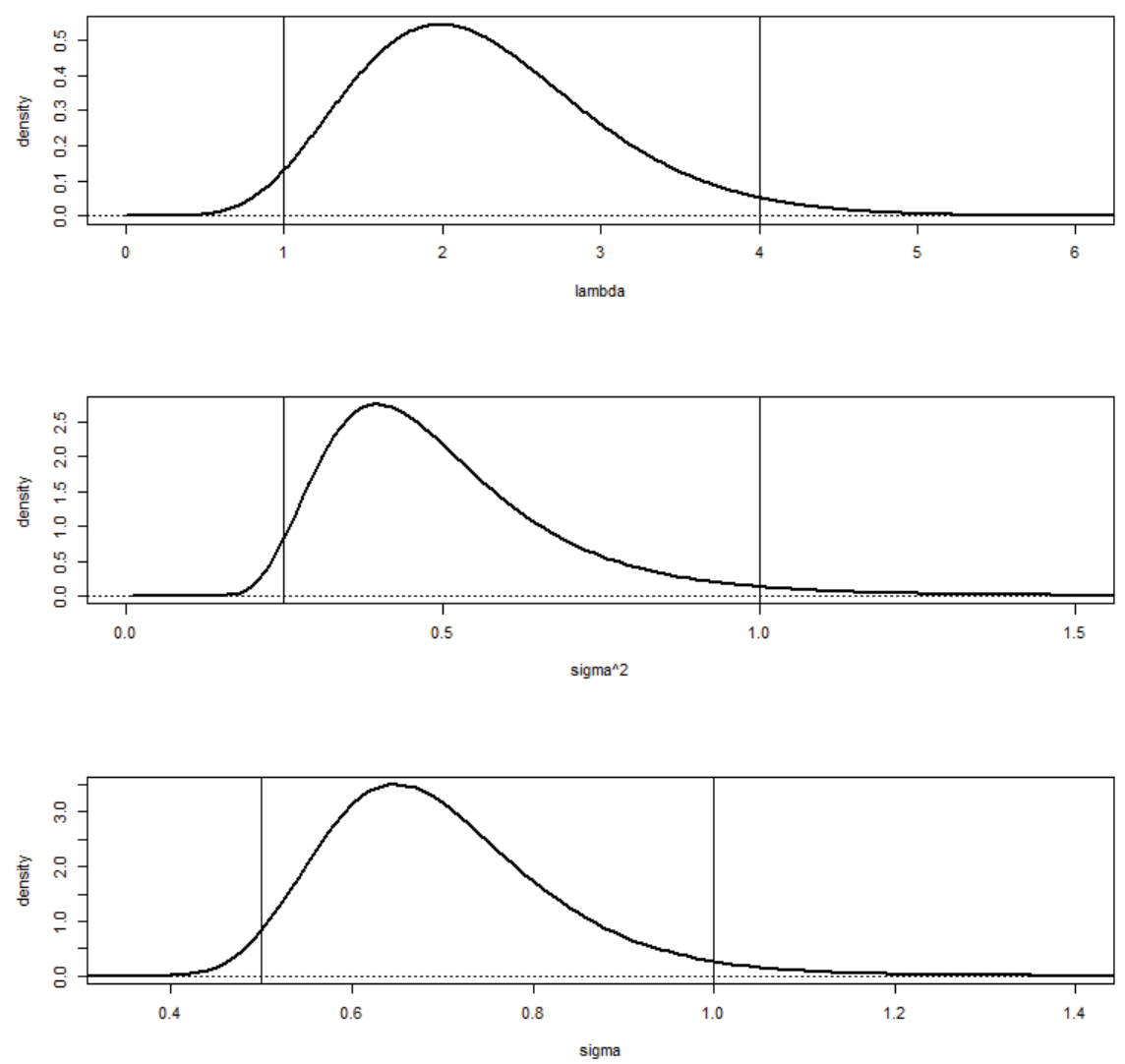


\section{R Code for Exercise 4.I I}

options(digits=5); $a=0.5 ; b=1 ;$ alp $=0.05$;

fun=function $(v, a \mid p=0.05, a=0.5, b=1)\{$

$\left(\text { pgamma }\left(1 / \mathrm{a}^{\wedge} 2, \mathrm{v}[1], \mathrm{v}[2]\right)-(1-\mathrm{alp} / 2)\right)^{\wedge} 2+$

$\left.\left(\text { pgamma }\left(1 / \mathrm{b}^{\wedge} 2, \mathrm{v}[1], \mathrm{v}[2]\right)-(\mathrm{alp} / 2)\right)^{\wedge} 2 \quad\right\}$

res $0=$ optim(par=c $(0.2,6)$, fn=fun $) \$ p$ par

res0 \# 8.47643 .7679

pgamma(c(1/b^2,1/a^2),res0[1],res0[2]) \# 0.0250480 .975104 Close

res=optim(par=res0,fn=fun)\$par; res \# 8.47483 .7654

pgamma(c(1/b^2,1/a^2),res[1],res[2]) \# 0.0250 .975 Correct

res2=optim(par=c(6,3),fn=fun)\$par; res2 \# 8.47533 .7655

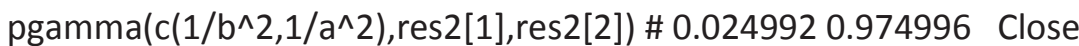

res3=optim(par=res2,fn=fun)\$par; res3 \# 8.47483 .7654

pgamma $\left(c\left(1 / b^{\wedge} 2,1 / a^{\wedge} 2\right)\right.$,res3[1],res3[2]) \# 0.0250 .975 Correct

$\operatorname{par}(\operatorname{mfrow}=c(3,1)) ; \mathrm{tv}=\mathrm{seq}(0,10,0.01)$

plot(tv, dgamma(tv, res[1],res[2]), type="l",Iwd=2, $x \lim =c(0,6)$, xlab="lambda",ylab="density"); abline $\left(v=c\left(1 / a^{\wedge} 2,1 / b^{\wedge} 2\right)\right)$; abline $(\mathrm{h}=0, \mathrm{lty}=3)$

plot(tv,dgamma(1/tv, res[1],res[2])/tv^2, type="l", Iwd=2, xlim=c(0,1.5), xlab="sigma^2",ylab="density");

abline $\left(v=c\left(a^{\wedge} 2, b^{\wedge} 2\right)\right)$; abline $(h=0$, tty $=3)$

plot(tv,dgamma(1/tv^2, res[1],res[2])*2/tv^3, type="l", Iwd=2, $x \lim =c(0.35,1.4), x l a b=" s i g m a ", y l a b=" d e n s i t y ") ;$

abline $(v=c(a, b))$; abline $(h=0$, Ity $=3$ )

\# Check areas under the last curve func=function( $t)\left\{\right.$ dgamma( $\left.\left(1 / \mathrm{t}^{\wedge} 2, \operatorname{res}[1], \operatorname{res}[2]\right) * 2 / \mathrm{t}^{\wedge} 3\right\}$ integrate(func,lower $=0$, upper $=$ Inf) $\$$ value \# 1 Correct integrate(func,lower $=0$,upper $=0.5$ ) $\$$ value \# 0.025 Correct integrate(func,lower=1,upper=Inf)\$value \# 0.025 Correct 


\section{CHAPTER 5}

\section{I Introduction}

The term Monte Carlo (MC) methods refers to a broad collection of tools that are useful for approximating quantities based on artificially generated random samples. These include the Monte Carlo integration (for estimating an integral using such a sample), the inversion technique (for generating the required sample), and Markov chain Monte Carlo methods (an advanced topic in Chapter 6). In principle, the approximation can be made as good as required simply by making the Monte Carlo sample size sufficiently large. As will be seen (further down), Monte Carlo methods are a very useful tool in Bayesian inference.

To illustrate the basic idea of Monte Carlo methods, consider Buffon's needle problem, where a needle of length $10 \mathrm{~cm}$ (say) is dropped randomly onto a floor with parallel lines being distance $10 \mathrm{~cm}$ apart. What is $p$, the probability of the needle crossing a line? The exact value of $p$ can be worked out analytically as $2 / \pi=0.63662$ (this is done in one of the exercises below). But this takes mathematical effort. If this analytical solution were not possible (or just too much work), we could instead estimate $p$ via Monte Carlo. The simplest way to do this would be to toss the needle onto the floor 1,000 times (randomly and independently). If the needle crosses a line 641 times (say), then the Monte Carlo estimate of $p$ is just $641 / 1,000=0.641$.

As a variation on this physical experiment (which could be rather laborious), we could toss the needle 1,000 times virtually, meaning that we simulate each drop (or rather the parameters of each drop) on a computer and each time determine whether the virtual needle has crossed a virtual line.

This method will be faster and more accurate; but it will also require at least some mathematical work to identify exactly what the parameters of each drop are and what configuration of those parameters correspond to the needle crossing a line (again, this is done in one of the exercises below). 
In this chapter, we will first discuss Monte Carlo methods and their usefulness under the assumption that we have available or can generate the required random samples. As we will see in the exercises and their solutions, such samples can often be obtained very easily using inbuilt $\mathrm{R}$ functions, e.g. runif() and rnorm().

After this we will describe special methods for generating a random samples, starting with the simplest, such as the inversion technique and rejection sampling. We reserve the more complicated techniques which involve Markov chain theory to the next and later chapters.

Also, as part of the structure of the present chapter, we will first discuss Monte Carlo methods and random number generation in a fully general setting. Only after we have finished our treatment of these two topics (to a certain level at least) will we discuss their application to Bayesian inference. Hopefully this format will minimise any confusion.

\subsection{The method of Monte Carlo integration for estimating means}

One of the most important applications of Monte Carlo methods is the estimation of means. Suppose we are interested in $\mu$, the mean of some distribution defined by a density $f(x)$ (or by a cumulative distribution function $F(x)$ ), but we are unable to calculate $\mu$ exactly (or easily), for example by applying the formula

$$
\mu=E x=\int x f(x) d x
$$

(or $\quad \mu=E x=\sum_{x} x f(x) \quad$ or $\quad \mu=E x=\int x d F(x)$ ).

Also suppose, however, that we are able to generate (or obtain) a random sample from the distribution in question. Denote this sample as

$$
x_{1}, \ldots, x_{J} \sim \text { iid } f(x)
$$

(or

$$
\left.x_{1}, \ldots, x_{J} \sim \text { iid } F(x)\right) \text {. }
$$

Then we may use this sample to estimate $\mu$ by

$$
\bar{x}=\frac{1}{J} \sum_{j=1}^{J} x_{j} .
$$


Also, a $1-\alpha$ confidence interval (CI) for $\mu$ given by

$$
C I=\left(\bar{x} \pm z_{\alpha / 2} s / \sqrt{J}\right),
$$

where

$$
s^{2}=\frac{1}{J-1} \sum_{j=1}^{J}\left(x_{j}-\bar{x}\right)^{2}
$$

is the sample variance of the random values.

In this context we refer to:

$\begin{array}{ll}X_{1}, \ldots, X_{J} & \begin{array}{l}\text { as the Monte Carlo sample values } \\ \text { or the Monte Carlo sample } \\ \bar{X}\end{array} \\ \text { as the Monte Carlo sample mean } \\ \text { or the Monte Carlo estimate } \\ \text { as the Monte Carlo } 1-\alpha \text { confidence interval } \\ \text { for } \mu \\ s^{2} & \text { as the Monte Carlo sample size } \\ s & \text { as the Monte Carlo sample variance } \\ s / \sqrt{J} & \text { as the Monte Carlo sample standard deviation } \\ & \text { as the Monte Carlo standard error (SE). }\end{array}$

Three important facts here are that:

- $\bar{X}$ is unbiased for $\mu$ (i.e. $E \bar{X}=\mu$ )

- the CI has coverage approximately $1-\alpha$, by the central limit theorem

- the width of the CI converges to zero as the MC sample size $J$ tends to infinity.

\section{Exercise 5.I Monte Carlo estimation of a known gamma mean}

(a) Use the $\mathrm{R}$ function rgamma() to generate a random sample of size $J=100$ from the $\operatorname{Gamma}(3,2)$ distribution, whose mean is $\mu=3 / 2=1.5$. Then use the method of Monte Carlo to produce a point estimate $\mu$ and a 95\% CI for $\mu$.

(b) Repeat (a) but with MC sample sizes of 1,000 and 10,000, and discuss the results. 
Note: In this exercise we are focusing on the integral

$$
\mu=\int x f(x) d x=\int_{0}^{\infty} x\left(\frac{2^{3} x^{3-1} e^{-2 x}}{\Gamma(3)}\right) d x,
$$

showing how it could be estimated via MC if it were not possible to evaluate analytically. Exactly the same approach could be applied if the integral were impossible to evaluate.

\section{Solution to Exercise 5.I}

(a) Applying the above procedure (see the $\mathrm{R}$ code below) we estimate $\mu$ by $\bar{x}=1.5170$. The Monte Carlo $95 \%$ confidence interval for $\mu$ is

$$
C I=\left(\bar{x} \pm z_{0.025} s / \sqrt{J}\right)=(1.3539,1.6800) .
$$

We note that $\bar{x}$ is 'close' to the true value, $\mu=1.5$, and the CI contains that true value.

(b) Repeating (a) with $J=1,000$ we obtain the point estimate 1.5199 and the interval estimate $(1.4658,1.5740)$.

Repeating (a) with $J=10,000$ we obtain the point estimate 1.4942 and the interval estimate $(1.4773,1.5110)$.

As in (a) we note in each case that $\bar{X}$ is 'close' to $\mu$, and the CI contains $\mu$. We also note that as $J$ increases the MC point estimate tends to get closer to $\mu$, and the $95 \%$ CI tends to get narrower. (The widths of the three CIs are $0.3261,0.1081$ and 0.0337.$)$

\section{R Code for Exercise 5. I}

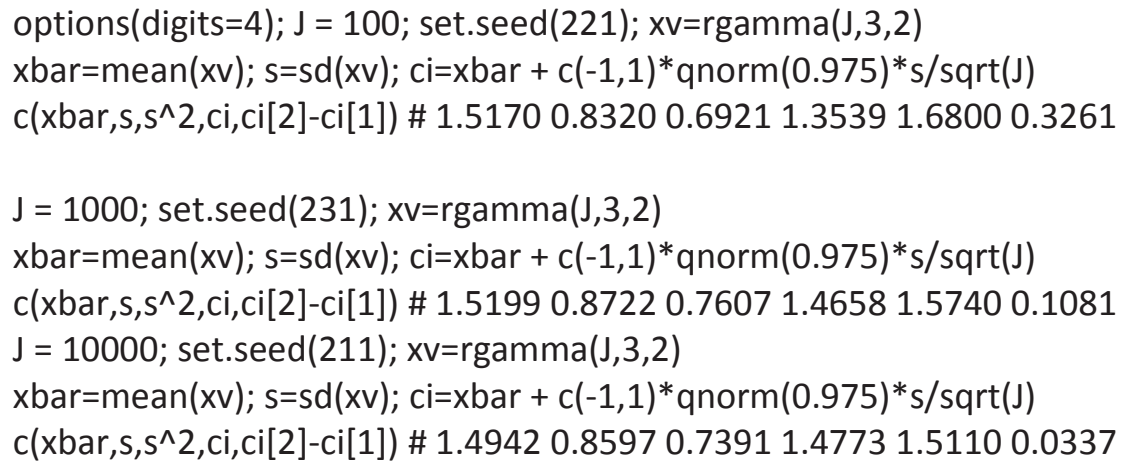




\subsection{Other uses of the MC sample}

Once a Monte Carlo sample $x_{1}, \ldots, x_{J} \sim$ iid $f(x)$ has been obtained, it can be used for much more than just estimating the mean of the distribution, $\mu=E x$. For example, suppose we are interested in the (lower) $p$-quantile of the distribution, namely

$$
q_{p}=F_{X}^{-1}(p)=\{\text { value of } x \text { such that } F(x)=p\} .
$$

The MC estimate of $q_{p}$ is simply $\hat{q}_{p}$, the empirical $p$-quantile of $x_{1}, \ldots, x_{J}$. For instance, the median $q_{1 / 2}$ can be estimated by the middle number amongst $x_{1}, \ldots, X_{J}$ after sorting in increasing order. This assumes that $J$ is odd. If $J$ is even, we estimate $q_{1 / 2}$ by the average of the two middle numbers. Thus we may write the MC estimate of $q_{1 / 2}$ as

$$
\hat{q}_{1 / 2}=\left\{\begin{array}{c}
x_{((J+1) / 2)}, J \text { odd } \\
\frac{x_{(J / 2)}+x_{((J+1) / 2)}}{2}, J \text { even, }
\end{array}\right.
$$

where $x_{(k)}$ is the $k$ th smallest value amongst $x_{1}, \ldots, x_{J} \quad(k=1, \ldots, J)$.

Also, we estimate the $1-\alpha$ central density region (CDR) for $x$, namely $\left(q_{\alpha / 2}, q_{1-\alpha / 2}\right)$, by $\left(\hat{q}_{\alpha / 2}, \hat{q}_{1-\alpha / 2}\right)$.

Further, suppose we are interested in the expected value of some function of $x$, say $y=g(x)$. That is, we wish to estimate the quantity/integral

$$
\psi=E y=\int y f(y) d y=E g(x)=\int g(x) f(x) d x .
$$

Then we simply calculate $y_{j}=g\left(x_{j}\right)$ for each $j=1, \ldots, J$. The result will be a random sample $y_{1}, \ldots, y_{J} \sim$ iid $f(y)$ to which the method of Monte Carlo can then be applied in the usual way. Thus, an estimate of $\psi$ is

$$
\bar{y}=\frac{1}{J} \sum_{j=1}^{J} y_{j} \quad \text { (the sample mean of the } y \text {-values), }
$$

and a $1-\alpha$ CI for $\psi$ is

$$
\left(\bar{y} \pm z_{\alpha / 2} \frac{s_{y}}{\sqrt{J}}\right)
$$


where $s_{y}^{2}=\frac{1}{J-1} \sum_{j=1}^{J}\left(y_{j}-\bar{y}\right)^{2}$ (the sample variance of the $y$-values).

This idea applies to even very complicated functions $y=g(x)$ for which the exact or even approximate value of $\psi=E y$ would otherwise be very difficult to obtain, either analytically or numerically using a deterministic technique such as numerical integration (or quadrature).

Also, the density $f(x)$ can be estimated by smoothing a probability histogram of $x_{1}, \ldots, x_{J}$. Likewise, the density $f(y)$ can be estimated by smoothing a probability histogram of $y_{1}, \ldots, y_{J}$. (This could be extremely useful if $y$ is a very complicated function of $x$.)

Note 1: As we will see later, it is often the case that we are able to sample from a distribution without knowing - or being able to derive - the exact form of its density function.

Note 2: Smoothing a histogram requires some arbitrary decisions to be made about the degree of smoothing and other smoothing parameters. So the MC estimate of a density is not uniquely defined.

\section{Exercise 5.2 Monte Carlo estimation of complicated quantities}

Suppose that $x \sim G(3,2)$. Use MC methods and a sample of size $J=1,000$ to estimate:

$$
\begin{aligned}
& \mu=E x \text {, the } 80 \% \operatorname{CDR} \text { for } x \text {, and } f(x) \\
& \psi=E y, \text { the } 80 \% \operatorname{CDR} \text { for } y \text {, and } f(y) \text {, where } y=\frac{x^{2} e^{-x}}{1+x+1 / x} .
\end{aligned}
$$

Present your results graphically, and wherever possible show the true values of the quantities being estimated. Then repeat everything but using a Monte Carlo sample size of $J=10,000$.

\section{Solution to Exercise 5.2}

The required graphs are shown in Figures 5.1 to 5.4. See the R code below for more details. 
Figure 5. I Histogram of $x$-value $(J=I, 000)$

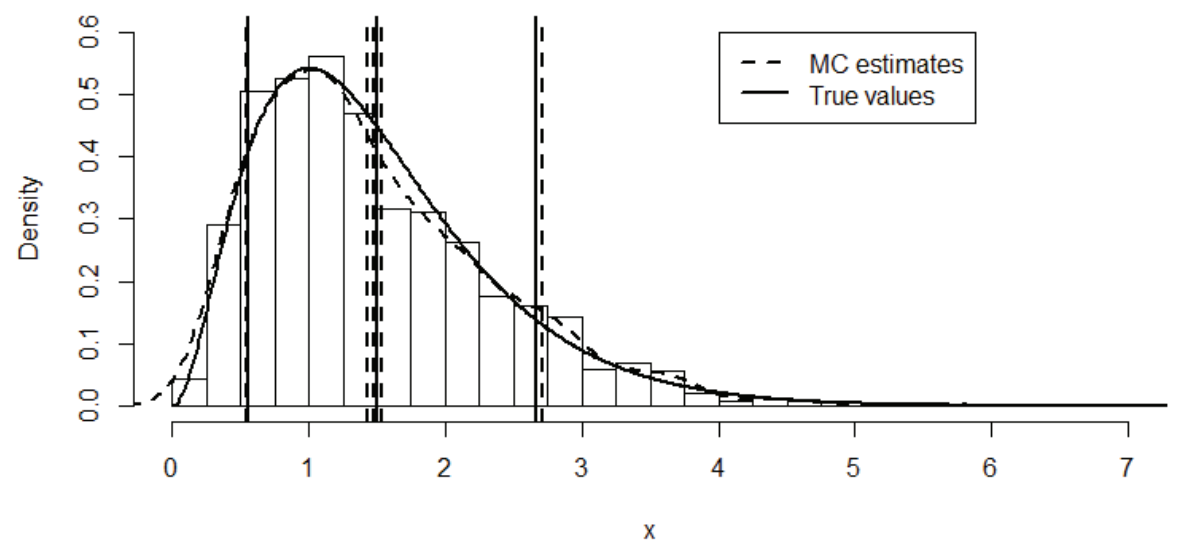

Figure 5.2 Histogram of $y$-value $(J=1,000)$

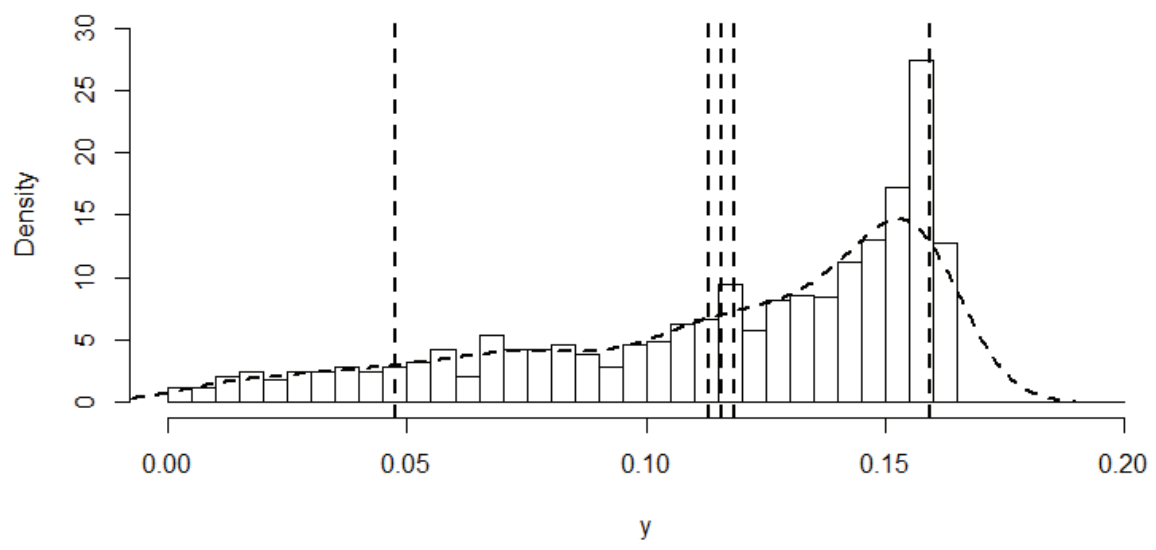


Figure 5.3 Histogram of $x$-value $(J=10,000)$

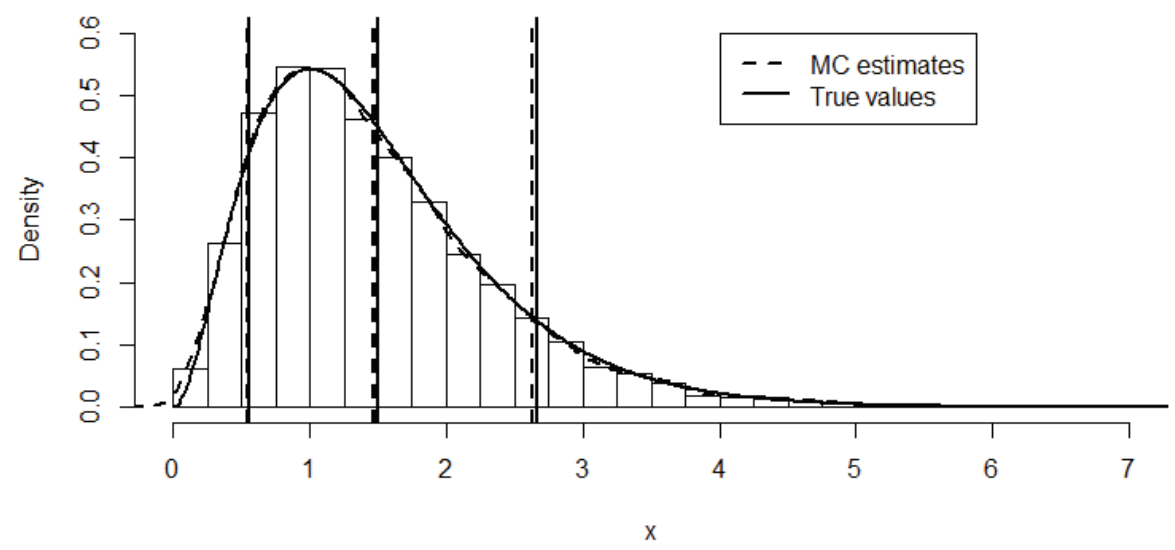

Figure 5.4 Histogram of $y$-value $(J=10,000)$

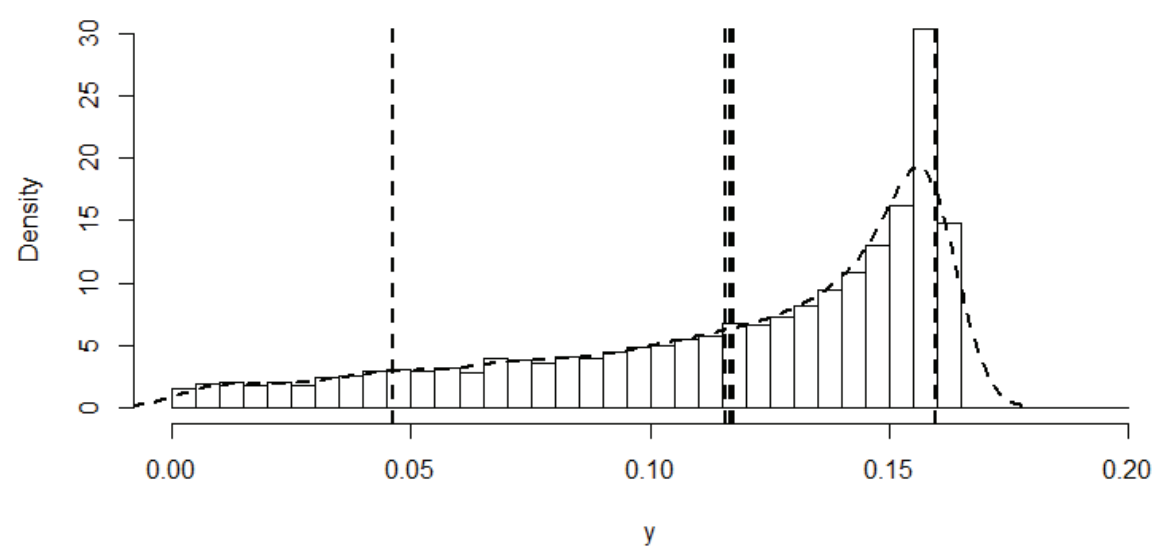

\section{R Code for Exercise 5.2}

$\mathrm{X} 11(\mathrm{w}=8, \mathrm{~h}=4.5) ; \operatorname{par}(\mathrm{mfrow}=\mathrm{c}(1,1))$; options(digits=4);

$\mathrm{J}=1000$; set.seed(221); $\mathrm{xV}=\mathrm{rgamma}(\mathrm{J}, 3,2)$

xbar=mean $(x v) ; x c i=x b a r+c(-1,1) *$ qnorm $(0.975) * s d(x v) / \operatorname{sqrt}(J)$

$x c d r=q u a n t i l e(x v, c(0.1,0.9)) ; x d e n=\operatorname{density}(x v)$

$y v=x v^{\wedge} 2 * \exp (-x v) /(1+x v+1 / x v)$

ybar=mean(yv); yci=ybar $+c(-1,1) *$ qnorm $(0.975) * s d(y v) / \operatorname{sqrt}(J)$

ycdr=quantile $(y v, c(0.1,0.9)) ; y d e n=d e n s i t y(y v)$ 
hist $(x v$, prob=T, breaks=seq $(0,7,0.25), x \lim =c(0,7), y \lim =c(0,0.6), x l a b=" x "$,

main=""); lines(xden,lty=2,lwd=2)

$x v e c=\operatorname{seq}(0,10,0.01)$; lines $(x v e c$, dgamma(xvec,3,2), Ity=1,lwd=2)

abline $(v=c(x b a r, x c i, x c d r), I t y=2, I w d=2)$

abline $(v=c(3 / 2$, qgamma $(c(0.1,0.9), 3,2))$, Ity=1, lwd=2)

legend(4,0.6,c("MC estimates","True values"), Ity=c(2,1), Iwd=c(2,2))

hist $(y v, p r o b=T$, breaks=seq(0,0.2,0.005), $x \lim =c(0,0.2), y \lim =c(0,30), x l a b=" y "$,

main=""); lines(yden, Ity=2,lwd=2)

abline( $v=c(y b a r, y c i, y c d r)$, Ity=2, Iwd=2)

legend(4,0.6,c("MC estimates","True values"), Ity=c(2,1), I $\mathrm{wd}=c(2,2))$

\# Repeat with J = 10000

$\mathrm{J}=$ 10000; set.seed(221); $x \mathrm{v}=\operatorname{rgamma}(\mathrm{J}, 3,2)$

xbar=mean $(x v) ; x c i=x b a r+c(-1,1) *$ qnorm $(0.975) * s d(x v) / \operatorname{sqrt}(J)$

$x c d r=q u a n t i l e(x v, c(0.1,0.9)) ; x d e n=\operatorname{density}(x v)$

$y v=x v^{\wedge} 2 * \exp (-x v) /(1+x v+1 / x v)$

ybar=mean(yv); yci=ybar $+c(-1,1) *$ qnorm $(0.975) * s d(y v) / \operatorname{sqrt}(J)$

ycdr=quantile $(y v, c(0.1,0.9)) ; y d e n=\operatorname{density}(y v)$

hist(xv,prob=T,breaks=seq(0,9,0.25),xlim=c(0,7),ylim=c(0,0.6),xlab="x",

main="'"); lines(xden,lty=2,lwd=2)

$x v e c=\operatorname{seq}(0,10,0.01)$; lines $(x v e c$, dgamma(xvec,3,2), Ity=1,lwd=2)

abline ( $v=c(x b a r, x c i, x c d r), I t y=2, I w d=2)$

abline $(v=c(3 / 2$, qgamma $(c(0.1,0.9), 3,2))$, Ity=1, lwd=2)

legend(4,0.6,c("MC estimates","True values"), Ity=c(2,1), $\operatorname{lwd}=c(2,2))$

hist(yv,prob=T, breaks=seq(0,0.2,0.005),xlim=c(0,0.2),ylim=c(0,30),xlab="y", main="")

lines(yden, Ity=2,Iwd=2); abline(v= c(ybar, yci, ycdr), Ity=2, Iwd=2)

legend(4,0.6,c("MC estimates","True values"), Ity=c(2,1), $\operatorname{lwd}=c(2,2))$

\subsection{Importance sampling}

When applying the method of MC to estimate an integral of the form

$$
\psi=E g(x)=\int g(x) f(x) d x,
$$

suppose it is impossible (or difficult) to sample from $f(x)$, but it is easy to sample from a distribution/density $h(x)$ which is 'similar' to $f(x)$. 
Then we may write

$$
\psi=\int\left(g(x) \frac{f(x)}{h(x)}\right) h(x) d x=\int w(x) h(x) d x,
$$

where

$$
w(x)=g(x) \frac{f(x)}{h(x)} .
$$

This suggests that we sample $x_{1}, \ldots, x_{J} \sim$ iid $h(x)$ and use MC to estimate $\psi$ by

$$
\hat{\psi}=\bar{w}=\frac{1}{J} \sum_{j=1}^{J} w_{j},
$$

where

$$
w_{j}=w\left(x_{j}\right)=g\left(x_{j}\right) \frac{f\left(x_{j}\right)}{h\left(x_{j}\right)} .
$$

This techniques is called importance sampling, and there are several issues to consider. As already indicated, the method works best if $h(x)$ is chosen to be very similar to $f(x)$.

Another issue is that $f(x)$ may be known only up to a multiplicative constant, i.e. where $f(x)=k(x) / c$, where the kernel $k(x)$ is known exactly but it is too difficult or impossible to evaluate the normalising constant $c=\int k(x) d x$. In that case, we may write

$$
\begin{aligned}
\psi=\int g(x) \frac{k(x)}{c} d x & =\frac{\int g(x) k(x) d x}{\int k(x) d x} \\
& =\frac{\int\left(g(x) \frac{k(x)}{h(x)}\right) h(x) d x}{\int\left(\frac{k(x)}{h(x)}\right) h(x) d x}=\frac{\int w(x) h(x) d x}{\int u(x) h(x) d x},
\end{aligned}
$$

where:

$$
\begin{aligned}
& w(x)=g(x) \frac{k(x)}{h(x)} \\
& u(x)=\frac{k(x)}{h(x)} .
\end{aligned}
$$


This suggests that we sample $x_{1}, \ldots, x_{J} \sim$ iid $h(x)$ (as before) and apply MC estimation to the means of $w(x)$ and $u(x)$, respectively (each with respect to the distribution defined by density $h(x))$ so as to obtain the estimate

$$
\hat{\psi}=\frac{\bar{w}}{\bar{u}}=\frac{\frac{1}{J} \sum_{j=1}^{J} w_{j}}{\frac{1}{J} \sum_{j=1}^{J} u_{j}}=\frac{w_{1}+\ldots+w_{J}}{u_{1}+\ldots+u_{J}},
$$

where $w_{j}=w\left(x_{j}\right)$ and $u_{j}=u\left(x_{j}\right)$.

\section{Exercise 5.3 Example of Monte Carlo with importance sampling}

We wish to find $\mu=E x$ where $x$ has density

$$
f(x) \propto \frac{1}{x+1} e^{-x}, x>0 .
$$

Use Monte Carlo methods and importance sampling to estimate $\mu$.

\section{Solution to Exercise 5.3}

Here, $k(x)=\frac{1}{x+1} e^{-x}$, and it is convenient to use $h(x)=e^{-x}, x>0$ (the standard exponential density, or $\operatorname{Gamma}(1,1)$ density). Then,

$$
\begin{aligned}
& \mu=E x=\int_{0}^{\infty} x f(x) d x=\frac{\int x k(x) d x}{\int k(x) d x} \\
& =\frac{\int\left(x \frac{k(x)}{h(x)}\right) h(x) d x}{\int\left(\frac{k(x)}{h(x)}\right) h(x) d x}=\frac{\int \frac{x}{x+1} h(x) d x}{\int \frac{1}{x+1} h(x) d x} . \\
& \text { So a MC estimate of } \mu \text { is } \hat{\mu}=\frac{\frac{1}{J} \sum_{j=1}^{J} \frac{x_{j}}{x_{j}+1}}{\frac{1}{J} \sum_{j=1}^{J} \frac{1}{x_{j}+1}} \text {, }
\end{aligned}
$$

where $x_{1}, \ldots, x_{J} \sim$ iid $G(1,1)$. 
Implementing this with $J=100,000$, we get $\hat{\mu}=\frac{0.40345}{0.59655}=0.67631$.

Note 1: For interest we use numerical techniques to get the exact answer,

$$
\mu=0.67687 \text {. }
$$

Thus the relative error is $-0.084 \%$. Figure 5.5 illustrates.

Note 2: The exact value of the normalising constant is

$$
c=\int k(x) d x \text { is } 0.596347 .
$$

From the above we see that our MC estimate of $c$ is 0.59655 (similar).

Figure 5.5 Illustration of importance sampling

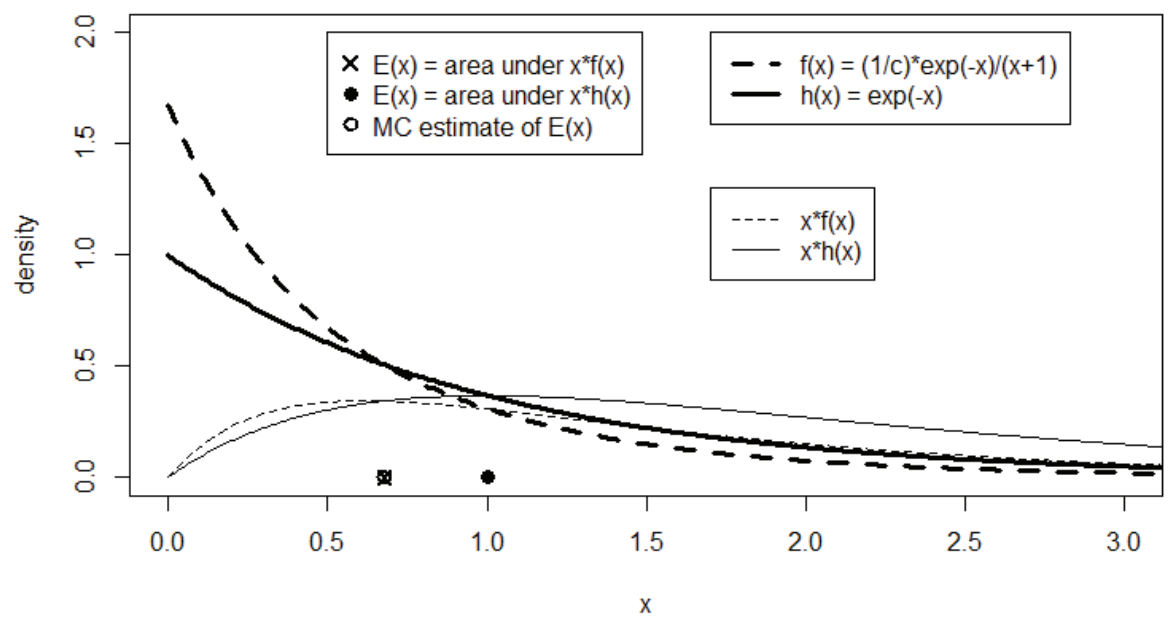

\section{R Code for Exercise 5.3}

options(digits $=10)$;

kfun=function $(x)\{\exp (-x) /(x+1)\}$

$c=$ integrate $(f=k f u n, l o w e r=0$,upper=Inf $) \$$ value; c \# 0.5963473624

ffun=function $(x)\{(1 / 0.5963473624) * \exp (-x) /(x+1)\}$

integrate $(f=f f u n, l o w e r=0$, upper=Inf)\$value; \# 0.9999999999

$x f f u n=$ function $(x)\left\{x^{*}(1 / 0.5963474) * \exp (-x) /(x+1)\right\}$

$m u=$ integrate $(f=x f f u n, l o w e r=0$,upper=Inf $) \$$ value; $m u \# 0.6768749849$ 
$\mathrm{J}=100000$; set.seed(413); $\mathrm{xV}=\mathrm{rgamma}(\mathrm{J}, 1,1)$

num=mean $(x v /(x v+1))$; den=mean $(1 /(x v+1))$

est=num/den; c(num, den, est) \# 0.40345106850 .59654893150 .6763084254

err=100* (est-mu)/mu; err \#-0.08370222467

$\operatorname{plot}(c(0,3), c(0,2)$,type="n",xlab="x",ylab="density"); $x v e c=s e q(0,5,0.01)$;

lines(xvec,dgamma(xvec,1,1), Ity =1, Iwd=3)

lines (xvec, xvec*dgamma(xvec,1,1), Ity=1, Iwd=1)

lines(xvec,ffun( $x v e c)$, Ity=2,Iwd=3); lines ( $x v e c, x v e c * f f u n(x v e c)$, Ity=2,Iwd=1)

points $(c(1, \mathrm{mu}, \mathrm{est}), c(0,0,0), \mathrm{pch}=c(16,4,1), \operatorname{lwd}=c(2,2,2), \operatorname{cex}=c(1.2,1.2,1.2))$

legend $\left(1.7,2, c\left(" f(x)=(1 / c)^{*} \exp (-x) /(x+1) ", " h(x)=\exp (-x) "\right)\right.$,

$\operatorname{lty}=c(2,1), \operatorname{Iwd}=c(3,3))$

legend $\left(1.7,1.3, c\left(" x^{*} f(x) ", " x^{*} h(x) "\right)\right.$, Ity=c(2,1), Iwd=c(1,1))

legend $\left(0.5,2, c\left(" E(x)=\right.\right.$ area under $x^{*} f(x) ", " E(x)=$ area under $x^{*} h(x) "$,

"MC estimate of $E(x) "), p c h=c(4,16,1)$,pt.Iwd=c(2,2,2),pt.cex $=c(1 \cdot 2,1 \cdot 2,1.2))$

\subsection{MC estimation involving two or more random variables}

All the examples so far have involved only a single random variable $x$. However, the method of Monte Carlo generalises easily to two or more random variables. In fact, the procedure for MC estimation of the mean of a function, as described above, is already valid in the case where $x$ is a vector. We will now focus on the bivariable case, but the same principles apply when three or more random variables are being considered simultaneously.

Suppose that we have a random sample from the bivariate distribution of two random variables $x$ and $y$, denoted $\left(x_{1}, y_{1}\right), \ldots,\left(x_{J}, y_{J}\right) \sim$ iid $f(x, y)$, and we are interested in some function of $x$ and $y$, say $r=g(x, y)$. Then we simply calculate $r_{j}=g\left(x_{j}, y_{j}\right)$ and perform MC inference on the resulting sample $r_{1}, \ldots, r_{J} \sim$ iid $f(r)$.

Note 1: This procedure applies whether or not the random variables $x$ and $y$ are independent. If they are independent then we simply sample $x_{j} \sim f(x)$ and $y_{j} \sim f(y)$.

Note 2: If $x$ and $y$ are dependent, it may not be obvious how to generate $\left(x_{j}, y_{j}\right) \sim f(x, y)$. 
Then, one approach is to apply the method of composition, as detailed below. If that fails, other methods are available, in particular ones which involve Markov chain theory. Much more will be said on these methods later in the course.

\subsection{The method of composition}

Suppose we wish to sample a vector $\left(x_{j}, y_{j}\right) \sim f(x, y)$. Often this can be done in two different ways via the method of composition, as follows.

One way is to first sample $x_{j} \sim f(x)$ and then sample $y_{j} \sim f\left(y \mid x_{j}\right)$. The result will be the desired $\left(x_{j}, y_{j}\right) \sim f(x, y)$. This follows by the identity (or 'composition')

$$
f(x, y)=f(x) f(y \mid x) \text {. }
$$

Note: Having obtained $\left(x_{j}, y_{j}\right) \sim f(x, y)$ in this manner, suppose we 'discard' $x_{j}$. Then this will leave behind a single number, $y_{j} \sim f(y)$. This could be useful if all we really want is a sample from $f(y)$ but sampling from this distribution/density directly is difficult.

Alternatively, first sample $y_{j} \sim f(y)$ and then sample $x_{j} \sim f\left(x \mid y_{j}\right)$. The result will again be $\left(x_{j}, y_{j}\right) \sim f(x, y)$. This follows by the identity

$$
f(x, y)=f(y) f(x \mid y) \text {. }
$$

Note: Having obtained $\left(x_{j}, y_{j}\right) \sim f(x, y)$ in this second manner, suppose that we 'discard' $y_{j}$. This will leave behind a single number, $x_{j} \sim f(x)$. This could be useful if all we really desire is a sample from $f(x)$ but sampling from this distribution/density directly is difficult.

This idea of composition generalises easily to higher dimensions. For example, one of several different ways to sample a triplet

$$
\left(x_{j}, y_{j}, z_{j}\right) \sim f(x, y, z)
$$

is first sample $y_{j} \sim f(y)$, then sample $x_{j} \sim f\left(x \mid y_{j}\right)$ and finally sample $z_{j} \sim f\left(z \mid x_{j}, y_{j}\right)$. This works because of the identity

$$
f(x, y, z)=f(y) f(x \mid y) f(z \mid x, y) .
$$




\section{Exercise 5.4}

Suppose that we are interested in the distribution of a random variable defined by $r=y /(x+\sqrt{|y|})$, where $x$ and $y$ have a joint distribution defined by the pdf $f(x, y)=f(x) f(y \mid x)$, and where $x \sim G(3,2)$ and $(y \mid x) \sim N(x, x)$.

Use the R functions rgamma() and rnorm() to generate a sample of size $J=1,000$ from the joint distribution of $x$ and $y$. Then use the method of MC to estimate $\psi=E r$, and report a 95\% CI for $\psi$. Also estimate the 80\% CDR for $r$ and $f(r)$. Present your results both graphically and numerically.

\section{Solution to Exercise 5.4}

Numerically, we estimate $\psi$ by 0.4256 , and our 95\% CI for $\psi$ is (0.4026, 0.4486). We also estimate the $80 \%$ CDR for $r$ by $(-0.1025$, 0.8339). The required graph is shown in Figure 5.6.

Figure 5.6 Histogram of $r$-values $(U=I, 000)$

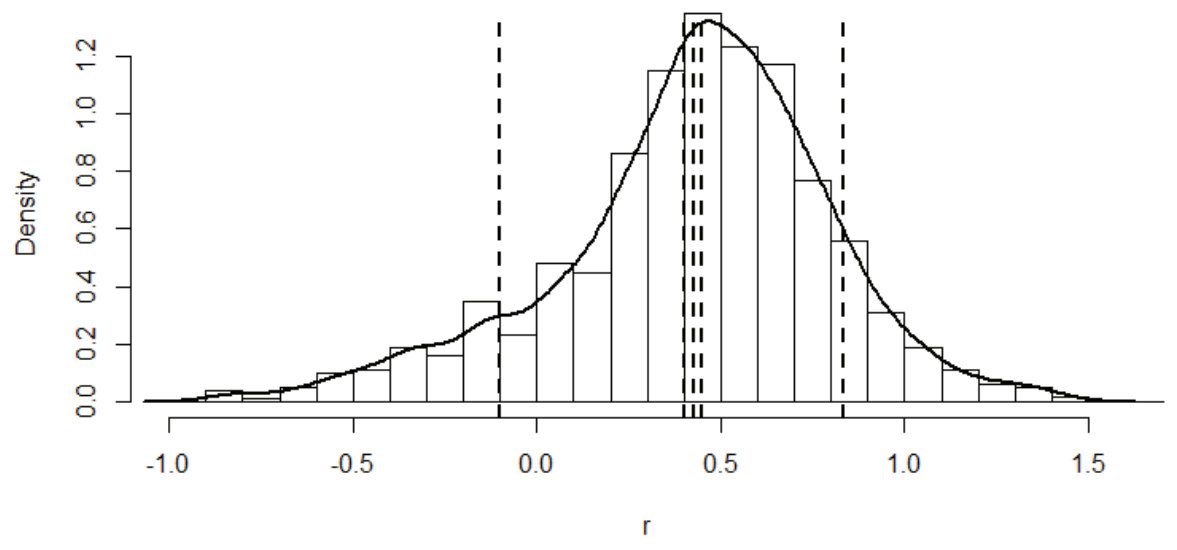




\section{R Code for Exercise 5.4}

$\mathrm{X} 11(\mathrm{w}=8, \mathrm{~h}=4.5) ; \operatorname{par}(\mathrm{mfrow}=\mathrm{c}(1,1)) ;$ options $($ digits $=4) ;$

$\mathrm{J}=1000 ;$ set.seed $(221) ; \mathrm{xV}=\mathrm{rgamma}(J, 3,2) ; \mathrm{yv}=\operatorname{rnorm}(\mathrm{xv}, \operatorname{sqrt}(\mathrm{xv}))$

$\mathrm{rv}=\mathrm{yv} /(\mathrm{xv}+\mathrm{sqrt}(\mathrm{abs}(\mathrm{yv})))$

rbar=mean (rv); rci=rbar $+c(-1,1) *$ qnorm $(0.975) *$ sd(rv)/sqrt(J)

$r c d r=q u a n t i l e(r v, c(0.1,0.9))$; rden=density $(r v)$

c(rbar,rci,rcdr) \# $0.4256 \quad 0.4026 \quad 0.4486-0.10250 .8339$

hist(rv, prob=T, breaks=seq(-1,1.8,0.1), $x \lim =c(-1,1.6), y \lim =c(0,1.3), x \mathrm{lab}=" r "$, main="'); lines(rden, Ity=1,lwd=2); abline(v= c(rbar, rci, rcdr), Ity=2, Iwd=2)

\subsection{Monte Carlo estimation of a binomial parameter}

Suppose we are interested in a binomial proportion (i.e. probability) $p$ but have difficulty calculating this quantity exactly. Then we may interpret $p$ as the mean $\mu$ of a Bernoulli distribution and directly apply the method of Monte Carlo in the usual way. In this special case, there are certain simplifications which result in slightly different-looking final formulae.

Explicitly, suppose we are able to generate

$$
x_{1}, \ldots, x_{J} \sim \text { iid Bernoulli }(p) \text {. }
$$

Then the MC estimate of $p$ is

$$
\bar{x}=\frac{1}{J} \sum_{j=1}^{J} x_{j} \quad \text { (the sample proportion of 1s in the sample), }
$$

and the MC sample variance is

$$
\begin{aligned}
s^{2} & =\frac{1}{J-1}\left(\sum_{j=1}^{J} x_{j}^{2}-\sqrt{X}^{2}\right) \\
& =\frac{1}{J-1}\left(J \bar{x}-\bar{X}^{2}\right) \quad \text { since } x_{j}^{2}=x_{j} \text { (because each } x_{j} \text { is } 0 \text { or } 1 \text { ) } \\
& =\frac{J}{J-1} \bar{x}(1-\bar{x}) .
\end{aligned}
$$

So the MC SE is $\frac{s}{\sqrt{J}}=\frac{1}{\sqrt{J}} \sqrt{\frac{J}{J-1} \bar{x}(1-\bar{x})}=\sqrt{\frac{\bar{x}(1-\bar{x})}{J-1}}$. 
It follows that a MC $1-\alpha$ CI for $p$ is

$$
\left(\bar{X} \pm z_{\alpha / 2} \frac{s}{\sqrt{J}}\right)=\left(\bar{x} \pm z_{\alpha / 2} \sqrt{\frac{\bar{x}(1-\bar{x})}{J-1}}\right) .
$$

The MC estimate $\bar{x}$ is often written as $\hat{p}$, and $J-1$ is often replaced by $J$ (for simplicity). These changes lead to the standard form of the MC $1-\alpha$ confidence interval for $p$,

$$
\left(\hat{p} \pm z_{\alpha / 2} \sqrt{\frac{\hat{p}(1-\hat{p})}{J}}\right) .
$$

Note 1: The above theory is really nothing other than the usual classical theory for estimating a binomial proportion. Thus, there are many other CIs that could be substituted, (e.g. the Wilson CI whose coverage is closer to $1-\alpha$, and the Clopper-Pearson CI whose coverage is always guaranteed to be at least $1-\alpha$ but which is typically wider).

Note 2: The above MC inference depends on the $x_{j}$ values only by way of the sample mean $\bar{X}$ or, equivalently, by way of the sample total $x_{T}=x_{1}+\ldots+x_{J}=\sqrt{X}$. A consequence of this is that exactly the same Monte Carlo inference can be performed if we observe only a single value of the total $x_{T}$, whose distribution is given by $x_{T} \sim \operatorname{Bin}(J, p)$.

Note 3: A common application of the theory here is where the binomial parameter is the probability of some event involving random variables, for example $p=P(x>1)$ and $p=P(x<y)$.

For the first example here, we generate $x_{1} \sim f(x)$, let $r_{1}=I\left(x_{1}>1\right)$, and then repeat independently many times so as to generate a random sample $r_{1}, \ldots, r_{J} \sim$ iid $\operatorname{Bern}(p)$. That sample can then be used for MC inference on $p=P(x>1)$.

The procedure for the second example is similar, except that it involves sampling $\left(x_{1}, y_{1}\right) \sim f(x, y)$ and determining $r_{1}=I\left(x_{1}<y_{1}\right)$, etc. 
Note 4: One use of MC CIs for a binomial proportion is to assess the coverage of MC CIs.

Often, the true coverage probability of a MC CI is not exactly the nominal level, say $95 \%$. This may be due to the MC sample size $J$ being insufficiently large or for some other reason.

If we are concerned about this, we may wish to estimate the true coverage of the MC CI by repeating the entire MC inference procedure itself a large number of times, say $M$. Each time we record an indicator $r$ for the MC CI containing the quantity of interest.

The result will be a sample $r_{1}, \ldots, r_{M} \sim$ iid $\operatorname{Bern}(p)$, where $p$ is the true coverage probability, which can then be estimated via MC methods in the usual way.

\section{Exercise 5.5 Estimating a probability via Monte Carlo}

Use MC to estimate $p=P\left(\sqrt{\frac{x}{x+1}}>0.3 e^{x}\right)$, where $x \sim \operatorname{Gamma}(3,2)$.

\section{Solution to Exercise 5.5}

With $J=20,000$, we sample $x_{1}, \ldots, x_{J} \sim$ iid $G(3,2)$ and let

$$
r_{j}=I\left(\sqrt{\frac{x_{j}}{x_{j}+1}}>0.3 e^{x_{j}}\right) .
$$

Thereby we obtain an estimate of $p$ equal to $\hat{p}=\frac{1}{J} \sum_{j=1}^{J} r_{j}=0.2117$

and a 95\% CI for $p$ equal to $\left(\hat{p} \pm 1.96 \sqrt{\frac{\hat{p}(1-\hat{p})}{200000}}\right)=(0.2060,0.2173)$.

Note 1: We may also view $p$ as $p=P(y>0.3)$, where $y=e^{-x} \sqrt{\frac{x}{x+1}}$ (for example). In that case, we sample $x_{1}, \ldots, x_{J} \sim$ iid $G(3,2)$, calculate 
$y_{j}=e^{-x_{j}} \sqrt{\frac{x_{j}}{x_{j}+1}}$, and then let $r_{j}=I\left(y_{j}>0.3\right)$. This leads to exactly the same results regarding $p$. As a by-product of this second approach, we obtain an estimate of the density function of the random variable $y=e^{-x} \sqrt{\frac{x}{x+1}}$, namely $f(y)$, which would be very difficult to obtain analytically. Figure 5.7 illustrates.

Note 2: The density() function in R used to smooth the histogram does not adequately capture the upper region of the density $f(y)$, nor the fact that $f(y)=0$ when $y<0$.

\section{Figure 5.7 Histogram of 20,000 values of $y$}

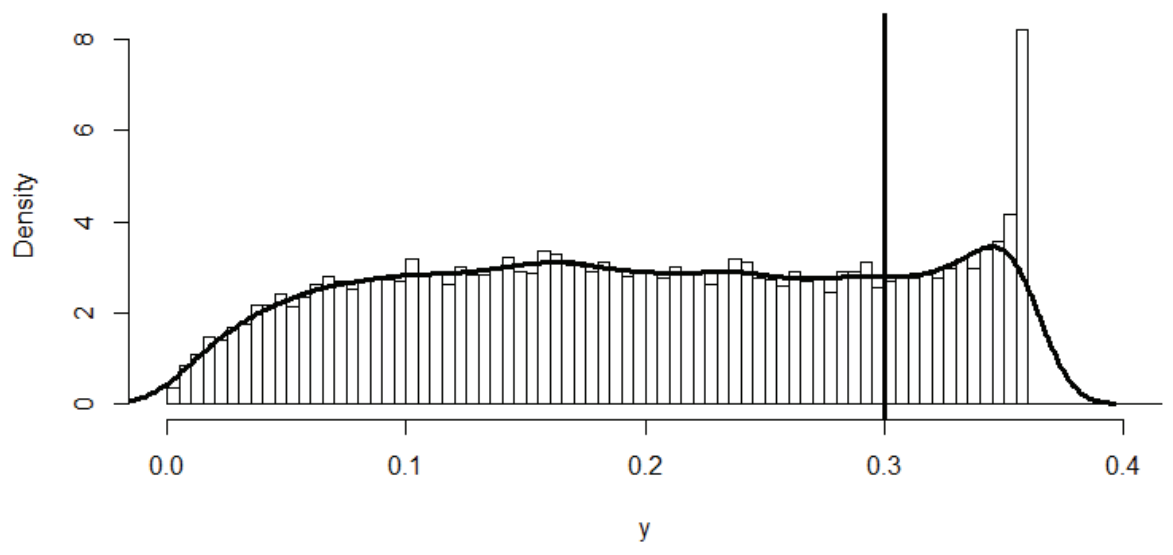

\section{R Code for Exercise 5.5}

$\mathrm{X} 11(\mathrm{w}=8, \mathrm{~h}=4.5) ; \operatorname{par}(\mathrm{mfrow}=\mathrm{c}(1,1)) ;$ options$($ digits=4)

$\mathrm{J}=20000$; set.seed(162); $\mathrm{xV}=\mathrm{rgamma}(\mathrm{J}, 3,2) ; \mathrm{ct}=0$

$y v=\operatorname{sqrt}(x v) * \exp (-x v) / \operatorname{sqrt}(x v+1)$

for(j in 1:J) if(yv[j] >0.3) ct=ct+1

phat=ct/J; ci=phat+c(-1,1)*qnorm(0.975)*sqrt(phat*(1-phat)/J)

c(phat,ci) \# 0.21170 .20600 .2173

hist(yv,prob=T, breaks=seq $(0,0.5,0.005), x \lim =c(0,0.4), x l a b=" y "$, main=" ")

abline(v=0.3,lwd=3); lines(density(yv), lwd=3) 


\section{Exercise 5.6 Buffon's needle problem}

A needle of length $10 \mathrm{~cm}$ is dropped randomly onto a floor with lines on it that are parallel and $10 \mathrm{~cm}$ apart.

(a) Analytically derive $p$, the probability that the needle crosses a line.

(b) Now forget that you know $p$. Estimate $p$ using Monte Carlo methods on a computer and a sample size of 1,000. Also provide a 95\% confidence interval for $p$. Then repeat with a sample size of 10,000 and discuss.

\section{Solution to Exercise $\mathbf{5 . 6}$}

(a) Let: $X$ = perpendicular distance from centre of needle to nearest line in units of $5 \mathrm{~cm}$

$Y=$ acute angle between lines and needle in radians

$C=$ 'The needle crosses a line'.

Then: $\quad X \sim U(0,1) \quad$ with density $\quad f(x)=1,0<x<1$

$Y \sim U\left(0, \frac{\pi}{2}\right)$ with density $f(y)=\frac{2}{\pi}, 0<y<\frac{\pi}{2}$

$X \perp Y \quad$ (i.e. $X$ and $Y$ are independent, so that

$$
\begin{gathered}
\left.f(x, y)=f(x) f(y)=1 \times \frac{2}{\pi}, 0<x<1,0<y<\frac{\pi}{2}\right) \\
C=\{X<\sin Y\}=\{(x, y): x<\sin y\} .
\end{gathered}
$$

Figure 5.8 illustrates this setup.

It follows that

$$
\begin{aligned}
p & =P(C)=P(X<\sin Y) \\
& \left.=\iint_{x<\sin y} f(x, y) d x d y=\frac{2}{\pi} \int_{y=0}^{\pi / 2} \int_{x=0}^{\sin y} d x\right) d y=\frac{2}{\pi} \int_{y=0}^{\pi / 2} \sin y d y \\
& =\frac{2}{\pi}\left[-\left.\cos y\right|_{0} ^{\pi / 2}\right]=\frac{2}{\pi}\left(-\cos \left(\frac{\pi}{2}\right)-(-\cos 0)\right) \\
& =\frac{2}{\pi}(-0-(-1))=\frac{2}{\pi}=0.63662 .
\end{aligned}
$$

Figure 5.9 illustrates the integration here. 
Figure 5.8 Illustration of Buffon's needle problem

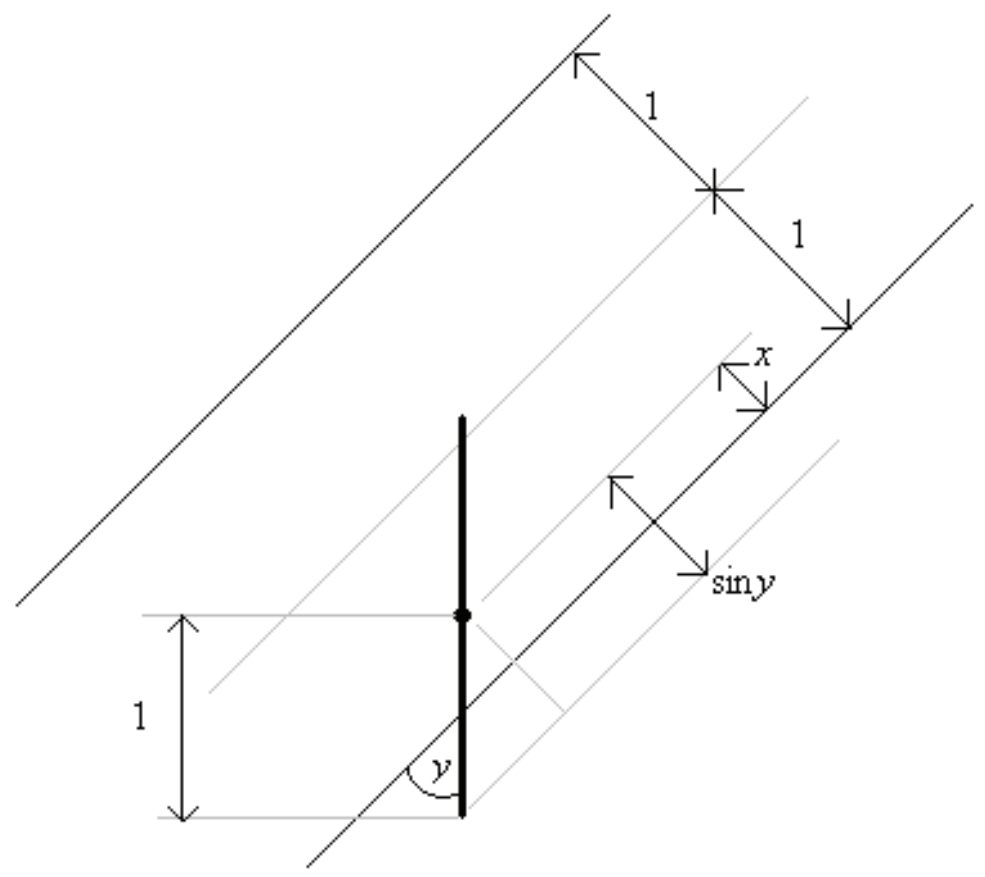

Figure 5.9 Illustration of the solution to Buffon's needle problem

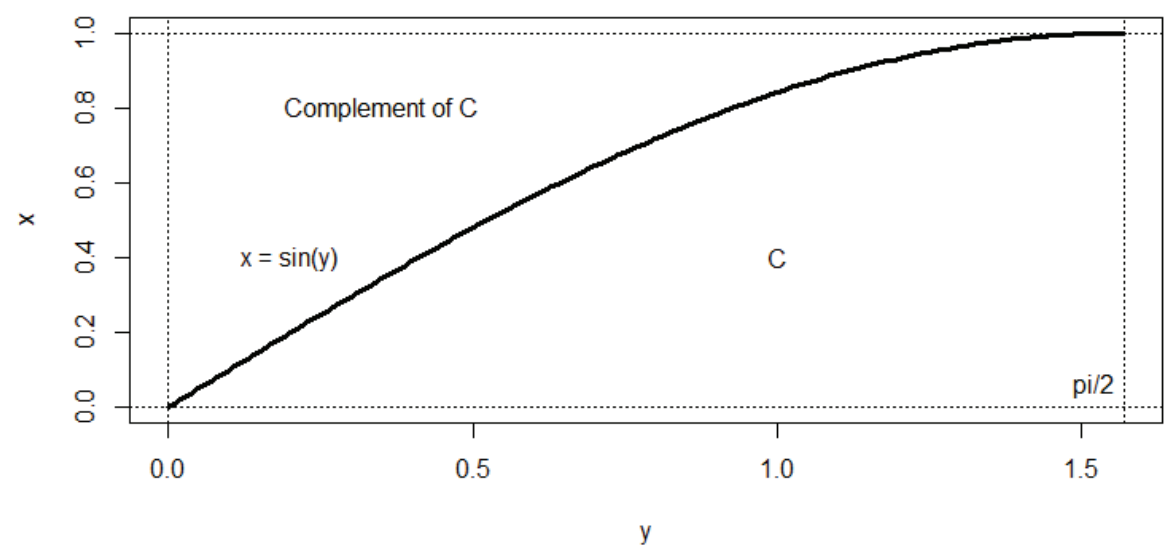


Note 1: Another way to express the above working is to first note that $P(C \mid y) \equiv P(C \mid Y=y)=P(X<\sin y \mid y)=P(X<\sin y)=\sin y$, since $(X \mid y) \sim X \sim U(0,1)$ with $\operatorname{cdf} F(x \mid y)=F(x)=x, 0<x<1$.

It follows that

$$
p=P(C)=E P(C \mid Y)=E \sin Y=\int_{0}^{\pi / 2}(\sin y) \frac{2}{\pi} d y=\frac{2}{\pi},
$$

as before.

Note 2: It can be shown that if the length of the needle is $r$ times the distance between lines, then the probability that the needle will cross a line is given by the formula

$$
p=\left\{\begin{array}{cc}
2 r / \pi, & r \leq 1 \\
1-\frac{2}{\pi}\left(\sqrt{r^{2}-1}-r+\sin ^{-1}\left(\frac{1}{r}\right)\right), & r>1 .
\end{array}\right.
$$

(b) For this part, we will make use of the analysis in (a) whereby

$$
C=\{(x, y): x<\sin y\},
$$

and where:

$$
x \sim U(0,1), \quad y \sim U\left(0, \frac{\pi}{2}\right), \quad X \perp Y .
$$

Note: We suppose that these facts are understood but that the integration required to then proceed on from these facts to the final answer (as in (a)) is too difficult.

We now sample $x_{1}, \ldots, x_{J} \sim$ iid $U(0,1)$ and $y_{1}, \ldots, y_{J} \sim$ iid $U(0, \pi / 2)$ (all independently of one another). Next, we obtain the indicators defined by

$$
r_{j}=I\left(x_{j}<\sin y_{j}\right)=\left\{\begin{array}{cc}
1 & \text { if } x_{j}<\sin y_{j} \\
0 & \text { otherwise. }
\end{array}\right.
$$

The result is the MC sample $r_{1}, \ldots, r_{J} \sim$ iid $\operatorname{Bern}(p)$ (i.e. a sample of size $J$ to be used for inference on $p$ ). (Equivalently, we may obtain $r_{T} \equiv r_{1}+\ldots+r_{J} \sim \operatorname{Bin}(J, p)$, which will lead to the same final results.) 
The MC estimate of $p$ is $\hat{p}=\bar{r}=\frac{1}{J} \sum_{j=1}^{J} r_{j}=\frac{r_{T}}{J}$, and a $95 \%$ CI for $p$ is $C I=\left(\hat{p} \pm z_{\alpha / 2} \sqrt{\frac{\hat{p}(1-\hat{p})}{J}}\right)$.

Carrying out this experiment in R with $J=1,000$ we get $\hat{p}=0.618$ and $C I=(0.588,0.648)$.

Then repeating, but with $J=10,000$ instead, we obtain

$$
\hat{p}=0.633 \text { and } C I=(0.624,0.643) \text {. }
$$

We see that increasing the MC sample size (from 1,000 to 10,000) has reduced the width of the MC CI from 0.060 to 0.019. Both intervals contain the true value, namely $2 / \pi=0.6366$.

\section{R Code for Exercise 5.6}

\section{\# (a)}

$\mathrm{X} 11(\mathrm{w}=8, \mathrm{~h}=4.5) ; \operatorname{par}(\operatorname{mfrow}=\mathrm{c}(1,1))$

plot(seq(0,pi/2,0.01), $\sin ($ seq( $(0, p i / 2,0.01))$, type="l",Iwd=3,xlab="y", ylab="x") abline $(v=c(0, p i / 2)$, Ity=3); abline $(h=c(0,1)$, Ity=3)

text(0.2,0.4," $x=\sin (y) ") ; \operatorname{text}(1,0.4, " C ") ; \operatorname{text}(0.35,0.8, "$ Complement of C") text(1.52,0.06,"pi/2")

\# (b)

$\mathrm{J}=1000$; set.seed(213); $\mathrm{xv}=\operatorname{runif}(\mathrm{J}, 0,1) ; \mathrm{yv}=\operatorname{runif}(\mathrm{J}, 0, \mathrm{pi} / 2) ; \mathrm{rv}=\operatorname{rep}(0, \mathrm{~J})$ options(digits=4); for(j in 1:J) if $(x v[j]<\sin (y v[j])) r v[j]=1$

phat=mean(rv); z=qnorm(0.975); pci=phat+c(-1,1)*z*sqrt(phat*(1-phat)/J) c(phat,pci,pci[2]-pci[1]) \# 0.618000 .587890 .648110 .06023

$\mathrm{J}=10000$; set.seed(215); $\mathrm{xV=runif(J,0,1);yv=runif(J,0,pi/2);rv=rep(0,J)}$ for(j in 1:J) if $(x v[j]<\sin (y v[j])) r v[j]=1$

phat=mean(rv); $z=$ qnorm(0.975); pci=phat+c(-1,1)*z*sqrt(phat*(1-phat)/J) c(phat,pci,pci[2]-pci[1]) \# 0.633200 .623750 .642650 .01889 


\section{Exercise 5.7 MC Cls for the coverage probabilities of $\mathrm{MC} \mathrm{Cls}$ for a gamma mean}

(a) Using the $\mathrm{R}$ function rgamma(), generate a random sample of size $J=100$ from the gamma distribution with parameters 3 and 2 and mean $\mu=3 / 2$. Then use the method of Monte Carlo to estimate $\mu$. In your estimation, include a $95 \% \mathrm{CI}$ for $\mu$ and the width of this CI. Also report whether the CI contains the true value of $\mu$.

(b) Repeat (a) but with $J=200,500,1,000,10,000$ and 100,000, respectively. Report the widths of the resulting CIs and, for each CI, state whether it contains $\mu$. Discuss any patterns that you see.

(c) Repeat (a) $M=100$ times and report the proportion of the resulting $M$ 95\% MC CIs which contain the true value of the mean. (In each case use $J=100$.) Hence calculate a 95\% CI for $p$, the true coverage probability of the $95 \%$ MC CI for $\mu$ based on a MC sample of size $J=100$ from the Gamma(3,2) distribution.

(d) Repeat (c), but with $M=200,500,1,000$ and 10,000, respectively. Discuss any patterns that you see.

\section{Solution to Exercise 5.7}

(a) Applying the procedure (see the $\mathrm{R}$ code below) we estimate $\mu$ by $\bar{X}=1.517$. The Monte Carlo 95\% confidence interval for $\mu$ is

$$
C I=\left(\bar{x} \pm z_{0.025} S / \sqrt{J}\right)=(1.354,1.680) .
$$

We observe that this interval has width 0.326 and contains $\mu$.

(b) Repeating (a) as required, we obtain:

$$
\begin{aligned}
& \bar{X}=1.471 \text { and } C I=(1.348,1.593) \text { with width } 0.245 \text { for } J=200 \\
& \bar{X}=1.430 \text { and } C I=(1.358,1.502) \text { with width } 0.144 \text { for } J=500 \\
& \bar{X}=1.475 \text { and } C I=(1.419,1.530) \text { with width } 0.111 \text { for } J=1,000 \\
& \bar{X}=1.490 \text { and } C I=(1.473,1.508) \text { with width } 0.0344 \text { for } J=10,000 \\
& \bar{X}=1.502 \text { and } C I=(1.497,1.507) \text { with width } 0.0107 \text { for } J=100,000 \text {. }
\end{aligned}
$$

We see that $\bar{X}$ appears to be converging towards $\mu=1.5$. The width of the CI appears to be decreasing as $J$ increases. Each of these five CIs contains $\mu$, just like the $\mathrm{CI}$ in (a). 
(c) Repeating (a) $M=100$ times leads to $M=100$ MC CIs of which 93 contain $\mu=1.5$. Thus $\hat{p}=93 \%$, which as expected is 'close' to the $95 \%$ nominal coverage of the $\mathrm{CI}$.

A $95 \%$ CI for $p$ is $\left(0.93 \pm 1.96 \sqrt{\frac{0.93(1-0.93)}{100}}\right)=\left(\begin{array}{ll}0.880 & 0.980\end{array}\right)$.

This is consistent with the MC 95\% CI for $\mu$ having coverage 95\%.

(d) Repeating (a) $M=200$ times leads to $\hat{p}=94.5 \%$ of the 200 CIs containing 1.5 , with a $95 \%$ CI for $p$,

$$
\left(0.945 \pm 1.96 \sqrt{\frac{0.945(1-0.945)}{200}}\right)=(0.913,0.977) \text {. }
$$

Repeating (a) $M=500$ times leads to $\hat{p}=94.2 \%$ of the 500 CIs containing 1.5 with a $95 \%$ CI for $p$,

$$
\left(0.942 \pm 1.96 \sqrt{\frac{0.942(1-0.942)}{500}}\right)=(0.922,0.962) \text {. }
$$

Repeating (a) $M=1,000$ times leads to $\hat{p}=93.5 \%$ of the 1,000 CIs containing 1.5 , with a $95 \%$ CI for $p$,

$$
\left(0.935 \pm 1.96 \sqrt{\frac{0.935(1-0.935)}{1,000}}\right)=(0.935,0.963) \text {. }
$$

Repeating (a) $M=10,000$ times leads to $\hat{p}=94.4 \%$ of the 10,000 CIs containing 1.5 , with a $95 \%$ CI for $p$,

$$
\left(0.944 \pm 1.96 \sqrt{\frac{0.94(1-0.94)}{10,000}}\right)=(0.940,0.949) \text {. }
$$

The widths of all five CIs for $p$ are: $0.100,0.063,0.041,0.027$ and 0.009 . We see that the CI for $p$ becomes narrower as $M$ increases. Also, the proportion of CIs containing 1.5 converges towards $95 \%$ as $M$ increases. The convergence does not seem to be uniform. This is because of Monte Carlo error. If we repeated the experiment again, we might find a slightly different pattern.

Each of the CIs for $p$ is consistent with $p=0.95$, except the one with $M=10,000$, which is the most reliable. In that case the CI for $p$ is 
(0.940, 0.949), which is entirely below 0.95 . This suggests that the true coverage probability of the $95 \%$ MC CI for $\mu$ is slightly less than $95 \%$.

The observed proportions appear to be converging to this limit rather than to $95 \%$ exactly. This is explainable by the fact that the MC sample size $J=100$ is far from infinity. If we repeated (d) with a larger value of $J$ in each case, say $J=1,000$, we would see the proportion of the $M$ CIs converge towards a limiting value which is even closer to $95 \%$. But then an even larger value of $M$ would be necessary to establish that there is in fact any difference between the limiting value and $95 \%$.

\section{R Code for Exercise 5.7}

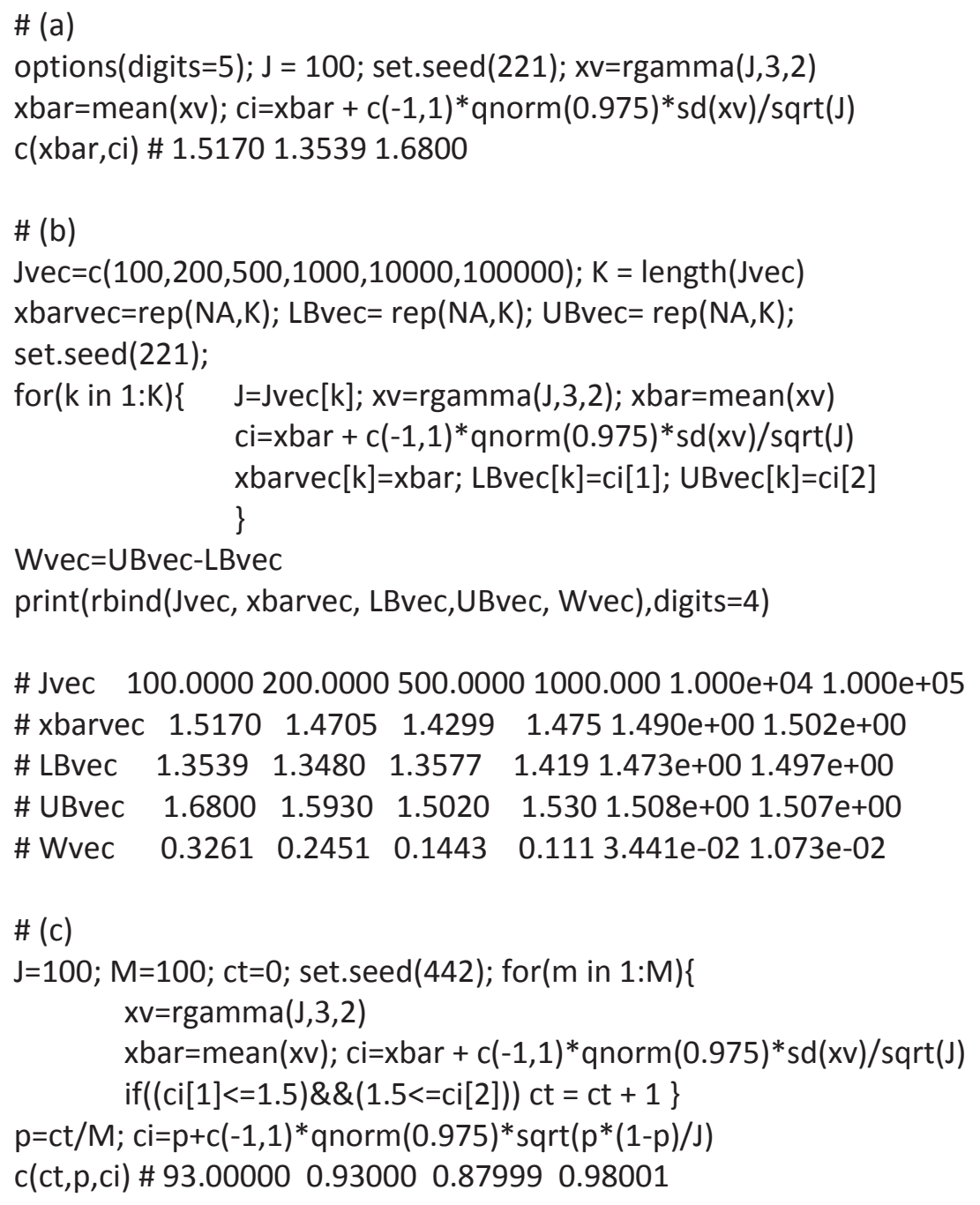




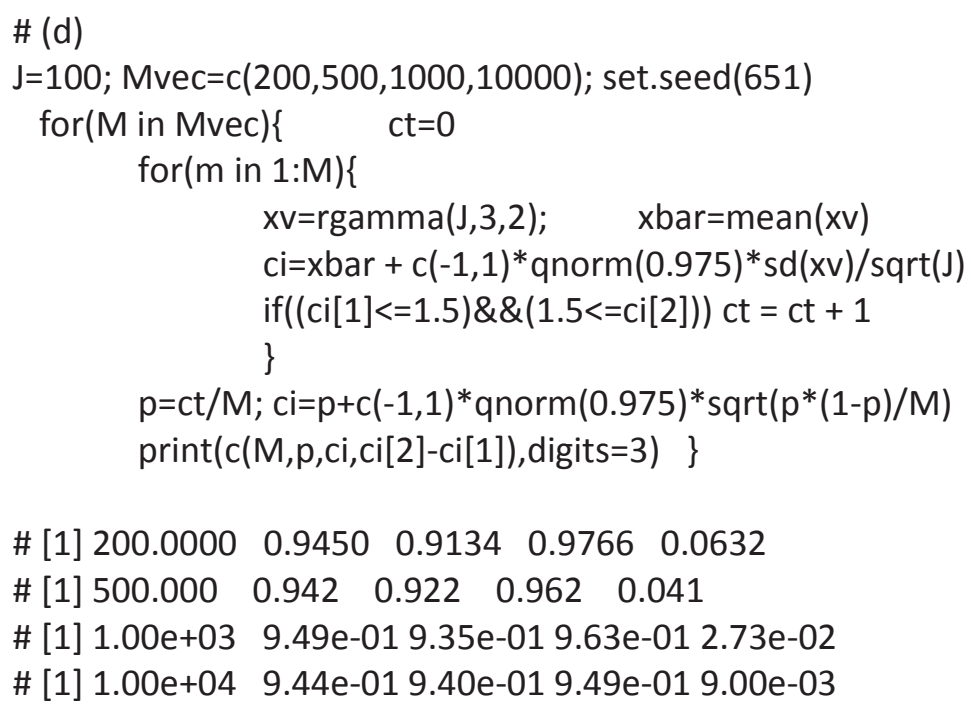

\subsection{Random number generation}

So far we have assumed the availability of the sample required for Monte Carlo estimation, such as $x_{1}, \ldots, x_{J} \sim$ iid $f(x)$. The issue was skipped over by making use of ready made functions in R such as runif(), rbeta() and rgamma(). However, many applications involve dealing with complicated distributions from which sampling is not straightforward.

So we will next discuss some basic techniques that can be used to generate the required Monte Carlo sample from a given distribution. More advanced techniques will be treated later. We will first treat the discrete case, which is the simplest, and then the continuous case. It will be assumed throughout that we can at least sample easily from the standard uniform distribution, i.e. that we can readily generate $u \sim U(0,1)$.

Note: This sampling is easily achieved using the runif() function in R. Alternatively, it can be done physically by using a hat with 10 cards in it, where these have the numbers $0,1,2, \ldots ., 9$ written on them. Three cards (say) are drawn out of the hat, randomly and with replacement. The three numbers thereby selected are written down in a row, and a decimal point is placed in front of them. The resulting number (e.g. $0.472,0.000$ or 0.970 ) is an approximate draw from the standard uniform distribution. Repeating the entire procedure several times results in a random sample from that distribution. Increasing 'three' above (to 'five', say) improves the approximation (e.g. yielding $0.47207,0.00029$ or 0.97010 ). 


\subsection{Sampling from an arbitrary discrete distribution}

Suppose we wish to sample a value $x \sim f(x)$ where $f(x)$ is a discrete pdf defined over the possible values $x=x_{1}, \ldots, x_{K}$. First define

$$
f_{k}=f\left(x_{k}\right)
$$

and

$$
F_{k}=f_{1}+\ldots+f_{k}(k=1, \ldots, K),
$$

noting that $F_{K}=1$.

Then sample $u \sim U(0,1)$, and finally return:

$$
\begin{array}{ll}
x=x_{1} & \text { if } 0 \leq u \leq F_{1} \\
x=x_{2} & \text { if } F_{1}<u \leq F_{2} \\
\ldots \ldots \ldots \ldots \ldots \ldots \ldots \ldots \ldots \ldots \ldots \ldots \ldots \ldots \ldots \ldots \ldots \ldots \ldots \ldots \ldots & \ldots \ldots \ldots
\end{array}
$$

One way to implement the above is to set $k=1$, to repeatedly increment $k$ by 1 until $F_{k-1}<u \leq F_{k}$, and then, using the final value of $k$ thereby obtained, to return $x=x_{k}$.

Note 1: We see that this procedure will work also in the case where $K$ is infinite. In that case a practical alternative is to redefine $K$ as a value $k$ for which $F_{k}$ is very close to 1 (e.g. 0.9999) and then approximate $f(x)$ by zero for all $x>x_{K}$.

Note 2: In R, an alternative to using $u \sim U(0,1)$ is to apply the function sample() with appropriate specifications of $x_{1}, \ldots, x_{K}$ and $f_{1}, \ldots, f_{K}$ (as illustrated in an exercise below).

\section{Exercise 5.8 Example of sampling from a simple discrete distribution}

Show that the above method works when applied to generating a value $x$ from the $\operatorname{Bin}(2,1 / 2)$ distribution, i.e. that it returns $x=0,1$ and 2 with probabilities 1/4, 1/2 and 1/4, respectively. 


\section{Solution to Exercise 5.8}

In this case, $K=3$ and: $\quad x_{1}=0, \quad F\left(x_{1}\right)=P(x \leq 0)=0.25$

$$
\begin{array}{ll}
x_{2}=1, & F\left(x_{2}\right)=P(x \leq 1)=0.75 \\
x_{3}=2, & F\left(x_{3}\right)=P(x \leq 2)=1.00 .
\end{array}
$$

Let $u \sim U(0,1)$. Then the method returns:

$$
\begin{array}{lllll}
x=x_{1}=0 & \text { if } & 0<u<F\left(x_{1}\right) & \text { i.e. if } & 0.00<u<0.25 \\
x=x_{2}=1 & \text { if } & F\left(x_{1}\right)<u<F\left(x_{2}\right) & \text { i.e. if } & 0.25<u<0.75 \\
x=x_{3}=2 & \text { if } & F\left(x_{2}\right)<u<F\left(x_{3}\right) & \text { i.e. if } & 0.75<u<1.00 .
\end{array}
$$

Thus, $x$ has: $\quad 0.25-0.00=0.25$ probability of being set to 0

$0.75-0.25=0.50$ probability of being set to 1

$1.00-0.75=0.25$ probability of being set to 2 (all correct).

\section{Exercise 5.9 Sampling from a complicated discrete distribution}

Consider the discrete distribution defined by the pdf

$$
f(x) \propto \frac{x^{3} e^{-x}}{1+\sqrt{x}}, x=1,3,5, \ldots
$$

Find the mean of the distribution by performing appropriate summations. Then generate a random sample from this distribution and use it to confirm the mean.

\section{Solution to Exercise 5.9}

Using $\mathrm{R}$ we calculate $k(x)=\frac{x^{3} e^{-x}}{1+\sqrt{x}}, x=1,3,5, \ldots, 41$ (here $k$ stands for kernel), noting that the last two values of $k(x)$ are tiny $(9.455201 \mathrm{e}-14$ and 1.454999e-14).

We then calculate the sum of the kernel values,

$$
c=k(1)+k(3)+\ldots+k(41)=1.051009 \text {, }
$$

and thereby normalise the kernel to obtain

$$
f(x)=\frac{k(x)}{c}, x=1,3,5, \ldots, 41 .
$$


The pdf may also be written as $f(x)=k(x) / c, x=x_{1}, \ldots, x_{K}$, where: $x_{k}=2 k-1 ; k=1, \ldots, K ; K=21$. The exact mean of the distribution is then evaluated numerically as

$$
\mu=\sum_{k=1}^{K} x_{k} f\left(x_{k}\right)=3.6527 \text {. }
$$

Note: Changing 41 to 101 here changes the approximation to 3.6527, i.e. makes no difference to 4 decimals. This suggests that taking the upper bound as 41 is good enough.

To sample $J=100,000$ values from the distribution we may write sample $(x=x v e c$, size $=J$, replace $=T R U E$, prob=fvec)

where xvec is a vector with values $1,3, \ldots, 41$ and fvec is a vector with the values $f(1), f(3), \ldots, f(41)$ (see the R Code below).

Note: We could also change fvec to kvec here, where kvec is a vector with the values $k(1), k(3), \ldots, k(41)$; both possibilities will work since sample() will automatically normalise the values in its parameter 'prob'.

The Monte Carlo estimate of $\mu$ works out as 3.6494 with 95\% CI (3.6374, 3.6615). We note that this CI contains the true value, 3.6527.

\section{R Code for Exercise 5.9}

kfun $=$ function $(x)\left\{x^{\wedge} 3^{*} \exp (-x) /(1+\operatorname{sqrt}(x))\right\} ;$ options(digits=5)

$x v e c=s e q(1,41,2) ; k v e c=k f u n(x v e c) ; c=s u m(k v e c) ; c$ \# 1.051

fvec=kvec/c; sum(fvec) \# 1

print(rbind(xvec,fvec) [,1:9], digits=3)

\# xvec $1.0003 .0005 .0007 .00009 .000011 .000013 .000001 .50 \mathrm{e}+011.70 \mathrm{e}+01$ \# fvec 0.1750 .4680 .2480 .08160 .02140 .00490 .00103 2.02e-04 3.78e-05 sum(xvec*kvec)/sum(kvec) \# 3.6527

\# Check that 41 is large enough:

$\mathrm{xvec}=\operatorname{seq}(1,101,2) ; \mathrm{kvec}=\mathrm{kfun}(\mathrm{xvec}) ; \operatorname{sum}\left(\mathrm{xvec}^{*} \mathrm{kvec}\right) / \mathrm{sum}(\mathrm{kvec})$

\# 3.6527 (same)

\# Sample from the distribution

xvec=seq(1,41,2); kvec=kfun(xvec); J=100000; set.seed(332);

samp = sample $(x=x v e c$, size $=J$, replace $=T R U E$, prob $=f v e c)$

est =mean $($ samp $) ; \operatorname{std}=s d(\operatorname{samp}) ;$ ci $=e s t+c(-1,1) *$ qnorm $(0.975) *$ std $/$ sqrt $(J)$

c(est,ci) \# 3.64943 .63743 .6615 


\section{I 0 The inversion technique}

Suppose we wish to sample $x$, a value of a continuous random variable $X$ with cdf $F_{X}(x)$. One way to do this is using the inversion technique, defined as follows, with the underlying theorem and proof shown below.

First derive the quantile function of $X$, denoted $F_{X}^{-1}(p)(0<p<1)$. (This can be done by setting $F_{X}(x)$ to $p$ and solving for $x$.)

Next, generate a random number $u$ from the standard uniform distribution. (It will be assumed that this can be done easily, e.g. using runif() in R.)

Then return $x=F_{X}^{-1}(u)$ as a value sampled from the distribution of $X$.

Theorem 5.I: Suppose that $X$ is a continuous random variable with cdf $F_{X}(x)$ and quantile function $F_{X}^{-1}(p)$. Let $U \sim U(0,1)$, independently of $X$, and define $R=F_{X}^{-1}(U)$. Then $R$ has the same distribution as $X$.

Proof of Theorem 5.I: Observe that $U$ has cdf $F_{U}(u)=u, 0<u<1$. This implies that $R$ has cdf

$$
F_{R}(r)=P(R \leq r)=P\left(F_{X}\left(F_{X}^{-1}(U)\right) \leq F_{X}(r)\right)=P\left(U \leq F_{X}(r)\right)=F_{X}(r) .
$$

Thus, $R$ has the same cdf as $X$ and therefore the same distribution.

Note: A complication with the inversion technique may arise if there is difficulty deriving the quantile function $F_{X}^{-1}(p)$. In that case, since the task is fundamentally to solve $F_{X}(x)=u$ for $x$, it may be useful to employ the Newton-Raphson algorithm to the problem of solving the equation $g(x)=0$, where $g(x)=F_{X}(x)-u$.

\section{Exercise 5.10 Practice at the inversion technique}

(a) Using $u=0.371$ as a value from the standard uniform distribution, obtain a value from the standard exponential distribution. Then generate a large random sample $u_{1}, \ldots, u_{J} \sim$ iid $U(0,1)$ (of size $J=1,000$ say) and use this to create a random sample of the same size from the standard exponential distribution. Check your results by calculating an estimate of the mean of that distribution and also a 95\% CI for that mean. Compare your results with the true value of that mean, namely 1 . 
(b) Using $u=0.371$ as a value from the standard uniform distribution, obtain a value from the gamma distribution with mean and variance both equal to 2 . Then generate a large random sample $u_{1}, \ldots, u_{K} \sim$ iid $U(0,1)$ (of size $J=1,000$, say) and use this to create a random sample of the same size from the said gamma distribution. Check your results by calculating an estimate of the mean of that distribution and also a 95\% CI for that mean. Compare your results with the true value, namely 2 .

\section{Solution to Problem 5. 10}

(a) Let $X \sim G(1,1)$ with density function $f(x)=e^{-x}, x>0$, and cdf $F(x)=\int_{0}^{x} e^{-t} d t=1-e^{-x}, x>0$. The quantile function here is the solution of $1-e^{-x}=p$, namely $F^{-1}(p)=-\log (1-p)$.

So a value from the standard exponential distribution is easily computed as $x=F^{-1}(u)=-\log (1-0.371)=0.463624$.

Taking $J=1,000$, we now generate $u_{1}, \ldots, u_{J} \sim$ iid $U(0,1)$ in $\mathrm{R}$ using the runif() function, and then calculate $x_{j}=-\log \left(1-u_{j}\right)$ for each $j=1, \ldots, J$.

This results in the required sample $X_{1}, \ldots, X_{J} \sim$ iid $G(1,1)$. Using this sample, the MC estimate of $\mu=E X$ is 0.9967 , and a $95 \%$ CI for $\mu$ is $(0.9322,1.0613)$. We see that the CI contains the true value being estimated (i.e. 1).

(b) Here, $X \sim G(2,1)$ with mean $2 / 1$, variance $2 / 1^{2}=2$, pdf $f(x)=x e^{-x}$ and cdf

$$
\begin{aligned}
F(x) & =\int_{0}^{x} t e^{-t} d t=\left[\left.t\left(-e^{-t}\right)\right|_{0} ^{x}\right]-\int_{0}^{x} 1\left(-e^{-t}\right) d t \\
& =-x e^{-x}+0+\left[-\left.e^{-t}\right|_{0} ^{x}\right]=-x e^{-x}-e^{-x}+1=1-(x+1) e^{-x} .
\end{aligned}
$$

We see that the quantile function of $X, F^{-1}(p)$, does not have a closed form expression, since it is the root of the function

$$
g(x)=F(x)-p=1-(x+1) e^{-x}-p
$$

(i.e. the solution of $g(x)=0)$. 
However, for any $p$ we can obtain that root using the Newton-Raphson algorithm by iterating

$$
\begin{aligned}
x_{j+1} & =x_{j}-\frac{g\left(x_{j}\right)}{g^{\prime}\left(x_{j}\right)} \quad \text { where } g^{\prime}(x)=F^{\prime}(x)-0=f(x)=x e^{-x} \\
& =x_{j}-\left(\frac{1-\left(x_{j}+1\right) e^{-x_{j}}-p}{x_{j} e^{-x_{j}}}\right) .
\end{aligned}
$$

With $p=u=0.371$ and starting arbitrarily at $x_{0}=1$, we get the sequence:

$$
1.0000,1.2902,1.2939,1.2939,1.2939,1.2939,1.2939 \ldots . .
$$

So we return 1.2939 as a value from the $G(2,1)$ distribution.

As a check, we use the pgamma() function in $\mathrm{R}$ to confirm that $F_{X}(1.2939)=0.371$ as follows:

pgamma(1.2939,2,1) \# 0.37101

Taking $K=1,000$, we now generate $u_{1}, \ldots, u_{K} \sim$ iid $U(0,1)$ in $\mathrm{R}$ using the runif() function, and then for $k=1, \ldots, K$ we solve

$$
1-\left(x_{k}+1\right) e^{-x_{k}}=u_{k} \text { for } x_{k}
$$

using the NR algorithm each time. This procedure results in the sample,

$$
x_{1}, \ldots, x_{K} \sim \text { iid } G(2,1) \text {. }
$$

Using this sample, an estimate of $\mu=E X$ is 1.9631, and a 95\% CI for $\mu$ is $(1.8815,2.0446)$. We see that the CI contains the true value, 2.

\section{R Code for Problem 5.10}

options(digits=5)

$\#($ a)

$-\log (1-0.371) \# 0.463624$

$\mathrm{J}=1000$; set.seed(221); uv=runif(J,0,1)

$\mathrm{xv}=-\log (1-\mathrm{uv})$ \# Generate a random sample of size 1000 from the $\mathrm{G}(1,1) \mathrm{dsn}$ est=mean(xv); std=sd(xv); ci=est+c(-1,1)*qnorm(0.975)*std/sqrt(J)

c(est,ci) \# 0.996730 .932161 .06130 
\# (b)

$\mathrm{u}=0.371 ; \mathrm{x}=1 ; \mathrm{xv}=\mathrm{x}$; for $(\mathrm{j}$ in $1: 7)\left\{\mathrm{x}=\mathrm{x}-\left(1-(\mathrm{x}+1)^{*} \exp (-\mathrm{x})-\mathrm{u}\right) /(\mathrm{x} * \exp (-\mathrm{x})) ; \mathrm{xv}=\mathrm{c}(\mathrm{xv}, \mathrm{x})\right\}$ $x v$ \# 1.00001 .29021 .29391 .29391 .29391 .29391 .29391 .2939

pgamma $(x, 2,1) \# 0.371 \quad$ Just checking that $F(1.293860)=0.371$

pgamma(1.2939,2,1) \# 0.37101

$\mathrm{K}=1000 ; \mathrm{xvec}=\operatorname{rep}(\mathrm{NA}, \mathrm{K})$; set.seed(332); for $(\mathrm{k}$ in $1: \mathrm{K})\{$

$\mathrm{u}=\operatorname{runif}(1) ; \mathrm{x}=1$; for $(\mathrm{j}$ in $1: 10) \mathrm{x}=\mathrm{x}-\left(1-(\mathrm{x}+1)^{*} \exp (-\mathrm{x})-\mathrm{u}\right) /\left(\mathrm{x}^{*} \exp (-\mathrm{x})\right)$

$x v e c[k]=x \quad$ \# Generate a random sample of size 1000 from the $G(2,1)$ dsn

est=mean(xvec); std=sd(xvec)

ci=est+c(-1,1)*qnorm(0.975)*std/sqrt(K)

c(est,ci) \# 1.96311 .88152 .0446

\section{I I Random number generation via compositions}

Sometimes the most convenient way to sample from a distribution is to express it as a function (or composition) of two or more random variables which are easy to sample from. For example, to obtain two independent values from the standard normal distribution we may use the well-known Box-Muller algorithm, as follows.

Sample $u_{1}, u_{2} \sim$ iid $U(0,1)$ and let:

$$
\begin{aligned}
& z_{1}=\sqrt{-2 \log u_{1}} \cos \left(2 \pi u_{2}\right) \\
& z_{2}=\sqrt{-2 \log u_{1}} \sin \left(2 \pi u_{2}\right) .
\end{aligned}
$$

It can be shown that $z_{1}, z_{2} \sim$ iid $N(0,1)$. If we only need one value from the standard normal distribution then we may arbitrarily discard $z_{2}$ and return only $z_{1}$.

\section{Exercise 5. I I Sampling from the double exponential distribution}

Suppose we wish to sample a value $x \sim f(x)$, where

$$
f(x)=(1 / 2) e^{-|x|}, x \in \mathfrak{R} \text {. }
$$

Describe how to obtain $x$ as a composition of two other values than can be easily sampled. 


\section{Solution to Exercise 5.I I}

Let $R$ and $Y$ be independent random variables such that $R \sim \operatorname{Bern}(0.5)$ and $Y \sim G(1,1)$. Then $U=(2 R-1) Y$ has the same distribution as $X$.

This is because $R$ is equally likely to be 0 as it is to be 1 , and so $2 R-1$ is equally likely to be -1 as it is to be +1 . So there is a $50 \%$ chance that $U$ will be exponential $(G(1,1))$ and a $50 \%$ chance that $U$ will be negative exponential. So, obviously $U$ has exactly the same distribution as $X$. For a formal proof, see the Note below.

We see that a method for obtaining a value $x \sim f(x)$ is to independently sample $r \sim \operatorname{Bern}(0.5)$ and $y \sim G(1,1)$, and then calculate $x=(2 r-1) y$.

Note: The cdf of $U=(2 R-1) Y$ is

$$
\begin{aligned}
F(u)= & P(U \leq u) \\
= & P((2 R-1) Y \leq u) \\
= & E P((2 R-1) Y \leq u \mid R) \\
= & P(R=0) P((2 R-1) Y \leq u \mid R=0) \\
& \quad+P(R=1) P((2 R-1) Y \leq u \mid R=1) \\
= & \frac{1}{2} P(-Y \leq u \mid R=0)+\frac{1}{2} P(+Y \leq u \mid R=1) \\
= & \frac{1}{2} P(Y \geq-u)+\frac{1}{2} P(Y \leq u) \\
= & \left\{\begin{array}{cc}
(1 / 2) e^{-(-u)}+(1 / 2)(0), \quad u<0 \\
(1 / 2)(1)+(1 / 2)\left(1-e^{-u}\right), \quad u \geq 0
\end{array}\right. \\
= & \left\{\begin{array}{c}
(1 / 2) e^{u}, \quad u<0 \\
1-(1 / 2) e^{-u}, \quad u \geq 0 .
\end{array}\right.
\end{aligned}
$$

So $U$ has pdf $f(u)=F^{\prime}(u)=\left\{\begin{array}{c}(1 / 2) e^{u}, \quad u<0 \\ 0-(1 / 2) e^{-u}(-1), \quad u \geq 0\end{array}\right\}$.

That is, $f(u)=\frac{1}{2} e^{-|u|},-\infty<u<\infty$, which is the same the pdf of $X$. 


\section{Exercise 5.I 2 Sampling from a triangular distribution}

Suppose we want to sample $x \sim f(x)$ where $f(x)=\left\{\begin{array}{cc}x, & 0<x<1 \\ 2-x, & 1<x<2\end{array}\right\}$.

Describe how two random variables can be combined to obtain $x$.

\section{Solution to Exercise 5.12}

Sample the two random variables $r \sim \operatorname{Bern}(0.5)$ and $y \sim \operatorname{Beta}(2,1)$. Then calculate $x=r y+(1-r)(2-y)$. This way, there is a $50 \%$ chance that $x$ will equal $y$, whose pdf is $f(y)=2 y, 0<y<1$, and a $50 \%$ chance that $x$ will equal $z=2-y$, whose pdf is $f(z)=2(2-z), 1<z<2$.

A second solution is as follows. Sample $u_{1}, u_{2} \sim$ iid $U(0,1)$ and calculate $x=u_{1}+u_{2}$. It can easily be shown that a value of $x$ formed in this way has the triangular pdf in question.

\section{I 2 Rejection sampling}

Some distributions are difficult to sample from using any of the already mentioned methods. For example, when applying the inversion technique, solving the equation $F(x)=u$ may be problematic even with the aid of the Newton-Raphson algorithm (e.g. due to instability unless starting at very close to the solution).

In such cases, one convenient and easy way to obtain a value from the distribution of interest may be via rejection sampling (also known as the rejection method or the acceptance-rejection method). This method works as follows.

Suppose we want to generate a random number from a target distribution with density $f(x)$. This target distribution may be continuous or discrete.

We must first decide on a suitable envelope distribution with envelope density $h(x)$. (These are also called the majorising distribution and majorising density.) Ideally, the chosen density $h(x)$ is similar in shape to $f(x)$ and relatively easy to sample from. 
We next define the following quantities:

$$
\begin{aligned}
& c=\max _{x}\left(\frac{f(x)}{h(x)}\right) \\
& p(x)=\frac{f(x)}{c h(x)} .
\end{aligned}
$$

The idea here is that $f(x)$ lies entirely beneath $\operatorname{ch}(x)$ except that it touches $\operatorname{ch}(x)$ at maybe only one point. Then $p(x)$, which is called the acceptance probability, appropriately lies between 0 and 1 (inclusive). Figure 5.10 illustrates this setup. The rejection algorithm is as follows:

1. Sample a proposed value (or candidate) $\quad x^{\prime} \sim h(x)$.

2. Calculate the acceptance probability $p=p\left(x^{\prime}\right)=\frac{f\left(x^{\prime}\right)}{\operatorname{ch}\left(x^{\prime}\right)}$.

3. Generate a standard uniform value $u \sim U(0,1)$.

4. Decide whether to accept or reject the candidate, as follows:

If $u<p$ then accept $x^{\prime}$, meaning return $x=x^{\prime}$ and STOP. If $u>p$ then reject $x^{\prime}$, meaning go to Step 1 and REPEAT.

Steps 1 to 4 are repeated as many times as necessary until an acceptance occurs, resulting in $x=x^{\prime}$. The finally accepted value $x$ is an observation from $f(x)$. Repeating the entire procedure above another $J-1$ times independently will result in a random sample of size $J$ from $f(x)$.

Figure 5.10 illustrates, with:

$$
\begin{aligned}
& f(x)=\text { density of the } \operatorname{Beta}(4,8) \text { distribution } \\
& h(x)=\text { density of the } \operatorname{Beta}(2,2) \text { distribution } \\
& c=\max _{x}\left(\frac{f(x)}{h(x)}\right)=2.45 \\
& x^{\prime}=0.4 \quad \text { (example of a candidate) } \\
& p=p\left(x^{\prime}\right)=\frac{f\left(x^{\prime}\right)}{c h\left(x^{\prime}\right)}=\frac{2.365}{3.524}=0.671 .
\end{aligned}
$$

In this case, if we sample $u=0.419$ (for example), then we accept $x^{\prime}$ and return $x=0.4$. If, however, we sample $u=0.705$ (say), then we reject $x^{\prime}$ and propose another $x^{\prime}$, etc. 


\section{Figure 5.10 Illustration of the rejection sampling algorithm}

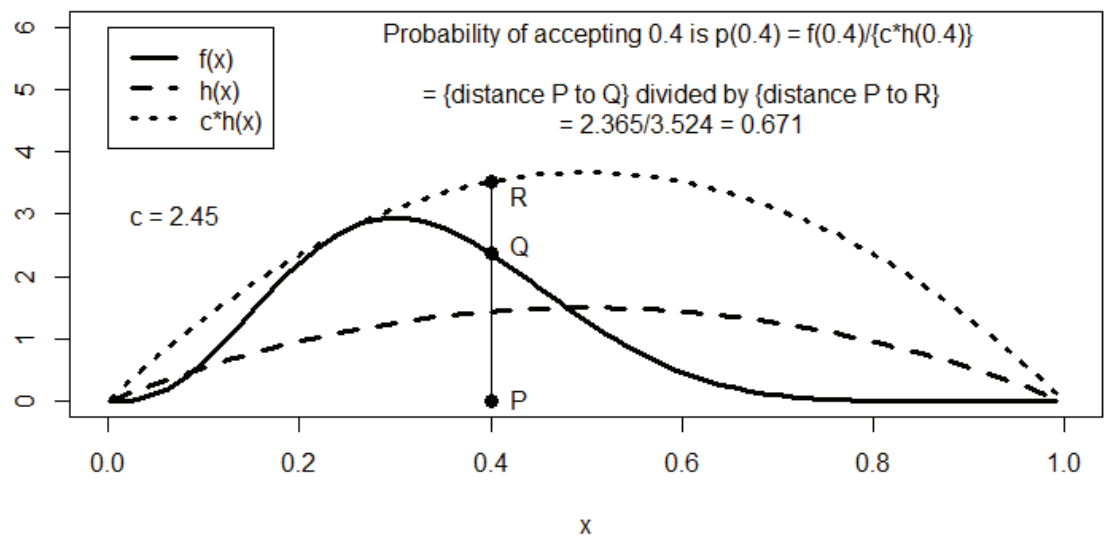

Note 1: The rejection sampling algorithm as defined here also works with $f(x)$ and $h(x)$ in the equations replaced by any kernels of the target and envelope distributions, respectively.

Note 2: The overall acceptance rate is the unconditional probability of acceptance and equals the area under $f(x)$ divided by the area under $\operatorname{ch}(x)$, which is obviously $1 / c$ (= 0.409 in our example).

The wastage may be defined as the overall probability of rejection, namely $1-1 / c$, and this is simply the area between $f(x)$ and $\operatorname{ch}(x)$ (= 0.591 in our example).

Note 3: If we consider the experiment of proposing values repeatedly until the next acceptance, then the number of candidates follows a geometric distribution with parameter $1 / c$, and so the expected number of candidates (until acceptance) is $1 /(1 / c)=c$.

Note 4: There are two basic principles which must be considered in rejection sampling:

(i) The envelope density $h(x)$ should be similar to the target density $f(x)$ since this will minimise wastage, i.e. minimise the average number of proposals per acceptance, $c$, and hence optimise the computer time required. 
(ii) The envelope distribution should be easy to sample from.

Note 5: The idea of rejection sampling can be used to give an intuitively appealing account of how Bayes' theorem works. In this regard, see Smith and Gelfand (1992).

Note 6: How rejection sampling works can most easily be explained by considering the case where $f(x)$ defines a simple discrete distribution. This is the subject of the next exercise.

\section{R Code for Section 5.12}

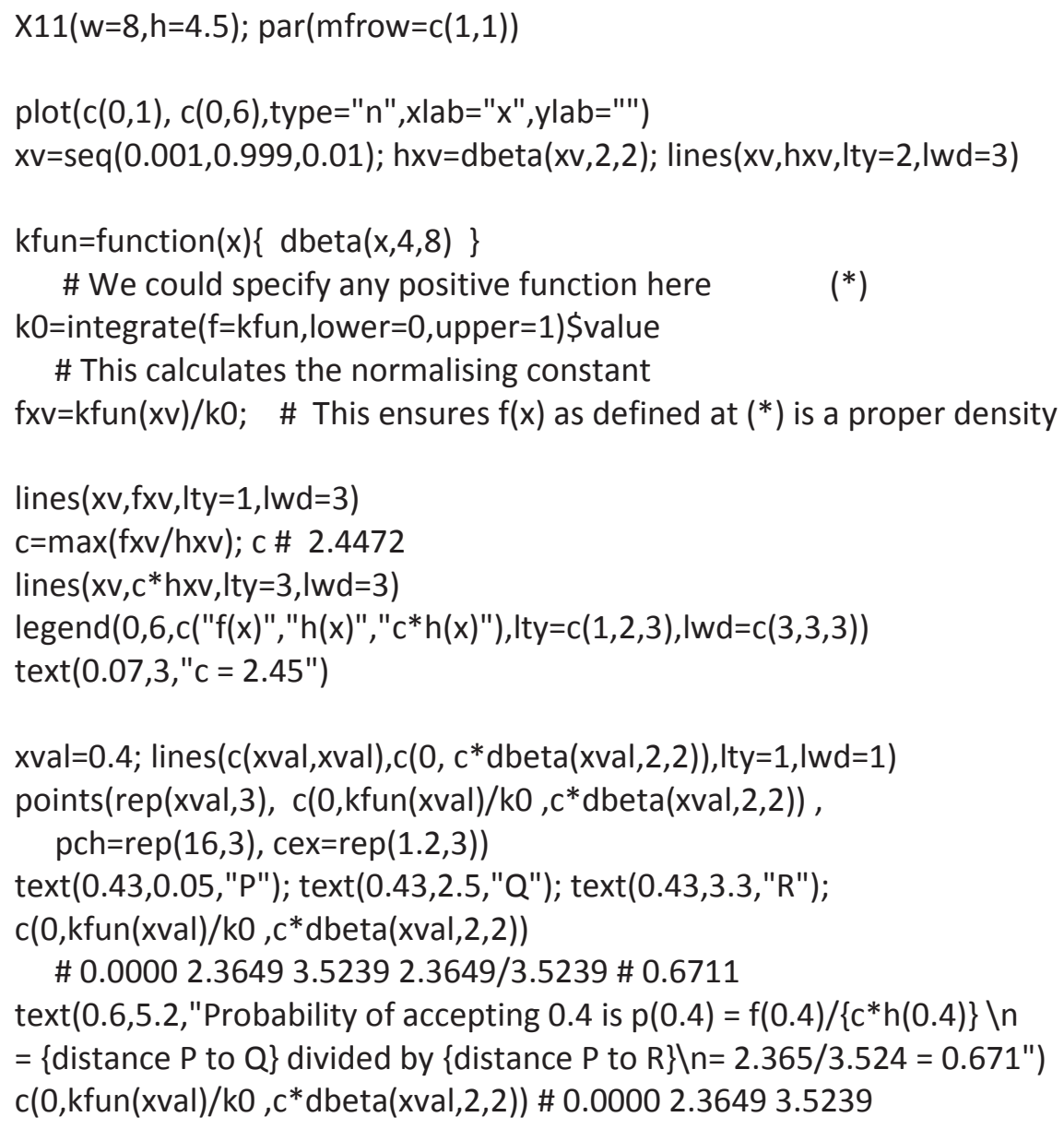




\section{Exercise 5.13 Illustration of rejection sampling}

Consider the $\operatorname{Bin}(2,1 / 2)$ distribution with pdf

$$
f(x)=\left\{\begin{array}{l}
1 / 4, x=0,2 \\
1 / 2, x=1
\end{array}\right\},
$$

and suppose we want to sample from this using rejection method envelope $g(x)=1 / 3, x=0,1,2$, i.e. the density of the discrete uniform distribution over the integers 0,1 and 2 . Show that the rejection sampling algorithm returns 0,1 and 2 with the correct probabilities.

\section{Solution to Exercise 5.13}

Here: $\quad c=\max _{x}\left(\frac{f(x)}{g(x)}\right)=\frac{1 / 2}{1 / 3}=\frac{3}{2}, \quad p(x)=\frac{f(x)}{c g(x)}=\left\{\begin{array}{c}1 / 2, x=0,2 \\ 1, \quad x=1\end{array}\right\}$.

Now, suppose that we propose a very large number of proposed values from $g(x)$. Then:

- about $1 / 3$ of these will be 0 , of which about $1 / 2$ will be accepted

- about $1 / 3$ of these will be 1 , of which (fully) all will be accepted

- about $1 / 3$ of these will be 2 , of which about $1 / 2$ will be accepted.

We see that about $2 / 3$ of all the proposed values will be accepted, and of these about $25 \%$ will be $0,50 \%$ will be 1 , and $25 \%$ will be 2 . About $1 / 3$ of the candidates will be rejected, about half of these being 0 and half being 2 . The overall acceptance rate is $1 / c=1 /(3 / 2)=2 / 3$, and the wastage is $1-1 / c=1 / 3$. On average, $c=1.5$ candidates will have to be proposed until an acceptance. Thus, generation of $1,000 \operatorname{Bin}(2,1 / 2)$ values (say) will require about 1,500 candidates.

\section{I3 Methods based on the rejection algorithm}

The rejection method may be used in conjunction with many other methods. For example, the Box-Muller algorithm (mentioned earlier) is a basis for the Marsaglia polar method for sampling from a normal distribution. This method involves generating

$$
u_{1}, u_{2} \sim \text { iid } U(0,1)
$$

repeatedly until

$$
s \equiv\left(2 u_{1}-1\right)^{2}+\left(2 u_{2}-1\right)^{2}<1
$$

and then returning $z_{i}=\left(2 u_{i}-1\right) \sqrt{-2(\log s) / s}, i=1,2$. 
The result will (eventually) be the required sample

$$
\mathrm{Z}_{1}, \mathrm{Z}_{2} \sim \text { iid } N(0,1) \text {. }
$$

This algorithm includes a condition for rejecting the sample values $u_{1}, u_{2}$ and involves iterating until these values are accepted (as a pair). The procedure may be less efficient than the Box-Muller algorithm (which does not involve rejection sampling and never requires more than two standard uniform variates) but avoids the computation of sines and cosines.

\section{I4 Monte Carlo methods in Bayesian inference}

Most of the ideas above in this chapter are directly applicable to Bayesian inference. Suppose we have derived a posterior distribution or density $f(\theta \mid x)$ but it is complicated and difficult to work with directly. Then we can try to generate a random sample from that posterior with a view to estimating all the required inferential quantities (e.g. point and interval estimates) via the method of Monte Carlo.

First, denote the Monte Carlo sample as $\theta_{1}, \ldots, \theta_{J} \sim$ iid $f(\theta \mid x)$. Then, the MC estimate of the posterior mean of $\theta$, namely

$$
\hat{\theta}=E(\theta \mid x)=\int \theta f(\theta \mid x) d \theta,
$$

is

$$
\bar{\theta}=\frac{1}{J} \sum_{j=1}^{J} \theta_{j} \text { (the MC sample mean), }
$$

and a $1-\alpha$ CI for $\hat{\theta}$ is

$$
\left(\bar{\theta} \pm z_{\alpha / 2} \frac{s_{\theta}}{\sqrt{J}}\right)
$$

where

$$
s_{\theta}^{2}=\frac{1}{J-1} \sum_{j=1}^{J}\left(\theta_{j}-\bar{\theta}\right)^{2} .
$$

Also, a MC estimate of the $1-\alpha$ CPDR for $\theta$ is $\left(\hat{q}_{\alpha / 2}, \hat{q}_{1-\alpha / 2}\right)$, where $\hat{q}_{p}$ is the empirical $p$-quantile of $\theta_{1}, \ldots, \theta_{J}$, and the MC estimate of the posterior median is $\hat{q}_{1 / 2}$, etc. 
Further, when the posterior density $f(\theta \mid x)$ does not have a closed form expression (as is often the case), it can be estimated by smoothing a probability histogram of $\theta_{1}, \ldots, \theta_{J}$.

Once an estimate of the posterior density has been obtained, the mode of that estimate defines the MC estimate of the posterior mode.

Suppose we are interested in some posterior probability

$$
p=P(\theta \in A \mid y)
$$

(where $A$ is a subset of the parameter space).

Then, the MC estimate of $p$ is

$$
\hat{p}=\frac{1}{J} \sum_{j=1}^{J} I\left(\theta_{j} \in A\right) \text {, }
$$

i.e. the proportion of the $\theta_{j}$ values which lie in $A$, and a $1-\alpha$ CI for $p$ is

$$
\left(\hat{p} \pm z_{\alpha / 2} \sqrt{\hat{p}(1-\hat{p}) / J}\right) .
$$

Suppose we are interested in a function of the parameter, $\psi=g(\theta)$. Then regardless of how complicated $g$ is, we can perform MC inference on $\psi$ easily. Simply calculate $\psi_{j}=g\left(\theta_{j}\right)$ for each $j=1, \ldots, J$. This results in a random sample from the posterior distribution of $\psi$, namely the values $\psi_{1}, \ldots, \psi_{J} \sim$ iid $f(\psi \mid x)$.

One may then apply any of the ideas above, just as before. For example, the posterior mean of $\psi$, namely

$$
\hat{\psi}=E(\psi \mid x)=\int \psi f(\psi \mid x) d \psi=\int g(\theta) f(\theta \mid x) d \theta,
$$

can be estimated by its MC estimate,

$$
\bar{\psi}=\frac{1}{J} \sum_{j=1}^{J} \psi_{j},
$$

and a $1-\alpha$ CI for $\hat{\psi}$ is

$$
\left(\bar{\psi} \pm z_{\alpha / 2} \frac{s_{\psi}}{\sqrt{J}}\right),
$$

where

$$
s_{\psi}^{2}=\frac{1}{J-1} \sum_{j=1}^{J}\left(\psi_{j}-\bar{\psi}\right)^{2} .
$$




\section{Exercise 5.I4 MC inference under the normal-normal-gamma model}

Recall the Bayesian model: $\quad\left(y_{1}, \ldots, y_{n} \mid \mu, \lambda\right) \sim$ iid $N(\mu, 1 / \lambda)$

$$
f(\mu, \lambda) \propto 1 / \lambda, \mu \in \mathfrak{R}, \lambda>0 \text {. }
$$

Suppose we observe the data vector $y=\left(y_{1}, \ldots, y_{n}\right)=(2.1,3.2,5.2,1.7)$.

(a) Generate $J=1,000$ values from the posterior distribution of $\mu$. Use this sample to perform MC inference on $\mu$. Illustrate your inferences with a suitable graph.

(b) Generate $J=1,000$ values from the posterior distribution of $\lambda$. Use this sample to perform MC inference on $\lambda$. Illustrate your inferences with a suitable graph.

(c) Use MC methods to estimate the signal to noise ratio (SNR), defined as $\gamma=\mu / \sigma=\mu \sqrt{\lambda}$. Illustrate your inferences with a suitable graph.

\section{Solution to Exercise 5.14}

(a) Recall that the marginal posterior distribution of $\mu$ is given by

$$
\left(\frac{\mu-\bar{y}}{s / \sqrt{n}} \mid y\right) \sim t(n-1) \text {. }
$$

So we generate $w_{1}, \ldots, w_{J} \sim$ iid $t(n-1)$ and then calculate

$$
\mu_{j}=\bar{y}+\frac{s}{\sqrt{n}} w_{j}, j=1, . ., J .
$$

We then use the sample $\mu_{1}, \ldots, \mu_{J} \sim$ iid $f(\mu \mid y)$ for MC inference on $\mu$. Thereby, we estimate $\mu$ 's posterior mean $\hat{\mu}=E(\mu \mid y)$ by $\bar{\mu}=3.077$ with $(3.001,3.153)$ as the $95 \%$ MC CI for $\hat{\mu}$. The MC estimate of $\mu$ 's 95\% CPDR is $(0.685,5.507)$.

We now compare the above estimates with the true values:

$\hat{\mu}=\bar{y}=3.050$

95\% CPDR for $\mu=\left(\bar{y} \pm t_{0.025}(n-1) \frac{s}{\sqrt{n}}\right)=(0.556,5.544)$. 
We observe that the true posterior mean is contained in the 95\% MC CI for that mean. Figure 5.11 provides a comparison of the above Monte Carlo and 'exact' inferences.

Note 1: The formula for the exact posterior density is

$$
\begin{gathered}
f(\mu \mid y)=f(w \mid y)\left|\frac{d w}{d \mu}\right|=f_{t(n-1)}\left(\frac{\mu-\bar{y}}{s / \sqrt{n}}\right) \times\left|\frac{1}{s / \sqrt{n}}\right| \\
=\frac{\Gamma\left(\frac{(n-1)+1}{2}\right)}{\Gamma\left(\frac{n-1}{2}\right) \sqrt{(n-1) \pi}}\left(1+\frac{\left(\frac{\mu-\bar{y}}{s / \sqrt{n}}\right)^{2}}{n-1}\right)^{-\left(\frac{(n-1)+1}{2}\right)} \times \frac{\sqrt{n}}{s}, \quad \mu \in \mathfrak{R} .
\end{gathered}
$$

Note 2: The MC sample $\mu_{1}, \ldots, \mu_{J} \sim$ iid $f(\mu \mid y)$ could also be obtained using the following results:

$$
\begin{aligned}
& (\lambda \mid y) \sim \text { Gamma }\left(\left(\frac{n-1}{2}\right),\left(\frac{n-1}{2}\right) s^{2}\right) \\
& (\mu \mid y, \lambda) \sim N\left(\bar{y}, \frac{1}{n \lambda}\right) .
\end{aligned}
$$

Thus, using the method of composition and the identity

$$
f(\mu, \lambda \mid y)=f(\lambda \mid y) f(\mu \mid y, \lambda),
$$

we first sample

$$
\lambda_{1}, \ldots, \lambda_{J} \sim \operatorname{Gamma}\left(\left(\frac{n-1}{2}\right),\left(\frac{n-1}{2}\right) s^{2}\right),
$$

and then sample

$$
\mu_{j} \sim N\left(\bar{y}, \frac{1}{n \lambda_{j}}\right) \text { for each } j=1, \ldots, J .
$$

The result of this procedure is

$$
\left(\mu_{1}, \lambda_{1}\right), \ldots,\left(\mu_{J}, \lambda_{J}\right) \sim \text { iid } f(\mu, \lambda \mid y),
$$

and thereby

$$
\mu_{1}, \ldots, \mu_{J} \sim \text { iid } f(\mu \mid y),
$$

as before, after discarding all of the $\lambda_{j}$ values. 


\section{Figure 5. I I Monte Carlo inference on the normal mean}

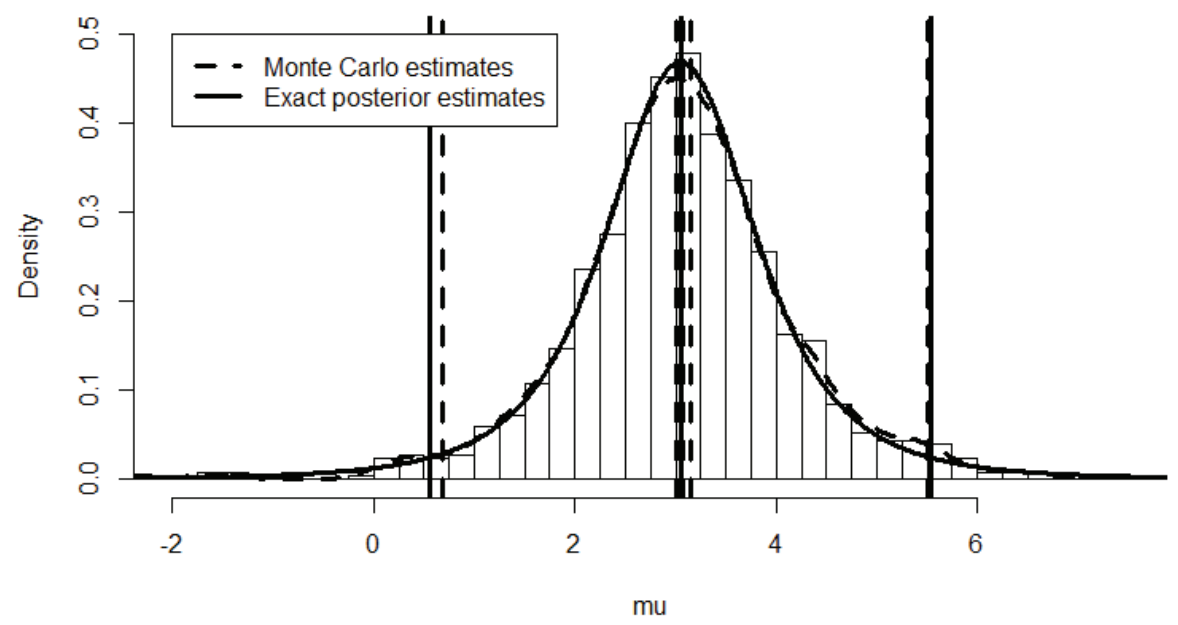

(b) One way to obtain a MC sample from the marginal posterior distribution of $\lambda$ is as indicated in Note 2 of part (a). Alternatively, we can make use of the result

$$
(\lambda \mid y, \mu) \sim \operatorname{Gamma}\left(\frac{n}{2}, \frac{n}{2} s_{\mu}^{2}\right) \text {, where } s_{\mu}^{2}=\frac{1}{n} \sum_{i=1}^{n}\left(y_{i}-\mu\right)^{2} .
$$

So, again by the method of composition, but this time using the identity

$$
f(\mu, \lambda \mid y)=f(\mu \mid y) f(\lambda \mid y, \mu),
$$

we make use of the sample already generated in (a) and sample

$$
\lambda_{j} \sim \operatorname{Gamma}\left(\frac{n}{2}, \frac{n}{2} s_{\mu_{j}}^{2}\right)
$$

for each $j=1, \ldots, J$. The result is $\left(\mu_{1}, \lambda_{1}\right), \ldots,\left(\mu_{J}, \lambda_{J}\right) \sim$ iid $f(\mu, \lambda \mid y)$, and thereby $\lambda_{1}, \ldots, \lambda_{J} \sim$ iid $f(\lambda \mid y)$ (after discarding all of the $\mu_{j}$ values).

Implementing this procedure (i.e. making use of the simulated values in (a)) we obtain the required sample, $\lambda_{1}, \ldots, \lambda_{J} \sim$ iid $f(\lambda \mid y)$, and use it for MC inference. Thereby we estimate $\lambda$ 's posterior mean $\hat{\lambda}=E(\lambda \mid y)$ by $\bar{\lambda}=0.3998$ with $(0.3804,0.4192)$ as the $95 \%$ MC CI for $\hat{\lambda}$. The MC estimate of $\lambda$ 's $95 \%$ CPDR is $(0.0347,1.2828)$.

We now compare the above estimates with the true values: 


$$
\begin{aligned}
\hat{\lambda}=\frac{1}{s^{2}}=0.4071 \\
\begin{aligned}
95 \% \mathrm{CPDR} & =\left(F_{G\left(\frac{n-1}{2}, \frac{n-1}{2} s^{2}\right)}^{-1}(0.025), F_{G\left(\frac{n-1}{2}, \frac{n-1}{2} s^{2}\right)}^{-1}(0.975)\right) \\
& =(0.0293,1.2684) .
\end{aligned}
\end{aligned}
$$

We see that the true posterior mean is contained in the 95\% MC CI for that mean. Figure 5.12 illustrates these Monte Carlo and 'exact' inferences.

Figure 5.I2 Monte Carlo inference on the precision parameter

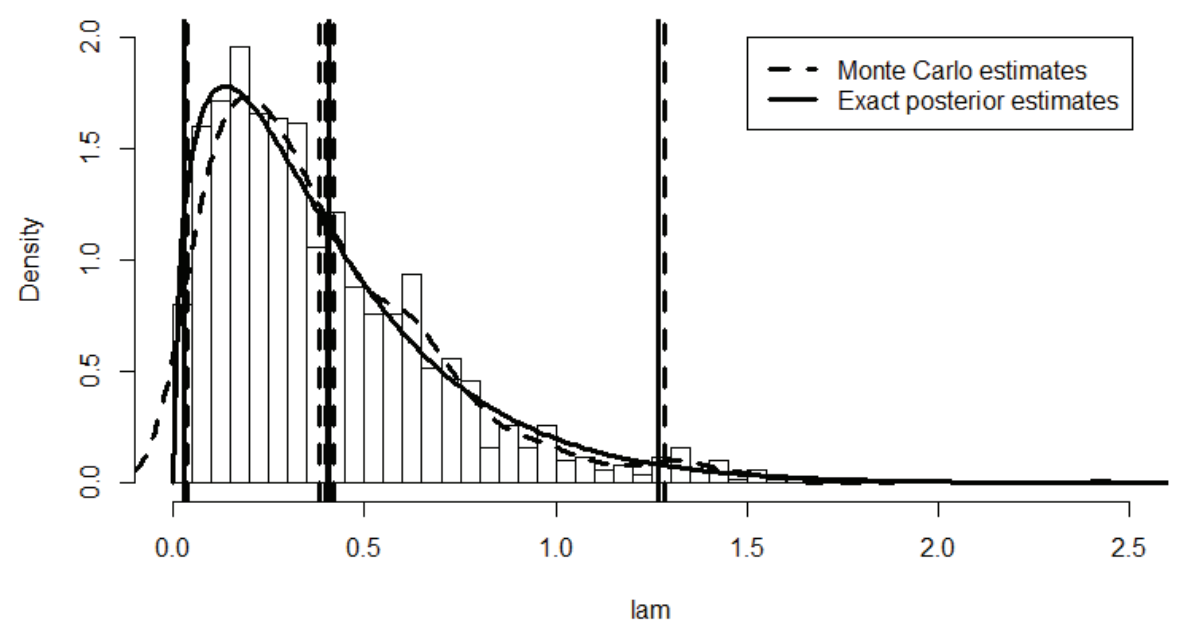

(c) Using the values sampled in (a) and (b), we now calculate $\gamma_{j}=\mu_{j} \sqrt{\lambda_{j}}$ for each $j=1, . ., J$, and hence obtain a MC sample $\gamma_{1}, \ldots, \gamma_{J} \sim$ iid $f(\gamma \mid y)$, which can then be used to perform MC inference on $\gamma$. (NB: The symbols ' $\gamma$ ' and ' $\gamma$ ' are typographically equivalent.) Implementing this strategy, we estimate $\gamma$ 's posterior mean by 1.800 , with $(1.7451 .854)$ as a $95 \%$ CI for that mean, and we estimate $\gamma$ 's 95\% CPDR as $(0.228,3.543)$.

Figure 5.13 illustrates these Monte Carlo estimates. Also shown are:

- the exact posterior mean of $\gamma$, which is $\hat{\gamma}=E(\gamma \mid y)=1.793$

- the exact 95\% CPDR for $\gamma$, which is (0.0733, 3.5952)

- the exact posterior density of $\gamma$

- the MLE of $\gamma$, which is $\tilde{\gamma}=\bar{y} / s=3.05 / 1.567=1.946$. 
See the Note and R Code below for details of these calculations.

Figure 5.13 Monte Carlo inference on the signal to noise ratio

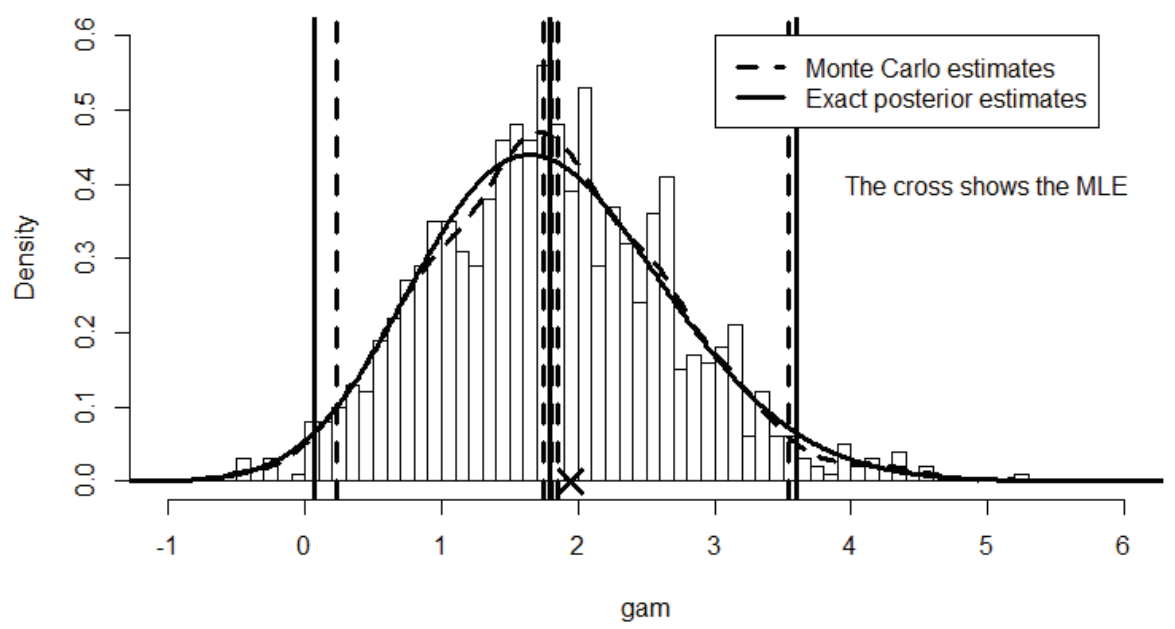

Note: The conditional posterior distribution of $\gamma=\mu \sqrt{\lambda}$ given $\lambda$ is

$$
(\gamma \mid y, \lambda) \sim N\left((\sqrt{\lambda}) \bar{y},(\sqrt{\lambda})^{2} 1 /(n \lambda)\right) \sim N(\bar{y} \sqrt{\lambda}, 1 / n) .
$$

This follows from the uninformative normal-normal model, i.e. from the fact that

$$
(\mu \mid y, \lambda) \sim N(\bar{y}, 1 /(n \lambda)) .
$$

So the posterior density of $\gamma$ may be obtained numerically according to

$$
f(\gamma \mid y)=E_{\lambda}\{f(\gamma \mid y, \lambda) \mid y\}=\int_{0}^{\infty} f(\gamma \mid y, \lambda) f(\lambda \mid y) d \lambda,
$$

where:

$$
\begin{aligned}
& f(\gamma \mid y, \lambda)=f_{N(\bar{y} \sqrt{\lambda}, 1 / n)}(\gamma)=\frac{n}{\sqrt{2 \pi}} e^{-\frac{n}{2}(\gamma-\bar{y} \sqrt{\lambda})^{2}}, \gamma \in \Re \\
& f(\lambda \mid y)=f_{G\left(\frac{n-1}{2}, \frac{n-1}{2} s^{2}\right)}(\lambda)=\frac{\left(\frac{n-1}{2} s^{2}\right)^{\frac{n-1}{2}} \lambda^{\frac{n-1}{2}-1} e^{-\lambda \frac{n-1}{2} s^{2}}}{\Gamma\left(\frac{n-1}{2}\right)}, \lambda>0 .
\end{aligned}
$$


Also (as shown in a previous exercise), the posterior mean of $\gamma$ is exactly

$$
\begin{aligned}
\hat{\gamma} & =E(\mu \sqrt{\lambda} \mid y)=E\{E(\mu \sqrt{\lambda} \mid y, \lambda) \mid y\} \\
& =\frac{\bar{y}}{s} \times \frac{\Gamma\left(\frac{n-1}{2}+\frac{1}{2}\right)}{\left(\frac{n-1}{2}\right)^{1 / 2} \Gamma\left(\frac{n-1}{2}\right)}=1.793
\end{aligned}
$$

(after some algebra).

The exact 95\% CPDR for $\gamma$ may be obtained by using the optim() function to minimise

$$
\begin{aligned}
g(L, U) & =\left(F_{\gamma}(U \mid y)-F_{\gamma}(L \mid y)-0.95\right)^{2}+\left(f_{\gamma}(U \mid y)-f_{\gamma}(L \mid y)\right)^{2} \\
& =\left(\int_{L}^{U}\left(\int_{0}^{\infty} f(\gamma \mid y, \lambda) f(\lambda \mid y) d \lambda\right) d \gamma-0.95\right)^{2} \\
+ & \left(\int_{0}^{\infty} f_{\gamma}(U \mid y, \lambda) f(\lambda \mid y) d \lambda-\int_{0}^{\infty} f_{\gamma}(L \mid y, \lambda) f(\lambda \mid y) d \lambda\right)^{2},
\end{aligned}
$$

with the result being $(L, U)=(0.0733,3.5952)$.

\section{R Code for Exercise 5.I4}

\# (a)

$y=c(2.1,3.2,5.2,1.7) ; n=$ length $(y) ; y b a r=m e a n(y) ; s=s d(y) ; s$ \# 1.567

$\mathrm{J}=1000$; set.seed(144); options(digits=4)

$\mathrm{wv}=\mathrm{rt}(\mathrm{J}, \mathrm{n}-1) ;$ muv=ybar+s*wv/sqrt(n)

mubar=mean (muv); muci=mubar $+c(-1,1) *$ qnorm $(0.975) *$ sd(muv)/sqrt $(J)$

mucpdr=quantile(muv, $c(0.025,0.975))$

c(mubar,muci,mucpdr) \# 3.07703 .00123 .15280 .68485 .5069

muhat=ybar; mucpdrtrue $=y b a r+(s / s q r t(n)) * q t(c(0.025,0.975), n-1)$

c(muhat,mucpdrtrue) \# 3.0500 .5565 .544

$X 11(w=8, h=5) ; \operatorname{par}(\operatorname{mfrow}=c(1,1))$

hist(muv, prob=T,xlab="mu",xlim=c(-2,7.5), ylim=c(0,0.5), main="", breaks=seq $(-20,20,0.25))$

muvec=seq $(-20,20,0.01)$;

postvec $=\mathrm{dt}($ (muvec-ybar)/(s/sqrt(n)), n-1 ) / (s/sqrt(n)) 
lines(muvec, postvec, Ity=1,Iwd=3)

lines(density(muv), Ity=2, Iwd=3)

abline $(v=c$ (mubar, muci, $m u c p d r), \mid t y=2, l w d=3$ )

abline ( $v=c(y b a r$, mucpdrtrue) , Ity $=1, \mid \mathrm{lwd}=3$ )

legend(-2,0.5,c("Monte Carlo estimates", "Exact posterior estimates"), Ity $=c(2,1), \operatorname{lwd}=c(3,3), \mathrm{bg}="$ white")

\# (b)

lamv=rep(NA,J); set.seed(332)

for(j in $1: J) \operatorname{lamv}[j]=\operatorname{rgamma}\left(1, n / 2,(n / 2)^{*} \operatorname{mean}\left((y-\operatorname{muv}[j])^{\wedge} 2\right)\right)$

lambar=mean(lamv); lamci=lambar $+\mathrm{c}(-1,1) *$ qnorm $(0.975) * \mathrm{sd}($ lamv $) /$ sqrt $(\mathrm{J})$

lamcpdr=quantile $($ lamv,$c(0.025,0.975))$

c(lambar, lamci, lamcpdr) \# 0.399800 .380400 .419200 .034651 .28283

lamhat $=1 / s^{\wedge} 2$; lamcpdrtrue $=$ qgamma $\left(c(0.025,0.975),(n-1) / 2,((n-1) / 2) * s^{\wedge} 2\right)$

c(lamhat, lamcpdrtrue) \# 0.407060 .029281 .26844

hist(lamv, prob=T,xlab="lam",xlim=c(0,2.5), ylim=c(0,2),main="",

breaks $=\operatorname{seq}(0,3,0.05))$

lamvec=seq(0,3,0.01) ; lampostvec= dgamma (lamvec, $\left.(\mathrm{n}-1) / 2,((\mathrm{n}-1) / 2) * \mathrm{~s}^{\wedge} 2\right)$

lines(lamvec, lampostvec, Ity=1, Iwd=3)

lines(density(lamv), Ity=2,lwd=3)

abline ( $v=c($ lambar, lamci, lamcpdr), Ity=2,lwd=3)

abline $\left(v=c\left(1 / s^{\wedge} 2\right.\right.$, lamcpdrtrue $)$, Ity $\left.=1, I w d=3\right)$

legend(1.5,2,c("Monte Carlo estimates","Exact posterior estimates"), Ity $=c(2,1), \operatorname{lwd}=c(3,3), \mathrm{bg}="$ white")

\# (c)

gamv=muv*sqrt(lamv)

gambar=mean(gamv); gamci=gambar $+c(-1,1) *$ qnorm $(0.975) *$ sd(gamv)/sqrt(J) gamcpdr=quantile (gamv, c(0.025,0.975))

c(gambar, gamci, gamcpdr) \# 1.79971 .74531 .85400 .22843 .5433

$\mathrm{mle}=\mathrm{ybar} / \mathrm{s} ; \mathrm{mle} \# 1.946$

gamhat $=(\mathrm{ybar} / \mathrm{s}) * \operatorname{gamma}(0.5+(\mathrm{n}-1) / 2) /(\operatorname{sqrt}((\mathrm{n}-1) / 2) * \operatorname{gamma}((\mathrm{n}-1) / 2))$

print(c(ybar,s,gamhat), digits=8) \# 3.05000001 .56737571 .7928178

intfun=function(lam,gam, ybar=3.05,s=1.5673757, $n=4)\{$

$\left.\operatorname{dnorm}(\operatorname{gam}, y b a r * \operatorname{sqrt}(\operatorname{lam}), 1 / \mathrm{sqrt}(\mathrm{n})) * \operatorname{dgamma}\left(\operatorname{lam},(\mathrm{n}-1) / 2, \mathrm{~s}^{\wedge} 2 *(\mathrm{n}-1) / 2\right) \quad\right\}$ 


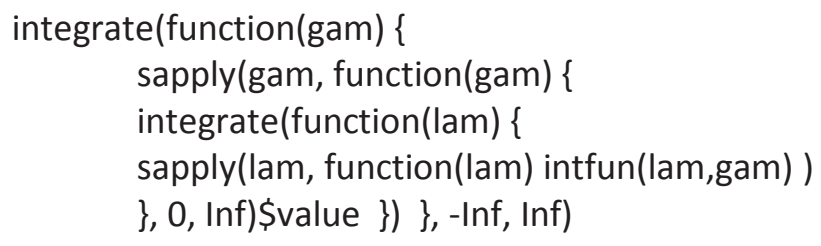

\# 1 with absolute error < 4.7e-07 OK (Just checking)

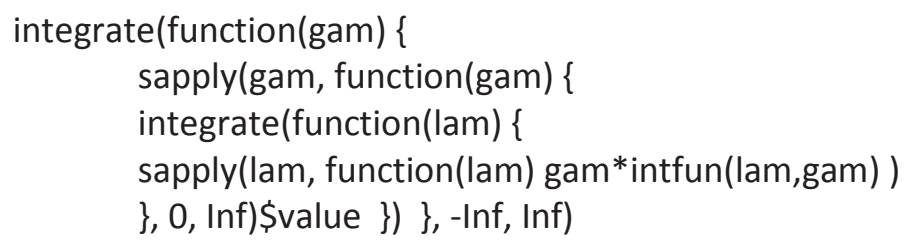

\# 1.793 with absolute error $<4.7$ e-06 OK (Agrees with exact calculation)

gamvec=seq $(-5,10,0.01) ;$ fgamvec=gamvec

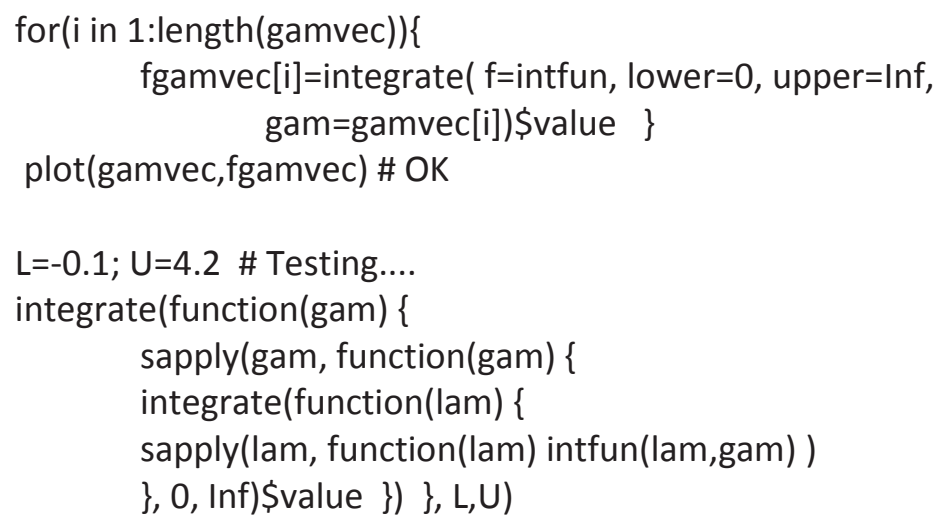




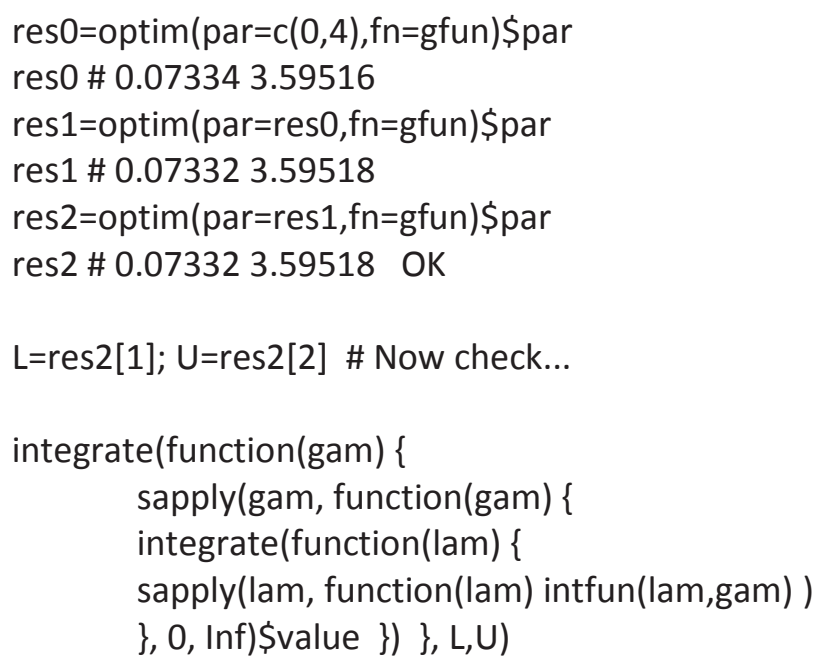

\section{I 5 MC predictive inference via the method of composition}

Suppose that in the context of a Bayesian model defined by $f(y \mid \theta)$ and $f(\theta)$, we wish to predict a value $x$ whose distribution is specified by $f(x \mid y, \theta)$. Recall that the posterior predictive density is

$$
f(x \mid y)=\int f(x \mid y, \theta) f(\theta \mid y) d \theta .
$$

If this density is complicated, we may choose to perform MC predictive inference on $x$ using a sample $x_{1}, \ldots, x_{J} \sim$ iid $f(x \mid y)$. The question then arises as to how such a sample may be obtained. 
One answer is to sample from $f(x \mid y)$ directly. But that may be difficult since $f(x \mid y)$ is complicated. Another answer is to apply the method of composition through the equation

$$
f(x, \theta \mid y)=f(x \mid y, \theta) f(\theta \mid y) .
$$

This means that we should first sample $\theta^{\prime} \sim f(\theta \mid y)$ and then sample $x^{\prime} \sim f\left(x \mid y, \theta^{\prime}\right)$, the result being $\left(x^{\prime}, \theta^{\prime}\right) \sim f(x, \theta \mid y)$. If we then discard $\theta^{\prime}$, the result is the required $x^{\prime} \sim f(x \mid y)$. Implementing this process a total of $J$ times results in the required sample, $x_{1}, \ldots, x_{J} \sim$ iid $f(x \mid y)$.

\section{Exercise 5.I5 Monte Carlo prediction in the binomial-beta model}

The probability of heads coming up on a bent coin follows a standard uniform distribution a priori. We toss the coin 50 times and get 28 heads. Estimate using Monte Carlo the probability that heads will come up on at least six of the next 10 tosses of the same bent coin.

\section{Solution to Exercise 5.15}

Recall that the binomial-beta model:

$$
\begin{aligned}
& (y \mid \theta) \sim \operatorname{Bin}(n, \theta) \\
& \theta \sim \operatorname{Beta}(\alpha, \beta),
\end{aligned}
$$

for which the posterior distribution is given by

$$
(\theta \mid y) \sim \operatorname{Beta}(\alpha+y, \beta+n-y) \text {. }
$$

Earlier we showed that if the future data $x$ has distribution defined by

$$
(x \mid y, \theta) \sim \operatorname{Bin}(m, \theta),
$$

then posterior predictive distribution is given by

$$
f(x \mid y)=\left(\begin{array}{c}
m \\
x
\end{array}\right) \frac{B(y+x+\alpha, n-y+m-x+\beta)}{B(y+\alpha, n-y+\beta)}, x=0, \ldots, m .
$$

Rather than trying to sample from this distribution directly, we may do the following:

$$
\begin{aligned}
& \text { Sample } \theta^{\prime} \sim \operatorname{Beta}(\alpha+y, \beta+n-y) \\
& \text { Sample } x^{\prime} \sim \operatorname{Bin}\left(m, \theta^{\prime}\right) .
\end{aligned}
$$

Discarding $\theta^{\prime}$, we obtain the required sample value, $x^{\prime} \sim f(x \mid y)$. In the situation here: $\alpha=\beta=1, n=50, y=32, m=10$. 
Implementing the above sampling strategy $J=10,000$ times with these specifications, we obtain a large MC sample, $x_{1}, \ldots, x_{J} \sim f(x \mid y)$.

It is found that 7,084 of the sample values are at least 6 . So we estimate $p=P(X \geq 6 \mid y)$ by $\hat{p}=0.7084$. A $95 \%$ CI for $p$ is then

$$
(\hat{p} \pm 1.96 \sqrt{\hat{p}(1-\hat{p}) / J})=(0.6995,0.7173) \text {. }
$$

For interest, we also work out the probability exactly as

$$
p=\sum_{x=6}^{10} f(x \mid y)=0.7030 \quad \text { (correct to } 4 \text { decimals) }
$$

and note that this value lies in the 95\% CI obtained using MC methods.

\section{R Code for Exercise 5.I5}

options(digits=5)

$n=50 ; y=32 ; \quad$ alp=1;bet=1; $\quad a=a l p+y ; b=b e t+n-y ; \quad m=10 ; \quad J=10000$

set.seed(443); tv=rbeta(J,a,b); $x v=r b i n o m(J, m, t v)$

phat=length $(x v[x v>=6]) / J$;

ci=phat+c(-1,1)*qnorm $(0.975) *$ sqrt(phat*(1-phat)/J)

c(phat,ci) \# 0.708400 .699490 .71731

$x v e c=0: m ;$ fxgiveny=

choose $(m, x v e c) *$ beta(y+xvec+alp, $n-y+m-x v e c+b e t) /$ beta $(y+a l p, n-y+b e t)$

sum(fxgiveny) \# 1 Just checking

sum(fxgiveny[xvec>=6]) \# 0.70296

\section{I 6 Rao-Blackwell methods for estimation and prediction}

Consider a Bayesian model with two parameters given by a specification of $f(y \mid \theta, \psi)$ and $f(\theta, \psi)$, and suppose that we obtain a sample from the joint posterior distribution of the two parameters, say

$$
\left(\theta_{1}, \psi_{1}\right), \ldots,\left(\theta_{J}, \psi_{J}\right) \sim \text { iid } f(\theta, \psi \mid y) .
$$

As we have seen, an unbiased Monte Carlo estimate of $\theta$ 's posterior mean, $\hat{\theta}=E(\theta \mid y)$, is $\bar{\theta}=(1 / J) \sum_{j=1}^{J} \theta_{j}$, with an associated MC $1-\alpha$ CI for $\hat{\theta}$ given by $\left(\bar{\theta} \pm z_{\alpha / 2} s_{\theta} / \sqrt{J}\right)$, where $s_{\theta}$ is the sample standard deviation of $\theta_{1}, \ldots, \theta_{J}$. 
Now observe that

$$
\hat{\theta}=E\{E(\theta \mid y, \psi) \mid y\}=\int E(\theta \mid y, \psi) f(\psi \mid y) d \psi .
$$

This implies that another unbiased Monte Carlo estimate of $\hat{\theta}$ is

$$
\bar{e}=\frac{1}{J} \sum_{j=1}^{J} e_{j},
$$

where

$$
e_{j}=E\left(\theta \mid y, \psi_{j}\right),
$$

and another $1-\alpha$ CI for $\hat{\theta}$ is

$$
\left(\bar{e} \pm z_{\alpha / 2} s_{e} / \sqrt{J}\right) \text {, }
$$

where $s_{e}$ is the sample standard deviation of $e_{1}, \ldots, e_{J}$.

If possible, this second method of Monte Carlo inference is preferable to the first because it typically leads to a shorter CI. We call this second method Rao-Blackwell (RB) estimation. The first (original) method may be called direct Monte Carlo estimation or histogram estimation.

The same idea extends to estimation of the entire marginal posterior density of $\theta$, because this can be written

$$
f(\theta \mid y)=\int f(\theta \mid y, \psi) f(\psi \mid y) d \psi=E_{\psi}\{f(\theta \mid y, \psi) \mid y\} .
$$

Thus, the Rao-Blackwell estimate of $f(\theta \mid y)$ is

$$
\tilde{f}(\theta \mid y)=\frac{1}{J} \sum_{j=1}^{J} f\left(\theta \mid y, \psi_{j}\right),
$$

as distinct from the ordinary histogram estimate obtained by smoothing a probability histogram of $\theta_{1}, \ldots, \theta_{J}$.

The idea further extends to predictive inference, where we are interested in a future quantity $x$ defined by a specification of $f(x \mid y, \theta, \psi)$.

The direct MC estimate of the predictive mean, namely

$$
\hat{x}=E(x \mid y) \text {, }
$$

is

$$
\bar{x}=(1 / J) \sum_{j=1}^{J} x_{j},
$$

where

$$
x_{1}, \ldots, x_{J} \sim \text { iid } f(x \mid y)
$$

(e.g. as obtained via the method of composition). 
A superior estimate is the Rao-Blackwell estimate given by

$$
\bar{E}=\frac{1}{J} \sum_{j=1}^{J} E_{j},
$$

where there is now a choice from the following:

$$
\begin{aligned}
E_{j} & =E\left(x \mid y, \theta_{j}, \psi_{j}\right) \\
\text { or } \quad E_{j} & =E\left(x \mid y, \psi_{j}\right) \\
\text { or } \quad E_{j} & =E\left(x \mid y, \theta_{j}\right) .
\end{aligned}
$$

This estimator $(\bar{E})$ is based on the identities

$$
\hat{x}=E\{E(x \mid y, \theta, \psi) \mid y\}=E\{E(x \mid y, \psi) \mid y\}=E\{E(x \mid y, \theta) \mid y\} .
$$

Note: The first of the three choices for $E_{j}$ is typically the easiest to calculate but also leads to the least improvement over the ordinary 'histogram' predictor, $\bar{x}=(1 / J) \sum_{j=1}^{J} x_{j}$.

Likewise, the Rao-Blackwell estimate of the entire posterior predictive density $f(x \mid y)$ is

$$
\tilde{f}(x \mid y)=\frac{1}{J} \sum_{j=1}^{J} f_{j}(x),
$$

where there is a choice from the following:

$$
\begin{aligned}
f_{j}(x) & =f\left(x \mid y, \theta_{j}, \psi_{j}\right) \\
\text { or } \quad f_{j}(x) & =f\left(x \mid y, \psi_{j}\right) \\
\text { or } f_{j}(x) & =f\left(x \mid y, \theta_{j}\right) .
\end{aligned}
$$

\section{Exercise 5.16 Practice at Rao-Blackwell estimation in the normal-normal-gamma model}

Recall the Bayesian model:

$$
\begin{aligned}
& \left(y_{1}, \ldots, y_{n} \mid \mu, \lambda\right) \sim \text { iid } N(\mu, 1 / \lambda) \\
& f(\mu, \lambda) \propto 1 / \lambda, \mu \in \mathfrak{R}, \lambda>0 .
\end{aligned}
$$

Suppose that we observe the vector $y=\left(y_{1}, \ldots, y_{n}\right)=(2.1,3.2,5.2,1.7)$.

Generate $J=100$ values from the joint posterior distribution of $\mu$ and $\lambda$ and use these values as follows. Calculate the direct Monte Carlo estimate and the Rao-Blackwell estimate of $\lambda$ 's marginal posterior mean. 
In each case, report the associated 95\% CI for that mean. Compare your results with the true value of that mean. Produce a probability histogram of the simulated $\lambda$-values. Overlay a smooth of this histogram and the Rao-Blackwell estimate of $\lambda$ 's marginal posterior density. Also overlay the exact density.

\section{Solution to Exercise 5.16}

Recall from Equation (3.3) in Exercise 3.11 that:

$$
\begin{aligned}
& (\lambda \mid y) \sim \text { Gamma }\left(\left(\frac{n-1}{2}\right),\left(\frac{n-1}{2}\right) s^{2}\right) \\
& (\mu \mid y, \lambda) \sim N\left(\bar{y}, \frac{1}{n \lambda}\right) .
\end{aligned}
$$

So we first sample

$$
\lambda^{\prime} \sim \text { Gamma }\left(\left(\frac{n-1}{2}\right),\left(\frac{n-1}{2}\right) s^{2}\right),
$$

and then we sample

$$
\mu^{\prime} \sim N\left(\bar{y}, \frac{1}{n \lambda^{\prime}}\right) .
$$

The result is

$$
\left(\mu^{\prime}, \lambda^{\prime}\right) \sim f(\mu, \lambda \mid y) .
$$

Repeating many times, we get

$$
\left(\mu_{1}, \lambda_{1}\right), \ldots,\left(\mu_{J}, \lambda_{J}\right) \sim \text { iid } f(\mu, \lambda \mid y) .
$$

The histogram estimate of $\hat{\lambda}=E(\lambda \mid y)$ works out as $\bar{\lambda}=0.4142$, with 95\% CI (0.4076, 0.4209).

Next let $e_{j}=E\left(\lambda \mid y, \mu_{j}\right)$.

Then the Rao-Blackwell estimate of $\hat{\lambda}$ is $\bar{e}=0.4073$, with associated 95\% CI $(0.4047,0.4100)$.

It will be observed that this second CI is narrower than the first (having width 0.0053 compared with 0.0133 ). It will also be observed that both CIs contain the true value, $\hat{\lambda}=1 / s^{2}=0.4071$. 
Figure 5.14 shows:

- a probability histogram of $\lambda_{1}, \ldots, \lambda_{J}$

- a smooth of that probability histogram

- the true marginal posterior density, namely

$$
f(\lambda \mid y)=f_{\text {Gammaa } \left.(n-1) / 2, s^{2}(n-1) / 2\right)}(\lambda)
$$

- the Rao-Blackwell estimate of $f(\lambda \mid y)$ as given by

$$
\tilde{f}(\lambda \mid y)=\frac{1}{J} \sum f_{\text {Gamma }\left(n / 2, s_{j}^{2} / 2\right)}(\lambda) \quad \text { where } \quad s_{j}^{2}=\frac{1}{n} \sum_{i=1}^{n}\left(y_{i}-\mu_{j}\right)^{2} .
$$

Note: The Rao-Blackwell estimate here is based on the result

$$
(\lambda \mid y, \mu) \sim \operatorname{Gamma}\left(\frac{n}{2}, \frac{n}{2}\left[\frac{1}{n} \sum_{i=1}^{n}\left(y_{i}-\mu\right)^{2}\right]\right) .
$$

It will be observed that the Rao-Blackwell estimate of $\lambda$ 's posterior density is fairly close. The histogram estimate is much less accurate and incorrectly suggests that $\lambda$ has some probability of being negative.

\section{Figure 5. I 4 Illustration of Rao-Blackwell estimation}

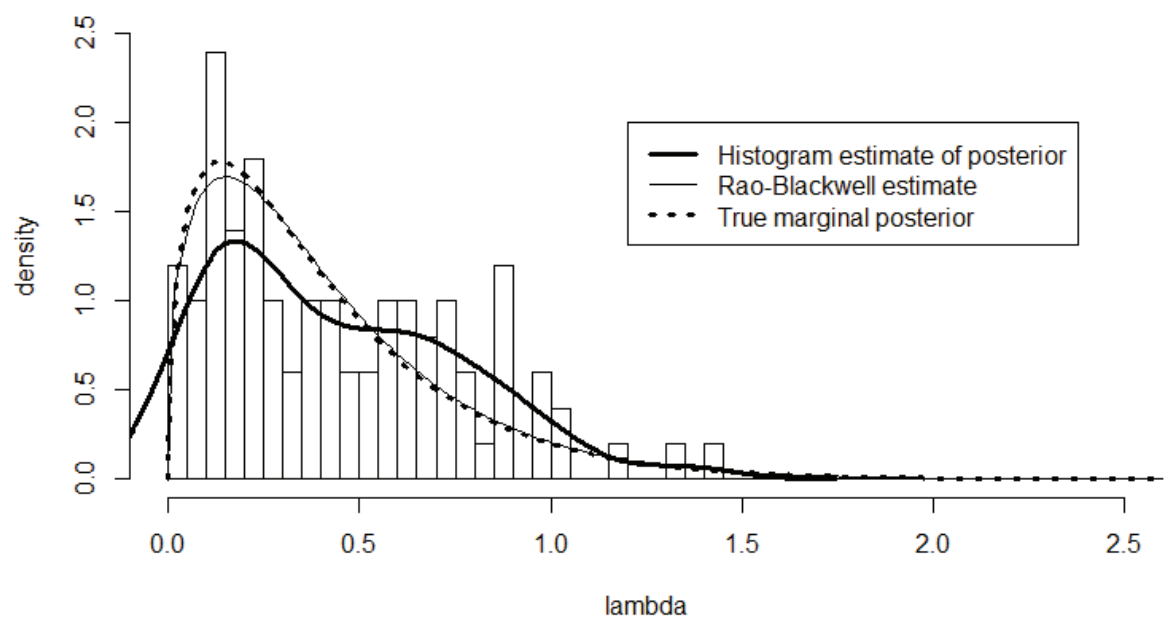




\section{R Code for Exercise 5.16}

options(digits=4)

\section{\# (a)}

$\mathrm{y}=\mathrm{c}(2.1,3.2,5.2,1.7) ; \mathrm{n}=$ length $(\mathrm{y}) ; \mathrm{ybar}=\operatorname{mean}(\mathrm{y}) ; \mathrm{s}=\mathrm{sd}(\mathrm{y}) ; \mathrm{s} 2=\mathrm{s}^{\wedge} 2$

$\mathrm{J}=100$; $\operatorname{set}$.seed(254); lamv=rgamma(J,(n-1)/2,s2*(n-1)/2);

muv=rnorm(J,ybar, $1 /$ sqrt( $n^{*}$ lamv)); est $0=1 / s^{\wedge} 2$

est1=mean(lamv); std1=sd(lamv); ci1=est1 +c(-1,1)*qnorm(0.975)*std1/sqrt(J)

ev=rep(NA,J); for(j in 1:J)\{ muval=muv[j]; ev[j] $=1 /$ mean $((y-m u v a l) \wedge 2)\}$

est2=mean(ev); std2=sd(ev); ci2=est2 +c(-1,1)*qnorm $(0.975) *$ std2/sqrt(J)

rbind( c(est0,NA,NA,NA), c(est1,ci1,ci1[2]-ci1[1]), c(est2,ci2,ci2[2]-ci2[1]) )

\#[1,] 0.4071 NA NA NA

\#[2,] 0.43960 .37670 .50260 .12589

\# [3,] 0.41500 .38920 .44080 .05166

\# (b)

$\mathrm{X} 11(\mathrm{w}=8, \mathrm{~h}=5) ; \operatorname{par}(\mathrm{mfrow}=\mathrm{c}(1,1))$

hist(lamv,xlab="lambda",ylab="density",prob=T,xlim=c(0,2.5),

ylim=c(0,2.5), main="",breaks=seq $(0,4,0.05))$

lines(density(lamv), Ity=1,lwd=3)

lamvec=seq(0,3,0.01); RBvec=lamvec; smu2v=1/ev

for $(k$ in 1:length (lamvec) $)\{\quad$ lamval=lamvec $[\mathrm{k}]$

RBvec $[k]=$ mean $($ dgamma $($ lamval, $n / 2,(n / 2) *$ smu2v) ) \}

lines(lamvec, RBvec, Ity=1, Iwd=1)

lines(seq(0,3,0.005),dgamma(seq(0,3,0.005),(n-1)/2,s2*(n-1)/2), Ity=3,Iwd=3) legend(1.2,2,c("Histogram estimate of posterior","Rao-Blackwell estimate",

"True marginal posterior"), Ity $=c(1,1,3), \operatorname{lwd}=c(3,1,3))$

\section{I 7 MC estimation of posterior predictive p-values}

Recall the theory of posterior predictive p-values whereby, in the context of a Bayesian model specified by $f(y \mid \theta)$ and $f(\theta)$, we test $H_{0}$ versus $H_{1}$ by choosing a suitable test statistic $T(y, \theta)$.

The posterior predictive $\mathrm{p}$-value is then

$$
p=P(T(x, \theta) \geq T(y, \theta) \mid y)
$$

(or something similar, e.g. with $\geq$ replaced by $\leq$ ), calculated under the implicit assumption that $H_{0}$ is true. 
If the calculation of $p$ is problematic, a suitable Monte Carlo strategy is as follows:

1. Generate a random sample from the posterior,

$$
\theta_{1}, \ldots, \theta_{J} \sim \text { iid } f(\theta \mid y) \text {. }
$$

2. Generate $x_{j} \sim \perp f\left(y \mid \theta_{j}\right), j=1, \ldots, J$ (so that $x_{1}, \ldots, x_{J} \sim$ iid $f(x \mid y)$ ).

3. For each $j=1, \ldots, J$ calculate $T_{j}=T\left(x_{j}, \theta_{j}\right)$ and $I_{j}=I\left(T_{j} \geq T\right)$, where $T=T(y, \theta)$.

4. Estimate $p$ by $\hat{p}=\frac{1}{J} \sum_{j=1}^{J} I_{j}$ with associated $1-\alpha$ CI

$$
\left(\hat{p} \pm z_{\alpha / 2} \sqrt{\frac{\hat{p}(1-\hat{p})}{J}}\right) .
$$

\section{Exercise 5.17 Testing for independence in a sequence of Bernoulli trials}

A bent coin has some chance of coming up heads whenever it is tossed. Our uncertainty about that chance may be represented by the standard uniform distribution.

The bent coin is tossed 10 times. Heads come up on the first seven tosses and tails come up on the last three tosses.

Using Bayesian methods, test that the 10 tosses were independent.

\section{Solution to Exercise 5.17}

The observed number of runs (of heads or tails in a row) is 2, which seems rather small.

Let $y_{i}$ be the indicator for heads on the ith toss, $(i=1, \ldots, n)(n=10)$, and let $\theta$ be the unknown probability of heads coming up on any single toss.

Also let $x_{i}$ be the indicator for heads coming up on the ith of the next $n$ tosses of the same coin, tossed independently each time. 
Further, let $y=\left(y_{1}, \ldots, y_{n}\right)$ and $x=\left(x_{1}, \ldots, x_{n}\right)$, and choose the test statistic as

$$
T(y, \theta)=R(y),
$$

defined as the number of runs in the vector $y$.

Then an appropriate posterior predictive p-value is

$$
p=P(R(x) \leq R(y) \mid y),
$$

where $y=(1,1,1,1,1,1,1,0,0,0)$ and $R(y)=2$.

Under the Bayesian model:

$$
\begin{aligned}
& \left(y_{1}, \ldots, y_{n} \mid \theta\right) \sim \text { iid } \operatorname{Bern}(\theta) \\
& \theta \sim U(0,1),
\end{aligned}
$$

the posterior is given by

$$
(\theta \mid y) \sim \operatorname{Beta}\left(y_{T}+1, n-y_{T}+1\right),
$$

where $y_{T}=y_{1}+\ldots+y_{n}=7$.

With $J=10,000$, we now generate

$\theta_{1}, \ldots, \theta_{J} \sim$ iid $\operatorname{Beta}(8,4)$.

After that, we do the following for each $j=1, \ldots, J$ :

1. Sample $x_{1}^{j}, \ldots, x_{n}^{j} \sim$ iid $\operatorname{Bern}\left(\theta_{j}\right)$ and form the vector

$$
x^{j}=\left(x_{1}^{j}, \ldots, x_{n}^{j}\right) \text {. }
$$

2. Calculate $R_{j}=R\left(x^{j}\right) \quad$ (i.e. calculate the number of runs in

$$
\left.\left(x_{1}^{j}, \ldots, x_{n}^{j}\right)\right) \text {. }
$$

3. Obtain $I_{j}=I\left(R_{j} \leq R\right)$, where $R=R(y)=2$.

Thereby we estimate $p$ by

$$
\hat{p}=\frac{1}{J} \sum_{j=1}^{J} I_{j}=0.0995
$$

with $95 \%$ CI

$$
\left(\hat{p} \pm 1.96 \sqrt{\frac{\hat{p}(1-\hat{p})}{J}}\right)=(0.0936,0.1054) .
$$


So the posterior predictive p-value is about 10 percent, which may be considered as statistically non-significant. That is, there is insufficient evidence (at the 5\% level of significance, say) to conclude that the 10 tosses of the coin were somehow dependent.

Note 1: Using a suitable formula from runs theory, the exact value of $p$ could be obtained as

$$
\begin{aligned}
p & =\int_{0}^{1} P(R(x) \leq 2 \mid \theta) f_{\text {Beta }(8,4)}(\theta) d \theta \\
& =\int_{0}^{1}\left\{\sum_{x_{T}=0}^{n} P\left(R(x) \leq 2 \mid \theta, x_{T}\right) f\left(x_{T} \mid \theta\right)\right\} f_{\text {Beta }(8,4)}(\theta) d \theta,
\end{aligned}
$$

where:

- $P(R(x) \leq 2 \mid \theta)$ is the exact probability that 2 or fewer runs will result on 10 Bernoulli trials if each has probability of success $\theta$

- $P\left(R(x) \leq 2 \mid \theta, x_{T}\right)$ is the probability of 2 or fewer runs will result when $x_{T}$ 1s and $n-x_{T}$ 0s are placed in a row

- $f\left(x_{T} \mid \theta\right)=\left(\begin{array}{c}n \\ x_{T}\end{array}\right) \theta^{x_{T}}(1-\theta)^{n-x_{T}}$ is the binomial density with parameters $n$ and $\theta$, evaluated at $x_{T}$.

Note 2: It is of interest to recalculate $p$ using data which seems even more 'extreme', for example,

$$
y=(1,1,1,1,1,1,1,1,1,1,1,1,1,1,0,0,0,0,0,0) .
$$

For this data, $R(y)=2$ again but with $n=20$ and $y=14$. In this case,

$$
(\theta \mid y) \sim \operatorname{Beta}\left(y_{T}+1, n-y_{T}+1\right) \sim \operatorname{Beta}(15,7),
$$

and we obtain the estimate $\hat{p}=0.0088$ with $95 \%$ CI $(0.00700 .0106)$.

Thus there is, as was to be expected, much stronger statistical evidence to reject the null hypothesis of independence. 


\section{R Code for Exercise 5.I7}

$\mathrm{R}=$ function(v) $\{\mathrm{m}=$ length(v); sum(abs $(\mathrm{v}[-1]-\mathrm{v}[-\mathrm{m}]))+1\}$

\# Calculates the runs in vector $\mathrm{v}$

$\mathrm{R}(\mathrm{c}(1,1,1,0,1)) \# 3$ testing ...

$\mathrm{R}(\mathrm{c}(1,1)) \# 1$

$\mathrm{R}(\mathrm{c}(1,0,1,0,1)) \# 5$

$\mathrm{R}(\mathrm{c}(0,0,1,1,1)) \# 2$

$R(c(1,0,0,1,1,0,0,1,1,1,1,0)) \# 6$ .... all OK

$\mathrm{n}=10 ; \mathrm{J}=10000 ; \mathrm{Iv}=\mathrm{rep}(0, \mathrm{~J})$; set.seed(214); tv=rbeta(J,8,4)

for $(j$ in $1: J)\{x j=\operatorname{rbinom}(n, 1, \operatorname{tv}[j])$; if $(R(x j)<=2) \operatorname{Iv}[j]=1\}$

$\mathrm{p}=$ mean(Iv); $\mathrm{ci}=\mathrm{p}+\mathrm{c}(-1,1) *$ qnorm $(0.975) * \operatorname{sqrt}(\mathrm{p} *(1-\mathrm{p}) / \mathrm{J})$

$c(p, c i) \# 0.099500 .093630 .10537$

n=20; J=10000; Iv=rep(0,J); set.seed(214); tv=rbeta(J,15,7)

for $(j$ in $1: J)\{x j=r b i n o m(n, 1, \operatorname{tv}[j]) ;$ if $(R(x j)<=2) \operatorname{Iv}[j]=1\}$

$p=\operatorname{mean}($ Iv); $c i=p+c(-1,1) *$ qnorm $(0.975) * \operatorname{sqrt}(p *(1-p) / J)$

$c(p, c i) \# 0.0088000 .0069690 .010631$ 


\section{CHAPTER 6}

\section{I Introduction}

Monte Carlo methods were introduced in the last chapter. These included basic techniques for generating a random sample and methods for using such a sample to estimate quantities such as difficult integrals. This chapter will focus on advanced techniques for generating a random sample, in particular the class of techniques known as Markov chain Monte Carlo (MCMC) methods. Applying an MCMC method involves designing a suitable Markov chain, generating a large sample from that chain for a burn-in period until stochastic convergence, and making appropriate use of the values following that burn-in period.

Like other iterative techniques such as the Newton-Raphson and Expectation-Maximisation algorithms, MCMC methods require an arbitrary starting point (or vector) and then involve iterating repeatedly until convergence. But MCMC methods are distinguished from these other methods by the fact that the update at each iteration is not deterministic but stochastic, with the probability distributions involved dependent on results from the previous iteration.

Typically, MCMC methods are used to sample from multivariate probability distributions rather than univariate ones. This is because a univariate distribution can usually be sampled from using simpler methods. Nevertheless, we will begin our discussion of MCMC methods with a description of the Metropolis algorithm for sampling from univariate distributions, because that algorithm constitutes a basic building block for the more advanced methods.

\subsection{The Metropolis algorithm}

Suppose that we wish to sample from a univariate distribution with pdf $f(x)$ for which rejection sampling and the other techniques described previously are problematic (say). Then another way to proceed is via the Metropolis algorithm. This is an example of Markov chain Monte Carlo (MCMC) methods. The Metropolis algorithm may be described as follows. 
As with the Newton-Raphson algorithm, we begin by specifying an initial value of $x$, call it $x_{0}$. We then also need to specify a suitable driver distribution which is easy to sample from, defined by a pdf, $g(t \mid x)$.

For now, we will assume the driver to be symmetric, in the sense that $g(t \mid x)=g(x \mid t)$,

or more precisely,

$$
g(t=a \mid \theta=b)=g(t=b \mid \theta=a) \quad \forall a, b \in \mathfrak{R} .
$$

Note: The driver distribution may also be non-symmetric, but this case will be discussed later.

We then do the following iteratively for each $j=1,2,3, \ldots, K$ (where $K$ is 'large'):

(a) Generate a candidate value of $x$ by sampling $x_{j}^{\prime} \sim g\left(t \mid x_{j-1}\right)$. We call $x_{j}^{\prime}$ the proposed value and $g\left(t \mid x_{j-1}\right)$ the proposal density.

(b) Calculate the acceptance probability as $p=\frac{f\left(x_{j}^{\prime}\right)}{f\left(x_{j-1}\right)}$.

Note: If $p>1$ then we take $p=1$. Also, if $x_{j}^{\prime}$ is outside the range of possible values for the random variable $x$, then $f\left(x_{j}^{\prime}\right)=0$ and so $p=0$.

(c) Accept the proposed value $x_{j}^{\prime}$ with probability $p$.

To determine if $x_{j}^{\prime}$ is accepted, generate $u \sim U(0,1)$ (independently). If $u<p$ then accept $x_{j}^{\prime}$, and otherwise reject $x_{j}^{\prime}$.

(d) If $x_{j}^{\prime}$ has been accepted then let $x_{j}=x_{j}^{\prime}$, and otherwise let $x_{j}=x_{j-1}$ (i.e. repeat the last value $x_{j-1}$ in the case of a rejection).

This procedure results in the realisation of a Markov chain, $x_{0}, x_{1}, x_{2}, \ldots, x_{K}$. The last value of this chain, $x_{K}$, may be taken as an 
observation from $f(x)$, at least approximately. The approximation will be extremely good if $K$ is sufficiently large.

If we want a random sample of size $J$ from $f(x)$, then the whole procedure can be repeated another $J-1$ times, each time using either the same starting value $x_{0}$ or a different one.

If $K$ is sufficiently large, stochastic convergence will be achieved within $K$ iterations, regardless of the point(s) from which the algorithm is started. Relabelling the last value, $x_{K}$, in the $j$ th chain as $x_{j}(j=1, \ldots, J)$ leads the required sample, namely $x_{1}, \ldots, x_{J} \sim$ iid $f(x)$.

Generating a chain of length $K$ a large number times $J$ may be considered wasteful of computer resources. So typically only one long chain is generated, of length $K=B+J$, where $B$ is sufficiently large for stochastic convergence to be achieved from the single starting value, $x_{0}$, and $J$ is again the required sample size. Discarding the results of the first $B$ iterations (called the burn-in, including also $x_{0}$ ) and relabelling the last $J$ values of the chain appropriately, the result will be the sample $x_{1}, \ldots, x_{J} \sim f(x)$.

A problem with this second method of generating the sample values is that they will be autocorrelated to some extent i.e. not a truly random (iid) sample from the distribution $f(x)$. We will later discuss this issue and how to deal with the problems that may arise from it. For the moment, we stress that $x_{1}, \ldots, x_{J}$ will be approximately a random sample from $f(x)$. Moreover, if $J$ is sufficiently large, then these values will be effectively independent. This means that a probability histogram of these values will in fact converge to $f(x)$ as $J$ tends to infinity.

\section{Exercise 6.I A simple application of the Metropolis algorithm}

Illustrate the Metropolis algorithm by generating a sample of size 400 from the distribution defined by the density

$$
f(x)=6 x^{5}, 0<x<1 .
$$

Note: This is just the Beta(6,1) density and could be sampled from easily in many other ways. 


\section{Solution to Exercise 6.I}

Let us specify the driver distribution as the uniform distribution from $x-c$ to $x+c$, where $c$ is a tuning parameter whose value is to be determined (as discussed further below). Thus the driver density is

$$
g(t \mid x)=\frac{1}{2 c}, x-c<t<x+c,
$$

or equivalently

$$
g(t \mid x)=\frac{1}{2 c} I(|t-x|<c) .
$$

Note: This driver is symmetric, since

$$
g(t=a \mid x=b)=g(t=b \mid x=a) \quad \forall a, b \in \mathfrak{R} .
$$

The $j$ th iteration of the algorithm involves first sampling a candidate value (or proposed value) from the driver distribution centred at the last value, namely

$$
x_{j}^{\prime} \sim U\left(x_{j-1}-c, x_{j-1}+c\right),
$$

and then accepting this candidate value with probability

$$
p=\frac{f\left(x=x_{j}^{\prime}\right)}{f\left(x=x_{j-1}\right)}=\frac{\not b x_{j}^{\prime 5}}{\not b x_{j-1}^{5}}=\left(\frac{x_{j}^{\prime}}{x_{j-1}}\right)^{5},
$$

where $p$ is taken to be:

0 in the case where $x_{j}^{\prime}<0$ or $x_{j}^{\prime}>1$

1 in the case where $x_{j-1}<x_{j}^{\prime} \leq 1$.

Note: The cancellation of $6 \mathrm{~s}$ in (6.1) illustrates an attractive feature of the Metropolis algorithm generally: only the kernel of the sampling density is needed. Here, the kernel of the sampling density $f(x)=6 x^{5}$ is $k(x)=x^{5}$. This fact can be very useful in more complicated situations where only the kernel of the sampling density is known.

Starting from $x_{0}=0.1$ and with $c=0.15$ (arbitrarily), we obtain a Markov chain of length $K=500$, with values as illustrated in Figure 6.1.

Some of the values of this chain are as follows: 


$$
\begin{aligned}
& x_{0}, \ldots, X_{10}, \ldots \ldots \ldots . . \\
& x_{301}, \ldots, x_{310}, \ldots \ldots \ldots . . . \\
& x_{491}, \ldots, x_{500}= \\
& 0.1000,0.1000,0.1000,0.1000,0.1000, \underline{0.1861}, 0.2650,0.2650 \text {, } \\
& \text { 0.4065, 0.4388, 0.4388, } \\
& \text { 0.9261, 0.9987, 0.9987, 0.9987, 0.9987, 0.9725, 0.8889, 0.8889, } \\
& \text { 0.9672, 0.9315, } \\
& \text { 0.8058, 0.6811, 0.6073, 0.4587, 0.4353, 0.3462, 0.3462, 0.4177, } \\
& 0.4177,0.4656 \text {. }
\end{aligned}
$$

Note: There were four rejections until the first acceptance, at iteration 5, where $x_{5}=x_{5}^{\prime}=0.1861$, as underlined above.

Figure 6.2 shows a probability histogram of the last $J=400$ values, together with the exact density of $x$. It would appear that stochastic equilibrium has been achieved by about iteration 50 . So we may, very conservatively, discard the first $B=100$ iterations as the burn-in.

The acceptance rate (AR) for this Markov chain is found to be $64 \%$, meaning that 320 of the 500 candidate values $x_{j}^{\prime}$ were accepted and $36 \%$ (or 180) were rejected.

Figure 6.I Trace of sample values with tuning constant $c=0.15$

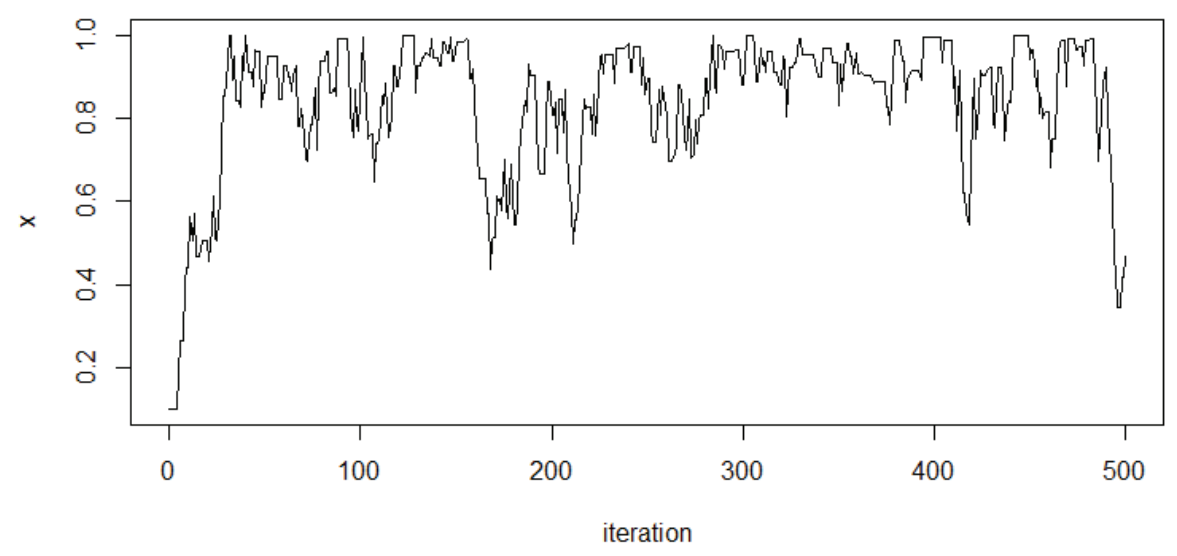


Figure 6.2 Probability histogram with tuning constant $c=0.15$

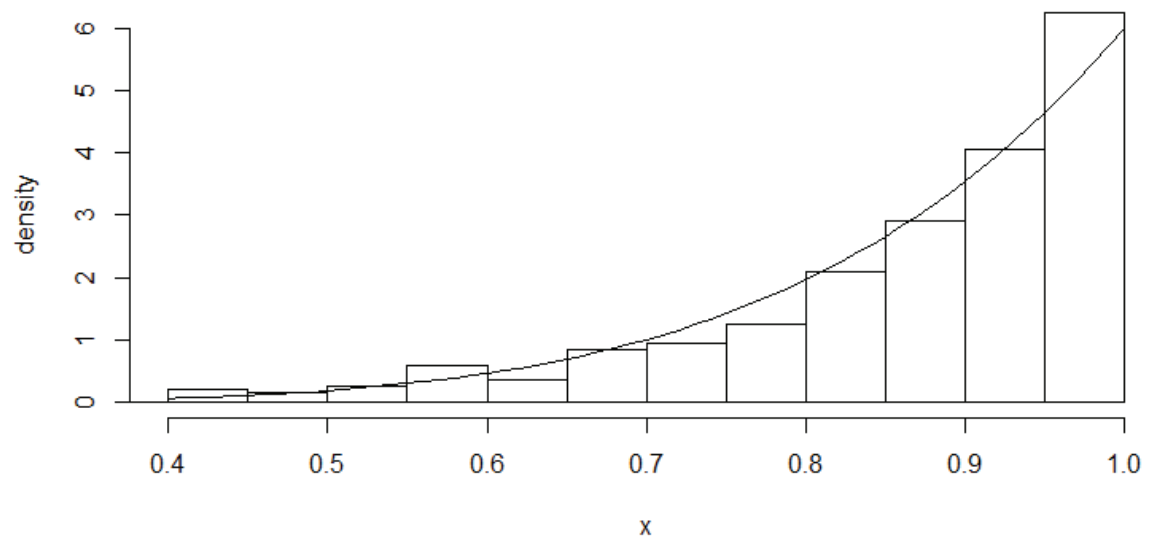

\section{Changing the tuning parameter}

What happens if we make the tuning parameter $c=0.15$ larger? Figures 6.3 and 6.4 are a repeat of Figures 6.1 and 6.2, respectively, but using simulated values from a run of the Metropolis algorithm with $c=0.65$.

In this case the acceptance rate is only $20.8 \%$ and the histogram is a poorer estimate of the true density (to which it would however converge as $J \rightarrow \infty)$. We say that the algorithm is now displaying poor mixing compared to results in the first run of 500 where $c=0.15$.

What happens if we make $c=0.15$ smaller? Figures 6.5 and 6.6 are a repeat of Figures 6.1 and 6.2, respectively, but using simulated values from a run of the Metropolis algorithm with $c=0.05$.

In this case the acceptance rate is higher at $83 \%$, there is greater autocorrelation, and the histogram is again a poorer estimate of the true density (to which it would however still converge as $J \rightarrow \infty$ ). We again say that the algorithm is mixing poorly.

It is important to stress that even if the algorithm is mixing poorly (whether this be due to the tuning constant being too large or too small), it will eventually (with a sufficiently large value of $J$ ) yield a sample that is useful for inference to the desired degree of precision. Tweaking the tuning constant is merely a device for optimising computational efficiency. 
Figure 6.3 Trace of sample values with tuning constant $c=0.65$

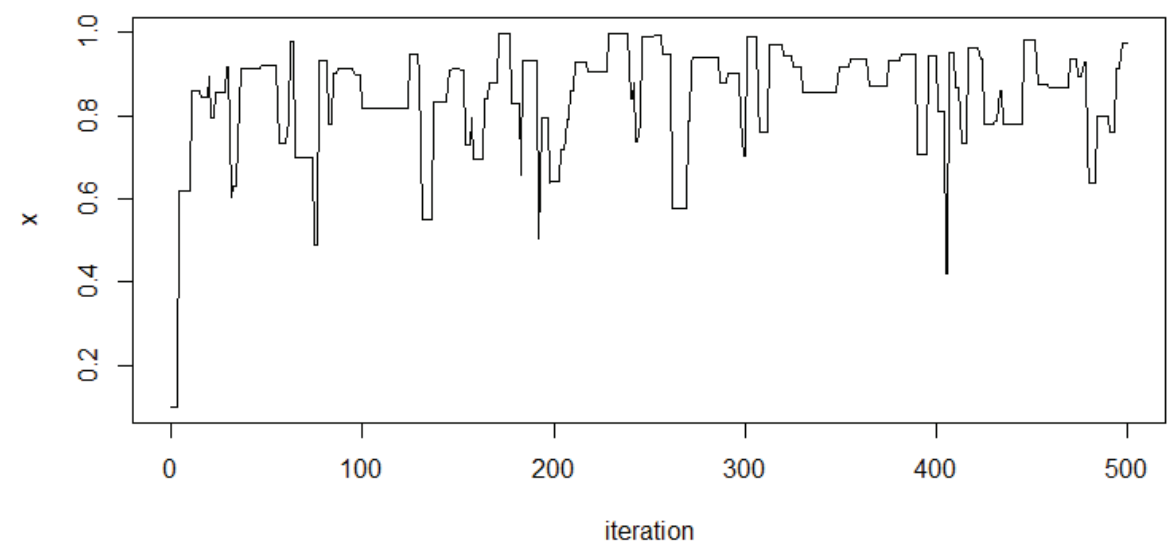

Figure 6.4 Probability histogram with tuning constant $c=0.65$

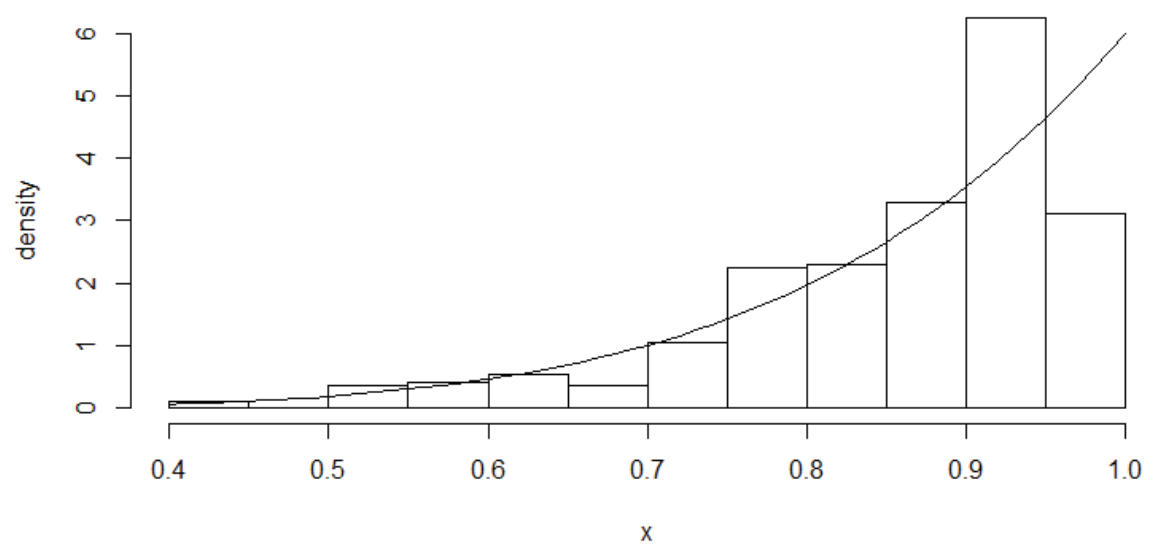


Figure 6.5 Trace of sample values with tuning constant $c=0.05$

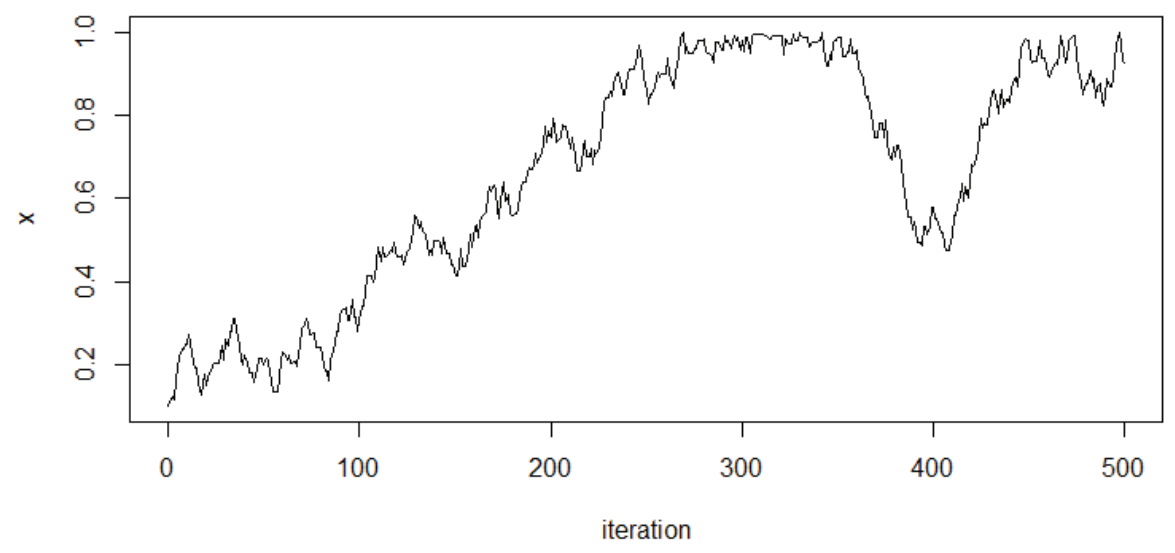

Figure 6.6 Probability histogram with tuning constant $c=0.05$

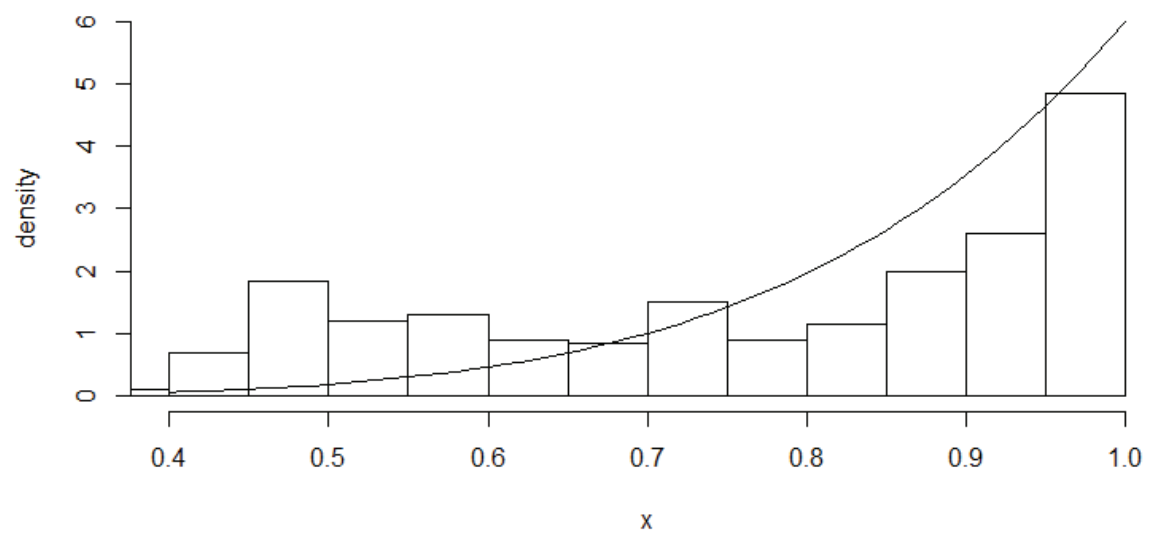

\section{R Code for Exercise 6.I}

MET <- function $(K, \mathrm{x}, \mathrm{C})\{$

\# This function performs the Metropolis algorithm for a simple model.

\# Inputs: $\quad K=$ total number of iterations

\# $\quad x=$ initial value of $x$

\# $\quad \mathrm{c}=$ tuning parameter.

\# Outputs: $\quad$ \$vec $=$ vector of $(K+1)$ values of $x$

\# $\quad$ \$ar = acceptance rate 
vec $<-\mathrm{x}$; ct $<-0$

for $(\mathrm{j}$ in $1: \mathrm{K})\{$

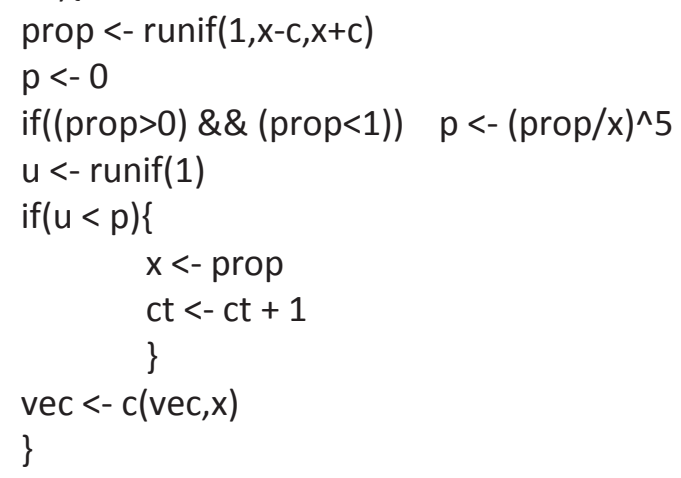




\section{Exercise 6.2 Sampling from a normal distribution via the Metropolis algorithm}

Use the Metropolis algorithm and a uniform driver to sample 10,000 values from the standard normal distribution.

Check your result by comparing the sample mean and sample standard deviation of your sample to the true theoretical values, 0 and 1 .

Calculate a Monte Carlo 95\% confidence interval for the normal mean, 0.

\section{Solution to Exercise 6.2}

Since $f(x) \propto e^{-\frac{1}{2} x^{2}}$, the acceptance probability at iteration $j$ is given by

$$
p=\frac{f\left(x=x_{j}^{\prime}\right)}{f\left(x=x_{j-1}^{\prime}\right)}=\frac{\frac{1 / e^{-\frac{1}{2}\left(x_{j}^{\prime}\right)^{2}}}{\sqrt{2 \pi}}}{\frac{1}{\sqrt{2 \pi}} e^{-\frac{1}{2}\left(x_{j-1}\right)^{2}}}=\exp \left\{\frac{\left(x_{j-1}\right)^{2}-\left(x_{j}^{\prime}\right)^{2}}{2}\right\} .
$$

Using the same uniform driver as in Exercise 6.1, $x_{0}=5$ and $c=2.5$ (where this tuning constant was chosen after some experimentation), we obtain a Markov chain of length $K=10,500$, as shown in Figure 6.7.

Figure 6.8 shows a histogram of the last $J=10,000$ values, together with the standard normal density overlaid.

We have very conservatively discarded the first $B=500$ iterations as the burn-in. The acceptance rate for this Markov chain is $56.1 \%$.

The average of the $J$ sampled values is 0.0355 (close to 0 ) and their sample standard deviation is 1.0047 (close to 1). These values lead to a 95\% CI for the normal mean equal to $(0.0158,0.0552)$. We note that this CI does not contain the true value, 0 , as one might expect. The underlying issue behind this fact will be discussed generally in the next section. 
Figure 6.7 Trace of sample values

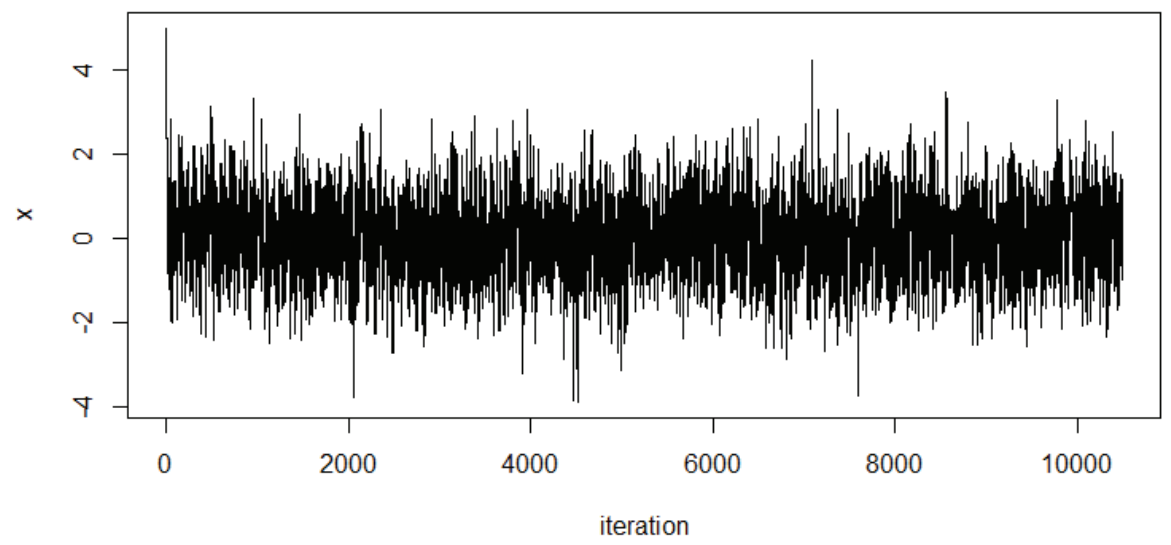

Figure 6.8 Probability histogram

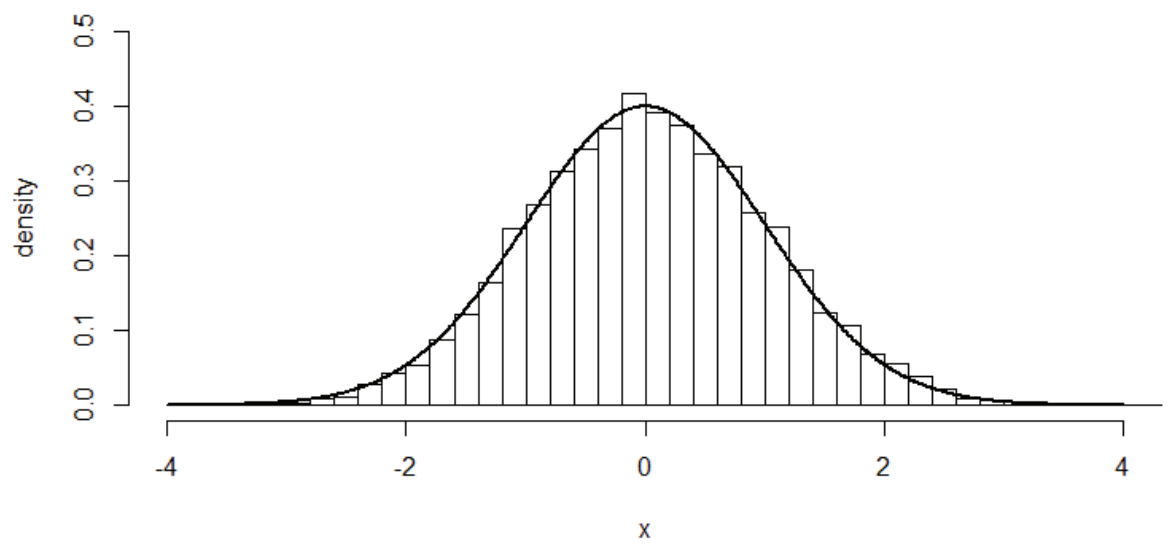

R Code for Exercise 6.2

MET <- function $(K, x, c)\{$

\# This function performs the Metropolis algorithm to sample from the \# standard normal dsn.

\# Inputs: $\quad K=$ total number of iterations

\# $\quad \mathrm{x}=$ initial value of $\mathrm{x}$

$\# \quad c=$ tuning parameter.

\# Outputs: $\quad \$ \mathrm{vec}=$ vector of $(\mathrm{K}+1)$ values of $\mathrm{x}$

\# $\quad$ \$ar $=$ acceptance rate. 


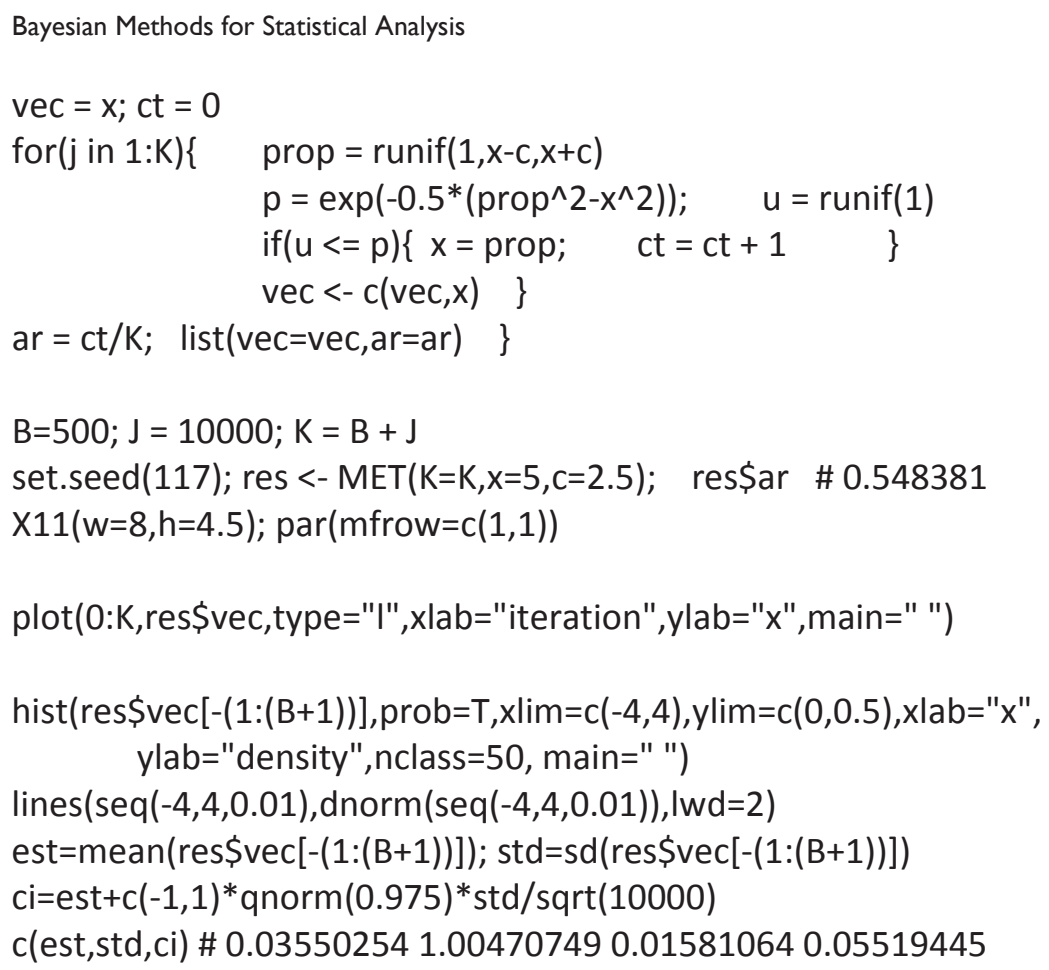

\subsection{The batch means method}

As stated earlier, the output from the Metropolis algorithm leads to a sample, $x_{1}, \ldots, x_{J}$, from the target density, $f(x)$, which exhibits some degree of positive autocorrelation.

This does not present a major problem when one is interested in calculating only point estimates. For example, if we wish to estimate the distribution mean $E X=\int x f(x) d x$, each sample value $x_{j}$ has expected value $E X$, and this is true regardless of how severely the simulated values are correlated (assuming that all the simulated values are collected after stochastic convergence). Therefore, the expected value of the Monte Carlo mean is also exactly $E X$ (or very nearly so).

However, when one uses a severely and positively autocorrelated Monte Carlo sample to calculate the standard $1-\alpha$ confidence interval for a quantity such as $E X$, the true coverage probability of that interval may be far less than the intended nominal value of $1-\alpha$.

One way of dealing with this problem is to generate $J$ independent chains and take the last value in each chain. Note that this was our original formulation of the Metropolis algorithm (i.e. for sampling a single value). 
Another option is to generate a single long chain, of length $K=B+10 \mathrm{~J}$ (say) and thin it out by recording only every 10th value in the chain after burn-in. Even so, there will still be some autocorrelation remaining in the $J$ resulting values. The autocorrelation could be reduced further by changing 10 to 100, say; but this would be at the cost of a 10-fold increase in computer time needed.

A more efficient solution to the autocorrelation problem is the batch means method. We will now describe how this works for when we wish to construct a $1-\alpha$ CI for $E X=\int x f(x) d x$ based on an autocorrelated sample $x_{1}, \ldots, x_{J} \dot{\sim}$ iid $f(x)$.

The batch means $C I$ will be different from the ordinary $C I$, namely $\left(\bar{x} \pm 1.96 s_{x} / \sqrt{J}\right)$, where $\bar{x}$ and $s_{x}$ are the sample mean and sample standard deviation of $x_{1}, \ldots, x_{J}$. The batch means CI is obtained as follows.

First, break up the $J$ sample values into $m$ batches of size $n$ each, so that:

Batch 1 contains values $1, \ldots, n$ (the first $n$ values)

Batch 2 contains values $n+1, \ldots, 2 n$ (the next $n$ values)

Batch $m$ contains values $(m-1) n+1, \ldots, J \quad$ (the last $n$ values).

Next: Let $y_{k}$ be the mean of the $n x_{j}$-values in the $k$ th batch $(k=1, \ldots, m)$. Let $s_{y}^{2}$ be the sample variance of $y_{1}, \ldots, y_{m}$. Note: Thus $s_{y}^{2}=\frac{1}{m-1} \sum_{k=1}^{m}\left(y_{k}-\bar{y}\right)^{2}$, where $\bar{y}=\frac{1}{m} \sum_{k=1}^{m} y_{k}=\bar{x}$ is the mean of the batch means and identical to the mean of all $J x_{j}$-values.

Finally, compute the $1-\alpha$ batch means CI for $E X$ as $\left(\bar{x} \pm 1.96 s_{y} / \sqrt{m}\right)$. 


\section{Discussion}

The rationale for the batch means method is as follows. If the batch size $n$ is sufficiently large then, by the central limit theorem,

$$
y_{1}, \ldots, y_{m} \dot{\sim} \text { iid } N\left(\mu, \sigma^{2} / n\right),
$$

where $\mu=E\left(x_{j}\right)$ and $\sigma^{2}=\operatorname{Var}\left(x_{j}\right)$.

Consequently,

$$
\bar{y} \dot{\sim} N\left(\mu, \frac{\sigma^{2} / n}{m}\right) \sim N\left(\mu, \frac{\sigma^{2}}{J}\right),
$$

since $J=m n$.

Therefore a $1-\alpha$ CI for $\mu$ is

$$
\left(\bar{y} \pm z_{\alpha / 2} r / \sqrt{J}\right) \text {, }
$$

where $r$ is an estimate of $\sigma$.

Now, an unbiased estimator of $\sigma^{2} / n$ is $s_{y}^{2}$.

So an unbiased estimator of $\sigma^{2}$ is $n s_{y}^{2}$.

It follows that a $1-\alpha \mathrm{CI}$ for $\mu$ is

$$
\left(\bar{x} \pm z_{\alpha / 2} \sqrt{n s_{y}^{2}} / \sqrt{J}\right)=\left(\bar{x} \pm z_{\alpha / 2} s_{y} / \sqrt{m}\right) .
$$

\section{Exercise 6.3 Testing the batch means method}

We wish to perform Monte Carlo estimation of the expected value of $X$ whose pdf is given by $f(x) \propto x^{2}, 0<x<2$.

Note: Here, $X \sim 2 \operatorname{Beta}(3,1)$ and so $E X=2 \times 3 /(3+1)=1.5$.

(a) Use the Metropolis algorithm to generate a sample of size $J=1,000$ from $X$ 's distribution after a burn-in of 100 .

Then use this sample to estimate $E X$, together with a 95\% confidence interval for $E X$. For this CI use the formula $(\bar{x} \pm 1.96 s / \sqrt{J})$, where $s^{2}$ is the sample variance of the $J$ sampled $X$-values. Also draw a histogram of the $J X$-values overlaid with the exact pdf of $X$. 
(b) Use the output from the Metropolis algorithm in (a) to construct another 95\% CI for $E X$, one using the batch means method, as follows:

Divide the $J=1,000$ iterations into $m=20$ consecutive batches, each having $n=50$ values of $X$.

Let $y_{k}$ be the average of the $n X$-values in the $k$ th batch $(k=1, \ldots, m)$.

Let $s_{y}^{2}$ be the sample variance of the $m$ batch means $y_{1}, \ldots, y_{m}$.

Let the confidence interval for $E X$ be $\left(\bar{X} \pm 1.96 s_{y} / \sqrt{m}\right)$.

(c) Conduct a Monte Carlo experiment to assess the quality of the two CIs for $E X$ in (a) and (b).

Do this by implementing the following three-step procedure a total of $R=100$ times:

(i) Run the Metropolis algorithm in (a) so as to generate $J=1,000$ observations from $f(x)$.

(ii) Calculate the CI in (a) and count 1 if 1.5 is in it.

(iii) Calculate the CI in (b) and count 1 if 1.5 is in it.

Now divide the total count from (ii) by $R$ to get an unbiased point estimate of the probability that the ordinary CI for $E X$ in (a) contains $E X$.

Similarly, divide the two total count from (iii) by $R$ to get an unbiased point estimate of the probability that the batch means CI for $E X$ in (b) contains $E X$.

Also produce 95\% CIs for the two probabilities just mentioned.

(d) Repeat the experiment in (c) but with the following in place of (i):

Generate $J=1,000$ observations from $X$ 's distribution using the rbeta() function. 


\section{Solution to Exercise 6.3}

(a) Let us specify a uniform driver centred at the last value and with halfwidth $h$. We now iterate as follows after choosing a suitable starting value of $x$ :

Sample $x^{\prime} \sim U(x-h, x+h)$.

If $x^{\prime}$ is outside the interval $(0,2)$ then automatically reject $x^{\prime}$.

Otherwise accept $x^{\prime}$ with probability $\min (1, p)$, where

$$
p=x^{\prime 2} / x^{2} \text {. }
$$

Starting from $x=1$ with $h=0.7$, we get an acceptance rate of $55 \%$ and simulated values as depicted in Figures 6.9 and 6.10.

Taking the last 1,000 values of $x$ as a random sample from $f(x)$ we estimate $E X$ as 1.539 , with ordinary $95 \%$ CI $(1.467,1.611)$. We note that this CI does not contain the true value, 1.5.

Figure 6.9 Trace of sample values

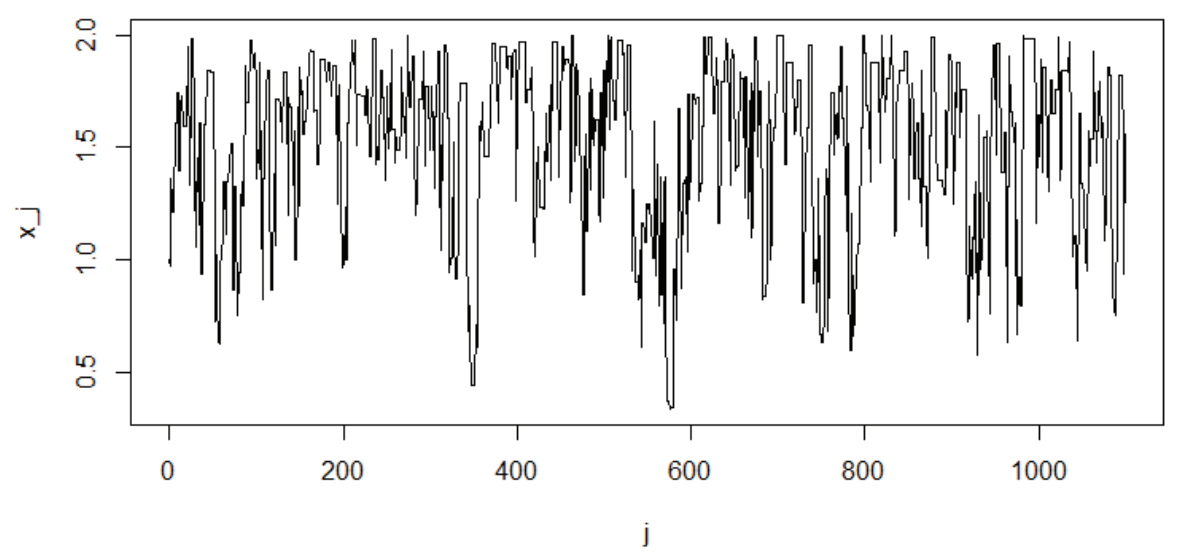




\section{Figure 6.10 Histogram of sample values}

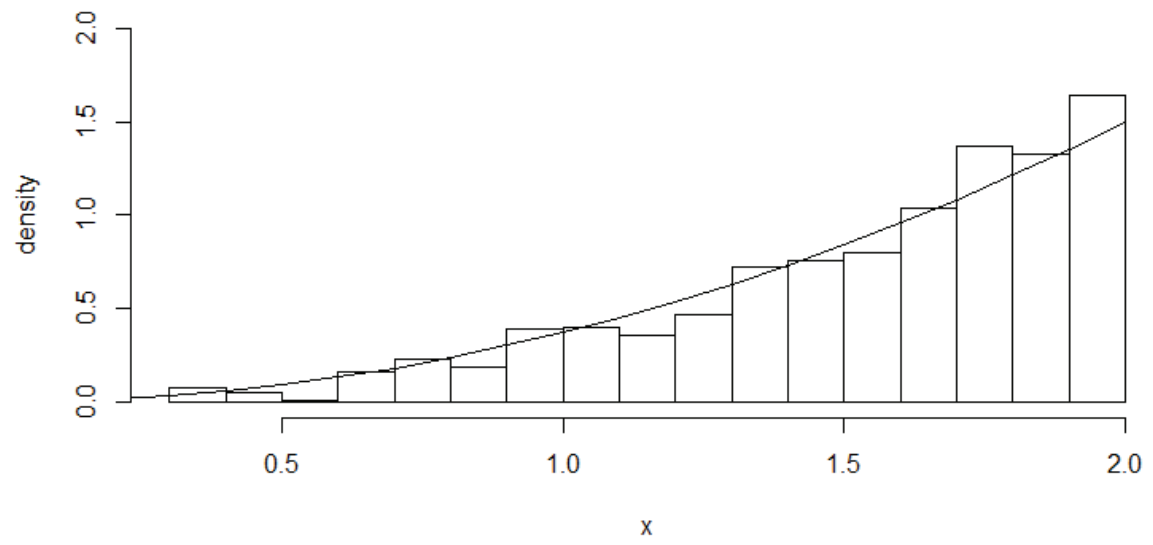

(b) Applying the batch means method with $m=20$ and $n=50$, we estimate $E X$ as 1.539 again, but with $95 \%$ CI $(1.467,1.611)$. Note that this CI is wider than the $\mathrm{CI}$ in (a) and does contain the true value, 1.5.

(c) After conducting the experiment we estimate $p_{1}$, the true probability content of the ordinary $95 \% \mathrm{CI}$ in (a), as $52.0 \%$, with $95 \%$ CI $42.2 \%$ to $61.8 \%$.

We also estimate $p_{2}$, the true probability content of the batch means $95 \%$ CI in (b) (with $m=20$ and $n=50$ ), as $90.0 \%$, with 95\% CI $84.1 \%$ to 95.9\%.

We see that in this example the batch means method has performed far better than the ordinary method for constructing 95\% CIs for EX from the output of a Metropolis algorithm.

(d) Generating each value of $X$ as twice a random number from the $\operatorname{Beta}(3,1)$ distribution, we estimate $p_{1}$ by $92.0 \%$, with $95 \%$ CI $86.7 \%$ to $97.3 \%$. We also estimate $p_{2}$ by $90.0 \%$, with $95 \%$ CI $84.1 \%$ to $95.9 \%$.

We see that the two CIs have performed about equally well when calculated using a truly random sample from $X$ 's distribution. In such situations, the batch means $\mathrm{CI}$ is in fact slightly inferior and the ordinary CI should be used. 


\section{R Code for Exercise 6.3}

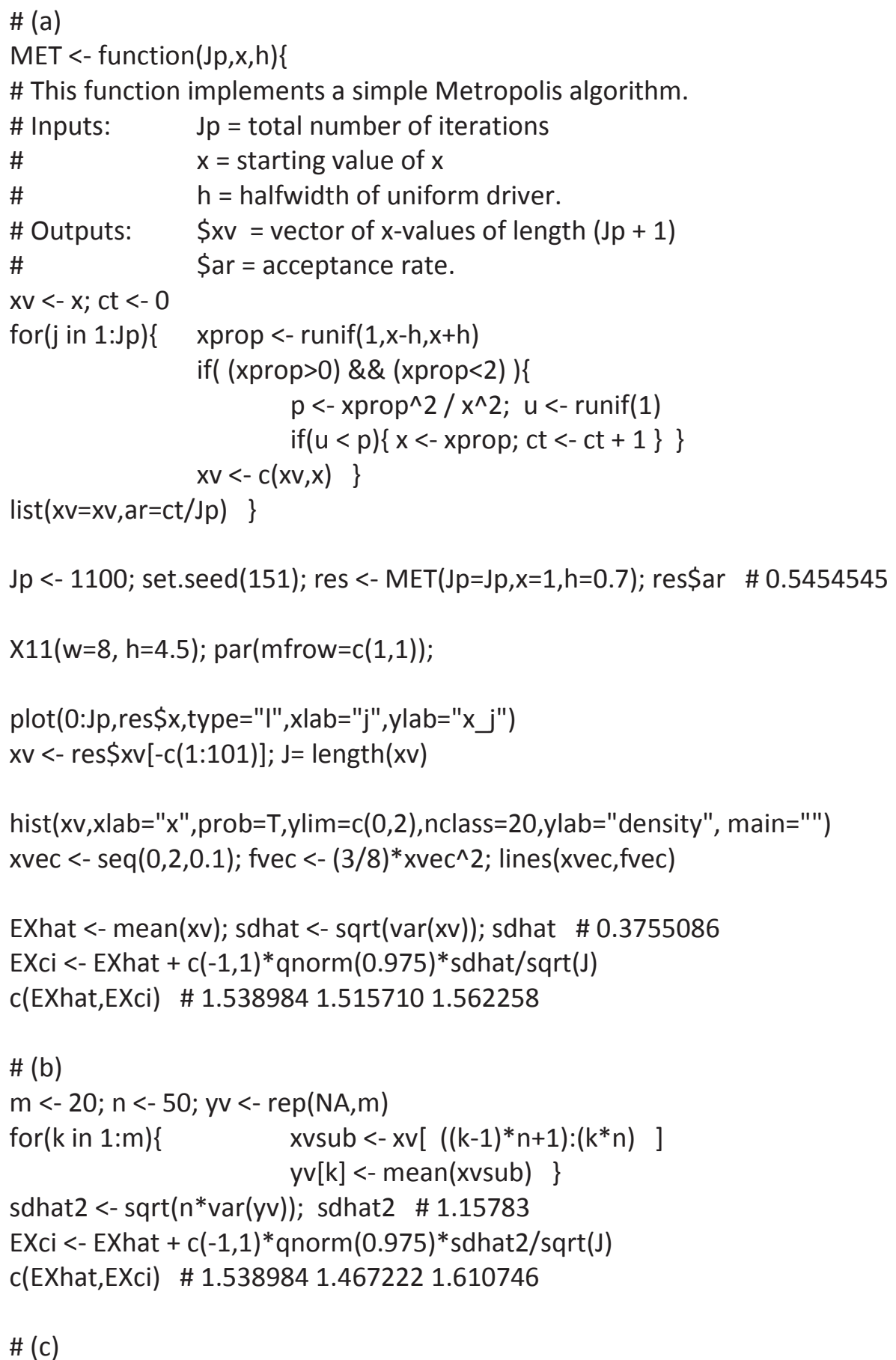

$\mathrm{R}<-100 ; \mathrm{m}<-$ 20; $\mathrm{n}<-$ 50; J <- 1000; burn <- 100; EX <- 1.5; ct1 <- 0 ; ct2 <- 0; yv <- rep(NA,m); set.seed(214) 


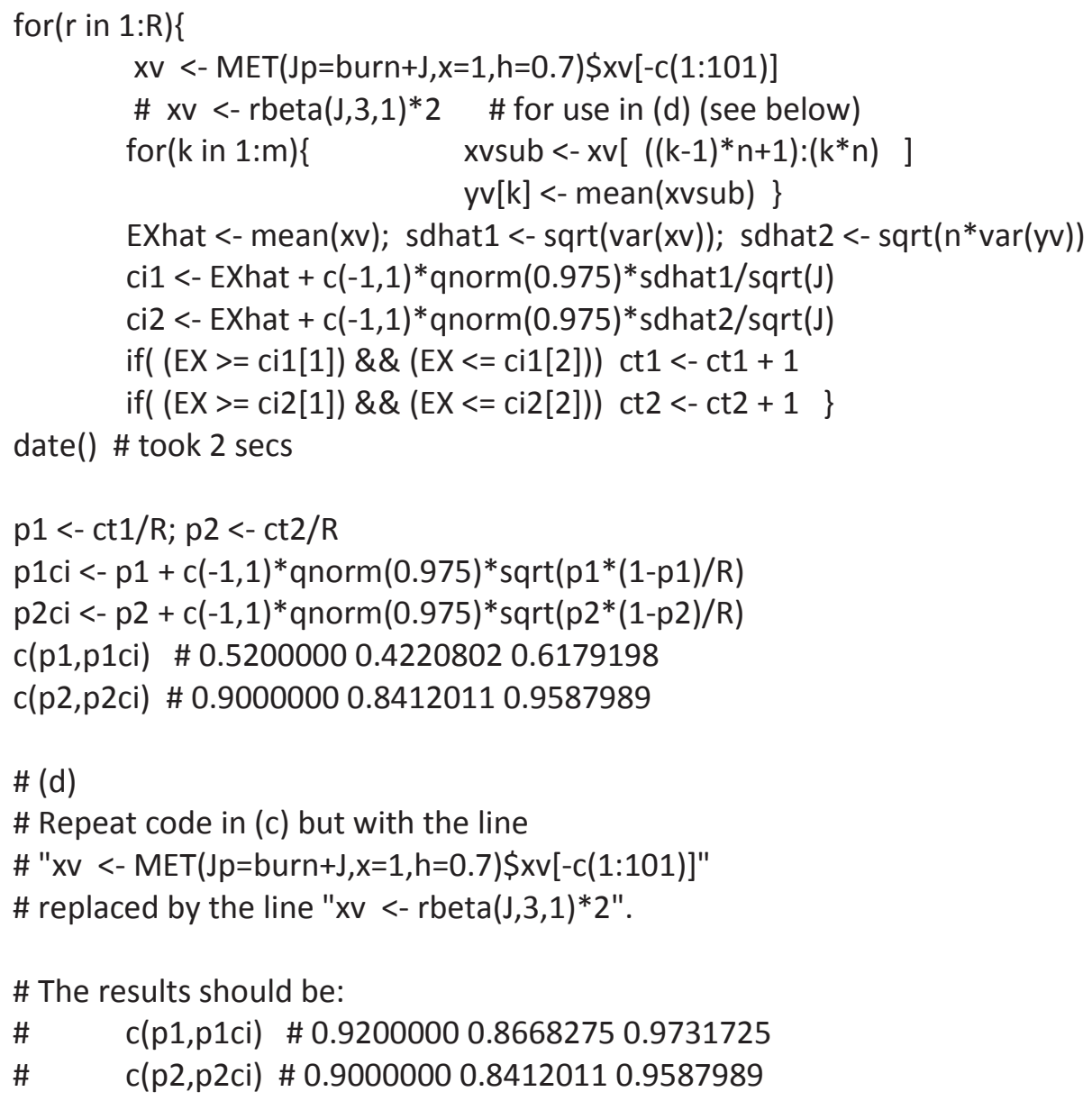

\section{Exercise 6.4 Bayesian inference via the Metropolis algorithm}

The prior on a normal mean $\mu$ is uniform from zero to infinity. Values are sampled repeatedly from the $N(\mu, 1)$ distribution until $n=4$ positive values have been observed, resulting in the data: $0.1,0.2,1.9,0.8$.

Find the posterior mean of $\mu$ in the following ways:

(a) exactly, using numerical integration in $\mathrm{R}$

(b) approximately, using a Monte Carlo method that does not involve Markov chains

(c) approximately, using the Metropolis algorithm with a normal driver. 


\section{Solution to Exercise 6.4}

(a) The posterior density of $\mu$ is

$$
f(\mu \mid y) \propto f(\mu) f(y \mid \mu) \propto 1 \times \prod_{i=1}^{n} \frac{e^{-\frac{1}{2}\left(y_{i}-\mu\right)^{2}}}{1-\Phi(-\mu)},
$$

since $P(y>0 \mid \mu)=1-P\left(z<\frac{0-\mu}{1}\right)=1-\Phi(-\mu)$.

Thus $f(\mu \mid y) \propto(1-\Phi(-\mu))^{-n} \exp \left(-\frac{1}{2} \sum_{i=1}^{n}\left(y_{i}-\mu\right)^{2}\right)$

$$
\begin{aligned}
& =(1-\Phi(-\mu))^{-n} \exp \left(-\frac{1}{2}\left[(n-1) s^{2}+n(\bar{y}-\mu)^{2}\right]\right) \\
& \propto(1-\Phi(-\mu))^{-n} \exp \left(-\frac{1}{2} n(\mu-\bar{y})^{2}\right) \\
& \equiv k(\mu), \quad \mu>0 \quad \text { (this is the kernel of the posterior density). }
\end{aligned}
$$

Thus $\hat{\mu}=E(\mu \mid y)=\frac{\int_{0}^{\infty} \mu^{1} k(\mu) d \mu}{\int_{0}^{\infty} \mu^{0} k(\mu) d \mu}=\frac{I_{1}}{I_{0}}$,

where $I_{q}=\int_{0}^{\infty} \mu^{q} k(\mu) d \mu, \quad q=0,1$.

Using integrate() in R we obtain $I_{0}=4.328041, I_{1}=2.328058$ and hence $\hat{\mu}=0.5379$.

$\int_{0}^{\infty} \mu^{1}(1-\Phi(-\mu))^{-n} h(\mu) d \mu$

(b) Observe that $\hat{\mu}=\frac{0}{\infty}$

$$
\int_{0}^{\infty} \mu^{0}(1-\Phi(-\mu))^{-n} h(\mu) d \mu
$$

where $h(\mu)=\frac{\frac{\sqrt{n}}{\sqrt{2 \pi}} \exp \left(-\frac{n}{2}(\mu-\bar{y})^{2}\right)}{1-\Phi\left(\frac{0-\bar{y}}{1 / \sqrt{n}}\right)}$. 
Note: $h(\mu)$ is the density of the $N(\bar{y}, 1 / n)$ distribution restricted to the positive real line.

Thus $\hat{\mu}=\frac{E_{1}}{E_{0}}$, where: $E_{q}=E\left\{\mu^{q}(1-\Phi(-\mu))^{-n}\right\}, \quad q=0,1$

$$
\mu \sim h(\mu) \sim N(\bar{y}, 1 / n) I(\mu>0) .
$$

Note: At this point we 'forget' about the posterior distribution of $\mu$.

We see that a non-Markov chain Monte Carlo estimate of $\hat{\mu}$ is

$$
\tilde{\mu}=\frac{\tilde{E}_{1}}{\tilde{E}_{0}},
$$

where: $\tilde{E}_{q}=\frac{1}{J} \sum_{j=1}^{J} \mu_{j}^{q}\left(1-\Phi\left(-\mu_{j}\right)\right)^{-n}$

$$
\mu_{1}, \ldots, \mu_{J} \sim \text { iid } h(\mu) \text {. }
$$

Note: To obtain the required sample here, we repeatedly sample $\mu \sim N(\bar{y}, 1 / n)$ until $J$ positive values have been achieved.

Implementing this strategy in R using the rnorm() function with a Monte Carlo sample size of $J=100,000$, we obtain $\tilde{E}_{0}=3.7059926$, $\tilde{E}_{1}=1.9900593$ and hence $\tilde{\mu}=0.5370$.

(c) Using the Metropolis algorithm and a normal driver distribution with standard deviation 0.5, we obtain a Markov chain of size 10,000 following a burn-in of size 100 . The acceptance rate is found to be $59 \%$.

Then taking every 10th value results in a very nearly uncorrelated sample of size 1,000 from the posterior distribution of $\mu$. Using these 1,000 values, leads to the estimate $\hat{\mu}$ by 0.5297 , with associated $95 \%$ CI equal to $(0.5047,0.5547)$.

We note that the true exact value calculated in (a), 0.5379 , is contained in this CI. 


\section{R Code for Exercise 6.4}

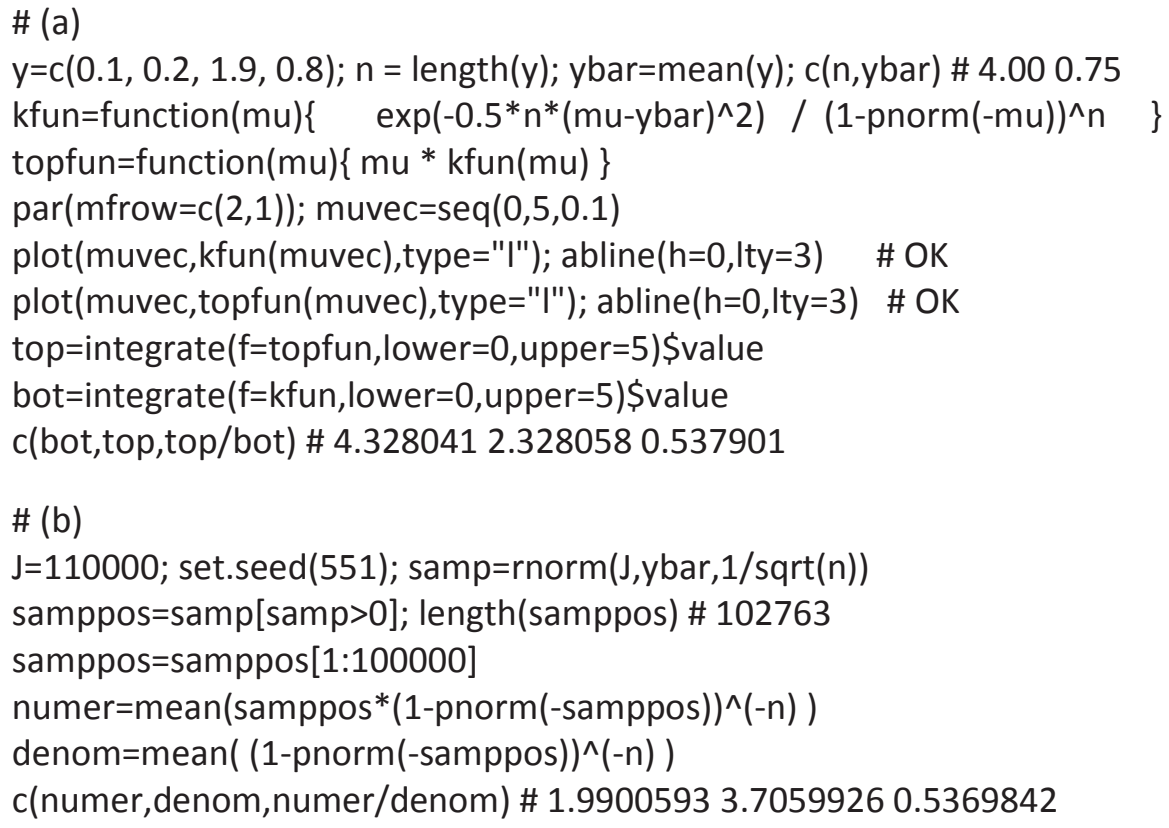


vec1=res\$muv[-(1:101)]

print(acf(vec1)\$acf[1:10], digits=2) \# Evidence of strong autocorrelation \# 1.000 .780 .610 .480 .390 .300 .240 .190 .140 .11

$v=v e c 1[\operatorname{seq}(10,10000,10)]$ \# Take every 10th value only print(acf(v)\$acf[1:10],digits=2) \# No apparent residual autocorrelation \# $1.00000 .05340 .00140 .0331-0.0089-0.00410 .00340 .00870 .01020 .0133$

J=length(v); J \# 1000

est=mean(v); std=sd(v); ci=est+c(-1,1)*qnorm(0.975)*std/sqrt(J)

c(est,std,ci) \# 0.52968870 .40392380 .50465370 .5547237

\subsection{Computational issues}

Numerical issues may arise when attempting to calculate the acceptance probability

$$
p=f\left(x_{j}^{\prime}\right) / f\left(x_{j-1}\right)
$$

due to $f\left(x_{j}^{\prime}\right)$ or $f\left(x_{j-1}\right)$ being too large or too small for $\mathrm{R}$ to handle.

One relevant fact here is that in $\mathrm{R}$ on most computers (at present), 5e-324 (meaning $5 \times 10^{-324}$ ) is the smallest representable non-zero number. This problem can often be resolved by calculating $p$ as

$$
p=\exp (q)
$$

after first computing

$$
q=\log f\left(x_{j}^{\prime}\right)-\log f\left(x_{j-1}\right),
$$

but even this formulation may not be sufficient in every situation.

It may sometimes also be necessary to replace the calculation of a function, say $h(r)$, by

$$
h(\max (r, 5 e-324))
$$

if that function requires a non-zero argument $r$ which is likely to be reported by $\mathrm{R}$ as 0 (because the exact value of $r$ is likely to be between 0 and $5 e-324)$.

Further, and by the same token, if

$$
0<h(\max (r, 5 \mathrm{e}-324))<5 \mathrm{e}-324
$$

then $\mathrm{R}$ will report a value of 0 . In that case, if a non-zero value of $h$ is absolutely required (for some subsequent calculation) then the code for $h(r)$ should be replaced by code which returns

$$
\max (h(\max (r, 5 \mathrm{e}-324)), 5 \mathrm{e}-324) \text {. }
$$




\subsection{Non-symmetric drivers and the general Metropolis algorithm}

In some cases, applying the Metropolis algorithm as described above may lead to poor mixing, even after experimentation to decide on the most suitable value of the tuning constant.

For example, if the random variable of interest is strictly positive with a pdf $f(x)$ which is positively skewed and highly concentrated just above 0 (for example, if $f(x) \rightarrow \infty$ as $x \downarrow 0$ ), proposing a value symmetrically distributed around the last value may lead to many candidate values which are negative and therefore automatically rejected.

In such cases, the support of $X$ may not be properly represented, and it may be preferable to choose a different type of driver distribution, one which adapts 'cleverly' to the current state of the Markov chain.

This can be achieved using the general Metropolis algorithm which allows for non-symmetric driver distributions. As before, let $g(t \mid x)$ denote a driver density, where $t$ denotes the proposed value and $x$ is the last value in the chain. Then at iteration $j$, after generating a proposed value from the driver distribution,

$$
x_{j}^{\prime} \sim g\left(t \mid x=x_{j-1}\right),
$$

the acceptance probability is

$$
p=\frac{f\left(x_{j}^{\prime}\right)}{f\left(x_{j-1}\right)} \times \frac{g\left(x_{j-1} \mid x_{j}^{\prime}\right)}{g\left(x_{j}^{\prime} \mid x_{j-1}\right)} .
$$

Note 1: Previously, when $g(t \mid x)$ was assumed to be symmetric,

$$
\frac{g\left(x_{j-1} \mid x_{j}^{\prime}\right)}{g\left(x_{j}^{\prime} \mid x_{j-1}\right)}=1 \text {. }
$$

Note 2: To calculate $p$, the best strategy is to let

$$
p=\exp (q)
$$

after first computing

$$
\begin{aligned}
q & =\log f\left(x_{j}^{\prime}\right)-\log f\left(x_{j-1}\right) \\
& +\log g\left(x_{j-1} \mid x_{j}^{\prime}\right)-\log g\left(x_{j}^{\prime} \mid x_{j-1}\right) .
\end{aligned}
$$




\section{Exercise 6.5 A Metropolis algorithm with a non-symmetric driver}

Generate a random sample of size 10,000 from the distribution defined by the pdf

$$
f(x)= \begin{cases}\frac{1}{4} x^{-1 / 2}, & 0<x<1 \\ \frac{1}{2} e^{-(x-1)}, & x>1\end{cases}
$$

using the Metropolis algorithm and a non-symmetric driver with density of the form

$$
g(t \mid x)=f_{G(x \delta, \delta)}(t)=\frac{\delta^{x \delta} t^{x \delta-1} e^{-x \delta}}{\Gamma(x \delta)}, t>0,
$$

or equivalently, a driver defined by

$$
(t \mid x) \sim G(x \delta, \delta) \text {. }
$$

Check your results by plotting a probability histogram of the sample values and overlaying the target density, $f(x)$. Also discuss why this driver is suitable in this situation.

\section{Solution to Exercise 6.5}

At each iteration $j$ the proposed value is generated by sampling

$$
x_{j}^{\prime} \sim G\left(x_{j-1} \delta, \delta\right) \text {. }
$$

The rationale for this choice of driver is that the proposed value is certainly positive, it has:

$$
\begin{array}{ll}
\text { mean } & x_{j-1} \delta / \delta=x_{j-1} \\
\text { variance } & x_{j-1} \delta / \delta^{2}=x_{j-1} / \delta .
\end{array}
$$

Thus the candidate $x_{j}^{\prime}$ is guaranteed to be in the appropriate range $\left(\mathfrak{R}^{+}\right)$, and it is centred at the last value $\left(x_{j-1}\right)$.

Also, its variance around that last value is proportional to it (by a factor of $1 / \delta$ ). This ensures that values near zero are appropriately 'explored' by the Markov chain. 
With this driver, the acceptance probability at iteration $j$ is

$$
p=\exp (q),
$$

where:

$$
\begin{aligned}
& q=\log f\left(x_{j}^{\prime}\right)-\log f\left(x_{j-1}\right)+\log g\left(x_{j-1} \mid x_{j}^{\prime}\right)-\log g\left(x_{j}^{\prime} \mid x_{j-1}\right) . \\
& \log f(x)=I(0<x<1)\{-0.5 \log x-\log 4\}+I(x>1)\{1-x-\log 2\} \\
& \log g(t \mid x)=x \delta \log \delta+(x \delta-1) \log t-x \delta-\log \Gamma(x \delta) .
\end{aligned}
$$

Even with this use of the logarithmic function, computational issues arose in $\mathrm{R}$ on account of limitations with the functions rgamma() and lgamma(). These limitations are acknowledged in the help files for these functions in $\mathrm{R}$.

To give an example:

set.seed(321)

$v=\operatorname{rgamma}(10000,0.001,0.001)$

\# Large sample from the $\mathrm{G}(0.001,0.001)$ distribution.

mean(v) \# 0.5827886

\# This is clearly wrong since the mean is $0.001 / 0.001=1$. length $(\mathrm{v}[\mathrm{v}==0])$ \# 4777

\# Almost HALF of the values are EXACTLY zero.

The R code was appropriately modified so that whenever very small but non-zero values were reported as zero by $\mathrm{R}$ (and problems ensued or potentially ensued because of this) those values were changed in the code to 5e-324 (the smallest representable non-zero number in R).

With the above specification and fixes, the Metropolis algorithm was run for 10,000 iterations following a burn-in of size 100 and starting at 1 . The value of $\delta$ used was 1.3 and this resulted in an acceptance rate of $53 \%$ as well as good mixing. Figure 6.11 shows the resulting trace of all 10,101 values of $x$, and Figure 6.12 shows the required probability histogram of the last 10,000 values, together with the exact density $f(x)$ overlaid.

Note: Applying a gamma driver here (in an attempt to improve the 'vanilla' version of the Metropolis algorithm) created problems, due to numerical issues in $\mathrm{R}$ associated with the gamma distribution. With some modifications, we were in the end able to make things work. Another choice of nonsymmetric driver distribution is the lognormal, and we leave it as an additional exercise to examine this option in detail. 
Figure 6. I I Trace of simulated values

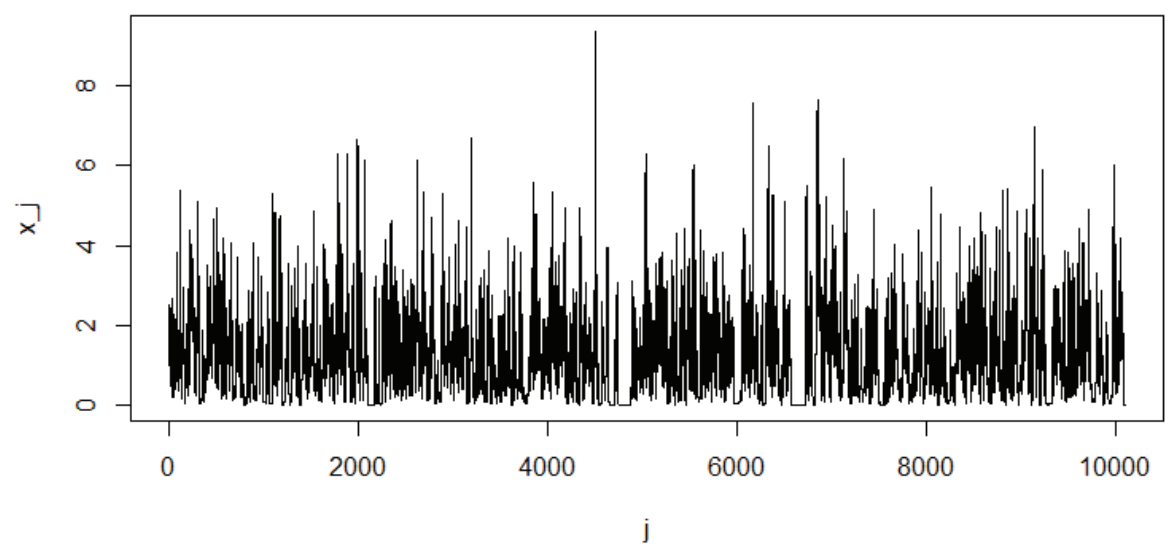

Figure 6. I 2 Histogram and true density

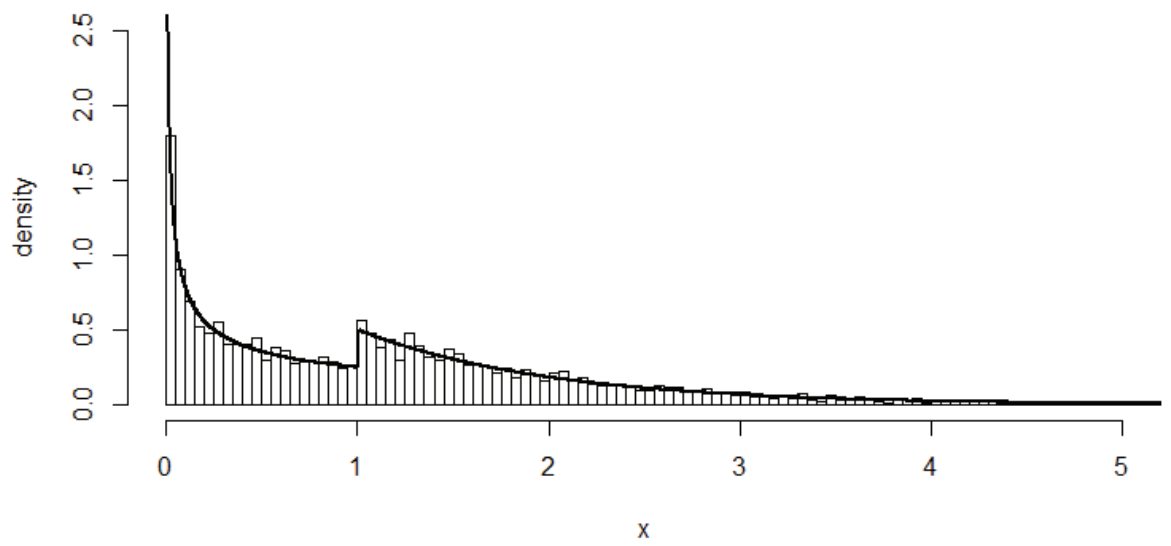

\section{R Code for Exercise 6.5}

set.seed(321); $\quad v=\operatorname{rgamma}(10000,0.001,0.001)$

\# Large sample from the $\mathrm{G}(0.001,0.001)$ distribution.

mean(v) \# 0.5827886 This is clearly wrong since the mean is 0.001/0.001 = 1 . length $(\mathrm{v}[\mathrm{v}==0])$ \# 4777 Almost HALF of the values are EXACTLY zero. $\operatorname{logffun}=$ function $(x)\left\{\right.$ res $=-0.5^{*} \log (x)-\log (4)$; if $(x>1)$ res=1-x-log(2); res $\}$ loggfun=function $(t, x, d e l)\{$

$$
x^{*} \text { del }{ }^{*} \log (\text { del })+\left(x^{*} \text { del-1)* } \log (\mathrm{t})-\mathrm{t} * \text { del-Igamma }\left(\max \left(x^{*} \text { del, 5e-324 }\right)\right)\right\}
$$


MET <- function $(K, x, d e l)\{$ \# This function implements a simple Metropolis alg. \# Inputs: $K=$ total number of iterations, $x=$ starting value of $x$, \# del = tuning constant in driver

\# Outputs: $\$ x v=$ vector of $x$-values of length $(K+1)$, \$ar = acceptance rate $x v=x ; \quad c t=0$

for $(j$ in $1: K)\left\{x p=\max \left(\operatorname{rgamma}\left(1, x^{*}\right.\right.\right.$ del,del $\left.), 5 e-324\right)$

$\log p=\operatorname{logffun}(x=x p)-\operatorname{logffun}(x=x)+$ $\operatorname{loggfun}(t=x, x=x p, d e l=d e l)-\operatorname{loggfun}(t=x p, x=x$, del $=$ del $)$

list $(\mathrm{xV}=\mathrm{xv}, \mathrm{ar}=\mathrm{ct} / \mathrm{K}) \quad\}$

$p=\exp (\log p) ; \quad u=\operatorname{runif}(1) ; \quad$ if $(u<p)\{x=x p ; c t=c t+1\}$ $\mathrm{xv}=\mathrm{c}(\mathrm{xv}, \mathrm{x})\}$

$\mathrm{X} 11(\mathrm{w}=8, \mathrm{~h}=4.5) ; \operatorname{par}(\operatorname{mfrow}=\mathrm{c}(1,1)) ; \mathrm{K}=10100$;

set.seed(319); res = MET(K=K, $\mathrm{x}=1, \mathrm{del}=1.3)$; res\$ar \# 0.5324752

plot(0:K,res\$xv,type="l",xlab="j",ylab="x_j")

$x v<-\operatorname{res} \$ x v[-c(1: 101)]$

hist(xv,xlab="x",prob=T,ylim=c(0,2.5),xlim=c(0,5), ylab="density", main="", breaks $=\operatorname{seq}(0,20,0.05)$ )

$x v e c=\operatorname{seq}(0,10,0.001)$; fvec $=x v e c$;

for(i in 1:length(xvec)) fvec[i]=exp(logffun(xvec[i]))

lines(xvec,fvec, Iwd=2)

summary(res\$xv)

\# Min. 1st Qu. Median Mean 3rdQu. Max.

\# 0.0042430 .3094001 .0340001 .2180001 .7380009 .356000 (OK, as Min > 0)

\subsection{The Metropolis-Hastings algorithm}

We have introduced Markov chain Monte Carlo methods with a detailed discussion of the Metropolis algorithm. As already noted, this algorithm is limited and rarely used on its own because it can only be used to sample from univariate distributions. Typically, other methods will be better suited to the task of sampling from a univariate distribution.

We now turn to the Metropolis-Hastings (MH) algorithm, a generalisation of the Metropolis algorithm that can be used to sample from a very wide range of multivariate distributions. This algorithm is very useful and has been applied in many difficult statistical modelling settings. 
First let us again review the Metropolis algorithm for sampling from a univariate density, $f(x)$. This involves choosing an arbitrary starting value of $x$, a suitable driver density $g(t \mid x)$ and then repeatedly proposing a value $x^{\prime} \sim g(t \mid x)$, each time accepting this value with probability

$$
p=\frac{f\left(x^{\prime}\right)}{f(x)} \times \frac{g\left(x \mid x^{\prime}\right)}{g\left(x^{\prime} \mid x\right)}
$$

(or $p=\frac{f\left(x^{\prime}\right)}{f(x)}$ in the case of a symmetric driver).

Each proposal and then either acceptance or rejection constitutes one iteration of the algorithm and may be referred to as a Metropolis step.

Performing $K$ iterations, each consisting of a single Metropolis step, results in a Markov chain of values which may be denoted $x^{(0)}, x^{(1)}, \ldots, x^{(K)}$.

Assuming that stochastic equilibrium has been attained within $B$ iterations ( $B$ standing for burn-in) the last $J=K-B$ values may be renumbered so as to yield the required sample, $x^{(1)}, \ldots, x^{(J)} \dot{\sim}$ iid $f(x)$.

The Metropolis-Hastings (MH) algorithm is a generalisation of this procedure to the case where $x$ is a vector of length $M$ (say) .

\section{The bivariate $\mathrm{MH}$ algorithm}

For simplicity we will first focus on the bivariate case $(M=2)$. Thus, suppose we wish to generate a random sample from the distribution of a random vector $X=\left(X_{1}, X_{2}\right)$ with pdf $f(x)$, where $x=\left(x_{1}, x_{2}\right)$ denotes a value of $X$.

First, choose an initial value of $x=\left(x_{1}, x_{2}\right)$.

Then choose two suitable driver distributions or densities:

$$
\begin{aligned}
& g_{1}\left(t \mid x_{1}, x_{2}\right) \\
& g_{2}\left(t \mid x_{1}, x_{2}\right) .
\end{aligned}
$$

Next perform the following two Metropolis steps: 
1. Propose a candidate value of $x_{1}$ by sampling

$$
x_{1}^{\prime} \sim g_{1}\left(t \mid x_{1}, x_{2}\right),
$$

and accept this value with probability

$$
p_{1}=\frac{f\left(x_{1}^{\prime} \mid x_{2}\right)}{f\left(x_{1} \mid x_{2}\right)} \times \frac{g_{1}\left(x_{1} \mid x_{1}^{\prime}, x_{2}\right)}{g_{1}\left(x_{1}^{\prime} \mid x_{1}, x_{2}\right)} .
$$

(In the case of an acceptance, let $x_{1}=x_{1}^{\prime}$,

and otherwise leave $x_{1}$ unchanged.)

2. Propose a candidate value of $x_{2}$ by sampling

$$
x_{2}^{\prime} \sim g_{2}\left(t \mid x_{1}, x_{2}\right),
$$

and accept this value with probability

$$
p_{2}=\frac{f\left(x_{2}^{\prime} \mid x_{1}\right)}{f\left(x_{2} \mid x_{1}\right)} \times \frac{g_{2}\left(x_{2} \mid x_{1}, x_{2}^{\prime}\right)}{g_{2}\left(x_{2}^{\prime} \mid x_{1}, x_{2}\right)} .
$$

(In the case of an acceptance, let $x_{2}=x_{2}^{\prime}$, and otherwise leave $x_{2}$ unchanged.)

This completes the first iteration of the MH algorithm.

The initial value of $x=\left(x_{1}, x_{2}\right)$ may be denoted

$$
x^{(0)}=\left(x_{1}^{(0)}, x_{2}^{(0)}\right),
$$

and the current value of the Markov chain may be denoted

$$
x^{(1)}=\left(x_{1}^{(1)}, x_{2}^{(1)}\right) .
$$

Performing another iteration of the MH algorithm as above (starting from $\left.x=x^{(1)}\right)$ leads to the next value,

$$
x^{(2)}=\left(x_{1}^{(2)}, x_{2}^{(2)}\right) \text {, }
$$

and so on.

Continuing in this fashion results in a Markov chain of vectors,

$$
x^{(0)}, x^{(1)}, \ldots, x^{(K)} \text {. }
$$

Assuming that stochastic equilibrium has been attained within $B$ iterations, the last $J=K-B$ vectors may be renumbered consecutively to yield the required sample,

$$
x^{(1)}, \ldots, x^{(J)} \dot{\sim} \text { iid } f(x),
$$

where $x^{(j)}=\left(x_{1}^{(j)}, x_{2}^{(j)}\right)$. 
Note 1: This multivariate sample can then be used to perform marginal inferences. For example, by discarding all the $x_{2}^{(j)}$ values, we obtain a sample from the marginal posterior distribution of $x_{1}$, namely

$$
x_{1}^{(1)} \ldots, x_{1}^{(J)} \dot{\sim} \text { iid } f\left(x_{1}\right) .
$$

This technique would be useful if obtaining a sample from $f\left(x_{1}\right)$ directly were for any reason problematic. For example, the marginal density

$$
f\left(x_{1}\right)=\int f\left(x_{1}, x_{2}\right) d x_{2}
$$

might be difficult to derive explicitly or sample from.

Note 2: Observe that

$$
\frac{f\left(x_{1}^{\prime} \mid x_{2}\right)}{f\left(x_{1} \mid x_{2}\right)}=\frac{f\left(x_{1}^{\prime}, x_{2}\right) / f\left(x_{2}\right)}{f\left(x_{1}, x_{2}\right) / f\left(x_{2}\right)} \text {, etc. }
$$

Thus the two acceptance probabilities could also be written as:

$$
\begin{aligned}
& p_{1}=\frac{f\left(x_{1}^{\prime}, x_{2}\right)}{f\left(x_{1}, x_{2}\right)} \frac{g_{1}\left(x_{1} \mid x_{1}^{\prime}, x_{2}\right)}{g_{1}\left(x_{1}^{\prime} \mid x_{1}, x_{2}\right)} \\
& p_{2}=\frac{f\left(x_{2}^{\prime}, x_{1}\right)}{f\left(x_{2}, x_{1}\right)} \times \frac{g_{2}\left(x_{2} \mid x_{1}, x_{2}^{\prime}\right)}{g_{2}\left(x_{2}^{\prime} \mid x_{1}, x_{2}\right)} .
\end{aligned}
$$

\section{The trivariate $\mathrm{MH}$ algorithm}

The Metropolis-Hastings algorithm for sampling from the trivariate distribution $(M=3)$ of a vector random variable $X=\left(X_{1}, X_{2}, X_{3}\right)$ involves choosing an initial value of the vector

$$
x=\left(x_{1}, x_{2}, x_{3}\right),
$$

specifying three driver densities:

$$
\begin{aligned}
& g_{1}\left(t \mid x_{1}, x_{2}, x_{3}\right) \\
& g_{2}\left(t \mid x_{1}, x_{2}, x_{3}\right) \\
& g_{3}\left(t \mid x_{1}, x_{2}, x_{3}\right),
\end{aligned}
$$

and repeatedly iterating three Metropolis steps as follows: 
1. Propose a candidate value of $x_{1}$ by sampling

$$
x_{1}^{\prime} \sim g_{1}\left(t \mid x_{1}, x_{2}, x_{3}\right),
$$

and accept this value with probability

$$
p_{1}=\frac{f\left(x_{1}^{\prime} \mid x_{2}, x_{3}\right)}{f\left(x_{1} \mid x_{2}, x_{3}\right)} \times \frac{g_{1}\left(x_{1} \mid x_{1}^{\prime}, x_{2}, x_{3}\right)}{g_{1}\left(x_{1}^{\prime} \mid x_{1}, x_{2}, x_{3}\right)}
$$

2. Propose a candidate value of $x_{2}$ by sampling

$$
x_{2}^{\prime} \sim g_{2}\left(t \mid x_{1}, x_{2}, x_{3}\right),
$$

and accept this value with probability

$$
p_{2}=\frac{f\left(x_{2}^{\prime} \mid x_{1}, x_{3}\right)}{f\left(x_{2} \mid x_{1}, x_{3}\right)} \times \frac{g_{2}\left(x_{2} \mid x_{1}, x_{2}^{\prime}, x_{3}\right)}{g_{2}\left(x_{2}^{\prime} \mid x_{1}, x_{2}, x_{3}\right)} .
$$

3. Propose a candidate value of $x_{3}$ by sampling

$$
x_{3}^{\prime} \sim g_{3}\left(t \mid x_{1}, x_{2}, x_{3}\right),
$$

and accept this value with probability

$$
p_{3}=\frac{f\left(x_{3}^{\prime} \mid x_{1}, x_{2}\right)}{f\left(x_{3} \mid x_{1}, x_{2}\right)} \times \frac{g_{3}\left(x_{3} \mid x_{1}, x_{2}, x_{3}^{\prime}\right)}{g_{3}\left(x_{3}^{\prime} \mid x_{1}, x_{2}, x_{3}\right)} .
$$

As before, continuing in this fashion until stochastic equilibrium has been achieved, and then for another $J$ iterations, leads to the random sample $x^{(1)}, \ldots, x^{(J)} \dot{\sim}$ iid $f(x)$, where now $x^{(j)}=\left(x_{1}^{(j)}, x_{2}^{(j)}, x_{3}^{(j)}\right)$.

Note: As before, the $x_{1}^{(j)}$ values on their own then constitute a sample from the marginal distribution of $x_{1}$, whose density is now

$$
f\left(x_{1}\right)=\iint f\left(x_{1}, x_{2}, x_{3}\right) d x_{2} d x_{3},
$$

and the three acceptance probabilities can also be expressed as

$$
p_{1}=\frac{f\left(x_{1}^{\prime}, x_{2}, x_{3}\right)}{f\left(x_{1}, x_{2}, x_{3}\right)} \frac{g_{1}\left(x_{1} \mid x_{1}^{\prime}, x_{2}, x_{3}\right)}{g_{1}\left(x_{1}^{\prime} \mid x_{1}, x_{2}, x_{3}\right)}, \text { etc. }
$$

\section{The general $\mathrm{MH}$ algorithm}

These ideas extend naturally and in an obvious fashion to higher values of $M$. Thus, for sampling from an $M$-variate distribution with density $f\left(x_{1}, \ldots, x_{M}\right)$, the $\mathrm{MH}$ algorithm involves choosing a starting value

$$
x=\left(x_{1}, \ldots, x_{M}\right) \text {, }
$$


specifying $M$ drivers,

$$
g_{m}\left(t \mid x_{1}, \ldots, x_{M}\right) \quad(m=1, \ldots, M),
$$

and repeatedly iterating $M$ steps as follows:

1. Propose a candidate value of $x_{1}$ by sampling

$$
x_{1}^{\prime} \sim g_{1}\left(t \mid x_{1}, \ldots, x_{M}\right),
$$

and accept this value with probability

$$
p_{1}=\frac{f\left(x_{1}^{\prime} \mid x_{2}, \ldots, x_{M}\right)}{f\left(x_{1} \mid x_{2}, \ldots, x_{M}\right)} \times \frac{g_{1}\left(x_{1} \mid x_{1}^{\prime}, \ldots, x_{M}\right)}{g_{1}\left(x_{1}^{\prime} \mid x_{1}, \ldots, x_{M}\right)}
$$

2. Propose a candidate value of $x_{2}$ by sampling

$$
x_{2}^{\prime} \sim g_{2}\left(t \mid x_{1}, \ldots, x_{M}\right),
$$

and accept this value with probability

$$
p_{2}=\frac{f\left(x_{2}^{\prime} \mid x_{1}, x_{3}, \ldots, x_{M}\right)}{f\left(x_{2} \mid x_{1}, x_{3}, \ldots, x_{M}\right)} \times \frac{g_{2}\left(x_{2} \mid x_{1}, x_{2}^{\prime}, x_{3}, \ldots, x_{M}\right)}{g_{2}\left(x_{2}^{\prime} \mid x_{1}, x_{2}, x_{3}, \ldots, x_{M}\right)}
$$

$\boldsymbol{M}$. Propose a candidate value of $x_{M}$ by sampling

$$
x_{M}^{\prime} \sim g_{M}\left(t \mid x_{1}, \ldots, x_{M}\right),
$$

and accept this value with probability

$$
p_{M}=\frac{f\left(x_{M}^{\prime} \mid x_{1}, \ldots, x_{M-1}\right)}{f\left(x_{M} \mid x_{1}, \ldots, x_{M-1}\right)} \times \frac{g_{M}\left(x_{M} \mid x_{1}, \ldots, x_{M-1}, x_{M}^{\prime}\right)}{g_{M}\left(x_{M}^{\prime} \mid x_{1}, \ldots, x_{M-1}, x_{M}\right)} .
$$

As before, continuing in this fashion until stochastic equilibrium and then for $J$ more iterations leads to the sample $x^{(1)}, \ldots, x^{(J)} \dot{\sim}$ iid $f(x)$, where now $x^{(j)}=\left(x_{1}^{(j)}, \ldots, x_{M}^{(j)}\right)$.

Note: Again, the $x_{1}^{(j)}$ values on their own then constitute a sample from the marginal distribution of $x_{1}$, whose density is now

$$
\left.f\left(x_{1}\right)=\int \cdots \int f\left(x_{1}, \ldots, x_{M}\right) d x_{2} \ldots d x_{M}\right),
$$

and the $M$ acceptance probabilities can also be expressed as

$$
p_{1}=\frac{f\left(x_{1}^{\prime}, \ldots, x_{M}\right)}{f\left(x_{1}, \ldots, x_{M}\right)} \frac{g_{1}\left(x_{1} \mid x_{1}^{\prime}, x_{2}, \ldots, x_{M}\right)}{g_{1}\left(x_{1}^{\prime} \mid x_{1}, x_{2}, \ldots, x_{M}\right)}, \text { etc. }
$$




\section{Exercise 6.6 $\mathrm{MH}$ algorithm applied to a bent coin which is tossed an unknown number of times}

Suppose that five heads have come up on an unknown number of tosses of a bent coin.

Before the experiment, we believed the coin was going to be tossed a number of times equal to $1,2,3, \ldots$, or 9 , with all possibilities equally likely. As regards the probability of heads coming up on a single toss, we deemed no value more or less likely than any other value. We also considered the probability of heads as unrelated to the number of tosses.

Find the marginal posterior distribution and mean of the number of tosses and of the probability of heads, respectively. Also find the number of heads we can expect to come up if the coin is tossed again the same number of times.

Do all this via Monte Carlo by designing and implementing a suitable $\mathrm{MH}$ algorithm.

Note: This problem was solved analytically in Exercise 3.10.

\section{Solution to Exercise 6.6}

As in Exercise 3.10, the relevant Bayesian model is:

$$
\begin{aligned}
& (y \mid \theta, n) \sim \operatorname{Binomial}(n, \theta) \\
& (\theta \mid n) \sim U(0,1) \\
& f(n)=1 / k, n=1, \ldots, k, \quad k=9,
\end{aligned}
$$

and the joint posterior density of the two parameters $n$ and $\theta$ is

$$
\begin{aligned}
f(n, \theta \mid y) & \propto f(n) f(\theta \mid n) f(y \mid n, \theta) \\
& \propto \frac{n ! \theta^{y}(1-\theta)^{n-y}}{(n-y) !} \\
& \equiv h(n, \theta), \quad 0<\theta<1, n=y, y+1, \ldots, k .
\end{aligned}
$$

Let us now specify the driver for $n$ as discrete uniform over the integers from $n-r$ to $n+r$, where $r$ is a tuning parameter.

Also let the driver for $\theta$ be uniform from $\theta-c$ to $\theta+c$, where $c$ is another tuning parameter. 
Note: These drivers may also be expressed by writing the distributions explicitly as:

$$
\begin{aligned}
n^{\prime} & \sim D U(n-r, n-r+1, \ldots, n+r) \\
\theta^{\prime} & \sim U(\theta-c, \theta+c),
\end{aligned}
$$

or by writing the driver densities explicitly as:

$$
\begin{aligned}
& g_{1}(t \mid n, \theta)=\frac{1}{2 r+1}, \quad t=n-r, n-r+1, \ldots, n+r \\
& g_{2}(t \mid n, \theta)=\frac{1}{2 c}, \quad \theta-c<t<\theta+c .
\end{aligned}
$$

Noting that both drivers are symmetric, a suitable $\mathrm{MH}$ algorithm may be defined by the following two steps at each iteration:

1. Propose a value

$$
n^{\prime} \sim D U(n-r, \ldots, n+r),
$$

and accept this value with probability

$$
\begin{aligned}
p_{1}=\frac{f\left(n^{\prime}, \theta \mid y\right)}{f(n, \theta \mid y)}=\frac{h\left(n^{\prime}, \theta\right)}{h(n, \theta)} & =\frac{n^{\prime} ! \theta^{y}(1-\theta)^{n^{\prime}-y} /\left(n^{\prime}-y\right) !}{n ! \theta^{y}(1-\theta)^{n-y} /(n-y) !} \\
& =\frac{n^{\prime} !(1-\theta)^{n^{\prime}} /\left(n^{\prime}-y\right) !}{n !(1-\theta)^{n} /(n-y) !}
\end{aligned}
$$

2. Propose a value

$$
\theta^{\prime} \sim U(\theta-c, \theta+c),
$$

and accept this value with probability

$$
\begin{aligned}
p_{2}=\frac{f\left(n, \theta^{\prime} \mid y\right)}{f(n, \theta \mid y)}=\frac{h\left(n, \theta^{\prime}\right)}{h(n, \theta)} & =\frac{n ! \theta^{\prime y}\left(1-\theta^{\prime}\right)^{n-y} /(n-y) !}{n ! \theta^{y}(1-\theta)^{n-y} /(n-y) !} \\
& =\frac{\theta^{\prime y}\left(1-\theta^{\prime}\right)^{n-y}}{\theta^{y}(1-\theta)^{n-y}} .
\end{aligned}
$$

Note: The proposed value $n^{\prime}$ should automatically be rejected if it is outside the set $\{5, \ldots, 9\}$ (because then $f\left(n^{\prime}, \theta \mid y\right)=0$ ), and otherwise automatically accepted if $p_{1}>1$. If $n^{\prime}=n$ then $p_{1}=1$, again leading to automatic acceptance.

Likewise, the proposed value $\theta^{\prime}$ should be automatically rejected if it is outside the interval $(0,1)$, and otherwise automatically accepted if $p_{2}>1$. 
Setting $c=0.3$ and $r=1$ (after some experimentation) and starting from $n=7$ and $\theta=0.5$, the $\mathrm{MH}$ algorithm converged very quickly, with acceptance rates of $73 \%$ for $n$ and $58 \%$ for $\theta$ over a total of 10,100 iterations.

The first 100 iterations were thrown away as the burn-in, and then every 20th value (only) was recorded so as to thereby yield an approximately random sample of size $J=500$ from the joint posterior distribution of $n$ and $\theta$, namely $\left(n_{1}, \theta_{1}\right), \ldots,\left(n_{J}, \theta_{J}\right) \sim$ iid $f(n, \theta \mid y)$.

Figures 6.13 and 6.14 (pages 299 and 300) show the traces for all 10,101 values of $n$ and $\theta$, respectively, and Figures 6.15 and 6.16 (pages 300 and 301) show the traces for the final 500 values of $n$ and $\theta$, respectively.

Figure 6.17 (page 301) shows the corresponding sample ACFs (autocorrelation functions), labelled nv0 and thv0 for the last 10,000 values of $n$ and $\theta$, respectively, and labelled nv and thv for the final 500 values of $n$ and $\theta$. The thinning process has dramatically reduced the high serial correlation.

The final bivariate sample of size $J=500$ was used for Monte Carlo inference in the usual way, with the following results.

The MC estimate of $\hat{n}=E(n \mid y)(=6.744)$ was $\bar{n}=6.708$, with 95\% CI (6.587, 6.829).

The Monte Carlo estimate of $\hat{\theta}=E(\theta \mid y)(=0.7040)$ was $\bar{\theta}=0.7097$, with $95 \%$ CI $(0.6943,0.7252)$. Also, the $95 \%$ CPDR estimate for $\theta$ was (0.3547, 0.9886).

Figure 6.18 (page 302) is a probability histogram of the almost random sample $n_{1}, \ldots, n_{J} \sim$ iid $f(n \mid y)$, and Figure 6.19 (page 302) is a probability histogram of the almost random sample $\theta_{1}, \ldots, \theta_{J} \sim$ iid $f(\theta \mid y)$.

Each histogram is overlaid with a nonparametric density estimate based on the histogram, as well as with the true marginal posterior density.

Each histogram also includes vertical lines showing the true distribution mean, the MC estimate of that mean, and the 95\% CI for that mean.

Figure 6.19 also displays the 95\% CPDR estimate for $\theta$. 
Note 1: The histogram of $n$-values in Figure 6.18 (page 302) is itself an estimate of $f(n \mid y)$. The short vertical lines in the histogram indicate the MC 95\% CIs for $f(n \mid y)$.

For example, the height of the bar above 6 is the proportion of sample values $n_{1}, \ldots, n_{J}$ equal to 6 , which is $117 / 500=0.234$, and the short vertical bar above 6 is the MC 95\% CI for $P(n=6 \mid y)$, which is $(0.234 \pm 1.96 \sqrt{0.234(1-0.234) / 500})=(0.1969,0.2711)$.

Note 2: The histogram of $\theta$-values in Figure 6.19 (page 302) in fact shows two posterior density estimates. The first and simplest estimate tapers towards zero as $\theta$ approaches 1 . The second estimate was obtained using a special mathematical device that was applied so as to 'force' the density estimate to be relatively high near 1 . For values of $\theta$ less than about 0.8 , the two density estimates are virtually identical. Details of said mathematical device can be found in the R code below.

\section{Figure 6.13 Trace of 10,101 n-values}

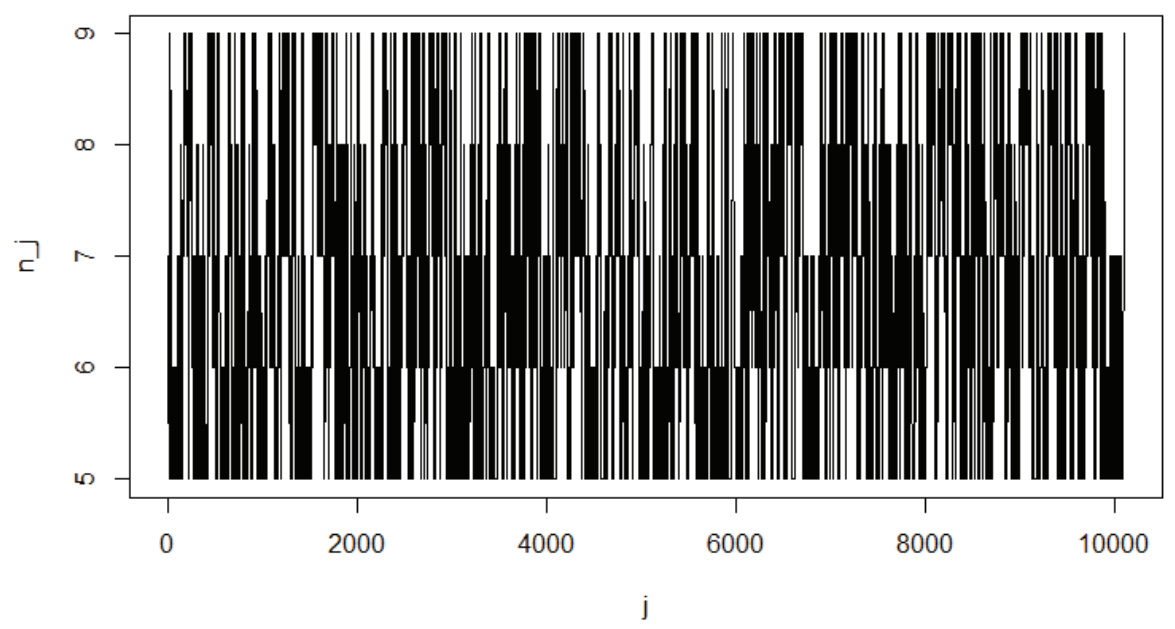


Figure 6.14 Trace of 10,10I $\theta$-values

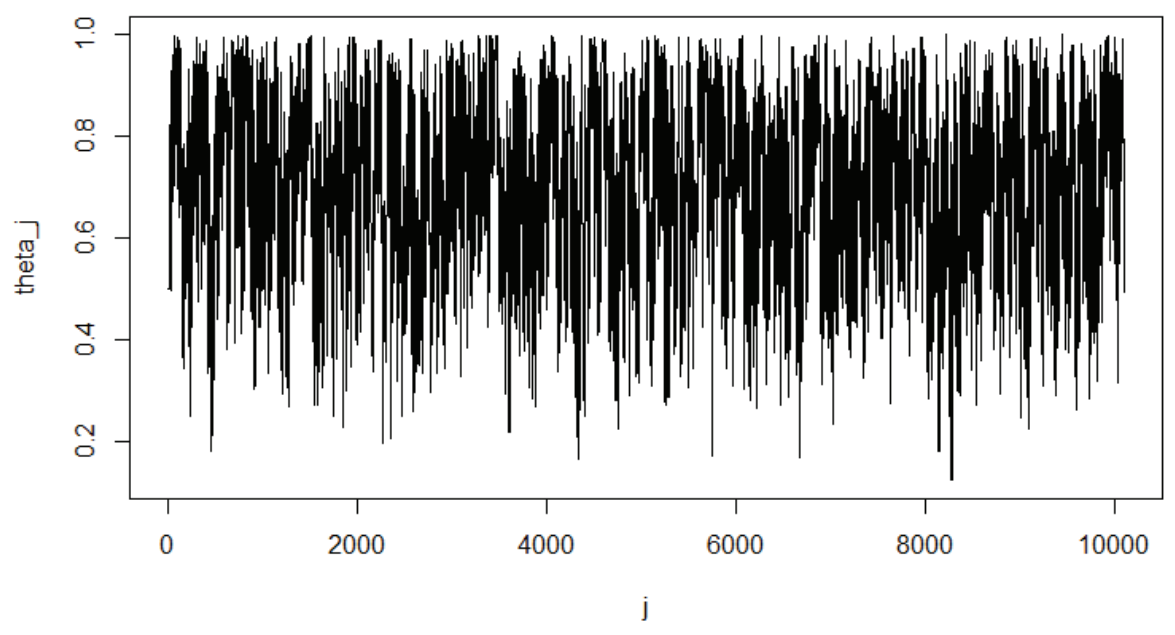

Figure 6.15 Trace of $500 n$-values

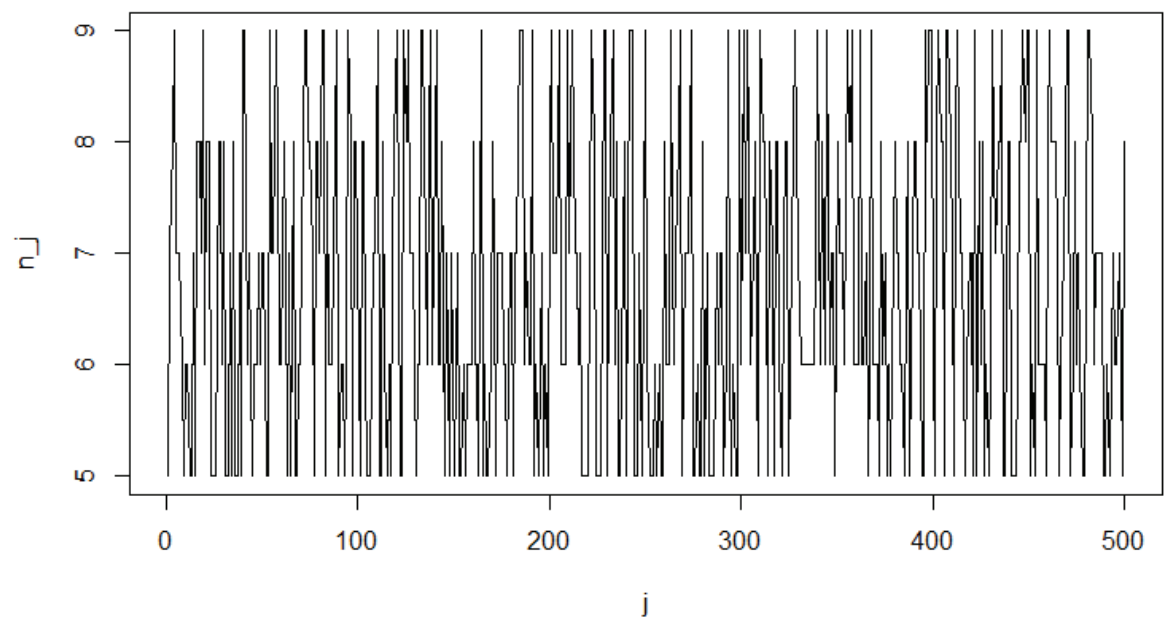


Figure 6.16 Trace of $500 \theta$-values

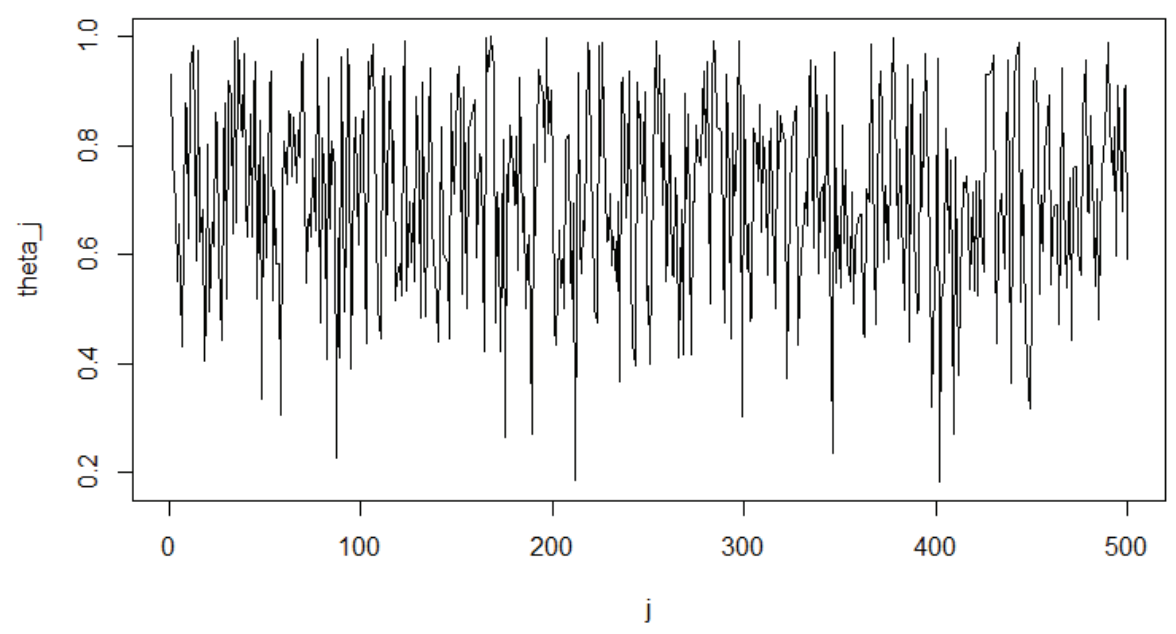

Figure 6.I7 Sample autocorrelation functions

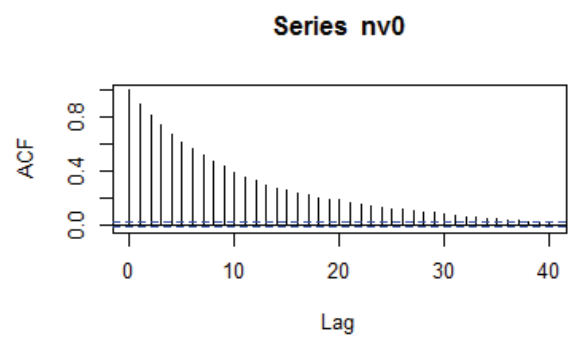

Series nv

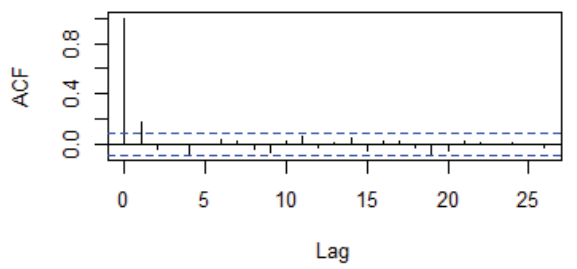

Series thv0

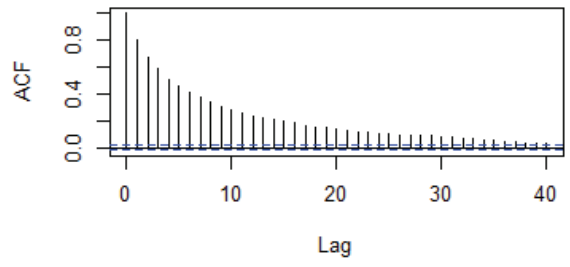

Series thv

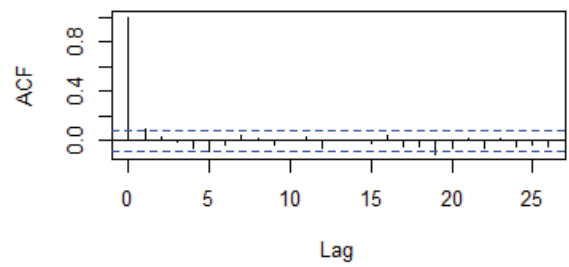




\section{Figure 6. I 8 Probability histogram of 500 n-values}

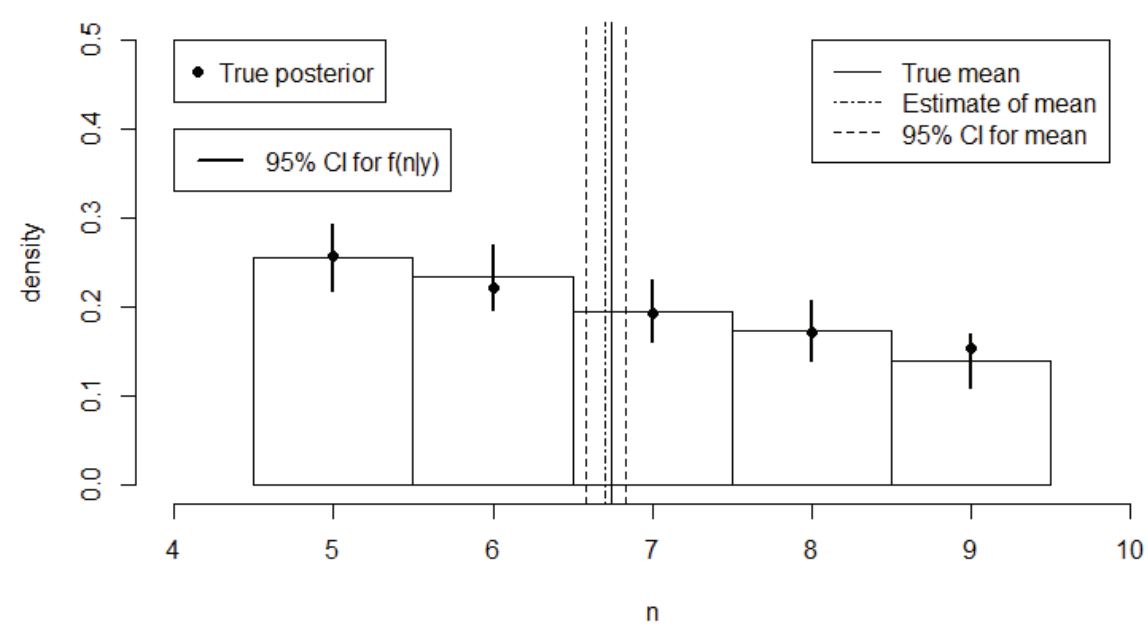

Figure 6.19 Probability histogram of $500 \theta$-values

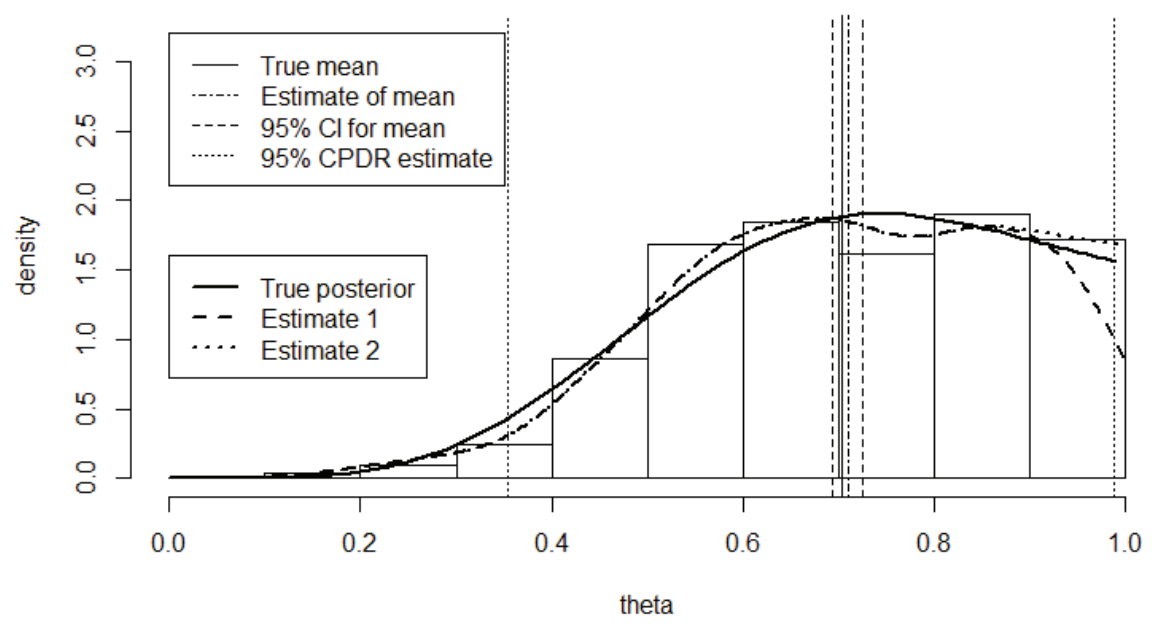




\section{R Code for Exercise 6.6}

\# NB: Some of this R Code was copied from a previous exercise

$\mathrm{y}<-5$; $\mathrm{k}<-$ 9; options(digits=4)

nvec <- y:k; avec <- 1/(nvec+1); sumavec <- sum(avec); sumavec \#0.6456

fny <- avec/sumavec; rbind(nvec,avec,fny)

\# nvec 5.00006 .00007 .00008 .00009 .0000

\# avec 0.16670 .14290 .12500 .11110 .1000

\# fny 0.25810 .22130 .19360 .17210 .1549

nhat <- sum(nvec*fny); nhat $\quad$ \# 6.744

thhat $<-\operatorname{sum}($ fny * $(y+1) /($ nvec +2$))$; thhat \# 0.704

xhat $<-$ sum $($ fny $*$ nvec* $(y+1) /($ nvec +2$)$ ); xhat \# 4.592

thvec <- seq $(0,0.99,0.01)$; fthyvec <- thvec

for(i in 1:length(thvec)) fthyvec[i] <- sum( fny * dbeta(thvec[i],y+1,nvec-y+1) )

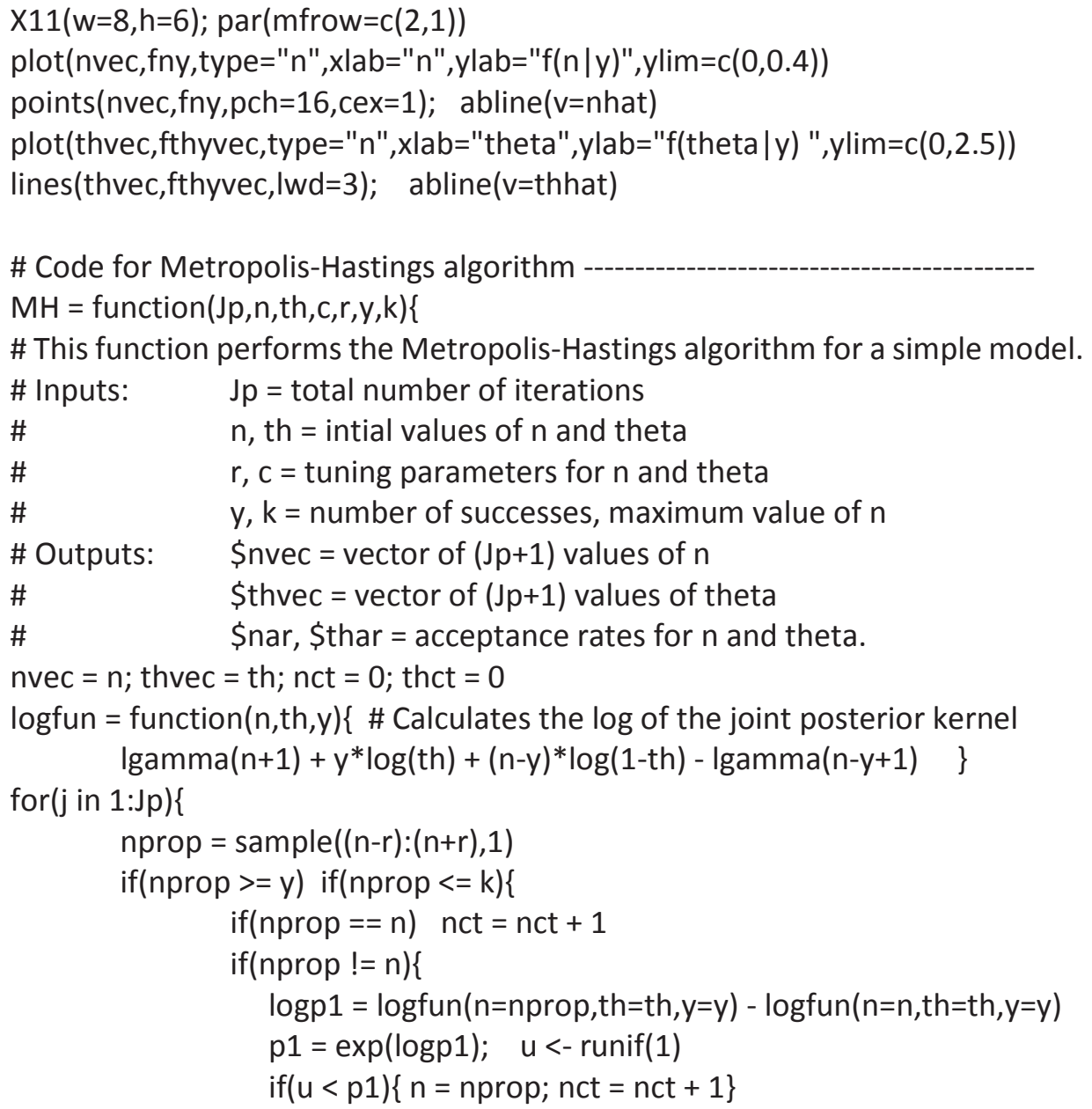




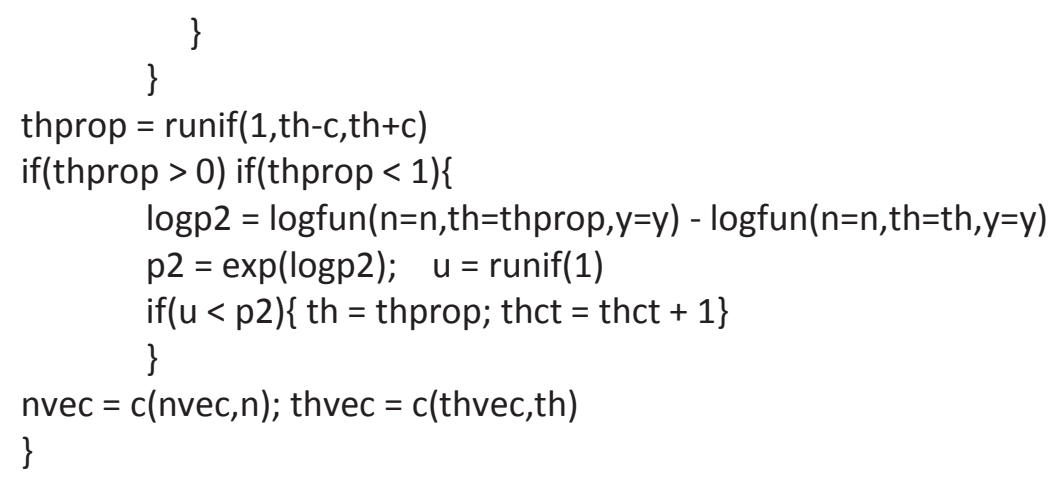




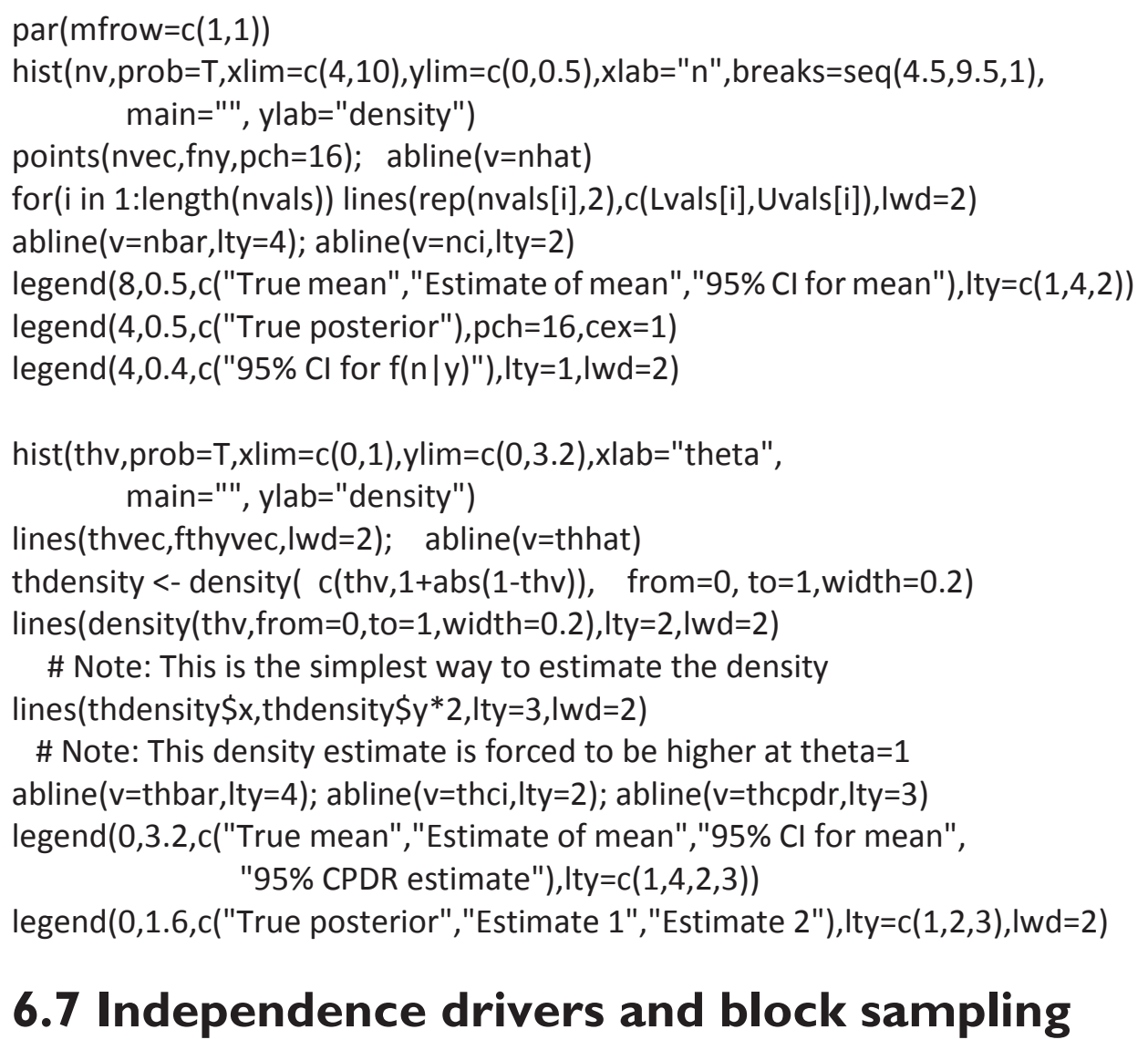

The Metropolis-Hastings algorithm is very flexible and allows for a lot of choice in the way it is designed. In any particular application, many different $\mathrm{MH}$ algorithms will work, but some may perform better than others, meaning they will result in better mixing and faster convergence towards stochastic equilibrium. This will have a lot to do with how the random variables involved are set up and parameterised, what driver distributions are specified, and which tuning parameters are then chosen for completely defining those driver distributions.

For example, the driver distribution for a component $x_{m}$ of the vector $x=\left(x_{1}, \ldots, x_{M}\right)$ may be chosen so that it depends only on the last value of itself. In that case, $g_{m}\left(t \mid x_{1}, x_{2}, \ldots, x_{M}\right)$ can also be written $g_{m}\left(t \mid x_{m}\right)$.

In fact, this is the norm in practice, and it was the case for both drivers in the last exercise. 
It is also permissible to choose the $m$ th driver so that it doesn't depend on any of the current values of the Markov chain, including itself. In that case, the driver $g_{m}\left(t \mid x_{1}, x_{2}, \ldots, x_{M}\right)$ may be written $g_{m}(t)$ and be referred to as an independence driver.

Also, one may 'bundle' any of the $M$ random variables into blocks and thereby reduce the number of actual Metropolis steps per iteration. For example, instead of doing a Metropolis step for each of $x_{3}$ and $x_{4}$ at each iteration, one may do a single Metropolis step as follows:

Create a candidate value of $\left(x_{3}, x_{4}\right)$ by sampling

$$
\left(x_{3}^{\prime}, x_{4}^{\prime}\right) \sim g_{34}\left(t, u \mid x_{3}, x_{4}\right) \text { (say), }
$$

and then accept this candidate $\left(x_{3}^{\prime}, x_{4}^{\prime}\right)$ with probability

$$
\begin{aligned}
p_{34}= & \frac{f\left(x_{1}, x_{2}, x_{3}^{\prime}, x_{4}^{\prime}, x_{5}, \ldots, x_{M}\right)}{f\left(x_{1}, x_{2}, x_{3}, x_{4}, x_{5}, \ldots, x_{M}\right)} \\
& \times \frac{g_{34}\left(x_{3}, x_{4} \mid x_{1}, x_{2}, x_{3}^{\prime}, x_{4}^{\prime}, x_{5}, \ldots, x_{M}\right)}{g_{34}\left(x_{3}^{\prime}, x_{4}^{\prime} \mid x_{1}, x_{2}, x_{3}, x_{4}, x_{5}, \ldots, x_{M}\right)} .
\end{aligned}
$$

This idea can be used to improve mixing and speed up the rate of convergence but may require more work sampling from the bivariate driver and determining the optimal tuning constant. Note that to sample $\left(x_{3}^{\prime}, x_{4}^{\prime}\right)$, it may be possible to do this in two steps via the method of composition according to

$$
g_{34}\left(t, u \mid x_{3}, x_{4}\right)=g_{3}\left(t \mid x_{3}, x_{4}\right) g_{4 \mid 3}\left(u \mid x_{3}, x_{4}, t\right) .
$$

\subsection{Gibbs steps and the Gibbs sampler}

One important possibility is to give the driver for $x_{m}$ exactly the same distribution as the conditional distribution of $x_{m}$ given all the other values.

In that case, the proposal density is

$$
g_{m}\left(t \mid x_{1}, \ldots, x_{M}\right)=f\left(x_{m}=t \mid x_{1}, \ldots, x_{m-1}, x_{m+1}, \ldots, x_{M}\right) .
$$

With this choice, the acceptance probability equals

$$
\begin{aligned}
p_{m} & =\frac{f\left(x_{m}^{\prime} \mid x_{1}, \ldots, x_{m-1}, x_{m+1}, \ldots, x_{M}\right)}{f\left(x_{m} \mid x_{1}, \ldots, x_{m-1}, x_{m+1}, \ldots, x_{M}\right)} \times \frac{f\left(x_{m} \mid x_{1}, \ldots, x_{m-1}, x_{m+1}, \ldots, x_{M}\right)}{f\left(x_{m}^{\prime} \mid x_{1}, \ldots, x_{m-1}, x_{m+1}, \ldots, x_{M}\right)} \\
& =1 \text { (that is, 100\%). }
\end{aligned}
$$


This means that the candidate value $x_{m}^{\prime}$ is definitely accepted at every iteration. In that case we call the $m$ th step of the Metropolis-Hastings algorithm a Gibbs step.

If all the Metropolis steps are Gibbs steps then the algorithm may also be called a Gibbs sampler.

Note: In the case $M=1$, the Gibbs sampler equates to sampling directly from the distribution of interest, with no stochastic dependence between values of the resulting chain.

Thus a Gibbs sampler for sampling from a multivariate distribution

$$
f(x)=f\left(x_{1}, \ldots, x_{M}\right)
$$

may be defined as iteratively sampling from the full conditional densities:

$$
\begin{aligned}
& f\left(x_{1} \mid x_{2}, x_{3}, \ldots, x_{M}\right) \\
& f\left(x_{2} \mid x_{1}, x_{3}, \ldots, x_{M}\right) \\
& \ldots\left(x_{M} \mid x_{1}, x_{2}, \ldots, x_{M-1}\right),
\end{aligned}
$$

where each of these is proportional to $f\left(x_{1}, \ldots, x_{M}\right)$, for example, where

$$
\begin{aligned}
f\left(x_{1} \mid x_{2}, x_{3}, \ldots, x_{M}\right)= & \frac{f\left(x_{1}, x_{2}, x_{3}, \ldots, x_{M}\right)}{f\left(x_{2}, x_{3}, \ldots, x_{M}\right)} \\
& \propto x_{1} \\
& \propto f\left(x_{1}, x_{2}, x_{3}, \ldots, x_{M}\right) .
\end{aligned}
$$

Note: We could also write the $m$ th conditional density as

$$
f\left(x_{m} \mid x_{-m}\right) \text {, }
$$

where

$$
X_{-m}=\left(x_{1}, \ldots, x_{m-1}, x_{m+1}, \ldots, x_{M}\right)
$$

denotes the vector $x$ with the $m$ th component removed.

In any case, the $m$ th distribution can be obtained by examining the joint density of all the variables seeing that joint density as a density function of only $x_{m}$.

An advantage of the Gibbs sampler is that it produces 'good mixing', on account of no 'wastage' due to rejections. A disadvantage is that sampling from all the required exact conditional distributions may not be easy or even possible. 
The Metropolis-Hastings algorithm is a very versatile tool that will work in almost every situation with the least amount of mathematical effort. The Gibbs sampler performs better but is practically feasible only in some special cases.

A general recommendation in any given situation is to begin by specifying a 'pure' Metropolis-Hastings algorithm, and then to examine each of its $M$ Metropolis steps with a view to converting it into a Gibbs step if that is not too much effort. If the resulting Metropolis-Hastings algorithm consists of at least one Gibbs step and at least one Metropolis step, it may also be referred to as a Metropolis-Hastings within Gibbs sampler.

\section{Example}

As an example of converting a Metropolis step into a Gibbs step, recall the joint posterior density in Exercise 6.5:

$$
f(n, \theta \mid y) \propto \frac{n ! \theta^{y}(1-\theta)^{n-y}}{(n-y) !}, 0<\theta<1, n=y, y+1, \ldots, k .
$$

This density was used as a basis for the following Metropolis step for $\theta$ at each iteration:

2. Propose a value $\theta^{\prime} \sim U(\theta-c, \theta+c)$, and accept this value with probability $p_{2}=\frac{\theta^{\prime y}\left(1-\theta^{\prime}\right)^{n-y}}{\theta^{y}(1-\theta)^{n-y}}$.

Instead of this Metropolis step at each iteration, it would be better and also easier to apply a Gibbs step which involves sampling the next value of $\theta$ directly from the $\operatorname{Beta}(y+1, n-y+1)$ distribution.

Equivalently, one could write that Gibbs step as:

2. Draw $\theta \sim \operatorname{Beta}(y+1, n-y+1)$.

Now consider the Metropolis step for $n$ in Exercise 6.5:

1. Propose a value $n^{\prime} \sim D U(n-r, \ldots, b+r)$, and accept this value with probability $p_{1}=\frac{f\left(n^{\prime}, \theta \mid y\right)}{f(n, \theta \mid y)}=\frac{n^{\prime} !(1-\theta)^{n^{\prime}} /\left(n^{\prime}-y\right) !}{n !(1-\theta)^{n} /(n-y) !}$. 
Unfortunately, the kernel of $f(n, \theta \mid y)$ when seen as a function of $n$ alone (i.e. $n !(1-\theta)^{n} /(n-y)$ !) does not suggest a well-known distribution. However, with a little effort, it is still possible to convert the Metropolis step for $n$ into a Gibbs step, as follows:

1. Calculate $q(n)=n !(1-\theta)^{n} /(n-y)$ ! for each $n=5, \ldots, 9$.

Calculate $q_{T}=q(5)+\ldots+q(9)$.

Hence obtain $f(n \mid y, \theta)=q(n) / q_{T}$.

Draw $n \sim f(n \mid y, \theta)$ (now easy).

\section{Exercise 6.7 Sampling from a normal-normal-gamma model via MCMC}

Consider the general normal-normal-gamma model given by:

$$
\begin{aligned}
& \left(y_{1}, \ldots, y_{n} \mid \mu, \lambda\right) \sim \text { iid } N(\mu, \lambda) \\
& (\mu \mid \lambda) \sim N\left(\mu_{0}, \sigma_{0}^{2}\right) \\
& \lambda \sim G(\alpha, \beta) .
\end{aligned}
$$

Suppose that $\mu_{0}=10, \sigma_{0}=2, \alpha=3, \beta=6$ and $n=40$.

(a) Generate $y=\left(y_{1}, \ldots, y_{n}\right)$ from the model using these constants.

(b) Design a suitable Metropolis-Hastings algorithm in this setting. Then apply it $y$ in (a) so as to generate a random sample of size $J=5,000$ from the bivariate posterior distribution of $\mu$ and $\lambda$. Illustrate the sample with appropriate trace plots and probability histograms.

(c) Repeat (b) but with a Gibbs sampler in place of the MH algorithm.

\section{Solution to Exercise 6.7}

(a) Using the specified values, we generated the parameters

$$
\lambda=0.1292 \text { and } \mu=11.95
$$

from their independent prior distributions.

We then generated $n=40$ values from the $N\left(\mu, \sigma^{2}\right)$ distribution with $\sigma=1 / \sqrt{\lambda}=2.782$. The sample mean and standard deviation of these values were 12.28 and 2.592. A histogram of the sample values is shown in Figure 6.20. Overlaid is the $N\left(\mu, \sigma^{2}\right)$ density. 


\section{Figure 6.20 Probability histogram of $40 y$-values}

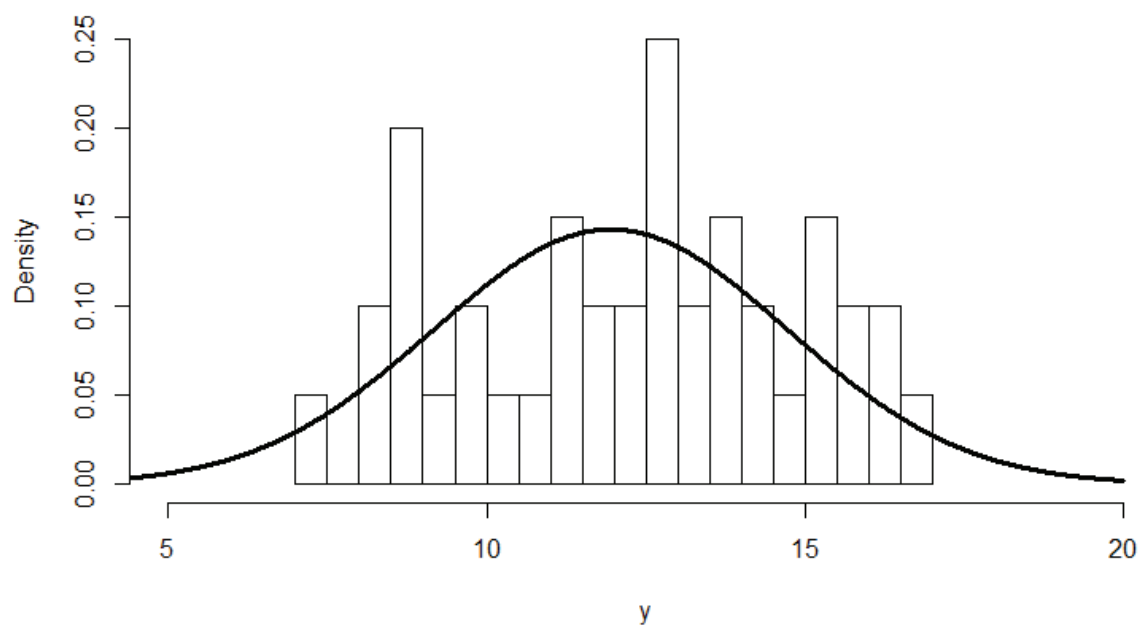

(b) The joint posterior density of $\mu$ and $\lambda$ is

$$
\begin{aligned}
f(\mu, \lambda \mid y) & \propto f(\lambda) f(\mu \mid \lambda) f(y \mid \lambda, \mu) \\
& \propto \lambda^{\alpha-1} e^{-\beta \lambda} \times e^{-\frac{1}{2 \sigma_{0}^{2}}\left(\mu-\mu_{0}\right)^{2}} \times \prod_{i=1}^{n} \lambda^{\frac{1}{2}} e^{-\frac{\lambda}{2}\left(y_{i}-\mu\right)^{2}} \\
& \propto \lambda^{\alpha+\frac{n}{2}-1} \exp \left\{-\lambda \beta-\frac{1}{2 \sigma_{0}^{2}}\left(\mu-\mu_{0}\right)^{2}-\frac{\lambda}{2} \sum_{i=1}^{n}\left(y_{i}-\mu\right)^{2}\right\} \\
& \equiv k(\mu, \lambda) .
\end{aligned}
$$

A suitable $\mathrm{MH}$ algorithm is then defined by the following two steps:

1. Draw a value $\mu^{\prime} \sim U(\mu-c, \mu+c)$

$$
\text { and accept it with probability } p_{1}=\frac{k\left(\mu^{\prime}, \lambda\right)}{k(\mu, \lambda)} \text {. }
$$

2. Draw a value $\lambda^{\prime} \sim U(\lambda-r, \lambda+r)$

and accept it with probability $p_{2}=\frac{k\left(\mu, \lambda^{\prime}\right)}{k(\mu, \lambda)}$.

Note: The best way to calculate the acceptance probabilities is as:

$$
p_{1}=\exp \left(q_{1}\right) \text { and } p_{2}=\exp \left(q_{2}\right) \text {, }
$$

after first deriving $q_{1}=l\left(\mu^{\prime}, \lambda\right)-l(\mu, \lambda)$ and $q_{2}=l\left(\mu, \lambda^{\prime}\right)-l(\mu, \lambda)$, 
where $l(\mu, \lambda)=\log k(\mu, \lambda)$

$$
=\left(\alpha+\frac{n}{2}-1\right) \log \lambda-\lambda \beta-\frac{1}{2 \sigma_{0}^{2}}\left(\mu-\mu_{0}\right)^{2}-\frac{\lambda}{2} \sum_{i=1}^{n}\left(y_{i}-\mu\right)^{2} .
$$

The $\mathrm{MH}$ algorithm was started at $\mu=0$ and $\lambda=1$ with tuning constants $c=0.1$ and $r=0.01$, and run for a total of 6,000 iterations. The resulting traces are shown in Figures 6.21 and 6.22.

The acceptance rates for $\mu$ and $\lambda$ were 92\% and 92\%. These rates were judged to be unduly high because they led to very strong serial correlation in the simulated values (i.e. poor mixing).

So the algorithm was run again from the same starting values but with $c=0.9$ and $r=0.08$ (both larger). This resulted in Figures 6.23 and 6.24 (pages 312 and 313), with much better mixing, faster convergence, and the better acceptance rates of $59 \%$ and $58 \%$.

The last 5,000 pairs of values from this second run of the algorithm were then collected and used to produce the two histograms in Figures 6.25 and 6.26 (pages 313 and 314). Each histogram is overlaid by a density estimate of the corresponding posterior and shows a dot indicating the true value of the parameter (which was initially sampled from its prior).

Figure 6.2I Trace for $\mu$

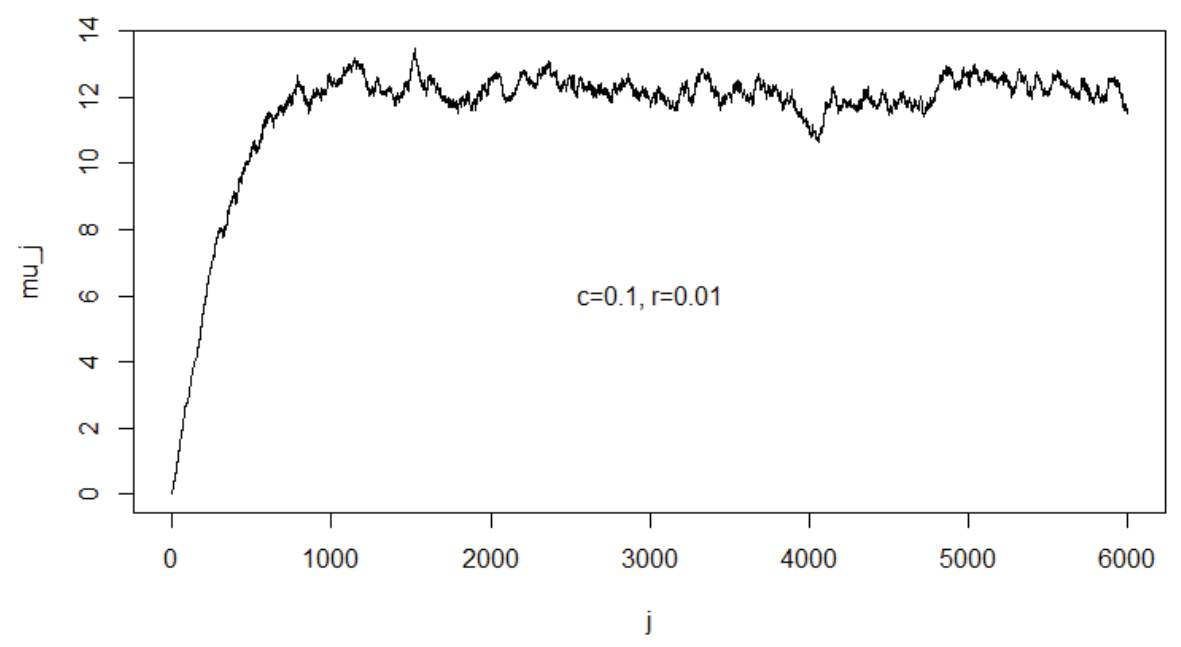


Bayesian Methods for Statistical Analysis

Figure 6.22 Trace for $\lambda$

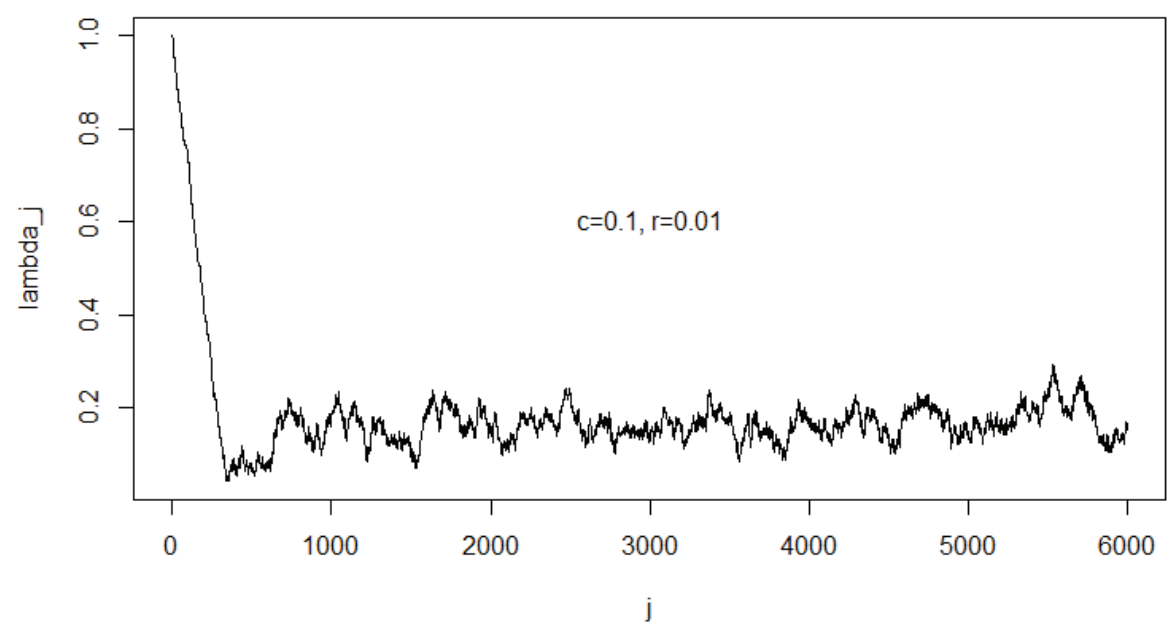

Figure 6.23 Improved trace for $\mu$

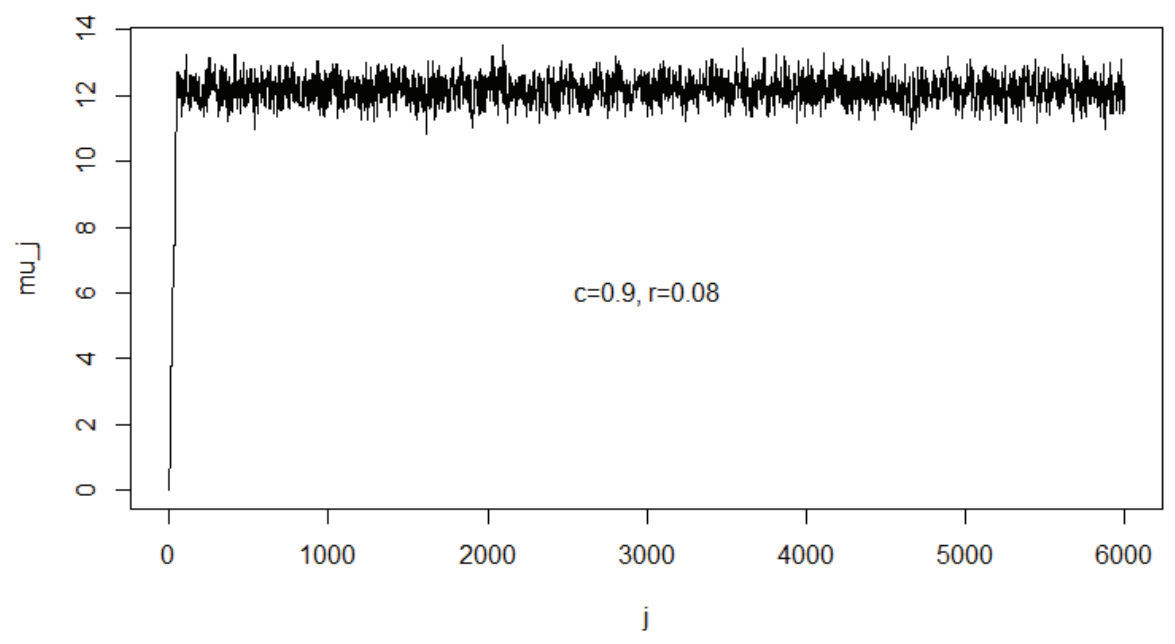


Figure 6.24 Improved trace for $\lambda$

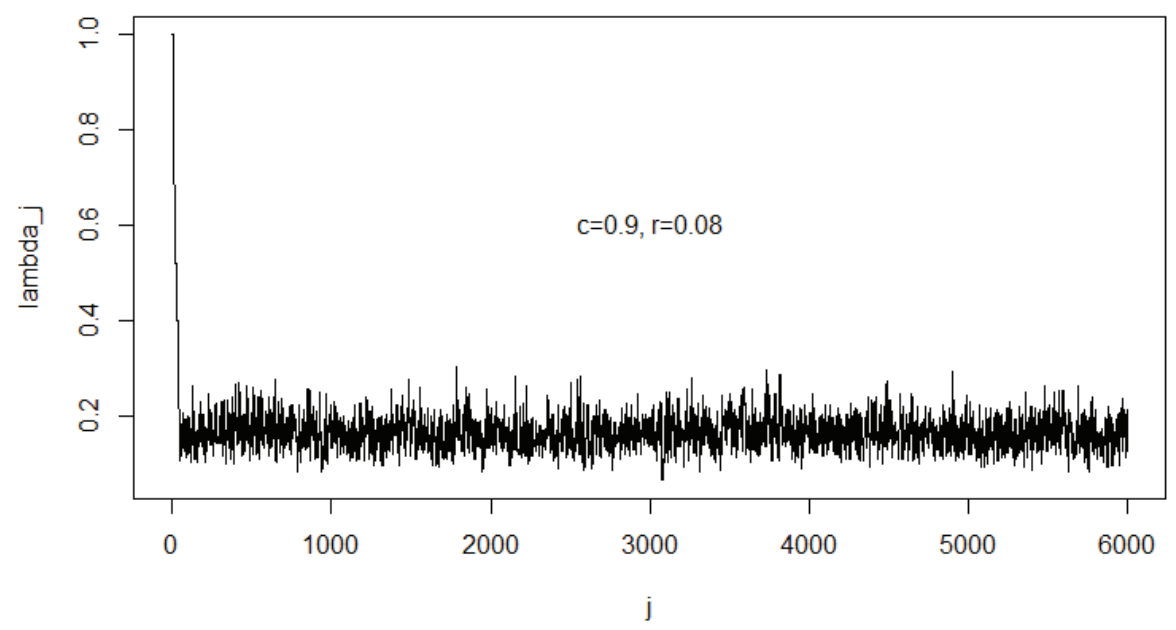

Figure 6.25 Histogram for $\mu$

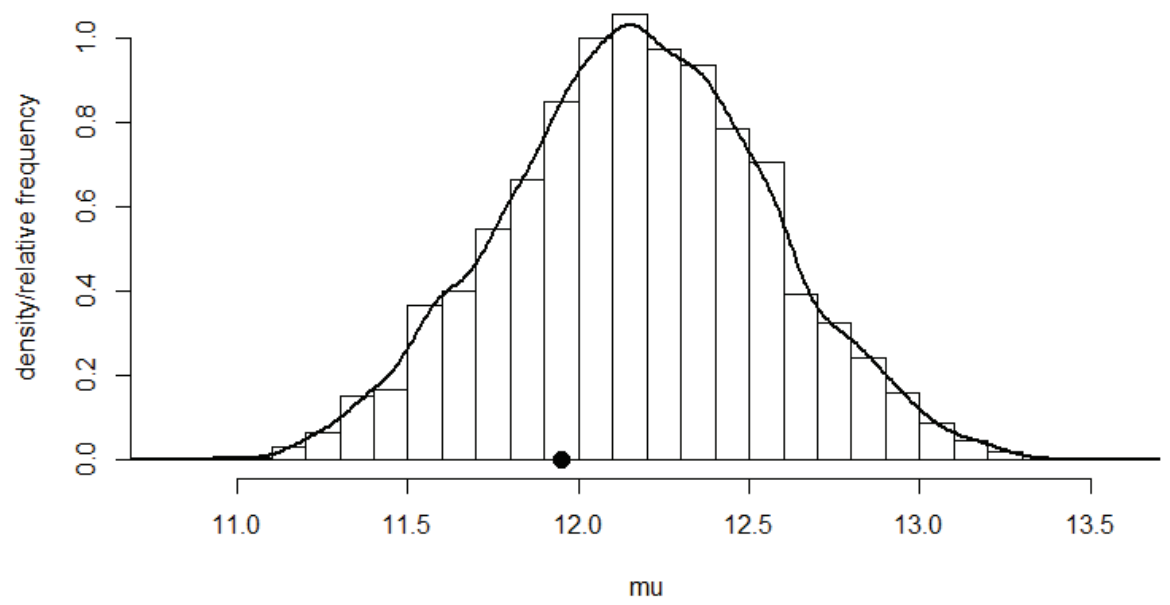




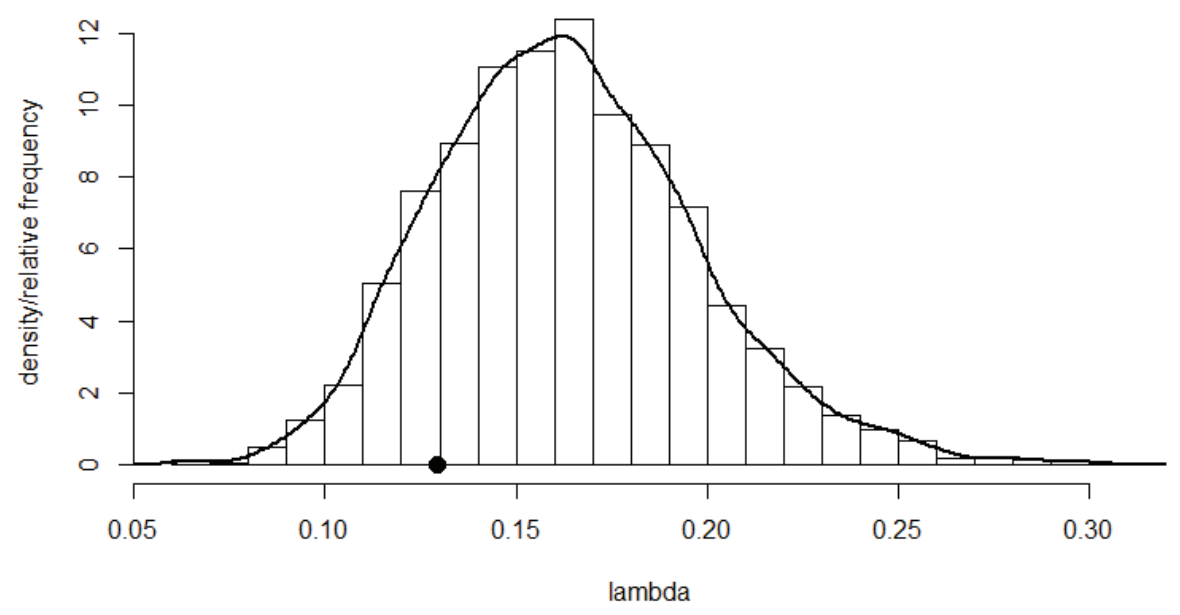

(c) Examining the kernel of the joint posterior in (b) and studying previous exercises (involving the normal-normal model and the normal-gamma model) we easily identify the two conditional distributions which define the Gibbs sampler. These are defined as follows:

1. Sample $\mu \sim f(\mu \mid y, \lambda) \sim N\left(\mu_{*}, \sigma_{*}^{2}\right)$, where: $\mu_{*}=(1-k) \mu_{0}+k \bar{y}$,

$$
\sigma_{*}^{2}=k \frac{\sigma^{2}}{n}=\frac{k}{n \lambda}, k=\frac{n}{n+\sigma^{2} / \sigma_{0}^{2}}=\frac{n}{n+\left(1 /\left(\lambda \sigma_{0}^{2}\right)\right)}, \sigma^{2} \equiv 1 / \lambda .
$$

2. Sample $\lambda \sim f(\lambda \mid y, \mu) \sim G\left(\alpha+\frac{n}{2}, \beta+\frac{1}{2}\left((n-1) s^{2}+n(\mu-\bar{y})^{2}\right)\right)$.

This Gibbs sampler was started at $\mu=0$ and $\lambda=1$ and run for a total of 6,000 iterations. The resulting traces are shown in Figures 6.27 and 6.28.

The last 5,000 pairs of values were then collected and used to produce the histograms in Figures 6.29 and 6.30 (page 316). Each histogram is overlaid by a density estimate of the corresponding posterior and shows a dot indicating the true value of the parameter.

We see that the Gibbs sampler has produced very similar output to that in (b) as obtained using the Metropolis-Hastings algorithm, but with less effort (e.g. no need to worry about tuning constants) and with arguably better results. 
By this we mean that the output from the Gibbs sampler exhibits far less serial correlation. This is evidenced clearly in Figure 6.31 (page 317), which shows the sample autocorrelation functions of the simulated values of $\mu$ and $\lambda$ in (b) (top two subplots) and in (c) (bottom two subplots).

Figure 6.27 Trace for $\mu$ from Gibbs sampler

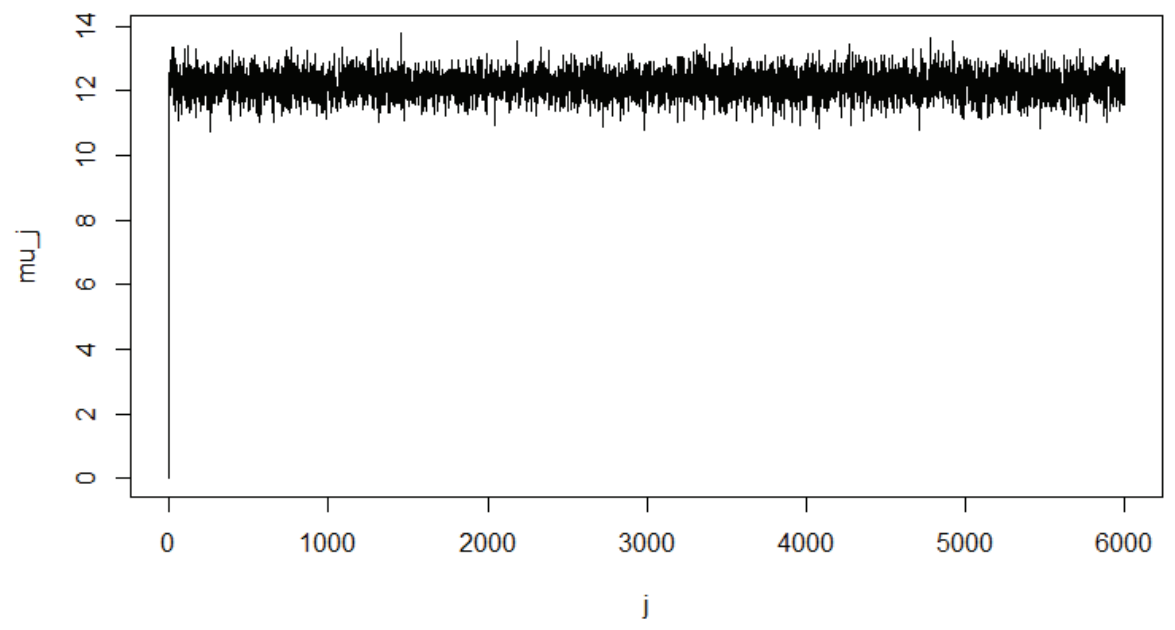

Figure 6.28 Trace for $\lambda$ from Gibbs sampler

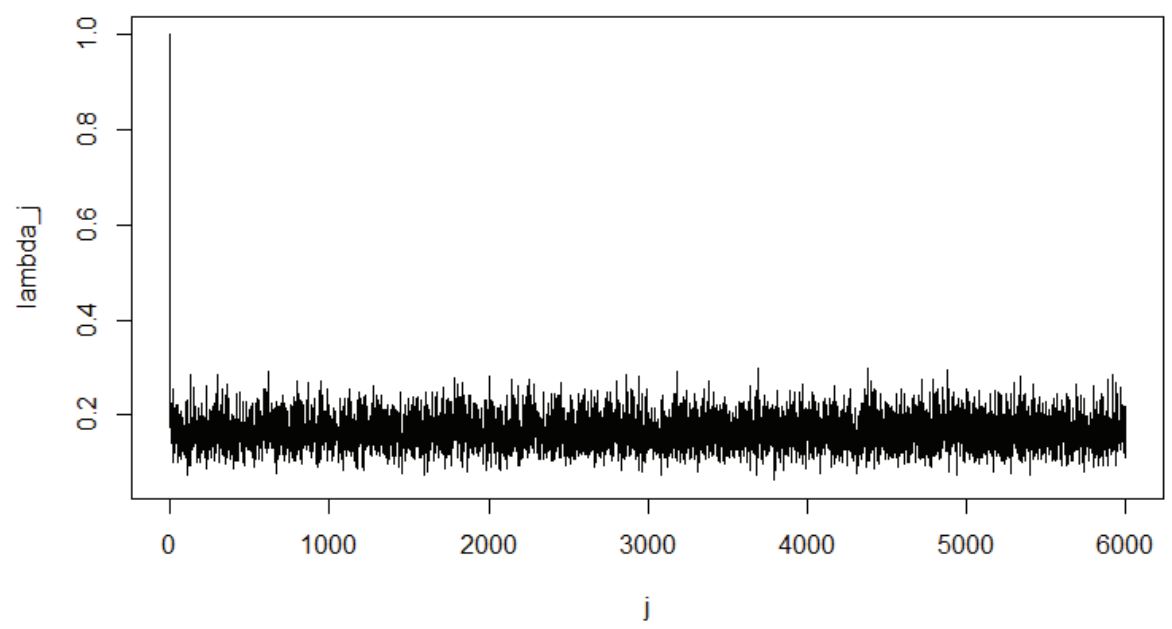


Figure 6.29 Histogram for $\mu$ from Gibbs sampler

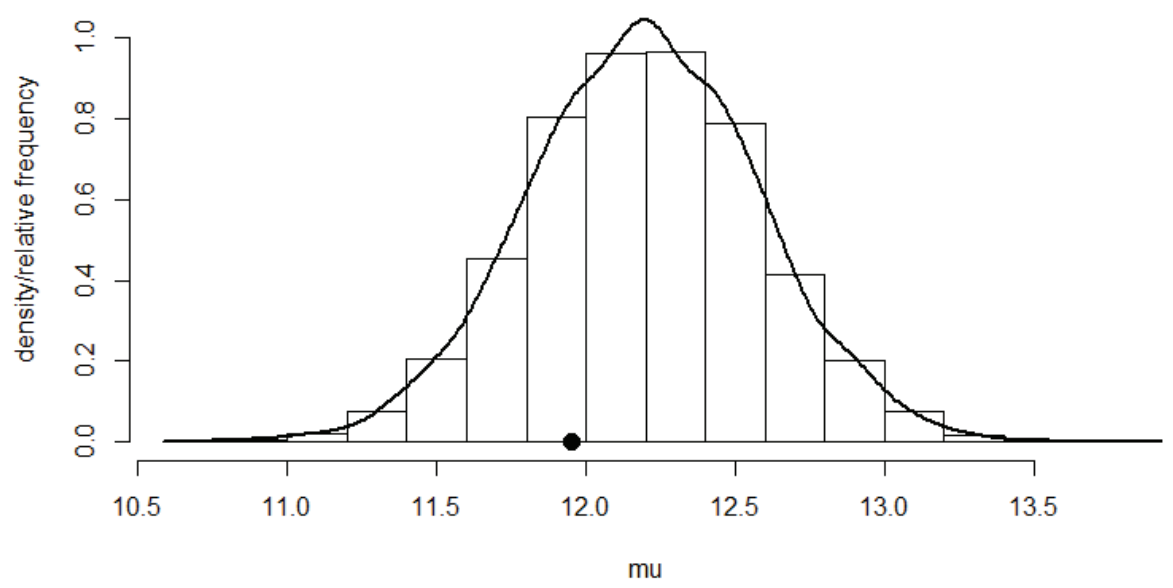

Figure 6.30 Histogram for $\lambda$ from Gibbs sampler

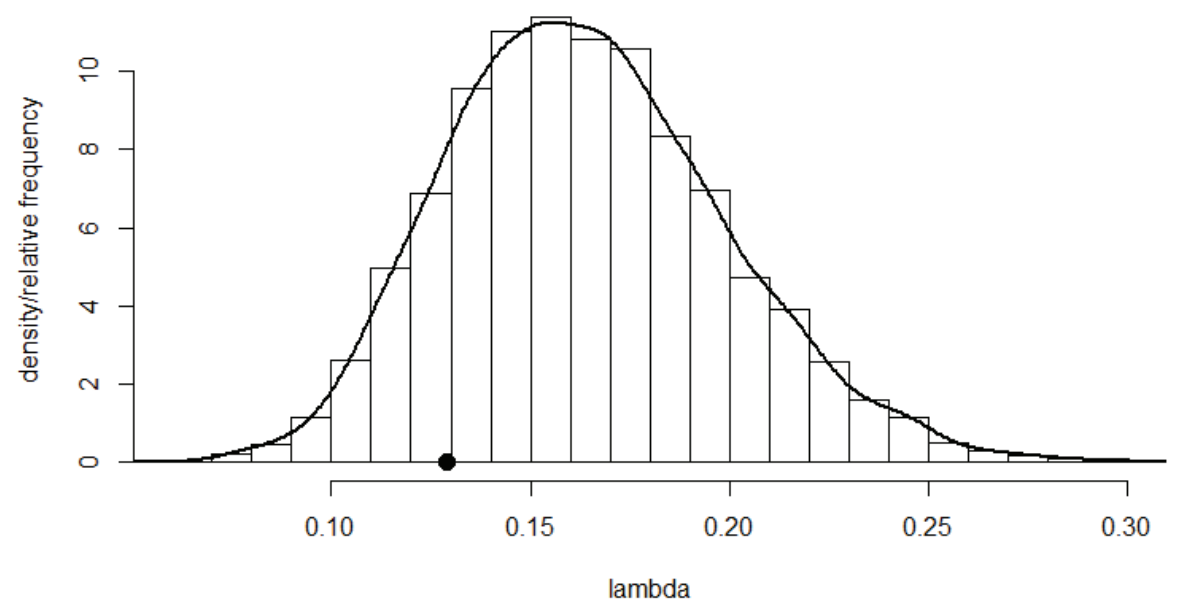


Figure 6.3 I Sample autocorrelations

Series muvb

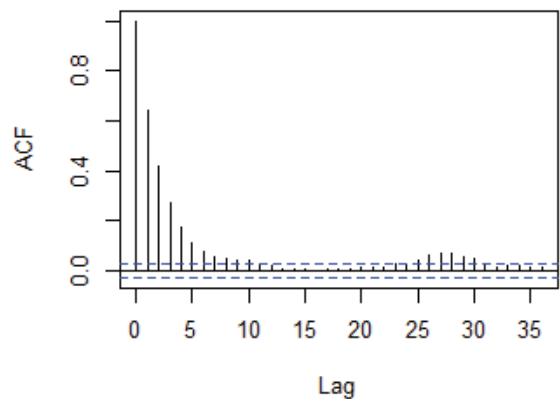

Series muvc

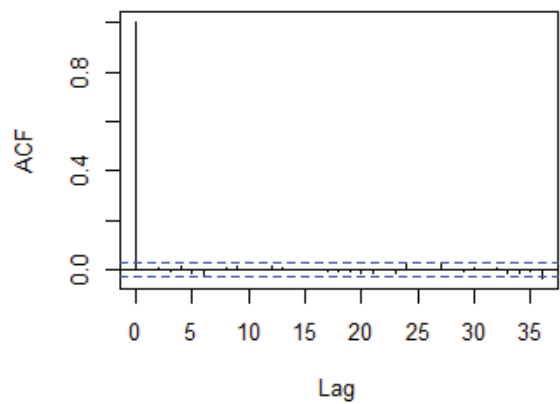

Series lamvb

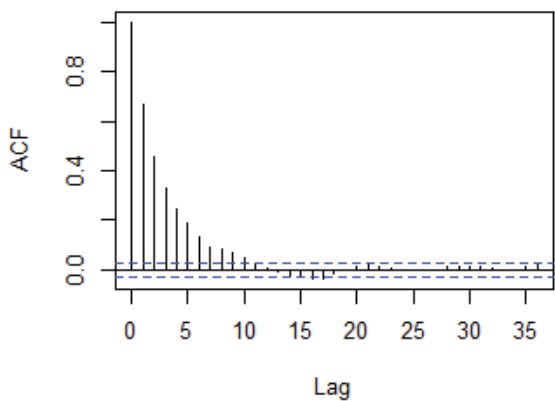

Series lamvc

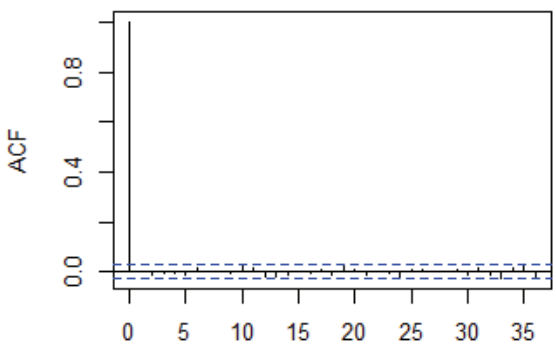

Lag

\section{R Code for Exercise 6.7}

\section{\# (a)}

mu0=10; sig0=2; alp=3; bet=6; $n=40 ;$ options (digits=4)

set.seed(226); lam=rgamma(1,alp, bet); mu=rnorm(1,mu0,sig0);

sig=1/sqrt(lam); y=rnorm(n,mu,sig)

c(lam, sig, sig^2, mu, mean(y), sd(y))

\# $0.1292 \quad 2.78227 .740511 .951112 .27682 .5919$

$\mathrm{X} 11(\mathrm{w}=8, \mathrm{~h}=5) ; \operatorname{par}(\operatorname{mfrow}=\mathrm{c}(1,1))$

hist $(y$, prob $=T, x \lim =c(5,20), y \lim =c(0,0.25)$,breaks=seq $(7,17,0.5)$, main=" ")

$\mathrm{yv}=\operatorname{seq}(0,20,0.01)$; lines(yv, dnorm(yv,mu,sig),lwd=3) 
\# (b)

$\mathrm{MH}<-$ function(Jp, mu, lam, y, c, $r$, alp=0, bet=0, mu0=0, sig0=10000 )\{ \# This function implements a Metropolis-Hastings algorithm for the general \# normal-normal-gamma model.

\# Inputs: $\quad$ Jp = total number of iterations

\# $\quad \mathrm{mu}$, lam = starting values of $\mathrm{mu}$ and lambda

\# $\quad \mathrm{y}=$ vector of $\mathrm{n}$ observations

\# $\quad$ c, $r=$ tuning parameters for mu and lambda

\# alp, bet = parameters of lambda's gamma prior (mean = alp/bet)

\# $\quad$ mu0, sig0 = mean and standard deviation of mu's normal prior

\# Outputs: $\quad$ \$muv, \$lamv $=(\mathrm{Jp}+1)$-vectors of values of mu and lambda

\#

\$muar, \$lamar = acceptance rates for mu and lambda.

muv <- mu; lamv <- lam; ybar <- mean(y); $\mathrm{n}<-$ length(y); muct <- 0; lamct <- 0

logpost <- function(n,y, mu,lam,alp, bet, mu0,sig0)\{

$($ alp $+n / 2-1) * \log (\operatorname{lam})-$ bet*lam -

for(j in 1:Jp)\{

$\left.0.5^{*} \operatorname{lam}^{*} \operatorname{sum}\left((y-m u)^{\wedge} 2\right)-0.5^{*}(\mathrm{mu}-\mathrm{mu} 0)^{\wedge} 2 / \operatorname{sig}^{\wedge} 2\right\}$

mup $<-\operatorname{runif}(1, \mathrm{mu}-\mathrm{c}, \mathrm{mu}+\mathrm{c}) \quad$ \# propose a value of $\mathrm{mu}$

q1<-

logpost $(n=n, y=y, m u=m u p, \quad$ lam=lam, alp=alp, bet=bet, $m u 0=m u 0$, sig0=sig0) -

$\operatorname{logpost}(n=n, y=y, m u=m u \quad, l a m=l a m, a l p=a l p, b e t=b e t, m u 0=m u 0$, sig0=sig0)

p1 <- exp(q1) \# acceptance probability

$\mathrm{u}<-\operatorname{runif}(1)$; if $(\mathrm{u}<\mathrm{p} 1)\{\mathrm{mu}<-$ mup; muct $<-$ muct +1$\}$

lamp $<-$ runif(1,lam-r,lam+r) \# propose a value of lambda

if(lamp $>0)\{\#$ automatically reject if lamp $<0$

q2 <-

logpost $(n=n, y=y, m u=m u, l a m=l a m p, a l p=a l p$, bet=bet, $m u 0=m u 0$, sig0=sig0)-

logpost( $n=n, y=y, m u=m u$, lam=lam ,alp=alp, bet=bet, mu0=mu0, sig0=sig0)

p2 <- exp(q2) \# acceptance probability

$\mathrm{u}<-\operatorname{runif}(1)$; if $(\mathrm{u}<\mathrm{p} 2)\{$ lam <- lamp; lamct <- lamct +1$\}$

\}

muv <- c(muv,mu); lamv <- c(lamv,lam)

\}

list(muv=muv,lamv=lamv, muar=muct/Jp,lamar=lamct/Jp)

\}

Jp <- 6000; set.seed(331)

res $<-\mathrm{MH}(J p=J p, \mathrm{mu}=0, \mathrm{lam}=1, \mathrm{y}=\mathrm{y}, \mathrm{c}=0.1, \mathrm{r}=0.01$, alp=3, bet=6, mu0 $=10$, sig0 $=2$ )

c(res\$muar,res\$lamar) \# 0.91930 .9165

plot(0:Jp,res\$muv,type="I",xlab="j",ylab="mu_j"); text(3000,6,"c=0.1, r=0.01") plot(0:Jp,res\$̧lamv,type="l",xlab="j",ylab="lambda_j");

$\operatorname{text}(3000,0.6, " c=0.1, r=0.01 ")$ 
res $<-\mathrm{MH}(\mathrm{Jp}=\mathrm{Jp}, \mathrm{mu}=0$, lam=1, $\mathrm{y}=\mathrm{y}, \mathrm{c}=0.9, \mathrm{r}=0.08, \mathrm{alp}=3$, bet=6, mu0 $=10$, sig0 $=2$ )

$c($ res\$muar,res\$lamar) \# 0.58900 .5757

plot(0:Jp,res\$muv,type="I",xlab="j",ylab="mu_j"); text(3000,6,"c=0.9, r=0.08")

plot(0:Jp,res\$lamv,type="l",xlab="j",ylab="lambda_j");

$\operatorname{text}(3000,0.6, " c=0.9, r=0.08 ")$

burn <- 1000; muv <- res\$muv[-(1:(burn+1))]; lamv <- res\$lamv[-(1:(burn+1))] hist(muv, prob=T,xlab="mu",nclass=20, main="", ylab="density/relative frequency"); lines(density(muv),lwd=2);

points(mu,0,pch=16,cex=1.5)

hist(lamv, prob=T,xlab="lambda",nclass=20,main="", ylab="density/relative frequency"); lines(density(lamv),lwd=2)

points (lam, 0, pch=16, cex=1.5)

\# acf(muv)\$acf[1:5] \# 1.00000 .64520 .41750 .27440 .1770

\# acf(lamv)\$acf[1:5] \# 1.00000 .66410 .45350 .33000 .2419

muvb= muv; lamvb=lamv \# For use later

\# (c)

GS = function(Jp, mu, lam, $y$, alp=0, bet=0, mu0=0, sig0=10000 )\{

\# This function implements a Gibbs Sampler for the general normal-normalgamma model.

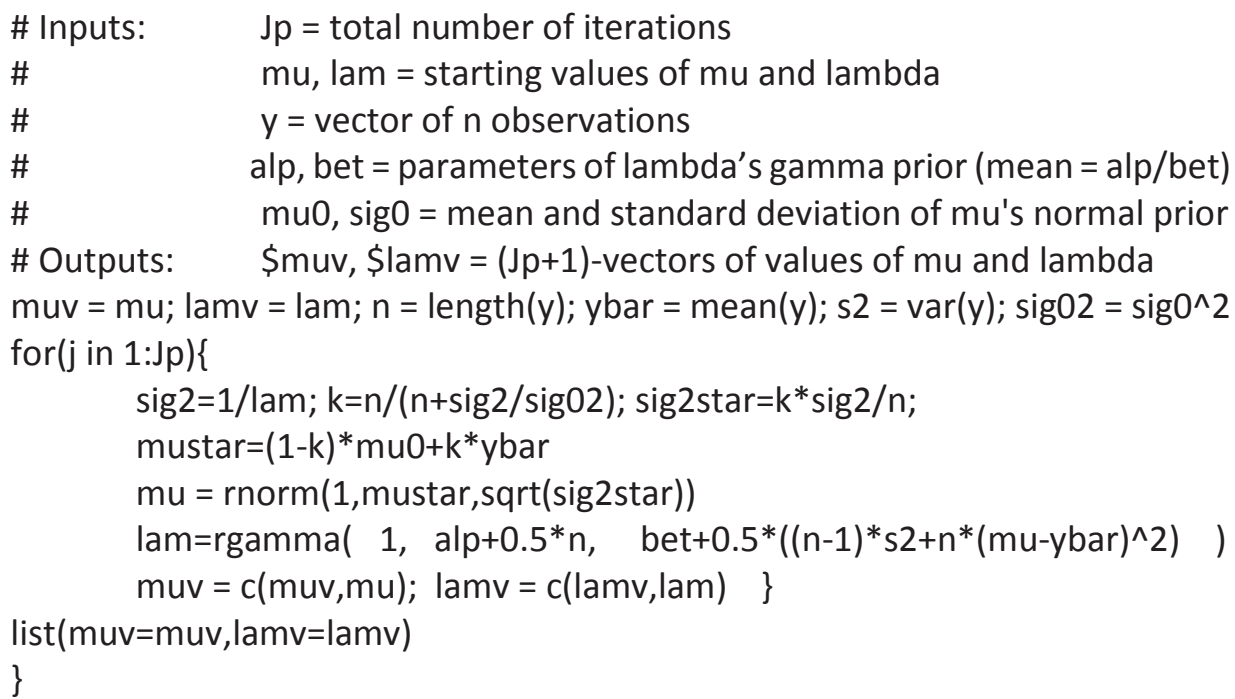


$J p=6000 ;$ set.seed $(331)$

res $=G S(J p=J p, \quad m u=0, \operatorname{lam}=1, y=y, \quad$ alp=3, bet=6, $m u 0=10$, sig0=2)

plot(0:Jp,res\$muv,type="l",xlab="j",ylab="mu_j");

plot(0:Jp,res\$lamv,type="l",xlab="j",ylab="lambda_j");

burn <- 1000; muv <- res\$muv[-(1:(burn+1))]; lamv <- res\$lamv[-(1:(burn+1))]

hist(muv,prob=T,xlab="mu",nclass=20, main="'",ylim=c(0,1.1), ylab="density/relative frequency"); lines(density(muv),lwd=2);

points(mu,0,pch=16,cex=1.5)

hist(lamv,prob=T,xlab="lambda",nclass=20,main="",

ylab="density/relative frequency"); lines(density(lamv),lwd=2)

points(lam,0,pch=16,cex=1.5)

muvc=muv; lamvc=lamv

$\mathrm{X} 11(\mathrm{w}=8, \mathrm{~h}=7) ; \operatorname{par}(\mathrm{mfrow}=\mathrm{c}(2,2))$

$\operatorname{acf}($ muvb)\$acf[1:5] \# 1.00000 .64520 .41750 .27440 .1770

acf(lamvb)\$acf[1:5] \# 1.00000 .66410 .45350 .33000 .2419

$\operatorname{acf(muvc)\$ acf[1:5]~\# ~} 1.0000000-0.00040310 .0079520-0.00735170 .0135979$

acf(lamvc)\$acf[1:5] \# $1.0000000 .002873-0.011504-0.006671-0.001769$ 


\section{MCMC Methods Part 2}

\section{I Introduction}

In the last chapter we introduced a set of very powerful tools for generating samples required for Bayesian Monte Carlo inference, namely Markov chain Monte Carlo (MCMC) methods. The topics we covered included the Metropolis algorithm, the Metropolis Hastings algorithm and the Gibbs sampler.

We now present one more topic, stochastic data augmentation, and provide some further exercises in MCMC. These exercises will illustrate how many statistical problem can be cast in the Bayesian framework and how easily inference can then proceed relative to the classical framework.

The examples below include simple linear regression, logistic regression (an example of generalised linear modelling and survival analysis), autocorrelated Bernoulli data, and inference on the unknown bounds of a uniform distribution.

\subsection{Data augmentation}

Data augmentation (DA) is a method for using unobserved data or latent variables so as to simplify and facilitate an iterative optimisation or sampling algorithm. There are two basic types of DA: deterministic DA and stochastic DA. An example of the former is the EM algorithm as described earlier. Stochastic DA is illustrated in the following example.

\section{Example of stochastic data augmentation}

Suppose we wish to sample from a univariate distribution defined by a density $f(x)$ but that this is difficult to do directly. But then, also suppose that we can factor this density as

where:

$$
f(x) \propto g(x) h(x),
$$

$$
g(x)=\int q(u \mid x) d u
$$




$$
\begin{array}{ll}
q(u \mid x) \quad \begin{array}{l}
\text { is the kernel of conditional density for a latent } \\
\text { random variable } u \text { given } x \text { which is easy to sample } \\
\text { from }
\end{array} \\
\begin{array}{l}
\text { defines the kernel of a conditional density for } x \\
\text { given } u \text { which is easy to sample from; call this } \\
\text { kernel } k(x \mid u)
\end{array}
\end{array}
$$

In such a situation we may define the joint distribution of $u$ and $x$ by the density

$$
f(u, x) \propto q(u \mid x) h(x) .
$$

Then, since both of the conditional distributions (of $u$ given $x$, and of $x$ given $u$ ) are easy to sample from, we may define a suitable Gibbs sampler by the following two steps:
(i) Sample $u^{\prime} \sim q(u \mid x)$
(ii) Sample $x^{\prime} \sim k\left(x \mid u^{\prime}\right)$.

Running this Gibbs sampler will eventually result in a random sample $\left(u_{1}, x_{1}\right), \ldots,\left(u_{J}, x_{J}\right) \sim$ iid $f(u, x)$.

Discarding the simulated latent variables $u_{1}, \ldots, u_{J}$ then yields the desired sample,

$$
x_{1}, \ldots, x_{J} \sim \text { iid } f(x) .
$$

This idea can be extended in a straightforward fashion to sampling from a multivariate distribution, i.e. where $x$ is a vector. In such cases, it may be necessary to define several latent variables in the fashion described above.

\section{Exercise 7.I Sampling with the aid of stochastic data augmentation}

We wish to find the mean of a random variable with density

$$
f(x) \propto \frac{e^{-x}}{x+1}, x>0 .
$$

(a) Calculate the exact value of $E X$ using numerical integration techniques.

(b) Estimate EX using a Monte Carlo sample obtained via rejection sampling. 
(c) Estimate EX using a Monte Carlo sample obtained via the Metropolis algorithm.

(d) Estimate $E X$ using a Monte Carlo sample obtained via a Gibbs sampler designed using the principles of data augmentation.

Note 1: We have already seen the above density $f(x)$ in the context of a previous exercise.

Note 2: The intent of this exercise is threefold:

(i) to illustrate stochastic data augmentation

(ii) to provide additional practice at several Monte Carlo techniques

(iii) to introduce an idea that will be useful later when attempting finite population inference under biased sampling without replacement.

\section{Solution to Exercise 7.I}

(a) Let the kernel be $k(x)=\frac{e^{-x}}{x+1}$.

Then, using the integrate() function in $\mathrm{R}$, we obtain

$$
\int_{0}^{\infty} k(x) d x=0.59635 \text { and } \int_{0}^{\infty} x k(x) d x=0.40365 .
$$

So $E X=0.40365 / 0.59635=0.6769$.

(b) A suitable envelope is the standard exponential density

$$
h(x)=e^{-x}, x>0,
$$

for which the acceptance probability is

where

$$
p(x)=\frac{k(x)}{\operatorname{ch}(x)},
$$

$$
c=\max \frac{k(x)}{h(x)}=\max \frac{e^{-x} /(x+1)}{e^{-x}}=1 .
$$


Thus $p(x)=\frac{1}{x+1}$.

Applying this algorithm we obtained a random sample of size $J=1,000$ using a total of 1,651 draws from the envelope. (Thus the acceptance rate was 1,000/1651 $=61 \%$.) Using this Monte Carlo sample, we estimated $E X$ as 0.6875 with $95 \%$ CI $(0.6402,0.7349)$.

Figure 7.1 shows a trace plot of the simulated values and (just for interest) the associated sample ACF of these values (showing the complete absence of autocorrelation), respectively.

Figure 7.I Trace plot and sample ACF

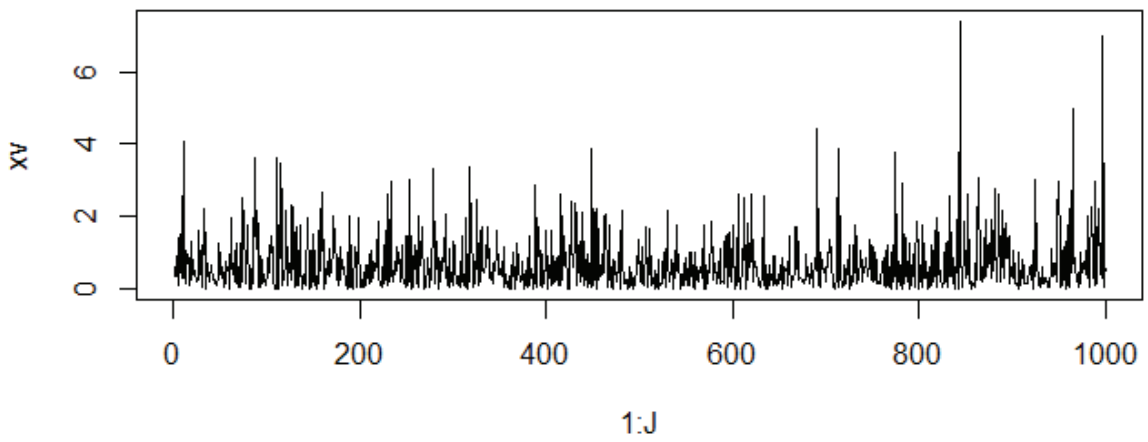

Series $\mathrm{xV}$

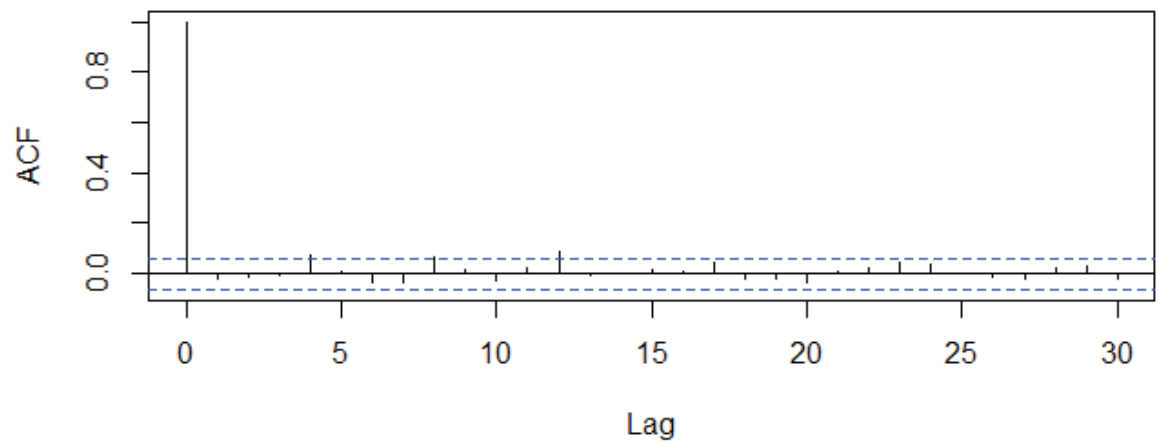


(c) Using a normal driver distribution centred at the last value and with standard deviation 0.6 we ran a Metropolis algorithm for 40,500 iterations, starting at $x=1$. We kept every 40th sampled value after first discarding the first 500 iterations as the burn-in. Using the resulting Monte Carlo sample of size 1,000, we estimated $E X$ as 0.7049 with $95 \%$ CI (0.6561, $0.7537)$. The overall acceptance rate of the algorithm was $58 \%$. Figure 7.2 shows a trace plot of all 40,500 simulated values, the sample ACF of those values (showing a very strong autocorrelation), a trace plot of the 1,000 values used for inference, and the sample ACF of those values (showing very little autocorrelation).

Figure 7.2 Trace plots and sample ACFs

Series res $\$ x v$
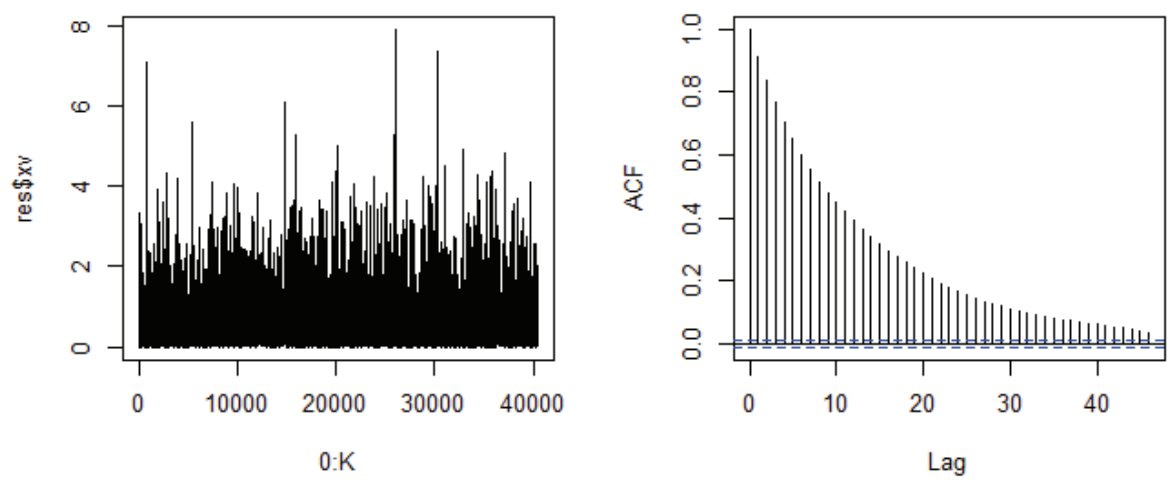

Series $\mathrm{xV}$
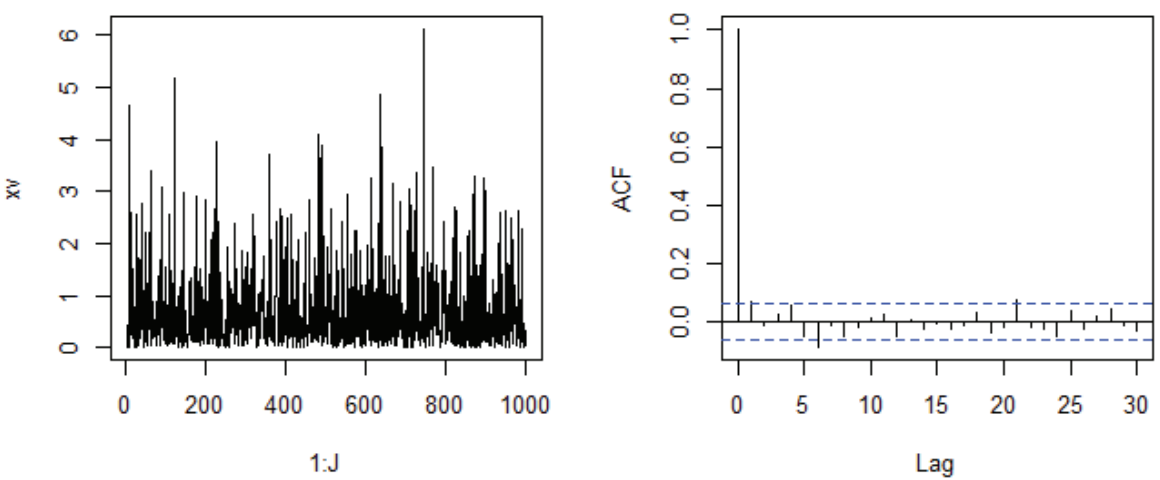
(d) Observe that $\frac{1}{x+1}=\int_{0}^{\infty} e^{-(x+1) w} d w$.

Therefore $f(x)=\frac{1}{x+1} e^{-x} \propto \int_{0}^{\infty} e^{-(x+1) w} e^{-x} d w$.

Hence we may define an artificial latent variable $w$ such that the joint density of $w$ and $x$ is

$$
f(w, x) \propto e^{-(x+1) w} e^{-x}, w>0, x>0 .
$$

We see that:

$$
\begin{aligned}
& f(w \mid x) \propto f(w, x) \propto)^{w} e^{-(x+1) w}, w>0 \\
& f(x \mid w) \propto f(w, x) \propto e^{-(w+1) x}, x>0 .
\end{aligned}
$$

So, a Gibbs sampler for sampling from $f(w, x)$ is defined by the two densities:

$$
\begin{aligned}
& f(w \mid x)=(x+1) e^{-(x+1) w}, w>0 \\
& f(x \mid w)=(w+1) e^{-(w+1) x}, x>0,
\end{aligned}
$$

or equivalently by the two steps:

Sample $w \sim \operatorname{Gamma}(1, x+1)$

Sample $x \sim \operatorname{Gamma}(1, w+1)$.

Starting at $x=1$, we ran this Gibbs sampler for 5,100 iterations. We then kept every 5th sampled value after first discarding the first 100 iterations as the burn-in. Using the resulting Monte Carlo sample of size 1,000 we estimated $E X$ as 0.7172 with $95 \%$ CI (0.6671, 0.7673).

Figure 7.3 shows a trace plot of all 5,100 simulated values, their sample ACF (showing a slight autocorrelation), a trace plot of the 1,000 values used for inference, and the sample ACF of these 1,000 values (showing very little autocorrelation).

Note that similar plots could also be produced for the simulated latent variable, $w$. Also note how data augmentation and a Gibbs sampler have resulted in a usable Monte Carlo sample more easily and effectively than the Metropolis algorithm. 
Figure 7.3 Trace plots and sample ACFs

Series res\$xv
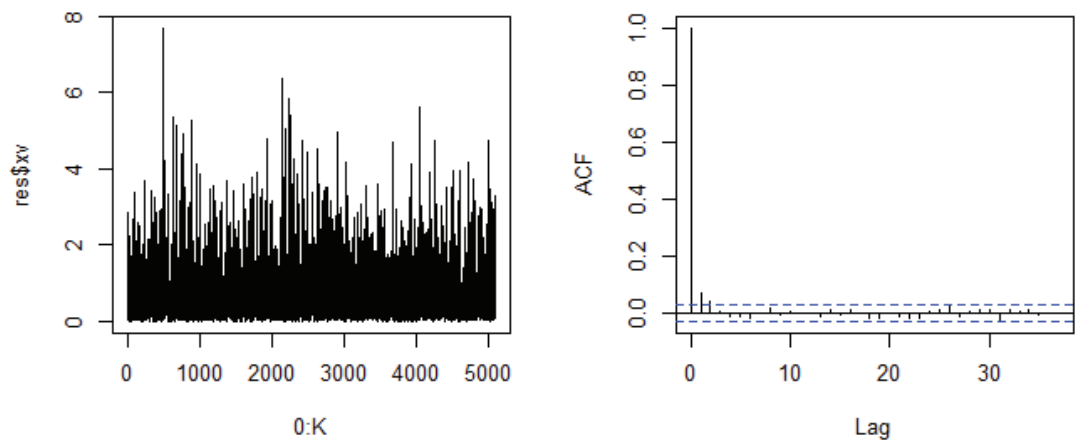

Series $\mathbf{x v}$
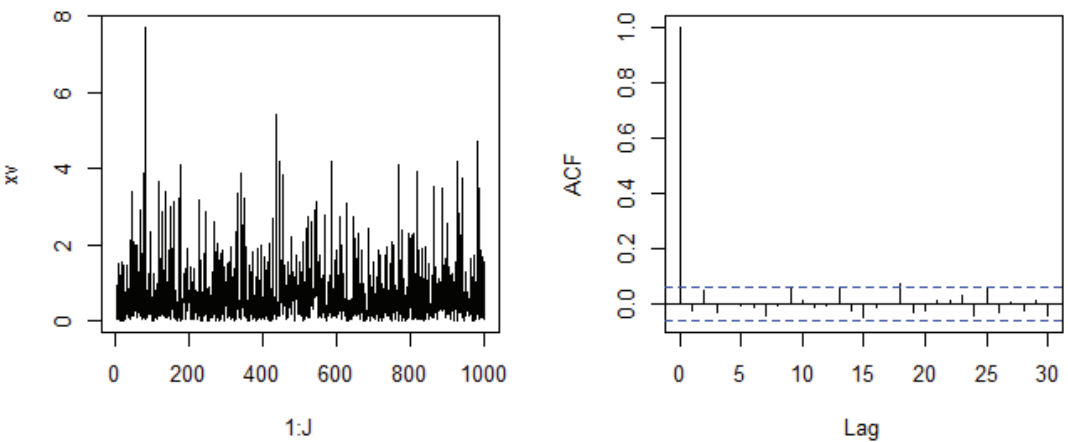

\section{R Code for Exercise 7.I}

\# (a)

options(digits $=5$ ); $k$ fun=function $(x)\{\exp (-x) /(x+1)\}$

$c=$ integrate $(f=k f u n$, lower=0,upper=Inf $) \$$ value; $c$ \# 0.59635

$\mathrm{xkfun}=$ function $(\mathrm{x})\left\{\mathrm{x}^{*} \exp (-\mathrm{x}) /(\mathrm{x}+1)\right\}$

top=integrate $(f=x k f u n, l o w e r=0$, upper $=\operatorname{lnf}) \$$ value; top \# 0.40365

$\mathrm{EX}=$ top/c; EX \# 0.67688

\# (b)

$\mathrm{J}=1000 ; \mathrm{xv}=\mathrm{rep}(\mathrm{NA}, \mathrm{J}) ; \mathrm{ct}=0$; $\operatorname{set} . \operatorname{seed}(331)$

for $(\mathrm{j}$ in $1: \mathrm{J})\{$ acc $=F$; while $(\mathrm{acc}==\mathrm{F})\{\mathrm{ct}=\mathrm{ct}+1$

$\mathrm{x}=\operatorname{rgamma}(1,1,1) ; \mathrm{p}=1 /(\mathrm{x}+1) ; \mathrm{u}=\operatorname{runif}(1) ; \operatorname{if}(\mathrm{u}<\mathrm{p})\{\operatorname{acc}=\mathrm{T} ; \mathrm{xv}[\mathrm{j}]=\mathrm{x}\}\}$

xbar=mean $(x v) ; c i=x b a r+c(-1,1) *$ qnorm $(0.975) * s d(x v) / \operatorname{sqrt}(J)$

$c(c t, x b a r, c i) \# 1651.00000 \quad 0.68754 \quad 0.64016 \quad 0.73492$

$\operatorname{par}(\operatorname{mfrow}=c(2,1)) ; \operatorname{plot}(1: \mathrm{J}, \mathrm{xv}$, type="I")

$\operatorname{acf}(x v) \$ a c f[1: 5]$ \# $1.0000000-0.0205516-0.0100987-0.00400180 .0732520$ 


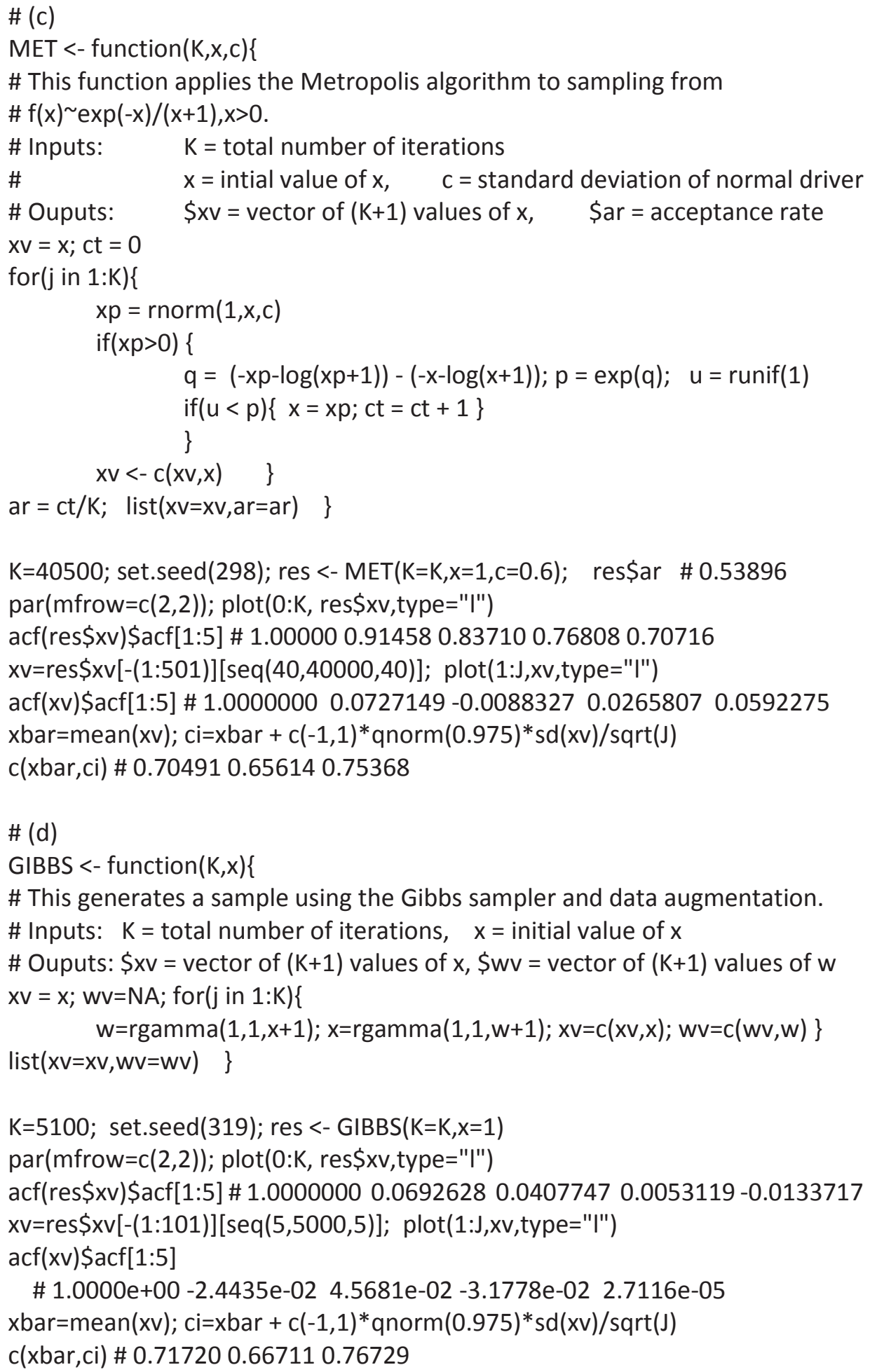




\section{Exercise 7.2 Comparison of classical and Bayesian simple linear regression (and practice at various statistical techniques)}

Consider the following simple linear regression model:

$$
Y_{i} \sim \perp N\left(\mu_{i}, \sigma^{2}\right), i=1, \ldots, n,
$$

where

$$
\mu_{i}=a+b x_{i}
$$

(linear predictor for a value with covariate $x_{i}$ ).

(a) Generate a data vector $y=\left(y_{1}, \ldots, y_{n}\right)$ from the model, using:

$$
n=10, a=5, b=2, \sigma=2,
$$

and with covariates

$$
x_{i}=i
$$

for all $i=1, \ldots, n$.

(b) Conduct a classical analysis of the data in (a). Report the MLEs and 95\% CIs for $a$ and $b$. Also create a single graph which shows:

- the data values

- the true regression line $E(Y \mid x)=a+b x$

- the fitted regression line $\hat{E}(Y \mid x)=\hat{a}+\hat{b} x$

- two lines showing the 95\% CI for the regression line

- two lines showing the 95\% prediction interval at each value of $x$.

(c) Perform a Bayesian analogue of the inference in (b) using the Metropolis-Hastings algorithm and a Monte Carlo sample of size $J=2,000$.

Use a suitable joint uninformative and improper prior for the three parameters in the model.

(d) Create a single graph showing all the information in the two graphs in (b) and (c).

Note: The Bayesian analysis in (c) could also be performed via the Gibbs sampler. 


\section{Solution to Exercise 7.2}

(a) The simulated data are shown in Table 7.1. Note that $x_{i}=i$.

\section{Table 7. I Simulated data}

\begin{tabular}{c|ccccc}
$i$ & 1 & 2 & 3 & 4 & 5 \\
\hline$y_{i}$ & 5.879 & 8.54 & 14.12 & 13.14 & 15.26 \\
\hline & & & & & \\
$i$ & 6 & 7 & 8 & 9 & 10 \\
\hline$y_{i}$ & 20.43 & 19.92 & 18.47 & 21.63 & 24.11 \\
\hline
\end{tabular}

(b) The MLE of $b$ is $\hat{b}=\frac{\sum_{i=1}^{n}\left(x_{i}-\bar{x}\right)\left(y_{i}-\bar{y}\right)}{\sum_{i=1}^{n}\left(x_{i}-\bar{x}\right)^{2}}=1.836$,

and the MLE of $a$ is then $\hat{a}=\bar{y}-\hat{b} \bar{x}=6.051$.

An unbiased estimate of $\sigma^{2}(=1 / \lambda=4)$ is

$$
s^{2}=\frac{1}{n-2} \sum_{i=1}^{n}\left(y_{i}-\left\{\hat{a}+\hat{b} x_{i}\right\}\right)^{2}=3.816
$$

Let: $\quad X=\left(\begin{array}{cc}1 & 1 \\ 1 & 2 \\ \vdots & \vdots \\ 1 & n\end{array}\right)$

$$
M=\left(\begin{array}{ll}
m_{11} & m_{12} \\
m_{21} & m_{22}
\end{array}\right)=\left(X^{\prime} X\right)^{-1} \text {. }
$$

A 95\% CI for $a$ is then

$$
\left(\hat{a} \pm t_{0.025}(8) s \sqrt{m_{11}}\right)=(1.340,2.332)
$$

and a 95\% CI for $b$ is

$$
\left(\hat{b} \pm t_{0.025}(8) s \sqrt{m_{22}}\right)=(2.973,9.128) \text {. }
$$


Also, a $95 \%$ CI for $E(Y \mid x)=a+b x$ is

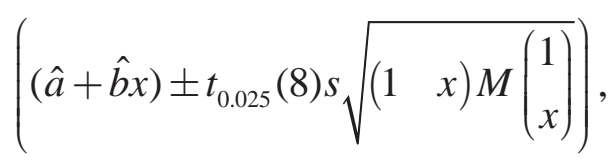

and a 95\% prediction interval for a new observation $Y$ with covariate $x$ is

$$
\left((\hat{a}+\hat{b} x) \pm t_{0.025}(8) s \sqrt{1+\left(\begin{array}{ll}
1 & x
\end{array}\right) M\left(\begin{array}{l}
1 \\
x
\end{array}\right)}\right) .
$$

The required graph is shown in Figure 7.4.

Figure 7.4 Classical inference

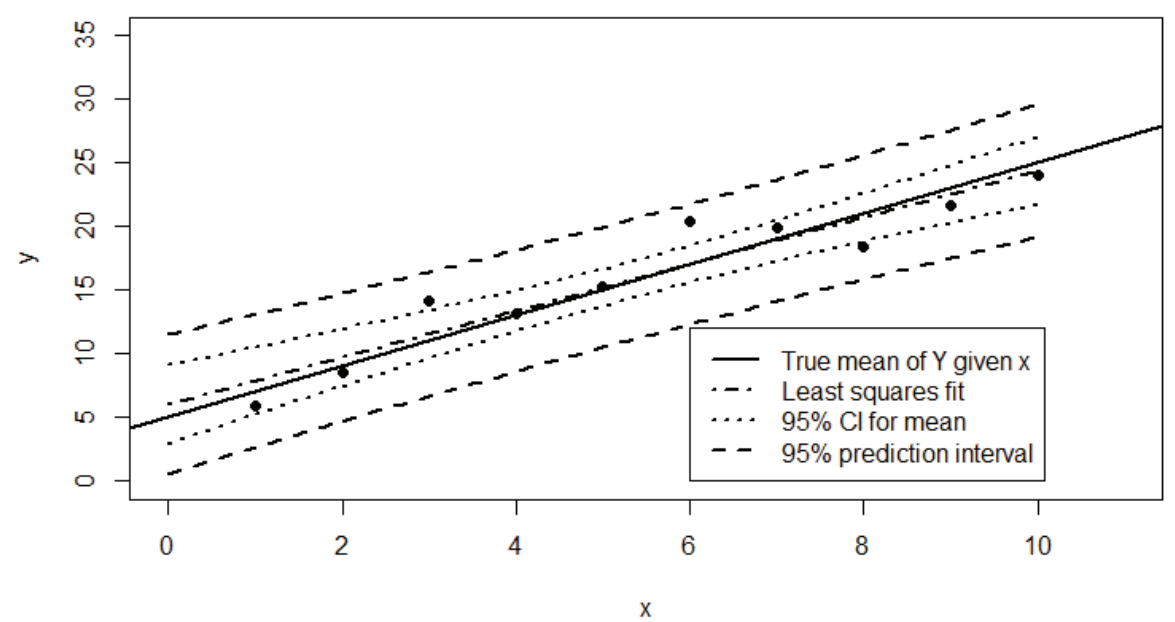

(c) A suitable Bayesian model is given by:

$$
\begin{aligned}
& \left(Y_{i} \mid a, b, \lambda\right) \sim \perp N\left(a+b x_{i}, 1 / \lambda\right), i=1, \ldots, n \\
& \left.f(a, b, \lambda) \propto 1 / \lambda, \quad a, b \in \Re, \quad \lambda>0 \quad \text { (where } \lambda=1 / \sigma^{2}\right) .
\end{aligned}
$$

Let us now solve this Bayesian model so as to estimate the posterior means and 95\% CPDRs for $a$ and $b$. The joint posterior density of the three model parameters is

$$
f(a, b, \lambda \mid y) \propto \frac{1}{\lambda} \times \prod_{i=1}^{n} \sqrt{\lambda} \exp \left\{-\frac{\lambda}{2}\left(y_{i}-\mu_{i}\right)^{2}\right\}
$$

(where $\mu_{i}=a+b x_{i}$ as already defined). 
Hence the joint log-posterior density (up to an additive constant) is

$$
l(a, b, \lambda)=\left(\frac{n}{2}-1\right) \log \lambda-\frac{\lambda}{2} \sum_{i=1}^{n}\left(y_{i}-\mu_{i}\right)^{2} .
$$

Applying the $\mathrm{MH}$ algorithm for 2,500 iterations, we obtain traces for the three parameters as shown in Figure 7.5. The horizontal lines show the true values of the three parameters. The fourth subplot (bottom right) is a histogram of the last 2,000 values of $b$ simulated.

Figure 7.5 Results of a MH algorithm
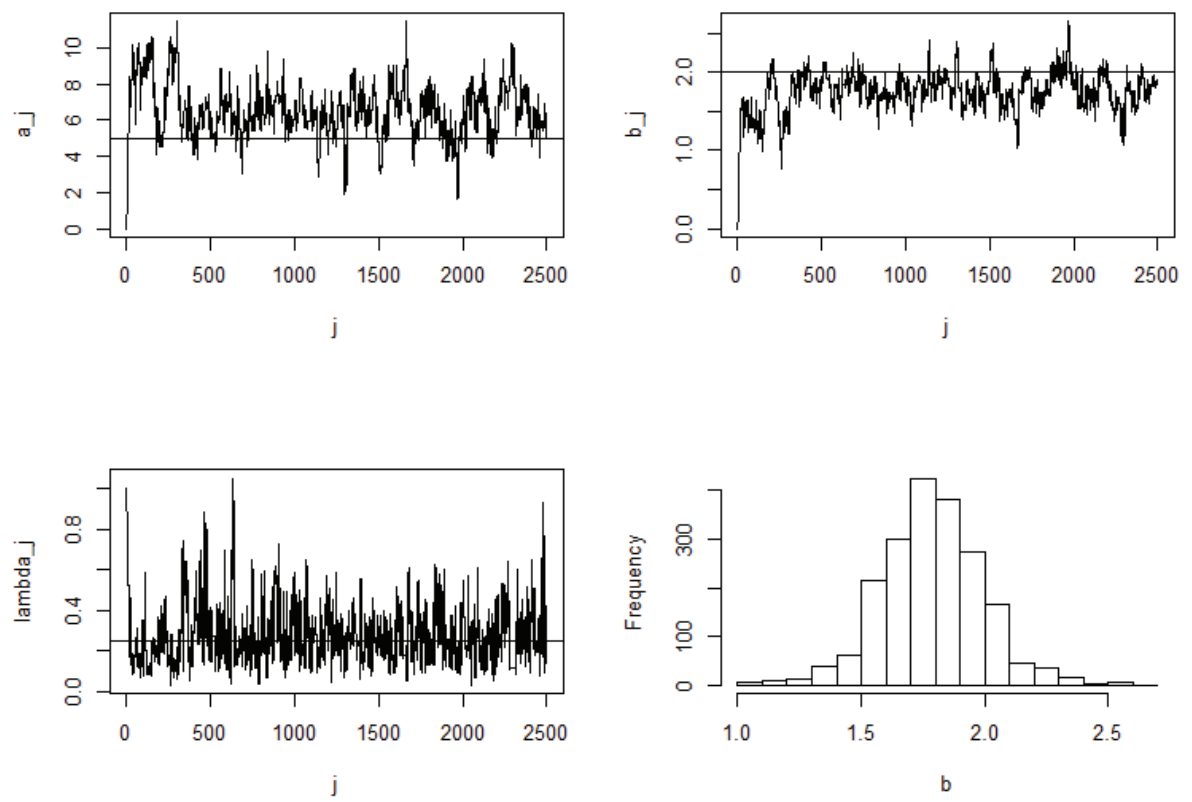

Using output from the last 2,000 iterations only, we estimate the posterior mean and 95\% CPDR for $a(=5)$ as 6.3445 and $(3.578,8.808)$, and the same for $b(=2)$ are about 1.7881 and $(1.392,2.234)$.

Figure 7.6 shows the Bayesian analogue of Figure 7.5 in part (b). 
Figure 7.6 Bayesian inference

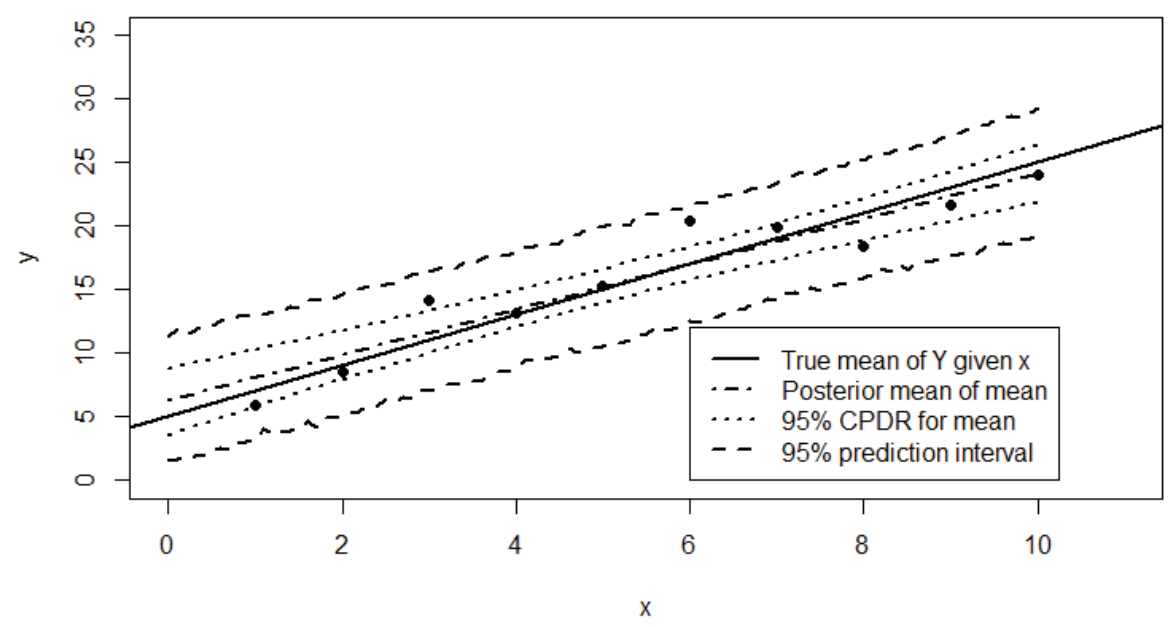

(d) The required graph is shown in Figure 7.7.

Figure 7.7 Comparison of inferences

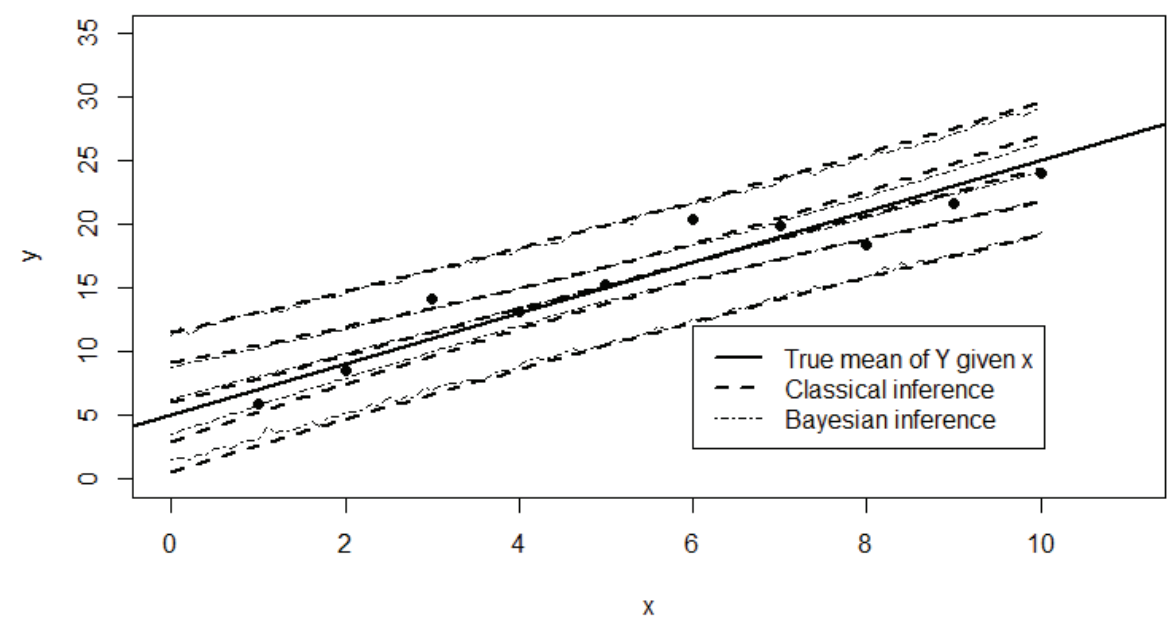




\section{R Code for Exercise 7.2}

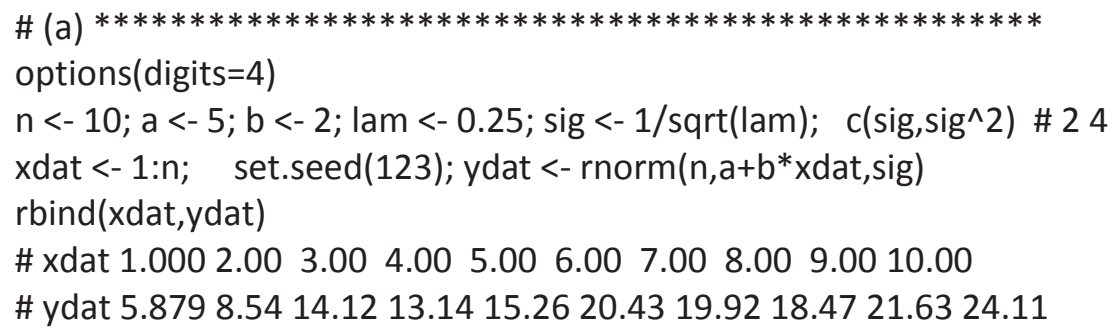


lines ( $x x v$, muhatub, Ity $=3, \mathrm{lwd}=2)$

lines $(x x v$, predlb, $\mid t y=2, l w d=2$ )

lines( $x x v$, predub, $I t y=2, I w d=2)$

legend(6,12,c("True mean of Y given $x "$,"Least squares fit","95\% Cl for mean", "95\% prediction interval"), Ity=c(1,4,3,2),Iwd=rep(2,4))

$\#$ (c)

MH.SLR <- function(Jp, $x, y, a, b$, lam, asd, bsd, lamsd)\{ \# This function implements a Metropolis Hastings algorithm for a \# simple linear regression model with uninformative priors.

\# Inputs: $\quad J p=$ total number of iterations

\# $\quad \mathrm{x}=$ vector of covariates

\# $\quad y=$ vector of observations

\# $\quad a, b$, lam = starting values of a,b,lambda

\# asd,bsd,lamsd = st. dev.s of drivers for a,b,lambda.

\# Outputs: $\quad$ \$av,\$bv,\$lamv = (Jp+1)-vectors of values of $a, b, l a m b d a$

\# \$aar,\$bar,\$lamar = acceptance rates for $a, b, l a m b d a$.

av <- a; bv <- b; lamv <- lam; ybar <- mean(y); $\mathrm{n}<-$ length(y)

act $<-0$; bct $<-0$; lamct $<-0$

logpost $<-$ function $(n, x, y, a, b$, lam $)\{\#$ logposterior

$\left.(n / 2-1) * \log (\operatorname{lam})-0.5 * \operatorname{lam} * \operatorname{sum}\left((y-a-b * x)^{\wedge} 2\right) \quad\right\}$

for(j in 1:Jp) \{

ap $<-\operatorname{rnorm}(1, a$ asd) \# propose a value of a

$k<-\quad \log \operatorname{post}(n=n, x=x, y=y, a=a p, \quad b=b, l a m=l a m)-$

logpost $(n=n, x=x, y=y, a=a, \quad b=b, l a m=l a m)$

$p<-\exp (k) \quad \#$ acceptance probability

$\mathrm{u}<-\operatorname{runif}(1) ; \quad$ if $(\mathrm{u}<\mathrm{p})\{\mathrm{a}<-\mathrm{ap} ;$ act $<-$ act +1$\}$

bp $<-\operatorname{rnorm}(1, b, b s d)$ \# propose a value of $b$

$k<-\quad \log \operatorname{cost}(n=n, x=x, y=y, a=a, b=b p, l a m=l a m)-$

$\log \operatorname{post}(n=n, x=x, y=y, a=a, b=b$, lam=lam)

$\mathrm{p}<-\exp (\mathrm{k}) \#$ acceptance probability

$u<-\operatorname{runif}(1) ; \operatorname{if}(u<p)\{b<-b p ;$ bct $<-$ bct +1$\}$

lamp <- rnorm(1, lam, lamsd) \# propose a value of lambda

if(lamp $>0)\{\#$ automatically reject if lamp $<0$

$k<-\operatorname{logpost}(n=n, x=x, y=y, a=a, b=b, l a m=l a m p)$ -

$\log \operatorname{post}(n=n, x=x, y=y, a=a, b=b$, lam=lam)

$\mathrm{p}<-\exp (\mathrm{k})$ \# acceptance probability

$\mathrm{u}<-\operatorname{runif}(1) ;$ if $(\mathrm{u}<\mathrm{p})\{$ lam $<-$ lamp; lamct $<-$ lamct +1$\}$

\}

av <- c(av, a); bv <- c(bv, b); lamv<- c(lamv, lam)

\}

list $(a v=a v, b v=b v$, lamv $=$ lamv, aar $=a c t / J p$, bar $=b c t / J p$, lamar $=$ lamct $/ J p)$

\} 
Jp <- 2500; set.seed(441)

$\mathrm{mh}<-\mathrm{MH} . \mathrm{SLR}(J p=J p, \quad x=x d a t, y=y d a t, a=0, b=0, l a m=1$, asd $=1.2$, bsd=0.2,lamsd=0.2)

c(mh\$aar,mh\$bar,mh\$lamar) \# 0.52280 .50080 .5132

$\mathrm{X} 11(\mathrm{w}=8, \mathrm{~h}=6) ; \operatorname{par}(\operatorname{mfrow}=\mathrm{c}(2,2))$ \# Figure

plot(0:Jp,mh\$av,xlab="j",ylab="a_j",type="l"); abline(h=a)

plot(0:Jp,mh\$bv,xlab="j",ylab="b_j", type="l"); abline(h=b)

plot(0:Jp,mh\$lamv,xlab="j",ylab="lambda_j", type="l"); abline(h=lam)

hist(mh\$̧bv[-(1:501)],main="",xlab="b")

burn <- 500; J <- Jp - burn; J \# 2000

av <- $\operatorname{mh} \$ \operatorname{av}[-c(1:($ burn+1))]; abar <-mean(av)

bv <- mh\$bv[-c(1:(burn+1))]; bbar <- mean(bv)

lamv <- mh\$lamv[-c(1:(burn+1))]; lambar <- mean(lamv)

sig2bar <- mean(1/lamv)

c(abar,bbar,lambar,sig2bar) \# 6.34451 .78810 .27584 .7505

quantile(av,c(0.025,0.975)) \# 3.5788 .808

quantile(bv,c(0.025,0.975)) \# 1.3922 .234

cpdrLBs <- xxv; cpdrUBs <- xxv; predLBs <- xxv; predUBs <- xxv; set.seed(171)

for(i in $1: \mathrm{nn})\{$

$$
\begin{aligned}
& \text { mus <- av + bv*xxv[i] } \\
& \text { cpdrLBs[i] <- quantile(mus,0.025) } \\
& \text { cpdrUBs[i] <- quantile(mus,0.975) } \\
& \text { sim <- rnorm(J,mus,1/sqrt(lamv)) } \\
& \text { predLBs[i] <- quantile(sim,0.025) } \\
& \text { predUBs[i] <- quantile(sim,0.975) } \\
& \}
\end{aligned}
$$

$\mathrm{X} 11(\mathrm{w}=8, \mathrm{~h}=5) ; \operatorname{par}(\operatorname{mfrow}=\mathrm{c}(1,1))$ \# Figure

plot $(x d a t, y d a t, p c h=16, x \lim =c(0,11), y \lim =c(0,35), x l a b=" x ", y l a b=" y " ~)$

abline $(c(a, b), \operatorname{lwd}=2)$; lines $(c(0, n), c(a b a r$, abar + bbar *n), Ity=4, Iwd=2);

lines (xxv,cpdrLBs, Ity=3,lwd=2)

lines( $x x v, c p d r U B s, I t y=3$, lwd $=2$ )

lines( $x x v$, predLBs, $|t y=2| w d=2$,

lines ( $x x v$, predUBs, Ity $=2$, Iwd $=2$ )

legend $(6,12, c(" T r u e ~ m e a n$ of $Y$ given $x "$, "Posterior mean of mean",

"95\% CPDR for mean","95\% prediction interval"),Ity=c(1,4,3,2), Iwd=rep(2,4)) 
$\#(d)$

$\mathrm{X} 11(\mathrm{w}=8, \mathrm{~h}=5) ; \operatorname{par}(\operatorname{mfrow}=\mathrm{c}(1,1))$ \# Figure

plot(xdat,ydat, $p c h=16, x \lim =c(0,11), y \lim =c(0,35), x l a b=" x ", y l a b=" y " ~)$

abline $(c(a, b), l w d=2)$ \# True regression line

\# Classical lines

lines $\left(c(0, n), c\left(\right.\right.$ fit $\$$ coef[1], fit $\$ \operatorname{coef}[1]+$ fit $\left.\left.\$ \operatorname{coef}[2]^{*} n\right), \operatorname{lty}=2, \operatorname{lwd}=2\right)$

lines(xxv, muhatlb, Ity=2, Iwd=2); lines(xxv, muhatub,lty=2, Iwd=2)

lines(xxv, predlb,Ity=2, Iwd=2); $\quad$ lines $(x x v$, predub, Ity=2, Iwd=2)

\# Bayesian lines

lines $(c(0, n), c($ abar, abar+n*bbar), Ity=4, Iwd=1)

lines $(x x v, c p d r L B s, \mid t y=4, I w d=1)$; $\quad$ lines $(x x v, c p d r U B s, \mid t y=4, I w d=1)$

lines $(x x v$, predLBs, Ity=4, Iwd=1); $\quad$ lines $(x x v$, predUBs, Ity=4, Iwd=1)

legend(6,12,c("True mean of $Y$ given $x "$,

"Classical inference", "Bayesian inference"), Ity $=c(1,2,4), \operatorname{lwd}=c(2,2,1))$

\section{Exercise 7.3 Comparison of classical and Bayesian logistic regression (an example of GLMs) (and practice at various statistical techniques)}

Table 7.2 shows data on the number of rats who died in each of $n=10$ experiments within one month of being administered a particular dose of radiation. For example in Experiment 3, a total of 40 rats were exposed to radiation for 3.6 hours, and 23 of them died within one month. Thus an estimate of the probability of a rat dying within one month if it is exposed to 3.6 hours of radiation is $23 / 40=57.5 \%$.

\section{Table 7.2 Rat mortality data}

\begin{tabular}{c|cccccc}
$i$ & $n_{i}$ & $x_{i}$ & $y_{i}$ & $y_{i} / n_{i}$ & $=$ & $\hat{p}_{i}$ \\
\hline 1 & 10 & 0.1 & 1 & $1 / 10$ & $=$ & 0.1 \\
2 & 30 & 1.4 & 0 & $0 / 30$ & $=$ & 0 \\
3 & 40 & 3.6 & 23 & $23 / 40$ & $=$ & 0.575 \\
4 & 20 & 3.8 & 12 & $12 / 20$ & $=$ & 0.6 \\
5 & 15 & 5.2 & 8 & $8 / 15$ & $=$ & 0.5333 \\
6 & 46 & 6.1 & 32 & $32 / 46$ & $=$ & 0.696 \\
7 & 12 & 8.7 & 10 & $10 / 12$ & $=$ & 0.833 \\
8 & 37 & 9.1 & 35 & $35 / 37$ & $=$ & 0.946 \\
9 & 23 & 9.1 & 19 & $19 / 23$ & $=$ & 0.826 \\
10 & 8 & 13.6 & 8 & $8 / 8$ & $=$ & 1 \\
\hline
\end{tabular}


Consider the following logistic regression model for these data:

$$
Y_{i} \sim \perp \operatorname{Bin}\left(n_{i}, p_{i}\right), \quad i=1, \ldots, n,
$$

where:

$$
\begin{aligned}
p_{i} & =\frac{1}{1+\exp \left(-z_{i}\right)} & & \text { (probability of a 'success' for experiment } i \text { ) } \\
z_{i} & =a+b x_{i} & & \text { (linear predictor). }
\end{aligned}
$$

(a) Find the ML estimates of $a$ and $b$ using the $\operatorname{glm}()$ function in R. For each parameter also calculate a suitable 95\% CI.

(b) Find the ML estimates and associated 95\% CIs in R using your own code for the Newton-Raphson algorithm and without using the $\operatorname{glm}()$ function.

(c) Find the ML estimates using a modification of the Newton-Raphson algorithm which does not require the inversion of matrices.

(d) Suppose that $a$ and $b$ are assigned independent flat priors over the whole real line. Thus consider the Bayesian model:

$$
\begin{array}{rlrl}
\left(Y_{i} \mid a, b\right) & \sim \operatorname{Bin}\left(n_{i}, p_{i}\right), \quad i=1, \ldots, n \\
p_{i} & =\frac{1}{1+\exp \left(-z_{i}\right)} \quad & \text { (probability of death for experiment } i \text { ) } \\
z_{i} & =a+b x_{i} & \text { (linear predictor) } \\
f(a, b) & \propto 1, \quad a, b \in \Re . &
\end{array}
$$

Use the MH algorithm to get a sample of $J=10,000$ observations from $f(a, b \mid y)$, where $y=\left(y_{1}, \ldots, y_{n}\right)$.

Hence estimate the posterior means of $a$ and $b$, together with 95\% MC CIs for these estimates, and also estimate the 95\% CPDRs.

Show graphs of the traces and histograms. Overlay the MC estimates and MLEs over the traces, together with 95\% CPDRs and CIs, respectively. Also, overlay kernel density estimates over the histograms.

(e) Use the sample in (d) to estimate $p(x)$, the probability of a rat dying if it is exposed to $x$ hours of radiation, for each $x=0,1,2, \ldots, 15$.

Graph these results with a line in a figure which also shows the $10 \hat{p}_{i}$ values. 
Also include:

- the MC 95\% CI for each estimate of $p(x)$ (i.e. for each $E\{p(x) \mid y\}$ )

- the MC 95\% CPDR for each $p(x)$

- the MLE of each $p(x)$ using standard GLM procedures, together with associated large-sample 95\% CIs.

(f) Suppose that 20 more rats are about to be exposed to exactly five hours of radiation. Use the sample in (d) to estimate how many of these 20 rats will die, together with a 95\% CI for your estimate. Also construct an approximate $95 \%$ prediction region for the number of rats that will die and report the estimated actual probability content of this region.

(g) Use the sample in (d) to estimate $L D 50$, the lethal dose of radiation at which $50 \%$ of rats die, together with a 95\% CPDR. Also compute an estimate and 95\% CI for LD50 using standard GLM techniques.

(h) Consider the Bayesian model and data in (d). Modify the model suitably so as to constrain the probability of death at a dose of zero to be exactly zero. Estimate the parameters in the new model and draw a graph similar to the one in (e) which shows the posterior probability of death for each dose $x$ from zero to 15, together with the associated 95\% CPDRs.

\section{Solution to Exercise 7.3}

(a) Using the glm() function in R, we find that the MLE and 95\% CI for $a$ are -2.156 and $(-2.9998,-1.3113)$. Also, the MLE and 95\% CI for $b$ are 0.5028 and $(0.3456,0.6601)$.

(b) Since the priors on $a$ and $b$ are flat, finding the maximum likelihood estimate of $(a, b)$ is the same as finding the posterior mode of $(a, b)$. Now, the posterior density of $a$ and $b$ is

$$
f(a, b \mid y) \propto \prod_{i=1}^{n} p_{i}^{y_{i}}\left(1-p_{i}\right)^{n_{i}-y_{i}} .
$$

So the log-posterior is

$$
l(a, b)=\log f(a, b \mid y)=\sum_{i=1}^{n} q_{i},
$$

where $q_{i}=y_{i} \log p_{i}+\left(n_{i}-y_{i}\right) \log \left(1-p_{i}\right)$

$$
=y_{i} z_{i}-n_{i} \log \left(1+\exp \left(z_{i}\right)\right) \text { (after some algebra). }
$$


Let: $\quad d_{1 i}=\frac{d q_{i}}{d a}=y_{i}-n_{i} p_{i}, \quad d_{2 i}=\frac{d q_{i}}{d b}=\left(y_{i}-n_{i} p_{i}\right) x_{i}$

$$
\begin{aligned}
& d_{11 i}=\frac{d^{2} q_{i}}{d a^{2}}=-n_{i} p_{i}\left(1-p_{i}\right), \quad d_{12 i}=\frac{d^{2} q_{i}}{d a d b}=-n_{i} p_{i}\left(1-p_{i}\right) x_{i} \\
& d_{22 i}=\frac{d^{2} q_{i}}{d b^{2}}=-n_{i} p_{i}\left(1-p_{i}\right) x_{i}^{2}, \quad d_{1}=\sum_{i=1}^{n} d_{1 i}, d_{2}=\sum_{i=1}^{n} d_{2 i} \\
& d_{11}=\sum_{i=1}^{n} d_{11 i}, \quad d_{22}=\sum_{i=1}^{n} d_{22 i}, \quad d_{12}=\sum_{i=1}^{n} d_{12 i} \\
& v=\left(\begin{array}{l}
a \\
b
\end{array}\right), \quad D=D(v)=\left(\begin{array}{l}
d_{1} \\
d_{2}
\end{array}\right), \quad M=M(v)=\left(\begin{array}{ll}
d_{11} & d_{12} \\
d_{12} & d_{22}
\end{array}\right) .
\end{aligned}
$$

Then the NR algorithm is defined by

$$
v_{t}=v_{t-1}-M\left(v_{t-1}\right)^{-1} D\left(v_{t-1}\right), \quad t=1,2,3, \ldots
$$

Starting from the origin, the iterates of $a$ and $b$ are as shown in Table 7.3.

Table 7.3 Results of a Newton-Raphson algorithm

\begin{tabular}{c|cccccc}
$t$ & 0 & 1 & 2 & 3 & 4 & 5 \\
\hline$a_{t}$ & 0 & -1.474 & -2.013 & -2.148 & -2.156 & -2.156 \\
$b_{t}$ & 0 & 0.3369 & 0.4670 & 0.5008 & 0.5028 & 0.5028 \\
\hline
\end{tabular}

Thus the MLEs of $a$ and $b$ are $\hat{a}=-2.156$ and $\hat{b}=0.5028$. This agrees perfectly with the results in (a).

A 95\% CI for $a$ is $\left(\hat{a} \pm t_{0.025}(8) s_{a}\right)$ and a $95 \%$ CI for $b$ is $\left(\hat{b} \pm t_{0.025}(8) s_{b}\right)$, where:

$$
t_{0.025}(8)=2.306
$$

$s_{a}^{2}$ is the top left element of $V$

$s_{b}^{2}$ is the bottom right element of $V$

$V=\phi\left(X^{\prime} W X\right)^{-1} \quad$ (a 2 by 2 matrix)

$$
\phi=1, X=\left(\begin{array}{cc}
1 & x_{1} \\
1 & x_{2} \\
\vdots & \vdots \\
1 & x_{n}
\end{array}\right), \quad W=\operatorname{diag}\left(w_{1}, \ldots, w_{n}\right), w_{i}=\frac{\omega_{i}}{V\left(\mu_{i}\right) g^{\prime}\left(\mu_{i}\right)^{2}}
$$




$$
\begin{aligned}
& \left.\omega_{i}=n_{i}, \mu_{i}=\hat{p}_{i}=\frac{1}{1+\exp \left(-\hat{z}_{i}\right)} \text { (MLE of the probability at } x=x_{i}\right) \\
& \hat{z}_{i}=\hat{a}+\hat{b} x_{i} \quad\left(\text { MLE of linear predictor at } x=x_{i}\right) \\
& V(\mu)=\mu(1-\mu), \quad g(\mu)=\log \left(\frac{\mu}{1-\mu}\right) \quad \text { (logit link function) } \\
& g^{\prime}(\mu)=\frac{1}{\mu^{2}(1-\mu)^{2}} .
\end{aligned}
$$

We find that $w_{i}=n_{i} \hat{p}_{i}\left(1-\hat{p}_{i}\right)$. Numerically, we find that 95\% CIs for $a$ and $b$ are $(-3.000,-1.311)$ and $(0.3456,0.6601)$, respectively. These results agree with those in (a).

(c) At each iteration $t=1,2,3,4, \ldots$, we:

1. Fix $b$ and perform a NR step towards maximising wrt $a$ :

$$
a_{t+1}=a_{t}-d_{1}\left(a_{t}\right) / d_{11}\left(a_{t}\right)
$$

2. Fix $a$ and perform a NR step towards maximising wrt $b$ :

$$
b_{t+1}=b_{t}-d_{2}\left(b_{t}\right) / d_{22}\left(b_{t}\right) \text {. }
$$

Starting from the origin $(a, b)=(0,0)$ we obtain the results in Table 7.4.

\section{Table 7.4 Results of a search algorithm}

\begin{tabular}{c|ccccc}
$t$ & 0 & 1 & 2 & 3 & 4 \\
\hline$a_{t}$ & 0 & 0.4564 & -0.45034 & -0.06132 & -0.7294 \\
$b_{t}$ & 0 & 0.1401 & 0.09223 & 0.20571 & 0.1690 \\
\hline \multicolumn{1}{l}{} & & 20 & 21 & 99 & 100 \\
\hline$t$ & & -1.8585 & -1.8619 & -2.1555 & -2.1555 \\
$a_{t}$ & & 0.4424 & 0.4532 & 0.5028 & 0.5028 \\
$b_{t}$ & & &
\end{tabular}

We see that this modified and simpler algorithm converges more slowly than plain NR. Also, it is less stable, as it fails to converge if started from $(a, b)=(0.3,0.3)$, unlike plain NR. Both algorithms fail to converge if started from $(0.5,0.5)$. (See the R code below for details.) 
(d) We apply the Metropolis Hastings algorithm with a burn-in of 500 and starting from the origin to get a sample of size of $J=10,000$ from $f(a, b \mid y)$. The acceptance rates were $37 \%$ for $a$ and $55 \%$ for $b$. The Markov chain was not thinned for subsequent inference, meaning that the CIs obtained below are perhaps narrower than they should be.

The MC estimate of $E(a \mid y)$ is -2.207 (similar to the MLE, -2.156), with 95\% CI $(-2.214,-2.199)$ and 95\% CPDR $(-2.963,-1.521)$.

The MC estimate of $E(b \mid y)$ is 0.5145 (similar to the MLE, 0.5028), with 95\% CI (0.5132, 0.5158) and 95\% CPDR (0.3895, 0.6605).

Traces and histograms of the sampled values of $a$ and $b$ are shown in Figure 7.8.

\section{Figure 7.8 Results of $\mathrm{MH}$ algorithm}

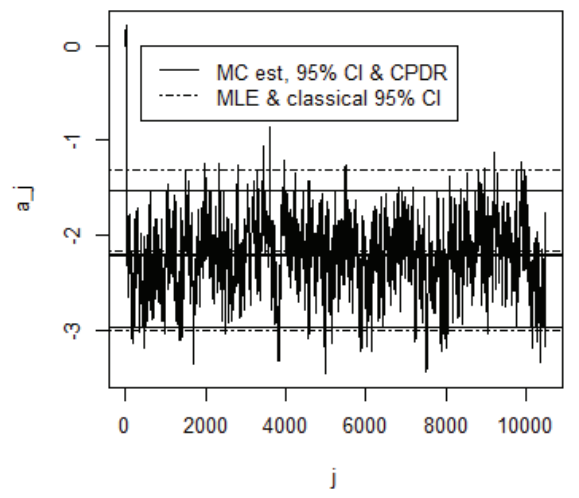

Histogram of av

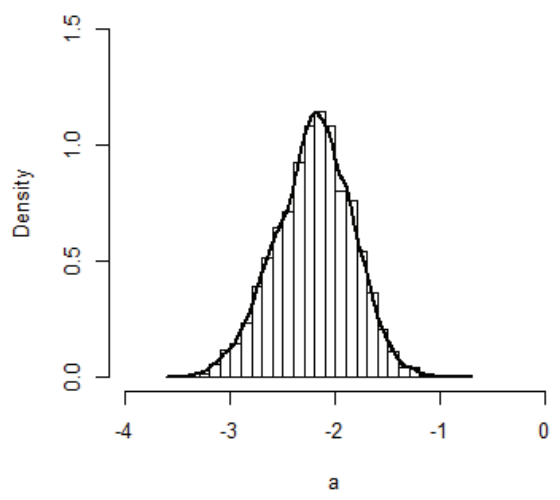

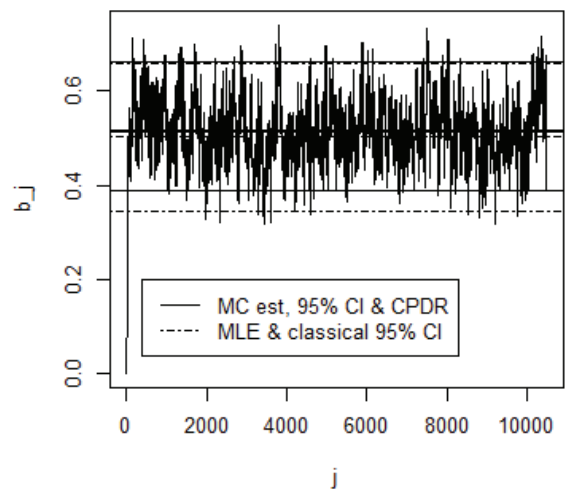

Histogram of bv

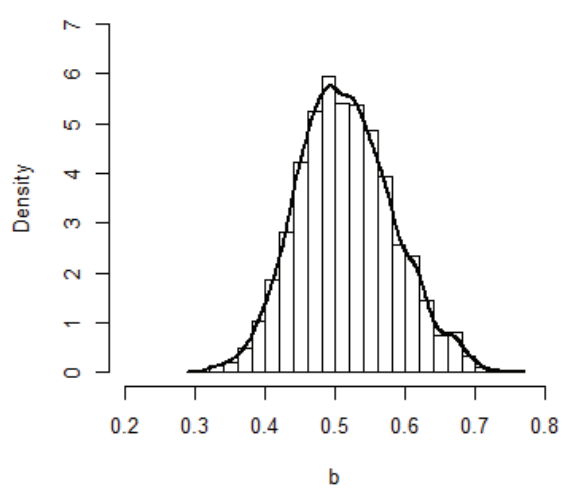


(e) The required results are shown in Figure 7.9.

Note: Figure 7.9 shows that the probability of a rat dying when given no radiation is about $10 \%$. We should interpret this result and the graph near $x=0$ with caution. Ideally, we would conduct another experiment with only small values of $x$ and a second logistic regression, perhaps using the $\log$ of $x$ as the explanatory variable. On the other hand, maybe the $10 \%$ figure is reasonable because rats could die within one month for reasons other than radiation. Alternatively, we could modify our model so as to force $p(0)=0$ (see (h) below).

\section{Figure 7.9 Mortality rate estimates}

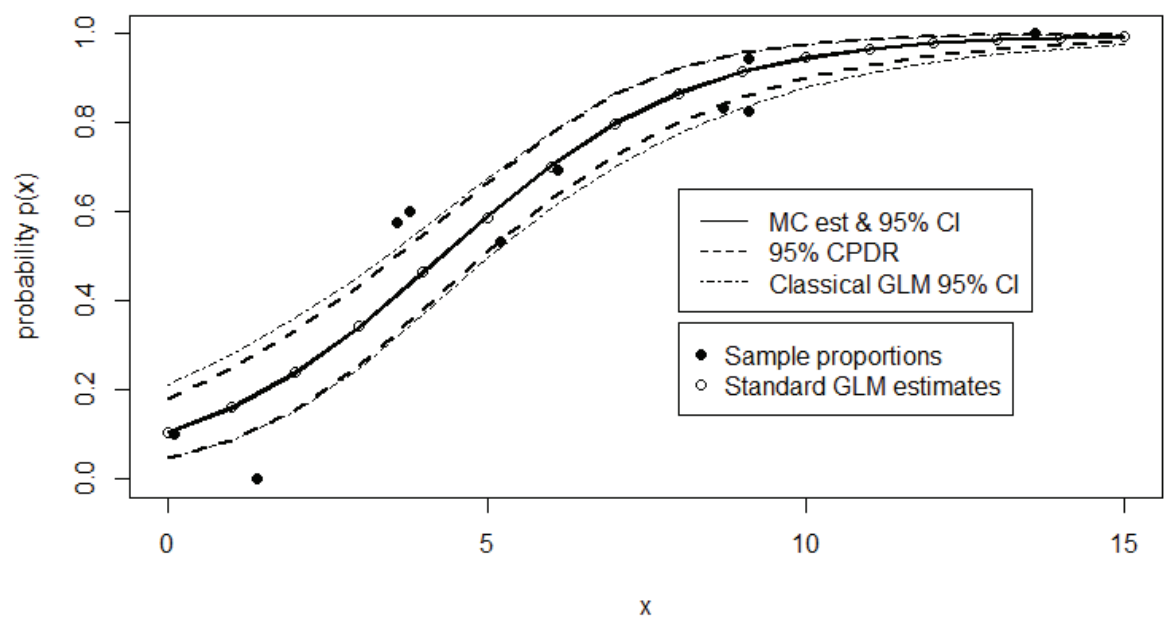

(f) Let $d$ be the number of rats which will die if exposed to radiation for five hours. Then

where

$$
(d \mid y, a, b) \sim \operatorname{Bin}(20, p(a, b)),
$$

$$
p(a, b)=1 /(1+\exp (-a-5 b)) .
$$

We can now apply the method of composition whereby

$$
f(d, a, b \mid y)=f(d \mid y, a, b) f(a, b \mid y) \text {. }
$$

Thus for each sampled $(a, b)$ we calculate $p(a, b)$ and sample from the binomial distribution of $d$ above. The frequencies of the resulting 10,000 values of $d$ are shown in Table 7.5 
Table 7.5 Simulated frequencies of rats dying

\begin{tabular}{c|cccccc}
$d$ & 3 & 4 & 5 & 6 & 7 & 8 \\
\hline frequency & 1 & 3 & 20 & 75 & 217 & 472 \\
\hline$d$ & 9 & 10 & 11 & 12 & 13 & 14 \\
\hline frequency & 845 & 1188 & 1562 & $1733^{*}$ & 1546 & 1123 \\
\hline \multicolumn{1}{c}{$d$} & 15 & 16 & 17 & 18 & 19 & \\
\hline frequency & 709 & 332 & 131 & 37 & 6 & \\
\hline
\end{tabular}

Using the 10,000 values of $d$, our estimate of $d$ is 11.81 (the average of the 10,000 values), with $(11.76,11.85)$ as the $95 \%$ MC CI for $d$ 's posterior mean. We feel about $95.1 \%$ confident that the number of rats which die will be between 8 and 16, inclusive (since $95.1 \%$ of the simulated $d$ values are in this range). Also, it is most likely that 12 of the 20 rats will die, because the MC estimate of $\operatorname{Mode}(d \mid y)$ is 12 (since $d=12$ above has the highest frequency, namely 1,733 , as marked by an asterisk).

(g) First observe that the $L D 50$ is the value of $x$ such that $p(x)=0.5$.

Solving $1 /(1+\exp (-a-b x))=0.5$, we get $x=L D 50=-a / b$.

Using the sample of 10,000 in part (f), we estimate the posterior mean of LD50 is 4.279, with 95\% MC CI $(4.273,4.286)$. The MC 95\% CPDR for LD50 is $(3.584,4.916)$. Thus we can be $95 \%$ confident that the dose required to kill half of a large number of rats is between 3.6 and 4.9.

Using standard GLM procedures and the delta method we estimate $L D 50$ as 4.287 (the MLE) with 95\% CI (3.532, 5.042). Thus we can be 95\% confident that the dose required to kill half of a large number of rats is between 3.5 and 5.0. We see that Bayesian and classical methods have resulted in inferences which are very similar.

(h) An alternative to the logistic model in (d), one with zero probability of death at zero dosage of radiation, is as follows:

$$
\begin{aligned}
\left(Y_{i} \mid a, b\right) & \sim \perp \operatorname{Bin}\left(n_{i}, p_{i}\right), \quad i=1, \ldots, n \\
p_{i} & =1-\exp \left(-z_{i}\right), \quad z_{i}=a x_{i}+b x_{i}^{2} \\
f(a, b) & \propto 1, \quad a, b>0 .
\end{aligned}
$$


Running a suitable modification of the $\mathrm{MH}$ algorithm in (d), we estimate $a$ and $b$ as 0.11 and 0.017 , with respective $95 \%$ CPDRs $(0.04,0.20)$ and $(0.004,0.030)$. The required graph is shown in Figure 7.10.

\section{Figure 7.10 Modified mortality rate estimates}

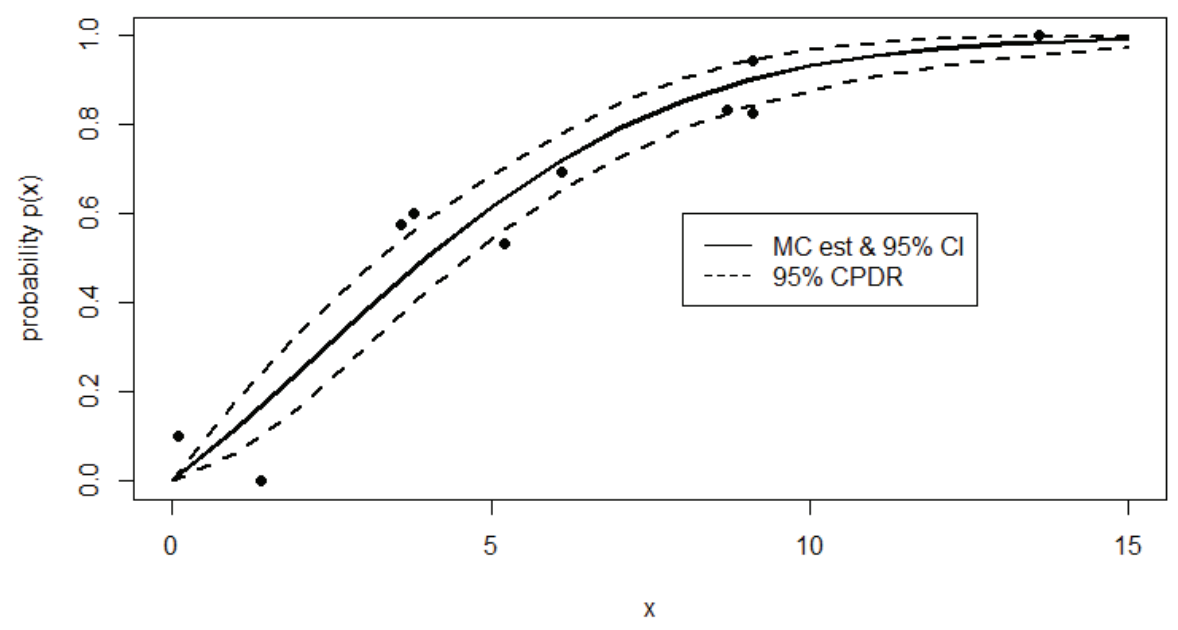

\section{R Code for Exercise 7.3}

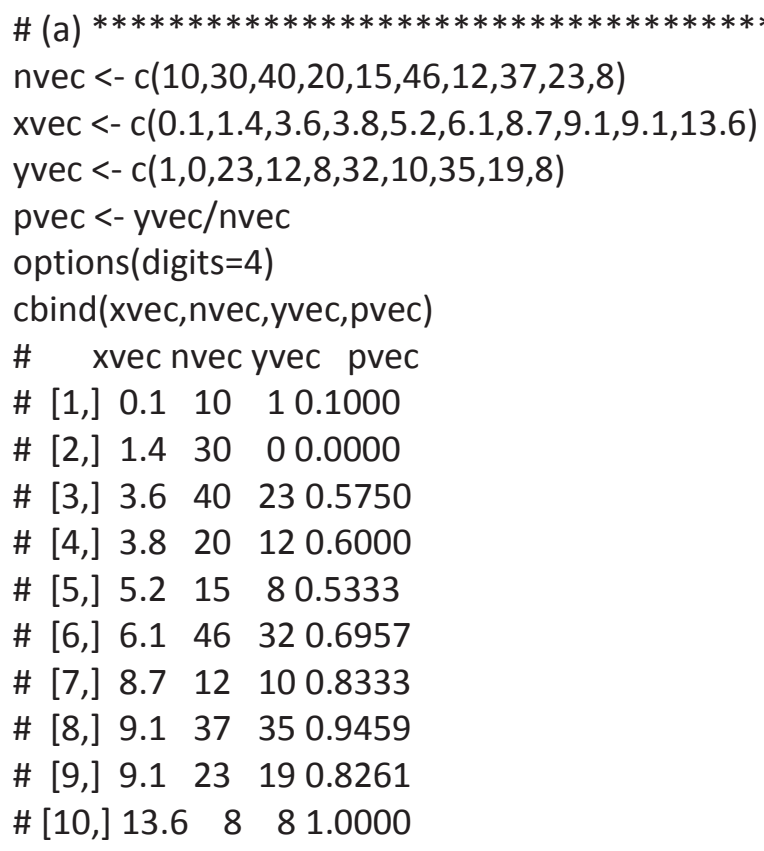


options(digits $=4$ )

nrres <- NR.LOGISTIC ( $m=20$, alp=0, bet $=0, x v=x v e c, n v=n v e c, y v=y v e c)$

nrres

\# \$alpv: [1] $0.000-1.474-2.013-2.148-2.156-2.156 \ldots$

\# Sbetv: [1] $0.00000 .33690 .46700 .50080 .50280 .5028 \ldots$

NR.LOGISTIC ( $m=20$,alp=0.3, bet=0.3, xv=xvec, nv=nvec,yv=yvec)

\# \$alpv: [1] $0.000-1.474-2.013-2.148-2.156-2.156 \ldots$.

\# Sbetv: [1] $0.00000 .33690 .46700 .50080 .50280 .5028 \ldots$

NR.LOGISTIC (m=20, alp=0.5, bet=0.5, xv=xvec, nv=nvec, $y v=y v e c)$

\# Error in solve.default(M) :

\# system is computationally singular: reciprocal condition

\# number $=9.01649 \mathrm{e}-18$

alpmle <- nrres\$alp[21]; betmle <- nrres\$bet[21]

$X<-$ cbind $(1, x v e c)$

zmle <- alpmle + betmle*xvec \# linear predictor

pmle $<-1 /(1+\exp (-z m l e))$

wtvec <- nvec*pmle*(1-pmle)

W <- diag(wtvec)

varmat <- solve(t(X) \%*\% W \%*\% X)

varmat

\# $\quad 0.13404 \quad-0.022442$

\# $\quad-0.02244 \quad 0.004651$

qt $(0.975,8) \quad \# 2.306$

alpmle $+c(-1,1) *$ qt $(0.975,8) *$ sqrt(varmat[1,1]) \# -3.000 -1.311

betmle $+c(-1,1)^{*}$ qt $(0.975,8) * \operatorname{sqrt}(\operatorname{varmat}[2,2]) \# 0.34560 .6601$

$\#(c)$

NRMOD.LOGISTIC <- function( $m$, alp, bet, $x v, n v, y v)\{$

\# Performs logistic regression via a modification of the Newton-Raphson

\# algorithm.

\# Inputs: $\quad m=$ number of iterations

\# alp, bet = starting values of alpha and beta

\# $\quad \mathrm{xv}, \mathrm{nv}, \mathrm{yv}=$ vectors of covariates, sample sizes and

\# numbers of successes, respectively.

\# Outputs: $\quad$ \$alpv $=$ vector of $(m+1)$ alpha values

\# $\quad$ \$betv $=$ vector of $(m+1)$ beta values

alpv <- alp; betv <- bet; ve <- c(alp, bet) 


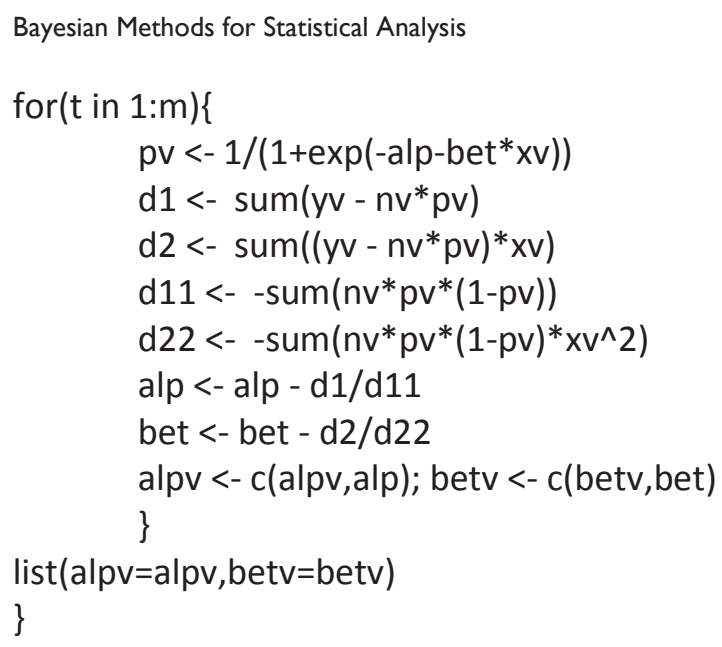




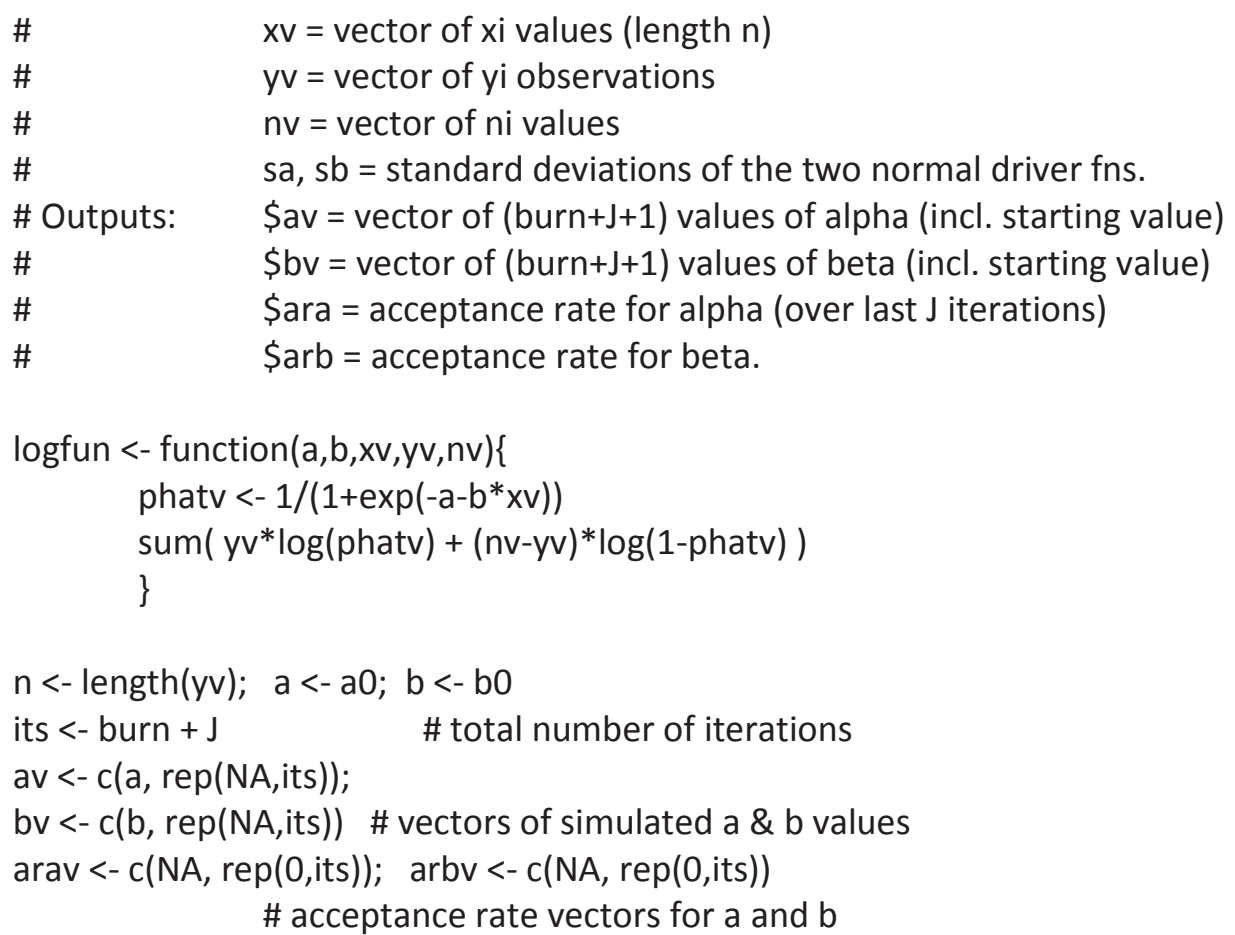

for(j in $1:$ its $)\{$

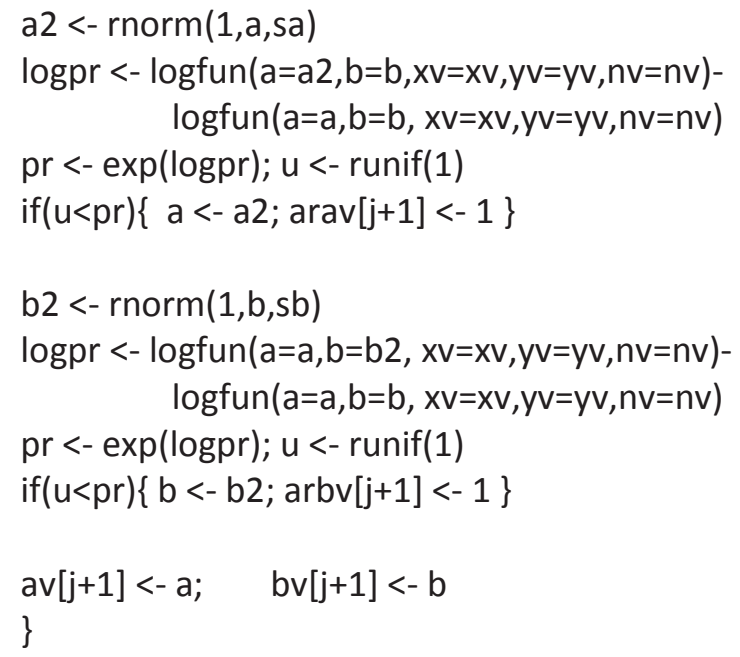


burn <- 500; K <- 10000; its <- burn + K; set.seed(221); date() \#

res <- MHLR(burn=burn, $\mathrm{J}=\mathrm{K}, \mathrm{a} 0=0, \mathrm{bO}=0, \mathrm{xv}=\mathrm{xv}$ data,

$\mathrm{yv}=\mathrm{yvdata}, \mathrm{nv}=\mathrm{nvdata}, \mathrm{sa}=0.5, \mathrm{sb}=0.05)$; date() \# 10000 Took 1 second c(res\$ara,res\$arb) \# \# 0.36500 .5544

$\operatorname{par}(\operatorname{mfrow}=c(2,1)) ; \operatorname{plot}(\operatorname{res} \$ a v, t y p e=" I ") ; \operatorname{plot}($ res\$bv,type="I") \# OK

options(digits=4); J = K; thin=1

\# thin=1 means no thinning (for experimentation)

av <- res\$av[-(1:(burn+1))][seq(thin,K,thin)]; length(av) \# 10000

acf(av)\$acf[1:5] \# 1.00000 .92830 .87560 .83240 .7945

\# (very high autocorrelation)

ahat <- mean(av); aci <- ahat + c(-1,1) * qnorm(1-0.05/2)*sqrt(var(av)/J)

acpdr $<-$ quantile $(a v, c(0.025,0.975))$

c(ahat,aci,acpdr) \# -2.207-2.214 -2.199 -2.963 -1.521

bv <- res\$bv[-(1:(burn+1))][seq(thin,K,thin)]; length(bv) \# 10000

acf(bv)\$acf[1:5] \# 1.00000 .93630 .88920 .84810 .8109

bhat <- mean(bv); bci <- bhat + c(-1,1) * qnorm(1-0.05/2)*sqrt(var(bv)/J)

bcpdr <- quantile(bv, c $(0.025,0.975))$

c(bhat,bci,bcpdr) \# 0.51450 .51320 .51580 .38950 .6605

dena <- density(av); $\quad$ denb <- density(bv)

fit $<-$ glm(pvdata $\sim$ xvdata, family=binomial(link=logit), weights=nvdata)

fit\$coef \# $\quad-2.1555 \quad 0.5028$

ase <- sqrt(summary(fit)\$cov.unscaled[1,1])

fitaci $<-$ fit $\$$ coef $[1]+c(-1,1) * q t(0.975,8) *$ ase

c(ase,fitaci) \# $0.3661-2.9998-1.3113$

bse <- sqrt(summary(fit)\$cov.unscaled[2,2])

fitbci <- fit coef $[2]+c(-1,1) *$ qt $(0.975,8) *$ bse

c(bse,fitbci) \# 0.06820 .34560 .6601

$\mathrm{X} 11(\mathrm{w}=8, \mathrm{~h}=8) ; \operatorname{par}(\operatorname{mfrow}=\mathrm{c}(2,2))$

plot(0:its,res\$av,type="l",xlab="j",ylab="a_j")

abline $(\mathrm{h}=\mathrm{c}(\mathrm{ahat}, \mathrm{aci}, \mathrm{acpdr}))$

abline( $h=c($ fit $\$$ coef[1],fitaci), Ity=4)

legend(400,0,c("MC est, 95\% Cl \& CPDR",

"MLE \& classical 95\% Cl"),Ity=c(1,4))

plot(0:its,res\$́bv,type="l", xlab="j",ylab="b_j")

abline $(\mathrm{h}=\mathrm{c}(\mathrm{bhat}, \mathrm{bci}, \mathrm{bcpdr}))$

abline( $\mathrm{h}=\mathrm{c}($ fit coef[2],fitbci), Ity=4)

legend(400,0.2,c("MC est, 95\% Cl \& CPDR",

"MLE \& classical 95\% Cl"),Ity=c(1,4)) 


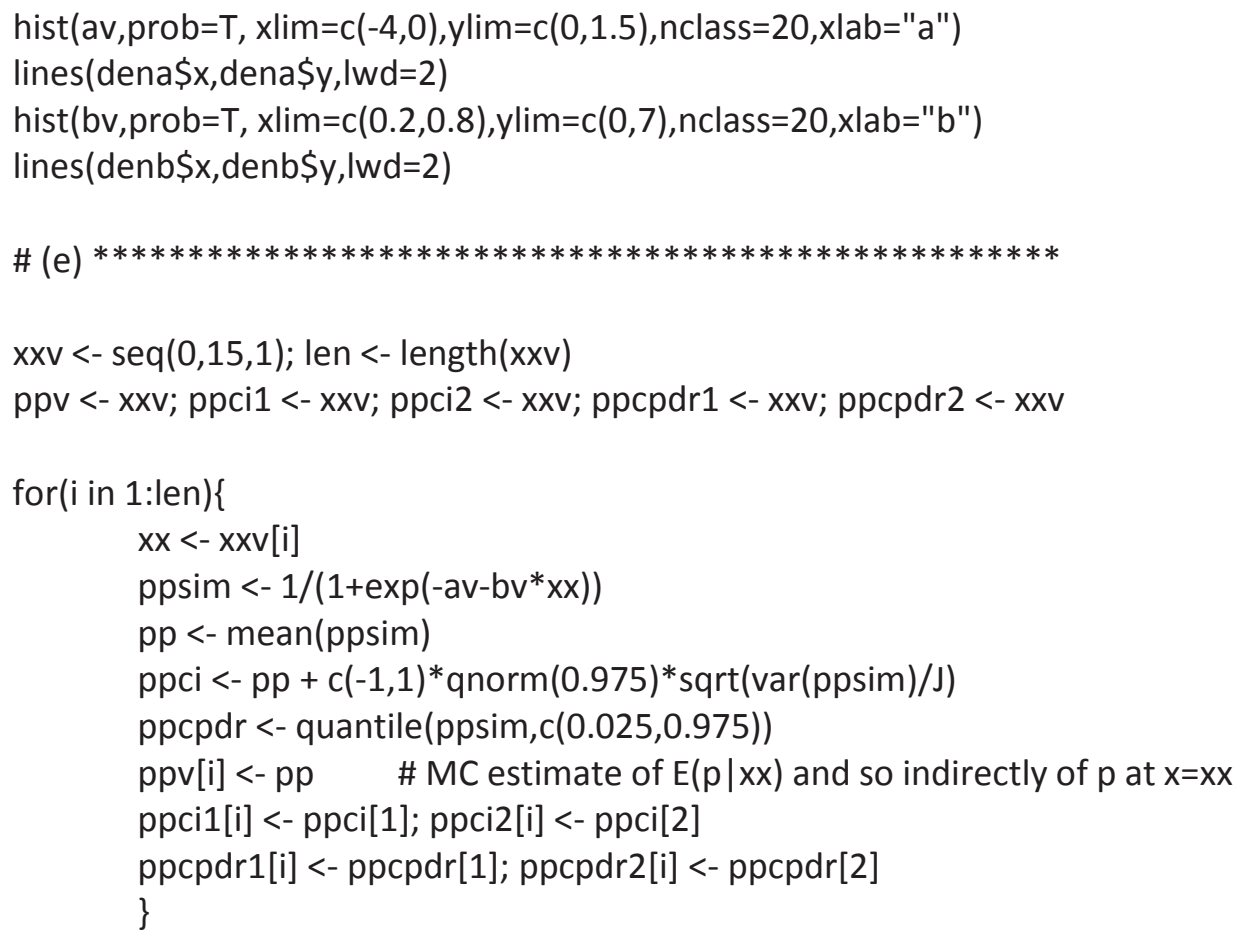


\# (f)

p5v <- 1/(1+exp(-av-bv*5)); set.seed(331); dv <- rbinom(J,20,p5v)

hist $(d v$, prob=T, breaks=seq $(-0.5,20.5,1))$

summary(as.factor(dv))

$\begin{array}{lllllllllllllllll}\text { \#3 } & 4 & 5 & 6 & 7 & 8 & 9 & 10 & 11 & 12 & 13 & 14 & 15 & 16 & 17 & 18 & 19\end{array}$

\#1 $320207521747284511881562173315461123709332131 \quad 37 \quad 6$

dhat <- mean(dv); dci <- dhat $+\mathrm{c}(-1,1) *$ qnorm(0.975)*sqrt(var(dv)/J)

dcpdr <- quantile(dv,c(0.025,0.975))

c(dhat,dci,dcpdr) \# $\quad$ \#11.81 11.7611 .857 .0016 .00

$\mathrm{dv} 2<-\mathrm{dv}[\mathrm{dv}>=7] ; \mathrm{dv} 3<-\mathrm{dv} 2[\mathrm{dv} 2<=16] ;$ length(dv3)/J \# 0.9727

$d v 2<-d v[d v>=8] ; d v 3<-d v 2[d v 2<=16] ;$ length(dv3)/J \# 0.951 OK (>= 95\%)

$d v 2<-d v[d v>=7] ; d v 3<-d v 2[d v 2<=15] ;$ length(dv3)/J \# 0.9395 (too small)

dhat2 <- mean(p5v) \# alternative method

qbinom(c(0.025,0.975),20,dhat2) \# 716

\# (g)

Lv <- -av/bv; Lhat <- mean(Lv); Lci <- Lhat + c(-1,1)* qnorm(0.975)*sqrt(var(Lv)/J) Lcpdr <- quantile(Lv, c(0.025,0.975))

c(Lhat,Lci,Lcpdr) \# 4.279 4.273 4.286 3.584 4.916

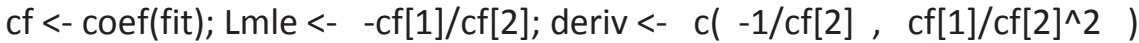

Lvar <- t(deriv) \%*\% summary(fit)\$cov.unscaled \%*\% deriv

Lci2 <- Lmle + c(-1,1)*qt $(0.975,8) *$ sqrt(Lvar)

c(Lmle,Lci2) \# $4.287 \quad 3.532 \quad 5.042$

\# (h)

xvdata <- c(0.1,1.4,3.6,3.8,5.2,6.1,8.7,9.1,9.1,13.6)

yvdata <- c(1,0,23,12,8,32,10,35,19,8)

nvdata <- c $(10,30,40,20,15,46,12,37,23,8)$

pvdata <- yvdata/nvdata 
MHLRZC <- function(burn, J,a0,b0,xv,yv,nv,sa,sb) \{

\# Performs the Metropolis-Hastings algorithm for a logistic regression model \# modified to have a zero constraint.

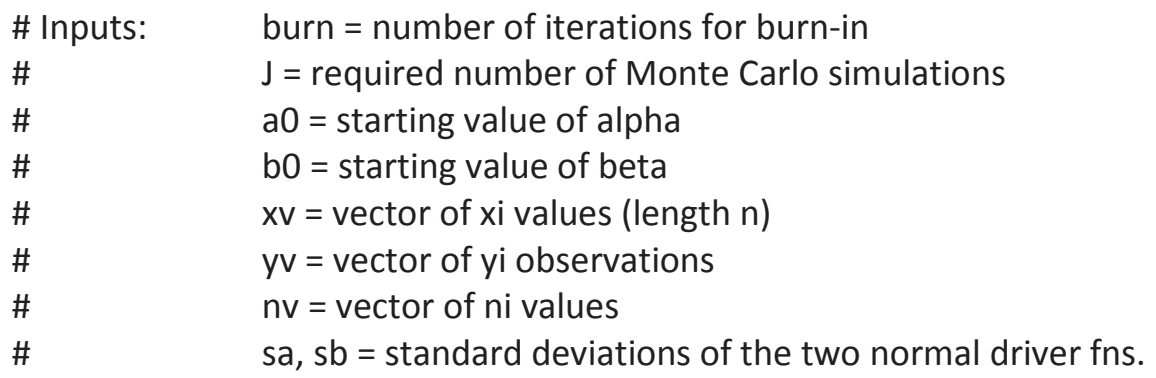
\# Outputs: $\quad \$ a v=$ vector of $($ burn $+J+1)$ values of alpha (incl. starting value) \# $\quad \$ b v=$ vector of (burn+J+1) values of beta (incl. starting value)
\# $\quad$ \$ara $=$ acceptance rate for alpha (over last J iterations)
$\# \quad \$ a r b=$ acceptance rate for beta.

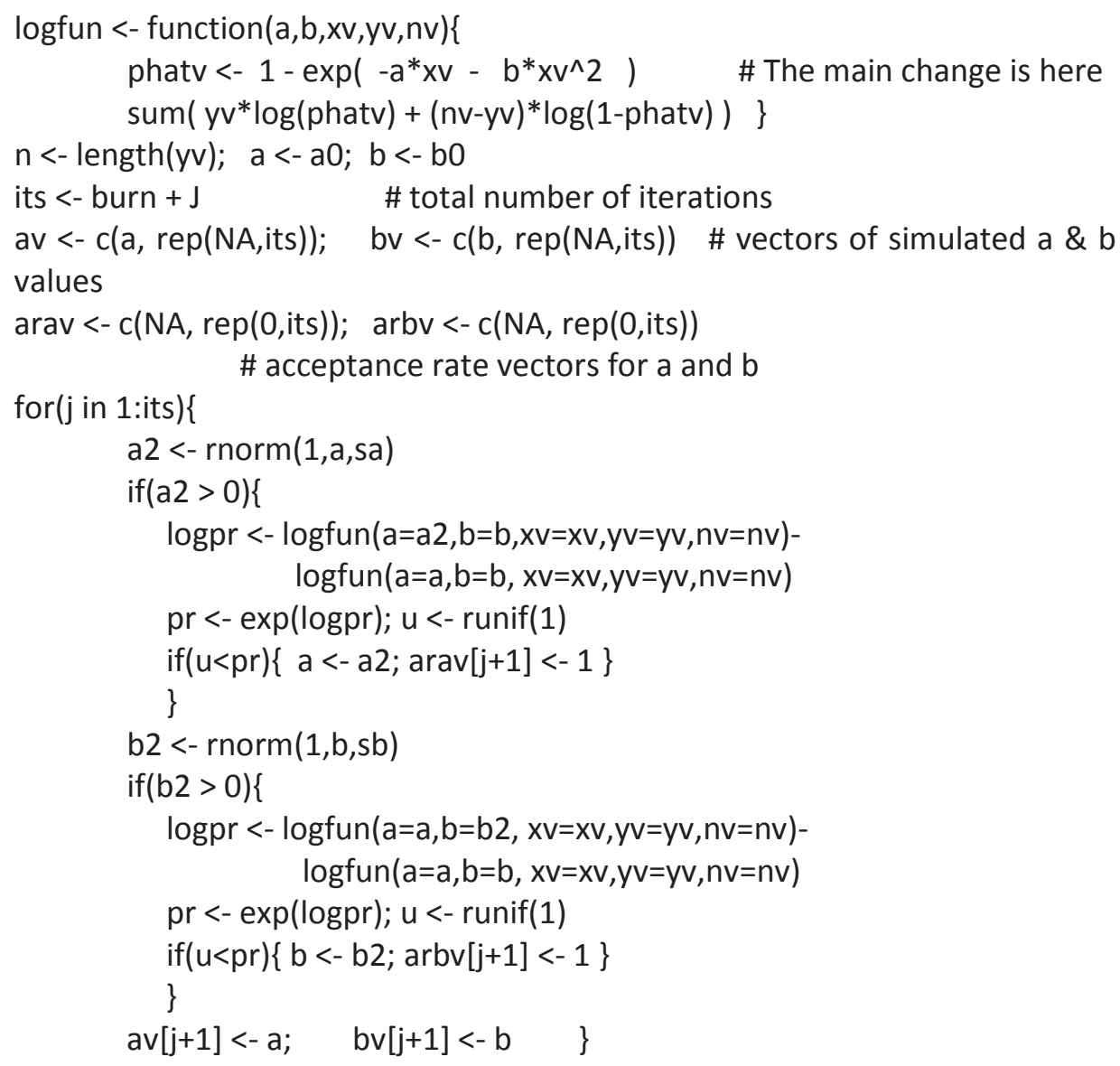




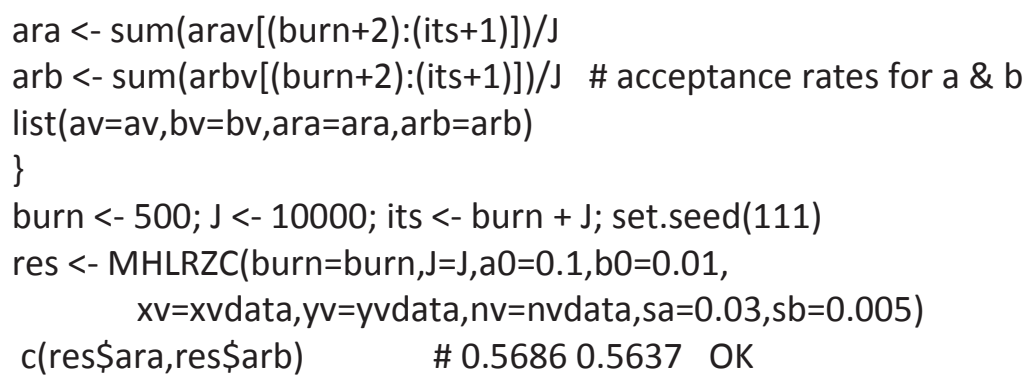

$\mathrm{X} 11(\mathrm{w}=8, \mathrm{~h}=5) ; \operatorname{par}(\mathrm{mfrow}=\mathrm{c}(1,1))$

plot $(c(0,15), c(0,1)$,type="n",xlab="x",ylab="probability $p(x) ")$

points(xvdata,pvdata,pch=16); lines(xxv,ppv)

lines(xxv,ppci1,lwd=2); lines( $x x v, p p c i 2, l w d=2)$

lines (xxv, ppcpdr1, Ity=2,lwd=2); lines(xxv, ppcpdr2, Ity=2,lwd=2)

legend(8,0.6, c("MC est \& 95\% Cl","95\% CPDR"), Ity=c(1,2)) 


\section{Exercise 7.4 Autocorrelated Bernoulli data (and practice at various statistical techniques)}

Consider the following Bayesian model for a sequence of identically distributed but possibly dependent and serially autocorrelated Bernoulli random variables $y_{i}$ :

$$
\begin{aligned}
& \left(y_{i} \mid a, b, y_{i-1}, y_{i-2}, y_{i-3}, \ldots\right) \sim \operatorname{Bernoulli}\left(p_{i}\right), \quad i=0, \pm 1, \pm 2, \ldots \\
& \quad p_{i}=\frac{1}{1+\exp \left\{-\left(a+b y_{i-1}\right)\right\}} \\
& f(a, b) \propto 1, \quad a, b \in \Re .
\end{aligned}
$$

Suppose that the data is $y=\left(y_{1}, \ldots, y_{n}\right)=(1,1,1,1,1,1,1,0,0,0)$.

Use the Metropolis-Hastings algorithm to generate a random sample of $J=10,000$ values from the joint posterior distribution of $a$ and $b$. Use this sample to estimate the posterior means and 95\% CPDRs for $a$ and $b$. Also estimate $P(b<0 \mid y)$.

\section{Solution to Exercise 7.4}

The first thing we need to do is work out the probability that $Y_{1}=1$ conditional on $a$ and $b$ but not conditional on $y_{0}$ (since $y_{0}$ is not known). With an implicit conditioning on $a$ and $b$, observe by the law of total probability that

$$
\begin{aligned}
P\left(Y_{1}=1\right) & =P\left(Y_{0}=0\right) P\left(Y_{1}=1 \mid Y_{0}=0\right)+P\left(Y_{0}=1\right) P\left(Y_{1}=1 \mid Y_{0}=1\right) \\
& =\left\{1-P\left(Y_{1}=1\right)\right\} P\left(Y_{1}=1 \mid Y_{0}=0\right)+P\left(Y_{1}=1\right) P\left(Y_{1}=1 \mid Y_{0}=1\right) .
\end{aligned}
$$

Solving for $P\left(Y_{1}=1\right)$, we get

$$
q_{1} \equiv P\left(Y_{1}=1 \mid a, b\right)=\frac{1+\exp (a+b)}{2+\exp (a+b)+\exp (-a)} .
$$

Hence, with $p_{i}=P\left(Y_{i}=1 \mid a, b, y_{i-1}\right)=\frac{1}{1+\exp \left(-a-b y_{i-1}\right)}$

(as already defined), the joint posterior pdf of $a$ and $b$ is

$$
\begin{aligned}
& \quad f(a, b \mid y) \propto f(a, b) f(y \mid a, b) \\
& \propto 1 \times f\left(y_{1} \mid a, b\right) \prod_{i=2}^{n} f\left(y_{i} \mid a, b, y_{i-1}\right)=q_{1}^{y_{1}}\left(1-q_{1}\right)^{1-y_{1}} \prod_{i=2}^{n} p_{i}^{y_{i}}\left(1-p_{i}\right)^{1-y_{i}} .
\end{aligned}
$$


So the log of the posterior density is given by

$$
\begin{aligned}
l(a, b) \equiv & \log f(a, b \mid y) \\
= & c+y_{1} \log q_{1}+\left(1-y_{1}\right) \log \left(1-q_{1}\right) \\
& +\sum_{i=2}^{n}\left\{y_{i} \log p_{i}+\left(1-y_{i}\right) \log \left(1-p_{i}\right)\right\} .
\end{aligned}
$$

Using normal drivers for both $a$ and $b$, we implement a MetropolisHastings algorithm and thereby, following a burn-in of size $B=1,000$, obtain an approximately random MC sample of size $J=10,000$, which we will denote by

$$
\left(a_{j}, b_{j}\right) \sim \text { iid } f(a, b \mid y), j=1, \ldots, J .
$$

From this MC sample we estimate $a$ by -2.337 with 95\% CPDR (-6.3980, 0.8313), and $b$ by 5.411 with $95 \%$ CPDR $(0.9098,11.8691)$. We also estimate $P(b<0 \mid y)$ by 0.081 .

The traces of $a$ and $b$ over all 11,000 iterations, and histograms of the last 10,000 values of $a$ and $b$, respectively, are shown in Figure 7.11, together with posterior density estimates.

Note: In an earlier exercise we considered a posterior predictive pvalue for the null hypothesis that the sequence in the present exercise consists of values that are iid.

That p-value was estimated as 0.0995 with 95\% CI (0.0936, 0.1054). The estimate 0.081 of $P(b<0 \mid y)$ in the present exercise may be interpreted in a similar way to the p-value 0.0995 .

In this case the appropriate p-value is one-sided.

If we wish to do a two-sided test, in the present context, $b=0$ versus $b \neq 0$, then the p-value may be calculated as twice the minimum of $P(b<0 \mid y)$ and $P(b>0 \mid y)$.

Clearly, if the posterior distribution of $b$ is well above or well below zero, then the resulting two-sided p-value will appropriately be very close to zero. 
Figure 7.I I Traces and histograms for $a$ and $b$
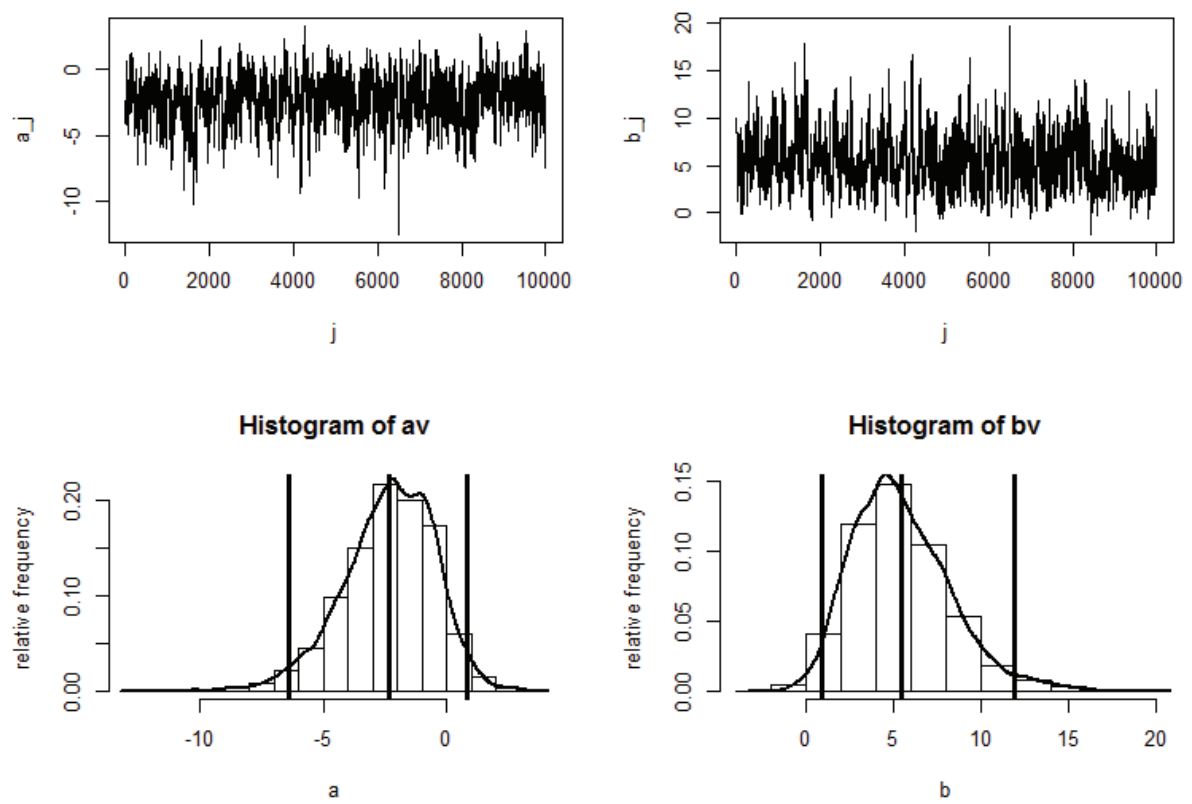

\section{R Code for Exercise 7.4}

yv <- c(1,1,1,1,1, 1,1,0,0,0); $\mathrm{n}<-$ length(yv); ybar <- mean(yv); ydot <- sum(yv)

MHBD <- function(K,a,b,yv,sa,sb) \{

\# Performs a Metropolis-Hastings algorithm for a binary dependence model.

\# Inputs: $\quad \mathrm{K}=$ total number of iterations

$\# \quad a, b=$ starting values of $a$ and $b$

\# $\quad \mathrm{yv}=$ vector of 0 -or-1 values $(\mathrm{y} 1, \ldots, \mathrm{yn})$

\# $\quad \mathrm{sa}, \mathrm{sb}=$ standard deviations of the two normal driver fns.

\# Outputs: $\quad$ \$av $=$ vector of $(\mathrm{K}+1)$ values of a (incl. starting value)

$\# \quad \$ b v=$ vector of $(K+1)$ values of $b$ (incl. starting value)

\# $\quad \$ a r a, \$ a r b=$ acceptance rates for $a$ and $b$.

$\mathrm{n}<-$ length(yv); av <- $\mathrm{a}$; bv <- b; cta $<-0$; ctb <- 0

logfun $<-$ function $(a, b, y v, n)\{$

$$
\begin{aligned}
& \mathrm{p} 1=(1+\exp (\mathrm{a}+\mathrm{b})) /(2+\exp (\mathrm{a}+\mathrm{b})+\exp (-\mathrm{a})) \quad \# \mathrm{p} 1 \\
& \text { p2ton }<-1 /\left(1+\exp \left(-a-b^{*} y v[-n]\right)\right) \quad \# p 2, \ldots, p n \\
& \mathrm{pv}<-\mathrm{c}(\mathrm{p} 1, \mathrm{p} 2 \text { ton }) \\
& \left.\operatorname{sum}\left(y v^{*} \log (p v)+(1-y v) * \log (1-p v)\right) \quad\right\} \\
& \# p 1, \ldots, p n
\end{aligned}
$$


for $(\mathrm{j}$ in $1: \mathrm{K})\{$

a2 <- rnorm(1,a,sa) \# proposed value of a

$\operatorname{logpr}<-\operatorname{logfun}(a=a 2, b=b, y v=y v, n=n)-\operatorname{logfun}(a=a, b=b, y v=y v, n=n)$

pr <- exp(logpr); u <- runif(1)

if $(u<p r)\{a<-a 2 ; c t a<-c t a+1\}$

if $(s b>0)\{$

b2 <- rnorm(1,b,sb) \# proposed value of $b$

$\operatorname{logpr}<-\operatorname{logfun}(a=a, b=b 2, y v=y v, n=n)-\operatorname{logfun}(a=a, b=b, y v=y v, n=n)$

pr <- exp(logpr); $u<-$ runif(1)

if $(u<p r)\{b<-b 2 ; c t b<-c t b+1\}$

\}

av <- c(av,a); bv <- c(bv, b)

\}

list(av=av, bv=bv, $a r a=c t a / K, a r b=c t b / K)$

\}

options(digits=4); set.seed(143); date() \# res <- $\operatorname{MHBD}(\mathrm{K}=11000, \mathrm{a}=0, \mathrm{~b}=0, \mathrm{yv}=\mathrm{yv}, \mathrm{sa}=1.5, \mathrm{sb}=2.2)$; date() \# Took 2 secs $c($ res\$ara,res\$arb) \# 0.55750 .5753 (acceptance rates for a and b) OK

$\mathrm{X} 11(\mathrm{w}=8, \mathrm{~h}=6) ; \operatorname{par}(\mathrm{mfrow}=\mathrm{c}(2,1)) ; \operatorname{plot}(\operatorname{res} \$ \mathrm{av}) ; \operatorname{plot}($ res\$bv) \# OK

av <- res\$av[1002:11001]; bv <- res\$bv[1002:11001]; J=1000

abar <- mean(av); bbar <- mean(bv);

acpdr <- quantile (av,c(0.025,0.975));

bcpdr <- quantile(bv,c(0.025,0.975))

rbind(c(abar,acpdr),c(bbar,bcpdr))

\# [1,] -2.337 -6.3980 0.8313

\# [2,] 5.4110 .909811 .8691

pr <- length $($ bv $[$ bv<0])/J; pr \# 0.081

$\mathrm{X} 11(\mathrm{w}=8, \mathrm{~h}=6) ; \operatorname{par}(\operatorname{mfrow}=c(2,2))$;

plot(av,type="l",xlab="j",ylab="a_j",cex=1.2)

plot(bv,type="l",xlab="j",ylab="b_j",cex=1.2)

hist(av, prob=T,xlab="a",ylab="relative frequency",cex=1.2);

abline ( $v=c(a b a r, a c p d r), ~ I t y=1, l w d=3)$; lines(density(av),lwd=2)

hist(bv, prob=T,xlab="b",ylab="relative frequency",cex=1.2);

abline ( $v=c($ bbar, $b c p d r)$, Ity=1,lwd=3); lines(density(bv), Iwd=2) 


\section{Exercise 7.5 Inference on the bounds of a uniform distribution}

Consider the following Bayesian model:

$$
\begin{aligned}
& \left(y_{1}, \ldots, y_{n} \mid a, b\right) \sim \text { iid } U(a, b) \\
& (a \mid b) \sim U(0, b) \\
& b \sim U(0,1) .
\end{aligned}
$$

Generate a random sample of size $n=20$ from the model with $a=0.6$ and $b=0.8$. Then apply MCMC methods to generate a random sample from the joint posterior of $a$ and $b$. Then use this sample to perform Monte Carlo inference on $m=E\left(y_{i} \mid a, b\right)=(a+b) / 2$.

\section{Solution to Exercise 7.5}

Rounding to four decimals, the generated sample values are as shown in Table 7.6.

\section{Table 7.6 Sample values}

\begin{tabular}{c|ccccc}
$i$ & 1 & 2 & 3 & 4 & 5 \\
\hline$y_{i}$ & 0.7846 & 0.7572 & 0.6381 & 0.7626 & 0.6105 \\
\hline
\end{tabular}

\begin{tabular}{c|ccccc}
$i$ & 6 & 7 & 8 & 9 & 10 \\
\hline$y_{i}$ & 0.6990 & 0.7728 & 0.7113 & 0.7314 & 0.7435 \\
\hline
\end{tabular}

\begin{tabular}{c|ccccc}
$i$ & 11 & 12 & 13 & 14 & 15 \\
\hline$y_{i}$ & 0.6324 & 0.7072 & 0.7493 & 0.7979 & 0.6182 \\
\hline
\end{tabular}

\begin{tabular}{c|ccccc}
$i$ & 16 & 17 & 18 & 19 & 20 \\
\hline$y_{i}$ & 0.7652 & 0.7883 & 0.7194 & 0.6211 & 0.6054 \\
\hline
\end{tabular}

Note: The range of this data is from 0.6054 to 0.7979 . This tells us immediately that $0 \leq a \leq 0.6054$ and $0.7979 \leq b \leq 1$. 
Now, the joint posterior density of $a$ and $b$ is

$$
\begin{aligned}
& f(a, b \mid y) \propto f(a, b, y)=f(b) f(a \mid b) f(y \mid a, b) \\
& \quad \propto \frac{I(0<b<1)}{1} \times \frac{I(0<a<b)}{b} \times \prod_{i=1}^{n} \frac{I\left(a<y_{i}<b\right)}{b-a} \\
& \quad=\frac{1}{b(b-a)^{n}}, \quad 0<a<b<1, \quad a<\min y_{i}<\max y_{i}<b .
\end{aligned}
$$

So the two conditional posterior distributions are defined by:

$$
\begin{aligned}
& f(a \mid y, b) \propto \frac{1}{(b-a)^{n}}, \quad 0<a<\min \left(y_{i}\right) \\
& f(b \mid y, a) \propto \frac{1}{b(b-a)^{n}}, \quad \max \left(y_{i}\right)<b<1 .
\end{aligned}
$$

Neither of these conditionals defines a well-known distribution. So we will apply a 'pure' Metropolis-Hastings algorithm (rather than a Gibbs sampler).

With $a^{\prime}$ and $b^{\prime}$ denoting the proposed values of $a$ and $b$, the acceptance probabilities at the two steps are:

$$
\begin{aligned}
& p_{a}=\frac{f\left(a^{\prime} \mid y, b\right)}{f(a \mid y, b)}=\frac{1 /\left(b-a^{\prime}\right)^{2}}{1 /(b-a)^{2}}=\left(\frac{b-a}{b-a^{\prime}}\right)^{n} \\
& p_{b}=\frac{f\left(b^{\prime} \mid y, a\right)}{f(b \mid y, a)}=\frac{1 /\left(b^{\prime}\left(b^{\prime}-a\right)^{2}\right)}{1 /\left(b(b-a)^{2}\right)}=\frac{b}{b^{\prime}}\left(\frac{b-a}{b^{\prime}-a}\right)^{n} .
\end{aligned}
$$

The following drivers were chosen:

$$
\begin{aligned}
a^{\prime} & \sim N\left(a, r^{2}\right) \\
b^{\prime} & \sim N\left(b, t^{2}\right) .
\end{aligned}
$$

Starting at $a=0.1$ and $b=0.9$, and using the tuning constants $r=0.008$ and $t=0.01$, the algorithm was run for 2,500 iterations. The resulting trace plots are shown in Figure 7.12.

We see that stochastic convergence was achieved within 500 iterations. The acceptance rates over the last 2,000 iterations were $62 \%$ and $58 \%$ for $a$ and $b$, respectively. 
Figure 7.12 Traces for $a$ and $b$
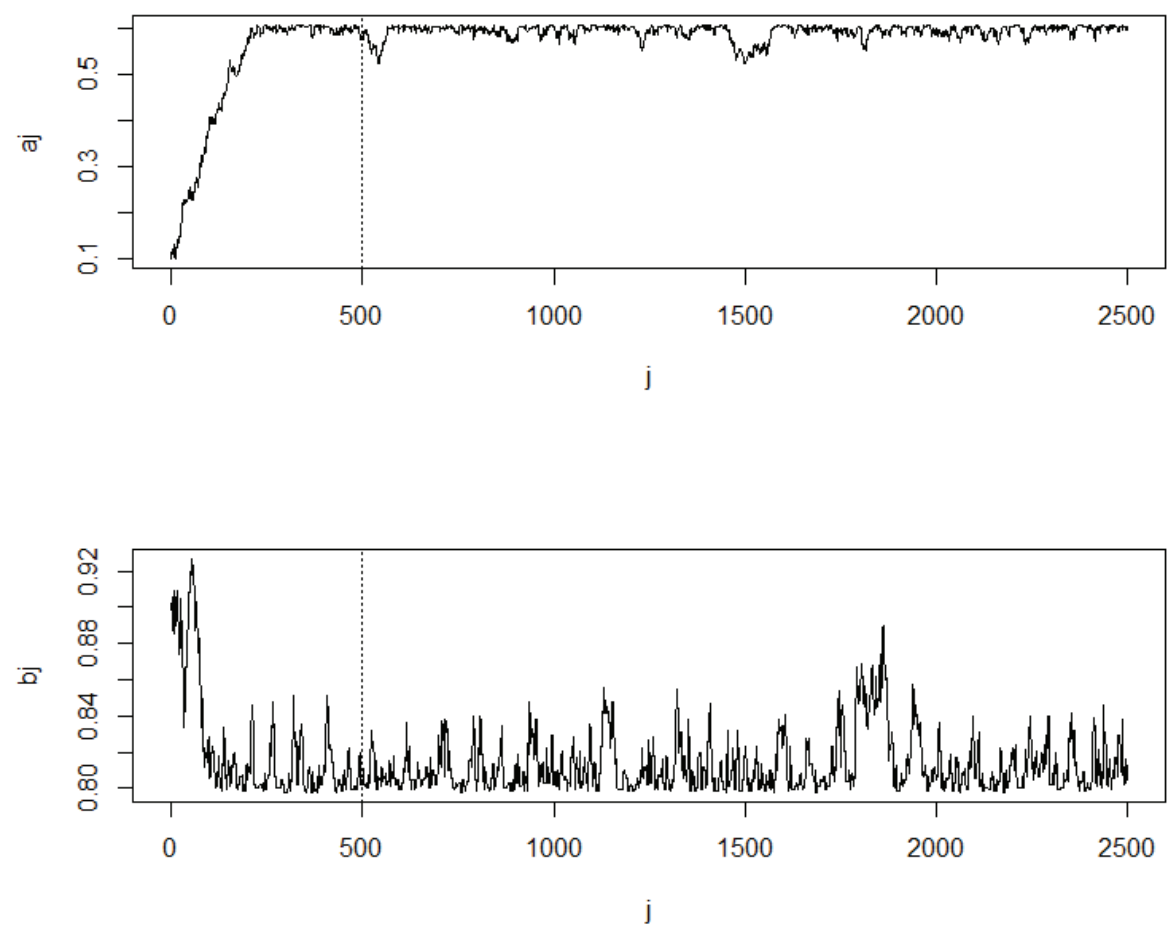

The algorithm was then run for a further 50,000 iterations, starting at the last values in the previous run ( $a=0.5979$ and $b=0.8123)$. The acceptance rates were now $61 \%$ and $54 \%$, and this second run took 14 seconds of computer time.

Then every 50th value was recorded so as to yield a final random sample of size $J=1,000$ from the joint posterior distribution of $a$ and $b$, i.e.

$$
\left(a_{1}, b_{1}\right), \ldots,\left(a_{J}, b_{J}\right) \sim \text { iid } f(a, b \mid y) .
$$

As a check, the sample ACF of each sample of size 1,000 was calculated. Figure 7.13 shows the ACF estimates for $a$ and $b$, and these provide no evidence for residual autocorrelation in either series. 


\section{Figure 7.13 Sample ACFs for $a$ and $b$}

Series av

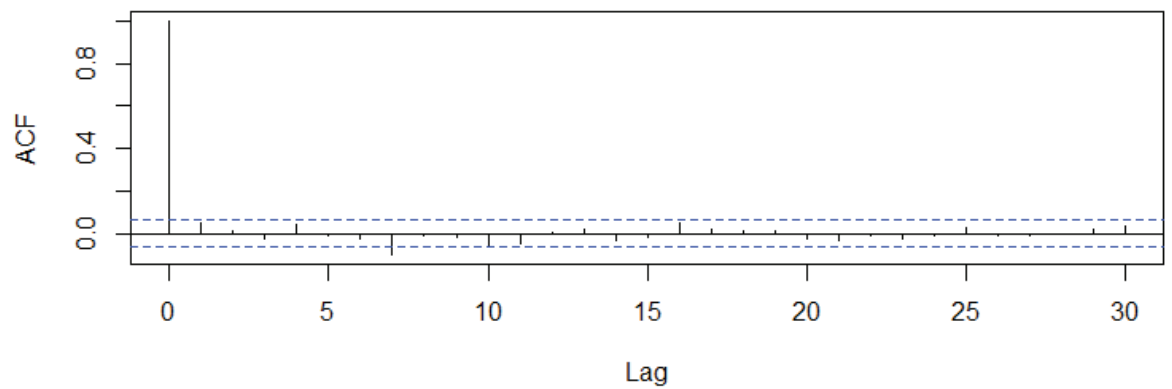

Series bv

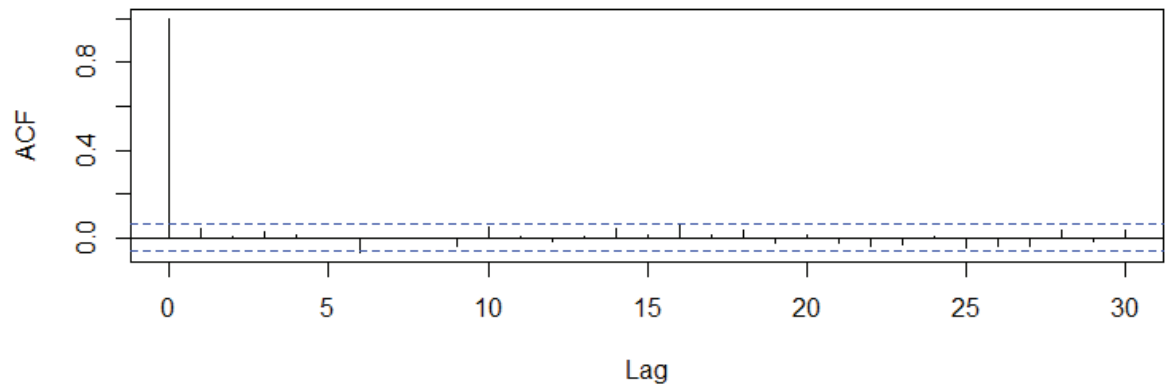

A random sample from the posterior distribution of the mean

$$
m=E\left(y_{i} \mid a, b\right)=(a+b) / 2
$$

was then formed by calculating

$$
m_{j}=\left(a_{j}+b_{j}\right) / 2 \text {. }
$$

We thereby obtained the random sample

$$
m_{1}, \ldots, m_{J} \sim \text { iid } f(m \mid y) .
$$

This Monte Carlo sample was used to estimate $\hat{m}=E(m \mid y)$ by 0.7013 , with 95\% CI $(0.7008,0.7019)$. The estimated 95\% CPDR for $m$ was (0.6837, 0.7173).

Figure 7.14 is a histogram of the 1,000 values of $m$, overlaid by a density estimate of $f(m \mid y)$, with the vertical lines showing the point and interval estimates reported above. 
Figure 7.14 Inference on $m=(a+b) / 2$

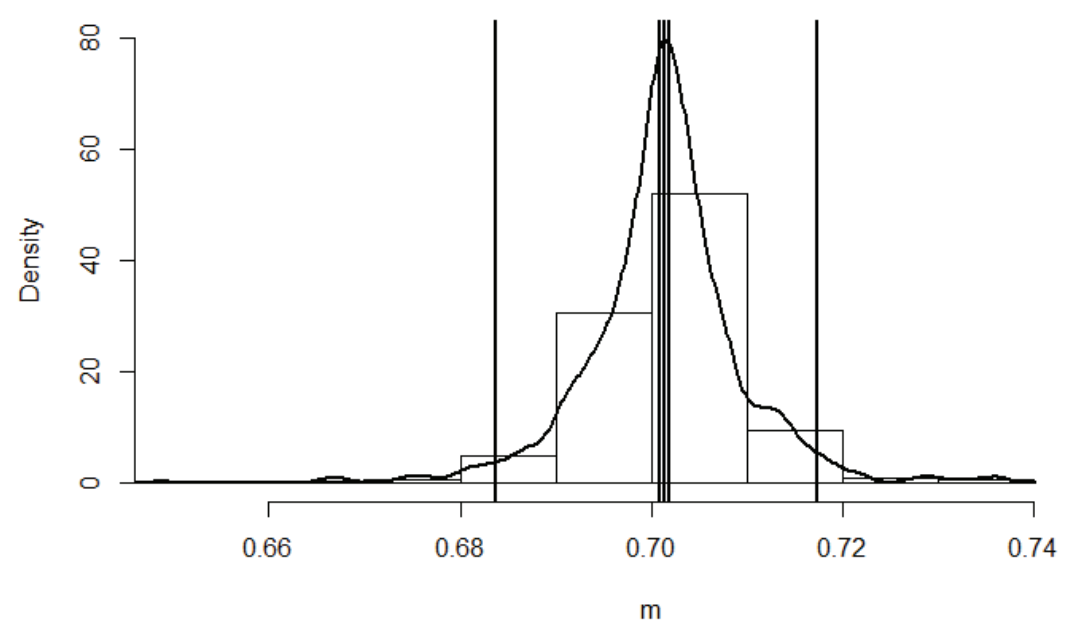

R Code for Problem 7.5

options(digits=4)

$\mathrm{MH}=$ function $(\mathrm{B}, \mathrm{J}=1000, \mathrm{y}, \mathrm{a}, \mathrm{b}, \mathrm{r}, \mathrm{t})\{$

\# This function performs a Metropolis-Hastings algorithm for a model involving 3 uniforms.

\# Inputs:

$B=$ burn-in length

$\#$

$\mathrm{J}=$ desired Monte Carlo size

$\#$

$\mathrm{y}=(\mathrm{y} 1, \ldots, \mathrm{yn})=$ data $(\mathrm{yi} \sim$ iid $\mathrm{U}(\mathrm{a}, \mathrm{b}))$

$\#$

$a=$ starting value of $a(a \sim U(0, b))$

\#

$b=$ starting value of $b(b \sim U(0,1))$

\#

$r, t=$ tuning constants for $a \& b$, respectively

\# Outputs:

$\$ a v=(1+B+J)$ vector of a-values

$\# \quad \$ b v=(1+b+J)$ vector of $b$-values

\# $\quad$ \$ar $=$ acceptance rate for a (over last $\mathrm{J}$ iterations)

\# $\quad \$ b r=$ acceptance rate for $b$ (over last $J$ iterations)

$a v=a ; b v=b ; a n=0 ; b n=0 ; \min y=\min (y) ; \operatorname{maxy}=\max (y) ; n=l e n g t h(y)$;

for $(j$ in $1:(B+J))\{$

$$
\begin{aligned}
& \text { ap }=\operatorname{rnorm}(1, a, r) \\
& \text { if }((0<a p) \& \&(a p<\operatorname{miny}))\{ \\
& \quad p=((b-a) /(b-a p))^{\wedge} n ; u=\operatorname{runif}(1) \\
& \quad \text { if }(u<p)\{a=a p ; \text { if }(j>B) a n=a n+1\}\} \\
& \text { bp }=\operatorname{rnorm}(1, b, t) \\
& \text { if }((\operatorname{maxy}<b p) \& \&(b p<1))\{ \\
& \quad q=(b / b p)^{*}((b-a) /(b p-a))^{\wedge} n ; v=\operatorname{runif}(1)
\end{aligned}
$$




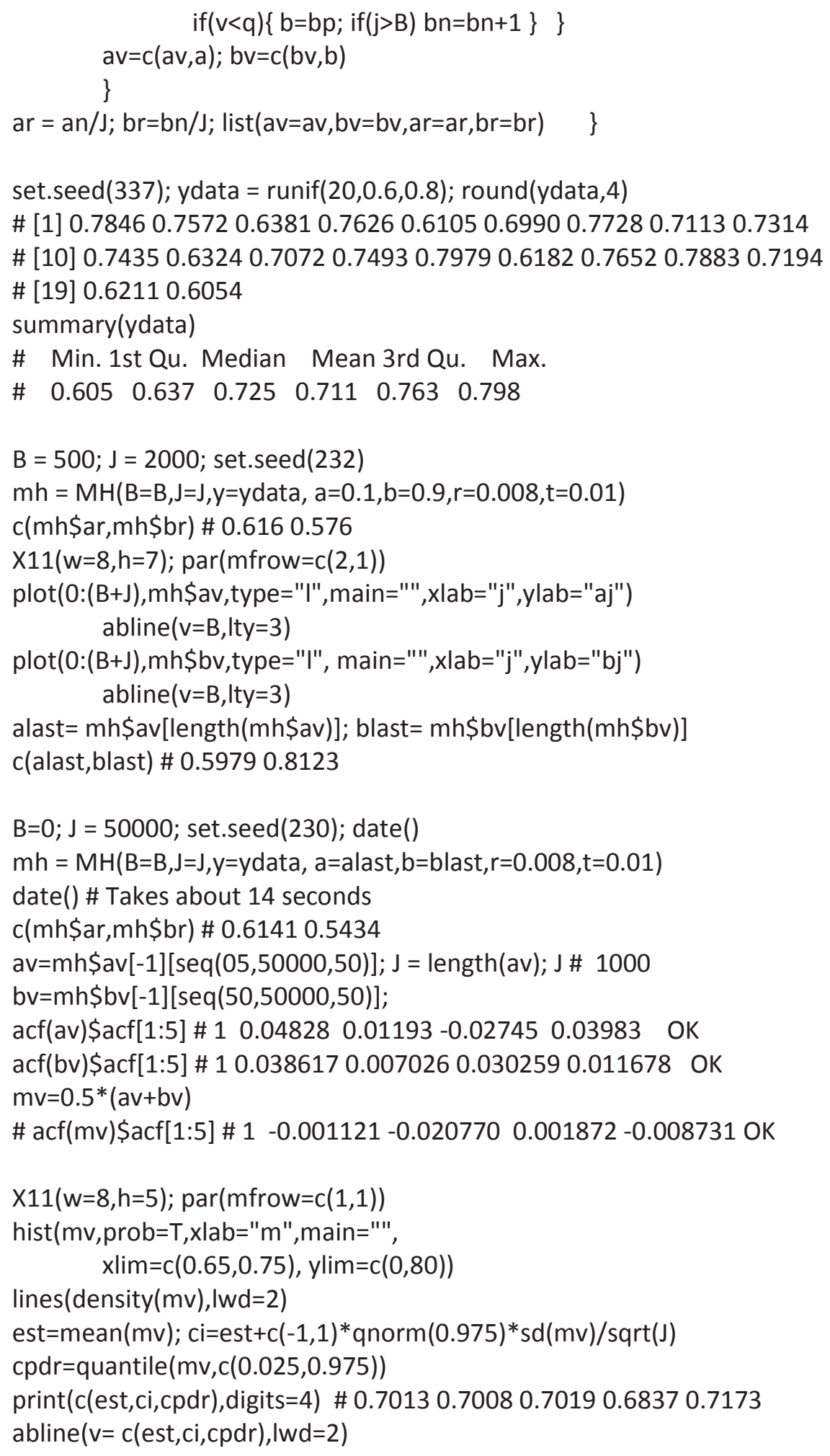




\section{Inference via WinBUGS}

\section{I Introduction to BUGS}

We have illustrated the usefulness of MCMC methods by applying them to a variety of statistical contexts. In each case, specialised $\mathrm{R}$ code was used to implement the chosen method. Writing such code is typically time consuming and requires a great deal of attention to details such as choosing suitable tuning constants in the Metropolis-Hastings algorithm.

A software package which can greatly assist with the application of MCMC methods is WinBUGS. This stands for:

Bayesian Inference Using Gibbs Sampling for Microsoft Windows.

The BUGS Project was started in 1989 by a team of statisticians in the UK (at the Medical Research Council Biostatistics Unit, Cambridge, and Imperial College School of Medicine, London) and developed until the latest version WinBUGS 1.4.3 was released in 2007.

WinBUGS 1.4.3 is a stable version of BUGS which is suitable for routine use, even today.

Since 2007, development of BUGS has focused on OpenBUGS, an open source version of the package. In what follows we will only refer to WinBUGS 1.4.3. This is freely available from the official website: http://www.mrc-bsu.cam.ac.uk/software/bugs/

Figure 8.1 shows this website (as it appeared on 18 February 2015).

Figure 8.2 shows the Wikipedia article on WinBUGS (on the same day): http://en.wikipedia.org/wiki/WinBUGS

The preferred reference for citing WinBUGS in scientific papers is:

Lunn, D.J., Thomas, A., Best, N., and Spiegelhalter, D. (2000). WinBUGS - A Bayesian modelling framework: Concepts, structure, and extensibility. Statistics and Computing, 10: 325-337. 


\section{Figure 8.I Official website for WinBUGS}

MRC $\mid \begin{aligned} & \text { Meaical } \\ & \text { Research } \\ & \text { Council }\end{aligned}$

\section{MRC Biostatistics Unit}

ABOUT BSU RESEARCH \& DEVELOPMENT PEOPLE PUBLISHED RESEARCH SOFTWARE TRAINING RECRUITMENT NEWS \& EVENTS

Home $>$ software $>$ The BuGS Project

Software
The Bugs
Project
Latest News
Contact us/BUGS
list
WinBUGS
New WinBUGS
examples
The BUGS Book
FAQs
DIC

\section{Welcome}

Background to BUGS

The BUGS (Bayesian inference Using Gibbs Sampling) project is concerned with flexible software for the Bayesian analysis of complex statistical models using Markov chain Monte Carlo (MCMC) methods. The project began in 1989 in the MRC Biostatistics Unit, Cambridge, and led initially to the 'Classic' BUGS program, and then onto the WinBUGS software developed jointly with the Imperial College School of Medicine at St Mary's, London.

Development is now focussed on the OpenBUGS project.

\section{WinBUGS 1.4.3}

This site at the MRC Biostatistics Unit is primarily concerned with the stand-alone WinBUGS 1.4.3 package.

\section{Figure 8.2 Wikipedia article on WinBUGS}

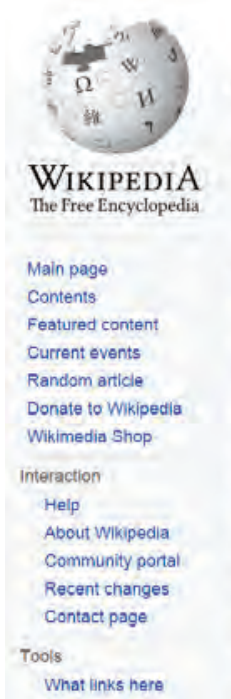

Article Talk

\section{WinBUGS}

From Wikipedia, the free encyclopedia

WinBUGS is statistical software for Bayesian analysis using Markov chain Monte Carlo (MCMC) methods.

It is based on the BUGS (Bayesian inference Using Gibbs Sampling) project started in 1989. It runs under Microsoft Windows, though it can also be run on Linux using Wine, ${ }^{(1)}$ It was developed by the BUGS Project, a team of UK researchers at the MRC Biostatistics Unit, Cambridge, and Imperial College School of Medicine, London.

The last version of WinBUGS was version 1.4.3, released in August 2007. Development is now focused on OpenBUGS, an open source version of the package. WinBUGS 1.4 .3 remains available as a stable version for routine use, but is no longer being developed [2][3]
Create account $\log$ ir

\begin{tabular}{l} 
WinBUGS \\
$\begin{array}{ll}\text { Developer(s) } & \text { The BUGS Project } \\
\text { Initial release } & 1997 \\
\text { Discontinued } & 1.4 .3 \text { / August } 6.2007: 7 \\
& \text { years ago } \\
\text { Operating system } & \text { Windows } \\
\text { Available in } & \text { English } \\
\text { Type } & \text { Statistical package } \\
\text { License } & \text { Freeware }\end{array}$ \\
\hline
\end{tabular}




\subsection{A first tutorial in BUGS}

Consider the following Bayesian model:

$$
\begin{aligned}
& y_{1}, \ldots, y_{n} \mid \mu, \tau \sim \text { iid } \operatorname{Normal}\left(\mu, \sigma^{2}\right) \quad\left(\tau=1 / \sigma^{2}\right) \\
& \mu \mid \tau \sim \operatorname{Normal}\left(\mu_{0}, \sigma_{0}^{2}\right) \\
& \tau \sim \operatorname{Gamma}(\alpha, \beta) \quad(E \tau=\alpha / \beta)
\end{aligned}
$$

where $\mu_{0}=0, \sigma_{0}^{2}=10,000$ and $\alpha=\beta=0.001$.

Suppose the data is $y=\left(y_{1}, \ldots, y_{n}\right)=(2.4,1.2,5.3,1.1,3.9,2.0)$, and we wish to find the posterior mean and 95\% posterior interval for each of $\mu$ and $\gamma=\mu \sqrt{\tau}$ (the signal to noise ratio).

To perform this in WinBUGS 1.4.3, open a new window (select 'File' and then 'New' in the BUGS toolbar), and type the following BUGS code:

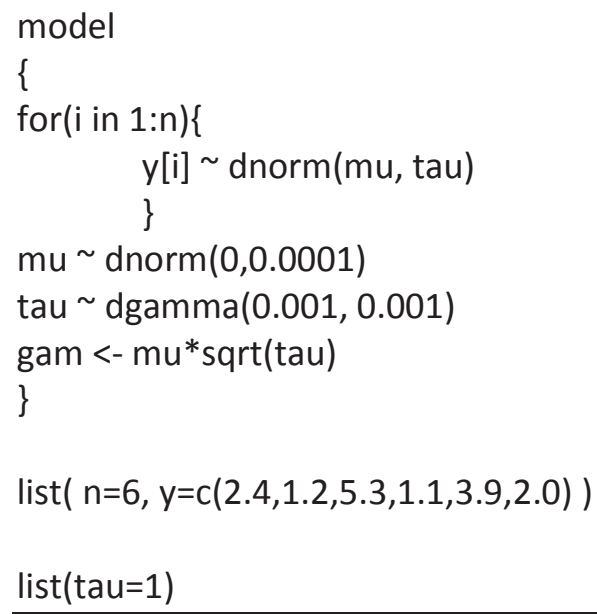

Alternatively, copy this text from a Word document into a Notepad file, and then copy the text from the Notepad file into the WinBUGS window.

Note: Do not copy text from Word to WinBUGS directly or you may get an error message.

The WinBUGS window should then look as depicted in Figure 8.3. 


\section{Figure 8.3 WinBUGS window with code}

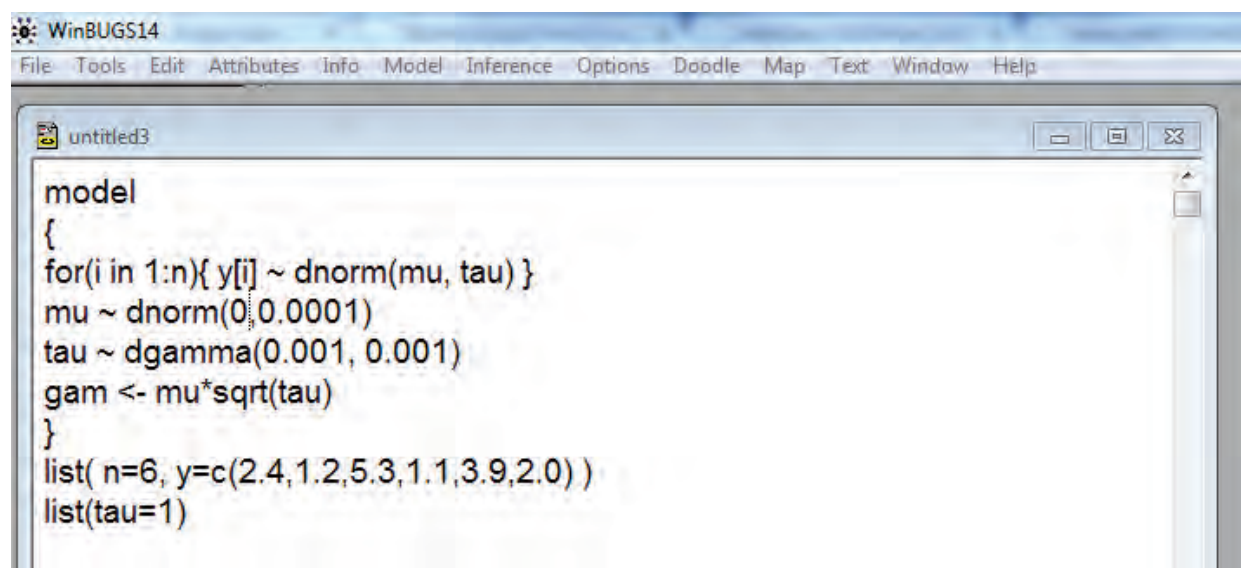

Next, select 'Model' (in the WinBUGS toolbar) and then 'Specification'.

Then highlight the word 'model' (in the BUGS code above) and click on 'check model' in the 'Specification Tool'.

Then highlight the first word 'list', click on 'load data' and click on 'compile'.

Then highlight the second word 'list', click on 'load inits' and click on 'gen inits'.

Next, select 'Inference' and then 'Samples'. Then, in the 'Sample Monitor Tool' which appears, type 'mu' in the 'node' box, click 'set', type 'gam' in the 'node' box and click 'set' again.

Then click 'Model' and 'Update'.

In the 'Update Tool' which appears, change '1000' to '1500' and click 'update'. This will implement 1,500 iterations of an MCMC algorithm.

Next type '*' (an asterisk) in the 'node' box, change ' 1 ' to ' 501 ' in the 'beg' box (meaning beginning) and click 'stats' (statistics).

This should produce something similar to what is shown in Figure 8.4 and Table 8.1. 
Figure 8.4 Tools and node statistics in WinBUGS

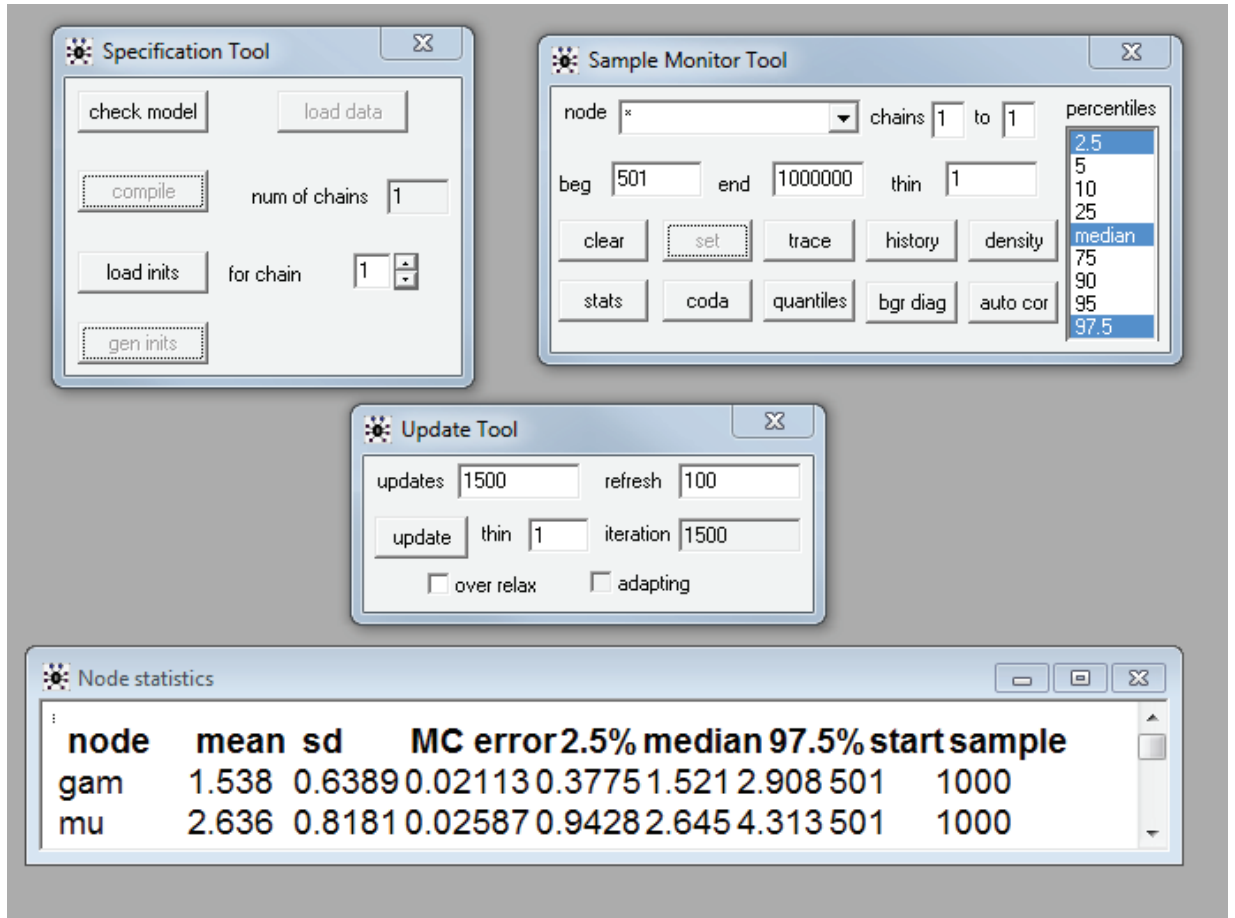

Table 8. I Node statistics in WinBUGS (as in Figure 8.4)

\begin{tabular}{lcccccccc} 
node & mean & sd & MC error & $\mathbf{2 . 5 \%}$ & median 97.5\% & start & sample \\
\hline gam & 1.538 & 0.6389 & 0.02113 & 0.3775 & 1.521 & 2.908 & 501 & 1000 \\
mu & 2.636 & 0.8181 & 0.02587 & 0.9428 & 2.645 & 4.313 & 501 & 1000 \\
\hline
\end{tabular}

From these results, we see that the posterior mean and 95\% posterior interval for $\mu$ are about 2.64 and $(0.94,4.31)$, and the same quantities for $\gamma$ are about 1.54 and $(0.38,2.91)$.

To obtain more precise inference we could repeat the above procedure with a larger Monte Carlo sample size (e.g. 10,000 rather than 1,000). 
Note: If $\sigma_{0}=\infty$ and $\alpha=\beta=0$, the posterior mean and 95\% CPDR for $\mu$ are exactly

$$
\bar{y}=2.65
$$

(i.e. the sample mean) and

$$
\left(\bar{y} \pm t_{0.025}(n-1) / \sqrt{n}\right)=(0.92,4.38)
$$

(where $s$ is the sample standard deviation).

The posterior mean and CPDR for $\gamma$ do not have such simple formulae.

To see line plots of the simulated values, click on 'history' (in the 'Sample Monitor Tool'), and to view smoothed histograms of them, click 'density'. Figure 8.5 illustrates.

\section{Figure 8.5 Line plots and smoothed histograms in WinBUGS}

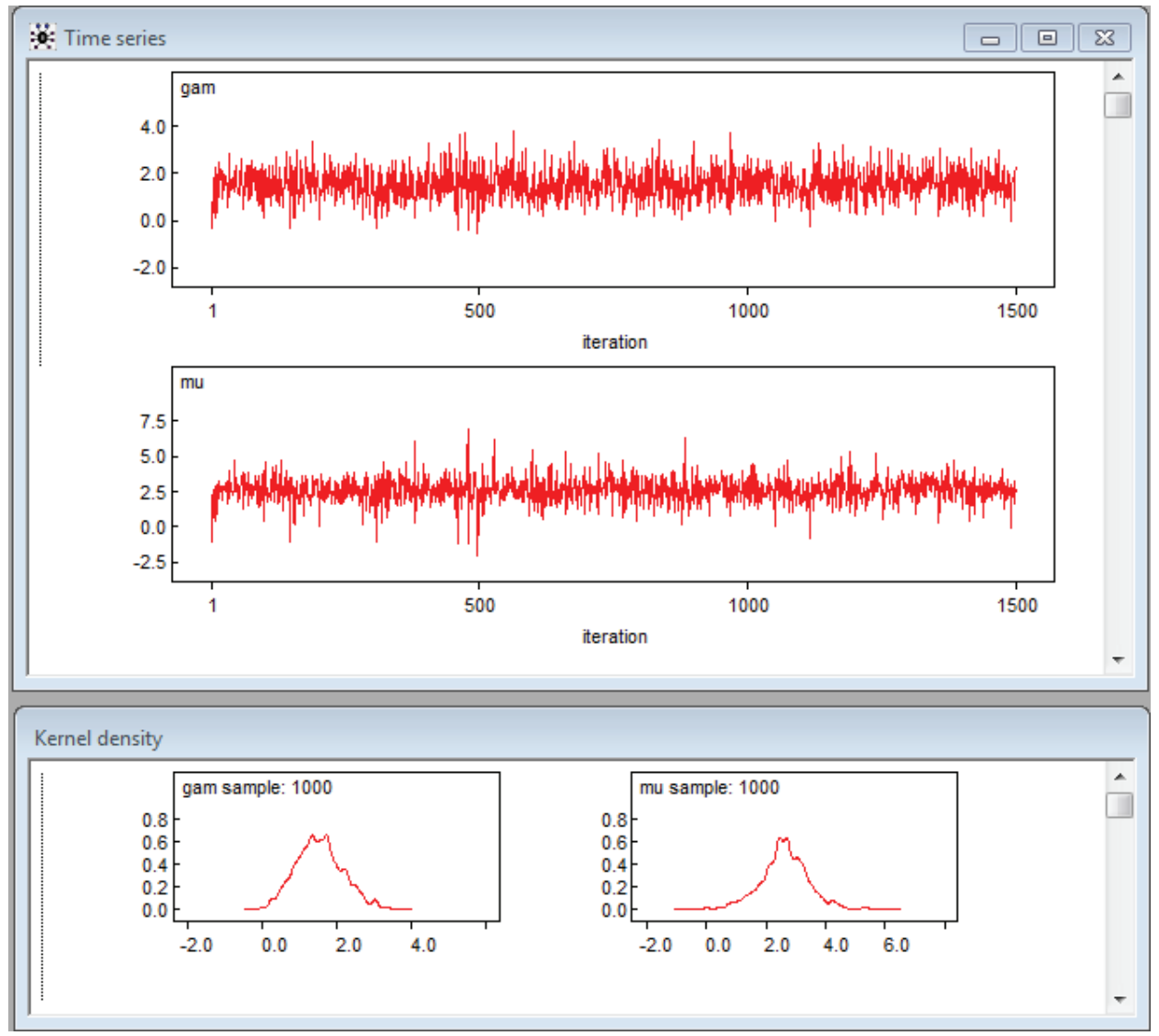


To transfer the simulated values from WinBUGS into $\mathrm{R}$ (for further analysis) click on 'coda'. Two boxes will appear, one called 'CODA index' with the following:

\begin{tabular}{lll}
\hline gam & 1 & 1000 \\
$\mathrm{mu}$ & 1001 & 2000 \\
\hline
\end{tabular}

The other box, called 'CODA for chain 1', should have two columns and 2,000 rows and look as follows:

\begin{tabular}{ll}
501 & 1.298 \\
502 & 1.307 \\
503 & 1.478 \\
& $\ldots \ldots \ldots \ldots$ \\
1498 & 0.8303 \\
1499 & 1.993 \\
1500 & 2.326 \\
501 & 1.812 \\
502 & 1.999 \\
503 & 2.8 \\
$\ldots \ldots \ldots \ldots \ldots \ldots \ldots \ldots \ldots \ldots$ \\
1498 & 1.628 \\
1499 & 2.161 \\
1500 & 2.748 \\
\hline
\end{tabular}

Next, copy the contents of 'CODA for chain 1' into a Notepad file called 'out.txt' (say). Save that file somewhere, e.g. onto the desktop. 
Then begin a session in $\mathrm{R}$ and proceed as follows:

out <- read.table(file=file.choose()) \# Navigate to and choose 'out.txt' $\operatorname{dim}$ (out) \# $2000 \quad 2$

gamv <- out[1:1000,2]; muv <- out[1001:2000,2]

$\operatorname{par}(\operatorname{mfrow}=c(2,1)) ;$ hist(muv, breaks=20); hist(gamv, breaks=20)

This should result in the graphs shown in Figure 8.6.

Figure 8.6 Histograms in R using output from WinBUGS

\section{Histogram of muv}

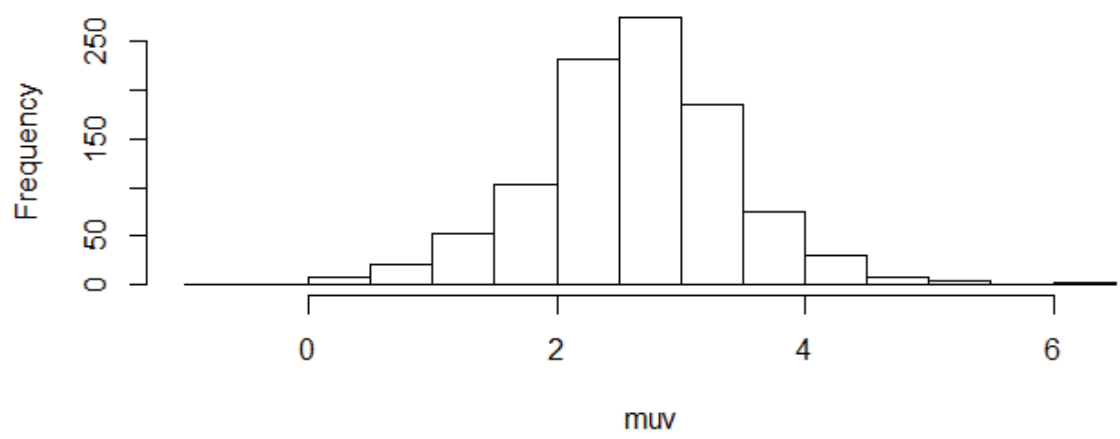

Histogram of gamv

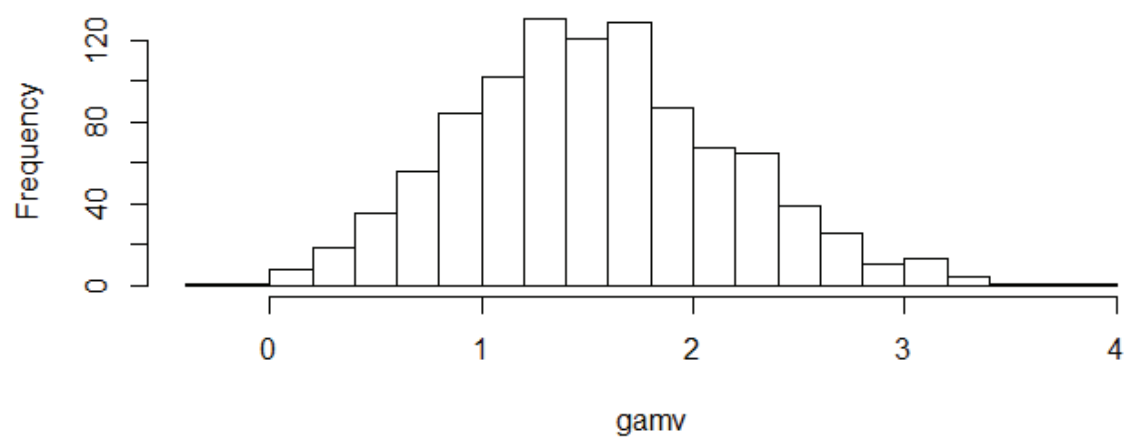


One can then use the MCMC output in many other ways, e.g. to simulate from a posterior predictive distribution via the method of composition.

As an alternative, it is possible to run WinBUGS directly from $\mathrm{R}$ after installing the appropriate packages. (This will be done in a future exercise). But this method is really only for production runs and is not recommended during the experimentation stage of an analysis.

For more information on BUGS, click on 'Help' and 'User manual' in the toolbar. Also see 'Examples Vol I' and 'Examples Vol II' for several dozen worked examples in BUGS. The examples are very user-friendly. They contain data, code and everything one needs to reproduce the results shown. Figure 8.7 shows various excerpts from these files.

\section{Figure 8.7 Exerpts from the WinBUGS I.4.3 User Manual} (several pages)

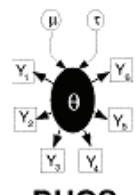

\section{WinBUGS User Manual}

Version 1.4, January 2003

Upgraded to:

Version 1.4.3

Auoust 6th. 2007

(please see here for details)

Beware: MCMC sampling can be dangerous!

\section{Contents}

Introduction $\Rightarrow \Leftrightarrow$

Compound Documents $\Rightarrow$

Model Specification $\Rightarrow \Leftrightarrow$

DoodleBUGS: The Doodle Editor $\Rightarrow \Leftrightarrow$

The Model Menu $\Rightarrow \Leftrightarrow$

The Info Menu $\Rightarrow \Leftrightarrow$

The Inference Menu $\Rightarrow \Leftarrow$

The info Menu $\Rightarrow \theta$

Batch-mode: Scripts

WinBUGS Graphics $\Rightarrow \Leftrightarrow$

Tutorial $\Rightarrow \Leftrightarrow$

The Options Menu $\Rightarrow \Leftrightarrow$

Tricks: Advanced Use of the BUGS Language $\Rightarrow \Leftrightarrow$

Tips and Troubleshooting $\Rightarrow \Leftrightarrow$

Changing MCMC Defaults (advanced users only) $\Rightarrow \theta$

Distributions $\Rightarrow \Leftrightarrow$

References 


\section{Distributions}

$\theta$

$Y_{3}, Y_{4}$

BUGS

\section{Contents}

$\begin{array}{ll}\text { Discrete Univariate } & \Rightarrow \varnothing \\ \text { Continuous Univariate } & \Rightarrow \Leftarrow \\ \text { Discrete Multivariate } & \Rightarrow \varnothing \\ \text { Continuous Multivariate } & \Rightarrow \Leftarrow\end{array}$

Discrete Univariate [ top | home ]

\section{Bernoulli}

$r \sim \operatorname{dbern}(p)$

\section{Binomial}

$r \sim \operatorname{dbin}(\mathrm{p}, \mathrm{n})$

\section{Categorical}

$r \sim \operatorname{dcat}(p[])$

\section{Negative Binomial}

$\mathrm{x} \sim$ dnegbin $(\mathrm{p}, \mathrm{r})$

\section{Poisson}

$r \sim \operatorname{dpois~(lambda)}$

Continuous Univariate

Beta

$p \sim \operatorname{dbeta}(a, b)$

\section{Chi-squared}

$\mathrm{x} \sim$ dchisqr $(\mathrm{k})$

$$
p^{r}(1-p)^{1-r} ; \quad r=0,1
$$

$$
\frac{n !}{r !(n-r) !} p^{r}(1-p)^{n-r} ; \quad r=0, \ldots, n
$$

$$
p[r] ; \quad r=1,2, \ldots, \operatorname{dim}(p) ; \quad \sum_{i} p[i]=1
$$

$$
\frac{(x+r-1) !}{x !(r-1) !} p^{r}(1-p)^{x} ; \quad x=0,1,2, \ldots
$$

$$
e^{-\lambda} \frac{\lambda^{r}}{r !} ; \quad r=0,1, \ldots
$$

[ top | home ]

$$
p^{a-1}(1-p)^{b-1} \frac{\Gamma(a+b)}{\Gamma(a) \Gamma(b)} ; \quad 0<p<1
$$

$$
\frac{2^{-k / 2} x^{k / 2-1} e^{-x / 2}}{\Gamma\left(\frac{k}{2}\right)} ; \quad x>0
$$




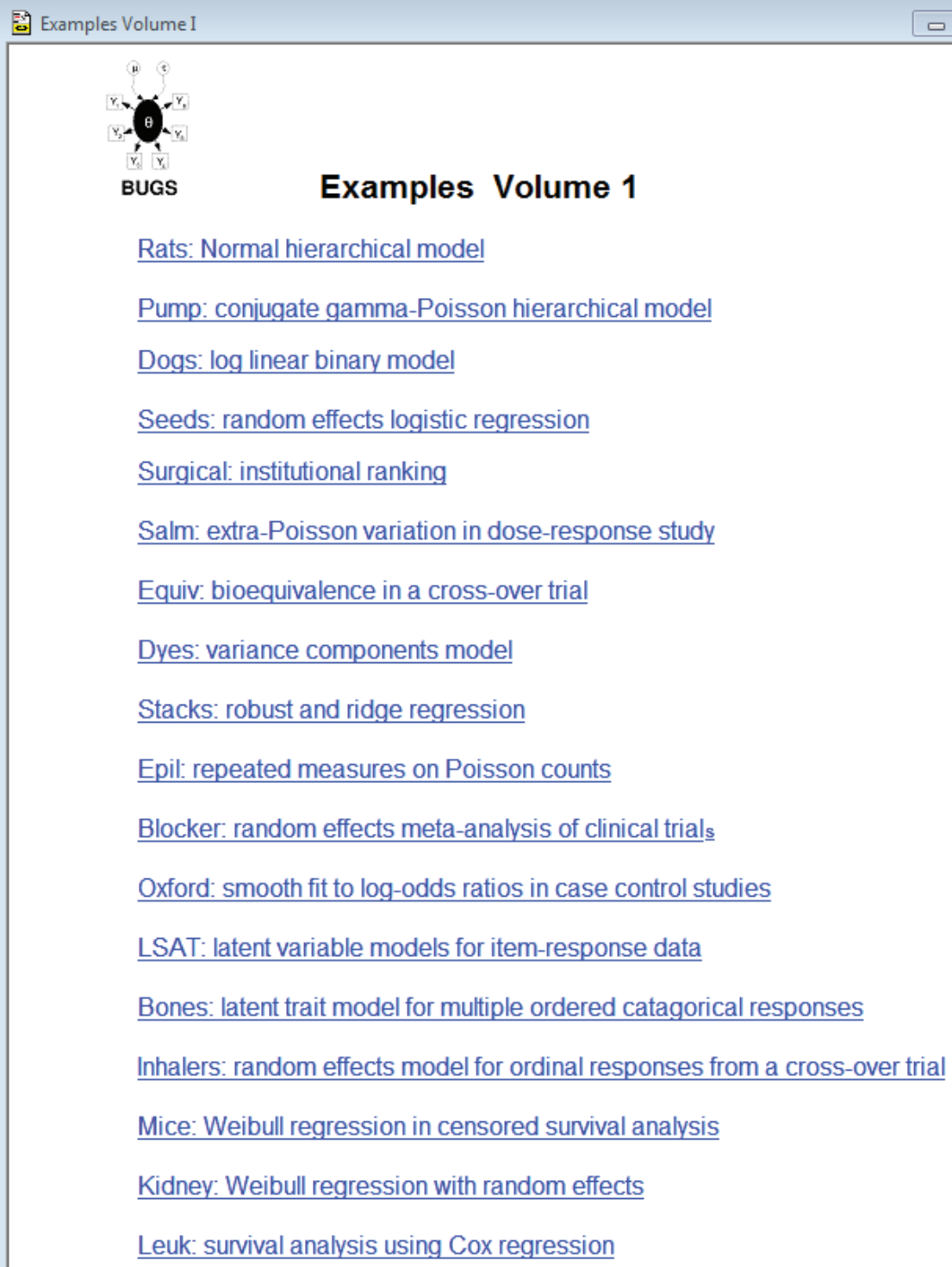

$\underline{\text { Cox regression with frailties }}$

\section{References:}

Sorry - an on-line version of the references is currently unavailable. Please refer to the existing Examples documentation available from http://ww . mrc-bsu . cam . ac. uk/bugs. 


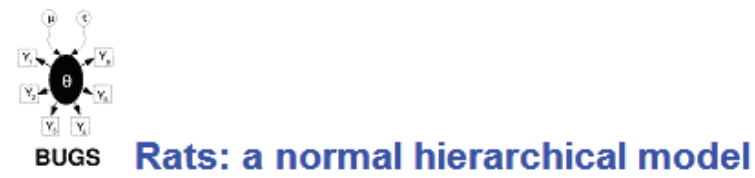

This example is taken from section 6 of Gelfand et al (1990), and concerns 30 young rats whose weights were measured weekly for five weeks. Part of the data is shown below, where $Y_{\mathrm{ij}}$ is the weight of the ith rat measured at age $\mathrm{x}_{\mathrm{j}-}$

\begin{tabular}{l|lllll} 
& \multicolumn{6}{|c}{ Weights $\mathrm{Y}_{\mathrm{ij}}$ of rat $\mathrm{i}$ on day $\mathrm{x}_{\mathrm{j}}$} \\
& $\mathrm{x}_{\mathrm{j}=8}$ & 15 & 22 & 29 & 36 \\
& & & & & \\
\hline Rat 1 & 151 & 199 & 246 & 283 & 320 \\
Rat 2 & 145 & 199 & 249 & 293 & 354 \\
$\ldots \ldots .$. & & & & & \\
Rat 30 & 153 & 200 & 244 & 286 & 324
\end{tabular}

A plot of the 30 growth curves suggests some evidence of downward curvature.

The model is essentially a random effects linear growth curve

$$
\begin{aligned}
& Y_{i j} \sim \operatorname{Normal}\left(\alpha_{i}+\beta_{f}\left(x_{j}-x_{\text {bar }}\right), \tau_{c}\right) \\
& \alpha_{i} \sim \operatorname{Normal}\left(\alpha_{\circ} \tau_{\alpha}\right) \\
& \beta_{i} \sim \operatorname{Normal}\left(\beta_{\circ} \tau_{\beta}\right)
\end{aligned}
$$

where $\mathrm{x}_{\mathrm{bar}}=22$, and $\tau$ represents the precision (1/variance) of a normal distribution. We note the absence of a parameter representing correlation between $\alpha_{i}$ and $\beta_{i}$ unlike in Gelfand et al 1990. However, see the Birats example in Volume 2 which does explicitly model the covariance between $\alpha_{i}$ and $\beta_{i}$. For now, we standardise the $x_{j}^{\prime}$ s around their mean to reduce dependence between $\alpha_{i}$ and $\beta_{i}$ in their likelihood: in fact for the full balanced data, complete independence is achieved. (Note that, in general, prior independence does not force the posterior distributions to be independent).

$\alpha_{c}, \tau_{\alpha}, \beta_{c}, \tau_{\beta}, \tau_{c}$ are given independent "noninformative" priors, with two alternatives considered for $\tau_{\alpha}$ and $\tau_{\beta}$ : prior 1 is uniform on the scale of the standard deviations $\sigma_{\alpha}=1 / \operatorname{sqr}\left(\tau_{\alpha}\right)$ and $\sigma_{\beta}=1 / \operatorname{sqr}\left(\tau_{\beta}\right)$, and prior 2 is a gamma $(0.001,0.001)$ on the precisions $\tau_{\alpha}$ and $\tau_{\beta}$. Interest particularly focuses on the intercept at zero time (birth), denoted $\alpha_{0}=\alpha_{c}-\beta_{c} x_{\text {bar }}$.

\section{Graphical model for rats example (using prior 1):}

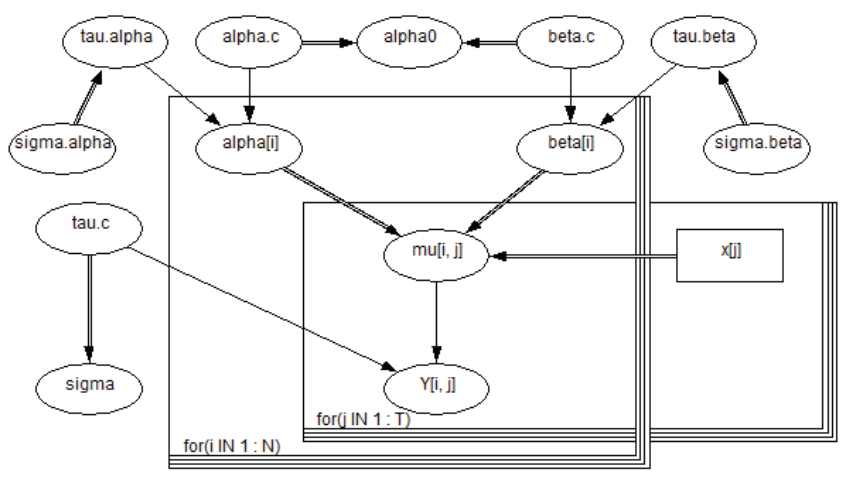


Note: The last graphic shown is called a Doodle. WinBUGS has a facility whereby the user can create such a diagram and have the code generated automatically.

BUGS language for rats example:

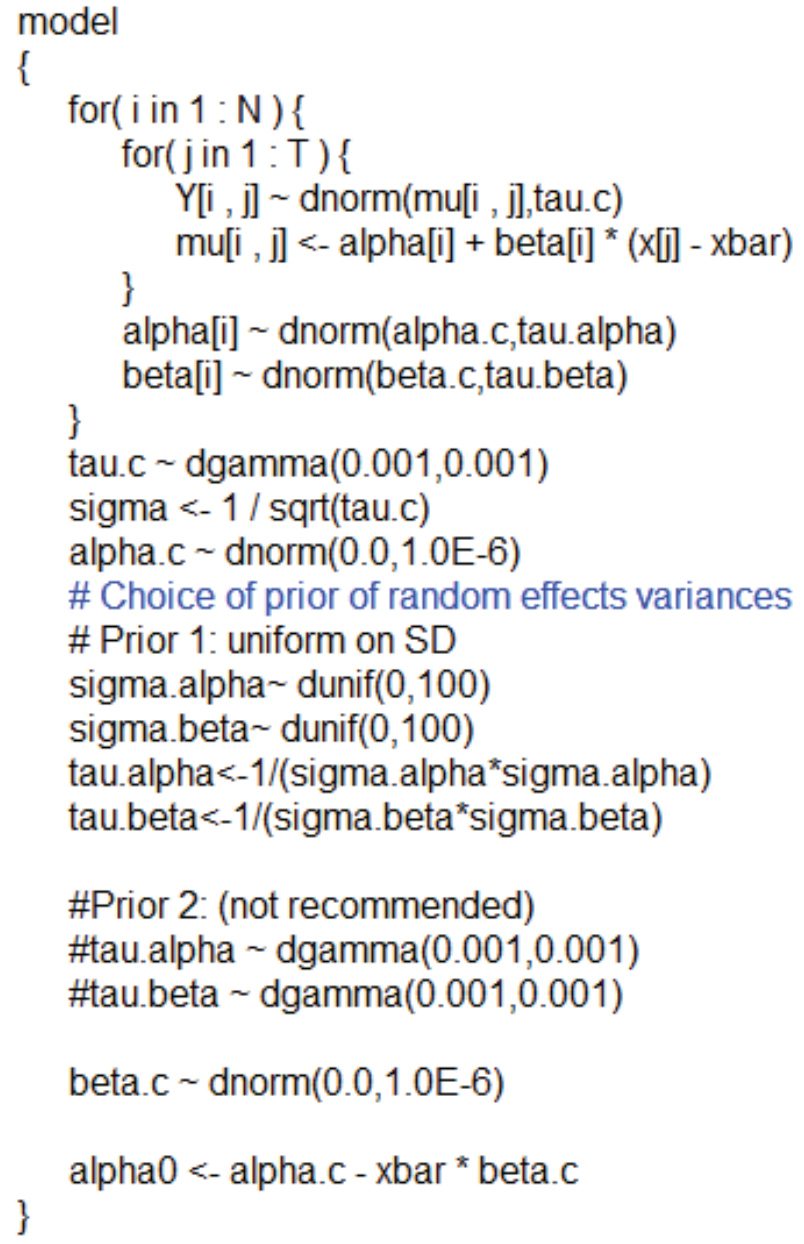


Data $\Rightarrow$ list $(x=c(8.0,15.0,22.0,29.0,36.0), x b a r=22, N=30, T=5$,

$$
\begin{aligned}
& Y=\text { structure }( \\
& \text { Data }=\mathrm{c}(151,199,246,283,320 \text {, } \\
& 145,199,249,293,354 \text {, } \\
& 147,214,263,312,328 \text {, } \\
& 155,200,237,272,297 \text {, } \\
& 135,188,230,280,323 \text {, } \\
& 159,210,252,298,331 \text {, } \\
& 141,189,231,275,305 \text {, } \\
& 159,201,248,297,338 \text {, } \\
& 177,236,285,350,376 \text {, } \\
& 134,182,220,260,296 \text {, } \\
& 160,208,261,313,352 \text {, } \\
& 143,188,220,273,314 \text {, } \\
& 154,200,244,289,325 \text {, } \\
& 171,221,270,326,358 \text {, } \\
& 163,216,242,281,312 \text {, } \\
& 160,207,248,288,324 \text {, } \\
& 142,187,234,280,316 \text {, } \\
& 156,203,243,283,317 \text {, } \\
& 157,212,259,307,336 \text {, } \\
& 152,203,246,286,321 \text {, } \\
& 154,205,253,298,334 \text {, } \\
& 139,190,225,267,302 \text {, } \\
& 146,191,229,272,302 \text {, } \\
& 157,211,250,285,323 \text {, } \\
& 132,185,237,286,331 \text {, } \\
& 160,207,257,303,345 \text {, } \\
& 169,216,261,295,333 \text {, } \\
& 157,205,248,289,316 \text {, } \\
& 137,180,219,258,291 \text {, } \\
& 153,200,244,286,324) \text {, } \\
& \operatorname{Dim}=\mathrm{c}(30,5)))
\end{aligned}
$$

Inits1 $\Rightarrow$ list(alpha $=c(250,250,250,250,250,250,250,250,250,250,250,250,250,250,250$, $250,250,250,250,250,250,250,250,250,250,250,250,250,250,250$ ), beta $=c(6,6,6,6,6,6,6,6,6,6,6,6,6,6,6$,

$6,6,6,6,6,6,6,6,6,6,6,6,6,6,6)$

alpha.c $=150$, beta.c $=10$,

tau.c $=1$, sigma. alpha $=1$, sigma $\cdot$ beta $=1) \Leftrightarrow$

\section{Results}

A 1000 update burn in followed by a further 10000 updates gave the parameter estimates:

$\begin{array}{cccllllll}\text { node } & \text { mean } & \text { sd } & \text { MC error } & \mathbf{2 . 5 \%} & \text { median } & \mathbf{9 7 . 5 \%} & \text { start } & \text { sample } \\ \text { alpha0 } & 106.6 & 3.65 & 0.04151 & 99.43 & 106.5 & 113.9 & 1001 & 10000 \\ \text { beta.c } & 6.185 & 0.1102 & 0.001294 & 5.967 & 6.185 & 6.404 & 1001 & 10000 \\ \text { sigma } & 6.074 & 0.4673 & 0.007724 & 5.247 & 6.044 & 7.068 & 1001 & 10000\end{array}$




\section{Predictions:}

\begin{tabular}{|l|l|l|l|l|l|l|l|l|} 
node & mean & sd & MC error & $\mathbf{2 . 5 \%}$ & median & $\mathbf{9 7 . 5 \%}$ & start & sample \\
$\mathrm{Y}[26,2]$ & 204.5 & 8.74 & 0.1159 & 187.0 & 204.4 & 221.7 & 1001 & 10000 \\
$\mathrm{Y}[26,3]$ & 250.0 & 10.27 & 0.1642 & 229.7 & 249.9 & 270.1 & 1001 & 10000 \\
$\mathrm{Y}[26,4]$ & 295.4 & 12.64 & 0.2092 & 270.3 & 295.3 & 320.3 & 1001 & 10000 \\
$\mathrm{Y}[26,5]$ & 340.6 & 15.32 & 0.284 & 310.2 & 340.5 & 370.5 & 1001 & 10000 \\
beta.c & 6.575 & 0.1507 & 0.003708 & 6.281 & 6.573 & 6.875 & 1001 & 10000
\end{tabular}

Trace plots and density estimates:
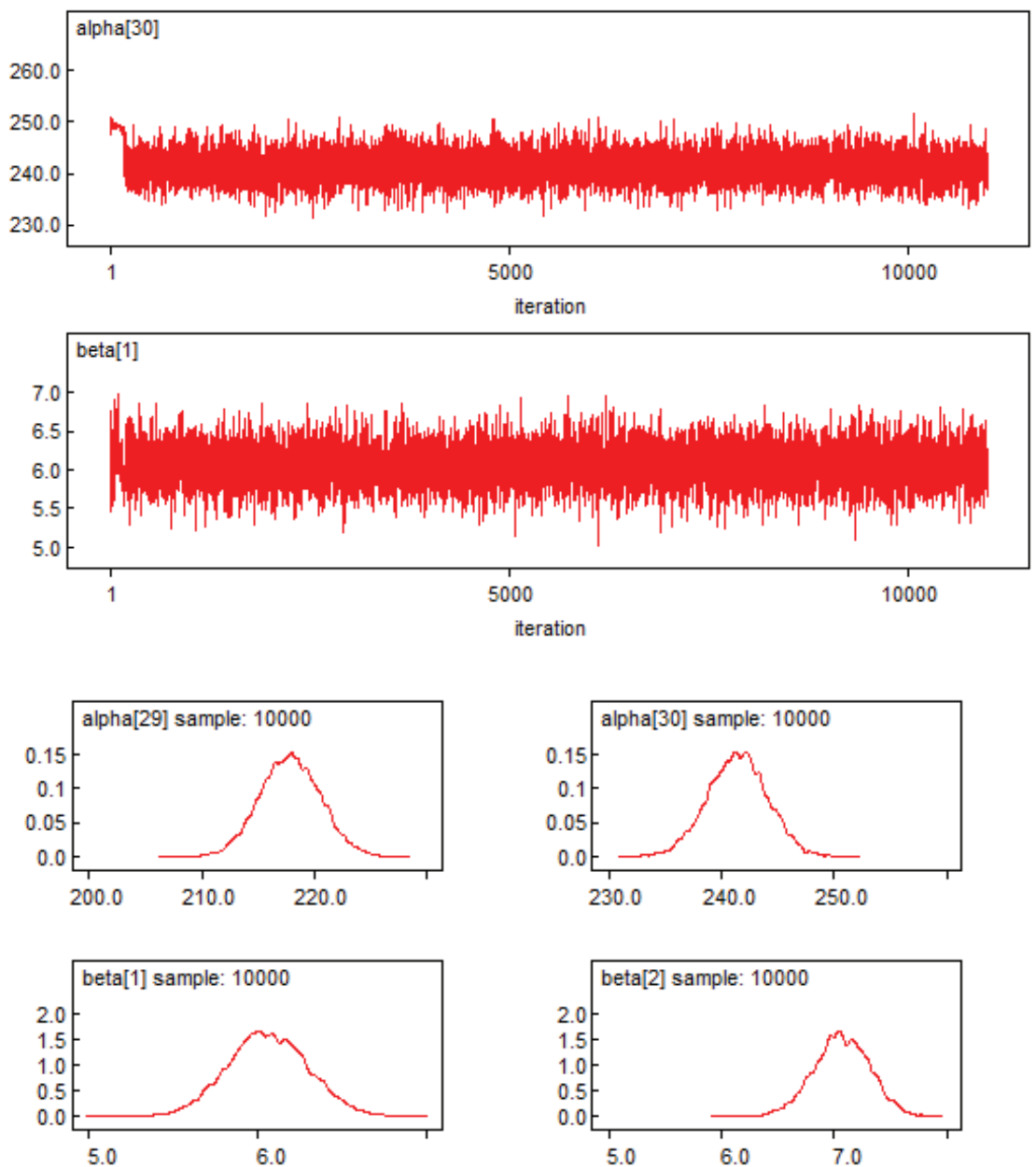

(End of Figure 8.7) 


\section{Exercise 8.I Simple linear regression via WinBUGS}

Use WinBUGS to perform a simple linear regression on the data in Table 8.2 (which is the same as Table 7.1 in Exercise 7.2).

\section{Table 8.2 Regression data}

\begin{tabular}{c|ccccc}
$x_{i}(=i)$ & 1 & 2 & 3 & 4 & 5 \\
\hline$y_{i}$ & 5.879 & 8.54 & 14.12 & 13.14 & 15.26 \\
\hline \multicolumn{1}{c}{$\mid c$} & 6 & 7 & 8 & 9 & 10 \\
\hline$y_{i}$ & 20.43 & 19.92 & 18.47 & 21.63 & 24.11 \\
\hline
\end{tabular}

\section{Solution to Exercise 8.I}

Using the following WinBUGS code, we obtain the results in Table 8.3:

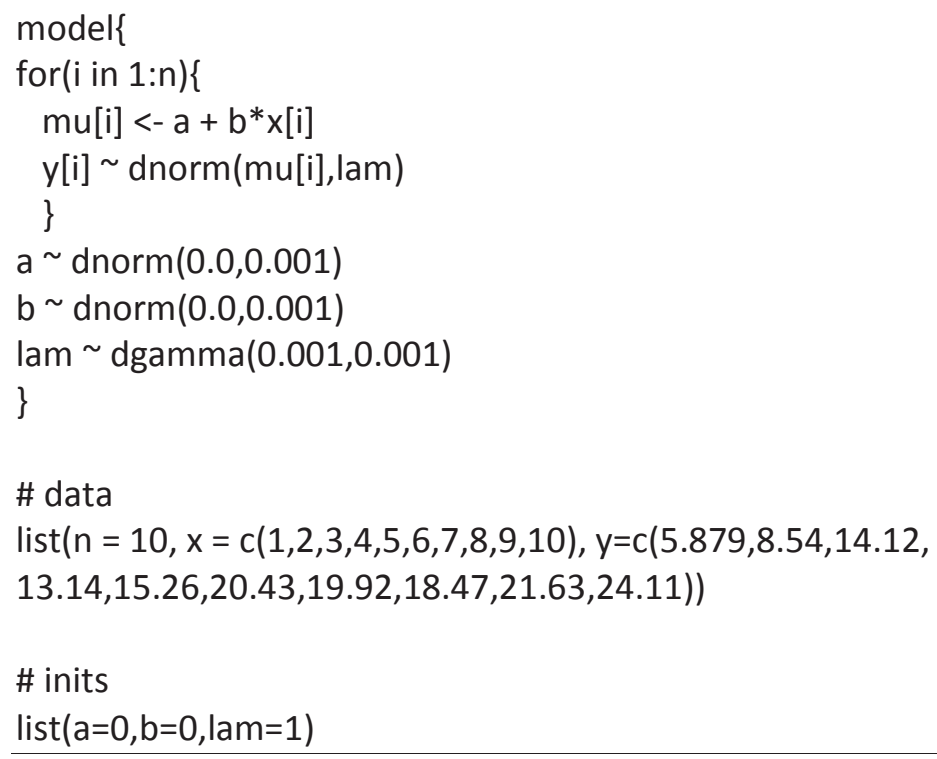

Table 8.3 Results of regression performed using WinBUGS

\begin{tabular}{|c|c|c|c|c|c|c|c|c|}
\hline node & mean & sd & MC error & $2.5 \%$ & median & $97.5 \%$ & start & sample \\
\hline $\bar{a}$ & 6.039 & 1.532 & 0.01646 & 2.955 & 6.051 & 9.107 & 1001 & 10000 \\
\hline$b$ & 1.836 & 0.247 & 0.00266 & 1.342 & 1.834 & 2.334 & 1001 & 10000 \\
\hline lam & 0.2625 & 0.1313 & 0.001602 & 0.07259 & 0.2404 & 0.5788 & 1001 & 10000 \\
\hline
\end{tabular}


Using the results in Table 8.3, we estimate $a$ by 6.039 with 95\% CPDR (2.955, 9.107), and we estimate $b$ by 1.836 with 95\% CPDR (1.342, 2.334).

It may be noted that these results are very similar to those obtained via classical techniques in an earlier exercise: 6.051 and $(2.973,9.128)$ for $a$, and 1.836 and $(1.340,2.332)$ for $b$.

Figure 8.8 shows trace plots and density estimates produced as part of the WinBUGS output.

Figure 8.8 Graphical output from WinBUGS regression
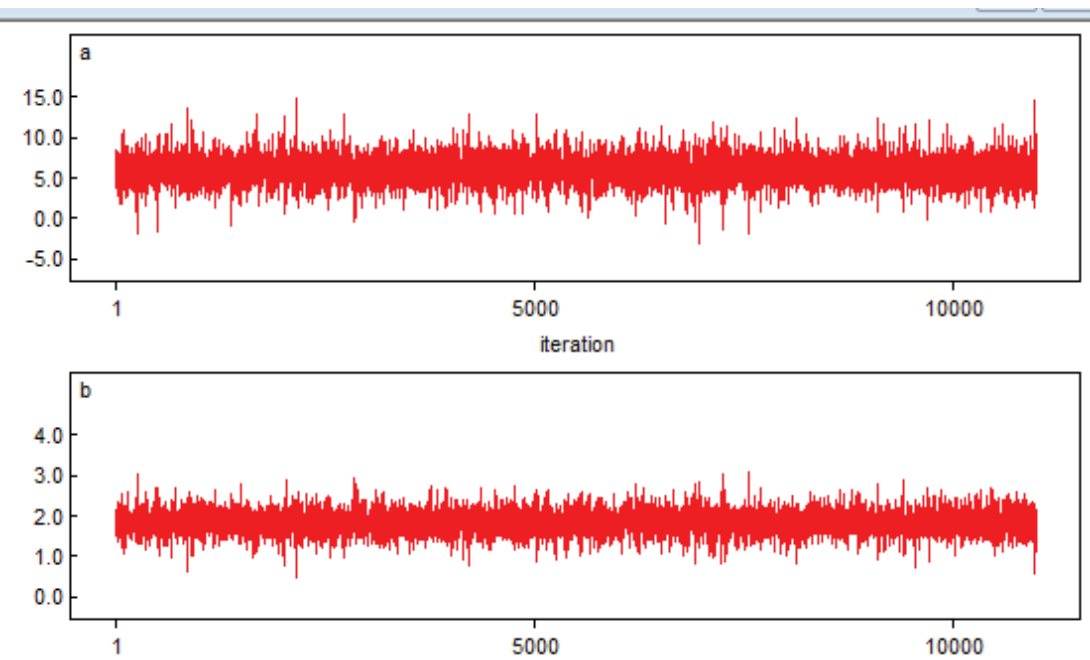

;: Kernel density
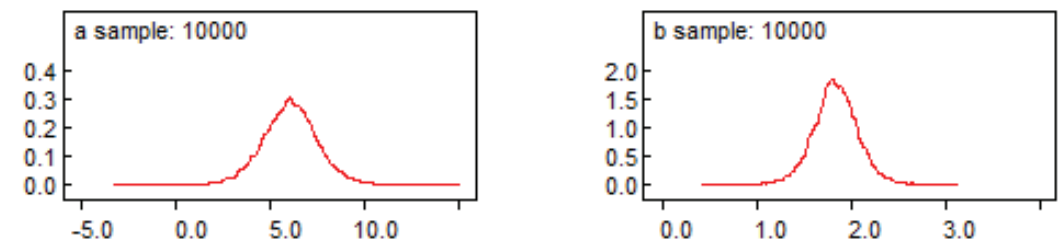


\section{Exercise 8.2 Logistic regression via WinBUGS}

Consider the data in Table 8.4, which is the same as in Table 7.2 of Exercise 7.3 (where, for example, in Experiment 3 a total of 40 rats were exposed to radiation for 3.6 hours, and 23 of them died within one month).

\section{Table 8.4 Rat mortality data}

\begin{tabular}{c|cccccc}
$i$ & $n_{i}$ & $x_{i}$ & $y_{i}$ & $y_{i} / n_{i}$ & $=$ & $\hat{p}_{i}$ \\
\hline 1 & 10 & 0.1 & 1 & $1 / 10$ & $=$ & 0.1 \\
2 & 30 & 1.4 & 0 & $0 / 30$ & $=$ & 0 \\
3 & 40 & 3.6 & 23 & $23 / 40$ & $=$ & 0.575 \\
4 & 20 & 3.8 & 12 & $12 / 20$ & $=$ & 0.6 \\
5 & 15 & 5.2 & 8 & $8 / 15$ & $=$ & 0.5333 \\
6 & 46 & 6.1 & 32 & $32 / 46$ & $=$ & 0.696 \\
7 & 12 & 8.7 & 10 & $10 / 12$ & $=$ & 0.833 \\
8 & 37 & 9.1 & 35 & $35 / 37$ & $=$ & 0.946 \\
9 & 23 & 9.1 & 19 & $19 / 23$ & $=$ & 0.826 \\
10 & 8 & 13.6 & 8 & $8 / 8$ & $=$ & 1 \\
\hline
\end{tabular}

Use WinBUGS to estimate the parameters in the following logistic regression model for these data:

$$
Y_{i} \sim \perp \operatorname{Bin}\left(n_{i}, p_{i}\right), \quad i=1, \ldots, n,
$$

where:

$$
\begin{aligned}
p_{i}=\frac{1}{1+\exp \left(-z_{i}\right)} & \text { (probability of a 'success' for experiment } i \text { ) } \\
z_{i}=a+b x_{i} & \text { (linear predictor). }
\end{aligned}
$$

In your results, also include inference on $L D 50$, the dose at which $50 \%$ of rats will die $(=-a / b)$, and on $d$, defined as the number of rats that will die out of 20 that are exposed to five hours of radiation. 


\section{Solution to Exercise 8.2}

Applying the following WinBUGS code, we obtain the results in Table 8.5:

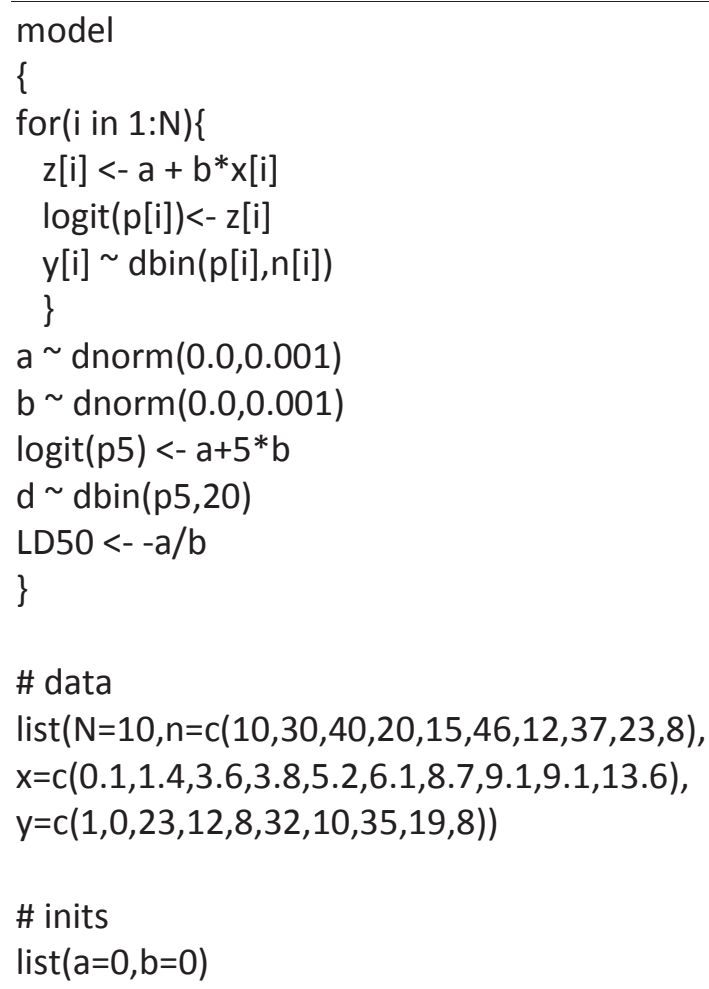

Table 8.5 Results of logistic regression performed using WinBUGS

\begin{tabular}{lrlllllll}
\multicolumn{2}{l}{ nodemean } & sd & MC error & $\mathbf{2 . 5 \%}$ & median & $\mathbf{9 7 . 5 \%}$ & start sample \\
\hline & & & & & & \\
\hline LD50 4.273 & 0.3373 & 0.00464 & 3.587 & 4.285 & 4.899 & 100110000 \\
a & -2.177 & 0.3726 & 0.01041 & -2.922 & -2.168 & -1.478 & 100110000 \\
b & 0.5082 & 0.06962 & 0.001964 & 0.3794 & 0.5059 & 0.6501 & 100110000 \\
d & 11.79 & 2.344 & 0.02447 & 7.0 & 12.0 & 16.0 & 100110000 \\
p5 & 0.5895 & 0.03946 & $3.174 E 4$ & 0.5125 & 0.5896 & 0.6664 & 100110000 \\
\hline
\end{tabular}


Thus, we estimate $a$ by -2.177 with 95\% CPDR $(-2.922,-1.478)$, etc.

These results are very similar to those obtained via classical techniques in Exercise 7.3 , namely -2.156 and $(-3.000,-1.311)$ for $a$, etc.

Figure 8.9 shows some traces and density estimates produced as part of the WinBUGS output. Here, 'p5' represents the probability of a rat dying within one month if exposed to five hours of radiation. We chose to monitor this node so as to estimate its posterior density

\section{Figure 8.9 Graphical output from WinBUGS logistic regression}
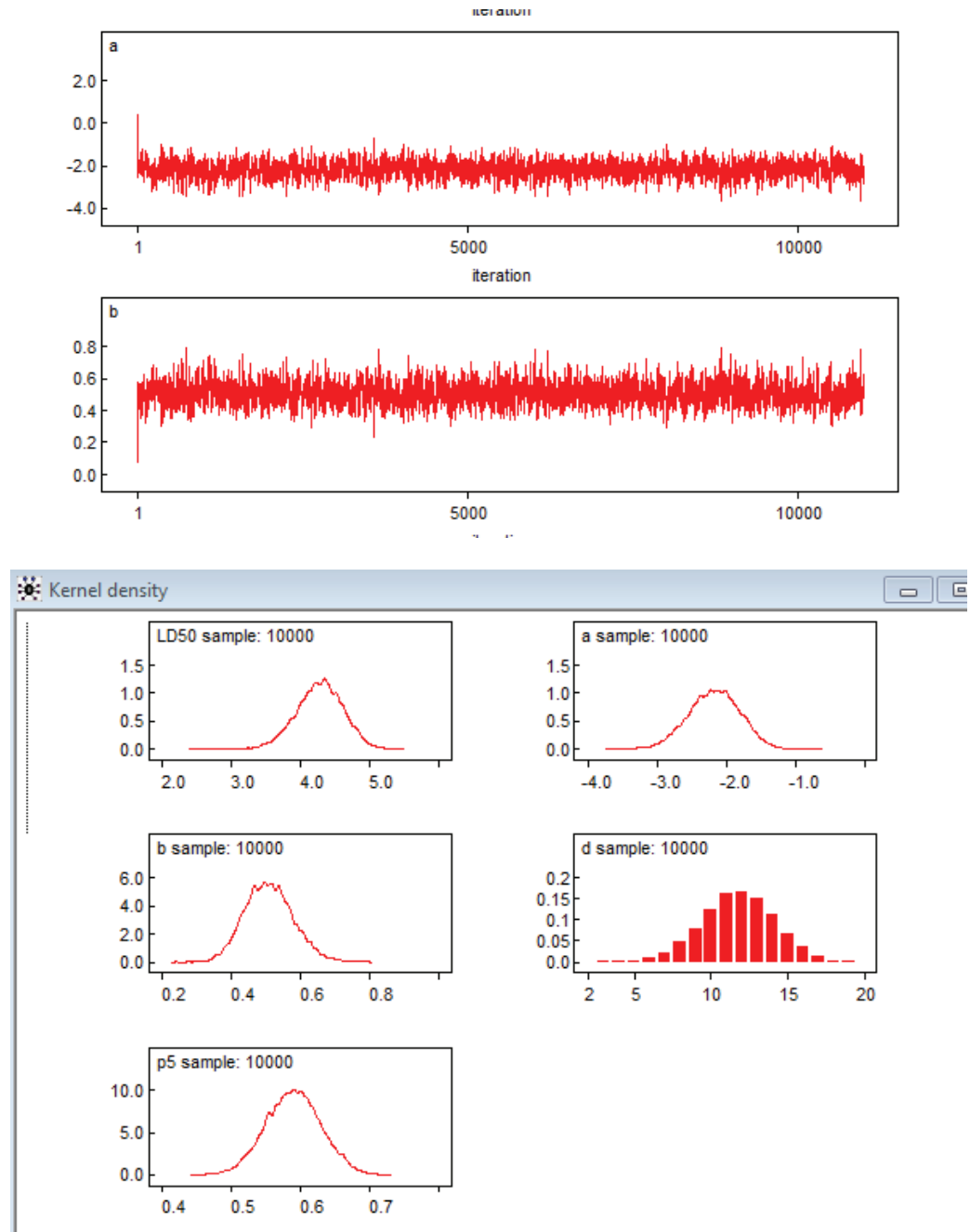


\section{Exercise 8.3 Inference on a uniform distribution via WinBUGS}

Consider the following Bayesian model:

$$
\begin{aligned}
& \left(y_{1}, \ldots, y_{n} \mid a, b\right) \sim \text { iid } U(a, b) \\
& (a \mid b) \sim U(0, b) \\
& b \sim U(0,1) .
\end{aligned}
$$

Suppose that $n=20$ data values from this model with $a=0.6$ and $b=0.8$ are as shown in Table 8.6 (which is the same as Table 7.6 in Exercise 7.5).

\section{Table 8.6 Sample values from a uniform distribution}

\begin{tabular}{c|ccccc}
$i$ & 1 & 2 & 3 & 4 & 5 \\
\hline$y_{i}$ & 0.7846 & 0.7572 & 0.6381 & 0.7626 & 0.6105 \\
\hline$i$ & 6 & 7 & 8 & 9 & 10 \\
\hline$y_{i}$ & 0.6990 & 0.7728 & 0.7113 & 0.7314 & 0.7435 \\
\hline$i$ & 11 & 12 & 13 & 14 & 15 \\
\hline$y_{i}$ & 0.6324 & 0.7072 & 0.7493 & 0.7979 & 0.6182 \\
\hline & & & & & \\
$i$ & 16 & 17 & 18 & 19 & 20 \\
\hline$y_{i}$ & 0.7652 & 0.7883 & 0.7194 & 0.6211 & 0.6054 \\
\hline
\end{tabular}

Use WinBUGS to generate a random sample from the joint posterior distribution of the parameters $a$ and $b$. Then use this sample to estimate the mean of the uniform distribution, namely

$$
m=E\left(y_{i} \mid a, b\right)=(a+b) / 2 \text {. }
$$




\section{Solution to Exercise 8.3}

Applying the following WinBUGS code we obtain the results in Table 8.7:

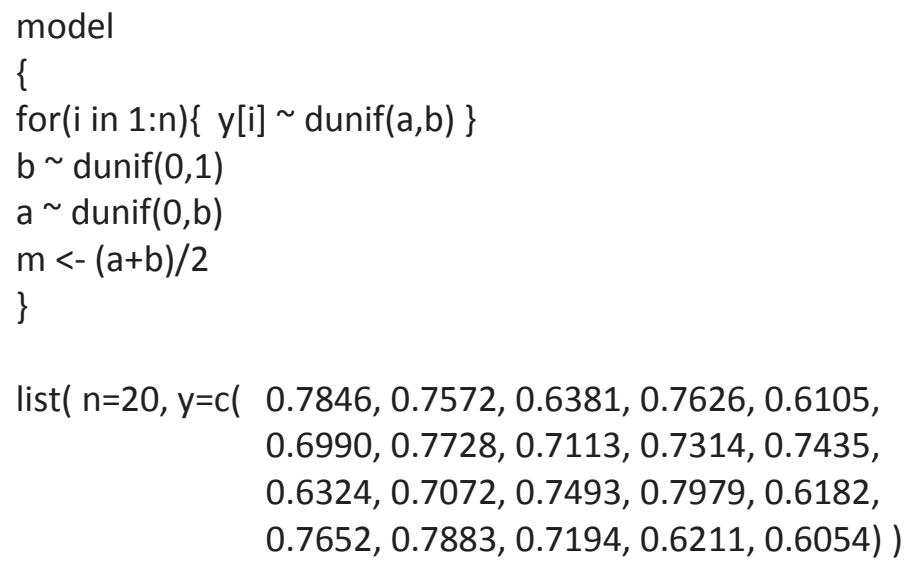

list $(a=0.1, b=0.9)$

Table 8.7 Results of WinBUGS analysis for a uniform distribution

\begin{tabular}{lllllllll} 
node & mean & sd & MC error & $\mathbf{2 . 5 \%}$ & median & $\mathbf{9 7 . 5 \%}$ & start & sample \\
\hline a & 0.594 & 0.01184 & $1.996 \mathrm{E}-4$ & 0.5623 & 0.5977 & 0.6051 & 1001 & 10000 \\
b & 0.8091 & 0.01187 & $2.004 \mathrm{E}-4$ & 0.7982 & 0.8054 & 0.841 & 1001 & 10000 \\
m & 0.7016 & 0.008201 & $1.388 \mathrm{E}-4$ & 0.6844 & 0.7015 & 0.7187 & 1001 & 10000 \\
\hline
\end{tabular}

Using the results in Table 8.7, we estimate $m$ by 0.7016 , with $95 \%$ CI $(0.7013,0.7019)$ for $m$ 's posterior mean.

We also estimate the 95\% CPDR for $m$ as $(0.6844,0.7187)$. 
Note 1: The CI here was obtained in R using the following code:

$$
0.7016+c(-1,1) * \text { qnorm }(0.975) * 0.0001388
$$

Another CI is $(0.7014,0.7018)$, obtained using the code:

$$
0.7016+c(-1,1) * \text { qnorm }(0.975) * 0.008201 / \operatorname{sqrt}(10000)
$$

But this second CI is 'inferior' to $(0.7013,0.7019)$ because it ignores the autocorrelation in the simulated values. The fact that the second CI is shorter corresponds to the fact that its true coverage probability is less than the nominal and desired 95\%.

Note 2: These inferences (above Note 1) are similar to those obtained in the solution to Exercise 7.5 using custom-written R code: 0.7013 with 95\% CI $(0.7008,0.7019)$ and $95 \%$ CPDR estimate $(0.6837,0.7173)$.

Note 3: The CI in Note 2 is wider than the CI $(0.7013,0.7019)$ because it is based on a smaller Monte Carlo sample size (of 1,000 rather than $10,000)$. If we use only iterations 1,001 to 2,000 from the WinBUGS output, we get

\section{m \\ $\begin{array}{llllllll}0.7016 & 0.008287 & 3.573 E-4 & 0.6833 & 0.7016 & 0.7194 & 1001 & 1000\end{array}$}

in place of the corresponding row of Table 8.7. Then, the 95\% CI for $m$ 's posterior mean becomes $(0.7009,0.7023)$, obtained via

$$
0.7016+c(-1,1) * \text { qnorm }(0.975) * 0.0003573
$$

This CI has a width of 0.0014 , which is greater than 0.0006 , the width of $(0.7013,0.7019)$, and closer to 0.0011 , the width of the CI in Note 2.

Figure 8.10 shows some traces and density estimates produced as part of the WinBUGS output. 
Figure 8.10 Graphs from WinBUGS analysis for a uniform distribution
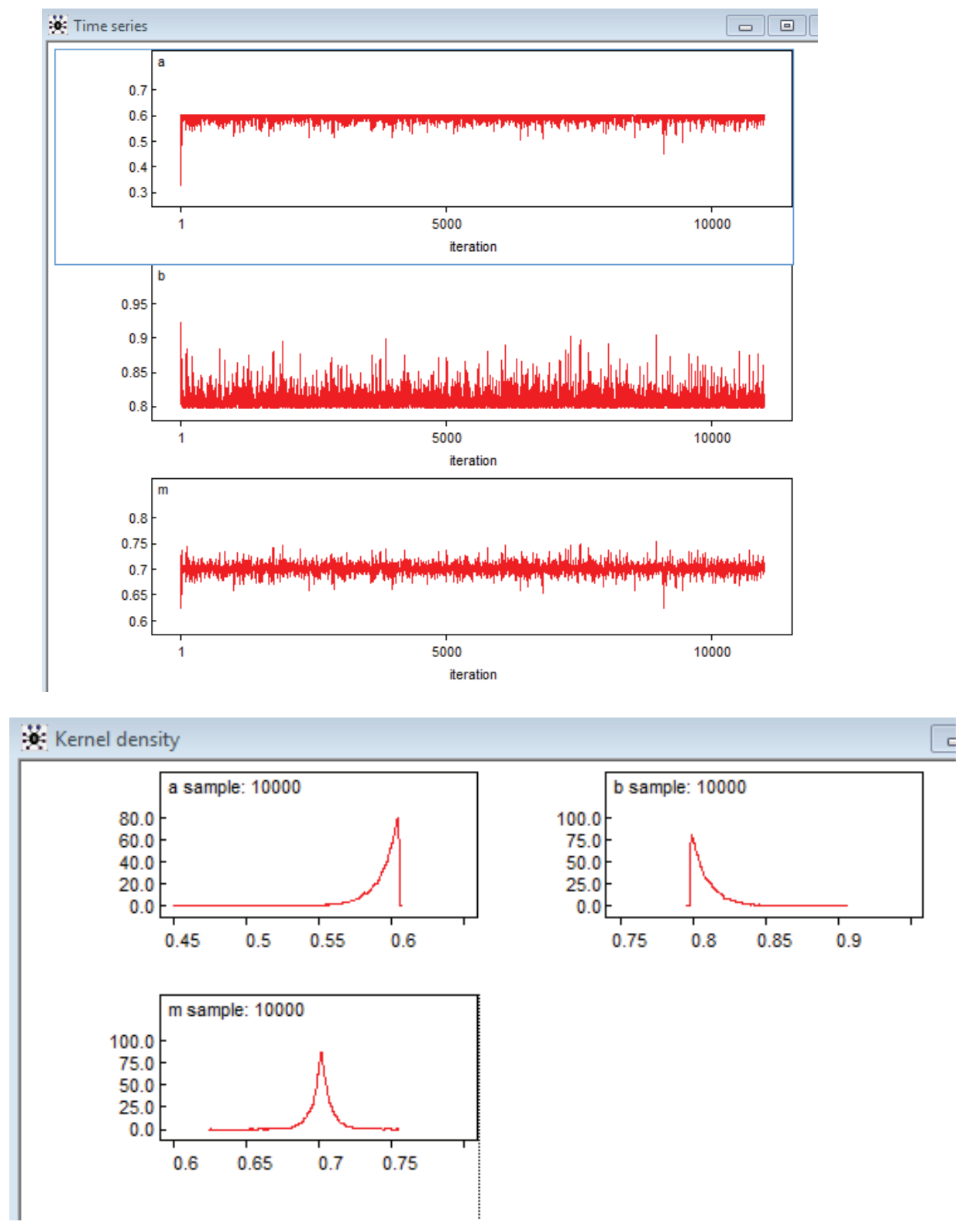


\subsection{Tutorial on calling BUGS in $\mathbf{R}$}

The following is a short tutorial on how WinBUGS can be called within an $\mathrm{R}$ session. Some of the details may need to be changed depending on the configuration of files and directories in the computer being used.

First, assume that R (v3.01) is installed in C:/R-3.0.1

Also assume that WinBUGS (v4.1.3) is installed in C:/WinBUGS14

Open R and type

install.packages("R2WinBUGS")

Note: You must have a connection to the internet for this to work. This command is required only once for each installed version of $\mathrm{R}$.

Next, select a CRAN mirror when prompted. 'Melbourne' should work.

You should then see something like the following:

package 'coda' successfully unpacked and MD5 sums checked

package 'R2WinBUGS' successfully unpacked and MD5 sums checked, etc.

Then type

library("R2WinBUGS")

Note: This loads the necessary functions and must be done at the beginning of each R session in which WinBUGS is to be called.

You should now see something like:

Loading required package: coda

Loading required package: lattice

Loading required package: boot, etc. 
Next, create a file called C:/R-3.0.1/BugsCode1.txt

which contains the following code for a simple Bayesian model:

model

\{

for(i in 1:n) $\{y[i] \sim \operatorname{dnorm}(m u$, tau $)\}$

$\mathrm{mu} \sim \operatorname{dnorm}(0,0.0001)$

tau dgamma(0.001, 0.001)

gam $<-$ mu*sqrt(tau)

\}

Next create a working directory, say C:/R-3.0.1/BugsOut/ and proceed in $\mathrm{R}$ as follows:

$y<-c(2.4,1.2,5.3,1.1,3.9,2.0)$

$\mathrm{n}<-$ length $(\mathrm{y})$

data <- list("n","y")

inits $<-$ function ()$\{$ list(mu=0, tau=1.0) $\}$

parameters <- c("mu", "gam")

sim <- bugs(data, inits, parameters,

model.file= "C:/R-3.0.1/BugsCode1.txt",

n.chains $=1$, n.iter $=1500$, n. burnin=500, DIC $=F A L S E$,

bugs.directory = "C:/WinBUGS14/",

working.directory = "C:/R-3.0.1/BugsOut/")

This sets things up, starts WinBUGS, runs the BUGS code, closes WinBUGS, and creates a number of files in the working directory, similar to the ones shown in Figure 8.11. 
Figure 8. I I Files created by running WinBUGS in $\mathbf{R}$

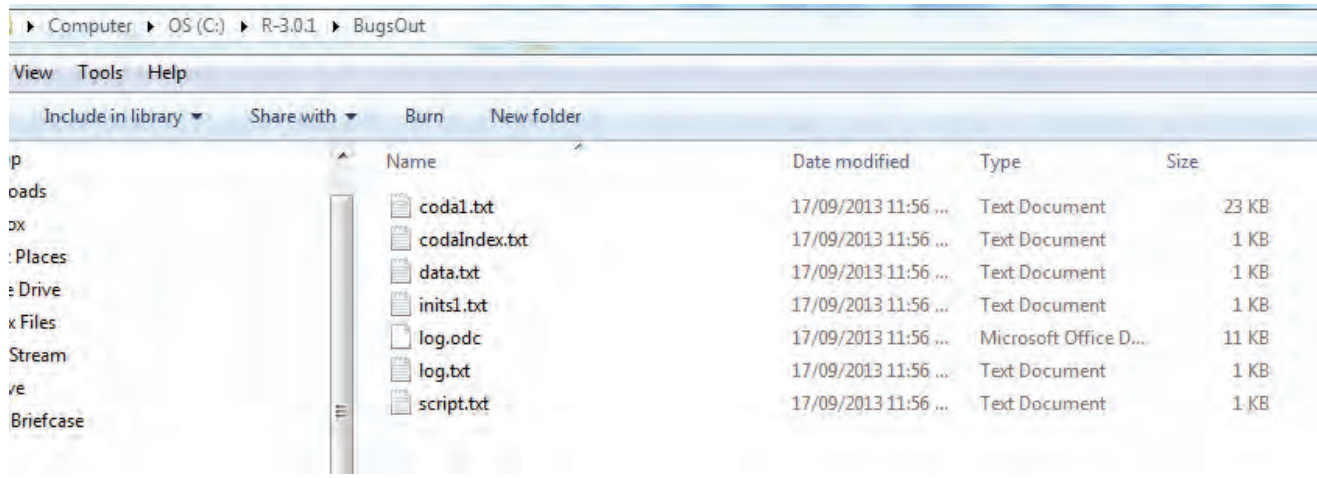

These files contain information which can then be accessed within $\mathrm{R}$, for example as follows:

print(sim, digits=4)

\# Inference for Bugs model at "C:/R-3.0.1/BugsCode1.txt", fit using WinBUGS,

\# 1 chains, each with 1500 iterations (first 500 discarded)

\# n.sims $=1000$ iterations saved

\# $\quad$ mean sd $2.5 \% \quad 25 \% \quad 50 \% \quad 75 \% \quad 97.5 \%$

\# mu 2.63580 .81850 .94242 .17602 .6453 .11754 .2984

\# gam 1.53800 .63920 .37741 .09351 .5211 .93602 .9061

$\operatorname{par}(\operatorname{mfrow}=c(2,1))$

hist(sim\$sims.list\$mu, breaks=20)

hist(sim\$sims.list\$gam, breaks=20)

After typing these commands, you should see two histograms similar to the ones shown in Figure 8.12. For more information on the bugs() function, simply type

help(bugs) 
Figure 8. 12 Histograms obtained in $\mathbf{R}$ after calling WinBUGS

Histogram of sim\$sims.list\$mu

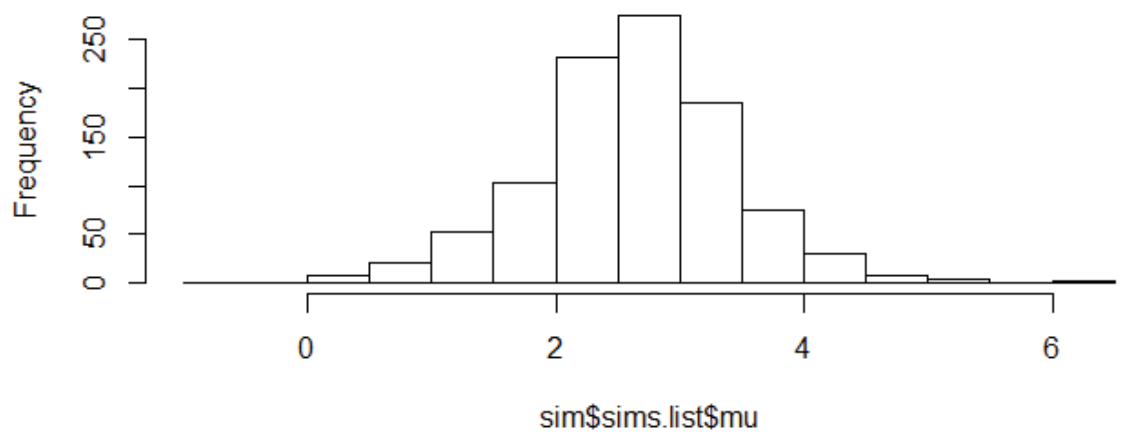

Histogram of sim\$sims.list\$gam

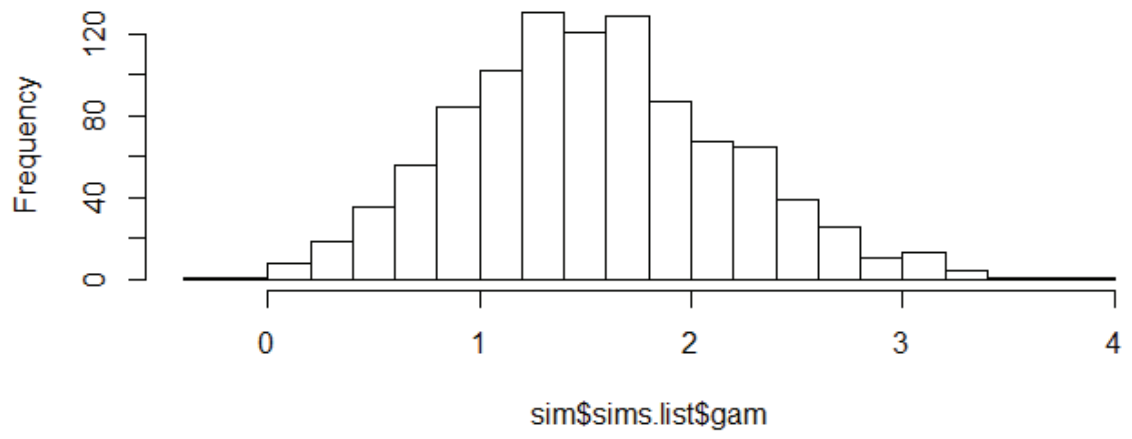

Note: If your WinBUGS code has an error, the procedure will crash, with little to tell you what went wrong. In that case, first iron out any 'bugs' directly in WinBUGS, and only then run your WinBUGS code in $\mathrm{R}$, as above. 


\section{Exercise 8.4 ARIMA modeling and forecasting with WinBUGS in $\mathbf{R}$}

Consider the well-known Total International Airline Passengers (TIAP) time series, as shown in Table 8.8. This series describes quarterly totals of international passengers for the period January 1949 to December 1960. (Here, Qtr1 refers to the period January-March, etc.)

Table 8.8 The TIAP time series

\begin{tabular}{c|cccc} 
Year & Qtr1 & Qtr2 & Qtr3 & Qtr4 \\
\hline 1949 & 362 & 385 & 432 & 341 \\
1950 & 382 & 409 & 498 & 387 \\
1951 & 473 & 513 & 582 & 474 \\
1952 & 544 & 582 & 681 & 557 \\
1953 & 628 & 707 & 773 & 592 \\
1954 & 627 & 725 & 854 & 661 \\
1955 & 742 & 854 & 1023 & 789 \\
1956 & 878 & 1005 & 1173 & 883 \\
1957 & 972 & 1125 & 1336 & 988 \\
1958 & 1020 & 1146 & 1400 & 1006 \\
1959 & 1108 & 1288 & 1570 & 1174 \\
1960 & 1227 & 1468 & 1736 & 1283 \\
\hline
\end{tabular}

Using classical methods, fit a suitable ARIMA model to this time series.

Then forecast the time series forward for one up to twelve quarters.

Then repeat your analysis and forecasts using WinBUGS called from R.

Also create a single graph which compares both sets of forecasts. 


\section{Solution to Exercise 8.4}

Figure 8.13 shows plots of the original times series $x_{t}$, its logarithm (showing stabilised variability), the difference of the logarithm (showing a removal of the trend), and $y_{t}$, the fourth seasonal difference of the first difference of the logarithm (showing that seasonality has been removed). The last two (bottom) plots are the sample ACF and sample PACF for $y_{t}$.

Figure 8.13 Plots for the TIAP time series
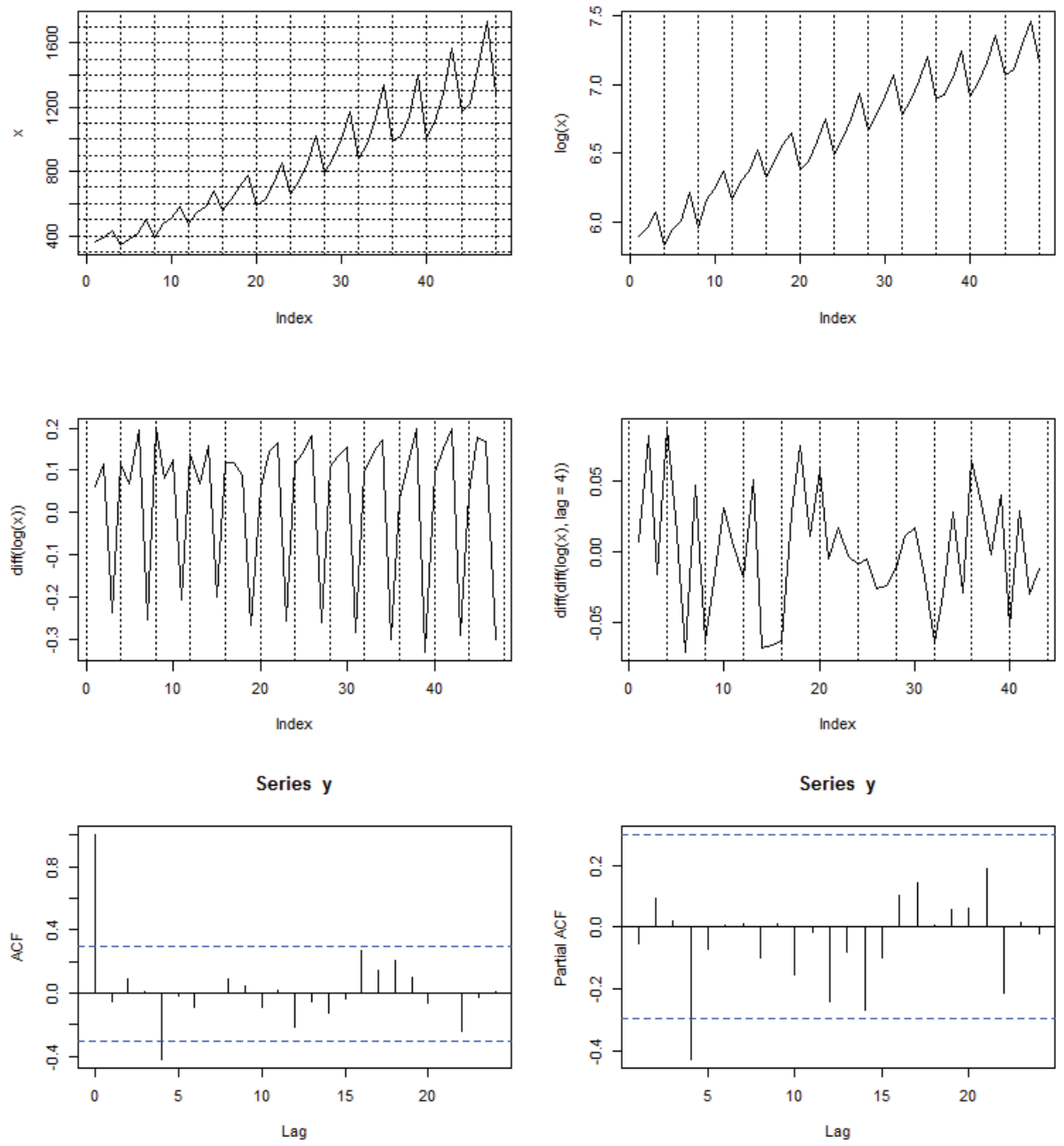
The last two plots in Figure 8.13 suggest SAR(1) or SMA(1) processes. Both fits pass standard diagnostic checks, the second being marginally better. Figure 8.14 shows some diagnostic plots for the SMA(1) fit (see the R Code below for further details).

Figure 8.I4 Diagnostics for the SMA(I) fit to the TIAP time series

Standardized Residuals

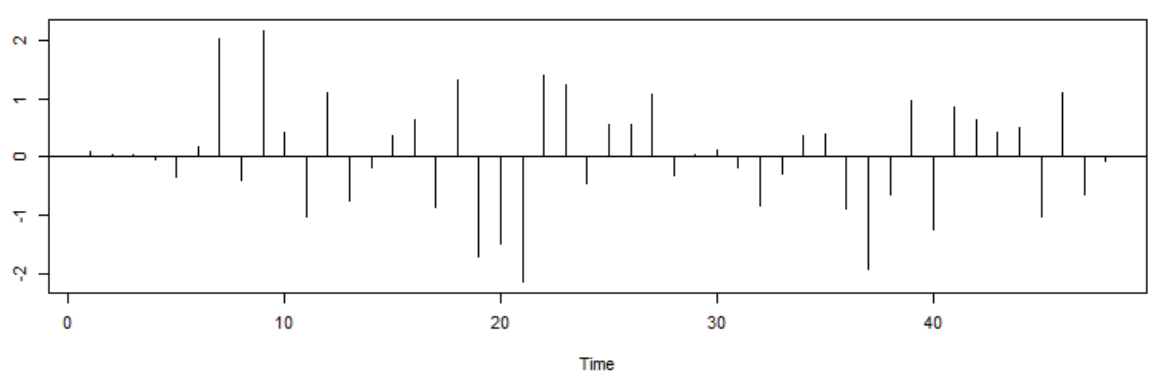

ACF of Residuals

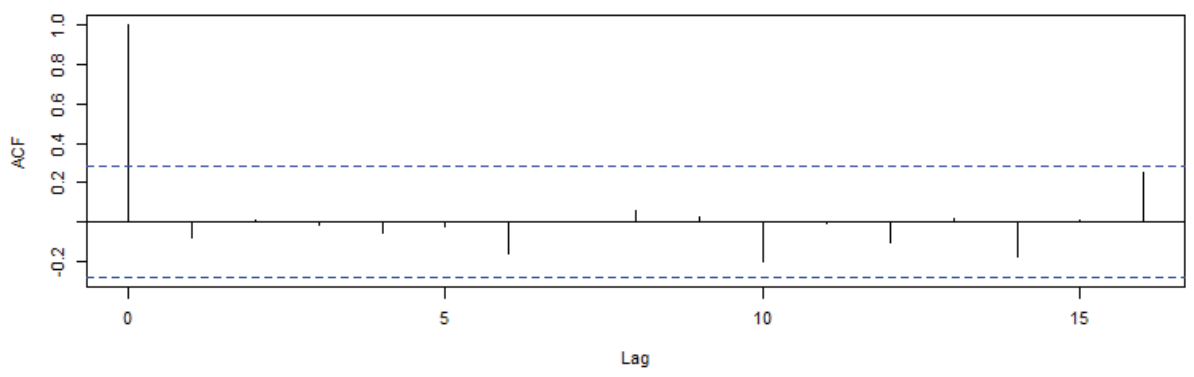

p values for Ljung-Box statistic

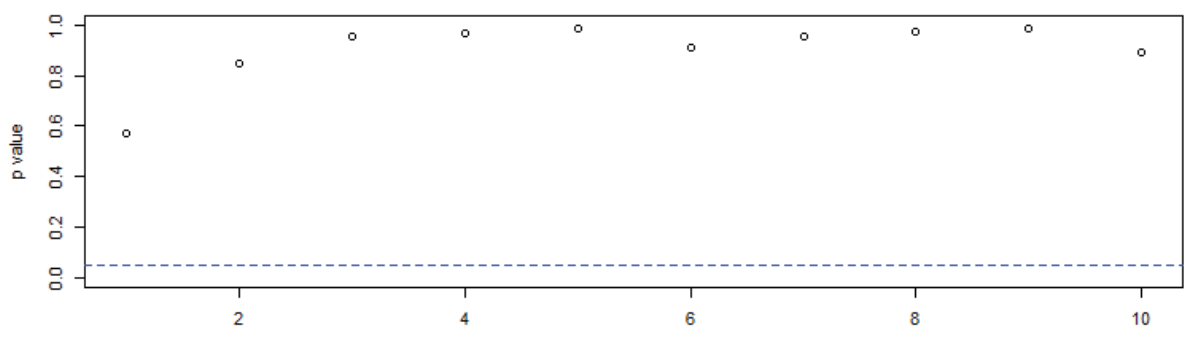




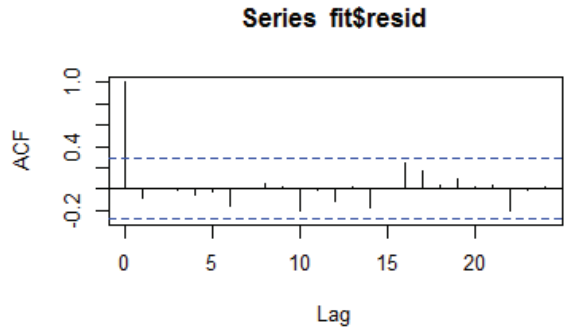

Normal Q-Q Plot

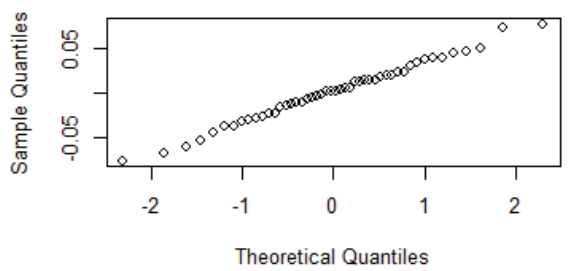

Series fit\$resid

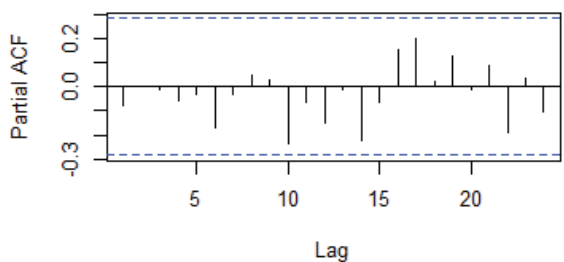

Histogram of fit\$resid

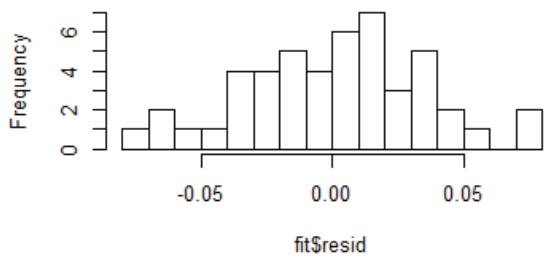

The chosen SMA(1) model for the TIAP time series $x_{t}$ may be expressed by writing

$$
y_{t}=\nabla_{4} \nabla \log x_{t}
$$

where

$$
y_{t}=w_{t}+\Theta_{1} w_{t-4}, \quad w_{t} \sim \text { iid } N\left(0, \sigma^{2}\right) .
$$

The parameter estimates for this model are:

$$
\begin{aligned}
& \hat{\Theta}_{1}=-0.4927(\mathrm{SE}=0.1201) \\
& \hat{\sigma}^{2}=0.0013 .
\end{aligned}
$$

Figure 8.15 shows the time series $x_{t}$ plus predictions 12 quarters ahead based on the above fitted model. The dashed lines show the 95\% prediction interval at each of the 12 future times points. (See the R code below for details regarding all calculations.) 
Figure 8. I5 Classical forecasts of the TIAP time series

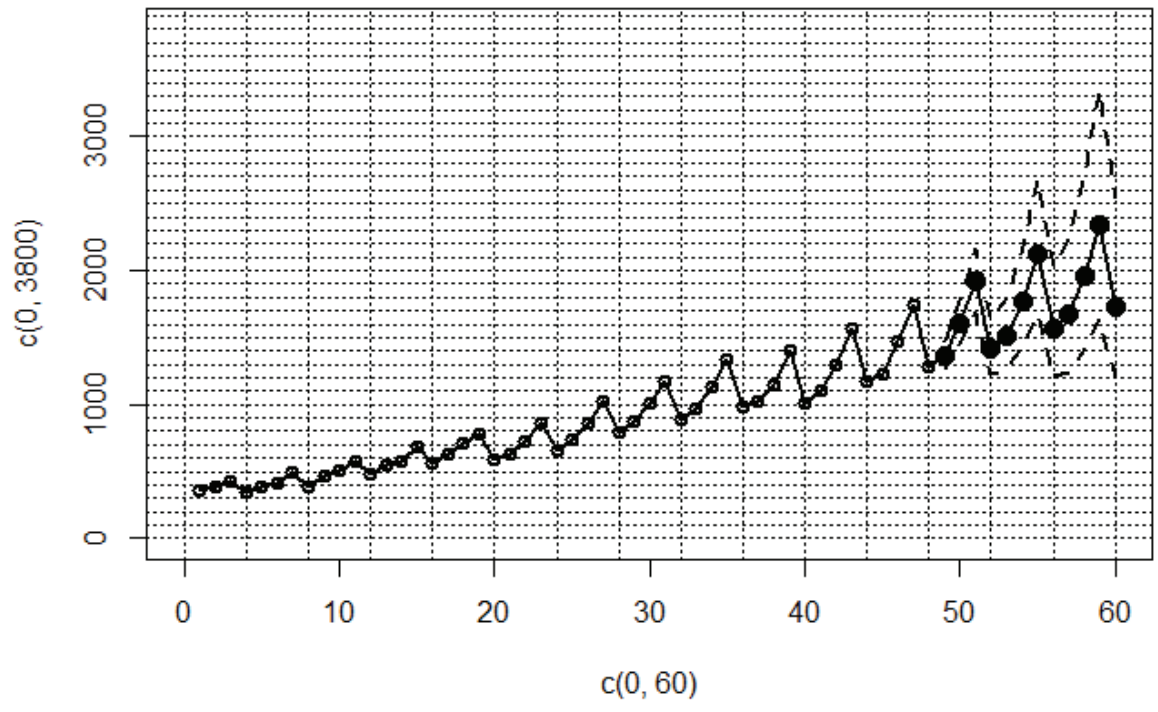

We now fit the same model to the time series but using MCMC via WinBUGS called from R. Some graphical output from the WinBUGS run is shown in Figure 8.16. (See the code below for details.)

Figure 8.17 shows the Bayesian analogue of the classical forecasts displayed in Figure 8.15.

To compare the classical and Bayesian analyses, we combine the two sets of forecasts into a single plot, as shown in Figure 8.18 (page 399). Figure 8.19 (page 399) is a detail in Figure 8.18. 
Figure 8.16 Output from an analysis of the TIAP series using WinBUGS

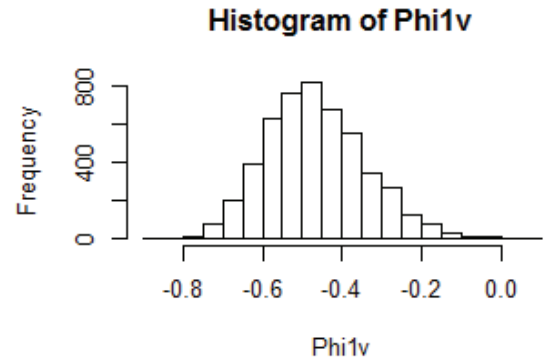

Histogram of $x m[, 1]$

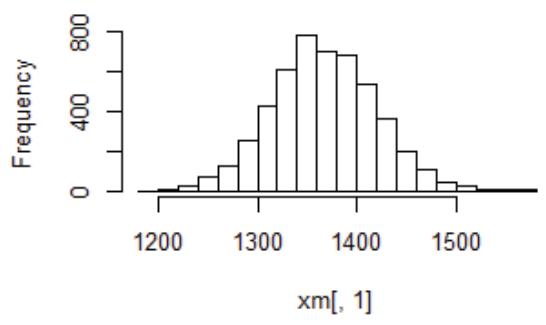

Histogram of sig2v

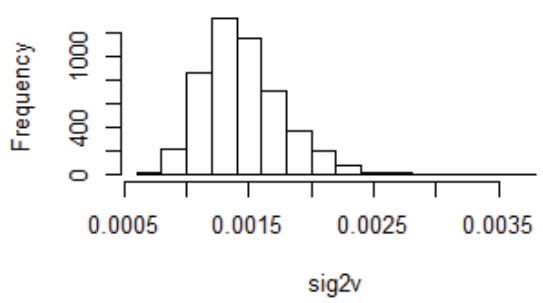

Histogram of $x m[, 2]$

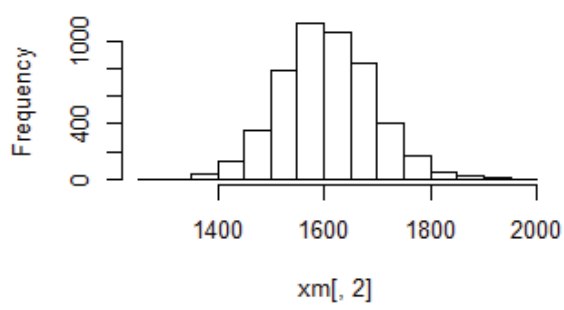

Figure 8.I7 Bayesian forecasts of the TIAP time series

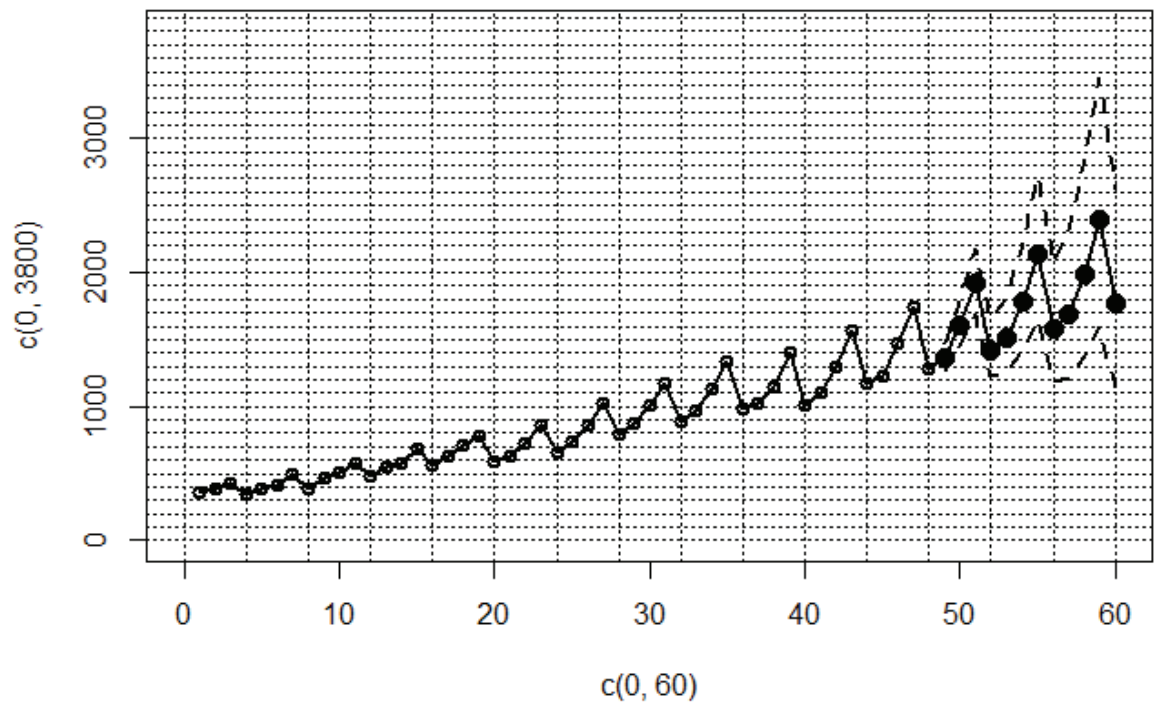


Figure 8. 18 Comparison of forecasts for the TIAP time series

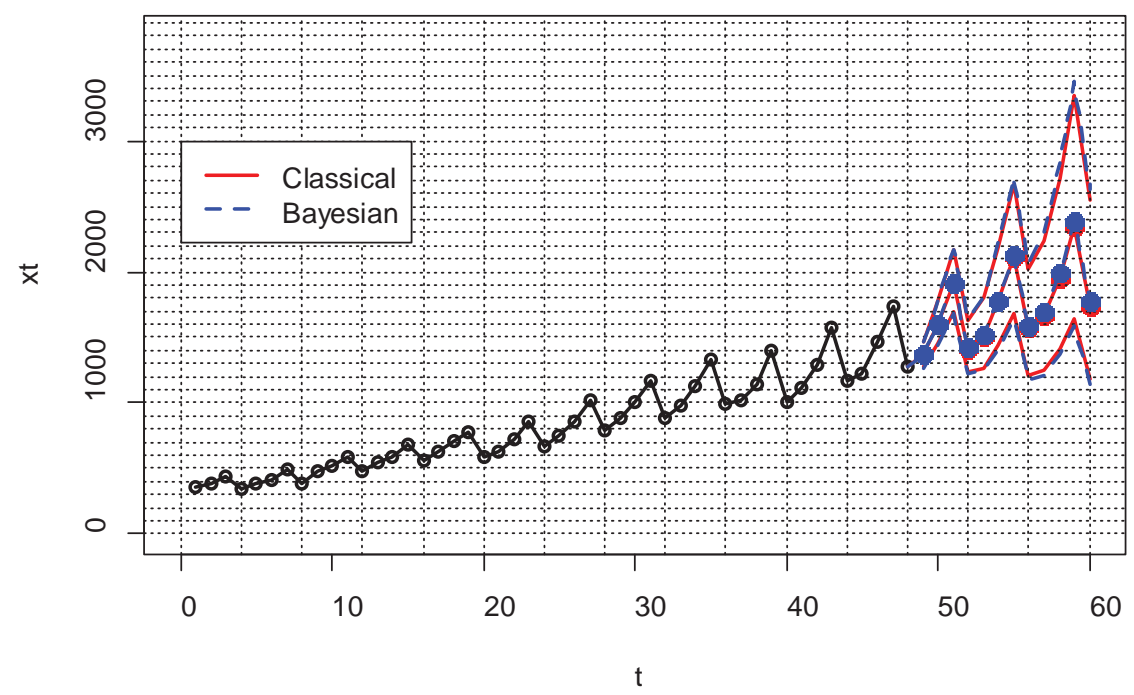

Figure 8.19 Detail in Figure 8.18

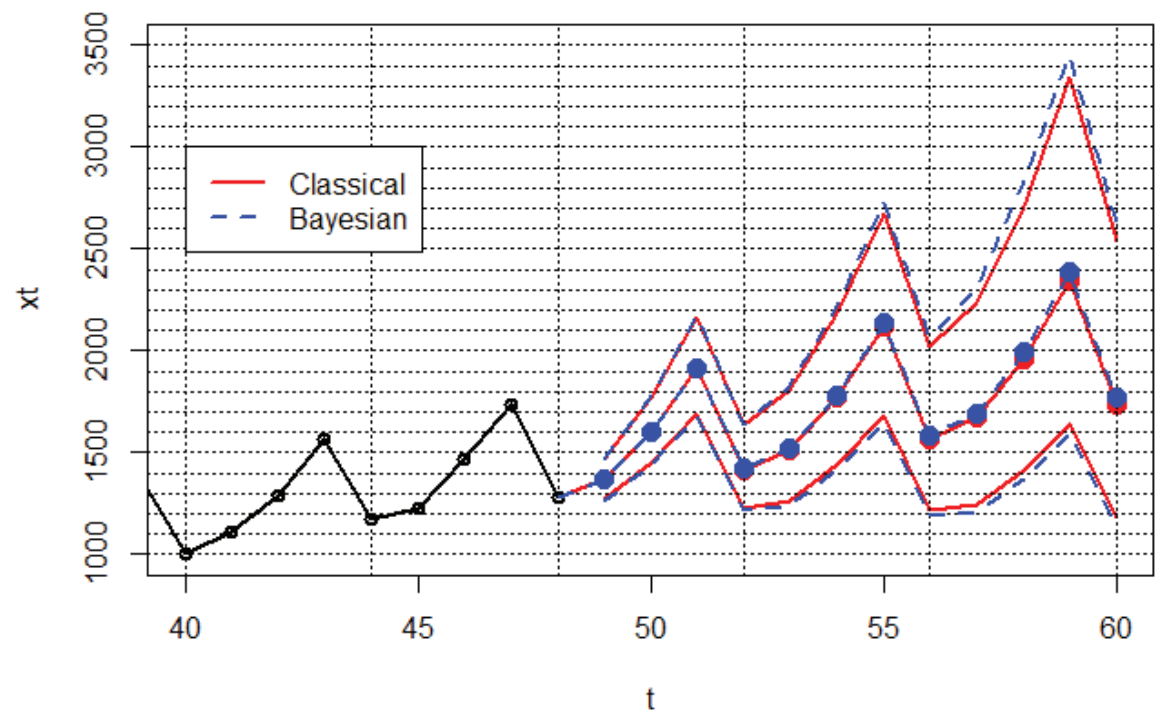


We see from Figures 8.18 and 8.19 that the two approaches to inference have yielded very similar results, at least as regards prediction.

The Bayesian approach has produced 95\% prediction intervals which are slightly wider than those obtained via the classical approach.

It may be argued that such wider intervals are more appropriate, since the classical approach makes forecasts without taking into account any uncertainty in the parameter estimates.

By contrast, the Bayesian approach to forecasting does take into account that uncertainty.

To conclude, we report that the fitted model for the TIAP time series $x_{t}$ is given by

$$
y_{t}=\nabla_{4} \nabla \log x_{t}
$$

with

$$
y_{t}=\hat{w}_{t}+\hat{\Theta}_{1} \hat{w}_{t-4}, \quad \hat{w}_{t} \sim \text { iid } N\left(0, \hat{\sigma}^{2}\right) \text {, }
$$

where, via classical analysis:

$$
\begin{aligned}
& \hat{\Theta}_{1}=-0.4927(\mathrm{SE}=0.1201) \\
& \hat{\sigma}^{2}=0.0013,
\end{aligned}
$$

and where, via Bayesian analysis:

$$
\begin{aligned}
& \hat{\Theta}_{1}=-0.4661(\mathrm{SE}=0.1266) \\
& \hat{\sigma}^{2}=0.0015 .
\end{aligned}
$$




\section{R and WinBUGS Code for Exercise 8.4}

\# Classical analysis in $\mathrm{R}$

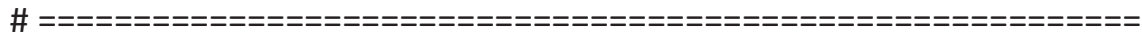

$x<-$

$c(362,385,432,341,382,409,498,387,473,513,582,474$,

$544,582,681,557,628,707,773,592,627,725,854,661,742$,

$854,1023,789,878,1005,1173,883,972,1125,1336,988,1020$,

$1146,1400,1006,1108,1288,1570,1174,1227,1468,1736,1283$ )

$\mathrm{n}<-$ length $(\mathrm{x}) ; \mathrm{n} \# 48$

$\mathrm{X} 11(\mathrm{w}=8, \mathrm{~h}=9) ; \operatorname{par}(\operatorname{mrrow}=\mathrm{c}(3,2))$

plot(x,type="I"); abline $(v=$ seq $(0,48,4), h=s e q(0,2000,100)$, Ity=3)

plot $(\log (x)$,type="l"); abline $(v=s e q(0,48,4)$, Ity=3)

plot(diff( $(\log (x))$,type="I"); abline $(v=\operatorname{seq}(0,48,4)$, Ity=3)

plot(diff(diff(log(x),lag=4)), type="I"); abline $(v=\operatorname{seq}(0,48,4)$, Ity=3)

$y<-\operatorname{diff}(\operatorname{diff}(\log (x), \operatorname{lag}=4))$

$\operatorname{acf}(y, \operatorname{lag}=24)$

$\operatorname{pacf}(y, \operatorname{lag}=24)$

fit1 <- $\operatorname{arima}(\log (x)$, order $=c(0,1,0)$, seasonal=list $(\operatorname{order}=c(1,1,0)$, period=4) $)$

tsdiag(fit1); fit1

\# sar1

$\# \quad-0.4990$

\# s.e. 0.1417

\# sigma^2 estimated as 0.001310: $\log$ lik. $=81.12$, aic $=-158.24$

fit2 $<-\operatorname{arima}(\log (x)$, order $=c(0,1,0)$, seasonal=list(order $=c(0,1,1)$, period $=4)$ ) tsdiag(fit2); fit2

\# sma1

\# $\quad-0.4927$

\# s.e. 0.1201

\# sigma^$^{\wedge} 2$ estimated as $\underline{0.001306}$ : log lik. $=81.2$, aic $=-158.4$

\# There's not much to distinguish the two fits.

\# The second one is marginally better.

\# Let's now display the diagnostics for that fit (again). 
fit <- fit2; tsdiag(fit)

\# We see that the residuals from the fit are well-behaved, \# and their sample ACF is consistent with that of white noise.

\# Let's also look at some other diagnostics. These turn out to be OK too.

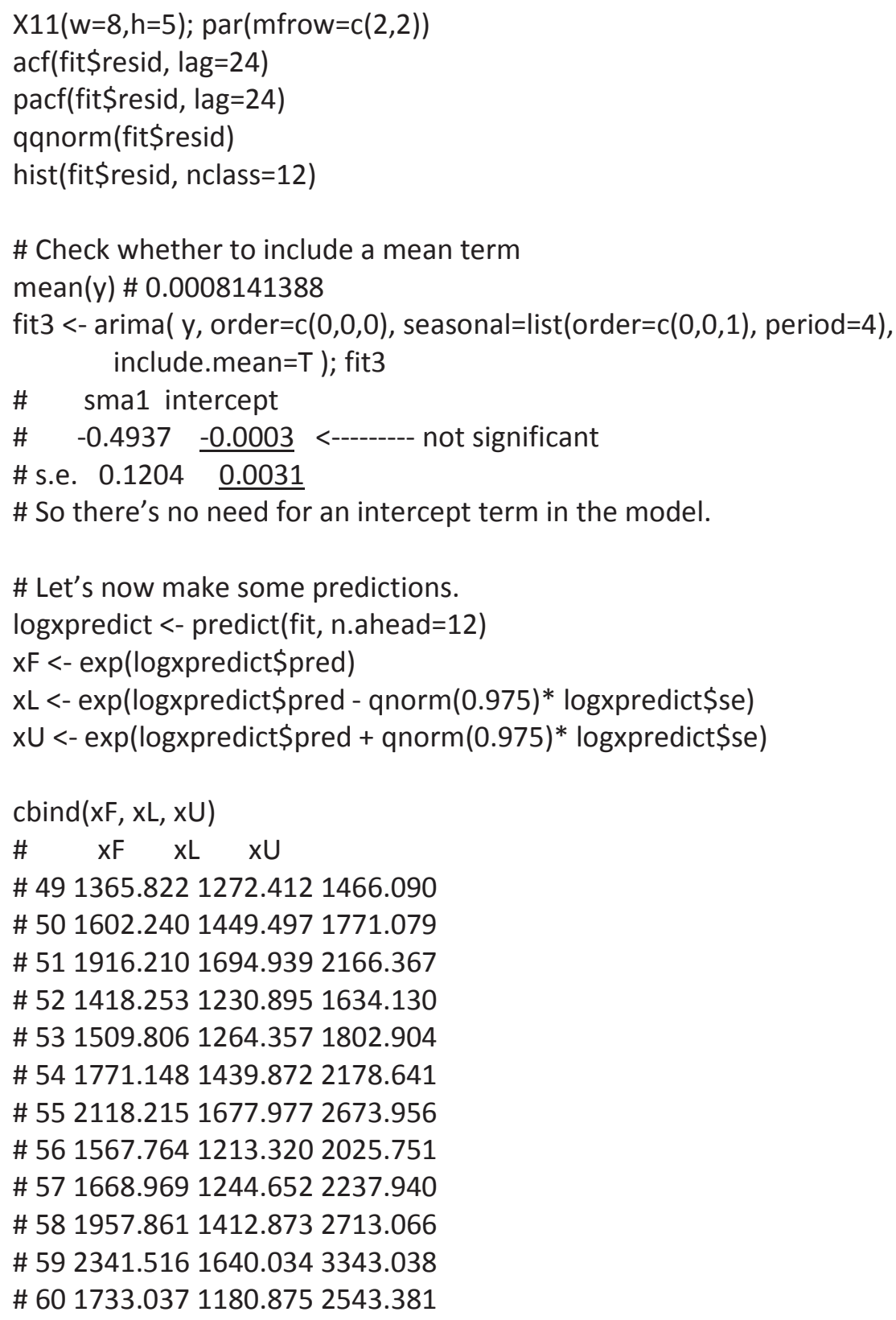


$\mathrm{X} 11(\mathrm{~h}=5)$; $\operatorname{par}(\operatorname{mfrow}=c(1,1)) ; \operatorname{plot}(\mathrm{c}(0,60), c(0,3800)$, type=" $\mathrm{n} ")$

lines $(x, \operatorname{lwd}=2)$; points $(x, \operatorname{lwd}=2)$;

points $((n+1):(n+12), x F, p c h=16$, cex $=1.5)$;

lines $(n:(n+12), c(x[n], x F)$, Ity=1,lwd=2)

\# points $((n+1):(n+12), x L, p c h=16)$;

lines $((n+1):(n+12), x L, \mid t y=2, I w d=2)$

\# points $((n+1):(n+12), x U, p c h=16)$;

lines $((n+1):(n+12), x U,|t y=2| l w d=2$,

abline $(v=\operatorname{seq}(0,100,4), h=\operatorname{seq}(0,4000,100)$, Ity=3) \# OK....

\# Bayesian reanalysis in $\mathrm{R}$ and WinBUGS

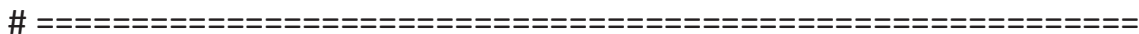

\# Assume that R (v3.0.1) is installed in C:/R-3.0.1

\# and WinBUGS (v4.1.3) is installed in C:/WinBUGS14

install.packages("R2WinBUGS") \# Not necessary if done previously

library("R2WinBUGS") \# Necessary every time R is started

\# Make the following directory exists: C:/R-3.0.1/BugsOut/

\# Create a file called C:/R-3.0.1/BugsCode2.txt with the following:

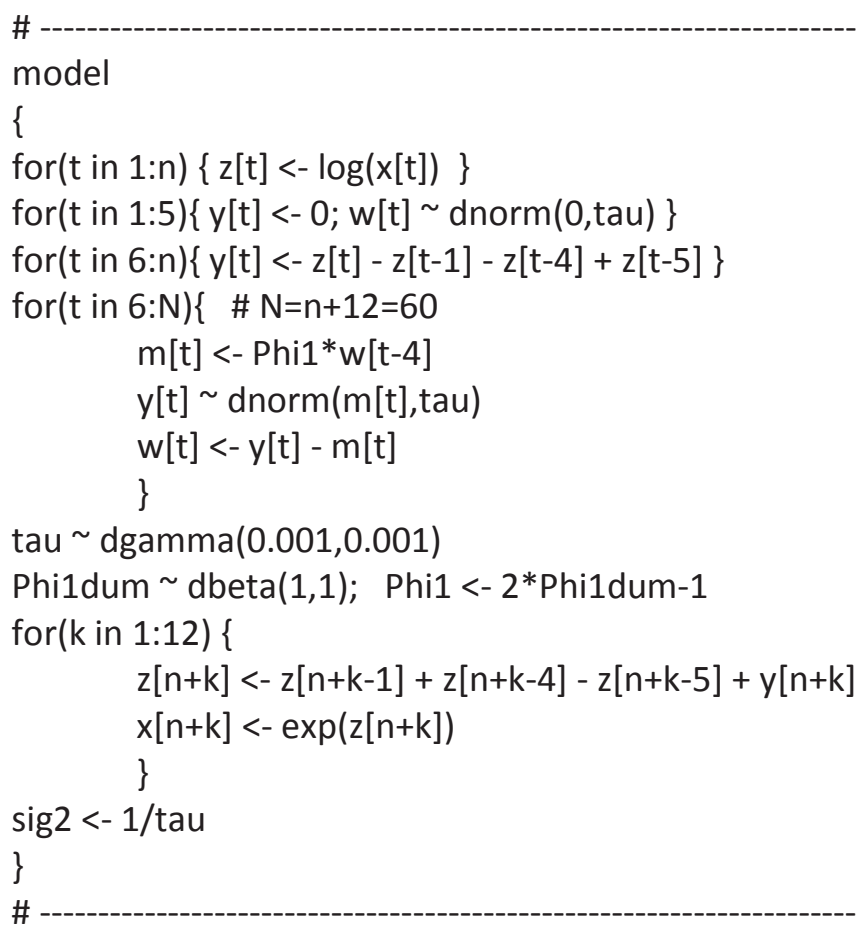


\# NB: We can't specify Phi1 dunif(-1,1). This causes an error. \# Update in March 2014: Phi1 dunif(-1,1) works in WinBUGS 1.4.3.

$x<-c(362,385,432,341,382,409,498,387,473,513,582,474$, $544,582,681,557,628,707,773,592,627,725,854,661,742$, $854,1023,789,878,1005,1173,883,972,1125,1336,988,1020$, $1146,1400,1006,1108,1288,1570,1174,1227,1468,1736,1283$, $\mathrm{NA}, \mathrm{NA}, \mathrm{NA}, \mathrm{NA}, \quad \mathrm{NA}, \mathrm{NA}, \mathrm{NA}, \mathrm{NA}, \mathrm{NA}, \mathrm{NA}, \mathrm{NA}, \mathrm{NA})$

$\mathrm{n}<-48 ; \mathrm{N}<-60 ;$ data <- list("n","N","x")

inits <- function()\{ list(tau=1, Phi1dum=0.5) $\}$

parameters <- c("sig2", "Phi1", "x")

sim <- bugs(data, inits, parameters, n.thin=1, model.file= "C:/R-3.0.1/BugsCode2.txt", n.chains $=1$, n.iter $=6000$, n. burnin=1000, DIC $=$ FALSE, bugs.directory = "C:/WinBUGS14/", working.directory = "C:/R-3.0.1/BugsOut/")

\# This starts WinBUGS, runs the BUGS code for 6000 iterations, closes \# WinBUGS, and creates a number of files in the working directory. These \# files contain information which can also be accessed within $\mathrm{R}$, as follows.

print(sim, digits=4)

\# Inference for Bugs model at "C:/R-3.0.1/BugsCode2.txt", fit using WinBUGS, \# 1 chains, each with 6000 iterations (first 1000 discarded) \# n.sims $=5000$ iterations saved

\# $\quad$ mean sd $2.5 \% \quad 25 \% \quad 50 \% \quad 75 \% \quad 97.5 \%$

$\begin{array}{lllllllll}\text { \# sig2 } & 0.0015 & 0.0003 & 0.0009 & 0.0012 & 0.0014 & 0.0016 & 0.0022\end{array}$ $\begin{array}{llllllll}\text { \# Phi1 } & -0.4661 & 0.1266 & -0.6910 & -0.5548 & -0.4740 & -0.3865 & -0.1944\end{array}$ \# x[49] 1367.182052 .61891265 .00001332 .00001365 .00001402 .0000 \# 1472.0000

\# x[50] 1605.974686 .27901443 .00001547 .00001603 .00001662 .0000 \# 1781.0000

\# x[51] 1918.2346 124.7788 1681.9750 1835.00001914.0000 2000.0000 \# 2172.0250

\# x[52] 1422.9222 107.4501 1220.97501350 .00001420 .00001491 .0000 \# 1641.0000 \# x[53] 1517.8472146 .01191247 .97501418 .74991514 .00001610 .0000 \# 1822.0000 \# x[54] 1783.4306201 .98341415 .00001645 .00001777 .00001908 .2500 \# 2217.0000 
\# x[55] 2133.7016273 .12911646 .97501946 .75002119 .00002306 .0000 \# 2724.0000

\# x[56] 1584.1955 223.5842 1187.97501431.00001576.00001720.2499 \# 2066.0000

\# x[57] 1693.4548 276.4929 1211.9750 1499.74991674.00001857.0000 \# 2309.0750

\# x[58] 1992.9153 364.38491370 .97501742 .74991968 .00002204 .0000 \# 2837.0999

\# x[59] 2388.4000 476.7169 1589.89992058.75002345.00002668.0000 \# 3453.0250

\# x[60] 1775.0647381.9082 1137.00001511.00001735.00001992.0000 \# 2628.1249

help(bugs) \# To get info on how to do the following...

Phi1v <- sim\$sims.list\$Phi1; sig2v <- sim\$sims.list\$sig2

$\mathrm{xm}<-\operatorname{sim} \$ \operatorname{sims}$. list $\$ \mathrm{x}$

$\operatorname{par}(\operatorname{mfrow}=\mathrm{c}(2,2))$

hist (Phi1v, breaks=20); hist(sig2v, breaks $=20)$

hist $(x m[, 1]$, breaks=20); hist( $x m[, 2]$, breaks $=20)$

\# Let's now make the forecasts of the series using the BUGS output.

$\mathrm{xF} 2<-\mathrm{xF} ; \mathrm{xL2}<-\mathrm{xL} ; \mathrm{xU} 2<-\mathrm{xU}$; for(t in $1: 12)\{$

$x F 2[t]<-\operatorname{mean}(x m[, t])$

$x \mathrm{~L} 2[\mathrm{t}]<-$ quantile $(\mathrm{xm}[\mathrm{t}], 0.025)$

$\mathrm{xU} 2[\mathrm{t}]<-$ quantile $(\mathrm{xm}[, \mathrm{t}], 0.975) \quad\}$ \# Calc. estimates

$\operatorname{par}(\operatorname{mfrow}=c(1,1)) ; \operatorname{plot}(c(0,60), c(0,3800)$, type $=" n ")$

lines $(x, \operatorname{lwd}=2)$; points $(x, \operatorname{lwd}=2)$

points $((n+1):(n+12), x F 2, p c h=16$, cex $=1.5)$;

lines $(n:(n+12), c(x[n], x F 2), \mid t y=1, l w d=2)$

lines $((n+1):(n+12), x L 2, \mid t y=2, I w d=2)$

lines $((n+1):(n+12), x \cup 2, \mid t y=2, I w d=2)$

abline $(\mathrm{v}=\mathrm{seq}(0,100,4), \mathrm{h}=\mathrm{seq}(0,4000,100), \mathrm{Ity}=3) \# \quad$ OK..... 
\# Next we graph both sets of forecasts together in a single plot, \# and then produce a close-up in that single plot, as follows:

$\mathrm{X} 11(\mathrm{~h}=5) ; \operatorname{par}(\operatorname{mfrow}=\mathrm{c}(1,1))$;

plot $(c(0,60), c(0,3800)$, type="n", xlab="t", ylab="xt")

lines $(x, \operatorname{lwd}=2)$; points $(x, \operatorname{lwd}=2)$

points $((n+1):(n+12), x F, p c h=16$, cex=1.5, col="red");

lines(n:(n+12), c(x[n],xF), Ity=1,lwd=2, col="red")

lines $((n+1):(n+12), x L$, lty=1, lwd=2, col="red")

lines $((n+1):(n+12), x U, \mid t y=1$, lwd=2, col="red")

abline $(v=\operatorname{seq}(0,100,4), h=\operatorname{seq}(0,4000,100)$, Ity=3)

points $((n+1):(n+12), x F 2, p c h=16$, cex=1.5, col="blue" );

lines(n: $(n+12), c(x[n], x F 2)$, Ity=2, Iwd=2, col="blue ")

lines $((n+1):(n+12), x L 2$, Ity=2, Iwd=2, col="blue ")

lines $((n+1):(n+12), x \cup 2$, Ity=2, Iwd=2, col="blue ")

legend(0,3000,c("Classical","Bayesian"), Ity=c(1,2),

I $w d=c(2,2)$, col=c("red", "blue"), bg="white" )

$\operatorname{par}(\operatorname{mfrow}=c(1,1))$

plot(c(40,60),c(1000,3500), type="n", xlab="t", ylab="xt")

lines $(x, \operatorname{lwd}=2)$; points $(x, \operatorname{lwd}=2)$

points $((n+1):(n+12), x F, p c h=16$, cex=1.5, col="red");

lines(n:(n+12), c(x[n],xF), Ity=1,lwd=2, col="red")

lines $((n+1):(n+12), x L$, lty=1, Iwd=2, col="red")

lines $((n+1):(n+12), x U,|t y=1| l w d=$,2 , col="red")

abline $(v=\operatorname{seq}(0,100,4), h=\operatorname{seq}(0,4000,100)$, Ity=3)

points $((n+1):(n+12), x F 2, p c h=16$, cex $=1.5$, col="blue" );

lines $(n:(n+12), c(x[n], x F 2)$, lty=2, I $w d=2$, col="blue ")

lines $((n+1):(n+12), x L 2, \mid$ ty $=2$, I $w d=2$, col="blue ")

lines $((n+1):(n+12), x \cup 2$, Ity=2, Iwd=2, col="blue ")

legend(40,3000,c("Classical", "Bayesian"), Ity=c(1,2),

lwd=c(2,2), col=c("red", "blue"), bg="white" ) 


\section{Bayesian Finite Population Theory}

\section{I Introduction}

In this chapter we will focus on the topic of Bayesian methods for finite population inference in the sample survey context. We have previously touched on this topic when considering posterior predictive inference of 'future' values in the context of the normal-normal-gamma model. The topic will now be treated more generally and systematically.

There are many and various ways in which Bayesian finite population inference can be categorised, for example:

- situations with and without prior information being available

- sampling with and without replacement

- Monte Carlo based methods versus deterministic (or 'exact') methods

- situations with and without auxiliary information being available

- scenarios where a superpopulation variance is known and where it is unknown

- sampling with equal probabilities versus unequal probabilities

- sampling mechanisms that are ignorable versus nonignorable (i.e. biased)

- cases where the order of sampling is known versus where that order is unknown

- cases with full response versus where some sampled units fail to respond.

Each of these categories can in turn be broken down further. For example, Monte Carlo based techniques may or may not require Markov chain Monte Carlo methods for generating the sample required for inference. We see there is potentially a vast subject ground to cover.

We will begin with a description of some basic general concepts, notation and terminology in relation to finite population modelling in the Bayesian framework, with a focus on simple random sampling without replacement (SRSWOR). We then illustrate these ideas by way of a series of exercises which also feature some other concepts such as simple random sampling 
with replacement (SRSWR), nonignorable sampling schemes, and covariate data. Some of these ideas will be taken up again in later chapters.

We defer discussion of Bayesian finite population models involving normal (i.e. Gaussian) data to the next chapter (Chapter 10), where such models are the focus and treated in detail. In Chapter 11 we will discuss data transformations, inference on non-standard quantities of interest, and frequentist properties of Bayesian estimators in a finite population context, including the notions of model bias and design bias. Chapter 12 will focus on the issues of biased sampling and nonignorable nonresponse.

The exposition in Chapters 9 to 12 is largely theoretical but does include mention of several real world applications, including on-site sampling of recreation parks, oil discovery, and correcting for self-selection bias in volunteer surveys. Further discussion of the role that Bayesian methods and prior information play in survey sampling and finite population inference can be found in Rao (2011). This paper also lists many other papers and books on this and related topics, for example Ericson (1969) and Särndal, Swensson and Wretman (1992).

\subsection{Finite population notation and terminology}

Consider a finite population of $N$ units labelled $i=1, \ldots, N$, and let $y_{i}$ be the value of the $i$ th unit for some observable variable of interest.

Define $y=\left(y_{1}, \ldots, y_{N}\right)$ as the population vector.

Suppose that $n$ units are selected from the finite population without replacement.

We refer to $n$ as the sample size and to $m=N-n$ as the nonsample size.

Let $s=\left(s_{1}, \ldots, s_{n}\right)$ be the vector of the ordered labels of the sampled units.

Also let $r=\left(r_{1}, \ldots, r_{m}\right)$ be the vector of the ordered labels of the nonsampled units, i.e. those remaining.

Define $y_{s}=\left(y_{s_{1}}, \ldots, y_{s_{n}}\right)$ to be the sample vector, and likewise define $y_{r}=\left(y_{r_{1}}, \ldots, y_{r_{m}}\right)$ to be the nonsample vector. 
Note 1: With the above definitions, it is always true that

$$
s_{1}<\ldots<s_{n}
$$

and

$$
r_{1}<\ldots<r_{m},
$$

irrespective of the order in which the population units may actually be sampled. Also,

$$
\left\{s_{1}, \ldots, s_{n}, r_{1}, \ldots, r_{m}\right\}=\{1, \ldots, N\} .
$$

Note 2: For mathematical convenience, the population, sample and nonsample vectors may later sometimes be defined as the column vectors

$$
y=\left(y_{1}, \ldots, y_{N}\right)^{\prime}=\left(\begin{array}{c}
y_{1} \\
\vdots \\
y_{N}
\end{array}\right), y_{s}=\left(y_{s_{1}}, \ldots, y_{s_{n}}\right)^{\prime} \text { and } y_{r}=\left(y_{r_{1}}, \ldots, y_{r_{m}}\right)^{\prime},
$$

respectively.

Also, the population vector may sometimes be written using upper case letters, as $Y=\left(Y_{1}, \ldots, Y_{N}\right)$ or $Y=\left(Y_{1}, \ldots, Y_{N}\right)^{\prime}$. For the remainder of this chapter, these alternative notations will not be used.

Example: Suppose that we select $n=3$ units from a finite population of size $N=7$ and obtain units 4, 5 and 2 (in that order, or any other order).

Then the nonsample size is $m=N-n=4$ and:

$$
\begin{array}{ll}
y=\left(y_{1}, \ldots, y_{7}\right) & \\
S=\left(s_{1}, s_{2}, s_{3}\right)=(2,4,5), & y_{s}=\left(y_{2}, y_{4}, y_{5}\right) \\
r=\left(r_{1}, r_{2}, r_{3}, r_{4}\right)=(1,3,6,7), & y_{r}=\left(y_{1}, y_{3}, y_{6}, y_{7}\right) .
\end{array}
$$

\subsection{Bayesian finite population models}

Consider a finite population vector $y$ which may be thought of as having been generated from some probability distribution which depends on a parameter $\theta$ (possibly a vector).

Also suppose that a sample of size $n$ is drawn from the finite population without replacement according to some probability distribution for $s$. 
This scenario may be expressed in terms of a Bayesian finite population model with the following form:
$f(s \mid y, \theta) \quad$ (the probability of obtaining sample $s$ for given values of $y$ and $\theta$ )
$f(y \mid \theta)$ (the model density of the finite population vector $f(\theta)$ given $\theta$ ) (the prior density of the parameter).

Suppose that we have data of the form $D=\left(s, y_{s}\right)$ and are interested in a quantity $Q=g(y, \theta)$, for some function $g$. Then the task is to determine the distribution of $Q$ given $D$.

This distribution will be based on the joint distribution of the two unobserved quantities $\theta$ and $y_{r}$, given the two observed quantities, namely:

$s \quad$ (which tells us which units are sampled); and

$y_{s} \quad$ (the vector of the values of the sampled units).

Thus, inference on the quantity of interest $Q=g(y, \theta)$ is based on the density $f(Q \mid D)$, which in turn is based on the density

$$
\begin{aligned}
f\left(\theta, y_{r} \mid s, y_{s}\right) & \propto f\left(\theta, y_{s}, y_{r}, s\right) \\
& =f(\theta) f\left(y_{s}, y_{r} \mid \theta\right) f\left(s \mid y_{s}, y_{r}, \theta\right) .
\end{aligned}
$$

Note 1: The values of $s$ and $r$ here are fixed at their observed values defined by the data. Thus, given $D=\left(s, y_{s}\right)$, we may always express $Q=g(y, \theta)$ as $h\left(\left(y_{s}, y_{r}\right), \theta\right)$ for some function $h$ (which will in many cases be the same function as $g$ ), and there should be no ambiguity in the meaning of quantities such as $f\left(y_{s}, y_{r} \mid \theta\right)$ in (9.1).

Note 2: We have specified the sampling mechanism in terms of the quantity $s$ which tells us which units are sampled but not the order in which they are sampled. In some cases it may be appropriate to replace $f(s \mid y, \theta)$ in the model by $f(L \mid y, \theta)$, where

$$
L=\left(L_{1}, \ldots, L_{n}\right)
$$

is the vector of the labels of the selected units in the order that they are sampled. $L$ provides more information than $s$, which is a function of $L$. 
Note 3: We have assumed that sampling is without replacement. If sampling is with replacement, it may be appropriate to replace $f(s \mid y, \theta)$ or $f(L \mid y, \theta)$ in the model by $f(I \mid y, \theta)$, where

$$
I=\left(I_{1}, \ldots, I_{N}\right) \text {, }
$$

and where $I_{i}$ is the number of times that population unit $i$ is sampled.

In this case it may be necessary to modify the notation to account for the number of distinct units sampled, previously the fixed constant $n$, due to the possibility of multiple selections under sampling with replacement.

Example 1: Suppose that we sample units 4, 5 and 2, in that order. Then $L=\left(L_{1}, L_{2}, L_{3}\right)=(4,5,2)$ and $s=(2,4,5)$. Note that $s$ is a function of $L$.

Example 2: Suppose we sample units 3, 5 and 3, in that order. Then $L=\left(L_{1}, L_{2}, L_{3}\right)=(3,5,3)$ and $I=(0,0,2,0,1)$. In this case, we write $s=\left(s_{1}, \ldots, s_{d}\right)=\left(s_{1}, s_{2}\right)=(3,5)$ as the ordered vector of distinct labels for the units sampled. Here, $d$ is the number of distinct units sampled (a random variable with realised value 2), in contrast to $n$, the total number of selections (a fixed constant equal to 3). Note that $d$ is a function of $s$, which is a function of $I$, which in turn is a function of $L$.

\subsection{Two types of sampling mechanism}

There are basically two types of sampling mechanism in the context of the above model, data and quantity of interest. These two types correspond to two distinct cases, as follows:

(i) where $f(Q \mid D)$ remains exactly the same if the sampling density $f\left(s \mid y_{s}, y_{r}, \theta\right)$ is omitted from the calculation at equation (9.1); in this case we say that the sampling mechanism is ignorable (or unbiased)

(ii) where $f(Q \mid D)$ changes in some way if the sampling density $f\left(s \mid y_{s}, y_{r}, \theta\right)$ is omitted from the calculation at equation (9.1); we then say the sampling mechanism is nonignorable (or biased). 
Perhaps the simplest example of an ignorable sampling mechanism is simple random sampling without replacement (SRSWOR), for which

$$
f(s \mid y, \theta)=\left(\begin{array}{c}
N \\
n
\end{array}\right)^{-1}, s \in S(s),
$$

where

$$
S(s)=\{(1, \ldots, n),(1,2, \ldots, n-1, n+1), \ldots .,(N-n+1, \ldots, N)\} .
$$

is the sample space for $s$ (the set of all possible combinations of $n$ integers taken from $N$ ).

In this case, $f(s \mid y, \theta)$ does not depend on $y$ or $\theta$ at all and so may also be written simply as $f(s)$. This then guarantees that

$$
f\left(s \mid y_{s}, y_{r}, \theta\right)=f(s)=\left(\begin{array}{c}
N \\
n
\end{array}\right)^{-1}
$$

at the single observed value of $s$, whatever that value may be.

Therefore, the joint density of the two unknowns is

$$
\begin{aligned}
f\left(\theta, y_{r} \mid s, y_{s}\right) & \propto f\left(\theta, y_{s}, y_{r}, s\right) \\
& =f(\theta) f\left(y_{s}, y_{r} \mid \theta\right) f\left(s \mid y_{s}, y_{r}, \theta\right) \\
& \propto f(\theta) f\left(y_{s}, y_{r} \mid \theta\right) \times 1,
\end{aligned}
$$

which is the same as (9.1) but with $f\left(s \mid y_{s}, y_{r}, \theta\right)$ omitted.

This result tells us that $f(Q \mid D)$ will be the same when the sampling mechanism density $f\left(s \mid y_{s}, y_{r}, \theta\right)$ is 'ignored' in the model, so to speak.

\subsection{Two types of inference}

There are basically two types of inference in the context of the above model, data and quantity of interest:

(a) where $Q$ does not depend on $y$, in which case inference is on $Q=g(\theta)$ (a function of only $\theta$ ) and may be called analytic inference or infinite population inference or superpopulation inference

(b) where $Q$ does not depend on $\theta$, in which case inference is on $Q=g(y)$ (a function of only $y$ ) and may be called descriptive inference or finite population inference or predictive inference. 


\subsection{Analytic inference}

In the case of analytic inference, this is based solely on the posterior density of the model parameter $\theta$, namely

$$
\begin{aligned}
f(\theta \mid D) & =f\left(\theta \mid s, y_{s}\right) \\
& \propto f\left(\theta, s, y_{s}\right) \\
& =\int f\left(\theta, s, y_{r}, y_{s}\right) d y_{r} \\
& =f(\theta) \int f\left(y_{s}, y_{r} \mid \theta\right) f\left(s \mid y_{s}, y_{r}, \theta\right) d y_{r} .
\end{aligned}
$$

Now suppose further that the sampling mechanism is ignorable. In that case,

$$
\begin{gathered}
f(\theta \mid D) \propto f(\theta) \int f\left(y_{s}, y_{r} \mid \theta\right) \times 1 d y_{r} \\
\text { since } f\left(s \mid y_{s}, y_{r}, \theta\right) \text { may be ignored } \\
=f(\theta) f\left(y_{s} \mid \theta\right) \int f\left(y_{r} \mid y_{s}, \theta\right) d y_{r} \\
\quad \text { since } f\left(y_{s}, y_{r} \mid \theta\right)=f\left(y_{s} \mid \theta\right) f\left(y_{r} \mid \theta, y_{s}\right) \\
=f(\theta) f\left(y_{s} \mid \theta\right) \\
\text { since } \int f\left(y_{r} \mid y_{s}, \theta\right) d y_{r}=1 \text { for all } \theta .
\end{gathered}
$$

Thus the posterior density of $\theta$ is obtained in exactly the same way as in previous chapters.

Note: As stressed earlier, it is to be understood that $s$ in $f\left(y_{s} \mid \theta\right)$ here is fixed at its observed value. With this understanding, we will sometimes abbreviate $f(\theta \mid D)=f\left(\theta \mid s, y_{s}\right)$ as $f\left(\theta \mid y_{s}\right)$.

Example: If $s=(2,4,5)$, then $y_{s}$ means specifically $\left(y_{2}, y_{4}, y_{5}\right)$. Thus, in this context, $y_{s}$ does not refer to the vector $\left(y_{s_{1}}, y_{s_{2}}, y_{s_{3}}\right)$ with the subscripts $s_{1}, s_{2}, s_{3}$ as random variables. 


\subsection{Descriptive inference}

In the case of descriptive inference, this is based solely on the predictive density of the nonsample vector $y_{r}$, namely

$$
\begin{aligned}
& f\left(y_{r} \mid D\right)=f\left(y_{r} \mid s, y_{s}\right) \propto f\left(s, y_{s}, y_{r}\right) \\
& \quad=\int f\left(\theta, s, y_{r}, y_{s}\right) d \theta=\int f(\theta) f\left(y_{s}, y_{r} \mid \theta\right) f\left(s \mid y_{s}, y_{r}, \theta\right) d \theta .
\end{aligned}
$$

Now suppose further that the sampling mechanism is ignorable. In that special case,

$$
\begin{aligned}
f\left(y_{r} \mid D\right) & \propto \int f(\theta) f\left(y_{s}, y_{r} \mid \theta\right) \times 1 d \theta \\
& \text { since } f\left(s \mid y_{s}, y_{r}, \theta\right) \text { may be ignored } \\
= & \int f\left(y_{r} \mid y_{s}, \theta\right) f(\theta) f\left(y_{s} \mid \theta\right) d \theta \\
\propto \int f\left(y_{r} \mid y_{s}, \theta\right) f\left(\theta \mid y_{s}\right) d \theta & \text { since } f\left(\theta \mid y_{s}\right) \propto f(\theta) f\left(y_{s} \mid \theta\right) .
\end{aligned}
$$

So the predictive density of $y_{r}$ is obtained in exactly the same way as in previous chapters.

Note: As before, it is to be understood that $s$ and $r$ in $f\left(y_{r} \mid y_{s}, \theta\right)$ and $f\left(\theta \mid y_{s}\right)$ are fixed at their observed values. With this understanding, we will sometimes write

$$
f\left(y_{r} \mid D\right)=f\left(y_{r} \mid s, y_{s}\right) \quad \text { as } \quad f\left(y_{r} \mid y_{s}\right) .
$$

More generally, we will sometimes write

$$
f\left(\theta, y_{r} \mid D\right)=f\left(\theta, y_{r} \mid s, y_{s}\right) \quad \text { as } \quad f\left(\theta, y_{r} \mid y_{s}\right)
$$

and

$$
f(Q \mid D)=f\left(Q \mid s, y_{s}\right) \quad \text { as } \quad f\left(Q \mid y_{s}\right) .
$$

Example: If $s=(2,4,5)$ and $N=7$ then $y_{s}$ means $\left(y_{2}, y_{4}, y_{5}\right)$ and $y_{r}$ means $\left(y_{1}, y_{3}, y_{6}, y_{7}\right)$. 


\section{Exercise 9.I A Bernoulli finite population model with ignorable sampling}

A finite population of size $N=4$ consists of values that are independently and identically distributed (iid) Bernoulli with parameter $\theta$, where $\theta$ is a priori equally likely to be $1 / 4$ or $1 / 2$ (with no other possibilities).

We sample $n=2$ units from the finite population according to SRSWOR.

Units 2 and 4 are sampled, and both have the value 1 .

(a) Find the posterior distribution of $\theta$.

(b) Find the predictive distribution of the finite population total, namely $y_{T}=y_{1}+\ldots+y_{N}$.

\section{Solution to Exercise 9.I}

(a) The Bayesian model here may be written:

$$
\begin{aligned}
& f(s \mid y, \theta)=\left(\begin{array}{l}
N \\
n
\end{array}\right)^{-1}=\left(\begin{array}{l}
4 \\
2
\end{array}\right)^{-1}=\frac{1}{6}, \\
& s=(1,2),(1,3),(1,4),(2,3),(2,4),(3,4) \\
& f(y \mid \theta)=\prod_{i=1}^{N} \theta^{y_{i}}(1-\theta)^{1-y_{i}} \\
& \quad \text { (the model density of the finite population values) } \\
& f(\theta)=1 / 2, \theta=1 / 4,1 / 2 \quad \text { (the prior density of the parameter). }
\end{aligned}
$$

The observed sample data is

$$
D=\left(s, y_{s}\right)=\left(\left(s_{1}, s_{2}\right),\left(y_{s_{1}}, y_{s_{2}}\right)\right)=\left((2,4),\left(y_{2}, y_{4}\right)\right)=((2,4),(1,1)),
$$

and the nonsample vector is $y_{r}=\left(y_{r_{1}}, y_{r_{2}}\right)=\left(y_{1}, y_{3}\right) \in\{0,1\}^{2}$.

The sampling mechanism is ignorable, and so

$$
\begin{aligned}
& f(\theta \mid D) \propto f(\theta) f\left(y_{s} \mid \theta\right) \propto 1 \times \prod_{i \in s} \theta^{y_{i}}(1-\theta)^{1-y_{i}} \\
& =\theta^{2} \quad \text { since } n=2 \text { and } y_{i}=1 \text { for all } i \in s \\
& =\left\{\begin{array}{l}
(1 / 4)^{2}=1 / 16, \theta=1 / 4 \\
(1 / 2)^{2}=4 / 16, \theta=1 / 2 .
\end{array}\right.
\end{aligned}
$$


It follows that $f(\theta \mid D)=\left\{\begin{array}{l}1 / 5, \theta=1 / 4 \\ 4 / 5, \theta=1 / 2\end{array}\right.$

(b) Next, observe that $f\left(y_{r} \mid D, \theta\right)=\left\{\begin{array}{cc}(1-\theta)^{2}, & y_{r}=(0,0) \\ (1-\theta) \theta, & y_{r}=(0,1) \\ \theta(1-\theta), & y_{r}=(1,0) \\ \theta^{2}, & y_{r}=(1,1) .\end{array}\right.$

This implies that

$$
\begin{aligned}
f\left(y_{r} \mid D\right)= & \sum_{\theta} f\left(y_{r} \mid D, \theta\right) f(\theta \mid D) \\
& =\left\{\begin{array}{l}
\left(1-\frac{1}{4}\right)^{2} \frac{1}{5}+\left(1-\frac{2}{4}\right)^{2} \frac{4}{5}=\frac{25}{80}, y_{r}=(0,0) \\
\left(1-\frac{1}{4}\right) \frac{1}{4} \frac{1}{5}+\left(1-\frac{2}{4}\right) \frac{2}{4} \frac{4}{5}=\frac{19}{80}, y_{r}=(0,1) \\
\frac{1}{4}\left(1-\frac{1}{4}\right) \frac{1}{5}+\frac{2}{4}\left(1-\frac{2}{4}\right) \frac{4}{5}=\frac{19}{80}, y_{r}=(1,0) \\
\left(\frac{1}{4}\right)^{2} \frac{1}{5}+\left(\frac{2}{4}\right)^{2} \frac{4}{5}=\frac{17}{80}, y_{r}=(1,1) .
\end{array}\right.
\end{aligned}
$$

The nonsample total is $y_{r T}=y_{1}+y_{3}$, with three possible possible values:

$$
\begin{aligned}
& 0+0=0 \\
& 0+1=1+0=1 \\
& 1+1=2
\end{aligned}
$$

Therefore $f\left(y_{r T} \mid D\right)=\left\{\begin{array}{l}25 / 80, y_{r T}=0 \\ 38 / 80, y_{r T}=1 \\ 17 / 80, y_{r T}=2\end{array}\right.$

The finite population total is $y_{T}=y_{S T}+y_{r T}$, where $y_{s T}=y_{2}+y_{4}=1+1$ $=2$ is the sample total. It follows that the required predictive density of the finite population total is

$$
f\left(y_{T} \mid D\right)=\left\{\begin{array}{c}
25 / 80, y_{T}=2+0=2 \\
38 / 80, y_{T}=2+1=3 \\
17 / 80, y_{T}=2+2=4 .
\end{array}\right.
$$




\section{Exercise 9.2 A Bernoulli finite population model with nonignorable sampling}

A finite population of size $N=4$ consists of values that are conditionally iid Bernoulli with parameter $\theta$, where $\theta$ is a priori equally likely to be $1 / 4$ or $1 / 2$ (with no other possibilities).

We sample $n=2$ units from the finite population without replacement in such a way that the probability of selecting a sample is proportional to the sum of the values in that sample.

Units 2 and 4 are sampled, and both have the value 1 .

(a) Find the posterior distribution of $\theta$.

(b) Find the predictive distribution of the finite population total, namely $y_{T}=y_{1}+\ldots+y_{N}$

(c) Find the conditional posterior distribution of $\theta$ given the nonsample vector, and then employ this distribution to check your answer to (a) using results in (b).

(d) Find the following probabilities of selection into the sample:
(i) $P(i \in s \mid y, \theta)$
(ii) $P(i \in s \mid y)$
(iii) $P(i \in s \mid \theta)$
(iv) $P(i \in s)$.

\section{Solution to Exercise $\mathbf{9 . 2}$}

(a) The Bayesian model here may be written:

$$
\begin{aligned}
& f(s \mid y, \theta) \propto y_{s T}, \quad s=(1,2),(1,3),(1,4),(2,3),(2,4),(3,4) \\
& f(y \mid \theta)=\prod_{i=1}^{N} \theta^{y_{i}}(1-\theta)^{1-y_{i}} \\
& \quad \text { (the model density of the finite population values) } \\
& f(\theta)=1 / 2, \theta=1 / 4,1 / 2 \quad \text { (the prior density of the parameter). }
\end{aligned}
$$

The observed sample data is

$$
D=\left(s, y_{s}\right)=\left(\left(s_{1}, s_{2}\right),\left(y_{s_{1}}, y_{s_{2}}\right)\right)=\left((2,4),\left(y_{2}, y_{4}\right)\right)=((2,4),(1,1)),
$$

and the nonsample vector is

$$
y_{r}=\left(y_{r_{1}}, y_{r_{2}}\right)=\left(y_{1}, y_{3}\right) \in\{0,1\}^{2} \text {. }
$$


In this case the sampling mechanism is nonignorable and the first thing we should do is determine the exact form of the sampling density of $s=\left(s_{1}, s_{2}\right)$. Now,

$$
f(s \mid y, \theta)=c y_{s T}=c\left(y_{s_{1}}+y_{s_{2}}\right)
$$

for some constant $c$ such that

$$
\begin{aligned}
1 & =\sum_{s} f(s \mid y, \theta) \\
& =c\left\{\left(y_{1}+y_{2}\right)+\left(y_{1}+y_{3}\right)+\left(y_{1}+y_{4}\right)+\left(y_{2}+y_{3}\right)+\left(y_{2}+y_{4}\right)+\left(y_{3}+y_{4}\right)\right\} \\
& =c\left\{3\left(y_{1}+y_{2}+y_{3}+y_{4}\right)\right\}=3 c y_{T} .
\end{aligned}
$$

We see that $c=1 /\left(3 y_{T}\right)$, and so

$$
f(s \mid y, \theta)=\frac{y_{s_{1}}+y_{s_{2}}}{3 y_{T}}, \quad s=\left(s_{1}, s_{2}\right)=(1,2),(1,3),(1,4),(2,3),(2,4),(3,4) \text {. }
$$

Note 1: This formula shows explicitly how the sampling mechanism depends on the values in the finite population vector $y$. It also shows that, conditional on $y$, the sampling mechanism does not depend on the superpopulation parameter $\theta$.

Note 2: This formula is only true when the finite population total $y_{T}$ is positive, i.e. when at least one of $y_{1}, \ldots, y_{N}$ is nonzero. In the case where all population values are zero, we have that $y_{s T}=y_{s_{1}}+y_{s_{2}}=0$ for all possible samples $s=\left(s_{1}, s_{2}\right)$, and consequently $f(s \mid y, \theta) \propto 0$, which must be understood to mean that that no sample actually gets drawn. The fact that a sample has been observed implies $f(s \mid y, \theta)>0$ for at least one value of $s$, which implies that at least one population value is positive, which in turn implies that $y_{T}>0$. This would be true even if all the sample values were zero; but as it happens, at least one of them is positive (in fact both are), which in itself implies that $y_{T}>0$.

We may now work out the joint density of all quantities in the model:

$$
\begin{aligned}
f\left(\theta, y_{s}, y_{r}, s\right) & =f(\theta) f\left(y_{s} \mid \theta\right) f\left(y_{r} \mid \theta\right) f\left(s \mid y_{s}, y_{r}, \theta\right) \\
& =\frac{1}{2} \times\left(\prod_{i \in s} \theta^{y_{i}}(1-\theta)^{1-y_{i}}\right) \times\left(\prod_{i \in r} \theta^{y_{i}}(1-\theta)^{1-y_{i}}\right) \times \frac{y_{s_{1}}+y_{s_{2}}}{3 y_{T}}
\end{aligned}
$$




$$
\begin{aligned}
& =\frac{1}{2} \times \theta^{2} \times \theta^{y_{1}+y_{3}}(1-\theta)^{2-y_{1}-y_{3}} \times \frac{1+1}{3\left(y_{1}+1+y_{3}+1\right)} \\
& \propto \frac{\theta^{2+y_{1}+y_{3}}(1-\theta)^{2-y_{1}-y_{3}}}{2+y_{1}+y_{3}} .
\end{aligned}
$$

So the posterior density of $\theta$ is

$$
\begin{aligned}
& f(\theta \mid D)=f\left(\theta \mid s, y_{s}\right) \\
& \propto f\left(\theta, s, y_{s}\right) \\
& =\sum_{y_{r}} f\left(\theta, s, y_{s}, y_{r}\right) \\
& \propto \theta^{2}(1-\theta)^{2} \sum_{y_{1}=0}^{1} \sum_{y_{3}=0}^{1}\left(\frac{\theta}{1-\theta}\right)^{y_{1}+y_{3}} \frac{1}{2+y_{1}+y_{3}} \\
& \propto \theta^{2}(1-\theta)^{2}\left\{\left(\frac{\theta}{1-\theta}\right)^{0+0} \frac{1}{2+0+0}+\left(\frac{\theta}{1-\theta}\right)^{0+1} \frac{1}{2+0+1}\right. \\
& \left.+\left(\frac{\theta}{1-\theta}\right)^{1+0} \frac{1}{2+1+0}+\left(\frac{\theta}{1-\theta}\right)^{1+1} \frac{1}{2+1+1}\right\} \\
& =\theta^{2}(1-\theta)^{2}\left\{\frac{1}{2}+\left(\frac{\theta}{1-\theta}\right) \frac{1}{3}+\left(\frac{\theta}{1-\theta}\right) \frac{1}{3}+\left(\frac{\theta}{1-\theta}\right)^{2} \frac{1}{4}\right\} \\
& =\frac{1}{12}\left\{6 \theta^{2}(1-\theta)^{2}+8 \theta^{3}(1-\theta)+3 \theta^{4}\right\} \\
& =\left\{\begin{array}{l}
\frac{1}{12}\left[6\left(\frac{1}{4}\right)^{2}\left(1-\frac{1}{4}\right)^{2}+8\left(\frac{1}{4}\right)^{3}\left(1-\frac{1}{4}\right)+3\left(\frac{1}{4}\right)^{4}\right], \quad \theta=\frac{1}{4} \\
\frac{1}{12}\left[6\left(\frac{2}{4}\right)^{2}\left(1-\frac{2}{4}\right)^{2}+8\left(\frac{2}{4}\right)^{3}\left(1-\frac{2}{4}\right)+3\left(\frac{2}{4}\right)^{4}\right], \quad \theta=\frac{2}{4}
\end{array}\right. \\
& =\left\{\begin{array}{cc}
\frac{1}{12(256)}[6(9)+8(3)+3(1)], & \theta=\frac{1}{4} \\
\frac{1}{12(256)}[6(16)+8(16)+3(16)], & \theta=\frac{2}{4}
\end{array}\right. \\
& \propto\left\{\begin{array}{c}
6(9)+8(3)+3(1)=81, \quad \theta=\frac{1}{4} \\
6(16)+8(16)+3(16)=272, \quad \theta=\frac{2}{4} .
\end{array}\right.
\end{aligned}
$$


Now $81+272=353$, and so

$$
f(\theta \mid D)=\left\{\begin{array}{rc}
81 / 353=0.22946, & \theta=1 / 4 \\
272 / 353=0.77054, & \theta=1 / 2 .
\end{array}\right.
$$

(b) The predictive density of the nonsample vector

$$
y_{r}=\left(y_{r_{1}}, y_{r_{2}}\right)=\left(y_{1}, y_{3}\right)
$$

is

$$
\begin{aligned}
& f\left(y_{r} \mid D\right)=f\left(y_{r} \mid s, y_{s}\right) \propto f\left(y_{r}, s, y_{s}\right) \\
& =\sum_{\theta} f\left(\theta, s, y_{s}, y_{r}\right) \\
& \propto \frac{1}{2+y_{1}+y_{3}} \sum_{\theta=1 / 4,2 / 4} \theta^{2+y_{1}+y_{3}}(1-\theta)^{2-y_{1}-y_{3}} \\
& =\frac{1}{2+y_{1}+y_{3}}\left\{\left(\frac{1}{4}\right)^{2+y_{1}+y_{3}}\left(1-\frac{1}{4}\right)^{2-y_{1}-y_{3}}+\left(\frac{2}{4}\right)^{2+y_{1}+y_{3}}\left(1-\frac{2}{4}\right)^{2-y_{1}-y_{3}}\right\} \\
& =\frac{1}{\left(2+y_{1}+y_{3}\right) 256}\left\{3^{2-y_{1}-y_{3}}+16\right\} \propto \frac{16+3^{2-y_{1}-y_{3}}}{2+y_{1}+y_{3}} \\
& \left(\frac{16+3^{2-0-0}}{2+0+0}=\frac{25}{2}=\frac{150}{12}, \quad\left(y_{1}, y_{3}\right)=(0,0)\right. \\
& \propto \begin{cases}\frac{16+3^{2-0-1}}{2+0+1}=\frac{19}{3}=\frac{76}{12}, & \left(y_{1}, y_{3}\right)=(0,1) \\
\frac{16+3^{2-1-0}}{2+1+0}=\frac{19}{3}=\frac{76}{12}, & \left(y_{1}, y_{3}\right)=(1,0)\end{cases} \\
& \frac{16+3^{2-1-1}}{2+1+1}=\frac{17}{4}=\frac{51}{12}, \quad\left(y_{1}, y_{3}\right)=(1,1) \\
& \propto \begin{cases}75, & \left(y_{1}, y_{3}\right)=(0,0) \\
38, & \left(y_{1}, y_{3}\right)=(0,1) \\
38, & \left(y_{1}, y_{3}\right)=(1,0) \\
24, & \left(y_{1}, y_{3}\right)=(1,1) .\end{cases}
\end{aligned}
$$

Now, $150+76+76+51=353$, and so

$$
f\left(y_{r} \mid D\right)=\left\{\begin{aligned}
150 / 353=0.42493, & y_{r}=(0,0) \\
76 / 353=0.21530, & y_{r}=(0,1) \\
76 / 353=0.21530, & y_{r}=(1,0) \\
51 / 353=0.14448, & y_{r}=(1,1) .
\end{aligned}\right.
$$


So the predictive density of the nonsample total,

$$
y_{r T}=y_{r_{1}}+y_{r_{2}}=y_{1}+y_{3} \text {, }
$$

is $\quad f\left(y_{r T} \mid D\right)= \begin{cases}150 / 353=0.42493, & y_{r T}=0 \\ 152 / 353=0.43059, & y_{r T}=1 \\ 51 / 353=0.14448, & y_{r T}=2 .\end{cases}$

So the predictive density of the finite population total,

$$
\begin{gathered}
y_{T}=y_{s T}+y_{r T}=(1+1)+y_{r T}, \\
\text { is } \quad f\left(y_{T} \mid D\right)= \begin{cases}150 / 353=0.42493, & y_{T}=2 \\
152 / 353=0.43059, & y_{T}=3 \\
51 / 353=0.14448, & y_{T}=4 .\end{cases}
\end{gathered}
$$

(c) The conditional posterior density of $\theta$ given $y_{r}$ is

$$
\begin{aligned}
f\left(\theta \mid y_{s}, y_{r}, s\right) & \propto f\left(\theta, y_{s}, y_{r}, s\right) \\
& \propto \theta^{2+y_{1}+y_{3}}(1-\theta)^{2-y_{1}-y_{3}} .
\end{aligned}
$$

We now need to consider all the possible values of $y_{r}$, one by one.

For $y_{r}=(0,0)$ :

$$
\begin{gathered}
f\left(\theta \mid y_{s}, y_{r}, s\right) \propto \theta^{2+0+0}(1-\theta)^{2-0-0}=\left\{\begin{array}{l}
\left(\frac{1}{4}\right)^{2}\left(1-\frac{1}{4}\right)^{2}=\frac{9}{256}, \quad \theta=\frac{1}{4} \\
\left(\frac{2}{4}\right)^{2}\left(1-\frac{2}{4}\right)^{2}=\frac{16}{256}, \quad \theta=\frac{2}{4}
\end{array}\right. \\
\Rightarrow f\left(\theta \mid y_{s}, y_{r}, s\right)= \begin{cases}9 / 25, & \theta=1 / 4 \\
16 / 25, & \theta=1 / 2 .\end{cases}
\end{gathered}
$$

For $y_{r}=(0,1)$ :

$$
\begin{gathered}
f\left(\theta \mid y_{s}, y_{r}, s\right) \propto \theta^{2+0+1}(1-\theta)^{2-0-1}=\left\{\begin{array}{l}
\left(\frac{1}{4}\right)^{3}\left(1-\frac{1}{4}\right)^{1}=\frac{3}{256}, \quad \theta=\frac{1}{4} \\
\left(\frac{2}{4}\right)^{3}\left(1-\frac{2}{4}\right)^{1}=\frac{16}{256}, \quad \theta=\frac{2}{4}
\end{array}\right. \\
\Rightarrow f\left(\theta \mid y_{s}, y_{r}, s\right)=\left\{\begin{aligned}
3 / 19, & \theta=1 / 4 \\
16 / 19, & \theta=1 / 2 .
\end{aligned}\right.
\end{gathered}
$$


For $y_{r}=(1,0)$ :

$$
\begin{gathered}
f\left(\theta \mid y_{s}, y_{r}, s\right) \propto \theta^{2+1+0}(1-\theta)^{2-1-0}=\left\{\begin{array}{l}
\left(\frac{1}{4}\right)^{3}\left(1-\frac{1}{4}\right)^{1}=\frac{3}{256}, \quad \theta=\frac{1}{4} \\
\left(\frac{2}{4}\right)^{3}\left(1-\frac{2}{4}\right)^{1}=\frac{16}{256}, \quad \theta=\frac{2}{4}
\end{array}\right. \\
\Rightarrow f\left(\theta \mid y_{s}, y_{r}, s\right)=\left\{\begin{aligned}
3 / 19, & \theta=1 / 4 \\
16 / 19, & \theta=1 / 2 .
\end{aligned}\right.
\end{gathered}
$$

For $y_{r}=(1,1)$ :

$$
\begin{aligned}
& f\left(\theta \mid y_{s}, y_{r}, s\right) \propto \theta^{2+1+1}(1-\theta)^{2-1-1}= \begin{cases}\left(\frac{1}{4}\right)^{4}=\frac{1}{256}, & \theta=\frac{1}{4} \\
\left(\frac{2}{4}\right)^{4}=\frac{16}{256}, & \theta=\frac{2}{4}\end{cases} \\
& \Rightarrow f\left(\theta \mid y_{s}, y_{r}, s\right)=\left\{\begin{array}{cc}
1 / 17, & \theta=1 / 4 \\
16 / 17, & \theta=1 / 2 .
\end{array}\right.
\end{aligned}
$$

Now,

$$
f\left(\theta \mid y_{s}, s\right)=\sum_{y_{r}} f\left(\theta, y_{r} \mid y_{s}, s\right)=\sum_{y_{r}} f\left(\theta \mid y_{s}, y_{r}, s\right) f\left(y_{r} \mid y_{s}, s\right) .
$$

So, using results in (b), we have that:

$$
\begin{aligned}
& f\left(\theta=1 / 4 \mid y_{s}, s\right)=\sum_{y_{r}} f\left(\theta=\frac{1}{4} \mid y_{s}, y_{r}, s\right) f\left(y_{r} \mid y_{s}, s\right) \\
& =\frac{9}{25} \times \frac{150}{353}+\frac{3}{19} \times \frac{76}{353}+\frac{3}{19} \times \frac{76}{353}+\frac{1}{17} \times \frac{51}{353}=0.22946 \\
& f\left(\theta=1 / 2 \mid y_{s}, s\right)=\sum_{y_{r}} f\left(\theta=\frac{1}{2} \mid y_{s}, y_{r}, s\right) f\left(y_{r} \mid y_{s}, s\right) \\
& =\frac{16}{25} \times \frac{150}{353}+\frac{16}{19} \times \frac{76}{353}+\frac{16}{19} \times \frac{76}{353}+\frac{16}{17} \times \frac{51}{353}=0.77054 \text {. }
\end{aligned}
$$

These results are all in agreement with those obtained in (a) using a different approach. 
(d) (i) The probability of selecting unit $i$ into the sample given $y$ and $\theta$ is the same for all $i$, in particular $i=1$, and so may be written

$$
\begin{aligned}
P(1 \in s \mid y, \theta)=\sum_{s: 1 \in s} f(s \mid y, \theta) & =\frac{1}{3 y_{T}}\left\{\left(y_{1}+y_{2}\right)+\left(y_{1}+y_{3}\right)+\left(y_{1}+y_{4}\right)\right\} \\
& =\frac{y_{T}+2 y_{1}}{3 y_{T}}=\frac{1}{3}+\frac{2 y_{1}}{3 y_{T}},
\end{aligned}
$$

assuming that $y_{T}>0$; otherwise, $P(1 \in s \mid y, \theta)=0$.

Thus, for each $i=1, \ldots, 4$ we have that $P(i \in s \mid y, \theta)=\left\{\begin{array}{c}\frac{1}{3}+\frac{2 y_{i}}{3 y_{T}}, \quad y_{T}>0 \\ 0, \quad y_{T}=0 .\end{array}\right.$

As a check, we may ask whether the sum of these inclusion probabilities equals $n=2$.

The answer is yes, assuming that $y$ is such that $y_{T}>0$; in that case,

$$
\sum_{i=1}^{N} P(i \in s \mid y, \theta)=\sum_{i=1}^{4}\left(\frac{1}{3}+\frac{2 y_{i}}{3 y_{T}}\right)=\frac{4}{3}+\frac{2\left(y_{1}+y_{2}+y_{3}+y_{4}\right)}{3 y_{T}}=2=n .
$$

(ii) Since $P(i \in s \mid y, \theta)$ does not depend on $\theta$, we also have

$$
P(i \in s \mid y)=\left\{\begin{array}{c}
\frac{1}{3}+\frac{2 y_{i}}{3 y_{T}}, \quad y_{T}>0 \\
0, \quad y_{T}=0
\end{array}\right.
$$

(iii) The probability of selecting unit $i$ into the sample given $\theta$ is the same for all $i$, in particular $i=1$, and so may be written

$$
\begin{aligned}
& P(i \in s \mid \theta)=P(1 \in s \mid \theta)=\sum_{y} P(1 \in s \mid \theta, y) f(y \mid \theta) \\
& =0 \times P(y=(0,0,0,0) \mid \theta)+\sum_{y: y_{T}>0}\left(\frac{1}{3}+\frac{2 y_{1}}{3 y_{T}}\right) \prod_{i=1}^{4} \theta^{y_{i}}(1-\theta)^{1-y_{i}} \\
& =\sum_{y: y_{T}>0}\left(\frac{1}{3}+\frac{2 y_{1}}{3 y_{T}}\right) \theta^{y_{T}}(1-\theta)^{4-y_{T}}= \begin{cases}0.34180, & \theta=1 / 4 \\
0.46875, & \theta=1 / 2 .\end{cases}
\end{aligned}
$$

These numbers were obtained by writing and implementing a suitable function in R (see the R code below). 
(iv) The unconditional probability that any particular population unit $i$ will be selected into the sample is

$$
\begin{aligned}
P(i \in s) & =\sum_{\theta} P(i \in s \mid \theta) f(\theta) \\
& =0.34180 \times \frac{1}{2}+0.46875 \times \frac{1}{2}=0.40527 .
\end{aligned}
$$

To check this result, we note that the sum of inclusion probabilities should in this case be identical to the expected sample size.

The first of these quantities is $\sum_{i=1}^{4} P(i \in s)=4 \times 0.40527=1.6211$.

The second of these quantities can be obtained by first noting that

$$
P\left(y_{T}=0 \mid \theta\right)=(1-\theta)^{4}=\left\{\begin{array}{l}
(3 / 4)^{4}=81 / 256, \theta=3 / 4 \\
(2 / 4)^{4}=16 / 256, \theta=2 / 4 .
\end{array}\right.
$$

This implies that

$$
\begin{aligned}
P\left(y_{T}=0\right)=\sum_{\theta} P\left(y_{T}=0 \mid \theta\right) f(\theta) & =\frac{81}{256} \times \frac{1}{2}+\frac{16}{256} \times \frac{1}{2}=\frac{97}{512} \\
& =0.18945 .
\end{aligned}
$$

The sample vector has size 2 if $y_{T}>0$, and size 0 if $y_{T}=0$. So its expected size is $0 \times 0.18945+2 \times(1-0.18945)=1.6211$, which is the same as $\sum_{i=1}^{4} P(i \in s)$ above.

\section{R Code for Exercise $\mathbf{9 . 2}$}

\# (a) \& (b)

options(digits=5)

kern=function(th,yr) $\left\{\operatorname{th}^{\wedge}(2+\operatorname{sum}(y r))^{*}(1-\text { th })^{\wedge}(2-\operatorname{sum}(y r)) /(2+\operatorname{sum}(\mathrm{yr}))\right\}$

kernth0.25 $=$ kern $($ th $=0.25, y r=c(0,0))+\operatorname{kern}(\operatorname{th}=0.25, y r=c(0,1))+$ kern $($ th $=0.25, y r=c(1,0))+\operatorname{kern}($ th $=0.25, y r=c(1,1))$

kernth0.5 $=\operatorname{kern}(\mathrm{th}=0.5, \mathrm{yr}=\mathrm{c}(0,0))+\operatorname{kern}(\mathrm{th}=0.5, \mathrm{yr}=\mathrm{c}(0,1))+$ kern $($ th $=0.5, y r=c(1,0))+\operatorname{kern}(\operatorname{th}=0.5, y r=c(1,1))$ 
postth $=c($ kernth0.25, kernth0.5)/( kernth0.25 + kernth0.5) postth \# 0.229460 .77054

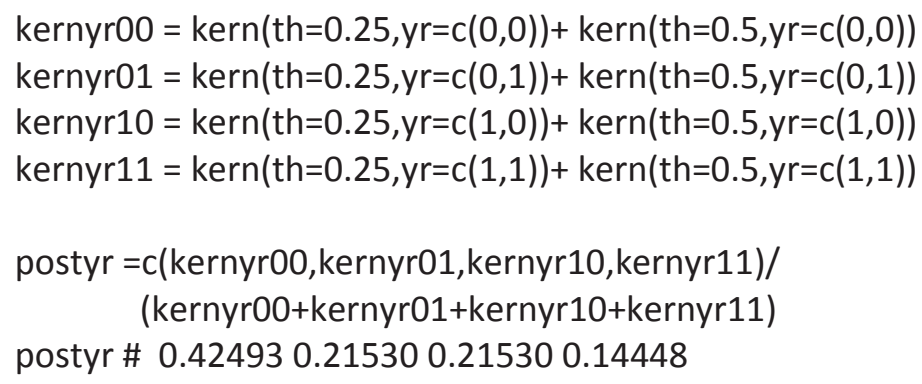

prob0.25=0; for(i in 1:16) prob0.25 = prob0.25 + probfun (y=ymat[i,],th=0.25) prob0.5=0; for(i in 1:16) prob0.5 = prob0.5 + probfun $(y=y m a t[i],, t h=0.5)$

c(prob0.25,prob0.5) \# 0.341800 .46875

(prob0.25+prob0.5)/2 \# 0.40527

4*(prob0.25+prob0.5)/2 \# 1.6211

c(97/512, 2*(1-97/512) ) \# 0.189451 .62109 


\section{Exercise 9.3 A finite population Bayesian model with SRSWOR}

We sample $n=2$ units from a finite population of $N=4$ via SRSWOR.

If $\theta=0$ then the finite population vector $y$ is equally likely to be each of the following:

$$
(0,0,0,0),(0,0,0,1),(0,0,1,1),(0,1,1,1) .
$$

If $\theta=1$ then the finite population vector $y$ is equally likely to be each of the following:

$$
\text { (1,1,1,1), (1,1,1,0), (1,1,0,0), }(1,0,0,0) \text {. }
$$

A priori, the parameter $\theta$ is equally likely to be 0 or 1 (e.g. according to the toss of a coin).

Suppose we sample units 2 and 3, with values 1 and 1, respectively.

(a) Find the posterior distribution of $\theta$.

(b) Find the predictive distribution of the finite population mean, namely $\bar{y}=\left(y_{1}+\ldots+y_{N}\right) / N$.

\section{Solution to Exercise 9.3}

The easiest way to do this exercise is to first identify eight equally likely possibilities to start with. These possibilities are:

$$
\begin{aligned}
\text { 1. } & \theta=0, y=(0,0,0,0) \text { with } \bar{y}=0 \\
\text { 2. } & \theta=0, y=(0,0,0,1) \text { with } \bar{y}=1 / 4 \\
\text { 3. } & \theta=0, y=(0,0,1,1) \text { with } \bar{y}=1 / 2 \\
\rightarrow \quad \text { 4. } & \theta=0, y=(0,1,1,1) \text { with } \bar{y}=3 / 4 \\
\rightarrow \text { 5. } & \theta=1, y=(1,1,1,1) \text { with } \bar{y}=1 \\
\rightarrow \quad \text { 6. } & \theta=1, y=(1,1,1,0) \text { with } \bar{y}=3 / 4 \\
\text { 7. } & \theta=1, y=(1,1,0,0) \text { with } \bar{y}=1 / 2 \\
\text { 8. } & \theta=1, y=(1,0,0,0) \text { with } \bar{y}=1 / 4 .
\end{aligned}
$$

After observing $y_{s}=\left(y_{2}, y_{3}\right)=(1,1)$, there are only three possibilities remaining (4, 5 and 6 in the list, each highlighted by an arrow). 
(a) Two possibilities out of the 3 correspond to $\theta=1$ (namely 5 and 6) and one to $\theta=0$ (namely 4); consequently, $f(\theta \mid D)=\left\{\begin{array}{l}1 / 3, \theta=0 \\ 2 / 3, \theta=1\end{array}\right\}$, or equivalently, $(\theta \mid D) \sim \operatorname{Bern}(2 / 3)$.

(b) Two possibilities out of the 3 correspond to $\bar{y}=3 / 4$ (namely 4 and 6) and one to $\bar{y}=1$ (namely 5); therefore $f(\bar{y} \mid D)=\left\{\begin{array}{ll}2 / 3, & \bar{y}=3 / 4 \\ 1 / 3, & \bar{y}=1\end{array}\right\}$.

\section{Alternative solution}

The above results can also be obtained by working through in the style of the solutions to previous exercises, as follows. Before the data is observed, the Bayesian model may be written:

$$
\begin{aligned}
& \begin{aligned}
f(s \mid y, \theta)=\left(\begin{array}{l}
N \\
n
\end{array}\right)^{-1}=\left(\begin{array}{l}
4 \\
2
\end{array}\right)^{-1}=\frac{1}{6}, \\
s=(1,2),(1,3),(1,4),(2,3),(2,4),(3,4)
\end{aligned} \\
& \begin{aligned}
& f(y \mid \theta)=\frac{1}{4}, \quad y=(\theta, \theta, \theta, \theta),(\theta, \theta, \theta, 1-\theta), \\
&(\theta, \theta, 1-\theta, 1-\theta),(\theta, 1-\theta, 1-\theta, 1-\theta) \\
& f(\theta)=1 / 2, \theta=0,1 \quad \text { (the prior density of the parameter). }
\end{aligned}
\end{aligned}
$$

The observed data is $D=\left(s, y_{s}\right)=((2,3),(1,1))$. At this particular value of the data:

$$
\begin{aligned}
& f(s \mid y, \theta)=\frac{1}{6}, s=(2,3) \quad \text { (the value of } s \text { actually observed) } \\
& f(y \mid \theta)=\frac{1}{4}, \quad y=(0,1,1,1) \text { and } \theta=0, \\
& y \in\{(1,1,1,1),(1,1,1,0)\} \text { and } \theta=1 \quad \text { (where we need } \\
& \text { only consider values of } y \text { consistent with the data) } \\
& \text { (since both values of } \theta \text { are still possible, } \\
& \text { i.e. consistent with the observed data). }
\end{aligned}
$$

With the quantities $s=(2,3), y_{s}=\left(y_{2}, y_{3}\right)=(1,1)$ and $y_{r}=\left(y_{1}, y_{4}\right)$ all fixed at these values, the joint density of all quantities in the model may be written 


$$
\begin{aligned}
& \quad f(\theta, s, y)=f\left(\theta, s, y_{s}, y_{r}\right)=f(\theta) f\left(y_{s}, y_{r} \mid \theta\right) f\left(s \mid y_{s}, y_{r}, \theta\right) \\
& =\frac{I(\theta \in\{0,1\})}{2} \times \frac{I(y=(0,1,1,1), \theta=0)+I(y \in\{(1,1,1,1),(1,1,1,0)\}, \theta=1)}{4} \times \frac{1}{6} \\
& \stackrel{\theta, y_{r}}{\propto} I\left(y_{r}=(0,1), \theta=0\right)+I\left(y_{r} \in\{(1,1),(1,0)\}, \theta=1\right) .
\end{aligned}
$$

(a) It follows that

$$
\begin{aligned}
f(\theta \mid D) \propto f\left(\theta, s, y_{s}\right) & =\sum_{y_{r}} f(\theta, s, y) \\
& \propto \begin{cases}\sum I\left(y_{r}=(0,1)\right)=1, & \theta=0 \\
\sum I\left(y_{r} \in\{(1,1),(1,0)\}\right)=2, & \theta=1 .\end{cases}
\end{aligned}
$$

After normalising, we see that $f(\theta \mid D)=\left\{\begin{array}{l}1 / 3, \theta=0 \\ 2 / 3, \theta=1\end{array}\right\}$.

(b) Also,

$$
\begin{gathered}
f\left(y_{r} \mid D\right) \propto f\left(y_{r}, s, y_{s}\right)=\sum_{\theta} f\left(\theta, s, y_{s}, y_{r}\right) \\
\propto\left\{\begin{array}{l}
\sum_{\theta=0}^{1}\left[I\left(y_{r}=(0,1), \theta=0\right)+I\left(y_{r} \in\{(1,1),(1,0)\}, \theta=1\right)\right]=1, \quad y_{r}=(0,1) \\
\sum_{\theta=0}^{1}\left[I\left(y_{r}=(0,1), \theta=0\right)+I\left(y_{r} \in\{(1,1),(1,0)\}, \theta=1\right)\right]=1, \quad y_{r}=(1,1) \\
\sum_{\theta=0}^{1}\left[I\left(y_{r}=(0,1), \theta=0\right)+I\left(y_{r} \in\{(1,1),(1,0)\}, \theta=1\right)\right]=1, \quad y_{r}=(1,0)
\end{array}\right.
\end{gathered}
$$

which implies that $f\left(y_{r} \mid D\right)=1 / 3, \quad y_{r}=(0,1),(1,1),(1,0)$.

Consequently, $f(y \mid D)=1 / 3, \quad y=(0,1,1,1),(1,1,1,1),(1,1,1,0)$.

Now, the values of $y$ listed here as possible given the observed data have means 3/4, 1 and 3/4, respectively.

It follows that the predictive density of the population mean is

$$
f(\bar{y} \mid D)=\left\{\begin{array}{ll}
2 / 3, & \bar{y}=3 / 4 \\
1 / 3, & \bar{y}=1
\end{array}\right\} \text { (as was obtained previously). }
$$




\section{Exercise 9.4 Length-biased with-replacement sampling from a Poisson finite population}

A finite population of size 9 consists of values that are conditionally iid Poisson with a mean whose prior distribution is gamma with both parameters zero (considered uninformative).

We sample 3 times from the finite population according to a withreplacement sampling scheme, where on each draw the probability of selecting a unit is proportional to its value.

Unit $\mathbf{2}$ is selected once and its value is $\mathbf{1}$.

Unit $\mathbf{4}$ is selected twice and its value is $\mathbf{3}$.

Find the posterior distribution of the Poisson mean and the predictive distribution of the nonsample total.

Also find these distributions under the (false) assumption that the sampling is SRSWR.

Then create two plots which suitably compare the four distributions indicated above.

Note: The concepts here involve a biased sampling mechanism and are relevant to on-site sampling, where for example we wish to estimate the total number of times that visitors (or potential visitors) to a recreational park actually visit there in some specified time period.

If we go to the site at random times to survey visitors, we are more likely to interview people who come very often relative to those who come only rarely. This means that we may end up over-estimating the popularity of the park-unless we make a suitable correction (downwards) to account for the (upwardly) biased sampling mechanism. If a potential visitor to the site doesn't come at all, then there is zero chance of sampling them.

If we wish to consider only the population of persons who actually visit the site in a given period (i.e. to exclude the potential visitors who do not visit), we may need to consider a truncated model involving the Poisson random variable conditional on it being non-zero. For further details and a discussion of the modelling issues here, see Shaw (1988). 


\section{Solution to Exercise 9.4}

Generally, we are considering a sample of size $n$ obtained with replacement from a finite population with values, $y_{1}, \ldots, y_{N}$ which are conditionally Poisson with some mean $\lambda$, where the prior distribution on $\lambda$ is gamma with parameters $\eta$ and $\tau$ (and mean $\eta / \tau$ ).

Let $I_{i}$ be the number of times population unit $i$ is sampled and define $I=\left(I_{1}, \ldots, I_{N}\right)$. Then let $d=\sum_{i=1}^{N} I\left(I_{i}>0\right)$ be the distinct sample size (the number of distinct population units sampled), and let $m=N-d$ be the nonsample size (the number of units not sampled).

In this scenario, we define the sample vector as $y_{s}=\left(y_{s_{1}}, \ldots, y_{s_{d}}\right)$, where $s=\left(s_{1}, \ldots, s_{d}\right)$ is the vector of the labels of the $d$ distinct units that are sampled, and we define the nonsample vector as $y_{r}=\left(y_{r_{1}}, \ldots, y_{r_{m}}\right)$, where $r=\left(r_{1}, \ldots, r_{m}\right)$ is the vector of the labels of the $m$ units that are not sampled.

Note: Here, $s$ is a function of $I$, and so the data in this situation could also be written as $D=\left(I, y_{s}\right)$.

Since we are interested in the nonsample values only by way of their total $y_{r T}$, a suitable Bayesian finite population model in this context is:

$$
\begin{aligned}
& f(I \mid y, \lambda)=\frac{n !}{\prod_{i=1}^{N} I_{i} !} \prod_{i=1}^{N}\left(\frac{y_{i}}{y_{T}}\right)^{I_{i}}, \\
& I \in\left\{\left(a_{1}, \ldots, a_{N}\right): a_{i} \in\{0,1, \ldots, n\} \forall i, a_{1}+\ldots+a_{N}=n\right\} \\
& f\left(y_{s}, y_{r T} \mid \lambda\right)=\left(\prod_{i \in s} \frac{e^{-\lambda} \lambda^{y_{i}}}{y_{i} !}\right) \times \frac{e^{-m \lambda}(m \lambda)^{y_{r T}}}{y_{r T} !} \\
& \lambda \sim G(\eta, \tau) .
\end{aligned}
$$

In our specific situation, $N=9, n=3$, and the data is

$$
D=\left(I, y_{s}\right)=((0,1,0,2,0,0,0,0,0),(1,3)) \text {, }
$$

meaning that unit 2 is selected once and its values is 1 , and unit 4 is selected twice and its value is 3 . Thus $d=2$ and $m=7$. Also, $\eta=0$ and $\tau=0$. 
On the basis of these specifications, we wish to make inferences about $\lambda$ and the nonsample total,

$$
y_{r T}=y_{1}+y_{3}+y_{5}+y_{6}+y_{7}+y_{8}+y_{9} .
$$

Note: The probability of sampling unit 2 once and unit 4 twice (as is assumed to have occurred) equals

$$
\frac{y_{2}}{y_{T}} \frac{y_{4}}{y_{T}} \frac{y_{4}}{y_{T}}+\frac{y_{4}}{y_{T}} \frac{y_{2}}{y_{T}} \frac{y_{4}}{y_{T}}+\frac{y_{4}}{y_{T}} \frac{y_{4}}{y_{T}} \frac{y_{2}}{y_{T}}=\frac{3 !}{1 ! 2 !}\left(\frac{y_{2}}{y_{T}}\right)^{1}\left(\frac{y_{4}}{y_{T}}\right)^{2}=\frac{3 !}{\prod_{i=1}^{9} I_{i} !} \prod_{i=1}^{9}\left(\frac{y_{i}}{y_{T}}\right)^{I_{i}}
$$

and so is consistent with

$$
f(I \mid y, \theta)=\frac{n !}{\prod_{i=1}^{N} I_{i}} \prod_{i=1}^{N}\left(\frac{y_{i}}{y_{T}}\right)^{I_{i}}
$$

as specified in the general model.

For this exercise we will first derive the predictive distribution of $y_{r T}$ and then use this to obtain the posterior distribution of $\lambda$ only afterwards. The predictive density of $y_{r T}$ is

$$
\begin{aligned}
& f\left(y_{r T} \mid D\right) \propto \int f\left(y_{r T}, y_{s}, I, \lambda\right) d \lambda \\
&= \int f(\lambda) f\left(y_{s} \mid \lambda\right) f\left(y_{r T} \mid \lambda\right) f\left(I \mid y_{s}, y_{r T}, \lambda\right) d \lambda \\
& \propto \int_{0}^{\infty} \lambda^{\eta-1} e^{-\tau \lambda} \times\left(\prod_{i \in s} e^{-\lambda} \lambda^{y_{i}}\right) \times \frac{e^{-m \lambda}(m \lambda)^{y_{r T}}}{y_{r T} !} \times \frac{1}{y_{T}^{n}} d \lambda \\
&\left.\quad \text { (note that } \prod_{i=1}^{N}\left(\frac{1}{y_{T}}\right)^{I_{i}}=\left(\frac{1}{y_{T}}\right)^{n}\right) \\
&=\frac{m^{y_{r T}}}{y_{r T} !} \frac{1}{y_{T}^{n}} \int_{0}^{\infty} \lambda^{\eta+y_{s T}+y_{r T}-1} e^{-\lambda(\tau+d+m)} d \lambda \\
&=\frac{m^{y_{r T}}}{y_{r T} !} \frac{1}{y_{T}^{n}} \times \frac{\Gamma\left(\eta+y_{s T}+y_{r T}\right)}{(\tau+d+m)^{\eta+y_{s T}+y_{r T}}} \times 1 .
\end{aligned}
$$


Thus

$$
f\left(y_{r T} \mid D\right)=\frac{k\left(y_{r T}\right)}{c}, y_{r T}=0,1,2, \ldots,
$$

where

$$
k\left(y_{r T}\right)=\left(\frac{N-d}{N+\tau}\right)^{y_{r T}} \frac{\Gamma\left(\eta+y_{s T}+y_{r T}\right)}{y_{r T} !\left(y_{s T}+y_{r T}\right)^{n}}
$$

and

$$
C=\sum_{y_{r T}=0}^{\infty} k\left(y_{r T}\right)
$$

Note: Here, $d+m=N$, and so

$$
(\tau+d+m)^{\eta+y_{s T}+y_{r T}}=(N+\tau)^{\eta+y_{s T}+y_{r T}} \stackrel{y_{r T}}{\propto}(N+\tau)^{y_{r T}} .
$$

We may approximate $f\left(y_{r T} \mid D\right)$ by calculating $k\left(y_{r T}\right)$ only for $y_{r T}=0,1,2, \ldots, M$ for some large integer $M$ (in practice we used 100) for which $k\left(y_{r T}\right)$ is sufficiently close to zero.

Using the predictive density of $y_{r T}$, we can now obtain the posterior density of $\lambda$ as

$$
f(\lambda \mid D)=\sum_{y_{r T=0}}^{\infty} f\left(\lambda \mid D, y_{r T}\right) f\left(y_{r T} \mid D\right),
$$

where

$$
\left(\lambda \mid D, y_{r T}\right) \sim G\left(\eta+y_{s T}+y_{r T}, \tau+N\right) .
$$

Note: This result is obvious but can also be obtained as follows:

$$
\begin{aligned}
& f\left(\lambda \mid D, y_{r T}\right)=f\left(\lambda \mid s, y_{s}, y_{r T}\right) \propto f\left(\lambda, s, y_{s}, y_{r T}\right) \propto f(\lambda) f\left(y_{s}, y_{r T} \mid \lambda\right) \\
& \propto \lambda^{\eta-1} e^{-\tau \lambda}\left(\prod_{i \in s} \frac{e^{-\lambda} \lambda^{y_{i}}}{y_{i} !}\right) \times \frac{e^{-m \lambda}(m \lambda)^{y_{r T}}}{y_{r T} !} \\
& \propto \lambda^{\eta-1} e^{-\tau \lambda} \times e^{-d \lambda} \lambda^{y_{s T}} \times e^{-m \lambda} \lambda^{y_{r T}} \\
&\left.=\lambda^{\eta+y_{s T}+y_{r T}-1} e^{-\lambda(\tau+N)} \quad \text { (since } d+m=N\right) .
\end{aligned}
$$


We see that $f(\lambda \mid D)$ is an infinite mixture of gamma densities where the weight assigned to each one is the corresponding (marginal) predictive density of $y_{r T}$.

Note: An alternative way to derive $f(\lambda \mid D)$ is using the equation

$$
f(\lambda \mid D) \propto \sum_{y_{r T}} f\left(y_{r T}, y_{s}, I, \lambda\right) .
$$

\section{The case of SRSWR}

In the case of SRSWR, the sampling density

changes to

$$
f(I \mid y, \lambda)=\frac{n !}{\prod_{i=1}^{N} I_{i}} \prod_{i=1}^{N}\left(\frac{y_{i}}{y_{T}}\right)^{I_{i}}
$$

$$
f(I \mid y, \lambda)=\frac{n !}{\prod_{i=1}^{N} I_{i}} \prod_{i=1}^{N}\left(\frac{1}{N}\right)^{I_{i}}=\frac{n !}{N^{n} \prod_{i=1}^{N} I_{i}},
$$

which we note does not depend on $\lambda$ or $y_{r T}$ and so can be 'ignored'.

The result is then almost the same as before, the only difference being that the term

$$
\prod_{i=1}^{N} y_{T}^{I_{i}}=y_{T}^{n}=\left(y_{s T}+y_{r T}\right)^{n}
$$

in

$$
k\left(y_{r T}\right)=\left(\frac{N-d}{N+\tau}\right)^{y_{r T}} \frac{\Gamma\left(\eta+y_{s T}+y_{r T}\right)}{y_{r T} !\left(y_{s T}+y_{r T}\right)^{n}}
$$

is replaced by 1 .

Thus under SRSWR we find that

where

$$
f\left(y_{r T} \mid D\right)=\frac{K\left(y_{r T}\right)}{C}, y_{r T}=0,1,2, \ldots,
$$

and

$$
K\left(y_{r T}\right)=\left(\frac{N-d}{N+\tau}\right)^{y_{r T}} \frac{\Gamma\left(\eta+y_{s T}+y_{r T}\right)}{y_{r T} ! \times 1}
$$

$$
C=\sum_{y_{r T}=0}^{\infty} K\left(y_{r T}\right) .
$$


As regards the posterior distribution of $\lambda$ under SRSWR, this need no longer be expressed as an infinite mixture of gamma distributions but simply as

$$
(\lambda \mid D) \sim G\left(\eta+y_{s T}, \tau+d\right) .
$$

Figure 9.1 shows the posterior density $f(\lambda \mid D)$ under the length-biased and SRSWR assumptions, respectively.

We see that the inference under the assumption of length-bias is the lower of the two. This is because it appropriately corrects for large finite population values being more likely to be selected. If we 'ignore' the fact that large values are more likely to be selected. then we will erroneously over-estimate the superpopulation mean, $\lambda$.

Figure 9.2 shows the predictive density $f\left(y_{r T} \mid D\right)$, again under the two assumptions.

As in Figure 9.1, we see that ignoring the length-biased sampling mechanism tends to bias the inference upwards.

As a check on our calculations, which omitted all terms corresponding to values of $y_{r T}$ greater than $M=100$ (see above), we calculate the predictive mean of $y_{r T}$ under the SRSWR assumption using the formula

$$
E\left(y_{r T} \mid D\right) \approx \frac{1}{C} \sum_{r T=0}^{M} y_{r T} K\left(y_{r T}\right)
$$

and obtain the value of 14 .

This may be compared with the theoretical value, which is exactly

$$
\begin{aligned}
E\left(y_{r T} \mid D\right) & =E\left\{E\left(y_{r T} \mid D, \lambda\right) \mid D\right\}=E(m \lambda \mid D) \\
& =m \times \frac{\eta+y_{s T}}{\tau+d}=7 \times \frac{0+(3+1)}{0+2}=14 .
\end{aligned}
$$


Figure 9.I Posterior densities of the Poisson mean

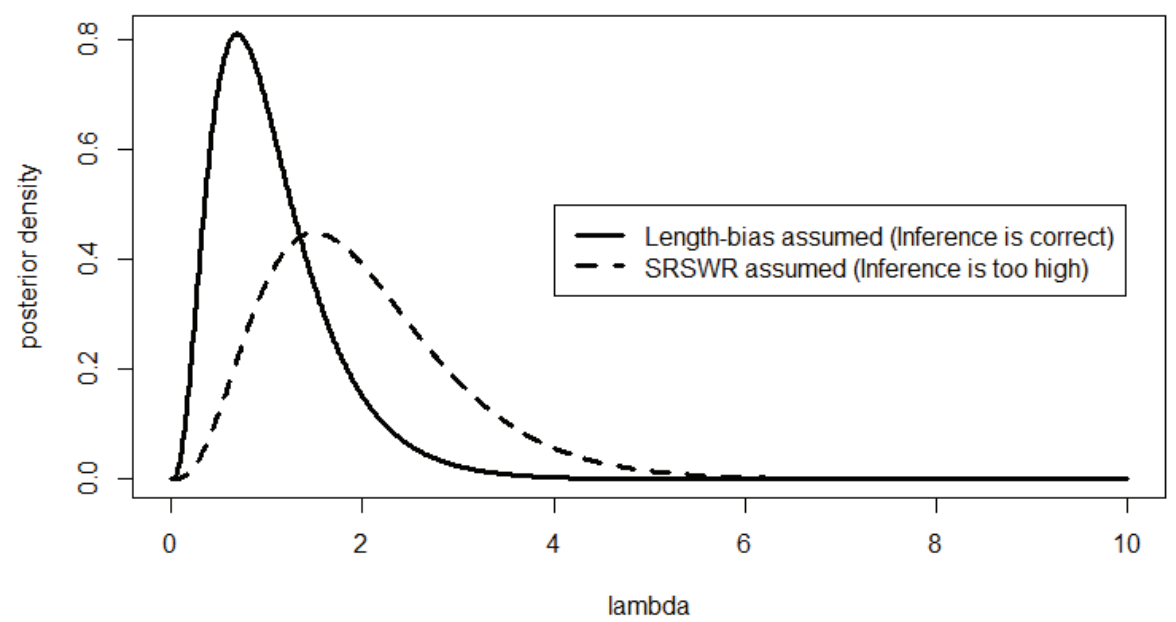

Figure 9.2 Predictive densities of the nonsample total

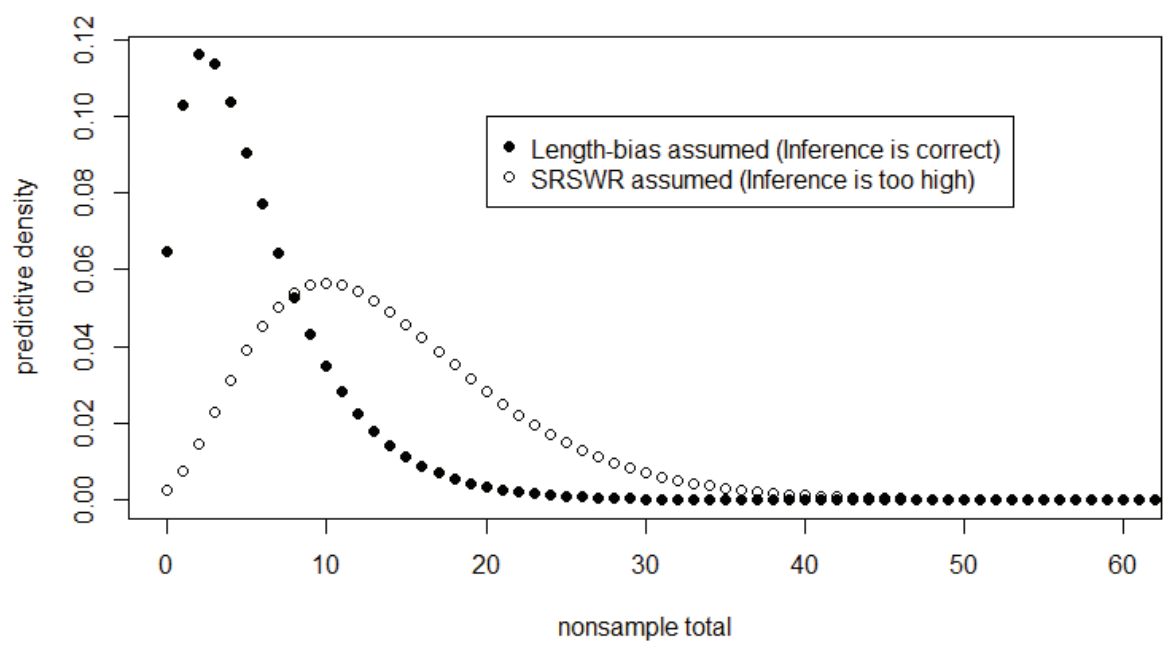




\section{R Code for Exercise 9.4}

options(digits=5); X11 $(w=8, h=5) ; \operatorname{par}(m f r o w=c(1,1))$

$\mathrm{N}=9 ; \mathrm{n}=3 ; \mathrm{ys}=\mathrm{c}(1,3) ; \mathrm{ys} \mathrm{T}=\operatorname{sum}(\mathrm{ys}) ; \mathrm{d}=2 ; \mathrm{m}=7$; eta=0; tau=0; $\mathrm{yrTv}=0: 100$

$\mathrm{kv}=((\mathrm{N}-\mathrm{d}) /(\operatorname{tau}+\mathrm{N}))^{\wedge} \mathrm{yrTv}$ *gamma $($ eta $+\mathrm{ys} T+\mathrm{yrTv}) /$

( factorial $(y r T v) *(y s T+y r T v)^{\wedge} n$ )

$c=\operatorname{sum}(k v) ; f v=k v / c$

plot(yrTv,fv,pch=16, xlab="nonsample total",

ylab="predictive density", $x \lim =c(0,60)$, main=" ")

kvigno $=((\mathrm{N}-\mathrm{d}) /($ tau $+\mathrm{N})) \wedge \mathrm{yrTv} *$ gamma $($ eta $+\mathrm{ys} T+\mathrm{yrTv}) /($ gamma $(\mathrm{yrTv}+1) * 1)$

cigno $=\operatorname{sum}(\mathrm{kvigno}) ;$ fvigno $=\mathrm{kvigno} / \mathrm{cigno}$

points(yrTv,fvigno, pch=1)

legend(20,0.1,c("Length-bias assumed (Inference is correct)",

"SRSWR assumed (Inference is too high)"),pch=c(16,1))

c(sum(yrTv*fv), sum(yrTv*fvigno) ) \# 5.630214 .0000

$\mathrm{m}^{*}($ eta+ysT)/(tau+d) \# 14

lamv=seq $(0,10,0.01) ;$ lamfv=lamv

for(i in 1:length (lamv)) lamfv[i]=sum(fv*dgamma(lamv[i],eta+ysT+yrTv,tau+N))

plot(lamv,lamfv,type="l", Ity=1, Iwd=3,

xlab="lambda",ylab="posterior density", main=" ")

lamfvigno=lamv

for(i in 1:length(lamv))

lamfvigno[i]=sum(fvigno*dgamma(lamv[i],eta+ysT+yrTv,tau+N))

\# lines(lamv,lamfvigno, Ity=2,Iwd=1) \# Can do as a check on calculations

lines (lamv, dgamma(lamv, eta+ysT, tau+d), Ity=2, I wd=3)

legend(4,0.5,c("Length-bias assumed (Inference is correct)",

"SRSWR assumed (Inference is too high)"), Ity=c(1,2), Iwd=c(3,3)) 


\section{Exercise 9.5 An exponential finite population model with a biased Poisson sampling scheme}

A sample is drawn from a finite population of size $N=7$ in such a way that unit $i$ has probability of inclusion $\pi_{i}$, independently of all the other units.

The values in the finite population are independent and identically distributed exponentials with mean $\mu=1 / \lambda$, where the prior distribution for $\lambda$ is given by

$$
f(\lambda) \propto 1 / \lambda, \lambda>0 \text {. }
$$

Units $\mathbf{3}$ and $\mathbf{5}$ are selected, and their values are $\mathbf{1 . 6}$ and $\mathbf{0 . 4}$, respectively.

Find and sketch the posterior density of the superpopulation mean $\mu$ and the predictive density of the finite population mean $\bar{y}$ under each of the following specifications:

(a) All the $\pi_{i}$ values are equal to $0.3(i=1, \ldots, N)$.

(b) All the $\pi_{i}$ values are equal to 0.3 except that:

$$
\begin{aligned}
& \pi_{5}=0.3 \text { if } y_{5}<1 \\
& \pi_{5}=0.9 \text { if } y_{5}>1
\end{aligned}
$$

(thus unit 5 is 3 times as likely to be sampled if its value exceeds 1 ).

(c) All the $\pi_{i}$ values are equal to 0.3 except that:

$$
\begin{aligned}
& \pi_{4}=0.3 \text { if } y_{4}<1 \\
& \pi_{4}=0.9 \text { if } y_{4}>1
\end{aligned}
$$

(thus unit 4 is 3 times as likely to be sampled if its value exceeds 1 ).

Note: Here, the sample size $n$ is not fixed and is a random variable. 


\section{Solution to Exercise 9.5}

(a) The relevant Bayesian model is:

$$
\begin{aligned}
& f(I \mid y, \lambda)=\prod_{i=1}^{N} f\left(I_{i} \mid y, \lambda\right)=\prod_{i=1}^{N} \pi_{i}^{I_{i}}\left(1-\pi_{i}\right)^{1-I_{i}}, \quad n=\sum_{i=1}^{N} I_{i} \\
& f(y \mid \lambda)=\prod_{i=1}^{N} \lambda e^{-\lambda y_{i}}, y_{i}>0 \forall i \\
& f(\lambda) \propto 1 / \lambda, \lambda>0 .
\end{aligned}
$$

Here,

$$
\pi_{1}=\ldots=\pi_{N}=0.3
$$

and the data is

$$
D=\left(I, y_{s}\right)=((0,0,1,0,1,0,0),(1.6,0.4)),
$$

with $n=2$ (the achieved sample size).

The sampling mechanism is ignorable and so

$$
\begin{aligned}
f(\lambda \mid D) & \propto f(\lambda) f\left(y_{s} \mid \lambda\right) \propto \frac{1}{\lambda} \prod_{i \in s} \lambda e^{-\lambda y_{i}}=\lambda^{n-1} e^{-\lambda y_{s T}} \\
& \Rightarrow(\lambda \mid D) \sim G\left(n, y_{s T}\right) \\
& \Rightarrow(\mu \mid D) \sim \operatorname{IG}\left(n, y_{s T}\right) .
\end{aligned}
$$

Next,

$$
\left(y_{r T} \mid \lambda\right) \sim G(m, \lambda),
$$

where $m=N-n=7-2=5$.

It follows that

$$
\begin{aligned}
f\left(y_{r T} \mid D\right) & =\int_{0} f\left(y_{r T} \mid D, \lambda\right) f(\lambda \mid D) d \lambda \\
& \propto \int_{0}^{\infty} \lambda^{m} y_{r T}^{m-1} e^{-\lambda y_{r T}} \times \lambda^{n-1} e^{-\lambda y_{s T}} d \lambda \\
& =y_{r T}^{m-1} \int_{0}^{\infty} \lambda^{n+m-1} e^{-\lambda\left(y_{r T}+y_{s T}\right)} d \lambda \\
& =\frac{y_{r T}^{m-1} \Gamma(n+m)}{\left(y_{r T}+y_{s T}\right)^{n+m}} \\
& \propto \frac{y_{r T}^{m-1}}{\left(y_{r T}+y_{s T}\right)^{N}}, y_{r T}>0 .
\end{aligned}
$$


Hence

$$
f(\bar{y} \mid D) \propto \frac{\left(\bar{y}-\frac{n}{N} \bar{y}_{s}\right)^{N-n-1}}{\bar{y}^{N}}, \bar{y}>\frac{n}{N} \bar{y}_{s}
$$

(using the fact that $y_{r T}=N \bar{y}-n \bar{y}_{s}$ ).

(b) In this case, inferences will be exactly the same as in (a). This is because, even though the sampling mechanism is potentially nonignorable due to $f(I \mid y, \lambda)$ depending on a population value $y_{5}$, that value happens to be known (since unit 5 is in the sample, i.e. $5 \in S$ ).

To clarify, we write

$$
\pi_{5}=\pi_{5}\left(y_{5}\right)=\left\{\begin{array}{l}
0.3, y_{5}<1 \\
0.9, y_{5}>1
\end{array}\right\}=0.3+0.6 I\left(y_{5}>1\right)
$$

Then, noting that $I_{5}=1$ and $y_{5}=0.4$, we have that

$$
f\left(I_{5} \mid y, \lambda\right)=\pi_{5}^{I_{5}}\left(1-\pi_{5}\right)^{1-I_{5}}=\pi_{5}=0.3+0.6 I\left(y_{5}>1\right)=0.3 .
$$

Thus

$$
f(I \mid y, \lambda)=\prod_{i=1}^{N} \pi_{i}^{I_{i}}\left(1-\pi_{i}\right)^{1-I_{i}}
$$

doesn't depend on $\lambda$ or $y_{r}$ and is completely known.

Therefore,

$$
\begin{aligned}
& f(\lambda \mid D) \propto f\left(\lambda, I, y_{s}\right)=\int f\left(\lambda, I, y_{s}, y_{r}\right) d y_{r} \\
= & \int f(\lambda) f\left(y_{s}, y_{r} \mid \lambda\right) f\left(I \mid y_{s}, y_{r}, \lambda\right) d y_{r} \\
\propto & f(\lambda) f\left(y_{s} \mid \lambda\right) \int f\left(y_{r} \mid \lambda\right) d y_{r} \propto f(\lambda) f\left(y_{s} \mid \lambda\right) \times 1 \text { as before in (a). }
\end{aligned}
$$

(c) In this case, the sampling mechanism is nonignorable and inferences will be different to those in (a), because $f(I \mid y, \lambda)$ depends on a 
population value $y_{4}$ which is unknown (since unit 4 is not in the sample, i.e. $4 \in r)$; that is, $f(I \mid y, \lambda)$ is unknown. To clarify, we write

$$
\pi_{4}=\pi_{4}\left(y_{4}\right)=\left\{\begin{array}{l}
0.3, y_{4}<1 \\
0.9, y_{4}>1
\end{array}\right\}=0.3+0.6 I\left(y_{4}>1\right) \text {. }
$$

Then, noting that $I_{4}=0$ and $y_{4}$ is unknown, we have that

$$
f\left(I_{4} \mid y, \lambda\right)=\pi_{4}^{I_{4}}\left(1-\pi_{4}\right)^{1-I_{4}}=1-\pi_{4}=0.7-0.6 I\left(y_{4}>1\right)
$$

(a function of $y_{4}$ ).

So $f(I \mid y, \lambda)=\prod_{i=1}^{N} f\left(I_{i} \mid y, \lambda\right)$ is unknown.

With this in mind, we now write

$$
\begin{aligned}
f(\lambda \mid D) \propto f\left(\lambda, I, y_{s}\right) & =\int f\left(\lambda, I, y_{s}, y_{r}\right) d y_{r} \\
& =\int f(\lambda) f\left(y_{s}, y_{r} \mid \lambda\right) f\left(I \mid y_{s}, y_{r}, \lambda\right) d y_{r} \\
& \propto f(\lambda) f\left(y_{s} \mid \lambda\right) W,
\end{aligned}
$$

where

$$
\begin{aligned}
W & =W(\lambda)=\int f\left(y_{r} \mid \lambda\right) f\left(I_{4} \mid y_{4}\right) d y_{r} \\
& =\left(\prod_{\substack{i \in r \\
i \neq 4}}^{\infty} f\left(y_{i} \mid \lambda\right) d y_{i}\right) \int f\left(y_{4} \mid \lambda\right) f\left(I_{4} \mid y_{4}\right) d y_{4} \\
& =\left(\prod_{\substack{i \in r \\
i \neq 4}} 1 \int_{0}^{\infty} \lambda e^{-\lambda y_{4}}\left[0.7-0.6 I\left(y_{4}>1\right)\right] d y_{4}\right. \\
& =0.7 \int_{0}^{\infty} \lambda e^{-\lambda y_{4}} d y_{4}-0.6 \int_{1}^{\infty} \lambda e^{-\lambda y_{4}} d y_{4} \\
& =0.7 \times 1-0.6 e^{-\lambda .} .
\end{aligned}
$$

Thus

$$
f(\lambda \mid D) \propto \lambda^{n-1} e^{-\lambda y_{s T}}\left(7-6 e^{-\lambda}\right)=7 \lambda^{n-1} e^{-\lambda y_{s T}}-6 \lambda^{n-1} e^{-\lambda\left(y_{s T}+1\right)} .
$$


Thus

$$
f(\lambda \mid D)=c\left\{\frac{7}{y_{s T}^{n}}\left(\frac{y_{s T}^{n} \lambda^{n-1} e^{-\lambda y_{s T}}}{\Gamma(n)}\right)-\frac{6}{\left(y_{s T}+1\right)^{n}}\left(\frac{\left(y_{s T}+1\right)^{n} \lambda^{n-1} e^{-\lambda\left(y_{s T}+1\right)}}{\Gamma(n)}\right)\right\},
$$

where

$$
\begin{gathered}
1=\int f(\lambda \mid D) d \lambda=c\left\{\frac{7}{y_{s T}^{n}}(1)-\frac{6}{\left(y_{s T}+1\right)^{n}}(1)\right\} \\
\Rightarrow c=\left\{\frac{7}{y_{s T}^{n}}-\frac{6}{\left(y_{s T}+1\right)^{n}}\right\}^{-1} .
\end{gathered}
$$

Note 1: The posterior $f(\lambda \mid D)$ is a weighted average of two gamma densities where one of the weights is negative.

Note 2: The posterior density of $\mu=1 / \lambda$ is given by

$$
f(\mu \mid D)=f(\lambda=1 / \mu \mid D) / \mu^{2} .
$$

We now turn our attention to the predictive distribution of the nonsample total. Observe that

$$
f\left(y_{r} \mid D\right)=\int f\left(y_{r} \mid D, \lambda\right) f(\lambda \mid D) d \lambda,
$$

where

$$
f\left(y_{r} \mid D, \lambda\right) \propto f\left(y_{r}, y_{s}, I, \lambda\right) \propto\left\{\left[7-6 I\left(y_{4}>1\right)\right] \lambda e^{-\lambda y_{4}}\right\} \times \prod_{\substack{i \in r \\ i \neq 4}} \lambda e^{-\lambda y_{i}} .
$$

This suggests that we decompose the nonsample total according to

$$
y_{r T}=y_{0}+y_{4}
$$

(where $y_{0}$ is the total of all values in $y_{r}$ except for $y_{4}$ ) and think about how we can use the following facts:

$$
\begin{aligned}
& \left(y_{0} \perp y_{4} \mid D, \lambda\right) \quad\left(y_{4}\right. \text { is independent of all other nonsample } \\
& \quad \text { units, given } D \text { and } \lambda) \\
& \left(y_{0} \mid D, \lambda\right) \sim G(m-1, \lambda) \quad \text { (a simple distribution) } \\
& f\left(y_{4} \mid D, \lambda\right) \propto\left[7-6 I\left(y_{4}>1\right)\right] \lambda e^{-\lambda y_{4}}, y_{4}>0
\end{aligned}
$$

(a complicated distribution). 
One strategy is to use these facts to obtain the $\operatorname{cdf} F\left(y_{4} \mid D, \lambda\right)$, hence $F\left(y_{r T} \mid D, \lambda\right)$, hence $f\left(y_{r T} \mid D, \lambda\right)=F^{\prime}\left(y_{r T} \mid D, \lambda\right)$, hence $f(\bar{y} \mid D, \lambda)$, and hence ultimately the required $f(\bar{y} \mid D)=\int f(\bar{y} \mid D, \lambda) f(\lambda \mid D) d \lambda$.

First, $\begin{aligned} F\left(y_{4} \mid D, \lambda\right) & \propto\left\{\begin{array}{lr}7 \int_{0}^{y_{4}} \lambda e^{-\lambda t} d t, & 0<y_{4}<1 \\ 7 \int_{0}^{y_{4}} \lambda e^{-\lambda t} d t-6 \int_{1}^{y_{4}} \lambda e^{-\lambda t} d t, & y_{4}>1\end{array}\right. \\ & =\left\{\begin{array}{lr}7\left(1-e^{-\lambda y_{4}}\right), & 0<y_{4}<1 \\ 7\left(1-e^{-\lambda y_{4}}\right)-6\left(e^{-\lambda 1}-e^{-\lambda y_{4}}\right), & y_{4}>1 .\end{array}\right.\end{aligned}$

Thus $F\left(y_{4} \mid D, \lambda\right)=\left\{\begin{array}{lc}k\left(7-7 e^{-\lambda y_{4}}\right), & 0<y_{4}<1 \\ k\left(7-e^{-\lambda y_{4}}-6 e^{-\lambda}\right), & y_{4}>1,\end{array}\right.$

where $k=k(\lambda)=1 /\left(7-6 e^{-\lambda}\right)$, since $1=F\left(y_{4}=\infty \mid D, \lambda\right)=k\left(7-6 e^{-\lambda}\right)$.

Check: Since $\left(y_{4} \mid D, \lambda\right)$ is continuous we would expect that

$$
F\left(y_{4}=1^{+} \mid D, \lambda\right)-F\left(y_{4}=1^{-} \mid D, \lambda\right)=0 \text {. }
$$

The left hand side here is

$$
k\left(7-e^{-\lambda 1}-6 e^{-\lambda}\right)-k\left(7-7 e^{-\lambda 1}\right)=k\left(7-7 e^{-\lambda}\right)-k\left(7-7 e^{-\lambda}\right)=0
$$

(which is correct).

Next, writing $a \equiv y_{r T}$ for notational convenience, we have that

$$
\begin{aligned}
F(a \mid D, \lambda) & =P\left(y_{0}+y_{4} \leq a \mid D, \lambda\right) \\
& =E\left\{P\left(y_{0}+y_{4} \leq a \mid D, \lambda, y_{0}\right) \mid D, \lambda\right\} \\
& =\int_{0}^{a} P\left(y_{4} \leq a-y_{0} \mid D, \lambda, y_{0}\right) f\left(y_{0} \mid D, \lambda\right) d y_{0} \\
& =\int_{0}^{a} F\left(y_{4}=a-y_{0} \mid D, \lambda\right) f_{G(m-1, \lambda)}\left(y_{0}\right) d y_{0} \\
& =\int_{0}^{a} k\left[7-7 e^{-\lambda\left(a-y_{0}\right)}\right] f_{G(m-1, \lambda)}\left(y_{0}\right) d y_{0}, \quad 0<a<1 .
\end{aligned}
$$


For the case $a>1$ we find that

$$
\begin{aligned}
F(a \mid D, \lambda)= & \int_{0}^{a-1} k\left[7-e^{-\lambda\left(a-y_{0}\right)}-6 e^{-\lambda}\right] f_{G(m-1, \lambda)}\left(y_{0}\right) d y_{0} \\
& +\int_{a-1}^{a} k\left[7-7 e^{-\lambda\left(a-y_{0}\right)}\right] f_{G(m-1, \lambda)}\left(y_{0}\right) d y_{0} .
\end{aligned}
$$

Note: If $a>1$ and $0 \leq y_{0} \leq a-1$ then $1 \leq a-y_{0} \leq a$.

If $a>1$ and $a-1 \leq y_{0} \leq a$ then $0 \leq a-y_{0} \leq 1$.

Check: Since $(a \mid D, \lambda)$ is continuous we would expect that

$$
F\left(a=1^{+} \mid D, \lambda\right)-F\left(a=1^{-} \mid D, \lambda\right)=0 .
$$

The LHS here is

$$
\begin{aligned}
& \left\{\int_{0}^{1-1} k\left[7-e^{-\lambda\left(1-y_{0}\right)}-6 e^{-\lambda}\right] f_{G(m-1, \lambda)}\left(y_{0}\right) d y_{0}\right. \\
& \left.+\int_{1-1}^{1} k\left[7-7 e^{-\lambda\left(1-y_{0}\right)}\right] f_{G(m-1, \lambda)}\left(y_{0}\right) d y_{0}\right\} \\
& -\underbrace{\int_{0}^{1} k\left[7-7 e^{-\lambda\left(1-y_{0}\right)}\right] f_{G(m-1, \lambda)}\left(y_{0}\right) d y_{0}}_{0}=0 \text { (which is correct). }
\end{aligned}
$$

We now consider Leibniz's rule for differentiating an integral:

$$
\begin{aligned}
\frac{d}{d y} \int_{a(y)}^{b(y)} f(x, y) d x=\int_{a(y)}^{b(y)} & \frac{\partial}{\partial y} f(x, y) d x \\
& +b^{\prime}(y) f(b(y), y)-a^{\prime}(y) f(a(y), y)
\end{aligned}
$$

(where the symbols here are not directly related to those in this exercise).

Applying this rule for the case $0<a<1$, we obtain

$$
\begin{array}{r}
f(a \mid D, \lambda)=\frac{d F(a \mid D, \lambda)}{d a}=\int_{0}^{a} k\left[0-7 e^{-\lambda\left(a-y_{0}\right)}(-\lambda)\right] f_{G(m-1, \lambda)}\left(y_{0}\right) d y_{0} \\
+\frac{d a}{d a} k\left[7-7 e^{-\lambda(a-a)}\right] f_{G(m-1, \lambda)}(a) \quad \text { (this is zero) } \\
-\frac{d 0}{d a} k\left[7-7 e^{-\lambda(a-0)}\right] f_{G(m-1, \lambda)}(0) \quad \text { (this is zero) }
\end{array}
$$




$$
\begin{aligned}
& =7 k \lambda e^{-\lambda a} \int_{0}^{a} e^{\lambda y_{0}}\left(\frac{\lambda^{m-1} y_{0}^{m-2} e^{-\lambda \sigma_{0}}}{\Gamma(m-1)}\right) d y_{0}=7 k \lambda e^{-\lambda a} \frac{\lambda^{m-1}}{(m-2) !} \int_{0}^{a} y_{0}^{m-2} d y_{0} \\
& =7 k \lambda e^{-\lambda a} \frac{\lambda^{m-1}}{(m-1) !} a^{m-1}=7 k\left(\frac{\lambda^{m} a^{m-1} e^{-\lambda a}}{(m-1) !}\right)=7 k f_{G(m, \lambda)}(a) .
\end{aligned}
$$

Likewise, for the case $a>1$, we obtain

$$
\begin{aligned}
& f(a \mid D, \lambda)=\frac{d F(a \mid D, \lambda)}{d a}=\int_{0}^{a-1} k\left[0-e^{-\lambda\left(a-y_{0}\right)}(-\lambda)-0\right] f_{G(m-1, \lambda)}\left(y_{0}\right) d y_{0} \\
& +\frac{d(a-1)}{d a} k\left[7-e^{-\lambda(a-(a-1))}-6 e^{-\lambda}\right] f_{G(m-1, \lambda)}(a-1) \\
& -\frac{d 0}{d a} k\left[7-e^{-\lambda(a-0)}-6 e^{-\lambda}\right] f_{G(m-1, \lambda)}(0) \text { (this is zero) } \\
& +\int_{a-1}^{a} k\left[0-7 e^{-\lambda\left(a-y_{0}\right)}(-\lambda)\right] f_{G(m-1, \lambda)}\left(y_{0}\right) d y_{0} \\
& +\frac{d a}{d a} k\left[7-7 e^{-\lambda(a-a)}\right] f_{G(m-1, \lambda)}(a) \text { (this is zero) } \\
& -\frac{d(a-1)}{d a} k\left[7-7 e^{-\lambda(a-(a-1))}\right] f_{G(m-1, \lambda)}(a-1) \\
& =k \lambda e^{-\lambda a} \int_{0}^{a-1} e^{\lambda y_{0}}\left(\frac{\lambda^{m-1} y_{0}^{m-2} e^{-\lambda \sigma_{0}}}{\Gamma(m-1)}\right) d y_{0}+k\left[7-e^{-\lambda}-6 e^{-\lambda}\right] f_{G(m-1, \lambda)}(a-1) \\
& +7 k \lambda e^{-\lambda a} \int_{a-1}^{a} e^{\lambda y_{0}}\left(\frac{\lambda^{m-1} y_{0}^{m-2} e^{-\lambda \sigma_{0}}}{\Gamma(m-1)}\right) d y_{0}-7 k\left[1-e^{-\lambda}\right] f_{G(m-1, \lambda)}(a-1) \\
& =k \lambda e^{-\lambda a} \frac{\lambda^{m-1}}{(m-1) !}(a-1)^{m-1}+7 k\left(1-e^{-\lambda}\right) f_{G(m-1, \lambda)}(a-1) \\
& +7 k \lambda e^{-\lambda a} \frac{\lambda^{m-1}}{(m-1) !}\left[a^{m-1}-(a-1)^{m-1}\right]-7 k\left(1-e^{-\lambda}\right) f_{G(m-1, \lambda)}(a-1) \\
& =k e^{-\lambda}\left(\frac{\lambda^{m}(a-1)^{m-1} e^{-\lambda(a-1)}}{(m-1) !}\right)+7 k\left(\frac{\lambda^{m} a^{m-1} e^{-\lambda a}}{(m-1) !}\right) \\
& -7 k e^{-\lambda}\left(\frac{\lambda^{m}(a-1)^{m-1} e^{-\lambda(a-1)}}{(m-1) !}\right) \\
& =k\left\{7 f_{G(m, \lambda)}(a)-6 e^{-\lambda} f_{G(m, \lambda)}(a-1)\right\} .
\end{aligned}
$$


In summary so far,

$$
f(a \mid D, \lambda)=k \times \begin{cases}7 f_{G(m, \lambda)}(a), & 0<a<1 \\ 7 f_{G(m, \lambda)}(a)-6 e^{-\lambda} f_{G(m, \lambda)}(a-1), & a>1 .\end{cases}
$$

Check: Here,

$$
\begin{aligned}
& \int f(a \mid D, \lambda) d a= \\
& k \times\left\{\frac{7 F_{\sigma(m, \lambda)}(1)}{}+7\left[1-F_{G(m, \lambda)}(1)\right]-6 e^{-\lambda}\left[1-F_{G(m, \lambda)}(1-1)\right]\right\} \\
& \quad=\frac{1}{7-6 e^{-\lambda}} \times\left\{7-6 e^{-\lambda}[1-0]\right\}=1
\end{aligned}
$$

(which is correct).

Next, using the relationship $\bar{y}=\left(n \bar{y}_{s}+y_{r T}\right) / N$, we obtain:

$$
\begin{aligned}
& f(\bar{y} \mid D, \lambda)=f_{1}(\bar{y}, \lambda) \\
& \equiv N k(\lambda) 7 f_{G(m, \lambda)}\left(N \bar{y}-n \bar{y}_{s}\right) \\
& \text { for } \frac{n \bar{y}_{s}}{N}<\bar{y}<\frac{n \bar{y}_{s}+1}{N} \\
& f(\bar{y} \mid D, \lambda)=f_{2}(\bar{y}, \lambda) \\
& \equiv N k(\lambda)\left\{7 f_{G(m, \lambda)}\left(N \bar{y}-n \bar{y}_{s}\right)-6 e^{-\lambda} f_{G(m, \lambda)}\left(N \bar{y}-n \bar{y}_{s}-1\right)\right\} \\
& \text { for } \bar{y}>\frac{n \bar{y}_{s}+1}{N} \text {, }
\end{aligned}
$$

where:

$$
\begin{aligned}
& \frac{n \bar{y}_{s}}{N}=0.2857 \\
& \frac{n \bar{y}_{s}+1}{N}=0.4286 \\
& k(\lambda)=\frac{1}{7-6 e^{-\lambda}} \text { (as before). }
\end{aligned}
$$


Thus we finally obtain the required posterior predictive density:

$$
\begin{array}{ll}
f(\bar{y} \mid D)=g_{1}(\bar{y}) \equiv \int_{0}^{\infty} f_{1}(\bar{y}, \lambda) f(\lambda \mid D) d \lambda, & \frac{n \bar{y}_{s}}{N}<\bar{y}<\frac{n \bar{y}_{s}+1}{N} \\
f(\bar{y} \mid D)=g_{2}(\bar{y}) \equiv \int_{0}^{\infty} f_{2}(\bar{y}, \lambda) f(\lambda \mid D) d \lambda, & \bar{y}>\frac{n \bar{y}_{s}+1}{N},
\end{array}
$$

where $\quad f(\lambda \mid D)=\left\{\frac{7}{y_{s T}^{n}}-\frac{6}{\left(y_{s T}+1\right)^{n}}\right\}^{-1}$

(as obtained earlier).

$$
\times\left\{\frac{7}{y_{s T}^{n}} f_{G\left(n, y_{s T}\right)}(\lambda)-\frac{6}{\left(y_{s T}+1\right)^{n}} f_{G\left(n, y_{s T}+1\right)}(\lambda)\right\}
$$

Figure 9.3 shows the two densities $f(\mu \mid D)$ and $f(\bar{y} \mid D)$ under each of the scenarios in (a) and (c).

We see that inferences under the length-biased sampling scheme in (c) are lower than those under SRSWR in (a). This is because, generally speaking, length bias makes larger units more likely to be selected, and not adjusting for that bias naturally leads to inferences that are too high.

These patterns are consistent with the following point estimates as obtained numerically (see the R code below for details of the calculation):

$$
\begin{aligned}
& E(\mu \mid D)=1.38 \text { in (c) } \quad<\quad E(\mu \mid D)=2.00 \text { in (a) } \\
& E(\bar{y} \mid D)=1.19 \text { in (c) } \quad<\quad E(\bar{y} \mid D)=1.71 \text { in (a). }
\end{aligned}
$$

Note 1: In (a),

$$
(\mu \mid D) \sim \operatorname{IG}\left(n, y_{s T}\right),
$$

and therefore

$$
E(\mu \mid D)=y_{S T} /(n-1)=2 /(2-1)=2 \text { (exactly). }
$$

Note 2: The posterior predictive mean of $\bar{y}$ in (c) was obtained numerically as follows:

$$
\begin{aligned}
\hat{\bar{y}}=E(\bar{y} \mid D) & =\int_{\frac{n \bar{y}_{s}}{N}}^{\frac{n \bar{y}_{s}+1}{N}} \bar{y} g_{1}(\bar{y}) d \bar{y}+\int_{\frac{n \bar{y}_{s}+1}{N}}^{\infty} \bar{y} g_{2}(\bar{y}) d \bar{y} \\
& =0.01140+1.17546=1.1869 .
\end{aligned}
$$


Figure 9.3 Posterior and predictive densities

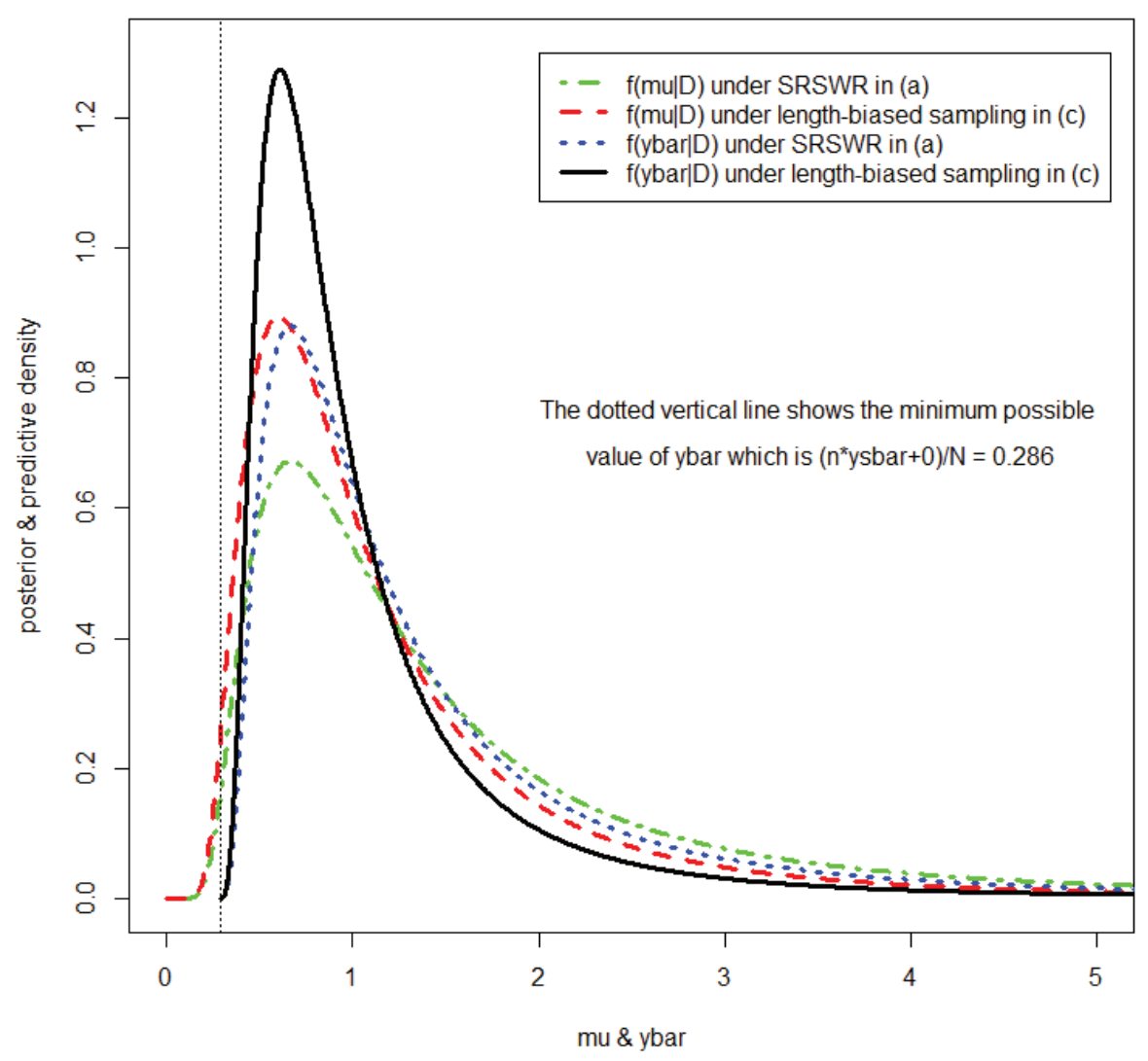

\section{R Code for Exercise 9.5}

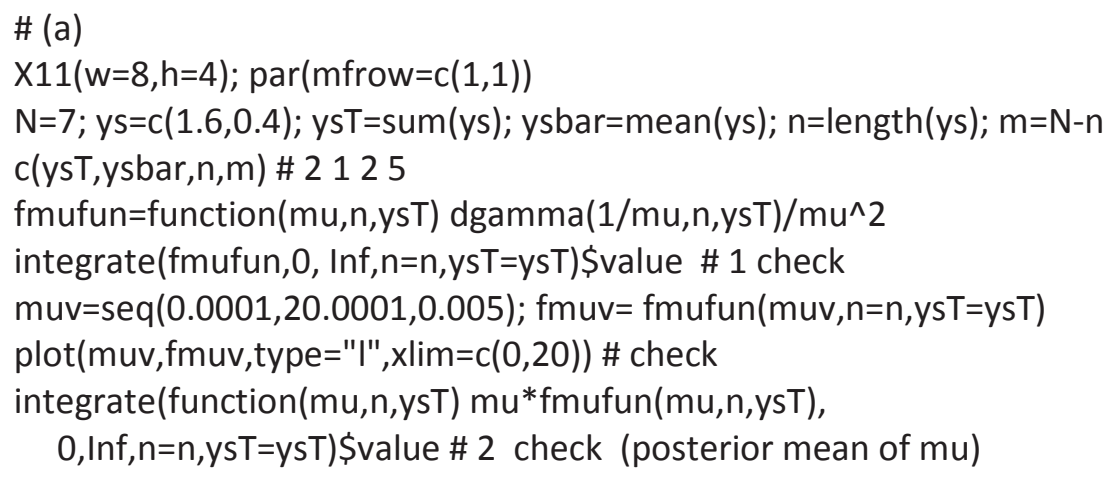


kybarfun=function(ybar,n,N,ysbar) $\quad\left(y \operatorname{bar}-(n / N)^{*} y s b a r\right)^{\wedge}(N-n-1) \quad / \quad \operatorname{ybar}^{\wedge} N$ const = integrate(kybarfun, $(\mathrm{n} / \mathrm{N}) *$ ysbar , Inf, $n=\mathrm{n}, \mathrm{N}=\mathrm{N}, \mathrm{ysbar}=\mathrm{ysbar}) \$$ value const \# 0.4083333 ybarv $=\operatorname{seq}((n / N) * y s b a r, \quad(n / N) * y s b a r+30,0.005)$ fybarv= kybarfun(ybarv, $n=n, N=N, y s b a r=y s b a r) /$ const plot(ybarv,fybarv, type="l",xlim=c(0,20)) \# check (1/const)*integrate(function(ybar,n,N,ysbar) ybar*kybarfun(ybar, n, N,ysbar), $(\mathrm{n} / \mathrm{N}) *$ ysbar, Inf, $\mathrm{n}=\mathrm{n}, \mathrm{N}=\mathrm{N}, \mathrm{ysbar}=\mathrm{ysbar}) \$$ value \# 1.714286 (predictive mean of ybar)

\# (c) $c=1 /\left(7 / y^{\wedge} T^{\wedge} n-6 /(y s T+1)^{\wedge} n\right) ; c \# 0.9230769$ flamfunc $=$ function $(\operatorname{lam}, \mathrm{n}, \mathrm{ysT}, \mathrm{c}) \mathrm{c}^{*}$

( (7/ysT^n)*dgamma(lam,n,ysT) - (6/(ysT+1)^n)*dgamma(lam,n,ysT+1) ) integrate(flamfunc, $0, \operatorname{lnf}, \mathrm{n}=\mathrm{n}, \mathrm{ys} \mathrm{T}=\mathrm{ys} \mathrm{T}, \mathrm{c}=\mathrm{c}) \$$ value \# 1 check lamv=seq $(0,20,0.01)$ plot(lamv, flamfunc(lamv, n=n,ysT=ysT,c=c), type="l") \# OK fmufunc=function $(m u, n, y s T, c) \quad c^{*}\left(1 / m u^{\wedge} 2\right)^{*}$

( (7/yst^n)*dgamma(1/mu,n,ysT) - (6/(ysT+1)^n)*dgamma(1/mu,n,ysT+1) )

integrate (fmufunc, $0, \operatorname{Inf}, \mathrm{n}=\mathrm{n}, \mathrm{ys} T=y s \mathrm{~T}, \mathrm{c}=\mathrm{c}) \$$ value \# 1 check integrate(function(mu,n,ysT,c) mu*fmufunc(mu,n,ysT,c),

$0, \operatorname{lnf}, \mathrm{n}=\mathrm{n}, \mathrm{ys}=\mathrm{ysT}, \mathrm{c}=\mathrm{c}) \$$ value $\# 1.384615 \quad$ (posterior mean of $\mathrm{mu}$ ) fmuvc=fmufunc(mu=muv, n=n,ysT=ysT,c); plot(muv,fmuvc) \# OK

ybarmin=ysT/N; ybarmin \# 0.2857143 Minimum possible value of ybar ybarcut=(ysT+1)/N; ybarcut \# 0.4285714 Cut-point for ybar

f1fun=function(ybar,lam,n,N,m,ysT) (N / (7-6*exp(-lam))) * $7 *$ dgamma( $N *$ ybar-ysT, $m$,lam)

f2fun=function(ybar,lam, n,N,m,ysT) (N / (7-6*exp(-lam))) * (7*dgamma(N*ybar-ysT,m,lam)-6*exp(-lam)*dgamma(N*ybar-ysT-1,m,lam) )

\# Check for particular values of lambda

lam $=0.764$ \# (example in the range ybarmin to ybarcut) p1 = integrate(f1fun, ybarmin,ybarcut, lam=lam, $n=n, N=N, m=m, y s T=y s T) \$$ value p2 = integrate(f2fun, ybarcut, Inf, lam=lam, $n=n, N=N, m=m, y s T=y s T) \$$ value $c(p 1, p 2, p 1+p 2) \# 0.0019218530 .9980781471 .000000000$ OK

lam=3.214 \# (example in the range ybarcut to infinity) p1 = integrate(f1fun, ybarmin,ybarcut, lam=lam, n=n,N=N,m=m,ysT=ysT) $\$$ value p2 = integrate(f2fun, ybarcut, Inf, lam=lam, $n=n, N=N, m=m, y s T=y s T) \$$ value $c(p 1, p 2, p 1+p 2) \# 0.22980260 .77019741 .0000000$ OK 


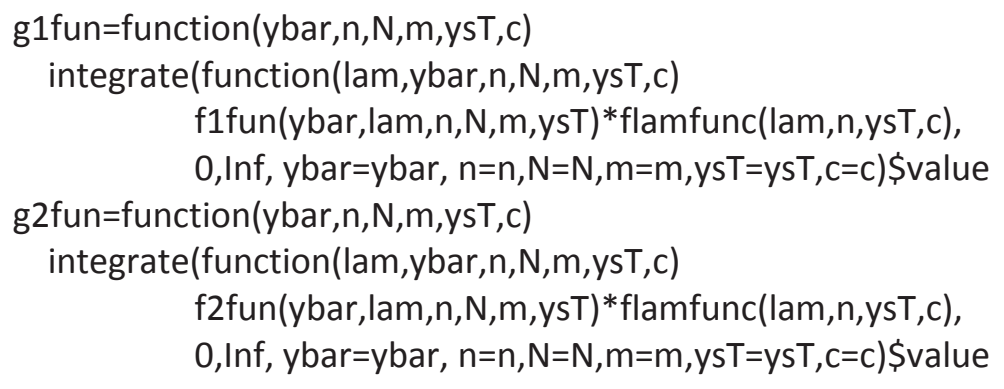

\# Check:

g1fun(ybar=0.4,n,N,m,ysT,c) \# 0.4119163 OK

g2fun(ybar=0.6,n, N,m,ysT,c) \# 1.274185 OK

ybarv1=seq(ybarmin,ybarcut,length.out=400); fybarv1=ybarv1

for $(j$ in 1 :length(ybarv1)) fybarv1[j] =

$$
\text { g1fun(ybar=ybarv1[j], n=n,N=N,m=m,ysT=ysT,c=c) }
$$

ybarv2=c( seq(ybarcut,1,length.out=200), seq(1,2,length.out=200), seq $(2,3$, length.out $=200)$, seq(3,5, length.out=200), seq $(5,10$, length.out $=200)$, seq $(10,50$, length.out $=200)$, seq $(50,1000$,length.out=200), seq $(1000,10000$,length.out=200) )

fybarv2=ybarv 2

for $(j$ in $1:$ length(ybarv2)) fybarv2[j] = g2fun(ybar=ybarv2[j], n=n, N=N,m=m,ysT=ysT,c=c)

$\operatorname{plot}(c(0,5), c(0,1.5)$, type $=" n ")$

lines(ybarv1, fybarv1, Ity=1,lwd=2)

lines(ybarv2, fybarv2, Ity=1,lwd=2) \# OK

\# Check

INTEG <- function(xvec, yvec, $a=\min (x v e c), b=\max (x v e c))\{$

\# Integrates numerically under a spline through the points given by \# the vectors $x v e c$ and yvec, from $a$ to $b$.

fit <- smooth.spline(xvec, yvec); spline.f <- function(x)\{predict(fit, $x) \$ y\}$ integrate(spline.f, $a, b) \$$ value \}

INTEG(seq $(0,1,0.01)$,seq $\left.(0,1,0.01)^{\wedge} 2,0,1\right)$ \# 0.3333333 check

prob1=INTEG(ybarv1,fybarv1,ybarmin,ybarcut)

prob2=INTEG(ybarv2,fybarv2,ybarcut,10000)

c(prob1,prob2,prob1+prob2) \# 0.028806590 .971193991 .00000058 OK

INTEG(c(ybarv1,ybarv2),c(fybarv1,fybarv2),ybarmin,10000) \# 1.000004 OK 
$\mathrm{X} 11(\mathrm{w}=8, \mathrm{~h}=6) ; \operatorname{par}(\operatorname{mfrow}=\mathrm{c}(2,1))$

plot(ybarv1, ybarv1* fybarv1, $x \lim =c(0,1))$ \# OK

plot(ybarv2, ybarv2* fybarv2, $x \lim =c(0,20))$ \# OK

term1 = INTEG(ybarv1, ybarv1*fybarv1,ybarmin,ybarcut)

term2 = INTEG(ybarv2, ybarv2*fybarv2,ybarcut,10000)

ybarhatc $=$ term $1+$ term $2 ; c($ term 1, term 2 , ybarhatc $)$

\# 0.011396011 .175462001 .18685801 (predictive mean of ybar)

$\mathrm{X} 11(\mathrm{w}=8, \mathrm{~h}=8) ; \operatorname{par}(\operatorname{mfrow}=\mathrm{c}(1,1)) \quad$ \# Produce final plots

$\operatorname{plot}(c(0,5), c(0,1.3)$, type="n",xlab="mu \& ybar",

ylab="posterior \& predictive density")

lines(muv, fmuv, Ity=4,lwd=3,col="green") \# mu under SRS

lines(muv,fmuvc,lty=2,lwd=3, col="red") \# mu under length-biased sampling lines(ybarv,fybarv, Ity=3,Iwd=3, col="blue") \# ybar under SRS

lines(ybarv1, fybarv1, Ity=1,Iwd=3); lines(ybarv2, fybarv2, Ity=1,lwd=3)

abline $(\mathrm{v}=(\mathrm{n} / \mathrm{N}) * \mathrm{ysbar}, \mathrm{Ity}=3) ;(\mathrm{n} / \mathrm{N}) * \mathrm{ysbar}$ \# 0.2857143

legend(2,1.3,c("f(mu|D) under SRSWR in (a)",

"f(mu|D) under length-biased sampling in (c)",

"f(ybar|D) under SRSWR in (a)",

"f(ybar|D) under length-biased sampling in (c)"),

Ity=c(4,2,3,1),Iwd=rep(3,4),col=c("green", "red","blue","black"))

text(3.5,0.75, "The dotted vertical line shows the minimum possible")

text(3.5,0.68," value of ybar which is $(n * y s b a r+0) / N=0.286 ")$

\section{Exercise 9.6 A Gibbs sampler for solving a length-biased with- replacement model}

Consider the Bayesian model in part (c) of Exercise 9.5, namely:

$$
\begin{aligned}
& f(I \mid y, \lambda)=\prod_{i=1}^{N} f\left(I_{i} \mid y, \lambda\right)=\prod_{i=1}^{N} \pi_{i}^{I_{i}}\left(1-\pi_{i}\right)^{1-I_{i}}, \quad n=\sum_{i=1}^{N} I_{i} \\
& f(y \mid \lambda)=\prod_{i=1}^{N} \lambda e^{-\lambda y_{i}}, \quad f(\lambda) \propto 1 / \lambda, \lambda>0,
\end{aligned}
$$

where: $N=7, \quad \pi_{i}=0.3 \forall i=1,2,3,5,6, \ldots, N$

$$
\begin{aligned}
& \pi_{4}=0.3 \text { if } y_{4}<1 \text { and } \pi_{4}=0.9 \text { if } y_{4}>1 \\
& D=\left(I, y_{s}\right)=((0,0,1,0,1,0,0),(1.6,0.4)), \quad n=2, \quad m=N-n=5 .
\end{aligned}
$$

Design and implement a suitable Gibbs sampler so as to obtain a random sample from the joint distribution of $\mu=1 / \lambda$ and $\bar{y}$. Illustrate your results with suitable plots and estimates. 


\section{Solution to Exercise 9.6}

Motivated by and using results in the previous exercise (Exercise 9.5), define $y_{0}=y_{r T}-y_{4}$ and then note that at the observed value of the data, the Bayesian model implies that:

$$
\begin{aligned}
& f\left(I \mid y_{s}, y_{0}, y_{4}, \lambda\right) \propto 7-6 I\left(y_{4}>1\right) \\
& f\left(y_{4} \mid y_{s}, y_{0}, \lambda\right) \sim G(1, \lambda) \\
& f\left(y_{0} \mid y_{s}, \lambda\right) \sim G(m-1, \lambda) \\
& f\left(y_{s} \mid \lambda\right) \propto \prod_{i=1}^{n} \lambda e^{-\lambda y_{i}} \\
& f(\lambda) \propto 1 / \lambda, \lambda>0 .
\end{aligned}
$$

So

$$
\begin{aligned}
f\left(I, y_{s}, y_{0}, y_{4}, \lambda\right) \propto \frac{1}{\lambda} \times\left(\prod_{i=1}^{n} \lambda e^{-\lambda y_{i}}\right) \times & \left(\lambda^{m-1} y_{0}^{m-2} e^{-\lambda y_{0}}\right) \\
& \times \lambda e^{-\lambda y_{4}} \times\left[7-6 I\left(y_{4}>1\right)\right] .
\end{aligned}
$$

We see that a suitable Gibbs sampler is defined by the following three conditionals:

1. $f\left(\lambda \mid I, y_{s}, y_{0}, y_{4}\right) \propto \lambda^{-1+n+m-1+1} e^{\lambda\left(y_{s T}+y_{0}+y_{4}\right)}=\lambda^{N-1} e^{-\lambda y_{T}}$

$$
\Rightarrow\left(\lambda \mid I, y_{s}, y_{0}, y_{4}\right) \sim G\left(N, y_{T}\right) \sim G\left(N, y_{s T}+y_{0}+y_{4}\right)
$$

2. $f\left(y_{0} \mid I, y_{s}, \lambda, y_{4}\right) \propto y_{0}^{m-2} e^{-\lambda y_{0}}$

$$
\Rightarrow\left(y_{0} \mid I, y_{s}, \lambda, y_{4}\right) \sim G(m-1, \lambda)
$$

3. $f\left(y_{4} \mid I, y_{s}, \lambda, y_{0}\right) \propto\left[7-6 I\left(y_{4}>1\right)\right] \lambda e^{-\lambda y_{4}}, \quad y_{4}>0$.

The first of these three conditionals are straightforward and easy to sample from. The third conditional can be sampled from via the inversion technique as follows.

First, for notational convenience, write the relevant random variable as $x$ with density

$$
f(x) \propto[7-6 I(x>1)] \lambda e^{-\lambda x}, \quad x>0 .
$$


Then the cdf of $x$ is

$$
\begin{aligned}
F(x) & =r\left\{\begin{array}{ll}
7 \int_{0}^{x} \lambda e^{-\lambda t} d t, & 0<x<1 \\
7 \int_{0}^{x} \lambda e^{-\lambda t} d t-6 \int_{1}^{x} \lambda e^{-\lambda t} d t, & x>1
\end{array}\right\} \\
& =r\left\{\begin{array}{cc}
7\left(1-e^{-\lambda x}\right), & \text { for some constant } r \\
7\left(1-e^{-\lambda x}\right)-6\left(e^{-\lambda 1}-e^{-\lambda x}\right), & x>1
\end{array}\right\} \\
& =r\left\{\begin{array}{cc}
7-7 e^{-\lambda x}, & 0<x<1 \\
7-e^{-\lambda x}-6 e^{-\lambda}, & x>1
\end{array}\right\},
\end{aligned}
$$

which equals $1=7-0-6 e^{-\lambda}$ in the limit as $x \rightarrow \infty$; so $r=1 /\left(7-6 e^{-\lambda}\right)$.

Now observe that $F(x=1)=\frac{7-7 e^{-\lambda}}{7-6 e^{-\lambda}}$.

This is a constant in the formula for the quantile function of $X$, obtained as follows.

First, if $p<\frac{7-7 e^{-\lambda}}{7-6 e^{-\lambda}}$ then we solve $p=r\left(7-7 e^{-x \lambda}\right)$

and thereby obtain $x=-\frac{1}{\lambda} \log \left(1-\frac{p}{7 r}\right)$.

Secondly, if $p>\frac{7-7 e^{-\lambda}}{7-6 e^{-\lambda}}$ then we solve $p=r\left(7-e^{-\lambda x}-6 e^{-\lambda}\right)$

and thereby obtain $x=-\frac{1}{\lambda} \log \left(7-6 e^{-\lambda}-\frac{p}{r}\right)$.

In summary, the quantile function of $x$ is given by

$$
Q(p)=\left\{\begin{array}{c}
x=-\frac{1}{\lambda} \log \left(1-\frac{p}{7}\left(7-6 e^{-\lambda}\right)\right), \quad p<\frac{7-7 e^{-\lambda}}{7-6 e^{-\lambda}} \\
x=-\frac{1}{\lambda} \log \left(7-6 e^{-\lambda}-p\left(7-6 e^{-\lambda}\right)\right), \quad p>\frac{7-7 e^{-\lambda}}{7-6 e^{-\lambda}}
\end{array}\right\} .
$$


So a procedure for sampling from the third conditional in the Gibbs sampler, namely

$$
f\left(y_{4} \mid I, y_{s}, \lambda, y_{0}\right) \propto\left[7-6 I\left(y_{4}>1\right)\right] \lambda e^{-\lambda y_{4}},
$$

is to draw $u \sim U(0,1)$ and then return $y_{4}=Q(u)$ as per equation (9.2).

Implementing the above Gibbs sampler for 20,000 iterations following a burn-in of 1,000 and then thinning out by a factor of 10 we obtained a random sample of size $J=2,000$ from the joint posterior/predictive distribution of $f\left(\lambda, y_{0}, y_{4} \mid I, y_{s}\right)$.

Figure 9.4 displays trace plots for the three unknowns, $\lambda, y_{0}, y_{4}$, sample ACFs for these over the last 20,000 iterations, and the three sample ACFs again over the final samples of size $J$. Figure 9.5 is a histogram of the $J$ simulated values of $\mu=1 / \lambda$ and Figure 9.6 is a histogram of the $J$ simulated values of $\bar{y}=\left(y_{s T}+y_{0}+y_{4}\right) / N$. In each histogram are shown a density estimate as well as three vertical lines for the Monte Carlo point estimate and 95\% CI for the mean.

The posterior density of $\mu$, i.e. $f(\mu \mid D)$, was estimated via RaoBlackwell as

where

$$
\hat{f}(\mu \mid D)=\frac{1}{J} \sum_{j=1}^{J} f_{I G(N, N \bar{y}(j))}(\mu),
$$

$$
\bar{y}^{(j)}=\left(y_{s T}+y_{0}^{(j)}+y_{4}^{(j)}\right) / N,
$$

using the fact that

$$
\left(\mu \mid I, y_{s}, y_{0}, y_{4}\right) \sim \operatorname{IG}\left(N, y_{s T}+y_{0}+y_{4}\right) \sim \operatorname{IG}\left(N, y_{T}\right) \sim \operatorname{IG}(N, N \bar{y}) .
$$

The posterior mean of $\mu$, i.e. $E(\mu \mid D)$, was also estimated via RaoBlackwell as

using the fact that

$$
\hat{\mu}=\frac{1}{J} \sum_{j=1}^{J} \frac{y_{s T}+y_{0}^{(j)}+y_{4}^{(j)}}{N-1}=\frac{1}{J} \sum_{j=1}^{J} \frac{N \bar{y}^{(j)}}{N-1}=1.41,
$$

$$
E\left(\mu \mid I, y_{s}, y_{0}, y_{4}\right)=\left(y_{s T}+y_{0}+y_{4}\right) /(N-1),
$$

with $95 \% \mathrm{CI}$ for the posterior mean equal to

$$
\left(\hat{\mu} \pm 1.96 \sqrt{\frac{1}{J}} \sqrt{\frac{1}{J-1} \sum_{j=1}^{J}\left(\frac{N \bar{y}^{(j)}}{N-1}-\hat{\mu}\right)^{2}}\right)=(1.34,1.47) .
$$


Note: This is consistent with the exact value, namely $E(\mu \mid D)=1.38$, as obtained in Exercise 9.5.

The predictive density of $\bar{y}$ was estimated by smoothing a probability histogram of the simulated values $\bar{y}^{(j)}$, and the predictive mean of $\bar{y}$, i.e. $E(\bar{y} \mid D)$, was estimated by

$$
\hat{\bar{y}}=\frac{1}{J} \sum_{j=1}^{J} \bar{y}^{(j)}=1.21,
$$

with $95 \%$ CI

$$
\left(\hat{\bar{y}} \pm 1.96 \sqrt{\frac{1}{J}} \sqrt{\frac{1}{J-1} \sum_{j=1}^{J}\left(\bar{y}^{(j)}-\hat{\bar{y}}\right)^{2}}\right)=(1.15,1.26) .
$$

Note 1: This is consistent with the exact value, $E(\bar{y} \mid D)=1.19$, as obtained in Exercise 9.5.

Note 2: We may be able to improve on the above 'histogram' estimation of $E(\bar{y} \mid D)$ using Rao-Blackwell methods. For example, observe that

$$
E\left(\bar{y} \mid D, \lambda, y_{4}\right)=\frac{1}{N}\left(y_{s T}+y_{4}+\frac{m-1}{\lambda}\right) \text {. }
$$

So we define

$$
e_{j}=E\left(\bar{y} \mid D, \lambda_{j}, y_{4}^{(j)}\right)=\frac{1}{N}\left(y_{s T}+y_{4}^{(j)}+\frac{m-1}{\lambda_{j}}\right) .
$$

The associated Rao-Blackwell estimate of $E(\bar{y} \mid D)$ is

$$
\bar{e}=\frac{1}{J} \sum_{j=1}^{J} e_{j}=1.21
$$

with $95 \%$ CI

$$
\left(\bar{e} \pm 1.96 \sqrt{\frac{1}{J}} \sqrt{\frac{1}{J-1} \sum_{j=1}^{J}\left(e_{j}-\bar{e}\right)^{2}}\right)=(1.16,1.26) .
$$

Note 3: In this case, applying Rao-Blackwell methods has only slightly narrowed the CI for $E(\bar{y} \mid D)$. 
Figure 9.4 Trace plots and sample ACFs

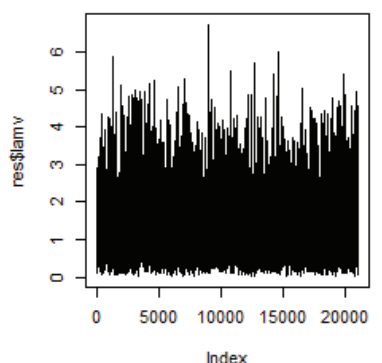

Series lamv

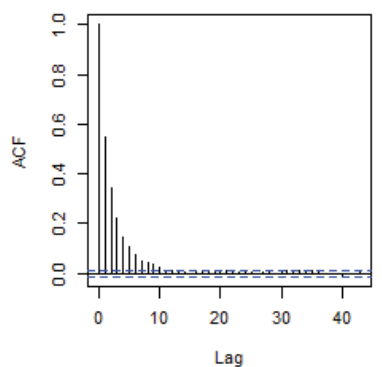

Series lamvec

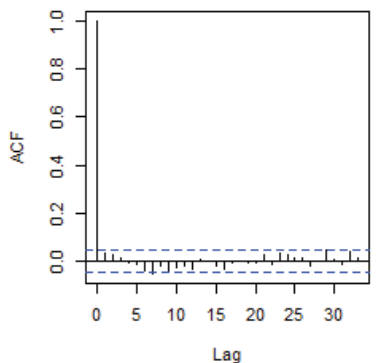

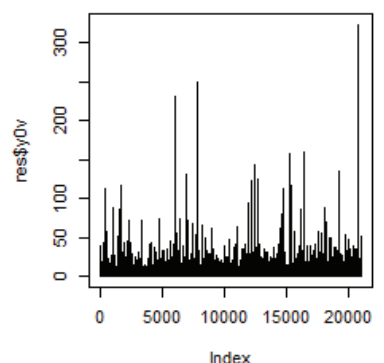

Series y0v

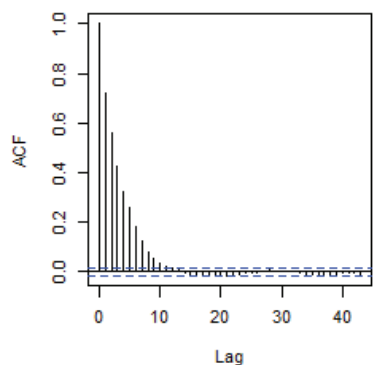

Series y0vec

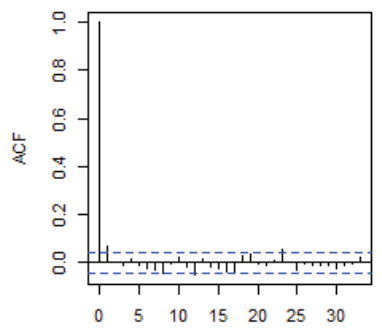

Lag

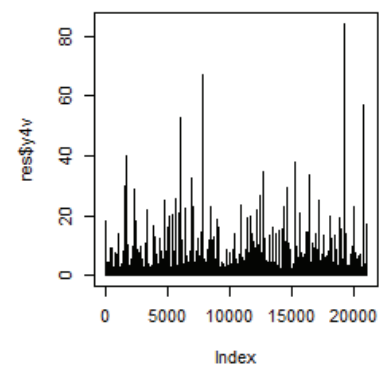

Series $y 4 v$

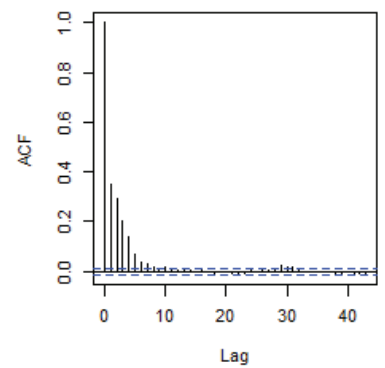

Series y4vec

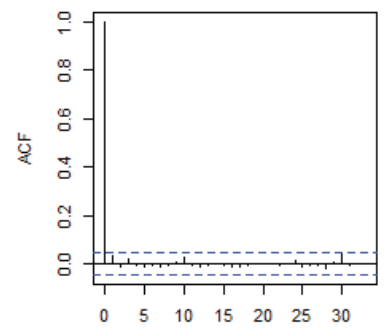

Lag 
Figure 9.5 Inference on the superpopulation mean

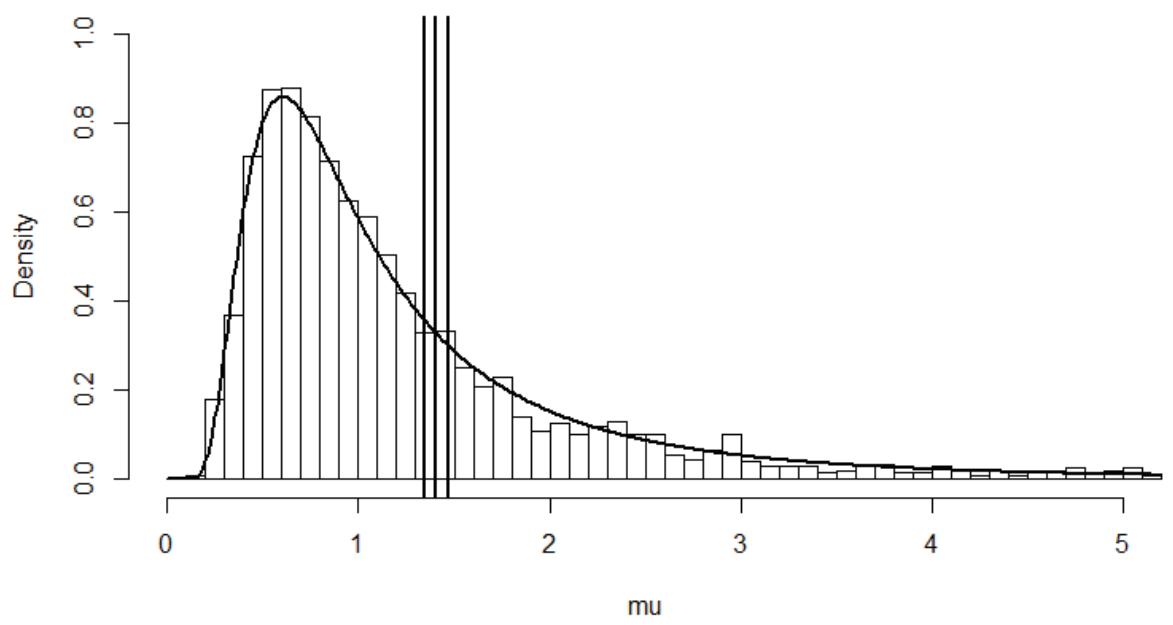

Figure 9.6 Inference on the finite population mean

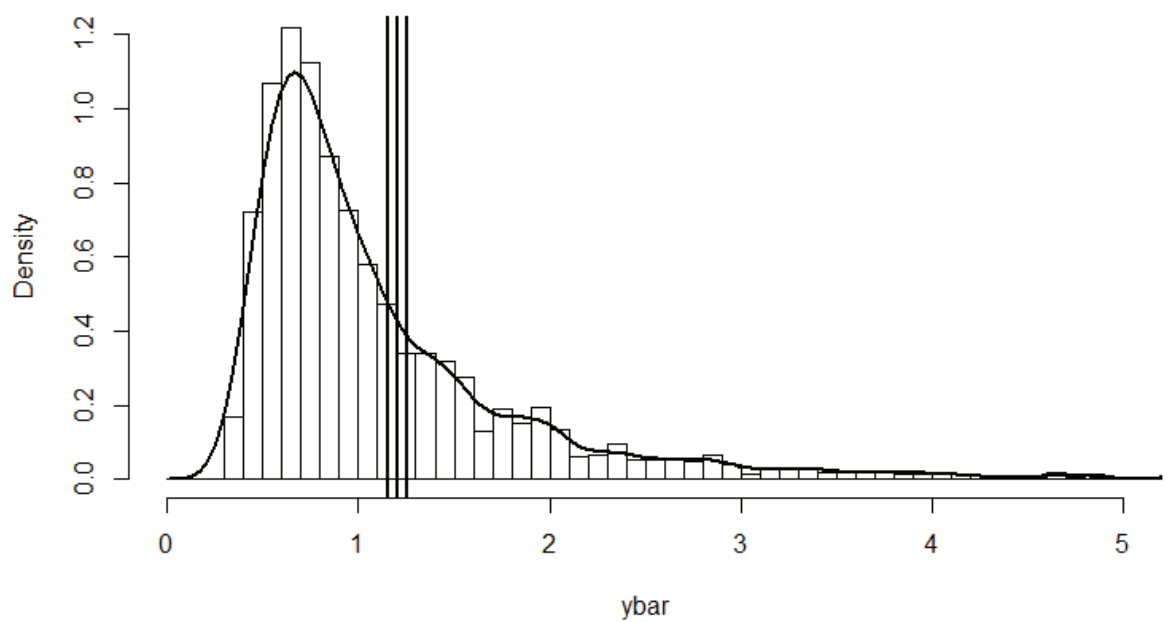




\section{R Code for Exercise 9.6}

$$
\begin{aligned}
\text { Qfun }= & \text { function }(p=0.5, \operatorname{lam}=1)\{ \\
& \mathrm{c1}=\left(7-7^{*} \exp (-\operatorname{lam})\right) /\left(7-6^{*} \exp (-\operatorname{lam})\right) \\
& \text { if }(p<=c 1) \mathrm{c} 2=1-(p / 7) *\left(7-6^{*} \exp (-\operatorname{lam})\right) \\
& \text { if }(p>c 1) c 2=7-6^{*} \exp (-\operatorname{lam})-p^{*}\left(7-6^{*} \exp (-\operatorname{lam})\right) \\
& -(1 / \operatorname{lam})^{*} \log (\mathrm{c} 2)
\end{aligned}
$$

\# Check:

pvec=seq(0,1,0.001); Qvec=pvec

for(i in 1:length(pvec)) Qvec[i] = Qfun(p=pvec[i],lam=1.3)

plot(pvec,Qvec); plot(Qvec,pvec) \# OK

$\mathrm{GS}=$ function $(J=1000, \mathrm{~N}=7, \mathrm{n}=2, \mathrm{~m}=5, \mathrm{ys} \mathrm{T}=2, \mathrm{lam}=1, \mathrm{y} 0=1, \mathrm{y} 4=1)\{$

lamv=lam; $y 0 v=y 0 ; y 4 v=y 4 ;$ for $(j$ in $1: J)\{$

$$
\begin{aligned}
& \text { lam=rgamma }(1, N, y s T+y 0+y 4) \\
& y 0=\operatorname{rgamma}(1, m-1, \operatorname{lam}) \\
& \text { u=runif(1); } y 4=Q f u n(p=u, l a m=l a m) \\
& \text { lamv=c(lamv,lam); } \mathrm{y} 0 v=c(y 0 v, y 0) ; y 4 v=c(y 4 v, y 4) \quad
\end{aligned}
$$

list (lamv=lamv, y0v=y0v, y4v=y4v) \}

$\mathrm{X} 11(\mathrm{w}=8, \mathrm{~h}=9) ; \operatorname{par}(\operatorname{mfrow}=\mathrm{c}(3,3)) ;$ set.seed(321); date()

res= $\mathrm{GS}(\mathrm{J}=21000, \mathrm{~N}=7, \mathrm{n}=2, \mathrm{~m}=5$, ys $\mathrm{T}=2$, lam=1,y0=1,y4=1); date() \# took 3 secs plot(res\$lamv,type="I"); plot(res\$y0v,type="I"); plot(res\$y4v,type="I") \# OK

lamv=res\$lamv[-(1:1001)]; y0v=res\$y0v[-(1:1001)]; y4v=res\$y4v[-(1:1001)]; acf(lamv); acf(yOv); acf(y4v) \# high serial correlation, so need to thin out inc= seq $(10,20000,10) ;$ lamvec=lamv[inc]; $y 0 v e c=y 0 v[i n c] ; y 4 v e c=y 4 v[i n c]$; acf(lamvec); acf(y0vec); acf(y4vec) \# OK $\mathrm{J}=$ length(lamvec); J \# 2000

$N=7 ; n=2 ; m=5 ; y s T=2 ;$ muvec $=1 /$ lamvec; $y$ barvec $=(1 / N) *(y s T+y 0 v e c+y 4 v e c)$ ybarhat=mean(ybarvec);

ybarci=ybarhat+c(-1,1)*qnorm(0.975)*sd(ybarvec)/sqrt(J)

c(ybarhat, ybarci, ybarci[2]-ybarci[1]) \# 1.2045191 .1516191 .2574190 .105800

evec $=(1 / N) *(y s T+y 4 v e c+(m-1) / l a m v e c)$

ebar=mean(evec); eci= ebar+c(-1,1)*qnorm(0.975)*sd(evec)/sqrt(J)

c(ebar,eci,eci[2]-eci[1]) \# 1.2091236 1.1581903 1.2600569 0.1018666

muhat $=(\mathrm{N} /(\mathrm{N}-1)) *$ ybarhat muci=muhat $+\mathrm{c}(-1,1) *$ qnorm $(0.975) * \mathrm{sd}\left((\mathrm{N} /(\mathrm{N}-1))^{*}\right.$ ybarvec $) / \operatorname{sqrt}(\mathrm{J})$ c(muhat, muci) \# 1.4052721 .3435561 .466989 
mugrid $=\operatorname{seq}(0.001,10.001,0.01)$

fmuhat=mugrid; for(i in 1:length(mugrid))

fmuhat $[i]=\operatorname{mean}\left(\operatorname{dgamma}\left(1 /\right.\right.$ mugrid $[i], N, N^{*}$ ybarvec $\left.) / \operatorname{mugrid}[i]^{\wedge} 2\right)$

$\mathrm{X} 11(\mathrm{w}=8, \mathrm{~h}=5)$

hist (muvec, prob=T, $x \lim =c(0,5), y \lim =c(0,1)$, breaks=seq $(0,80,0.1)$,

$$
x l a b=" m u ", \text { main="") }
$$

lines(mugrid,fmuhat, $l w d=2)$; abline $(v=c$ (muhat, muci), $I w d=2$ )

hist $(y b a r v e c$, prob $=T, x \lim =c(0,5), y \lim =c(0,1.2)$, breaks=seq $(0,80,0.1)$, xlab="ybar", main=" ")

lines(density(ybarvec), Iwd=2); abline(v=c(ybarhat, ybarci), lwd=2)

\section{Exercise 9.7 Gibbs sampler for a length-biased without- replacement sampling model}

Earlier we defined $L=\left(L_{1}, \ldots, L_{n}\right)$ as the vector of the labels of the selected units in the order in which they are sampled.

Now consider the following Bayesian finite population model:

$$
\begin{aligned}
& f(L \mid y, \lambda)=\prod_{i=1}^{n} \frac{y_{L_{i}}}{y_{T}-\sum_{j=1}^{i-1} y_{L_{j}}}, L=\left(L_{1}, \ldots, L_{n}\right) \in\left\{\left(a_{1}, \ldots, a_{n}\right):\right. \\
& \left.a_{i} \in\{1, \ldots, N\} \forall i \in\{1, \ldots, n\} \& a_{i} \neq a_{j} \forall i, j \in\{1, \ldots, n\}\right\} \\
& f(y \mid \lambda)=\prod_{i=1}^{N} \lambda e^{-\lambda y_{i}}, y_{i}>0 \forall i \\
& f(\lambda) \propto 1 / \lambda, \lambda>0 .
\end{aligned}
$$

Design and implement a suitable Gibbs sampler so as to obtain a random sample from the joint distribution of $\mu=1 / \lambda$ and $\bar{y}$ in the case where

$$
N=7, n=3, m=N-n=4
$$

and when the observed data is

$$
D=\left(L, y_{s}\right)=((4,3,6),(1.6,0.4,0.7)) \text {. }
$$

Illustrate your results with suitable plots and estimates. 


\section{Solution to Exercise 9.7}

The sampling mechanism here is defined by the model density of $L$, which may also be written as

$$
\begin{gathered}
f\left(L_{1}, \ldots, L_{n} \mid y, \lambda\right)=\frac{y_{L_{1}}}{y_{T}} \times \frac{y_{L_{2}}}{y_{T}-y_{L_{1}}} \times \frac{y_{L_{3}}}{y_{T}-y_{L_{1}}-y_{L_{2}}} \times \ldots \times \frac{y_{L_{n}}}{y_{T}-y_{L_{1}}-y_{L_{2}}-\ldots-y_{L_{n-1}}} \\
\text { for } L=(1, \ldots, n),(1,3,2, \ldots, n), \ldots,(N, N-1, \ldots, N-n+1) .
\end{gathered}
$$

This pdf implies that units are selected from the finite population, one by one and without replacement, in such a way that the probability of selecting a unit on any given draw is its value divided by the sum of the values of all units which have not yet been sampled at that point in time. We call this procedure length-biased sampling without replacement.

Note: This is an example of a sampling mechanism that is nonignorable but known. If $f(L \mid y, \lambda)$ depended on $\lambda$, or on some other unknown quantity, then we would say that the sampling mechanism is nonignorable and unknown.

In the present case it is convenient to relabel the population units-after sampling —in such a way that $L=(1,2, \ldots, n)$ and so also $s=(1, \ldots, n)$ and $r=(n+1, \ldots, N)$. Assuming that this is done, we may write the density of the sampling mechanism in various other and simpler ways, for example:

$$
\begin{aligned}
f(L \mid y, \lambda) & =\frac{y_{1}}{y_{T}} \times \frac{y_{2}}{y_{T}-y_{1}} \times \frac{y_{3}}{y_{T}-y_{1}-y_{2}} \times \ldots \times \frac{y_{n}}{y_{T}-y_{1}-y_{2}-\ldots-y_{n-1}} \\
& =\frac{y_{1}}{y_{1}+\ldots+y_{N}} \times \frac{y_{2}}{y_{2}+\ldots+y_{N}} \times \frac{y_{3}}{y_{3}+\ldots+y_{N}} \times \ldots \times \frac{y_{n}}{y_{n}+\ldots+y_{N}} \\
& =\prod_{i=1}^{n} \frac{y_{i}}{y_{i}+\ldots+y_{N}}=\prod_{i=1}^{n} \frac{y_{i}}{\sum_{j=i}^{N} y_{j}}, \text { etc. }
\end{aligned}
$$

Note: We have not previously relabelled population units in this manner because doing so would have provided only marginal notational convenience and may have obscured the nature of the sampling mechanisms we were trying to illustrate. In the next chapter, we will again make use of a convenient relabelling scheme similar to the one applied here.

With the above relabelling in place, and noting that 


$$
\left(y_{r T} \mid \lambda\right) \sim G(m, \lambda),
$$

the joint posterior density of $\lambda$ and $y_{r T}$ (given the data, $D=\left(L, y_{s}\right)$ ) may now be written as

$$
\begin{aligned}
f\left(\lambda, y_{r T} \mid D\right) & \propto f\left(\lambda, y_{r T}, y_{s}, L\right)=f(\lambda) f\left(y_{s} \mid \lambda\right) f\left(y_{r T} \mid \lambda\right) f\left(L \mid y_{s}, y_{r T}\right) \\
& \propto \frac{1}{\lambda} \times\left(\prod_{i=1}^{n} \lambda e^{-\lambda y_{i}}\right) \times\left(\lambda^{m} y_{r T}^{m-1} e^{-\lambda y_{r T}}\right) \times \prod_{i=1}^{n} \frac{1}{y_{i}+\ldots+y_{n}+y_{r T}} .
\end{aligned}
$$

This joint density suggests a Metropolis-Hastings algorithm with a Gibbs step defined by the conditional posterior distribution

$$
\left(\lambda \mid D, y_{r T}\right) \sim G\left(N, y_{s T}+y_{r T}\right)
$$

and a Metropolis step defined by a rather complicated conditional predictive density defined by

$$
f\left(y_{r T} \mid D, \lambda\right) \propto y_{r T}^{m-1} e^{-\lambda y_{r T}} \prod_{i=1}^{n} \frac{1}{\left(y_{i}+\ldots+y_{n}\right)+y_{r T}} .
$$

At this point it is useful to recall a data augmentation technique based on the identity

$$
1=\int_{0}^{\infty} x e^{-x w} d w
$$

or equivalently

$$
\frac{1}{x}=\int_{0}^{\infty} e^{-x w} d w
$$

which can be applied here so as to yield the identity

$$
\prod_{i=1}^{n} \frac{1}{y_{i}+\ldots+y_{n}+y_{r T}}=\prod_{i=1}^{n} \int_{0}^{\infty} e^{-\left(y_{i}+\ldots+y_{n}+y_{r T}\right) w_{i}} d w_{i} .
$$

This suggests that we introduce an artificial or latent random variable $w=\left(w_{1}, \ldots, w_{n}\right)$ into our model which is defined in such a way that the joint posterior density of $\lambda, y_{r T}$ and $w$ is given by

$$
f\left(\lambda, y_{r T}, w \mid D\right) \propto \frac{1}{\lambda} \times\left(\prod_{i=1}^{n} \lambda e^{-\lambda y_{i}}\right) \times\left(\lambda^{m} y_{r T}^{m-1} e^{-\lambda y_{r T}}\right) \times \prod_{i=1}^{n} e^{-\left(y_{i}+\ldots+y_{n}+y_{r T}\right) w_{i}} .
$$

Note: If we integrate this joint density with respect to $w$ then we recover $f\left(\lambda, y_{r T} \mid D\right)$ as above. 
The above expression for $f\left(\lambda, y_{r T}, w \mid D\right)$ now suggests a 'pure' Gibbs sampler defined by the following $n+2$ conditional distributions:

$$
\begin{aligned}
& \left(\lambda \mid D, y_{r T}, w\right) \sim G\left(N, y_{s T}+y_{r T}\right) \\
& \left(y_{r T} \mid D, \lambda, w\right) \sim G\left(m, \lambda+w_{T}\right) \quad \text { where } \quad w_{T}=w_{1}+\ldots+w_{n} \\
& \left(w_{i} \mid D, \lambda, y_{r T}\right) \sim \perp G\left(1, y_{i}+\ldots+y_{n}+y_{r T}\right), \quad i=1, \ldots, n .
\end{aligned}
$$

This Gibbs sampler can be used to generate a random sample

$$
\left(\lambda_{j}, y_{r T}^{(j)}, w^{(j)}\right) \sim \operatorname{iid} f\left(\lambda, y_{r T}, w \mid D\right), j=1, \ldots, J,
$$

where

$$
w^{(j)}=\left(w_{1}^{(j)}, \ldots, w_{n}^{(j)}\right) .
$$

This sample can then be used for Monte Carlo inference on the quantities of interest, namely $\mu=1 / \lambda$ and $\bar{y}=\left(y_{s T}+y_{r T}\right) / N$.

Applying the above Gibbs sampler (with a suitable burn-in and thinning) we obtained a random sample of size $J=2,000$ from the joint posterior distribution of $\lambda, y_{r T}$ and $w=\left(w_{1}, \ldots, w_{n}\right)$.

The posterior density of $\mu$ was estimated via Rao-Blackwell as

$$
\hat{f}(\mu \mid D)=\frac{1}{J} \sum_{j=1}^{J} f_{I G(N, N \bar{y}(j))}(\mu),
$$

where

$$
\bar{y}^{(j)}=\left(y_{s T}+y_{r T}^{(j)}\right) / N,
$$

using the fact that

$$
\left(\mu \mid I, y_{s}, y_{r T}, w\right) \sim \operatorname{IG}\left(N, y_{s T}+y_{r T}\right) \sim \operatorname{IG}\left(N, y_{T}\right) \sim \operatorname{IG}(N, N \bar{y}) .
$$

The posterior mean of $\mu$ was also estimated via Rao-Blackwell as

$$
\hat{\mu}=\frac{1}{J} \sum_{j=1}^{J} \frac{y_{s T}+y_{r T}^{(j)}}{N-1}=\frac{1}{J} \sum_{j=1}^{J} \frac{N \bar{y}^{(j)}}{N-1}=0.619,
$$

using the fact that

$$
E\left(\mu \mid I, y_{s}, y_{r T}, w\right)=\left(y_{s T}+y_{r T}\right) /(N-1),
$$

with $95 \%$ CI

$$
\left(\hat{\mu} \pm 1.96 \sqrt{\frac{1}{J}} \sqrt{\frac{1}{J-1} \sum_{j=1}^{J}\left(\frac{N \bar{y}^{(j)}}{N-1}-\hat{\mu}\right)^{2}}\right)=(0.614,0.624) .
$$


The predictive density of $y_{r T}$ was likewise estimated via Rao-Blackwell as

$$
\hat{f}\left(y_{r T} \mid D\right)=\frac{1}{J} \sum_{j=1}^{J} f_{G\left(m, \lambda_{j}+w_{T}^{(j)}\right)}\left(y_{r T}\right),
$$

where

$$
w_{T}^{(j)}=w_{1}^{(j)}+\ldots+w_{n}^{(j)} .
$$

The predictive mean of $y_{r T}$ was also estimated via Rao-Blackwell as

$$
\hat{y}_{r T}=\frac{1}{J} \sum_{j=1}^{J} \frac{m}{\lambda_{j}+w_{T}^{(j)}}=1.013
$$

using the fact that

$$
E\left(y_{r T} \mid I, y_{s}, \lambda, w\right)=m /\left(\lambda+w_{T}\right),
$$

with $95 \%$ CI

$$
\left(\hat{y}_{r T} \pm 1.96 \sqrt{\frac{1}{J}} \sqrt{\frac{1}{J-1} \sum_{j=1}^{J}\left(\frac{m}{\lambda_{j}+w_{T}^{(j)}}-\hat{y}_{r T}\right)^{2}}\right)=(0.993,1.033) .
$$

These Rao-Blackwell estimates for $y_{r T}$ were then transformed into estimates for $\bar{y}$ via the equation

$$
\bar{y}=\left(y_{s T}+y_{r T}\right) / N \text {. }
$$

In this way, we estimated $\bar{y}$ 's posterior mean by 0.530 , with $95 \% \mathrm{CI}$ $(0.614,0.624)$.

Figure 9.7 shows trace plots for $\lambda, y_{r T}$ and $w_{1}$, sample ACFs for these quantities over the last 10,000 iterations, and these three sample ACFs again but calculated using only the final smaller samples of size $J=2,000$.

Figures 9.8 and 9.9 (page 464) show two histograms, of the $J$ simulated values of $\mu=1 / \lambda$, and of the $J$ simulated values of $\bar{y}=\left(y_{s T}+y_{r T}\right) / N$. In each histogram are shown a density estimate and three vertical lines representing the Monte Carlo point estimate and 95\% CI for the posterior mean.

Note 1: The type of sampling mechanism which features in this exercise has applications in the analysis of oil discovery data. For further details, see West (1996). 
Note 2: In this chapter, we have presented several examples of how Bayesian methods can be used to perform inference on an exponential finite population under biased sampling. For another such example, see Puza and O’Neill (2005).

Figure 9.7 Trace plots and sample ACFs for samples obtained via MCMC

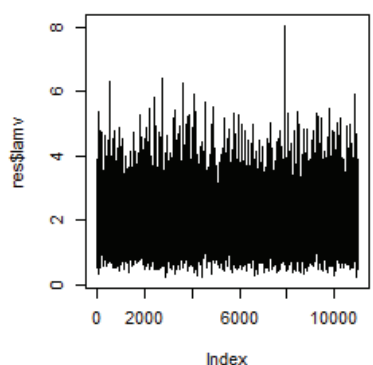

Series lamv

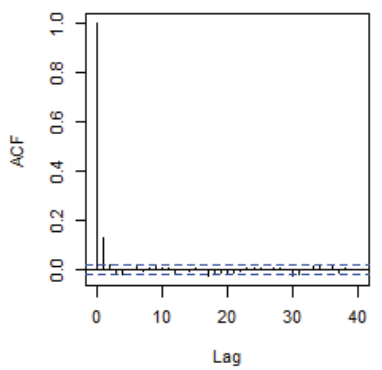

Series lamvec

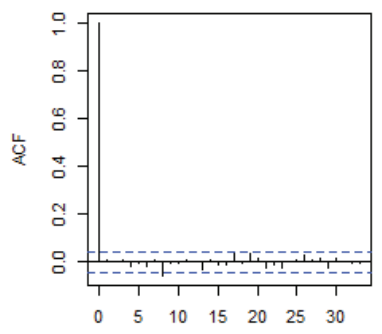

Lag

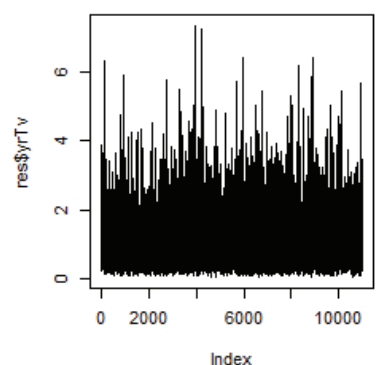

Series yrTv

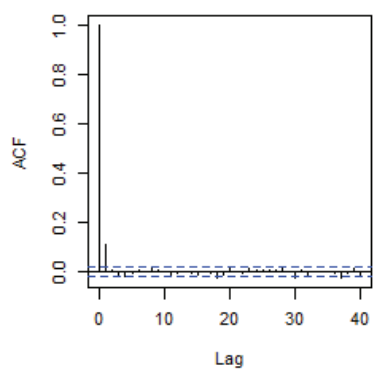

Series yrTvec

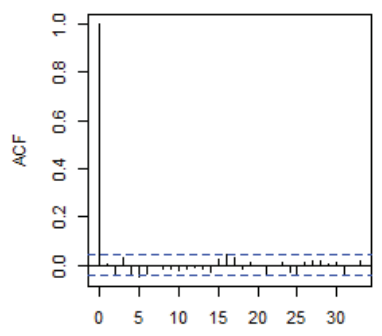

Lag

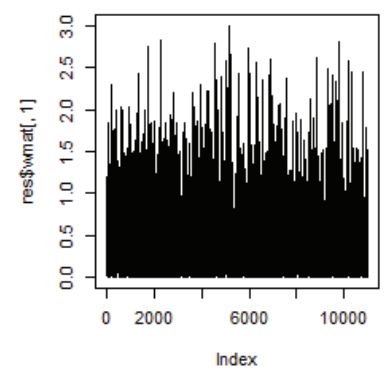

Series wmat[, 1]

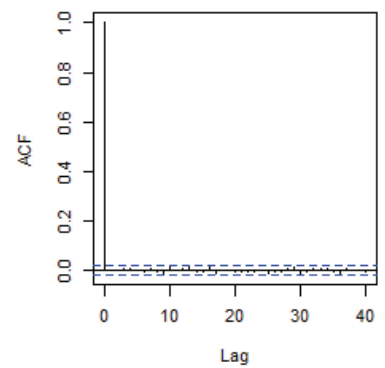

Series wmatrix[, 1]

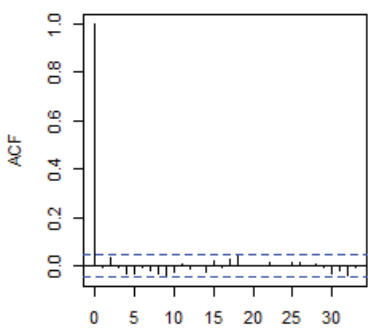

Lag 
Figure 9.8 Inference on the superpopulation mean via MCMC

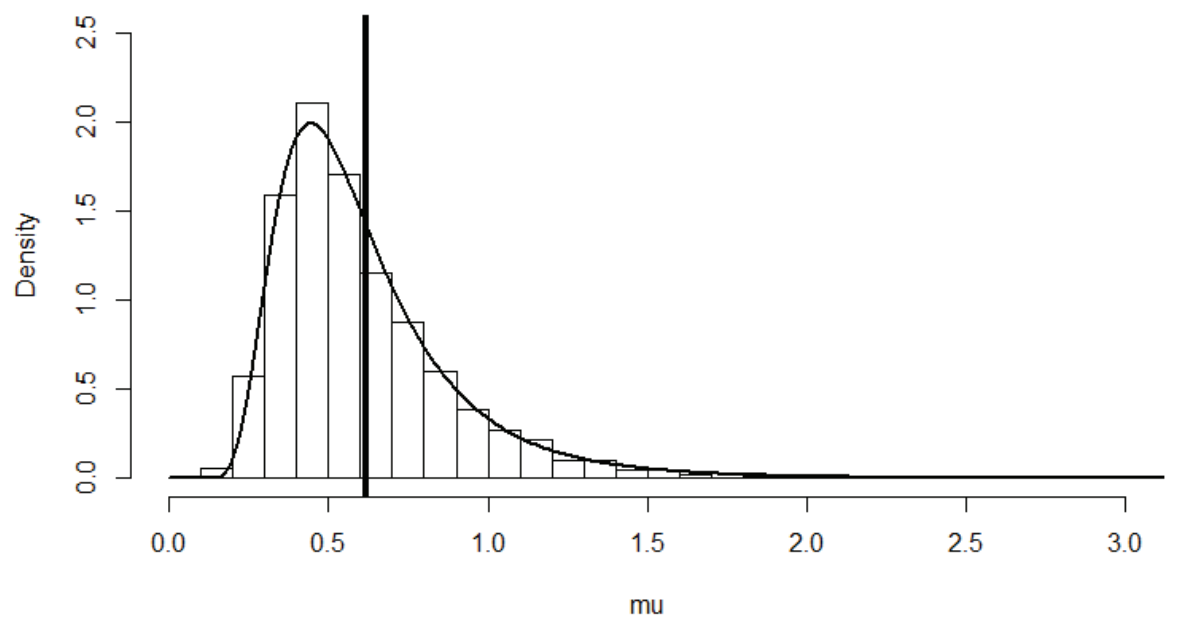

Figure 9.9 Inference on the finite population mean via MCMC

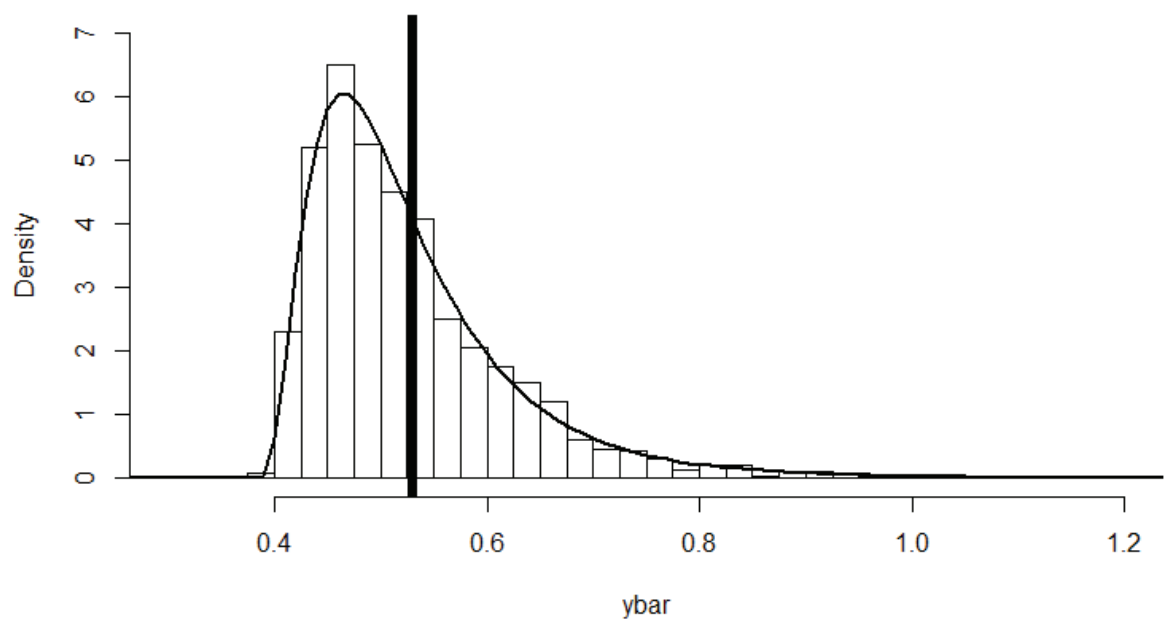




\section{R Code for Exercise 9.7}

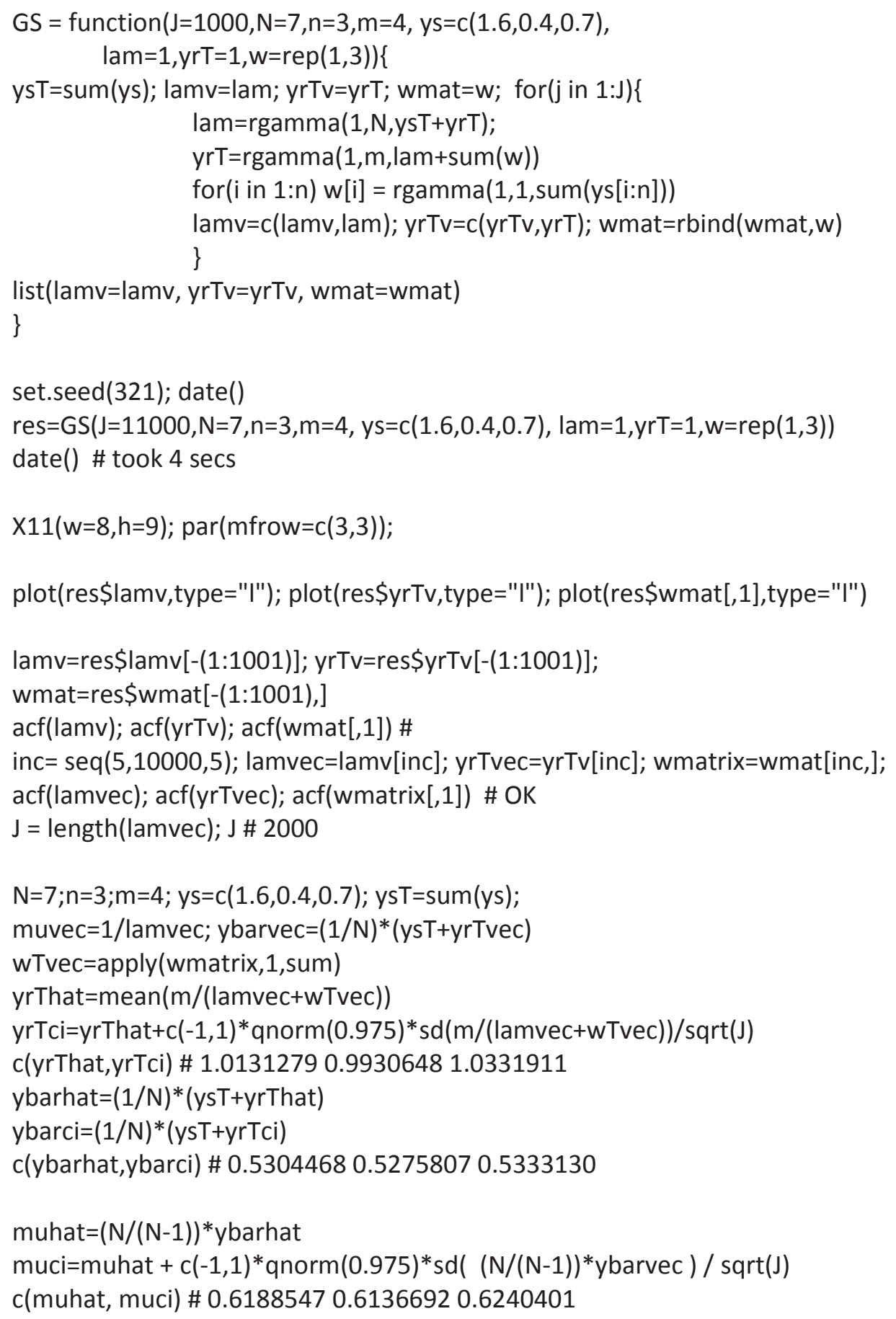


mugrid=seq $(0.001,10.001,0.01)$

fmuhat=mugrid; for(i in 1:length(mugrid))

fmuhat $[i]=\operatorname{mean}\left(\operatorname{dgamma}\left(1 /\right.\right.$ mugrid$[i], N, N^{*}$ ybarvec $\left.) / \operatorname{mugrid}[i]^{\wedge} 2\right)$

ybargrid=seq $(0,10,0.01)$

fybarhat= ybargrid; for(i in 1:length(ybargrid))

fybarhat $[\mathrm{i}]=$ mean $\left(\operatorname{dgamma}\left(\mathrm{N}^{*}\right.\right.$ ybargrid $[\mathrm{i}]-\mathrm{ys} \mathrm{T}, \mathrm{m}$, lamvec+wTvec $) * \mathrm{~N}$ )

$\mathrm{X} 11(\mathrm{w}=8, \mathrm{~h}=5) ; \operatorname{par}(\operatorname{mfrow}=c(1,1))$

hist(muvec, prob=T, $x \lim =c(0,3), y \lim =c(0,2.5)$, breaks=seq $(0,80,0.1)$, xlab="mu", main="")

lines(mugrid,fmuhat, $l w d=2)$; abline( $v=c$ (muhat, muci), $\mid w d=2$ )

hist(ybarvec, prob=T, $x \lim =c(0.3,1.2), y \lim =c(0,7)$, breaks $=$ seq $(0,80,0.025)$, xlab="ybar", main="")

lines(ybargrid, fybarhat, $l w d=2)$; abline( $v=c(y b a r h a t, y b a r c i), ~ I w d=2)$ 


\section{Normal Finite Population Models}

\section{0. I The basic normal-normal finite population model}

Consider a finite population of $N$ values $y_{1}, \ldots, y_{N}$ from the normal distribution with unknown mean $\mu$ and known variance $\sigma^{2}$. Assume we have prior information about $\mu$ which may be expressed in terms of a normal distribution with mean $\mu_{0}$ and variance $\sigma_{0}^{2}$.

Suppose that we are interested in the finite population mean, namely $\bar{y}=\left(y_{1}+\ldots+y_{N}\right) / N$, and wish to perform inference on $\bar{y}$ based on the observed values in a sample of size $n$ taken from this finite population via simple random sampling without replacement (SRSWOR).

For convenience, we will in what follows label (or rather relabel) the $n$ sample units as $1, \ldots, n$ and the $m=N-n$ nonsample units as $n+1, \ldots, N$. This convention simplifies notation and allows us to write the finite population vector, originally defined by $y=\left(y_{1}, \ldots, y_{N}\right)$, as

$$
y=\left(\left(y_{1}, \ldots, y_{n}\right),\left(y_{n+1}, \ldots, y_{N}\right)\right)=\left(y_{s}, y_{r}\right) \text {. }
$$

Example: Suppose that we sample units 2, 3 and 5 from a finite population of size 7 . Then we change the labels of units 2,3 and 5 to 1 , 2 and 3, respectively, and we change the labels of units 1, 4, 6 and 7 to 4, 5, 6 and 7, respectively.

Thereby, instead of writing $y_{s}=\left(y_{2}, y_{3}, y_{5}\right)$ and $y_{r}=\left(y_{1}, y_{4}, y_{6}, y_{7}\right)$, we may write $y_{s}=\left(y_{1}, y_{2}, y_{3}\right)$ and $y_{r}=\left(y_{4}, y_{5}, y_{6}, y_{7}\right)$, respectively.

We will also implicitly condition on $s=\left(s_{1}, \ldots, s_{n}\right)$ at its fixed value and suppress $s$ from much of the notation. Thus we will sometimes write $f\left(\bar{y} \mid s, y_{s}\right)$ as $f\left(\bar{y} \mid y_{s}\right)$, with an understanding that $s$ refers to the particular units which were actually sampled. 
Our inferential problem may be thought of as prediction of $\bar{y}_{r}$ given the data, $y_{s}$ (and s), since $\bar{y}=\left(y_{s T}+m \bar{y}_{r}\right) / N$. Considering the various distributions that are involved, a suitable Bayesian model is:

$$
\begin{aligned}
& \left(\bar{y}_{r} \mid y_{s}, \mu\right) \sim N\left(\mu, \sigma^{2} / m\right) \\
& \quad \text { (the model distribution of the nonsample mean) } \\
& \left(y_{1}, \ldots, y_{n} \mid \mu\right) \sim N\left(\mu, \sigma^{2}\right) \\
& \text { (the model distribution of the sample values) } \\
& \mu \sim N\left(\mu_{0}, \sigma_{0}^{2}\right) \quad \text { (the prior distribution). }
\end{aligned}
$$

This model will be called the basic normal-normal finite population model. By results for the normal-normal model reported earlier, we see that the posterior distribution of the superpopulation mean is given by

$$
\left(\mu \mid y_{s}\right) \sim N\left(\mu_{*}, \sigma_{*}^{2}\right),
$$

where: $\mu_{*}=(1-k) \mu_{0}+k \bar{y}_{s}$ (the posterior mean as a credibility estimate)

$$
\sigma_{*}^{2}=k \frac{\sigma^{2}}{n} \quad \text { (the posterior variance), } k=\frac{n}{n+\sigma^{2} / \sigma_{0}^{2}}
$$

(the credibility factor and weight given to the MLE, $\bar{y}_{s}$ ).

It will be recalled that in this context the predictive density of the nonsample mean is

$$
f\left(\bar{y}_{r} \mid y_{s}\right)=\int f\left(\bar{y}_{r} \mid y_{s}, \mu\right) f\left(\mu \mid y_{s}\right) d \mu \text {. }
$$

But this is the integral of the exponent of a quadratic equation in $\mu$ and $\bar{y}_{r}$, and so equals the exponent of a quadratic equation in $\bar{y}_{r}$. It follows that

$$
\left(\bar{y}_{r} \mid y_{s}\right) \sim N\left(a, b^{2}\right),
$$

where: $a=E\left(\bar{y}_{r} \mid y_{s}\right)=E\left\{E\left(\bar{y}_{r} \mid y_{s}, \mu\right) \mid y_{s}\right\}=E\left\{\mu \mid y_{s}\right\}=\mu_{*}$

$$
\begin{aligned}
b^{2}=V\left(\bar{y}_{r} \mid y_{s}\right) & =V\left\{E\left(\bar{y}_{r} \mid y_{s}, \mu\right) \mid y_{s}\right\}+E\left\{V\left(\bar{y}_{r} \mid y_{s}, \mu\right) \mid y_{s}\right\} \\
& =V\left\{\mu \mid y_{s}\right\}+E\left\{\frac{\sigma^{2}}{m} \mid y_{s}\right\}=\sigma_{*}^{2}+\frac{\sigma^{2}}{m} .
\end{aligned}
$$

It follows that $\left(\bar{y} \mid y_{s}\right) \sim N\left(c, d^{2}\right)$, where: 


$$
\begin{aligned}
c=E\left(\bar{y} \mid y_{s}\right)=E\left(\frac{n \bar{y}_{s}+m \bar{y}_{r}}{N} \mid y_{s}\right) & =\frac{n \bar{y}_{s}+m E\left(\bar{y}_{r} \mid y_{s}\right)}{N} \\
& =\frac{n \bar{y}_{s}+m a}{N}=\frac{n \bar{y}_{s}+m \mu_{*}}{N} \\
d^{2}=V\left(\bar{y} \mid y_{s}\right)=V\left(\frac{n \bar{y}_{s}+m \bar{y}_{r}}{N} \mid y_{s}\right) & =\left(\frac{m}{N}\right)^{2} V\left(\bar{y}_{r} \mid y_{s}\right) \\
& =\frac{m^{2}}{N^{2}} b^{2}=\frac{m^{2}}{N^{2}}\left(\sigma_{*}^{2}+\frac{\sigma^{2}}{m}\right) .
\end{aligned}
$$

Then, the $1-\alpha$ central predictive density region (CPDR) for $\bar{y}$ is given by $\left(c \pm z_{\alpha / 2} d\right)$.

Summary: For the basic normal-normal finite population model:

$$
\begin{aligned}
& \left(\bar{y}_{r} \mid y_{s}, \mu\right) \sim N\left(\mu, \frac{\sigma^{2}}{N-n}\right) \\
& \left(y_{1}, \ldots, y_{n} \mid \mu\right) \sim \text { iid } N\left(\mu, \sigma^{2}\right), \mu \sim N\left(\mu_{0}, \sigma_{0}^{2}\right),
\end{aligned}
$$

the posterior distribution of the superpopulation mean $\mu$ is given by

$$
\left(\mu \mid y_{s}\right) \sim N\left(\mu_{*}, \sigma_{*}^{2}\right),
$$

where: $\mu_{*}=(1-k) \mu_{0}+k \bar{y}_{s}, \quad \sigma_{*}^{2}=k \frac{\sigma^{2}}{n}, k=\frac{n}{n+\sigma^{2} / \sigma_{0}^{2}}$.

The predictive distribution of the nonsample mean $\bar{y}_{r}$ is given by

$$
\left(\bar{y}_{r} \mid y_{s}\right) \sim N\left(a, b^{2}\right) \text {, }
$$

where: $a=\mu_{*}, b^{2}=\sigma_{*}^{2}+\frac{\sigma^{2}}{m}, m=N-n$.

The $1-\alpha$ CPDR for $\bar{y}_{r}$ is $\left(a \pm z_{\alpha / 2} b\right)$.

The predictive distribution of the finite population mean $\bar{y}$ is given by

$$
\left(\bar{y} \mid y_{s}\right) \sim N\left(c, d^{2}\right) \text {, }
$$

where: $c=\frac{n \bar{y}_{s}+m \mu_{*}}{N}, d^{2}=\frac{m^{2}}{N^{2}}\left(\sigma_{*}^{2}+\frac{\sigma^{2}}{m}\right)$ (with $\mu_{*}$ and $\sigma_{*}^{2}$ as above).

The $1-\alpha$ CPDR for $\bar{y}$ is $\left(c \pm z_{\alpha / 2} d\right)$. 


\section{Exercise 10.I Practice with the basic normal-normal finite population model}

Consider the Bayesian model given by:

$$
\begin{aligned}
& \left(\bar{y}_{r} \mid y_{s}, \mu\right) \sim N\left(\mu, \sigma^{2} / m\right) \\
& \left(y_{1}, \ldots, y_{n} \mid \mu\right) \sim \text { iid } N\left(\mu, \sigma^{2}\right) \\
& \mu \sim N\left(\mu_{0}, \sigma_{0}^{2}\right) .
\end{aligned}
$$

(a) Express the predictive mean of the finite population mean $\bar{y}$ as a credibility estimate with a suitable credibility factor. Then also express the predictive variance and distribution in terms of that credibility factor. Use your results to answer parts (b) through (e) below.

(b) What is the predictive distribution in the case of very weak prior information?

(c) What is the predictive distribution in the case of very strong prior information?

(d) What is the predictive distribution in the case of a very large sample size?

(e) What is the predictive distribution in the case of a census?

(f) Suppose we believe with a priori probability $95 \%$ that $\mu$ lies between 7.0 and 13.0. We sample the values 5.7, 9.6 and 8.3 from a finite population of seven units. Find the predictive mean and 95\% highest predictive density region for the average of all seven values in the finite population if the superpopulation standard deviation is 2.0.

Create a graph showing:

(i) the likelihood function for the superpopulation mean

(ii) the prior density of the superpopulation mean

(iii) the posterior density of the superpopulation mean

(iv) the prior density of the nonsample mean

(v) the predictive density of the nonsample mean

(vi) the prior density of the finite population mean

(vii) the predictive density of the finite population mean.

In your graph indicate the predictive mean and 95\% highest predictive density region for the average of all seven values in the finite population. 


\section{Solution to Exercise I 0.I}

(a) It is easy to show that the predictive mean of $\bar{y}$, namely

$$
c=E\left(\bar{y} \mid y_{s}\right)=\frac{n \bar{y}_{s}+m \mu_{*}}{N}=\frac{n \bar{y}_{s}+(N-n)\left[(1-k) \mu_{0}+k \bar{y}_{s}\right]}{N},
$$

may also be written as the credibility estimate

$$
c=(1-q) \mu_{0}+q \bar{y}_{s},
$$

where

$$
q=\frac{n+(N-n) k}{N}
$$

is the credibility factor, meaning the weight assigned to $\bar{y}_{s}$ (the direct data estimate of $\bar{y}$ ), and where $1-q$ is the weight assigned to $\mu_{0}$ (the prior estimate of $\bar{y}$ ).

It can then also be shown that the predictive variance of $\bar{y}$, namely

$$
d^{2}=V\left(\bar{y} \mid y_{s}\right)=\frac{m^{2}}{N^{2}}\left(\sigma_{*}^{2}+\frac{\sigma^{2}}{m}\right),
$$

may be expressed as

$$
\frac{(N-n)^{2}}{N^{2}}\left(k \frac{\sigma^{2}}{n}+\frac{\sigma^{2}}{N-n}\right)=q \frac{\sigma^{2}}{n}\left(1-\frac{n}{N}\right) .
$$

Thus we may also write the predictive distribution of the finite population mean as

$$
\left(\bar{y} \mid y_{s}\right) \sim N\left((1-q) \mu_{0}+q \bar{y}_{s}, q \frac{\sigma^{2}}{n}\left(1-\frac{n}{N}\right)\right),
$$

where:

$$
\begin{gathered}
q=\frac{n+(N-n) k}{N} \\
k=\frac{n}{n+\sigma^{2} / \sigma_{0}^{2}} .
\end{gathered}
$$

Note: If the original credibility factor $k$ equals 1 then the second credibility factor $q$ also equals 1 . This then implies that we estimate $\bar{y}$ by

$$
c=(1-1) \mu_{0}+1 \bar{y}_{s}=\bar{y}_{s} \text {. }
$$


This makes sense because if the sample data values are given 'full credibility' then their straight average should intuitively be used to estimate the finite population mean.

On the other hand, if $k=0$ then $q=n / N$ (the sampling fraction). This then implies that we estimate $\bar{y}$ by

$$
c=(1-n / N) \mu_{0}+(n / N) \bar{y}_{s}=\left((N-n) \mu_{0}+n \bar{y}_{s}\right) / N .
$$

This also makes sense because if the sample data are given 'zero credibility' then each of the $N-n$ nonsampled values should intuitively be estimated by the prior mean of the superpopulation mean $\mu$.

(b) In the case of very weak prior information we have (in the limit) that $\sigma_{0}=\infty$, hence $k=1$, and hence $q=1$. Consequently

$$
\left(\bar{y} \mid y_{s}\right) \sim N\left((1-1) \mu_{0}+1 \bar{y}_{s}, 1 \frac{\sigma^{2}}{n}\left(1-\frac{n}{N}\right)\right) \sim N\left(\bar{y}_{s}, \frac{\sigma^{2}}{n}\left(1-\frac{n}{N}\right)\right) .
$$

This implies a posterior mean and $1-\alpha$ CPDR for $\bar{y}$ of $\bar{y}_{s}$ and $\left(\bar{y}_{s} \pm z_{\alpha / 2} \frac{\sigma}{\sqrt{n}} \sqrt{1-\frac{n}{N}}\right)$.

Note: This is the same inference one would make via classical techniques after substituting the sample standard deviation

$$
s=\sqrt{\frac{1}{n-1} \sum_{i=1}^{n}\left(y_{i}-\bar{y}_{s}\right)^{2}}
$$

for $\sigma$, assuming that $n$ is 'large'.

(c) In the case of very strong prior information we have (in the limit) that $\sigma_{0}=0$, hence $k=0$, and hence $q=n / N$. Consequently,

$$
\begin{aligned}
&\left(\bar{y} \mid y_{s}\right) \sim N\left(\left(1-\frac{n}{N}\right) \mu_{0}+\frac{n}{N} \bar{y}_{s}, \frac{n}{N} \frac{\sigma^{2}}{n}\left(1-\frac{n}{N}\right)\right) \\
& \sim N\left(\frac{(N-n) \mu_{0}+n \bar{y}_{s}}{N}, \frac{\sigma^{2}}{N}\left(1-\frac{n}{N}\right)\right) .
\end{aligned}
$$


(d) In the case of a very large sample size we have (in the limit) that $n=\infty$, hence $k=1$, and hence $q=1$. Consequently (just as in (b) for the case of very weak prior information),

$$
\begin{aligned}
\left(\bar{y} \mid y_{s}\right) & \sim N\left((1-1) \mu_{0}+1 \bar{y}_{s}, 1 \frac{\sigma^{2}}{n}\left(1-\frac{n}{N}\right)\right) \\
& \sim N\left(\bar{y}_{s}, \frac{\sigma^{2}}{n}\left(1-\frac{n}{N}\right)\right) .
\end{aligned}
$$

(e) In the case of $a$ census we have $n=N$, hence

$$
q=\frac{N+(N-N) k}{N}=1,
$$

and therefore

$$
\begin{aligned}
\left(\bar{y} \mid y_{s}\right) & \sim N\left((1-1) \mu_{0}+1 \bar{y}_{s}, 1 \frac{\sigma^{2}}{N}\left(1-\frac{N}{N}\right)\right) \\
& \sim N\left(\bar{y}_{s}, 0\right),
\end{aligned}
$$

meaning that $\bar{y}=\bar{y}_{s}$ with posterior probability 1 (obviously).

Note: Some of the equations developed previously implicitly assume that $n<N$.

(f) The given specifications imply that:

$$
\begin{aligned}
& n=3, \quad N=7, \quad m=N-n=4, \quad \sigma=2 \\
& \bar{y}_{s}=\frac{1}{3}(5.7+9.6+8.3)=7.8667 \\
& \mu_{0}=10, \sigma_{0}=\frac{3}{1.96}=1.53064 \\
& \mu \sim N\left(\mu_{0}, \sigma_{0}^{2}\right) \\
& k=\frac{n}{n+\sigma^{2} / \sigma_{0}^{2}}=0.63731 \\
& \mu_{*}=(1-k) \mu_{0}+k \bar{y}_{s}=8.6404, \sigma_{*}=\sqrt{k \frac{\sigma^{2}}{n}}=0.9218141 \\
& \left(\mu \mid y_{s}\right) \sim N\left(\mu_{*}, \sigma_{*}^{2}\right)
\end{aligned}
$$




$$
\begin{aligned}
& a=\mu_{*}=8.6404, \quad b=\sqrt{\sigma_{*}^{2}+\sigma^{2} / m}=1.3601 \\
& \left(\bar{y}_{r} \mid y_{s}\right) \sim N\left(a, b^{2}\right) \\
& q=\frac{n+(N-n) k}{N}=0.79275 \\
& c=\frac{n \bar{y}_{s}+m \mu_{*}}{N}=(1-q) \mu_{0}+q \bar{y}_{s}=8.3088 \\
& d=\sqrt{\frac{m^{2}}{N^{2}} b^{2}}=0.77717 \\
& \left(\bar{y} \mid y_{s}\right) \sim N\left(c, d^{2}\right) .
\end{aligned}
$$

So the predictive mean of $\bar{y}$, the average of all 7 values in the finite population, is $c=8.3088$, and the $95 \%$ highest predictive density region for that average is $(c \pm 1.96 d)=(6.7856,9.8320)$. Figure 10.1 shows:

(i) the likelihood function for the superpopulation mean, $L(\mu)$, equal to the posterior density of $\mu$ under a flat prior; thus $L(\mu)=f_{N\left(\bar{y}_{s}, \sigma^{2} / n\right)}(\mu)$

(ii) the prior density of the superpopulation mean,

$$
f(\mu)=f_{N\left(\mu_{0}, \sigma_{0}^{2}\right)}(\mu)
$$

(iii) the posterior density of the superpopulation mean,

$$
f\left(\mu \mid y_{s}\right)=f_{N\left(\mu_{*}, \sigma_{*}^{2}\right)}(\mu)
$$

(iv) the prior density of the nonsample mean,

$$
f\left(\bar{y}_{r}\right)=f_{N\left(\mu_{0}, \sigma_{0}^{2}+\sigma^{2} / m\right)}\left(\bar{y}_{r}\right)
$$

(v) the predictive density of the nonsample mean,

$$
f\left(\bar{y}_{r} \mid y_{s}\right)=f_{N\left(a, b^{2}\right)}\left(\bar{y}_{r}\right)
$$

(vi) the prior density of the finite population mean,

$$
f(\bar{y})=f_{N\left(\mu_{0}, \sigma_{0}^{2}+\sigma^{2} / N\right)}(\bar{y})
$$

(vii) the predictive density of the finite population mean,

$$
f\left(\bar{y} \mid y_{s}\right)=f_{N\left(c, d^{2}\right)}(\bar{y}) .
$$


Figure 10.I Various densities in Exercise 10.I

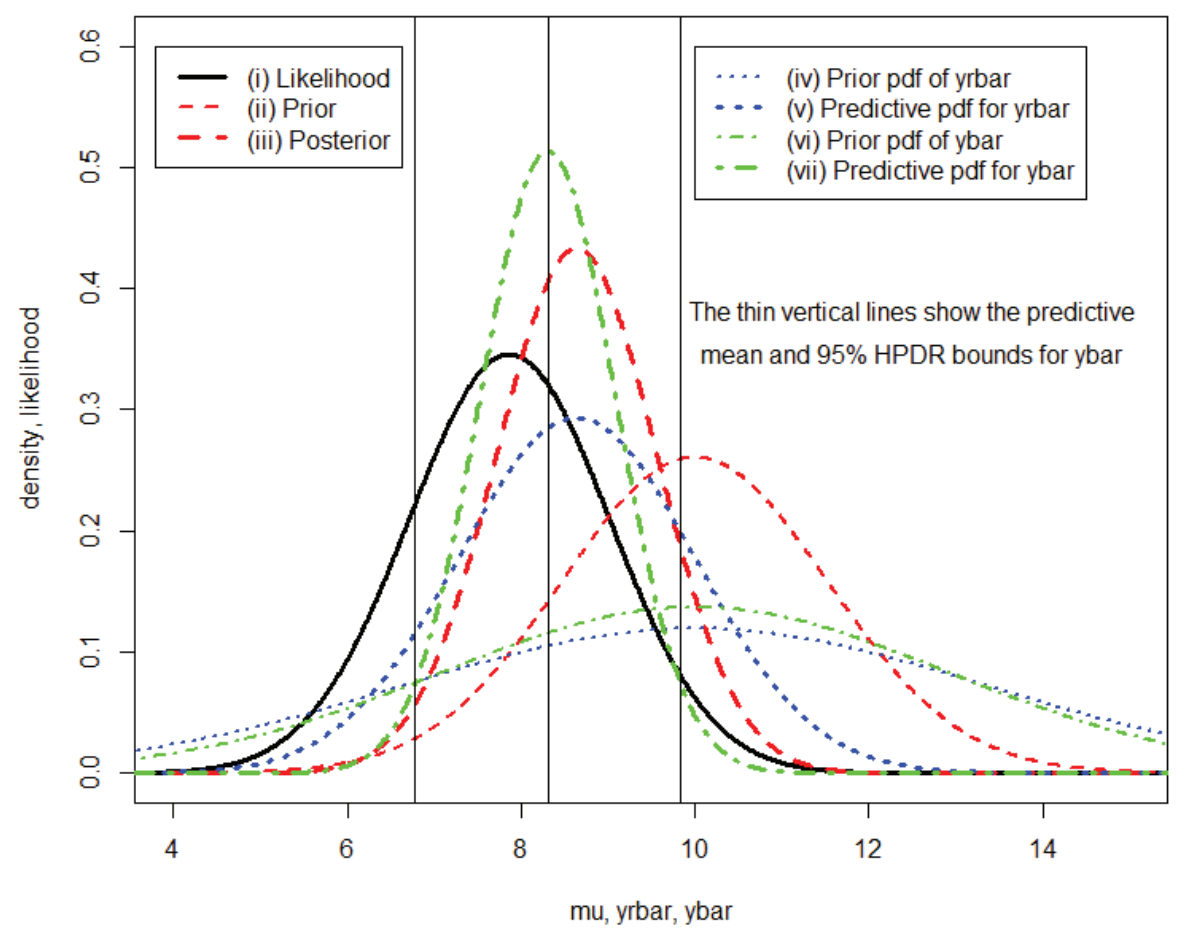

In Figure 10.1, we may observe how the prior densities of $\mu, \bar{y}_{r}$ and $\bar{y}$ are all centred around the prior mean $\mu_{0}=10$. The line for $\mu$ is most highly concentrated about 10 because it represents the prior density of the mean of a hypothetically infinite number of population values. The line for $\bar{y}_{r}$ is the least focused about 10 because it represents the prior density of the mean of only 4 such values (compared with the line for $\bar{y}$ which is the prior pdf for the mean of 7 such values).

Each of the posterior/predictive densities for $\mu, \bar{y}_{r}$ and $\bar{y}$ is located somewhere between the corresponding prior density and the likelihood function. The posterior/predictive densities for $\mu$ and $\bar{y}_{r}$ are centred at the same values, namely the posterior mean, $\mu_{*}=8.6404$, whereas the predictive density for $\bar{y}$ is centred closer to the likelihood mode, $\bar{y}_{s}=7.8667$. This is because the second credibility factor is larger than the first $(q=0.79275>k=0.63731)$. 


\section{R Code for Exercise I0.I}

ys=c(5.7,9.6,8.3); ysbar=mean(ys); ysbar \# 7.866667

sig=2; $n=3 ; N=7 ; m=N-n ; m u 0=10 ; \operatorname{sig} 0=3 /$ qnorm(0.975);

$k=n /\left(n+\operatorname{sig}{ }^{\wedge} 2 / \operatorname{sig} 0^{\wedge} 2\right) ; q=(n+m * k) / N$

$c(\mathrm{~m}, \mathrm{mu0}$, sig0,k,q) \# 4.000000010 .00000001 .53064040 .63730600 .7927463

mustar $=(1-k) *$ mu0+k*ysbar; sigstar $2=k^{*} \operatorname{sig} \wedge 2 / n$

c(mustar,sqrt(sigstar2)) \# 8.6404139 0.9218141

$a=$ mustar; b2=sigstar2+sig^2/m; c=(n*ysbar+m*a)/N; d2=(m/N)^2*b2

$c(a, s q r t(b 2), c, s q r t(d 2)) \# 8.64041391 .36005198 .30880800 .7771725$

HPDR $=c+c(-1,1) *$ qnorm(0.975)*sqrt(d2); HPDR \# 6.7855789 .832038

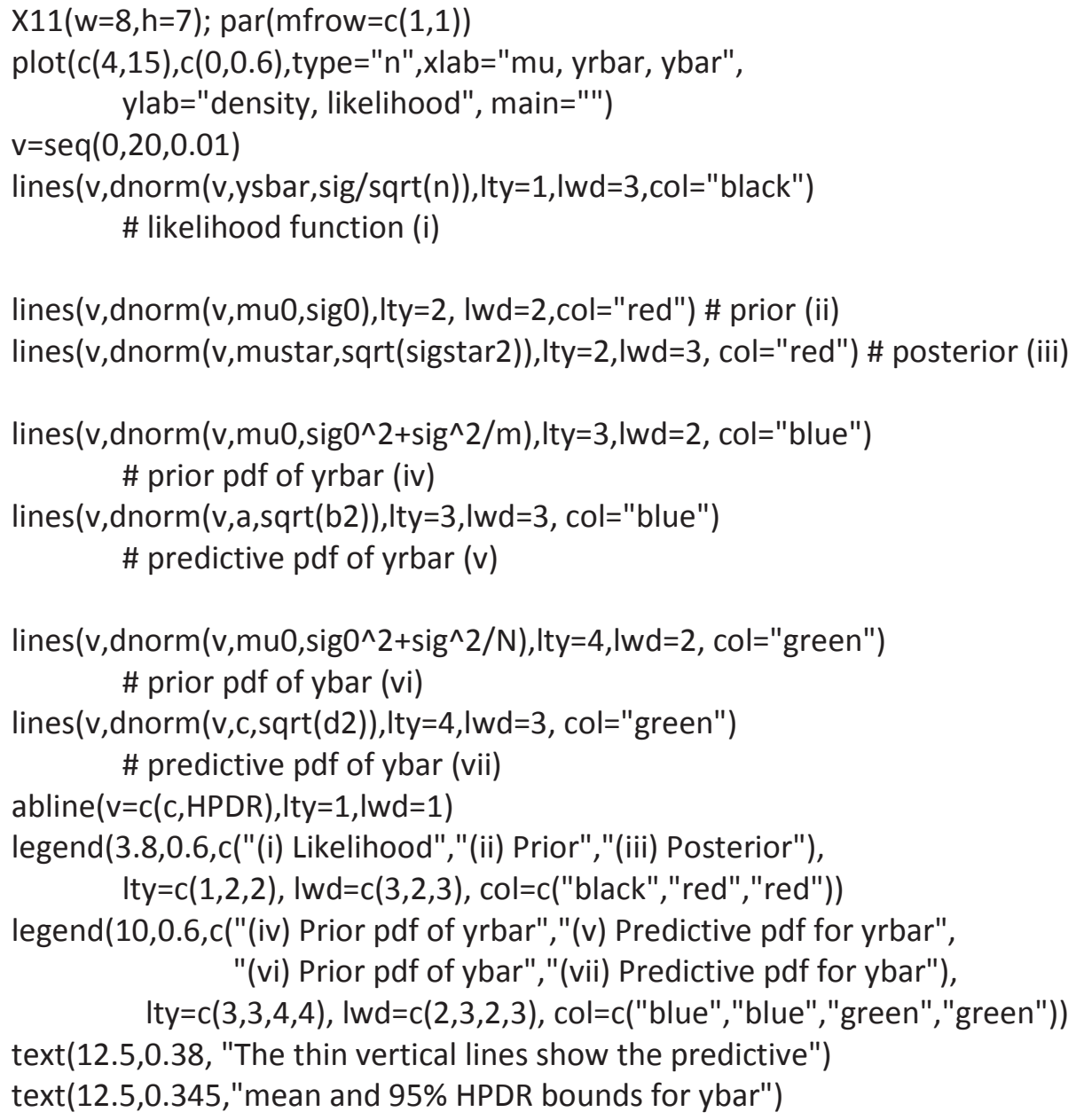




\section{0.2 The general normal-normal finite population model}

The basic normal-normal finite population model examined in the previous section assumes that:

- all $N$ values in the finite population are conditionally normal and iid

- we are interested only in the nonsample mean $\bar{y}_{r}$ and functions of $\bar{y}_{r}$ (such as the finite population mean $\bar{y}$ ).

We will now examine a generalisation of this basic model which allows for:

- non-independence of values

- covariate information

- inference on the entire nonsample vector and linear combinations thereof.

We will continue to assume that the values in the population are all (conditionally) normally distributed, and that the (conditional) variance of each value in the finite population is known. We will now also assume that all the covariance terms between these values are known. (These assumptions will be relaxed at a later stage.)

First, define the (finite) population vector in column form as

$$
\left.y=\left(\begin{array}{c}
y_{s} \\
y_{r}
\end{array}\right)=\left(\begin{array}{c}
\left(\begin{array}{c}
y_{1} \\
\vdots \\
y_{n}
\end{array}\right) \\
y_{n+1} \\
\vdots \\
y_{N}
\end{array}\right)\right)=\left(\begin{array}{c}
y_{1} \\
\vdots \\
y_{N}
\end{array}\right) .
$$

Next, suppose that auxiliary information is available in the form of an $N$ by $p$ matrix

$$
X=\left(\begin{array}{c}
x_{1}^{\prime} \\
\vdots \\
x_{N}^{\prime}
\end{array}\right)=\left(X_{1}, \ldots, X_{p}\right)=\left(\begin{array}{ccc}
x_{11} & \cdots & x_{1 p} \\
\vdots & \vdots & \vdots \\
x_{N 1} & \cdots & x_{N p}
\end{array}\right),
$$

where 


$$
x_{i}=\left(\begin{array}{c}
x_{i 1} \\
\vdots \\
x_{i p}
\end{array}\right)
$$

is the covariate vector for the $i$ th population unit $(i=1, \ldots, N)$ and

$$
X_{j}=\left(\begin{array}{c}
x_{1 j} \\
\vdots \\
x_{N j}
\end{array}\right)
$$

is the population vector for the $j$ th explanatory variable $(j=1, \ldots, p)$.

Also suppose that the finite population vector $y$ has a known variancecovariance structure in the form of an $N$ by $N$ positive definite matrix

$$
\Sigma=\left(\begin{array}{ccc}
\sigma_{11} & \cdots & \sigma_{1 N} \\
\vdots & \ddots & \vdots \\
\sigma_{N 1} & \cdots & \sigma_{N N}
\end{array}\right),
$$

where: $\sigma_{i j}=C\left(y_{i}, y_{j}\right)=\sigma_{j i}$

$$
\sigma_{i i}=V y_{i} \equiv \sigma_{i}^{2}
$$

with the covariance and variance operations here $(C$ and $V)$ implicitly conditional on all model parameters.

In the above context, the Bayesian model we will focus on is:

$$
\begin{aligned}
& (y \mid \beta) \sim N_{N}(X \beta, \Sigma) \\
& \beta \sim N_{p}(\delta, \Omega) .
\end{aligned}
$$

This model will be called the general normal-normal finite population model. Here,

$$
\beta=\left(\begin{array}{c}
\beta_{1} \\
\vdots \\
\beta_{p}
\end{array}\right)
$$

is the vector of regression coefficients, whose prior distribution is multivariate normal with (specified) mean

$$
\delta=\left(\begin{array}{c}
\delta_{1} \\
\vdots \\
\delta_{p}
\end{array}\right)
$$


and (specified) variance-covariance matrix

$$
\Omega=\left(\begin{array}{ccc}
\omega_{11} & \cdots & \omega_{1 p} \\
\vdots & \ddots & \vdots \\
\omega_{p 1} & \cdots & \omega_{p p}
\end{array}\right),
$$

where: $\omega_{i j}=C\left(\beta_{i}, \beta_{j}\right)=\omega_{j i}$

$$
\omega_{i i}=V \beta_{i} \equiv \omega_{i}^{2} \text {, }
$$

with the covariance and variance operations here $(C$ and $V)$ implicitly unconditional, thereby reflecting prior belief regarding the $\beta_{i}$ values.

We will assume interest lies generally in the nonsample vector $y_{r}$ and functions of that vector, and specifically in the finite population mean $\bar{y}$ (a simple function of $y_{r}$ and of the known quantities $y_{s}, n$ and $N$ ). Thus the regression coefficient vector $\beta$ will be treated as a nuisance parameter and inference will be based on the predictive distribution of $y_{r}$ given $y_{s}$.

Note: The basic normal finite population model as considered previously is a special case of the just-defined general normal finite population model with:

$$
\begin{aligned}
& p=1, \quad \beta=\left(\beta_{1}\right)=\mu, \quad \delta=\left(\delta_{1}\right)=\mu_{0}, \quad \Omega=\left(\omega_{11}\right)=\sigma_{0}^{2} \\
& X=1_{N}=(1, \ldots, 1)^{\prime}=\left(\begin{array}{c}
1 \\
\vdots \\
1
\end{array}\right) \quad \text { (a column vector of } N \text { ones) } \\
& \Sigma=\sigma^{2} I_{N}=\left(\begin{array}{cccc}
\sigma^{2} & 0 & \cdots & 0 \\
0 & \sigma^{2} & & 0 \\
\vdots & & \ddots & \vdots \\
0 & 0 & 0 & \sigma^{2}
\end{array}\right) \\
& \text { (where } I_{N} \text { is the } N \text { by } N \text { identity matrix). }
\end{aligned}
$$

Thus, the previous normal finite population model could also be written as:

$$
\begin{aligned}
& (y \mid \mu) \sim N_{N}\left(\mu 1_{N}, \sigma^{2} I_{N}\right) \\
& \mu \sim N_{1}\left(\mu_{0}, \sigma_{0}^{2}\right) .
\end{aligned}
$$




\section{0.3 Derivation of the predictive distribution of the nonsample vector}

Observe that the unconditional (or prior) distribution of the entire finite population vector $y$ is given by the density

$$
f(y)=\int f(y, \beta) d \beta=\int f(y \mid \beta) f(\beta) d \beta .
$$

Now, the integrand of this multiple integral is a quadratic in the $y_{i}$ and $\beta_{j}$ values. This implies that the value of the integral has the form of a quadratic in the $y_{i}$ values alone. This then implies that the prior (or unconditional) distribution of $y$ is also multivariate normal. It then remains to find the mean and covariance vector of that prior distribution, as follows:

$$
\begin{aligned}
& E y=E E(y \mid \beta)=E(X \beta)=X \delta \\
& V y=E V(y \mid \beta)+V E(y \mid \beta)=E \Sigma+V(X \beta)=\Sigma+X \Omega X^{\prime} .
\end{aligned}
$$

Thus, $\quad y \sim N_{N}\left(X \delta, \Sigma+X \Omega X^{\prime}\right)$.

This result may also be written as

$$
\left(\begin{array}{c}
y_{s} \\
y_{r}
\end{array}\right) \sim N_{N}\left(\left(\begin{array}{c}
X_{s} \delta \\
X_{r} \delta
\end{array}\right),\left(\begin{array}{ll}
\Sigma_{s s}+X_{s} \Omega X_{s}^{\prime} & \Sigma_{s r}+X_{s} \Omega X_{r}^{\prime} \\
\Sigma_{r s}+X_{r} \Omega X_{s}^{\prime} & \Sigma_{r r}+X_{r} \Omega X_{r}^{\prime}
\end{array}\right)\right),
$$

where we partition $X$ and $\Sigma$ according to

$$
X=\left(\begin{array}{l}
X_{s} \\
X_{r}
\end{array}\right) \text { and } \Sigma=\left(\begin{array}{cc}
\Sigma_{s s} & \Sigma_{s r} \\
\Sigma_{r s} & \Sigma_{r r}
\end{array}\right) .
$$

Thus, $X_{s}=\left(\begin{array}{c}X_{1}^{\prime} \\ \vdots \\ X_{n}^{\prime}\end{array}\right)$ is a submatrix consisting of the first $n$ rows of $X$, etc.

It follows by standard multivariate normal theory (see below) that

$$
\left(y_{r} \mid y_{s}\right) \sim N_{m}\left(E_{*}, V_{*}\right),
$$

where:

$$
\begin{aligned}
E_{*} & =X_{r} \delta+\left(\Sigma_{r s}+X_{r} \Omega X_{s}^{\prime}\right)\left(\Sigma_{s s}+X_{s} \Omega X_{s}^{\prime}\right)^{-1}\left(y_{s}-X_{s} \delta\right) \\
V_{*} & =\left(\Sigma_{r r}+X_{r} \Omega X_{r}^{\prime}\right)-\left(\Sigma_{r s}+X_{r} \Omega X_{s}^{\prime}\right)\left(\Sigma_{s s}+X_{s} \Omega X_{s}^{\prime}\right)^{-1}\left(\Sigma_{s r}+X_{s} \Omega X_{r}^{\prime}\right) .
\end{aligned}
$$


Note: We have here used the following result (e.g. see equation (81.2.11) in Rao, 1973):

$$
\begin{aligned}
& \left(\begin{array}{l}
X_{1} \\
X_{2}
\end{array}\right) \sim N_{n_{1}+n_{2}}\left(\left(\begin{array}{l}
\mu_{1} \\
\mu_{2}
\end{array}\right),\left(\begin{array}{ll}
\Sigma_{11} & \Sigma_{12} \\
\Sigma_{21} & \Sigma_{22}
\end{array}\right)\right) \\
& \quad \Rightarrow\left(X_{2} \mid X_{1}\right) \sim N_{n_{2}}\left(\mu_{2}+\Sigma_{21} \Sigma_{11}^{-1}\left(X_{1}-\mu_{1}\right), \Sigma_{22}-\Sigma_{21} \Sigma_{11}^{-1} \Sigma_{12}\right) .
\end{aligned}
$$

\section{0.4 Alternative formulae for the predictive distribution of the nonsample vector}

Another way to obtain the distribution of $\left(y_{r} \mid y_{s}\right)$ (already derived above) is as follows. First, the posterior density of $\beta$ is

$$
\begin{aligned}
& f\left(\beta \mid y_{s}\right) \propto f(\beta) f\left(y_{s} \mid \beta\right) \\
\propto & \exp \left\{-\frac{1}{2}(\beta-\delta)^{\prime} \Omega^{-1}(\beta-\delta)\right\} \exp \left\{-\frac{1}{2}\left(y_{s}-X_{s} \beta\right)^{\prime} \Sigma_{s s}^{-1}\left(y_{s}-X_{s} \beta\right)\right\} \\
= & \exp \left(-\frac{1}{2} Q_{1}\right),
\end{aligned}
$$

where

$$
Q_{1}=(\beta-\delta)^{\prime} \Omega^{-1}(\beta-\delta)+\left(y_{s}-X_{s} \beta\right)^{\prime} \Sigma_{s s}^{-1}\left(y_{s}-X_{s} \beta\right) .
$$

We see that $f\left(\beta \mid y_{s}\right)$ is proportional to the exponent of a quadratic form in $\beta$. This implies that

$$
\left(\beta \mid y_{s}\right) \sim N_{p}(\hat{\beta}, D)
$$

for some $\hat{\beta}$ and $D$ to be determined.

Now observe that

$$
f\left(\beta \mid y_{s}\right) \propto \exp \left(-\frac{1}{2} Q_{2}\right),
$$

where

$$
\begin{aligned}
Q_{2} & =(\beta-\hat{\beta})^{\prime} D^{-1}(\beta-\hat{\beta}) \\
& =\beta^{\prime} D^{-1} \beta-\beta^{\prime} D^{-1} \hat{\beta}-\hat{\beta}^{\prime} D^{-1} \beta+\text { constant }
\end{aligned}
$$

(where the constant does not depend on $\beta$ ). 
But $Q_{1}=\beta^{\prime} \Omega^{-1} \beta-\beta^{\prime} \Omega^{-1} \delta-\delta^{\prime} \Omega^{-1} \beta-y_{s}^{\prime} \Sigma_{s s}^{-1} X_{s} \beta$

$$
\begin{gathered}
-\beta^{\prime} X_{s}^{\prime} \Sigma_{s s}^{-1} y_{s}+\beta^{\prime} X_{s}^{\prime} \Sigma_{s s}^{-1} X_{s} \beta+\text { constant } \\
=\beta^{\prime}\left(\Omega^{-1}+X_{s}^{\prime} \Sigma_{s s}^{-1} X_{s}\right) \beta-\beta^{\prime}\left(\Omega^{-1} \delta+X_{s}^{\prime} \Sigma_{s s}^{-1} y_{s}\right)-\left(\delta \Omega^{-1}+y_{s}^{\prime} \Sigma_{s s}^{-1} X_{s}\right) \beta \\
+ \text { constant. }
\end{gathered}
$$

Equating (10.3) and (10.4) we see that:

$$
\begin{aligned}
& D^{-1}=\Omega^{-1}+X_{s}^{\prime} \Sigma_{s s}^{-1} X_{s} \\
& D^{-1} \hat{\beta}=\Omega^{-1} \delta+X_{s}^{\prime} \Sigma_{s s}^{-1} y_{s} .
\end{aligned}
$$

It follows that:

$$
\begin{aligned}
& D=\left(\Omega^{-1}+X_{s}^{\prime} \Sigma_{s s}^{-1} X_{s}\right)^{-1} \\
& \hat{\beta}=D\left(\Omega^{-1} \delta+X_{s}^{\prime} \Sigma_{s s}^{-1} y_{s}\right) .
\end{aligned}
$$

We can now use the result

$$
\left(\beta \mid y_{s}\right) \sim N_{p}(\hat{\beta}, D)
$$

to find the predictive mean and variance of $y_{r}$.

First, observe that

$$
(y \mid \beta) \sim N_{N}(X \beta, \Sigma)
$$

may also be written

$$
\left(\left(\begin{array}{c}
y_{s} \\
y_{r}
\end{array}\right) \mid \beta\right) \sim N_{N}\left(\left(\begin{array}{c}
X_{s} \beta \\
X_{r} \beta
\end{array}\right),\left(\begin{array}{cc}
\Sigma_{s s} & \Sigma_{s r} \\
\Sigma_{r s} & \Sigma_{r r}
\end{array}\right)\right),
$$

which implies that

$$
\left(y_{r} \mid y_{s}, \beta\right) \sim N_{m}\left(X_{r} \beta+\Sigma_{r s} \Sigma_{s s}^{-1}\left(y_{s}-X_{s} \beta\right), \Sigma_{r r}-\Sigma_{r s} \Sigma_{s s}^{-1} \Sigma_{s r}\right) .
$$

It follows that:

$$
\begin{aligned}
& E\left(y_{r} \mid y_{s}\right)=E\left\{E\left(y_{r} \mid y_{s}, \beta\right) \mid y_{s}\right\} \\
& =E\left\{X_{r} \beta+\Sigma_{r s} \Sigma_{s s}^{-1}\left(y_{s}-X_{s} \beta\right) \mid y_{s}\right\} \\
& =X_{r} \hat{\beta}+\Sigma_{r s} \Sigma_{s s}^{-1}\left(y_{s}-X_{s} \hat{\beta}\right) \\
& V\left(y_{r} \mid y_{s}\right)=E\left\{V\left(y_{r} \mid y_{s}, \beta\right) \mid y_{s}\right\}+V\left\{E\left(y_{r} \mid y_{s}, \beta\right) \mid y_{s}\right\} \\
& =E\left\{\Sigma_{r r}-\Sigma_{r s} \Sigma_{s s}^{-1} \Sigma_{s r} \mid y_{s}\right\}+V\left\{X_{r} \hat{\beta}+\Sigma_{r s} \Sigma_{s s}^{-1}\left(y_{s}-X_{s} \hat{\beta}\right) \mid y_{s}\right\} \\
& =\Sigma_{r r}-\Sigma_{r s} \Sigma_{s s}^{-1} \Sigma_{s r}+V\left\{\left(X_{r}-\Sigma_{r s} \Sigma_{s s}^{-1} X_{s}\right) \hat{\beta} \mid y_{s}\right\} \\
& =\Sigma_{r r}-\Sigma_{r s} \Sigma_{s s}^{-1} \Sigma_{s r}+\left(X_{r}-\Sigma_{r s} \Sigma_{s s}^{-1} X_{s}\right) D\left(X_{r}-\Sigma_{r s} \Sigma_{s s}^{-1} X_{s}\right)^{\prime} \text {. }
\end{aligned}
$$


Note: The expression for $E_{*}$ at (10.1) must be the same as that for $E\left(y_{r} \mid y_{s}\right)$ at (10.5), and likewise the expression for $V_{*}$ at (10.2) must be the same as that for $V\left(y_{r} \mid y_{s}\right)$ at (10.6). This equivalence can also be shown with some algebra by making use of the formula

$$
\left(\Sigma_{s s}+X_{s} \Omega X_{s}^{\prime}\right)^{-1}=\Sigma_{s s}^{-1}\left\{I_{s}-X_{s}\left(\Omega^{-1}+X_{s} \Sigma_{s s}^{-1} X_{s}\right)^{-1} X_{s}^{\prime} \Sigma_{s s}^{-1}\right\},
$$

which in turn follows from the general matrix identity

$$
\left(A-U W^{-1} V\right)^{-1}=A^{-1}+A^{-1} U\left(W-V A^{-1} U\right)^{-1} V A^{-1} \text {. }
$$

Here, $I_{s}$ is the $n$ by $n$ identity matrix and could also be written $I_{n}$.

\section{0.5 Prediction of the finite population mean and other linear combinations}

We may now write down a general expression for the predictive distribution of the finite population mean. That mean may be expressed as the linear combination

$$
\bar{y}=\frac{y_{s T}+m \bar{y}_{r}}{N}=\frac{1}{N}\left(y_{s T}+1_{r}^{\prime} y_{r}\right) .
$$

Note: Here, $1_{r}^{\prime}$ denotes the row vector with $m=N-n$ ones. This vector could also be written $1_{m}^{\prime}$ or $1_{N-n}^{\prime}$ or $(1, \ldots, 1)$.

Therefore the predictive distribution of $\bar{y}$ given $y_{s}$ is normal with mean $e_{*}=\frac{y_{s T}+1_{r}^{\prime} E_{*}}{N}$ and variance $v_{*}=\frac{1_{r}^{\prime} V_{*} 1_{r}}{N^{2}}$.

So the $1-\alpha$ CPDR for $\bar{y}$ is $\left(e_{*} \pm z_{\alpha / 2} \sqrt{v_{*}}\right)$.

More generally, the predictive distribution of the linear combination

$$
\psi=c_{0}+\left(c_{1} y_{1}+\ldots+c_{n} y_{n}\right)+\left(c_{n+1} y_{n+1}+\ldots+c_{N} y_{N}\right)
$$

is normal with mean $e_{\#}=c_{0}+c_{s}^{\prime} y_{s}+c_{r}^{\prime} E_{*}$ and variance $v_{\#}=\frac{c_{r}^{\prime} V_{*} c_{r}}{N^{2}}$, where $c_{s}=\left(c_{1}, \ldots, c_{n}\right)^{\prime}$ and $c_{r}=\left(y_{n+1}, \ldots, c_{N}\right)^{\prime}$.

So the $1-\alpha$ CPDR for $\psi$ is $\left(e_{\#} \pm z_{\alpha / 2} \sqrt{v_{\#}}\right)$. 
Summary: For the general normal-normal finite population model:

$$
\begin{aligned}
& (y \mid \beta) \sim N_{N}(X \beta, \Sigma) \\
& \beta \sim N_{p}(\delta, \Omega),
\end{aligned}
$$

the posterior distribution of the regression vector $\beta$ is given by

$$
\left(\beta \mid y_{s}\right) \sim N_{p}(\hat{\beta}, D),
$$

where: $\hat{\beta}=D\left(\Omega^{-1} \delta+X_{s}^{\prime} \Sigma_{s s}^{-1} y_{s}\right), \quad D=\left(\Omega^{-1}+X_{s}^{\prime} \Sigma_{s s}^{-1} X_{s}\right)^{-1}$.

The predictive distribution of the nonsample vector $y_{r}$ is given by

$$
\left(y_{r} \mid y_{s}\right) \sim N_{m}\left(E_{*}, V_{*}\right) \quad(m=N-n),
$$

where: $E_{*}=X_{r} \delta+\left(\Sigma_{r s}+X_{r} \Omega X_{s}^{\prime}\right)\left(\Sigma_{s s}+X_{s} \Omega X_{s}^{\prime}\right)^{-1}\left(y_{s}-X_{s} \delta\right)$

$$
\begin{gathered}
=X_{r} \hat{\beta}+\Sigma_{r s} \Sigma_{s s}^{-1}\left(y_{s}-X_{s} \hat{\beta}\right) \\
V_{*}=\left(\Sigma_{r r}+X_{r} \Omega X_{r}^{\prime}\right)-\left(\Sigma_{r s}+X_{r} \Omega X_{s}^{\prime}\right)\left(\Sigma_{s s}+X_{s} \Omega X_{s}^{\prime}\right)^{-1}\left(\Sigma_{s r}+X_{s} \Omega X_{r}^{\prime}\right) \\
=\Sigma_{r r}-\Sigma_{r s} \Sigma_{s s}^{-1} \Sigma_{s r}+\left(X_{r}-\Sigma_{r s} \Sigma_{s s}^{-1} X_{s}\right) D\left(X_{r}-\Sigma_{r s} \Sigma_{s s}^{-1} X_{s}\right)^{\prime} .
\end{gathered}
$$

The predictive distribution of the finite population mean $\bar{y}$ is given by

$$
\left(\bar{y} \mid y_{s}\right) \sim N\left(e_{*}, v_{*}\right) \text {, where } e_{*}=\frac{y_{s T}+1_{r}^{\prime} E_{*}}{N} \text { and } v_{*}=\frac{1_{r}^{\prime} V_{*} 1_{r}}{N^{2}} \text {, }
$$

with $1-\alpha$ CPDR for $\bar{y}$ given by $\left(e_{*} \pm z_{\alpha / 2} \sqrt{v_{*}}\right)$.

The predictive distribution of any linear combination of the form $\psi=c_{0}+c_{s}^{\prime} y_{s}+c_{r}^{\prime} y_{r}$ is given by

$$
\left(\psi \mid y_{s}\right) \sim N\left(e_{\#}, v_{\#}\right),
$$

where $e_{\#}=c_{0}+c_{s}^{\prime} y_{s}+c_{r}^{\prime} E_{*}$ and $v_{\#}=\frac{c_{r}^{\prime} V_{*} c_{r}}{N^{2}}$,

with $1-\alpha$ CPDR for $\psi$ given by $\left(e_{\#} \pm z_{\alpha / 2} \sqrt{v_{\#}}\right)$.

\section{0.6 Special cases including ratio estimation}

In the context of the above general normal-normal finite population model, suppose that $p=1$ (i.e. there is a single covariate) and the population values are conditionally independent, the $i$ th one having mean $x_{i} \beta$ and variance $x_{i}^{2 \gamma} \sigma^{2}$, where $\gamma \in \mathfrak{R}$ and $\sigma^{2}>0$ are known. 
Also, suppose that the prior distribution on the single regression coefficient $\beta$ is normal with mean $\delta$ and variance $\omega^{2}$. Then:

$$
\begin{aligned}
& (y \mid \beta) \sim N_{N}(X \beta, \Sigma) \\
& \beta \sim N_{p}(\delta, \Omega),
\end{aligned}
$$

where: $p=1, \quad X=\left(\begin{array}{c}x_{1} \\ x_{2} \\ \vdots \\ x_{N}\end{array}\right), \Sigma=\left(\begin{array}{cccc}x_{1}^{2 \gamma} & 0 & \cdots & 0 \\ 0 & x_{2}^{2 \gamma} & & 0 \\ \vdots & \vdots & \ddots & \vdots \\ 0 & 0 & \cdots & x_{N}^{2 \gamma}\end{array}\right) \sigma^{2}, \quad \Omega=\omega^{2}$.

The model may also be written in non-matrix form as:

$$
\begin{aligned}
& \left(y_{i} \mid \beta\right) \sim \perp\left(x_{i}^{\prime} \beta, x_{i}^{2 \gamma} \sigma^{2}\right), i=1, \ldots, N \\
& \beta \sim N\left(\delta, \omega^{2}\right) .
\end{aligned}
$$

Under this model it can be shown that the predictive distribution of the finite population mean is given by

$$
\left(\bar{y} \mid y_{s}\right) \sim N\left(A, B^{2}\right)
$$

where:

$$
\begin{aligned}
& A=\frac{n}{N} \bar{y}_{s}+\left(1-\frac{n}{N}\right) \bar{x}_{r}\left\{\frac{\delta \sigma^{2}+\omega^{2} \sum_{i=1}^{n} y_{i} x_{i}^{1-2 \gamma}}{\sigma^{2}+\omega^{2} \sum_{i=1}^{n} x_{i}^{2-2 \gamma}}\right\} \\
& B^{2}=\frac{\sigma^{2}}{N^{2}}\left\{\sum_{i=n+1}^{N} x_{i}^{2 \gamma}+\frac{m^{2} \omega^{2} \bar{x}_{r}^{2}}{\sigma^{2}+\omega^{2} \sum_{i=1}^{n} x_{i}^{2-2 \gamma}}\right\} \\
& \bar{x}_{r}=\frac{1}{m} \sum_{i=n+1}^{N} x_{i} \text { (average of the covariate values in the nonsample). }
\end{aligned}
$$

Now suppose it is believed that the variances of the population values are exactly proportional to the covariate values, i.e. $V\left(y_{i} \mid \beta\right)=x_{i} \sigma^{2}$.

Then $\gamma=1 / 2$, and we find that:

$$
\begin{aligned}
& A=\frac{n}{N} \bar{y}_{s}+\left(1-\frac{n}{N}\right) \bar{x}_{r}\left\{\frac{\omega^{2} \bar{y}_{s}+\delta \sigma^{2} / n}{\omega^{2} \bar{x}_{s}+\sigma^{2} / n}\right\} \\
& B^{2}=\frac{\sigma^{2}}{n} \bar{x}_{r}\left(1-\frac{n}{N}\right)\left\{\frac{n}{N}+\left(1-\frac{n}{N}\right) \frac{\omega^{2} \bar{x}_{r}}{\omega^{2} \bar{x}_{s}+\sigma^{2} / n}\right\} \\
& \bar{x}_{s}=\frac{1}{n} \sum_{i=1}^{n} x_{i} \text { (the average of the covariate values in the sample). }
\end{aligned}
$$


If there is a priori ignorance regarding $\beta$ we may further set $\omega=\infty$, and in that case:

$$
\begin{aligned}
& A=\frac{n}{N} \bar{y}_{s}+\left(1-\frac{n}{N}\right) \bar{x}_{r} \frac{\bar{y}_{s}}{\bar{X}_{s}}=\bar{y}_{s}\left\{\frac{n}{N}+\left(1-\frac{n}{N}\right) \frac{\bar{x}_{r}}{\bar{x}_{s}}\right\} \\
& =\bar{y}_{s}\left\{\frac{n \bar{x}_{s}+(N-n) \bar{x}_{r}}{N \bar{x}_{s}}\right\}=\frac{\bar{y}_{s}}{\bar{x}_{s}} \bar{x} \\
& B^{2}=\frac{\sigma^{2}}{n} \bar{x}_{r}\left(1-\frac{n}{N}\right)\left\{\frac{n}{N}+\left(1-\frac{n}{N}\right) \frac{\bar{x}_{r}}{\bar{x}_{s}}\right\}=\frac{\sigma^{2}}{n}\left(1-\frac{n}{N}\right) \frac{\bar{x}_{r}}{\bar{x}_{s}} \bar{X} \\
& \bar{x}=\frac{1}{N} \sum_{i=1}^{N} x_{i} \text { (average of covariate values in the finite population). }
\end{aligned}
$$

As regards this last special case, we see that the predictive mean $A$ is identical to the common design-based ratio estimator.

Also, the predictive variance $B^{2}$, although not identical to any designbased formula, is the same as a model-based analogue (e.g. see Brewer, 1963, and Royall, 1970). The formula for $B^{2}$ suggests a purposive sampling scheme whereby units with the largest covariate values should be selected.

Note 1: If units with relatively large $y$-values are selected, then $\bar{x}_{s}$ will likely be larger than $\bar{x}_{r}$, so that then $\frac{\bar{X}_{r}}{\bar{x}_{s}}$ will likely be small, and thereby $B^{2}=V\left(\bar{y} \mid y_{s}\right)=\frac{\sigma^{2}}{n}\left(1-\frac{n}{N}\right) \frac{\bar{x}_{r}}{\bar{x}_{s}} \bar{x}$ will also likely be small.

Note 2: The same formulae as derived in the last special case will also apply approximately when the sample size $n$ is very large. This makes sense because the effect of a very large sample size is the same as that of a very diffuse prior. Note that in the case of a census, $n=N$ and we find that the above formulae correctly yield $A=\bar{y}_{s}$ and $B^{2}=0$.

In a way similar to the above, it is possible to obtain analogues of other common design-based and model-based results, such as regression and stratified estimators, together with their associated variances (see Ericson, 1969 and 1988). 


\section{Exercise 10.2 Derivation of the Bayesian ratio estimator}

Consider the Bayesian model given by:

$$
\begin{aligned}
& \left(y_{i} \mid \beta\right) \sim \perp N\left(x_{i} \beta, x_{i} \sigma^{2}\right), i=1, \ldots, N \\
& f(\beta) \propto 1, \beta \in \mathfrak{R} .
\end{aligned}
$$

Derive the predictive distribution of the finite population mean given data of the form $D=\left(s, y_{s}\right)$.

\section{Solution to Exercise I 0.2}

The Bayesian model is:

$$
\begin{aligned}
& (y \mid \beta) \sim N_{N}(X \beta, \Sigma) \\
& \beta \sim N_{p}(\delta, \Omega),
\end{aligned}
$$

where:

$$
\begin{gathered}
p=1, \quad \delta=0, \quad \Omega=\infty, \quad X=x=\left(\begin{array}{c}
x_{1} \\
\vdots \\
x_{N}
\end{array}\right) \\
\Sigma=\sigma^{2} \operatorname{diag}\left(x_{1}, \ldots, x_{N}\right)=\sigma^{2}\left(\begin{array}{ccc}
x_{1} & & \\
& \ddots & \\
& & x_{N}
\end{array}\right) .
\end{gathered}
$$

Note: Here, $\sigma^{-2} \Sigma$ is a matrix with zeros everywhere except for $x_{1}, \ldots, x_{N}$ along the main diagonal.

Using general results derived previously we first have that

$$
\left(\beta \mid y_{s}\right) \sim N(\hat{\beta}, D),
$$

where:

$$
\begin{aligned}
D & =\left(\Omega^{-1}+X_{s}^{\prime} \Sigma_{s s}^{-1} X_{s}\right)^{-1} \\
& =\left\{\infty^{-1}+\left(\begin{array}{lll}
x_{1} & \cdots & x_{n}
\end{array}\right) \frac{1}{\sigma^{2}}\left(\begin{array}{ccc}
x_{1}^{-1} & & \\
& \ddots & \\
& & x_{n}^{-1}
\end{array}\right)\left(\begin{array}{c}
x_{1} \\
\vdots \\
x_{n}
\end{array}\right)\right\}^{-1} \\
& =\sigma^{2}\left(\sum_{i=1}^{n} x_{i} x_{i}^{-1} x_{i}\right)^{-1}=\frac{\sigma^{2}}{x_{s T}}
\end{aligned}
$$


Bayesian Methods for Statistical Analysis

$$
\begin{aligned}
\hat{\beta} & =D\left(\Omega^{-1} \delta+X_{s}^{\prime} \Sigma_{s s}^{-1} y_{s}\right) \\
& =D\left\{0+\left(\begin{array}{lll}
x_{1} & \cdots & x_{n}
\end{array}\right) \frac{1}{\sigma^{2}}\left(\begin{array}{ccc}
x_{1}^{-1} & & \\
& \ddots & \\
& & x_{n}^{-1}
\end{array}\right)\left(\begin{array}{c}
y_{1} \\
\vdots \\
y_{n}
\end{array}\right)\right\} \\
& =\frac{\sigma^{2}}{x_{s T}} \frac{1}{\sigma^{2}} \sum_{i=1}^{n} x_{i} x_{i}^{-1} y_{i}=\frac{y_{s T}}{x_{s T}}=\frac{\bar{y}_{s}}{\bar{x}_{s}} .
\end{aligned}
$$

Next,

$$
\left(y_{r} \mid y_{s}\right) \sim N_{m}\left(E_{*}, V_{*}\right),
$$

where:

$$
\begin{aligned}
& m=N-n \\
& E_{*}=X_{r} \hat{\beta}+\Sigma_{r s} \Sigma_{s s}^{-1}\left(y_{s}-X_{s} \hat{\beta}\right)=\left(\begin{array}{c}
x_{n+1} \\
\vdots \\
x_{N}
\end{array}\right) \hat{\beta}+0=\left(\begin{array}{c}
x_{n+1} \\
\vdots \\
x_{N}
\end{array}\right) \frac{\bar{y}_{s}}{\bar{X}_{s}} \\
& V_{*}=\Sigma_{r r}-\Sigma_{r s} \Sigma_{s s}^{-1} \Sigma_{s r}+\left(X_{r}-\Sigma_{r s} \Sigma_{s s}^{-1} X_{s}\right) D\left(X_{r}-\Sigma_{r s} \Sigma_{s s}^{-1} X_{s}\right)^{\prime} \\
& =\sigma^{2}\left(\begin{array}{ccc}
x_{n+1} & & \\
& \ddots & \\
& & x_{N}
\end{array}\right)-0+\left(\left(\begin{array}{c}
x_{n+1} \\
\vdots \\
x_{N}
\end{array}\right) 1_{r}-0\right) \frac{\sigma^{2}}{X_{S T}}\left(\left(\begin{array}{lll}
x_{n+1} & \cdots & x_{N}
\end{array}\right)-0\right) \\
& =\sigma^{2}\left\{\left(\begin{array}{ccc}
x_{n+1} & & \\
& \ddots & \\
& & x_{N}
\end{array}\right)+\frac{1}{x_{s T}}\left(\begin{array}{ccc}
x_{n+1} x_{n+1} & \cdots & x_{n+1} x_{N} \\
\vdots & \ddots & \vdots \\
x_{N} x_{n+1} & \cdots & x_{N} x_{n+1}
\end{array}\right)\right\} \text {. }
\end{aligned}
$$

Thus finally we have that

$$
\left(\bar{y} \mid y_{s}\right) \sim N\left(e_{*}, v_{*}\right)
$$

where:

$$
\begin{aligned}
& e_{*}=\frac{y_{s T}+1_{r}^{\prime} E_{*}}{N}=\frac{1}{N}\left\{y_{s T}+\left(\begin{array}{lll}
1 & \cdots & 1
\end{array}\right)\left(\begin{array}{c}
x_{n+1} \\
\vdots \\
x_{N}
\end{array}\right) \frac{\bar{y}_{s}}{\bar{x}_{s}}\right\} \\
& =\frac{1}{N}\left\{y_{s T}+x_{r T} \frac{y_{s T}}{x_{s T}}\right\}=\frac{y_{s T}}{N}\left\{\frac{x_{s T}+x_{r T}}{x_{s T}}\right\}=\frac{y_{s T}}{x_{s T}} \frac{x_{T}}{N}=\frac{\bar{y}_{s}}{\bar{x}_{s}} \bar{x}
\end{aligned}
$$




$$
\begin{aligned}
& V_{*}=\frac{1_{r}^{\prime} V_{*} 1_{r}}{N^{2}}=\frac{1}{N^{2}} \sigma^{2}\left(\begin{array}{lll}
1 & \cdots & 1
\end{array}\right) \\
& \times\left\{\left(\begin{array}{ccc}
x_{n+1} & & \\
& \ddots & \\
& & x_{N}
\end{array}\right)+\frac{1}{x_{s T}}\left(\begin{array}{ccc}
x_{n+1} x_{n+1} & \cdots & x_{n+1} x_{N} \\
\vdots & \ddots & \vdots \\
x_{N} x_{n+1} & \cdots & x_{N} x_{n+1}
\end{array}\right)\right\}\left(\begin{array}{c}
1 \\
\vdots \\
1
\end{array}\right) \\
& =\frac{1}{N^{2}} \sigma^{2}\left\{\left(\begin{array}{lll}
x_{n+1} & \cdots & x_{N}
\end{array}\right)+\frac{1}{x_{s T}}\left(\begin{array}{lll}
\sum_{i=n+1}^{N} x_{i} x_{n+1} & \cdots & \sum_{i=n+1}^{N} x_{i} x_{N}
\end{array}\right)\right\}\left(\begin{array}{c}
1 \\
\vdots \\
1
\end{array}\right) \\
& =\frac{1}{N^{2}} \sigma^{2}\left\{\left(x_{n+1}+\ldots+x_{N}\right)+\frac{1}{x_{s T}}\left(x_{n+1} \sum_{i=n+1}^{N} x_{i}+\ldots+x_{N} \sum_{i=n+1}^{N} x_{i}\right)\right\} \\
& =\frac{1}{N^{2}} \sigma^{2}\left\{x_{r T}+\frac{1}{x_{s T}} x_{r T}^{2}\right\}=\frac{x_{r T}}{N^{2}} \sigma^{2}\left\{\frac{x_{s T}+x_{r T}}{x_{s T}}\right\} \\
& =\sigma^{2} \times \frac{1}{N} \times \frac{(N-n) \bar{x}_{r}}{n \bar{x}_{s}} \times \frac{x_{s T}+x_{r T}}{N}=\frac{\sigma^{2}}{n}\left(1-\frac{n}{N}\right) \frac{\bar{x}_{r}}{\bar{x}_{s}} \bar{x} .
\end{aligned}
$$

\section{Exercise 10.3 Practice with the general normal-normal finite population model}

Consider a superpopulation model in which all values are independent and normally distributed with mean $\mu$, and where each value $y_{i}$ has a variance which is either:

$$
\begin{aligned}
& \sigma_{0}^{2} \text { if the corresponding covariate value } x_{i} \text { is } 0 \text {, or } \\
& \sigma_{1}^{2} \text { if } x_{i}=1 \text { (the only other possibility). }
\end{aligned}
$$

Suppose that $\sigma_{0}^{2}, \sigma_{1}^{2}$ and all $N$ covariate values $X_{i}$ are given. Also suppose there is a priori ignorance regarding $\mu$.

Find a simple expression for the predictive distribution of the finite population mean $\bar{y}$. Then calculate the predictive mean and 95\% predictive interval for $\bar{y}$ if:

$$
\begin{aligned}
& \sigma_{0}=0.08, \sigma_{1}=1.2, y_{s}=(2.1,4.9,2.3,2.0,0.2)^{\prime} \\
& x=(0,1,0,0,1, \quad 1,1,1,0,0, \quad 1,1,1,1,0, \quad 0,1)^{\prime} .
\end{aligned}
$$


Note: We have here defined a type of stratification; the finite population is assumed to consist of two strata with different variances but the same underlying mean in both strata.

\section{Solution to Exercise 10.3}

Let $n_{0}$ denote the number of covariate values $x_{i}$ in the sample (of size $n$ ) which are 0 , and let $n_{1}$ be the number which are 1 . Likewise, let $m_{0}$ denote the number of covariate values $x_{i}$ in the nonsample (of size $m=N-n$ ) which are 0 , and let $m_{1}$ be the number which are 1 .

(Thus, $n_{1}=\sum_{i=1}^{n} x_{i}, n_{0}=n-n_{1}, m_{1}=\sum_{i=n+1}^{N} x_{i}$ and $m_{0}=m-m_{1}$.)

Then, without loss of generality, re-order the finite population values in such a way that $x_{s}=(0, \ldots, 0,1, \ldots, 1)^{\prime}$ and $x_{r}=(0, \ldots, 0,1, \ldots, 1)^{\prime}$.

(Thus, in each of the sample and nonsample vectors, place the values with covariate 0 first, and place the values with covariate 1 last.)

With this setup, the Bayesian model is:

$$
\begin{aligned}
& (y \mid \beta) \sim N_{N}(X \beta, \Sigma) \\
& \beta \sim N_{p}(\delta, \Omega),
\end{aligned}
$$

where:

$$
\begin{aligned}
& p=1, \beta \equiv \mu, \delta=0, \Omega=\infty \\
& X=1_{N} \text { (since the covariates do not affect the means) } \\
& \Sigma=\operatorname{diag}\left(\sigma_{0}^{2} 1_{n_{0}}^{\prime}, \sigma_{1}^{2} 1_{n_{1}}^{\prime}, \sigma_{0}^{2} 1_{m_{0}}^{\prime}, \sigma_{1}^{2} 1_{m_{1}}^{\prime}\right)
\end{aligned}
$$

(a matrix with zeros everywhere except for

$$
\sigma_{0}^{2}, \ldots, \sigma_{0}^{2}, \sigma_{1}^{2}, \ldots, \sigma_{1}^{2}, \sigma_{0}^{2}, \ldots, \sigma_{0}^{2}, \sigma_{1}^{2}, \ldots, \sigma_{1}^{2}
$$

along the main diagonal).

Then

$$
\left(\beta \mid y_{s}\right) \sim N_{p}(\hat{\beta}, D),
$$

where: 


$$
\begin{aligned}
& D=\left(\Omega^{-1}+X_{s}^{\prime} \Sigma_{s s}^{-1} X_{s}\right)^{-1}=\left(\infty^{-1}+1_{s}^{\prime} \Sigma_{s s}^{-1} 1_{s}\right)^{-1}
\end{aligned}
$$

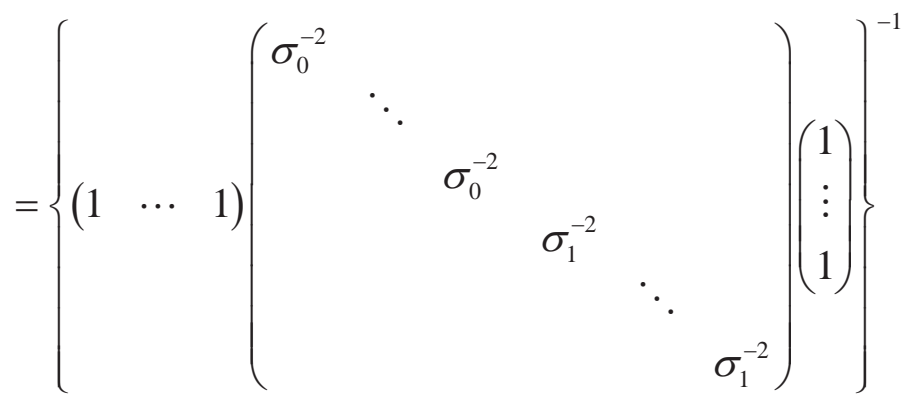

$$
\begin{aligned}
& \left.=\left\{\begin{array}{llllll}
\sigma_{0}^{-2} & \cdots & \sigma_{0}^{-2} & \sigma_{1}^{-2} & \cdots & \sigma_{1}^{-2}
\end{array}\right)\left(\begin{array}{c}
1 \\
\vdots \\
1
\end{array}\right)\right\}^{-1} \\
& =\frac{1}{n_{0} \sigma_{0}^{-2}+n_{1} \sigma_{1}^{-2}}
\end{aligned}
$$

$$
\hat{\beta}=D\left(\Omega^{-1} \delta+X_{s}^{\prime} \Sigma_{s s}^{-1} y_{s}\right)=D\left(\infty^{-1} 0+1_{s}^{\prime} \Sigma_{s s}^{-1} y_{s}\right)
$$

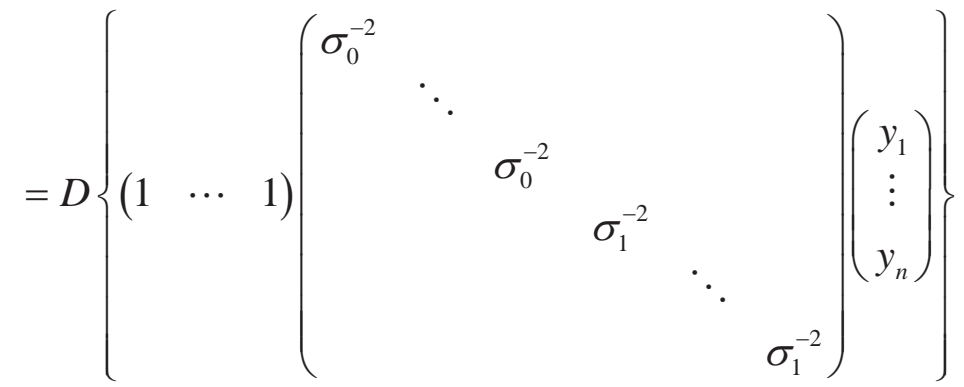

$$
\begin{aligned}
& =D\left(\sigma_{0}^{-2} y_{s 0 T}+\sigma_{1}^{-2} y_{s 1 T}\right) \text {. }
\end{aligned}
$$

Note: Here,

$$
y_{s 0 T}=\sum_{i=1}^{n_{0}} y_{i}
$$

denotes the total of the sample values with covariate $x_{i}=0$, and

$$
y_{s 1 T}=\sum_{i=n_{0}+1}^{n} y_{i}
$$

denotes the total of the sample values with covariate $X_{i}=1$. 
Next, $\left(y_{r} \mid y_{s}\right) \sim N_{m}\left(E_{*}, V_{*}\right)$, where $m=N-n$ and:

$$
\begin{aligned}
& E_{*}=X_{r} \hat{\beta}+\Sigma_{r s} \Sigma_{s s}^{-1}\left(y_{s}-X_{s} \hat{\beta}\right)=1_{r} \hat{\beta}+0=1_{r} \hat{\beta} \\
& V_{*}=\Sigma_{r r}-\Sigma_{r s} \Sigma_{s s}^{-1} \Sigma_{s r}+\left(X_{r}-\Sigma_{r s} \Sigma_{s s}^{-1} X_{s}\right) D\left(X_{r}-\Sigma_{r s} \Sigma_{s s}^{-1} X_{s}\right)^{\prime}
\end{aligned}
$$

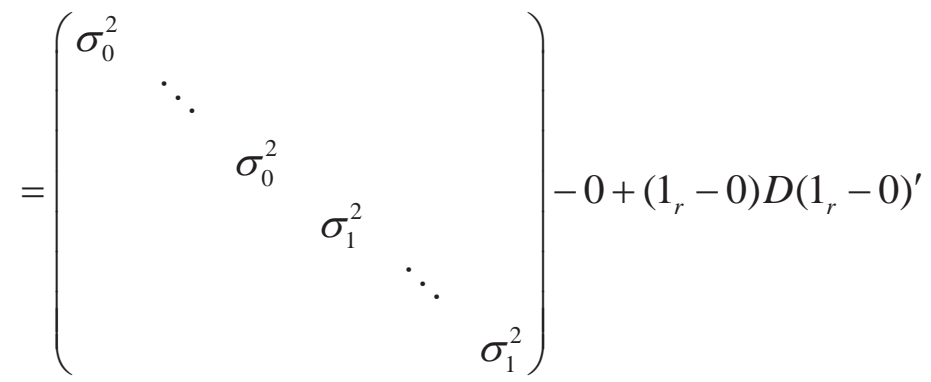

$$
\begin{aligned}
& =\left(\begin{array}{cccccc}
\sigma_{0}^{2} & & & & & \\
& \ddots & & & & \\
& & \sigma_{0}^{2} & & & \\
& & & \sigma_{1}^{2} & & \\
& & & & \ddots & \\
& & & & & \sigma_{1}^{2}
\end{array}\right)-D\left(\begin{array}{ccc}
1 & \cdots & 1 \\
\vdots & \ddots & \vdots \\
1 & \cdots & 1
\end{array}\right) .
\end{aligned}
$$

Thus $\left(\bar{y} \mid y_{s}\right) \sim N\left(e_{*}, v_{*}\right)$, where:

$$
\begin{aligned}
& e_{*}=\frac{y_{s T}+1_{r}^{\prime} E_{*}}{N}=\frac{1}{N}\left\{y_{s T}+\left(\begin{array}{lll}
1 & \cdots & 1
\end{array}\right) 1_{r} \hat{\beta}\right\}=\frac{1}{N}\left\{y_{s T}+m \hat{\beta}\right\} \\
& v_{*}=\frac{1_{r}^{\prime} V_{*} 1_{r}}{N^{2}}=\frac{1}{N^{2}}\left(\begin{array}{lll}
1 & \cdots & 1
\end{array}\right) \\
& \times\left[\left(\begin{array}{cccccc}
\sigma_{0}^{2} & & & & & \\
& \ddots & & & & \\
& & \sigma_{0}^{2} & & & \\
& & & \sigma_{1}^{2} & & \\
& & & & \ddots & \\
& & & & & \sigma_{1}^{2}
\end{array}\right)-D\left(\begin{array}{ccc}
1 & \cdots & 1 \\
\vdots & \ddots & \vdots \\
1 & \cdots & 1
\end{array}\right)\right]\left(\begin{array}{c}
1 \\
\vdots \\
1
\end{array}\right)
\end{aligned}
$$

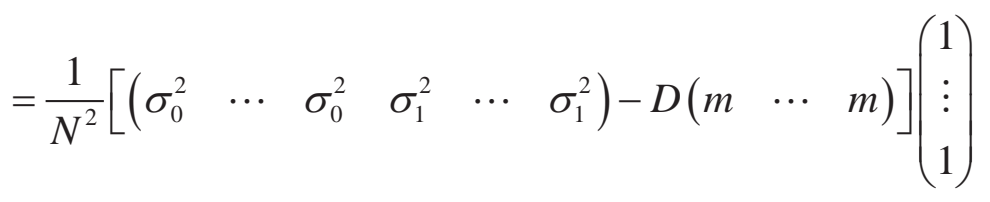

$$
\begin{aligned}
& =\frac{1}{N^{2}}\left(m_{0} \sigma_{0}^{2}+m_{1} \sigma_{1}^{2}-D m^{2}\right) .
\end{aligned}
$$


In summary, we have that $\left(\bar{y} \mid y_{s}\right) \sim N\left(e_{*}, v_{*}\right)$, where:

$$
\begin{aligned}
& e_{*}=\frac{y_{s T}+m \hat{\beta}}{N}, \quad m=N-n, \quad \hat{\beta}=D\left(\sigma_{0}^{-2} y_{s 0 T}+\sigma_{1}^{-2} y_{s 1 T}\right) \\
& D=\frac{1}{n_{0} \sigma_{0}^{-2}+n_{1} \sigma_{1}^{-2}}, \quad v_{*}=\frac{m_{0} \sigma_{0}^{2}+m_{1} \sigma_{1}^{2}-m^{2} D}{N^{2}} .
\end{aligned}
$$

Numerically, we are given:

$$
\begin{aligned}
& \sigma_{0}=0.08, \quad \sigma_{1}=1.2, \quad y_{s}=(2.1,4.9,2.3,2.0,0.2)^{\prime} \quad(\text { thus } n=5) \\
& x=(0,1,0,0,1, \quad 1,1,1,0,0,1,1,1,1,0,0,1)^{\prime} \\
& \quad \text { (thus } m=12 \text { and } N=n+m=17) \\
& x_{s}=(0,1,0,0,1)^{\prime}, \quad x_{r}=(1,1,1,0,0,1,1,1,1,0,0,1)^{\prime} .
\end{aligned}
$$

We now re-order the sample and nonsample values appropriately and so redefine:

$$
\begin{aligned}
& y_{s}=(2.1,2.0,2.3,4.9,0.2)^{\prime} \\
& x_{s}=(0,0,0,1,1)^{\prime} \\
& x_{r}=(0,0,0,0,1,1,1,1,1,1,1,1)^{\prime} .
\end{aligned}
$$

Note: We have merely swapped units 2 and 4 in both $y_{s}$ and $x_{s}$, respectively, so that all units with covariate 0 appear first and all units with covariate 1 appear last. We have also written the nonsample covariate vector $x_{r}$ with all four zero values listed at the beginning.

We see that:

$$
\begin{aligned}
& n_{0}=3, n_{1}=2, \quad m_{0}=4, m_{1}=8 \\
& y_{s 0 T}=2.1+2.0+2.3=6.4, \quad y_{s 1 T}=4.9+0.1=5.1, \\
& y_{s T}=6.4+5.1=11.5 \\
& \bar{y}_{s 0}=6.4 / 3=2.1333, \quad \bar{y}_{s 1}=5.1 / 2=2.55, \\
& \bar{y}_{s}=11.5 / 5=2.3 .
\end{aligned}
$$

Thereby we obtain $\left(\bar{y} \mid y_{s}\right) \sim N\left(e_{*}, v_{*}\right)$, where:

$$
D=\frac{1}{n_{0} \sigma_{0}^{-2}+n_{1} \sigma_{1}^{-2}}=\frac{1}{3 / 0.08^{2}+2 / 1.2^{2}}=0.0021270
$$




$$
\begin{aligned}
\begin{aligned}
\hat{\beta} & =D\left(\sigma_{0}^{-2} y_{s 0 T}+\sigma_{1}^{-2} y_{s 1 T}\right)=0.0021270\left(6.4 / 0.08^{2}+5.1 / 1.2^{2}\right) \\
& =2.1345
\end{aligned} \\
\begin{aligned}
e_{*} & =\frac{y_{s T}+m \hat{\beta}}{N}=\frac{11.5+12 \times 2.1345}{17}=2.1832 \\
V_{*} & =\frac{m_{0} \sigma_{0}^{2}+m_{1} \sigma_{1}^{2}-m^{2} D}{N^{2}}=\frac{4 \times 0.08^{2}+8 \times 1.2^{2}-12^{2} \times 0.0021250}{17^{2}} \\
& =0.038890 .
\end{aligned}
\end{aligned}
$$

Thus the predictive mean of the finite population mean $\bar{y}$ is $\hat{\beta}=2.13$, and the $95 \%$ predictive interval for $\bar{y}$ is $\left(e_{*} \pm 1.96 \sqrt{v_{*}}\right)=(1.80,2.57)$.

\section{R Code for Exercise 10.3}

options(digits $=4$ )

sig0=0.08; sig1=1.2; ys = $c(2.1,2.0,2.3,4.9,0.2) ; n=$ length(ys)

$x s=c(0,0,0,1,1) ; x r=c(0,0,0,0,1,1,1,1,1,1,1,1) ; m=$ length $(x r) ; N=n+m$

$\mathrm{n} 1=\operatorname{sum}(\mathrm{xs}) ; \mathrm{n} 0=\mathrm{n}-\mathrm{n} 1 ; \quad \mathrm{m} 1=\operatorname{sum}(\mathrm{xr}) ; \mathrm{m0}=\mathrm{m}-\mathrm{m} 1$

$c(n, n 0, n 1, \quad m, m 0, m 1, N) \# \begin{array}{lllllll}3 & 2 & 12 & 4 & 17\end{array}$

ys $T=\operatorname{sum}(y s) ; y s 1 T=s u m(y s * x s) ; y s 0 T=y s T-y s 1 T$

ysbar=ysT/n; ys1bar=ys1T/n1; ysObar=ys0T/n0

c(ys0T,ys1T,ysT, ys0bar,ys1bar,ysbar)

\# $6.400 \quad 5.10011 .500 \quad 2.133 \quad 2.5502 .300$

$\mathrm{D}=1 /\left(\mathrm{n0} / \operatorname{sig} 0^{\wedge} 2+\mathrm{n} 1 / \operatorname{sig} 1^{\wedge} 2\right) ;$ betahat $=\mathrm{D}^{*}\left(\mathrm{ys} 0 \mathrm{~T} / \operatorname{sig} 0^{\wedge} 2+\mathrm{ys} 1 \mathrm{~T} / \operatorname{sig} 1^{\wedge} 2\right)$

estar $=(1 / \mathrm{N}) *\left(\mathrm{ys} T+\mathrm{m}^{*}\right.$ betahat $)$;

$\operatorname{vstar}=\left(1 / \mathrm{N}^{\wedge} 2\right) *\left(\mathrm{~m} 0^{*} \operatorname{sig} 0^{\wedge} 2+\mathrm{m} 1^{*} \operatorname{sig} 1^{\wedge} 2-\mathrm{D}^{*} \mathrm{~m}^{\wedge} 2\right)$

c(D,betahat,estar,vstar) \# 0.0021272 .1345642 .1832220 .038890

hpdr=estar+c(-1,1)*qnorm(0.975)*sqrt(vstar); c(hpdr) \# 1.7972 .570

\section{0.7 The normal-normal-gamma finite population model}

For the models so far considered in this chapter, the superpopulation variance $\sigma^{2}$ parameter or variance-covariance matrix parameter $\Sigma$ has been assumed to be known.

If this parameter were unknown, as might typically be the case in practice, then an estimate could be computed from the data via some 
method (which need not necessarily be Bayesian) and substituted into the equations derived.

This strategy, which may be considered an example of empirical Bayes techniques, may sometimes work well, especially if based on a sufficiently large sample size.

For example, recall that in the case of no covariates, with the superpopulation variance $\sigma^{2}$ known, the $1-\alpha$ CPDR for $\bar{y}$ is

$$
\left(\bar{y}_{s} \pm z_{\alpha / 2} \frac{\sigma}{\sqrt{n}} \sqrt{1-\frac{n}{N}}\right) .
$$

Now suppose that $n$ is large and we estimate $\sigma^{2}$ by the sample variance,

$$
s^{2}=\frac{1}{n-1} \sum_{i=1}^{n}\left(y_{i}-\bar{y}_{s}\right)^{2} .
$$

Then the result is the same as the classical design-based CI one would use in the same situation of a large sample size.

However, this strategy will not work well generally. For example, if $n$ is small then it will lead to an interval which has a frequentist coverage well below the intended level of $1-\alpha$. In such cases, the problem could be addressed to some extent by applying an adjustment which reflects uncertainty regarding the unknown variance parameter. However, the nature of this type of adjustment would be ad hoc and lead to possibly other problems with the inference.

Perhaps the best way to deal with uncertainty regarding the variance parameter is to incorporate it into the finite population model as yet another random variable with its own prior distribution, i.e. to add another level to the hierarchical structure of that model. This is the approach we will now take. Note that parts of the exposition below will be a review of material already covered in previous chapters.

With the above in mind, and with quantities as defined previously, we define the normal-normal-gamma finite population model as follows:

$$
\begin{aligned}
& (y \mid \beta, \lambda) \sim N_{N}(X \beta, \Sigma / \lambda) \\
& (\beta \mid \lambda) \sim N_{p}(\delta, \Omega) \\
& \lambda \sim G(\eta, \tau) .
\end{aligned}
$$


A problem with this model is that is involves an additional nuisance parameter to deal with relative to the normal-normal finite population model, namely $\lambda$. This means that the predictive pdf of the nonsample vector cannot be obtained so easily.

That density is now

$$
f\left(y_{r} \mid y_{s}\right)=\iint f\left(y_{r}, \beta, \lambda \mid y_{s}\right) d \beta d \lambda \propto \iint f(y, \beta, \lambda) d \beta d \lambda,
$$

where $f(y, \beta, \lambda)=f(\lambda) f(\beta \mid \lambda) f(y \mid \beta, \lambda)$

$$
\begin{aligned}
& \propto \lambda^{\eta-1} e^{-\tau \lambda} \times \exp \left\{-\frac{1}{2}(\beta-\delta)^{\prime} \Omega^{-1}(\beta-\delta)\right\} \\
& \quad \times \lambda^{N / 2} \exp \left\{-\frac{1}{2} \lambda(y-X \beta)^{\prime} \Sigma^{-1}(y-X \beta)\right\}
\end{aligned}
$$

is the joint density of all random variables involved in the model, namely the $N$ finite population values, $y_{1}, \ldots, y_{N}$, and the $p+1$ model parameters, namely $\lambda, \beta_{1}, \ldots, \beta_{p}$.

In an attempt to perform the second double integral at (10.7) (which is actually a $(p+1)$-fold integral), we may first integrate with respect to $\lambda$ and obtain

$$
f\left(y_{r} \mid y_{s}\right) \propto \int_{-\infty}^{\infty} \frac{\exp \left\{-(1 / 2)(\beta-\delta)^{\prime} \Omega^{-1}(\beta-\delta)\right\}}{\left[\tau+(1 / 2)(y-X \beta)^{\prime} \Sigma^{-1}(y-X \beta)\right]^{\eta+N / 2}} d \beta
$$

(after recognising a gamma density in $\lambda$ ), or first integrate with respect to $\beta$ and obtain

$$
\begin{aligned}
& f\left(y_{r} \mid y_{s}\right) \propto \int_{0}^{\infty} \frac{\lambda^{\eta+N / 2-1}}{\operatorname{det}\left(\Omega^{-1}+\lambda X^{\prime} \Sigma^{-1} X\right)} \times \exp \left\{-\lambda\left(\tau+\frac{1}{2} y^{\prime} \Sigma^{-1} y\right)\right. \\
& \left.+\left(\Omega^{-1} \delta+\lambda X^{\prime} \Sigma^{-1} y\right)^{\prime}\left(\Omega^{-1}+\lambda X^{\prime} \Sigma^{-1} X\right)^{-1}\left(\Omega^{-1} \delta+\lambda X^{\prime} \Sigma^{-1} y\right)\right\} d \lambda
\end{aligned}
$$

(after recognising a multivariate normal density in $\beta$ ).

Either way, the remaining integral is in general impossible to perform analytically, and the posterior predictive distributions of the nonsample vector and linear combinations of that vector (such as the finite population mean and total) are not normally distributed. However, there is an important special case which simplifies matters considerably. 


\section{0.8 Special cases of the normal-normal- gamma finite population model}

Theorem 10.1: Suppose there is priori ignorance regarding $\beta$ and it is appropriate to set $\delta=0$ and $\Omega=\infty$, so that

$f(\beta \mid \lambda) \propto f(\beta) \propto 1, \beta \in \Re$.

Then the predictive distribution of the finite population mean is given by

$$
\left(\frac{\bar{y}-a}{b} \mid y_{s}\right) \sim t(2 \eta+n-p) \text {, }
$$

where: $a=\frac{y_{s T}+1_{r}^{\prime}\left[X_{r} \hat{\beta}+\Sigma_{r s} \Sigma_{s s}^{-1}\left(y_{s}-X_{s} \hat{\beta}\right)\right]}{N}$

$$
\begin{aligned}
& b^{2}=\frac{1_{r}^{\prime}\left[\Sigma_{r r}-\Sigma_{r s} \Sigma_{s s}^{-1} \Sigma_{s r}+A D A^{\prime}\right] 1_{r}\left[2 \tau+\left(y_{s}-X_{s} \hat{\beta}\right)^{\prime} \Sigma_{s s}^{-1}\left(y_{s}-X_{s} \hat{\beta}\right)\right]}{(2 \eta+n-p) N^{2}} \\
& \hat{\beta}=D X_{s}^{\prime} \Sigma_{s s}^{-1} y_{s}, \quad D=\left(X_{s}^{\prime} \Sigma_{s s}^{-1} X_{s}\right)^{-1}, \quad A=X_{r}-\Sigma_{r s} \Sigma_{s s}^{-1} X_{s} .
\end{aligned}
$$

Note: Here, $\hat{\beta}$ is the MLE of $\beta$, and also the posterior mean of $\beta$ under the simpler normal-normal finite population model with improper prior $f(\beta) \propto 1, \beta \in \mathfrak{R}$ (and $\sigma^{2}$ known).

Theorem 10.1 can be proved by first noting that:

(a) $\left(\lambda \mid y_{s}\right)$ is gamma (with parameters that can be obtained by integrating $f\left(\beta, \lambda \mid y_{s}\right)$ with respect to $\left.\beta\right)$, and

(b) $\left(\bar{y} \mid y_{s}, \lambda\right)$ is normal (with parameters that can be obtained by examining the normal-normal finite population model above).

Using these two distributions, one can solve for the predictive density of the finite population mean via the identity

$$
f\left(\bar{y} \mid y_{s}\right)=\int f\left(\bar{y}, \lambda \mid y_{s}\right) d \lambda=\int f\left(\bar{y} \mid y_{s}, \lambda\right) f\left(\lambda \mid y_{s}\right) d \lambda .
$$

A special case of Theorem 10.1 which assumes a priori ignorance of $\lambda$ by way of setting $\eta=\tau=0$ can be found in Royall and Pfeffermann (1982). 
If we further assume conditional independence (which may expressed by writing $\Sigma=I_{N}$ ) and no auxiliary information ( $p=1$ and $X=1_{N}$ ), the result in Theorem 10.1 reduces to

$$
\left(\frac{\bar{y}-\bar{y}_{s}}{\left(s_{s} / n\right) \sqrt{1-n / N}} \mid y_{s}\right) \sim t(n-1),
$$

where $s_{s}^{2}=\frac{1}{n-1} \sum_{i=1}^{n}\left(y_{i}-\bar{y}_{s}\right)^{2}$ (the sample variance).

This result was already proved in a previous chapter without the involvement of vectors and matrices. Again note that the result leads to point estimates and interval estimates which are identical to those which one might construct using a design-based approach (see Cochran, 1977, Section 2.8).

\section{Exercise 10.4 Proof of Theorem I}

Prove Theorem 10.1 above.

\section{Solution to Exercise I 0.4}

Using the procedure outlined above, we first derive the unconditional pdf of $\lambda$ as follows:

$$
f\left(\lambda \mid y_{s}\right)=\int f\left(\beta, \lambda \mid y_{s}\right) d \beta \propto \int \lambda^{\eta-1} e^{-\tau \lambda} \times 1 \times \lambda^{\frac{n}{2}} \exp \left(-\frac{\lambda}{2} Q_{1}\right) d \beta,
$$

where

$$
\begin{aligned}
Q_{1} & =\left(y_{s}-X_{s} \beta\right)^{\prime} \Sigma_{s s}^{-1}\left(y_{s}-X_{s} \beta\right) \\
& =y_{s}^{\prime} \Sigma_{s s}^{-1} y_{s}-y_{s}^{\prime} \Sigma_{s s}^{-1} X_{s} \beta-\beta^{\prime} X_{s}^{\prime} \Sigma_{s s}^{-1} y_{s}+\beta^{\prime} X_{s}^{\prime} \Sigma_{s s}^{-1} X_{s} \beta .
\end{aligned}
$$

Now equate $Q_{1}$ with

$$
\begin{aligned}
Q_{2} & =(\beta-T)^{\prime} M(\beta-T)^{\prime}+R \quad \text { (where } R \text { stands for 'remainder') } \\
& =\beta^{\prime} M \beta-\beta^{\prime} M T-T^{\prime} M \beta+T^{\prime} M T+R .
\end{aligned}
$$

We see that

$$
M=X_{s}^{\prime} \Sigma_{s s}^{-1} X_{s} \text { and } M T=X_{s}^{\prime} \Sigma_{s s}^{-1} y_{s},
$$

so that

$$
T=M^{-1}(M T)=\left(X_{s}^{\prime} \Sigma_{s s}^{-1} X_{s}\right)^{-1} X_{s}^{\prime} \Sigma_{s s}^{-1} y_{s} .
$$


Note: Here, $T$ is the same as $\hat{\beta}$ in Theorem 10.1 .

Also, $R=y_{s}^{\prime} \Sigma_{s s}^{-1} y_{s}-T^{\prime} M T=\left(y_{s}-X_{s} T\right)^{\prime} \Sigma_{s s}^{-1}\left(y_{s}-X_{s} T\right)$.

Note: This is easily proved by noting that the RHS here is

$$
y_{s}^{\prime} \Sigma_{s s}^{-1} y_{s}-y_{s}^{\prime} \Sigma_{s s}^{-1} X_{s} T-T^{\prime} X_{s}^{\prime} \Sigma_{s s}^{-1} y_{s}+T^{\prime} X_{s}^{\prime} \Sigma_{s s}^{-1} X_{s} T
$$

where

$$
y_{s}^{\prime} \Sigma_{s s}^{-1} X_{s} T=\left(y_{s}^{\prime} \Sigma_{s s}^{-1} X_{s} T\right)^{\prime}=T^{\prime} X_{s}^{\prime} \Sigma_{s s}^{-1} y_{s}
$$

(since $y_{s}^{\prime} \Sigma_{s s}^{-1} X_{s} T$ is a scalar quantity), and where

$$
T^{\prime} X_{s}^{\prime} \Sigma_{s s}^{-1} X_{s} T=T^{\prime} M T=T^{\prime} X_{s}^{\prime} \Sigma_{s s}^{-1} X_{s}\left(X_{s}^{\prime} \Sigma_{s s}^{-1} X_{s}\right)^{-1} X_{s}^{\prime} \Sigma_{s s}^{-1} y=T^{\prime} X_{s}^{\prime} \Sigma_{s s}^{-1} y,
$$

so that the RHS equals

$$
y_{s}^{\prime} \Sigma_{s s}^{-1} y_{s}-T^{\prime} M T-T^{\prime} M T+T^{\prime} M T=y_{s}^{\prime} \Sigma_{s s}^{-1} y_{s}-T^{\prime} M T .
$$

Thus

$$
\begin{aligned}
f\left(\lambda \mid y_{s}\right) & \propto \int \lambda^{\eta-1} e^{-\tau \lambda} \times 1 \times \lambda^{\frac{n}{2}} \exp \left(-\frac{\lambda}{2}\left[(\beta-T)^{\prime} M(\beta-T)^{\prime}+R\right]\right) d \beta \\
& =\lambda^{\eta+\frac{n}{2}-1} \exp \left(-\lambda\left(\tau+\frac{R}{2}\right)\right) \times I,
\end{aligned}
$$

where

$$
\begin{aligned}
I & =\int \exp \left(-\frac{1}{2}(\beta-T)^{\prime}\left(\frac{M^{-1}}{\lambda}\right)^{-1}(\beta-T)^{\prime}\right) d \beta \\
& =(2 \pi)^{\frac{p}{2}}\left\{\operatorname{det}\left(\frac{M^{-1}}{\lambda}\right)\right\}^{-\frac{1}{2}}
\end{aligned}
$$

(using standard multivariate normal theory)

$\propto \lambda^{\frac{p}{2}} \quad$ (since $M=X_{s}^{\prime} \Sigma_{s s}^{-1} X_{s}$ is a $p$ by $p$ matrix).

It follows that

$$
f\left(\lambda \mid y_{s}\right) \propto \lambda^{\eta+\frac{n}{2}+\frac{p}{2}-1} \exp \left(-\lambda\left(\tau+\frac{R}{2}\right)\right)=\lambda^{\frac{A}{2}-1} \exp \left(-\frac{B}{2} \lambda\right),
$$

where:

$$
A=2 \eta+n-p, \quad B=2 \tau+R, \quad R=\left(y_{s}-X_{s} T\right)^{\prime} \Sigma_{s s}^{-1}\left(y_{s}-X_{s} T\right) .
$$


We thereby arrive at the required distribution,

$$
\left(\lambda \mid y_{s}\right) \sim G(A / 2, B / 2),
$$

which may also be expressed by writing

$$
\left(B \lambda \mid y_{s}\right) \sim G(A / 2,1 / 2)=\chi^{2}(A) \text {. }
$$

Having derived the posterior dsn of $\lambda$, we now observe that

$$
\left(\bar{y} \mid y_{s}, \lambda\right) \sim N\left(e_{0}, v_{0}\right) \text {, }
$$

where:

$$
\begin{array}{ll}
e_{0}=\frac{1}{N}\left(y_{s T}+1_{r}^{\prime} E_{0}\right), & E_{0}=X_{r} T+\Sigma_{r s} \Sigma_{s s}^{-1}\left(y_{s}-X_{s} T\right) \\
v_{0}=\frac{1_{r}^{\prime} V_{0} 1_{r}}{N^{2} \lambda} \equiv \frac{w_{0}}{\lambda}, & w_{0}=\frac{1_{r}^{\prime} V_{0} 1_{r}}{N^{2}}, \\
V_{0}=G+A M^{-1} A^{\prime} & \\
G=\Sigma_{r r}-\Sigma_{r s} \Sigma_{s s}^{-1} \Sigma_{s r}, & A=X_{r}-\Sigma_{r s} \Sigma_{s s}^{-1} X_{s} .
\end{array}
$$

Note: We have here simply applied the theory of the normal-normal finite population model with $\Omega=\infty$ and with quantities such as $\Sigma_{s r}$ and $\Sigma_{s s}$ replaced by $\Sigma_{s r} / \lambda$ and $\Sigma_{s s} / \lambda$, etc.

Therefore

$$
\begin{aligned}
f\left(\bar{y} \mid y_{s}\right) & =\int f\left(\bar{y} \mid y_{s}, \lambda\right) f\left(\lambda \mid y_{s}\right) d \lambda \\
& \propto \int \lambda^{\frac{1}{2}} \exp \left\{-\frac{\lambda}{2 w_{0}}\left(\bar{y}-e_{0}\right)^{2}\right\} \times \lambda^{\frac{A}{2}-1} \exp \left(-\frac{B}{2} \lambda\right) d \lambda \\
& =\int \lambda^{\frac{A+1}{2}-1} \exp \left\{-\lambda\left[\frac{B}{2}+\frac{\left(\bar{y}-e_{0}\right)^{2}}{2 w_{0}}\right]\right\} d \lambda \\
\propto & \propto\left[\frac{B}{2}+\frac{\left(\bar{y}-e_{0}\right)^{2}}{2 w_{0}}\right]^{-\left(\frac{A+1}{2}\right)} \propto\left[1+\frac{\left(\bar{y}-e_{0}\right)^{2}}{B w_{0}}\right]^{-\left(\frac{A+1}{2}\right)} \\
\left.\propto\left[1+\frac{\left(\bar{y}-e_{0}\right)^{2}}{B w_{0} / A}\right\}\right]^{-\left(\frac{A+1}{2}\right)} & \propto\left[\frac{\left(\frac{\bar{y}-e_{0}}{\sqrt{B w_{0} / A}}\right)^{2}}{A}\right]^{-\left(\frac{A+1}{2}\right)} .
\end{aligned}
$$


It follows that $\left(\frac{\bar{y}-e_{0}}{h_{0}} \mid y_{s}\right) \sim t(A)$, where $h_{0}^{2}=\frac{B w_{0}}{A}$.

Here: $A=2 \eta+n-p \quad$ (which is the same as the degrees of freedom in the $t$ distribution in Theorem 10.1)

$$
\begin{gathered}
e_{0}=\frac{1}{N}\left(y_{s T}+1_{r}^{\prime} E_{0}\right)=\frac{y_{s T}+1_{r}^{\prime}\left[X_{r} T+\Sigma_{r s} \Sigma_{s s}^{-1}\left(y_{s}-X_{s} T\right)\right]}{N} \\
\text { (which is the same as } a \text { in Theorem 10.1). } \\
h_{0}^{2}=\frac{B}{A} w_{0}=\frac{2 \tau+R}{2 \eta+n-p} \times \frac{1_{r}^{\prime} V_{0} 1_{r}}{N^{2}} \\
=\frac{\left[2 \tau+\left(y_{s}-X_{s} T\right)^{\prime} \Sigma_{s s}^{-1}\left(y_{s}-X_{s} T\right)\right]}{(2 \eta+n-p) N^{2}} 1_{r}^{\prime}\left(G+A M^{-1} A^{\prime}\right) 1_{r} \\
=\frac{\left[2 \tau+\left(y_{s}-X_{s} T\right)^{\prime} \Sigma_{s s}^{-1}\left(y_{s}-X_{s} T\right)\right]}{(2 \eta+n-p) N^{2}} \\
\times 1_{r}^{\prime}\left\{\Sigma_{r r}-\Sigma_{r s} \Sigma_{s s}^{-1} \Sigma_{s r}+\left(X_{r}-\Sigma_{r s} \Sigma_{s s}^{-1} X_{s}\right)\left(X_{s}^{\prime} \Sigma_{s s}^{-1} X_{s}\right)^{-1}\left(X_{r}-\Sigma_{r s} \Sigma_{s s}^{-1} X_{s}\right)^{\prime}\right\} 1_{r}
\end{gathered}
$$

(which is the same as $b^{2}$ in Theorem 10.1).

That completes the proof of Theorem 10.1.

\subsection{The case of an informative prior on the regression parameter}

If there is some prior information available regarding the regression parameter $\beta$ then $\Omega<\infty$ and Theorem 10.1 above cannot be applied. So the problem of inference on the finite population mean $\bar{y}$ becomes much more difficult.

However, that difficulty can be easily 'sidestepped' via Monte Carlo methods based on a random sample from the predictive distribution of $\bar{y}$, namely

$$
\bar{y}^{(1)}, \ldots, \bar{y}^{(J)} \sim \text { iid } f\left(\bar{y} \mid y_{s}\right) .
$$


With such a sample we can, for example, estimate $\bar{y}$ 's predictive mean, namely $\hat{\bar{y}}=E\left(\bar{y} \mid y_{s}\right)$, by the average of $\bar{y}^{(1)}, \ldots, \bar{y}^{(J)}$, and estimate $\bar{y}$ 's 95\% CPDR by the empirical 0.025 and 0.975 quantiles of $\bar{y}^{(1)}, \ldots, \bar{y}^{(J)}$.

This then raises the question of how the Monte Carlo sample can be obtained. In this context, we may employ the method of composition via the equation

$$
f\left(\bar{y}, \beta, \lambda \mid y_{s}\right)=f\left(\bar{y} \mid y_{s}, \beta, \lambda\right) f\left(\beta, \lambda \mid y_{s}\right) .
$$

Thus, we first generate a sample from the joint posterior distribution the two parameters,

$$
\left(\beta^{(1)}, \lambda^{(1)}\right), \ldots,\left(\beta^{(J)}, \lambda^{(J)}\right) \sim \text { iid } f\left(\beta, \lambda \mid y_{s}\right) .
$$

and then for each $j=1, \ldots, J$ we sample

$$
\bar{y}^{(j)} \sim f\left(\bar{y} \mid y_{s}, \beta^{(j)}, \lambda^{(j)}\right) \sim N\left(\frac{y_{s T}+1_{r}^{\prime} X_{r} \beta^{(j)} 1_{r}}{N}, \frac{1_{r}^{\prime} \Sigma_{r} 1_{r}}{N^{2} \lambda^{(j)}}\right) .
$$

This in turn raises the question of how to obtain the sample from $f\left(\beta, \lambda \mid y_{s}\right)$. In this case an ideal solution is to apply a Gibbs sampler defined by the following conditional distributions:

1. $\left(\beta \mid y_{s}, \lambda\right) \sim N_{p}(\tilde{\beta}, D)$, where: $\quad \tilde{\beta}=D\left(\Omega^{-1} \delta+\lambda X_{s}^{\prime} \Sigma_{s s}^{-1} y_{s}\right)$

$$
D=\left(\Omega^{-1}+\lambda X_{s}^{\prime} \Sigma_{s s}^{-1} X_{s}\right)^{-1}
$$

2. $\left(\lambda \mid y_{s}, \beta\right) \sim G\left(\eta+\frac{n}{2}, \tau+\frac{1}{2}\left(y_{s}-X_{s} \beta\right)^{\prime} \Sigma_{s s}^{-1}\left(y_{s}-X_{s} \beta\right)\right)$.

Note: The first of these distributions derives directly from the normalnormal finite population model with $\Sigma_{s r}$ and $\Sigma_{s s}$ replaced by $\Sigma_{s r} / \lambda$ and $\Sigma_{s s} / \lambda$, etc.

The second conditional is obtained by noting that

$$
\begin{aligned}
f\left(\lambda \mid y_{s}, \beta\right) & \propto f\left(\lambda, \beta \mid y_{s}\right) \\
& \propto f\left(\lambda, \beta, y_{s}\right)
\end{aligned}
$$




$$
\begin{aligned}
& =f(\lambda) f(\beta \mid \lambda) f\left(y_{s} \mid \lambda, \beta\right) \\
& \propto \lambda^{\eta-1} e^{-\tau \lambda} \times \exp \left(-\frac{1}{2}(\beta-\delta)^{\prime} \Omega^{-1}(\beta-\delta)\right) \\
& \times \lambda^{\frac{n}{2}} \exp \left(-\frac{\lambda}{2}\left(y_{s}-X_{s} \beta\right)^{\prime} \Sigma_{s s}^{-1}\left(y_{s}-X_{s} \beta\right)\right) \\
& \stackrel{\lambda}{\propto} \lambda^{\eta+\frac{n}{2}-1} \exp \left(-\lambda\left\{\tau+\frac{1}{2}\left(y_{s}-X_{s} \beta\right)^{\prime} \Sigma_{s s}^{-1}\left(y_{s}-X_{s} \beta\right)\right\}\right) \text {. }
\end{aligned}
$$

\section{Exercise 10.5 Practice with the normal-normal-gamma finite population model}

In the context of the normal-normal-gamma finite population model, suppose we obtain a sample of size $n=5$, with values given by

$$
y_{s}=\left(y_{1}, \ldots, y_{n}\right)^{\prime}=(5.6,2.3,8.4,5.1,4.3)^{\prime}
$$

via SRSWOR from a finite population of size $N=15$.

Find the predictive mean and 95\% central predictive density region for the finite population mean $\bar{y}$ in each of the following scenarios.

(a) There are no covariates, the population values are conditionally iid and there is no prior information available regarding the model parameters.

(b) The population values are conditionally independent, the ith population value has mean $x_{i} \beta$ and variance $x_{i} / \lambda(i=1, \ldots, N)$, the population covariate vector is

$$
\begin{aligned}
x=\left(x_{1}, \ldots, x_{N}\right)^{\prime}= & (9.3,4.6,15.0,11.2,7.8,2.4,6.6,3.0,2.1,7.3, \\
& 5.5,8.0,2.4,4.2,5.5)^{\prime},
\end{aligned}
$$

and there is no prior information regarding the model parameters.

(c) There are no covariates, the population values are conditionally iid, the prior on the normal mean is normal with mean 10 and variance 2.25, and (independently) the prior on the normal precision parameter (inverse of the normal variance) is gamma with mean 2 and variance 1/2 (or equivalently, gamma with parameters 8 and 4 ). 


\section{Solution to Exercise I 0.5}

(a) In this case, Theorem 10.1 reduces to

$$
\left(\frac{\bar{y}-\bar{y}_{s}}{\left(s_{s} / n\right) \sqrt{1-n / N}} \mid y_{s}\right) \sim t(n-1),
$$

where: $\bar{y}_{s}=\frac{1}{n}\left(y_{1}+\ldots+y_{n}\right)=5.140$

$$
s_{s}^{2}=\frac{1}{n-1} \sum_{i=1}^{n}\left(y_{i}-\bar{y}\right)^{2}=4.9030 \text {. }
$$

So the required predictive mean and 95\% predictive interval of $\bar{y}$ are

$$
\bar{y}_{s}=5.140 \text { and }\left(\bar{y}_{s} \pm t_{\alpha / 2}(n-1) \frac{s_{s}}{n} \sqrt{1-\frac{n}{N}}\right)=(2.8951,7.3849) \text {. }
$$

(b) In this case (a variation of Bayesian ratio estimation as discussed earlier) we apply Theorem 10.1 with:

$$
p=1, \quad \eta=\tau=0, X=x, \quad \Sigma=\operatorname{diag}(x)=\left(\begin{array}{ccc}
x_{1} & & \\
& \ddots & \\
& & x_{N}
\end{array}\right) .
$$

Instead of deriving a 'simple' general algebraic expression for the predictive distribution of the finite population mean in this case, we can obtain the specific required result more quickly by directly applying the formulae in Theorem 10.1 using R. An advantage of this approach is that it leads us to write a general algorithm in $\mathrm{R}$ which can be straightaway used in other situations requiring Theorem 10.1. Also, the algorithm can be used to check our answer to part (a).

Thereby we obtain the result that

$$
\left(\frac{\bar{y}-a}{b} \mid y_{s}\right) \sim t(c),
$$

where $a=3.3945, b=0.1159$ and $c=2 \eta+n-p=4$.

So the required predictive mean and 95\% predictive interval of $\bar{y}$ are $\bar{y}_{s}=3.3945$ and $\left(a \pm t_{\alpha / 2}(c) b\right)=(3.0725,3.7164)$. 
Note: This inference is lower than that in (a) because the mean of the covariate values in the nonsample is 4.7 , which is much lower than their mean in the sample, 9.58. The regression coefficient $\beta$ in our model is estimated as 0.5365 , reflecting the positive linear relationship between the $x$ and $y$ values in the sample.

(c) In this case, a good option is to first employ the Gibbs sampler to generate a random sample from the joint posterior distribution of $\beta$ and $\lambda$, with:

$$
p=1, \delta=10, \Omega=9, \eta=8, \tau=4, X=1_{N}, \Sigma=\operatorname{diag}\left(1_{N}\right) .
$$

The two conditional distributions are:

1. $\left(\beta \mid y_{s}, \lambda\right) \sim N_{p}(\tilde{\beta}, D)$, where:

$$
\begin{aligned}
& \tilde{\beta}=D\left(\Omega^{-1} \delta+\lambda X_{s}^{\prime} \Sigma_{s s}^{-1} y_{s}\right) \\
& D=\left(\Omega^{-1}+\lambda X_{s}^{\prime} \Sigma_{s s}^{-1} X_{s}\right)^{-1}
\end{aligned}
$$

2. $\left(\lambda \mid y_{s}, \beta\right) \sim G\left(\eta+\frac{n}{2}, \tau+\frac{1}{2}\left(y_{s}-X_{s} \beta\right)^{\prime} \Sigma_{s s}^{-1}\left(y_{s}-X_{s} \beta\right)\right)$.

But, by analogy with the simpler normal-normal model and normalgamma model, these conditionals must be equivalent to:

1. $\left(\beta \mid y_{s}, \lambda\right) \sim N\left(\beta_{\lambda}, \sigma_{\lambda}^{2}\right)$, where:

$$
\begin{aligned}
& \beta_{\lambda}=\left(1-k_{\lambda}\right) \beta_{0}+k_{\lambda} \bar{y}_{s} \\
& \sigma_{\lambda}^{2}=\frac{k_{\lambda}}{n \lambda}, k_{\lambda}=\frac{n}{n+1 /\left(\lambda \sigma_{0}^{2}\right)} \\
& \beta_{0}=10, \quad \sigma_{0}=3
\end{aligned}
$$

2. $\left(\lambda \mid y_{s}, \beta\right) \sim G\left(\eta+\frac{n}{2}, \tau+\frac{n}{2} s_{\beta}^{2}\right)$,

where

$$
s_{\beta}^{2}=\frac{1}{n} \sum_{i=1}^{n}\left(y_{i}-\beta\right)^{2} .
$$


Either way, implementing this Gibbs sampler for 10,100 iterations with a burn-in of 100 we obtain the trace plots and histograms for $\beta$ and $\lambda$ in Figure 10.2. (The two subplots on the left are for $\beta$, and the two on the right are for $\lambda$. The histograms do not include the first 100 iterations.)

Thinning the last 10,000 values of each parameter by a factor of 10 we obtain an approximately random sample of size $J=1,000$ from the joint posterior distribution of the two parameters, namely

$$
\left(\beta_{j}, \lambda_{j}\right) \sim \text { iid } f\left(\beta, \lambda \mid y_{s}\right), j=1, \ldots, J \text {. }
$$

The sample ACFs over the entire sample of 10,000 and over the thinned sample of 1,000 are shown for each of $\beta$ and $\lambda$ in Figure 10.3. (E.g. the top-left subplot is for $\beta$ over the entire sample of 10,000.) The thinning process has virtually eliminated all signs of autocorrelation.

\section{Figure 10.2 Trace plots and histograms}

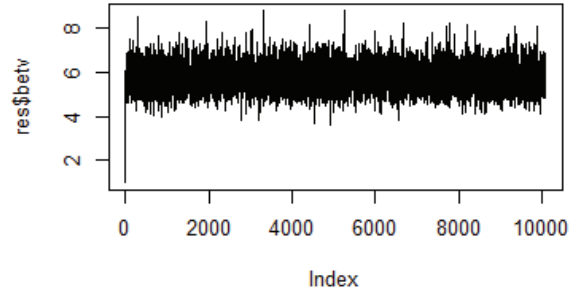

Histogram of res\$betv[-c(1:101)]

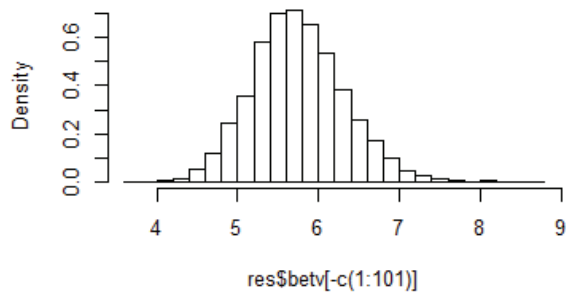

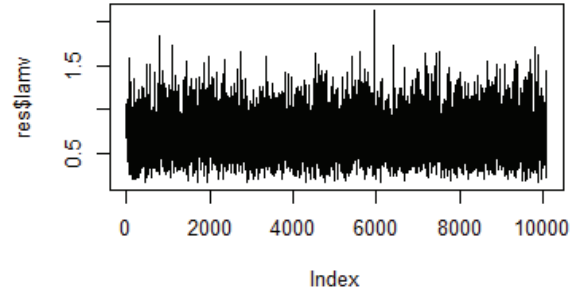

Histogram of res\$lamv[-c(1:101)]

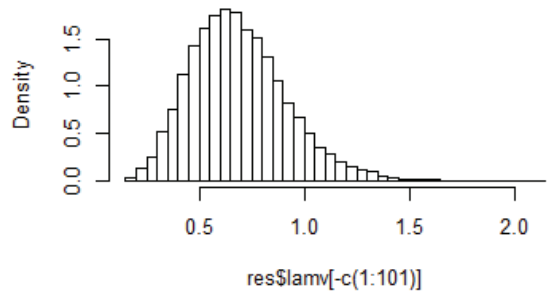


Figure 10.3 Sample ACFs

(Top two: $J=10,000$; Bottom two: $J=1,000$ )

Series res\$betv

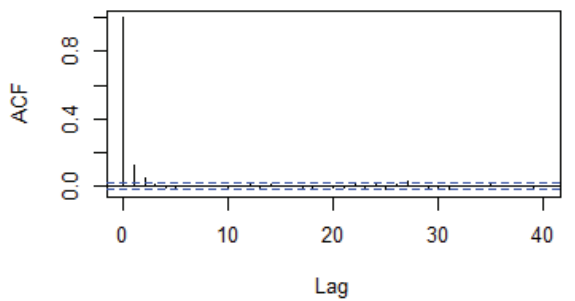

Series betvec

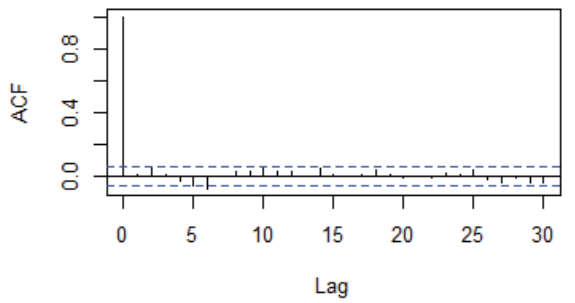

Series res\$lamv

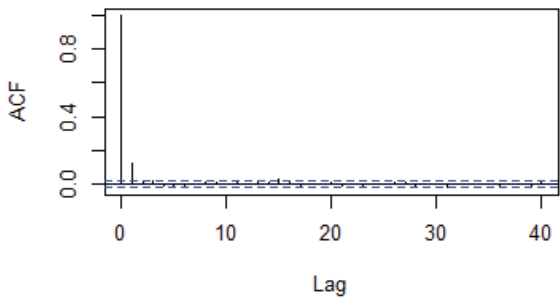

Series lamvec

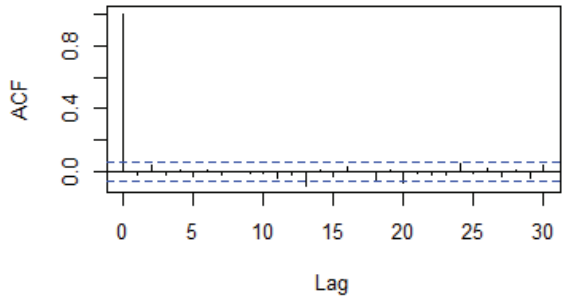

Using our sample from the joint posterior of the two parameters we now generate a sample from the predictive distribution of the nonsample mean by drawing

$$
\bar{y}_{r}^{(j)} \sim f\left(\bar{y}_{r} \mid y_{s}, \beta_{j}, \lambda_{j}\right) \sim N\left(\beta_{j}, \frac{1}{(N-n) \lambda_{j}}\right) \text { for each } j=1, \ldots, J .
$$

Note: The result is

$$
\bar{y}_{r}^{(1)}, \ldots, \bar{y}_{r}^{(J)} \sim \text { iid } f\left(\bar{y}_{r} \mid y_{s}\right),
$$

by virtue of the method of composition and the equation

$$
f\left(\bar{y}_{r}, \beta, \lambda \mid y_{s}\right)=f\left(\bar{y}_{r} \mid y_{s}, \beta, \lambda\right) f\left(\beta, \lambda \mid y_{s}\right) \text {. }
$$

We next form a random sample from the predictive distribution of the finite population mean by calculating

$$
\bar{y}^{(j)}=\frac{1}{N}\left(n \bar{y}_{s}+(N-n) \bar{y}_{r}^{(j)}\right) \text { for each } j=1, \ldots, J .
$$

Note: The result is $\bar{y}^{(1)}, \ldots, \bar{y}^{(J)} \sim$ iid $f\left(\bar{y} \mid y_{s}\right)$. 
We now estimate $\bar{y}$ (and $\bar{y}$ 's predictive mean, $\hat{\bar{y}}=E\left(\bar{y} \mid y_{s}\right)$ ) by

$$
\overline{\bar{y}}=\frac{1}{J} \sum_{j=1}^{J} \bar{y}^{(j)}=5.555,
$$

with $95 \%$ CI for $\hat{\bar{y}}$ equal to

$$
\left(\overline{\bar{y}} \pm 1.96 \sqrt{\frac{1}{J(J-1)} \sum_{j=1}^{J}\left(\overline{\bar{y}}-\bar{y}^{(j)}\right)^{2}}\right)=(5.526,5.584) .
$$

We also estimate the 95\% CPDR for $\bar{y}$ by $(4.685,6.633)$, where the bounds of this interval are the empirical 0.025 and 0.975 quantiles of $\bar{y}^{(1)}, \ldots, \bar{y}^{(J)}$.

Another approach to performing Monte Carlo inference on $\bar{y}$ is via Rao-Blackwell methods. This approach does not require the sample $\bar{y}_{r}^{(1)}, \ldots, \bar{y}_{r}^{(J)}$ and should provide more accurate Monte Carlo estimates.

The idea is based on the identities:

$$
\begin{aligned}
& \begin{aligned}
f\left(\bar{y} \mid y_{s}\right) & =\int f\left(\bar{y}, \beta, \lambda \mid y_{s}\right) d \beta d \lambda \\
& =\int f\left(\bar{y} \mid y_{s}, \beta, \lambda\right) f\left(\beta, \lambda \mid y_{s}\right) d \beta d \lambda
\end{aligned} \\
& \hat{\bar{y}}=E\left(\bar{y} \mid y_{s}\right)=E_{\beta, \lambda}\left\{E\left(\bar{y} \mid y_{s}, \beta, \lambda\right) \mid y_{s}\right\} \\
& f\left(\bar{y} \mid y_{s}\right)=E_{\beta, \lambda}\left\{f\left(\bar{y} \mid y_{s}, \beta, \lambda\right) \mid y_{s}\right\} .
\end{aligned}
$$

Now note once again that:

$$
\begin{aligned}
& \bar{y}=\frac{1}{N}\left(n \bar{y}_{s}+(N-n) \bar{y}_{r}\right) \\
& \left(\bar{y}_{r} \mid y_{s}, \beta, \lambda\right) \sim N\left(\beta, \frac{1}{(N-n) \lambda}\right) .
\end{aligned}
$$

So we now define:

$$
\begin{aligned}
e(\beta, \lambda) & =E\left(\bar{y} \mid y_{s}, \beta, \lambda\right) \\
& =\frac{1}{N}\left(n \bar{y}_{s}+(N-n) E\left(\bar{y}_{r} \mid y_{s}, \beta, \lambda\right)\right) \\
& =\frac{1}{N}\left(n \bar{y}_{s}+(N-n) \beta\right)
\end{aligned}
$$




$$
\begin{aligned}
& v(\beta, \lambda)=V\left(\bar{y} \mid y_{s}, \beta, \lambda\right) \\
& =\frac{(N-n)^{2}}{N^{2}} V\left(\bar{y}_{r} \mid y_{s}, \beta, \lambda\right) \\
& =\frac{(N-n)^{2}}{N^{2}} \times \frac{1}{(N-n) \lambda}=\frac{N-n}{N^{2} \lambda} \\
& e_{j}=e\left(\beta_{j}, \lambda_{j}\right)=\frac{1}{N}\left(n \bar{y}_{s}+(N-n) \beta_{j}\right) \\
& v_{j}=v\left(\beta_{j}, \lambda_{j}\right)=\frac{N-n}{N^{2} \lambda_{j}} .
\end{aligned}
$$

Note: Since $e(\beta, \lambda)$ does not depend on $\lambda$, we may also write $e(\beta, \lambda)$ as $e(\beta)$. Likewise, since $v(\beta, \lambda)$ does not depend on $\beta$, we may also write $v(\beta, \lambda)$ as $v(\lambda)$.

Then the Rao-Blackwell estimate of $\bar{y}$ (and $\hat{\bar{y}}=E\left(\bar{y} \mid y_{s}\right)$ ) is

$$
\bar{e}=\frac{1}{J} \sum_{j=1}^{J} e_{j}=5.557,
$$

with $95 \%$ CI for $\hat{\bar{y}}$ working out as

$$
\left(\bar{e} \pm 1.96 \sqrt{\frac{1}{J(J-1)} \sum_{j=1}^{J}\left(\bar{e}-e_{j}\right)^{2}}\right)=(5.534,5.581) .
$$

Note: The width of this Rao-Blackwell CI is $5.581-5.534=0.046$, which (as could be expected) is less than that of the earlier CI, namely $5.584-5.526=0.058$.

We can now also obtain the Rao-Blackwell estimate of the CPDR for $\bar{y}$.

First, the Rao-Blackwell estimate of the predictive density of $\bar{y}$ (that is, of $\left.f\left(\bar{y} \mid y_{s}\right)\right)$ is

$$
\begin{aligned}
\bar{f}\left(\bar{y} \mid y_{s}\right) & =\frac{1}{J} \sum_{j=1}^{J} f\left(\bar{y} \mid y_{s}, \mu_{j}, \theta_{j}\right) \\
& =\frac{1}{J} \sum_{j=1}^{J} \frac{1}{\sqrt{v_{j}} \sqrt{2 \pi}} \exp \left\{-\frac{1}{2 v_{j}}\left(\bar{y}-e_{j}\right)^{2}\right\} .
\end{aligned}
$$


Note: The simplest and most 'basic' estimate of $f\left(\bar{y} \mid y_{s}\right)$ is the 'histogram' estimate, $\hat{f}\left(\bar{y} \mid y_{s}\right)$, obtained by smoothing a histogram of the sampled values $\bar{y}^{(1)}, \ldots, \bar{y}^{(J)} \sim$ iid $f\left(\bar{y} \mid y_{s}\right)$.

The Rao-Blackwell estimate of the 95\% CPDR of $\bar{y}$ is $(L, U)$, where $L$ and $U$ satisfy:

$$
\begin{aligned}
& \int_{-\infty}^{L} \frac{1}{J} \sum_{j=1}^{J} \frac{1}{\sqrt{v_{j}} \sqrt{2 \pi}} \exp \left\{-\frac{1}{2 v_{j}}\left(\bar{y}-e_{j}\right)^{2}\right\} d \bar{y}=0.025 \\
& \int_{-\infty}^{U} \frac{1}{J} \sum_{j=1}^{J} \frac{1}{\sqrt{v_{j}} \sqrt{2 \pi}} \exp \left\{-\frac{1}{2 v_{j}}\left(\bar{y}-e_{j}\right)^{2}\right\} d \bar{y}=0.975 .
\end{aligned}
$$

To obtain $L$ we rewrite the first of these two equations as

$$
\frac{1}{J} \sum_{j=1}^{J} P\left(X_{j}<L\right)=0.025
$$

where $X_{j} \sim N\left(e_{j}, v_{j}\right)$, or equivalently as

$$
\frac{1}{J} \sum_{j=1}^{J} \Phi\left(\frac{L-e_{j}}{\sqrt{v_{j}}}\right)=0.025 \quad \text { (where } \Phi \text { is the standard normal cdf). }
$$

We can now solve this equation in a number of ways, for example by minimising the function

$$
g(L)=\left\{\frac{1}{J} \sum_{j=1}^{J} \Phi\left(\frac{L-e_{j}}{\sqrt{v_{j}}}\right)-0.025\right\}^{2}
$$

e.g. using the optim() function in R.

$$
\text { (whose minimum is } 0 \text { at the required } L \text { ), }
$$

Likewise we can obtain $U$ by using optim() to minimise

$$
h(U)=\left\{\frac{1}{J} \sum_{j=1}^{J} \Phi\left(\frac{L-e_{j}}{\sqrt{v_{j}}}\right)-0.975\right\}^{2}
$$

(whose minimum is 0 at the required $U$ ).

Note: We could also obtain $L$ and $U$ using trial and error or the Newton-Raphson algorithm. 
Implementing the above procedure we arrive at the required RaoBlackwell estimate of the central predictive region for the finite population mean: $(L, U)=(4.707,6.542)$.

Note: This is similar to the previous 'histogram' estimate of the CPDR, (4.685, 6.633).

Figure 10.4 shows a histogram of the $J=1,000$ simulated values $\bar{y}^{(1)}, \ldots, \bar{y}^{(J)} \sim$ iid $f\left(\bar{y} \mid y_{s}\right)$, together with the histogram estimate $\overline{\bar{y}}$ and the Rao-Blackwell estimate $\bar{e}$ of $\hat{\bar{y}}=E\left(\bar{y} \mid y_{s}\right)$. Also shown are the two corresponding 95\% CIs for $\hat{\bar{y}}$. The histogram is overlaid with the histogram estimate $\hat{f}\left(\bar{y} \mid y_{s}\right)$ and the Rao-Blackwell estimate $\bar{f}\left(\bar{y} \mid y_{s}\right)$ of $f\left(\bar{y} \mid y_{s}\right)$. It will be observed that the Rao-Blackwell estimate provides the smoother result.

Figure 10.4 Inferences on the finite population mean

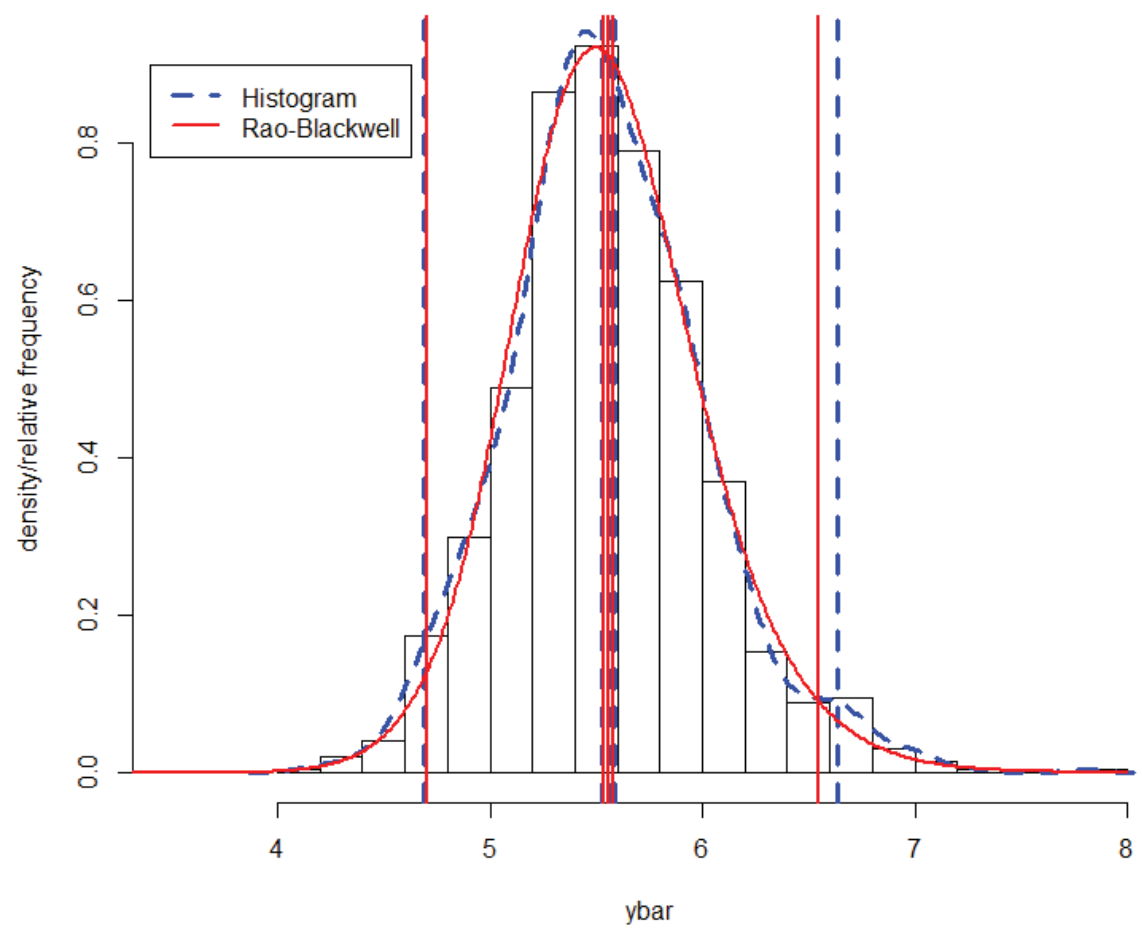




\section{R Code for Exercise 10.5}

\# (a)

options(digits=4); $\mathrm{N}=15 ; \mathrm{ys}=\mathrm{c}(5.6,2.3,8.4,5.1,4.3) ; \mathrm{n}=$ length(ys)

est=mean(ys); ss2=var(ys); varybar $=(\mathrm{ss} 2 / \mathrm{n}) *(1-\mathrm{n} / \mathrm{N}) ; \quad$ tval= qt $(0.975, \mathrm{n}-1)$

cpdr=est+c(-1,1)*tval*sqrt(varybar)

c(est,ss2,sqrt(ss2), varybar, sqrt(varybar), tval, cpdr)

\# 5.14004 .90302 .21430 .65370 .80852 .77642 .89517 .3849

\# (b)

NNGFPM $=$ function(eta $=0$, tau $=0$, alp $=0.05$, $y s=c(5.6,2.3,8.4,5.1,4.3), X=r e p(1,15), N=15, \operatorname{sigma}=\operatorname{diag}(\operatorname{rep}(1, N)) \quad)$ \{

\# This function performs inference under the normal-normal-gamma \# finite population model.

\# Inputs: eta, tau, alp, ys, $\mathrm{X}, \mathrm{N}$, sigma

\# Outputs: A list with $\$ a, \$ b$ and $\$ c$ indicating $(y b a r-a) / b$ given ys $\sim t(c)$

$p=n \operatorname{col}(\operatorname{cbind}(N A, X))-1 ; \quad n=$ length(ys); $\quad c=2 *$ eta $+n-p$

ysT=sum(ys); Xs=cbind(NA,X)[1:n,][,-1]; Xr=cbind(NA,X)[(n+1):N,][,-1]

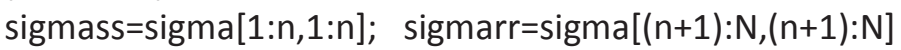

sigmasr=sigma[1:n, $(n+1): N]$; sigmars $=t($ sigmasr)

$\mathrm{D}=$ solve(t(Xs)\%*\%solve(sigmass)\%*\%(X)

beta=D\%*\%t(Xs)\%*\%solve(sigmass)\%*\%ys

$A=X r$-sigmars\%*\%solve(sigmass)\%*\%Xs; oner=rep $(1, N-n)$

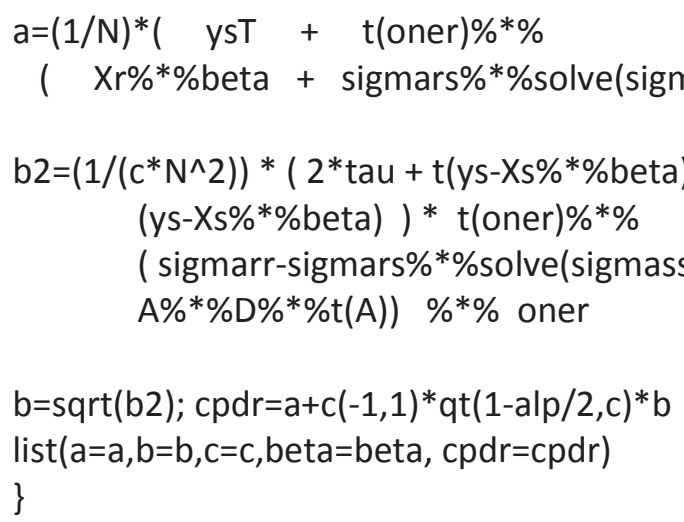


\# Test function by using it to check (a):

res= NNGFPM(); c(res\$a,res\$b,res\$c,res\$beta, res\$cpdr)

\# 5.1400 0.8085 4.0000 5.1400 2.8951 7.3849 Same as in (a) OK

\# Apply function with covariate info:

$x v e c=c(9.3,4.6,15.0,11.2,7.8, \quad 2.4,6.6,3.0,2.1,7.3, \quad 5.5,8.0,2.4,4.2$, 5.5)

res $=$ NNGFPM $(X=x v e c$, sigma $=\operatorname{diag}(x v e c))$

$c($ res $\$ a$, res $\$ b$, res $\$ c$, res $\$$ beta, res $\$ c p d r)$

\# 3.39450 .11594 .00000 .53653 .07253 .7164

c(mean(xvec), mean(xvec[1:5]), mean(xvec[6:15]) ) \# 6.3279 .5804 .700

$\#(c)$

$y s=c(5 \cdot 6,2 \cdot 3,8.4,5 \cdot 1,4.3) ; y s b a r=m e a n(y s) ; n=5 ; N=15 ;$ options(digits=4)

GIBBS = function $(J=1000$,ys $=c(5 \cdot 6,2 \cdot 3,8 \cdot 4,5 \cdot 1,4.3)$, bet $=1$, lam $=1$, bet $0=10$, sig $0=1.5$, eta $=8, \operatorname{tau}=4$ )

\{

betv=bet; lamv=lam; sig02=sig0^2; $\mathrm{n}=$ length(ys); ysbar=mean(ys);

for(j in $1: J)\{$

$\mathrm{klam}=\mathrm{n} /(\mathrm{n}+1 /(\operatorname{lam} * \operatorname{sig} 02)) ; \quad$ sig2lam=klam/(n*lam)

betlam $=(1-$ klam $) *$ bet0+klam*ysbar;

bet=rnorm (1,betlam,sqrt(sig2lam))

s2 bet=mean ((ys-bet)^2); lam=rgamma(1,eta $+n / 2$, tau $+n * s 2$ bet/2)

betv $=c($ betv, bet $)$; lamv $=c(l a m v, l a m) \quad\}$

list(betv=betv,lamv=lamv)

\}

set.seed(641); res=GIBBS(J=10100); X11(w=8,h=5.5); $\operatorname{par}(\operatorname{mfrow}=c(2,2))$

plot(res\$betv,type="l"); plot(res\$lamv,type="l")

hist(res\$betv[-c(1:101)], prob=T,nclass=30);

hist(res\$lamv[-c(1:101)],prob=T,nclass=30) \# Fig. 10.2

betvec=res\$betv[-c(1:101)][seq(10,10000,10)]; J = length(betvec); J \# 1000 lamvec=res\$lamv[-c(1:101)][seq(10,10000,10)]

acf(res\$betv); acf(res\$lamv); acf(betvec); acf(lamvec) \# Fig. 10.3

betbar=mean(betvec); betci=betbar+c(-1,1)*qnorm $(0.975) *$ sd(betvec)/sqrt(J)

c(betbar,betci) \# 5.7665 .7315 .801 
set.seed(121); yrbarvec=rnorm(J, betvec, 1/sqrt((N-n)*(lamvec)) )

yrbarbar=mean(yrbarvec);

yrbarci $=$ yrbarbar+c(-1,1)*qnorm $(0.975) *$ sd(yrbarvec)/sqrt(J)

yrbarcpdr=quantile(yrbarvec, $c(0.025,0.975))$

c(yrbarbar,yrbarci,yrbarcpdr) \# 5.762 5.718 5.806 4.458 7.380

ybarvec $=(1 / N) *(n * y s b a r+(N-n) * y r b a r v e c)$

ybarbar=mean(ybarvec);

ybarci $=$ ybarbar $+c(-1,1) *$ qnorm $(0.975) *$ sd $($ ybarvec $) / \operatorname{sqrt}(\mathrm{J})$

ybarcpdr=quantile(ybarvec, $c(0.025,0.975))$

c(ybarbar,ybarci,ybarcpdr) \# 5.5555 .5265 .5844 .6856 .633

ybarci[2]-ybarci[1] \# 0.05849

evec $=(1 / N) *(n * y s b a r+(N-n) *$ betvec $) ; \quad$ vvec $=(N-n) /\left(N^{\wedge} 2 *\right.$ lamvec $)$

ebar=mean(evec); eci=ebar+c(-1,1)*qnorm(0.975)*sd(evec)/sqrt(J)

Lfun=function $(L)\left\{(0.025-\text { mean(pnorm( (L-evec)/sqrt(vvec) ) ) })^{\wedge} 2\right\}$

$\mathrm{L}=\operatorname{optim}($ par=3,fn=Lfun)\$par; L \# 4.707 (ignore warning message)

mean( pnorm( (L-evec)/sqrt(vvec) )) \#0.025 OK

Ufun=function(U)\{(0.975-mean(pnorm( (U-evec)/sqrt(vvec) ) ) $\left.)^{\wedge} 2\right\}$

$U=$ optim(par=7,fn=Ufun)\$par; U \# 6.542 (ignore warning message)

mean( pnorm( (U-evec)/sqrt(vvec) )) \#0.975 OK

ecpdr=c(L,U); c(ebar,eci,ecpdr) \# 5.557 5.534 5.581 4.707 6.542

eci[2]-eci[1] \# 0.04642

$\mathrm{X} 11(\mathrm{w}=8, \mathrm{~h}=7) ; \operatorname{par}(\operatorname{mfrow}=\mathrm{c}(1,1))$

hist(ybarvec, prob=T,nclass=20, $\operatorname{xim}=c(3.5,8)$,

xlab="ybar",ylab="density/relative frequency",main="")

lines(density(ybarvec), Ity=2,lwd=3,col="blue")

abline(v=c(ybarbar,ybarci,ybarcpdr), Ity=2,lwd=3,col="blue")

ybarv=seq(3,8,0.01); fv=rep(NA,length(ybarv))

for(i in 1:length(ybarv)) fv[i] = mean(dnorm(ybarv[i], evec, sqrt(vvec)))

lines(ybarv, fv, Ity=1, Iwd=2,col="red")

abline(v=c(ebar,eci,ecpdr), Ity=1,lwd=2,col="red")

legend(3.4,0.9,c("Histogram","Rao-Blackwell"),

Ity=c(2,1), Iwd=c(3,2), col=c("blue","red"), bg="white") 


\section{Transformations and Other Topics}

\section{I.I Inference on complicated quantities}

So far, in the context of Bayesian finite population models specified by:

$f(\xi \mid y, \theta)$ where $\xi$ is $s$ or $I$ or $L$ (as discussed earlier) $f(y \mid \theta)$ where

$y=\left(y_{s}, y_{r}\right)=\left(\left(y_{1}, \ldots, y_{n}\right),\left(y_{n+1}, \ldots, y_{N}\right)\right)=\left(y_{1}, \ldots, y_{N}\right)$

$f(\theta)$ where $\theta=\left(\theta_{1}, \ldots, \theta_{q}\right)$,

we have been focusing primarily on two finite population quantities, the finite population total $y_{T}=y_{1}+\ldots+y_{N}$ and the finite population mean $\bar{y}=\left(y_{1}+\ldots+y_{N}\right) / N=y_{T} / N$.

These are special cases of the class of linear combinations of the $N$ population values

$$
\tilde{y}=c_{0}+c y_{1}+\ldots+c_{N} y_{N},
$$

for which inference is often straightforward, such as in the context of the general normal-normal-gamma finite population model.

We will now consider other inferential targets.

Generally, suppose we are interested in the quantity $\psi=g(\theta, y)$, where $g$ is a potentially very complicated function of all $q$ model parameters and all $N$ finite population values. In such cases, we may adopt the following four-step strategy.

Step 1. Obtain a sample from the posterior distribution of $\theta=\left(\theta_{1}, \ldots, \theta_{q}\right)$, that is $\theta^{(1)}, \ldots, \theta^{(J)} \sim$ iid $f(\theta \mid D)$, where $\theta^{(j)}=\left(\theta_{1}^{(j)}, \ldots, \theta_{q}^{(j)}\right)$ and where $D$ is the data, typically defined as $\left(s, y_{s}\right)$ or $(I, s)$ or $\left(L, y_{s}\right)$ as discussed previously, and whichever the case may be.

Make use of special techniques if suitable, e.g. the method of composition and MCMC methods like the Gibbs sampler. 
Step 2. Use the sample in Step 1 to generate a random sample from the predictive distribution of the nonsample vector $y_{r}=\left(y_{n+1}, \ldots, y_{N}\right)$, that is $y_{r}^{(1)}, \ldots, y_{r}^{(J)} \sim$ iid $f\left(y_{r} \mid D\right)$, where $y_{r}^{(j)}=\left(y_{n+1}^{(j)}, \ldots, y_{N}^{(j)}\right)$.

Make use of special techniques if required.

Often, the sample can be obtained easily via the method of composition and the identity

$$
f\left(y_{r}, \theta \mid D\right)=f\left(y_{r} \mid D, \theta\right) f(\theta \mid D),
$$

namely by sampling

$$
y_{r}^{(j)} \sim f\left(y_{r} \mid D, \theta^{(j)}\right)
$$

for each $j=1, \ldots, J$.

In many cases, each sampled nonsample vector $y_{r}^{(j)}$ here can obtained by sampling

$$
y_{i}^{(j)} \sim \perp f\left(y_{i} \mid D, \theta^{(j)}\right), \quad i=n+1, \ldots, N,
$$

and then forming the vector according to

$$
y_{r}^{(j)}=\left(y_{n+1}^{(j)}, \ldots, y_{N}^{(j)}\right) .
$$

Step 3. Form the completed population vector

$$
y^{(j)}=\left(y_{s}, y_{r}^{(j)}\right)=\left(y_{1}, \ldots, y_{n}, y_{n+1}^{(j)}, \ldots, y_{N}^{(j)}\right)
$$

and then calculate

$$
\psi^{(j)}=g\left(y^{(j)}, \theta^{(j)}\right)
$$

for each $j=1, \ldots, J$.

The result will be a sample from the posterior/predictive distribution of $\psi$, namely

$$
\psi^{(1)}, \ldots, \psi^{(J)} \sim \text { iid } f(\psi \mid D) .
$$

Step 4. Use the sample obtained in Step 3 to perform Monte Carlo inference on $\psi$ in the usual way. Thus, estimate the posterior/predictive mean of $\psi$, namely

$$
\hat{\psi}=E(\psi \mid D)=\int \psi f(\psi \mid D) d \psi
$$

(which may be impossible to obtain analytically), by the Monte Carlo sample mean $\bar{\psi}=\frac{1}{J} \sum_{j=1}^{J} \psi^{(j)}$ (which is unbiased, in that $E(\bar{\psi} \mid D)=\hat{\psi}$ ). 
Also calculate the $1-\alpha$ CI for $\hat{\psi}$ given by

$$
\left(\bar{\psi} \pm z_{\alpha / 2} \frac{s_{\psi}}{\sqrt{J}}\right) \text {, where } s_{\psi}^{2}=\frac{1}{J-1} \sum_{j=1}^{J}\left(\psi^{(j)}-\bar{\psi}\right)^{2} \text {. }
$$

Also, estimate the $1-\alpha$ central posterior/predictive density region (CPDR generally) for $\psi$ by $\left(Q_{\alpha / 2}, Q_{1-\alpha / 2}\right)$, where $Q_{p}$ is the empirical $p$ quantile of the sample $\psi^{(1)}, \ldots, \psi^{(J)}$.

Also, estimate the entire posterior/predictive density of $\psi$, namely $f(\psi \mid D)$, by $\hat{f}(\psi \mid D)$, a smooth of a histogram of $\psi^{(1)}, \ldots, \psi^{(J)}$ (obtained by adjusting the smooth parameters).

Use Rao-Blackwell methods to improve precision, if possible and practicable. For example, suppose that $q=2, \theta=\left(\theta_{1}, \theta_{2}\right), \psi=g\left(y, \theta_{2}\right)$, and $f\left(\psi \mid D, \theta_{1}\right)$ has a simple form. Then, instead of using a 'histogram estimate' $\hat{f}(\psi \mid D)$ to estimate $f(\psi \mid D)$, use the Rao-Blackwell estimate $\bar{f}(\psi \mid D)=\frac{1}{J} \sum_{j=1}^{J} f\left(\psi \mid D, \theta^{(j)}\right)$.

\section{Exercise I I.I Estimation of nonstandard target quantities}

(a) Suppose that 2.1, 5.2, 3.0, 7.7 and 9.3 constitute a random sample from a normal finite population of size 20 whose mean and variance are unknown. We are interested in the finite population median. Estimate this quantity using a suitable Bayesian model.

(b) Repeat (a) but for the quantity:

average percentage increase between subsequent ordered population values greater than 4 .

(c) Repeat (a) but for the quantity:

sum of finite population values in the upper quartile of the normal superpopulation. 


\section{Solution to Exercise I I.I}

The Bayesian model here is:

$$
\begin{aligned}
& f(s \mid y, \mu, \lambda)=\left(\begin{array}{c}
N \\
n
\end{array}\right)^{-1}, \\
& \quad s=(1, \ldots, n),(1, \ldots, n-1, n+1), \ldots,(N-n+1, \ldots, N) \text { (SRSWOR) } \\
& \left(y_{1}, \ldots, y_{N} \mid \mu, \lambda\right) \sim \text { iid } N(\mu, 1 / \lambda) \\
& f(\mu, \lambda) \propto 1 / \lambda, \mu \in \mathfrak{R}, \lambda>0,
\end{aligned}
$$

where $N=20, n=5$, and where the data is

$$
D=\left(s, y_{s}\right)=((1, \ldots, n),(2.1,5.2,3.0,7.7,9.3)) \text {. }
$$

Note 1: This data is presented according to a convenient reordering of population labels, after sampling, so that the sampled values are listed at the beginning of the finite population vector (as discussed earlier).

Note 2: The superpopulation parameter in the model may be thought of as the vector

$$
\theta=\left(\theta_{1}, \theta_{2}\right)=(\mu, \lambda)
$$

in which case the model could also be written:

$$
\begin{aligned}
& (s \mid y, \theta) \sim \operatorname{SRSWOR}(N, n) \\
& (y \mid \theta) \sim N_{N}\left(\theta_{1} 1_{N}, I_{N} / \theta_{2}\right) \\
& f(\theta) \propto 1 / \theta_{2}, \theta_{1} \in \Re, \theta_{2}>0 .
\end{aligned}
$$

For the purposes of this exercise, let $y_{(i)}$ denote the ith finite population order statistic, meaning the $i$ th value amongst $y_{1}, \ldots, y_{N}$ after these are ordered from smallest to largest. We are interested in three finite population quantities, as follows:
(a) $\psi_{1}=g_{1}(y, \theta)=g_{1}(y)=\frac{y_{(N / 2)}+y_{(N / 2)+1}}{2}$
(b) $\psi_{2}=g_{2}(y, \theta)=g_{2}(y)=100 \frac{\sum_{i=2}^{N}\left(\frac{y_{(i)}-y_{(i-1)}}{y_{(i-1)}}\right) I\left(y_{(i-1)}>4\right)}{\sum_{i=2}^{N} I\left(y_{(i-1)}>4\right)}$
(c) $\psi_{3}=g_{3}(y, \theta)=\sum_{i=1}^{N} y_{i} I\left(y_{i}>\mu+\frac{1}{\sqrt{\lambda}} \Phi^{-1}(0.75)\right)$. 
Note 1: The median $\psi_{1}$ is the average of the middle two values, since $N=20$ is even.

Note 2: In general, $\psi_{2}$ is defined only if at least two of the finite population values are greater than 4 . For our data, there is no problem with the definition because the observed sample already contains three such values. If there were a problem, then $\psi_{2}=g_{2}(y)$ could be defined as zero (say) in the case where the number of population values is only 0 or 1 , i.e. if $\sum_{i=1}^{N} I\left(y_{i}>4\right)<2$.

Note 3: As regards $\psi_{3}$, if $c$ is the upper quartile of the normal superpopulation then

$$
\begin{aligned}
0.75= & P\left(y_{i}<c \mid \theta\right)=P\left(\frac{y_{i}-\mu}{\sigma}<\frac{c-\mu}{\sigma} \mid \theta\right) \\
& \Rightarrow \frac{c-\mu}{\sigma}=\Phi^{-1}(0.75) \\
& \Rightarrow c=\mu+\sigma \Phi^{-1}(0.75)=\mu+\frac{1}{\sqrt{\lambda}} \Phi^{-1}(0.75) .
\end{aligned}
$$

In each case, the inferential target has a posterior/predictive distribution which cannot be obtained analytically. One way to proceed is as follows:

Step 1. Generate $\lambda_{1}, \ldots, \lambda_{J} \sim$ iid $f(\lambda \mid D) \sim G\left(\frac{n-1}{2}, \frac{n-1}{2} s_{s}^{2}\right)$, where $s_{s}^{2}=\frac{1}{n} \sum_{i=1}^{n}\left(y_{i}-\bar{y}\right)^{2}$.

(This step derives from results for the normal-normal-gamma model.)

Step 2. Generate $\mu_{j} \sim f\left(\mu \mid D, \lambda_{j}\right) \sim N\left(\bar{y}_{s}, \frac{1}{n \lambda_{j}}\right)$ for each $j=1, \ldots, J$.

(This step derives from results for the normal-normal model). 
Step 3. For each $j=1, \ldots, J$ :

- Generate $y_{n+1}^{(j)}, \ldots, y_{n+1}^{(j)} \sim$ iid $f\left(y_{i} \mid D, \mu_{j}, \lambda_{j}\right) \sim N\left(\mu_{j}, \frac{1}{\lambda_{j}}\right)$

- Form $y_{r}^{(j)}=\left(y_{n+1}^{(j)}, \ldots, y_{N}^{(j)}\right)$ and $y^{(j)}=\left(y_{s}, y_{r}^{(j)}\right)=\left(y_{1}, \ldots, y_{n}, y_{n+1}^{(j)}, \ldots, y_{N}^{(j)}\right)$

- Calculate $\psi^{(j)}=g\left(y^{(j)}, \theta^{(j)}\right)$, where $\theta^{(j)}=\left(\mu_{j}, \lambda_{j}\right)$.

Step 4. Use the values $\psi^{(1)}, \ldots, \psi^{(J)} \sim$ iid $f(\psi \mid D)$ for Monte Carlo inference on $\psi$ in the usual way.

Note 1: Steps 1 and 2 result in the sample

$$
\left(\mu_{1}, \lambda_{1}\right), \ldots,\left(\mu_{J}, \lambda_{J}\right) \sim \text { iid } f(\mu, \lambda \mid D) \text {. }
$$

Note 2: In the above, Steps 1 and 2 could be replaced as follows:

Step 1'. Generate $\mu_{1}, \ldots, \mu_{J} \sim f(\mu \mid D)$ for each $j=1, \ldots, J$. Do this by first sampling $w_{1}, \ldots, w_{J} \sim$ iid $t(n-1)$ and then forming

$$
\mu_{j}=\bar{y}_{s}+w_{j} s_{s} / \sqrt{n} \text { for each } j=1, \ldots, J
$$

(using results from the normal-normal-gamma model).

Step 2'. Generate $\lambda_{j} \sim \perp f\left(\lambda \mid D, \mu_{j}\right) \sim G\left(\frac{n}{2}, \frac{n}{2} s_{\mu_{j}}^{2}\right)$, where

$$
s_{\mu_{j}}^{2}=\frac{1}{n} \sum_{i=1}^{n}\left(y_{i}-\mu_{j}\right)^{2}
$$

(using results from the normal-gamma model).

These modified steps will also result in the sample

$$
\left(\mu_{1}, \lambda_{1}\right), \ldots,\left(\mu_{J}, \lambda_{J}\right) \sim \text { iid } f(\mu, \lambda \mid D) \text {. }
$$

Applying the above four-step procedure (using the original Steps 1 and 2) with Monte Carlo sample size $J=1,000$, we obtain Table 11.1 which shows numerical estimates for the three quantities of interest: $\psi=\psi_{1}, \psi_{2}$ and $\psi_{3}$, respectively. Figure 11.1 shows histograms which illustrate these inferences. 
Table 11.1 and Figure 11.1 also contain analogous results for a fourth quantity of interest which may be defined as

$$
\begin{gathered}
\psi_{4}=g_{4}(y, \theta)=\left(\psi_{3} \mid \psi_{3} \neq 0\right) \\
=\left\{\left[\sum_{i=1}^{N} y_{i} I\left(y_{i}>\mu+\frac{1}{\sqrt{\lambda}} \Phi^{-1}(0.75)\right)\right] \mid\left[\sum_{i=1}^{N} I\left(y_{i}>\mu+\frac{1}{\sqrt{\lambda}} \Phi^{-1}(0.75)\right)\right]>0\right\} .
\end{gathered}
$$

The relevant posterior/predictive density may also be written

$$
f\left(\psi_{4} \mid D\right)=f\left(\psi_{3} \mid D, \psi_{3} \neq 0\right) .
$$

Inferences on $\psi_{4}$ were obtained using the 960 values of $\psi_{3}$ which were non-zero. It was meaningful to perform this additional inference because there were 40 simulations amongst the 1,000 for which the upper quartile of the normal distribution lay above the largest finite population value, resulting in the sum $\psi_{3}$ being equal to 0 exactly.

Note 1: From the above, we see that $\psi_{3}$ is neither a discrete nor a continuous random variable but one with a mixed distribution.

The discrete part of this mixed distribution is the probability that $\psi_{3}=0$ exactly, and this we estimated via MC as 40/1,000 $=0.04$.

Note 2: We also see that neither $\psi_{3}$ nor $\psi_{4}$ is necessarily positive.

This is because it might be the case that the upper quartile of the normal distribution is negative and many of the finite population values happen (by a very small chance) to lie between that negative quartile and zero. 


\section{Table I I.I Point and interval estimates for four quantities}

Quantity of interest:

$\psi_{1}$

Posterior mean estimate:

5.842

9.975

58.31

60.74

95\% CI for posterior mean:
(5.790, 5.893)
$(9.775,10.175)$
(56.48 60.15) (58.99, 62.49)

Posterior mode estimate:
5.528
8.150
62.29
62.45

Posterior median estimate:
5.769
9.377
59.48
60.59

95\% CPDR estimate:
$(4.308,7.528)$
$(5.522,17.770)$
$(0.00114 .87) \quad(11.72,114.96)$

Figure I I.I Four histograms and sets of inferences
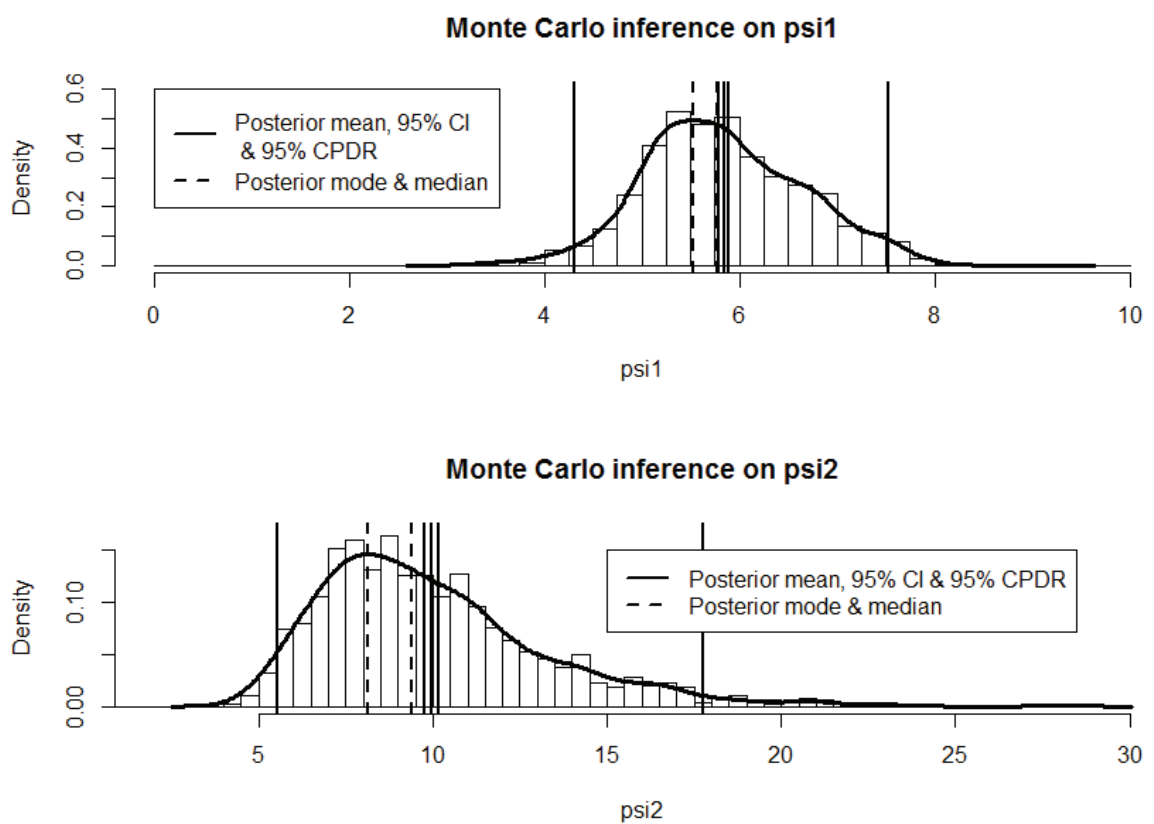


\section{Monte Carlo inference on psi3}

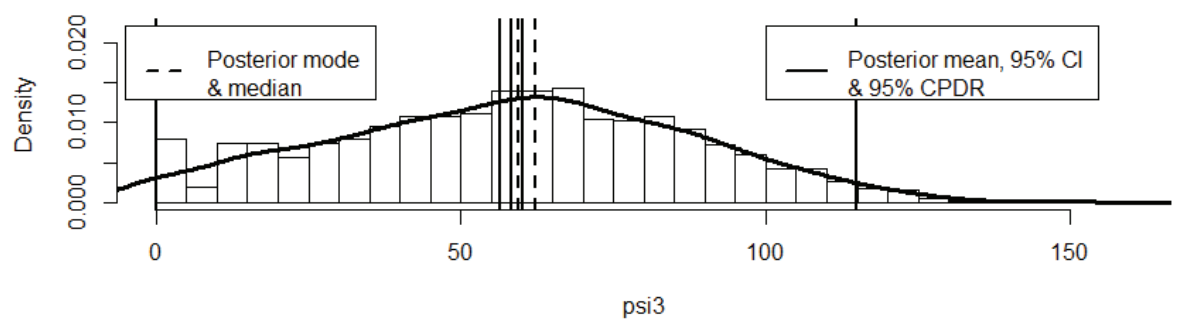

Monte Carlo inference on psi4 $=(p s i 3$ given psi3 $!=0)$

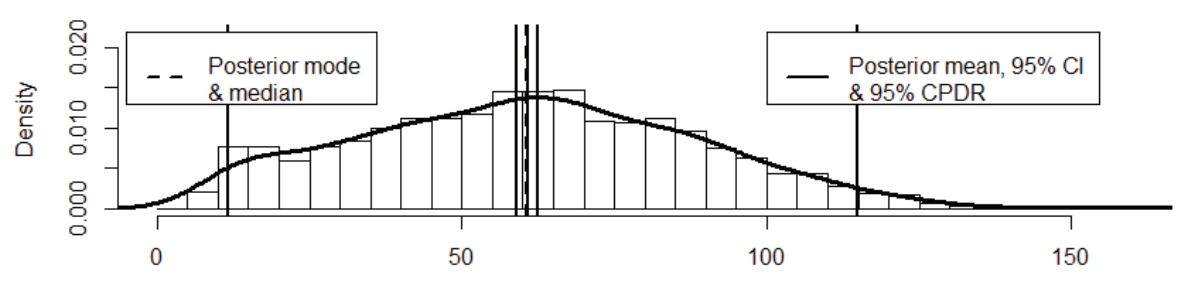

psi3, psi4

\section{R Code for Exercise I I.I}

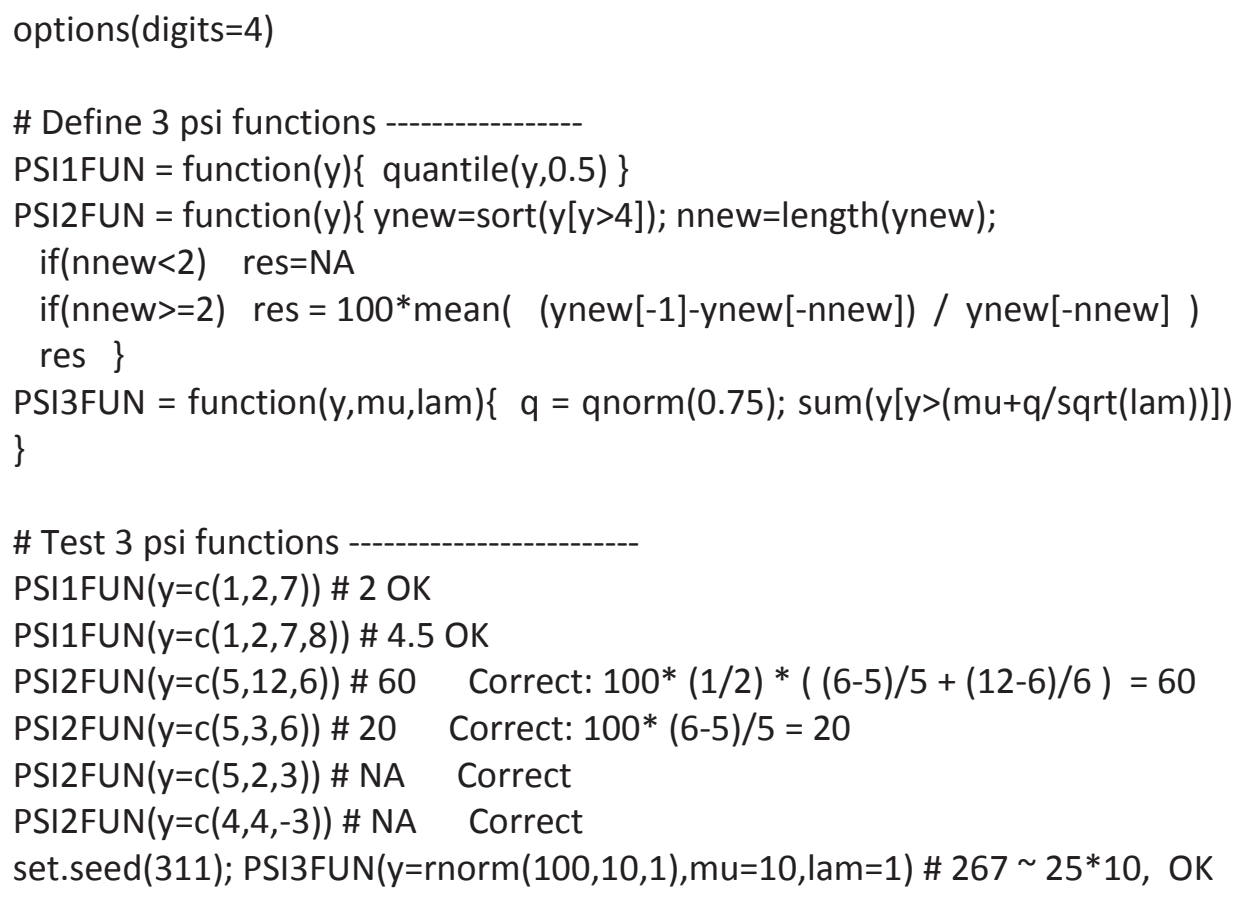


\# Perform inference on 3 psi functions

$y s=c(2.1,5.2,3.0,7.7,9.3) ; y s b a r=m e a n(y s) ; n=l e n g t h(y s) ; ~ s s 2=v a r(y s) ; N=20$

options(digits=4); J=1000; set.seed(232)

lamvec=rgamma( J, (n-1)/2, ((n-1)/2)*ss2)

muvec $=\operatorname{rnorm}(\mathrm{J}, \mathrm{ysbar}, 1 / \mathrm{sqrt}(\mathrm{n} *$ lamvec $))$

yrmat=matrix (NA, nrow=J, ncol=N-n)

for(j in 1:J) yrmat[j,] = rnorm(N-n, muvec,1/sqrt(lamvec))

psi1vec=rep(NA,J); psi2vec=rep(NA,J); psi3vec=rep(NA,J)

for $(j$ in 1:J)\{ yrj = yrmat[j,]

$\operatorname{psi1vec}[j]=\operatorname{PSI} 1 F U N(y=c(y s, y r j))$

psi2vec $[\mathrm{j}]=\operatorname{PSI} 2 \mathrm{FUN}(\mathrm{y}=\mathrm{c}(\mathrm{ys}, \mathrm{yrj}))$

$\operatorname{psi3vec}[j]=\operatorname{PSI} 3 F U N(y=c(y s, y r j), \operatorname{mu}=\operatorname{muvec}[j]$, lam=lamvec[j]) $\}$

cbind( summary(psi1vec), summary(psi2vec),

summary(psi3vec), summary(psi3vec[psi3vec!=0]) )

$\begin{array}{llll}\text { \# Min. } 3.14 & 4.44 & 0.0 & 9.3\end{array}$

\# 1st Qu. 5.287 .6537 .940 .3

\# Median 5.779 .3859 .560 .6

\# Mean 5.849 .9758 .360 .7

\# 3rd Qu. 6.4111 .5079 .680 .7

\# Max. 9.0928 .10156 .0156 .0

$\mathrm{X} 11(\mathrm{w}=9, \mathrm{~h}=6.5) ; \operatorname{par}(\operatorname{mfrow}=\mathrm{c}(2,1))$

psivec=psi1vec; $\mathrm{J}=$ length(psivec)

psibar=mean (psivec); psici=psibar+c(-1,1)*qnorm $(0.975) *$ sd $($ psivec $) /$ sqrt $(J)$

$\mathrm{fpsi}=\operatorname{density}(p s i v e c) ; p \operatorname{pimode}=f p s i \$ x[f p s i \$ y==\max (f p s i \$ y)]$

psimedian=quantile $(p \operatorname{sivec}, 0.5) ; p \operatorname{psicpdr}=q u a n t i l e(p \operatorname{sivec}, c(0.025,0.975))$

c(psibar,psici,psimode,psimedian,psicpdr)

\# 5.8425 .7905 .8935 .5285 .7694 .3087 .528

hist(psivec, prob=T, xlab="psi1",xlim=c(0,10),ylim=c(0,0.6),

breaks=seq(0,10,0.25), main="Monte Carlo inference on psi1")

lines(fpsi,lwd=3)

abline $(v=c($ psibar, psici, psicpdr, psimedian, psimode) ,

Ity $=c(1,1,1,1,1,2,2), \operatorname{lwd}=\operatorname{rep}(2,7))$

legend(0,0.6,

c("Posterior mean, 95\% Cl \n \& 95\% CPDR", "Posterior mode \& median"), Ity $=c(1,2), \operatorname{lwd}=c(2,2), \mathrm{bg}="$ white")

psivec=psi2vec; $\mathrm{J}=$ length $(\mathrm{psivec})$

psibar=mean(psivec); psici=psibar+c(-1,1)*qnorm(0.975)*sd(psivec)/sqrt(J)

$\mathrm{fpsi}=\operatorname{density}(p s i v e c) ; p \operatorname{pimode}=f p s i \$ x[f p s i \$ y==\max (f p s i \$ y)]$

psimedian=quantile(psivec,0.5); psicpdr=quantile $(p \operatorname{pivec}, c(0.025,0.975))$

c(psibar,psici,psimode,psimedian,psicpdr)

\# 9.9759 .77510 .1758 .1509 .3775 .52217 .770 
hist(psivec, prob=T, xlab="psi2",xlim=c(2,30),ylim=c(0,0.17),

breaks=seq(0,30,0.5), main="Monte Carlo inference on psi2")

lines(fpsi,lwd=3)

abline $(v=c(p s i b a r, p s i c i, p s i c p d r$, psimedian, psimode $)$,

Ity $=c(1,1,1,1,1,2,2)$, Iwd=rep(2,7))

legend(15,0.15,

c("Posterior mean, 95\% Cl \& 95\% CPDR","Posterior mode \& median"), Ity=c(1,2), Iwd=c(2,2), bg="white") \# End of first 2 graphs

psivec=psi3vec \# Start of next 2 graphs

psibar=mean(psivec); psici=psibar+c(-1,1)*qnorm $(0.975) *$ sd(psivec)/sqrt(J)

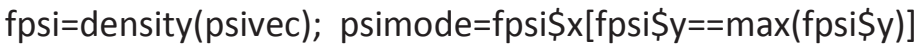

psimedian=quantile(psivec,0.5); psicpdr=quantile $(p \operatorname{pivec}, c(0.025,0.975))$

c(psibar,psici,psimode,psimedian,psicpdr)

\# $58.3156 .48 \quad 60.15 \quad 62.2959 .48 \quad 0.00114 .87$

hist(psivec, prob=T, xlab="psi3",xlim=c(0,160),ylim=c(0,0.022),

breaks=seq(0,200,5), main="Monte Carlo inference on psi3")

lines(fpsi, $I w d=3$ )

abline $(v=c(p s i b a r, p s i c i, p s i c p d r$, psimedian, psimode) ,

Ity $=c(1,1,1,1,1,2,2)$, Iwd=rep(2,7))

legend(100,0.022,

c("Posterior mean, 95\% Cl \n\& 95\% CPDR"), Ity=1,lwd=2,bg="white") legend(-5,0.022,c("Posterior mode \n\& median"), Ity=2, Iwd=2, bg="white")

length(psi3vec[psi3vec!=0]) \# 960

length (psi3vec[psi3vec==0]) \# $40 \quad 40 / 1000=4 \%$

psivec=psi3vec[psi3vec!=0]; J=length(psivec); J \# 960 Condition on psi >0

psibar=mean(psivec); psici=psibar+c(-1,1)*qnorm $(0.975) *$ sd(psivec)/sqrt(J)

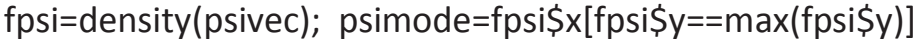

psimedian=quantile(psivec,0.5); psicpdr=quantile $(p \operatorname{pivec}, c(0.025,0.975))$

c(psibar,psici,psimode,psimedian,psicpdr)

\# 60.7458 .9962 .4962 .4560 .5911 .72114 .96

hist(psivec, prob=T, xlab="psi3, psi4",xlim=c(0,160),ylim=c(0,0.022),

breaks=seq $(0,200,5)$,

main="Monte Carlo inference on psi4 = (psi3 given psi3 != 0)")

lines(fpsi,lwd=3)

abline $(v=c(p s i b a r, p s i c i, p s i c p d r, p s i m e d i a n, p s i m o d e)$,

Ity $=c(1,1,1,1,1,2,2)$, Iwd=rep(2,7))

legend(100,0.022,

c("Posterior mean, 95\% Cl \n\& 95\% CPDR"), Ity=1,Iwd=2,bg="white") legend(-5,0.022,c("Posterior mode \n\& median"), Ity=2, Iwd=2, bg="white") 


\section{I.2 Data transformations}

In statistical analysis, a common practice is to first transform the data before applying a model. For example, if the data values are strictly positive and highly right skewed, it may be worthwhile taking natural logarithms before applying a normal model.

In the classical setting, e.g. in the design-based survey sampling, this idea may work well for purposes of analytical inference (i.e. estimation of model parameters) but can be problematic for prediction. This is because the quantity requiring prediction (e.g. the nonsample total) does not typically have a simple distribution on the untransformed scale. Although prediction can be performed easily on the transformed scale there is no way to translate results back onto the original scale. By contrast, this issue does not create any special problems within the Bayesian framework.

Suppose that we are interested in some finite population quantity which is denoted $\psi=g(y)$, e.g. $\bar{y}=1_{N}^{\prime} y / N$.

Also suppose that there is no convenient superpopulation model for the finite population values $y_{i}, i=1, \ldots, N$, but there does exist such a model for some function of those values, say $z_{i}=h\left(y_{i}\right)$ for a function $h$.

In that case we may consider a Bayesian model specified in terms of:

$f(\xi \mid z, \theta)$ where $\xi$ is $s$ or $I$ or $L$ (as discussed earlier)

$f(z \mid \theta)$ where $z=\left(z_{s}, z_{r}\right)=\left(\left(z_{1}, \ldots, z_{n}\right),\left(z_{n+1}, \ldots, z_{N}\right)\right)=\left(z_{1}, \ldots, z_{N}\right)$

$f(\theta)$ where $\theta=\left(\theta_{1}, \ldots, \theta_{q}\right)$.

We now use Monte Carlo methods (perhaps MCMC methods if needed) to generate a random sample from the predictive distribution of the nonsample vector for the $z$ variable (i.e. $z_{r}$ ), given the data $D$ (for example $\left(s, y_{s}\right),(I, s)$ or $\left.\left(L, y_{s}\right)\right)$. Let us call this sample

$$
z_{r}^{(1)}, \ldots, z_{r}^{(J)} \sim \text { iid } f\left(z_{r} \mid D\right) .
$$

We next calculatate $y_{i}^{(j)}=h^{-1}\left(z_{i}^{(j)}\right)$ for each $i=n+1, \ldots, N$ and each $j=1, \ldots, J$. Thus, we untransform the simulated individual data values back to the original scale. 
Next, we form the vectors

$$
y_{r}^{(j)}=\left(y_{n+1}^{(j)}, \ldots, y_{N}^{(j)}\right)
$$

and

$$
y^{(j)}=\left(y_{s}, y_{r}^{(j)}\right)
$$

for each $j=1, \ldots, J$.

This results in the samples

$$
y_{r}^{(1)}, \ldots, y_{r}^{(J)} \sim \text { iid } f\left(y_{r} \mid D\right)
$$

and

$$
y^{(1)}, \ldots, y^{(J)} \sim \text { iid } f(y \mid D) .
$$

Finally, we calculate

$$
\psi^{(j)}=g\left(y^{(j)}\right)
$$

for each $j=1, \ldots, J$.

This results in

$$
\psi^{(1)}, \ldots, \psi^{(J)} \sim \text { iid } f(\psi \mid D),
$$

namely a sample from the predictive distribution of the finite population quantity of interest, on the original scale required for that quantity. This sample can then be used for Monte Carlo inference on $\psi$ in the usual way.

Note: We may think of this topic as an example and special application of the last topic, that is, Bayesian inference on complicated functions of the finite population vector.

\section{Exercise I I.2 Finite population inference using data transformation}

Consider the following random sample of size 50 from a finite population of size 200:

$28.374,69.857,22.721,57.593,126.965, \quad 17.816,16.078,0.803,3.164,3.544$, 2.123, 2.353, 184.539, 59.856, 63.701, 585.684, 29.094, 79.245, 18.105, 1.623, $5.513,1.629,63.654,22.060,187.463, \quad 5.051,34.299,27.475,0.746,34.016$, $8.547,1.081,3.151,55.569,2.593$, $522.377,1.660,130.435,1.246,169.462$, $3.444,6.376,18.735,51.312,33.920$, $350.346,475.795,4.972,24.451,86.987$.

Use Bayesian methods with a suitable transformation to estimate the finite population mean. 


\section{Solution to Exercise I I.2}

We create a histogram of the sample values and see that the underlying distribution is highly right skewed. However, a histogram of the natural logarithm of the sample values is consistent with a normal superpopulation model. The histograms are shown in Figure 11.2.

Therefore we posit the following Bayesian model involving an uninformative prior and the logarithms of the finite population values, $z_{i}=h\left(y_{i}\right)=\log y_{i}, i=1, \ldots, N(N=200)$ :

$$
\begin{aligned}
& (s \mid z, \mu, \lambda) \sim \text { SRSWOR } \\
& \left(z_{1}, \ldots, z_{N} \mid \mu, \lambda\right) \sim \text { iid } N(\mu, 1 / \lambda) \\
& f(\mu, \lambda) \propto 1 / \lambda, \quad \mu \in \mathfrak{R}, \lambda>0 .
\end{aligned}
$$

Figure I I.2 Histograms of the sample data

Sample values

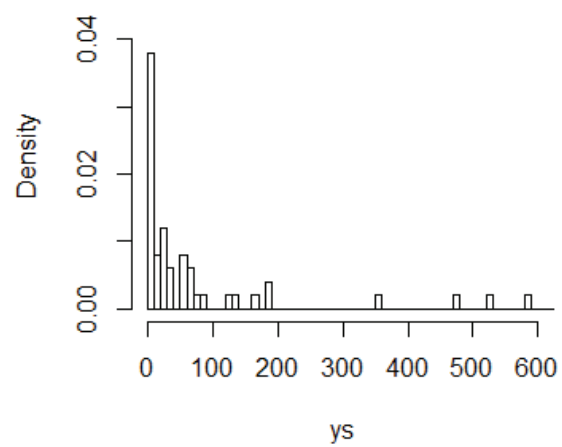

Log of sample values

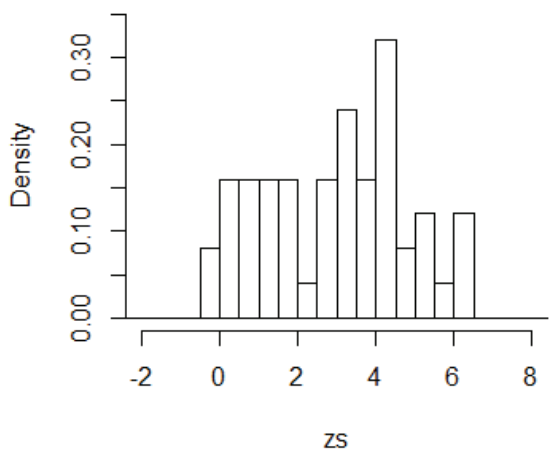

The data is $D=\left(s, z_{s}\right)=((1, \ldots, 50),(28.374,69.857, \ldots, 86.987))$ (after a convenient ordering), and the quantity of interest is

$$
\bar{y}=\frac{1}{N} \sum_{i=1}^{N} y_{i}=g(z)=\frac{1}{N} \sum_{i=1}^{N} h^{-1}\left(z_{i}\right)=\frac{1}{N} \sum_{i=1}^{N} \exp \left(z_{i}\right) .
$$

So we generate

$$
\left(\mu_{1}, \lambda_{1}\right), \ldots,\left(\mu_{J}, \lambda_{J}\right) \sim \text { iid } f(\mu, \lambda \mid D)
$$

(using methods detailed previously). 
Then for each $j=1, \ldots, J$ we sample

and calculate

$$
z_{n+1}^{(j)}, \ldots, z_{N}^{(j)} \sim \text { iid } N\left(\mu_{j}, 1 / \lambda_{j}\right)
$$

$$
\begin{aligned}
\bar{y}^{(j)} & =\frac{1}{N}\left(\left\{y_{1}+\ldots+y_{n}\right\}+\left\{\exp \left(z_{n+1}^{(j)}\right)+\ldots+\exp \left(z_{N}^{(j)}\right)\right\}\right) \\
& =\frac{1}{N}\left(y_{s T}+\sum_{i=n+1}^{N} \exp \left(z_{i}^{(j)}\right)\right) .
\end{aligned}
$$

The result is

$$
\bar{y}^{(1)}, \ldots, \bar{y}^{(J)} \sim \text { iid } f(\bar{y} \mid D),
$$

which can then be used for Monte Carlo inference.

Applying the above procedure with a Monte Carlo sample size of $J=1,000$ we estimate $\bar{y}$ 's posterior mean, $\hat{\bar{y}}=E(\bar{y} \mid D)$, and so also $\bar{y}$ itself, by

$$
\overline{\bar{y}}=\frac{1}{J} \sum_{j=1}^{J} \bar{y}^{(j)}=110.83
$$

with $95 \%$ CI for $\hat{\bar{y}}$

$$
\left(\overline{\bar{y}} \pm 1.96 \sqrt{\frac{1}{J}} \sqrt{\frac{1}{J-1} \sum_{j=1}^{J}\left(\bar{y}^{(j)}-\overline{\bar{y}}\right)^{2}}\right)=(104.64,117.02) .
$$

We also estimate the bounds of the 95\% CPDR for $\bar{y}$ by 49.26 and 302.05, where these are the empirical 0.025 and 0.975 quantiles of $\bar{y}^{(1)}, \ldots, \bar{y}^{(J)}$.

Figure 11.3 shows a histogram of the simulated values of $\bar{y}$, together with the above five numbers, as well as a 'histogram estimate' of the predictive density $f(\bar{y} \mid D)$. In this histogram the dot shows the true value of the finite population mean, $\bar{y}=114.2$, which was known prior to the generation of the sample data. 
Figure II.3 Inference on the finite population mean

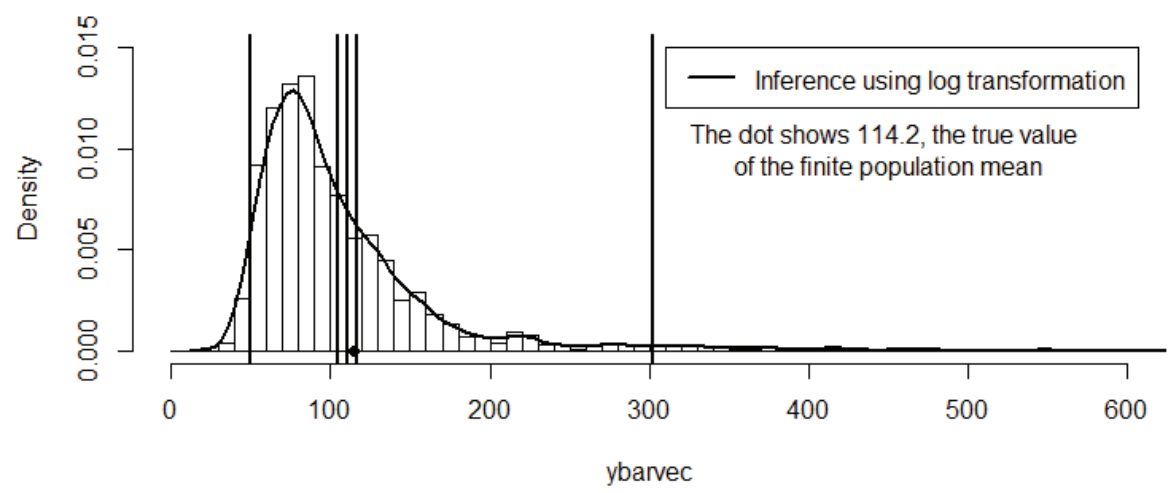

\section{Discussion}

Figure 11.4 shows histograms of the values $Z_{1}, \ldots, Z_{N}$ which were in fact drawn from the normal distribution with mean 3 and standard deviation 2 (left plot), and the values of $y_{1}=\exp \left(z_{1}\right), \ldots, y_{N}=\exp \left(z_{N}\right)$ (right plot), together with the true underlying superpopulation densities of the variables $z_{i}$ and $y_{i}$.

\section{Figure I I.4 Histogram of all $N$ values of $z$ and $y$}
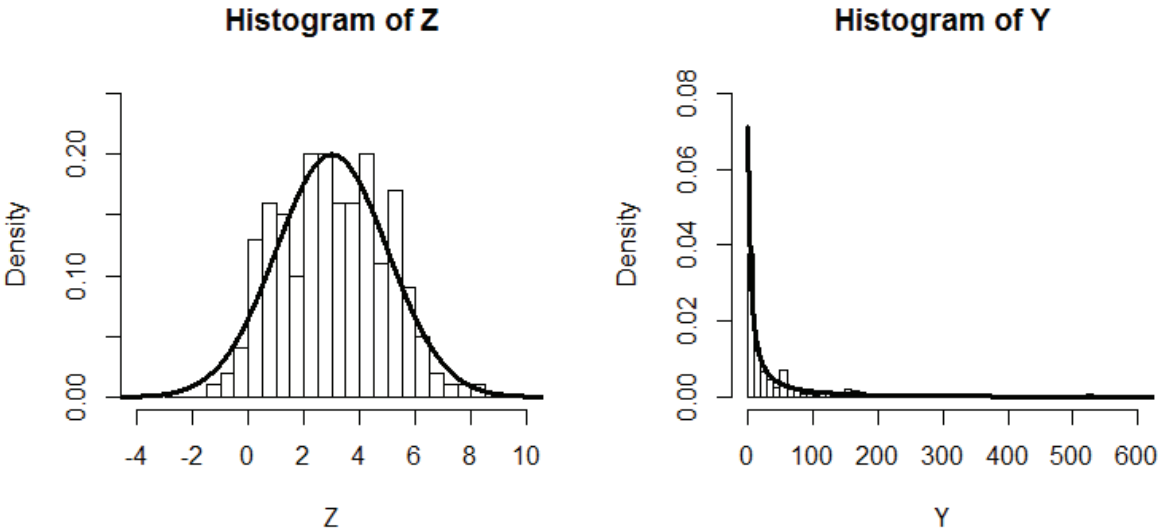
For comparison we repeat the above inference on the original scale of the data and 'exactly' (since there is then no need for Monte Carlo methods).

In that case-where we replace $z$ by $y$ in the Bayesian model-we find that the predictive mean of $\bar{y}$ is $\hat{\bar{y}}=E(\bar{y} \mid D)=\bar{y}_{s}=74.15$ (the average of the raw data values), and the 95\% CPDR for $\bar{y}$ is exactly (41.36, 106.94). We see that this inference does much worse at estimating $\bar{y}$, whose true value is 114.2 .

Note: This second set of inference is the same as design-based inference since it is based on the result

$$
\left(\frac{\bar{y}-\bar{y}_{s}}{\frac{s_{s}}{\sqrt{n}} \sqrt{1-\frac{n}{N}}} \mid D\right) \sim t(n-1) \text {, where } s_{s}^{2}=\frac{1}{n-1} \sum_{i=1}^{n}\left(y_{i}-\bar{y}_{s}\right)^{2} \text {. }
$$

Figure 11.5 shows the original data values (untransformed) and both sets of inferences above. It highlights the value of performing an appropriate prior transformation for purposes of estimating the finite population mean.

\section{Figure I I.5 Comparison of two sets of inference}

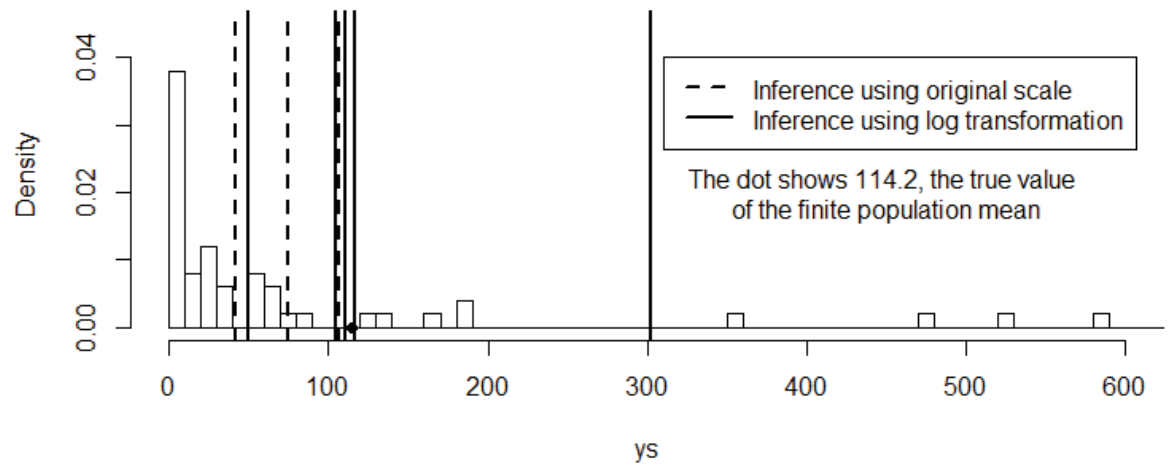


For interest, we repeat the above simulations and comparison with a $N(2,1)$ model for the $z_{i} \mathrm{~s}$ (rather than a $N(3,4)$ model). Figure 11.6 shows the analogue of the last figure above.

We see, of course, that the benefits of applying the log transformation to the data diminishes as the skewness of the sample data decreases.

\section{Figure I I.6 Comparison of two sets of inference with less skewed data}

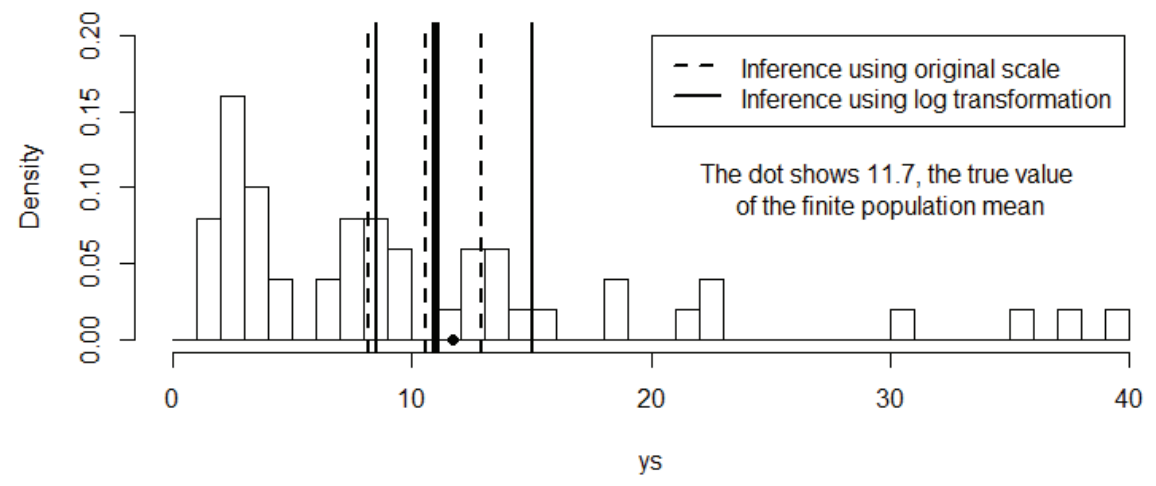

Note 1: Using the formula for sample skewness given by

$$
g=\frac{(1 / n) \sum_{i=1}^{n}\left(y_{i}-\bar{y}_{s}\right)^{3}}{\left\{(1 / n) \sum_{i=1}^{n}\left(y_{i}-\bar{y}_{s}\right)^{2}\right\}^{3 / 2}},
$$

we obtained a value of $g=2.662$ for the first set of data and a value of $g=1.549$ for the second set of data.

Note 2: For another example of finite population inference via Bayesian and MCMC methods which involves the logarithmic transformation, see Puza (2002). This other example also features the use of covariate information.

Note 3: It can be shown (mathematically) that $\hat{\bar{y}}=E(\bar{y} \mid D)=\infty$ (exactly). This seems somewhat counterintuitive in light of the fact that our Monte Carlo estimate $\overline{\bar{y}}=110.83$ is very close to the actual finite population mean, $\bar{y}=114.2$. 


\section{R Code for Exercise I I.2}

\# Data generation used to set up exercise

options(digits=4); $\mathrm{X} 11(\mathrm{w}=8, \mathrm{~h}=6) ; \operatorname{par}(\operatorname{mfrow}=\mathrm{c}(2,2))$

$\mathrm{N}=200 ; \mathrm{n}=50$; set.seed(432); $Z=\operatorname{rnorm}(N, 3,2) ; S=\operatorname{sample}(1: N, n)$

$\mathrm{ZS}=\mathrm{Z}[\mathrm{S}] ; \mathrm{Y}=\exp (\mathrm{Z}) ; \mathrm{YS}=\exp (\mathrm{ZS}) ; \mathrm{YBAR}=\operatorname{mean}(\mathrm{Y}) ; \mathrm{YBAR} \# 114.2$

hist(Z,prob=T); hist(Y,prob=T); hist(ZS,prob=T); hist(YS,prob=T)

\# preliminary plots

$\mathrm{X} 11(\mathrm{w}=8, \mathrm{~h}=4) ; \operatorname{par}(\operatorname{mfrow}=\mathrm{c}(1,2))$

hist (Z,prob=T,xlim=c(-4,10), ylim=c(0,0.25), breaks=seq $(-3,12,0.5))$

lines(seq(-5,12,0.01), dnorm(seq(-5,12,0.01),3,2),lwd=3)

$\operatorname{hist}(Y, \operatorname{prob}=T, x \lim =c(0,600), y \lim =c(0,0.08)$, breaks $=s e q(0,5000,10))$;

yg=seq(0.1,700,0.5); lines(yg ,dnorm( $\log (y g), 3,2) / y g, ~ I w d=3)$

format(list(YS=YS), digits=3) \# "28.374, 69.857, 22.721, ..., 24.451, 86.987"

\# Look at given data and the log of that data (load data etc.)

$\mathrm{N}=200 ; \mathrm{n}=50 ; \mathrm{m}=\mathrm{N}-\mathrm{n}$; options(digits=4)

ys $=c(28.374,69.857,22.721,57.593,126.965$,

$17.816,16.078,0.803,3.164,3.544$,

$2.123,2.353,184.539,59.856,63.701$,

$585.684,29.094,79.245,18.105,1.623$,

$5.513,1.629,63.654,22.060,187.463$,

5.051, 34.299, 27.475, 0.746, 34.016,

8.547, 1.081, 3.151, 55.569, 2.593,

$522.377,1.660,130.435,1.246,169.462$,

3.444, 6.376, 18.735, 51.312, 33.920,

$350.346,475.795,4.972,24.451,86.987)$

summary(ys)

\# Min. 1st Qu. Median Mean 3rd Qu. Max.

$\begin{array}{lllllll}\# & 0.7 & 3.5 & 23.6 & 74.2 & 63.7 & 586.0\end{array}$

skewness=mean( (ys-mean(ys) $\left.)^{\wedge} 3\right) /\left(\operatorname{mean}\left((y s-m e a n(y s))^{\wedge} 2\right)\right)^{\wedge}(3 / 2)$

skewness \# 2.662

$z s=\log (y s) ; \operatorname{par}(\operatorname{mfrow}=c(1,2))$

hist(ys, prob=T); hist(zs, prob=T) \# preliminary plots

hist(ys, prob $=T, x \lim =c(0,600), y \lim =c(0,0.045)$,

breaks=seq $(0,700,10)$, main="Sample values");

hist(zs, prob $=T, x \lim =c(-2,8)$, ylim $=c(0,0.35)$,

breaks=seq $(-3,10,0.5)$, main="Log of sample values"); 
\# Finite population inference using original scale and design-based approach \# (same as the 'exact' Bayesian approach without Monte Carlo) ysbar=mean(ys); sy=sd(ys); ybarhat=ysbar ybarci=ybarhat+c(-1,1)*qt $(0.975, n-1) *($ sy/sqrt(n)) * sqrt(1-n/N) inf.original=c(ybarhat,ybarci); c(inf.original, YBAR) \# $74.15 \quad 41.36106 .94 \quad 114.24$

\# Finite population inference via Bayesian approach using log transformation \# (and a 'crude' approach which makes no use of Rao-Blackwell ideas etc.) ----

zsbar=mean(zs); sz=sd(zs); J=1000; $\operatorname{set} . \operatorname{seed}(142) ;$

lamvec=rgamma $\left(J,(n-1) / 2,\left(s z^{\wedge} 2\right) *(n-1) / 2\right)$

muvec=rnorm(J,zsbar,1/sqrt(n*lamvec)); yrbarvec=rep(NA,J)

for $(j$ in $1: J)\{\quad z r=\operatorname{rnorm}(m$, muvec[j], 1/sqrt(lamvec[j]) )

$y r=\exp (z r) ; \quad$ yrbarvec[j] = mean(yr) $\quad\}$

ybarvec $=(1 / N) *(n * y s b a r+m * y r b a r v e c) ; \quad$ ybarhat=mean(ybarvec)

ybarci=ybarhat+c(-1,1)* qnorm(0.975)*sd(ybarvec)/sqrt(J)

ybarcpdr=quantile(ybarvec,c(0.025,0.975))

inf.transform = c(ybarhat,ybarci,ybarcpdr)

c(inf.transform,YBAR) \# 110.83104 .64117 .0249 .26302 .05114 .24

summary(ybarvec)

\# Min. 1st Qu. Median Mean 3rd Qu. Max.

\# $\quad 37.0 \quad 70.6 \quad 89.4 \quad 111.0 \quad 122.0 \quad 2080.0$

$\operatorname{par}(\operatorname{mfrow}=\mathrm{c}(1,1))$; hist $(\mathrm{ybarvec}, \mathrm{prob}=\mathrm{T})$ \# preliminary plot

hist(ybarvec, prob=T,xlim=c(0,600),ylim=c(0,0.015),

breaks=seq $(0,3000,10)$, main $="$ ");

abline(v=inf.transform, Ity=1,Iwd=2); points(YBAR,0,pch=16)

legend(310,0.015,c("Inference using log transformation"), Ity=c(1),Iwd=c(2))

text $(450,0.01$,

"The dot shows 114.2, the true value \nof the finite population mean")

lines(density(ybarvec), Iwd=2)

$\operatorname{par}(\operatorname{mfrow}=\mathrm{c}(1,1)) ;$ hist $(\mathrm{ys}, \mathrm{prob}=\mathrm{T}) \#$ preliminary plot

hist(ys, prob=T,xlim=c(0,600),ylim=c(0,0.045), breaks=seq $(0,700,10)$, main=" ");

abline(v=inf.original, Ity=2,lwd=2); abline(v=inf.transform, Ity=1,lwd=2)

points(YBAR,0,pch=16)

legend(310,0.04,c("Inference using original scale",

"Inference using log transformation"), Ity $=c(2,1), \operatorname{lwd}=c(2,2))$

text $(450,0.02$,

"The dot shows 114.2, the true value (nof the finite population mean") 
\# Repeat with 'less extreme' lognormal data

$N=200 ; n=50$; set.seed(432); Z=rnorm(N,2,1); S=sample(1:N,n) \#<- difference $\mathrm{ZS}=\mathrm{Z}[\mathrm{S}] ; \mathrm{Y}=\exp (\mathrm{Z}) ; \mathrm{YS}=\exp (\mathrm{ZS}) ; \mathrm{YBAR}=\operatorname{mean}(\mathrm{Y})$

$\mathrm{X} 11(\mathrm{w}=8, \mathrm{~h}=6) ; \operatorname{par}(\operatorname{mfrow}=\mathrm{c}(2,2))$;

hist(Z,prob=T); hist(Y,prob=T); hist(ZS, prob=T); hist(YS, prob=T)

\# preliminary plots

$\mathrm{ys}=\mathrm{YS} ; \quad \mathrm{zs}=\log (\mathrm{ys})$;

skewness=mean( (ys-mean(ys) $\left.)^{\wedge} 3\right) /\left(\text { mean }\left((y s-m e a n(y s))^{\wedge} 2\right)\right)^{\wedge}(3 / 2)$

skewness \# 1.549

ysbar=mean(ys); sy=sd(ys); ybarhat=ysbar

ybarci=ybarhat+c(-1,1)*qt $(0.975, n-1) *(\operatorname{sy} / \operatorname{sqrt}(n)) * \operatorname{sqrt}(1-n / N)$

inf.original $=c(y b a r h a t, y b a r c i)$;

c(inf.original, YBAR) \# 10.541 8.177 12.90611 .698

zsbar=mean(zs); sz=sd(zs); J=1000; set.seed(142);

lamvec=rgamma(J,(n-1)/2,(sz^2)*(n-1)/2)

muvec=rnorm $(\mathrm{J}, z$ sbar, $1 / \mathrm{sqrt}(\mathrm{n} *$ lamvec $)) ; \quad$ yrbarvec=rep(NA,J)

for(j in 1:J)\{ $\quad z r=r n o r m(m, \operatorname{muvec}[j], 1 / \operatorname{sqrt}($ lamvec[j]) )

$y r=\exp (z r) ; \quad$ yrbarvec $[j]=\operatorname{mean}(y r) \quad\}$

ybarvec $=(1 / N) *(n * y s b a r+m * y r b a r v e c) ; \quad$ ybarhat=mean $(y b a r v e c)$

ybarci=ybarhat+c(-1,1)*qnorm $(0.975) *$ sd (ybarvec)/sqrt(J)

ybarcpdr=quantile(ybarvec, $c(0.025,0.975))$

inf.transform $=c(y b a r h a t, y b a r c i, y b a r c p d r)$

c(inf.transform,YBAR) \# 11.00610 .90411 .1088 .47815 .01611 .698

$\mathrm{X} 11(\mathrm{w}=8, \mathrm{~h}=4) ; \operatorname{par}(\mathrm{mfrow}=\mathrm{c}(1,1))$

hist(ys,prob=T) \# preliminary plot

hist(ys, $\operatorname{prob}=T, x \lim =c(0,40), y \lim =c(0,0.2)$, breaks $=\operatorname{seq}(0,40,1)$, main=" ");

abline( $v=$ inf.original, Ity=2,lwd=2); abline( $v=$ inf.transform, Ity=1,lwd=2)

points(YBAR, 0, pch $=16$ )

legend(20,0.2,

c("Inference using original scale", "Inference using log transformation"), $\operatorname{lty}=c(2,1), \operatorname{lwd}=c(2,2))$

text(30,0.1,

"The dot shows 11.7, the true value (nof the finite population mean") 


\section{I.3 Frequentist properties of Bayesian finite population estimators}

We have previously studied the frequentist characteristics of Bayesian estimators. That was in the context of analytic inference (i.e. inference on model parameters) and based on a random sample from a hypothetically infinite population (e.g. a normal distribution). We will now generalise those ideas in the broader framework of a Bayesian finite population model.

As before, we are primarily interested in the frequentist characteristics of Bayesian estimators which are based on uninformative priors and used as proxies for classical or design based estimators. Nevertheless we will consider both types of prior (informative and uninformative).

Consider a Bayesian finite population model specified in terms of:

$f(\xi \mid y, \theta)$ where $\xi$ is $s$ or $I$ or $L$ (as discussed earlier)

$f(y \mid \theta)$ where $y=\left(y_{s}, y_{r}\right)=\left(\left(y_{1}, \ldots, y_{n}\right),\left(y_{n+1}, \ldots, y_{N}\right)\right)=\left(y_{1}, \ldots, y_{N}\right)$

$f(\theta)$ where $\theta=\left(\theta_{1}, \ldots, \theta_{q}\right)$.

Also suppose that the data is

$$
D=\left(s, y_{s}\right) \text { or }(I, s) \text { or }\left(L, y_{s}\right)
$$

(as the case may be), and the quantity of interest is

$$
\psi=g(\theta, y)
$$

(generally) or $\psi=g(\theta)$ (as considered previously for 'pure' analytic inference) or $\psi=g(y)$ (the case of 'pure' finite population inference).

Now suppose that in the context of this general model, data and quantity of interest, we derive a point estimate for $\psi$ (such as the posterior mean, mode or median) of the form

$$
\hat{\psi}=\hat{\psi}(D)
$$

and a $1-\alpha$ interval estimate for $\psi$ (such as the CPDR or HPDR) of the form

$$
I=(L, U)=I(D)=(L(D), U(D)) .
$$

Note: If the sampling mechanism is defined in terms of $I=\left(I_{1}, \ldots, I_{N}\right)$, the vector of inclusion counters, there is a conflict of notation and one of these quantities needs a different symbol. 
In the above context, there may be interest in the frequentist bias of $\hat{\psi}$ and the frequentist coverage probabilities of the interval $I$, especially if these estimators are intended as proxies for classical ones.

However, because there is now an extra level in the Bayesian model hierarchy relative to previously, in the form of the density defining the sampling mechanism, namely

$$
f(\xi \mid y, \theta) \text {, }
$$

there are two ways (at least) of defining the required frequentist characteristics:

- model-based, meaning conditional on $\theta$ and $\xi$

- design-based, meaning conditional on $\theta$ and $y$.

For definiteness, suppose that the data is $D=\left(s, y_{s}\right)$. Then we define:

- the model bias of $\hat{\psi}$ as

$$
B_{\theta, s}=E_{y}\left\{\left(\hat{\psi}\left(s, y_{s}\right)-\psi(y, \theta) \mid \theta, s\right\}\right.
$$

- the relative model bias of $\hat{\psi}$ as

$$
R_{\theta, s}=E_{y}\left\{\frac{\hat{\psi}\left(s, y_{s}\right)-\psi(y, \theta)}{\psi(y, \theta)} \mid \theta, s\right\}
$$

- the model coverage probability of $I$ as

$$
C_{\theta, s}=P_{y}\left\{\psi(y, \theta) \in I\left(s, y_{s}\right) \mid \theta, s\right\} .
$$

Also, we define:

- the design bias of $\hat{\psi}$ as

$$
B_{\theta, y}=E_{s}\left\{\left(\hat{\psi}\left(s, y_{s}\right)-\psi(y, \theta) \mid \theta, y\right\}\right.
$$

- the relative design bias of $\hat{\psi}$ as

$$
R_{\theta, y}=E_{s}\left\{\frac{\hat{\psi}\left(s, y_{s}\right)-\psi(y, \theta)}{\psi(y, \theta)} \mid \theta, y\right\}
$$

- the design coverage probability of $I$ as

$$
C_{\theta, y}=P_{s}\left\{\psi(y, \theta) \in I\left(s, y_{s}\right) \mid \theta, y\right\} .
$$


Note 1: Each of the three model-based characteristics is an expectation with respect to the distribution of $y$ given $\theta$ and $s$. Each of the three design-based characteristics is an expectation with respect to the distribution of $s$ given $\theta$ and $y$.

Note 2: Analogous definitions apply if $D=\left(I, y_{s}\right)$ or $D=\left(L, y_{s}\right)$, etc., noting that $s$ is a function of $I$ and $L$, there is a one-to-one correspondence between $I$ and $s$ under sampling without replacement, etc. For instance, if $D=\left(I, y_{s}\right)$, we define the model bias of $\hat{\psi}$ as

$$
B_{\theta, I}=E_{y}\left\{\left(\hat{\psi}\left(I, y_{s}\right)-\psi(y, \theta) \mid \theta, I\right\},\right.
$$

and when $D=\left(L, y_{s}\right)$, we define the model bias of $\hat{\psi}$ as

$$
B_{\theta, L}=E_{y}\left\{\left(\hat{\psi}\left(L, y_{s}\right)-\psi(y, \theta) \mid \theta, L\right\}\right. \text {, etc. }
$$

Note 3: If a model-based characteristic such as the model bias $B_{\theta, s}$ is be the same for all possible samples $s$, then $s$ may be dropped from the subscript; e.g. we may instead write $B_{\theta}$. Likewise, if a design-based characteristic such as the design bias $B_{\theta, y}$ is the same for all possible values of the model parameter $\theta$, then $\theta$ may be dropped; e.g. we may write $B_{y}$.

Note 4: If a model-based or design based characteristic cannot be evaluated analytically then it may be possible to estimate via a Monte Carlo simulation. This idea features in the next exercise below.

Note 5: The model bias of $\hat{\psi}$ above is a generalisation of the frequentist bias of an estimator as defined earlier and based on a random sample from an infinite population (e.g. a normal distribution). The following argument illustrates. Suppose that $\psi=\theta, \hat{\psi}=\bar{y}_{s}$ (the sample mean) and the sampling mechanism is SRSWOR. Then, by the above definitions, the model bias of $\hat{\psi}$ is

$$
\begin{aligned}
B_{\theta, s} & =E_{y}\left\{\left(\hat{\psi}\left(s, y_{s}\right)-\psi(y, \theta) \mid \theta, s\right\} \quad\right. \text { (generally) } \\
& =E_{y}\left(\bar{y}_{s}-\theta \mid \theta, s\right)=E_{y}\left(\bar{y}_{s} \mid \theta, s\right)-\theta,
\end{aligned}
$$

where 


$$
E_{y}\left(\bar{y}_{s} \mid \theta, s\right)=\int \bar{y}_{s} f(y \mid \theta, s) d y .
$$

Now, in this case,

$$
f(s \mid y, \theta)=f(s)=\left(\begin{array}{c}
N \\
n
\end{array}\right)^{-1} \text { for all } s=(1, \ldots, n), \ldots,(N-n+1, \ldots, N),
$$

so that

$$
f(y \mid \theta, s) \stackrel{y}{\propto} f(y, \theta, s)=f(s \mid y, \theta) f(y \mid \theta) f(\theta) \stackrel{y}{\propto} 1 \times f(y \mid \theta) \times 1,
$$

and therefore

$$
f(y \mid \theta, s)=f(y \mid \theta)=f\left(y_{r}, y_{s} \mid \theta\right)=f\left(y_{r} \mid \theta, y_{s}\right) f\left(y_{s} \mid \theta\right),
$$

with $s$ fixed at its observed value.

From these observations we see that

$$
\begin{aligned}
E_{y}\left(\bar{y}_{s} \mid \theta, s\right) & =\iint \bar{y}_{s} f\left(y_{r} \mid \theta, y_{s}\right) f\left(y_{s} \mid \theta\right) d y_{r} d y_{s} \\
& =\int f\left(y_{r} \mid \theta, y_{s}\right) d y_{r} \times \int \bar{y}_{s} f\left(y_{s} \mid \theta\right) d y_{s} \\
& =1 \times E\left(\bar{y}_{s} \mid \theta\right) .
\end{aligned}
$$

Therefore $B_{\theta, s}=E\left(\bar{y}_{s} \mid \theta\right)-\theta=E\left(\bar{y}_{s}-\theta \mid \theta\right)$.

We have shown that the model bias here is the same as the bias of $\bar{y}_{s}$ in the earlier non-finite population context (where $s$ did not feature in the notation).

This is an example of where $s$ could be dropped from the subscript in $B_{\theta, s}$, i.e. where this could also be written $B_{\theta}$.

If the sampling mechanism in this illustration were nonignorable, with $f(s \mid y, \theta)$ depending on $y$ in some way, then the simplifications above might not be possible and the bias might need to be evaluated, with more difficulty, according to the formula

$$
B_{\theta, s}=-\theta+\int \bar{y}_{s} f(y \mid \theta, s) d y=-\theta+\int \bar{y}_{s} \frac{f(y, \theta, s)}{f(\theta, s)} d y
$$

where: $f(\theta, s)=\int f(y, \theta, s) d y$

$$
f(y, \theta, s)=f(s \mid y, \theta) f(y \mid \theta) f(\theta), \text { etc. }
$$


Note 6: The design bias of $\hat{\psi}$ above is a generalisation of the bias of an estimator in the classical survey sampling context where a sample is drawn from a finite population of values which are thought of as constants. The following argument illustrates. Suppose that $\psi=\bar{y}$ (the finite population mean), $\hat{\psi}=\bar{y}_{s}$ (the sample mean) and the sampling mechanism is SRSWOR. Then, by the above definitions, the design bias of $\hat{\psi}$ is

$$
\begin{aligned}
B_{\theta, y} & =E_{s}\left\{\left(\hat{\psi}\left(s, y_{s}\right)-\psi(y, \theta) \mid \theta, y\right\} \quad\right. \text { (generally) } \\
& =E_{s}\left\{\bar{y}_{s}-\bar{y} \mid \theta, y\right\}=E_{s}\left(\bar{y}_{s} \mid \theta, y\right)-\bar{y} .
\end{aligned}
$$

Now, $\quad E_{s}\left(\bar{y}_{s} \mid \theta, y\right)=\sum_{s} \bar{y}_{s} f(s \mid y, \theta)=\frac{1}{k n} \sum_{s}\left(y_{s_{1}}+\ldots+y_{s_{n}}\right)$

$$
\begin{gathered}
\text { where } f(s \mid y, \theta)=\frac{1}{k} \text { and } k=\left(\begin{array}{c}
N \\
n
\end{array}\right) \\
=\frac{1}{k n}\left\{\left(y_{1}+\ldots+y_{n}\right)+\ldots\left(y_{N-n+1}+\ldots+y_{N}\right)\right\} .
\end{gathered}
$$

Here, expression \{\} contains a total of $k n$ terms, with each of $y_{1}, \ldots, y_{N}$ is represented equally often and therefore $\mathrm{kn} / \mathrm{N}$ times.

We see that \{\}$=\frac{k n}{N}\left(y_{1}+\ldots+y_{N}\right)=k n \bar{y}$.

Thus $E_{s}\left(\bar{y}_{s} \mid \theta, y\right)=\frac{1}{k h} k h \bar{y}=\bar{y}$,

and so $B_{\theta, y}=E_{s}\left(\bar{y}_{s} \mid \theta, y\right)-\bar{y}=\bar{y}-\bar{y}=0$.

We have here simply followed through with our general definitions and notation to show that under SRSWOR the sample mean is unbiased for the population mean.

If the sampling mechanism were nonignorable, with $f(s \mid y, \theta)$ depending on $y$ in some way, then the bias of the sample mean might need to be evaluated, with more difficulty, according to the formula

$$
B_{\theta, y}=-\bar{y}+\sum_{s} \bar{y}_{s} f(s \mid y, \theta)=-\bar{y}+\sum_{s} \bar{y}_{s} \frac{f(s, y, \theta)}{f(y, \theta)},
$$

where $f(y, \theta)=\sum_{s} f(s, y, \theta), f(s, y, \theta)=f(s \mid y, \theta) f(y \mid \theta) f(\theta)$, etc. 


\section{Exercise I I.3 Frequentist properties of Bayesian estimators in a normal finite population model}

Consider a sample of size $n=20$ taken from a finite population of size $N=100$ according to SRSWOR, where the population values are normal with mean $\mu=10$ and variance $\sigma^{2}=1 / \lambda=4$, with prior given by

$$
f(\mu, \lambda) \propto 1 / \lambda, \mu \in \mathfrak{R}, \lambda>0 \text { (uninformative). }
$$

(a) Using these specifications, generate a finite population vector $y=\left(y_{1}, \ldots, y_{N}\right)$, take the sample vector as $y_{s}=\left(y_{1}, \ldots, y_{n}\right)$, and then use Monte Carlo (MC) methods with a sample size of $J=1,000$ to estimate the superpopulation signal to noise ratio defined by $\gamma=\mu / \sigma$.

Report a point estimate of $\gamma$ in the form of a MC estimate of the posterior mean $\hat{\gamma}=E(\gamma \mid D)$ where $D=\left(s, y_{s}\right)$ is the data, and an interval estimate in the form of a MC estimate of the 95\% CPDR for $\gamma$. (Do not bother to calculate a 95\% CI for $\hat{\gamma}$.)

What is the difference between your point estimate and $\gamma$ ? Does $\gamma$ lie inside the interval? Calculate $\tilde{\gamma}$, the MLE of $\gamma$ and report the difference between $\tilde{\gamma}$ and $\gamma$.

Illustrate your inferences by drawing a suitable histogram of the simulated values of $\gamma$, marked over with the various estimates.

(b) Perform the procedure in (a) $K=100$ times independently, with $K$ different finite populations but the sample always consisting of the first $n$ values in that finite population.

Based on your results, estimate the model bias and relative model bias of your point estimator, and the model coverage of your interval estimator. Also estimate the model bias and relative model bias of the MLE $\tilde{\gamma}$.

Illustrate your results by drawing a suitable histogram of the $K$ simulated MC estimates, marked over with the various relevant quantities.

(c) Repeat (b) but with $K=5,000$ and discuss. 
(d) Generate a finite population, vector $y=\left(y_{1}, \ldots, y_{N}\right)$, and then take a sample from the finite population via SRSWOR. Then use MC methods with sample size $J=1,000$ to estimate the finite population ratio of largest value to median, which is given by the formula

$$
\psi=\frac{y_{(100)}}{\left(y_{(50)}+y_{(51)}\right) / 2},
$$

where $y_{(i)}$ is the ith order statistic for the $N$ population values $y_{1}, \ldots, y_{N}$.

Report a point estimate of $\psi$ in the form of a MC estimate of the posterior mean $\hat{\psi}=E(\psi \mid D)$ and an interval estimate in the form of a MC estimate of the 95\% CPDR for $\psi$. (Do not bother to calculate a $95 \%$ CI for $\hat{\psi}$.)

What is the difference between your point estimate and $\psi$ ? Does $\psi$ lie inside the interval?

Illustrate your inferences by drawing a suitable histogram of the simulated values of $\psi$, marked over with the various estimates.

(e) Perform the procedure in (d) $K=100$ times independently, with $K$ different samples taken from the same finite population.

Based on your results, estimate the design bias and relative design bias of your point estimator, and the design coverage of your interval estimator.

Illustrate your results by drawing a suitable histogram of the $K$ simulated MC estimates, marked over with the various relevant quantities.

(f) Repeat (e) using two other point estimators, respectively.

\section{Solution to Exercise II.3}

(a) A finite population of size $N=100$ from the $N\left(\mu=10, \sigma^{2}=4\right)$ distribution was generated. The sample mean and standard deviation of the 100 finite population values were $\bar{y}=9.932$ and $s_{y}=1.907$. Figure 11.7 shows a histogram of these values. 
Figure I I.7 Histogram of $\mathbf{N}=100$ finite population values

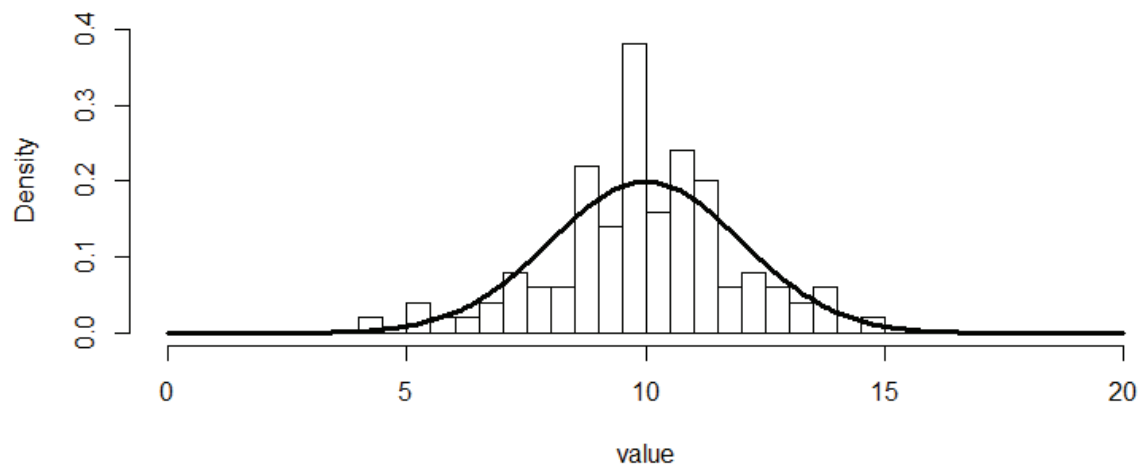

Then the first $n=20$ values were taken as a sample from the finite population. Figure 11.8 shows a histogram of these sample values. The sample mean and standard deviation of the sample values were $\bar{y}_{s}=10.516$ and $s_{s}=1.749$. So the MLE of $\gamma=\mu / \sigma$ was calculated as $\tilde{\gamma}=\tilde{\mu} / \tilde{\sigma}=\bar{y}_{s} / s_{s}=6.011$.

Figure I I.8 Histogram of $n=20$ sample values

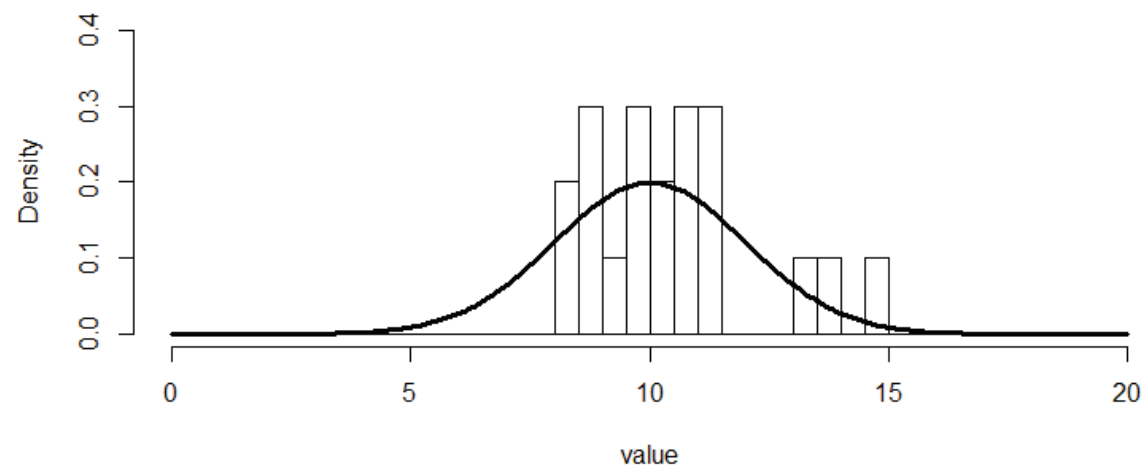

Then a Monte Carlo sample of size $J=1,000$ was taken from the joint posterior distribution of $\mu$ and $\lambda=1 / \sigma^{2}$, i.e. from $f(\mu, \lambda \mid D)$ where $D=\left(s, y_{s}\right)$. Hence a MC sample of size $J$ was obtained from the posterior distribution of $\gamma$, namely $\gamma_{1}, \ldots, \gamma_{J} \sim$ iid $f(\gamma \mid D)$. 
Note: As explained in previous exercises, this was done by:

- first sampling $\lambda_{1}, \ldots, \lambda_{J} \sim$ iid $G\left(\frac{n-1}{2}, \frac{n-1}{2} s_{s}^{2}\right)$

- then sampling $w_{1}, \ldots, w_{J} \sim$ iid $t(n-1)$

- next forming $\mu_{j}=\bar{y}_{s}+w_{j} s / \sqrt{n}$

- finally calculating $\gamma_{j}=\mu_{j} \sqrt{\lambda_{j}}$.

The MC sample from $\gamma$ 's posterior was used to calculate the point estimate

$$
\bar{\gamma}=\frac{1}{J} \sum_{j=1}^{J} \gamma_{j}=5.925
$$

(the MC estimate of $\gamma$ 's posterior mean) and the interval estimate

$$
I=(4.115,7.963)
$$

(formed by the empirical 0.025 and 0.975 quantiles of $\gamma_{1}, \ldots, \gamma_{J}$ ).

Figure 11.9 shows a histogram of the simulated values $\gamma_{1}, \ldots, \gamma_{J}$ overlaid by an estimate of $\gamma$ 's posterior density $f(\gamma \mid D)$. Also shown in the figure are the Bayesian estimates (3 vertical lines), the MLE $\hat{\gamma}=6.011$, and the true value of $\gamma$, namely $\gamma=\mu / \sigma=10 / 2=5$. We see that the true value of $\gamma$ lies in the Bayesian interval estimate, and the difference between the Bayesian estimate and the true value is $5.925-5=0.925$. Likewise, the MLE is in 'error' by $6.011-5=1.011$.

Figure II.9 Inference on $\gamma$ based on a MC sample $(J=I, 000)$

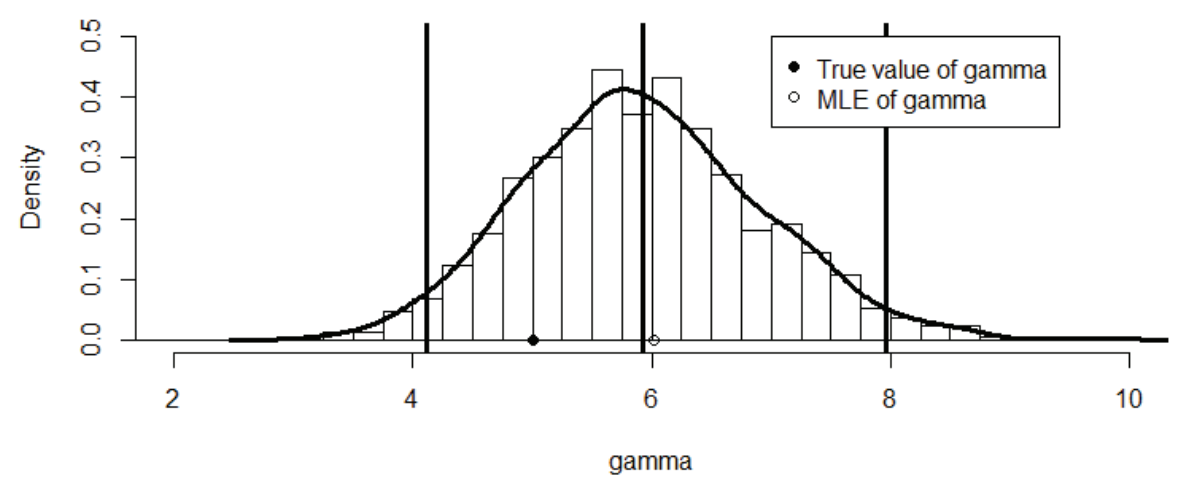


(b) The procedure in (a) was repeated so as to yield a total of $K=100$ Bayesian estimates $\bar{\gamma}_{1}, \ldots, \bar{\gamma}_{K}$, as well as $K$ Bayesian interval estimates $I_{1}, \ldots, I_{K}$ and $K$ MLEs $\tilde{\gamma}_{1}, \ldots, \tilde{\gamma}_{K}$.

From these results we estimated the model mean of the Bayesian estimate $\bar{\gamma}$ by

$$
\overline{\bar{\gamma}}=\frac{1}{K} \sum_{k=1}^{K} \bar{\gamma}_{k}=5.2226,
$$

with 95\% CI (for that mean) of

$$
\left(\overline{\bar{\gamma}} \pm 1.96 \sqrt{\frac{1}{K(K-1)} \sum_{k=1}^{K}\left(\bar{\gamma}_{k}-\overline{\bar{\gamma}}\right)^{2}}\right)=(4.9986,5.4466) \text {. }
$$

Hence we estimated the model bias of $\bar{\gamma}$ by $\overline{\bar{\gamma}}-\gamma=0.2226$ with 95\% CI $(-0.0014,0.4466)$.

Likewise, we estimated the model mean of the MLE $\tilde{\gamma}$ by

$$
\overline{\tilde{\gamma}}=\frac{1}{K} \sum_{k=1}^{K} \tilde{\gamma}_{k}=5.298,
$$

with 95\% CI (for that mean) of

$$
\left(\overline{\tilde{\gamma}} \pm 1.96 \sqrt{\frac{1}{K(K-1)} \sum_{k=1}^{K}\left(\tilde{\gamma}_{k}-\overline{\tilde{\gamma}}\right)^{2}}\right)=(5.070,5.526) .
$$

Hence we estimate the model bias of $\tilde{\gamma}$ by $\overline{\tilde{\gamma}}-\gamma=0.298$ with $95 \% \mathrm{CI}$ (0.0705, 0.5255).

Thus we also estimate the relative model biases of $\bar{\gamma}$ and $\tilde{\gamma}$ by

$$
\begin{aligned}
& (\overline{\bar{\gamma}}-\gamma) / \gamma=0.0445 \text { with 95\% CI } \quad(-0.0003,0.0893) \\
& (\overline{\tilde{\gamma}}-\gamma) / \gamma=0.0596 \quad \text { with 95\% CI } \quad(0.0141,0.1051) .
\end{aligned}
$$

Note: These could also be reported as the percentages (\%):

$$
\begin{aligned}
& (\overline{\bar{\gamma}}-\gamma) / \gamma=4.5 \text { with } 95 \% \text { CI }(-0.03,8.9) \\
& (\overline{\tilde{\gamma}}-\gamma) / \gamma=6.0 \quad \text { with 95\% CI }(1.4,10.5) .
\end{aligned}
$$

Also, exactly 91 of the 100 Bayesian interval estimates $I_{1}, \ldots, I_{K}$ actually contained the true value $\gamma=5$. 
So we estimate the model coverage of the 95\% CPDR estimate of $\bar{\gamma}$ (based on a MC sampled size of specifically $J=1,000$ ) as 0.91 , with 95\% CI (for that coverage)

$$
(0.91 \pm 1.96 \sqrt{0.91(1-0.91) / 100})=(0.854,0.966) \text {. }
$$

Figure 11.10 shows a histogram of the $K$ simulated values of $\bar{\gamma}_{1}, \ldots, \bar{\gamma}_{K}$ and related quantities.

We see that the Bayesian inference appears to have slightly outperformed the MLE as regards model bias.

Note that this applies in a very particular situation, namely one with $N=100, n=20, \mu=10, \sigma=2$, and a MC estimation scheme as described above with specifically $J=1,000$.

Note: If we were to use a different common sample from each finite population (e.g. $\left.y_{s}=\left(y_{2}, y_{14}, y_{15}, \ldots, y_{87}\right)\right)$, or a different sample each time, the results would be the same, subject to Monte Carlo variation. This might not be the case in a situation where the sampling mechanism is nonignorable or where there are covariate values. But as a matter of form when calculating model-based properties, we must condition on the sample being taken, i.e. on $s$.

\section{Figure I I. I 0 Distribution of $K=100$ estimates}

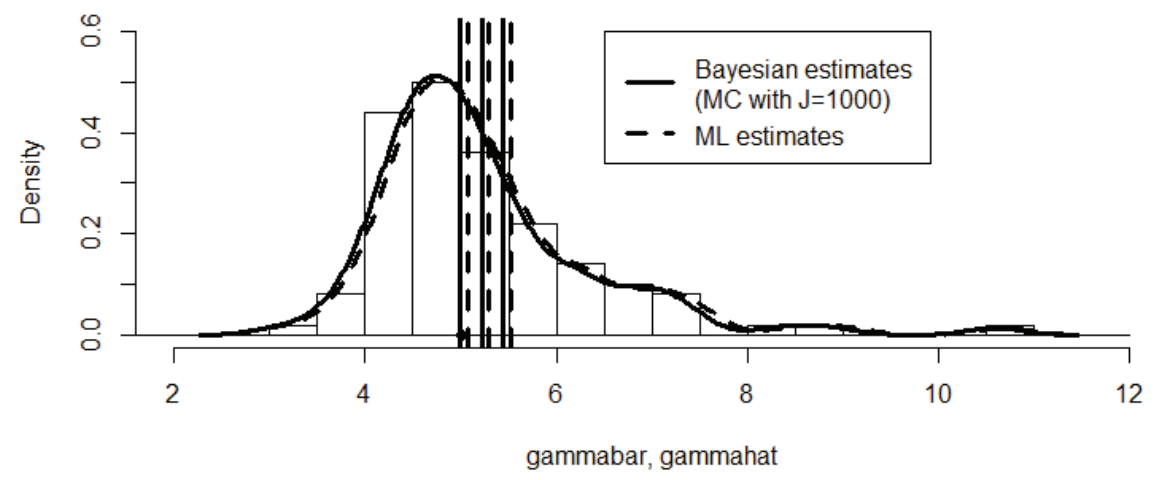


(c) Repeating (a) and (b) with $K=5,000$, we obtained the following results:

Estimate of model bias of $\bar{\gamma}$ is 0.1616 with $95 \%$ CI $(0.1359,0.1872)$

Estimate of model bias of $\tilde{\gamma}$ is 0.2301 with $95 \%$ CI $(0.2041,0.2561)$

Estimate of relative model bias of $\bar{\gamma}$ is 3.2 with 95\% CI $(2.7,3.7)$ (\%)

Estimate of relative model bias of $\tilde{\gamma}$ is 4.6 with 95\% CI $(4.1,5.1)$ (\%).

Exactly 4,755 of the 5,000 Bayesian interval estimates $I_{1}, \ldots, I_{K}$ actually contained the true value $\gamma=5$.

So we estimate the model coverage of the 95\% CPDR estimate of $\bar{\gamma}$ (based on a MC sample of size $J=1,000$ ) as 4,755/5,000 $=0.951$, with 95\% CI (for that coverage),

$$
(0.951 \pm 1.96 \sqrt{0.951(1-0.951) / 5,000})=(0.945,0.957) .
$$

From these results it appears that both the Bayesian and ML estimators are indeed positively biased by several percent, with the Bayesian estimator slightly outperforming the MLE.

It also appears that the model coverage of the Bayesian interval estimate is very close to the nominal $95 \%$.

Figure 11.11 shows a histogram of the 5,000 simulated Bayesian estimates and related information. A detail in this figure is shown as Figure 11.12.

Figure I I. I I Distribution of $K=5,000$ estimates

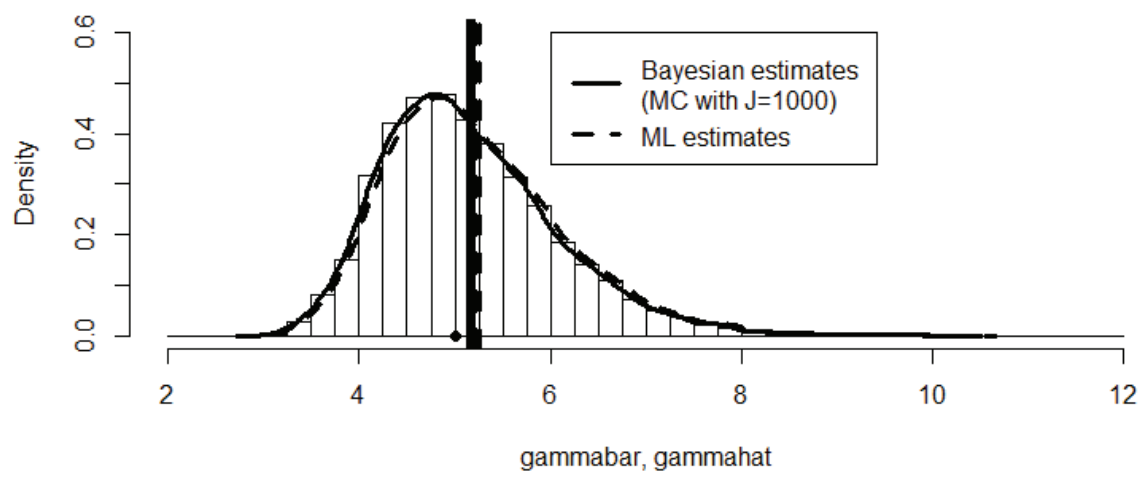




\section{Figure I I.I 2 Detail in Figure I I.I I}

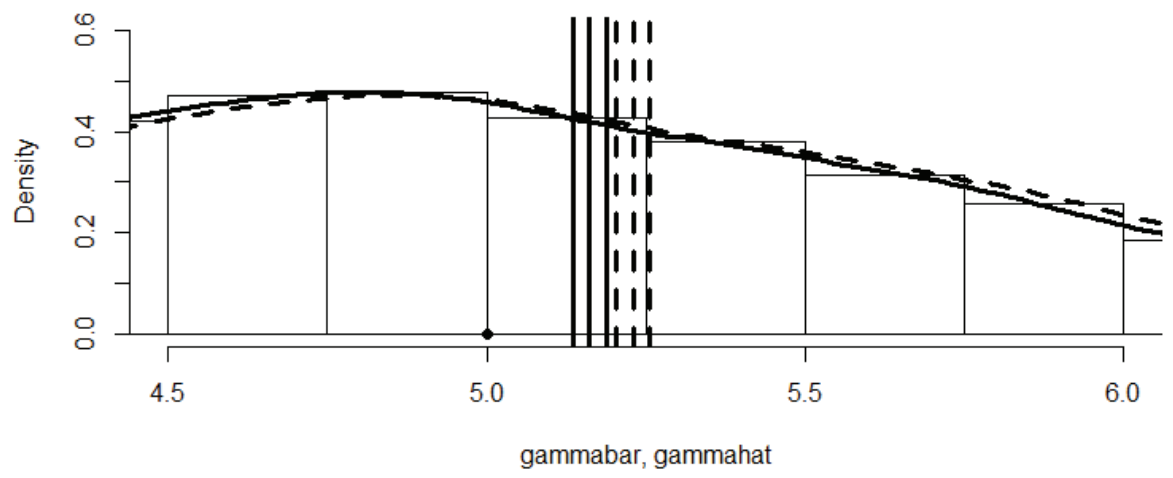

(d) A finite population of size $N=100$ from the $N\left(\mu=10, \sigma^{2}=4\right)$ distribution was generated. The sample mean and standard deviation of the 100 finite population values were $\bar{y}=9.675$ and $s_{y}=2.159$.

A histogram of the values is shown in Figure 11.13. The true value of the ratio requiring inference was in this case calculated as

$$
\psi=\frac{y_{(100)}}{\left(y_{(50)}+y_{(51)}\right) / 2}=\frac{15.622}{10.171}=1.536 .
$$

Figure I I. 13 Histogram of $N=100$ finite population values

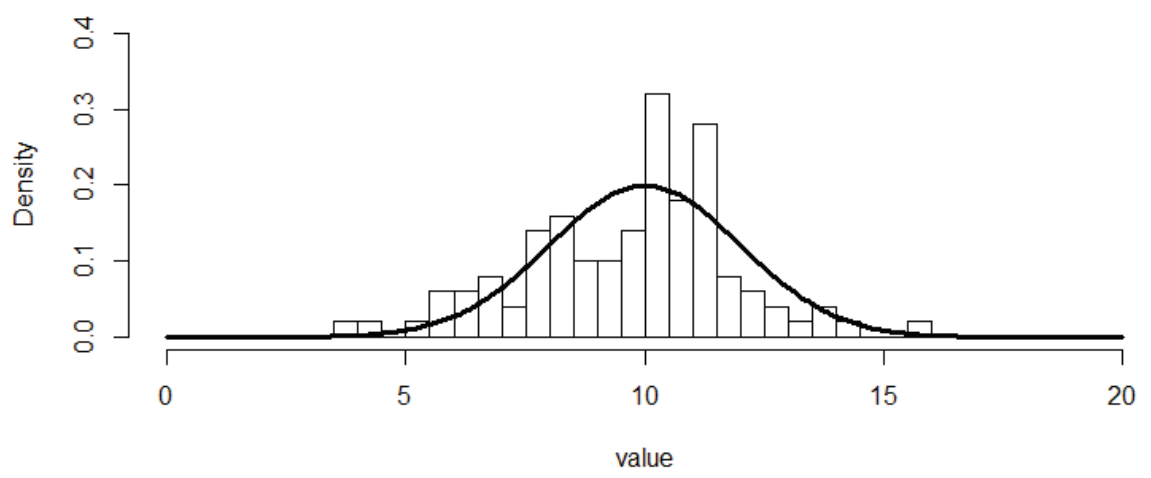


Then a sample of size $n=20$ values was taken from the finite population. The sample mean and standard deviation of the sampled values were $\bar{y}_{s}=9.438$ and $s_{s}=2.448$. A histogram of the sample values is shown in Figure 11.14.

\section{Figure I I.I4 Histogram of $n=20$ sample values}

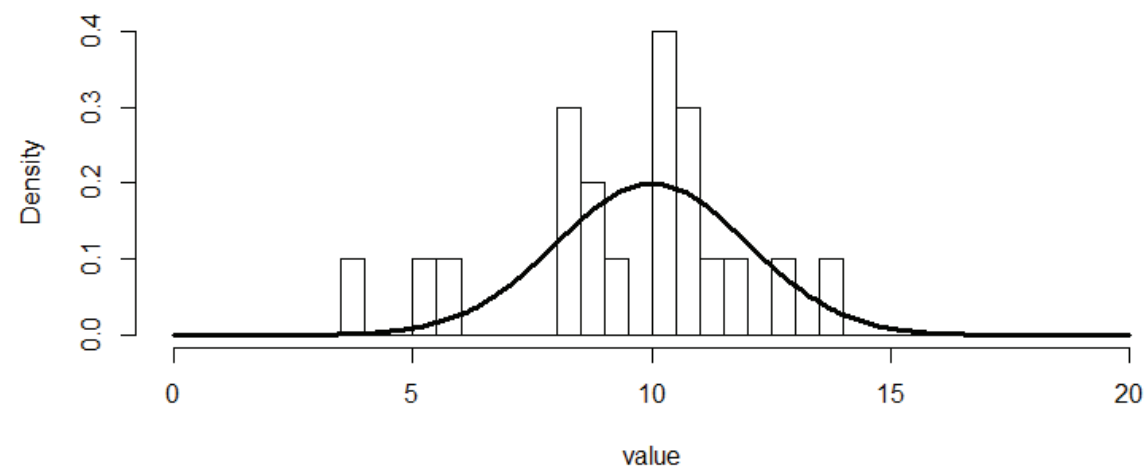

Then a MC sample of size $J=1,000$ was taken from the joint posterior distribution of $\mu$ and $\lambda=1 / \sigma^{2}$, i.e. from $f(\mu, \lambda \mid D)$ with $D=\left(s, y_{s}\right)$. Hence a MC sample of size $J$ was obtained from the predictive distribution of $\psi$, namely $\psi_{1}, \ldots, \psi_{J} \sim$ iid $f(\psi \mid D)$.

Note: As explained in previous exercises, this was done by doing the following for each $j=1, \ldots, J$ :

- first sample $y_{i}^{(j)} \sim$ iid $N\left(\mu_{j}, 1 / \lambda_{j}\right), i \in r$

- then form $y_{r}^{(j)}=\left(y_{r_{1}}^{(j)}, \ldots, y_{r_{N-n}}^{(j)}\right)$

- finally calculate $\psi_{j}$ from $\left(y_{s}, y_{r}^{(j)}\right)$.

The MC sample from $\psi$ 's predictive distribution was used to calculate the point estimate

$$
\bar{\psi}=\frac{1}{J} \sum_{j=1}^{J} \psi_{j}=1.715
$$

(the MC estimate of $\psi$ 's predictive mean) and the interval $I=(1.456$, 2.078) formed by the empirical 0.025 and 0.975 quantiles of $\psi_{1}, \ldots, \psi_{J}$. 
Figure 11.15 shows a probability histogram of the simulated values $\psi_{1}, \ldots, \psi_{J}$ overlaid by an estimate of $\psi$ 's predictive density $f(\psi \mid D)$. Also shown are the Bayesian estimates (represented by three vertical lines), and the true value of $\psi$, which is 1.536 (represented by the dot).

We note that the true value of $\psi$ lies in the Bayesian interval estimate, and the difference between the Bayesian estimate and the true value is $1.715-1.536=0.179$.

Figure I I.I5 Inference on $\psi$ based on a MC sample $(J=I, 000)$

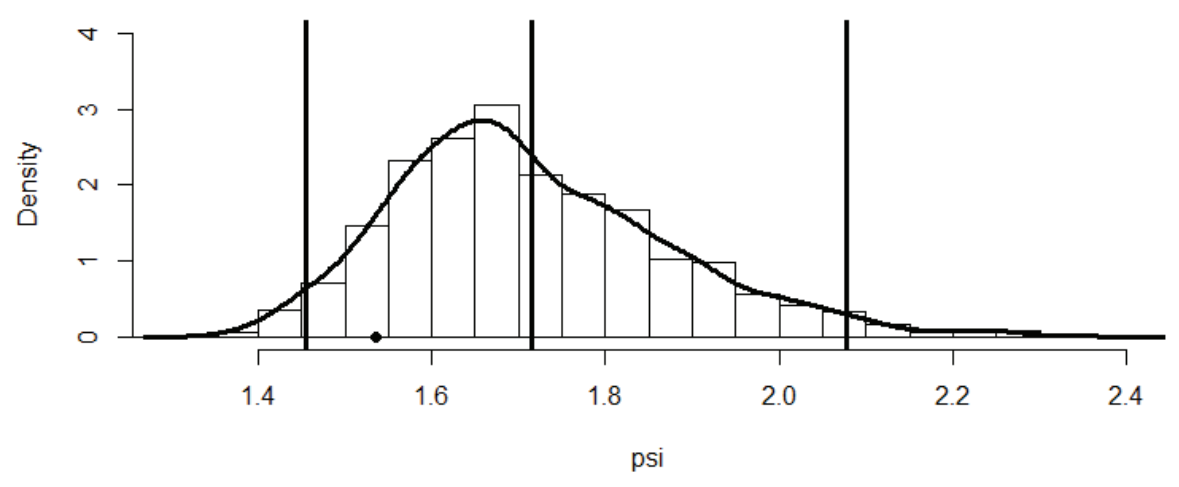

(e) The procedure in (d) was repeated so as to yield a total of $K=100$ Bayesian estimates $\bar{\psi}_{1}, \ldots, \bar{\psi}_{K}$ and $K$ corresponding Bayesian interval estimates $I_{1}, \ldots, I_{K}$. From these results we estimate the design mean of the Bayesian predictive mean estimate $\bar{\psi}$ by

$$
\overline{\bar{\psi}}=\frac{1}{K} \sum_{k=1}^{K} \bar{\psi}_{k}=1.6168,
$$

with 95\% CI (for that mean)

$$
\left(\overline{\bar{\psi}} \pm 1.96 \sqrt{\frac{1}{K(K-1)} \sum_{k=1}^{K}\left(\bar{\psi}_{k}-\overline{\bar{\psi}}\right)^{2}}\right)=(1.5962,1.6374) .
$$

Hence we estimate the design bias of $\bar{\psi}$ by $\overline{\bar{\psi}}-\psi=0.0808$, with $95 \%$ CI $(0.0602,0.1014)$. Thus we also estimate the relative design bias of $\bar{\psi}$ by $(\overline{\bar{\psi}}-\psi) / \psi=5.3$, with $95 \%$ CI $(3.9,6.6)$ (\%). 
Also, 91 of the 100 Bayesian interval estimates $I_{1}, \ldots, I_{K}$ contained the true value, $\psi=1.536$. So we estimate the design coverage of the $95 \%$ CPDR estimate of $\bar{\psi}$ (based on a MC sample with size $J=1,000$ ) as 0.91 , with $95 \%$ CI $(0.91 \pm 1.96 \sqrt{0.91(1-0.91) / 100})=(0.8539,0.9661)$.

Figure 11.16 shows a probability histogram of the $K$ simulated values $\bar{\psi}_{1}, \ldots, \bar{\psi}_{K}$ and related quantities.

Figure I I.I 6 Distribution of $K=100$ estimates of $\psi$

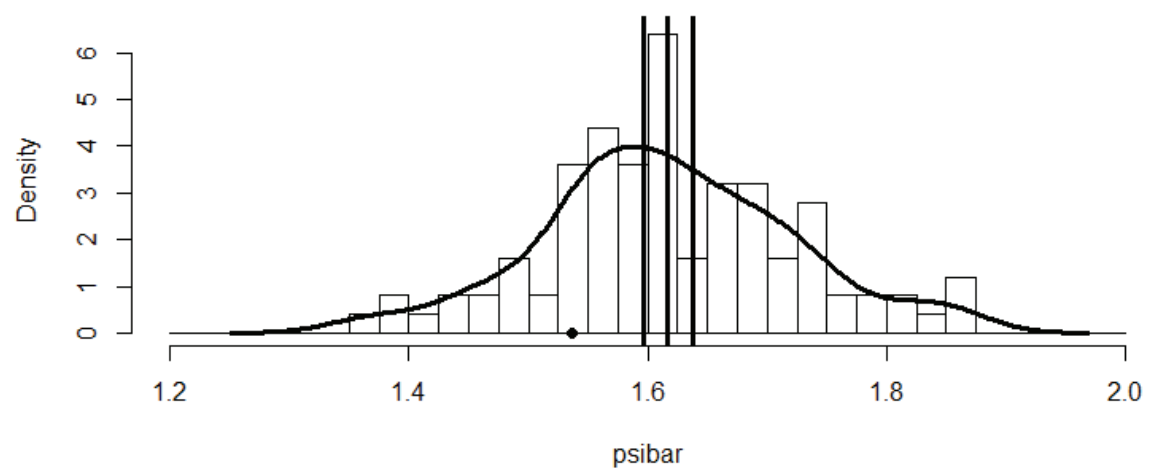

(f) Figure 11.17 is an analogue of Figure 11.16 but obtained by replacing the Monte Carlo sample mean estimate $\bar{\psi}=\left(\psi_{1}+\ldots+\psi_{J}\right) / J$ by the empirical median of $\psi_{1}, \ldots, \psi_{J}$.

Likewise, Figure 11.18 is an analogue of Figure 11.16 but obtained by replacing the posterior mean estimate by the empirical mode of $\psi_{1}, \ldots, \psi_{J}$.

Note: The empirical mode was obtained using the R function density().

We see that the design bias of the empirical mode appears to be smaller than that of the empirical median, which in turn is smaller than that of the posterior mean. The biases of the Monte Carlo predictive mean, median and mode estimates (based on a Monte Carlo sample size of $J=1,000)$ are estimated as $+5.3 \%,+3.8 \%$ and $+1.4 \%$. 
Note: From Figure 11.15 in (d) we may have already guessed that the posterior mode is better than the posterior mean as an estimate of $\psi$ (whose true value is 1.536, as shown by the dot in Figures 11.15-18).

Figure I I.I7 Distribution of $K=100$ estimates of $\psi$

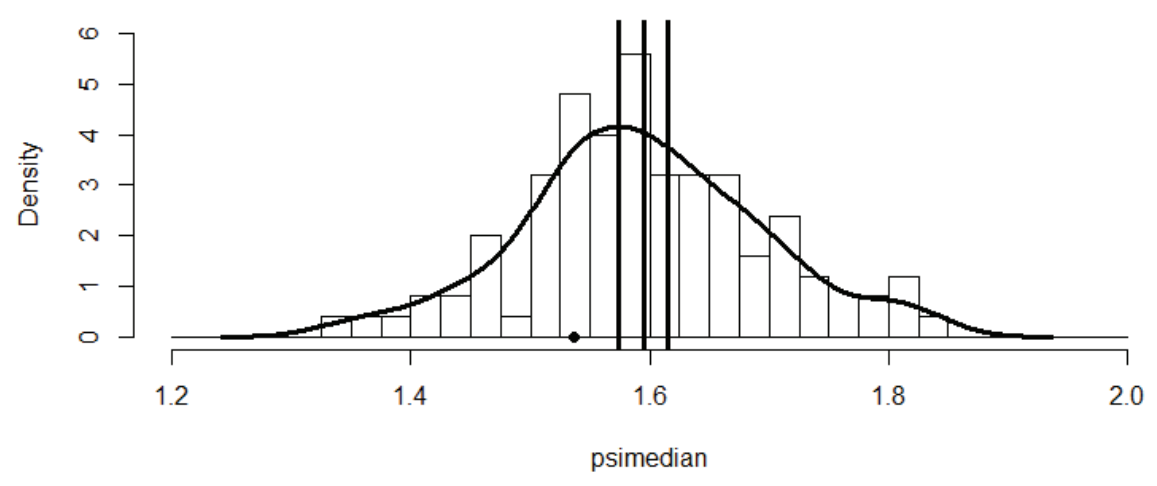

Figure I I. I 8 Distribution of $K=100$ estimates of $\psi$

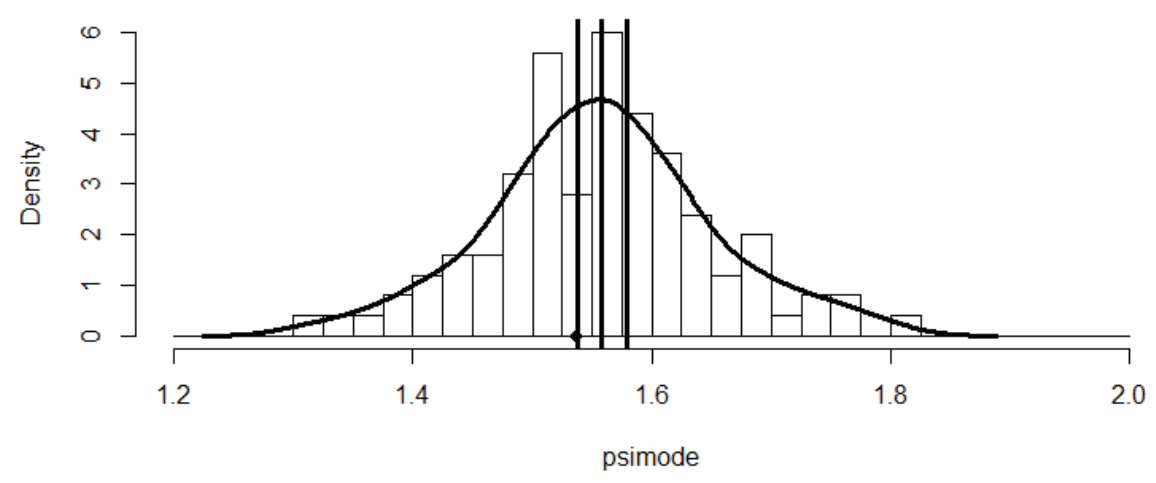




\section{R Code for Exercise II.3}

\# (a)

$\mathrm{X} 11(\mathrm{w}=8, \mathrm{~h}=4) ; \operatorname{par}(\mathrm{mfrow}=\mathrm{c}(1,1)) ;$ options $($ digits $=4)$

$\mathrm{N}=100 ; \mathrm{n}=20 ; \mathrm{mu}=10 ; \mathrm{sig}=2 ; \operatorname{lam}=1 / \mathrm{sig}^{\wedge} 2 ;$ gam=mu/sig

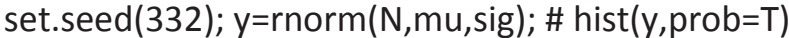

hist $(y, p r o b=T, x l a b=" v a l u e ", x \lim =c(0,20), y \lim =c(0,0.4)$, breaks=seq $(0,20,0.5)$, main=" ")

lines(seq(0,20,0.1), dnorm(seq(0,20,0.1),mu,sig),lwd=3)

$\mathrm{ys}=\mathrm{y}[1: \mathrm{n}]$

hist(ys,prob=T,xlab="value", $x \lim =c(0,20), \operatorname{ylim}=c(0,0.4)$, breaks=seq $(0,20,0.5)$, main=" ")

lines(seq(0,20,0.1),dnorm(seq(0,20,0.1), mu,sig),lwd=3)

ysbar=mean(ys); sys=sd(ys); gammle=ysbar/sys

ybar=mean(y); sy=sd(y); ygam=ybar/sy; c(ybar,sy,ygam) \# 9.932 1.907 5.207

c(lam,ysbar,sys, gam, gammle) \# 0.250 10.516 1.7495 .0006 .011

$\mathrm{J}=1000$; set.seed(171);

lamv=rgamma $\left(J,(n-1) / 2\right.$, sys $\left.^{\wedge} 2 *(n-1) / 2\right) ;$ muv=rnorm $(J, y s b a r, 1 / \operatorname{sqrt}((n * \operatorname{lamv})))$

gamv=muv*sqrt(lamv)

gambar=mean (gamv); gamint=quantile $($ gamv, $c(0.025,0.975))$

c(gambar, gamint) \# 5.925 4.115 7.963

hist(gamv, prob=T,xlab="gamma", $x \lim =c(2,10), y \lim =c(0,0.5)$, breaks=seq $(0,12,0.25)$, main=" ")

abline(v=c(gambar, gamint), lwd=3); lines(density(gamv), lwd=3)

points(c(gam,gammle),c(0,0),pch=c(16,1))

legend(7,0.5,c("True value of gamma","MLE of gamma"), pch=c(16,1),bg="white")

\# (b) Follows on from (a)

$\mathrm{K}=100 ; \mathrm{J}=1000$; gambarvec=rep(NA,K); gammlevec=rep(NA,K);

gamlie=rep $(0, \mathrm{~K})$; 
set.seed(143); for( $\mathrm{k}$ in 1:K)\{

$\mathrm{y}=\operatorname{rnorm}(\mathrm{N}, \mathrm{mu}, \mathrm{sig}) ; \mathrm{s}=1: \mathrm{n} ; \mathrm{ys}=\mathrm{y}[\mathrm{s}] ; \mathrm{ysbar}=\operatorname{mean}(\mathrm{ys}) ; \mathrm{sys}=\mathrm{sd}(\mathrm{ys})$

lamv $=\operatorname{rgamma}\left(J,(n-1) / 2\right.$, sys $\left.^{\wedge} 2 *(n-1) / 2\right)$;

muv=rnorm $(J, y s b a r, 1 / \operatorname{sqrt}((n * l a m v)))$

gamv=muv*sqrt(lamv); gambar=mean(gamv);

gammlevec $[k]=y s b a r / s y s$

gamint=quantile (gamv,c(0.025,0.975)); gambarvec[k]=gambar if((gamint[1]<=gam)\&\&(gam<=gamint[2])) gamlie[k]=1 \}

Eest=mean(gambarvec);

Eci=Eest+c(-1,1)*qnorm(0.975)*sd(gambarvec)/sqrt(K)

Cest=mean(gamlie); Cci=Cest+c(-1,1)*qnorm(0.975)*sqrt(Cest*(1-Cest)/K)

c(Eest,Eci,Cest,Cci) \# 5.22264 .99865 .44660 .91000 .85390 .9661

Emleest=mean(gammlevec)

Emleci=Emleest+c(-1,1)*qnorm(0.975)*sd(gammlevec)/sqrt(K)

c(Emleest,Emleci) \# 5.2985 .0705 .526

Biasest=Eest-gam; Biasci=Eci-gam

Biasmleest=Emleest-gam; Biasmleci=Emleci-gam

c(Biasest,Biasci, Biasmleest,Biasmleci)

\# 0.222583 -0.001418 0.4465830 .2980190 .0704930 .525544

c(Biasest,Biasci, Biasmleest,Biasmleci)/gam

\# 0.0445165 -0.0002836 0.08931660 .05960370 .01409860 .1051088

\# hist(gambarvec,prob=T)

hist(gambarvec, prob=T,xlab="gammabar, gammahat", $x \lim =c(2,12)$, ylim=c(0,0.6), breaks=seq $(0,12,0.5)$, main= "'")

abline( $v=c($ Eest,Eci), Ity=1, Iwd=3); abline( $v=c($ Emleest,Emleci), Ity=2, Iwd=3)

lines(density(gambarvec), Ity=1,Iwd=3); lines(density(gammlevec), Ity=2,lwd=3)

points(gam,0,pch=16)

legend(6.5,0.6,c("Bayesian estimates \n(MC with J=1000)", "ML estimates"), Ity $=c(1,2), \operatorname{lwd}=c(3,3))$

\# (c)

$\mathrm{K}=5000 ; \mathrm{J}=1000$; gambarvec=rep(NA,K);

gammlevec=rep(NA,K); gamlie=rep(0,K); 
set.seed(213); for(k in 1:K)\{ \# Takes a few seconds

$\mathrm{y}=\operatorname{rnorm}(\mathrm{N}, \mathrm{mu}, \mathrm{sig}) ; \mathrm{s}=1: \mathrm{n} ; \mathrm{ys}=\mathrm{y}[\mathrm{s}] ; \mathrm{ysbar}=\operatorname{mean}(\mathrm{ys}) ; \mathrm{sys}=\mathrm{sd}(\mathrm{ys})$

lamv=rgamma(J,(n-1)/2, sys^2*(n-1)/2);

muv=rnorm $\left(J, y s b a r, 1 / \operatorname{sqrt}\left(\left(n^{*}\right.\right.\right.$ lamv $\left.\left.)\right)\right)$

gamv=muv*sqrt(lamv);

gambar=mean(gamv); gammlevec[k]=ysbar/sys

gamint=quantile (gamv,c(0.025,0.975)); gambarvec[k]=gambar if((gamint[1]<=gam)\&\&(gam<=gamint[2])) gamlie[k]=1 \}

Eest=mean(gambarvec);

Eci=Eest+c(-1,1)* qnorm(0.975)*sd(gambarvec)/sqrt(K)

Cest=mean(gamlie); Cci=Cest+c(-1,1)*qnorm(0.975)*sqrt(Cest*(1-Cest)/K)

c(Eest,Eci,Cest,Cci) \# 5.162 5.136 5.187 0.951 0.945 0.957

Emleest=mean(gammlevec)

Emleci=Emleest+c(-1,1)*qnorm(0.975)*sd(gammlevec)/sqrt(K)

c(Emleest,Emleci) \# 5.230 5.204 5.256

Biasest=Eest-gam; Biasci=Eci-gam

Biasmleest=Emleest-gam; Biasmleci=Emleci-gam

c(Biasest,Biasci, Biasmleest,Biasmleci)

\# 0.16160 .13590 .18720 .23010 .20410 .2561

c(Biasest,Biasci, Biasmleest,Biasmleci)/gam

\# 0.032310 .027180 .037450 .046020 .040810 .05122

\# hist(gambarvec,prob=T)

hist(gambarvec,prob=T,xlab="gammabar, gammahat", $x \lim =c(2,12)$, ylim=c(0,0.6), breaks=seq $(2,12,0.25)$, main= "')

abline( $v=c($ Eest,Eci), Ity=1, Iwd=3); abline( $v=c($ Emleest,Emleci), Ity=2, Iwd=3)

lines(density(gambarvec), Ity=1,Iwd=3); lines(density(gammlevec), Ity=2,lwd=3) points(gam,0,pch=16)

legend(6,0.6,c("Bayesian estimates \n(MC with J=1000)", "ML estimates"), Ity $=c(1,2), \operatorname{lwd}=c(3,3))$

hist(gambarvec,prob=T,xlab="gammabar, gammahat", $x \lim =c(4.5,6)$, ylim=c(0,0.6), breaks=seq $(2,12,0.25)$, main= "")

abline ( $v=c($ Eest,Eci), Ity=1, Iwd=3); abline( $v=c($ Emleest,Emleci), Ity=2, Iwd=3) lines(density(gambarvec), Ity=1,lwd=3); lines(density(gammlevec), Ity=2,lwd=3) points(gam,0,pch=16) 
\# (d)

psifun=function(y) $\{\max (\mathrm{y}) /$ median(y) $\}$ \# Function for the quantity of interest $\mathrm{N}=100 ; \mathrm{n}=20 ; \mathrm{mu}=10 ;$ sig=2; set.seed(119); $\mathrm{y}=\mathrm{rnorm}(\mathrm{N}, \mathrm{mu}, \mathrm{sig})$

ybar=mean $(y) ; s y=s d(y) ; p s i=p s i f u n(y=y)$

$c(y b a r, s y, \min (y), \max (y), \operatorname{median}(\mathrm{y}), \mathrm{psi})$

\# 9.6752 .1593 .67815 .62210 .1711 .536

hist(y,prob=T,xlab="value", $x \lim =c(0,20), y \lim =c(0,0.4)$, breaks=seq $(0,20,0.5)$, main="")

lines(seq(0,20,0.1),dnorm(seq(0,20,0.1),mu,sig),Iwd=3)

set.seed(421); ys=sample(y,n)

ys=y[s]; ysbar=mean(ys); sy=sd(ys); sy2=var(ys)

c(ysbar,sy, sy2) \# 9.438 2.4485 .994

hist(ys, prob=T,xlab="value", $x \lim =c(0,20), \operatorname{ylim}=c(0,0.4)$, breaks=seq $(0,20,0.5)$, main="")

lines(seq(0,20,0.1),dnorm(seq(0,20,0.1), mu,sig),lwd=3)

set.seed(323); J=1000;

lamv=rgamma $(J,(n-1) / 2$, sy $2 *(n-1) / 2) ;$ muv=rnorm $(J, y s b a r, 1 / \operatorname{sqr}((n * \operatorname{lamv})))$

psiv=rep(NA,J);

for(j in 1:J)\{ yrsim=rnorm(N-n,muv,1/sqrt(lamv)); ysim=c(ys,yrsim); $\operatorname{psiv}[\mathrm{j}]=\operatorname{psifun}(\mathrm{y}=\mathrm{ysim}) \quad\}$

psibar=mean(psiv); psiint=quantile $(p \operatorname{siv}, c(0.025,0.975))$

c(psibar,psiint) \# 1.7151 .4562 .078

summary(psiv)

\# Min. 1st Qu. Median Mean 3rd Qu. Max.

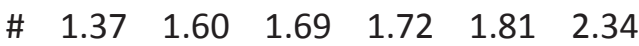

\# $\quad$ hist(psiv, prob=T)

hist(psiv,prob=T,xlab="psi", $x \lim =c(1.3,2.4)$, ylim=c(0,4),breaks=seq(1,2.5,0.05), main="")

abline(v=c(psibar,psiint), lwd=3); den=density(psiv)

lines(den,lwd=3); points(psi,0,pch=16)

psimedian=median (psiv)

psimode $=\operatorname{den} \$ x[\operatorname{den} \$ y==\max (\operatorname{den} \$ y)]$

c(psibar,psimedian,psimode) \# 1.7151 .6881 .659 
\# (e) Follows on from (d)

$K=100 ; \mathrm{J}=1000 ;$ psibarvec=rep(NA,K); LBvec= psibarvec; UBvec=LBvec;

alp $=0.05$

set.seed(411);

date() \#

for( $k$ in $1: K)\{$

ys=sample(y,n); ysbar=mean(ys); sy2=var(ys)

lamv=rgamma $(J,(n-1) / 2$, sy $2 *(n-1) / 2)$;

muv=rnorm(J,ysbar,1/sqrt ((n*lamv)))

psiv=rep(NA,J); for $(j$ in $1: J)\{$

yrsim=rnorm(N-n,muv,1/sqrt(lamv))

ysim=c(ys,yrsim)

$\operatorname{psiv}[j]=p \operatorname{sifun}(y=y \operatorname{sim})$

\}

psibarvec $[\mathrm{k}]=\operatorname{mean}(\mathrm{psiv})$;

LBvec[k]=quantile(psiv,alp/2); UBvec[k]=quantile(psiv,1-alp/2) \} ;

date() \# Simulation with $\mathrm{K}=100$ \& J=1000 takes 12 seconds

$c t=0 ;$ for $(k$ in $1: K)$ if $(($ LBvec $[k]<=p s i) \& \&(p s i<=U B v e c[k])) c t=c t+1$

\# hist(psibarvec,prob=T)

hist(psibarvec, prob=T,xlab="psibar", $x \lim =c(1.2,2), \operatorname{ylim}=c(0,6.5)$, breaks=seq $(1.2,2,0.025)$, main= "')

points(psi,0,pch=16)

\# Characteristics of posterior mean estimate

Eest=mean(psibarvec); Eci=Eest+c(-1,1)*qnorm(0.975)*sd(psibarvec)/sqrt(K)

Cest=ct/K; Cci=Cest+c(-1,1)*qnorm(0.975)*sqrt(Cest*(1-Cest)/K)

c(Eest,Eci,Cest,Cci) \# 1.61681 .59621 .63740 .91000 .85390 .9661

Biasest=Eest-psi; Biasci=Eci-psi; c(Biasest,Biasci) \# 0.080840 .060240 .10144

c(Biasest,Biasci)/psi \# 0.052630 .039220 .06604

abline( $v=c($ Eest,Eci), Ity=1, Iwd=3); lines(density(psibarvec), Ity=1,Iwd=3)

\# (f) Follows on from (e)

$\mathrm{K}=100 ; \mathrm{J}=1000 ; \mathrm{LBvec}=\operatorname{rep}(\mathrm{NA}, \mathrm{K}) ;$ UBvec=LBvec; $\mathrm{alp}=0.05$

psimodevec= LBvec; psimedianvec= LBvec; set.seed(411);

date() \# 
for $(k$ in $1: K)\{$

ys=sample(y,n); ysbar=mean(ys); sy2=var(ys)

lamv=rgamma(J,(n-1)/2,sy2*(n-1)/2);

muv=rnorm (J,ysbar, $1 / \operatorname{sqrt}\left(\left(n^{*}\right.\right.$ lamv $\left.\left.)\right)\right)$

psiv=rep(NA,J); for(j in 1:J)\{

yrsim=rnorm(N-n,muv,1/sqrt(lamv))

ysim=c(ys,yrsim)

$\operatorname{psiv}[\mathrm{j}]=\operatorname{psifun}(\mathrm{y}=\mathrm{ysim})$

\}

psimedianvec $[\mathrm{k}]=\operatorname{median}(\mathrm{psiv})$

den=density(psiv); psimodevec[k]=den\$x[den\$y==max(den\$y)]

LBvec[k]=quantile(psiv,alp/2); UBvec[k]=quantile(psiv,1-alp/2)

\}

date() \# Simulation with $\mathrm{K}=100$ \& J=1000 takes 12 seconds

$\mathrm{ct}=0$; for $(\mathrm{k}$ in $1: \mathrm{K})$ if $((\mathrm{LBvec}[\mathrm{k}]<=\mathrm{psi}) \& \&(\mathrm{psi}<=\mathrm{UBvec}[\mathrm{k}])) \mathrm{ct}=\mathrm{ct}+1$

\# hist(psimedianvec, prob=T)

hist(psimedianvec, prob=T,xlab="psimedian", $x \lim =c(1.2,2)$, ylim=c(0,6),breaks=seq $(1.2,2,0.025)$, main= "')

points(psi,0,pch=16)

\# Characteristics of posterior median estimate

Eest $=$ mean (psimedianvec);

Eci=Eest+c(-1,1)*qnorm $(0.975) *$ sd(psibarvec)/sqrt(K)

Cest=ct/K; Cci=Cest+c(-1,1)* qnorm(0.975)*sqrt(Cest*(1-Cest)/K)

c(Eest,Eci,Cest,Cci) \# 1.59471 .57411 .61530 .91000 .85390 .9661

Biasest=Eest-psi; Biasci=Eci-psi; c(Biasest,Biasci) \# 0.058730 .038130 .07934

c(Biasest,Biasci)/psi \# 0.038240 .024830 .05165

abline ( $v=c($ Eest,Eci), Ity=1, Iwd=3); lines(density(psimedianvec), Ity=1,Iwd=3)

\# hist(psimodevec,prob=T)

hist(psimodevec, prob=T,xlab="psimode", $x \lim =c(1.2,2)$,

ylim=c(0,6), breaks=seq(1.2,2,0.025), main= "")

points(psi,0,pch=16)

\# Characteristics of posterior mode estimate

Eest=mean(psimodevec); Eci=Eest+c(-1,1)*qnorm $(0.975) * s d(p s i b a r v e c) / s q r t(K)$

Cest=ct/K; Cci=Cest+c(-1,1)*qnorm(0.975)*sqrt(Cest*(1-Cest)/K)

c(Eest,Eci,Cest,Cci) \# 1.55791 .53731 .57850 .91000 .85390 .9661

Biasest=Eest-psi; Biasci=Eci-psi; c(Biasest,Biasci)

\# 0.0219330 .0013320 .042534

c(Biasest,Biasci)/psi \# 0.01427950 .00086720 .0276917

abline $(v=c($ Eest,Eci), Ity=1, Iwd=3); lines(density(psimodevec), Ity=1,lwd=3) 


\section{Biased Sampling and Nonresponse}

\section{2. I Review of sampling mechanisms}

We have already discussed the topic of ignorable and nonignorable sampling in the context of Bayesian finite population models. To be definite, let us now focus on the model defined by:
$f(s \mid y, \theta) \quad$ (the probability of obtaining sample $s$ for given values of $y$ and $\theta$ )
$f(y \mid \theta) \quad$ (the model density of the finite population vector)
$f(\theta) \quad$ (the prior density of the parameter),

where the data is $D=\left(s, y_{s}\right)$ and the quantity of interest is some functional $\psi=g(\theta, y)$, e.g. a function of two components of $\theta$ or a function of $y$ only, etc.

We say that the sampling mechanism is ignorable if

$$
f\left(\psi \mid s, y_{s}\right)=f\left(\psi \mid y_{s}\right)
$$

for all values of $\psi$, where $s$ is fixed at its observed value, or equivalently, if the posterior distribution of $\psi$ is exactly the same when it is calculated solely on the basis of the 'reduced model' as given by:

$$
\begin{array}{ll}
f(y \mid \theta) & \text { (same as before) } \\
f(\theta) & \text { (same as before), }
\end{array}
$$

that is, with $f(s \mid y, \theta)$ effectively being 'ignored'. Otherwise, we say that the sampling mechanism is nonignorable (or biased).

Equivalently, the sampling mechanism is ignorable if

$$
f\left(\psi \mid s, y_{s}\right)=f\left(\psi \mid y_{s}\right)
$$

for all $\psi$, and the sampling mechanism is nonignorable if

$$
f\left(\psi \mid s, y_{s}\right) \neq f\left(\psi \mid y_{s}\right)
$$

for at least one value of $\psi$.

Recall that in some situations, whether the sampling mechanism is ignorable may depend on which particular units happen to be sampled. 
For example if $f(s \mid y, \theta)$ is a function of only $N, n$ and $y_{3}$ (say), then (typically) the sampling mechanism is ignorable if and only if unit 3 is sampled (and thereby observed).

Also, recall that analogous definitions apply if the sampling mechanism is alternatively specified in terms of

$$
f(I \mid y, \theta)
$$

or in terms of

$$
f(L \mid y, \theta),
$$

rather than in terms of

$$
f(s \mid y, \theta) \text {. }
$$

Here, as previously, $I=\left(I_{1}, \ldots, I_{N}\right)$ denotes the vector of inclusion counters, i.e. the numbers of times units $1, \ldots, N$ are sampled (possibly more than once in the case of sampling with replacement), and $L=\left(L_{1}, \ldots, L_{n}\right)$ is the vector of the labels of the units sampled in the temporal order in which they are sampled.

\section{2.2 Nonresponse mechanisms}

An issue related to nonignorable sampling is nonignorable nonresponse. Once a sample has been taken, some of the units may then fail to respond. This may be for whatever reason, but the underlying issue is that the values of the nonresponding units will then be unobserved, with possibly serious consequences to the resulting inference.

This issue can be addressed by introducing another variable and level into the modelling equation. Let $R_{i}$ denote the $i$ th response indicator, meaning the indicator variable for the $i$ th population unit responding.

Thus $R_{i}=1$ if unit $i$ responds, and $R_{i}=0$ otherwise $(i=1, \ldots, N)$.

Now let $R=\left(R_{1}, \ldots, R_{N}\right)$ (or the transpose of this) be called the population response vector, and likewise, define:

$$
\begin{aligned}
& R_{s}=\left(R_{S_{1}}, \ldots, R_{S_{n}}\right) \text { as the sample response vector } \\
& R_{r}=\left(R_{r_{1}}, \ldots, R_{r_{N-n}}\right) \text { as the nonsample response vector. }
\end{aligned}
$$


With these definitions we may now augment our 'base model' above with a new level in the hierarchy, typically in between $y$ and $s$, as follows:

$$
\begin{array}{ll}
f(s \mid R, y, \theta) & \text { (the probability of obtaining sample } s \text { for given } \\
& \text { values of } R, y \text { and } \theta \text { ) } \\
f(R \mid y, \theta) & \text { (the probability of units responding as indicated } \\
& \text { by } R \text {, given } y \text { and } \theta \text { ) } \\
f(y \mid \theta) & \text { (same as before) } \\
f(\theta) & \text { (same as before) }
\end{array}
$$

Note 1: This general formulation, with $f(s \mid R, y, \theta)$ a function of $R$, means that which units are sampled could potentially depend on which units would respond if sampled. However, typically it will be reasonable to assume that the sampling and response mechanisms are independent in the model, meaning that $f(s \mid R, y, \theta)=f(s \mid y, \theta)$.

Note 2: The statistical literature contains many different and sometimes inconsistent treatments of nonignorable nonresponse. For a review of the term 'missing at random', which relates to but does not feature in the exposition here, see Seaman et al. (2013).

In the context of this model, let

$$
n_{o}=R_{s_{1}}+\ldots+R_{s_{n}}=1_{n}^{\prime} R_{s}
$$

be the number of values in the sample that respond (have a value that is observed), and let

$$
n_{u}=n-n_{o}
$$

be the number of units in the sample that do not respond (have a value that is unobserved).

Then define

$$
o=\left(o_{1}, \ldots, o_{n_{o}}\right)
$$

as the observed vector (the vector of the labels of the units sampled and observed), and define

$$
u=\left(u_{1}, \ldots, u_{n_{u}}\right)
$$

as the unobserved vector (the vector of the labels of the units sampled and unobserved). 
Note: In each of these vectors, the values (labels) are assumed to be in increasing order.

Then define the observed sample vector as

$$
y_{o}=\left(y_{o_{1}}, \ldots, y_{o_{n_{o}}}\right)
$$

and the unobserved sample vector as

$$
y_{u}=\left(y_{u_{1}}, \ldots, y_{u_{n_{u}}}\right) \text {. }
$$

With these definitions, the data has the general form

$$
D=\left(s, R_{s}, y_{o}\right)
$$

and also the quantity of interest has the general form

$$
\psi=g(\theta, y, R) \text {. }
$$

Note 1: The function $g$ defining $\psi$ takes into account the possibility there may be interest in whether some of the nonsampled units would have responded had they been sampled.

Note 2: As mentioned previously, it is often convenient to re-label the $N$ finite population values in such a way that

$$
\begin{aligned}
y=\left(y_{s}, y_{r}\right) & =\left(y_{o}, y_{u}, y_{r}\right) \\
& =\left(\left(y_{1}, \ldots, y_{n_{o}}\right),\left(y_{n_{o}+1}, \ldots, y_{n}\right),\left(y_{n+1}, \ldots, y_{N}\right)\right) \\
& =\left(y_{1}, \ldots, y_{N}\right) .
\end{aligned}
$$

In the context of the general four-level Bayesian finite population model given by (12.1) above (which involves $s, R, y$ and $\theta$ ), we may make the following definitions:

- The sampling mechanism is ignorable if

$$
f\left(\psi \mid s, R_{s}, y_{o}\right)=f\left(\psi \mid R_{s}, y_{o}\right) \forall \psi
$$

with $s$ fixed at its observed value (note that $o$ is a function of $s$ and $R_{s}$ ); otherwise the sampling mechanism is nonignorable.

- The response mechanism is ignorable if

$$
f\left(\psi \mid s, R_{s}, y_{o}\right)=f\left(\psi \mid s, y_{o}\right) \forall \psi
$$

with $o$ fixed at its observed value; otherwise the response mechanism is nonignorable. 
These two basic definitions then lead to four general cases, defined as follows:

- The sampling mechanism and response mechanism are both ignorable if

$$
f\left(\psi \mid s, R_{s}, y_{o}\right)=f\left(\psi \mid y_{o}\right) \forall \psi
$$

with $o$ fixed at its observed values.

- The sampling mechanism is ignorable and the response mechanism is nonignorable if

$$
f\left(\psi \mid s, R_{s}, y_{o}\right)=f\left(\psi \mid R_{s}, y_{o}\right) \forall \psi
$$

with $s$ fixed at its observed value, and

$$
f\left(\psi \mid R_{s}, y_{o}\right) \neq f\left(\psi \mid y_{o}\right)
$$

for at least one value of $\psi$.

- The response mechanism is ignorable and the sampling mechanism is nonignorable if

$$
f\left(\psi \mid s, R_{s}, y_{o}\right)=f\left(\psi \mid s, y_{o}\right) \forall \psi
$$

with $o$ fixed at its observed value and

$$
f\left(\psi \mid s, y_{o}\right) \neq f\left(\psi \mid y_{o}\right)
$$

for at least one value of $\psi$.

- The sampling mechanism and response mechanism are both nonignorable if

$$
f\left(\psi \mid s, R_{s}, y_{o}\right) \neq f\left(\psi \mid R_{s}, y_{o}\right)
$$

for at least one value of $\psi$ and

$$
f\left(\psi \mid s, R_{s}, y_{o}\right) \neq f\left(\psi \mid s, y_{o}\right)
$$

for at least one value of $\psi$. 


\section{Exercise 12.I A model with sampling and response mechanisms that are both ignorable}

Consider a Bayesian finite population model defined by:

$$
\begin{aligned}
& f(s \mid R, y, \theta) \\
& f(R \mid y, \theta) \\
& f(y \mid \theta) \\
& f(\theta),
\end{aligned}
$$

where the data is

$$
D=\left(s, R_{s}, y_{o}\right)
$$

and the quantity of interest is

$$
\psi=g(\theta, y, R)=1_{N}^{\prime} y=\sum_{i=1}^{N} y_{i}=y_{T} \quad \text { (finite population total). }
$$

Suppose that in this context:

- the sample of $n$ units is taken from the $N$ in the population via SRSWOR

- each unit in the population has the same probability of response, $\pi$

- the population values in the model are iid, each with a distribution which depends only on a single parameter $\mu$

- the model parameter vector is

$$
\theta=(\mu, \pi)
$$

with $\mu \perp \pi$ (thus the two model parameters are independent, a priori).

Show that the sampling mechanism and response mechanism are both ignorable, and that this is true for all possible values of the data. 


\section{Solution to Exercise I 2. I}

Observe that for all s, $R, y$ and $\theta$ :

$$
\begin{aligned}
& f(s \mid R, y, \theta)=f(s)=\left(\begin{array}{c}
N \\
n
\end{array}\right)^{-1} \\
& f(R \mid y, \theta)=f(R)=\prod_{i=1}^{N} \pi^{R_{i}}(1-\pi)^{1-R_{i}} \\
& y_{T}=y_{o T}+y_{u T}+y_{r T},
\end{aligned}
$$

where:

$$
\begin{aligned}
& y_{o T}=1_{o}^{\prime} y_{o}=\sum_{i \in o} y_{i} \text { is the total of the observed sample values } \\
& y_{u T}=1_{u}^{\prime} y_{u}=\sum_{i \in u} y_{i} \text { is the total of the unobserved sample values } \\
& y_{r T}=1_{r}^{\prime} y_{r}=\sum_{i \in r} y_{i} \text { is the total of the nonsample values. }
\end{aligned}
$$

Note: Here, $1_{o}^{\prime}$ denotes a column vector of $n_{o}$ ones, etc.

Consequently, the relevant predictive density of the quantity of interest, namely

$$
f(\psi \mid D)=f\left(y_{T} \mid s, R_{s}, y_{o}\right),
$$

is derived from the joint predictive density of all unobserved and nonsampled values, namely

$$
f\left(y_{u}, y_{r} \mid s, R_{s}, y_{o}\right) \text {. }
$$

We will now proceed to show that

$$
f\left(y_{u}, y_{r} \mid s, R_{s}, y_{o}\right)=f\left(y_{u}, y_{r} \mid y_{o}\right)
$$

with $o$ fixed at its observed value, and that this is true for all possible values of $y_{u}, y_{r}, s, R_{s}$ and $y_{o}$.

If this can be shown then also

$$
f\left(y_{T} \mid s, R_{s}, y_{o}\right)=f\left(y_{T} \mid y_{o}\right) \text {, }
$$

for all possible values of $y_{T}, s, R_{s}$ and $y_{0}$.

It will thereby be established that the sampling mechanism and response mechanism are both ignorable, and that this is true for all possible values of the data $D=\left(s, R_{s}, y_{o}\right)$. 
Observe that for any $y_{u}, y_{r}, s, R_{s}$ and $y_{o}$, it is true that

$$
\begin{aligned}
& f\left(y_{u}, y_{r} \mid s, R_{s}, y_{o}\right) \propto f\left(y_{u}, y_{r}, s, R_{s}, y_{o}\right) \\
& =\sum_{R_{r}} \iint f\left(y_{u}, y_{r}, s, R_{s}, y_{o}, R_{r}, \mu, \pi\right) d \mu d \pi \\
& =\sum_{R_{r}} \iint f(\mu) f(\pi) f\left(y_{o} \mid \mu\right) f\left(y_{u}, y_{r} \mid \mu, y_{o}\right) \\
& \times f\left(R_{s} \mid \pi\right) f\left(R_{r} \mid \pi\right) f(s) d \mu d \pi \\
& =f(s) \times \int f\left(y_{u}, y_{r} \mid \mu, y_{o}\right)\left\{f(\mu) f\left(y_{o} \mid \mu\right)\right\} d \mu \\
& \times\left[\int f(\pi) f\left(R_{s} \mid \pi\right) \sum_{R_{r}} f\left(R_{r} \mid \pi\right) d \pi\right] \\
& \text { where }[\cdot]=\int f\left(\pi, R_{s}\right) \times 1 d \pi=f\left(R_{s}\right)
\end{aligned}
$$

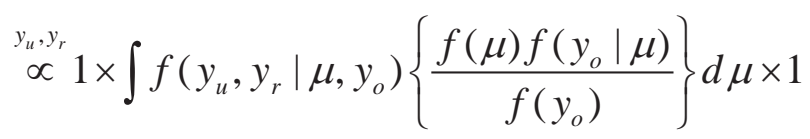

$$
\begin{aligned}
& =\int f\left(y_{u}, y_{r} \mid \mu, y_{o}\right) f\left(\mu \mid y_{o}\right) d \mu \\
& =\int f\left(y_{u}, y_{r}, \mu \mid y_{o}\right) d \mu \\
& =f\left(y_{u}, y_{r} \mid y_{o}\right) \text {. }
\end{aligned}
$$

That is,

$$
f\left(y_{u}, y_{r} \mid s, R_{s}, y_{o}\right)=f\left(y_{u}, y_{r} \mid y_{o}\right),
$$

as required. 


\section{Exercise I 2.2 An ignorable sampling mechanism with a nonignorable response mechanism}

A finite population consists of $N=500$ values that are modelled as normally distributed with unknown mean $\mu$ and unknown variance $\sigma^{2}=1 / \lambda$. A sample of size $n=100$ is taken from this population via SRSWOR. We find that only $n_{o}=34$ values are observed, with values:

$12.57,13.35,11.47,14.81,13.25, \quad 14.09,11.55,11.32,13.2,11.28$,

$9.7,12.18,11.49,10.52,9.93, \quad 11.84,12.2,10.57,11.9,14.75$,

$10.34,14.37,12.13,8.56,11.91, \quad 11.79,11.45,14.98,10.57,12.28$,

$9.91,10.94,13.28,11.43$.

(a) Assuming that the response mechanism is ignorable, estimate the finite population mean.

(b) A follow-up sample of size $n_{f}=15$ is taken from the $n_{u}=66$ nonresponding units via SRSWOR, and these $n_{f}$ units are observed (by 'force'), yielding the values:

$5.4,9.41,7.03,8.88,11.47$,

$7,9.44,8.58,9.27,8.18$

$8.62,8.73,7.33,9.81,9.88$.

Thus there remain $n-n_{o}-n_{f}=n_{u}-n_{f}=51$ nonresponding sample units with unknown values.

Assuming that the response mechanism is ignorable, use all of the available data to re-estimate the finite population mean.

(c) Repeat (b) but using a suitable Bayesian model which takes into account the response mechanism and appropriately incorporates it into the inferential procedure.

\section{Solution to Exercise I 2.2}

(a) We estimate $\bar{y}$ by the average of the $n_{o}=34$ observed values, which is $\bar{y}_{o}=11.94$. The sample standard deviation of these $n_{o}$ values is equal to $s_{o}=1.552$. So a 95\% CPDR for $\bar{y}$ is

$$
\left(\bar{y}_{o} \pm t_{0.025}\left(n_{o}-1\right) \frac{s_{o}}{\sqrt{n_{o}}} \sqrt{1-\frac{n_{o}}{N}}\right)=(11.42,12.46) \text {. }
$$


(b) We estimate $\bar{y}$ by the average of all $n_{\text {of }}=n_{o}+n_{f}=34+15=49$ observed values, which is equal to $\bar{y}_{\text {of }}=10.92$. The sample standard deviation of these $n_{\text {of }}$ values is $s_{\text {of }}=2.168$. So a 95\% CPDR for $\bar{y}$ is

$$
\left(\bar{y}_{\text {of }} \pm t_{0.025}\left(n_{\text {of }}-1\right) \frac{s_{\text {of }}}{\sqrt{n_{\text {of }}}} \sqrt{1-\frac{n_{\text {of }}}{N}}\right)=(10.33,11.51) \text {. }
$$

(c) Figure 12.1 is a histograms of the $n_{o}=34$ initially observed values and the $n_{f}=15$ follow-up values, respectively. We see that the 'forced' follow-up values which initially failed to respond seem to be smaller on average than the values of the units which responded. This suggests a biased or nonignorable nonresponse mechanism whereby units with large values are more likely to respond than units with small values.

\section{Figure I 2. I Initially observed and and follow-up sample values}

Initially observed

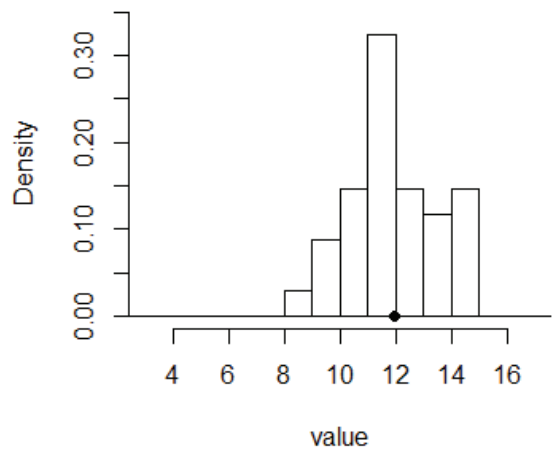

Follow-up

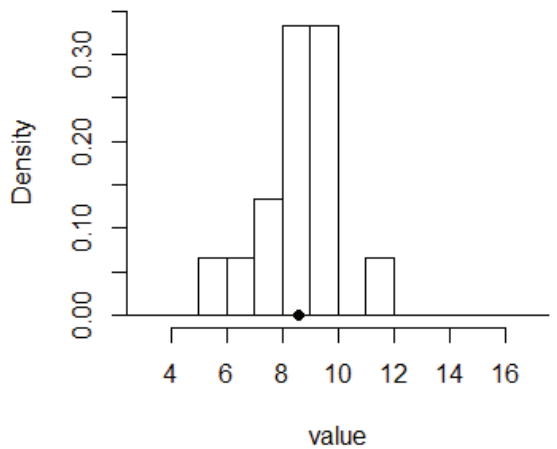

One way (amongst several) to model such a response mechanism is via the formulation

$$
\left(R_{i} \mid y, \mu, \lambda\right) \sim \perp \operatorname{Bernoulli}\left(p_{i}\right), \quad i=1, \ldots, N,
$$

where

$$
\log \left(\frac{p_{i}}{1-p_{i}}\right)=a+b y_{i}
$$

is the logit of the probability of unit $i$ responding. 
Noting that the sampling mechanism is ignorable, and that the response mechanism would be ignorable if all $n$ sample values were known, we posit a suitable Bayesian model as follows:

$$
\begin{aligned}
& \left(\bar{y} \mid y_{r T}, R_{s}, y_{s}, \mu, \lambda\right) \sim\left(\bar{y} \mid y_{r T}, y_{s}\right)=\frac{1}{N}\left(y_{s T}+y_{r T}\right) \\
& \left(y_{r T} \mid R_{s}, y_{s}, \mu, \lambda\right) \sim\left(y_{r T} \mid \mu, \lambda\right) \sim N\left((N-n) \mu, \frac{N-n}{\lambda}\right) \\
& f\left(R_{s} \mid y_{s}, \mu, \lambda\right)=f\left(R_{s} \mid y_{s}\right)=\prod_{i \in s} p_{i}^{R_{i}}\left(1-p_{i}\right)^{1-R_{i}} \\
& \quad \text { where } p_{i}=\frac{1}{1+e^{-\left(a+b y_{i}\right)}} \\
& f\left(y_{s} \mid \mu, \lambda\right)=\prod_{i \in s} \frac{\sqrt{\lambda}}{\sqrt{2 \pi}} e^{-\frac{\lambda}{2}\left(y_{i}-\mu\right)^{2}} \\
& f(\mu, \lambda) \propto 1 / \lambda, \mu \in \mathfrak{R}, \lambda>0 .
\end{aligned}
$$

Note: There is no need to include the nonsample response vector $R_{r}$ in the model.

Let $m=s-o-f=u-f$ be the vector of labels for the units which are sampled but still 'missing' after the follow-up sample has been observed.

Then the joint posterior/predictive density of all the relevant unknowns in the model may be written

$$
\begin{aligned}
& f\left(y_{r T}, \mu, \lambda, y_{m} \mid R_{s}, y_{o}, y_{f}\right) \propto f\left(y_{r T}, \mu, \lambda, y_{m}, R_{s}, y_{o}, y_{f}\right) \\
&=f(\mu, \lambda) \times\left\{f\left(y_{o} \mid \mu, \lambda\right) f\left(y_{f} \mid \mu, \lambda\right) f\left(y_{m} \mid \mu, \lambda\right\}\right. \\
& \times\left\{f\left(R_{o} \mid y_{s}\right) f\left(R_{f} \mid y_{s}\right) f\left(R_{m} \mid y_{s}\right)\right\} \times f\left(y_{r T} \mid \mu, \lambda\right) \\
& \propto \frac{1}{\lambda} \times \prod_{i \in o} \frac{\sqrt{\lambda}}{\sqrt{2 \pi}} e^{-\frac{\lambda}{2}\left(y_{i}-\mu\right)^{2}} \prod_{i \in f} \frac{\sqrt{\lambda}}{\sqrt{2 \pi}} e^{-\frac{\lambda}{2}\left(y_{i}-\mu\right)^{2}} \prod_{i \in m} \frac{\sqrt{\lambda}}{\sqrt{2 \pi}} e^{-\frac{\lambda}{2}\left(y_{i}-\mu\right)^{2}} \\
& \times \prod_{i \in o} p_{i}^{1}\left(1-p_{i}\right)^{1-1} \prod_{i \in f} p_{i}^{0}\left(1-p_{i}\right)^{1-0} \prod_{i \in m} p_{i}^{0}\left(1-p_{i}\right)^{1-0} \\
& \times \frac{\sqrt{\lambda}}{\sqrt{N-n} \sqrt{2 \pi}} e^{-\frac{\lambda}{2(N-n)}\left(y_{i}-(N-n) \mu\right)^{2}} .
\end{aligned}
$$


This joint density defines a suitable Metropolis-Hastings algorithm with Gibbs steps that could be run to obtain a Monte Carlo sample from the predictive distribution of the finite population mean $\bar{y}$.

One way to proceed is to implement this algorithm using WinBUGS and the code shown below (underneath the $\mathrm{R}$ Code below). Some of the results are as shown in Table 12.1. These inferences are based on $J=10,000$ iterations of a WinBUGS run, following an initial burn-in of size 1,000 .

\section{Table I 2.I Results of WinBUGS analysis}

\begin{tabular}{lllllll} 
node & mean & sd & MC error & $\mathbf{2 . 5 \%}$ & median & $\mathbf{9 7 . 5 \%}$ \\
\hline a & -17.86 & 4.582 & 0.4184 & -26.96 & -17.79 & -10.31 \\
b & 1.676 & 0.4535 & 0.04136 & 0.9301 & 1.672 & 2.586 \\
lam & 0.1921 & 0.04236 & 0.001112 & 0.118 & 0.189 & 0.2828 \\
mu & 9.688 & 0.3508 & 0.01358 & 8.976 & 9.693 & 10.35 \\
ps[1] & 0.9348 & 0.07378 & 0.006256 & 0.7572 & 0.959 & 0.997 \\
ps[2] & 0.9721 & 0.0535 & 0.004619 & 0.8664 & 0.9886 & 0.9996 \\
$\ldots \ldots \ldots \ldots \ldots \ldots \ldots \ldots \ldots \ldots \ldots \ldots \ldots \ldots \ldots \ldots \ldots \ldots$ & $\ldots \ldots \ldots \ldots \ldots \ldots \ldots \ldots \ldots \ldots \ldots \ldots \ldots \ldots$ & $\ldots \ldots \ldots \ldots$ \\
ps[99] & 0.1417 & 0.2097 & 0.003545 & $1.224 \mathrm{E}-5$ & 0.04017 & 0.7787 \\
ps[100] & 0.1423 & 0.2101 & 0.003883 & $1.12 \mathrm{E}-5$ & 0.03954 & 0.7731 \\
ybar & 9.687 & 0.3353 & 0.01329 & 9.013 & 9.696 & 10.32 \\
yrT & 3874.0 & 147.9 & 5.408 & 3573.0 & 3878.0 & 4156.0 \\
\hline
\end{tabular}

From Table 12.1, we estimate the posterior mean of $\bar{y}$ by 9.69 and we estimate the 95\% CPDR for $\bar{y}$ as $(9.01,10.32)$. It will be noted that this inference is significantly lower than the inferences in (a) and (b) where the response mechanism was taken as ignorable. Some of the graphical output from the WinBUGS run are shown in Figure 12.2

\section{Figure I 2.2 Graphical output from WinBUGS}

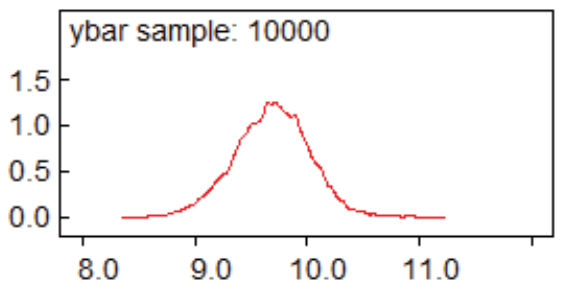




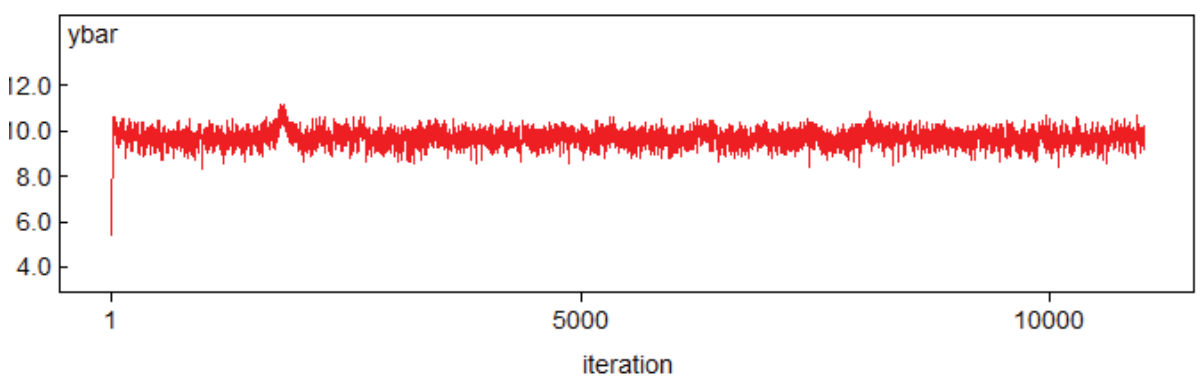

\section{Discussion}

It is instructive to now reveal that the data values in this exercise were in fact generated as follows.

First, a finite population of size $N=500$ was generated from the normal distribution with mean $\mu=10$ and standard deviation $\sigma=2$. The mean of the finite population values was calculated as $\bar{y}=10.10$.

Note: We see that the CPDR in (c), $(9.013,10.32)$, contains this true value of $\bar{y}$, whereas the CPDRs in (a) and (b), (11.42, 12.46) and (10.33, 11.51), do not. This suggests the analysis in (c) was on the right track.

Then a random sample of size $n=100$ was taken from the finite population according to SRSWOR. The sample mean was calculated as $\bar{y}_{s}=9.91$.

Note: Thus, if there had been no nonresponse then the finite population mean (with true value 10.10) would have been estimated by 9.91 .

Figure 12.3 shows histograms of the population and sample values, each overlaid by the superpopulation density. The dots in the two subplots show $\bar{y}=10.10$ and $\bar{y}_{s}=9.91$, respectively. 


\section{Figure I2.3 Histograms of the population and sample values}

Population

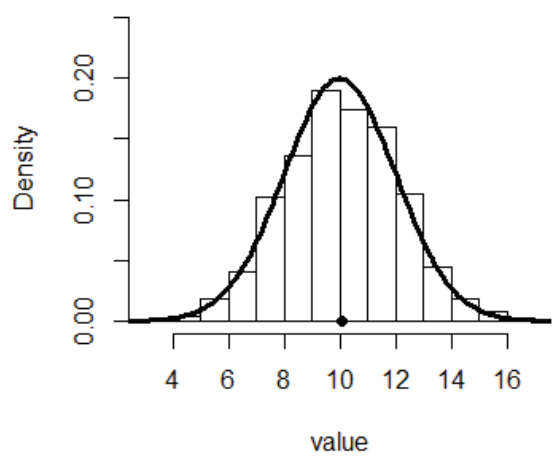

Sample

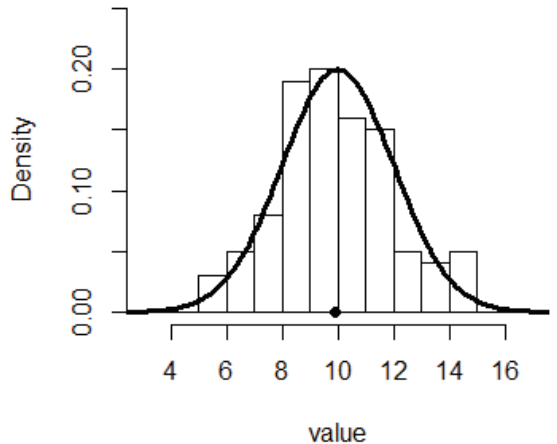

Then the probabilities of response were calculated as

$$
p_{i}=\frac{1}{1+e^{-\left(a+b y_{i}\right)}}
$$

with $a=-15$ and $b=1.4$ (set in advance).

Using these probabilities, it was next determined which units would respond, by sampling

$$
R_{i} \sim \operatorname{Bernoulli}\left(p_{i}\right)
$$

for each $i=1, \ldots, N$.

Thereby it was established which sample units would respond and which would not. Figure 12.4 shows histograms of these two groups (of size $n_{o}=34$ and $n_{u}=66$ ), each overlaid by the superpopulation density. The dots in the left and right subplots show $\bar{y}_{o}$ and $\bar{y}_{u}$, respectively, and each histogram is overlaid by the superpopulation density.

We see how the respondent values are systematically larger than the nonrespondent values. This reflects the fact that units with larger values were more likely to respond. 
Figure I 2.4 Observed and unobserved (non-responding) sample values
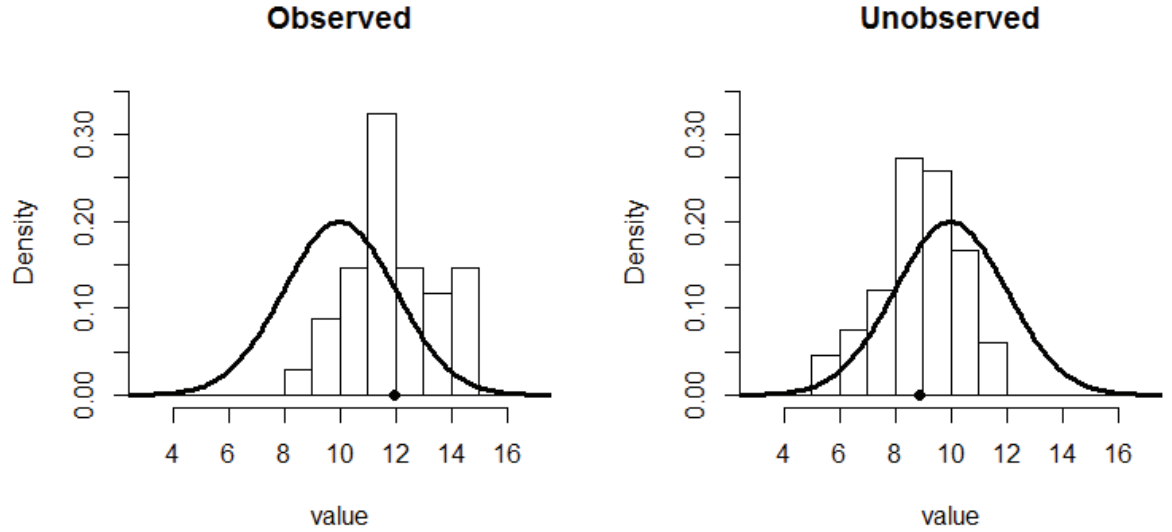

Figure 12.5 shows all $N$ probabilities of response $p_{1}, \ldots, p_{N}$ plotted against the population values $y_{1}, \ldots, y_{N}$. The crosses indicate population units which would not respond if sampled, and these naturally tend to be the units with the smallest values.

Figure I 2.5 Probabilities of response in the population

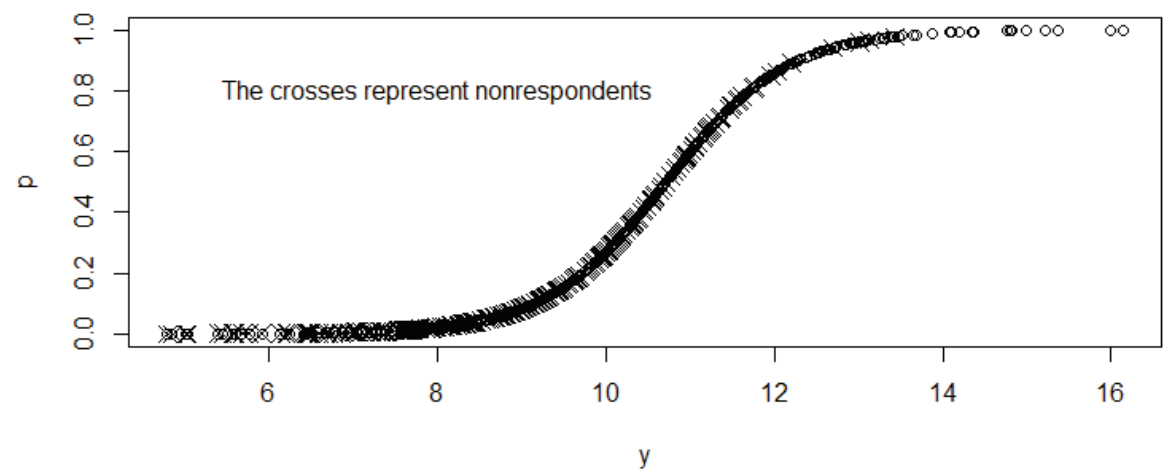

Likewise, Figure 12.6 shows the $n$ probabilities of response in the sample plotted against the sample values. The crosses indicate sample units which did not respond in actuality, and these tend to be the units with the smallest values. The solid dots indicate the 15 units which were selected for 'forced' follow-up according to SRSWOR (from the 66 nonresponding sample units). Without these 15 'representative' follow-up 
values it would have been impossible to appropriately address the nonignorable nonresponse problem and correct the biased inference in (a) and (b) downward.

\section{Figure I2.6 Probabilities of response in the sample}

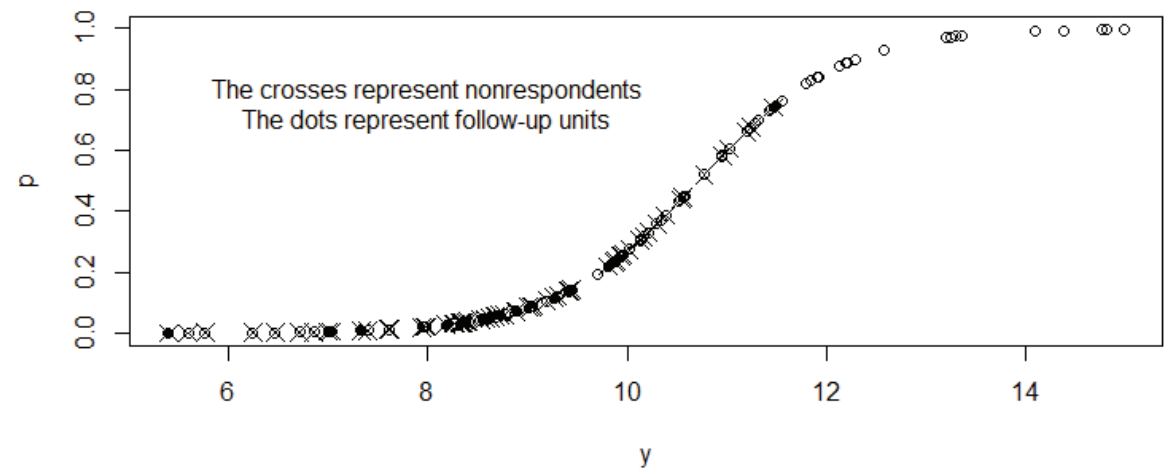

\section{R Code for Exercise 12.2}

\# Preliminary: Data generation and description ===========

$\mathrm{X} 11(\mathrm{w}=8, \mathrm{~h}=4) ; \operatorname{par}(\operatorname{mfrow}=\mathrm{c}(1,1)) ; \quad$ options$($ digits $=4)$;

$\mathrm{N}=500 ; \mathrm{n}=100 ; \mathrm{mu}=10 ; \mathrm{sig}=2 ; \mathrm{a}=-15 ; \mathrm{b}=1.4$;

set.seed(421); $\mathrm{y}=\mathrm{rnorm}(\mathrm{N}, \mathrm{mu}, \mathrm{sig}) \quad \# \mathrm{~N}$ finite population values

$\mathrm{p}=1 /\left(1+\exp \left(-\left(a+b^{*} y\right)\right)\right)$ \# N probabilities of response (logistic)

$\operatorname{plot}(\mathrm{y}, \mathrm{p})$ \# OK

set.seed(123); $R=$ rbinom( $N, 1, p)$ \# $N$ response indicators

set.seed(421); s=sort(sample(1:N,n)) \# n sample labels

$r=(1: N)[-s] \quad \# N-n$ nonsample labels

$\mathrm{ys}=\mathrm{y}[\mathrm{s}] \quad \# \mathrm{n}$ sample values

$\mathrm{yr}=\mathrm{y}[\mathrm{r}] \quad$ \#N-n nonsample values

$\mathrm{Rs}=\mathrm{R}[\mathrm{s}] \quad$ \# $\mathrm{n}$ sample response indicators

$\mathrm{Rr}=\mathrm{R}[\mathrm{r}] \quad$ \# N-n nonsample response indicators

$n o=\operatorname{sum}(R s) ; n u=n-n o ; c(n o, n u)$

\# 3466 numbers of observed and unobserved units

$\mathrm{o}=\mathrm{s}[\mathrm{Rs}==1] \quad$ \# labels of observed sample values

$\mathrm{u}=\mathrm{s}[\mathrm{Rs}==0] \quad$ \# labels of unobserved sample values 
rbind(s[1:10],Rs[1:10])

$\begin{array}{llllllllllll}\#[1,] & 6 & 7 & 14 & 17 & 22 & 37 & 39 & 48 & 66 & 69 \\ \#[2,] & 0 & 0 & 1 & 0 & 1 & 0 & 0 & 0 & 1 & 1 & \\ o[1: 5] & \# 14 & 22 & 66 & 69 & 78 & \text { Correct } & & \\ u[1: 5] & \# 6 & 7 & 17 & 37 & 39 & \text { Correct }\end{array}$

$\mathrm{yo}=\mathrm{y}[\mathrm{o}] ; \mathrm{yu}=\mathrm{y}[\mathrm{u}]$

ybar=mean(y); ysbar=mean(ys); yrbar=mean $(y r)$;

yobar=mean(yo); yubar=mean(yu)

c(ybar,ysbar,yrbar,yobar,yubar) \# 10.0959 .90710 .14311 .9388 .860

\# Plot population and sample values

$\operatorname{par}(\operatorname{mfrow}=c(1,2))$

hist(y,prob=T,xlab="value", main="Population", $x \lim =c(3,17), y \lim =c(0,0.25)$, breaks $=\operatorname{seq}(0,20,1))$

lines(seq(0,20,0.1), dnorm(seq(0,20,0.1), mu,sig), Iwd=3)

points(ybar, 0, pch $=16)$

hist(ys, prob=T,xlab="value", main="Sample", $x \lim =c(3,17), y \lim =c(0,0.25)$, breaks=seq $(0,20,1))$

lines(seq(0,20,0.1),dnorm(seq(0,20,0.1), mu,sig), Iwd=3)

points(ysbar,0,pch=16)

\# Plot observed and unobserved sample values

$\operatorname{par}(\operatorname{mfrow}=\mathrm{c}(1,2))$

hist(yo, prob=T,xlab="value", main="Observed", $x \lim =c(3,17), y \lim =c(0,0.35)$, breaks $=\operatorname{seq}(0,20,1))$

lines(seq(0,20,0.1),dnorm(seq(0,20,0.1), mu,sig), Iwd=3)

points (yobar, 0, pch=16)

hist(yu,prob=T,xlab="value", main="Unobserved", $x \lim =c(3,17), y \lim =c(0,0.35)$, breaks=seq $(0,20,1))$

lines(seq $(0,20,0.1)$,dnorm(seq $(0,20,0.1)$, mu,sig), lwd=3)

points(yubar, 0, pch $=16$ )

\# Plot probabilities of response in population

$\operatorname{par}(\mathrm{mfrow}=\mathrm{c}(1,1))$

plot(y,p,xlab="y",ylab="p",main="")

points $(y[R==0], p[R==0], p c h=4, c e x=1.5$ )

text(8,0.8,"The crosses represent nonrespondents")

\# Plot probabilities of response in sample and follow-up subsample

$\operatorname{par}(m f r o w=c(1,1)) ; \operatorname{plot}(y s, p[s], x l a b=" y ", y l a b=" p "$ "main="")

points(ys[Rs==0], $p[s][R s==0], p c h=4, \operatorname{cex}=1.5$ ) 
$\mathrm{nf}=15$; set.seed(112); followup = sort(sample(1:nu, nf)) \# Follow up sample $f=u[f o l l o w u p] ~ \#$ pop. labels of follow-up units

$\mathrm{yf}=\mathrm{y}[\mathrm{f}] \quad$ \# The follow-up sample vector

yfbar=mean(yf); yfbar \# 8.601 mean of follow-up values

points(yf, p[f], pch=16) \# OK

text $(8,0.8$, ,The crosses represent nonrespondents")

text(8,0.7,"The dots represent follow-up units")

\# Print data

s \#[1] $6 \quad 7 \quad 14 \quad 172237394866 \quad 697377 \quad 78103105106117 \ldots \ldots \ldots$.

o \#[1] $1422666978141152156172228230232 \ldots \ldots$.

f \# [1] 177377128145163187196253271318357436438481

paste(as.character(round(yo,2)), collapse=", ")

\# 12.57, 13.35, 11.47, 14.81, 13.25, 14.09, 11.55, 11.32,13.2,11.28,9.7,12.18, \# 11.49, 10.52, 9.93, 11.84, 12.2, 10.57, 11.9, 14.75, 10.34, 14.37, 12.13, 8.56, \# 11.91, 11.79, 11.45, 14.98, 10.57, 12.28, 9.91, 10.94, 13.28, 11.43 paste(as.character(round(yf,2)), collapse=", ")

\# 5.4, 9.41, 7.03, 8.88, 11.47, 7, 9.44, 8.58,9.27,8.18,8.62,8.73,7.33,9.81, 9.88

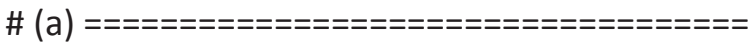

yo $=c(12.57,13.35,11.47,14.81,13.25,14.09,11.55,11.32,13.2,11.28,9.7$, $12.18,11.49,10.52,9.93,11.84,12.2,10.57,11.9,14.75,10.34,14.37,12.13$, $8.56,11.91,11.79,11.45,14.98,10.57,12.28,9.91,10.94,13.28,11.43)$ no=length(yo); $\mathrm{N}=500 ;$ ybarhata = mean(yo); so=sd(yo) ybarcpdra=ybarhata+c(-1,1)*qt(0.975,no-1)*(so/sqrt(no))*sqrt(1-no/N) c(no,so,ybarhata, ybarcpdra) \# 34.0001 .55211 .93911 .41612 .461

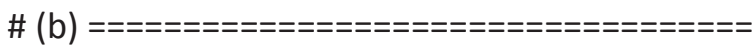

yf $=c(5.4,9.41,7.03,8.88,11.47,7,9.44,8.58,9.27,8.18,8.62,8.73,7.33,9.81,9.88)$

yof =c(yo,yf); nof=no+nf; ybarhatb = mean(yof);sof=sd(yof)

ybarcpdrb=ybarhatb+c(-1,1)*qt(0.975, nof-1)*(sof/sqrt(nof))*sqrt(1-nof/N) c(nof,sof,ybarhatb, ybarcpdrb) \# 49.0002 .16810 .91710 .32611 .509

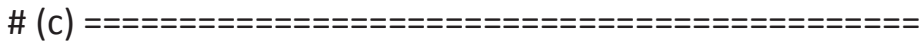

\# Plot observed and follow-up sample values separately $\operatorname{par}(\operatorname{mfrow}=\mathrm{c}(1,2))$

hist(yo,prob=T,xlab="value", main="Initially observed", $x \lim =c(3,17), y \lim =c(0,0.35)$, breaks=seq $(0,20,1))$;

points(mean(yo),0,pch=16);

hist(yf,prob=T,xlab="value", main="Follow-up", $x \lim =c(3,17), y \lim =c(0,0.35)$, breaks=seq $(0,20,1)) ;$

points(mean(yf),0,pch=16) 


\section{WinBUGS code for Exercise I $\mathbf{2 . 2}$}

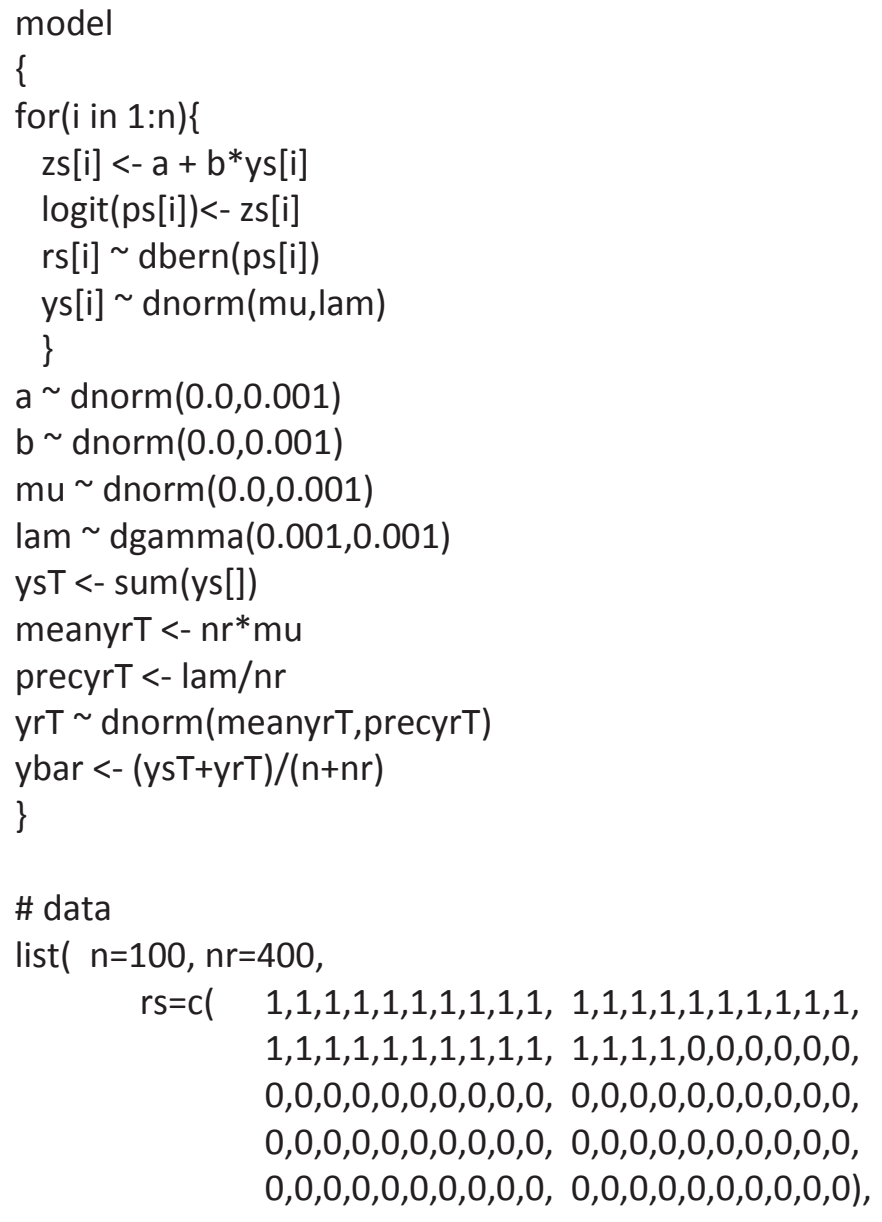

\# inits

list $(a=0, b=0, m u=0$,lam $=1)$ 


\section{2.3 Selection bias in volunteer surveys}

Volunteer surveys are common nowadays, with the main mediums being the telephone and Internet. However, they can be misleading on account of selection bias, and this has been known for a long time. For example, in 1983 a major television network in the US conducted a phone-in (or dial-in) poll. Viewers were invited to phone the network and answer the following question:

Should the United Nations continue to be based in the United States?

Of the 185,000 phones calls subsequently registered, 33\% were from persons answering yes, and $67 \%$ from persons answering no. The question then arose as to how reliable these figures are when applied to the American population as a whole. Many factors could affect said reliability, for example whether some people phoned in more than once.

A key concern is that maybe yes-respondents were more, or less, likely to phone in than no-respondents. For example, if yes-respondents were less likely to phone in, then the sample almost certainly contained an unrepresentatively low proportion of yes-responses. Consequently, the figure $33 \%$ is biased and too low when taken as an estimate of the percentage of all Americans in favour of the UN being based in the US.

Concerned about the accuracy of its phone-in polls generally, the TV network conducted an independent survey of the entire American population using proper probability sampling techniques. A SRSWOR of 1,000 persons yielded $72 \%$ yes-responses to the same question and $28 \%$ no-responses.

From these results, we may suspect that yes-respondents were indeed less likely to phone in than no-respondents. This prompts us to now study the issue in more depth, starting with the following model. This model and parts of the subsequent exposition can also be found in Puza and O’Neill (2006).

\section{2.4 A classical model for self-selection bias}

Suppose that there are a large number $N$ units in the population (e.g. persons in the US) and each unit has the same probability $p$ of having a particular characteristic in question (e.g. being in favour of the UN being based in the US). 
Then define:

$y_{i}$ as the indicator for pop. unit $i$ having the characteristic ( 0 or 1 )

$\pi_{i}$ as the probability that unit $i$ will be sampled (e.g. phone in to answer the question)

$I_{i}$ as the indicator that population unit $i$ is sampled.

In this context the data is $D=\left(n, y_{s T}\right)$, where:

$n=I_{1}+\ldots+I_{N}$ is the observed sample size

$y_{s T}=y_{s_{1}}+\ldots+y_{s_{n}}$ is the number of yes-respondents in the sample.

Now, a 'naïve' or 'base' model here is

$$
y_{s T} \sim \operatorname{Bin}(n, p) \text {, }
$$

and this leads to the straight sample proportion

$$
\bar{y}_{s}=y_{s T} / n
$$

as an estimate of $p$.

We now wish to generalise this model to account for the possibility that $\bar{y}_{s}$ may be biased. To this end, suppose each $\pi_{i}$ can be one of two values:

$\phi_{1}$ if that unit has the characteristic in question, i.e. if $y_{i}=1$

$\phi_{0}$ if that unit does not have the characteristic, i.e. if $y_{i}=0$.

Note: We may then write $\pi_{i}=\phi_{y_{i}}$.

Next, suppose that a unit with the characteristic in question is $\lambda$ times as likely to respond as a unit without the characteristic. Thus

$$
\phi_{1}=\lambda \phi_{0} \text {. }
$$

Also, write $\phi_{0}$ simply as $\phi$. Then,

$$
\pi_{i}=\left\{\begin{array}{c}
\phi \text { if } y_{i}=0 \\
\lambda \phi \text { if } y_{i}=1
\end{array}\right\}=\phi \lambda^{y_{i}} .
$$


With the above definitions, we now consider the probability of a respondent having the characteristic (as distinct from the probability of a nonrespondent having the characteristic):

$$
P\left(y_{i}=1 \mid I_{i}=1\right)=\frac{P\left(y_{i}=1\right) P\left(I_{i}=1 \mid y_{i}=1\right)}{P\left(I_{i}=1\right)}
$$

(note that we are applying Bayes’ rule here)

$$
\begin{aligned}
& =\frac{P\left(y_{i}=1\right) P\left(I_{i}=1 \mid y_{i}=1\right)}{P\left(y_{i}=0\right) P\left(I_{i}=1 \mid y_{i}=0\right)+P\left(y_{i}=1\right) P\left(I_{i}=1 \mid y_{i}=1\right)} \\
& =\frac{p \phi_{1}}{(1-p) \phi_{0}+p \phi_{1}}=\frac{p \phi \lambda}{(1-p) \phi+p \phi \lambda}=\frac{p \lambda}{1-p+p \lambda} .
\end{aligned}
$$

Note: Observe how one of the parameters, namely $\phi$, cancels out here.

We may now write $y_{s T} \sim \operatorname{Bin}(n, \omega)$, where $\omega=\frac{p \lambda}{1-p+p \lambda}$.

Next, the MLE and method of moments estimator of $\omega$ is $\bar{y}_{s}=y_{s T} / n$.

Also, solving $\omega=\frac{p \lambda}{1-p+p \lambda}$ for $p$ yields $p=\frac{\omega}{\lambda-\lambda \omega+\omega}$.

It follows that the MLE and MOME of $p$ is $\hat{p}=\frac{\bar{y}_{s}}{\lambda-\lambda \bar{y}_{s}+\bar{y}_{s}}$.

Also, $(L, U)=\left(\bar{y}_{s} \pm z_{\alpha / 2} \sqrt{\frac{\bar{y}_{s}\left(1-\bar{y}_{s}\right)}{n}}\right)$ is a $1-\alpha$ CI for $\omega$

Therefore, a $1-\alpha$ CI for $p$ is $\left(\frac{L}{\lambda-\lambda L+L}, \frac{U}{\lambda-\lambda U+U}\right)$.

It is of interest to now discuss the biases of the two estimators mentioned above. First, the bias of $\bar{y}_{s}$ is

$$
B\left(\bar{y}_{s}\right)=\omega-p=p\left(1-\frac{\lambda}{1-p+p \lambda}\right)=p \frac{(1-p)(1-\lambda)}{1-p(1-\lambda)} .
$$


This is not zero but reduces to zero when $\lambda=1$, i.e. when $\pi_{1}=\pi_{0}$.

Also, the bias of $\hat{p}$ is $B(\hat{p})=E\left(\frac{\bar{y}_{s}}{\lambda-\lambda \bar{y}_{s}+\bar{y}_{s}}\right)-p$.

Just like $B\left(\bar{y}_{s}\right), B(\hat{p})$ is nonzero but reduces to zero when $\lambda=1$. But unlike $B\left(\bar{y}_{s}\right), B(\hat{p})$ converges to zero as the sample size $n$ tends to infinity, this being true for all $\lambda$.

That is, $\hat{p}=\frac{\bar{y}_{s}}{\lambda-\lambda \bar{y}_{s}+\bar{y}_{s}}$ is asymptotically unbiased for $p$ as $n \rightarrow \infty$.

Note: This is obvious by construction. But just to check, we note that $E \bar{y}_{s}=\frac{p \lambda}{1-p+p \lambda}$ and $V \bar{y}_{s}<\infty$. Therefore

$$
B(\hat{p}) \rightarrow\left(\frac{\left[\frac{p \lambda}{1-p+p \lambda}\right]}{\lambda-\lambda\left[\frac{p \lambda}{1-p+p \lambda}\right]+\left[\frac{p \lambda}{1-p+p \lambda}\right]}\right)-p=0 \text { as } n \rightarrow \infty .
$$

\section{Example I2.I Application to the US TV network scenario (a classical analysis)}

Observe that $\omega=\frac{p \lambda}{1-p+p \lambda}$ implies $\lambda=\frac{\omega(1-p)}{p(1-\omega)}$.

Then recall that the phone-in poll conducted by the TV network yielded an estimate of 0.33 , and that the parallel scientifically designed (and 'proper') survey yielded an estimate of 0.72 .

Thus we may estimate $\lambda=\pi_{1} / \pi_{0}$ by

$$
\hat{\lambda}=\frac{\hat{\omega}(1-\hat{p})}{\hat{p}(1-\hat{\omega})}=\frac{0.33(1-0.72)}{0.72(1-0.33)}=0.19 \text {. }
$$

This estimate being less than unity is consistent with our earlier intuition that the phone-in poll estimate might be too low due to yes-respondents being less likely to phone in than no-respondents. 


\section{Example I 2.2 Inference on $p$ in a flag poll (a classical analysis)}

On 28 January 2000 an Internet poll was conducted by the Nine TV Network in Australia with the question:

Should the Australian flag be replaced by a new one?

To this poll there were 4,941 yes-responses and 4,512 no-responses, thus a proportion of

$$
4,941 /(4,941+4,512)=4,941 / 9,453=0.523 \text { yeses. }
$$

A similar question was asked in the Australian Constitutional Referendum Study, 1999 (Gow et al., 2000), and this proper survey yielded 829 yes-responses and 1,394 no-responses, thus a proportion of $829 /(829+1,394)=829 / 2,223=0.373$ yeses .

Hence, for the 28 January Internet poll we may estimate $\lambda=\pi_{1} / \pi_{0}$ by

$$
\hat{\lambda}=\frac{\hat{\omega}(1-\hat{p})}{\hat{p}(1-\hat{\omega})}=\frac{0.523(1-0.373)}{0.373(1-0.523)}=1.84 \text {. }
$$

This suggests that persons who wanted the flag replaced were almost twice as likely to register their opinion via the Internet poll as persons who were happy with the old flag.

\section{Example I 2.3 Inference on $p$ in a currency poll (a classical analysis)}

On 4 June 2000 an Internet poll was conducted by the Nine TV Network with the question:

Should the Queen's image be removed from our currency?

To this there were 2,544 yes-responses and 1,755 no-responses, thus a proportion of

$$
2,544 /(2,544+1,755)=2,544 / 4,299=0.592 \text { yeses. }
$$

Now recall Example 12.2. Clearly there is some similarity between the two polls. Both were conducted on the Internet by the same organisation within the same half-year, and the two questions asked both relate to changing something about Australia's heritage. This similarity suggests that 1.84 may be a plausible value of $\lambda=\pi_{1} / \pi_{0}$ to be used in the 4 June poll here. 
If so, we may estimate the true proportion of Australians in favour of removing the Queen's image from our currency as

$$
\hat{p}=\frac{\bar{y}_{s}}{\lambda-\lambda \bar{y}_{s}+\bar{y}_{s}}=\frac{0.592}{1.84-1.84 \times 0.592+0.592}=0.441 \text {. }
$$

Then, a 95\% CI for $\omega=\frac{p \lambda}{1-p+p \lambda}$ (the probability of a yes-response for a respondent) is

$$
\begin{aligned}
(L, U)=\left(\bar{y}_{s} \pm z_{\alpha / 2} \sqrt{\frac{\bar{y}_{s}\left(1-\bar{y}_{s}\right)}{n}}\right) & =\left(0.592 \pm 1.96 \sqrt{\frac{0.592(1-0.592)}{4,299}}\right) \\
& =(0.577,0.607) .
\end{aligned}
$$

Therefore, a $1-\alpha$ CI for $p$ is

$$
\begin{aligned}
& \left(\frac{L}{\lambda-\lambda L+L}, \frac{U}{\lambda-\lambda U+U}\right) \\
& \quad=\left(\frac{0.577}{1.84-1.84 \times 0.577+0.577}, \frac{0.607}{1.84-1.84 \times 0.607+0.607}\right) \\
& \quad=(0.426,0.456) .
\end{aligned}
$$

\section{2.5 Uncertainty regarding the sampling mechanism}

In Example 12.3 above, the value of $\lambda$ was taken to be exactly 1.84 . However, there is in fact uncertainty about $\lambda$ which ought to be taken into account and perhaps lead to a wider CI for $p$ than the one reported.

With this in mind we now postulate the following Bayesian model:

$$
\begin{aligned}
& \left(y_{s T} \mid p, \lambda\right) \sim \operatorname{Bin}(n, \omega) \quad \text { where } \omega=\frac{p \lambda}{1-p+p \lambda} \text { (as before) } \\
& (p \mid \lambda) \sim \operatorname{Beta}(\alpha, \beta) \\
& \lambda \sim \operatorname{Gamma}(\eta, \tau)
\end{aligned}
$$

Note: This model implicitly conditions on the sample size $n$. 


\section{Example I 2.4 Bayesian re-analysis of poll data Example I 2.2}

Recall the 28 January 2000 Internet poll yielding 4,941 yeses out of 9,453 responses and the related properly conducted probability survey yielding 829 yeses and 1,394 nos.

This suggests we apply the Bayesian model (12.2) in WinBUGS to estimate $\lambda$, with:

$$
\begin{aligned}
& \eta=\tau=0.000001 \quad \text { (implying an uninformative prior on } \lambda \text { ) } \\
& \alpha=829+1=830, \beta=1,394+1=1,395
\end{aligned}
$$

(the posterior of $p$ implied by the proper survey in a binomial-beta model and then fed here as the prior for $p$ )

$$
n=9,453, y_{s T}=4,941
$$

(the observed data in the self-selected sample).

Using suitable WinBUGS code (see below) and a sample size of 10,000 after a burn-in of 1,000, we obtained results shown in Table 12.2. Figure 12.7 shows some of the graphical output from WinBUGS.

\section{Table I 2.2 Results of WinBUGS analysis}

\begin{tabular}{lllllll} 
node & mean & sd & MC error & $\mathbf{2 . 5 \%}$ & \multicolumn{2}{c}{ median $\mathbf{9 7 . 5 \%}$} \\
\hline lam & 1.843 & 0.08879 & 0.00271 & 1.677 & 1.841 & 2.026 \\
$\mathrm{p}$ & 0.373 & 0.01022 & $3.15 \mathrm{E}-4$ & 0.3529 & 0.373 & 0.393 \\
\hline
\end{tabular}

We see that $\lambda$ has been estimated as 1.84 again, but now with some measure of uncertainty: the $95 \%$ posterior interval estimate for $\lambda$ is $(1.68,2.03)$. 
Figure I 2.7 Graphical output from WinBUGS
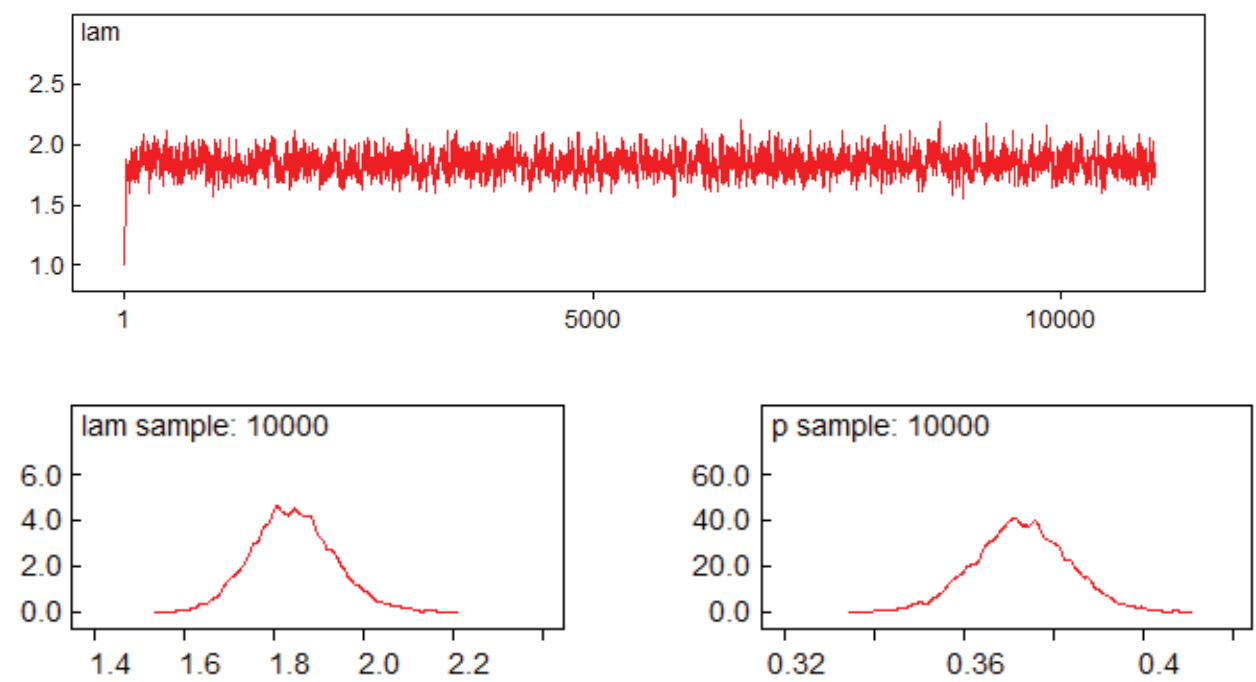

Equating the sample mean and sample variance of the 10,000 simulated values with the theoretical mean and variance of the $\operatorname{Gamma}(\eta, \tau)$, namely $\eta / \tau$ and $\eta / \tau^{2}$, respectively, we may approximate the posterior distribution of $\lambda$ as $\operatorname{Gamma}(\eta, \tau)$ with $\eta=431$ and $\tau=234$.

Figure 12.8 shows a histogram of the simulated values overlaid by the gamma density defined by these parameters. We see that the gamma posterior approximation fits quite well.

Figure I 2.8 Histogram of simulated values and fitted gamma density

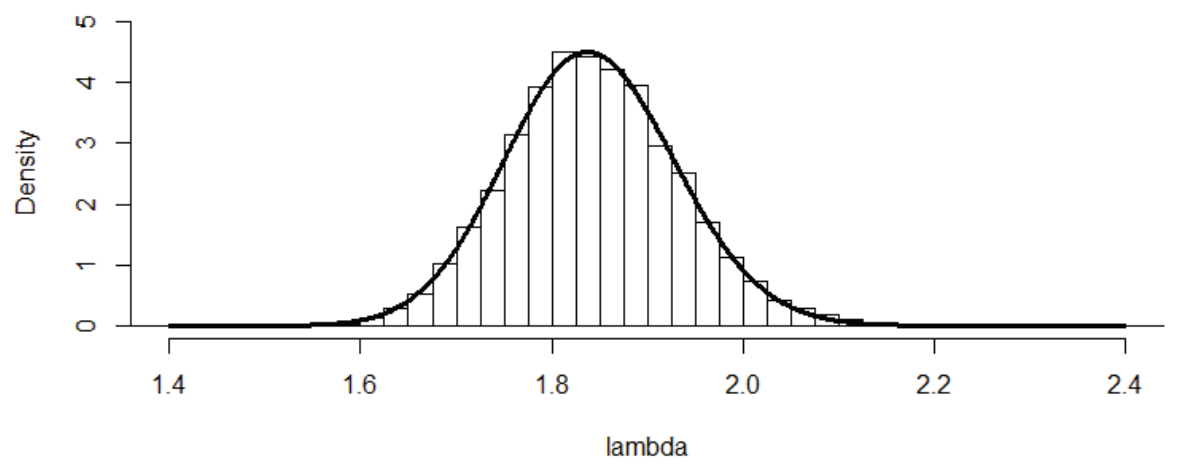




\section{WinBUGS Code for Example I 2.4}

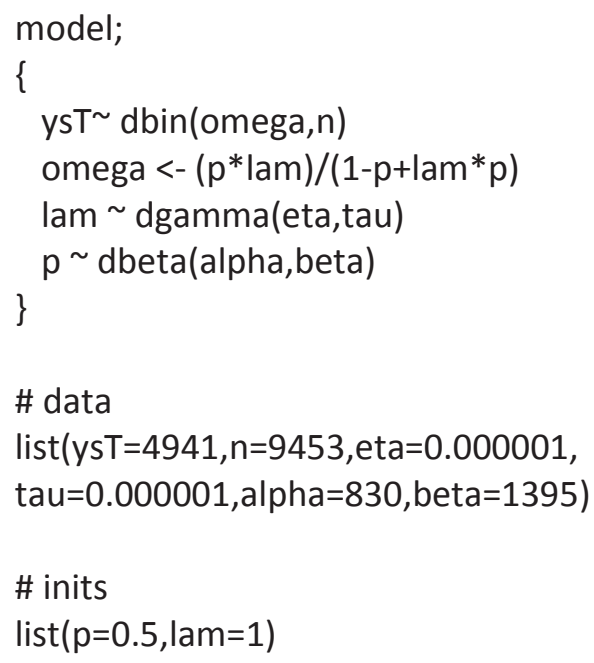

\section{R Code for Example I 2.4}

\# Need to run BUGS code above first, using coda to create output in data.txt

options(digits=3); $0.33 * 0.28 /(0.72 * 0.67) \# 0.192$

$0.523 *(1-0.373) /(0.373 *(1-0.523)) \# 1.84$

$0.592 /(1.84-1.84 * 0.592+0.592) \# 0.441$

Clomega $=0.592+c(-1,1) * 1.96 * \operatorname{sqrt}(0.592 *(1-0.592) / 4299)$

$\mathrm{Clp}=($ Clomega $/(1.84-1.84 *$ Clomega + Clomega $))$

c(Clomega, Clp) \# 0.577 0.607 0.4260.456

out=read.table(file=file.choose()) \# choose data.txt from BUGS run lamvec = out $[1: 10000,2] ;$ options (digits=5)

lambar=mean(lamvec); lamvar=var(lamvec) taufit=lambar/lamvar; etafit=lambar*taufit

c(lambar, lamvar, etafit, taufit)

\# 1.8432e+00 7.8849e-03 4.3087e+02 2.3376e+02

summary(lamvec)

\# Min. 1st Qu. Median Mean 3rd Qu. Max.

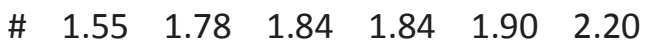

$\mathrm{X} 11(\mathrm{w}=8, \mathrm{~h}=4) ; \operatorname{par}(\mathrm{mfrow}=\mathrm{c}(1,1))$

lamv <- seq $(1.4,2.4,0.001)$

$\mathrm{fv}<-$ dgamma(lamv,431,234)

hist(lamvec, prob=T,xlim=c(1.4,2.4),ylim=c(0,5),xlab="lambda",cex=1.5, breaks=seq(1,3,0.025), main="")

lines(lamv, fv,lwd=3) 


\section{Example I 2.5 Bayesian re-analysis of poll data in Example I 2.3 using results in Example I 2.4}

Recall the 4 June 2000 poll yielding 2,544 yeses out of 4,299 responses, leading to 0.441 as an estimate of $p$, with $95 \%$ CI $(0.426,0.456)$, based on $\lambda$ being exactly equal to 1.84 . This suggests we apply our Bayesian model in WinBUGS to estimate $p$ with:

$$
\begin{aligned}
& \eta=431, \tau=234 \\
& \text { (using the posterior for } \lambda \text { in Example } 4 \text { as the prior) } \\
& \alpha=\beta=1 \quad \text { (implying an uninformative prior for } p \text { ) } \\
& n=4,299, \quad y_{s T}=2,544 \\
& \text { (the observed data in the self-selected sample). }
\end{aligned}
$$

Using suitable WinBUGS code (see below), we obtained the results shown in Table 12.3. Some of the graphical output is shown in Figure 12.9 .

\section{Table I 2.3 Results of WinBUGS analysis}

\begin{tabular}{lllllll} 
node & mean & sd & MC error & $\mathbf{2 . 5 \%}$ & median & $\mathbf{9 7 . 5 \%}$ \\
\hline lam & 1.841 & 0.08801 & 0.001991 & 1.67 & 1.84 & 2.014 \\
$\mathrm{p}$ & 0.4409 & 0.01408 & $3.18 \mathrm{E}-4$ & 0.414 & 0.4406 & 0.4698 \\
\hline
\end{tabular}

We see that $p$ has been estimated as 0.441 again, with 95\% interval estimate $(0.414,0.470)$. It will be noted that this interval is wider than the one in Example 12.3; this may be attributed to the fact that in Example 12.3 uncertainty regarding $\lambda$ was not properly taken into account. For more information on the topic in this section, see Puza and O’Neill (2006).

Note: The posterior for $\lambda$ is virtually the same as the prior for $\lambda$. This was to be expected, since-unlike in Example 12.4 - the data here does not contain any structure which could tell us anything about the relationship between the sampling propensities $\pi_{0}$ and $\pi_{1}$. 
Figure I 2.9 Graphical output from WinBUGS
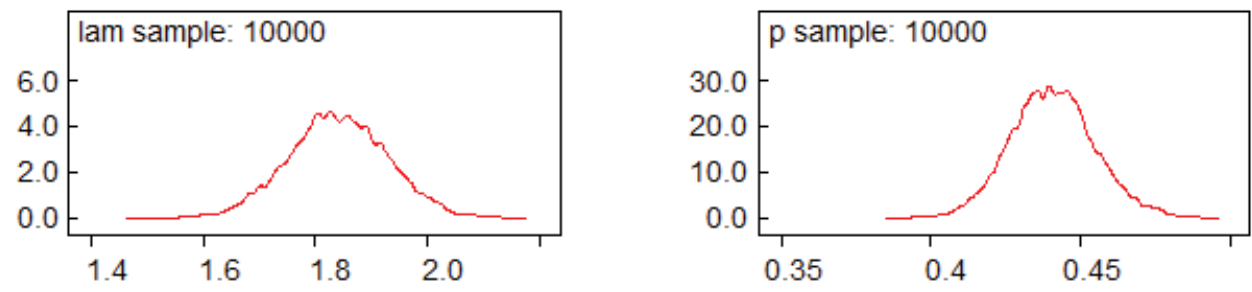

\section{WinBUGS Code for Example I2.5}

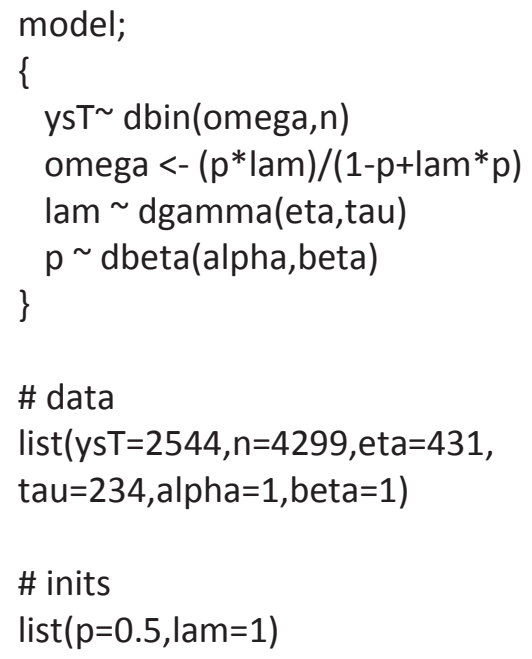

\section{2.6 Finite population inference under selection bias in volunteer surveys}

In the last section on selection bias in volunteer surveys, the finite population size $N$ was introduced at the beginning, but then seemed to disappear from the notation. The Bayesian model subsequently developed did not feature $N$ at all.

This is a clue to the fact that the Bayesian model in that section is only useful for infinite population inference, in particular on the superpopulation parameter $p$, and cannot be used for inference on finite population quantities, in particular the finite population mean

$$
\bar{y}=\left(y_{1}+\ldots+y_{N}\right) / N \text {. }
$$


This is not an issue when $N$ is very large (as it was assumed there), since in that case inference on $\bar{y}$ is, by the law of large numbers, virtually identical to inference on the superpopulation mean $p$.

The following exercise develops a 'true' Bayesian finite population model in the same setting, one which could be useful in scenarios where $N$ is not so large as to be effectively infinity.

\section{Exercise I 2.3 A Bayesian finite population self-selection model}

Consider a finite population of $N$ units, where each unit has common probability $p$ of having some characteristic, independently of all the other units, and where our prior beliefs regarding $p$ can be represented by way of a beta distribution with parameters $\alpha$ and $\beta$.

A sample is selected from the finite population in such a way that every unit without the characteristic has probability $\phi$ of being sampled, and every unit with the characteristic has probability $\lambda \phi$ of being selected. Every unit that is sampled has its value fully observed.

The prior on $\phi$ is beta with parameters $\delta$ and $\gamma$ but evenly spread over the interval $(0, c)$, where $c<1$ is a specified constant representing an absolute upper bound for what the value of $\phi$ could possibly be. (Examples of a potentially suitable values of $c$ are 0.1, 0.2 and 0.5.)

Also, the prior on $\lambda$ is beta with parameters $\eta$ and $\tau$ but evenly spread over the interval $(0,1 / c)$, so as to permit a suitably wide range of possible values for the ratio of sampling propensities $\pi_{1}=\lambda \phi$ to $\pi_{0}=\phi$. (For example, if $c=0.2$ then that ratio could be anything from 0 to 5.)

(a) Write down a Bayesian model which comprehensively represents the above situation. Assume that all of the model parameters are independent a priori. Clearly identify the data.

(b) Suppose we are interested in both the superpopulation mean (i.e. the common probability of a unit having the characteristic, $p$ ) and the finite population mean (i.e. proportion of the $N$ finite population units which have the characteristic, $\bar{y}$ ). Write down a formula for the joint posterior (and predictive) density of all quantities which are relevant to and could used be as a basis for the desired inference. 
(c) Use the density in (a) to construct a suitable Metropolis-Hastings algorithm. Then run the algorithm in $\mathrm{R}$ so as to redo the analyses in Examples 12.4 and 12.5. Perform each new analysis thrice, assuming the finite population size $N$ is 200,000,400,000 and 40,000, respectively.

(d) Modify the $\mathrm{MH}$ algorithm in (c) so that its output features only the three model parameters and none of the nonsample values. (NB: The idea here is to design a superior $\mathrm{MH}$ algorithm, one with better 'mixing' than the one in (c).)

(e) Describe a procedure whereby the output from the algorithm in (d) could be used to obtain a sample from the predictive distribution of the nonsample mean. Then run that algorithm and implement the procedure so as to produce results intended to be equivalent to those in the reanalysis of Example 5 in (c) with $N=200,000$.

\section{Solution to Exercise I 2.3}

(a) With $y=\left(y_{1}, \ldots, y_{N}\right)$ and $I=\left(I_{1}, \ldots, I_{N}\right)$, the Bayesian model may be written as follows:

$$
\begin{aligned}
& \left(I_{i} \mid y, p, \lambda, \phi\right) \sim \perp \operatorname{Bernoulli}\left(\phi \lambda^{y_{i}}\right), \quad i=1, \ldots, N \\
& \left(y_{1}, \ldots, y_{N} \mid p, \lambda, \phi\right) \sim \text { iid Bernoulli }(p) \\
& (p \mid \lambda, \phi) \sim \operatorname{Beta}(\alpha, \beta), \quad(\lambda \mid \phi) \sim(1 / c) \times \operatorname{Beta}(\eta, \tau) \\
& \phi \sim c \times \operatorname{Beta}(\delta, \gamma) \quad(0<c<1) .
\end{aligned}
$$

Note: The sampling mechanism here is nonignorable and unknown, since $f(I \mid y, p, \lambda, \phi)$ depends on the unknown quantities $\phi$ and $\lambda$. If $\lambda$ were equal to 1 then the sampling mechanism would again be unknown but in that case ignorable, since $\phi \perp p$ a priori.

The data here may be written as $D=\left(n, y_{s T}\right)$, where:

$$
\begin{aligned}
& n=\sum_{i=1}^{N} I_{i} \quad \text { is the sample size } \\
& y_{s T}=\sum_{i \in s} y_{i} \text { is the number of sampled units with the characteristic. }
\end{aligned}
$$


Since the data is a function of $\left(I, y_{s}\right)$, the relevant joint posterior/ predictive density is

$$
\begin{aligned}
& f\left(\phi, \lambda, p, y_{r} \mid I, y_{s}\right) \propto f\left(\phi, \lambda, p, \lambda, y_{r}, I, y_{s}\right) \\
&= f\left(\phi, \lambda, p, y_{r}, I_{s}, I_{r}, y_{s}\right) \\
&= f(\phi) f(\lambda) f(p) \times f\left(y_{s} \mid p\right) f\left(y_{r} \mid p\right) \\
& \times f\left(I_{s} \mid y_{s}, \phi, \lambda\right) f\left(I_{r} \mid y_{r}, \phi, \lambda\right) \\
&=\frac{(\phi / c))^{\delta-1}(1-\phi / c)^{\gamma-1}}{} \times \frac{c(c \lambda)^{\eta-1}(1-c \lambda)^{\tau-1}}{B(\eta, \tau)} \times \frac{p^{\alpha-1}(1-p)^{\beta-1}}{B(\alpha, \beta)} \\
& \times\left(\prod_{i \in s} p^{y_{i}}(1-p)^{1-y_{i}}\right)\left(\prod_{i \in r} p^{y_{i}}(1-p)^{1-y_{i}}\right) \times\left(\prod_{i \in s}\left(\phi \lambda^{y_{i}}\right)^{I_{i}}\left(1-\phi \lambda^{y_{i}}\right)^{1-I_{i}}\right) \\
& \times\left(\prod_{i \in r}\left(\phi \lambda^{y_{i}}\right)^{I_{i}}\left(1-\phi \lambda^{y_{i}}\right)^{1-I_{i}}\right) \\
& \propto \phi^{\delta-1}(1-\phi / c)^{\gamma-1} \times \lambda^{\eta-1}(1-c \lambda)^{\tau-1} \times p^{\alpha-1}(1-p)^{\beta-1} \\
& \times p^{y_{s T}}(1-p)^{n-y_{s T}} p^{y_{r T}}(1-p)^{N-n-y_{r T}} \\
& \times\left(\prod_{i \in s}\left(\phi \lambda^{y_{i}}\right)^{1}\left(1-\phi \lambda^{y_{i}}\right)^{1-1}\right)\left(\prod_{i \in r}\left(\phi \lambda^{y_{i}}\right)^{0}\left(1-\phi \lambda^{y_{i}}\right)^{1-0}\right) \\
&= \phi^{\delta-1}(1-\phi / c)^{\gamma-1} \times \lambda^{\eta-1}(1-c \lambda)^{\tau-1} \times p^{\alpha-1}(1-p) \tau^{\beta-1} \\
& \times p^{y_{s T}+y_{r T}}(1-p)^{N-y_{s T}-y_{r T}} \times \phi^{n} \lambda^{y_{s T}}(1-\phi \lambda)^{y_{r T}}(1-\phi)^{N-n-y_{r T}}
\end{aligned}
$$

Note 1: In all of the above e.g. (12.3), $s$ and $r$ are fixed at their observed values.

Note 2: In the step from (12.4) to (12.5), be aware that $I_{i}=1 \forall i \in S$ and $I_{i}=0 \forall i \in r$. 
Note 3: In the step form (12.3) to (12.4), $f(\phi)$ is derived as follows.

If $w \equiv \frac{\phi}{c} \sim \operatorname{Beta}(\delta, \gamma)$ then $f(w)=\frac{w^{\delta-1}(1-w)^{\gamma-1}}{B(\delta, \gamma)}$.

Therefore

$$
f(\phi)=f(w)\left|\frac{d w}{d \phi}\right|=\frac{(\phi / c)^{\delta-1}(1-\phi / c)^{\gamma-1}}{c B(\delta, \gamma)} .
$$

A similar logic can be used to derive the density

$$
f(\lambda)=\frac{c(c \lambda)^{\eta-1}(1-c \lambda)^{\tau-1}}{B(\eta, \tau)}
$$

(b) Examining the density in (a), in particular (12.6), we see that:

$$
\begin{gathered}
f\left(y_{r T} \mid D, \phi, \lambda, p\right) \propto[p(1-\phi \lambda)]^{y_{r T}}[(1-p)(1-\phi)]^{N-n-y_{r T}} \\
\Rightarrow\left(y_{r T} \mid D, \phi, \lambda, p\right) \sim \operatorname{Bin}(N-n, q), \\
\text { where } q=\frac{p(1-\phi \lambda)}{p(1-\phi \lambda)+(1-p)(1-\phi)} \\
f\left(p \mid D, \phi, \lambda, y_{r T}\right)=p^{\alpha+y_{s T}+y_{r T}-1}(1-p) \tau^{\beta+N-n-y_{s T}-y_{r T}-1} \\
\Rightarrow\left(p \mid D, \phi, \lambda, y_{r T}\right) \sim \operatorname{Beta}\left(\alpha+y_{s T}+y_{r T}, \beta+N-y_{s T}-y_{r T}\right) .
\end{gathered}
$$

Also:

$$
\begin{aligned}
& f\left(\phi \mid D, y_{r T}, \lambda, p\right) \propto \phi^{\delta+n-1}(1-\phi / c)^{\gamma-1}(1-\phi \lambda)^{y_{r T}}(1-\phi)^{N-n-y_{r T}} \\
& f\left(\lambda \mid D, y_{r T}, \phi, p\right) \propto \lambda^{\eta+y_{s T}-1}(1-c \lambda)^{\tau-1}(1-\phi \lambda)^{y_{r T}}
\end{aligned}
$$

The above implies a suitable $\mathrm{MH}$ algorithm with two Gibbs steps as defined at (12.7) and (12.8) and two Metropolis steps as defined by (12.9) and (12.10). 
(c) The $\mathrm{MH}$ algorithm in (b) was applied with the following specifications so as to redo the analysis in Example 12.4:

$$
\begin{aligned}
& N=200,000, \quad n=9453, \quad y_{s T}=4941, \quad c=0.2 \\
& \alpha=830, \quad \beta=1395, \quad \eta=\tau=1, \quad \delta=\gamma=1 .
\end{aligned}
$$

A run with burn-in 2,000 followed by $J=10,000$ iterations for inference was performed. Numerical results from this run are shown in Table 12.4.

\section{Table I 2.4 Monte Carlo inferences using $N=\mathbf{2 0 0 , 0 0 0}$}

\begin{tabular}{lcccl} 
phi, $\phi$ & lam, $\lambda$ & $p$ & ybar, $\bar{y}$ & \\
\hline 0.03597 & 1.84686 & 0.37259 & 0.37259 & mean of simulated values \\
0.08789 & 0.08789 & 0.01017 & 0.01022 & sample standard deviation \\
0.03449 & 1.68272 & 0.35266 & 0.35250 & LB of 95\% CPDR estimate \\
0.03749 & 2.02311 & 0.39190 & 0.39202 & UB of 95\% CPDR estimate \\
\hline
\end{tabular}

Our point and interval estimates for $\lambda$ are 1.85 and (1.68, 2.02), which are very similar to 1.84 and $(1.68,2.03)$ in Example 12.4.

Note: The primary object here is estimation of $\lambda$, not of $p$ or $\bar{y}$. But it will be noted that the estimates of these other two quantities ( $p$ or $\bar{y}$ ) are very alike, which is as one might expect.

Repeating the above but with finite population sizes 400,000 and 40,000, respectively, we obtain the corresponding results shown in Tables 12.5.

\section{Table I 2.5 Inferences using different $\mathbf{N}$ (same details as in

\begin{tabular}{|c|c|c|c|c|c|c|c|}
\hline \multicolumn{4}{|c|}{$N=400,000$} & \multicolumn{4}{|c|}{$N=40,000$} \\
\hline phi, $\phi$ & lam, $\lambda$ & $p$ & ybar, $\bar{y}$ & phi, $\phi$ & $\operatorname{lam}, \lambda$ & & ybar, $\bar{y}$ \\
\hline 0.0180 & 1.83548 & .3739 & 0.373948 & 0.18123 & 31.81588 & 37569 & 0.375834 \\
\hline & & & & 0.07579 & & & 939 \\
\hline 0.017 & 1.6840 & 354 & 113 & 0.17492 & 1.669220 & 35735 & 60.357050 \\
\hline 0.01878 & 2.00923 & .39122 & 0.391193 & 0.18813 & 3 1.972080 & 39396 & 90.394500 \\
\hline
\end{tabular} Table 12.4)}

Note: The three sets of inferences in Tables 12.4 and 12.5 have yielded different estimates of $\phi$ but very similar results for the other three quantities, in particular the object of this study, $\lambda$. 
Figure 12.10 shows graphical output from the first of the three Metropolis-Hastings algorithms (i.e. the one with $N=200,000$ ).

Figure I 2. I 0 Graphical output from run with $N=200,000$
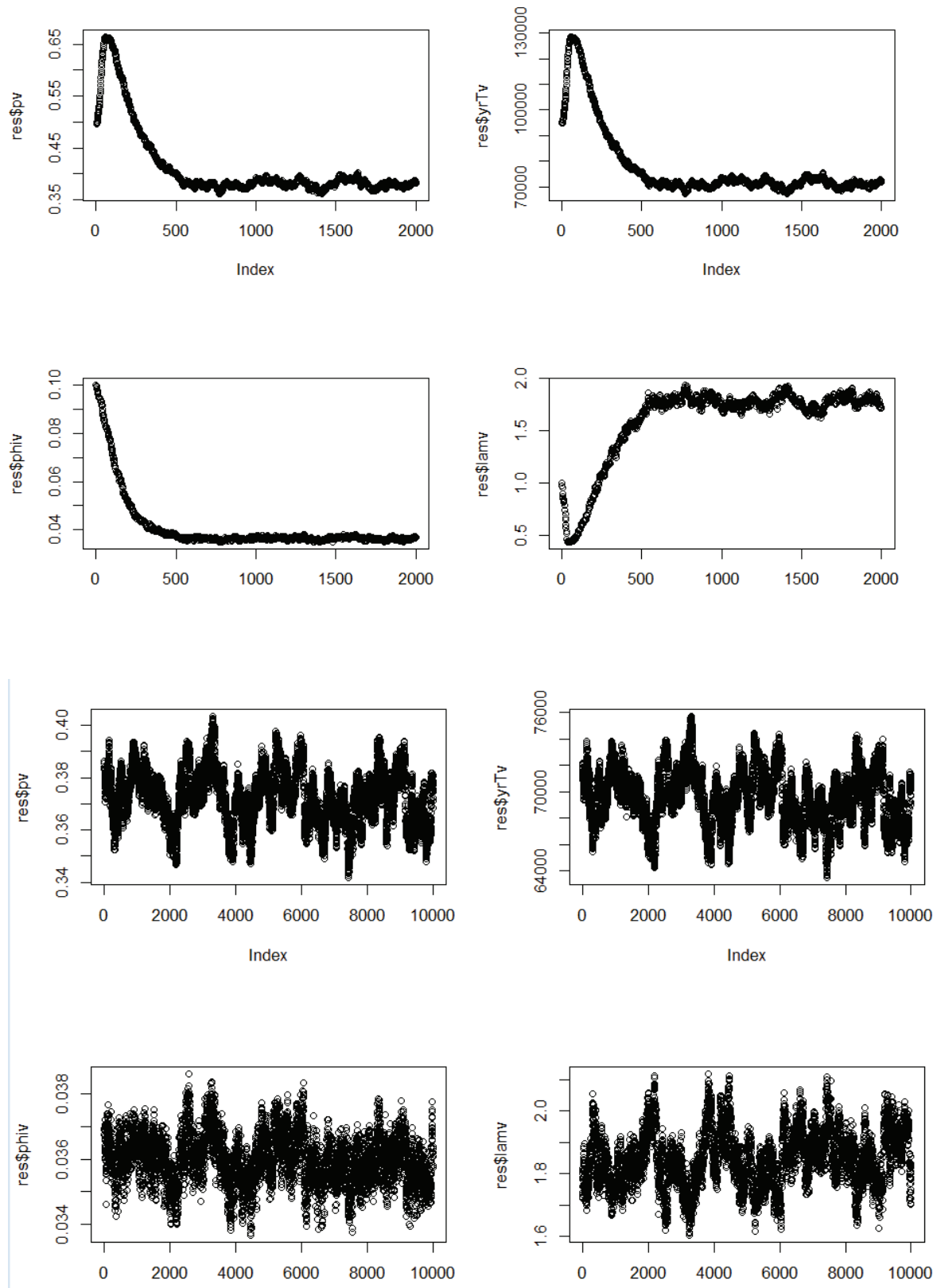
Histogram of pv

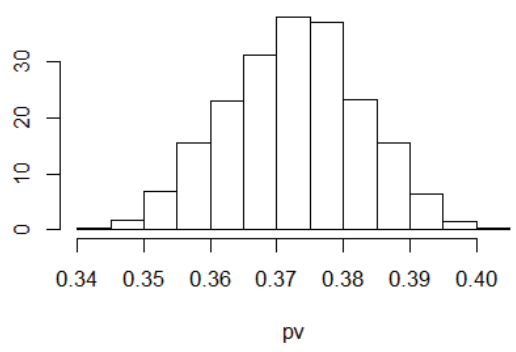

Histogram of phiv

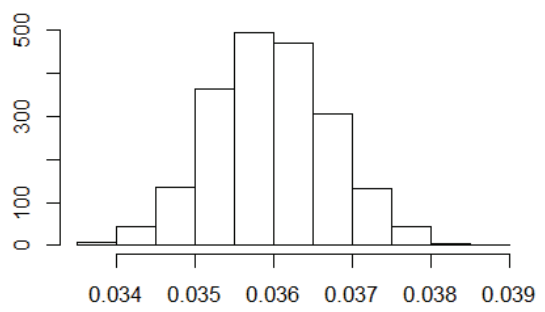

Histogram of yrTv

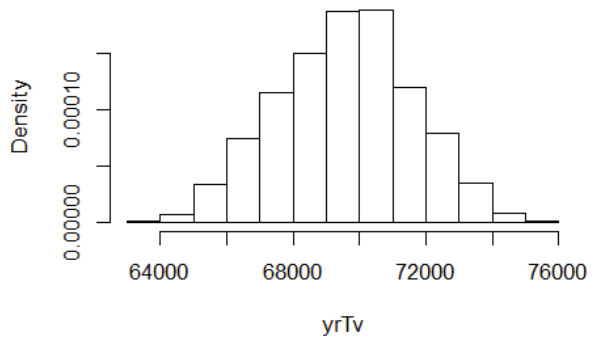

Histogram of lamv

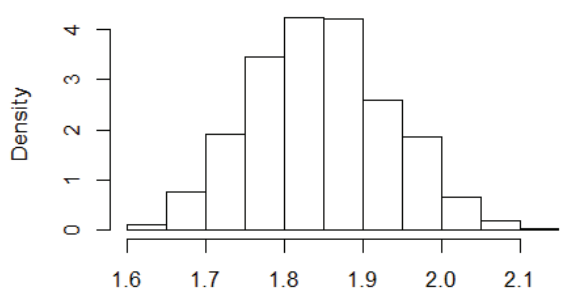

Next, a beta distribution was fitted to the 10,000 simulated values of $\lambda$ above (taken from the run with $N=200,000$ ) so as to define the approximate posterior given by

$$
(\lambda \mid D) \sim(1 / c) \times \operatorname{Beta}\left(\eta^{\prime}, \tau^{\prime}\right),
$$

where $\eta^{\prime}=278.1$ and $\tau^{\prime}=474.8$ (with $c=0.2$ as before).

This posterior for $\lambda$ was then fed in as the prior for $\lambda$ so as to redo the analysis in Example 12.5.

Accordingly, the $\mathrm{MH}$ algorithm in (b) was next applied once again but with the following specifications:

$$
\begin{aligned}
& N=200,000, \quad n=4299, \quad y_{s T}=2544, \quad c=0.2 \\
& \alpha=1, \quad \beta=1, \quad \eta=278.1, \quad \tau=474.8, \quad \delta=\gamma=1 .
\end{aligned}
$$

The relevant numerical estimates are as shown in Table 12.6. 
Table 1 2.6: Inferences using $N=\mathbf{2 0 0 , 0 0 0}$ and a fitted beta prior

\begin{tabular}{lcccl} 
phi, $\phi$ & lam, $\lambda$ & $p$ & ybar, $\bar{y}$ & \\
\hline 0.01570 & 1.84272 & 0.44049 & 0.45248 & mean of simulated values \\
0.08792 & 0.08792 & 0.01408 & 0.01403 & sample standard deviation \\
0.01495 & 1.67553 & 0.41344 & 0.42555 & LB of 95\% CPDR estimate \\
0.01656 & 2.01139 & 0.46602 & 0.47799 & UB of 95\% CPDR estimate \\
\hline
\end{tabular}

Thus point and interval estimates for $p$ are 0.440 and $(0.413,0.466)$, which we note are similar to 0.441 and $(0.414,0.470)$ in Example 12.5 .

Also point and $95 \%$ interval estimates for $\bar{y}$ are 0.452 and $(0.426$, 0.478).

Note 1: The inference on $\bar{y}$ here was not possible using the theory in the section just above the present exercise, i.e. using the infinite population models developed in that section.

Note 2: The posterior for $\lambda$ is very similar to its prior, which is as one might expect, since the data now has no structure which could tell us anything further about that parameter.

Repeating the above but with finite population sizes 400,000 and 40,000, respectively, we obtain the corresponding results shown in Tables 12.7.

Table I 2.7 Inferences using different $\mathbf{N}$ (same details as in Table I 2.6)

\begin{tabular}{|c|c|c|c|c|c|c|}
\hline \multicolumn{3}{|c|}{$N=400,000$} & \multicolumn{4}{|c|}{$N=40,000$} \\
\hline phi, $\phi$ & lam, $\lambda$ & ybar, $\bar{y}$ & phi, $\phi$ & lam, $\lambda$ & $p$ & ybar, $\bar{y}$ \\
\hline 0.00 & 1.83516 & 0.441930 .44792 & 0.07888 & 1.82895 & 0.4422 & 0.50220 \\
\hline 0.0877 & 0.08776 & 0.013750 .01372 & 0.08162 & 20.08162 & & 0.01337 \\
\hline 0.007 & 1.6680 & 0.415630 .42160 & 0.07538 & 81.66402 & 0.41 & 0.47517 \\
\hline 0.008 & 92.00048 & 0.468190 .47409 & 0.08278 & 81.99275 & 0.4700 & 0.52985 \\
\hline
\end{tabular}




\section{Discussion}

Something to be noted above is that estimation of $\bar{y}$ appears to increase slightly as $N$ decreases, whereas estimation of $p$ remains about the same.

Estimation of $\phi$ also increases as $N$ decreases. This could present a 'problem' if $N$ is 'too small'. Figures 12.11, 12.12 and 12.13 (pages 598 and 599) show histograms of the simulated values when $N=200,000$, 20,000 and 15,000, respectively.

We see no problem in the first two of these three cases. But for $N=15,000$, the estimation of $\phi$ appears to be artificially restricted by our arbitrary choice of $c$ as 0.2. (Observe that the simulated values are strongly 'bunched up' at just below 0.2.)

Repeating the MCMC run with $N=15,000$ but with $c$ also changed to 0.5 appears to solve this problem. Results are shown in Figure 12.14 (page 599). We note that estimation of $\lambda$ has changed from about 2 to less than 1 . This suggests that we might get very similar results with $c$ even larger, e.g. $c=1$.

But when we do this, we get very different results (not shown). Why?

Because when we changed $c$ from 0.2 to 0.5 , we forgot to reconfigure the prior for $\lambda$, which also involves $c$.

Note: The prior for $\phi$ also involves $c$ but does not need reconfiguring (because that prior is uniform for all values of $c$, since $\delta=\gamma=1$ ).

Thus, Figure 12.14 (the case of $N=15,000$ and $c=0.5$ ) in fact illustrates output which is 'flawed' (in this sense) and so should be disregarded.

Although these technical issues could satisfactorily be resolved with some effort, we will leave that task as an avenue of investigation for further research and move on to answering part (d). 
Figure I 2. I I Histograms using $\mathbf{N}=\mathbf{2 0 0 , 0 0 0}$ and $c=0.2$

Histogram of pv

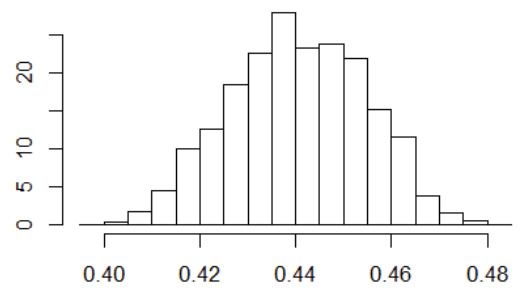

pv

Histogram of phiv

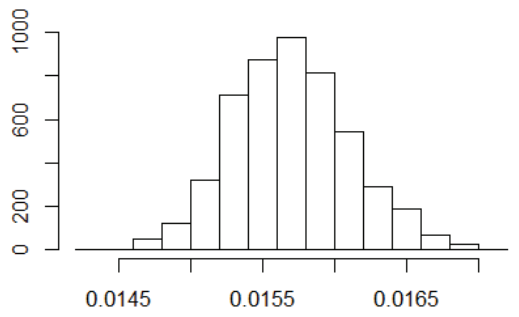

Histogram of yrTv

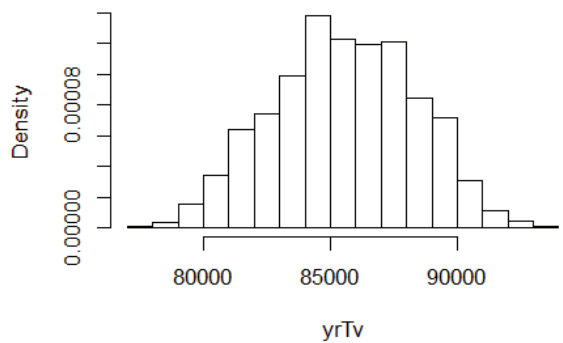

Histogram of lamv

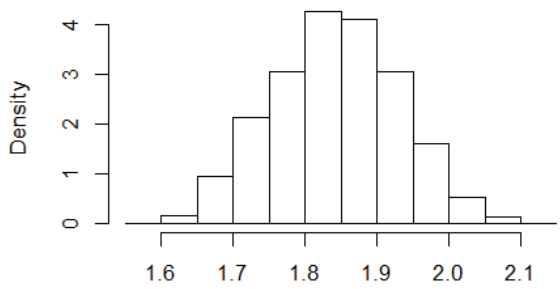

Figure I2.1 2 Histograms using $N=20,000$ and $c=0.2$

Histogram of pv

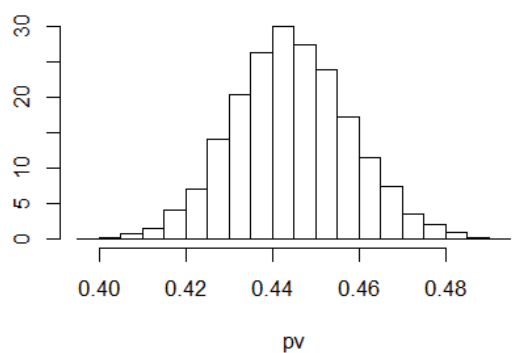

Histogram of phiv

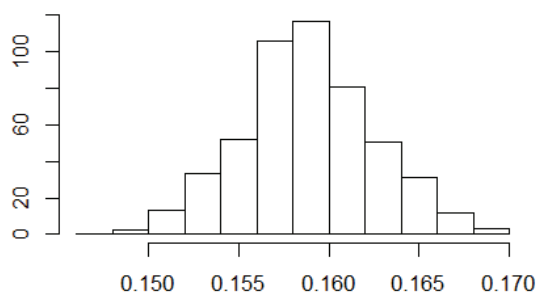

Histogram of yrTv

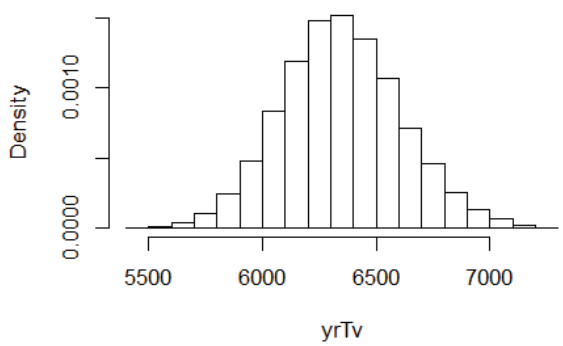

Histogram of lamv

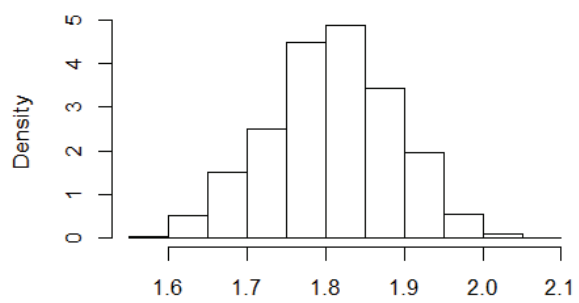


Figure I 2. I 3 Histograms using $N=\mid 5,000$ and $c=0.2$

Histogram of pv

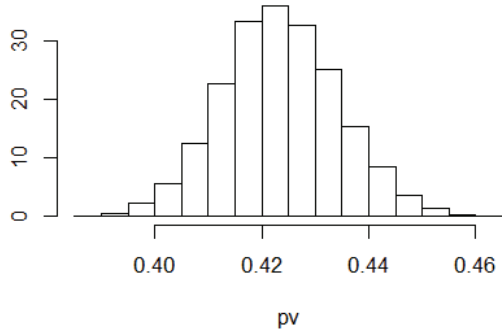

Histogram of phiv

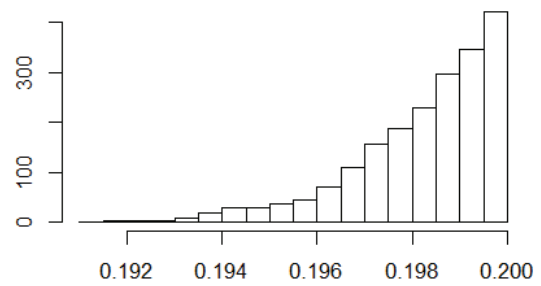

Histogram of yrTv

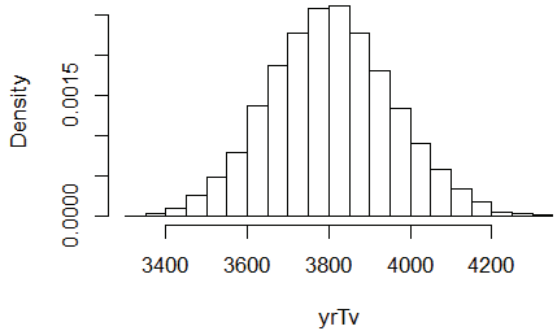

Histogram of lamv

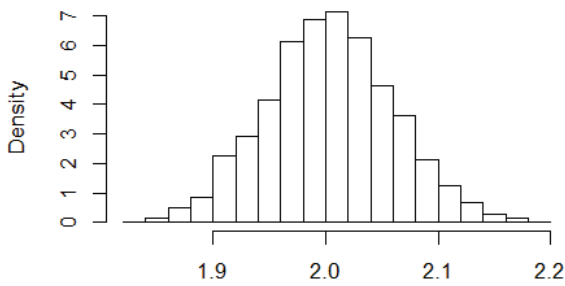

Figure I 2. I 4 Histograms using $N=\mid$ 5,000 and $c=0.5$

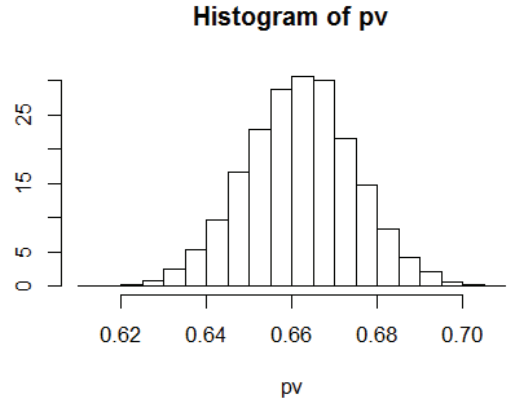

Histogram of phiv

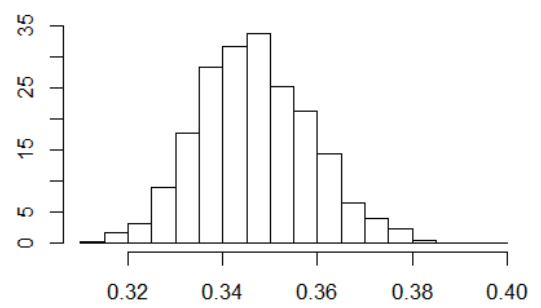

Histogram of yrTv

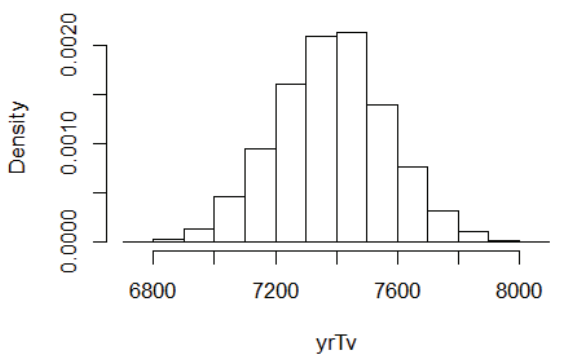

Histogram of lamv

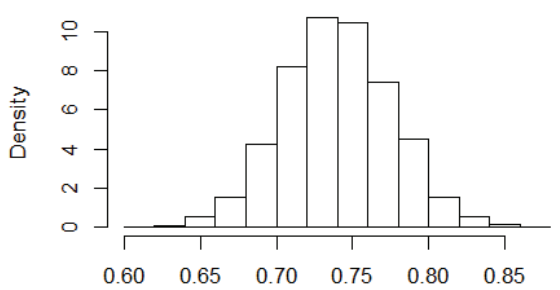


(d) Recall the joint density (12.6). This density may also be written as:

$$
\begin{aligned}
f\left(\phi, \lambda, p, y_{r} \mid I, y_{s}\right) \propto f(\phi, & , p) p^{y_{S T}+y_{r T}}(1-p)^{N-y_{S T}-y_{r T}} \\
& \times \phi^{n} \lambda^{y_{s T}}(1-\phi \lambda)^{y_{r T}}(1-\phi)^{N-n-y_{r T}},
\end{aligned}
$$

where $f(\phi, \lambda, p) \propto \phi^{\delta-1}(1-\phi / c)^{\gamma-1} \times \lambda^{\eta-1}(1-c \lambda)^{\tau-1} \times p^{\alpha-1}(1-p)^{\beta-1}$.

Now observe that

$$
f\left(\phi, \lambda, p, y_{r} \mid I, y_{s}\right) \propto f(\phi, \lambda, p) \times\left\{p^{y_{S T}}(1-p)^{n-y_{s T}} \phi^{n} \lambda^{y_{s T}}\right\} \times \xi,
$$

where: $\xi=[p(1-\phi \lambda)]^{y_{r T}}[(1-p)(1-\phi)]^{N-n-y_{r T}}$

$$
\begin{aligned}
& =[p(1-\phi \lambda)+(1-p)(1-\phi)]^{N-n} \times \prod_{i \in r} z^{y_{i}}(1-z)^{1-y_{i}} \\
Z & =\frac{p(1-\phi \lambda)}{p(1-\phi \lambda)+(1-p)(1-\phi)} .
\end{aligned}
$$

Further observe that

$$
\sum_{y_{r}} \prod_{i \in r} z^{y_{i}}(1-z)^{1-y_{i}}=\prod_{i \in r} \sum_{y_{i}=0}^{1} z^{y_{i}}(1-z)^{1-y_{i}}=1
$$

(since the first product is the joint pdf of $N-n$ iid Bernoulli(z) variables).

It follows that

$$
\begin{aligned}
f\left(\phi, \lambda, p \mid I, y_{s}\right) & =\sum_{y_{r}} f\left(\phi, \lambda, p, y_{r} \mid I, y_{s}\right) \\
\propto f(\phi, \lambda, p) & \times\left\{p^{y_{s T}}(1-p)^{n-y_{s T}} \phi^{n} \lambda^{y_{s T}}\right\} \\
& \times[p(1-\phi \lambda)+(1-p)(1-\phi)]^{N-n} .
\end{aligned}
$$

The above defines a $\mathrm{MH}$ algorithm with three steps based on the following conditionals:

$$
\begin{aligned}
& f(\phi \mid D, \lambda, p) \propto \phi^{\delta+n-1}(1-\phi / c)^{\gamma-1}[p(1-\phi \lambda)+(1-p)(1-\phi)]^{N-n} \\
& f(\lambda \mid D, \phi, p) \propto \lambda^{\eta+y_{s T}-1}(1-c \lambda)^{\tau-1}[p(1-\phi \lambda)+(1-p)(1-\phi)]^{N-n} \\
& f(p \mid D, \phi, \lambda) \propto p^{\alpha+y_{s T}-1}(1-p)^{\beta+n-y_{s T}-1}[p(1-\phi \lambda)+(1-p)(1-\phi)]^{N-n} .
\end{aligned}
$$


(e) From the working in (d) we see that

$$
\left(y_{r T} \mid I, y_{s}, \phi, \lambda, p\right) \sim \operatorname{Bin}(N-n, z),
$$

where

$$
z=\frac{p(1-\phi \lambda)}{p(1-\phi \lambda)+(1-p)(1-\phi)} .
$$

So, to get a sample from the predictive distribution of $\bar{y}$ we do as follows:

1. Obtain $\left(\phi_{j}, \lambda_{j}, p_{j}\right) \sim$ iid $f\left(\phi, \lambda, p \mid I, y_{s}\right), j=1, \ldots, J$ using the $\mathrm{MH}$ algorithm in (d)

2. Sample $y_{r T}^{(j)} \sim \operatorname{Bin}\left(N-n, z_{j}\right)$, where

$$
z_{j}=\frac{p_{j}\left(1-\phi_{j} \lambda_{j}\right)}{p_{j}\left(1-\phi_{j} \lambda_{j}\right)+\left(1-p_{j}\right)\left(1-\phi_{j}\right)}, j=1, \ldots, J \text { (from (12.11)) }
$$

3. Calculate $\bar{y}^{(j)}=\frac{1}{N}\left(y_{s T}+y_{r T}^{(j)}\right), j=1, \ldots, J$.

We now perform the $\mathrm{MH}$ algorithm in (d) and the above procedure with:

$$
\begin{aligned}
& N=200,000, \quad n=4299, \quad y_{s T}=2544, \quad c=0.2 \\
& \alpha=1, \quad \beta=1, \quad \eta=278.1, \quad \tau=474.8, \quad \delta=\gamma=1 .
\end{aligned}
$$

We thereby obtain the inferences shown in Table 12.8 .

\section{Table I 2.8 Results obtained in part (e)}

\begin{tabular}{ccccl} 
phi, $\phi$ & lam, $\lambda$ & $p$ & ybar, $\bar{y}$ & \\
\hline 0.01567 & 1.8491 & 0.43973 & 0.43973 & mean of simulated values \\
0.08660 & 0.0866 & 0.01387 & 0.01382 & sample standard deviation \\
0.01491 & 1.6844 & 0.41331 & 0.41346 & LB of 95\% CPDR estimate \\
0.01650 & 2.0278 & 0.46689 & 0.46673 & UB of 95\% CPDR estimate \\
\hline
\end{tabular}

We see that inferences are very similar to those in the reanalysis of Example 12.5 in (c) with $N=200,000$ (where $\bar{y}$ was estimated as 0.45248). But the results here should in fact be considered more accurate because they are based on a MH algorithm with fewer components. 
Note 1: The inferences on $\bar{y}$ could be further improved via RaoBlackwell arguments which obviate the need to sample values of $y_{r T}$ at all. In particular, the Rao-Blackwell estimate of the predictive mean of the finite population mean, $\hat{\bar{y}}=E(\bar{y} \mid D)$, is

$$
\bar{z}=\frac{1}{J} \sum_{j=1}^{J} z_{j}=0.4364,
$$

with $95 \%$ CI for $\hat{\bar{y}}$

$$
\left(\bar{z} \pm 1.96 \sqrt{\frac{1}{J(J-1)} \sum_{j=1}^{J}\left(z_{j}-\bar{z}\right)^{2}}\right)=(0.4361,0.4367) .
$$

Actually, this is not quite right, since $\bar{z}$ is the Rao-Blackwell estimate of $\hat{\bar{y}}_{r}=E\left(\bar{y}_{r} \mid D\right)$, and the $95 \% \mathrm{CI}$ is for $\hat{\bar{y}}_{r}$. To see this, refer to (12.3).

Thus, since

$$
\bar{y}=\frac{1}{N}\left(y_{s T}+(N-n) \bar{y}_{r}\right),
$$

the RB estimate of $\hat{\bar{y}}$ is actually

$$
\frac{1}{N}\left(y_{s T}+(N-n) \bar{z}\right)=0.440,
$$

with a $95 \%$ confidence interval for $\hat{\bar{y}}$ equal to

$$
\left(\frac { 1 } { N } \left(y_{s T}+(N-n) 0.4361, \frac{1}{N}\left(y_{s T}+(N-n) 0.4367\right)=(0.439,0.440)\right.\right. \text {. }
$$

Note 2: The Monte Carlo 95\% confidence intervals reported here are unduly narrow (i.e. will have less than $95 \%$ actual coverage). This is because we did not address the problem of the very strong serial correlation amongst the values outputted from the Metropolis-Hastings algorithm, for example by way of thinning or the batch means method.

But this remark only applies to confidence intervals for mean estimates and not to posterior or predictive interval estimates, such as $(0.413$, $0.467)$ for $\bar{y}$ in Table 12.8 . 


\section{R Code for Exercise I 2.3}

$\mathrm{MH}=$ function $(\mathrm{J}=100, \mathrm{n}=9453, \mathrm{ysT}=4941, \quad$ alp=830, bet=1395, $\mathrm{p}=0.5$, $\quad$ phi $0=0.1, \operatorname{lam} 0=1, \quad$ phisd $=0.1$, lamsd $=0.1$, eta $=1$, tau $=1, \quad$ del=1, gam $=1, \quad c=0.2, \quad N=200000)\{$

phi=phi0; lam=lam0; phiv=phi; lamv=lam; phict=0; lamct=0; $p v=N A ; y r T v=N A$ for(j in $1: J)\{$

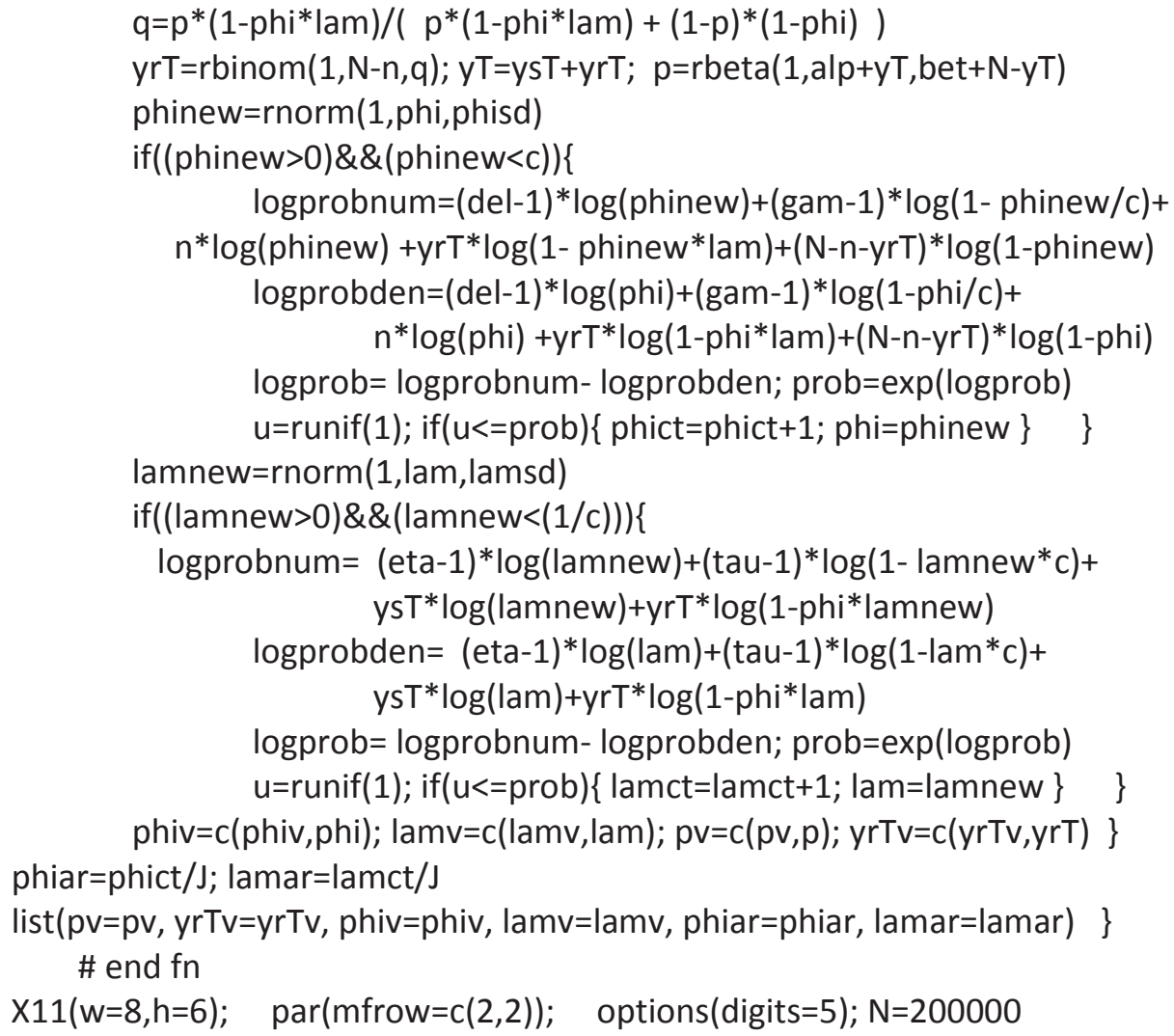

\# record last values 
set.seed(131); $\mathrm{K}=10000$; date() \#

res $=\mathrm{MH}(\mathrm{J}=\mathrm{K}, \mathrm{n}=9453$, ysT $=4941, \quad$ alp $=830$, bet $=1395$, $\mathrm{p}=\mathrm{p0}, \quad$ phi $0=$ phi0, $\operatorname{lam} 0=\operatorname{lam} 0, \quad$ phisd $=0.0006$, lamsd=0.04, eta=1, tau=1, del=1, gam=1, c=0.2, N=N ); date() \#

c(res\$phiar,res\$lamar) \# 0.55480 .5707 OK

plot(res\$pv); plot(res\$yrTv); plot(res\$phiv); plot(res\$lamv) \# OK

\# Example of optional thinning to reduce serial correlation:

\# acf(res\$pv[-1]); acf (res\$yrTv[-1]); acf (res\$phiv[-1]); acf (res\$lamv[-1])

\# skip=10; inc=1+seq(skip,K,skip); J=length(inc); J \# 1000

$\# p v=r e s \$ p v[i n c] ; y r T v=r e s \$ y r T v[i n c] ;$ phiv=res\$phiv[inc]; lamv=res\$lamv[inc] \# acf(pv); acf(yrTv); acf(phiv); acf(lamv) \# better

skip=1; inc=1+seq(skip,K,skip); J=length(inc); J \# 10000 (Just use whole sample)

$p v=\operatorname{res} \$ p v[i n c] ; y r T v=\operatorname{res} \$ y r T v[i n c] ; p h i v=r e s \$ p h i v[i n c] ;$ lamv=res\$lamv[inc] hist(pv,prob=T); hist(yrTv, prob=T); hist(phiv, prob=T); hist(lamv, prob=T); \# OK

\# Calculate estimates (Note we could improve these via Rao-Blackwell):

phat $=$ mean $(p v) ; p c p d r=q u a n t i l e(p v, c(0.025,0.975)) ; p s e=s d(p v)$

lamhat=mean(lamv); lamcpdr=quantile (lamv,c(0.025,0.975)); lamse=sd(lamv)

phihat=mean(phiv); phicpdr=quantile(phiv, $c(0.025,0.975))$; phise=sd $($ lamv $)$

$\mathrm{n}=9453 ; \mathrm{ysT}=4941 ; \mathrm{ybarv}=(1 / \mathrm{N}) *(\mathrm{ys} T+\mathrm{yrTv})$;

ybarhat=mean(ybarv); ybarcpdr=quantile(ybarv,c(0.025,0.975));

ybarse $=$ sd(ybarv)

print(cbind(c(phihat, phise ,phicpdr), c(lamhat, lamse ,lamcpdr),

$c($ phat, pse,pcpdr), c(ybarhat,ybarse, ybarcpdr)), digits=4)

\# B

\begin{tabular}{|c|c|c|c|c|c|}
\hline \# & phi & lam & $p$ & ybar & \\
\hline \# & 0.03597 & 1.84686 & 0.37259 & 0.37259 & mean \\
\hline \# & 0.08789 & 0.08789 & 0.01017 & 0.01022 & se \\
\hline \# 2.5\% & 0.03449 & 1.68272 & 0.35266 & 0.35250 & LB \\
\hline \# 97.5\% & 0.03749 & 2.02311 & 0.39190 & 0.39202 & UB \\
\hline
\end{tabular}

\# Repeat above exactly from A to B but after setting $\mathrm{N}=400000$. Results:

$\begin{array}{lllll}\# & 0.01803 & 1.83548 & 0.37394 & 0.373948 \\ \# & 0.08546 & 0.08546 & 0.00981 & 0.009832 \\ \# 2.5 \% & 0.01731 & 1.68407 & 0.35413 & 0.354113 \\ \# \text { \# } 97.5 \% & 0.01878 & 2.00923 & 0.39122 & 0.391193\end{array}$


\# Repeat above exactly from A to B but after setting N=40000. Results:

\begin{tabular}{|c|c|c|c|c|}
\hline \# & 0.18123 & 1.81588 & 0.375693 & 0.375834 \\
\hline \# & 0.07579 & 0.07579 & 0.009203 & 0.009399 \\
\hline \# 2.5\% & 0.17492 & 1.66922 & 0.357356 & .357050 \\
\hline \# 97.5\% & \% 0.18813 & 1.97208 & 0.393969 & 0.39450 \\
\hline
\end{tabular}

\# Now calculate new prior from posterior of lambda (based on 1st run above): c(lamhat,lamse) \# 1.8468640 .087889

fun=function(etatau, $c=0.2$, est=lamhat, se=lamse) \{ (est-(1/c)*etatau[1]/sum(etatau) $)^{\wedge} 2+$ $\left.\left(\operatorname{se}^{\wedge} 2-\left(1 / c^{\wedge} 2\right)^{*} \operatorname{prod}(\text { etatau }) /\left(\operatorname{sum}(\text { etatau })^{\wedge} 2 *(1+\operatorname{sum}(\text { etatau }))\right)\right)^{\wedge} 2\right\}$ etataunew0 = optim(par=c(2,5), fn=fun)\$par etataunew $=$ optim $($ par $=$ etataunew0, fn=fun $) \$ p a r$

etanew=etataunew[1]; taunew=etataunew[2] c(etanew, taunew) \# 278.10474 .79 $(1 / 0.2) *$ etanew/(etanew+taunew) \# 1.8469 $\operatorname{sqrt}\left(\left(1 / 0.2^{\wedge} 2\right)^{*} \text { etanew*taunew/((etanew+taunew }\right)^{\wedge} 2^{*}($ etanew+taunew +1$\left.\left.)\right)\right)$ \# 0.087889 OK

\# Now run MCMC with new prior and data: $\operatorname{par}(\operatorname{mfrow}=c(2,2)) ; \quad \mathrm{N}=200000$

\# C

set.seed(531); res=MH(J=2000, $\mathrm{n}=4299$, ysT=2544, alp=1, bet $=1$, $\mathrm{p}=0.5$, $\quad$ phi0 $=0.1, \quad \operatorname{lam} 0=1, \quad$ phisd $=0.0007$, lamsd $=0.04$, eta=etanew, tau=taunew, del=1, gam $=1, \quad c=0.2, \quad N=N$ ) c(res\$phiar,res\$lamar) \# 0.42950 .5485 OK plot(res\$pv); plot(res\$yrTv); plot(res\$phiv); plot(res\$lamv) \# Has burnt in OK p0=res\$pv[2001]; lam0=res\$lamv[2001]; phi0=res\$phiv[2001]

\# record last values

set.seed(131); K=10000; date() \# res $=\mathrm{MH}(J=K, \mathrm{n}=4299, \mathrm{ysT}=2544$, alp=1, bet $=1$, $\mathrm{p}=\mathrm{p0}, \quad$ phi0 $=$ phi0, $\operatorname{lam} 0=\operatorname{lam} 0, \quad$ phisd $=0.0004, \operatorname{lamsd}=0.05$, eta= etanew, tau=taunew, del=1, gam=1, c=0.2, N=N ); date() \# c(res\$phiar,res\$lamar) \# 0.54730 .5908 OK plot(res\$pv); plot(res\$yrTv); plot(res\$phiv); plot(res\$lamv) \# OK

skip=1; inc=1+seq(skip,K,skip); J=length(inc); J \# 10000 (Just use whole sample)

$p v=\operatorname{res} \$ p v[i n c] ; y r T v=\operatorname{res} \$ y r T v[i n c] ; p h i v=r e s \$ p h i v[i n c] ; l a m v=r e s \$ l a m v[i n c]$ hist(pv,prob=T); hist(yrTv,prob=T); hist(phiv,prob=T); hist(lamv, prob=T); \# OK 
\# Calculate estimates (Note we could improve these via Rao-Blackwell):

phat=mean $(p v) ; p c p d r=q u a n t i l e(p v, c(0.025,0.975)) ; p s e=s d(p v)$ lamhat=mean(lamv); lamcpdr=quantile (lamv, $c(0.025,0.975))$; lamse=sd(lamv) phihat=mean(phiv); phicpdr=quantile(phiv, $c(0.025,0.975))$; phise=sd (lamv) $\mathrm{n}=$ 9453; ysT=4941; ybarv=(1/N)*(ysT+yrTv); ybarhat=mean(ybarv); ybarcpdr=quantile $(y b a r v, c(0.025,0.975))$; ybarse $=$ sd(ybarv) print(cbind(c(phihat, phise ,phicpdr), c(lamhat, lamse ,lamcpdr), $c($ phat, pse,pcpdr), c(ybarhat,ybarse, ybarcpdr)), digits=4)

\# D

$\begin{array}{lllll}\# & \text { phi } & \text { lam } & \text { p } & \text { ybar } \\ \# & 0.01570 & 1.84272 & 0.44049 & 0.45248 \text { mean } \\ \# & 0.08792 & 0.08792 & 0.01408 & 0.01403 \text { se } \\ \# 2 ~ 2.5 \% & 0.01495 & 1.67553 & 0.41344 & 0.42555 \text { LB } \\ \# \quad 97.5 \% & 0.01656 & 2.01139 & 0.46602 & 0.47799 \text { UB }\end{array}$

\# Repeat above exactly from $\mathrm{C}$ to $\mathrm{D}$ but with $\mathrm{N}=400000$. Results:

$\begin{array}{lrlll}\# & 0.007863 & 1.83516 & 0.44193 & 0.44792 \\ \# & 0.087755 & 0.08776 & 0.01375 & 0.01372 \\ \# \text { 2 2.5\% } & 0.007482 & 1.66809 & 0.41563 & 0.42160 \\ \# \text { \# } 97.5 \% & 0.008299 & 2.00048 & 0.46819 & 0.47409\end{array}$

\# Repeat above exactly from C to D but with $\mathrm{N}=40000$. Results:

$\begin{array}{lrlll}\# & 0.07888 & 1.82895 & 0.44228 & 0.50220 \\ \# & 0.08162 & 0.08162 & 0.01359 & 0.01337 \\ \# 2.5 \% & 0.07538 & 1.66402 & 0.41490 & 0.47517 \\ \# \text { A } 97.5 \% & 0.08278 & 1.99275 & 0.47007 & 0.52985\end{array}$

\# Repeat above exactly from C to D but with $\mathrm{N}=20000$ and 15000 to produce \# extra graphs. We omit the code for the case $\mathrm{N}=15000, \mathrm{c}=0.5$ and the case $\# \mathrm{~N}=15000, \mathrm{c}=1$

\# (e)

$\mathrm{MH} 2=$ function $(J=100, \mathrm{n}=9453, \mathrm{ysT}=4941$, alp=830, bet=1395, $\mathrm{p} 0=0.5$, phi0 $=0.1, \operatorname{lam} 0=1, \quad \mathrm{psd}=0.1$, phisd $=0.1$, lamsd=0.1, eta $=1$, tau $=1, \quad$ del=1, gam $=1, \quad c=0.2, \quad N=200000)\{$

$\mathrm{p}=\mathrm{p0}$; phi=phi0; lam=lam0; $\mathrm{pv}=\mathrm{p}$; phiv=phi; lamv=lam; pct=0; phict=0; lamct=0; 
for(j in 1:J)\{

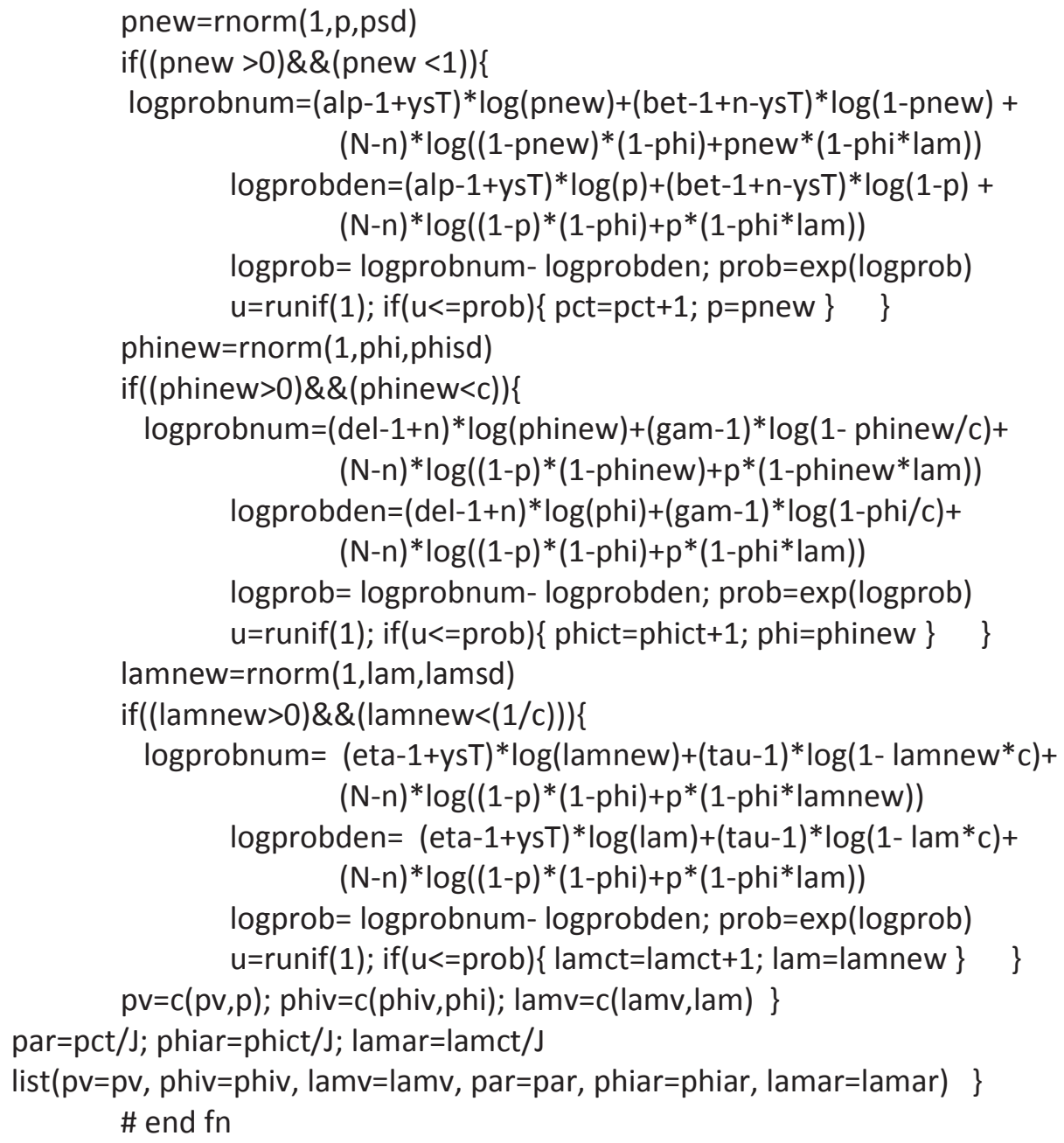


set.seed(131); K=10000; $\operatorname{par}(\operatorname{mfrow}=c(2,2)) ;$ date() \#

res $=\mathrm{MH} 2(\mathrm{~J}=\mathrm{K}, \mathrm{n}=4299$, ysT $=2544$, alp=1, bet $=1$,

$\mathrm{p} 0=\mathrm{p} 0, \quad$ phi $0=$ phi0, $\operatorname{lam} 0=\operatorname{lam} 0, \mathrm{psd}=0.008, \quad$ phisd $=0.0006$, lamsd=0.04,

eta= etanew, tau=taunew, del=1, gam=1, c=0.2, N=N ); date() \# c(res\$par, res\$phiar,res\$lamar) \# 0.68250 .43150 .6643 OK

plot(res\$pv); plot(res\$phiv); plot(res\$lamv) \# OK

skip=1; inc=1+seq(skip,K,skip); J=length(inc); J

\# 10000 (Just use whole sample)

$p v=\operatorname{res} \$ p v[i n c] ;$ phiv=res\$phiv[inc]; lamv=res\$lamv[inc]

$\operatorname{par}(\operatorname{mfrow}=c(2,2))$; hist $(\mathrm{pv}, \operatorname{prob}=\mathrm{T})$; hist(phiv, prob=T); hist(lamv, prob=T);

\# OK

\# Calculate estimates

phat $=$ mean $(p v) ; p c p d r=q u a n t i l e(p v, c(0.025,0.975)) ; p s e=s d(p v)$

lamhat=mean(lamv); lamcpdr=quantile $(\operatorname{lamv,c}(0.025,0.975))$; lamse=sd(lamv)

phihat=mean(phiv); phicpdr=quantile(phiv,c(0.025,0.975)); phise=sd(lamv)

\# Generate sample from predictive dsn of finite population mean $z v=p v^{*}\left(1-p h i v^{*}\right.$ lamv)/( pv*(1-phiv*lamv) + (1-pv)*(1-phiv) )

set.seed(331); yrTv = rbinom $(J, N-n, z v) ; \quad \operatorname{ybarv}=(1 / N) *(y s T+y r T v)$ ybarhat=mean(ybarv); ybarcpdr=quantile(ybarv,c(0.025,0.975));

ybarse $=s d($ ybarv $)$

\# Print out inferences

print(cbind(c(phihat, phise ,phicpdr), c(lamhat, lamse, lamcpdr), c(phat, pse,pcpdr), c(ybarhat,ybarse, ybarcpdr)), digits=4)

$\begin{array}{lrlll}\# & 0.01567 & 1.8491 & 0.43973 & 0.43973 \\ \# & 0.08660 & 0.0866 & 0.01387 & 0.01382 \\ \# \text { 2.5\% } & 0.01491 & 1.6844 & 0.41331 & 0.41346 \\ \# \text { 97.5\% } & 0.01650 & 2.0278 & 0.46689 & 0.46673\end{array}$

RBest=mean(zv); RBci=RBest+c(-1,1)* qnorm(0.975)*sd(zv)/sqrt(J) c(RBest,RBci) \# 0.436390 .436120 .43667

$(1 / N) *(y s T+(N-n) * R B e s t) \quad \# 0.43973$

$(1 / N) *(y s T+(N-n) * R B c i) \# 0.439460 .44000$ 


\section{Additional Exercises}

\section{Exercise A.I Practice with the Metropolis algorithm}

(a) Sample a value $m$ from the standard exponential distribution. Then randomly sample $n=100$ values from the normal distribution with mean $m$ and variance $v=m^{2}$.

Then design and implement a Metropolis algorithm so as to obtain a random sample of size $J=1,000$ from the posterior of $m$.

Use this sample to perform Monte Carlo inference on $\mathrm{m}$. Be sure to provide a $95 \%$ CI for the posterior mean of $m$, an estimate of the $95 \%$ central posterior density region for $m$, and an estimate of the entire marginal posterior density of $m$.

Then predict $c$, the average of a future independent sample of size $k=10$ from the normal distribution with the same mean $m$ and variance $v$.

Be sure to provide a 95\% CI for the predictive mean of $c$, an estimate of the $95 \%$ central predictive density region for $c$, and an estimate of the entire posterior predictive density of $c$.

Illustrate your results with suitable figures (for example, trace plots and histograms).

(b) Consider the following values in a sample obtained via SRSWOR from a finite population of size $N=50$ :

$$
3.4,6.3,1.0,2.9,1.8, \quad 2.0,0.5,7.9,4.8,6.5 \text {. }
$$

Suppose we model the finite population values as normal with (unknown) mean $m$ and variance $v=m^{2}$, with a standard exponential prior on $m$.

Using MCMC methods, estimate the finite population mean and provide a suitable $95 \%$ interval estimate. 


\section{Solution to Exercise A.I}

(a) The sampled value of $m$ was 0.7071 . A histogram of the 100 sampled normal values is shown in Figure A.1(a) (page 612). This histogram is overlaid by the (known) normal distribution with mean $m$ and variance $v=m^{2}=0.5$.

The posterior density of $m$ is

$$
\begin{aligned}
f(m \mid y) & \propto f(m) f(y \mid m) \\
& \propto e^{-m} \prod_{i=1}^{n} \frac{1}{m} \exp \left\{-\frac{1}{2 m^{2}}\left(y_{i}-m\right)^{2}\right\} \\
& =e^{-m} m^{-n} \exp \left\{-\frac{1}{2 m^{2}} \sum_{i=1}^{n}\left(y_{i}-m\right)^{2}\right\} .
\end{aligned}
$$

So the log-posterior is

$$
l(m)=\log f(m \mid y)=-m-n \log m-\frac{1}{2 m^{2}} \sum_{i=1}^{n}\left(y_{i}-m\right)^{2} .
$$

A suitable Metropolis algorithm is one which at each iteration proposes a value

$$
m^{\prime} \sim U(m-\delta, m+\delta),
$$

where $\delta$ is a tuning constant, and accepts this value with probability

$$
p=e^{q},
$$

where

$$
q=l\left(m^{\prime}\right)-l(m) .
$$

Implementing this algorithm we obtained the 10,100 values of $m$, whose trace is shown in Figure A.1(b) (page 612). Stochastic convergence appears to have been attained immediately, and so the burn-in was conservatively taken to be 100 .

The last 10,000 of these 10,100 values are highly autocorrelated, as evidenced by the sample ACF in Figure A.1(c) (page 612). However, thinning out by a factor of 10 removes almost all of the autocorrelation, as seen in the sample ACF in Figure A.1(d) (page 612), and yields the required random sample

$$
m_{1}, \ldots, m_{J} \sim \text { iid } f(m \mid y),
$$

where $J=1,000$. 
A histogram of these 1,000 values of $m$ is shown in Figure A.1(e).

The dashed line in this subplot is a histogram estimate of $f(m \mid y)$, and the solid line is the true posterior density. The vertical lines show the posterior mean estimate, $\bar{m}=0.7377$, the $95 \%$ CI for the posterior mean, (0.7350, 0.7404), and the 95\% CPDR estimate for $m$, (0.6620, 0.8298).

The dots show the true posterior mean, $\hat{m}=E(m \mid y)=0.7393$, and the true 95\% CPDR for $m$. The cross shows the true value of $m, 0.7071$.

The Monte Carlo sample was used to generate a random sample from the predictive distribution of

$$
c=\left(y_{n+1}+\ldots+y_{n+10}\right) / 10
$$

by sampling

$$
c_{j} \sim N\left(m_{j}, m_{j}^{2} / 10\right), j=1, \ldots, J .
$$

A histogram of these $c$-values is shown in Figure A.1(f).

The dashed line in this subplot is a histogram estimate of $f(c \mid y)$, and the solid line is the Rao-Blackwell estimate

$$
\bar{f}(c \mid y)=\frac{1}{J} \sum_{j=1}^{J} \frac{1}{m_{j} \sqrt{2 \pi}} \exp \left\{-\frac{1}{2 m_{j}^{2}}\left(c-m_{j}\right)^{2}\right\} .
$$

The vertical lines show the predictive mean estimate, $\bar{c}=0.741$, the $95 \%$ CI for the predictive mean, (0.7270, 0.7549), and the 95\% CPDR estimate for $c,(0.3063,1.1893)$.

The dot shows the Rao-Blackwell estimate of $\hat{c}=E(c \mid y)$, which is the same as $\bar{m}=0.7377$.

The Rao-Blackwell 95\% CI for $\hat{c}$ is the same as the 95\% CI $(0.7350$, 0.7404 ) reported earlier. 
Figure A.I Graphical results for part (a)

(a) Histogram of $100 \mathrm{y}$-values

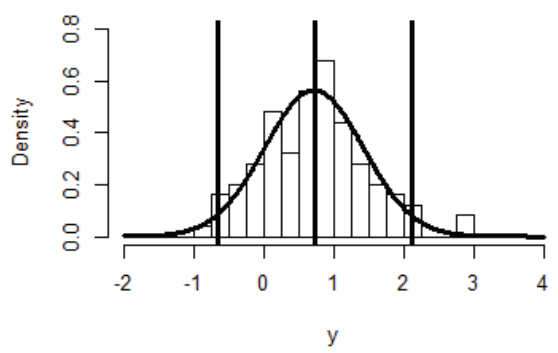

(c) Sample ACF of 10000 m-values

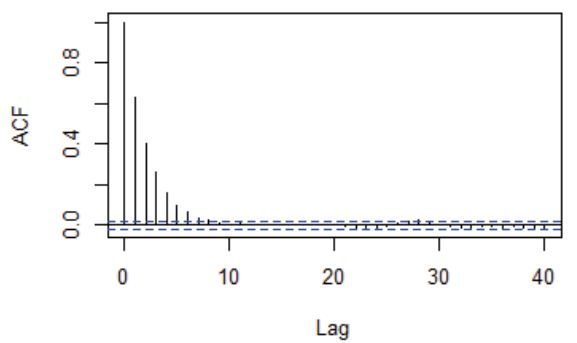

(b) Trace of $10100 \mathrm{~m}$-values

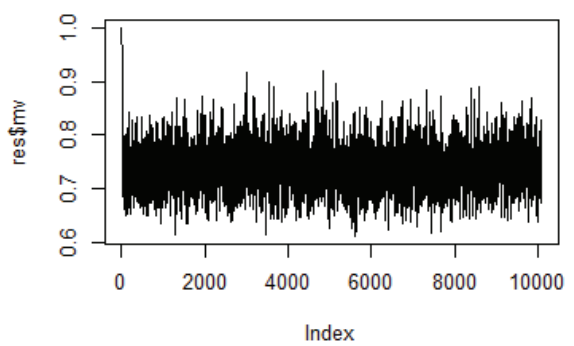

(d) Sample ACF of $1000 \mathrm{~m}$-values

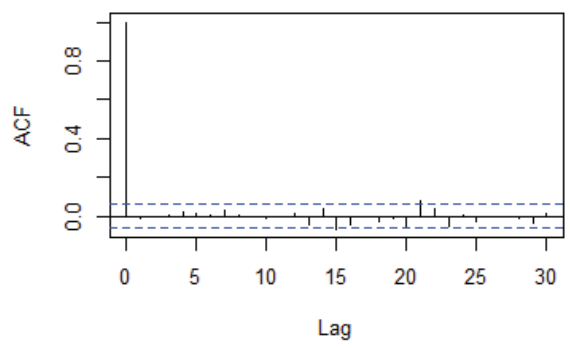

(e) Histogram of 1000 m-values

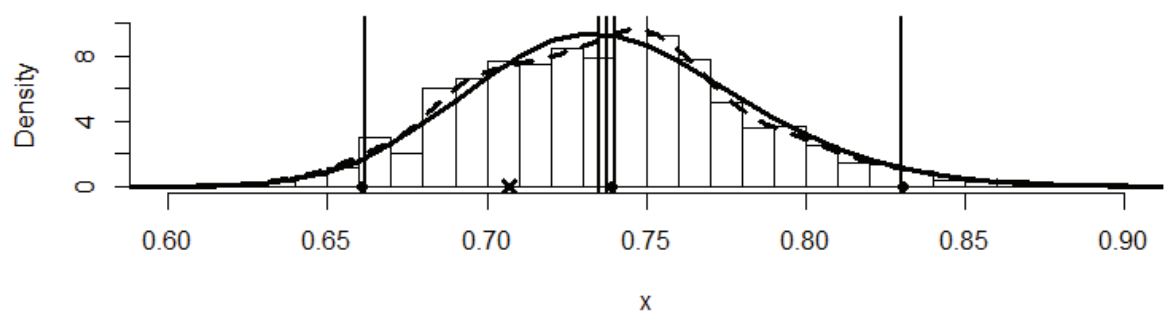

(f) Histogram of $1000 \mathrm{c}$-values

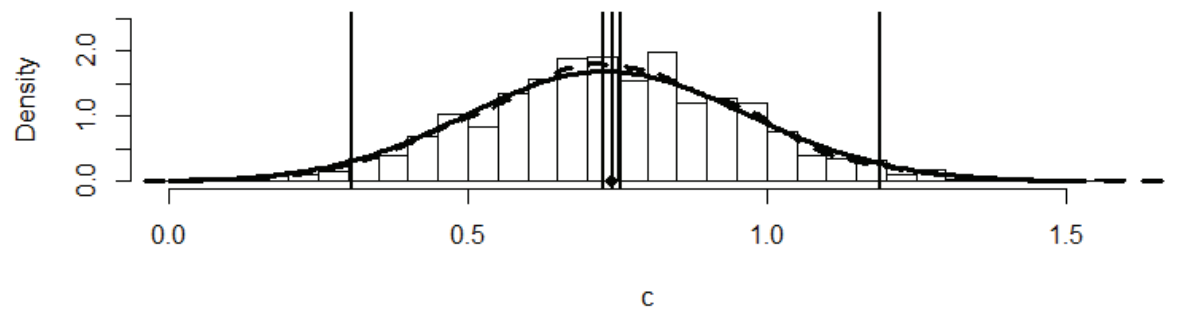


(b) Here we repeat the procedure in part (a), but:

- with $n=10$ (rather than 100)

- using the 10 given sample values, whose mean is 3.71

(instead of the 100 generated values, as previously)

- with $c=\frac{1}{40}\left(y_{11}+\ldots+y_{50}\right) \quad$ (instead of $\left.c=\frac{1}{10}\left(y_{101}+\ldots+y_{110}\right)\right)$.

Figure A.2 is an analogue of Figure A.1, except that subplot (a) does not have a normal density overlaid, and there is an extra subplot (g) that shows inference on the finite population mean, which may be denoted here by

$$
a=\frac{1}{50}(10 \times 3.71+40 c) \text {. }
$$

Figure A.2 Graphical results for part (b)

(a) Histogram of $10 y$-values

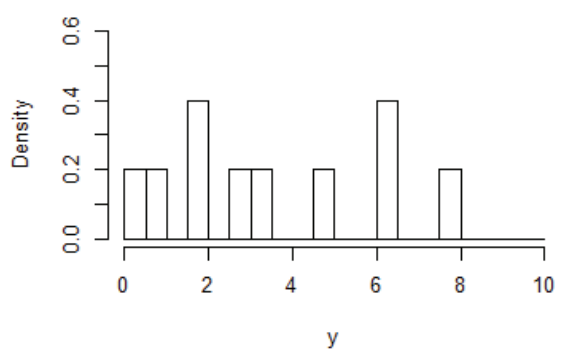

(c) Sample ACF of 10000 m-values

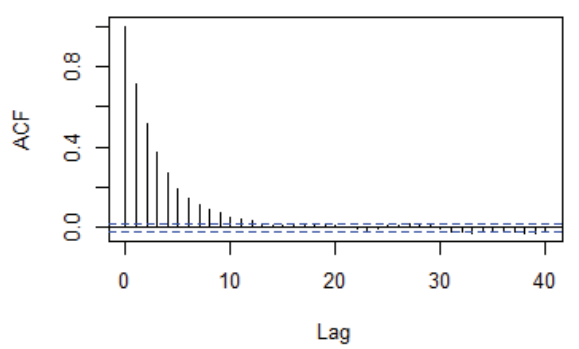

(b) Trace of $10100 \mathrm{~m}$-values

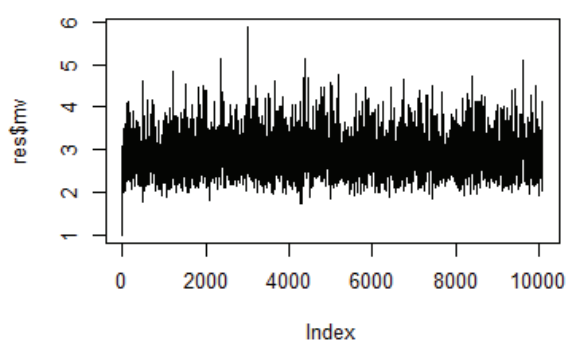

(d) Sample ACF of $1000 \mathrm{~m}$-values

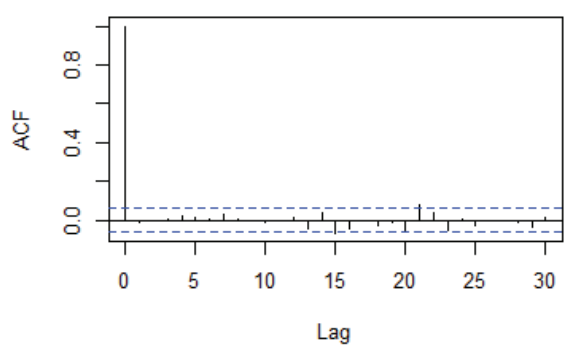


(e) Histogram of $\mathbf{1 0 0 0}$ m-values

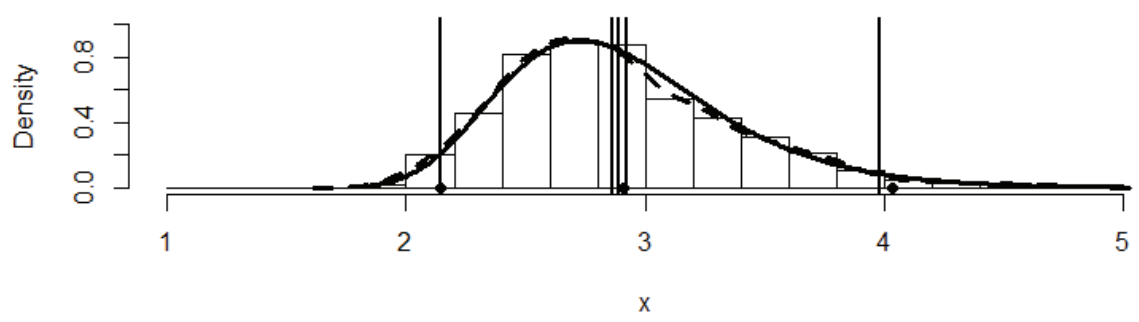

(f) Histogram of $1000 \mathrm{c}$-values

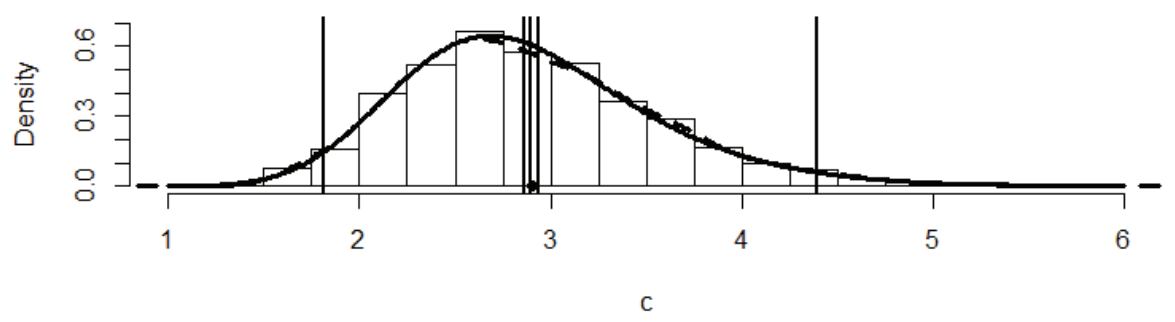

(g) Histogram of 1000 a-values (finite population mean)

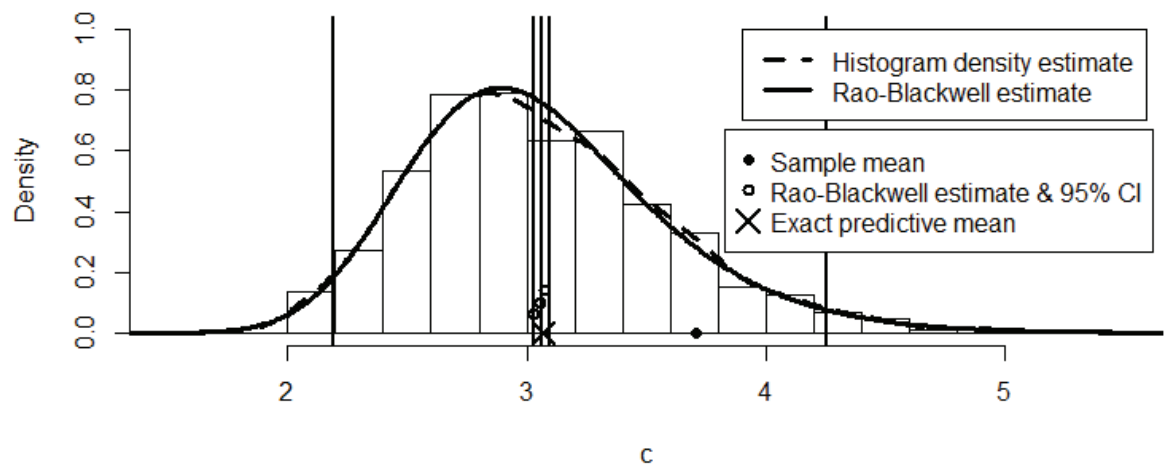

Some of the estimates and quantities shown in the last subplot (g) are as follows. The histogram estimate of $a$ 's predictive mean is $\bar{a}=3.061$ with 95\% CI $(3.028,3.094)$. The Rao-Blackwell estimate of $a$ 's predictive mean is $(10 \times 3.71+40 \bar{m}) / 50=3.055$, with $95 \%$ CI $(3.031,3.078)$. The exact predictive mean of $a$ is the same as the posterior mean of $m$ and equal to 3.068. The 95\% CPDR estimate for $a$ is 2.190 4.256. 


\section{R Code for Exercise A.I}

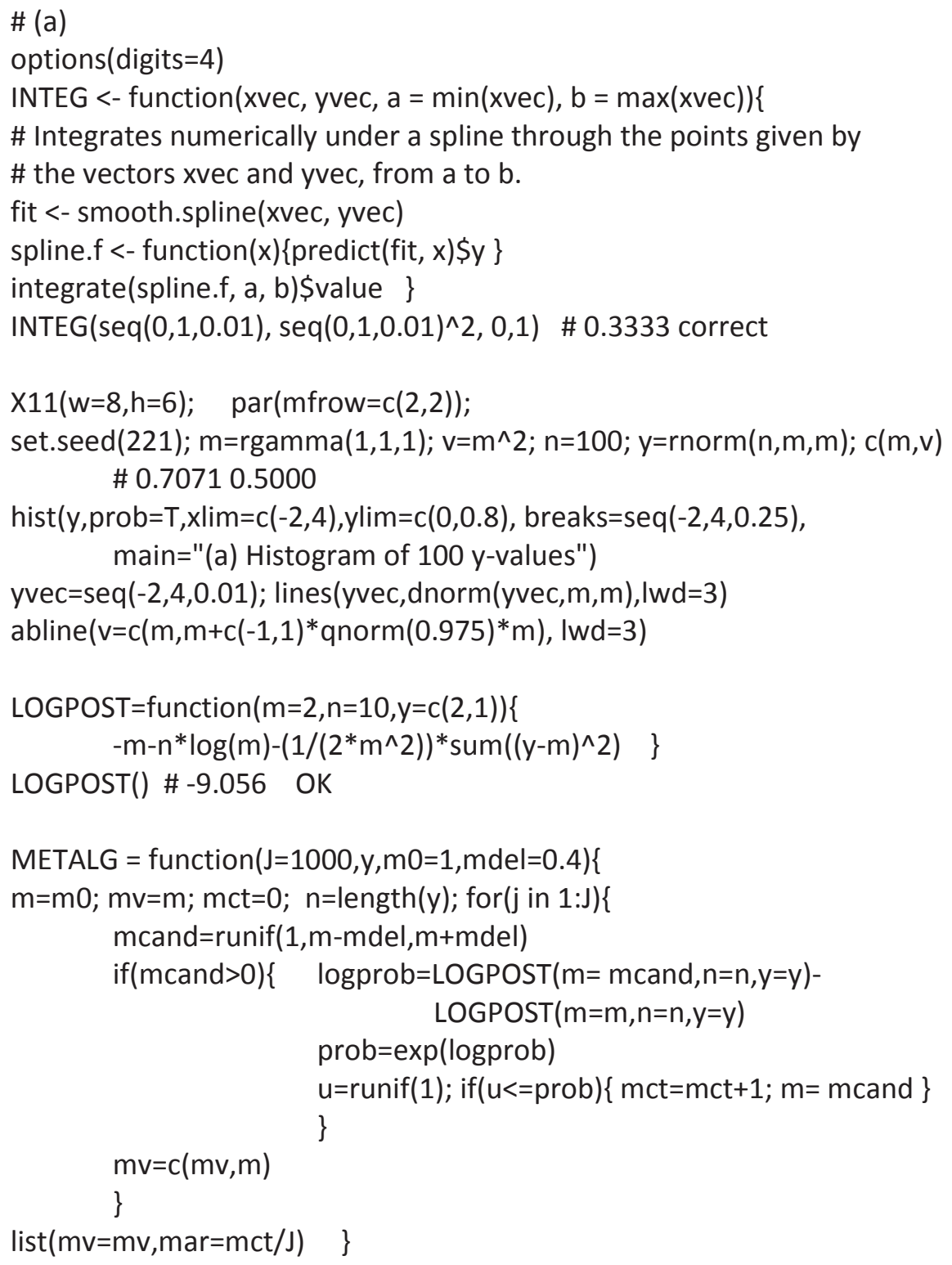


mbar=mean $(\mathrm{mv}) ; \operatorname{mci}=\mathrm{mbar}+\mathrm{c}(-1,1) *$ qnorm $(0.975) * \mathrm{sd}(\mathrm{mv}) / \mathrm{sqrt}(\mathrm{J})$ mcpdr=quantile $(m v, c(0.025,0.975))$;

mvec=seq $(0.5,1,0.01) ; \mathrm{kvec}=\mathrm{mvec}$;

for $(\mathrm{i}$ in 1:length $(\mathrm{mvec})) \mathrm{kvec}[\mathrm{i}]=\exp (\operatorname{LOGPOST}(\mathrm{m}=\mathrm{mvec}[\mathrm{i}], \mathrm{n}=\mathrm{n}, \mathrm{y}=\mathrm{y}))$

k0=INTEG(mvec,kvec); postvec=kvec/k0; k0 \# 6.269e-11

mhat=INTEG(mvec, mvec* postvec);

c(mbar,sd(mv),mhat,mci,mcpdr)

\# 0.737690 .043050 .739350 .735020 .740360 .661970 .82984

fun=function $\left.(q, p=0.025)\{(\text { INTEG (mvec,postvec, } 0, q)-p)^{\wedge} 2\right\}$

LBO = optim(par=0.5,fn=fun)\$par; LB = optim(par= LBO,fn=fun)\$par

fun=function $(q, p=0.975)\left\{(\text { INTEG }(\text { mvec, postvec, } 0, q)-p)^{\wedge} 2\right\}$

UBO = optim(par=0.8,fn=fun)\$par; UB = optim(par= UBO,fn=fun)\$par

c(LB,UB) \# 0.66090 .8305

INTEG(mvec, postvec,0,LB) \# 0.025

INTEG(mvec,postvec,UB,1) \# 0.025 OK (Ignore all the warnings)

$\operatorname{par}(\operatorname{mfrow}=c(2,1))$

hist $(m v, \operatorname{prob}=T, x \lim =c(0.6,0.9), y \lim =c(0,10)$, breaks=seq $(0.5,1,0.01)$, $x l a b=" x "$ main="(e) Histogram of 1000 m-values")

lines(mvec, postvec, Ity=1,lwd=3)

lines(density(mv), Ity=2,lwd=3)

abline $(v=c(m b a r, m c i, m c p d r), l w d=2)$

points $(\mathrm{c}(\mathrm{mhat}, \mathrm{LB}, \mathrm{UB}), \mathrm{c}(0,0,0), \mathrm{pch}=16)$

points $(m, 0, p c h=4, l w d=3)$

\# Prediction of $\mathrm{c}$

set.seed(332); cv=rnorm(J,mv,mv/sqrt(10))

cbar=mean(cv); cci=cbar+c(-1,1)*qnorm $(0.975) *$ sd(cv)/sqrt(J)

ccpdr=quantile(cv,c(0.025,0.975))

c(cbar,sd(cv),cci,ccpdr) \# 0.7410 0.2253 0.7270 0.75490.3063 1.1893

hist(cv,prob=T,xlim=c(0,1.6), ylim=c(0,2.5), breaks=seq $(0,1.6,0.05)$, xlab="c",main="(f) Histogram of 1000 c-values")

$\mathrm{cvec}=\operatorname{seq}(0,1.5,0.01) ; \mathrm{fcvec}=\operatorname{seq}(0,1.5,0.01)$; for $(\mathrm{i}$ in $1:$ length $(\mathrm{cvec}))$ fcvec[i]=mean (dnorm(cvec[i],mv,mv/sqrt(10)))

lines (cvec, fcvec, Ity=1, Iwd=3)

lines(density(cv), Ity=2,lwd=3)

abline $(v=c(c b a r, c c i, c c p d r), l w d=2)$

points(mhat, $0, p c h=16$ ) 
\# (b)

$\mathrm{X} 11(\mathrm{w}=8, \mathrm{~h}=6) ; \quad \operatorname{par}(\mathrm{mfrow}=\mathrm{c}(2,2))$;

$y=c(3.4,6.3,1.0,2.9,1.8,2.0,0.5,7.9,4.8,6.5) ; n=10 ; y \operatorname{bar}=\operatorname{mean}(y)$;

ybar \# 3.71

hist(y,prob=T,xlim=c(0,10),ylim=c(0,0.6), breaks=seq $(0,10,0.5)$, main="(a) Histogram of 10 y-values")

set.seed(312); res=METALG(J=10100,y=y,m0=1, $\mathrm{mdel}=1)$; res\$mar \# 0.5954

plot(res\$mv,type="I",main="(b) Trace of 10100 m-values");

acf(res\$mv, main="(c) Sample ACF of 10000 m-values")

acf(res\$mv,plot=F)[1:5] \# 0.710 0.513 0.374 0.270 0.195

$\operatorname{acf}(m v$, main="(d) Sample ACF of 1000 m-values")

$m v=r e s \$ m v[-(1: 101)][\operatorname{seq}(10,10000,10)] ;$

acf(mv,plot=F)[1:5] \# $0.0560 .001-0.006-0.027 \quad 0.035$

J=length(mv); J \# 1000

mbar=mean $(m v) ; m c i=m b a r+c(-1,1) * q n o r m(0.975) * s d(m v) / s q r t(J)$

mcpdr=quantile $(m v, c(0.025,0.975))$;

mvec=seq $(1.8,5,0.01)$; $k v e c=m v e c$;

for $(\mathrm{i}$ in 1:length $(\mathrm{mvec})) \operatorname{kvec}[\mathrm{i}]=\exp (\operatorname{LOGPOST}(\mathrm{m}=\mathrm{mvec}[\mathrm{i}], \mathrm{n}=\mathrm{n}, \mathrm{y}=\mathrm{y}))$

k0=INTEG(mvec,kvec); postvec=kvec/k0; k0 \# 3.317e-08

mhat=INTEG(mvec, mvec* postvec);

$\mathrm{c}(\mathrm{mbar}, \mathrm{sd}(\mathrm{mv})$, mhat, mci, mcpdr)

\# 2.89070 .48232 .90712 .86082 .92062 .14563 .9827

fun=function $\left.(q, p=0.025)\{(\text { INTEG (mvec,postvec, } 1.8, q)-p)^{\wedge} 2\right\}$

LBO = optim (par=2.1,fn=fun)\$par; LB = optim(par= LBO,fn=fun)\$par

fun=function( $\left.q, p=0.975)\{(\text { INTEG (mvec,postvec, } 1.8, q)-p)^{\wedge} 2\right\}$

UBO = optim(par=4.1,fn=fun)\$par; UB = optim(par= UBO,fn=fun)\$par

c(LB,UB) \# 2.1434 .033

INTEG(mvec, postvec,1.8,LB) \# 0.025

INTEG(mvec, postvec,UB,5) \# 0.025 OK (Ignore all the warnings)

$\operatorname{par}(\operatorname{mfrow}=c(2,1))$

$\operatorname{hist}(\mathrm{mv}, \operatorname{prob}=\mathrm{T}, \mathrm{xlim}=c(1,5), \mathrm{ylim}=c(0,1)$, breaks=seq $(1,5,0.2)$, $x l a b=" x "$,main="(e) Histogram of 1000 m-values")

lines(mvec, postvec, Ity=1,lwd=3)

lines(density (mv), Ity=2,lwd=3)

abline $(v=c(m b a r, m c i, m c p d r), l w d=2)$

points (c(mhat, LB,UB), c(0,0,0),pch=16)

points $(m, 0, p c h=4, \operatorname{lwd}=3$ ) 
\# Prediction of $c=(1 / 40)(y 11+\ldots+y 50) \quad$ (new definition) set.seed(332); cv=rnorm(J,mv,mv/sqrt(40))

cbar=mean(cv); cci=cbar+c(-1,1)*qnorm $(0.975) *$ sd(cv)/sqrt(J)

ccpdr=quantile(cv,c(0.025,0.975))

c(cbar,sd(cv),cci,ccpdr) \#2.8985 0.6594 2.8577 2.93941 .81054 .3925

hist(cv,prob=T,xlim=c(1,6), ylim=c(0,0.7), breaks=seq $(1,6,0.25)$, $x l a b=" c "$,main="(f) Histogram of 1000 c-values")

cvec=seq $(1,6,0.01)$; $f$ cvec=seq $(1,6,0.01)$; for(i in 1:length(cvec)) fcvec[i]=mean(dnorm(cvec[i],mv,mv/sqrt(40)))

lines(cvec, fcvec, Ity=1,lwd=3)

lines(density (cv), Ity=2,lwd=3)

abline $(v=c(c b a r, c c i, c c p d r), l w d=2)$

points(mhat, 0, pch=16)

\# Now perform inference on the finite population mean,

\# $\mathrm{a}=(1 / 50) *(10 * \mathrm{ybar}+40 * \mathrm{c})$

$\mathrm{av}=(1 / 50) *(10 * \mathrm{ybar}+40 * \mathrm{cv})$

abar=mean(av); aci=abar+c(-1,1)*qnorm(0.975)*sd(av)/sqrt(J)

acpdr=quantile $(a v, c(0.025,0.975))$

c(abar,sd(av),aci,acpdr) \# 3.06080 .52763 .02813 .09352 .19044 .2560

$(1 / 50) *(10 *$ ybar+40*mbar) \# 3.055 RB estimate of predictive mean of a

$(1 / 50) *(10 * y b a r+40 * \mathrm{mci})$ \# $3.0313 .078 \mathrm{RB} \mathrm{Cl}$ for predictive mean of a

$(1 / 50) *(10 *$ ybar $+40 *$ mhat $)$ \# 3.068 Exact predictive mean of a

$\mathrm{X} 11(\mathrm{w}=8, \mathrm{~h}=4) ; \operatorname{par}(\operatorname{mrow}=\mathrm{c}(1,1))$

hist(av, prob=T, $x \lim =c(1.5,5.5)$, ylim=c(0,1), breaks=seq $(1,6,0.2), x l a b=" c "$, main="(g) Histogram of 1000 a-values (finite population mean)")

avec=seq(1,6,0.01); favec=seq $(1,6,0.01)$; for(i in 1:length(avec))

favec $[\mathrm{i}]=$

mean( dnorm( avec[i], (1/50)*( 10*ybar+40*mv), mv*sqrt(40)/50 ) )

lines(avec,favec, Ity=1,lwd=3); lines(density(av), Ity=2,lwd=3)

abline $(v=c($ abar, $a c i, a c p d r), l w d=2)$

points( $(1 / 50) *(10 *$ ybar+40*mbar), 0.1, pch=1, cex=1, Iwd=2)

points $(1 / 50) *(10 * y b a r+40 * \mathrm{mci}), c(0.06,0.14), \mathrm{pch}=1, \mathrm{cex}=1, \mathrm{lwd}=2)$

points( $(1 / 50) *(10 * y b a r+40 *$ mhat $), 0, p c h=4, l w d=2$, cex $=2)$

points (ybar,0,cex=1,lwd=2,pch=16)

legend(3.9,1, c("Histogram density estimate","Rao-Blackwell estimate"), Ity $=c(2,1), \operatorname{lwd}=c(3,3), \mathrm{bg}="$ white")

legend(3.83,0.67,c("Sample mean","Rao-Blackwell estimate \& 95\% Cl",

"Exact predictive mean"),

pch $=c(16,1,4)$, pt.cex $=c(1,1,2), p t . \operatorname{lwd}=c(2,2,2), \quad b g="$ white" $)$ 


\section{Exercise A.2 Practice with the MH algorithm}

(a) Sample a value $a$ from the standard exponential distribution and a value $b$ from the uniform distribution between 0 and 10 (independently).

Then randomly sample $n=100$ values from the gamma distribution with mean $m=a / b$ and variance $v=a / b^{2}$.

Then design and implement a Metropolis-Hastings algorithm so as to generate a random sample of size $J=1,000$ from the joint posterior distribution of $a$ and $b$.

Use this sample to perform Monte Carlo inference on $m$.

Be sure to provide a $95 \% \mathrm{CI}$ for the posterior mean of $m$, an estimate of the $95 \%$ central posterior density region for $m$, and an estimate of the entire marginal posterior density of $m$.

Then predict $c$, the average of a future independent sample of size $k=10$ from the gamma distribution with the same mean $m$ and variance $v$.

Be sure to provide a 95\% CI for the predictive mean of $c$, an estimate of the $95 \%$ central predictive density region for $c$, and an estimate of the entire posterior predictive density of $c$.

Illustrate your results with suitable figures (e.g. trace plots and histograms).

(b) Consider the following values in a sample obtained via SRSWOR from a finite population of size $N=30$ :

$$
0.4,3.3,1.0,2.9,1.8,4.1 \text {. }
$$

Suppose we model the finite population values as gamma with mean $m=a / b$ and variance $v=a / b^{2}$, with a standard exponential prior on $m$ and a uniform prior on $b$ between 0 and 10 .

Using MCMC methods, estimate/predict the finite population mean absolute deviation about the superpopulation mean, equivalently referred to as the MAD for short, and defined by

$$
\psi=\frac{1}{N} \sum_{i=1}^{N}\left|y_{i}-m\right| .
$$




\section{Solution to Exercise A.2}

The sampled values of $a$ and $b$ were 1.463 and 5.528. So the value of $m$ was $a / b=0.2647$. The 100 sampled gamma values are shown in Figure A.3(a) (page 621).

Next, the posterior density of the two parameters $a$ and $b$ is

$$
\begin{aligned}
f(a, b \mid y) & \propto f(a, b) f(y \mid a, b) \\
& \propto e^{-a} \prod_{i=1}^{n} \frac{b^{a} y_{i}^{a-1} e^{-b y_{i}}}{\Gamma(a)}=\frac{e^{-a} b^{n a}\left(\prod_{i=1}^{n} y_{i}\right)^{a-1} e^{-b y_{T}}}{\Gamma(a)^{n}} .
\end{aligned}
$$

So the log-posterior is

$$
\begin{aligned}
l(a, b) & =\log f(a, b \mid y) \\
& =-a+n a \log b+(a-1) \sum_{i=1}^{n} \log y_{i}-b y_{T}-n \log \Gamma(a) .
\end{aligned}
$$

A suitable Metropolis algorithm is one which at each iteration:

1. Proposes a value

$$
a^{\prime} \sim U\left(a-\delta_{a}, a+\delta_{a}\right),
$$

where $\delta_{a}$ is a tuning constant, and accepts this value with probability $p=e^{q}$, where $q=l\left(a^{\prime}, b\right)-l(a, b)$

2. Proposes a value

$$
b^{\prime} \sim U\left(b-\delta_{b}, b+\delta_{b}\right),
$$

where $\delta_{b}$ is a tuning constant, and accepts this value with probability $p=e^{q}$, where $q=l\left(a, b^{\prime}\right)-l(a, b)$.

Implementing this algorithm we obtained the required $J=1,000$ values

$$
\left(a_{1}, b_{1}\right), \ldots,\left(a_{J}, b_{J}\right) \sim \text { iid } f(a, b \mid y)
$$

and hence

$$
m_{1}, \ldots, m_{J} \sim \text { iid } f(m \mid y)
$$

by calculating $m_{j}=a_{j} / b_{j}$ for each $j=1, \ldots, J$.

A histogram of these simulated $m$-values is shown in Figure A.3(b) (page $622)$. 
The dashed line is a histogram estimate of $f(m \mid y)$. The vertical lines show the posterior mean estimate, $\bar{m}=0.3017$, the 95\% CI for the posterior mean, $(0.3001,0.3033)$, and the 95\% CPDR estimate for $m$, $(0.2566,0.3570)$. The cross shows the true value of $m, 0.7071$.

The Monte Carlo sample was then used to generate a random sample from the predictive distribution of

$$
c=\left(y_{n+1}+\ldots+y_{n+10}\right) / 10 \text {. }
$$

This was done by sampling

$$
y_{n+1}^{(j)}, \ldots, y_{n+10}^{(j)} \sim \text { iid } G\left(a_{j}, b_{j}\right)
$$

and forming

$$
c_{j}=\left(y_{n+1}^{(j)}, \ldots, y_{n+10}^{(j)}\right) / 10, j=1, \ldots, J .
$$

A histogram of the $c$-values is shown in Figure A.3(c). The dashed line is a histogram estimate of $f(c \mid y)$. The vertical lines are the predictive mean estimate, $\bar{c}=0.2981$, the $95 \%$ CI for the predictive mean, $(0.2929$, $0.3033)$, and the $95 \%$ CPDR estimate for $c,(0.1584,0.4878)$.

Figure A.3 Graphical results for part (a)

(a) Histogram of $100 y$-values

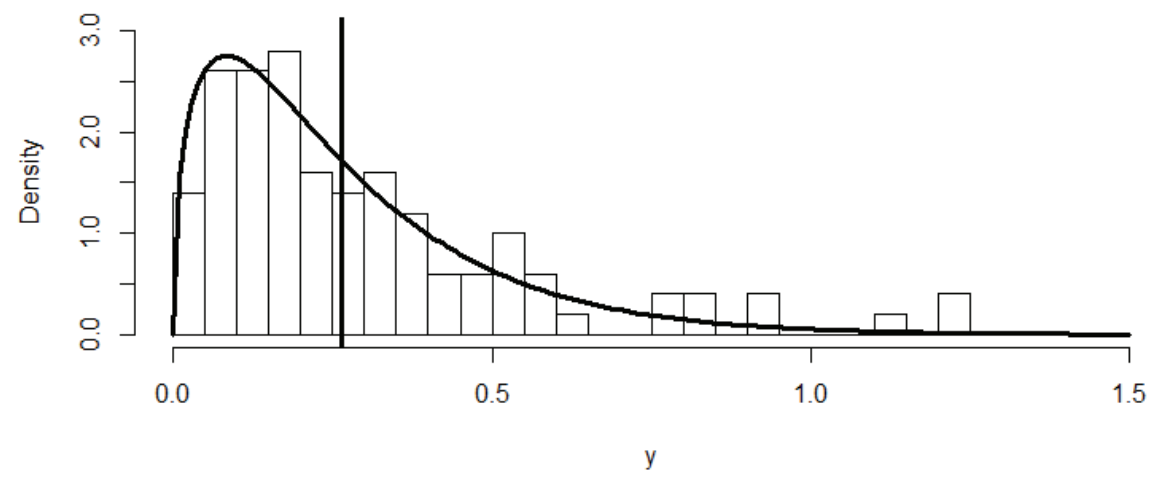


(b) Histogram of 1000 m-values

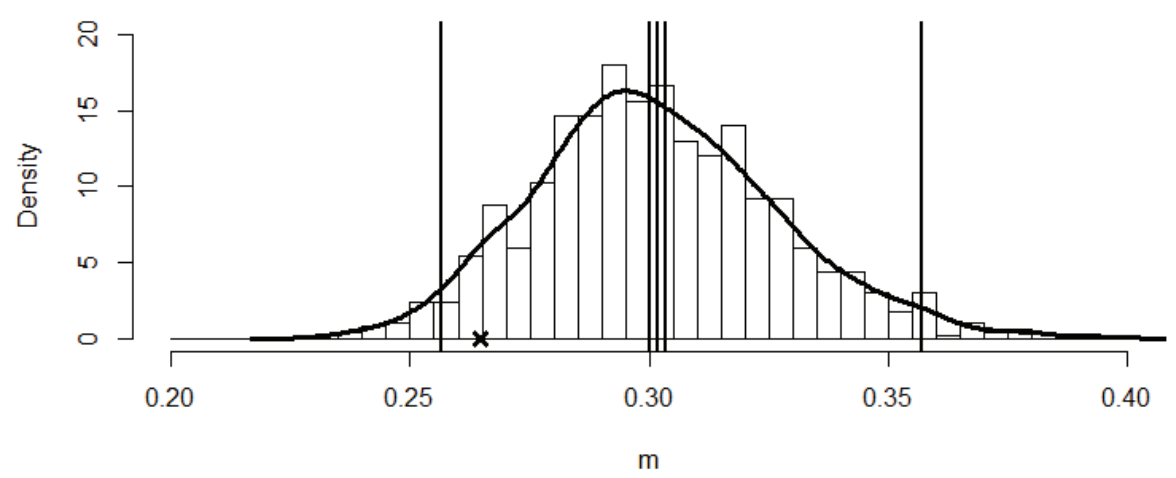

(c) Histogram of $1000 \mathrm{c}$-values

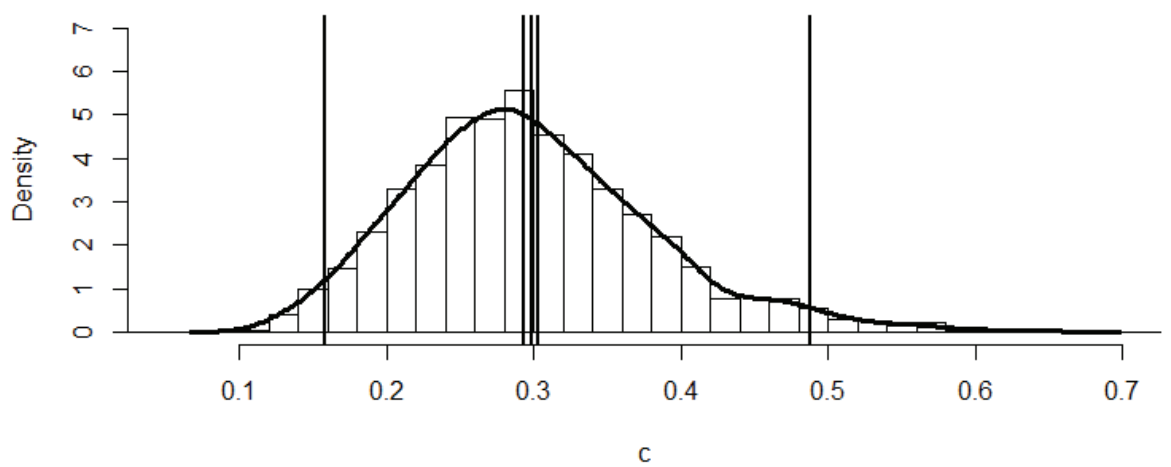

(b) Here we repeat the procedure in (a) but using $n=6$ (rather than 100), and the 6 given sample values whose mean is 2.25 (instead of the 100 generated values as before), so as to generate a Monte Carlo sample of size $J=1,000$ from the posterior distribution of $a$ and $b$.

We then use each pair of values, $a_{j}$ and $b_{j}$, to generate 24 values which are iid from the gamma distribution with parameters $a_{j}$ and $b_{j}$.

Then for each $j$ we calculate the associated value of the MAD, namely

$$
\psi_{j}=\frac{1}{N} \sum_{i=1}^{N}\left|y_{i}-\frac{a_{j}}{b_{j}}\right| .
$$

We then use the resulting $J$ values of the MAD, i.e. $\psi_{1}, \ldots, \psi_{J}$, for Monte Carlo inference in the usual way. 
Figure A.4 shows a histogram of these $J$ values and related information.

Numerically, we estimate $\psi$ 's posterior/predictive mean by 1.307 with $95 \%$ CI $(1.27,1.34)$, and we estimate $\psi$ 's CPDR by $(0.75,2.73)$.

\section{Figure A.4 Histogram of I,000 MAD values}

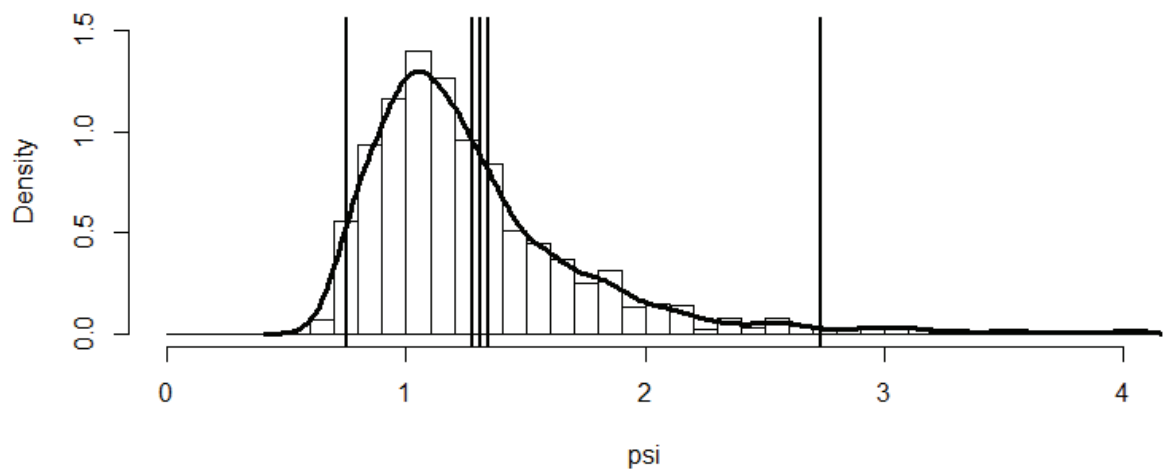

\section{R Code for Exercise A.2}

\# (a)

options(digits=4); $\mathrm{n}=100 ; \mathrm{X} 11(\mathrm{w}=8, \mathrm{~h}=4) ; \quad \operatorname{par}(\operatorname{mfrow}=\mathrm{c}(1,1))$;

set.seed(192); a=rgamma(1,1,1); b=runif(1,0,10); $y=\operatorname{rgamma}(n, a, b)$;

$\mathrm{m}=\mathrm{a} / \mathrm{b} ; \mathrm{v}=\mathrm{a} / \mathrm{b}^{\wedge} 2 ; \mathrm{c}(\mathrm{a}, \mathrm{b}, \mathrm{m}, \mathrm{v}) \# 1.463215 .527630 .264710 .04789$

hist $(y, \operatorname{prob}=T, x \lim =c(0,1.5), y \lim =c(0,3)$, breaks=seq $(0,1.5,0.05)$, main="(a) Histogram of $100 \mathrm{y}$-values")

yvec=seq(0,1.5,0.01); lines(yvec, dgamma(yvec,a,b),Iwd=3)

abline $(v=m, l w d=3)$

sumlogy=sum $(\log (y)) ;$ sumy=sum( $(y)$ \# sufficient statistics

LOGPOST=function ( $a=1, b=1, n=3$, sumlogy $=2$, sumy $=2)\{$

$-a+n * a * \log (b)+(a-1) *$ sumlogy-b*sumy-n*Igamma(a) \}

LOGPOST() \#-3 OK 


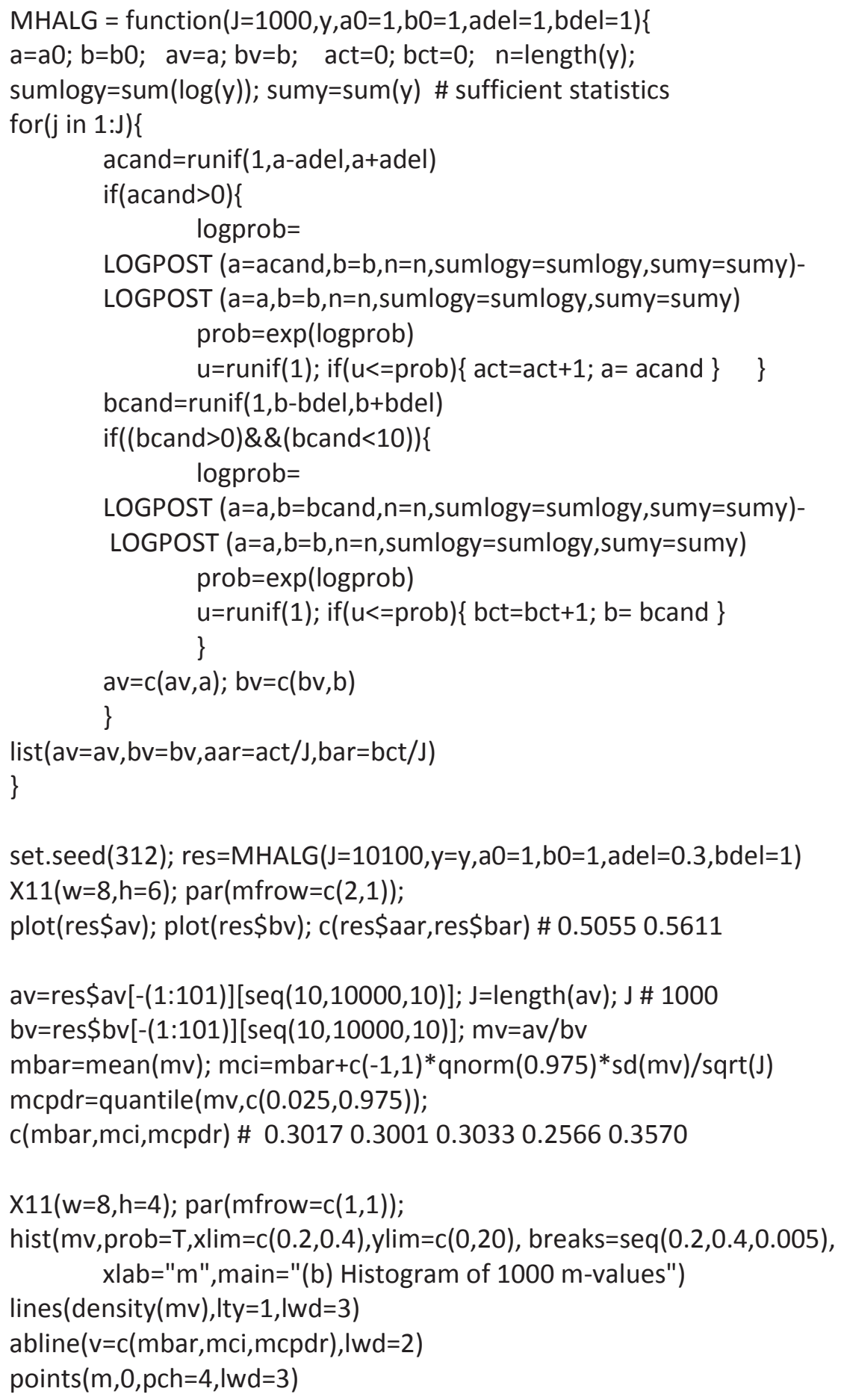


\# Prediction of c

set.seed(332); cv=rep(NA,J); for(j in 1:J) cv[j]=mean(rgamma(10,av[j],bv[j]))

cbar=mean(cv); cci=cbar+c(-1,1)*qnorm $(0.975) *$ sd(cv)/sqrt(J)

ccpdr=quantile(cv,c(0.025,0.975))

c(cbar,sd(cv),cci,ccpdr) \# 0.298120.08356 0.292940.303290.158430.48783

hist(cv,prob=T,xlim=c(0.05,0.7),ylim=c(0,7), breaks=seq $(0,1.6,0.02)$,

xlab="c",main="(c) Histogram of 1000 c-values")

lines(density(cv),lty=1,Iwd=3); abline(v=c(cbar,cci,ccpdr),lwd=2)

\# (b)

$y=c(0.4,3.3,1.0,2.9,1.8,4.1) ; X 11(w=8, h=6) ; \operatorname{par}(\operatorname{mfrow}=c(2,1))$;

$\mathrm{n}=$ length $(\mathrm{y})$; sumlogy=sum $(\log (\mathrm{y}))$; sumy=sum(y) \# sufficient statistics

set.seed(312); res=MHALG(J=10100,y=y,a0=1,b0=1, adel=1.3,bdel=0.7)

plot(res\$av); plot(res\$bv); c(res\$aar,res\$bar) \# 0.51290 .5094

av=res\$av[-(1:101)][seq(10,10000,10)]; J=length(av); J \# 1000

$b v=r e s \$ b v[-(1: 101)][s e q(10,10000,10)] ; m v=a v / b v$

mbar=mean (mv); mci=mbar+c(-1,1)*qnorm(0.975)*sd(mv)/sqrt(J)

mcpdr=quantile $(\mathrm{mv}, \mathrm{c}(0.025,0.975))$;

c(mbar,mci,mcpdr) \# 2.256 2.208 2.305 1.148 4.188

$\mathrm{X} 11(\mathrm{w}=8, \mathrm{~h}=4) ; \operatorname{par}(\operatorname{mfrow}=\mathrm{c}(1,1))$;

hist (mv, prob=T, $x \lim =c(0,7), y \lim =c(0,0.8)$, breaks $=\operatorname{seq}(0,10,0.5)$,

$x l a b=" x "$,main="Histogram of 1000 simulated $m$-values")

lines(density(mv),Ity=2,lwd=3); abline(v=c(mbar,mci, $m c p d r), l w d=2)$

\# Prediction of psi

set.seed(332); $p$ siv=rep(NA,J);

for $(j$ in $1: J)\{\quad$ yrem=rgamma(24,av[j],bv[j])

yall = c(y,yrem); psiv[j]=mean $((\operatorname{abs}($ yall-mv[j]) )) \}

psibar=mean(psiv); psici =psibar+c(-1,1)*qnorm $(0.975) * \operatorname{sd}($ psiv $) / \operatorname{sqrt}(J)$

psicpdr=quantile(psiv,c(0.025,0.975))

c(psibar,sd(psiv),psici,psicpdr) \# 1.30680 .54111 .27321 .34030 .74972 .7349

hist(psiv, prob=T, $x \lim =c(0,4), y \lim =c(0,1.5)$, breaks=seq $(0,7,0.1)$, xlab="psi",main="")

lines(density(psiv),Ity=1,lwd=3); abline(v=c(psibar, psici,psicpdr),lwd=2) 


\section{Exercise A.3 Practice with a Bayesian finite population regression model}

(a) Generate a population of covariates

$$
x_{1}, \ldots, x_{N} \sim \text { iid } U(10,20),
$$

where $N=100$.

Then generate a population of values

$$
y_{i} \sim N\left(a+b x_{i}, \sigma^{2}\right), i=1, \ldots, N,
$$

where $a=3, b=0.5, \sigma=2$.

Then select a random sample of size $n=20$ from the $N$ units in the finite population, without replacement.

Plot the $y$ values against the $x$ values, over the population and over the sample, respectively. Draw the true regression line $y=a+b x$ and the two least squares regression lines estimated using the population data and sample data, respectively.

(b) Consider the following Bayesian model:

$$
\begin{aligned}
& \left(y_{i} \mid a, b, \lambda\right) \sim \perp N\left(a+b x_{i}, 1 / \lambda\right), \quad i=1, \ldots, N \\
& f(a, b, \lambda) \propto 1 / \lambda ; \quad a, b \in \mathfrak{R} ; \quad \lambda>0 .
\end{aligned}
$$

Generate a random sample of size $J=1,000$ from the joint posterior distribution of $a, b$ and $\lambda$, given the sample data generated in (a).

Then use this sample and $\mathrm{R}$ to estimate each of the following quantities:

$$
\begin{aligned}
& m=a+16 b \quad \begin{array}{l}
\text { (average of a hypothetically infinite number of } \\
\text { values with covariate } 16)
\end{array} \\
& \bar{y}=\frac{y_{1}+\ldots+y_{N}}{N} \quad \text { (the finite population mean) } \\
& \psi=\frac{2 y_{(100)}}{y_{(50)}+y_{(51)}} \quad \text { (ratio of maximum to median of the } 100 \text { finite } \\
& \text { population values). }
\end{aligned}
$$

Assume that all $N$ covariate values in the population are known.

(c) Repeat the inferences in (b) but using WinBUGS and a sample size of $J=10,000$. 


\section{Solution to Exercise A.3}

(a) The required plot and regression lines are shown in the Figure A.5.

Figure A.5 Graphical results for part (a)

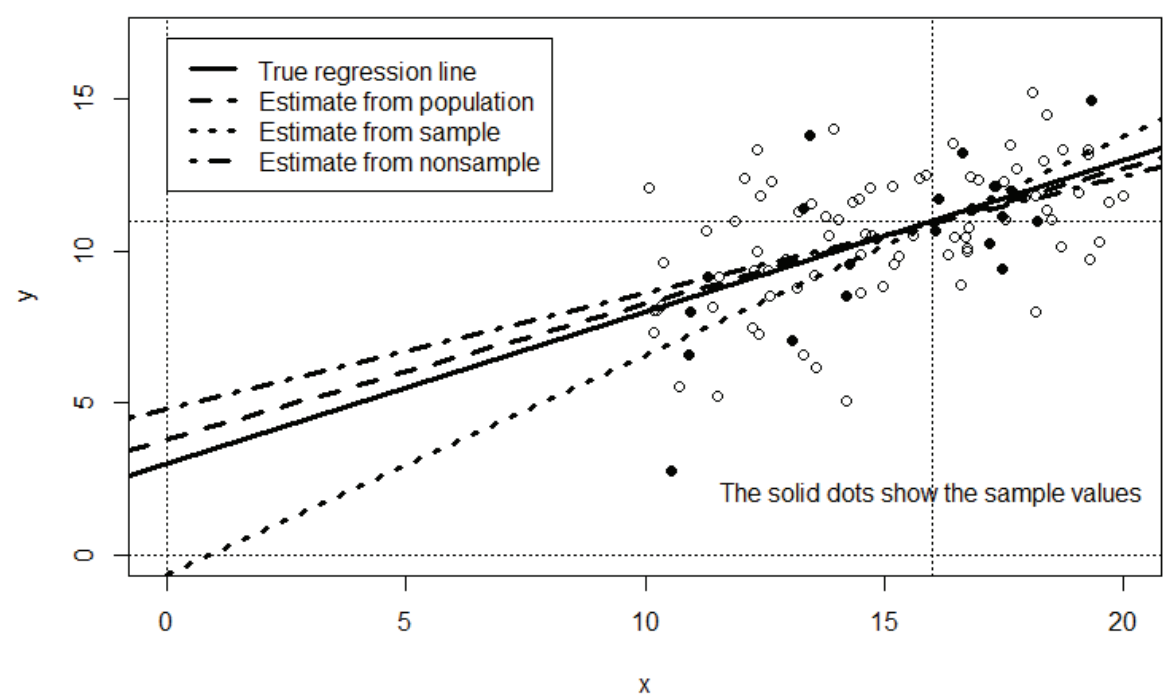

(b) Denote the sample values by $s_{1}, \ldots, s_{n} \in\{1, \ldots, N\}$, where $s_{1}<\ldots<s_{n}$, and define $s=\left(s_{1}, \ldots, s_{n}\right)$.

Then define the population vector as $y=\left(y_{1}, \ldots, y_{N}\right)^{\prime}$ and the sample vector as $y_{s}=\left(y_{s_{1}}, \ldots, y_{s_{n}}\right)^{\prime}$.

Also define $r=\left(r_{1}, \ldots, r_{N-n}\right)=\{1, \ldots, N\}-s$ in such a way that $r_{1}<\ldots<r_{N-n}$, and define the nonsample vector as $y_{r}=\left(y_{r_{1}}, \ldots, y_{r_{N-n}}\right)^{\prime}$.

Likewise, define the population covariate vector as $x=\left(x_{1}, \ldots, x_{N}\right)^{\prime}$, the sample covariate vector as $x_{s}=\left(x_{s_{1}}, \ldots, x_{s_{n}}\right)^{\prime}$, and the nonsample covariate vector as $x_{r}=\left(x_{r_{1}}, \ldots, X_{r_{N-n}}\right)^{\prime}$. 
Also consider all of $x_{1}, \ldots, x_{N}$ as known constants, and define $D=\left(s, y_{s}\right)$ as the data. Also let:

$$
\beta=\left(\begin{array}{l}
a \\
b
\end{array}\right), X_{s}=\left(1_{n}, X_{s}\right), X_{r}=\left(1_{N-n}, X_{r}\right), \quad \Sigma_{s s}=I_{n}, \quad \Sigma_{r r}=I_{N-n} .
$$

Then, from the theory of the normal-normal-gamma finite population model, we have that:

$$
\begin{aligned}
& \left(y_{r} \mid D, \beta, \lambda\right) \sim N_{N-n}\left(X_{r} \beta, \Sigma_{r r} / \lambda\right) \\
& (\beta \mid D, \lambda) \sim N_{2}(T, D / \lambda), \\
& \quad \text { where } D=\left(X_{s}^{\prime} \Sigma_{s s}^{-1} X_{s}\right)^{-1} \text { and } T=\left(X_{s}^{\prime} \Sigma_{s s}^{-1} X_{s}\right)^{-1} X_{s}^{\prime} \Sigma_{s s}^{-1} y_{s} \\
& (\lambda \mid D) \sim G(A / 2, B / 2), \\
& \quad \text { where } A=n-2 \text { and } B=\left(y_{s}-X_{s} T\right)^{\prime} \Sigma_{s s}^{-1}\left(y_{s}-X_{s} T\right) .
\end{aligned}
$$

Thus, to do the required inference, first carry out the following steps:

1. Relabel the population units so that $y_{s}=\left(y_{1}, \ldots, y_{n}\right)^{\prime}$,

$$
\begin{aligned}
& x_{s}=\left(x_{1}, \ldots, x_{n}\right)^{\prime}, y_{r}=\left(y_{n+1}, \ldots, y_{N}\right)^{\prime}, x_{r}=\left(x_{n+1}, \ldots, x_{N}\right)^{\prime}, \text { etc., } \\
& \text { so that } y=\left(y_{s}^{\prime}, y_{r}^{\prime}\right)^{\prime} \text {, etc. }
\end{aligned}
$$

2. Calculate $A, B, D$ and $T$ as per the above

3. Generate $\lambda_{1}, \ldots, \lambda_{J} \sim$ iid $G(A / 2, B / 2)$ (easy)

4. Generate $\beta^{(j)} \sim \perp N_{2}\left(T, D / \lambda_{j}\right)$, for $j=1, \ldots, J \quad$ (easy)

5. Generate $y_{r}^{(1)}, \ldots, y_{r}^{(J)} \sim N_{N-n}\left(X_{r} \beta^{(j)}, \Sigma_{r r} / \lambda_{j}\right)$, for $j=1, \ldots, J$ (e.g. for each $j$, generate $y_{i}^{(j)} \sim \perp N\left(a_{j}+b_{j} x_{i}, 1 / \lambda_{j}\right)$, $i=n+1, \ldots, N$, and form $y_{r}^{(j)}=\left(y_{n+1}^{(j)}, \ldots, y_{N}^{(j)}\right)^{\prime}$

6. Form $y^{(j)}=\left(y_{s}^{\prime}, y_{r}^{(j)^{\prime}}\right)^{\prime}$ for each $j=1, \ldots, J$.

Now calculate

$$
m_{j}=a_{j}+16 b_{j}
$$

and perform Monte Carlo inference on $m$, using the fact that

$$
m_{1}, \ldots, m_{J} \sim \text { iid } f(m \mid D) \text {. }
$$

(For example, estimate $m$ by $\bar{m}=J^{-1} \sum_{j=1}^{J} m_{j}$.)

Likewise, calculate $\bar{y}^{(j)}=1_{N}^{\prime} y^{(j)} / N$ and perform Monte Carlo inference on $\bar{y}$ in the usual way, using the fact that $\bar{y}^{(1)}, \ldots, \bar{y}^{(J)} \sim$ iid $f(\bar{y} \mid D)$. 
Finally, calculate

$$
\psi_{j}=\frac{2 y_{(100)}^{(j)}}{y_{(50)}^{(j)}+y_{(51)}^{(j)}}
$$

and perform Monte Carlo inference on $\psi$, using the fact that

$$
\psi_{1}, \ldots, \psi_{J} \sim \text { iid } f(\psi \mid D) .
$$

Optionally, we may improve on some of the above 'basic' inferences by considering Rao-Blackwell techniques, e.g. estimate $m$ by its exact posterior mean, $\hat{m}=E(m \mid D)=(1,16) T$.

Figure A.6 shows histograms of the simulated values of $m$ (subplot (a)), $\bar{y}$ (subplot (b)) and $\psi$ (subplot (c)), with each subplot overlaid by various points, interval and density estimates.

Subplot (d) (page 631) illustrates 'exact' inference on $\bar{y}$ based on the theory of the normal-normal-gamma finite population model, and subplot (e) (page 631) is a detail in subplot (d). Each plot features a cross showing the true value of the quantity being estimated.

Figure A.6 Graphical results for part (b)

(a) Histogram of 1000 m-values

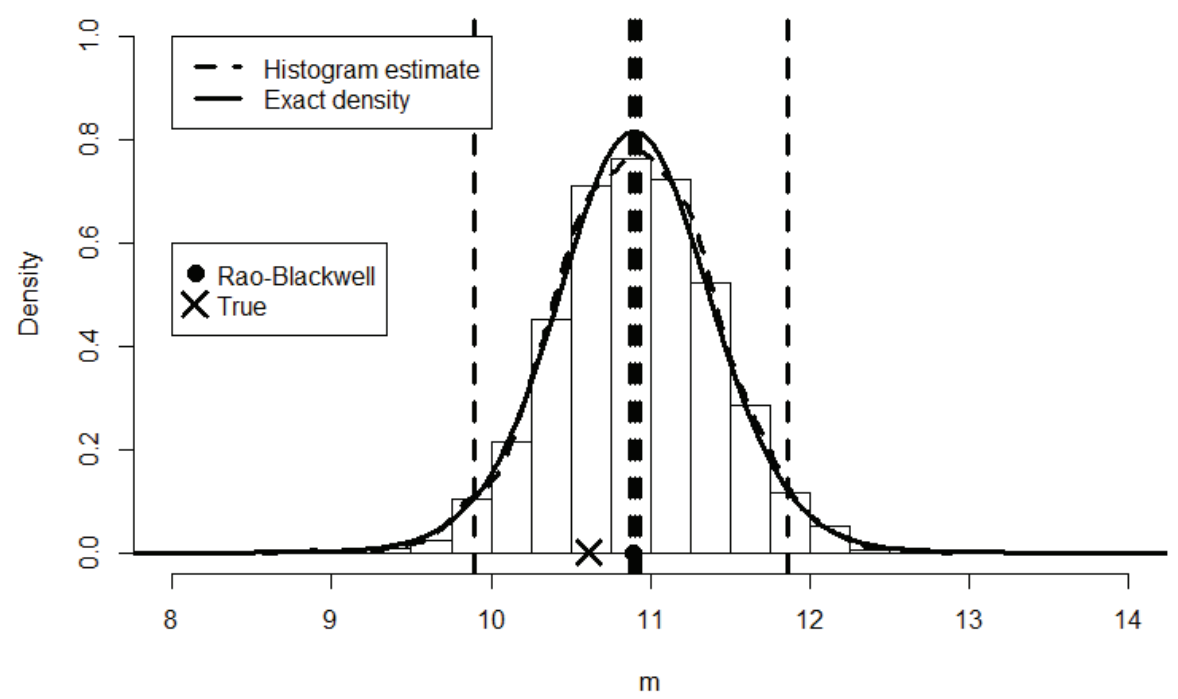


(b) Histogram of 1000 ybar-values

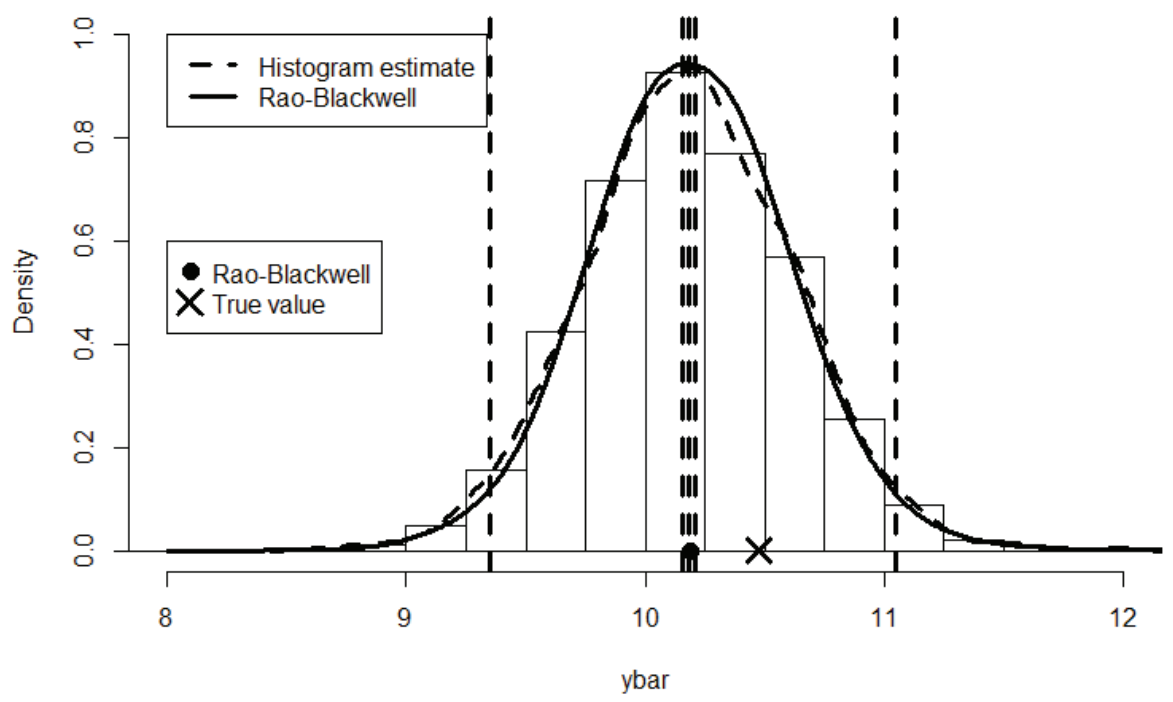

(c) Histogram of 1000 psi-values

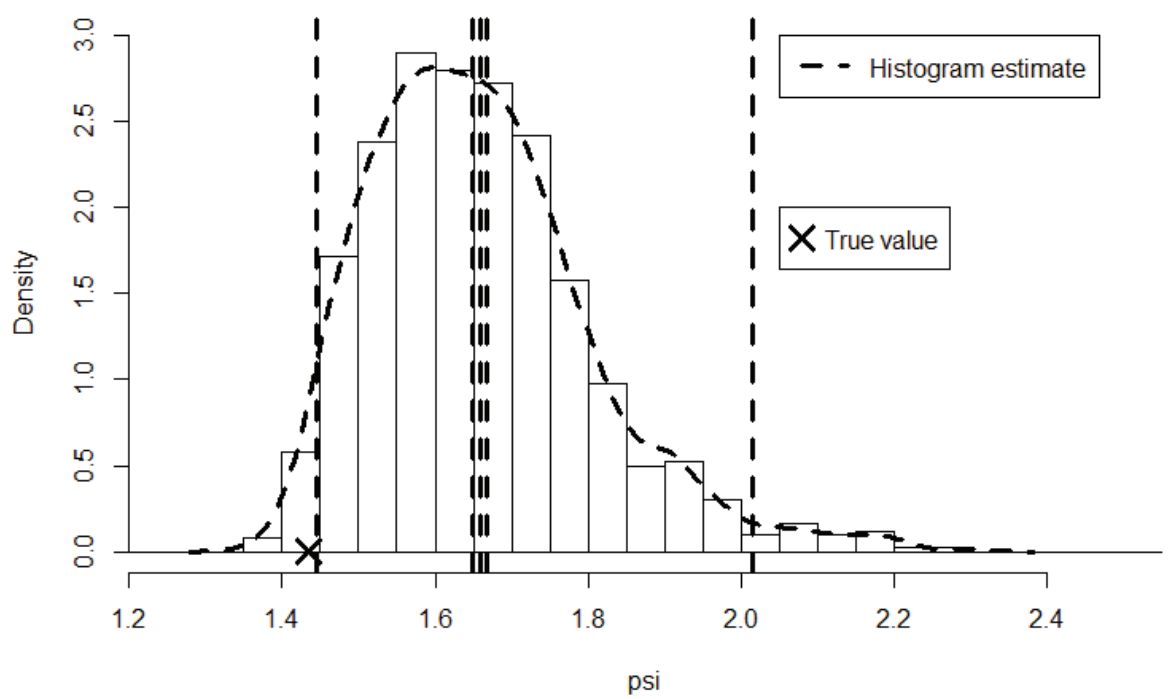


(d) Histogram of 1000 ybar-values

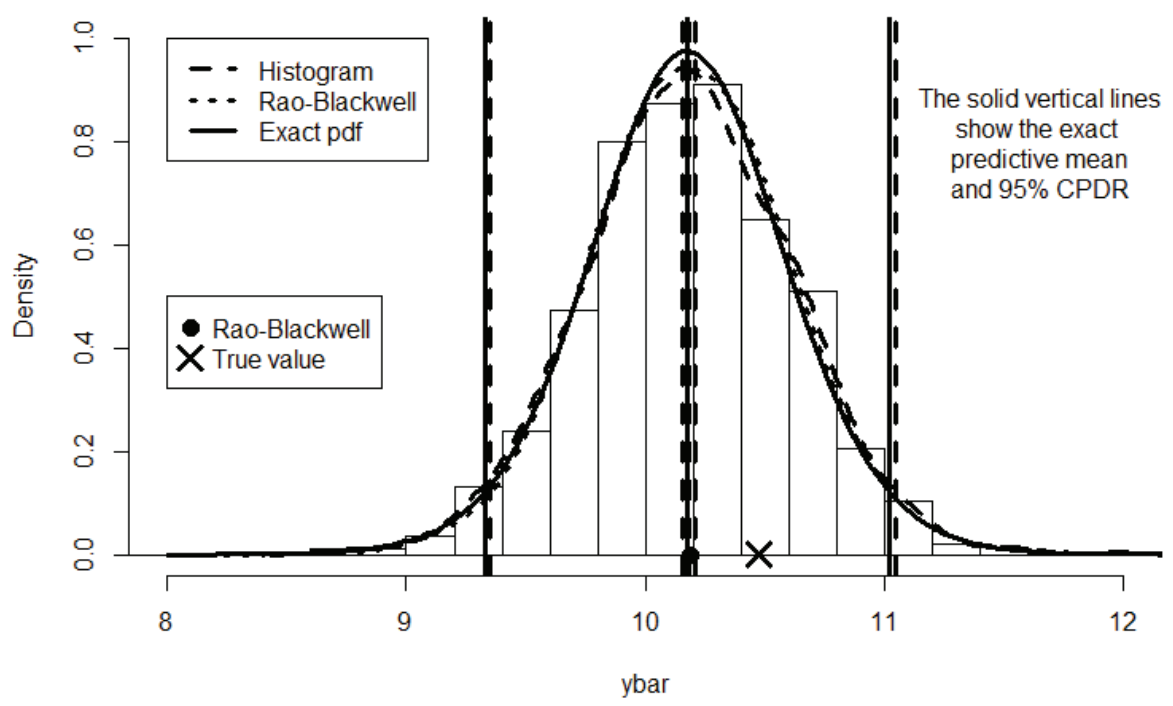

(e) Detail in subplot (d)

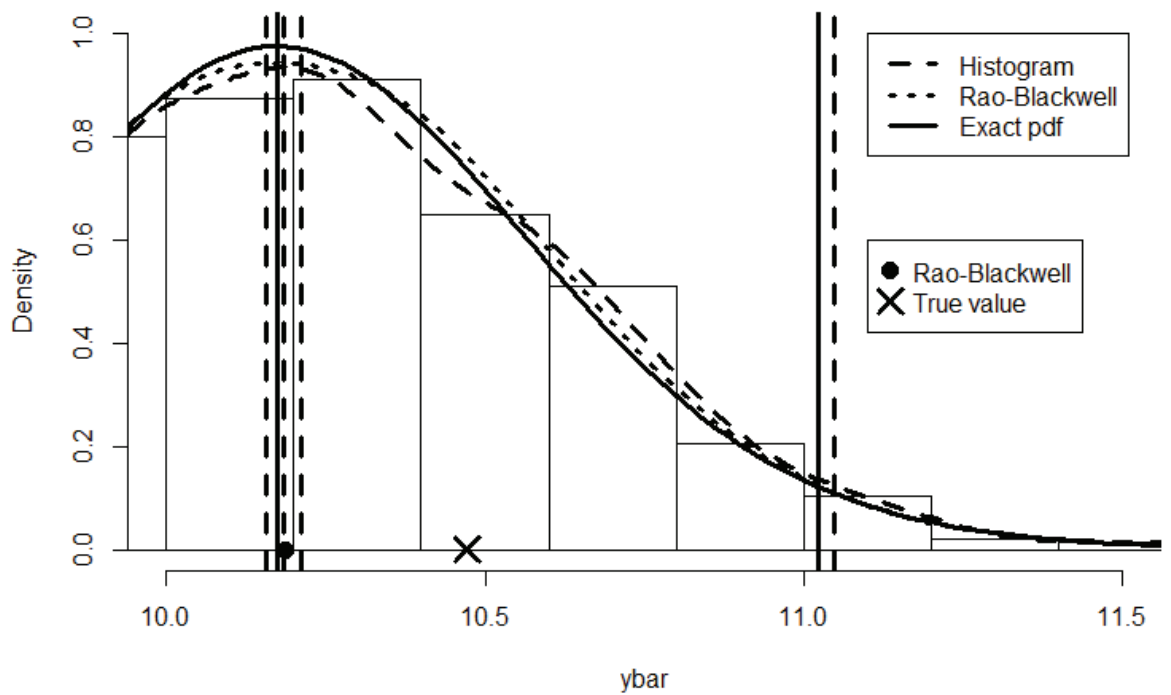

Table A.1 shows some of the true values and corresponding numerical estimates featuring in Figure A.6.

(c) Using the WinBUGS code below we obtained results as shown in Figure A.7. It will be noted that these are consistent with those in Table A.1 


\section{Table A.I Numerical results for part (b)}

\begin{tabular}{clcccc} 
Quantity & $\begin{array}{l}\text { True } \\
\text { value }\end{array}$ & $\begin{array}{c}\text { Posterior } \\
\text { mean }\end{array}$ & $\begin{array}{c}\mathrm{MC} \\
\text { estimate }\end{array}$ & $\begin{array}{c}95 \% \mathrm{Cl} \text { for } \\
\text { post. mean }\end{array}$ & $\begin{array}{c}\text { MC estimate } \\
\text { of 95\% CPDR }\end{array}$ \\
\hline$m$ & 11.000 & 10.895 & 10.906 & $(10.875,10.937)$ & $(9.893,11.863)$ \\
$\bar{y}$ & 10.473 & 10.174 & 10.185 & $(10.158,10.211)$ & $(9.353,11.049)$ \\
$\psi$ & 1.435 & NA & 1.659 & $(1.650,1.668)$ & $(1.444,2.014)$ \\
\hline
\end{tabular}

\section{Figure A.7 Output from WinBUGS run}

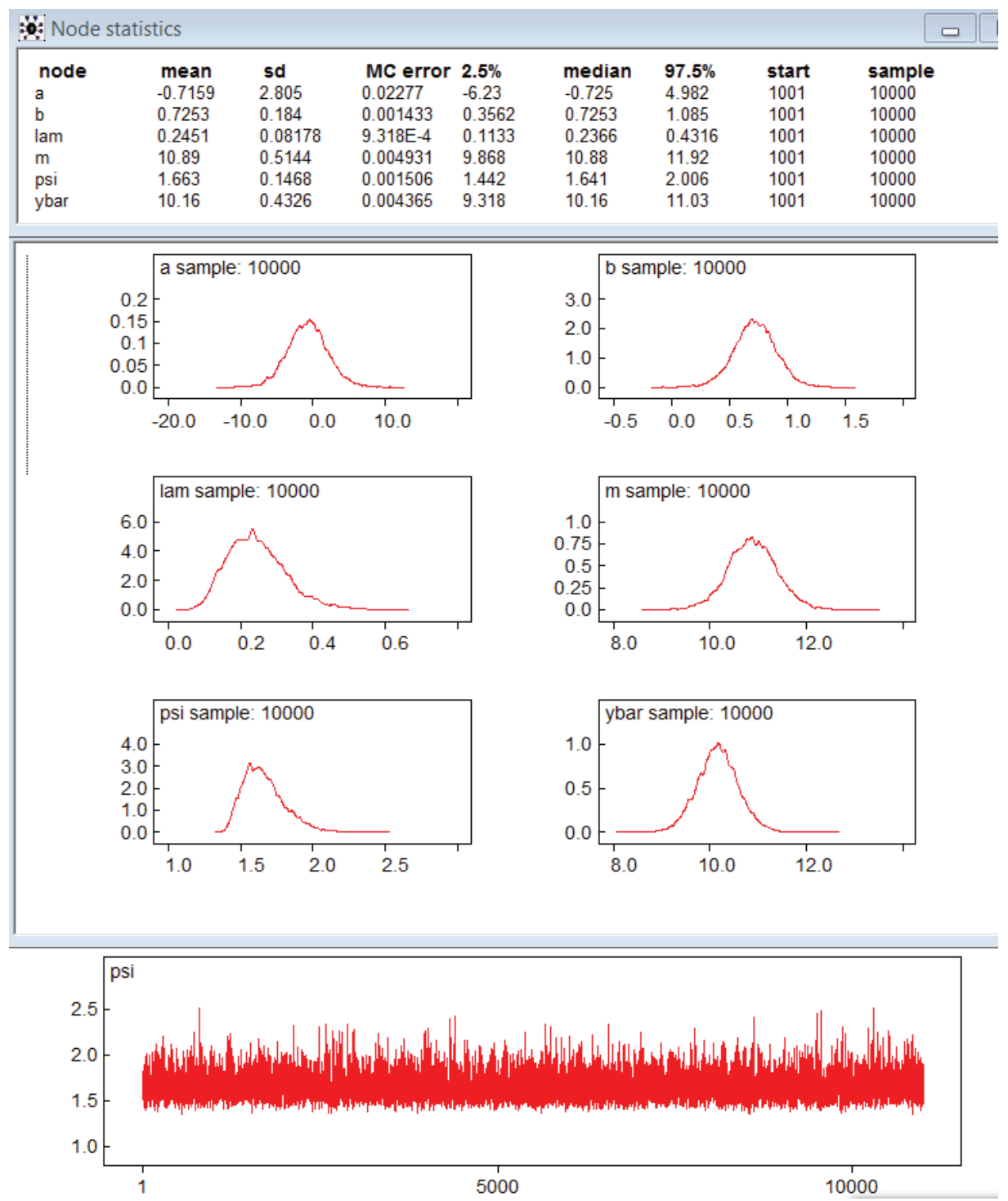




\section{R Code for Exercise A.3}

\# (a)

$\mathrm{X} 11(\mathrm{w}=8, \mathrm{~h}=5.5) ; \quad \operatorname{par}(\operatorname{mfrow}=\mathrm{c}(1,1))$; options(digits $=4)$

$\mathrm{N}=100 ; \mathrm{n}=20 ; a=3 ; b=0.5 ; \operatorname{sig}=2$; $\operatorname{set}$.seed(312); $\mathrm{x}=\operatorname{runif}(\mathrm{N}, 10,20)$;

$y=\operatorname{rnorm}(N, a+b * x, \operatorname{sig}) ; s=\operatorname{sort}(\operatorname{sample}(1: N, n)) ; x s=x[s] ; y s=y[s]$;

$r=(1: N)[-s] ; \quad x r=x[r] ; \quad y r=y[r] ; \quad y T=\operatorname{sum}(y) ; y s T=\operatorname{sum}(y s) ; y r T=\operatorname{sum}(y r)$

ybar=mean(y); ysbar=mean(ys); $y r b a r=m e a n(y r) ;$

$x T=\operatorname{sum}(x) ; x s T=\operatorname{sum}(x s) ; x r T=\operatorname{sum}(x r)$

$\operatorname{xbar}=\operatorname{mean}(\mathrm{x})$; $x$ sbar=mean $(\mathrm{xs})$; $\operatorname{xrbar}=\operatorname{mean}(\mathrm{xr})$;

$\mathrm{m}=\mathrm{a}+16 * \mathrm{~b} ; \mathrm{psi}=\max (\mathrm{y}) / \operatorname{median}(\mathrm{y})$

c(m, ybar,max(y),median(y),psi) \#11.000 10.473 15.23410 .6161 .435

$\operatorname{plot}(x, y, x \lim =c(0,20), y \lim =c(0,17))$;

points $(x s, y s, p c h=16)$; abline $(v=0$, Ity=3); abline $(h=0, \mid t y=3)$; abline $(v=16$, tty=3);

abline $(h=a+16 * b$, lty $=3)$;

abline $(a, b, l w d=3)$;

abline $(\operatorname{Im}(y \sim x), \operatorname{lty}=2, \operatorname{lwd}=3)$; abline $\left(\operatorname{Im}\left(y s^{\sim} x s\right), \operatorname{lty}=3, \operatorname{lwd}=3\right)$;

abline $\left(\operatorname{lm}\left(y r^{\sim} x r\right), I t y=4, I w d=3\right)$

legend $(0,17, b g="$ white", c("True regression line", "Estimate from population",

"Estimate from sample","Estimate from nonsample"),

Ity $=1: 4, \operatorname{lwd}=\operatorname{rep}(3,4)$ )

text(16,2,"The solid dots show the sample values")

\# (b) Follows on from (a)....

\# Packages, Load package, MASS (for use further down)

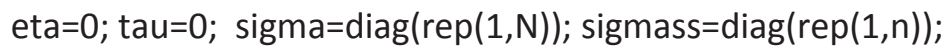

sigmarr $=\operatorname{diag}(\operatorname{rep}(1, \mathrm{~N}-\mathrm{n}))$;

$\mathrm{p}=2 ; \mathrm{c}=2 *$ eta+n-p; $\mathrm{Xs}=\mathrm{cbind}(1, \mathrm{xs}) ; \mathrm{Xr}=\mathrm{cbind}(1, \mathrm{xr}) ; \mathrm{X}=\mathrm{rbind}(\mathrm{Xs}, \mathrm{Xr})$

$\mathrm{D}=$ solve(t(Xs)\%*\%solve(sigmass)\%*\%Xs)

$\mathrm{T}=\mathrm{D} \%$ *\%t(Xs)\%*\%solve(sigmass)\%*\%ys; $\mathrm{t}(\mathrm{T})$ \# -0.66370 .7224$

$\mathrm{A}=2 *$ eta $+n-\mathrm{p} ; \mathrm{B}=2 *$ tau $+\mathrm{t}(\mathrm{ys}-\mathrm{Xs} \% * \% \mathrm{~T}) \% * \%$ solve(sigmass) $\% * \%$ (ys-Xs\%*\%T)

$\mathrm{J}=1000$; set.seed(5); lamvec=rgamma(J,A/2,B/2);

betamat=matrix (NA, nrow $=2$, ncol=J)

for $(j$ in 1:J) betamat[,j] = mvrnorm $(n=1, \operatorname{mu}=T$, Sigma=D/lamvec[j] )

avec=betamat[1,]; bvec=betamat[2,]

ahat=mean(avec); bhat=mean(bvec); c(ahat,bhat) \# -0.5742 0.7175

yrmat=matrix (NA, nrow $=\mathrm{N}-\mathrm{n}, \mathrm{ncol}=\mathrm{J})$

set.seed(334); for(j in 1:J)

$\operatorname{yrmat}[, j]=\operatorname{rnorm}(N-n, a v e c[j]+b v e c[j] * x r, 1 / \operatorname{sqrt}($ lamvec$[j]))$ 
\# Use simulated values of beta and yr to do inference mvec=avec+16*bvec; ybarvec=rep(NA,J); psivec=rep(NA,J)

for $(j$ in $1: J)\{\quad y s i m=c(y s, y r m a t[, j])$ ybarvec $[\mathrm{j}]=\operatorname{mean}(\mathrm{ysim})$ $\operatorname{psivec}[j]=\max (y \operatorname{sim}) / \operatorname{median}(y \operatorname{sim}) \quad\}$

mhat $=$ mean $(\mathrm{mvec}) ; \mathrm{mci}=$ mhat $+\mathrm{c}(-1,1) *$ qnorm $(0.975) * \mathrm{sd}(\mathrm{mvec}) / \operatorname{sqrt}(\mathrm{J})$ mcpdr=quantile (mvec, $c(0.025,0.975))$

ybarhat=mean(ybarvec);

ybarci $=$ ybarhat $+c(-1,1) *$ qnorm $(0.975) *$ sd(ybarvec)/sqrt(J) ybarcpdr=quantile (ybarvec, $c(0.025,0.975))$

psihat $=$ mean $($ psivec $) ;$ psici $=$ psihat $+c(-1,1) *$ nnorm $(0.975) * s d(p s i v e c) /$ sqrt $(J)$ psicpdr=quantile $(p s i v e c, c(0.025,0.975))$

hist(mvec, prob=T, $x \lim =c(8,14)$,ylim=c $(0,1)$, breaks=seq $(7,14,0.25)$, $x l a b=" m "$,main="(a) Histogram of 1000 m-values") \# Ignore warnings lines(density(mvec), Ity=2,lwd=3) \# Histogram estimate abline $(v=c(m h a t, m c i, m c p d r), I t y=2, l w d=3)$ \# Histogram estimates mhat2=c(1,16)\%*\%T; points(mhat2,0, pch=16,cex=1.5) \# Exact posterior mean mvarterm2=c $(1,16) \% * \% \mathrm{D} \% \% c(1,16) ;$ msdterm2=sqrt(mvarterm2) $\mathrm{mv}=\mathrm{seq}(6,16,0.05) ; \mathrm{fmv} 2=\mathrm{mv}$ for( $k$ in 1 :length $(m v))$

$\mathrm{fmv} 2[\mathrm{k}]=\operatorname{mean}(\mathrm{dnorm}(\mathrm{mv}[\mathrm{k}]$, mhat2, msdterm2/sqrt(lamvec))) lines $(m v, f m v 2, I w d=3)$; \# Exact posterior density of $m$ points(median(y),0, pch=4,cex=2,lwd=3 ) \# True value of $m$ legend(8,1,c("Histogram estimate", "Exact density"), Ity=c(2,1), Iwd=c(3,3), bg="white") legend(8,0.6,c("Rao-Blackwell","True"),pch=c(16,4), pt.cex $=c(1.5,2)$, pt.lwd=c(1,3), bg="white")

hist(ybarvec, prob $=T, x \lim =c(8,12), y \lim =c(0,1)$, breaks=seq $(3,18,0.25)$, xlab="ybar",main="(b) Histogram of 1000 ybar-values") lines(density(ybarvec), Ity=2,Iwd=3) \# Histogram estimate abline $(v=c(y b a r h a t, y b a r c i, y b a r c p d r), I t y=2, I w d=3)$ \# Histogram estimates ybarv=seq $(8,13,0.02)$; fybarhatv=ybarv; meanvalvec $=(1 / N) *(y s T+(N-n) *($ avec + bvec $* x r b a r) ~)$ varvalvec $=(\mathrm{N}-\mathrm{n}) /\left(\right.$ lamvec* $\left.\mathrm{N}^{\wedge} 2\right)$ for(k in 1:length(ybarv))\{

fybarhatv[k]= mean( dnorm(ybarv[k], meanvalvec, sqrt(varvalvec) )) \} lines(ybarv, fybarhatv, Ity=1,lwd=3) \# Rao-Blackwell points(mean(meanvalvec),0,pch=16,cex=1.5) \# Rao-Blackwell points(ybar, 0, pch=4,cex=2,lwd=3 ) \# True value of ybar legend(8,1,c("Histogram estimate","Rao-Blackwell"), Ity $=c(2,1), \operatorname{lwd}=c(3,3), \mathrm{bg}="$ white") 
legend(8,0.6,c("Rao-Blackwell","True value"),pch=c(16,4),

pt.cex $=c(1.5,2)$, pt.lwd=c(1,3), bg="white")

hist $(p s i v e c, p r o b=T, x \lim =c(1.25,2.5), y \lim =c(0,3)$, breaks=seq $(0,10,0.05)$, xlab="psi",main="(c) Histogram of 1000 psi-values")

den=density(psivec); lines(den, Ity=2,lwd=3)

abline $(v=c(p s i h a t, p s i c i, p s i c p d r), \mid t y=2, l w d=3)$

\# psimode=den\$x[(1:length(den\$x))[den\$y==max(den\$y)]] \# optional extras....

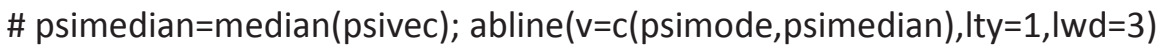

points(psi, 0, pch=4,cex=2,lwd=3 ) \# True value of psi legend(2.05,3,c("Histogram estimate"), Ity=c(2),lwd=c(3), bg="white") legend(2.05,2,c("True value"),pch=c(4), pt.cex=c(2), pt.lwd=c(3), bg="white")

\# Perform exact inference on ybar using a function from a previous exercise: NNGFPM $=$ function(eta $=0$, tau=0, $a l p=0.05$, $y s=c(5.6,2.3,8.4,5.1,4.3), X=\operatorname{rep}(1,15), N=15, \operatorname{sigma}=\operatorname{diag}(\operatorname{rep}(1, N)) \quad$ ～ \# This function performs inference under the normal-normal-gamma \# finite population model.

\# Inputs: eta, tau, alp, ys, X, N, sigma

\# Outputs: A list with $\$ a, \$ b$ and $\$ c$ indicating (ybar-a)/b given ys $\sim t(c)$

$\mathrm{p}=\mathrm{ncol}(\mathrm{cbind}(\mathrm{NA}, \mathrm{X}))-1 ; \quad \mathrm{n}=$ length$(\mathrm{ys}) ; \quad \mathrm{c}=2^{*}$ eta $+\mathrm{n}-\mathrm{p}$

ysT=sum(ys); Xs=cbind(NA,X)[1:n,][,-1]; Xr=cbind(NA,X)[(n+1):N,][,-1]

sigmass $=\operatorname{sigma}[1: n, 1: n] ; \quad$ sigmarr $=\operatorname{sigma}[(n+1): N,(n+1): N]$

sigmasr=sigma[1:n,(n+1):N]; sigmars=t(sigmasr)

$\mathrm{D}=$ solve $(\mathrm{t}(\mathrm{Xs}) \% * \%$ solve(sigmass) $\% * \% \mathrm{Xs}$ )

beta=D\%*\%t(Xs)\%*\%solve(sigmass)\%*\%ys

$\mathrm{A}=\mathrm{Xr}$-sigmars\%*\%solve(sigmass)\%*\%Xs; oner=rep(1,N-n)

$a=(1 / N) *(y s T+t$ (oner) $\% * \%$

( Xr\%*\%beta + sigmars\%*\%solve(sigmass)\%*\%(ys-Xs\%*\%beta) ) )

b2 $=\left(1 /\left(c^{*} N^{\wedge} 2\right)\right) *(2 *$ tau $+\mathrm{t}(\mathrm{ys}-\mathrm{Xs} \% * \%$ beta $) \% * \%$ solve(sigmass)\%*\%

(ys-Xs\%*\%beta) ) * t(oner)\%*\%

(sigmarr-sigmars\%*\%solve(sigmass)\%*\%sigmasr +A\%*\%D\%*\%t(A)) \%*\% oner

$b=\operatorname{sqrt}(b 2) ; c p d r=a+c(-1,1) * q t(1-a l p / 2, c) * b$

list $(a=a, b=b, c=c$, beta=beta, $c p d r=c p d r)\}$

res $=$ NNGFPM( eta $=0$, tau=0, alp=0.05, ys=ys, $\mathrm{X}=\mathrm{X}, \mathrm{N}=\mathrm{N}$, sigma=sigma )

$c($ res\$a,res\$b,res\$c, res\$cpdr) \# 10.17440 .403518 .00009 .326711 .0221

\# Plot for inference on ybar again

hist(ybarvec, prob=T,xlim=c(8,12),ylim=c(0,1), breaks=seq $(3,18,0.2)$, xlab="ybar",main="(d) Histogram of 1000 ybar-values") 
abline $(v=c(y b a r h a t, y b a r c i, y b a r c p d r), I t y=2, I w d=3)$ \# Histogram point estimates points(mean(meanvalvec), 0, pch=16,cex=1.5)

\# Rao-Blackwell estimate of predictive mean

abline(v=c(res\$a,res\$cpdr), Ity=1, Iwd=3) \# \# Exact point estimates

points(ybar, 0, pch=4,cex=2,lwd=3 ) \# True value of ybar

lines(density(ybarvec), Ity=2,lwd=3) \# Histogram estimate of predictive pdf lines(ybarv, fybarhatv, Ity=3,lwd=3) \# Rao-Blackwell estimate of pdf lines(ybarv, dt ((ybarv-res\$a)/res\$b,c)/res\$b,lty=1,lwd=3) \# Exact predictive pdf legend(8,1,c("Histogram","Rao-Blackwell","Exact pdf"),

$$
\text { Ity }=c(2,3,1), \operatorname{lwd}=c(3,3,3))
$$

legend(8,0.5,c("Rao-Blackwell","True value"),

$$
\text { pch }=c(16,4) \text {, pt.cex }=c(1.5,2), p t . I w d=c(1,3))
$$

text(11.65,0.8,

"The solid vertical lines\nshow the exact \npredictive mean\nand 95\% CPDR")

\# Detail in last figure

hist(ybarvec, prob=T,xlim=c(10,11.5),ylim=c(0,1), breaks=seq $(3,18,0.2)$,

xlab="ybar",main="(e) Detail in subplot (d)")

abline $(v=c(y b a r h a t, y b a r c i, y b a r c p d r), I t y=2, I w d=3)$ \# Histogram point estimates points(mean(meanvalvec), $0, p c h=16, c e x=1.5$ )

\# Rao-Blackwell estimate of predictive mean abline $(v=c($ res $\$ a, r e s \$ c p d r), I t y=1, I w d=3)$ \# \# Exact point estimates points(ybar, 0, pch=4,cex=2,lwd=3 ) \# True value of ybar lines(density(ybarvec), Ity=2,lwd=3) \# Histogram estimate of predictive pdf lines(ybarv, fybarhatv,lty=3,lwd=3) \# Rao-Blackwell estimate of pdf lines(ybarv, dt ((ybarv-res\$a)/res\$b,c)/res\$b,lty=1,lwd=3) \# Exact predictive pdf legend(11.1,1,c("Histogram","Rao-Blackwell",

"Exact pdf"), Ity=c(2,3,1), Iwd=c(3,3,3))

legend(11.1,0.6,c("Rao-Blackwell","True value"), pch $=c(16,4), p t \cdot c e x=c(1.5,2), p t . \operatorname{lwd}=c(1,3))$

\# Exact values of the quantities of interest and summary estimates $\mathrm{c}(\mathrm{m}, \mathrm{mhat} 2$, mhat, mci,mcpdr)

\# 11.00010 .89510 .90610 .87510 .9379 .89311 .863

c(ybar,res\$a,ybarhat,ybarci,ybarcpdr)

\# 10.47310 .17410 .18510 .15810 .2119 .35311 .049

c(psi,psihat,psici,psicpdr) \# 1.4351 .6591 .6501 .6681 .4442 .014

\# Preparation of data for input to WinBUGS

paste(as.character(round(ys,2)), collapse=",")

\# 14.98,10.99,9.58,6.56,13.83,......, 10.66,10.41"

paste(as.character(round(c(xs,xr),2)), collapse=",")

\# 19.34,18.2,14.27,10.91,13.45,.....,12.57,10.36,19.49 


\section{WinBUGS Code for Exercise A.3}

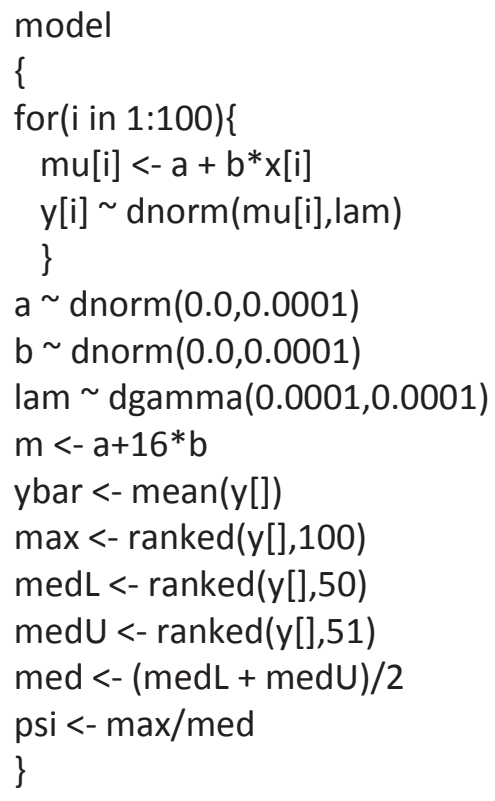

\# inits

list $(a=0, b=0$, lam $=1)$ 


\section{Exercise A.4 Case study in Bayesian finite population models with biased sampling}

A finite population of size $N=4$ consists of values $y_{1}, \ldots, y_{4}$ that are iid Bernoulli with parameter $\theta$.

A priori, $\theta$ is equally likely to be $1 / 4$ or $3 / 4$ (with no other possibilities).

We are interested in two quantities:

$$
\begin{aligned}
& \text { the superpopulation mean } \quad \theta=E\left(y_{i} \mid \theta\right) \\
& \text { the finite population mean } \quad \bar{y}=\frac{y_{1}+\ldots+y_{N}}{N} .
\end{aligned}
$$

We sample $n=2$ units from the finite population without replacement in such a way that

every sample is equally likely to be selected, apart from one exception, as follows:

if the value of unit 1 is 1 then each sample with unit 1 is twice as likely to be selected as each sample without unit 1.

We observe the values of the two sampled units (each being 0 or 1 ) as well as the labels identifying them (each being $1,2,3$ or 4 ).

(a) Write down a suitable Bayesian model for the above scenario in terms of the densities of the parameter $\theta$, the finite population vector, $y=\left(y_{1}, \ldots, y_{N}\right)$, and the sample, $s=\left(s_{1}, \ldots, s_{n}\right)$.

Your formulae may involve only these variables, as well as $n, N$, and the vector of inclusion counters, $I=\left(I_{1}, \ldots, I_{N}\right)$, where $I_{i}=1$ if the ith unit is in the sample, and $I_{i}=0$ otherwise. (Note that there is a one-to-one correspondence between $s$ and $I$ in this exercise.)

(b) Identify a condition which determines whether the sampling mechanism is ignorable or nonignorable. Then write down an expression for the density of $s$ in each of these two cases. 
(c) Derive the posterior density and mean of $\theta$ generally.

(d) Find the model bias of the posterior mean of $\theta$ if:

(i) $\theta=1 / 4$ and $s=(1,3)$

(ii) $\theta=1 / 4$ and $s=(2,3)$.

(e) Find the design bias of the posterior mean of $\theta$ if:

(i) $\theta=1 / 4$ and $y=(0,0,1,1)$

(ii) $\theta=1 / 4$ and $y=(1,0,1,1)$.

(f) Derive the predictive mean of $\bar{y}$ generally.

(g) Find the model bias of the predictive mean of $\bar{y}$ if:

(i) $\theta=1 / 4$ and $s=(1,3)$

(ii) $\theta=1 / 4$ and $s=(2,3)$.

(h) Find the design bias of the predictive mean of $\bar{y}$ if:

(i) $\theta=1 / 4$ and $y=(0,0,1,1)$

(ii) $\theta=1 / 4$ and $y=(1,0,1,1)$.

(i) Design and run a Gibbs sampler to check the posterior mean of $\theta$ in (c) and the predictive mean of $\bar{y}$ in (f).

(j) Use Monte Carlo methods to check the two design biases in (h).

(k) Find the mean of the predictive mean of the finite population mean. Then apply Monte Carlo methods to check your answer. 


\section{Solution to Exercise A.4}

(a) Part of the Bayesian model is:

$$
\begin{gathered}
f(y \mid \theta)=\prod_{i=1}^{N} \theta^{y_{i}}(1-\theta)^{1-y_{i}} \\
f(\theta)=1 / 2, \theta=1 / 4,3 / 4 .
\end{gathered}
$$

As regards the sampling mechanism, if $y_{1}=0$ then

$$
f(s \mid y, \theta)=f(s)=\left(\begin{array}{c}
N \\
n
\end{array}\right)^{-1}, \quad s=(1,2),(1,3),(1,4),(2,3),(2,4),(3,4) \text {. }
$$

Also, if $y_{1}=1$ then

$$
\begin{aligned}
f(s \mid y, \theta)=f\left(s \mid y_{1}\right) & =\left\{\begin{array}{cc}
c, & i \notin s \\
2 c, & i \in s
\end{array}\right\} \\
& =\left\{\begin{array}{cc}
c, & s=(1,2),(1,3),(1,4) \\
2 c, & s=(2,3),(2,4),(3,4)
\end{array}\right\} .
\end{aligned}
$$

To find the value of $c$, we may equate

$$
1=\sum_{s} f\left(s \mid y_{1}\right)=c \times 3+(2 c) \times 3=9 c .
$$

We thereby obtain $c=1 / 9$.

Note 1: Alternatively, we may observe that

$$
f\left(s \mid y_{1}\right)=c\left(1+I_{i}\right) \text {, }
$$

where

$$
I_{i}=I(i \in s) .
$$

Hence

$$
\begin{aligned}
& 1=\sum_{s} f\left(s \mid y_{1}\right)=c \sum_{s}\left(1+I_{i}\right)=c\left\{\sum_{s} 1+\sum_{s} I(i \in s)\right\} \\
&=c\left\{\sum_{s} 1+\sum_{s: i \in s} 1\right\}=c\left\{\left(\begin{array}{l}
N \\
n
\end{array}\right)+\left(\begin{array}{c}
N-1 \\
n-1
\end{array}\right)\right\}=c\left\{\left(\begin{array}{l}
4 \\
2
\end{array}\right)+\left(\begin{array}{l}
3 \\
1
\end{array}\right)\right\} \\
&=c(6+3)=9 c
\end{aligned}
$$

$\Rightarrow c=1 / 9$. 
Note 2: There are a total of $\left(\begin{array}{c}N-1 \\ n-1\end{array}\right)$ samples $s$ which contain any given particular unit $i$. So if $y_{1}=1$ then

$$
f(s \mid y, \theta)=f\left(s \mid y_{1}\right)=\left\{\begin{array}{ll}
1 / 9, & s=(1,2),(1,3),(1,4) \\
2 / 9, & s=(2,3),(2,4),(3,4)
\end{array}\right\} .
$$

Putting together the two cases above ( $y_{1}=0$ and 1 ), we see that the sampling mechanism is given generally by

$$
\begin{aligned}
f(s \mid y, \theta) & =f\left(s \mid y_{1}\right) \\
& =\frac{1+I_{1} y_{1}}{\left(\begin{array}{c}
N \\
n
\end{array}\right)+\left(\begin{array}{c}
N-1 \\
n-1
\end{array}\right) y_{1}} \\
& =\frac{1+I_{1} y_{1}}{6+3 y_{1}}, \quad s=(1,2),(1,3),(1,4),(2,3),(2,4),(3,4),
\end{aligned}
$$

where of course $I_{1}=I(s \in\{(1,2),(1,3),(1,4)\})$.

As a check, it is useful to list all of the values produced by this formula. These values are as shown in Table A.2. Observe that the sum of $f\left(s \mid y_{1}\right)$ over all values of $s$ is equal to 1 , both when $y_{1}=0$ and when $y_{1}=1$.

From Table A.2 we may also confirm that, as specified in the problem:

every sample is equally likely to be selected, apart from one exception, as follows: if the value of unit 1 is 1 then each sample with unit 1 is twice as likely to be selected as each sample without unit 1.

\section{Table A.2 All possible values of $s$ and their probabilities}

\begin{tabular}{lcccccc} 
Sample, $s:$ & $(1,2)$ & $(1,3)$ & $(1,4)$ & $(2,3)$ & $(2,4)$ & $(3,4)$ \\
\hline$I_{1}=I(1 \in s):$ & 1 & 1 & 1 & 0 & 0 & 0 \\
$f\left(s \mid y_{1}=0\right):$ & $1 / 6$ & $1 / 6$ & $1 / 6$ & $1 / 6$ & $1 / 6$ & $1 / 6$ \\
$f\left(s \mid y_{1}=1\right):$ & $2 / 9$ & $2 / 9$ & $2 / 9$ & $1 / 9$ & $1 / 9$ & $1 / 9$ \\
\hline
\end{tabular}


(b) If unit 1 is selected $\left(1 \in s, I_{i}=1\right)$ then $y_{1}=0$ or 1 is known and so the sampling mechanism is ignorable. In that case,

$$
\begin{aligned}
f(s \mid y, \theta)=\frac{1+y_{1}}{6+3 y_{1}}=\left\{\begin{array}{l}
1 / 6, y_{1}=0 \\
2 / 9, y_{1}=1
\end{array}\right\} & =\left\{\begin{array}{l}
3 / 18, y_{1}=0 \\
4 / 18, y_{1}=1
\end{array}\right\}=\frac{3+y_{1}}{18}, \\
s & =(1,2),(1,3),(1,4) .
\end{aligned}
$$

Conversely, if unit 1 is not selected $\left(1 \notin s, I_{i}=0\right)$ then $y_{1}=0$ or 1 is unknown and so the sampling mechanism is nonignorable.

In that case:

$$
\begin{aligned}
& f(s \mid y, \theta)=\frac{1}{6+3 y_{1}}=\left\{\begin{array}{l}
1 / 6, y_{1}=0 \\
1 / 9, y_{1}=1
\end{array}\right\}=\left\{\begin{array}{l}
3 / 18, y_{1}=0 \\
2 / 18, y_{1}=1
\end{array}\right\}=\frac{3-y_{1}}{18}, \\
& s=(2,3),(2,4),(3,4) .
\end{aligned}
$$

(c) The posterior distribution of $\theta$ given data $D=\left(s, y_{s}\right)$ can now be derived by considering the two cases in the note above.

First, if unit 1 happens to be sampled then the value of the sampling density $f(s \mid y, \theta)$ is known, and so the sampling mechanism is ignorable.

Explicitly, we find in that case,

$$
\begin{aligned}
& f(\theta \mid D)=f\left(\theta \mid s, y_{s}\right) \propto f\left(\theta, s, y_{s}\right)=\sum_{y_{r}} f\left(\theta, s, y_{s}, y_{r}\right) \\
&= \sum_{y_{r}} f(\theta) f\left(y_{s}, y_{r} \mid \theta\right) f\left(s \mid y_{s}, y_{r}, \theta\right) \\
&= \sum_{y_{r}} f(\theta) f\left(y_{s} \mid \theta\right) f\left(y_{r} \mid \theta\right) f\left(s \mid y_{1}\right) \\
&= f(\theta) f\left(y_{s} \mid \theta\right) f\left(s \mid y_{1}\right) \sum_{y_{r}} f\left(y_{r} \mid \theta\right) \\
& \quad \text { ince } f(s \mid y, \theta)=f\left(s \mid y_{1}\right), \text { where } s \text { is fixed at its } \\
& \text { observed value, } s=\left(s_{1}, s_{2}\right)=(1,2),(1,3) \text { or }(1,4) \\
& \theta \propto f(\theta) f\left(y_{s} \mid \theta\right) \times 1 \times 1 \\
& \quad \text { since } f\left(s \mid y_{1}\right) \text { does not depend on } \theta .
\end{aligned}
$$

Note: This is the point at which $f(s \mid y, \theta)$ can be 'ignored'. 
Thus we have that

$$
\begin{aligned}
f(\theta \mid D) & \propto 1 \times \prod_{i \in s} \theta^{y_{i}}(1-\theta)^{1-y_{i}} \\
& =\theta^{y_{S T}}(1-\theta)^{2-y_{S T}} \\
& =\left\{\begin{array}{ll}
\left(\frac{1}{4}\right)^{y_{S T}}\left(\frac{3}{4}\right)^{y_{s T} 2-y_{S T}}, & \theta=1 / 4 \\
\left(\frac{3}{4}\right)^{y_{S T}}\left(\frac{1}{4}\right)^{y_{S T} 2-y_{s T}}, & \theta=3 / 4
\end{array}\right\} \\
& \propto\left\{\begin{array}{cc}
3^{2-y_{S T}}, & \theta=1 / 4 \\
3^{y_{S T}}, & \theta=3 / 4
\end{array}\right\} \\
& \propto\left\{\begin{array}{cc}
3^{2}, & \theta=1 / 4 \\
3^{y_{S T}}+y_{S T}, & \theta=3 / 4
\end{array}\right\} \\
& \left\{\begin{array}{cc}
9, & \theta=1 / 4 \\
9^{y_{S T}}, & \theta=3 / 4
\end{array}\right\} .
\end{aligned}
$$

That is (if $1 \in s$ ),

$$
f(\theta \mid D)=\left\{\begin{array}{c}
\frac{9}{9+9^{y_{s T}}}, \theta=1 / 4 \\
\frac{9^{y_{s T}}}{9+9^{y_{s T}}}, \theta=3 / 4
\end{array}\right\}=\left\{\begin{array}{l}
\left\{\begin{array}{ll}
9 / 10, & \theta=1 / 4 \\
1 / 10, & \theta=3 / 4
\end{array}\right\}, y_{s T}=0 \\
\left\{\begin{array}{ll}
1 / 2, & \theta=1 / 4 \\
1 / 2, & \theta=3 / 4
\end{array}\right\}, y_{s T}=1 \\
\left\{\begin{array}{ll}
1 / 10, & \theta=1 / 4 \\
9 / 10, & \theta=3 / 4
\end{array}\right\}, y_{s T}=2 .
\end{array}\right.
$$

So then also (if $1 \in s$ ) the posterior mean of $\theta$ is

$$
\hat{\theta}=E(\theta \mid D)=\left\{\begin{aligned}
\frac{1}{4}\left(\frac{9}{10}\right)+\frac{3}{4}\left(\frac{1}{10}\right)=\frac{3}{10}, & y_{s T}=0 \\
\frac{1}{4}\left(\frac{1}{2}\right)+\frac{3}{4}\left(\frac{1}{2}\right)=\frac{1}{2}, & y_{s T}=1 \\
\frac{1}{4}\left(\frac{1}{10}\right)+\frac{3}{4}\left(\frac{9}{10}\right)=\frac{7}{10}, & y_{s T}=2 .
\end{aligned}\right.
$$

Note: This could also be written as $\hat{\theta}=\frac{3+2 y_{s T}}{10} \quad$ (if $1 \in S$ ). 
Next, suppose that unit 1 is not sampled. Then the value of unit 1 is unknown and so the sampling mechanism is nonignorable.

In that case, we see from (b) that

$$
f(s \mid y, \theta)=f\left(s \mid y_{1}\right)=\frac{3-y_{1}}{18} \propto 3-y_{1}, \quad s=(2,3),(2,4),(3,4),
$$

where $y_{1}$ is an unknown value in the nonsample vector $y_{r}=\left(y_{1}, y_{k}\right)$ where $k=2$, 3 or 4 .

Working through as before,

$$
\begin{aligned}
f(\theta \mid D) & =f\left(\theta \mid s, y_{s}\right) \\
& \propto f\left(\theta, s, y_{s}\right) \\
& =\sum_{y_{r}} f\left(\theta, s, y_{s}, y_{r}\right) \\
& =\sum_{y_{r}} f(\theta) f\left(y_{s}, y_{r} \mid \theta\right) f\left(s \mid y_{s}, y_{r}, \theta\right) \\
& =\sum_{y_{r}} f(\theta) f\left(y_{s} \mid \theta\right) f\left(y_{r} \mid \theta\right) f\left(s \mid y_{1}\right) \\
& =f(\theta) f\left(y_{s} \mid \theta\right) \sum_{y_{r}} f\left(s \mid y_{1}\right) f\left(y_{r} \mid \theta\right) \\
& =f(\theta) f\left(y_{s} \mid \theta\right) q(\theta),
\end{aligned}
$$

where

$$
\begin{aligned}
q(\theta) & \propto \sum_{y_{r}}(3-\theta) f\left(y_{r} \mid \theta\right) \\
& =E_{y_{r}}(3-\theta \mid \theta) \\
& =3-\theta .
\end{aligned}
$$

Note: We could also have written

$$
\begin{aligned}
q(\theta) & \propto \sum_{y_{1}=0}^{1} \sum_{y_{k}=0}^{1}\left(3-y_{1}\right)\left[\theta^{y_{1}}(1-\theta)^{1-y_{1}}\right]\left[\theta^{y_{k}}(1-\theta)^{1-y_{k}}\right] \\
& =\left(\sum_{y_{k}=0}^{1} \theta^{y_{k}}(1-\theta)^{1-y_{k}}\right) \sum_{y_{1}=0}^{1}\left(3-y_{1}\right) \theta^{y_{1}}(1-\theta)^{1-y_{1}} \\
& =1 \times\left\{(3-0) \theta^{0}(1-\theta)^{1-0}+(3-1) \theta^{1}(1-\theta)^{1-1}\right\} \\
& =3(1-\theta)+2 \theta \\
& =3-\theta .
\end{aligned}
$$


Having shown (in the case $1 \notin s$ ) that

$$
f(\theta \mid D) \propto f(\theta) f\left(y_{s} \mid \theta\right)(3-\theta),
$$

it now follows that

$$
\begin{aligned}
f(\theta \mid D) \propto\left\{\begin{array}{ll}
\left(\frac{1}{4}\right)^{y_{S T}}\left(\frac{3}{4}\right)^{y_{S T} 2-y_{s T}}\left(3-\frac{1}{4}\right), & \theta=1 / 4 \\
\left(\frac{3}{4}\right)^{y_{S T}}\left(\frac{1}{4}\right)^{y_{s T} 2-y_{s T}}\left(3-\frac{3}{4}\right), & \theta=3 / 4
\end{array}\right\} \\
\propto\left\{\begin{array}{cc}
3^{2-y_{S T}} \times 11, & \theta=1 / 4 \\
3^{y_{S T}} \times 9, & \theta=3 / 4
\end{array}\right\} \\
\propto\left\{\begin{array}{cc}
3^{2} \times 11, & \theta=1 / 4 \\
3^{y_{S T}+y_{S T}} \times 9, & \theta=3 / 4
\end{array}\right\} \\
=\left\{\begin{array}{cc}
11, & \theta=1 / 4 \\
9^{y_{S T}}, & \theta=3 / 4
\end{array}\right\} .
\end{aligned}
$$

Thus (if $1 \notin s$ ), we have that

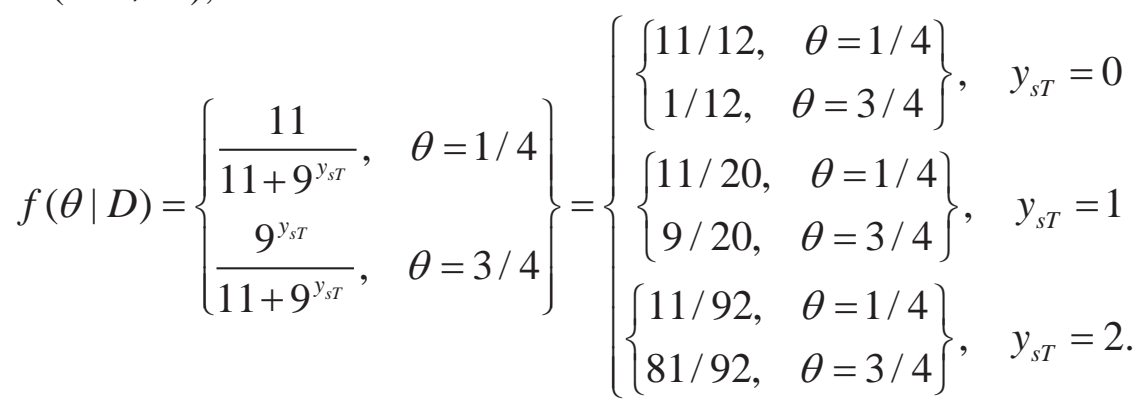

So then also (if $1 \notin S$ ) the posterior mean of $\theta$ is

$$
\begin{aligned}
\hat{\theta} & =E(\theta \mid D) \\
& =\left\{\begin{array}{l}
\frac{1}{4}\left(\frac{11}{12}\right)+\frac{3}{4}\left(\frac{1}{12}\right)=\frac{14}{48}=\frac{7}{24}=\frac{805}{2760}=0.2917, \quad y_{s T}=0 \\
\frac{1}{4}\left(\frac{11}{20}\right)+\frac{3}{4}\left(\frac{9}{20}\right)=\frac{38}{80}=\frac{19}{40}=\frac{1311}{2760}=0.4750, \quad y_{s T}=1 \\
\frac{1}{4}\left(\frac{11}{92}\right)+\frac{3}{4}\left(\frac{81}{92}\right)=\frac{254}{368}=\frac{127}{184}=\frac{1905}{2760}=0.6902, \quad y_{s T}=2 .
\end{array}\right.
\end{aligned}
$$


Note: This mean may also be written as

$$
\left.\hat{\theta}=\frac{805+462 y_{s T}+44 y_{s T}^{2}}{2760} \text { (if } 1 \notin s\right) .
$$

This alternative formula was obtained by solving the equation

$$
a+b x+c x^{2}=\left\{\begin{array}{ll}
805, & x=0 \\
1311, & x=1 \\
1905, & x=2
\end{array}\right\}
$$

for $a, b$ and $c$.

Putting the two cases together we find that the posterior mean of $\theta$ is given generally by:

$$
\begin{aligned}
\hat{\theta} & =E(\theta \mid D)=\hat{\theta}(D)=\hat{\theta}\left(s, y_{s}\right) \\
& =\left(\begin{array}{cl}
3 / 10=0.3000 & \text { if } 1 \in s \text { and } y_{s T}=0 \\
1 / 2=0.5000 & \text { if } 1 \in s \text { and } y_{s T}=1 \\
7 / 10=0.7000 & \text { if } 1 \in s \text { and } y_{s T}=2 \\
7 / 24=0.2917 & \text { if } 1 \notin s \text { and } y_{s T}=0 \\
19 / 40=0.4750 & \text { if } 1 \notin s \text { and } y_{s T}=1 \\
127 / 184=0.6902 & \text { if } 1 \notin s \text { and } y_{s T}=2,
\end{array}\right.
\end{aligned}
$$

or equivalently, by

$$
\hat{\theta}=\left(\frac{3+2 y_{s T}}{10}\right) I_{1}+\left(\frac{805+462 y_{s T}+44 y_{s T}^{2}}{2760}\right)\left(1-I_{1}\right) .
$$

Note: Here:

$$
\begin{aligned}
& 1 \in S \quad \Leftrightarrow \quad I_{1}=1 \quad \Leftrightarrow \quad s=(1,2),(1,3) \text { or }(1,4) \\
& 1 \notin S \quad \Leftrightarrow \quad I_{1}=0 \quad \Leftrightarrow \quad s=(2,3),(2,4) \text { or }(3,4) .
\end{aligned}
$$

Also:

$$
\begin{aligned}
& y_{s T}=0 \text { iff both sampled values are } 0 \\
& y_{s T}=1 \text { iff one sampled value is } 0 \text { and the other is } 1 \\
& y_{s T}=2 \text { iff both sampled values are } 1 .
\end{aligned}
$$


(d)(i) If $\theta=1 / 4$ and $s=(1,3)$ then $1 \in S$ and $I_{1}=1$, and so

$$
\hat{\theta}=\left(\frac{3+2 y_{s T}}{10}\right) 1+\left(\frac{805+462 y_{s T}+44 y_{s T}^{2}}{2760}\right)(1-1)=\frac{3+2 y_{s T}}{10} .
$$

So the model mean of $\hat{\theta}$ is

$$
E(\hat{\theta} \mid \theta, s)=\frac{1}{10}\left\{3+2 E\left(y_{s T} \mid \theta, s\right)\right\} .
$$

Now,

where:

$$
f(y \mid \theta, s)=\frac{f(y, s \mid \theta)}{f(s \mid \theta)},
$$

$$
\begin{gathered}
f(y, s \mid \theta)=f(s \mid y, \theta) f(y \mid \theta)=\frac{3+y_{1}}{18} \prod_{i=1}^{4} \theta^{y_{i}}(1-\theta)^{1-y_{i}} \\
\text { (using the result in (b) that } f(s \mid y, \theta)=\frac{3+y_{1}}{18} \text { if } 1 \in s \text { ) } \\
\begin{aligned}
f(s \mid \theta)=\sum_{y} f(y, s \mid \theta)=\sum_{y} f(s \mid y, \theta) f(y \mid \theta) & =E_{y}\{f(s \mid y, \theta) \mid \theta\} \\
=E_{y}\left(\frac{3+y_{1}}{18} \mid \theta\right) & =\frac{3+\theta}{18} .
\end{aligned}
\end{gathered}
$$

Therefore

$$
\begin{aligned}
f(y \mid \theta, s) & =\frac{\left(\frac{3+y_{1}}{18} \prod_{i=1}^{4} \theta^{y_{i}}(1-\theta)^{1-y_{i}}\right)}{\left(\frac{3+\theta}{18}\right)} \\
& =\left(\frac{3+y_{1}}{3+\theta} \theta^{y_{1}}(1-\theta)^{1-y_{1}}\right) \prod_{i=2}^{4} \theta^{y_{i}}(1-\theta)^{1-y_{i}} .
\end{aligned}
$$

We see that

$$
\left(y_{i} \mid \theta, s\right) \sim \perp \operatorname{Bernoulli}\left(\pi_{i}\right), i=1,2,3,4,
$$

where:

$$
\begin{aligned}
& \pi_{2}=\pi_{3}=\pi_{4}=\theta \\
& \pi_{1}=\frac{3+1}{3+\theta} \theta^{1}(1-\theta)^{1-1}=\frac{4 \theta}{3+\theta} .
\end{aligned}
$$


Check: $\frac{3+0}{3+\theta} \theta^{0}(1-\theta)^{1-0}=\frac{3(1-\theta)}{3+\theta}=1-\frac{4 \theta}{3+\theta}=1-\pi_{1}$.

It follows that

$$
\begin{aligned}
& E\left(y_{s T} \mid \theta, s\right)=E\left(y_{1} \mid \theta, s\right)+E\left(y_{3} \mid \theta, s\right) \\
& \quad=\pi_{1}+\pi_{3}=\theta+\frac{4 \theta}{3+\theta}=\frac{\theta(7+\theta)}{3+\theta}=\frac{\frac{1}{4}\left(7+\frac{1}{4}\right)}{\left(3+\frac{1}{4}\right)}=\frac{29 / 16}{13 / 4}=\frac{29}{52} .
\end{aligned}
$$

Hence

$$
E(\hat{\theta} \mid \theta, s)=\frac{1}{10}\left\{3+2\left(\frac{29}{52}\right)\right\}=\frac{107}{260}=0.4115 .
$$

So, if $\theta=1 / 4$ and $s=(1,3)$, then the model bias of $\hat{\theta}$ is

$$
E(\hat{\theta}-\theta \mid \theta, s)=\frac{107}{260}-\theta=\frac{107}{260}-\frac{1}{4}=\frac{21}{130}=0.1615 \text {. }
$$

Note: We can also report the relative model bias of $\hat{\theta}$ as

$$
E\left(\frac{\hat{\theta}-\theta}{\theta} \mid \theta, s\right)=\frac{21 / 130}{1 / 4}=\frac{42}{65}=+64.6 \% \text {. }
$$

(d)(ii) If $\theta=1 / 4$ and $s=(2,3)$ then $1 \in r$ and $I_{1}=0$, and so

$$
\begin{aligned}
\hat{\theta} & =\left(\frac{3+2 y_{s T}}{10}\right) 0+\left(\frac{805+462 y_{s T}+44 y_{s T}^{2}}{2760}\right)(1-0) \\
& =\frac{805+462 y_{s T}+44 y_{s T}^{2}}{2760} .
\end{aligned}
$$

So the model mean of $\hat{\theta}$ is

$$
E(\hat{\theta} \mid \theta, s)=\frac{805+462 E\left(y_{s T} \mid \theta, s\right)+44 E\left(y_{s T}^{2} \mid \theta, s\right)}{2760} .
$$


In this case,

as before, but with

$$
f(y \mid \theta, s)=\frac{f(y, s \mid \theta)}{f(s \mid \theta)},
$$

$$
f(y, s \mid \theta)=f(s \mid y, \theta) f(y \mid \theta)=\frac{3-y_{1}}{18} \prod_{i=1}^{4} \theta^{y_{i}}(1-\theta)^{1-y_{i}}
$$

(using the result in (b) that $f(s \mid y, \theta)=\frac{3-y_{1}}{18}$ if $1 \notin s$ ).

Thus,

$$
\begin{aligned}
f(s \mid \theta) & =\sum_{y} f(y, s \mid \theta)=\sum_{y} f(s \mid y, \theta) f(y \mid \theta) \\
& =E_{y}\{f(s \mid y, \theta) \mid \theta\}=E_{y}\left(\frac{3-y_{1}}{18} \mid \theta\right) \\
& =\frac{3-\theta}{18} .
\end{aligned}
$$

So

$$
\begin{aligned}
f(y \mid \theta, s) & =\frac{\left(\frac{3-y_{1}}{18} \prod_{i=1}^{4} \theta^{y_{i}}(1-\theta)^{1-y_{i}}\right)}{\left(\frac{3-\theta}{18}\right)} \\
& =\left(\frac{3-y_{1}}{3-\theta} \theta^{y_{1}}(1-\theta)^{1-y_{1}}\right) \prod_{i=2}^{4} \theta^{y_{i}}(1-\theta)^{1-y_{i}} .
\end{aligned}
$$

We see that

$$
\left(y_{i} \mid \theta, s\right) \sim \perp \operatorname{Bernoulli}\left(\pi_{i}\right), i=1,2,3,4,
$$

where:

$$
\begin{aligned}
& \pi_{2}=\pi_{3}=\pi_{4}=\theta \\
& \pi_{1}=\frac{3-1}{3-\theta} \theta^{1}(1-\theta)^{1-1}=\frac{2 \theta}{3-\theta} .
\end{aligned}
$$

Check: $\frac{3-0}{3-\theta} \theta^{0}(1-\theta)^{1-0}=\frac{3(1-\theta)}{3-\theta}=1-\frac{2 \theta}{3-\theta}=1-\pi_{1}$. 
It follows that

$$
\begin{aligned}
E\left(y_{s T} \mid \theta, s\right) & =E\left(y_{2} \mid \theta, s\right)+E\left(y_{3} \mid \theta, s\right) \\
& =\pi_{2}^{\prime}+\pi_{3}^{\prime}=\theta+\theta=\frac{1}{4}+\frac{1}{4}=\frac{1}{2} .
\end{aligned}
$$

Equivalently,

$$
\left(y_{s T} \mid \theta, s\right) \sim \operatorname{Bin}(2, \theta),
$$

and so

$$
E\left(y_{s T} \mid \theta, s\right)=2 \theta \text {. }
$$

By the same token,

$$
\begin{aligned}
E\left(y_{s T}^{2} \mid \theta, s\right) & =V\left(y_{s T} \mid s, \theta\right)+\left\{E\left(y_{s T} \mid s, \theta\right)\right\}^{2} \\
& =2 \theta(1-\theta)+(2 \theta)^{2}=2 \theta(1+\theta)=2 \times \frac{1}{4}\left(1+\frac{1}{4}\right)=\frac{5}{8} .
\end{aligned}
$$

Hence

$$
E(\hat{\theta} \mid \theta, s)=\frac{805+462\left(\frac{1}{2}\right)+44\left(\frac{5}{8}\right)}{2760}=\frac{2127}{5520}=0.3853
$$

So, if $\theta=1 / 4$ and $s=(2,3)$, then the model bias of $\hat{\theta}$ is

$$
E(\hat{\theta}-\theta \mid \theta, s)=E(\hat{\theta} \mid \theta, s)-\theta=\frac{2127}{5520}-\frac{1}{4}=\frac{747}{5520}=0.1353 .
$$

Note: As regards the model bias of $\hat{\theta}$, there are a total of 4 cases, corresponding to whether $1 \in S$ or $1 \notin s$, and to whether $\theta=1 / 4$ or $\theta=3 / 4$. We have covered two of these four cases.

(e)(i) If $\theta=1 / 4$ and $y=(0,0,1,1)$ then $y_{1}=0$. So in that particular case the sampling mechanism is definitely SRSWOR and ignorable. Without further thought, the posterior density of $\theta$ can be obtained as follows:

$$
\begin{aligned}
f(\theta \mid D)=f\left(\theta \mid s, y_{s}\right) & =f\left(\theta \mid y_{s}\right) \\
& \propto f(\theta) f\left(y_{s} \mid \theta\right) \\
& \propto 1 \times \prod_{i \in s} \theta^{y_{i}}(1-\theta)^{1-y_{i}} .
\end{aligned}
$$


Recalling (c), note that

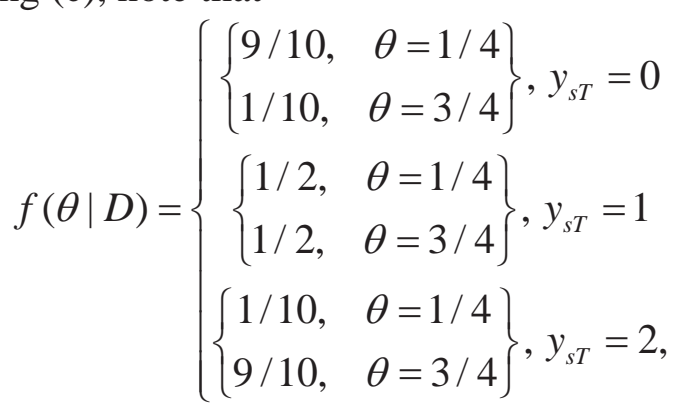

and

$$
\hat{\theta}=E(\theta \mid D)=\left\{\begin{array}{cc}
3 / 10, & y_{s T}=0 \\
1 / 2, & y_{s T}=1 \\
7 / 10, & y_{s T}=2
\end{array}\right\}=\frac{3+2 y_{s T}}{10}
$$

The design mean of $\hat{\theta}$ is therefore

where

$$
E(\hat{\theta} \mid \theta, y)=\frac{3+2 E\left(y_{s T} \mid \theta, y\right)}{10},
$$

$$
E\left(y_{s T} \mid \theta, y\right)=n E\left(\bar{y}_{s} \mid \theta, y\right)=n \sum_{s} \bar{y}_{s} f(s \mid \theta, y)=n \bar{y},
$$

since (making use of basic results in the classical theory)

$$
f(s \mid \theta, y)=f(s)=\left(\begin{array}{c}
N \\
n
\end{array}\right)^{-1}=2 \times \frac{0+0+1+1}{4}=1 .
$$

Therefore the design mean of $\hat{\theta}$ is

$$
E(\hat{\theta} \mid \theta, y)=\frac{3+2 \times 1}{10}=\frac{1}{2} .
$$

So the design bias of $\hat{\theta}$ is

$$
E(\hat{\theta}-\theta \mid \theta, y)=\frac{1}{2}-\theta=\frac{1}{2}-\frac{1}{4}=0.25 \text {. }
$$

Note: In the above, $E(\hat{\theta} \mid \theta, y)$ does not depend on $\theta$. So, for the case $\theta=3 / 4$ and $y=(0,0,1,1)$, the design bias of $\hat{\theta}$ is $\frac{1}{2}-\frac{3}{4}=-0.25$. 
(e)(ii) If $\theta=1 / 4$ and $y=(1,0,1,1)$ then $y_{1}=1$, and so the sampling mechanism is potentially nonignorable (depending on which sample $s$ happens to be drawn).

Recall from (c) that the posterior mean of $\theta$ is a function of the data given generally by

$$
\hat{\theta}=\hat{\theta}\left(s, y_{s}\right)=\left(\begin{array}{cc}
3 / 10=0.3000 & \text { if } 1 \in S \text { and } y_{s T}=0 \\
1 / 2=0.5000 & \text { if } 1 \in S \text { and } y_{s T}=1 \\
7 / 10=0.7000 & \text { if } 1 \in S \text { and } y_{s T}=2 \\
7 / 24=0.2917 & \text { if } 1 \notin S \text { and } y_{s T}=0 \\
19 / 40=0.4750 & \text { if } 1 \notin S \text { and } y_{s T}=1 \\
127 / 184=0.6902 & \text { if } 1 \notin S \text { and } y_{s T}=2 .
\end{array}\right.
$$

Also recall from (b) that

$$
f(s \mid y, \theta)=\left\{\begin{array}{l}
\frac{3+y_{1}}{18}, s=(1,2),(1,3),(1,4) \\
\frac{3-y_{1}}{18}, s=(2,3),(2,4),(3,4)
\end{array}\right\} .
$$

The design bias of $\hat{\theta}$ can now be worked out according to

$$
E(\hat{\theta} \mid \theta, y)=\sum_{s} \hat{\theta}\left(s, y_{s}\right) f(s \mid \theta, y) .
$$

Now, suppose that we draw the sample $s=(1,2)$.

Then $y_{s}=\left(y_{1}, y_{2}\right)=(1,0)$.

Thus $1 \in s$ and $y_{s T}=1$, and so by the above,

$$
\hat{\theta}\left(s, y_{s}\right) f(s \mid \theta, y)=\frac{1}{2} \times \frac{3+1}{18}=\frac{1}{9} \text {. }
$$

Likewise:

If $s=(1,3)$ then $y_{s}=\left(y_{1}, y_{3}\right)=(1,1)$ and so

$$
\hat{\theta}\left(s, y_{s}\right) f(s \mid \theta, y)=\frac{7}{10} \times \frac{3+1}{18}=\frac{7}{45} \text {. }
$$


If $s=(1,4)$ then $y_{s}=\left(y_{1}, y_{4}\right)=(1,1)$ and so

$$
\hat{\theta}\left(s, y_{s}\right) f(s \mid \theta, y)=\frac{7}{10} \times \frac{3+1}{18}=\frac{7}{45} \text {. }
$$

If $s=(2,3)$ then $y_{s}=\left(y_{2}, y_{3}\right)=(0,1)$ and so

$$
\hat{\theta}\left(s, y_{s}\right) f(s \mid \theta, y)=\frac{19}{40} \times \frac{3-1}{18}=\frac{19}{360} \text {. }
$$

If $s=(2,4)$ then $y_{s}=\left(y_{2}, y_{4}\right)=(0,1)$ and so

$$
\hat{\theta}\left(s, y_{s}\right) f(s \mid \theta, y)=\frac{19}{40} \times \frac{3-1}{18}=\frac{19}{360} \text {. }
$$

If $s=(3,4)$ then $y_{s}=\left(y_{3}, y_{4}\right)=(1,1)$ and so

It follows that

$$
\hat{\theta}\left(s, y_{s}\right) f(s \mid \theta, y)=\frac{127}{184} \times \frac{3-1}{18}=\frac{127}{1656} \text {. }
$$

$$
\begin{aligned}
E(\hat{\theta} \mid \theta, y) & =\sum_{s} \hat{\theta}\left(s, y_{s}\right) f(s \mid \theta, y) \\
& =(1 / 9)+(7 / 45)+(7 / 45)+(19 / 360)+(19 / 360)+(127 / 1656) \\
& =0.6045 .
\end{aligned}
$$

Thus, if $\theta=1 / 4$ and $y=(1,0,1,1)$, then the design bias of $\hat{\theta}$ is

$$
E(\hat{\theta}-\theta \mid \theta, y)=0.6045-\frac{1}{4}=0.3545 \text {. }
$$

Note 1: Also, if $\theta=3 / 4$ and $y=(1,0,1,1)$, then the design bias of $\hat{\theta}$ is

$$
0.6045-\frac{3}{4}=-0.1455 \text {. }
$$

Note 2: As regards the design bias of $\hat{\theta}$, there are a total of $2 \times 4 \times 2=16$ cases to be considered, corresponding to:

$$
\begin{array}{lll}
y_{1} & \text { being either } 0 \text { or } 1 & \text { (2 possibilities) } \\
y_{T}-y_{1} & \text { being } 0 \text { or } 1 \text { or } 2 \text { or } 3 & \text { (4 possibilities) } \\
\theta & \text { being either } 1 / 4 \text { or } 3 / 4 & \text { (2 possibilities). }
\end{array}
$$

We have covered four of these 16 cases. 
(f) Recall from (c) that

$$
\left(y_{i} \mid \theta, s\right) \sim \perp \operatorname{Bernoulli}\left(\pi_{i}\right), i=1,2,3,4,
$$

where:

$$
\begin{aligned}
& \pi_{2}=\pi_{3}=\pi_{4}=\theta \\
& \pi_{1}=\left\{\begin{array}{ll}
\frac{4 \theta}{3+\theta}, & 1 \in s \\
\frac{2 \theta}{3-\theta}, & 1 \notin s
\end{array}\right\} .
\end{aligned}
$$

Therefore

where

$$
E\left(y_{r T} \mid \theta, s, y_{s}\right)=E\left(y_{r T} \mid \theta, s\right)=\left\{\begin{array}{ll}
\theta+\theta, & 1 \in s \\
\theta+\phi, & 1 \notin s
\end{array}\right\},
$$

$$
\phi=\frac{2 \theta}{3-\theta} .
$$

So

where

$$
\begin{aligned}
E\left(y_{r T} \mid s, y_{s}\right) & =E\left\{E\left(y_{r T} \mid \theta, s, y_{s}\right) \mid s, y_{s}\right\} \\
& =\left\{\begin{array}{c}
E(2 \theta \mid D), \quad 1 \in s \\
E(\theta \mid D)+E(\phi \mid D), \quad 1 \notin s
\end{array}\right\} \\
& =\left\{\begin{array}{cc}
2 \hat{\theta}, & 1 \in s \\
\hat{\theta}+\hat{\phi}, & 1 \notin s
\end{array}\right\},
\end{aligned}
$$

$$
\begin{aligned}
\hat{\phi}=E(\phi \mid D) & =E_{\theta}\left(\frac{2 \theta}{3-\theta} \mid D\right) \\
& =\sum_{\theta=1 / 4,3 / 4}\left(\frac{2 \theta}{3-\theta}\right) f(\theta \mid D) .
\end{aligned}
$$

The finite population mean is

$$
\bar{y}=\frac{1}{4}\left(y_{s T}+y_{r T}\right),
$$

and so the predictive mean of $\bar{y}$ may be expressed as

$$
\hat{\bar{y}}=E\left(\bar{y} \mid s, y_{s}\right)=\frac{1}{4}\left(y_{s T}+E\left(y_{r T} \mid s, y_{s}\right)\right) .
$$


Using suitable $\mathrm{R}$ functions, we find that $\hat{\phi}$ and $\hat{\bar{y}}$ are as follows:

If $1 \in S$ and $y_{s T}=0$ then $\hat{\phi}=0.2303030$ and $\hat{\bar{y}}=0.1500000$

If $1 \in S$ and $y_{S T}=1$ then $\hat{\phi}=0.4242424$ and $\hat{\bar{y}}=0.5000000$

If $1 \in s$ and $y_{s T}=2$ then $\hat{\phi}=0.6181818$ and $\hat{\bar{y}}=0.8500000$

If $1 \notin S$ and $y_{s T}=0$ then $\hat{\phi}=0.2222222$ and $\hat{\bar{y}}=0.1284722$

If $1 \notin s$ and $y_{s T}=1$ then $\hat{\phi}=0.4000000$ and $\hat{\bar{y}}=0.4687500$

If $1 \notin s$ and $y_{s T}=2$ then $\hat{\phi}=0.6086957$ and $\hat{\bar{y}}=0.8247283$.

Note: Working through the above equation using exact fractions, it can be shown that

$$
\hat{\bar{y}}=\hat{\bar{y}}\left(s, y_{s}\right)= \begin{cases}3 / 20, & 1 \in s, y_{s T}=0 \\ 1 / 2, & 1 \in s, y_{s T}=1 \\ 17 / 20, & 1 \in s, y_{s T}=2 \\ 37 / 288, & 1 \notin s, y_{s T}=0 \\ 15 / 32, & 1 \notin s, y_{s T}=1 \\ 607 / 736, & 1 \notin s, y_{s T}=2 .\end{cases}
$$

The following are details of the working for 37/288, 15/32 and 607/736.

Observe that

$$
E\left(y_{r T} \mid \theta, s, y_{s}\right)=\theta+\frac{2 \theta}{3-\theta}=\frac{\theta(5-\theta)}{3-\theta} .
$$

Therefore

$$
\hat{y}_{r T}=E\left\{E\left(y_{r T} \mid s, y_{s}, \theta\right) \mid s, y_{s}\right\}=E\left\{\frac{\theta(5-\theta)}{3-\theta} \mid s, y_{s}\right\} .
$$

So, if $y_{s T}=0$ then

$$
\hat{y}_{r T}=E\left\{\frac{\theta(5-\theta)}{3-\theta} \mid D\right\}=\frac{\frac{1}{4}\left(5-\frac{1}{4}\right)}{3-\frac{1}{4}} \frac{11}{12}+\frac{\frac{3}{4}\left(5-\frac{3}{4}\right)}{3-\frac{3}{4}} \frac{1}{12}
$$




$$
\begin{aligned}
& =\frac{\frac{1}{\not}\left(\frac{19}{4}\right)}{\frac{\mathrm{N}}{\not}} \frac{\mathrm{N}}{12}+\frac{\frac{3}{\not}\left(\frac{17}{4}\right)}{\frac{9}{\not}} \frac{1}{12}=\frac{1}{48}\left\{19+\frac{17}{3}\right\} \\
& =\frac{1}{48}\left\{\frac{57+17}{3}\right\}=\frac{74}{48 \times 3}=\frac{37}{72} .
\end{aligned}
$$

Also, if $y_{s T}=1$ then

$$
\begin{gathered}
\hat{y}_{r T}=E\left\{\frac{\theta(5-\theta)}{3-\theta} \mid D\right\}=\frac{\frac{1}{4}\left(5-\frac{1}{4}\right)}{3-\frac{1}{4}} \frac{11}{20}+\frac{\frac{3}{4}\left(5-\frac{3}{4}\right)}{3-\frac{3}{4}} \frac{9}{20} \\
=\frac{\frac{1}{4}\left(\frac{19}{4}\right)}{\frac{1}{4}} \frac{\lambda}{20}+\frac{\frac{3}{4}\left(\frac{17}{4}\right)}{\frac{9}{4}} \frac{9}{20}=\frac{1}{80}\{19+51\}=\frac{7}{8} .
\end{gathered}
$$

And if $y_{s T}=2$ then

$$
\begin{aligned}
\hat{y}_{r T}=E & \left\{\frac{\theta(5-\theta)}{3-\theta} \mid D\right\}=\frac{\frac{1}{4}\left(5-\frac{1}{4}\right)}{3-\frac{1}{4}} \frac{11}{92}+\frac{\frac{3}{4}\left(5-\frac{3}{4}\right)}{3-\frac{3}{4}} \frac{81}{92} \\
= & \frac{\frac{1}{\not}\left(\frac{19}{4}\right)}{\frac{\mathrm{N}}{4}} \frac{\mathrm{N}}{92}+\frac{\frac{3}{\not}\left(\frac{17}{4}\right)}{\frac{9}{\not}} \frac{81}{92} \\
& =\frac{1}{368}\{19+27 \times 17\}=\frac{478}{368}=\frac{239}{184} .
\end{aligned}
$$

Thus (for $1 \notin s$ ) we have that

$$
\hat{y}_{r T}= \begin{cases}37 / 72, & y_{s T}=0 \\ 7 / 8, & y_{s T}=1 \\ 239 / 184, & y_{s T}=2 .\end{cases}
$$


Hence

$$
\hat{y}_{T}=y_{s T}+\hat{y}_{r T}=\hat{y}_{r T}=\left\{\begin{array}{cc}
0+37 / 72=37 / 72, & y_{s T}=0 \\
1+7 / 8=15 / 8, & y_{s T}=1 \\
2+239 / 184=607 / 184, & y_{s T}=2 .
\end{array}\right.
$$

Thus, finally (for $1 \notin s$ ), we obtain

$$
\hat{\bar{y}}=\frac{\hat{y}_{T}}{4}= \begin{cases}37 / 288, & y_{s T}=0 \\ 15 / 32, & y_{s T}=1 \\ 607 / 736, & y_{s T}=2\end{cases}
$$

A similar logic can be used to obtain the fractions 3/20, 1/2 and 17/20.

(g)(i) Suppose that $\theta=1 / 4$ and $s=(1,3)$. Then $1 \in s$ and so

$$
\left(y_{1}, \ldots, y_{4} \mid \theta, s\right) \sim \perp \operatorname{Bernoulli}\left(\pi_{i}\right),
$$

where:

$$
\begin{aligned}
& \pi_{1}=\frac{4 \theta}{3+\theta}=\frac{4\left(\frac{1}{4}\right)}{3+\left(\frac{1}{4}\right)}=\frac{4}{13} \\
& \pi_{i}=\theta=\frac{1}{4}, i>1 .
\end{aligned}
$$

In this case,

$$
y_{s T}=y_{1}+y_{3},
$$

and so:

$$
\begin{aligned}
& P\left(y_{s T}=0 \mid \theta, s\right)=\frac{9}{13} \times \frac{3}{4}=\frac{27}{52} \\
& P\left(y_{s T}=2 \mid \theta, s\right)=\frac{4}{13} \times \frac{1}{4}=\frac{4}{52} \\
& P\left(y_{s T}=1 \mid \theta, s\right)=1-\frac{27}{52}-\frac{4}{52}=\frac{21}{52} .
\end{aligned}
$$


So the model mean of $\hat{\bar{y}}$ is

$$
\begin{aligned}
E(\hat{\bar{y}} \mid \theta, s) & =E\left\{E\left(\hat{\bar{y}} \mid \theta, s, y_{s T}\right) \mid \theta, s\right\} \\
& =E\left\{\hat{\bar{y}}\left(s, y_{s T}\right) \mid \theta, s\right\} \\
& =\sum_{y_{s T}=0}^{2} \hat{\bar{y}}\left(s, y_{s T}\right) f\left(y_{s T} \mid \theta, s\right) \\
& =0.15(27 / 52)+0.5(21 / 52)+0.85(4 / 52) \\
& =0.3451923 .
\end{aligned}
$$

Also, the model mean of $\bar{y}$ is

$$
\begin{aligned}
E(\bar{y} \mid \theta, s)=\frac{1}{4}\left(\pi_{1}+\ldots+\pi_{4}\right) & =\frac{1}{4}\left(\frac{4}{13}+\frac{1}{4}+\frac{1}{4}+\frac{1}{4}\right) \\
& =55 / 208=0.2644231 .
\end{aligned}
$$

So the model bias of $\hat{\bar{y}}$ is

$$
E(\hat{\bar{y}}-\bar{y} \mid \theta, s)=0.3451923-0.2644231=0.08077 \text {. }
$$

(g)(ii) Suppose that $\theta=1 / 4$ and $s=(2,3)$. Then $1 \notin s$ and so

$$
\left(y_{1}, \ldots, y_{4} \mid \theta, s\right) \sim \perp \operatorname{Bernoulli}\left(\pi_{i}\right),
$$

where:

$$
\begin{aligned}
& \pi_{1}=\frac{2 \theta}{3-\theta}=\frac{2\left(\frac{1}{4}\right)}{3-\left(\frac{1}{4}\right)}=\frac{2}{11} \\
& \pi_{i}=\theta=\frac{1}{4}, i>1 .
\end{aligned}
$$

In this case,

and so:

$$
y_{s T}=y_{2}+y_{3},
$$

$$
\begin{aligned}
& P\left(y_{s T}=0 \mid \theta, s\right)=\frac{3}{4} \times \frac{3}{4}=\frac{9}{16} \\
& P\left(y_{s T}=2 \mid \theta, s\right)=\frac{1}{4} \times \frac{1}{4}=\frac{1}{16} \\
& P\left(y_{s T}=1 \mid \theta, s\right)=1-\frac{9}{16}-\frac{1}{16}=\frac{6}{16} .
\end{aligned}
$$


So (using results in (g)(i)) the model mean of $\hat{\bar{y}}$ is

$0.1284722(9 / 16)+0.46875(6 / 16)+0.8247283(1 / 16)=0.2995924$.

Also, the model mean of $\bar{y}$ is

$$
\begin{aligned}
E(\bar{y} \mid \theta, s)=\frac{1}{4}\left(\pi_{1}+\ldots+\pi_{4}\right) & =\frac{1}{4}\left(\frac{2}{11}+\frac{1}{4}+\frac{1}{4}+\frac{1}{4}\right) \\
& =41 / 176=0.2329545 .
\end{aligned}
$$

So the model bias of $\hat{\bar{y}}$ is

$$
E(\hat{\bar{y}}-\bar{y} \mid \theta, s)=0.2995924-0.2329545=0.06664 \text {. }
$$

(h)(i) Suppose that $\theta=1 / 4$ and $y=(0,0,1,1)$. Then $y_{1}=0$ and so the sampling mechansim is definitely SRSWOR and ignorable.

Explicitly, we have that

$$
f(s \mid \theta, y)=f(s)=1 / 6 \text {. }
$$

So the design mean of $\hat{\bar{y}}$ is

$$
\begin{aligned}
& E(\hat{\bar{y}} \mid \theta, y)=E\{E(\hat{\bar{y}} \mid \theta, y, s) \mid \theta, y\} \\
&=\sum_{s} \hat{\bar{y}}\left(s, y_{s}\right) f(s \mid \theta, y)=\frac{1}{6} \sum_{s} \hat{\bar{y}}\left(s, y_{s}\right) \\
&=\frac{1}{6}\{\hat{\bar{y}}((1,2),(0,0))+\hat{\bar{y}}((1,3),(0,1))+\hat{\bar{y}}((1,4),(0,1)) \\
&+\hat{\bar{y}}((2,3),(0,1))+\hat{\bar{y}}((2,4),(0,1))+\hat{\bar{y}}((3,4),(1,1))\} \\
&=(1 / 6)(0.15+0.5+0.5+0.46875+0.46875+0.8247283) \\
&= 0.4853714 .
\end{aligned}
$$

Also, the design mean of $\bar{y}$ is

$$
E(\bar{y} \mid \theta, y)=(0+0+1+1) / 4=0.5 \text {. }
$$

So the design bias of $\hat{\bar{y}}$ is

$$
E(\hat{\bar{y}}-\bar{y} \mid \theta, y)=0.4853714-0.5=-0.01463 \text {. }
$$

Note: The derivation of this result did not involve $\theta$. So for the case $\theta=3 / 4$ and $y=(0,0,1,1)$, the design bias of $\hat{\bar{y}}$ is also -0.01463 . 
(h)(ii) Suppose that $\theta=1 / 4$ and $y=(1,0,1,1)$. Then $y_{1}=1$ and so the sampling mechansim is possibly nonignorable, with

$$
f(s \mid y, \theta)=\left\{\begin{array}{l}
\frac{3+y_{1}}{18}=\frac{3+1}{18}=\frac{2}{9}, s=(1,2),(1,3),(1,4) \\
\frac{3-y_{1}}{18}=\frac{3-1}{18}=\frac{1}{9}, s=(2,3),(2,4),(3,4)
\end{array}\right\} .
$$

So the design mean of $\hat{\bar{y}}$ is

$$
\begin{aligned}
& E(\hat{\bar{y}} \mid \theta, y)=E\{E(\hat{\bar{y}} \mid \theta, y, s) \mid \theta, y\}=\sum_{s} \hat{\bar{y}}\left(s, y_{s}\right) f(s \mid \theta, y) \\
& =\hat{\bar{y}}((1,2),(1,0)) \frac{2}{9}+\hat{\bar{y}}((1,3),(1,1)) \frac{2}{9}+\hat{\bar{y}}((1,4),(1,1)) \frac{2}{9} \\
& \quad+\hat{\bar{y}}((2,3),(0,1)) \frac{1}{9}+\hat{\bar{y}}((2,4),(0,1)) \frac{1}{9}+\hat{\bar{y}}((3,4),(1,1)) \frac{1}{9} \\
& =(2 / 9)(0.5+0.85+0.85)+(1 / 9)(0.46875+0.46875+0.8247283) \\
& =0.684692 .
\end{aligned}
$$

Also, the design mean of $\bar{y}$ is $E(\bar{y} \mid \theta, y)=(1+0+1+1) / 4=0.75$.

So the design bias of $\hat{\bar{y}}$ is $E(\hat{\bar{y}}-\bar{y} \mid \theta, y)=0.684692-0.75=-0.06531$.

Note: The derivation of this result did not involve $\theta$. So for the case $\theta=3 / 4$ and $y=(1,0,1,1)$, the design bias of $\hat{\bar{y}}$ is also -0.06531 .

(i) A suitable Gibbs sampler is based on the joint density

$$
f(s, y, \theta)=f(\theta) f(y \mid \theta) f(s \mid y, \theta) \propto 1 \times \prod_{i=1}^{4} \theta^{y_{i}}(1-\theta)^{1-y_{i}} \times \frac{1+I_{1} y_{1}}{6+3 y_{1}} .
$$

We can identify three conditional distributions here. First observe that

$$
\begin{gathered}
f(\theta \mid s, y) \propto \prod_{i=1}^{4} \theta^{y_{i}}(1-\theta)^{1-y_{i}}=\theta^{y_{T}}(1-\theta)^{1-y_{T}}, \quad \theta=1 / 4,3 / 4 \\
=\left\{\begin{array}{c}
(3 / 4)^{y_{T}}(1-1 / 4)^{1-y_{T}}, \theta=1 / 4 \\
(1 / 4)^{y_{T}}(1-3 / 4)^{1-y_{T}}, \theta=3 / 4 .
\end{array}\right.
\end{gathered}
$$

Next, recall from (d)(ii) that

$$
\left(y_{i} \mid \theta, s\right) \sim \perp \operatorname{Bernoulli}\left(\pi_{i}\right), i=1,2,3,4,
$$


where: $\pi_{2}=\pi_{3}=\pi_{4}=\theta$

$$
\pi_{1}=\frac{3-1}{3-\theta} \theta^{1}(1-\theta)^{1-1}=\frac{2 \theta}{3-\theta} .
$$

Now, the second component of $r=\left(r_{1}, r_{2}\right)$ must be 2,3 or 4 .

Therefore

$$
\left(y_{r_{2}} \mid \theta, s, y_{s}, y_{r_{1}}\right) \sim \operatorname{Bernoulli}(\theta) .
$$

However, there are two possibilities for $y_{r_{1}}$. If the data is such that $s_{1}=1$ then

$$
\left(y_{r_{1}} \mid \theta, s, y_{s}, y_{r_{2}}\right) \sim \operatorname{Bernoulli}(\theta) .
$$

On the other hand, if the data is such that $s_{1}>1$ then $r_{1}=1$, and this implies that

$$
\left(y_{r_{1}} \mid \theta, s, y_{s}, y_{r_{2}}\right) \sim \text { Bernoulli }\left(\frac{2 \theta}{3-\theta}\right) .
$$

Equations (A.1), (A.2), (A.3) and (A.4) imply three conditional distributions which define a suitable Gibbs sampler (for $\theta, y_{r_{1}}$ and $y_{r_{2}}$ ).

Note: At (15.4), the ratio of probabilities of $y_{r_{1}}=0$ to $y_{r_{1}}=1$ is

$$
\frac{\left(1-\frac{2 \theta}{3-\theta}\right)}{\left(\frac{2 \theta}{3-\theta}\right)}=\frac{3(1-\theta)}{2 \theta}=\frac{3}{2} \times\left(\frac{1-\theta}{\theta}\right),
$$

which is exactly $3 / 2$ times the ratio of the probabilities of $y_{r_{1}}=0$ to $y_{r_{1}}=1$ at (A.3). (This observation provided some assistance when formulating the required $\mathrm{R}$ code, as detailed below.)

Implementing the above Gibbs sampler, we obtained a random sample

$$
\left(\theta_{1}, \bar{y}^{(1)}\right), \ldots,\left(\theta_{10000}, \bar{y}^{(10000)}\right) \sim \text { iid } f(\theta, \bar{y} \mid D)
$$

for each of the six possible data configurations in (c) and (f).

The respective sample means for $\theta$ were:

$0.3007,0.4924,0.6997,0.2952,0.4764,0.6925$. 
It will be observed that these numbers are very close to the corresponding values obtained in (c), namely

$$
\hat{\theta}=\left(\begin{array}{cc}
3 / 10=0.3000 & \text { if } 1 \in S \text { and } y_{s T}=0 \\
1 / 2=0.5000 & \text { if } 1 \in S \text { and } y_{s T}=1 \\
7 / 10=0.7000 & \text { if } 1 \in S \text { and } y_{s T}=2 \\
7 / 24=0.2917 & \text { if } 1 \notin s \text { and } y_{s T}=0 \\
19 / 40=0.4750 & \text { if } 1 \notin S \text { and } y_{s T}=1 \\
127 / 184=0.6902 & \text { if } 1 \notin s \text { and } y_{s T}=2 .
\end{array}\right.
$$

The respective sample means for $\bar{y}$ were:

$0.1518,0.4929,0.8485,0.1308,0.4719,0.8269$.

It will be noted that these are very close to the corresponding values obtained in ( $\mathrm{f}$ ), namely:

$0.15, \quad 0.5,0.85,0.1284722,0.4687500,0.8247283$.

(j) To check the design bias in (h)(i) we note that for $y=(0,0,1,1)$ the sampling mechanism is ignorable.

So proceed as follows. Simply select one of the 6 possible samples randomly. Then calculate the corresponding value of $\hat{\bar{y}}$. Repeat another $J-1$ times, independently. Then take the mean of the simulated $\hat{\bar{y}}$ values and subtract $\bar{y}=2 / 4$.

Implementing this procedure with $J=10,000$ yielded a point estimate of -0.01562 with $95 \%$ CI $(-0.01945,-0.01179)$. This is consistent with the result -0.01463 in $(\mathrm{h})(\mathrm{i})$.

To check the design bias in (h)(ii) we note that for $y=(1,0,1,1)$ the sampling mechanism is nonignorable with each sample containing unit 1 twice as likely as each unit not containing unit 1.

So, select a sample $s$ from $(1,2),(1,3),(1,4),(2,3),(2,4),(3,4)$, in such a way that each of the first three of these has probability $2 / 9$ and each of the last three has probability $1 / 9$. Then calculate the corresponding value of $\hat{\bar{y}}$. Repeat another $J-1$ times, independently. Then take the mean of the simulated $\hat{\bar{y}}$ values and subtract $\bar{y}=3 / 4$. 
Implementing this procedure with $J=10,000$ yielded a point estimate of -0.06592 with $95 \% \mathrm{CI}(-0.06944,-0.06239)$. This is consistent with the result -0.06531 in (h)(ii).

(k) The mean of the predictive mean of the finite population mean is the same as the unconditional mean of the finite population mean, which is the same as the prior mean of the superpopulation mean, which in our case equals $1 / 2$. Mathematically,

$$
\begin{array}{rlrl}
E \hat{\bar{y}} & =E E\left(\bar{y} \mid s, y_{s}\right) & & \text { by the definition of } \hat{\bar{y}} \\
& =E \bar{y} & & \text { by the law of conditional expectation } \\
& =E E(\bar{y} \mid \theta) & & \text { by the law of conditional expectation } \\
& =E \theta & & \text { since } E(\bar{y} \mid \theta)=\frac{1}{4} \sum_{i=1}^{4} E\left(y_{i} \mid \theta\right)=\frac{1}{4} \sum_{i=1}^{4} \theta=\theta \\
& =\sum_{\theta} \theta f(\theta)=\frac{1}{4} \times \frac{1}{2}+\frac{3}{4} \times \frac{1}{2}=\frac{1}{2} .
\end{array}
$$

To verify this obvious result via Monte Carlo is a good final check on previous calculations.

To this end, simulate $\theta$, then simulate $y$, then simulate $s$, hence obtain the data $\left(s, y_{s}\right)$, then calculate the associated $\hat{\bar{y}}$. Then repeat all of the above independently another $J-1$ times.

Implementing this procedure with $J=10,000$ yielded a point estimate of 0.4992 with $95 \%$ CI $(0.4938,0.5047)$. This is consistent with the answer of $1 / 2$ above.

\section{R Code for Exercise A.4}

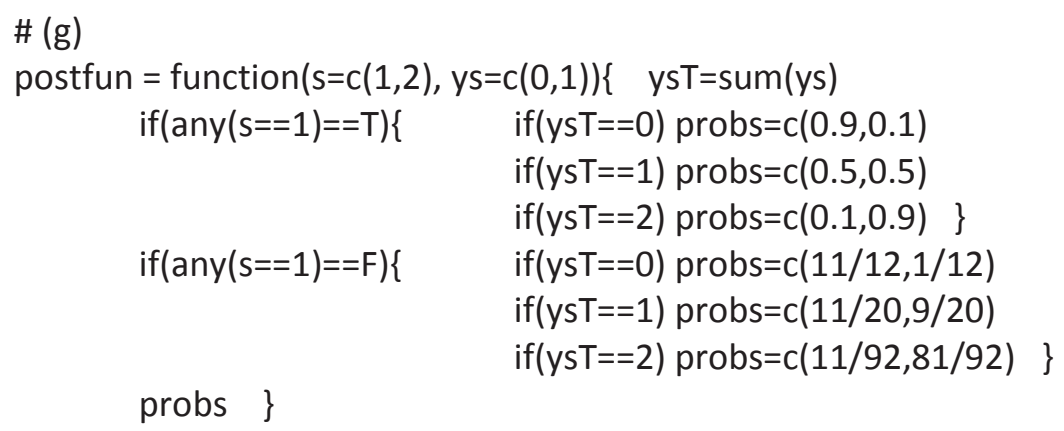

postfun() \# 0.50 .5 Just testing 
postfun $(s=c(2,4), y s=c(1,1)) \# 0.11956520 .8804348$

thetahatfun $=$ function $(s=c(1,2), y s=c(0,1))\{$ probs= postfun $(s=s, y s=y s)$; thetavals $=c(1,3) / 4$; sum( thetavals * probs ) $\}$

thetahatfun() \# 0.5 Just testing

thetahatfun $(s=c(2,4), y s=c(1,1)) \# 0.6902174$

phihatfun=function $(s=c(1,2), y s=c(0,1))\{$ probs=postfun $(s=s, y s=y s)$;

thetavals $=c(1,3) / 4$; phivals $=2 *$ thetavals $/(3$-thetavals)

sum( phivals * probs ) \}

phihatfun() \# 0.4242424 Just testing

phihatfun $(s=c(2,4), y s=c(1,1)) \# 0.6086957$

yrThatfun=function $(s=c(1,2), y s=c(0,1))\{$ thetahat=thetahatfun(s=s,ys=ys)

$$
\text { if }(\text { any }(s==1)==T)\{\quad \text { res }=2 * \text { thetahat }\}
$$$$
\text { if }(\text { any }(s==1)==F)\{
$$

phihat=phihatfun(s=s,ys=ys); res = thetahat + phihat $\}$ res $\}$

yrThatfun() \# 1 Just testing

yrThatfun $(s=c(2,4), y s=c(1,1)) \# 1.298913$

ybarhatfun=function $(s=c(1,2), y s=c(0,1))\{\quad$ EyrT= yrThatfun $(s=s, y s=y s)$

$(\operatorname{sum}(y s)+E y r T) / 4 \quad\}$

ybarhatfun() \# 0.5 Just testing

ybarhatfun $(s=c(2,4), y s=c(1,1)) \quad \# 0.8247283$

smat=matrix $(c(1,2,1,2,1,2,1,2,2,3,2,3,2,3,2,3)$, byrow=T,nrow=8, ncol=2)

ysmat $=$ matrix $(c(0,0,0,1,1,0,1,1,0,0,0,1,1,0,1,1)$, byrow $=T$, nrow $=8$, ncol=2)

thetahatvec=rep(NA,8); phihatvec=rep(NA,8); ybarhatvec=rep(NA,8);

for $(k$ in $1: 8)\{\quad$ thetahatvec[k]= thetahatfun(s=smat[k,,$y s=y s m a t[k]$,

phihatvec $[k]=$ phihatfun $(s=s m a t[k],, y s=y s m a t[k]$,

ybarhatvec[k]= ybarhatfun(s=smat[k,],ys=ysmat[k,]) \}

cbind(smat,NA,ysmat,NA,thetahatvec, NA, phihatvec, NA, ybarhatvec)

\# thetahatvec phihatvec ybarhatvec

\# [1,] 12 NA 00 NA 0.3000000 NA 0.2303030 NA 0.1500000

\#[2,] 12 NA 01 NA 0.5000000 NA 0.4242424 NA 0.5000000

\#[3,] 12 NA 10 NA 0.5000000 NA 0.4242424 NA 0.5000000 repeat OK

\#[4,] 12 NA 11 NA 0.7000000 NA 0.6181818 NA 0.8500000

\#[5,] 23 NA 00 NA 0.2916667 NA 0.2222222 NA 0.1284722 
\#[6,] 23 NA 01 NA 0.4750000 NA 0.4000000 NA 0.4687500

\#[7,] 23 NA 10 NA 0.4750000 NA 0.4000000 NA 0.4687500 repeat OK

\#[8,] 23 NA 11 NA 0.6902174 NA 0.6086957 NA 0.8247283

$0.15 *(27 / 52)+0.5 *(21 / 52)+0.85 *(4 / 52) \# 0.3451923$

$0.1284722 *(9 / 16)+0.46875 *(6 / 16)+0.8247283 *(1 / 16) \# 0.2995924$

\# (h)

$(1 / 6) *(0.15+0.5+0.5+0.46875+0.46875+0.8247283) \# 0.4853714$

$(2 / 9) *(0.5+0.85+0.85)+(1 / 9) *(0.46875+0.46875+0.8247283) \# 0.684692$

\# (i) Check posterior means and predcitive means via Gibbs sampler options(digits $=4$ )

GS=function $(J=1000, \quad s=c(1,2), y s=c(1,0), \quad$ theta $=1 / 4)\{$

thetav=rep(NA,J); yrTv=rep(NA,J); yTv=rep(NA,J)

yrmat=matrix(NA, nrow=J,ncol=2); ysT=sum(ys)

for(j in $1: J)\{$

probsyi $=c(1$-theta, theta $)$

yr2=sample $(x=c(0,1)$, size $=1$, prob=probsyi $)$

if $(s[1]==1)$ yr1=sample $(x=c(0,1)$, size $=1$, prob=probsyi) else yr1=sample $(x=c(0,1)$, size $=1, \operatorname{prob}=c(3,2) *$ probsyi $)$

$y r=c(y r 1, y r 2) ; y r T=s u m(y r) ; y T=y s T+y r T$

probstheta $=c\left((1 / 4)^{\wedge} y T^{*}(3 / 4)^{\wedge}(4-y T),(3 / 4)^{\wedge} y T^{*}(1 / 4)^{\wedge}(4-y T)\right)$

theta $=$ sample $(x=c(1 / 4,3 / 4)$, size $=1$, prob $=$ probstheta $)$

thetav[j]=theta; $y r T v[j]=y r T ; \quad y T v[j]=y T ; y r m a t[j]=,y r$

\}

list(thetav=thetav, yrTv=yrTv, yTv=yTv, ybarv=yTv/4, yrmat=yrmat) $\}$

set.seed(111); J = 10000; thetahatvec=rep(NA,6); ybarhatvec=rep(NA,6)

res $=\mathrm{GS}(\mathrm{J}=\mathrm{J}, \mathrm{s}=\mathrm{c}(1,2), \mathrm{ys}=\mathrm{c}(0,0))$

thetahatvec[1] = mean(res\$thetav); ybarhatvec[1] = mean(res\$ybarv);

res $=\mathrm{GS}(\mathrm{J}=\mathrm{J}, \mathrm{s}=\mathrm{c}(1,2), \mathrm{ys}=\mathrm{c}(0,1))$

thetahatvec[2] = mean(res\$thetav); ybarhatvec[2] = mean(res\$ybarv);

res $=\mathrm{GS}(\mathrm{J}=\mathrm{J}, \mathrm{s}=\mathrm{c}(1,2), \mathrm{ys}=\mathrm{c}(1,1))$

thetahatvec[3] = mean(res\$thetav); ybarhatvec[3] = mean(res\$ybarv);

res $=\mathrm{GS}(\mathrm{J}=\mathrm{J}, \mathrm{s}=\mathrm{c}(2,3), \mathrm{ys}=\mathrm{c}(0,0))$

thetahatvec[4] = mean (res\$thetav); ybarhatvec[4] = mean(res\$ybarv);

res $=\mathrm{GS}(\mathrm{J}=\mathrm{J}, \mathrm{s}=\mathrm{c}(2,3), \mathrm{ys}=\mathrm{c}(0,1))$

thetahatvec[5] = mean (res\$thetav); $y$ barhatvec[5] = mean(res\$ybarv);

res $=\mathrm{GS}(\mathrm{J}=\mathrm{J}, \mathrm{s}=\mathrm{c}(2,3), \mathrm{ys}=\mathrm{c}(1,1))$

thetahatvec[6] = mean(res\$thetav); ybarhatvec[6] = mean(res\$ybarv);

thetahatvec \# 0.3007 0.4924 0.6997 0.2952 0.47640.6925

\# All very close to results in (c) 
ybarhatvec \# 0.15180 .49290 .84850 .13080 .47190 .8269

\# All very close to results in ( $f$ )

\# (j) Check design bias of predictive mean of ybar if theta $=1 / 4$ and $y=(0,0,1,1)$ smatrix=matrix $(c(1,2,1,3,1,4,2,3,2,4,3,4)$, byrow=T, nrow=6, ncol=2) $y=c(0,0,1,1) ; J=10000 ; y b a r h a t s i m v=r e p(N A, J)$; set.seed(413)

for $(\mathrm{j}$ in 1:J)\{ indexsim=sample $(1: 6,1, \operatorname{prob}=c(1,1,1,1,1,1))$ ssim=smatrix[indexsim,]; yssim $=y[\mathrm{ssim}]$ ybarhatsimv[j] = ybarhatfun(s=ssim,ys=yssim) \}

est=mean(ybarhatsimv)-0.5;

$c i=e s t+c(-1,1) *$ qnorm $(0.975) * s d(y b a r h a t s i m v-0.5) /$ sqrt $(J)$ c(est,ci) \# -0.01562 -0.01945 -0.01179 Consistent with -0.01463 in (h)(i)

\# Check design bias of predictive mean of ybar if theta $=1 / 4$ and $y=(1,0,1,1)$ $\mathrm{y}=\mathrm{c}(1,0,1,1) ; \mathrm{J}=10000 ; \mathrm{ybarhatsimv}=\mathrm{rep}(\mathrm{NA}, \mathrm{J})$; set. $\operatorname{seed}(442)$

for $(\mathrm{j}$ in 1:J)\{ indexsim=sample $(1: 6,1, \operatorname{prob}=c(2,2,2,1,1,1))$ ssim=smatrix[indexsim,]; yssim $=y[\operatorname{ssim}]$ ybarhatsimv[j] = ybarhatfun(s=ssim,ys=yssim) \}

est=mean(ybarhatsimv)-0.75;

ci=est+c(-1,1)* qnorm(0.975)*sd(ybarhatsimv-0.5)/sqrt(J) c(est,ci) \# -0.06592 -0.06944 -0.06239 Consistent with -0.06531 in (h)(ii)

\# (k) Check mean of predictive mean of finite population mean smatrix=matrix $(c(1,2,1,3,1,4,2,3,2,4,3,4)$, byrow=T,nrow=6, ncol=2) $\mathrm{J}=$ 10000; ybarhatsimv=rep(NA,J); set.seed(102);

for $(j$ in $1: J)\{$ thetasim=sample(c(1/4,3/4),1); ysim=rbinom(4,1,thetasim) if $(y \operatorname{sim}[1]==0)$ indexsim $=$ sample $(1: 6,1, \operatorname{prob}=c(1,1,1,1,1,1))$ if $(y \operatorname{sim}[1]==1)$ indexsim $=$ sample $(1: 6,1, \operatorname{prob}=c(2,2,2,1,1,1))$ ssim=smatrix[indexsim,]; yssim=ysim[ssim]; ybarhatsimv[j]= ybarhatfun(s=ssim,ys=yssim) \}

est $=$ mean(ybarhatsimv);

$\mathrm{ci}=$ est+c(-1,1)* qnorm $(0.975) *$ sd(ybarhatsimv)/sqrt(J) c(est,ci) \# 0.49920 .49380 .5047 Consistent with 0.5 


\section{Distributions and Notation}

Below are several probability distributions which feature in this book. The purpose of this appendix is to provide a brief guide to the style of notation and terminology used throughout. It is not intended to be a comprehensive listing. Some of the notation introduced here is repeated in Appendix C.

\section{B.I The normal distribution}

A random variable (rv) $X$ has the normal distribution with parameters $\mu$ and $\sigma^{2}$ if its probability density function (pdf), or density, has the form

$$
f(x)=\frac{1}{\sigma \sqrt{2 \pi}} \exp \left\{-\frac{1}{2 \sigma^{2}}(x-\mu)^{2}\right\}, \mu \in \Re .
$$

We then write $X \sim N\left(\mu, \sigma^{2}\right)$. To be more explicit, we will sometimes write $f(x)$ as $f_{X}(x)$ or $f_{N\left(\mu, \sigma^{2}\right)}(x)$. To avoid subscripting notation and so aid legibility, $f_{N\left(\mu, \sigma^{2}\right)}(x)$ may sometimes be written as $f\left(x, N\left(\mu, \sigma^{2}\right)\right)$. Likewise for other functions and expressions which contain subscripts.

If $X \sim N\left(\mu, \sigma^{2}\right)$ then $E X=\operatorname{Mode}(X)=\operatorname{Median}(X)=\mu$ and $V X=\sigma^{2}$.

The cumulative distribution function (cdf) of $X$ is

$$
F(x)=P(X \leq x)=F_{N\left(\mu, \sigma^{2}\right)}(x)=F\left(x, N\left(\mu, \sigma^{2}\right)\right)=\int_{-\infty}^{x} f_{N\left(\mu, \sigma^{2}\right)}(t) d t .
$$

The (lower) $p$-quantile of $X$ is the value of $x$ such that $F(x)=p$.

Thus the $p$-quantile of $X$ is the inverse cdf of $X$. This may also be written

$$
F^{-1}(p)=F_{X}^{-1}(p)=F_{N\left(\mu, \sigma^{2}\right)}^{-1}(p)=F \operatorname{Inv}\left(p, N\left(\mu, \sigma^{2}\right)\right) .
$$

If $Z \sim N(0,1)$, we say that $Z$ has the standard normal distribution. The pdf, cdf, (lower) $p$-quantile and upper $p$-quantile of $Z$ may be denoted by $\phi(z)$, $\Phi(z), \Phi^{-1}(p)$, and $z_{p}=\Phi^{-1}(1-p)$, respectively. 
This notation means that if $X \sim N\left(\mu, \sigma^{2}\right)$, then we may write:

$$
f(x)=\frac{1}{\sigma} \phi\left(\frac{x-\mu}{\sigma}\right), \quad F(x)=\Phi\left(\frac{x-\mu}{\sigma}\right), \quad F_{X}^{-1}(p)=\mu+z_{1-p} \sigma .
$$

Note: We sometimes use upper and lower case letters interchangeably. Thus $X \sim N\left(\mu, \sigma^{2}\right)$ may also be written $x \sim N\left(\mu, \sigma^{2}\right)$. The pdf of a rv $X$ when evaluated at $c$ may also be denoted by $f(x=c)$.

\section{B.2 The gamma distribution}

A random variable $X$ has the gamma distribution with parameters $a$ and $b$ if its pdf has the form

$$
f_{X}(x)=\frac{b^{a} x^{a-1} e^{-b x}}{\Gamma(a)}, x>0 .
$$

We then write $X \sim \operatorname{Gamma}(a, b)$ or $X \sim \operatorname{Gam}(a, b)$ or $X \sim G(a, b)$. We may also write $f_{X}(x)$ as $f(x)$ or $f_{G(a, b)}(x)$ or $f(x, G(a, b))$.

The cdf of $X$ may be written $F_{X}(x)=F_{G(a, b)}(x)=F(x, G(a, b))$, and $X$ 's $p$-quantile is $F_{X}^{-1}(p)=F_{G(a, b)}^{-1}(p)=F^{-1}(p, G(a, b))=F \operatorname{Inv}(p, G(a, b))$.

If $X \sim G(a, b)$ then:

$$
\begin{aligned}
& \operatorname{Mode}(X)=(a-1) / b \text { if } a>1 \\
& \operatorname{Mode}(X)=0 \text { if } a \leq 1 \\
& E X=a / b, \quad V X=a / b^{2} \\
& \left.E X^{k}=\frac{\Gamma(a+k)}{b^{k} \Gamma(a)} \text { (the kth raw moment of } X\right) .
\end{aligned}
$$

The last result may be proved by writing

$$
E X^{k}=\int_{0}^{\infty} x^{k} \frac{b^{a} x^{a-1} e^{-b x}}{\Gamma(a)} d x=\frac{b^{a} \Gamma(a+k)}{b^{a+k} \Gamma(a)} \int_{0}^{\infty} \frac{b^{a+k} x^{a+k-1} e^{-b x}}{\Gamma(a+k)} d x
$$

and noting that the last integral is equal to unity.

The definition of the gamma distribution involves the gamma function,

$$
\Gamma(k)=\int_{0}^{\infty} t^{k-1} e^{-t} d t .
$$


Some properties of the gamma function are as follows:

$$
\begin{aligned}
& \Gamma(k) \rightarrow \infty \text { as } k \rightarrow \infty \text { or } k \rightarrow 0 \\
& \Gamma(k)=(k-1) \Gamma(k-1) \text { for } k>1 \\
& \Gamma(k)=(k-1) ! \text { if } k \in\{1,2,3, \ldots\}(\text { with } 0 !=1) \\
& \Gamma(1 / 2)=\sqrt{\pi} .
\end{aligned}
$$

Note: There is an alternative definition of the gamma distribution, whereby $X \sim G(a, b)$ means $f(x)=b^{-a} x^{a-1} e^{-x / b} / \Gamma(a), x>0$, so that $E X=a b$. This alternative definition is not used in this book.

\section{B.3 The exponential distribution}

If $X \sim G(1, b)$ then $X$ has the exponential distribution with parameter $b$, and we write $X \sim \operatorname{Exponential(b)}$ or $X \sim \operatorname{Expo(b)}$.

Note: We do not write $X \sim \operatorname{Exp}(b)$ because this could more easily be confused with $X=\exp (b)=e^{b}$ (where exp is the exponential function).

The pdf of $X$, namely $f(x)=b e^{-b x}, x>0$, may also be written as $f_{\operatorname{Expo(b)}}(x)$ or $f(x, \operatorname{Expo}(b))$.

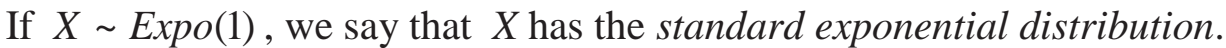

\section{B.4 The chi-squared distribution}

If $X \sim G(m / 2,1 / 2)$ then $X$ has the chi-squared distribution with parameter $m$ (called the degrees of freedom, abbreviated dof).

We then write $X \sim \chi^{2}(m)$ or $X \sim \operatorname{Chisq}(m)$, and denote the pdf of $X$ by $f_{\chi^{2}(m)}(x)$ or $f(x, \operatorname{Chis} q(m))$.

The upper $p$-quantile of the $\chi^{2}(m)$ distribution may be written

$$
\chi_{p}^{2}(m)=F_{\chi^{2}(m)}^{-1}(1-p)=\operatorname{FInv}(1-p, \operatorname{Chis} q(m)) .
$$


A useful result is that if $Y=r X$, where $X \sim \operatorname{Gamma}(\mathrm{m} / 2, r / 2)$, then $Y \sim G(m / 2,1 / 2) \sim \chi^{2}(m)$. This result can be proved easily using the transformation rule, as follows:

$$
f(y)=f(x)\left|\frac{d x}{d y}\right| \stackrel{y}{\propto}\left(\frac{y}{r}\right)^{\frac{m}{2}-1} e^{-\frac{r}{2} \times \frac{y}{r}}\left|\frac{1}{r}\right| \stackrel{y}{\propto} y^{\frac{m}{2}-1} e^{-\frac{1}{2} y} .
$$

Note: The symbol $\stackrel{y}{\propto}$ here denotes 'proportionality with respect to $y$ '. The statement $g \stackrel{t}{\propto} h$ means $g=c \times h$, where $c$ is a constant that does not depend on $t$. E.g. if $g=5 t^{2} r^{3}$, we may write: $g \stackrel{t}{g} t^{2}, g \stackrel{r}{g} \stackrel{\text { r }}{r}$, $g \propto t^{t, r} \propto t^{2} r^{3}, g \not c t, g \not d^{r} r^{4}$, etc. By default, $g(t) \propto t^{5}$ means $g(t) \stackrel{t}{\propto} t^{5}$, and $g(t \mid u) \propto t^{5}$ means $g(t \mid u) \stackrel{t}{\propto} t^{5}$ (not $g(t \mid u) \stackrel{t, u}{\propto} t^{5}$ ).

\section{B.5 The inverse gamma distribution}

If $X \sim G(a, b)$, then $Y=1 / X$ has the inverse gamma distribution with parameters $a$ and $b$. In that case, we write $Y \sim \operatorname{InverseGamma(a,b)\text {or}}$ $Y \sim \operatorname{IGam}(a, b)$ or $Y \sim \operatorname{IG}(a, b)$.

By the transformation rule, the pdf of $Y$ is

$$
f(y)=f(x)\left|\frac{d x}{d y}\right|=\frac{b^{a}(1 / y)^{a-1} e^{-b(1 / y)}}{\Gamma(a)}\left|-\frac{1}{y^{2}}\right|=\frac{b^{a} y^{-(a+1)} e^{-b / y}}{\Gamma(a)}, y>0,
$$

which may also be written $f_{\operatorname{IG}(a, b)}(y)$ or $f(y, \operatorname{IG}(a, b))$.

Some other properties of $Y$ are as follows:

$$
\begin{aligned}
& E Y=b /(a-1) \text { if } a>1, \quad E Y=\infty \text { if } a \leq 1 \\
& V Y=b^{2} /\left\{(a-1)^{2}(a-2)\right\} \text { if } a>2, \quad \operatorname{Mode}(Y)=b /(a+1) \text {. }
\end{aligned}
$$

\section{B.6 The $t$ distribution}

A random variable $X$ has the $t$ distribution with parameter $m$ if

$$
f(x)=\frac{\Gamma((m+1) / 2)}{\Gamma(m / 2) \sqrt{m \pi}}\left(1+\frac{x^{2}}{m}\right)^{-\frac{1}{2}(m+1)},-\infty<x<\infty .
$$

In that case, we write $X \sim t(m)$ and denote the density of $X$ by $f_{t(m)}(x)$ or $f(x, t(m))$. The cdf of $X$ is denoted $F_{t(m)}(x)$ or $F(x, t(m))$, and the 
upper $p$-quantile may be written $t_{p}(m)=F_{t(m)}^{-1}(1-p)=F \operatorname{Inv}(1-p, t(m))$. We call $m$ the degrees of freedom parameter.

An equivalent definition of the $t$ distribution is as follows. If $Z \sim N(0,1)$, $Y \sim \chi^{2}(m)$ and $Z \perp Y$, then $X=Z / \sqrt{Y / m} \sim t(m)$.

Note: The symbol $\perp$ here denotes independence. Thus, the statement $A \perp B$ means that $A$ and $B$ are independent random variables. Likewise, $(A \perp B \mid C)$ means that $A$ and $B$ are independent conditional on $C$.

\section{B.7 The F distribution}

Suppose that $U \sim \chi^{2}(a), W \sim \chi^{2}(b)$ and $U \perp W$. Then $X=\frac{U / a}{W / b}$ has the $F$ distribution with parameters $a$ and $b$. We then write $X \sim F(a, b)$. The pdf and cdf of $X$ (both omitted here) may be denoted $f_{F(a, b)}(x)$ and $F_{F(a, b)}(x)$, respectively. We call $a$ the numerator degrees of freedom and $b$ the denominator degrees of freedom. The upper $p$-quantile of $X$ may be denoted as $F_{p}(a, b)$ or $F_{F(a, b)}^{-1}(1-p)$ or $\operatorname{Finv}(1-p, F(a, b))$.

\section{B.8 The (continuous) uniform distribution}

A random variable $X$ has the (continuous) uniform distribution with parameters $a$ and $b$ if its pdf is $f(x)=1 /(b-a), a<x<b$.

We then write $X \sim U(a, b)$ and $f(x)=f_{U(a, b)}(x)=f(x, U(a, b))$.

The cdf of $X$ is $F_{U(a, b)}(x)=F(x, U(a, b))=(x-a) /(b-a), a<x<b$.

The mean and variance of $X$ are $(a+b) / 2$ and $(b-a)^{2} / 12$.

\section{B.9 The discrete uniform distribution}

A random variable $X$ has the discrete uniform distribution with parameters $a_{1}, \ldots, a_{K}$ if its density is $f(x)=1 / K, x=a_{1}, \ldots, a_{K}$.

We then write $X \sim D U\left(a_{1}, \ldots, a_{K}\right)$. The density $f(x)$ may also be written as $f_{D U\left(a_{1}, \ldots, a_{K}\right)}(x)$ or $f\left(x, D U\left(a_{1}, \ldots, a_{K}\right)\right)$. 
Equivalently, we may describe $X$ as having the discrete uniform distribution with parameter $a=\left(a_{1}, \ldots, a_{K}\right)$ (a vector). In that case, we may write $X \sim D U(a)$ and denote $f(x)$ by $f_{D U(a)}(x)$ or $f(x, D U(a))$.

Note: Because $X$ here is discrete, $f(x)$ may more aptly be called the probability mass function (pmf) of $X$. But for simplicity, we usually use the term probability density function (pdf) or density in reference to any type of random variable (continuous, discrete or mixed).

\section{B.I0 The binomial distribution}

A rv $X$ has the binomial distribution with parameters $n$ and $p$ if its density has the form

$$
f(x)=\left(\begin{array}{l}
n \\
x
\end{array}\right) p^{x}(1-p)^{n-x}, x=0,1, \ldots, n .
$$

We then write $X \sim \operatorname{Bin}(n, p)$. The density $f(x)$ may also be denoted by $f_{\operatorname{Bin}(n, p)}(x)$ or $f(x, \operatorname{Bin}(n, p))$. The mean and variance of $X$ are $n p$ and $n p(1-p)$. We call $n$ the number of trials and $p$ the probability of success (equivalently, the binomial parameter or the binomial proportion).

\section{B.I I The Bernoulli distribution}

If $X \sim \operatorname{Bin}(1, p)$ then we say that $X$ has the Benoulli distribution with parameter $p$. We then write $X \sim \operatorname{Bernoulli}(p)$ or $X \sim \operatorname{Bern}(p)$.

\section{B.I 2 The geometric distribution}

A random variable is said to have the geometric distribution with parameter $p$ if its pdf has the form

$$
f(x)=(1-p)^{x-1} p, x=1,2,3, \ldots
$$

We then write $X \sim \operatorname{Geo}(p)$. The pdf of $X$ may be denoted by $f_{G e o(p)}(x)$ or $f(x, \operatorname{Geo}(p))$. The mean and variance of $X$ are $1 / p$ and $(1-p) / p^{2}$. The cdf of $X$ is given by

$$
F_{G e o(p)}(x)=F(x, G e o(p))=P(X \leq x)=1-(1-p)^{x}, x=1,2,3, \ldots
$$




\section{Abbreviations and Acronyms}

Below are some of the abbreviations and acronyms used in this book. The list may not be comprehensive. Some of the expressions listed have more than one meaning, depending on the context.

ACF

AELF

AR

ARMA

$B$

Bern

Beta

BF

BUGS
Bin, Binom

autocorrelation function

absolute error loss function

autoregressive (process); acceptance rate

autoregressive moving average (process)

beta function; bias

Bernoulli distribution

beta distribution

Bayes factor

binomial distribution

Bayesian inference Using Gibbs Sampling (software environment for performing MCMC)

C, Cov

cdf

CDR

Chisq

CI

CNR

CPDI

CPDR

cts

$D$

DA

df

dof

dsn

$D U$ covariance operator

cumulative distribution function (same as $\mathrm{df}$ )

central density region

chi-squared distribution (equivalent to $\chi^{2}$ )

confidence interval

conditional Newton-Raphson (algorithm)

central posterior (or predictive) density interval

central posterior (or predictive) density region

continuous

data

data augmentation (algorithm)

distribution function (same as cdf)

degrees of freedom

distribution

discrete uniform distribution 


$\begin{array}{ll}E & \text { expectation operator } \\ e & \text { Euler's number (2.71828) } \\ \text { ECM } & \text { Expectation-Conditional-Maximisation (algorithm) } \\ \text { ELF } & \text { error loss function } \\ \text { EM } & \text { Expectation-Maximisation (algorithm) } \\ \text { E-Step } & \text { Expectation Step (in EM algorithm) } \\ \text { exp } & \text { exponential function (e raised to a power) } \\ \text { Expo } & \text { exponential distribution }\end{array}$

F $\quad$ F distribution; (cumulative) distribution function

$f$

FCP pdf or pmf (same as $p$ ); finite population correction factor

FInv frequentist coverage probability

fpc finite population correction (factor) inverse distribution function (equivalent to $F^{-1}$ )

G, Gam gamma distribution (not to be confused with the gamma function, which is denoted by the Greek letter $\Gamma$ )

Geo geometric distribution

GLM generalised linear model

GS Gibbs sampler/sampling

HPDI

HPDR highest posterior (or predictive) density interval Hyp highest posterior (or predictive) density region

I

id

IELF

IG, IGam

iid ind, indep

$J$

$L$

LIC

LIE

LIV

$\ln , \log$ hypergeometric distribution

standard indicator function; vector of sample inclusion indicators (or counters); Fisher information identically distributed (not necessarily independent) indicator error loss function inverse gamma distribution independent and identically distributed (as) independent (not necessarily identically distributed)

Monte Carlo sample size

loss function; lower bound; ordered sample (vector of the labels of selected units in the order that they are sampled) law of iterated covariance:

$$
C(X, Y)=E C(X, Y \mid Z)+C\{E(X \mid Z), E(Y \mid Z)\}
$$

law of iterated expectation: $E X=E E(X \mid Z)$

law of iterated variance: $V X=E V(X \mid Z)+V E(X \mid Z)$

natural logarithm (to base $e$ ) 


\begin{tabular}{|c|c|}
\hline$m$ & nonsample size $(m=N-n)$ \\
\hline MA & moving average (process); Metropolis algorithm \\
\hline MAD & $\begin{array}{l}\text { mean absolute deviation; finite population mean } \\
\text { absolute deviation about the superpopulation mean }\end{array}$ \\
\hline $\max$ & maximum/maximise \\
\hline MC & Monte Carlo (method); Markov chain \\
\hline MCMC & Markov chain Monte Carlo (method) \\
\hline $\mathrm{MH}$ & Metropolis-Hastings (algorithm) \\
\hline $\operatorname{lin}$ & minimum/minimise \\
\hline IL & maximum likelihood (method) \\
\hline ILE & maximum likelihood estimate/estimator/estimation \\
\hline OME & method of moments estimate/estimator/estimation \\
\hline I-Step & Maximisation Step (in EM algorithm) \\
\hline$N$ & normal (or Gaussian) distribution; finite population size \\
\hline$n$ & sample size \\
\hline NG & normal-gamma (Bayesian model) \\
\hline NN & normal-normal (Bayesian model) \\
\hline NNG & normal-normal-gamma (Bayesian model) \\
\hline NR & Newton-Raphson (algorithm) \\
\hline P, Pr, Prob & probability function \\
\hline$p$ & binomial proportion; pdf or pmf (same as $f$ ) \\
\hline PACF & partial autocorrelation function \\
\hline PDF & portable document format (file) \\
\hline pdf & $\begin{array}{l}\text { probability density function (used for all types of rvs: } \\
\text { continuous, discrete and mixed); used instead of pmf }\end{array}$ \\
\hline PEL & posterior expected loss (function) \\
\hline pmf & probability mass function (rarely used; see pdf) \\
\hline Poi & Poisson distribution \\
\hline $\mathrm{POO}$ & posterior odds \\
\hline рор & population \\
\hline post & posterior \\
\hline ppp-value & posterior predictive $\mathrm{p}$-value \\
\hline pr, prob & probability \\
\hline pred & predictive/prediction/predictor \\
\hline PRO & prior odds \\
\hline pt & point \\
\hline$Q$ & $\begin{array}{l}\text { quantity of interest; quantile function; Q-function (in the } \\
\text { EM algorithm) }\end{array}$ \\
\hline & quadratic error loss function \\
\hline
\end{tabular}




\begin{tabular}{|c|c|}
\hline $\mathrm{R}$ & R (software environment for statistical computing) \\
\hline$R$ & $\begin{array}{l}\text { relative bias; risk function (not to be confused with } \mathfrak{R} \text {, } \\
\text { which denotes the whole real line) }\end{array}$ \\
\hline$r$ & $\begin{array}{l}\text { Bayes risk; nonsample (vector of the labels of the units } \\
\text { that are not sampled) }\end{array}$ \\
\hline $\begin{array}{l}\mathrm{RB} \\
\mathrm{rV}\end{array}$ & $\begin{array}{l}\text { Rao-Blackwell (estimate/estimator/estimation or method) } \\
\text { random variable }\end{array}$ \\
\hline$s$ & $\begin{array}{l}\text { sample standard deviation; sample (vector of the labels of } \\
\text { the units that are sampled) }\end{array}$ \\
\hline SD, sd & standard deviation \\
\hline SE, se & standard error (estimate of standard deviation) \\
\hline SMA & seasonal moving average (process) \\
\hline SRS & simple random sampling (with or without replacement) \\
\hline SRSWOR & simple random sampling without replacement \\
\hline SRSWR & simple random sampling with replacement \\
\hline st & such that \\
\hline$T$ & random variable with the $t$ distribution \\
\hline$t$ & t distribution; upper quantile of the t distribution \\
\hline TIAP & Total International Airline Passengers (time series) \\
\hline$U$ & $\begin{array}{l}\text { (continuous) uniform distribution; random variable with } \\
\text { the standard uniform distribution; upper bound }\end{array}$ \\
\hline$V$, Var & variance operator \\
\hline $\begin{array}{l}\text { WinBUGS } \\
\text { wrt }\end{array}$ & $\begin{array}{l}\text { BUGS for Microsoft Windows (see BUGS) } \\
\text { with respect to }\end{array}$ \\
\hline$X$ & finite population covariate vector (of $N$ values) \\
\hline$x$ & sample covariate vector (of $n$ values) \\
\hline$Y$ & $\begin{array}{l}\text { random variable or vector of random variables; } \\
\text { finite population vector (of } N \text { values) }\end{array}$ \\
\hline$y$ & $\begin{array}{l}\text { realised value of a random variable or vector of } \\
\text { random variables; sample vector (of } n \text { values); } \\
\text { sometimes used interchangeably with } Y\end{array}$ \\
\hline$Z$ & standard normal random variable \\
\hline & upper quantile of the standard normal distribution \\
\hline
\end{tabular}




\section{Bibliography}

Albert, J. (2009). Bayesian Computation with R, 2nd Edition. New York: Springer.

Bolstad, W.M. (2009). Computational Bayesian Statistics. Hoboken NJ: Wiley.

Box, G.E.P, and Tiao, G.C. (1992). Bayesian Inference in Statistical Analysis by Box and Tiao (1973). Reading: Addison-Wesley.

Brooks, S., Gelman, A., Jones, G.L., and Meng, X.-L. (Eds.) (2011). Handbook of Markov Chain Monte Carlo. London: Chapman \& Hall/CRC.

Bühlmann, H. (1967). Experience rating and credibility. ASTIN Bulletin. Website: www.casact.org/library/astin/vol4no3/199.pdf

Byrne, A.P., and Dracoulis, G.D. (1985). Monte Carlo calculations for asymmetric $\mathrm{NaI}(\mathrm{Tl})$ and BGO Compton suppression shields, Nuclear Instruments and Methods in Physics Research, A234: 281-287.

Cochran, W.G. (1977). Sampling Techniques, 3rd Edition. New York: Wiley.

Ericson, W.A. (1969). Subjective Bayesian models in sampling finite populations. Journal of the Royal Statisticial Society, Series B, 31: 195-224.

Ericson, W.A. (1988). Bayesian inference in finite populations. In Handbook of Statistics, Vol. 6, P.R. Krishnaiah and C.R. Rao (Eds.), pp 213-246. Amserdam: North Holland.

Gelman, A., Carlin, J.B., Stern, H.S., and Rubin, D.B. (2004). Bayesian Data Analysis, 2nd Edition. New York: Chapman and Hall.

Gilks, W.R., Richardson, S., and Spiegelhalter, D.J. (1996). Markov Chain Monte Carlo in Practice. New York: Chapman \& Hall.

Hobert, J.P. and Casella, G. (1996). The effect of improper priors on Gibbs sampling in hierarchical linear mixed models. Journal of the American Statistical Association, 91: 1461-1473.

Jeffreys, H. (1961). Theory of Probability, 3rd Edition. Oxford: Oxford University Press.

Kéry, M., and Schaub, M. (2012). Bayesian Population Analysis Using WinBUGS. New York: Elsevier.

Lachlan, G.J., and and Krishnan, T. (2008). The EM Algorithm and Extensions. Hoboken, NJ: John Wiley \& Sons. 
Leonard, T., and Hsu, J.S.J. (1999). Bayesian Methods: An Analysis for Statisticians and Interdisciplinary Researchers. Cambridge:

Cambridge University Press.

Lee, P. (1997). Bayesian Statistics: An Introduction. New York: Oxford University Press.

Lunn, D.J., Thomas, A., Best., N., and Spiegelhalter, D. (2000). WinBUGS - A Bayesian modelling framework: Concepts, structure, and extensibility. Statistics and Computing, 10: 325-337.

Maindonald, J., and Braun, W.J. (2010). Data Analysis and Graphics Using R: An Example-Based Approach, 3rd Edition. Cambridge: Cambridge University Press.

Meng, X.-L. (1994). Posterior predictive p-values. The Annals of Statistics, 22: 1142-1160.

Ntzoufras, I. (2009). Bayesian Modeling Using WinBUGS. Hoboken NJ: Wiley.

O’Hagan, A, and Forster, J. (2004). Kendall's Advanced Theory of Statistics, Second Edition, Volume 2B, Bayesian Inference. London: Arnold.

Puza, B. (1995). Monte Carlo Methods for Finite Population Inference. Internal document. Canberra: Australian Bureau of Statistics.

Puza, B.D. (2002). 'Postscript: Bayesian methods for estimation' and 'Appendix C: Details of calculations in the Postscript'. In Combined Survey Sampling Inference: Weighing Basu's Elephants, by K. Brewer, London: Arnold, 2002, pp 293-296 and 299-302.

Puza, B.D., and O’Neill, T.J. (2005). Length-biased, with-replacement sampling from an exponential finite population. Journal of Statistical Computation and Simulation, 75: 159-174.

Puza, B. and O’Neill, T.J. (2006). Selection bias in binary data from volunteer surveys. The Mathematical Scientist, 31: 85-94.

Rao, C.R. (1973). Linear Statistical Inference and its Applications, 2nd Edition. New York: Wiley.

Rao, J.N.K. (2011). Impact of frequentist and Bayesian methods on survey sampling practice: a selective appraisal. Statistical Science, 26: $240-256$.

Särndal, C.-E., Swensson, B., and Wretman, J. (1992). Model Assisted Survey Sampling. New York: Springer.

Seaman, S., Galati, J., Jackson, D., and Carlin, J. (2013). What is meant by 'Missing at Random'? Statistical Science, 28(2): 257-268.

Shaw D, (1988). On-site samples' regression: Problems of non-negative integers, truncation, and endogenous stratification. Journal of Econometrics, 37: 211-223. 
Smith, A.F.M., and Gelfand, A.E. (1992). Bayesian statistics without tears: A sampling-resampling perspective. The American Statistician, 46(2): 84-88.

Wackerly, D.D., Mendenhall III, W., and Scheaffer, R.L. (2008). Mathematical Statistics with Applications, 7th edition. Duxbury: Thomson, Brooks/Cole.

West, M. (1996). Inference in successive sampling discovery models. Journal of Econometrics, 75: 217-238. 